\title{
Restauradores en Canton Ticino entre Ottocento y Novecento. Catalogación y gestión de datos.
}

\section{Restauratori in Canton Ticino fra Ottocento e Novecento.}

Catalogazione e gestione dati.

\author{
Tesis doctoral europea \\ Ester Giner Cordero \\ Dirigida por: \\ Mápilar Roig Picazo y \\ Mํㅡㄴ Antonia Zalbidea Muñoz
}

Facultad de Bellas Artes de San Carlos

Departamento de Conservación y Restauración de Bienes Culturales

PROGRAMA DE DOCTORADO:

Conservación y restauración del patrimonio histórico artístico

En colaboración con el "Dipartimento Ambiente Costruzioni e Design" de la

"Scuola Universitaria Professionale della Svizzera Italiana" 

"El viento me ha traído tu nombre en la mañana;

el eco de tus pasos repite la montaña... No te verán mis ojos; imi corazón te aguarda!".

(Antonio Machado, Amada, el áurea dice...)

A mi padre, 



\section{PREFAZIONE}

La presente tesi di dottorato, interessata alla scoperta delle più rilevanti personalità nel campo della conservazione e del restauro dei dipinti murali, la cui attività si svolge nel Canton Ticino (Svizzera) tra la fine dell'Ottocento e la prima metà del Novecento, è nata dalla collaborazione di formazione e di ricerca fra il Departamento de Conservación y Restauración de los Bienes Culturales all'Università Politecnica di Valencia (CRBC-UPV) ed il Dipartimento Ambiente Costruzioni e Design (DACD) della Scuola Universitaria Professionale della Svizzera Italiana (SUPSI) a Lugano.

La ricerca fu iniziata nel 2006, ed i risultati preliminari ottenuti da essa consentirono di essere presentati alla fine dell'anno scorso in forma di DEA (Diploma de Estudios Avanzados $^{1}$ ) all'Università Politecnica di Valencia. Lungo gli ultimi sette mesi la medesima ricerca è stata sviluppata per la realizzazione di questa tesi di dottorato, grazie alla concessione in settembre 2007 di una "Borsa di studio per studenti stranieri" della Confederazione Federale Svizzera ${ }^{2}$.

Il Canton Ticino, ed in particolare la storia del restauro in questo territorio, sono stati scelti come oggetto di analisi in consapevolezza della mancanza di studi svolti in precedenza. Questo ha fatto del lavoro un argomento di difficile esecuzione oltreché di enorme interesse per quanto innovativo e utile. La scarsa documentazione, la difficile ed in alcuni casi impossibile accessibilità a certi archivi privati ed altri dispersi, così come la problematica aggiuntiva di occuparmi dello studio di un paese e di una cultura, che fino a tre anni fa era da me completamente sconosciuta, non ha impedito ad ogni modo di svolgere gli obbiettivi che inizialmente mi ero proposta. Questo comunque non significa che già dal momento attuale, avendo completato la stesura della tesi, non abbia il desidero di approfondire molti degli argomenti qui presentati, rimasti poveri per limitazioni di tempo e impegni professionali. Ad ogni modo il materiale raccolto in questi anni mediante l'esaustiva ricerca bibliografica e di archivio, ha permesso di ricostruire in linee generali la storia della tutela dei monumenti storici nel Canton Ticino, ed in particolare sono stati individuati nomi come Edoardo Berta, Emilio Ferrazzini, Tita Pozzi, Carlo Cotti, Carlo Mazzi, Mario Moglia, Teodor Hallisch e Pompeo Maino, tutti pittori ticinesi la cui attività professionale ha occupato, inoltre, il campo del restauro dei dipinti

\footnotetext{
${ }^{1}$ Titolo ottenuto mediante la lettura in giorno 25 Novembre 2007 della tesi di specializzazione in conservazione e restauro del patrimonio storico artistico (Diploma de Estudios Avanzados, DEA): Giner Cordero E."Restauradores en Cantón Ticino entre Ottocento y Nocento. Catalogación y gestión de datos”, dipositata nella biblioteca del Departamento de Conservación y Restauración de Bienes Culturales dell'Università Politecnica de Valencia (non pubblicata).

II periodo finanziato dalla "Federal Commission for scholarships for foreign students" FCS è stato dal 16 settembre 2007 al 15 Giugno 2008.
} 
murali. Assieme a queste figure interagirono restauratori provenienti dalla Svizzera interna, come Henri Boissonas, Christian Schmidt ed Emilio Dillena, o dalla più prossima e influente regione lombarda come Mario Rossi e Francesco Annoni da Milano, il celebre Mauro Pellicioli ed i fratelli Steffanoni da Bergamo o Bruno Abbiati di Ponte Tresa, oltreché personalità come Ernesto Rusca e Ovidio Fonti, rispettivamente da Rancate e Miglieglia (Ticino), la cui attività si svolge più ampiamente in Italia, me ebbero in qualunque modo opportunità di intervenire nel cantone svizzero di loro provenienza.

La consultazione del materiale inerente ai nomi sopraelencati si è vista arricchita da una interessante polemica svolta all'interno di diversi giornali ticinesi (Gionale del Popolo e Rivista Storica Ticinesa, principalmente), tra gli anni Quaranta e Cinquanta del Novecento; essa, apparsa da articoli critici, mette in evidenza non solo le figure più importanti coinvolte a suo tempo nei più rilevanti interventi di restauro del momento, ma definisce anche un limite di tempo fondamentale. Tanto è vero che se le circostanze storiche scelte, per definire il periodo di studio all'interno della ricerca, sono state la creazione della prima legge di protezione dei monumenti nel 1909 e le trasformazioni organiche e politiche avvenute all'interno della Commissione Cantonale dei Monumenti Storici, diventata Ufficio dei Monumenti Storici nel 1959, per ultimo il "climax" storico è stato fissato nel momento in cui avvenne la suddetta polemica. Le contrapposizioni di opinione originatesi in questo periodo, nel settore del restauro dei beni storico-artistici, rappresentano se non altro, le necessità di cambio, e suppongono un tempo di meditazione nel quale si dibattono i lavori svolti nel primo cinquantennio. Quello che si analizza è un complesso di eventi, che hanno costituito le basi dell'attuale movimento di recupero dell'identità del paese, cioè della rivalorizzazione del proprio patrimonio. II legato tramandato dai promotori del medesimo movimento: storici come Emilio Motta, critici come Aldo Crivelli, pittori come Edoardo Berta, professori ed scrittori come Francesco Chiesa, sono usciti alla luce aprendo le scatole conservate nei diversi archivi consultati. Le fonti manoscritte e dattiloscritte, della corrispondenza fra gli enti pubblici e privati ed i pittori-restauratori, quali lettere, cartoline, perizie, preventivi o relazioni di restauro, presero vita per la elaborazione di questa ricerca. Essi hanno mostrato gli aspetti fondamentali per determinare gli ideali teorici del restauro tra i secoli XIX e XX nonché le prassi esecutive ed i materiali di restauro impiegati da ognuno dei pittori-restauratori individuati. II materiale raccolto, dove si elencano le opere d'arte intervenute ed i tipi di interventi eseguiti, è stato schedato per restauratore e vuole servire da strumento a tutti quei professionisti, responsabili in materie di conservazione dei beni culturali, principalmente nel contesto ticinese. Inoltre con la presente ricerca ho voluto promuovere la conservazione e divulgazione della memoria. Con questo scopo si sono messi a confronto alcuni dei sistemi 
informatici innovativi attualmente disponibili a livello internazionale e particolarmente in Italia, dove per prossimità territoriale, culturale e linguistica sarebbe più fattibile la collaborazione ed il riversamento dei dati relativi al contesto ticinese. Ma non è solo un ideale giacché con l'informazione ricuperata nella presente ricerca si sono relazionati due banche dati in concreto: il database AR.I.S.T.O.S (Archivio informatico per la storia della tutela della opere storiche artistiche) impiegato dal Ministero per i Beni e le Attività Culturali in Italia e la banca dati del Servizio Inventario dell'Ufficio dei Beni Culturali del Canton Ticino in Svizzera. Questa è stata una opportunità ideale per iniziare l'impostazione di un progetto di standardizzazione e di normalizzazione, specialmente utile per la divulgazione di una memoria storica sulla conservazione ed il restauro del patrimonio storico artistico.

E.G.C.

Lugano, 18 dicembre 2008 



\section{RINGRAZIAMENTI}

Sono tante le persone senza le quali sarebbe stata impossibile la realizzazione di questa tesi dottorale, ai quali devo una immensa riconoscenza. Vorrei ringraziare tutti loro perché l'appoggio e l'incoraggiamento procurato, in forme e modi diversi, mi hanno accompagnata in ogni momento in questi anni di lavoro.

In special modo vorrei esprimere la mia gratitudine alle persone sotto elencate:

alla Dott.ssa architetto Giacinta Jean, responsabile del corso di laurea in conservazione e restauro al Dipartimento Ambiente Costruzioni e Design (DACD) della Scuola Universitaria Professionale della Svizzera Italiana (SUPSI), che mi ha accolta tre anni fa all'interno della loro sede scolastica e prestato tutto l'aiuto necessario per lo svolgimento della ricerca dottorale, nonché per il suo personale sostegno;

- $\quad$ assieme a lei, tutti i colleghi della SUPSI, e sono davvero tanti, che mi hanno aiutata nella stesura della tesi: dal Dott. Albert Jornet, all'interno del Laboratorio Tecnico Sperimentale (LTS) per la indispensabile guida nell'esecuzione delle analisi chimiche e stratigrafiche, il restauratore Jacopo Gilardi per il coordinamento professionale ed accompagnamento alle indagini sui monumenti studiati, la Dott.sa Chiara Lumia per la sua fondamentale orientazione all'inizio del lavoro di ricerca, il collaboratore tecnico Ezio Pesenti per il supporto in laboratorio, al Dott. Christian Paglia, responsabile dell'LTS, al Dott. Angelo Bernasconi direttore del DACD ed a Franco Gervasoni direttore della SUPSI che hanno creduto nel sostegno a questa tesi dottorale;

- $\quad$ per offrirmi la possibilità di impiegare AR.I.S.T.O.S. all'interno degli studi svolti per la ricerca dottorale, esprimo la mia enorme riconoscenza alla Dott.sa Donata Levi e Clara Baracchini ed in particolare Martina Visentin e Marco Mozzo che mi hanno formato nell'utilizzo del programma e corretto il lavoro eseguito al suo interno;

- per l'aiuto alla ricerca di archivio un sincero ringraziamento a Carlotta Bortolloti, collaboratrice dell'Archivio dell'Ufficio dei Beni Culturali di Bellinzona (AUBCBZ); a Giuseppe Chiesi, Giulio Foletti, Katja Bigger, Patrizio Pedrioli e Lara Caldelari dell'Ufficio dei Beni Culturali e alla Sig.ra Doris Amacher, segretaria dell'Archivio Federale dei Monumenti Storici a Berna.

- all'avvocato Giovanni Pozzi ed a sua moglie, di Massagno, per le preziose indicazioni e per tutto il materiale benevolmente prestato riguardo la persona del padre, il pittore-restauratore Tita Pozzi; alla Sig.ra Silvia Mina-Mazzi di Tegna per la disponibilità e la fiducia dimostratami consentendo la consultazione del materiale di archivio del padre, il pittore-restauratore Carlo Mazzi; al restauratore Attilio 

Abbiati per l'appoggio e per il prestito di quattro album fotografici che testimoniano il lavoro eseguito dal padre, a sua volta restauratore Bruno Abbiati, studiato anche egli in questa ricerca;

a Maria Pilar Roig Picazo direttrice del Departamento de Conservación y Restauración de los Bienes Culturales all'Università Politecnica di Valencia (UPV) e direttrice di questa tesi; ai miei cari docenti alla Facoltà di Belle Arti di San Carlo a Valencia ed ai corsi di dottorato, la cui influenza è stata indispensabile, ed in speciale a Maria Antonia Zalbidea co-direttrice del presente lavoro che non solo è stata una mia referente professionale ma anche personale, durante tutto il periodo;

a Fabio, mio amico e fedele compagno, che ha saputo amorevolmente equilibrare i miei alti bassi nonché aiutarmi alla stesura della tesi.

Per ultimo grazie a mia madre Isabel ed a mio fratello Jordi: sin vuestro cariño sería imposible realizar cualquier cosa.

Dedico questa tesi a mio padre Vicente, come piccolo gesto di tutto ciò che a lui devo. 



\section{RIASSUNTO}

L'obiettivo della presente tesi dottorale intitolata: "Restauratori in Canton Ticino fra Ottocento e Novecento. Catalogazione e gestione dati" è quello di individuare e classificare i personaggi più rilevanti promotori della rivalutazione del patrimonio storico artistico nel loro paese, nello specifico nell'ambito del restauro dei dipinti murali, dai pittori-restauratori più conosciuti come Edoardo Berta, Emilio Ferrazzini e Tita Pozzi ad altri meno distinti in questo campo come Carlo Cotti, Ottorino Olgiati, Mario Moglia, Nino Facchinetti, Carlo Mazzi e Pompeo Maino.

L'informazione ottenuta e selezionata fra l'eterogeneità dei documenti che costituiscono archivi pubblici, archivi privati e biblioteche è stata analizzata e ordinata con l'obiettivo di caratterizzare la prassi esecutiva di ognuno dei personaggi individuati ed allo stesso tempo servire allo sviluppo di future ricerche, a restauratori professionisti, a storici ed a conservatori. A tale scopo sono state studiate, inoltre, i programmi di banca dati più recenti, fattibili a contenere, relazionare e visualizzare la documentazione elaborata, rispettando requisiti indispensabili quali l'impiego di una terminologia normalizzata, la concessione del libero acceso ai dati inseriti e, conseguentemente, la creazione di un'ampia rete "viva" che favorisca la divulgazione della memoria.

Restauratori in Canton Ticino fra Ottocento e Novecento. Catalogazione e gestione dati. SVIZZERA

Ester Giner Cordero. Dipartimento di Conservazione e Restauro dei beni Culturali Università Politecnica di Valencia.

Parole chiave: patrimonio, dipinti murali, restauratori, restauro, banca dati. 



\section{RESUMEN}

El objetivo de esta tesis doctoral titulada: "Restauradores en Canton Ticino entre Ottocento y Novecento. Catalogación y gestión de datos" es el de individualizar y clasificar los personajes más relevantes promotores de la revalorización del patrimonio históricoartístico en su país, concretamente en el campo de la restauración de las pinturas murales, desde los pintores-restauradores más conocidos como Edoardo Berta, Emilio Ferrazzini y Tita Pozzi a otros menos distinguidos como Carlo Cotti, Ottorino Olgiati, Mario Moglia, Nino Facchinetti, Carlo Mazzi y Pompeo Maino.

La información recogida entre la hetereogeneidad de documentos que componen archivos públicos, archivos privados y bibliotecas ha sido analizada y ordenada con la finalidad de caracterizar la praxis ejecutiva de cada uno de los personajes individualizados, así como para servir a futuras investigaciones, a restauradores profesionales, a historiadores y a conservadores entre otros. También por este último motivo han sido estudiados los más recientes programas de base de datos factibles a contener, relacionar y visualizar la documentación elaborada, atendiendo a requisitos indispensables como el uso de una terminología normalizada, permitir el libre acceso a los datos y la creación de una red amplia y "viva" que favorezca la difusión de la memoria.

\section{Restauradores en Canton Ticino entre Ottocento y Novecento. Catalogación y gestión de} datos. SUIZA

Ester Giner Cordero. Departamento de Conservación y Restauración de los Bienes Culturales - Universidad Politecnica de Valencia.

Palabras clave: patrimonio, pintura mural, restauradores, restauración, base de datos. 



\section{RESUM}

L'objectiu d'aquesta tesi doctoral titulada: "Restauradors en Canton Ticino entre Ottocento i Novecento. Catalogació i gestió de dades" és el d'individualitzar i classificar els personatges més rellevants promotors de la revaloració del patrimoni històric-artístic en el seu país, concretament en el camp de la restauració de les pintures murals, des dels pintors-restauradors més coneguts com Edoardo Berta, Emilio Ferrazzini i Tita Pozzi a altres menys distingits com Carlo Cotti, Ottorino Olgiati, Mario Moglia, Nino Facchinetti, Carlo Mazzi i Pompeo Maino.

L'informació recollida entre la hetereogeneitat dels documents que componen arxius públics, arxius privats i biblioteques s'ha analitzada i ordenada amb la finalitat de caracteritzar la praxi executiva de cadascun dels personatges individualitzats, així com per a servir a futures investigacions, a restauradors professionals, a historiadors i a conservadors entre altres. També per aquest últim motiu s'han estudiat els més recents programes de base de dades factibles a contenir, relacionar i visualitzar la documentació elaborada, atenent a requisits indispensables com l'ús d'una terminologia normalitzada, permetre el lliure accés a les dades i la creació d'una xarxa àmplia i "viva" que afavoreixi la difusió de la memòria.

Restauradors en Canton Ticino entri Ottocento i Novecento. Catalogació i gestió de dades. SUÏSSA

Ester Giner Cordero. Departament de Conservació i Restauració dels Béns Culturals Universitat *Politecnica de València.

Paraules clau: patrimoni, pintura mural, restauradors, restauració, base de dades. 



\section{SUMMARY}

The purpose of this docthoral thesis, titled "Restorers in Canton Ticino between Ottocento and Novecento. Classification and data management.", is to recognize and classify the most relevant characters, promoters of the historical and artistic patrimony revaluation in their canton, specifically in the field of wall paintings, from the most known painters and restorers like Edoardo Berta, Emilio Ferranzzini and Tita Pozzi, to the least known like Carlo Cotti, Ottorino Olgiati, Mario Mogila, Nino Facchinetti, Carlo Mazzi and Pompeo Maino.

The information gathered from the heterogeneous set of documents that form the public records, private records and libraries has been analyzed and arranged in order to characterize the executive praxis of each restorer, and to serve the future research, professional restorers, historians and conservators. To achieve this, the newest database programs able to contain, relate and visualize that information have been studied, paying attention to essential requirements like the use of normalized terminology, allow free access to data and the creation of a wide and 'alive' net that favours memory diffusion.

Restorers in Canton Ticino between Ottocento and Novecento. Classification and data management. SWITZERLAND

Ester Giner Cordero. Department of Conservation and Restoration of Cultural Heritage Polithecnic University of Valencia.

Keywords: heritage, wall painting, restorers, restoration, databases. 



\section{ABBREVIAZIONI}

AFMS Archivio Federale dei Monumenti Storici

ARISTOS Archivio Informatico per la Storia della Tutela degli Oggetti Storico Artistici

ASRI Archivio Storico Nazionale e Banca Dati dei Restauratori Italiani.

ASTi Archivio di Stato della Repubblica e Cantone Ticino

AUBCBZ Archivio dell'Ufficio dei Beni Culturali, Bellinzona

BCLU Biblioteca Cantonale di Lugano

BNS Biblioteca Nazionale Svizzera

CdS Consiglio di Stato

CdT Corriere del Ticino

CCMS Commissione Cantonale dei Monumenti Storici

CFMS Commissione Federale dei Monumenti Storici

DFI Dipartimento Federale dell'Interno

DPE Dipartimento della Pubblica Educazione

GdP Giornale del Popolo

LIDA Laboratorio informatico per la documentazione storico-artistica

NDT Nota del traduttore

OSMA Opera Svizzera dei Monumenti Storici e Artistici

SBT Sistema bibliotecario ticinese

SPSAS Società Pittori Scultori ed Architetti Svizzeri

STBA Società Ticinese delle Belle Arti

SUPSI Scuola Universitaria Professionale della Svizzera Italiana

TSI Televisione della Svizzera Italiana

UBC Ufficio dei Beni Culturali

UFC Ufficio Federale della Cultura

UPV Universidad Politécnica de Valencia 



\section{INDICE}

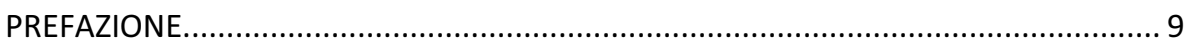

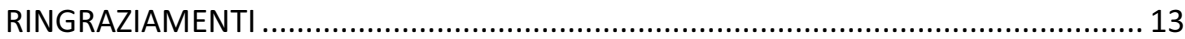

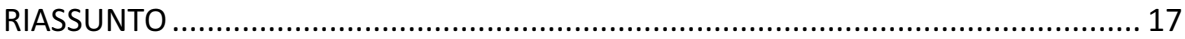

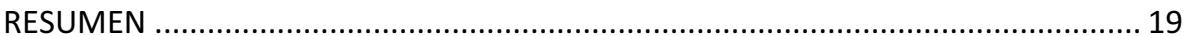

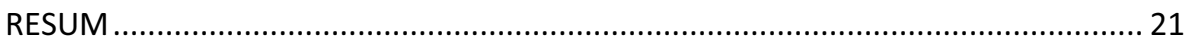

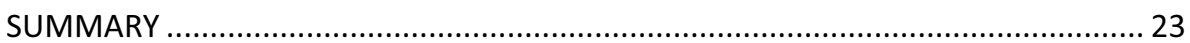

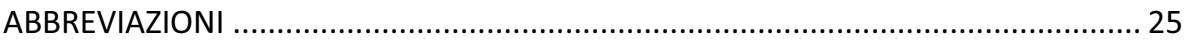

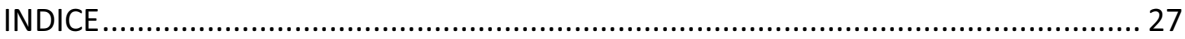

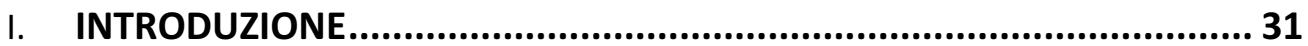

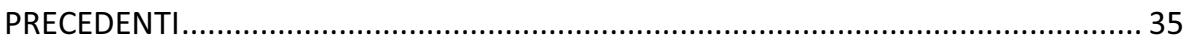

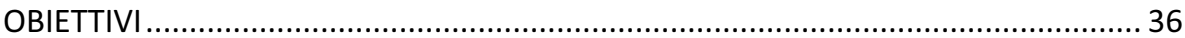

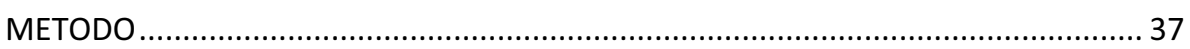

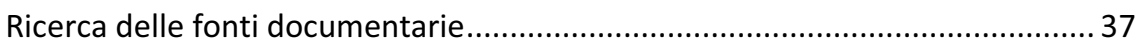

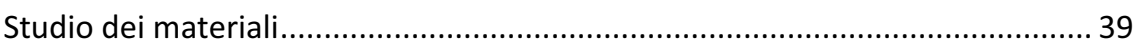

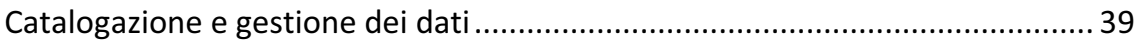

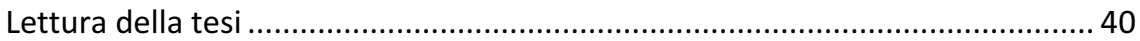

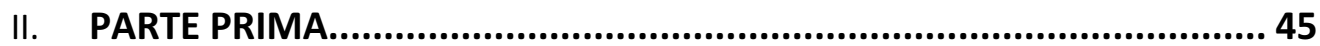

LA TUTELA DEI BENI CULTURALI IN SVIZZERA ….............................................. 47

LA COMMISSIONE CANTONALE DEI MONUMENTI STORICI. GLI ESORDI. ..................53

LA TUTELA DEI BENI CULTURALI IN CANTON TICINO ............................................. 61

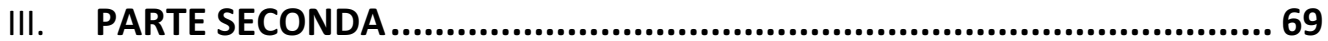

$\begin{array}{ll}\text { EDOARDO BERTA } & 73\end{array}$

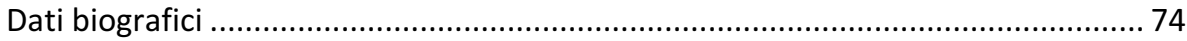

Chiesa di S. Maria degli Angioli, Lugano (1910 - 1930) ......................................... 83

Chiesa di S. Biagio, Ravecchia, (1913 - 1915) ..................................................... 122

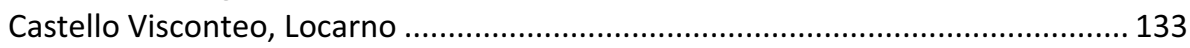

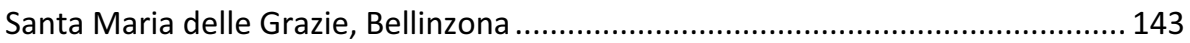

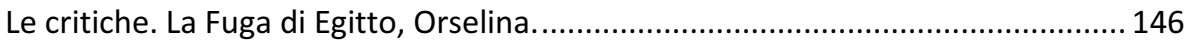

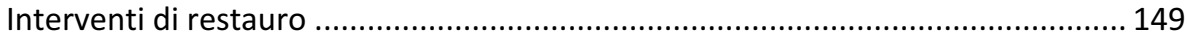

$\begin{array}{ll}\text { EMILIO FERRAZZINI } & 167\end{array}$

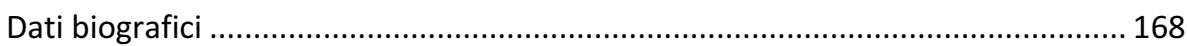

Le polemiche giornalistiche di Emilio Ferrazzini............................................... 172

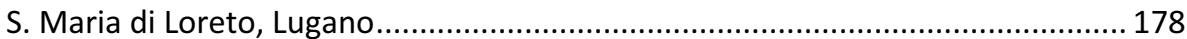

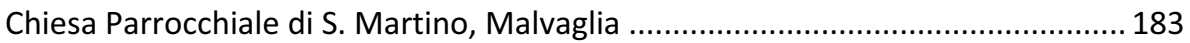

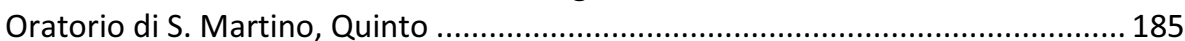




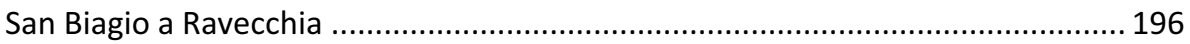

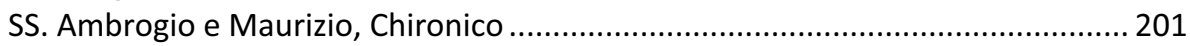

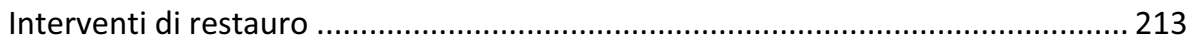

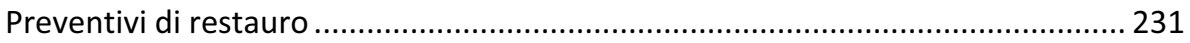

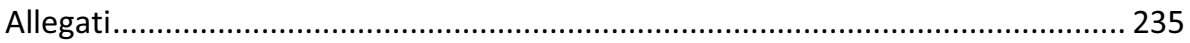

POZZI BATTISTA “Tita” 243

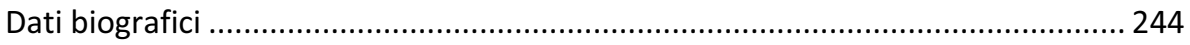

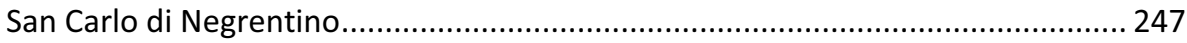

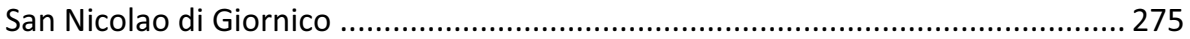

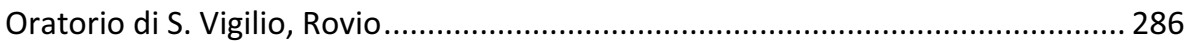

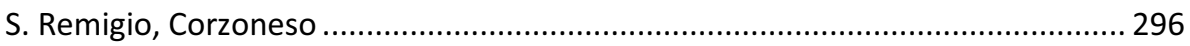

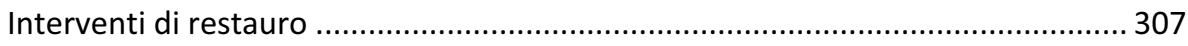

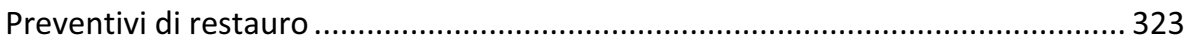

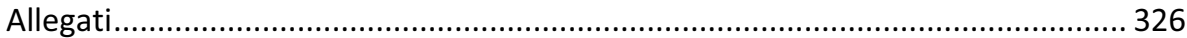

$\begin{array}{ll}\text { CARLO COTTI } & 335\end{array}$

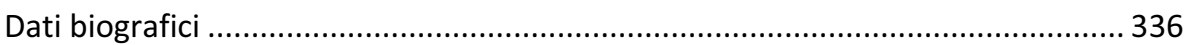

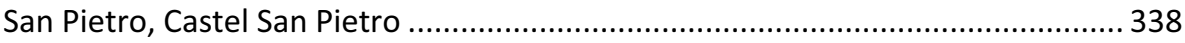

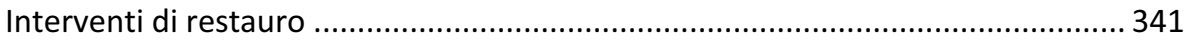

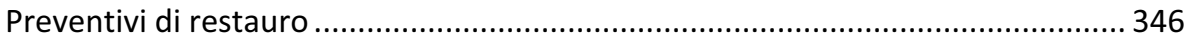

NINO FACCHINETTI 353

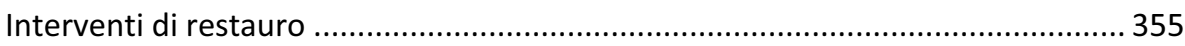

$\begin{array}{ll}\text { BRUNO ABBIATI } & 365\end{array}$

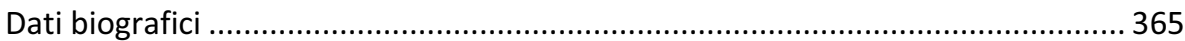

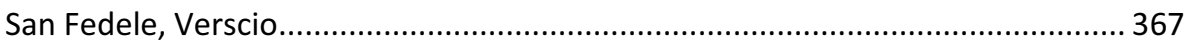

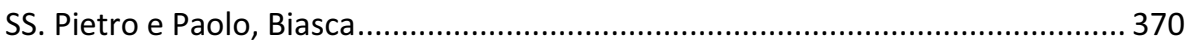

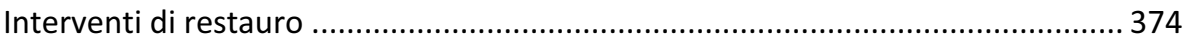

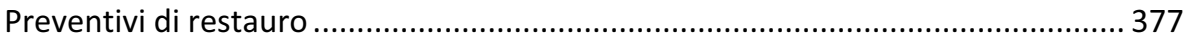

MARIO MOGLIA $\quad 385$

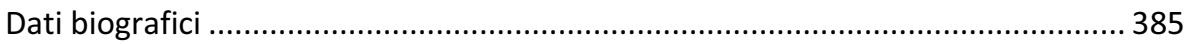

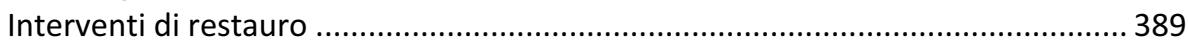

OTTORINO OLGIATI $\quad 405$

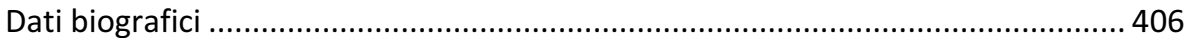

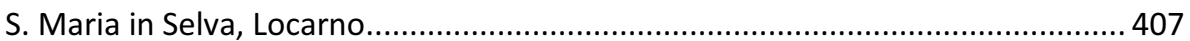

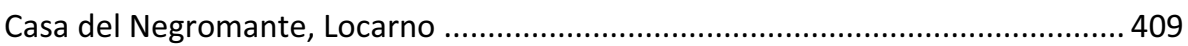

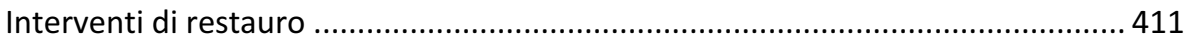

$\begin{array}{ll}\text { CARLO MAZZI } & 419\end{array}$

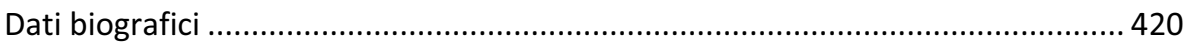

II pittore ticinese "specializzato" in restauro ....................................................... 421 


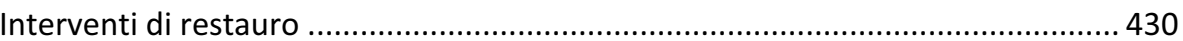

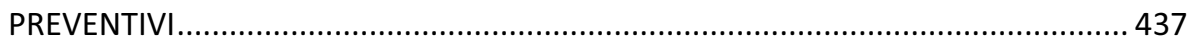

GIUSEPPE STEFFANONI E FIGLI $\quad 445$

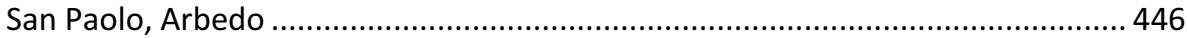

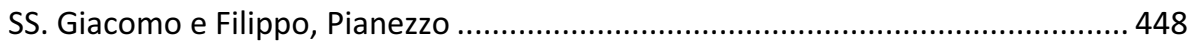

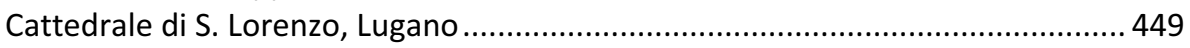

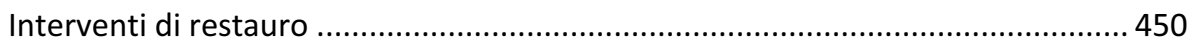

$\begin{array}{ll}\text { MAURO PELLICIOLI } & 457\end{array}$

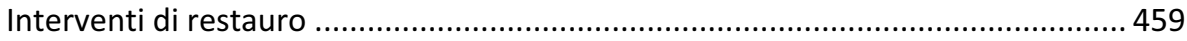

$\begin{array}{ll}\text { FRANCESCO ANNONI, MILANO } & 473\end{array}$

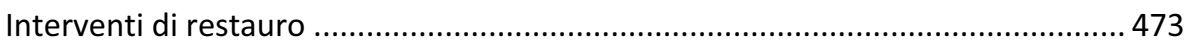

$\begin{array}{ll}\text { MARIO ROSSI } & 481\end{array}$

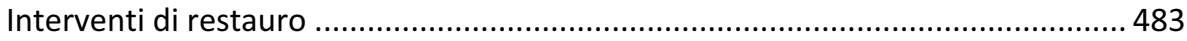

BOISSONAS HENRI-PAUL $\quad 493$

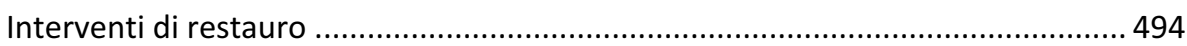

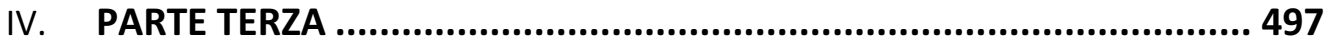

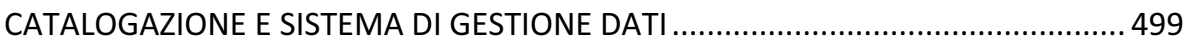

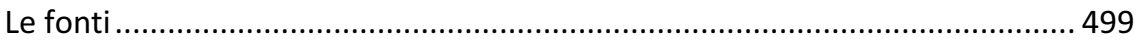

Archivio Federale dei Monumenti Storici, Berna........................................... 499

L'Archivio dell'Ufficio dei beni culturali, Bellinzona......................................... 501

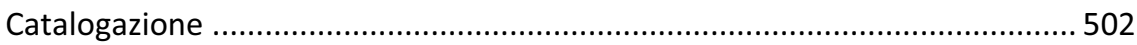

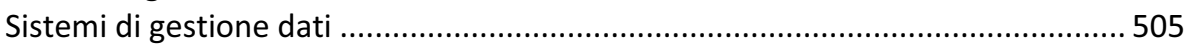

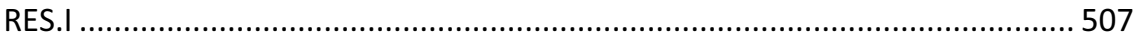

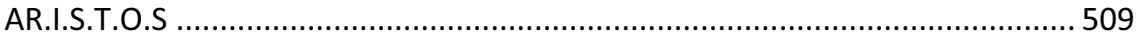

Banca dati UBC, Bellinzona .................................................................... 516

V. FUTURE LINEE DI RICERCA ........................................................ 519

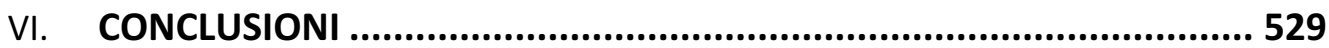

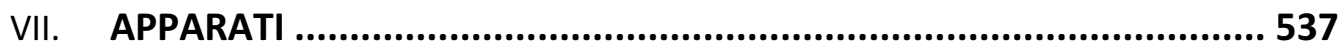

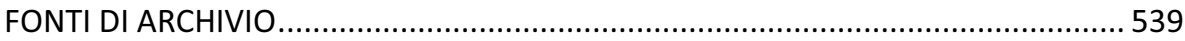

Archivio federale dei monumenti storici, Berna , AFMS BNS ......................... 539

Archivio di Stato del Canton Ticino, Bellinzona ................................................. 539

Archivio Ufficio dei beni culturali, Bellinzona ............................................... 541

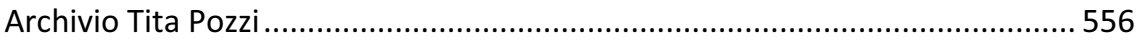

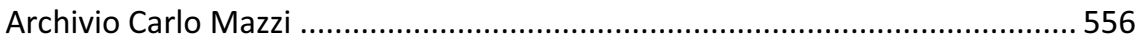




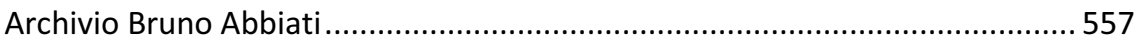

Archivio della Radiotelevisione svizzera …..................................................55 557

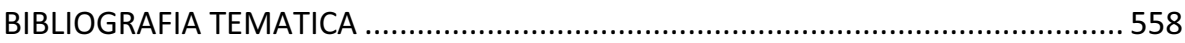

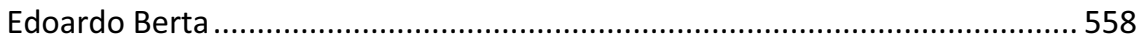

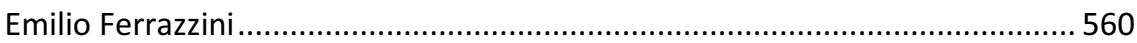

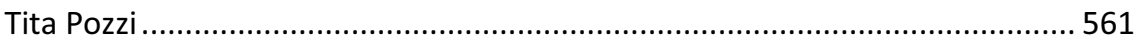

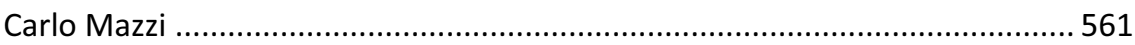

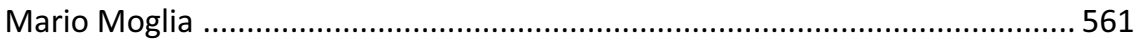

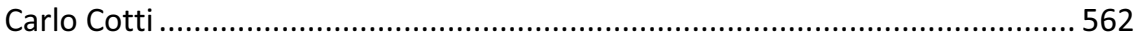

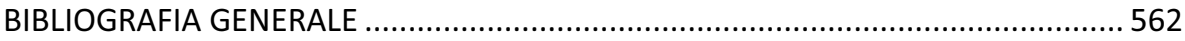

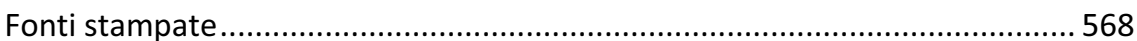

INDICE DEI NOMI DI PERSONA, DI LUOGO E DI ASSOCIAZIONI .............................571

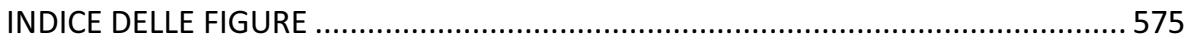

ARTICOLI PUBBLICATI DALLA RICERCA DOTTORALE...........................................589

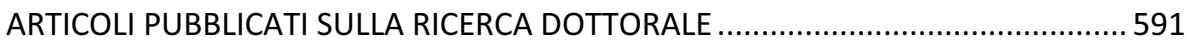

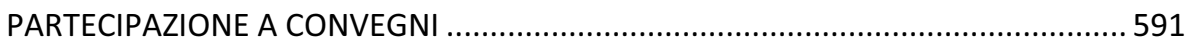


INTRODUZIONE 
[...]Vogliamo in questa miscellanea, portare a conoscenza dei nostri lettori quanto si è fatto nei diversi campi, strettamente collegati alla archeologia, alla storia, alle belle arti, alle bellezze naturali, affinché si possa constatare, e soprattutto far constatare, ciò che questo piccolo popolo, con esiguità dei mezzi, sa compiere per proteggere $e$ difendere il patrimonio che è legato alla sua terra come il sangue è connesso alla nostra vita. $[\ldots]^{3}$.

[...]Queremos en esta miscelánea, dar a conocer a nuestros lectores todo lo que se ha hecho en diversos campos, estrechamente legados a la arqueología, a la historia, a las bellas artes, a las bellezas naturales, para que se pueda constatar, y sobre todo hacer constatar, lo que este pequeño pueblo, con los mínimos medios, sabe hacer para proteger y defender el patrimonio que está legado a su tierra como el sangre está unido a nuestra vida.[...].

${ }^{3}$ (Crivelli, 1944, p. 961) 
La storia del restauro è stata creata da quelle persone che hanno creduto nella necessità di, in un modo o nell'altro, tramandare dei valori contenuti in un determinato oggetto perche identificativi di una cultura collettiva. Questo significa che per conoscere gli eventi passati in materia di conservazione e di restauro dobbiamo assolutamente conoscere queste persone, il loro contesto culturale, geografico, le loro ideologie ed i loro metodi di lavoro. In tal senso possiamo capire meglio il momento attuale e rispondere al perché un certo elemento è stato fino al momento conservato o per il contrario perché altri in passato furono cancellati, con che mezzi è stata permessa la salvaguardia di questo oggetto o qualli, invece, hanno peggiorato il suo stato di conservazione.

Ho voluto con questa tesi identificare ed studiare i personaggi di maggiore rilevanza, che fecero si che nel territorio del Canton Ticino si introducessero i concetti di "salvaguardia", di "monumento storico" e di "restauratore" alla fine del XIX sec. ed a tutti loro che con la collaborazione o con la critica, aprirono il passo allo studio ed al riconoscimento di un settore necessario per la sopravivenza della propria cultura fino ai primi cambiamenti politici e teorici in materia, intorno al 1960.

In particolare le figure analizzate in profondità sono state pittori-restauratori attivi nel territorio svizzero nella conservazione dei dipinti murali nel periodo compresso tra la fine dell'Ottocento e la prima metà del Novecento. Questo studio ha consentito di ricostruire parte della storia del restauro nel Canton Ticino con lo scopo di facilitare ulteriore ricerche, nonché analizzare la fattibilità di introdurre l'informazione raccolta all'interno di una banca dati di libero e facile acceso a tutti gli utenti.

In seguito si espongono approfonditamente i precedenti di questa ricerca ed i fattori che hanno incentivato la sua elaborazione, gli obiettivi principali prefissati, e le strategie adottate per lo svolgimento e la risoluzione finale di essi. Ho voluto manifestare attraverso questi punti la concretezza dei traguardi e la ampiezza di interesse che caratterizza il lavoro svolto, a suo tempo compiuto grazie al coordinamento di discipline ben diverse come la storia e l'informatica. 


\section{PRECEDENTI}

Lo studio della storia della tutela, ma concretamente dei professionisti dedicati al restauro dei dipinti murali nel Canton Ticino, è praticamente inesistente. Pochi sono stati fino al momento i testi e le ricerche concluse in questo ambito e quelli esistenti sono molto recenti. La bibliografia fondamentale riguardante la tutela dei monumenti storici è costituita dalle tre pubblicazioni elaborate dallo Stato del Canton Ticino con lo scopo di riassumere le attività svolte, dalla messa in vigore nel 1909 della prima legge di protezione dei monumenti e dalla creazione della Commissione Cantonale dei Monumenti Storici il medesimo anno. Si tratta dei testi: "Monumenti storici e artistici nel Canton Ticino restaurati dal 1910 al 1945"4, "Cinquant'anni di protezione dei monumenti storici artistici della repubblica e cantone del Ticino" ${ }^{5}$ "Settantacinque anni della commissione dei monumenti storici e artistici nel Canton Ticino" ${ }^{6}$.

A livello svizzero per il contrario possono elencarsi diversi saggi elaborati con lo scopo di promuovere il concetto di restauro e le prassi esecutive. Essi disegnano il profilo del restauratore e conservatore del patrimonio storico-artistico; si tratta dei testi di Johann Rudolf Rahn "Istruzioni per la salvaguardia dei monumenti e per il loro restauro", "Das Restaurieren" ${ }^{8}$ di Joseph Zemp e "Restaurierungspraxis und Kunsterbe in der Schweiz" ${ }^{\text {di }}$ Linus Birchler.

Oltre a queste pubblicazioni di origine nazionali, il precedente primario per lo studio dei restauratori attivi in Canton Ticino è stato il progetto europeo Archivio Storico dei Restauratori Europei- Rete europea per lo studio e la documentazione delle vicende conservative del Patrimonio Culturale promosso dall'Associazione Secco Suardo da Bergamo (Italia). A suo tempo questo progetto è nato in conseguenza ai risultati ottenuti con il programma RES.I (Restauratori italiani) della medesima associazione.

Attualmente, sono diversi i piani di lavori in corso che hanno come obiettivo ricostruire la storia del restauro e dei sui artifici. Ad esempio, con motivo del centenario della nascita dell'illustre teorico del restauro Cesare Brandi, nel 2006 si presenta il progetto europeo Cesare Brandi (1906-1988) II suo pensiero e il dibattito in Europa nel XX secolo, come proseguimento degli studi presentati nel 2003 al Convegno Internazionale "La teoria del restauro nel Novecento da Riegl a Brandi" la cui risoluzioni sono state radunate in una

\footnotetext{
${ }^{4}$ Chiesa F., Monumenti storici e artistici nel Canton Ticino restaurati dal 1910 al 1945. Bellinzona : Grassi, istituto d'arti grafiche ed editoriale, 1946.

5 Dipartimento della Pubblica Educazione, Cinquant'anni di protezione dei monumenti storici artistici della repubblica e cantone del Ticino 1909-1959. Edizione dello stato; Bellinzona, 1959.

${ }^{6}$ Dipartimento dell'ambiente, Settantacinque anni della Commissione dei monumenti storici ed artistici del Cantone Ticino. Dipartimento dell'ambiente - Ufficio e commissione cantonale dei monumenti storici; quaderni d'informazione, 10. Bellinzona, 1984.

(Rahn, 1876)

8 (Zemp, 1907)

${ }^{9}$ (Birchler, 1948)
} 
pubblicazione curata dalla professoressa Maria Andaloro ${ }^{10}$, allieva del medesimo teorico italiano, oggigiorno docente di Storia dell'arte medievale presso la Facoltà di Conservazione dei Beni Culturali dell'Università degli Studi della Tuscia di Viterbo.

Tutti questi eventi fanno si che la presente tesi si incorpori agli attuali progetti di carattere europeo ed internazionali, nonché servire a suo tempo di precedente a futuri studi per la ricostruzione della storia dei restauratori in Canton Ticino.

\section{OBIETTIVI}

La tesi qui presentata è stata concepita con lo scopo di individuare i pittori-restauratori più rilevanti che operarono in Canton Ticino (Svizzera) tra la fine dell'Ottocento e la prima metà del Novecento. In questo senso gli obiettivi principali proposti sono stati la ricompilazione delle informazioni disperse nei diversi archivi pubblici e privati in Svizzera, il disegno del profilo professionale dei pittori-restauratori ticinesi individualizzati, ed attraverso essi la conoscenza della storia conservativa delle opere trattate, le prassi esecutive impiegate nel periodo di studio (tecniche, materiali ed strumenti), nonché l'evoluzione delle teorie, dei lessici tecnici e delle politiche in materia di restauro.

Questo ha significato concretamente:

- $\quad$ la conformazione delle biografie dei pittori-restauratori;

- la ricostruzione dei diversi eventi che hanno definito la storia conservativa dell'opera, mediante la descrizione delle operazione di restauro, perizie, preventivi ed indagini eseguitesi dai pittori-restauratori individuati, indicando tutti quei dati che configurano l'evento: persone coinvolte, enti, stato conservativo dell'opera, ed operazione eseguite;

- definizione degli ideali teorici e delle prassi esecutive adottate dai pittorirestauratori ed i diversi ruoli a cui aderiscono;

- $\quad$ identificazione delle possibile influenze dall'estero.

Eseguendo adeguatamente questi primi presupposti, il materiale raccolto, riordinato e strutturato, si offre ai ricercatori come uno strumento informativo, che vuole migliorare l'approccio critico verso le opere storico-artistiche e la qualità dei futuri progetti conservativi.

\footnotetext{
${ }^{10}$ Andaloro Maria (a cura di), La teoria del restauro nel Novecento da Riegl a Brandi. Atti del Convegno Internazionale di Studi. Viterbo 12- 15 novembre 2003. Nardini editore, 2006.
} 


\section{METODO}

Per lo sviluppo degli obiettivi precedentemente elencati è stata impiagata una metodologia di indagini multidisciplinare, combinando la ricerca documentaria in archivi e biblioteche, con i supporti informatici.

\section{Ricerca delle fonti documentarie}

In primo luogo fu eseguita una ampia indagine bibliografica, identificando i principali testi riguardo la storia della tutela e della conservazione del patrimonio storico artistico in Svizzera, nel Canton Ticino ed in Italia. Si sono ricercati e poi analizzati con questo scopo, attraverso la rete bibliotecaria ticinese (SBT): libri, giornali, materiale audiovisivo e sonoro. In concreto sono state visitate le Biblioteche cantonali di Bellinzona (BCBZ) e Lugano (BCLU), la Biblioteca del Museo Cantonale di Lugano (MCALU), la Biblioteca Salita dei Frati a Lugano (BSFLU) e la Biblioteca del Centro Professionale di Trevano, (CPTCA). Sono stati fondamentali per indirizzare il lavoro e la conoscenza della cultura conservativa in Italia, il testo di Alessandro Conti "Storia del restauro e della conservazione delle opere d'arte"11, ed i due primi volumi dei "Quaderni dell'Archivio Storico Nazionale e Banca Dati dei restauratori Italiani"12 fino al momento pubblicati dalla Associazione Giovanni Secco Suardo a Bergamo, che raccolgono i risultati del progetto basato sui medesimi obiettivi di questa ricerca, nel contesto italiano. La lettura dei testi: "Monumenti storico artistici del Cantone Ticino restaurati dal 1910 al 1945"13, "Cinquant'anni di protezione dei monumenti storici artistici della repubblica e cantone del Ticino 1909-1959"14 e "Settantacinque anni della Commissione dei monumenti storici ed artistici del Cantone Ticino."15, dove si raccolgono i dati specifici delle tutela dei beni culturali in Canton Ticino, dalla prima legge per la protezione dei monumenti elaborata nel 1909, ha consentito inoltre di elaborare un primo elenco di persone, enti ed interventi suscettibili ad essere studiati ed approfonditi. In seguito alla raccolta dei primi dati furono localizzati i più importanti archivi, pubblici e privati, per la indagine documentaria mirata. Si sono consultati a questo scopo: I'Archivio di Stato del Canton Ticino (ASTi), l'Archivio dell'Ufficio dei Beni Culturali del Canton Ticino a Bellinzona (AUBCBZ), l'Archivio della Curia Vescovile a Lugano (ACVLU), e per ultimo I'Archivio Federale dei Monumenti Storici a Berna (AFMS). Nel primo, (ASTi) si sono analizzati due disordinati fascicoli costituiti da fonti manoscritte appartenenti al XIX sec. ed a inizi del XX sec. Si tratta di una raccolta di lettere, verbali, rapporti e preventivi

${ }^{11}$ (Conti, 2002)

${ }^{12}$ Le due pubblicazioni a cui si fa riferimento sono: Associazione Giovanni Secco Suardo, Basile Giuseppe (a cura di), Restauratori e restauri in archivio. Profili di restauratori italiani tra XVII e XX secolo. Quaderni dell'Archivio Storico Nazionale e Banca Dati dei restauratori Italiani. Volume 1. Nardini Editore, 2003; e Associazione Giovanni Secco Suardo, Basile Giuseppe (a cura di), Restauratori e restauri in archivio. Nuovi profili di restauratori italiani tra XIX e XX secolo. Quaderni dell'Archivio Storico Nazionale e Banca Dati dei restauratori Italiani. Volume 2. Nardini Editore, 2005.

${ }^{13}$ (Chiesa, 1946)

${ }^{14}$ (DPE, 1959)

${ }^{15}$ (Dipartimento dell'ambiente, 1984) 
principalmente, della corrispondenza emessa o diretta al Dipartimento della Pubblica Educazione del Canton Ticino (DPE), incaricato della sezione "Monumenti" prima e dopo l'impostazione legislativa. Questi documenti sono stati fondamentali per identificare ai personaggi della cultura ticinese attivi alla fine del Ottocento principalmente nell'ambito architettonico e artistico. Ad esempio si sono trovate fonti che documentano l'opera dell'architetto ticinese Augusto Guidini in favore della salvaguardia dei monumenti presenti nel territorio Svizzero, lettere che mostrano la collaborazione del Cantone con restauratori italiani, come i celebri fratelli Steffanoni da Bergamo, specialisti nella tecnica dello strappo degli affreschi, l'illustre Luigi Cavenaghi o con gli architetti Luca Beltrami ed Ambrogio Annoni.

All'Archivio dell'Ufficio dei Beni Culturali a Bellinzona (AUBCBZ) si è sviluppato il più interessante e complesso lavoro di ricerca documentaria. A partire dalle informazioni stratte dai sopracitati libri del Dipartimento della Pubblica Educazione per i Cinquant'anni ed i "Settantacinque anni di attività della Commissione cantonale dei monumenti storici", si sono esaminati uno ad uno i fascicoli dei monumenti intervenuti all'epoca, preselezionati. Lo stesso è stato eseguito all'Archivio Federale dei Monumenti Storici a Berna (AFMS) ed all'Archivio della Curia Vescovile a Lugano (ACVLU).

Inoltre, una volta sono stati individuati i personaggi di interesse alla presente ricerca, si è tentata la localizzazione degli Archivi personali dei medesimi artisti. Dalle quindici figure trattate nella tesi sono stati ritrovati e consultati unicamente tre archivi privati: quello di Tita Pozzi, custodito dal figlio avvocato Giovanni Pozzi di Massagno, l'archivio personale di Carlo Mazzi, conservato nella casa natale dalla figlia Silvia Mina-Mazzi, e l'archivio grafico del restauratore Bruno Abbiati curato anche questo dal figlio Attilio Abbiati. È stato impossibile ritrovare ulteriori archivi senza la localizzazione dei famigliare ed eredi degli artisti, d'altra parte, alcuni di essi che sono stati localizzati non hanno concesso la consultazione del materiale.

Sono stati indagati sistematicamente anche gli archivi grafici all'interno dell'AFMS e dell'AUBCBZ. La documentazione grafica è una base fondamentale del presente lavoro in quanto testimonianza visuale della storia dell'opera. Il confronto di essa con lo stato attuale dei dipinti murali esaminati permette di riconoscere trasformazioni, attuazioni di restauro ed ulteriori degradi.

La vasta indagine documentaria qui descritta è stata opportunamente riordinata in base agli interessi della ricerca, cioè l'identificazione dei dati inerenti alla storia della conservazione e restauro dei dipinti murali.

La riorganizzazione si è strutturata in tabelle, in base ai pittori-restauratori identificati attraverso l'analisi documentario e gli interventi eseguiti. Purtroppo, la dispersione delle fonti e la mancanza assoluta di informazione in altri casi, ha impossibilitato approfondire idoneamente tutti i campi che identificano l'evento "intervento". 


\section{Studio dei materiali}

L'esaustiva ricerca storica di archivio preliminare ha consentito oltre che identificare i pittori-restauratori che operarono nella conservazione dei dipinti murali nel Canton Ticino tra la fine del XIX sec. e la prima metà del XX sec., disegnare il loro profilo professionale definendo gli interventi eseguiti, il margine cronologico e geografico dello sviluppo della loro attività nonché le prassi esecutive impiegate nel restauro: le tecniche ed i materiali. Bisogna avvertire alla difficoltà anzitutto di completare questo specifico campo, poiché solo dopo gli anni Cinquanta del Novecento si ebbe la consapevolezza della necessità di documentare i diversi interventi. Inoltre alla fine dell'Ottocento ed inizi del Novecento i materiali impiegati furono quelli tradizionali e solitamente le prassi di lavoro erano le stesse per tutti i restauri. In questo modo abbiamo trovato più informazione che descrivono il ripristino architettonico di quello pittorico.

\section{Catalogazione e gestione dei dati}

Per promuovere la protezione e diffusione della storia della tutela e del restauro del patrimonio storico-artistico nel Canton Ticino, la presente tesi si è confrontata con i sistemi informatici innovativi usati a questo scopo. Sono stati analizzati i requisiti necessari per la catalogazione e la idonea divulgazione dei dati raccolti nella presente ricerca, in modo da permettere inoltre, che essi siano facilmente accessibili grazie alla nuove tecnologie.

Ho seguito i passi ad esempio della Associazione Secco Suardo a Bergamo, con il progetto Archivio Storico Nazionale e Banca Dati dei Restauratori Italiani, ed ora Archivio Storico (e Banca Dati) dei Restauratori Europei ${ }^{16}$, dell'Istituto LIDA $^{17}$ con il progetto AR.I.S.T.O.S (Archivio Informatico per la Storia della Tutela delle Opere Storico Artistiche), promosso da Clara Baracchini ${ }^{18}$, Ivan Boscaino ${ }^{19}$, Donata Levi ${ }^{20}$ y Andrea Maffei $^{21}$, del programma europeo MICHAEL (Multilingual inventory of Cultural Heritage in Europe) e della banca dati inventariale sviluppata attualmente dal Servizio inventariato dell'Ufficio dei Beni Culturali del Canton Ticino.

\footnotetext{
${ }^{16}$ La asociazione Giovanni Secco-Suardo con il progetto "Restauratori Europei" vuole costituire una rete europea per lo studio e la documentazione all'interno dell'Archivio Storico dei Restauratori Europei, di interventi di Conservazione e Restauro del patrimonio storico artistico, con lo scopo in futuro di creare una piattaforma comune di scambio e diffusione dei dati raccolti nei diversi paesi partecipi allo studio.

${ }^{17}$ Laboratorio Informatico per la Documentazione Storico Artistica (LIDA), Istituito presso il Dipartimento di Storia e Tutela dei Beni Culturali dell'Università di Udine, si occupa del trattamento informatico di fonti e materiali storico artistici, sia testuali che visivi, con particolare riferimento ai settori disciplinari della storia della critica d'arte, della museología, della storia del collezionismo, del restauro e della fortuna delle opere d'arte. http://test.infofactory.it/lida/

${ }^{18}$ Soprintendenza Beni Ambientali, Architettonici, Artistici e Storici per le province di Pisa, Livorno, Lucca e Massa Carrara.

${ }^{19}$ Università degli studi di Pisa

${ }^{20}$ Università degli studi di Udine

${ }^{21}$ Università degli studi di Pisa
} 
Le diverse banche di dati sono state confrontate ed analizzate, in modo da identificare quelli che possono in miglior modo rispondere alle necessità ideali per la gestione dei dati inerenti alla storia del restauro, nonché alla sua idonea diffusione.

Inoltre è stato possibile l'utilizzo di AR.I.S.T.O.S. per l'inserimento di parte delle fonti che conformano i dati dalla presente tesi e creare, grazie a questo lavoro, una coordinazione fra la base dei dati del laboratorio LIDA e la banca dati di prossima applicazione creata dall'Ufficio dei Beni Culturali del Canton Ticino. Il collegamento fra ambi due programmi sarà possibile per il momento vincolando i codici delle opere artistiche.

\section{Lettura della tesi}

La presente tesi dottorale è stata strutturata in tre parti fondamentali. La così denominata "Parte prima" ha lo scopo di introdurre il lettore alla storia della tutela nel Canton Ticino, analizzando gli esordi dell'attuale sistema istituzionale di protezione dei beni culturali, così come le personalità e meccanismi che hanno costituito le basi legislative e hanno definito la cultura della conservazione e del restauro nella prima metà del sec. XX. Inoltre si descrivono in linee generali le leggi che nell'attualità definiscono la politica di tutela in Svizzere e nel Canton Ticino. Questo capitolo si conclude con una tabella dove si elencano i membri che nel primo cinquennio hanno costituito la Commissione Cantonale dei Monumenti Storici, con lo scopo di mostrare l'organizzazione di essa nel periodo temporale studiato nella tesi. Assieme a questa tabella si è allegata una cartina del Canton Ticino, dove si segnala la disposizione geografica degli interventi analizzati nella tesi, restaurati tra il 1909 ed il 1959 circa.

La "Parte seconda" della tesi tratta le biografie dei pittori-restauratori individualizzati nella ricerca. A partire della raccolta dei dati e della sua gestione, come riferito nella "Parte prima", si strutturano dei profili professionali di nove restauratori: Edoardo Berta, Emilio Ferrazzini, Tita Pozzi, Carlo Cotti, Nino Facchinetti, Bruno Abbiati, Carlo Mazzi, Ottorino Olgiati e Mario Moglia. Essi sono stati esposti secondo un ordine logico di lettura basato nella cronologia della loro attività di restauro e nella notorietà. In questo modo il primo ad essere descritto è Edoardo Berta, direttore dei primi grandi interventi di restauro nel territorio ticinese come membro della Commissione Cantonale dei Monumenti Storici. Egli segna le basi teoriche e metodologiche dei restauri successivi alla sua morte, maestro e guida spirituale di artisti come Emilio Ferrazzini e Tita Pozzi. La lettura della tesi scorre in questo modo confermando la prasi esecutiva nel restauro dei dipinti murali, stabilite dal professore Berta nel XX sec. nel Canton Ticino, analizzando gli interventi e la personalità dei pittori-restauratori Ferrazzini e Pozzi. Inoltre si descrive l'attività di pittori con minore ripercussione nel campo del restauro, come Carlo Cotti o Nino Facchinetti, seguiti dal restauratore Bruno Abbiati, italiano "accolto" in territorio svizzero, e Carlo Mazzi che segnerà un'importante differenza cronologica con certi cambiamenti nella concezione del restauro. 
I capitoli, divisi per restauratore, sono stati organizzati mediante la ricostruzione iniziale della biografia, con gli eventi elementari che hanno definito la personalità della figura analizzata. In seguito, con lo scopo di ricostruire il profilo professionale di ogni restauratore, si sono scelti gli interventi più significativi da loro eseguiti, analizzandosi gli ideali e le prassi esecutive nonché i fattori che caratterizzano l'evento, come le diverse persone coinvolte, gli enti e le fonti di analisi fondamentali. Alla fine di ogni capitolo sono state allegate le tabelle che comprendono la catalogazione degli interventi identificati nella presente ricerca, e sulle quali si trova inoltre aggiunto il codice corrispondente al "Bene Artistico", al "Bene storico-artistico", all'"Ente" o alla "Persona", impiegato nella banca dati AR.I.S.T.O.S. per quelle voce già introdotte all'interno del programma. Assieme a queste tabelle si sono aggiunte delle cartine che consentono di visualizzare la disposizione geografica di questi restauri nel Canton Ticino.

Per ultimo sono stati descritti brevemente quei restauratori italiani (fratelli Steffanoni, Mauro Pellicioli, Francesco Annoni, Mario Rossi) e svizzeri (Henri-Paul Boissonas) la cui attività ha raggiunto il territorio ticinese. In particolare sono state allegate le tabelle con la catalogazione degli interventi svolti e la cartina. Bisogna avvertire che una descrizione più ampia dell'attività di queste personalità è stata trattata nei capitoli precedenti. Questo si deve a che la loro è stata principalmente una collaborazione, per questo si è creduto conveniente illustrare questa interazione all'interno dei capitoli che trattano le personalità ticinesi con le quali si crea la cooperazione. Ad esempio le prassi esecutive di Mauro Pellicioli sono state analizzate all'interno del capitolo corrispondente a Edoardo Berta giacché l'incursione più importante del restauratore bergamasco in Svizzera avviene all'interno del cantiere alla chiesa di Santa Maria degli Angioli a Lugano, diretto dal medesimo Berta negli anni Trenta.

La "Parte terza" di questa tesi, per ultimo, vuole analizzare i sistemi di catalogazione e di gestione dati mediante la descrizione degli archivi più importanti consultati per la ricerca, così come i sistemi di banca dati attualmente in uso in Europa che promuovono la diffusione dell'informazione contenuta nei medesimi archivi.

Per ultimo sono state sviluppate la conclusioni in basi ai risultati ottenuti.

Il lettore troverà alla fine del lavoro la descrizione della ampia bibliografia impiegata per la realizzazione di questa tesi. 


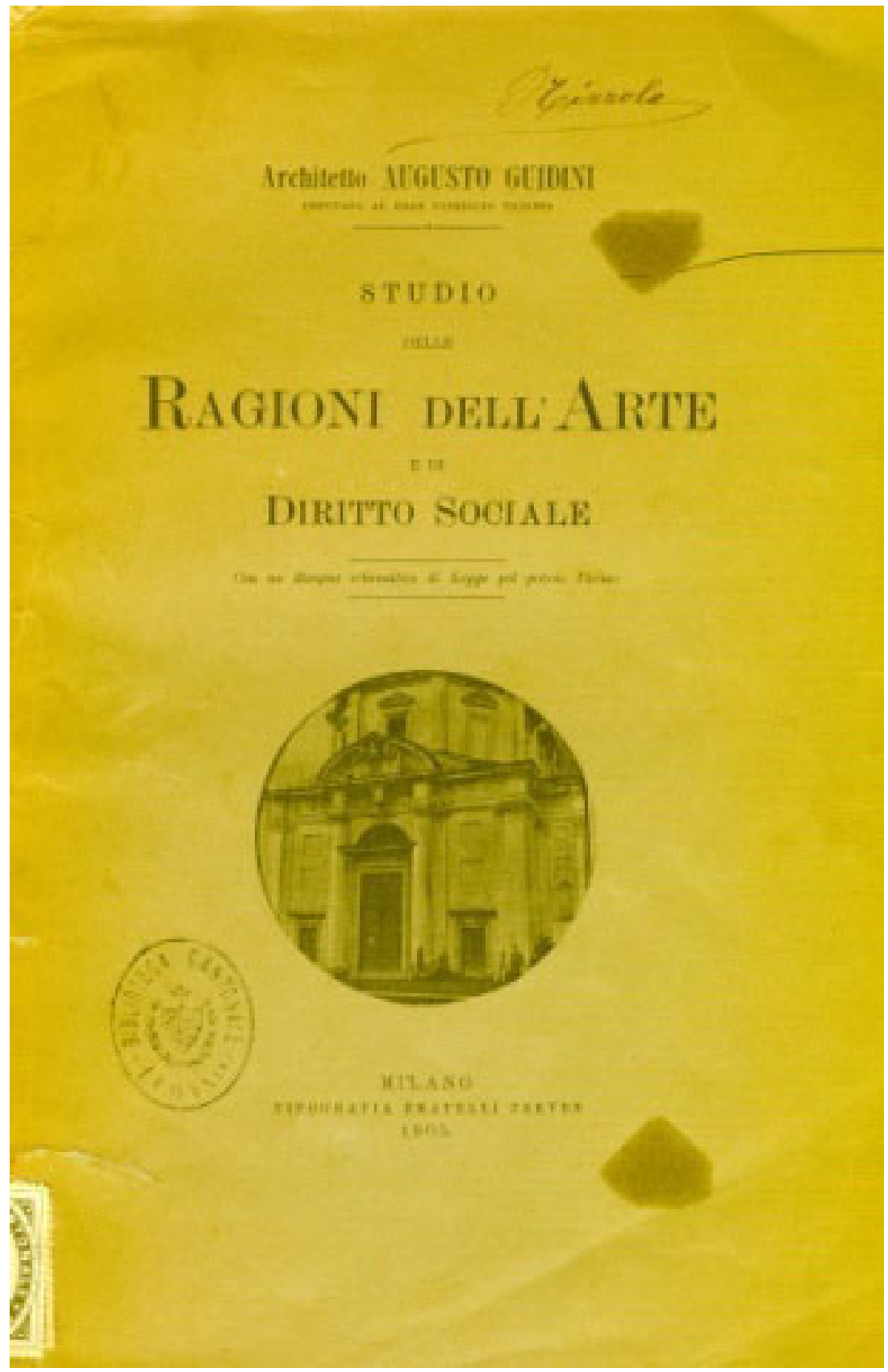

Figura 1 Guidini Augusto, Copertina di Studio della Ragioni dell'Arte e del Diritto Sociale. Milano 1905. Lo si trova nella "Libreria Patria" della Biblioteca Cantonale di Lugano. 


PARTE PRIMA

La tutela dei beni culturali 


\section{LA TUTELA DEI BENI CULTURALI IN SVIZZERA}

[...]II ritmo naturale dell'evoluzione tende a sostituire le cose vecchie con quelle nuove; probabilmente rimarrebbero unicamente palazzi di vetro e metallo se non ci fosse la legge cantonale sulla protezione dei monumenti introdotta nel 1909.[...] l'apparato per la protezione delle più importanti opere storiche ed artistiche del Canton Ticino sembra essersi assestato[... $]^{22}$

[...]El ritmo natural de la evolución tiende a sostituir las cosas viejas por las nuevas; probablemente quedarían solo edificios de cristal e metal si no existiese la ley cantonal de protección de los monumentos introducida en el 1909.[...] la comisión encargada de la protección de las obras histórico artísticas más importantes del Canton Ticino parece haberse consolidado.[...].

Queste parole pronunciate nel 1967 da Taddeo Carloni, presidente dell'allora Ufficio dei Monumenti Storici del Canton Ticino, esprimono quello che significò per il territorio svizzero la creazione, agli inizi del XX secolo, della legislazione che finalmente permetterebbe di regolare le operazioni per la salvaguardia dei monumenti storici ed il loro restauro.

Poco prima di questo evento, esattamente nel 1887, il Consiglio federale decise di creare la Commissione Federale dei Monumenti Storici (CFMS), conformata da esperti in materia di conservazione, con lo scopo di rendere operativa la protezione del patrimonio svizzero. Venne pubblicato proprio in questo periodo il primo testo in Svizzera che informerebbe sulle "Istruzioni per la salvaguardia dei monumenti e per il loro restauro" ${ }^{23}$ dello storico d'arte Johann Rudolf Rahn, al quale successero equivalenti testi scritti dai presidenti della CFMS: "Das Restaurieren" di Josef Zemp, nel $1907^{24}$ e "Restaurierungspraxis und Kunsterbe in der Schweiz" di Linus Birchler, nel $1948^{25}$. Fino ad oggi si è cercato di promuovere a questo scopo rapporti biennali e triennale che definiscono le basi di attuazioni comuni per le istituzioni ed operatori responsabili.

Si può affermare che la nascita del Consiglio di Esperti Federali prima, e delle commissioni cantonali con le leggi di protezione dei monumenti dopo, dimostrò la consapevolezza da parte degli enti della necessità di preservare certi oggetti, perché essi avevano la capacità di testimoniare le loro identità, le loro emozioni: si tratta di ciò che attualmente denominiamo "patrimonio culturale" ${ }^{26}$ e incorpora la scienza, la tecnologia, l'arte, le tradizioni, i monumenti, le abitudini e le pratiche sociali di diversa indole. Valori culturalmente acquisiti dallo stesso monumento o dall'opera d'arte, che gli ultimi studi

\footnotetext{
${ }^{22}$ RTSI, 1967.

${ }^{23}$ (Rahn, Anleitung zur Erhaltung von Baudenkmälern und zu ihrer Wiederherstellung, 1893)

${ }^{24}$ (Zemp, 1907)

${ }^{25}$ (Birchler, 1948)

${ }^{26}$ La UNESCO ha definito la cultura come "l'insieme degli aspetti spirituali, materiali, intellettuali ed emozionali unici nel loro genere che contraddistinguono una società o un gruppo sociale. Essa non comprende solo l'arte e la letteratura, ma anche i modi di vita, i diritti fondamentali degli esseri umani, i sistemi di valori, le tradizioni e le credenze". Citato in: Conferenza mondiale sulle politiche culturali. Rapporto finale della conferenza internazionale organizzata dall'UNESCO a Città del Messico dal 26 luglio al 6 agosto 1982. Pubblicato dalla Commissione UNESCO tedesca. Monaco di Baviera: K. G. Saur 1983 (Rapporti delle conferenze dell'UNESCO, n. 5), p. 121.
} 
antropologici hanno descritto come un complesso di simboli frutto del processo creativo, dinamico e multidimensionale, attraverso il quale la società arricchisce e protegge la propria cultura.

[...] La Svizzera è disseminata di monumenti culturali: singoli edifici, insediamenti e città. Queste testimonianze del passato subiscono trasformazioni: la società le abita, le demolisce e le sostituisce con altre costruzioni. Le loro tracce, però, rimangono. Esse costituiscono punti di riferimento sul territorio della Confederazione e sono pietre miliari della storia nazionale. Monumenti in lotta contro l'amnesia, i beni culturali lasciati alla posterità si presentano in diverse forme, provengono da epoche diverse e si trovano sia in superficie che nel sottosuolo [...] Nel loro insieme, essi rappresentano gli artefatti edificati della storia del popolo $[\ldots]^{27}$.

Le basi ideologiche e di attuazione attuali della Commissione Federale dei Monumenti Storici, sono state riepilogate nei "Principi per la tutela dei monumenti storici in Svizzera" ${ }^{28}$ il 22 marzo dell'anno 2006 secondo la decisione dell'assemblea convocata dalla suddetta Commissione il 21 gennaio 2004, e presentate al pubblico per il Consigliere federale Pascal Couchepin il 29 maggio 2007 nella torre campanaria della cattedrale di Berna.

Un gruppo di lavoro diretto dal presidente della CFMS, l'architetto Bernard Fürrer, assieme a François Guex, Nina Mekacher, Georg Mörsch e Beatrice Sendner, ebbe il compito di definire i precetti di tutela, sulla base degli ultimi più importanti documenti in materia elaborati dalle istituzioni internazionali come: la "Carta internazionale sulla conservazione ed il restauro dei monumenti e insieme architettonici" (Venecia, 1964), la "Carta per la salvaguardia dei giardini storici" (Firenze 1981), la "Carta per la salvaguardia delle città storiche" (Washington 1987), la "Convenzione per la salvaguardia del patrimonio architettonico d'Europa" (Granada 1985), la "Carta per la gestione del patrimonio archeologico" (Losanna, 1990) e per ultimo la "Convenzione europea per la tutela del patrimonio archeologico" (La Valletta, 1992) ${ }^{29}$.

Il testo, elaborato dall'ente federale, dovrebbe servire di strumento di lavoro e di consultazione a politici, committenti, architetti e specialisti del settore, favorendo la conoscenza del significato di "bene culturale", senza la quale sarebbe impossibile svolgere dei progetti idonei, fattibili a garantire alla loro conservazione.

A questi effetti il primo articolo del documento federale definisce "monumento storico" come [...]Un oggetto del passato con particolare carattere di testimonianza [...] attraverso il riconoscimento de parte della società $[. . .]^{30}$. II monumento storico, assieme agli oggetti mobili e testimonianze intangibili quali le lingue, la musica e le usanze formano parte del

\footnotetext{
${ }^{27}$ (Mürner, 2004, p. 2)

${ }^{28}$ Eidgenössische Kommission für Denkmalpflege, Principi per la tutela dei monumenti storici in Svizzera; vdf, Zurich 2007

${ }^{29}$ Le convenzioni e carte internazionali citate sono tutte consultabili all'interno del sito internet del Ufficio federale della cultura svizzero, esattamente nel link:

http://www.nb.admin.ch/bak/themen/kulturpflege/00513/00524/index.html?lang=it. (s.d.e.).

${ }^{30}$ EKD, 2007, p.61
} 
patrimonio della memoria umana e come tali devono essere conservati. Essi permettono l'identificazione individuale e collettiva e servono di base per l'impostazione sociale futura, perciò è obbligatoria, secondo i principi internazionali, la conservazione dei beni che documentino eventi positivi dell'attività umana, di sviluppi storici, di prestazioni artistiche di istituzioni sociali e di successi tecnici ma anche quelli che richiamano alla memoria ingiustizie o sofferenze.

Inoltre, i monumenti storici dovranno essere conservati mantenendo la loro integrità materica [...]con tutte le trace lasciate dal tempo [...] per cui le generazioni presenti ma anche quelle future, possono riconoscere le complesse stratificazioni quindi interpretarle[... $]^{31}$. Questo punto serve a ricordare una delle pratiche abituali nel territorio svizzero e nel resto di Europa, dalla fine dell'Ottocento fino all'avanzato XX sec., secondo la quale il monumento storico restaurato doveva essere riportato alle sue condizioni originarie, cancellando perciò qualsiasi aggiunta che la storia più recente avessi creato in conformità con i cambiamenti dei gusti estetici ed artistici. II presente fatto conferma, ancora una volta, la vulnerabilità dei valori dei monumenti ed in definitiva del bene culturale, poiché questo venne imposto dal proprio riconoscimento sociale. Attualmente le istituzione sono consapevoli di questo, e perciò la conservazione è obbligata a tutti quei oggetti, anche più recenti, che possano essere testimonianze future. Grazie ai presenti precetti, non si permetterebbe di commettere il passato errore che in Svizzera, ed in particolare in Canton Ticino, provocò I'annullazione della maggior parte dei monumenti ed elementi artistici del XIX sec. Nel punto 1.4. Valore di testimonianza del monumento storico la CFMS spiega appunto come: [...]Alle testimonianze storiche d'epoca recente può essere attribuito un valore monumentale di pari rango rispetto a quello attribuiti ad oggetti più antichi. Le parti più antiche di un monumento non sono, a priori, più preziose di quelle più recenti. Anche i restauri effettuati in precedenza possono costituire testimonianze significative del lato storiche[... $]^{32}$, e nel punto 1.6. Percezione del monumento storico si conferma che se [...]un oggetto del passato si trasforma in monumento storico attraverso l'identificazione e l'interpretazione da parte dell'essere umano[...] Vi è quindi il rischio di limitarsi all'interpretazione contemporanea, alla suggestione, alla semplificazione e alla distorsione. Per questo motivo il monumento deve essere conservato nella sua materialità considerata storicamente rilevante[...] ed anche per questo[...] Gli oggetti storici ipotizzati o rilevati con metodi storici, archeologici o scientifici, ma che non possono essere riconosciuti $e$ interpretati completamente, sono da trattare come monumenti storici[... $]^{33}$.

Attraverso la curata definizione di monumento storico, i "Principi per la tutela dei monumenti storici in Svizzera" della CFMS elenca sette punti che determinano la gestione di questo:

\footnotetext{
${ }^{31}$ EKD, 2007. pp.61-62

${ }^{32}$ EKD, 2007. pp.62

${ }^{33}$ EKD, 2007. pp.63
} 
1. I'identificazione del monumento storico come parte dell'ambiente la cui salvaguardia è garantita delle istituzioni per la tutela e la protezioni come base della vita umana;

2. i monumenti storici formano parte della vita contemporanea, per cui la loro conservazione e restauro sono altrimenti parte dell'odierna cultura

3. la società ha il dovere di proteggere il monumento storico assieme ai proprietari a cui essi sono stati affidati (sia pubblico o privato), e la disciplina di tutela tollererà la diversità di approcci che la collettività possa avere nel confronto del monumento.

4. è compito della società studiare il monumento servendosi possibilmente di metodi non invasivi, e nel caso di necessitare lo studio invasivo degli oggetti per completare delle informazioni, questo deve essere realizzato con il minimo intervento indispensabile.

5. i monumenti storici riconosciuti saranno designati pubblicamente mediante la messa sotto tutela, l'inventariato, le liste, gli elenchi, ecc. entro i limiti della salvaguardia dell'oggetto, sempre che la pubblicità eccessiva non sia per la conservazione di questo un pregiudizio.

6. la tutela dei monumenti storici è sommessa a della basi giuridiche

7. i risultati dallo studio dei monumenti devono essere accessibili al pubblico secondo il principio della trasparenza con la eccezione di particolari casi di proprietà privata.

Più avanti, l'articolo sulle regole $d^{\prime}$ 'intervento sul monumento storico ${ }^{34}$ descrivono: la sostenibilità delle azioni, che permettano alle generazioni future il massimo di opzioni per la scelta del trattamento più idoneo del monumento e per la sua conoscenza; l'utilizzo ragionato del monumento allo scopo di favorire la conservazione; la manutenzione regolare del monumento come misura meno invasiva e riduttiva dei costi nella conservazione; le misure preventive che devono servire a salvaguardare i monumenti il cui stato di tutela e protezione non sia stato ancora deciso; l'indispensabilità dello studio del monumento previo a determinare le condizioni appropriate di tutela; l'interdisciplinarietà necessaria nell'elaborazione delle più idonee misure di protezione; il piano di misura determinato mediante gli specialisti sarà verificato e adeguato in modo costante durante I'intervento per consentire la fattibilità di questo; la salvaguardia della sostanza storica mediante l'impiego dei metodi conservativi che modifichino questa nella minor misura, tenendo conto in questo punto dell'impossibilità della reversibilità delle azioni e dei materiali; e per ultimo, la necessità e obbligo da parte delle persone responsabili di fornire una completa documentazione adeguata alla singolarità dell'oggetto e dell'intervento.

I principi fino a qui presentati sono tuttavia gli stessi che determinano le basi della tutela dei beni culturali di carattere internazionale, di fatto la pubblicazione ha suscitato l'attenzione dei paesi limitrofi, poiché i precetti espressi possono essere applicati in linee generali a contesti culturali, politici e organizzativi, diversi della Svizzera. Non per altro al presente si tratta il modo di introdurre questi principi nei più importanti manuali di Germania e Italia.

Diverge sicuramente in alcuni punti dal resto dei paesi, e per questo è più interessante da analizzare nella presente ricerca, l'organizzazione della tutela nel sistema svizzero, ripresa

${ }^{34}$ EKD, 2007. pp.67 
nella seconda parte dei "Principi per la tutela dei monumenti storici in Svizzera". Secondo le leggi politiche del paese, nella tutela dei beni culturali sono i proprietari, privati o pubblici, a dover garantire la conservazione del monumento storico e gli specialisti incaricati dai proprietari ad svolgere un intervento, responsabili del suo successo. Inoltre in Svizzera esistono numerose organizzazioni di diritto privato, politicamente indipendenti, che appoggiano a livello nazionale, regionale o locale la sensibilizzazione sociale in materia, e possono contestare le decisioni delle autorità. E questo, nel mondo della globalizzazione in cui viviamo, è molto utile. Se in passato furono i letterati, artisti, architetti, storici, ed intellettuali in genere, a combattere la decadenza del proprio patrimonio storico-artistico, disposti a svegliare in questo modo l'anima di un popolo determinante, ma ferito dopo gli ultimi eventi storici, oggi siamo stati spettatori della risposta emanata dalla propria popolazione. Di fronte ai meccanismi di mercato, allo sfruttamento massivo del territorio, alle difficoltà degli enti pubblici a prendere decisioni disinteressate, senza interporre interessi economici e politici in beneficio della difesa delle proprie forme d'identità, la società ora accompagna, raccomanda ed esige agli enti interessati, di trovare la misura fra lo sviluppo e la preservazione della memoria.

L'articolo 78 della Costituzione federale della Confederazione Svizzera determina che la protezione della natura e del paesaggio - insediamenti, luoghi storici, rarità naturali, monumenti culturali ed i compiti dell'archeologia e della tutela- compete ai Cantoni; per questo motivo, considerando le condizioni politiche, il personale e le risorse economiche di ogni Cantone, esse cambiano da uno ad altro, e questi si reggono in modo indipendente. I Cantoni sono dotati di servizi specializzati per l'esecuzione delle misure di tutela, dispongono di mezzi finanziari a dipendenza delle singole esigenze. Essi possono convocare commissioni di esperti o delegare la responsabilità ai servizi specializzati comunali. La Confederazione intervenne come sostenitrice dei Cantoni operando sui problemi che questi non possono risolvere autonomamente, allo stesso tempo che $i$ Cantoni collaborano con I'Ufficio federale della cultura, nelle questioni tecniche riguardanti beni di protezione federale.

Il sopracitato Ufficio (UFC) è il servizio specializzato della Confederazione che sostiene la protezione del patrimonio culturale. Fra le sue prestazioni si possono elencare, inoltre, l'assegnazione di sussidi per la conservazione, l'acquisto, la salvaguardia, la ricerca, la documentazione di insediamenti, luoghi storici o monumenti culturali, il sostenimento di organizzazioni d'importanza nazionale, così come della formazione e della relazioni pubbliche, nomina esperti incaricati ai servizi specializzati per richiesta dei Cantoni o della Commissione Federale dei Monumenti Storici e gestisce la segreteria di quest'ultima.

La CFMS invece, è la commissione di esperti consultiva del Dipartimento Federale dell'Interno in materia di archeologia, conservazione dei monumenti e protezione degli insediamenti, nell'applicazione della "Legge federale sulla protezione della natura e del paesaggio" e nella relativa ordinanza, allo stesso tempo che è l'incaricata di elaborare e aggiornare gli inventari corrispondenti ad oggetti d'importanza nazionale -"I'Inventario 
degli insediamenti svizzeri da proteggere" ${ }^{35}$ (ISOS); “I'Inventario federale dei paesaggi, siti e monumenti naturali di importanza nazionale" ${ }^{36}$ (IFP) e "I'Inventario delle vie di comunicazione storiche in Svizzera" (IVS) ancora in elaborazione-, di realizzare perizie su questioni riguardanti il patrimonio storico-artistico allo scopo di chiamare l'attenzione sulle autorità federali e di curare l'interdisciplinarietà scientifica.

Questi principi, così come gli organismi federali e cantonali qui sopra analizzati, lottano per permettere alla società svizzera di conservare quella cultura che la caratterizza, inoltre al Canton Ticino in concreto, come ad ogni territorio, permette di identificarsi, relazionarsi e di confrontarsi con il resto mediante la trasmissione di queste testimonianze fisichesimboliche.

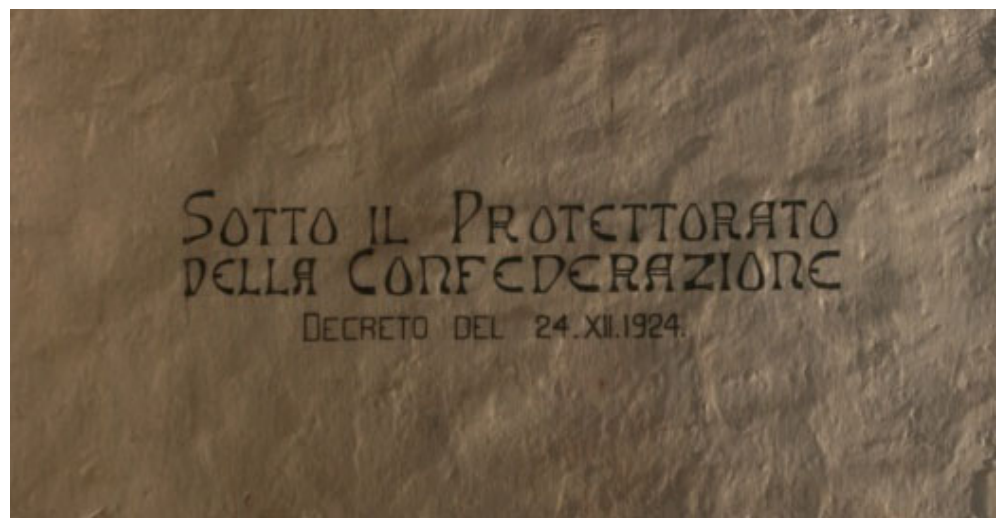

Figura 2 S. Maria del Castello, Mesocco; Targa commemorativa della Confederazio dei monumenti storici (1924)

\footnotetext{
35 L'Inventario degli insediamenti svizzeri da proteggere (ISOS) comprende più di 1'000 siti di importanza nazionale elencati ed inoltre studia i nessi architettonici e spaziali di insediamenti, villaggi e città ed i risultati vengono analizzati e completati con indicazioni per la pianificazione. L'Inventario delle vie di comunicazione storiche in Svizzera (IVS), invece è un progetto unico al mondo. Il suo compito è quello di eseguire un elenco cartografico e descrittivo delle strade e dei sentieri riconosciuti di importanza nazionale in base al loro significato storico come vie di collegamento. II risultato del lavoro realizzato è un strumento di protezione, conservazione di elementi fondamentali per il nostro paesaggio e per la storia, nonché un materiale imprescindibile di ricerca.

${ }^{36}$ L'Inventario federale dei paesaggi, siti e monumenti naturali di importanza nazionale (IFP) è stato il primo elenco realizzato dal Consiglio Federale. Comprende tre tipi di oggetti: oggetti unici in Svizzera o in Europa per la loro bellezza, particolarità, importanza scientifica, ecologica o geografica e culturale; i paesaggi tipici o paesaggi culturali nel verde che presentano superfici particolarmente caratteristiche per una determinata regione, elementi culturali, storici e spazi vitali importanti per la flora e la fauna; e per ultimo i monumenti naturali quali elementi imprescindibili nella configurazione del paesaggio.
} 


\section{LA COMMISSIONE CANTONALE DEI MONUMENTI STORICI. GLI ESORDI.}

[...]Con questo restauro si è segnato un esempio di ricupero e concessione di pregevoli ed antiche pitture: esempio che speriamo non abbia a restare isolato. Scarso è purtroppo già diventato il patrimonio artistico del Ticino, per le secolari incurie e per più colpevoli vandalismi. $E$ se codesto periodo nostro segnerà l'aprirsi di un'era intellettuale $e$ cosciente di cure e conservazione dei monumenti dell'arte ticinese, e d'ogni meritevole e storico documento, saremo lieti d'aver potuto onorati della fiducia degli odierni Reggitori dello stato - segnare il modesto avviamento. $[. . .]^{37}$.

[...]Con esta restauración se ha definido un ejemplo de recuperación de preciadas y antiguas pinturas: ejemplo que esperamos no se trate únicamente de un caso aislado. El patrimonio artístico del Ticino, a causa de las continuas negligencias y vandalismos ocasionados a lo largo de los siglos pasados, es ya reducido. Y si el presente período señalará la apertura hacia una era intelectual y sensible a la necesidad del cuidado y de la conservación de los monumentos artísticos ticineses, y de cada uno de los documentos históricos de valor, seremos felices por haber honorado la fidelidad de los actuales governantes [...].

\footnotetext{
${ }^{37}$ Guidini Augusto, Relazione e Collaudo. Chiesa di S. Paolo in Arbedo e di Pianezzo.Antiche Pitture scoperte e restaurate. Rapporto indirizzato al Lodevole DPE, Bellinzona. Milano, 15 settembre 1899. AN Bern, KT A-M №79.
} 
Queste parole, scritte dall'architetto Augusto Guidini nel 1899 per commemorare i restauri alla chiesa di S. Paolo in Arbedo ed alla chiesa di Pianezzo, esprimono al meglio le ambizioni dell'architetto milanese riguardo il futuro della storia della tutela nel Canton Ticino $^{38}$. Egli motivò ed aiutò con diverse pubblicazioni, la sensibilizzazione degli organismi politici del territorio svizzero sulla necessità di conformare un sistema organico, normalizzato, con il quale difendere gli aspetti fondamentali per la salvaguardia dei monumenti storici del Canton Ticino.

[...]Poche altre terre della regione prealpina abbondano quanto la nostra di cose pregevoli per ragioni $d^{\prime}$ arte e d'antichità: santuari, case, pietre scolpite, pareti affrescate [...]Cose che non tutte meriteranno il titolo di monumento d'alta importanza intrinseca: alcune sì, e tutte improntate $d^{\prime}$ ' un carattere, testimonianze d' una civiltà che si prosegue, non mai interrotta dalle sue remote origini. Ricco il suolo di cospicue reliquie archeologiche; copioso il patrimonio mobile, oggetti d'arte, arredi, carte. Copioso, quando non ancora disperso o decimato. Chiunque era libero di frugare nella terra $e$ $d^{\prime}$ asportare i più cospicui ritrovamenti; libere le Parrocchie di vendere al miglior offerente i loro monumentali altari. E non buona la condizione a cui erano venuti decadendo anche i monumenti di natura più resistente. $[\ldots]^{39}$.

II richiamo di Augusto Guidini fu sentito il 17 gennaio del 1905 quando si stabilì il primo progetto per la regolazione degli interventi sui monumenti storici, che si era basato in alcuni punti sulla legislazione vigente in Romania del 17 novembre 1892. La medesima legge, trovatosi incompleta, fu ritirata appena un mese dopo. In cambio furono impostate, ancora dal Guidini, le norme elementari sugli scavi archeologici nel decreto legislativo del 19 maggio 1905, e fu effettivamente l'antecedente alla legge che più tardi stipulerebbe le prassi per la tutela dei monumenti storici nel Cantone in un secondo progetto presentato il 10 giugno del 1908 ed approvato il 14 gennaio del 1909 con il titolo di "Legge sulla conservazione dei monumenti storici ad artistici del Cantone Ticino".

Più tardi sarebbe stata creata la Commissione Cantonale dei Monumenti Storici incaricata di far si che la suddetta legge fosse compiuta. La sua nascita fu conseguenza del lavoro dei personaggi che credevano, e tenevano a cuore, la conservazione del patrimonio del loro paese, così come dall'insostenibile situazione che fino al momento aveva caratterizzato gli sporadici e disorganizzati interventi, promossi per evitare il decadimento ancora maggiore di alcuni monumenti. Altrimenti favorevole alla creazione dell'evento furono, secondo il parere di Francesco Chiesa nelle sue riflessione pubblicate ai Cinquant'anni della $\mathrm{CCMS}^{40}$ : il clima sociale e politico che nell'ultimo decennio si caratterizzava da una poco abituale

\footnotetext{
${ }^{38}$ Dei 26 cantoni in cui è divisa la Svizzera, il Canton Ticino è il più meridionale, a sud delle alpi confina a est, ovest e sud con I'Italia, al nord-ovest con il Canton Vallese, a nord con il Canton Uri ed a nord-est con il Canton Grigioni. Occupa 2' $812 \mathrm{~km} 2$ dei 41'285 km2 della superficie totale della Svizzera. La lingua ufficiale è l'italiano in confronto alle altre tre lingue riconosciute nel paese: il tedesco, il francese ed il romancio. La religione predominante è il cattolicismo. La politica si regge da un sistema parlamentare democratico.

${ }^{39}$ (DPE, 1959, p. 10)

${ }^{40}$ (DPE, 1959)
} 
calma $^{41}$, l'esempio del resto della Svizzera e del prossimo paese italiano e l'influenza di figure come lo storico ticinese Emilio Motta $^{42}$ che tentò sempre di mantenere viva la memoria storica e motivò altri storici.

Nella nuova prospettiva politica, culturale e lenta miglioria economica, finalmente uno sguardo dallo Stato fu rivolto a lottare per la salvaguardia del proprio patrimonio storicoartistico, rivalorizzando le opere e combattendo l'amnesia della collettività che doveva ancora essere, anche essa, sensibilizzata. Così nel 1893 il Cantone promosse la traduzione di "I monumenti artistici del Medio Evo nel Canton Ticino" di Johann Rudolf Rahn ${ }^{43}$, ed alcuni monumenti furono restaurati con il sussidio della Confederazione, come ad esempio la cappella Camuzio della chiesa di Santa Maria degli Angioli, a Lugano (1891), la chiesa di San Carlo di Negretino ripristinata architettonicamente nel 1899, il Castello di Montebello a Bellinzona (1903), la Cattedrale di San Lorenzo a Locarno (1905) e la chiesa di San Paolo ad Arbedo e di Pianezzo (1899).

II secolo XIX aveva lasciato decadere numerosi monumenti, privi della loro simbologia. Alcuni di essi a causa della soppressione delle congregazioni, come ad esempio la Chiesa di S. Francesco a Locarno, e del Battistero e della chiesa di Santa Croce a Riva San Vitale, erano stati usati come magazzini, spogliati del loro arredo, del loro significato, e altri al contrario erano stati trasformati in occasione di sfortunati abbellimenti.

La fondazione nel 1909 della Commissione Cantonale dei Monumenti Storici (CCMS) non era stata considerata nella legge del 1909, ma venne istantaneamente imposta dal Gran Consiglio. Questo nuovo ente, dipendente dal Dipartimento della Pubblica Educazione del Canton Ticino aveva, come oggigiorno, il dovere della sorveglianza dei monumenti tutelati procedendo con la ricerca, inventariato, controllo della manutenzione o del loro restauro e dei sussidi che lo fanno possibile.

La CCMS era costituita da importanti figure dell'ambito culturale ed artistico ma anche ecclesiastico: scrittori, avvocati, pittori e preti $^{44}$.

Dalla sua fondazione nel 1909 al $1923^{45}$ la CCMS era conformata unicamente da tre membri, essi furono gli illustri Francesco Chiesa, Emilio Motta e Edoardo Berta; dal 1924 al

\footnotetext{
${ }^{41}$ Lungo il XIX, fino al 1890 la situazione politica del Canton Ticino è caratterizzata da continue lotte per il potere fra liberali e conservatori, la maggioranza di queste costituite da rivoluzioni violente. La Costituzione del 1830 elaborata da Stefano Franscini invece non è soggetta a cambiamenti fino al 1997. Nel 1893 si crea in Ticino, per la prima volta in Svizzera un sistema elettivo proporzionale all'esecutivo. II sistema governativo moderno del Canton Ticino fu completato con la nascita del movimento socialista, costituendosi il primo partito nel 1900 che nel 1920 entra finalmente al governo cantonale.

${ }^{42}$ Motta Emilio (1855-1920), ingegnere e storico, fonda nel 1879 il Bollettino Storico della Svizzera Italiana ed è responsabile dell'Archivio Storico Cantonale.

${ }^{43}$ (Rahn, 1976)

${ }^{44}$ II consigliere di Stato, l'avvocato. Brenno Galli, fu direttore del Dipartimento della Pubblica Educazione del Canton Ticino, nel periodo compreso fra il 1947 ed il 1959, rimpiazzando il Consigliere Giuseppe Lepori. Egli descrive, nell'introduzione al libro "Cinquant'anni di protezione dei monumenti storici artistici delle Repubblica e Cantone Ticino 1909-1959" la struttura della Commissione cantonale dei monumenti storici: [...] non necessariamente una commissione di tecnici del restauro: essa si varrà degli studiosi, per compire l'opera di tutela che le è affidata[...] (DPE, 1959, p. 7)
} 
1927 fu costituita da quattro membri, dal 1928 al 1939 da cinque e finalmente dal 1940 al 1997 con la messa in vigore della nuova legge di protezione, la CCMS venne conformata da sette membri ${ }^{46}$. In concreto le figure a incaricarsi del suddetto ente furono gli scrittori Francesco Chiesa, che la presiede dal 1909 al 1959, Agusto Ugo Tarabori (1940-1959), Carlo Speziali (1955-1959), Piero Bianconi (1940-1959), Ugo Donati (1944-1947) e Giuseppe Martinola (1956-1959), i pittori Edoardo Berta (1909-1930), Emilio Ferrazzini (1930-1945), Pietro Salati (1946-1959) ed Emilio Maria Beretta (1947-1954), gli ingegneri Emilio Motta (1909-1920) e Luigi Brentani (1909-1920/ 1957-1959), I'avvocato Angelo Martignoni (1921-1943), gli architetti Otto Maraini (1924-1942), Pietro Giovannini (19441956) ed Alberto Camenzind (1951-1957) e per ultimo i vescovi Giovanni Noseda (19281937), Davide Sesti (1940-1943), Alfonso Codadhengo (1944-1950), ed Agostino Robertini (1955-1959).

All'interno della CCMS funzionò per alcuni anni il così chiamato "Ispettorato dei Monumenti" incaricato dei lavori di segretariato, responsabile dell'esecuzione dei restauri condotti sui monumenti protetti nonché dei servizi archeologici e museali. L'organismo venne annullato nel 1943, assumendo le sue funzioni il presidente dalla CCMS, Francesco Chiesa. Solo nel 1958 I'IMS fu re instituito, lo stesso anno che la CCMS si trasforma in Ufficio dei Monumenti Storici e si definiscono nuovi incarichi in risposta alle recenti necessità, come ad esempio la cura dell'archivio storico che cominciava ad accrescere, l'esecuzione ed aggiornamento dell'inventario ed il tramandare dell'informazione documentata al Dipartimento della pubblica educazione del Canton Ticino. L'Ispettore responsabile doveva essere partecipe alle riunioni convocate dall'UMS, sorvegliare i restauri in corso e ugualmente la idonea conservazione dei monumenti sotto la protezione dello Stato.

[...] Che cosa si è fatto in questo lungo periodo? Quale è stata l'attività della Commissione? È riuscita valida e soddisfacente l'opera dello Stato a tutela dei monumenti?[... $]^{47}$

Nel primo Cinquennio di attività della CCMS furono più di cento i lavori di intervento di restauro eseguiti sui monumenti storici protetti dallo Stato, specie per la salvaguardia di edifici ed opere di arte considerati di interesse [...] quali ad esempio San Biagio di Ravecchia, il Castello di Locarno, Santa Maria delle Grazie in Bellinzona, Santa Maria degli Angioli in Lugano, la chiesa parrocchiale di Malvaglia, la chiesa parrocchiale di Morcote e gli edifici annessi, Santa Croce $e$ il Battistero di Riva San Vitale, San Nicolao di Giornico, San Pietro di Castel San Pietro, Sant'Ambrogio di Cademario, San Remigio di Corzoneso, la Madonna di Ponte a Brissago [...] aggiungendo gli edifici

\footnotetext{
${ }^{45}$ Dal 1909 al 1920 i membri che costituirono la CCMS furono il prof. Francesco Chiesa, il pittore Edoardo Berta, e l'ing. Emilio Motta; dal 1921 al 1923: il prof. F. Chiesa, E. Berta e l'avv. Angelo Martignoni. Dal 1924 al 1927 il prof. F. Chiesa, E. Berta, I'avv. Martignoni, e l'arch. Otto Maraini; dal 1928 al 1939: il prof. F. Chiesa, E. Berta, I'avv. Martignoni, l'arch. Maraini, e Mons. Giovanni Noseda; dal 1931 al 1937: il prof. F.Chiesa, I'avv. Martignoni, l'arch. Maraini, Mons. Noseda, ed il pittore Emilio Ferrazzini; dal 1938 al 1939: il prof. F. Chiesa, l'avv. Martignoni, I'arch. Maraini, E. Ferrazzini e Mons. Davide Sesti. (Chiesa, 1946, p. 128)

${ }^{46}$ Secondo un decreto esecutivo del 22 dicembre del 1939 che modifica il primo articolo della legge del 29 settembre del 1909.

${ }^{47}$ (DPE, 1959, p. 10)
} 
minori, gli affreschi consolidati o strappati, le tele riparate $[. . .]^{48}$ in favore della preservazione della memoria culturale del paese.

Nel 1944 fu completata la legge riguardo la tutela dei ritrovamenti archeologici sull'esame critico e le proposte di Aldo Crivelli nel 1940, aggiungendo inoltre delle norme per la conservazione dei Musei storici ed archeologici che prevedeva l'istituzione dell'Ufficio d'Ispettore degli scavi e dei musei.

Fra questi cambiamenti anche la "Legge sulla conservazione dei monumenti storici ed artistici del Cantone Ticino" del 1909 fu rivista ed approvata dal Gran Consiglio il 15 aprile $1946^{49}$ ed entrò in vigore il 7 gennaio 1947 adottando il titolo di "Legge per la protezione dei monumenti". Il cambiamento nell'appellativo indicava una trasformazione anche nell'impostazione della legislazione. Mentre il precedente regolamento imponeva le norme per la conservazione, cioè la manutenzione delle opere a carico dello Stato come principio base, ed il restauro supponeva una seconda esigenza che doveva essere realizzata dal proprietario dietro autorizzazione, la nuova legge prevedeva invece il restauro come operazione fondamentale, che poteva essere eseguita dal proprietario o dallo Stato con l'approvazione e la vigilanza di quest'ultimo.

Purtroppo, il sacrificio fu molto grande e le ricompense apparentemente poche, giacché il periodo conclusosi con delle intense polemiche giornalistiche che turbavano il ricordo di essi all'inizio proclamatesi come dei restauri esemplari. Di fatto, la storia del restauro moderno nel Canton Ticino come d'altra parte nel resto della Europa degli anni Venti, era appena iniziata e le divergenze fra gli intellettuali ed i professionisti in quanto ai concetti teorici, così come nel approccio pratico all'opera, erano numerose.

L'architetto Cino Chiesa, figlio dell'illustre scrittore e presidente della CCMS Francesco Chiesa, scriveva nel 1943 un articolo per la "Rivista Tecnica della Svizzera Italiana" sull'importanza della "Carta del restauro" enunciata in Italia nel 1931 per la gestione degli interventi realizzati sui monumenti protetti. Questa, secondo l'architetto Chiesa, era un testo di grande portata intellettuale e morale, e proclamava le basi per il restauro moderno secondo la quale si stabilivano prioritarie in ogni intervento: le operazioni di manutenzione e di consolidamento, la ristrutturazione non doveva realizzarsi se non sulla base documentata, non si dovevano eliminare dal complesso architettonico o decorativo elementi di importanza o con un significato identificativo della collettività, le aggiunte moderne dovevano manifestarsi evitando falsi storici e si dovevano adoperare i mezzi scientifici moderni in quanto possibile, fra le indicazioni più importanti.

In contrappunto, la teoria moderna a rimpiazzare il "restauro empirico" e "dottrinale", il "conservatore" e "romantico", secondo la quale ogni intervento doveva adattarsi alle intrinseche necessità dell'elemento senza regole a priori, non fece altro che creare maggiore confusione fra i responsabili della CCMS di decidere e sorvegliare le operazione

\footnotetext{
${ }^{48}$ (DPE, 1959, p. 11)

${ }^{49}$ La legge fu approvata dal Vice-presidente del Gran Consiglio Francesco Masina e dai consiglieri segretari, gli avvoccati A. Lanfranchi ed A. Borella. (DPE, 1959, p. 23)

${ }^{50}$ Chiesa Cino, Revista Tecnica della Svizzera Italiana, 1943.
} 
di restauro nei monumenti tutelati. La indecisione e la concezione ancora vaga del significato di "restauro" e di "conservazione" non aiutò ad evitare le critiche, e la CCMS ebbe bisogno di confermare dei metodi e criteri di restauri che la legge del 14 gennaio del 1909 non stabiliva.

Fra il 1950 ed il 1970 il Ticino comincia a trasformarsi perdendo le proprie caratteristiche rurali per dare luogo alla costruzione di moderni edifici di "vetro e metallo"- ricordando le parole del nuovo presidente dell'UMS dopo il 1959, Taddeo Carloni ${ }^{51}$. Nel 1962, di fronte alla situazione di confusione fra gli organismi che compongono l'UMS ancora debole, indefinito nella politica di tutela e ferito, lo Stato decise di creare l'OSMA, Opera svizzera dei monumenti d'arte, con la collaborazione della Società Svizzera di Storia dell'Arte (SSAS). Si trattava di una guida-inventario sorta a partire dei modelli che stavano contemporaneamente funzionando nel resto della Svizzera, e che ancora oggi venne pubblicata nel territorio con ampio successo. In Ticino il responsabile dell'istituto OSMA fu lo storico d'arte Virgilio Gilardoni. Questo pretendeva succedere agli inventari precedentemente pubblicati in Ticino: il primo fu realizzato nel 1909 dai membri fondatori della CCMS, lo scrittore Francesco Chiesa, lo storico ed archivista Emilio Motta ed il pittore Edoardo Berta, con lo scopo d'identificare i beni mobili ed immobili protetti dallo Stato. Più avanti sorsero "I'Inventario delle cose d'arte e di antichità I", edito dal Cantone Ticino ed introdotto dal Consigliere di Stato Breno Galli, "Le Tre Valli superiori Leventina, Blenio, Riviera" di Piero Bianconi (1948) e "I'Inventario delle cose d'arte e di antichità" di Virgilio Gilardoni (1955).

Tuttavia bisogna avvertire che un primo elemento di catalogazione si trova nel testo del professor Johann Rudolf Rahn che elabora uno studio dei monumenti medievali del Canton Ticino ${ }^{52}$, analizzati attraverso splendidi disegni ed acquarelli che oggi testimoniano molto onestamente lo stato dei monumento illustrati. Si tratta di un strumento di ricerca fondamentali nato dalla consapevolezza del Rahn della necessità di documentare e di tramandare i suoi analisi e conoscenza, che inoltre risultano sinceri ed estremamente obiettivi: sia questo il principio dell'inventario.

Le funzioni dell'OSMA furono in primo luogo, la raccolta e il riordino di tutta la documentazione storica, archeologica, tecnica, archivistica, biografica e bibliografica già esistente riguardante "i monumenti e le cose d'arte" del Ticino, per comune e distretto, e in secondo luogo, la documentazione raccolta fu completata con nuove ricerche regionali d'archivio e l'esame di bibliografia locale ed estera. I risultati ottenuti anni più tardi furono tre pubblicazioni dedicate al locarnese: "Locarno e il suo circolo (Locarno, Solduno, Muralto e Orselina)" nel 1972; "L'Alto Verbano I. II circolo delle Isole (Ascona, Ronco, Losone e Brissago)" nel 1979 e "L'Alto Verbano II. I circoli del Gambarogno e della Navegna", nel 1983.

\footnotetext{
${ }^{51}$ (RTSI, 1967).

Rudolf Rahn, Johann, I monumenti artistici del Medio Evo nel Canton Ticino. Società ticinese per la conservazione delle bellezze naturali ed artistiche. Lugano, 1976
} 
Con la perdita nel 1969 del presidente Taddeo Carloni, Pierangelo Donati (1937-1993) fu nominato capo dell'UMS, assumendo a suo tempo l'incarico di Ispettore dei Monumenti Storici, e pubblica l'inventario: "Elenco dei monumenti storici e artistici del Canton Ticino". La storia della rivoluzione dell'UMS negli anni successivi fu strettamente legata a Pierangelo Donati. Egli motiverà l'esercitazione di una nuova politica di tutela dei beni culturali, estendendo le azioni di protezione agli edifici rurali, strade storiche, siti archeologici e documenti, che hanno anche loro un significato essenziale nella configurazione del patrimonio culturale. Donati ebbe il compito di iniziare in un momento critico per l'UMS, come citato alcune righe più avanti, ancora colpito dagli eventi del primo quinquennio di vita della vecchia CCMS. II direttore dell'Ufficio si trovava ad agire di fronte all'incremento dell'attività edilizia pubblica e privata, alla legislazione federale e cantonale che regolava la pianificazione moderna territoriale e alle nuove circostanze liturgiche decretate dal II Concilio del Vaticano, che richiedevano una attuazione immediata.

Nel 1976 I'UMS si aggrega al Dipartimento dell'Ambiente e da questa nuova posizione verrà inoltre incaricata della gestione dell'approvata "Legge federale per la protezione dei beni culturali in caso di conflitto armato o in caso di catastrofe".

Anni più tardi si creano il Servizio archeologico ed il Servizio monumenti, assieme al già esistente Servizio inventario.

Nel 1989 furono nuovamente definite le aree funzionali del Dipartimento dell'Istruzione e della Cultura. All'interno di questo si creano la sezione dei beni ambientali e monumentali che a suo tempo comprendeva l'Ufficio Protezione della Natura, l'Ufficio dei Monumenti Storici, I'Opera Svizzera dei Monumenti Storici e Artistici e l'Ufficio Cantonale dei Musei. La partecipazione della Svizzera ai convegni internazionali del XX secolo, in materia di protezione e tutela dei beni culturali, è stata fondamentale per la trasformazione definitiva del vecchio sistema organizzativo e ideologico anche in Canton Ticino.

Indispensabili furono la Convenzione culturale europea del 19 dicembre 1954 che obbliga il paese ad adottare le misure necessarie per la salvaguardia del patrimonio culturale comune in Europa. Lo stesso anno, la Convenzione dell'Aia crea le basi internazionali per la protezione dei beni culturali in caso di conflitto armato o catastrofe che a livello nazionale è disciplinata dalla Legge federale (Legge sulla PBC del 1966) e dalla relativa Ordinanza (1984). Nel 1972 la Convenzione dell'Unesco per la protezione del patrimonio culturale e naturale, identifica e definisce in ogni paese i beni culturali e naturali propri assicurando la loro protezione e rivalorizzazione; inoltre l'apertura della Svizzera verso gli accordi internazionali si conferma con la firma del "Trattato per l'importazione di oggetti per fini educativi, scientifici e culturali, promovendo gli scambi culturali di questi beni mobili" del 22 novembre del 1950.

Dalla creazione della CCMS fino all'impostazione nel 1997 della nuova legge di protezione basata sui più importanti convegni internazionali sopraccitati, gli edifici e manufatti iscritti nell'elenco cantonale dei monumenti storici ed artistici, valutati secondo la legge del 1909 furono in totale circa 1525 oggetti. Essi venivano differenziati in: 85 manufatti architettonici del tipo: cappella, cimitero, colonna, croce cimiteriale, edicola funeraria, 
fontana, mura, muro, ossario, ponte, porta, pozzo, ruderi e vasca; 415 edifici (battistero, biblioteca, cappella, casa, castello, chiesa, collegio, convento, edificio, fornaci, fortini della fame, monastero, museo, oratorio, ospedale, ospizio, palazzo, palazzo comunale, palazzo del pretorio, palazzo vescovile, rustico, santuario, teatro, torba, torchio, torre, villa); 192 elementi architettonici (architrave, arco, balaustra, balcone, caminiera, camino, cancellata, cancello, capitello, chiave d'arco, colonna, cornice, facciata, finestra, inferriate, insegna di ferro battuto, lunetta, mensola, porta, portale, serraglia di volta, sopracamino, soffitto, spalle di portale, travatura); 79 corpi architettonici (abside, altana, androne, campanile, cappella, chiostro, coro, cortile, loggia, loggiato, nicchia, portico, presbiterio, sacrestia, sala, scalinata); 9 spazi urbani (parco, particelle, piazza, sagrato); 347 dipinti (affresco, ex voto, graffito, medaglione, olio su rame, ovale, stemma, tavola, tela); 128 sculture (ancona, bassorilievo, busto, caminiera, capitello, colonna, formella, gruppo ligneo, lapide, serraglia di volta, statua, statuette da presepio, stucchi) - alcuni di loro già inventariati fra gli elementi architettonici-; 11 documenti (album, antifonario, codice manoscritto, martirologio, messale, statuti manoscritti); 75 suppellettile sacra (bacile, calice, cassetta, croce astile, croce processionale, croce stazionale, ostensorio, piatto per offerte, portacero, recipiente per la questua, reliquiario, scatola sacramentaria, servizio d'altare, stendardo); 1 suppellettile civile (bandiera); 136 arredo sacro (acquasantiera, altare, armadio, campana, ciborio, confessionale, coro, croce d'altare, crocifisso, dossale d'altare, dossale del coro, fonte battesimale, lampada pendula, leggio, mobile, organo, paliotto, piedistallo, pila dell'acquasanta, poltrona, pulpito, scanno, stalli, tabernacolo, trittico, valve d'organo); 15 arredo civile (armadio, cassapanca, mobile, pigna di pietra ollare, pila da giardino, tavolo); 8 paramenti sacri (paramento in terza, pianeta); e 24 oggetti archeologici (ara, cippo, coperchio di sarcofago, lapide, masso cuppellare, oggetti di scavo, sarcofago, urna) $)^{53}$.

Le caratteristiche di questo elenco denunciano perfettamente quanto doveva ancora essere fatto dall'aspetto teorico e pratico.

\footnotetext{
53 II citato elenco è stato stratto dal testo di Giulio Foletti, Capo del Servizio Inventario all’Ufficio dei Beni Culturali: "L’'nventario dei beni culturali" in Dipartimento delle finanze e dell'economia (a cura di) "Dati", n. 1, 2003, pp. 40-42.
} 


\section{LA TUTELA DEI BENI CULTURALI IN CANTON TICINO}

[...]E come vecchio uomo, il quale ha avuto modo di conoscere quali erano, sullo scorcio del secolo passato ed agli inizi del presente, le condizioni del nostro patrimonio storico e artistico, posso affermare che qualche cosa di buono e di importante si è fatto[... $]^{54}$.

[...]Y como un viejo hombre, que ha tenido la oportunidad de conocer cuales fueron, a finales del siglo pasado y a principios del presente, las condiciones de nuestro patrimonio histórico - artístico, puedo afirmar que alguna cosa buena e importante se ha hecho[...].

Il 13 ottobre del 1997 la Confederazione svizzera approva la "Legge sulla protezione dei beni culturali" del 13 maggio dello stesso anno, decretata dal Gran Consiglio della Repubblica e Cantone del Ticino. Conformata da 55 articoli in contrapposizione ai solo 26 che costituivano il decreto legislativo del 14 gennaio del 1909, essa venne a sostituire definitivamente la prima legge per la protezione, e regola la tutela attuale dei beni culturali nel Cantone italiano.

Secondo questa legge, come più avanti si approfondirà, il così chiamato Ufficio dei Beni Culturali venne sostenuto dalla Commissione dei beni culturali e realizza tre servizi: il Servizio monumenti, il Servizio archeologia ed il Servizio dell'inventario.

Il primo, il Servizio monumenti, è incaricato della sorveglianza del patrimonio storicoartistico del Cantone e le sue prestazioni sono quelle di controllare e autorizzare i progetti di restauro dei beni mobili e immobili tutelati dallo Stato, assieme alla Commissione dei beni culturali. Ha inoltre l'obbligo di fornire consulenza a ricercatori e committenti pubblici e privati e di commissionare perizie o analisi di indagini specialistiche.

II Servizio archeologia ha lo scopo di tutelare i resti di antiche rovine e le testimonianze scoperte negli scavi, conservati nel sottosuolo e promuove le ricerche per la loro documentazione.

Per ultimo, il Servizio dell'inventario, è stato creato con l'integrazione al suo interno dell'Opera svizzera dei monumenti d'arte (OSMA), creata nel 1966 da Virgilio Gilardoni, ed è incaricato della consulenza e della realizzazione dell'inventario dei beni culturali sparsi nel territorio del Canton Ticino.

La sede attuale dell'Ufficio dei Beni Culturali ${ }^{55}$ del Canton Ticino si trova a Bellinzona, condividendo l'edificio con la Biblioteca Cantonale e l'Archivio di Stato. Nello stesso complesso si trova inoltre il Centro della Dialettologia e di etnografia della Svizzera Italiana (CDSI).

L'UBC si trova oggi sotto la Sezione dello sviluppo territoriale della Divisione dello sviluppo territoriale e della mobilità del Dipartimento del Territorio ${ }^{56}$.

\footnotetext{
54 (DPE, 1959, p. 10).

${ }^{55}$ Lo storico Giuseppe Chiesi è dal 1995 capo ufficio dell’Ufficio dei Beni Culturali del Canton Ticino.

${ }^{56}$ Attualmente il Consigliere di Stato avv. Marco Borradori (Lugano, 6 giugno 1959) è direttore del Dipartimento territorio e responsabile della gestione, pianificazione e sviluppo del territorio cantonale.
} 
A designare la sue prestazioni è stata, come precedentemente citato, la Legge sulla protezione del 1997 che più avanti analizziamo, supponendo importanti novità all'organizzazione primitiva regolata da una legge che aveva ormai quasi novant'anni.

I primi articoli del Titolo I, riguardanti le Disposizioni generali (1-5) descrivono gli obiettivi della suddetta legge, le definizioni di bene culturale, di bene culturale protetto, delle istituzioni culturali riconosciute e della responsabilità degli enti di attuazione.

In questo punto è da considerare rilevante per capire la trasformazione avvenuta nel contesto svizzero la alterazione del lessico: i "monumenti" passano ad essere "beni", mobili o immobili, che si definiscono come essi poiché di interesse collettivo in quanto testimonianze dell'attività creativa dell'uomo; essi hanno un simbolo che non corrispondeva alla vecchia concezione e definizione dei monumenti come "cosa d'arte e di antichità". Di fatto questo si dimostra perché, l'oggetto tutelato non è più unicamente l'opera d'arte ma qualsiasi elemento anche intangibile se la collettività riconosce in questo un valore identificato. Questo mutamento ha fatto riordinare gli inventari degli organismi di tutela del Canton Ticino, nonché collaborare strettamente con altri enti (Musei regionali, Ufficio dei musei etnografici, la Protezione civile, comuni stessi nell'ambito dell'allestimento del Piano Regolatore, fondazioni, associazioni come ad esempio l'APAV per la protezione del patrimonio artistico e architettonico della Valmaggia o con singoli cittadini.

II Titolo II elenca le misure di promozione per la protezione dei beni, secondo le quali il Consiglio di Stato e il Municipio devono favorire l'avanzamento della conoscenza ed il rispetto dei beni culturali, l'informazione e la consulenza; il Consiglio di Stato deve partecipare finanziariamente ai costi di manutenzione regolare, di conservazione e di restauro dei beni culturali protetti dei beni identificati di interesse cantonale sempre che $i$ lavori progettati non possano essere supportati da altri mezzi finanziari. II Comune, in proporzione alle sue capacità economiche, parteciperà alle spese in misura di protezione, sempre che non rispondano altri enti locali, così come potrà farlo in casi eccezionali il Cantone, quando i contributi del proprietario e della collettività locale non siano sufficienti o venga compromessa la salvaguardia dell'opera.

II Titolo III si divide in Protezione preventiva dove si decretano, fra la misure di protezione, l'obbligo da parte della società ad informare gli enti responsabili sull'esistenza di un bene culturale degno di protezione che fino al momento non fosse stato scoperto; in questo modo se il ritrovamento avviene durante l'esecuzione di lavori nell'ambito dell'opera, essi dovranno essere sospesi temporaneamente fino ad ottenere nuove indicazioni. Sarà pure un diritto delle autorità competenti l'esame regolare dei beni culturali.

Sulle istituzioni ed effetti della protezione, si è voluto evidenziare l'Art. 24 che dichiara che gli interventi su beni protetti avranno bisogno dell'autorizzazione e consenso da parte del Consiglio di Stato, a meno che il proprietario del bene mobile sia una istituzione culturale riconosciuta così come il fatto che prima dell'elaborazione di un progetto d'intervento dettagliato il proprietario consulterà la Commissione dei beni culturali. Inoltre si elencano le procedure in caso di alienazioni o di cambiamenti di ubicazioni di beni mobili, situazioni 
che dovranno essere assolutamente comunicate al Consiglio di Stato. Più avanti il Titolo III della legge termina con il capitolo 3 sull'acquisto di beni culturali da parte di enti pubblici ed il diritto da parte del Comune o del Cantone di espropriare immobili protetti di eccezionale importanza culturale per la collettività; il capitolo 4 sulla protezione speciale dei beni archeologici, la cui responsabilità e competenza è unicamente del Cantone; i capitolo 5 sulla Protezione in caso di conflitto armato o di catastrofe.

II Titolo IV decreta le Disposizioni organizzative come l'inventario che deve essere allestito ed aggiornato dal Consiglio di Stato, facendo la distinzione fra i beni di interesse cantonale, locale e quelli che dovranno essere protetti in caso di conflitto armato o di catastrofe, accompagnati questi da una breve scheda informativa; Le Competenze e organizzazioni, stipulano il ruolo del Consiglio di Stato, incaricato della vigilanza sulla protezione dei beni culturali, regolando le collaborazioni fra i diversi servizi dipartimentali competenti, mentre la Commissione dei beni culturali sarà composta da un presidente, un vice-presidente e da cinque a nove membri nominati dal medesimo Consiglio di Stato.

II Titolo V decreta le attuazioni in caso di inosservanza della legge e finalmente, il Titolo VI le Norme transitorie e finali.

Se fino al 1997, i beni culturali iscritti nell'elenco cantonale dei monumenti storici ed artistici erano circa 1'525 (480 mobili, 1'045 immobili di cui 415 erano tutelati e 271 sotto protezione parziale), attualmente il numero si è visto incrementato fino all'incirca 100'000 oggetti. Questo si deve alle nuove considerazioni e diversità d'approccio verso il "bene culturale": il recente inventario aggiunge ai monumenti riconosciuti come tali dalla legge del 1909, l'architettura civile e religiosa antica, moderna e contemporanea, l'architettura rurale, l'architettura industriale, i nuclei fuori zona edificabile, le vie storiche, i manufatti stradali e ferroviari, i manufatti della civiltà rurale (ponti, strade, ecc.), i giardini storici, i beni mobili appartenenti a enti pubblici come archivi comunali e patriziali, i fondi librari parrocchiali, ecc. i quali non erano stati presi in considerazione fino al 1997 con la nuova "Legge di protezione". Fra questi oltre 100'000 beni, 475 sono insediamenti da proteggere, $25^{\prime} 000$ edifici, 5'000 beni immobili in edifici civili, 15'000 edifici rurali fuori zona edificabile, 259 chiesa parrocchiali, 646 oratori e cappelle, 35'000 beni mobili conservati in chiese, 10 '000 beni mobili legati a chiese, 1'500 manufatti architettonici e artistici, 435 opere di difesa militare, 2'000 vie storiche, 500 ponti, 1'000 giardini storici, 200 sagrati, 2'000 edicole votive, 1'000 beni mobili conservati in edifici civili privati e pubblici, 300 cimiteri, 3'000 monumenti cimiteriali, 1'000 zone archeologiche, 57 fondi bibliotecari, 608 fondi archivistici e 50 collezioni museali. Tutti questi, ad eccezione dei beni appartenenti alle collezioni museali pubbliche e ai fondi bibliotecari ed archivistici la cui catalogazione corrisponde ai musei o agli organismi archivistici, sono stati schedati e informatizzati nella banca dati sviluppata dal Servizio inventario dell'Ufficio dei Beni Culturali del Canton Ticino. 
Tavola dei componenti della Commissione Cantonale dei Monumenti Storici dal 1909 al 1959

\begin{tabular}{|c|c|c|c|c|c|c|c|c|}
\hline & $\begin{array}{l}\text { Professore } \\
\text { presidente }\end{array}$ & Segretario & Professore & Pittore & Ingegnere & Avvocato & Architetto & Mons. \\
\hline 1909- & Francesco & Luigi & & Edoardo & Emilio Motta & & & \\
\hline 1920 & Chiesa & Brentani & & Berta & & & & \\
\hline 1921- & Francesco & Edoardo & & Edoardo & & Angelo & & \\
\hline 1923 & Chiesa & Berta & & Berta & & Martignoni & & \\
\hline 1924- & Francesco & Edoardo & & Edoardo & & Angelo & Otto & \\
\hline 1927 & Chiesa & Berta & & Berta & & Martignoni & Maraini & \\
\hline 1928- & Francesco & Edoardo & & Edoardo & & Angelo & Otto & Giovanni \\
\hline 1930 & Chiesa & Berta & & Berta & & Martignoni & Maraini & Noseda \\
\hline 1931- & Francesco & Augusto Ugo & & Emilio & & Angelo & Otto & Giovanni \\
\hline 1937 & Chiesa & Tarabori & & Ferrazzini & & Martignoni & Maraini & Noseda \\
\hline 1938- & Francesco & Augusto Ugo & & Emilio & & Angelo & Otto & Davide Sesti \\
\hline 1939 & Chiesa & Tarabori & & Ferrazzini & & Martignoni & Maraini & \\
\hline 1940- & Francesco & \multicolumn{3}{|c|}{ Augusto Ugo Augusto Ugo Emilio } & & Angelo & Otto & Davide Sesti \\
\hline 1942 & Chiesa & Tarabori & $\begin{array}{l}\text { Tarabori/ } \\
\text { Piero } \\
\text { Bianconi }\end{array}$ & Ferrazzini & & Martignoni & Maraini & \\
\hline 1943 & Francesco & Augusto Ugo & Augusto Ugo & Emilio & & Angelo & & Davide Sesti \\
\hline & Chiesa & Tarabori & $\begin{array}{l}\text { Tarabori/ } \\
\text { Piero } \\
\text { Bianconi }\end{array}$ & Ferrazzini & & Martignoni & & \\
\hline 1944- & Francesco & \multicolumn{3}{|c|}{ Augusto Ugo Augusto Ugo Emilio } & & & Pietro & Alfonso \\
\hline 1945 & Chiesa & Tarabori & $\begin{array}{l}\text { Tarabori/ } \\
\text { Piero } \\
\text { Bianconi/ } \\
\text { Ugo Donati }\end{array}$ & Ferrazzini & & & Giovannini & Codadhengo \\
\hline 1946- & Francesco & \multicolumn{3}{|c|}{ Augusto Ugo Augusto Ugo Pietro Salati } & & & Pietro & Alfonso \\
\hline 1947 & Chiesa & Tarabori & $\begin{array}{l}\text { Tarabori/ } \\
\text { Piero } \\
\text { Bianconi/ } \\
\text { Ugo Donati }\end{array}$ & & & & Giovannini & Codadhengo \\
\hline
\end{tabular}




\begin{tabular}{|c|c|c|c|c|c|c|c|c|}
\hline & $\begin{array}{l}\text { Professore } \\
\text { presidente }\end{array}$ & Segretario & Professore & Pittore & Ingegnere & Avvocato & Architetto & Mons. \\
\hline $\begin{array}{l}1947- \\
1950\end{array}$ & $\begin{array}{l}\text { Francesco } \\
\text { Chiesa }\end{array}$ & $\begin{array}{l}\text { Augusto Ugc } \\
\text { Tarabori }\end{array}$ & $\begin{array}{l}\text { Augusto Ugo } \\
\text { Tarabori/ } \\
\text { Piero } \\
\text { Bianconi }\end{array}$ & $\begin{array}{l}\text { Pietro Salati/ } \\
\text { Emilio } \\
\text { Beretta }\end{array}$ & & & $\begin{array}{l}\text { Pietro } \\
\text { Giovannini }\end{array}$ & $\begin{array}{l}\text { Alfonso } \\
\text { Codadhengo }\end{array}$ \\
\hline $\begin{array}{l}1951- \\
1954\end{array}$ & $\begin{array}{l}\text { Francesco } \\
\text { Chiesa }\end{array}$ & $\begin{array}{l}\text { Augusto Ugc } \\
\text { Tarabori }\end{array}$ & $\begin{array}{l}\text { Augusto Ugo } \\
\text { Tarabori/ } \\
\text { Piero } \\
\text { Bianconi }\end{array}$ & $\begin{array}{l}\text { Pietro Salati/ } \\
\text { Emilio } \\
\text { Beretta }\end{array}$ & & & $\begin{array}{l}\text { Pietro } \\
\text { Giovannini/ } \\
\text { Alberto } \\
\text { Camenzind }\end{array}$ & \\
\hline $\begin{array}{l}1955- \\
1956\end{array}$ & $\begin{array}{l}\text { Francesco } \\
\text { Chiesa }\end{array}$ & $\begin{array}{l}\text { Carlo } \\
\text { Speziali }\end{array}$ & $\begin{array}{l}\text { Augusto Ugo } \\
\text { Tarabori/ } \\
\text { Piero } \\
\text { Bianconi }\end{array}$ & Pietro Salati & & & $\begin{array}{l}\text { Pietro } \\
\text { Giovannini/ } \\
\text { Alberto } \\
\text { Camenzind }\end{array}$ & $\begin{array}{l}\text { Agostino } \\
\text { Robertini }\end{array}$ \\
\hline $\begin{array}{l}1956- \\
1957\end{array}$ & $\begin{array}{l}\text { Francesco } \\
\text { Chiesa }\end{array}$ & $\begin{array}{l}\text { Carlo } \\
\text { Speziali }\end{array}$ & $\begin{array}{l}\text { Augusto Ugo } \\
\text { Tarabori/ } \\
\text { Piero } \\
\text { Bianconi/ } \\
\text { Giuseppe } \\
\text { Martinola }\end{array}$ & Pietro Salati & & & $\begin{array}{l}\text { Alberto } \\
\text { Camenzind }\end{array}$ & $\begin{array}{l}\text { Agostino } \\
\text { Robertini }\end{array}$ \\
\hline $\begin{array}{l}1957- \\
1959\end{array}$ & $\begin{array}{l}\text { Francesco } \\
\text { Chiesa }\end{array}$ & $\begin{array}{l}\text { Carlo } \\
\text { Speziali }\end{array}$ & $\begin{array}{l}\text { Augusto Ugo } \\
\text { Tarabori/ } \\
\text { Piero } \\
\text { Bianconi/ } \\
\text { Giuseppe } \\
\text { Martinola }\end{array}$ & Pietro Salati & $\begin{array}{l}\text { Luigi } \\
\text { Brentani }\end{array}$ & & & $\begin{array}{l}\text { Agostino } \\
\text { Robertini }\end{array}$ \\
\hline
\end{tabular}




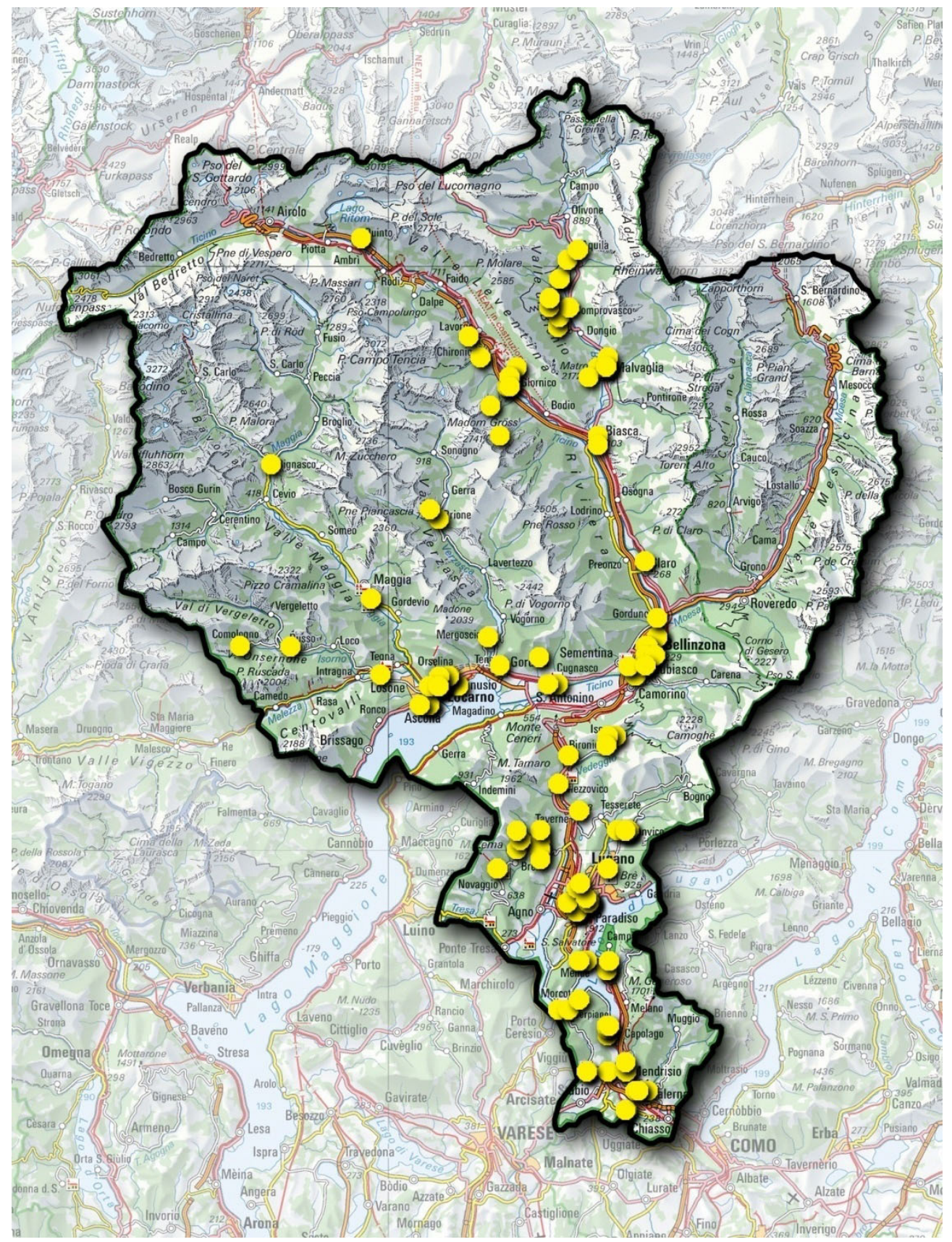

Figura 3 Cartina degli interventi di restauro murale esegiti fra il periodo 1909 - 1959 in Canton Ticino. 


PARTE SECONDA Biografie e interventi 
Show 2. Fosta 
\$सेदि

(4)
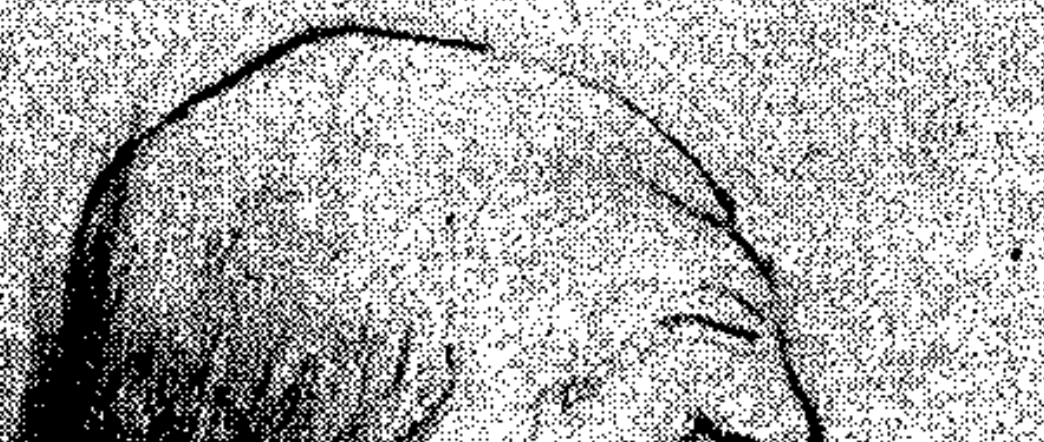

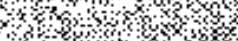

.

(x)

$x^{3}+3^{3}$

(3)

ses.

8े

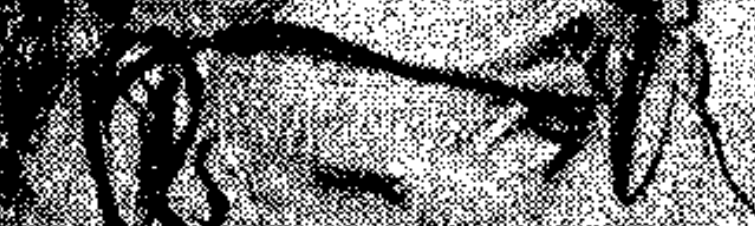

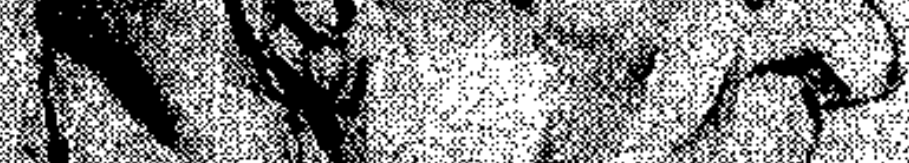

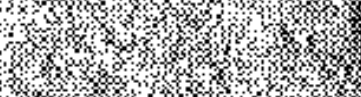

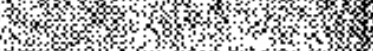

४ै

४४

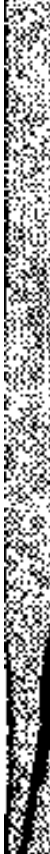

$8 \%$

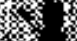

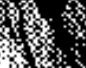

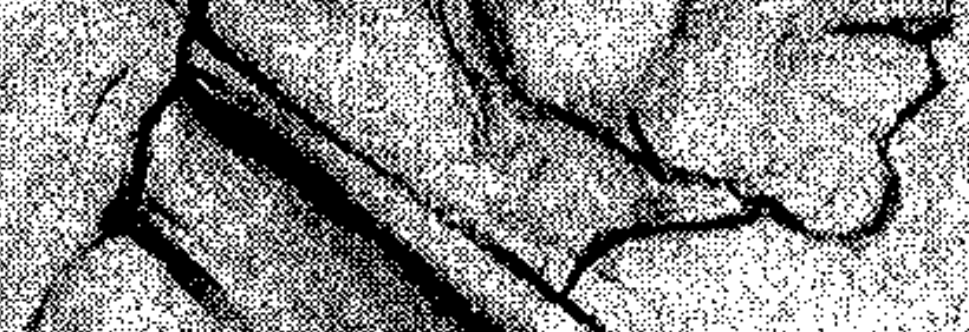

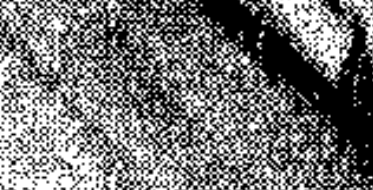

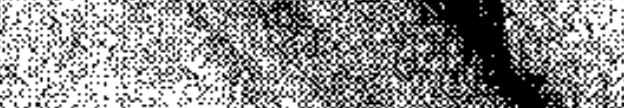

४

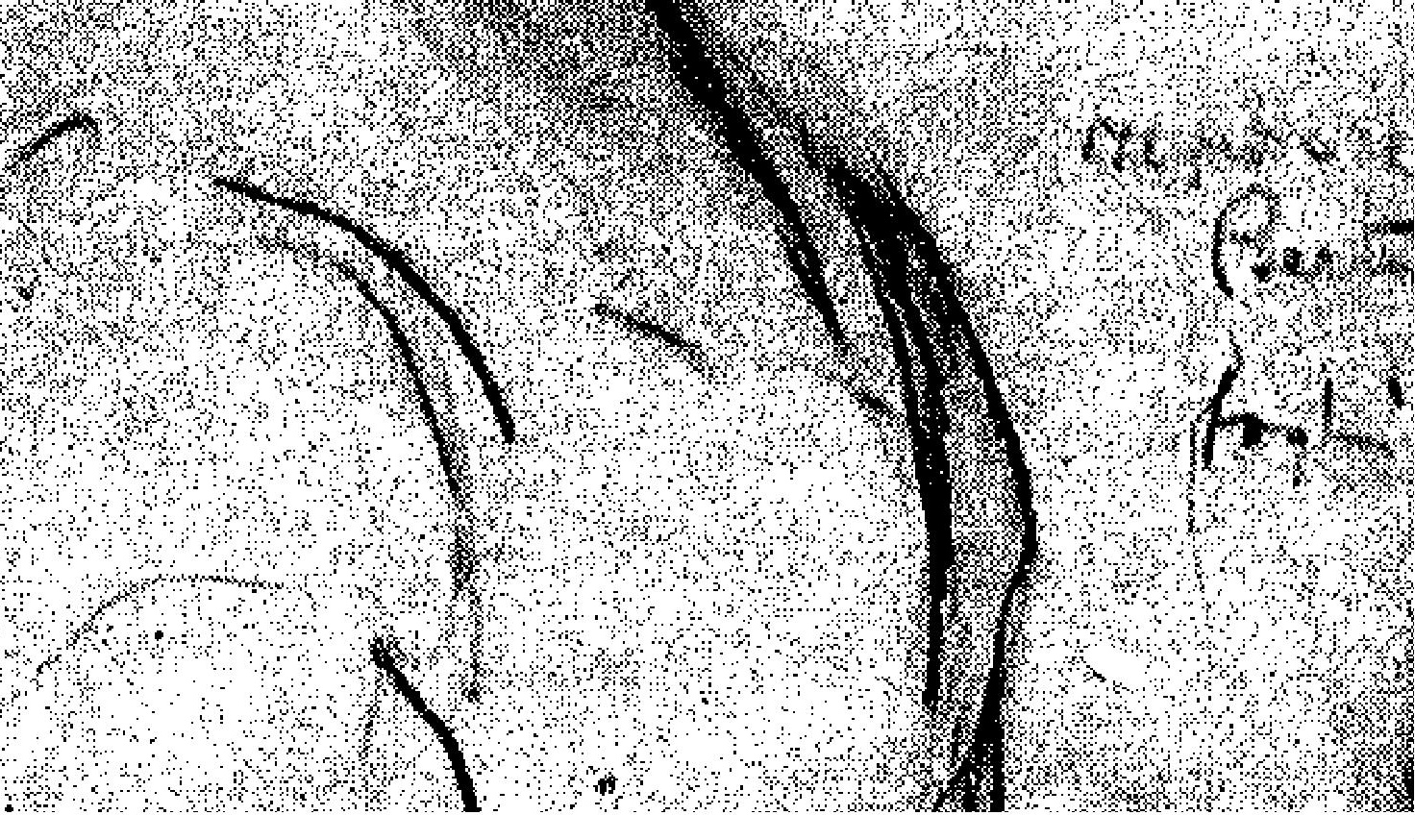




\section{EDOARDO BERTA}

[...]Edoardo Berta, il pittore insigne che per l'amore del suo Ticino si fece archeologo profondo e ricostruttore genialissimo, scrittore d'arte eminente onde tutto ciò che nel Canton Ticino era testimonianza d'arte e di storia patria si salvasse ad ogni costo dai travolgimenti e dalle rovine minacciate delle invadenze d'oltralpe. $[. . .]^{57}$.

[...]Magnifico pintor que por amor al Ticino se convirtió en un profundo arqueólogo y genialísimo reconstructor, escritor de arte eminente por que todo lo que fuese testimonio de arte y de historia patria en el Canton Ticino se salvase de cualquier manera de los cambios y las ruinas amenazadas por las invasiones más allá de los alpes.[...].

Pagina precedente:

Figura 4 Foglia Giuseppe ${ }^{58}$, Edoardo Berta. Disegno a mattita su carta.

\footnotetext{
${ }^{57}$ (Ventarini, 1932)

${ }^{58}$ Foglia Giuseppe (Lugano 1888-1950). Disegnatore, pittore, scultore e giornalista di corrente realista espressionista. Studiò nella scuola d'Arte e mestieri di Lugano, a Roma, a Milano ed a Parigi, rientrando in Ticino in 1914 con l'esplosione della Prima Guerra Mondiale. Fu amico di Edoardo Berta a chi realizza questo ritratto insieme a tanti altri di scrittori ed artisti ticinesi che saranno pubblicati sul Corriere del Ticino. Oggi il Museo di Villa dei Cedri di Bellinzona ha raggruppato un importante fondo con numerose opere, documenti e carte d'archivio. Più informazioni presso la pagina web on-line: http://www.sikart.ch sul sito "Artisti" (Istituto svizzero di studi d'arte (ISSA))
} 


\section{Dati biografici}

Sarà difficile descrivere un ruolo tanto complesso come quello che personificò Edoardo Berta all'interno del panorama artistico e culturale del Canton Ticino durante il primo decennio del XIX sec. ed $\mathrm{i}$ due primi decenni del $X X$ sec., prima della sua morte il 23 giugno 1931. Si è voluto approfondire nella sua figura dalla conoscenza completa in tutti i suoi campi di sviluppo professionali, per arrivare alla formazione di una concreta concezione delle sue caratteristiche come restauratore.

Edoardo Berta, figlio di Battista Berta e Francesca Regli nasce a Giubiasco, paese d'origine del padre situato ad appena tre chilometri della città di Bellinzona, il 29 marzo 1867. Una famiglia semplice, di poche risorse, che però non impedirà che il Berta ancora ragazzo si trasferisca dal 1881 al 1887 al Palazzo di Brera a Milano ${ }^{59}$, sede dell'Accademia di Belle Arti, dopo aver concluso gli studi obbligatori contempora-

\footnotetext{
${ }^{59}$ L'Accademia di belle arti di Brera di Milano è la tradizionale scuola in questa competenza dal 1776. II Palazzo dove ha sede l'Accademia sorge su un antico convento degli Umiliati che passò in mani dei Gesuit nel 1572, fino alla soppressione della Compagnia in 1772. Nel 1774, ai già presenti Osservatorio Astronomico e la Biblioteca dei frati Gesuiti $s$ aggiunge I'Orto Botanico e finalmente, come prima indicato, I'Accademia di Belle Arti. Informazione presso il sito (Brera). L'intenso rapporto esistente fra gli artisti ticinesi e l'Accademia di Belle Arti di Brera concluderà negli anni novanta con la collaborazione tra il pittore Giuseppe Mentessi assistente all'Accademia ed il pittore Pietro Chiesa, fratello d Francesco Chiesa, che diventeranno amici.
}

neamente alla frequenza della Scuola serale di disegno di Bellinzona ${ }^{60}$.

Sarà uno dei numerosi pittori obbligati ad emigrare nella vicina Lombardia in cerca di un apprendimento artistico assente in forma istituzionale in patria ${ }^{61}$.

Una lettera ${ }^{62}$ al Gran Consiglio di Bellinzona emessa il 19 aprile 1887 da trentaquattro

\footnotetext{
${ }^{60}$ Questa stessa scuola fu eseguita da pittori come Augusto Sartori (dal 1893 al 1894) prima di frequentare l'Accademia di Brera di Milano.

${ }^{61}$ Per approfondire nell'argomento sulle scuole di disegno in Ticino, e sull'ambizione per la creazione di una Accademia di Belle Arti nel proprio territorio sono fondamentali i capitoli "La forza dell'ideologia: I'utopia dell'Accademia di Belle Arti nel Ticino" e "Le scuole di disegno" presenti nella pubblicazione "Arte nel Ottocento" di Giulio Foletti (Foletti, 2002, p. 4151)
}

${ }^{62}$ (AA.VV. Lettera Circa l'istituzione d'una scuola per le Belle Arti nel nostro Cantone. Milano 19 aprile 1887. ASTi, Fondo DPE- Diversi 1905 - 1955, Segnatura 1.1.4.2.12.)

Firmano la lettera gli artisti: Bernasconi Ferdinando, Carona (studente architettura); Bianchi Paolo, Vacallo (studente scultura); Boschetti Pietro, Fescoggia (studente ingegnere); Bruni Vincenzo, Olivone (studente architettura); Chiaverio Emilio, Mendrisio (studente architettura); Chiesa Bernardo, Chiasso (studente architettura), Donati Domenico, Astano (studente architettura); Ferroni Bernardo, Gravesano (studente Maestro di Disegno); Fontana Gottardo, Cabbio (studente architettura); Galetti Adolfo, Lugano (studente architettura); Garlani Ercole, Russo, Vallemaggia (studente scultura); Ghirlanda Pietro, Dino (studente architettura); Gianini Giuseppe, Lugano (studente scultura); Gobbi Achille, Stabio (studente architettura); Guggeri Luigi, Vacallo (studente architettura); Lepori Francesco, Origlio (studente architettura); Maggiori Enrico, Aurigeno (studente pittura); Mercoli Giacomo, Mugena (studente architettura); Pellandini Augusto, Arbedo (studente pittura); Quadri Ernesto, Lugaggia (studente architettura); Regazzoni Ammellio, Chiasso (studente scultura); Soldati Achille, Lugano (studente architettura); Vassalli Luigi, Lugano (studente scultura); Zanetti Enrico, Sessa (studente scultura); Beretta Modesto, Lugano (studente scultura); Ceppi Dante, Mendrisio (studente?); Lubini Pietro, Lugano 
artisti ticinesi residenti a Milano esprime chiaramente la loro situazione. Fra questi firmava anche Edoardo Berta "di Giubiasco, studente pittore":

[...]l sottoscritti, studenti ticinesi, nell'Accademia di Belle Arti in Milano, interpreti del desiderio e dei voti di tutti i loro compagni e patrioti, espongono il vivissimo desiderio e fanno voti al sullodato Consiglio, a volersi interessare, ed appoggiare il progetto da tempo ideato e di cui ora più che mai si sente il desiderio anzi il bisogno d'effettuare. Un'Accademia di Belle Arti nel nostro amato Ticino, è l'ideale di tutti gli artisti suoi figli, un tempio d'arte che c'indirizzi nella nostra carriera è ciò che varrà anche a levarci dalle critiche condizioni in cui ci troviamo noi studenti presso un'accademia d'altra nazione. $\grave{E}$ umiliante per noi ticinesi, che vantiamo delle grandi celebrità nel campo dell'arte, e che possiamo avere insigni maestri, ed iniziatori d'una grande avvenire, è umiliante d'avvero l'esser costretti a limosinare in altri paesi un'educazione che il nostro patrio Ticino non sa offrirci. Di più scoraggiante è la nostra posizione presso un'Accademia estera quali diritti possiamo non avere? Certamente non possiamo partecipare degli stessi privilegi di cui godono i nazionali! Ecco perché tante volte noi siamo esclusi dai concorsi, siamo privati d'incoraggiamenti che molta parte possono avere sul nostro avvenire. Aggiungasi che coi nuovi regolamenti lo straniero non può aspirare alla patente di Maestro da disegno presso le accademie italiane per cui non ha il diritto ad un attestato che gli possa valere e che confermi i suoi studi. Questo nostro Lodevole Consiglio s'interessi a dunque dei suoi giovani artisti! Si organizzi nel nostro Ticino un'Accademia Federale di Belle Arti, e si vedrà come noi tutti

(studente architettura); Berta Edoardo, Giubiasco (studente pittura); Maggi Antonio, Mendrisio (studente ingegnere); Bonalina Luigi, Bellinzona (studente pittura); Botta Rodolfo, Genestrerio (studente scultura); Quadri Giovanni, Lugaggia (studente architettura). chiamati alla carriere dell'arte, concorreremo con ardore, con entusiasmo per farla fiorire, si vedrà come sapremo tener alta la bandiera dell'arte nella nostra patria.[... $]^{63}$.

Insieme a questa lettera, lo stesso Consigliere nazionale Johann Friedrich Riniker (1841 - 1892) allegava una sua propria denuncia sulla necessità di attualizzare le scuole di disegno nate nel 1840, secondo le idee dello scultore Vincenzo Vela e del Consigliere di Stato Giorgio Casella, ${ }^{64}$ direttore del Dipartimento della Pubblica Educazione del Canton Ticino. Si intendevano creare nella regione delle scuole secondarie superiori di belle arti in modo da evitare l'uscita per un minimo di sei anni degli artisti ticinesi all'estero, soggiornando in paesi come la vicina Italia o la Germania, principalmente, dove l'offerta educativa in questo specifico campo era maggiore.

Come si illustrava precedentemente, Edoardo Berta fu effettivamente uno dei pittori costretto a espatriare per formarsi nella sua grande vocazione, la pittura, ma una volta ristabilito in Ticino dopo il 1902, sarà lui stesso ad occuparsi delle riforme educative che si proponevano ai giovani artisti, allo stesso tempo che in contro posizione contemplerà e sarà vittima della chiusura delle frontiere con il resto $d^{\prime}$

\footnotetext{
(AA.VV. Lettera Circa l'istituzione d'una scuola per le Belle Arti nel nostro Cantone. Milano 19 aprile 1887. ASTi, Fondo DPE- Diversi 1905 - 1955, Segnatura 1.1.4.2.12.)

${ }^{64}$ Casella Giorgio (1847-1929). Medico e Consigliere di Stato per quattro legislature consecutive dal 1893 al 1909, presidente del Gran Consiglio insieme al fratello Giovanni Casella ed a Arnaldo Bolla. Fu il fondatore in 1917 della Sezione luganese della Croce Rossa.
} 
Europa quando nel 1914 scoppiò la prima guerra mondiale.

A Brera le doti di pittore di Edoardo Berta furono presto riconosciute dal maestro Giuseppe Bertini ${ }^{65}$, considerato il "paterfamilias"66 dell'Accademia. Egli animerà il giovane artista ticinese a recarsi a Milano, però, malgrado il suo evidente talento e non poco discreto successo artistico, più tardi il Berta abbandonerà i pennelli per amore a "la terra", alla sua terra, ai paesaggi che rappresenterà numerosi nei sui quadri, ai monumenti caduti in abbandono e, con questo, al recupero ed alla rivalorizzazione di una identità, di una memoria locale: quella della gente delle sue strade, quella dei sui paesi, quella che diventa innocenza nei visi dei bambini che ritrae, che è bontà ed estetica, e che guarda al futuro dall'ammirazione del passato, ricreandolo.

[...] Compresi che il meglio che potevo fare era di cercare me stesso, attraverso le impressioni ed emozioni vive che provo davanti a certi angoli remoti della natura del mio paese, che mi fermano, mi incatenano e mi parlano un linguaggio amico, pieno di carezzevole intimità $[\ldots]^{67}$.

Fu questa la più grande opera di restauro che Berta fece in diffusione del patrimonio tradizionale, della bellezza e della armonia

\footnotetext{
${ }^{65}$ Bertini Giuseppe (Milano 1825-1898) Professore dell'Accademia di Brera di Milano da 1860, si deve a lui la massiva presenza di artista ticinesi che arrivano all'Accademia chiamati del moderno e rivoluzionario insegnamento del maestro italiano, che si caratterizzava per prodigare il confronto nella pittura con il vero, nel paesaggio, nella pittura di genere, dando luogo ad una eterogeneità di stile che verrà poi chiaramente rappresentata da i suoi allievi.

${ }^{66}$ (Foletti, 2002, p. 144).I

${ }^{67}$ (Matteo Bianchi, 2000. p.23).
}

come esempio per una nuova generazione; come cita lo storico Giulio Foletti, di un [...] paese senza nerbo che gradualmente stava diventando adulto [...]; anche se come restauratore non avessi le conoscenze o fosse [...]troppo artista per essere un bravo restauratore $[. . .]^{68}$. Non si trattava di metodologia ma degli ideali che la muovevano.

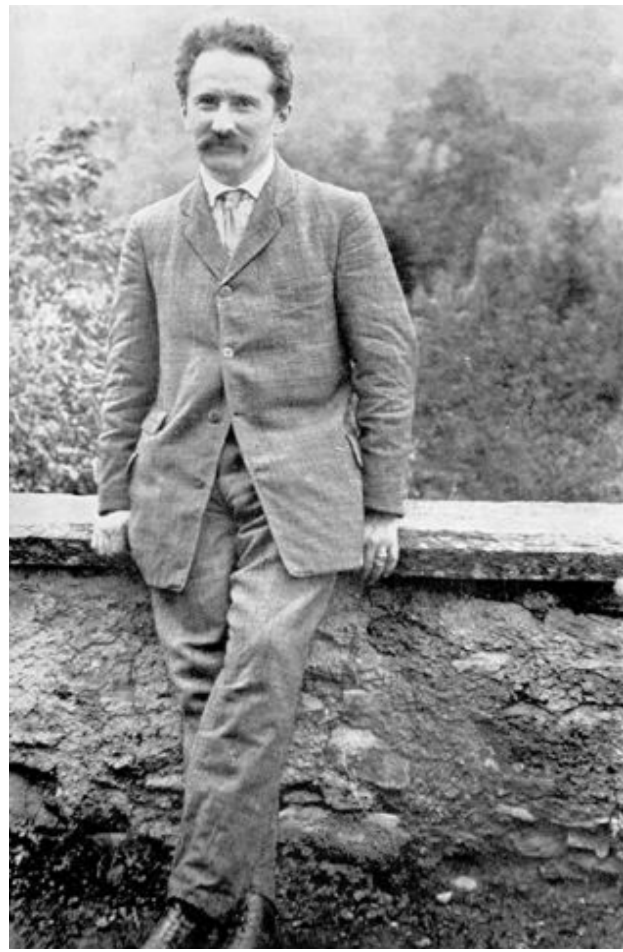

Figura 5 Francesco Chiesa. Fotografia pubblicata in Del Bove Milko, II Ticino delle lettere e nelle lettere, 14 dicembre 2006; [in linea].

Francesco Chiesa, così come gli altri biografi che ricordano il pittore, lo fanno descrivendoci una personalità ribelle, appassionata, influenzata dalla sensualità,

\footnotetext{
${ }^{68}$ (Foletti, 2002, p. 141-148).
} 
dalla semplicità e da una estrema sensibilità, con intenso rifiuto per tutto ciò che si manifestasse disonesto e falso.

[...] Poiché per lui ciò che contava nella vita, come nell'arte, era la coscienza e la sincerità; non ho mai conosciuto un uomo di studio così esclusivamente dominato dal compito a cui attendeva. Si alzava di notte per andare a riscontrare un particolare, a tentare colla mano uno scrostamento, a provare l'effetto d'una variante. Anche lontano dal lavoro, vi pensava continuamente [...]Quella sapienza ch'egli aveva assottigliato di giorno in giorno, di anno in anno: quell'intuito e quel gusto che, dai lavori d'arte, egli aveva trasferito nei lavori di restauro $[\ldots]^{69}$.

Amico di Giuseppe Pellizza da Volpedo ${ }^{70}$, Berta frequentò dopo Brera ${ }^{71}$ I'Accademia $^{\prime}$ di Carrara a Bergamo ${ }^{72}$, dove si conoscono

\footnotetext{
${ }^{69}$ (Chiesa, Edoardo Berta conmemorato per Francesco Chiesa, 1931)

70 Pellizza da Volpedo Giuseppe (Volpedo, 18681907) rappresentante del divisionismo italiano e della pittura socialmente compromessa. Studiò in diverse accademie come Brera a Milano dal 1884 al 1887, all'Accademia di San Luca ed all'Accademia di Francia in Roma nel 1887, all'Accademia di Belle Arti di Firenze nel 1888, all'Accademia di Carrara come allievo di Cesare Tallone dal 1888 al 1889. Dopo gli studi decise di ritornare alla sua terra natale $d$ Volpedo dove si sposa e comincerà a lavorare per le prime mostre, trovando la maturità artistica proprio con I'inizio del XX sec. In 1900 espose a Parigi il quadro Lo specchio della vita, opera fondamentale per il simbolismo, ed un anno più tarde termina // Quarto Stato che espose in 1902 alla Quadriennale Torinese. La morte in 1907 lo emergerà in una profonda depressione, togliendosi la vita il 14 giugno dello stesso anno.

${ }^{71}$ Insieme a E. Berta sono da segnalare i compatrioti Filippo Franzoni, Giuseppe Chiattone e Luigi Vassalli, amici e colleghi che studieranno con il pittore alla scuola di Brera. (Foletti, 2002, p. 142)

${ }^{72}$ Fondata in 1796, l'Accademia de Carrara de Bergamo si trova fra la più antiche d'Italia. È finanziata dal municipio di Bergamo e legalmente
}

ed imparano dal Maestro Cesare Tallone ${ }^{73}$, che insegnò agli allievi a creare con uno stile più libero di quello che fino al momento potevano imparare a Brera dal professor Bertini.

L'artista rappresentò la corrente divisionista come lo fecero in Italia Giovanni Segantini o il medesimo Pellizza da Volpedo. Insieme a quest'ultimo pittore ed altri colleghi della scuola bergamasca, Berta viaggiò a Parigi in occasione dell'Esposizione Universale del 1889, ma la relazione fra i due pittori sarà ancora, per tanti anni dopo le lezioni all'Accademia, molto legata e intima, con una forte costanza nello scambio di lettere che vanno dal 1887 al $1903^{74}$ e dove si rivela altrettanto importante il rapporto esistente fra le loro famiglie. Fortunatamente la vita del pittore ticinese seguirà una strada meno tragica di quella del magnifico artista italiano.

Edoardo Berta è stato non per casualità il primo personaggio ad uscire alla luce fra $i$ numerosi cartacei e libri consultati per questa ricerca, poiché la sua passione e dedicazione, precedentemente descritte, lo portano ad occupare i campi dell'arte come

riconosciuta dal 17 maggio del 1988. Informazione preso: Burzi Francesco, Accademia Carrara di Belle Arti Bergamo, sito "Storia" pubblicato il 21 settembre 2005,

http://www.accademiabellearti.bg.it/web/modules.p hp? name=Content\&pa=showpage\&pid=1. [Consulta 25 novembre 2007].

3 Tallone Cesare (Savona, 1853 - Milano, 1919). Pittore caratterizzato dall'impressionismo plastico, studia nella scuola dello stesso Giuseppe Bertini nella Accademia di Brera a Milano dove più tardi, dopo l'esperienza a Carrara, esercita anche lui la professione di docente nel momento della avanguardie.

${ }^{74}$ (Berta, 2001) 
pittore, restauratore, archeologo ma, sopratutto, come uomo di cultura massimo promotore di codesta.

Nell'ambito pittorico affrontato da tutti i suoi ricercatori, fu coetaneo ad artisti ticinesi come ad esempio Luigi Rossi ${ }^{75}$, Adolfo Feragutti Visconti ${ }^{76}$, Filippo Franzoni $^{77}$, Pietro Anastasio ${ }^{78}$, Antonio Barzaghi Cattaneo ${ }^{79}$, Luigi Vassalli ${ }^{80}$, Antonio $^{81}$ e Giuseppe Chiattone ${ }^{82}$, Luigi Monteverde $^{83}$ e Gioacchino Galbusera ${ }^{84}$. Ebbe il massimo successo nel 1901-1902 quando venne premiato per il progetto di decorazione del Museo nazionale Svizzero a Zurigo, e si dedicherà in modo regolare la realizzazione di mostre fra Italia e Svizzera con opere caratterizzate dallo stile

\footnotetext{
Rossi Luigi (Lugano, 1853 - Tesserete, 1923). Professore, pittore e illustratore, si formò nell'Accademia di Brera con il professore Giuseppe Bertini, sviluppando più tardi la sua professione professionale in Parigi dove s'esercita nell'illustrazione. Come Edoardo Berta la sua coscienza sociale verrà espressa nei suoi quadri ed in diverse attività didattiche di cui oggi sono testimonianza i due rapporti del 1893 e del 1897 realizzati insieme ad Augusto Guidini, per i riordinamento delle scuole di disegno ticinese, sulla base delle teorie di John Ruskin e Morris dell'unificazione delle arti. L'informazione è stata stratta dal sito internet: http://adhikara.com/capriasca/luigi_rossi.htm.

${ }^{76}$ Ferragutti Visconti Giuseppe Adolfo (Pura, 1850 Milano, 1924)

${ }^{77}$ Franzoni Filippo (Locarno, 1857 - Mendrisio, 1911). Fu pittore principalmente di paesaggi, ritratti e di soggeti mitolgici oltreché fotografo e musicista.

${ }^{78}$ Anastasio Pietro (Lugano, 1859 - Morcote, 1913)

${ }^{79}$ Barzaghi Cattaneo Antonio (Lugano, 1834 - 1922)

${ }^{80}$ Vassalli Luigi (Lugano, 1867 - 1933)

${ }^{81}$ Chiattone Antonio (Lugano, 1856 - 1904)

${ }^{82}$ Chiattone Giuseppe (Lugano, 1863 - 1954)

83 Monteverde Luigi, detto il Raffaello dell'uva. (Lugano, 1841 - 1923)

${ }^{84}$ Galbusera Gioacchino (Milano, 1871 - Lugano, 1944)
}

divisionista nato ufficialmente in Italia nel 1890 quando i primi quadri di questa corrente vengono esposti alla mostra Triennale di Brera. Le opere si caratterizzavano per accentuare l'oggetto della natura da un approccio intimista, simbolico e sincero.

Come studioso, invece, il primo contributo di Edoardo Berta alla storia dell'arte avviene nel 1903 con l'organizzazione dell'Esposizione di arte sacra a Bellinzona, che risulta un primo inventario dei tesori d'arte del Ticino raccolti fra i percorsi di chiese e cappelle. Più tardi, nel 1905, Berta viaggia a Venezia in qualità di delegato del Dipartimento della pubblica Educazione, per partecipare al Congresso artistico internazionale $^{85}$ che lo motiverà a realizzare la riforma delle Scuole di disegno ticinese, sulle basi teoriche apprese, insieme a Giuseppe Mentessi ${ }^{86}$. II progetto

\footnotetext{
85 (Berta, Relazione sul Congresso artistico internazionale : tenuto in Venezia nel settembre 1905, 1906). Nel simposio internazionale della Biennale di Venezia dei 9-12 dicembre 2005, il discorso del Presidente, David Croft, faceva riferimento agli origini di questa mostra, ricordando cent'anni prima quando un convegno internazionale d'artisti, al che partecipava Edoardo Berta, insieme al presidente della biennale allora anche sindaco della città di Venezia, Filippo Grimani, ed al segretario della Biennale Antonio Fradeletto, discutono sullo stato e le prospettive dell'arte contemporaneo. Si fecce nel congresso un particolare omaggio alla persona del pittore, poeta e critico d'arte inglese John Ruskin (1819 - 1900), precursore dell'Art Nouveaux, e gran influenzatore nelle teorie del restauro architettonico romantico, di negazione d'intervento sui monumenti o sulle opere decorative lasciate al deperimento del tempo, in confronto alle teorie dell'architetto francese Viollet-le-Duc (1814-1879) chi promoveva il restauro assoluto dell'opera ad uno stato forse mai esistito. (Venezia, 2006)

${ }^{86}$ Mentessi Giuseppe (Ferrara, 1857 - Milano 1931), aiutante dall'assistente Luca Beltrami e poi professore
} 
venne ultimato nel 1907, dopodiché realizza il riordino del Museo storico di Lugano di cui fu membro dalla sua fondazione nel 1903 traslocandolo agli spazi di Villa Ciani, nella stessa città.

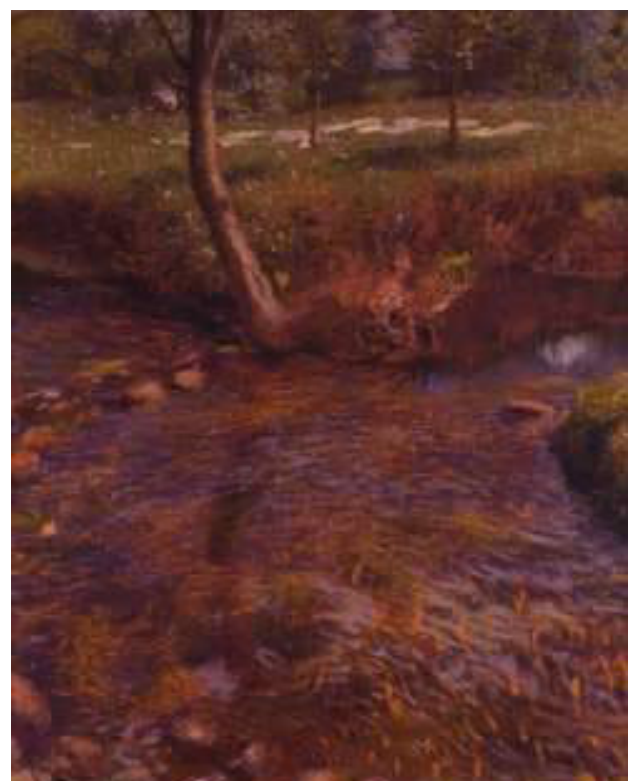

Figura 6 Berta Edoardo, Ruscello. Olio su tela. Museo Villa dei Cedri, Bellinzona.

Dall'anno 1909 Berta formerà parte della prima Commissione Cantonale per la conservazione dei monumenti storicoartistici, chiamato a partecipare dal presidente, il professore Francesco Chiesa $^{87}$ e dallo storico Emilio Motta.

di prospettiva alla Accademia di Belle arti di Brera dal 1880. Partecipa alle mostre triennali di Brera, compartendo gli ideali sociali di coetanei come Pellizza, Berta, e Filippo Turati.

${ }^{87}$ Chiesa Francesco (Sagno, 1871 - Lugano 1973), professore, pittore ed scrittore. Figlio primogenito di Innocente Chiesa, pittore-decoratore e di Maddalena Bagutti, nipote di Giovanni Battista Bagutti di Rovio (1742 - 1823). Questo ultimo, pittore di tele e di
La sua attività come membro della suddetta commissione sarà condivisa con quella di docente di pittura decorativa a Lugano, per poi diventare ispettore per l'insegnamento del disegno nelle Scuole medie.

All'interno della Commissione Cantonale dei Monumenti Storici elabora il primo inventario di opere d'arte del Canton Ticino appoggiato sugli appunti e disegni archeologici dello storico Johann Rudolf

affreschi di un gran numero di edifici di importanza nella città così come di famiglie prestigiose della località. Studiò nell'Accademia di Belle Arti di Parma, lo stile di pittura neoclassica che più tardi verrà influenzata dalla barocca. Fra le sue opere più rilevanti, altari, dipinti murale, trasparenti ed ex-voto, i principali in Ticino nel Museo d'Arte di Mendrisio, nella chiesa di san Giovanni, nella stessa località, la chiesa di san Sisinio), in Lugano (Museo cantonale d'arte), e nelle chiese parrocchiali di Rancate, Sala Capriasca e Riva San Vitale. Mentre il fratello di Francesco Chiesa, Pietro, continua la carriera del nonno e del padre come ritrattista e pittore, Francesco studia linguistica ed ancora in fasi di studio vive il primo successo con l'opera titolata Brindisi, poche settimane dopo pubblicata sulla Gazzetta Ticinese del 25 maggio. Otterrà la laurea in giurisprudenza ed insieme all'avvocato Emilio Bossi (Milesba) fonda un nuovo giornale l'idea moderna che elabora una serie di obbiettivi di coscienza sociale nel sistema legale. Nel primo numero pubblicato, fra gli obiettivo si cita(Lugano, 10 gennaio 1895, p.1), coltivare il sentimento artistico, cosi vivo, spontaneo e poco apprezzato nel nostro paese. In 1905 riceve l'incarico di direttore della Biblioteca cantonale di Lugano e cinque anni dopo la presidenza nella Commissione Cantonale dei Monumenti storici in Canton Ticino, carico che condividere da 1940 a 1961 con quello di presidente della Commissione per la protezione delle bellezze naturali e del paesaggio. Sarà uno dei massimi diffusori della cultura italiana nel Cantone, dirigendo la Scuola Ticinese di Cultura italiana fondata in 1917. Sarà premiato per il suo contributo alla cultura ticinese tanto in Italia come in Svizzera, nominato doctor honoris causa da tre università, Losanna, 1927; Roma, 1928 e Pavia, 1967. (Mazzarello, 2006) 
Rahn $^{88}$ e con la collaborazione degli altri membri storici. Dirigerà una importante pubblicazione di fascicoli dedicati ai "Monumenti storici ed artistici del Canton Ticino" ${ }^{\prime 89}$, in collaborazione con la famosa editoriale Hoepli ${ }^{90}$, che si inaugura con il primo volume del 1911 sulle chiese romaniche delle Leventina. Per servire la didattica scolastica il Berta inserì altri volumi, pubblicati sempre dalla editoriale milanese, in cui si potessero riprodurre modelli artistici basati nelle tradizioni locali sui lavori in pietra ${ }^{91}$, in legno ${ }^{92}$ e in ferro battuto $^{93}$, così come altri più generici sulle "Case tipiche ticinesi" ${ }^{\prime \prime 4}$, sul "Cenacolo di

\footnotetext{
${ }^{88}$ Rahn Johann Rudolf (Zurigo, 1841 - 1912)

${ }^{89}$ (Berta, Monumenti storici ed artistici del Ticino, 1912)

90 Hoepli Ulrico (Tuttwil, 1847 - Milano, 1935). Comincia la sua attività come editore in 1871 insieme a quella di libraio, con la pubblicazione di I prim elementi di lingua francese di G.S. Martin. Vivrà e lavorerà in Italia pubblicando collezioni di gran successo come l'importante Storia dell'arte Italiana. En Svizzera crea la "Fondazione Ulrico Hoepli" d'appoggio alle istituzioni e iniziative pubbliche o di promozione delle scienze e dell'arte in Svizzera insieme al pittore E. Berta, con chi editerà la collezione Monumenti Storici ed Artistici del Cantone Ticino. Sull'editore hanno scritto, fra altri: (Joseph Jung, 1997) (Decleva, 2001).

${ }^{91}$ I diversi volumi dedicati al lavoro in pietra sono divisi in due volumi, il primo (Janner, La pietra : camin e caminiere gotiche, del rinascimento e barocche, 1912) analizzava la produzione delle caminiere mentre il secondo (Berta, La pietra : sculture diverse, bassorilievi rappresentativi, 1914) lo faceva con le sculture.

92 II primo volume dedicato al lavoro in legno fu realizzato dal professor Arminio Janner a cura di Edoardo Berta, analizzando la produzione dei soffito (Janner, II legno : soffitti dei secoli XV, XVI e XVII, 1912); mentre il secondo trattava gli altari lignei (Hugelshofer, 1927)

${ }^{93}$ (Janner, II metallo, 1912 - 1914)

${ }^{94}$ (Berta, Case tipiche ticinesi : il Luganese, 19131914);
}

Ponte Capriasca" e su "San Biagio di Ravecchia" ${ }^{\prime \prime 66}$, come il suo primo lavoro di ricerca nel restauro. Un'altra interessante opera venne stampata nel fascicolo di gennaio del 1924 del "Heimatschutz" sulla conferenza tenutasi a Lugano, Locarno e nella Svizzera interna su: "il Ticino nelle sue abitazioni e costumi popolari" ${ }^{\prime 97}$ dove ancora una volta Edoardo Berta manifesta l'interesse per la sua terra e per le sue tradizioni.

Da 1911 a 1914 forma parte della Commissione Federale della Belle Arti e dal 1915 al 1918 della Società Svizzera dei monumenti storici in contemporaneità alla sua attività nella CCMS. Si occupò fra 1918 e 1919 della riorganizzazione del Museo Vela ${ }^{98}$ nella località di Ligornetto con il suo compagno pittore Luigi Rossi.

Da 1919 occuperà il ruolo di ispettore cantonale dei monumenti, incaricandosi del ripristino ed il controllo dei lavori su una grande diversità di opere, fra loro, le più importanti che più avanti saranno trattate sono la chiesa di San Biagio in Ravecchia, Bellinzona (1910 - 1929), primo lavoro di restauro, il Castello - Visconteo de Locarno (1922 - 1928), la chiesa di S. Maria degli Angioli in Lugano (1924 - 1931)

\footnotetext{
95 (Berta, Pitture murali e decorazioni pittoriche : ॥ cenacolo di Ponte Capriasca, 1912)

${ }^{96}$ (Berta, Relazione sui lavori di restauro della Chiesa di San Biagio in Ravecchia, 1915)

${ }^{97}$ (Berta, Casa e paessaggio nel Canton Ticino, 1924); (Berta, II Ticino nell sue costruzioni e costumi popolari: conferenza con 100 proiezioni originali, 1924)

${ }^{98}$ |l Museo Vela a Ligornetto è proprietà della Confederazione dal 1898, anno in cui Spartaco Vela, dona la residenza costruita per suo padre, l'artista Vincenzo Vela. In questa si conservano le opere d'arte di padre e figlio, così come di altri artisti lombardi e piemontesi amici della famiglia.
} 
e Santa Maria delle Grazie a Bellinzona, tutte loro dotate di un gran valore patrimoniale ticinese e svizzero, sussidiate dalla Confederazione.

Per tutto questo la sua attività e persona sarà elogiata e riconosciuta in un sentimentale addio dopo la sua morte. Nel libro pubblicato in sua memoria nel $1931 \mathrm{si}$ riprendono le numerose lettere e telegrammi di condoglianza con una premessa del 23 giugno 1931 del Dipartimento Federale dell'Interno in cui si elogiano i servizi del defunto artista per il Cantone, in speciale per il suo contributo alla rivalorizzazione del patrimonio ticinese. Alcuni degli scritti alla famiglia del pittore $^{99}$ furono spediti da personaggi rilevanti delle Autorità, come il Consigliere Albert Meyer ${ }^{100}$, Capo del Dipartimento Federale dell'Interno:

[...] i servizi resi al suo Cantone non saranno mai dimenticati, giacché egli è stato promotore ed esecutore di tante belle opere di restauro che rammenteranno sempre alla posterità $i$ suoi meriti $[\ldots]^{101}$.

Fernando Pedrini, presidente del Gran Consiglio del Canton Ticino:

[...] II Gran Consiglio del Canton Ticino esprime le cordiali condoglianze di tutto il popolo ticinese ricordando come E. Berta abbia intelligentemente illustrato, ricostruendolo il

\footnotetext{
${ }^{99}$ (dell'Interno, 1931, p. 33)

${ }^{100}$ Meyer Albert, (Fällanden 1870 - Zurigo 1953). II politico fu scelto il 12 dicembre del 1929 per il consiglio federale come rappresentante di Zurigo ne PLR, carica dalla quale si dimise nel 1938. Informazione preso il sito internet http://admin.ch/ch/i/cf/br/57.html

${ }^{101}$ Pedrini Fernando, presidente del Gran Consiglio del Canton Ticino (dell'Interno, 1931, p. 42)
}

patrimonio storico artistico del nostro paese $[\ldots]^{102}$.

Ambrogio Annoni, Soprintendente dei Monumenti della Lombardia:

[...]Edoardo Berta, artista fine, studioso acuto, strenuo difensore appassionato vivificatore monumento storici del Ticino $[. . .]^{103}$.

Plinio Bolla, Giudice federale:

[...]Nemico dei compartimenti stagni che separano i ticinesi fin dall'adolescenza, Edoardo Berta li combatteva nel modo più efficace: mostrando con la sua quotidiana attività in difesa del nostro patrimonio artistico e storico, una delle molte ragioni che abbiamo di vivere $[\ldots]^{104}$.

Dr. Sacchi ${ }^{105}$, presidente del Museo Civico di Bellinzona:

[...]Dolorosamente colpito dalla immature perdita del carissimo amico, a nome mio e della Commissione del civico Museo di Bellinzona, che ho l'onore di presiedere, presento le più sentite e sincere condoglianza. Se vi furono alcuni dissensi di vedute e di interpretazione fra la Commissione Museo e il caro Estinto, unanime fu la cooperazione e la collaborazione per condurre a termine i lavori di restauro ai nostri castelli ed alle nostre murate.

In Edoardo Berta il Ticino perde il massimo esponente della sua rivalutazione artistica in quanto che, se Motta fu il restauratore ed il promotore degli studi storici, Berta fu l'apostolo entusiasta e convinto della necessità assoluta di salvaguardare il nostro patrimonio artistico [...].

\footnotetext{
102 (dell'Interno, 1931, p. 42)

${ }^{103}$ (dell'Interno, 1931, p. 40)

${ }^{104}$ (dell'Interno, 1931, p. 40)

105 Luigi? Sacchi (Bellinzona 1856 - ?) (dell'Interno, 1931, p. 40)
} 


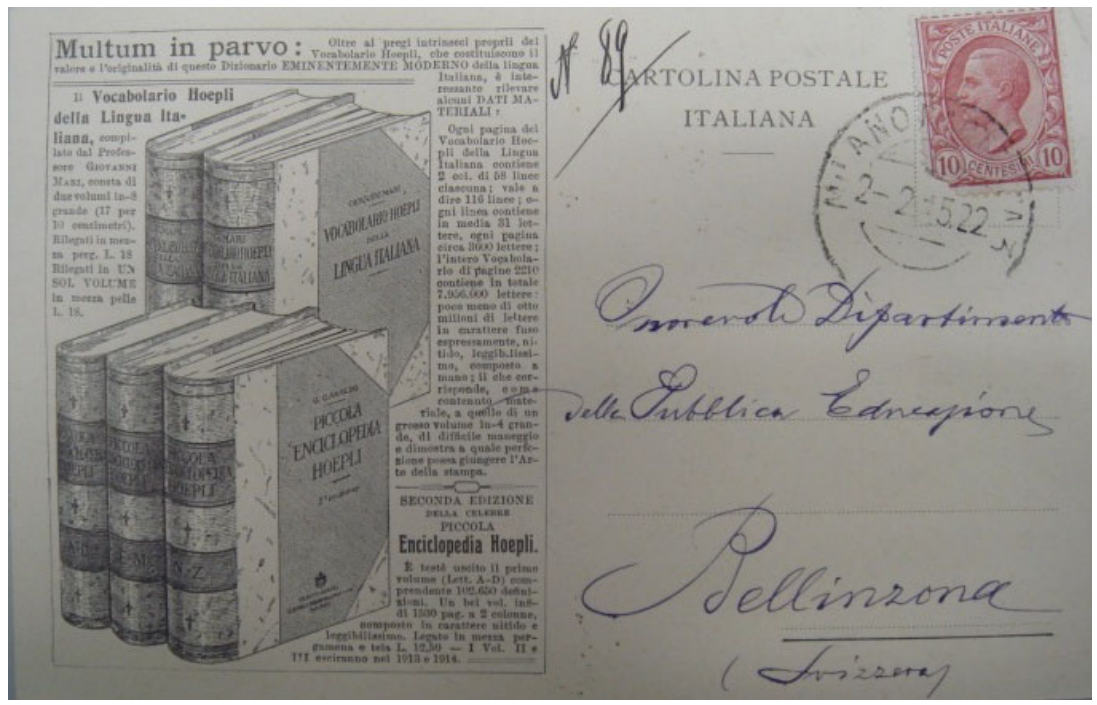

Figura 7 Cartolina da Ulrico Hoepli indirizzata al Dipartimento della Pubblica Educazione di Bellinzona, Milano 2 febbraio 1915. ASTi, Fondo DPE- Diversi 1905 - 1955, Segnatura 1.1.4.2.12.

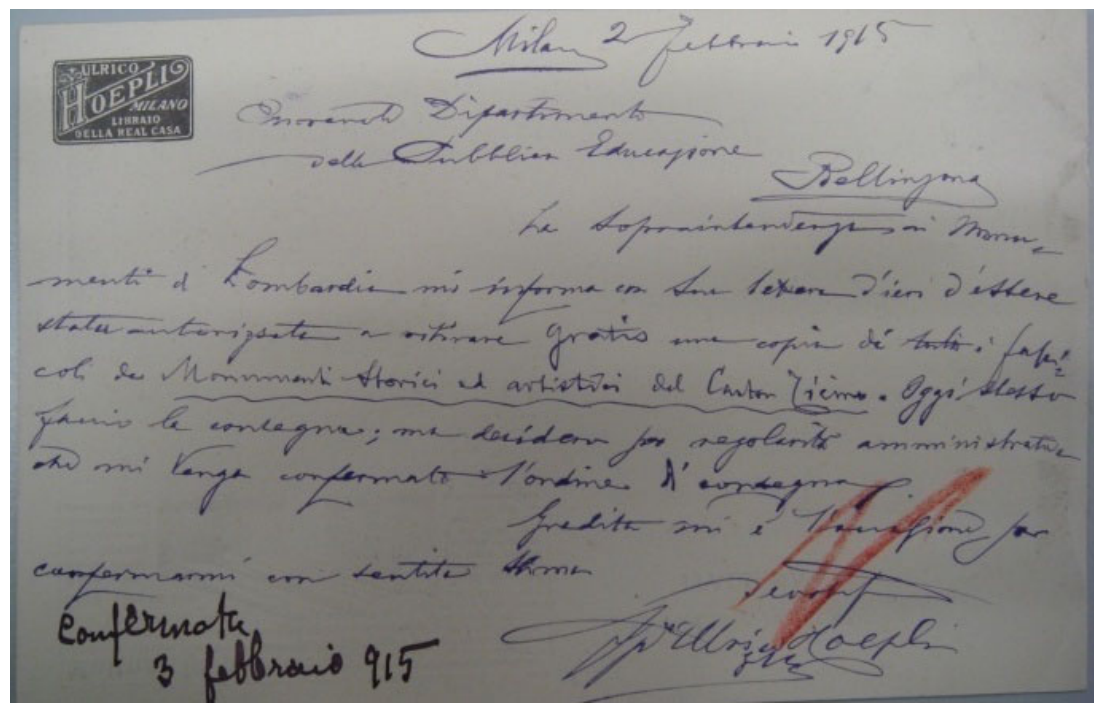

Figura 8 Cartolina da U. Hoepli indirizzata al DPE, Milano 2 febbraio 1915. ASTI, Fondo DPE- Diversi 1905 - 1955, Segnatura 1.1.4.2.12. Nella cartolina si legge: [...] La Soprintendenza dei monumenti della Lombardia mi informa con sua lettera di ieri di essere stata autorizzata a ritirare gratis i fascicoli dei Monumenti Storici ed Artistici del Canton Ticino. Oggi stesso fanno la consegna; ma desidero per regolarità amministrativa che mi venga confermato l'ordine di consegna[...]. 


\section{Chiesa di S. Maria degli Angioli, Lugano (1910 - 1930)}

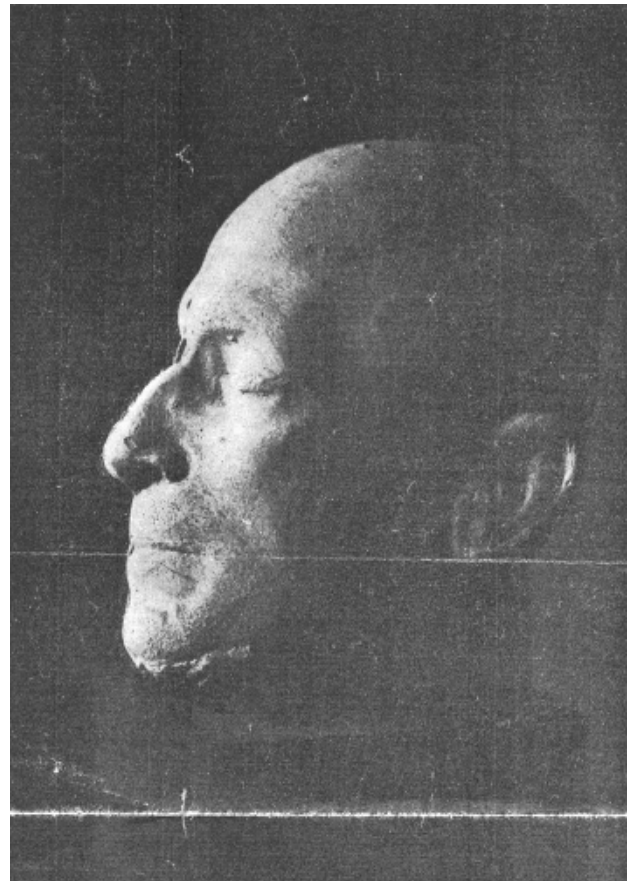

Figura 9 Foglia Giuseppe, Maschera di Edoardo Berta eseguita sul letto di morte, 1931. Fotografia Himmelsbach.
[...]Consentiva, e si capisce, all'idea che il restauratore si faceva in quel tempo: si ammetteva la possibilità di colmare $i$ vuoti, d'intonare gli elementi autentici con le aggiunte, di confondere il tutto in un'apparenza d'unità"contentar l'occhio" -si diceva - insomma, era concessa una libertà assai grande al restauratore $[. . .]^{106}$.

[...]Permitía, y se entiende, que se ejecutaran los ideales en los que el restaurador creía en este período: se admitía colmar los vacíos, igualar los elementos originales con los añadidos, confundir el todo un una apariencia única, "contentar a la vista" - se decía - en definitiva, se concedía un libertad muy grande al restaurador[...]

\footnotetext{
${ }^{106}$ Francesco Chiesa citato da Isidoro Marcionetti (Marcionetti, 1975, p. 218)
} 
Poco prima di morire il 23 giugno 1931 Edoardo Berta presenta al pubblico il restauro di uno dei monumenti più importanti e preziosi del Canton Ticino, la chiesa di Santa Maria degli Angeli, chiamata anche degli Angioli, a Lugano.

L'edificio rinascimentale, antica proprietà del convento dei Padri minori osservanti di San Francesco ${ }^{107}$ fu costruito nel 1499 grazie al progetto dell'architetto e ingegnere Giovanni Solari ${ }^{108}$ di Carona, e fu consacrato sedici anni più tardi nel 1515 . La fabbrica risultò scollegata dal convento per cui fu creata, al quale si univa a sud ed a sud-ovest, quando questo venne soppresso nel 1848 passando nelle mani dello Stato. In sua sostituzione Giacomo Ciani $^{109}$ fece erigere fra il 1852 e il 1854 l'Hotel du Parc nell'area adiacente alla chiesa, su progetto dell'architetto milanese Luigi Clerichetti. Questo edificio diventò nel 1903 il famoso Grand Hotel Palace ${ }^{110}$ di proprietà della ditta Bucher-Durrer, elevandosi in altezza di due piani su progetto dall'architetto Emil Vogt e dell'ingegnere Alfred Bucher. Si

\footnotetext{
107 || convento dei Francescani minoriti fu fondato a Lugano in 1490. (B. Anderes, 1998)

${ }^{108}$ Solari Giovanni (Carona 1400 circa - Milano, 1482) Architetto e ingegnere particolarmente attivo in Italia dove in 1452 ottenne la patente d'ingegnere ducale dedicandosi alla direzione del cantiere del Duomo di Milano fino al 1469.

${ }^{109}$ Ciani Giacomo (Milano 1776- Lugano 1868), fu bancario come il padre, stabilito con il fratello, Filippo $(1778$ - 1867) a Lugano dal 1830, dove insieme a lui diventa una delle figure più importanti del mondo imprenditoriale e politico alla fine dell'Ottocento, trasformando la città ticinese in un centro attivo, moderno e turistico

${ }^{110}$ L'ex-Hotel è oggi soggetto di ristrutturazione per la realizzazione del nuovo centro culturale della Città $\mathrm{d}$ Lugano, in un progetto che ha previsto i mantenimento delle facciate meridionale e orientale.
}

sottovalutarono in questo modo le norme regolatrici di costruzione e protezione del paesaggio, ma non solo, poiché già allora si cominciarono a temere i danni che i diversi momenti costruttivi potessero provocare alla chiesa e la conservazione degli importanti dipinti murali esistenti al suo interno ${ }^{111}$.

La chiesa di Santa Maria degli Angioli ${ }^{112}$, di tipo conventuale lombardo è, insieme alla chiesa di Santa Maria delle Grazie a Bellinzona, una delle poche attualmente in Svizzera che conserva il transetto interno divisorio dello spazio di utilizzo dei fedeli e quello dei monaci. Su questo muro di $110 \mathrm{~m}^{2}$ troviamo l'opera più importante della chiesa, la Passione e Crocifissione di

\footnotetext{
${ }^{11}$ Attualmente l'ex Hotel Palace è oggetto di diversi lavori che pretendono convertirlo nel "Nuovo centro culturale" della città di Lugano. Essi lavori hanno protetto la facciata ottocentesca demolendo il resto dell'edificio. I cantieri sono sotto continuo controllo monitorando i possibili movimenti occasionati all'edificio contiguo, è a dire, la Chiesa di Santa Maria degli Angioli. Oltre al controllo continuo della fabbrica in sé, dal 2007 il Cantone in collaborazione con il Laboratorio Tecnico Sperimentale della SUPSI, esamina lo stato di conservazione dell'affresco della Crocifissione del Luini. La tecnica impiegata è quella della battitura a mano di tutto la superficie pittorica, che per il momento, fortunatamente, non ha rivelato problemi di adesione dell'intonaco. (CdT, 2008)

112 Sono molto interessanti le descrizioni del monumenti negli scritti del maestro J. Rudolf Rahn: (Rahn, 1876, p. 548) e (Rahn, Die mittelalterlichen Wandgemälde in der italienischen Schweiz, 1881, p. 143). Abbiamo ritenuto citare $i$ volumi originali in tedesco che erano l'epoca quelli accessibili agli studiosi. Solo nel 1892 l'editoria Tip. e Litografia Eredi C. Colombi a Bellinzona pubblica in versione italiana il testo "I dipinti del Rinascimento nella Svizzera Italiana" tradotto da Giorgio Simona e nel 1894 "I monumenti artistici del Medio Evo nel Cantone Ticino" tradotta dallo storico Eligio Pometta.
} 
Cristo, che il pittore Bernardino Luini ${ }^{113}$ realizza nel 1529, e sopra la quale intervenne il prestigioso restauratore di Bergamo Mauro Pellicioli nel $1929^{114}$, raccomandato al Cantone dalla Soprintendenza dei Beni Culturali di Milano.

Sopra la immensa parete riccamente affrescata si raffigura la scena principale della Crocifissione con intorno una successione di soldati a cavallo e diversi personaggi fra i quali San Giovanni e la Maddalena inginocchiata, a sinistra; in basso osserviamo un gruppo di donne che sostengono la Vergine Maria addolorata ed un gruppo di soldati che gioca ai dadi sulla parte opposta. La croce di Cristo è circondata nella parte in alto da un insieme di angeli mentre nel piano medio si sviluppano le scene dell'Incontro con

\footnotetext{
113 Luini Bernardino (Dumenza 1481 circa - Milano 1532). Di cognome Schapis. Dopo essersi formato come pittore nel Veneto dal 1504, ritorna nel 1509 a Milano stabilendosi definitivamente in Lombardia dove troverà la fama e la maturità artistica con opere di carattere classicista influenzate dalle pitture $d$ Leonardo da Vinci, Vincenzo Foppa, Bartolomeo Suardi detto il Bramantino e Bernardo Zenale. In Svizzera lasciò una particolare testimonianza con l'elaborazione dell'affresco della Crocifissione e passione di Cristo nel 1529, la Crocifissione con la Madonna e i Santi Giovanni, Pietro, Paolo, Andrea e Pietro Martire nella cappella di San Pietro delle Erbette nel quartiere luganese di Cassarate, nella lunetta della parete frontale del coro, I'affresco Crocifisso con la Madonna, San Giovanni e due angel originariamente nel distrutto convento di Santa Maria degli Angeli, ora collocato nella chiesa di San Nazario di Dino, gli affreschi Crocifisso con due angeli che ne raccolgono il sangue con alla destra "Santo Stefano" e a sinistra Sant'Abbondio nella cappella di Sant'Andrea ad Agnuzzo (Muzzano), databile al 1525 circa, senza firme, ma di stile simile al capolavoro in Santa Maria degli Angeli.

${ }^{114}$ Pellicioli Mauro (Bergamo 1887 - 1974), ved. Parte III, Mauro Pellicioli. pp. 463, 465
}

Tommaso e della Derisione e la rappresentazione dell'Andata al Calvario in fondo al paesaggio; nella parte a sinistra, la Preghiera nell'orto degli ulivi e in centro I'Ascensione di Gesù. Sopra le arcate vengono rappresentate i Santi Rocco e Sebastiano.

Sono dello stesso artista l'affresco dell'Ultima Cena presente attualmente nella navata della chiesa, la lunetta raffigurante la Madonna con il Bambino e San Giovannino, sulla parete orientale della prima cappella e l'affresco Cristo in Pietà coi Santi Francesco e Bernardino sul pilastro destro della seconda. In tutte e tre le opere si evidenza l'influenza leonardesca propria della scuola di Bernardino Luini. ${ }^{115}$ Sulla seconda cappella è pure situata la statua di Sant'Antonio di Padova, una tela di Giuseppe Antonio Petrini ${ }^{116}$ raffigurante San Francesco (1728) e sopra I'arcata una Santa Chiara e una Immacolata della fine del XVI sec. Sull'arcosolio invece si osserva l'affresco raffigurante la Madonna fra i SS. Carlo e Francesco di Paola ed i membri della famiglia Castagna.

Gli affreschi sopracitati del pittore Bernardino Luini, rappresentanti I'Ultima

\footnotetext{
${ }^{115}$ I tre quadri strappati insieme ad altre tre opere di proprietà della chiesa di Massagno e del Vescovo di Lugano furono assicurati durante i lavori di restauro delle chiesa fino agli anni 1931 per un totale di Fr. 260.000 dal Municipio di Lugano. A lavori finiti l'assicurazione passò alla Chiesa di S. Maria come nuova proprietaria dal 21 marzo 1931. (AUBCBZ s. 143 Lugano).

${ }^{116}$ Petrini, Giuseppe Antonio (Carona 1677 - 1759) pittore svizzero-italiano, formatosi a Genova e a Torino particolarmente influenzato dai pittori caravaggeschi come Andrea Pozzo e Giovanni Serodine di Ascona e dal 1740 dagli svizzeri Carlo Innocenzo Carloni e Paolo Pagani, con una pittura caratterizzata da un impattante colorismo.
} 
Cena e la lunetta della Madonna, furono staccati nel marzo del 1851 dal pittore bresciano Bernardo Gallizioli e da Pietro Tatti $^{117}$, vice-assistente delle opere del Duomo, rispettivamente. Questo evento avviene dopo un primo sopraluogo del direttore della Pinacoteca di Brera (Milano) Ambrogio Nava ${ }^{118}$ nel dicembre del 1850. Egli propose ai proprietari e committente dell'opera, i fratelli Ciani ed il Governo del Canton Ticino, lo stacco dal muro della lunetta raffigurante la Madonna col bambino e lo strappo, per il contrario, del Cenacolo che doveva essere idoneamente riportato su tela. Essi lavori furono iniziati già nel marzo del 1951, da Pietro Tatti e da Giuseppe Knoller, restauratore all'Accademia di Brera. Mentre lo stacco della Madonna eseguito dal primo ebbe un grande successo, e l'opera si costata anche nei giorni nostri in un discreto stato di conservazione, lo strappo iniziato dal Knoller non riusci inizialmente, per cui l'operatore, in modo onesto, rinunciò sollecitamente all'incarico. I problemi furono provocati, secondo le spiegazione di Luca Beltrami riportate da Giuseppe Martinola nel suo scritto "Il distacco e il restauro degli affreschi del Luini a Lugano nel secolo scorso"119, dall'umidità del muro esistente durante i lavori, che avrebbe dovuto occasionare la perdita ritrovata nella parte inferiore del primo elemento staccato a sinistra. Attualmente sappiamo, grazie ancora ai ragionamenti dal

\footnotetext{
${ }^{117}$ (Atti degli strappi, Bollettino storico, 1944. p.39)

118 Nava Ambrogio (Milano, 1791 - 1862), pittore e mecenate. Era stato amministratore al Duomo di Milano e restauratore nel 1844 della guglia maggiore del medesimo edificio. Fu direttore dell'Accademia di Belle Arti di Brera.

${ }^{119}$ (Martinola, 1944).
}

Martinola nel sopracitato articolo, che la medesima lacuna fu sicuramente mancante già in inizio, prima dei lavori di strappo.

Dopo i tentativi di Giuseppe Knoller, il lavoro fu affidato a Pietro Tatti il quale pretendeva procedere questa volta con il metodo dello stacco a massello dell'affresco raffigurante il Cenacolo. II preventivo presentato da quest'ultimo ai committente era troppo elevato e si chiese nuovamente ad Ambrogio Nava raccomandazione su una persona capacitata per realizzare i sopracitati lavori. Fu Bernardino Gallizioli, nel mese di settembre 1851, a finire in solo dieci giorni I'incarico. II restauratore bresciano, distaccò la pittura in tre parti con la tradizionale tecnica dello strappo riportandogli su panelli rinforzati di telai ad angoli fissi di spessore circa $5 \mathrm{~cm}$. II risultato, conservato per oltre un secolo in uno stato buono, era riuscito secondo i metodi che nell'epoca si ritenevano esemplari. Non per altro le doti del Gallizioli erano apprezzate dai grandi maestri ed in particolare da Giovanni Secco Suardo. Alessandro Conti cita a quest'ultimo definendo gli interventi del così chiamto "estratista" bresciano come quelli ideali poiché [...]sottili, pieghevoli $e$ leggeri, dove non vi appare nessun resto di resina ed a tergo, una tinta cinereccia nasconde il metodo di applicazione degli strappi alla nuova tela $[. . .]^{120}$ cioè perché gli strappi si adattano alle nuove caratteristiche del supporto, in questo caso della tela. II successo di questa tecnica fu riconosciuto nel 1847, anno in cui il Gallizzioli vince il premio dell'industria per l'affresco

\footnotetext{
(Conti, 2002, p. 261).
} 
strappato del Refettorio dalla Vetabbia a Milano $^{121}$ per usare a questo scopo una "tecnica innovativa". In qualunque modo la tecnica dello strappo, sviluppata dal metodo di stacco a massello era conosciuta già nel XVIII sec., e sicuramente ancora prima. La differenzia avviene dal fatto che in passato, questa come altre maestrie svolgete nelle botteghe dei pittori furono mantenute in segreto all'interno di essi, e solo a metà dell'Ottocento con la pubblicazioni dei primi manuali "I'alchimia" e la "magia"122 dei sistemi di ricupero delle opere artistiche saranno messe a conoscenza di tutti.

[...]Molti altri restauratori proseguirono a perfezionare questa importante arte del distacco e del riattacco su tela delle pitture a fresco.[...] Però quasi tutti coloro che si dedicarono a questo particolare ramo di tecnica sui freschi seguirono processi differenti da loro stessi immaginati, sforzandosi di mantenere il più rigoroso segreto intorno al loro sistema.[...]. ${ }^{123}$

Fra gli interventi eseguiti dal Gallizioli, Conti cita ${ }^{124}$ : la Madonna degli Angeli del Bergognone, a Brera, trasportata nel 1847

\footnotetext{
${ }^{121}$ (Collezioni degli atti delle solenni distribuzioni dei premi dell'industria fatti in Milano e Venezia 1840 1852, 1852). Formato html, disponibile in Internet sulla banca dati on-line di libri elettronici: http://books.google.ch/books.

$122 \mathrm{NdA}$. Ho voluto utilizzare queste definizione trovate nel testo di Giorgio Bonsanti per I'Introduzione alla pubblicazione Giovanni Secco Suardo alle origini del restauro moderno, curata da Cristina Gianini, (Gianini, 2006, p. 7), poiché esprimono perfettamente la visione che del restauro si doveva tenere al di fuori delle botteghe, d'un mestiere appreso dalla tradizione e nascosto alla scienza.

${ }^{123}$ (Piva, 1988, p. 88).

${ }^{124}$ (Conti, 2002, p. 261)
}

da Santa Maria dei Servi a Milano, i Ferramola di Casa Borgondio, della Torre a Brescia trasportati nel 1845, il grande affresco del Victoria and Albert Museum con una scena di torneo nella piazza di Brescia e gli strappi dei Profeti del Moretto da palazzo Martinengo, premiati all'Esposizione Italiana del 1861.

Il primo elemento strappato dall'Ultima Cena di Bernardino Luini nella chiesa di Santa Maria degli Angioli in Lugano, ebbe una misura di $91 \times 200 \mathrm{~cm}$ e compresse le figure di Bartolomeo, Giacomo Minore e Andrea; il secondo strappo, di 91,5 x 188,5 $\mathrm{cm}$, raffigura da sinistra a destra Pietro, Giuda e Giovanni, il Cristo in centro, Tommaso, Giacomo Maggiore e Filippo, mentre l'ultimo gruppo, di $91 \times 182 \mathrm{~cm}$, rappresenta le figure di Matteo, Simone e Taddeo. I tre elementi furono incassati nella parete sinistra della navata della Chiesa di Santa Maria degli Angioli dopo i lavori di ritocco pittorico degli affreschi, anche se non ne abbiamo notizie del momento in cui avviene questa ricollocazione.

All'interno della chiesa di S. Maria degli Angioli, sul pilastro fra l'arco della prima e la seconda cappella possiamo oggi leggere inciso su una lastra di marmo:

"La Chiesa di S. Maria degli Angioli è stata restaurata, sotto la direzione del Pittore Edoardo Berta, dall'anno 1922 all'anno 1930, per iniziativa ed a spese dello Stato del Canton Ticino col concorso della Confederazione e col contributo finanziario della Città di Lugano e di enti privati. Le opere di consolidamento furono eseguite sotto la sorveglianza dell'arch. Otto Maraini e dell'ing. Francesco Riva. In data 23 ottobre 1922, il Governo del Cantone firmò gli impegni di legge verso la Confederazione circa la conservazione dell'edificio." 


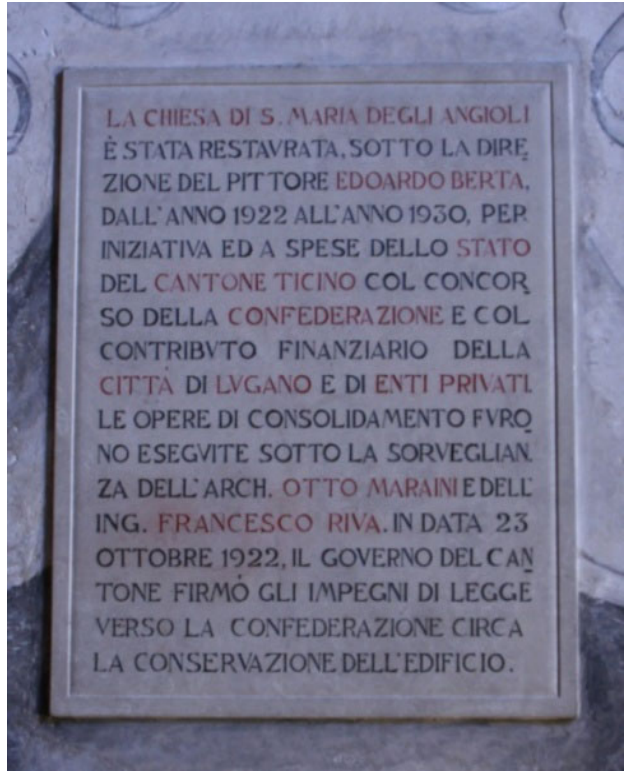

Figura 8 Lastra di marmo con l'iscrizione di testimonianza del restauro. Giner E., 2007.

L'impresa cominciò nel mese di settembre dell'anno $1891^{125}$ con la casuale scoperta nella Cappella dell'Immacolata o Cappella Camuzio, anteriormente citata, di interessanti dipinti sotto lo scialbo a calce applicato nel restauro del 1650 circa.

Gli affreschi trovati durante i lavori di muratore e di ristrutturazione della chiesa, furono datati dal pittore Andrea Demicheli $^{126}$ e dall'architetto Costantino Maselli $^{127}$ originari del 1524-1525,

\footnotetext{
125 Riguardo la scoperta degli affreschi alla chiesa di Santa Maria degli Angioli, come allo stesso tempo dei ritrovamenti effettuati alla chiesa di Ascona e di Mairengo ne scrive il professor Johann Rudolf Rahn in (Rahn J. R., 1893).

${ }^{126}$ De Micheli, Andrea (Lebensdaten 1860, Lugano 1930). Pittore e professore nella Scuola di Disegno di Lugano. Padre dell'artista Giovanni Micheli. (SIK/SEA, 2001) .

${ }^{127}$ Maselli Costantino. Fra le opere più important dell'architetto troviamo la Chiesa di San Carlo
}

contemporanei all'opera della Crocifissione di Bernardino Luini sul tramezzo. Più tardi essi furono attribuiti al così chiamato Maestro Camuzio, per considerarsi unico autore dell'insieme di decorazioni presenti nell'omonima cappella. Questa, fondata dal medico Ludovico Camuzio, era stata dedicata a San Giuseppe, e fra i sui dipinti si scoprì un ciclo di affreschi con quattro episodi dell'infanzia di Gesù: la Fuga in Egitto, copia della tavola del Bramantino ${ }^{128}$ presente nella chiesa della Madonna del Sasso in Orselina (Locarno) ed altri basati nelle xilografie di Albrecht Dürrer ${ }^{129}$, con angeli musicanti sulle lunette, quattro grandi angeli sulla volta e medaglioni con profeti sull'intradosso dell'arco.

II "Maestro della Cappella Camuzio" fu a suo tempo esecutore dei dipinti raffiguranti S. Francesco e Cosme (o Damiano) del 1523 circa, esistenti sul pilastro destro della terza cappella.

Effettuato lo studio sullo stato di conservazione della chiesa e dei diversi dipinti murali, che lo Stato aveva commissionato al sopracitato architetto C. Maselli, si eseguirono le operazioni sulla Cappella con l'intervento del pittore-

Borromeo a Cernesio (1891-1895), la progettazione del campanile neoromanico della Chiesa di Sant'Ambrogio di Barbengo (1883 ca.).

${ }^{128}$ Omonima opera del pittore e architetto italiano Bartolomeo Suardi, chiamato Bramantino (Milano, 1465 - 1530) situata nella navata meridionale della chiesa della Madonna Assunta al convento della Madonna del Sasso in Orselina. (Simona Martinoli, 2007, p. 301).

${ }^{129}$ Dürrer Albrecht (Norimberga 1471 - 1528). Pittore e incisore, massimo esponente del rinascimento germanico. 
decoratore Augusto Cometta ${ }^{130}$ che lavorò in un primo momento nello descialbo delle pitture. L'attività fu fermata in novembre dello stesso anno del 1892 a causa di assenza di sussidio economico. II pittore, non ricevendo da mesi lo stipendio accordato un compenso per il suo lavoro, abbandona l'attività all'interno della chiesa e si rivela al Consiglio Parrocchiale che minaccia di non riportare le chiavi della cappella se prima non riceveva i ritardi economici.

[...] La Confraternita mandò due volte dal signore Pittore Cometta per avere la chiave della Cappella, ma n'ebbe in risposta che la chiave sarà restituita quando il pittore sarà pagato. Per le quali cose prego all'Onorevole Sig. Consigliere ad occuparsi prima perché il sig. Pittore Cometta ci restituisca la chiave della Cappella e secondo perché se non s'intende proseguire i lavori per mettere in rilievo gli antichi affreschi si rimettano in modo convenevole le pareti per mano del pittore. Taluno ha osservato il poco pregio artistico delle figure in mostra a la poca correttezza morale delle medesime, è meglio seppellirle di nuovo sotto un'altro intonaco di calce. $[. . .]^{131}$.

Di fronte a questa situazione, la parrocchia deve esporre alle autorità il grave problema estetico in cui era rimasta l'opera, incompleta e disomogenea dopo l'interruzione dei lavori di pulitura e si proponeva nascondere nuovamente $\mathrm{i}$ dipinti sotto uno strato di calce. Questo perché il consiglio ecclesiastico pensava

\footnotetext{
${ }^{130}$ Cometta, Augusto (Lebensdaten 1863 - Lugano 1918) Pittore-decoratore. Professore dal 1895 alla Scuola di Disegno di Mendrisio. (SIK/SEA, 2001).

${ }^{131}$ (Pretore della Confraternita degli Angeli. Lettera indirizzata al Dipartimento della Pubblica Educazione del Canton Ticino, Lugano 23 Novembre 1892. AUBCBZ s. 143 Lugano, S. Maria degli Angioli).
}

che questi affreschi non avessero un valore artistico né conservativo tale da fare eseguire operazioni di restauro, o ancora peggio, permettere l'abbandono estetico anche se temporaneo.

Ripetute perizie sulle operazione di consolidamento della chiesa furono presentate il 29 agosto 1895 per l'architetto Costantino Maselli e nel 1897 per l'architetto Augusto Guidini al Dipartimento della Pubblica Educazione Svizzero. Le difficoltà economiche anteriormente esposte, tanto dello Stato del Canton Ticino come della Confederazione, sommato alla mancanza di una legge di protezione dei Beni Culturali, ritardò l'esecuzione delle misure conservative fino all'anno 1909 quando sotto la direzione dell'architetto Paolo Zanini si realizza la riparazione del tetto della chiesa, danneggiato in diversi punti per infiltrazione d'acqua.

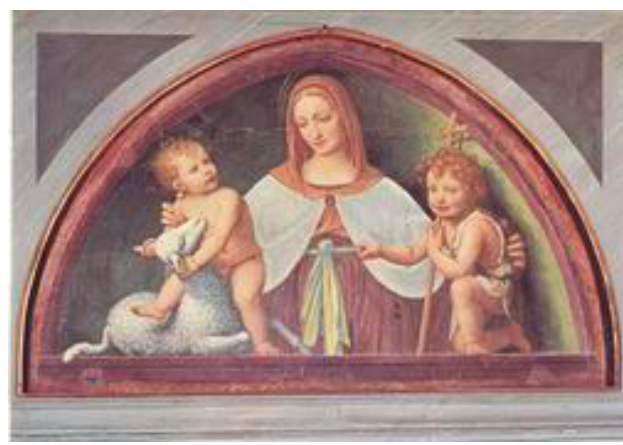

Figura 10 Luini Bernardino, Madonna con il Bambino e San Giovanni. Stacco. Chiesa di S. Maria degli Angeli, Lugano. (Marcionetti, 1975). 
La rivalutazione dei dipinti murali scoperti sulla Cappella Camuzio si deve agli scritti, analisi e ricerche che esegue in 1903 I'architetto Luca Beltrami ${ }^{132}$ di Milano, e più tardi dopo i primi lavori di restauro, in 1924, Edoardo Berta ${ }^{133}$.

A questo fine, già il 22 febbraio 1913, gli allievi dell'Accademia di disegno di Lugano dove impartiva docenza Berta, realizzano la ricostruzione completa in tempera su tela delle pitture. Questo disegno doveva cedersi al Museo Civico di Lugano, e fu il primo passo per l'elaborazione di discreti interventi di restauro e di conservazione, con i pochi contributi economici dello Stato. ${ }^{134}$

[...]Schema di progetto-preventivo per la ricostruzione completa della Cappella dei Camuzi nel Museo Civico di Lugano.[...] lo potrei proporre al lodevole Dipartimento della Pubblica Educazione che mi autorizzi di eseguire tale lavoro con $i$ miei allievi che poi potrebbe figurare come saggio scolastico alla Esposizione nazionale di Berna del 1914 e poi cederlo al Museo Civico di Lugano al prezzo di Fr. 1500. Sarebbe cosi possibile di avere una ricostruzione completa di tale capolavoro e di arricchire il nostro Museo. $[. . .]^{135}$.

\footnotetext{
${ }^{132}$ (Beltrami, 1903).

133 (Berta, Pitture murali e decorazioni pittoriche. Pitture del Rinascimento. La cappella Camuzio nella chiesa di S. M. degli Angioli in Lugano, 1924).

${ }^{134} \mathrm{Fu}$ eseguito anche il restauro dei dipinti della Madonna col Bambino fra i Santi $(170 \times 230 \mathrm{~cm})$, il S. Michele $(175 \times 230 \mathrm{~cm})$ ed il quadro di S. Antonio (110 $x 135 \mathrm{~cm}$ ), ai quali furono sostituiti i telai. L'intervento fu realizzato dal restauratore Francesco Annoni d Milano. (Annoni Francesco, preventivo di restauro indirizzato alla Commissione Cantonale dei Monumenti Storici Artistici. Milano 30 agosto 1930 AUBCBZ s. 143 Lugano. S. Maria degli Angioli.)

${ }^{135}$ (Berta Edoardo. Schema di progetto-preventivo per la ricostruzione della Cappella dei Camuzi nel Museo
}

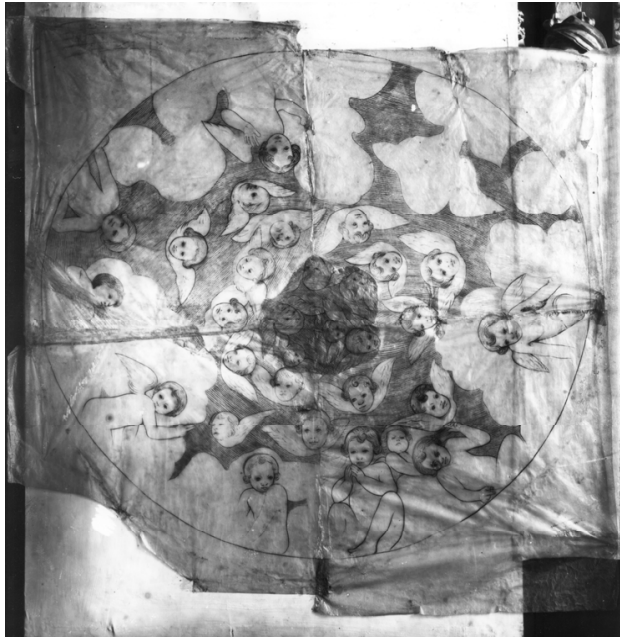

Figura 11 Studio su carta dei dipinti della volta della Cappella Camuzio. 1913 post. Lugano. AUBCBZ Archivio gráfico, Lugano, S. Maria degli Angioli.

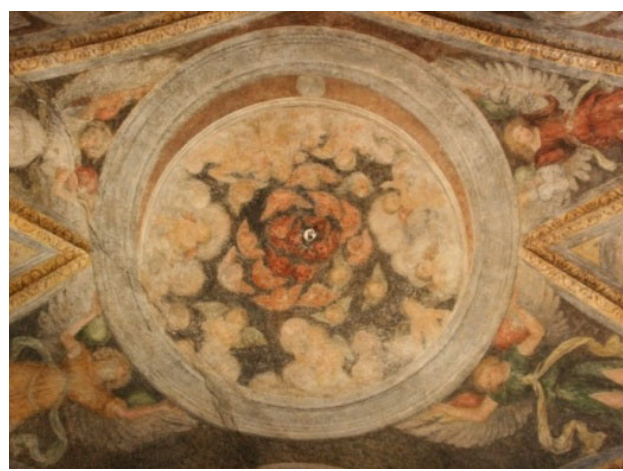

Figura 12 Volta della Cappella Camuzio. Chiesa di Santa Maria degli Angioli, Lugano. Giner Cordero E. 2008.

Civico di Lugano indirizzato alla Commissione dei Monumenti Storici ed Artistici del Cantone Ticino. Lugano 22 febbraio 1913. AUBCBZ s. 143 Lugano, S. Maria degli Angioli). 
I lavori verranno nuovamente interrotti, in questo caso per cause belliche fino al 1920, momento in cui si riprenderanno con l'incremento dei sussidi da parte della Commissione dei Monumenti Storici.

La copia di una lettera che Albert Naef ${ }^{136}$, presidente della Commissione Federale dei Monumenti, dirige al Dipartimento Federale dell'Interno il 27 agosto 1921, ci offre una descrizione molto analitica sugli ideali d'intervento che furono scelti.

Lo stesso giorno in cui venne scritta la relazione d'ispezione, Naef visitava la chiesa insieme al pittore Edoardo Berta, direttore dei lavori, al presidente della commissione cantonale Francesco Chiesa ed al pittore Emilio Maccagni ${ }^{137}$,

\footnotetext{
${ }^{136}$ Naef Albert (1862 - 1936, Losanna), conservatore di monumenti, architetto, storico d'arte ed archeologo. Fu presidente dal 1917 della Commissione Federale dei Monumento Storici ed artistici in Svizzera, fino a 1935 assumendo l'incarico d'esecutore di pratiche relative ai restauri, perizie, ispezioni, inventari e documentazione fotografica. I suoi ideali di restauro conservativo propongono la manutenzione delle parti originali dell'edificio con il rifiuto dei restauri pesanti ed illusionisti. II suo metodo di lavoro fu quello d'imporre durante la ricostruzione la chiara differenzazione fra le part originali e le aggiunte mediante righe di matton distanziati. Queste idee furono anteriormente adottate dall'architetto Adalbert Stiffer, chi nel saggio Nachsommer del 1857 raccomandava la differenzazione degli originali con l'utilizzo di lastre metalliche informative. (Gurtner, 2004).

${ }^{137}$ Maccagni, Emilio (Rivera 1888 - 1955). Allievo di Edoardo Berta nella Scuola di Disegno di Bellinzona nel 1900 e nel 1927 nella Scuola di Decorazione d Lugano. Sarà sempre molto legato al suo professore con chi collaborerà in progetti di restauro come i citato in Santa Maria degli Angioli a Lugano, Santa Maria delle Grazie a Bellinzona allo stesso tempo che si dedica ai lavori di decorazione in diverse case $d$ Bellinzona, Rivera, Bironico e dipingendo nel vecchio studio di Berta, la casa Landvogti.
}

riconoscendo, ottimista, un riuscito lavoro di restauro:

[...] II signor Berta ha adottato i principi che seguono: completare discretamente le decorazione lineare ed architettonica, là dove cadde, in maniera che l'assieme decorativo risulti, che le scene figurative appaiono inquadrate, che l'ossatura architettonica, la quale forma la base di tutta la composizione, sia messa in valore e si comprenda. Per quanto concerne le scene figurative delle pareti e della volta, non intaccare le figure umane, ma nei luoghi dove il colore impallidi talmente che a distanza, queste per così dire, spariscono, ritoccare parzialmente i fondi, in maniera di farli risaltare, nei luoghi dove la pittura o l'intonaco stesso sono caduti - ed è questa la questione più delicata e difficile - mettere delle gradazioni grigie, più o meno distinte, più o meno spiccate, più o meno sbiadite, le quali senza nulla ricostruire, mantengono o piuttosto ridiano all'assieme il suo carattere armonico, il suo elevato, elevatissimo, valore artistico. - il sig. Naef, espone più avanti i suoi desideri, dal punto di vista tecnico - in certi luoghi rinforzare le gradazioni oscure del fondo, in maniera di far risaltare maggiormente le teste, le mani, quando sono in stretta intima unione con l'architettura decorativa; murare la finestra laterale, vuoto abbastanza moderno, che guasta l'armonia della composizione e della decorazione. $[\ldots]^{138}$.

I consigli di Naef sono influenzati dagli ideali di restauro che si dibatterono in Italia nella transizione fra l'Ottocento e il Novecento al mettere in confronto le

138 (Naef Albert, Copia del rapporto d'ispezione Chiesa di S. Maria degli Angioli a Lugano indirizzato al Lodevole Dipartimento Federale dell'Interno, Berna. Berna 27 agosto 1921. AUBCBZ s. 143 Lugano, S. Maria degli Angioli).

Fonte ARISTOS. Id. 50790. (ved. allegato $\mathrm{x}$ ) http://aristos.mbigroup.it/search/index.php?entita=F onte\&id=50790\#top. 
teorie sentimentaliste degli interventi conservativi moderni, che in Lombardia ebbe il massimo esponente nella figura di un maturo e consapevole decoratorerestauratore Luigi Cavenaghi ${ }^{139}$ e nell'architetto Camillo Boito, ambi due docenti all'Accademia di Brera. Boito dichiarava la sua concezione del restauro la cui definizione promosse nel 1883 , al IV Congresso degli ingegneri e degli architetti a Roma, adottando una soluzione intermedia fra gli ideali di "rovina" di John Ruskin e l'unità stilistica di Viollet-le-Duc, cioè la conservazione delle parti originali dalla conoscenza storica ma, in questo caso, giustificandosi il ritocco pittorico e le velature di zone molto concrete per conferire all'insieme un carattere omogeneo, assumendo la storia della stessa opera e la patina, [...] la quale fa diventare il quadro ancor più armonioso di come l'aveva fatto il suo autore $[. . .]^{140}$, prodotta dal tempo, anzi, riproducendola. [...]Nell'ambito di questa sensibilità al dipinto intonato $e$ patinato dal tempo, la pulitura artistica insiste più o meno secondo l'effetto che il restauratore crede opportuno di raggiungere, con un'interpretazione soggettiva affidata ad un apprezzamento squisitamente figurativo[... $]^{141}$. Questo può confrontarsi con la metodica nel restauro architettonico dello stesso Albert Naef, al quale interessava una differenzazione fra le parti aggiunte e le

\footnotetext{
139 Cavenaghi Luigi (Caravaggio 1844- Roma 1918). Pittore e decoratore di stile, fu discepolo di Bertini e di Molteni all'Accademia di Belle Arti di Brera, qui si era iniziato nella corrente di restauro amatoriale per arrivare con la maturità professionale ad un approccio di maggiore sensibilità e rispetto per l'opera originale che dimostrò ad esempio nel restauro del Cenacolo $d$ Leonardo da Vinci. (AA.VV., 2007)

${ }^{140}$ (Conti, 2002, p. 237).

${ }^{141}$ (Conti, 2002, p. 240).
}

parti originali della fabbrica con l'utilizzo di righe di mattoni posti per differenziarsi dall'originale. Nella pittura, invece, i mattoni sono tinte grigiastre che ricoprono le lacune in modo neutro, ma come soprascritto, accettando dei veli sui fondi decorativi o sui contorni delle figure per farle risaltare. Non trattandosi comunque ancora del tipo di concezione di restauro purista che affronteranno personaggi come Giovanni Battista Cavalcaselle o Gaetano Previati $^{142}$, le reintegrazioni cromatiche, rifacimenti e velature furono in qualunque caso numerose. Questo si deve allo stato in cui si trovavano le pitture sotto lo strato di calce ma anche, in gran parte, dai meccanismi di pulitura utilizzati all'epoca. In una prima indagine macroscopica sono facilmente distinguibili i segni lasciati dai ferri, dalle martelline e le abrasioni provocate per lo sfregamento di spazzole di ferro nel tentativo di rimuovere lo scialbo.

Nel trattato del conte Giovanni Secco Suardo intitolato "Manuale ragionato per la parte meccanica dell'Arte del Restauratore di dipinti", pubblicato a sue spese nel 1866 e posteriormente rieditato dagli eredi come "II Ristauratore di dipinti $^{\prime 143}$ a Milano nel 1894, si avverte la necessaria differenziazione dei tipi di dipinto prima di progettare il metodo di

\footnotetext{
2 Previati Gaetano (Ferrara 1852-Lavagna 1920). II pittore italiano, allievo di Bertini come Edoardo Berta, fu uno dei massimi esponenti della corrente artistica divisionista, passando dalla scapigliatura, insieme ai già menzionati Pellizza da Volpedo e Giovanni Segantini.

Nella penultima edizione del 1979 il testo del Secco-Suardo è introdotto dal pittore-restauratore Gaetano Previati e consta da commenti di L. de Jasienski sul restauro moderno (Secco-Suardo, 1979).
} 
pulitura. Secco Suardo distingue fra i dipinti "antichi", che lo studioso data prima del $X V I$ sec. e quelli più recenti nella pulitura dello scialbo, poiché per togliere la suddetta imbiancatura bisogna conoscere le particolarità della superficie su cui fu applicata, così egli raccomanda, sui dipinti "moderni", dove gli impasti di colori venivano fatti più densi e dove I'imbiancatura si aderiva più fortemente, un sistema da operare diverso. Sui dipinti antichi consigliava l'uso del sistema di "percussione", il quale consisteva nell'applicazione di piccoli colpi di martellina sullo scialbo, per farlo staccare dal dipinto con l'aiuto, se necessario in qualche punto, di una "spatoletta di corno sottilissima", introdotta fra lo strato dello scialbo ed il dipinto da scoprire in modo di supportarsi con questo strumento nello stacco dello scialbo. Nelle zone più dure si utilizzerebbe per il contrario uno scalpello d'ebanista a modo di raschiatoio [...]maneggiato come il barbiere maneggia il rasoio e muovendolo d'alto in basso[... $]^{144}$. Se possibile, sulle zone suscettibili carenti di ritocchi, il Secco Suardo raccomandava utilizzare l'azione dell'acqua sulla calce, per bagnare uno spazio di un metro quadro. Una volta asciutto, al penetrare fra il dipinto e il bianco di calce, questo si stacca con maggiore facilità.

Un altro metodo citato dal restauratore bergamasco sono le "pezzuole" di tela aderite con colla di farina e poi strappate, metodo che doveva usarsi unicamente quando l'affresco fosse stato ripetutamente imbiancato e con la certezza di non trovare ritocchi a secco, poiché questi potrebbero venire via insieme allo strato di calce. Non è certo quest'ultimo il metodo usato nella cappella Camuzio poiché si sarebbe rischiato eccessivamente un facile strappo della parte dipinta, ma condivide certi aspetti con il procedimento proposto da Ulisse Forni nel suo trattato. Secondo il Forni, come metodo di pulitura dell'imbiancatura, invece di utilizzare le carte o tele incollate (pezzuole), lo strato di calce si staccherebbe dal dipinto originale con dei tamponi di cera prodotti nel seguente modo:

[...] si pone dentro a un vaso di terra vetriato 20 3 chilogrammi di cera vergine; a lento fuoco si fa sciogliere, e sciolta che sia, vi si aggiungono 160 o 200 grammi di trementina di Venezia, menando di quando in quando la massa con una mestola di legno, onde l'una e l'altra si combinino insieme; fuse che siano, si lascia freddare la composizione. Con questa cera così preparata si fanno dei pastelli non più grossi d'un uovo, i quali dal lato maggiore si battono sopra un marmo piano. La parte spianata $e$ compressa di questi si applica sulla superficie imbiancata, e premendola forte e poi ritirandola, staccherà tanto bianco dal muro quanto è quello abbracciato dalla cera.[... $]^{145}$.

Per Ulisse Forni è un sistema meno invasivo a quello dei ferri, che secondo lui è praticato da inesperti.

Se nessuno dei metodi sopracitati sono fattibili o non rendono dei risultati, il conte bergamasco raccomandava di procedere ad operare con gli acidi, in particolare I'acido acetico che secondo lui non altera facilmente i colori come invece lo fanno il nitrico ed il solforico. II modo d'utilizzo descritto è il seguente:

[...] si dovrà anzitutto lavare accuratamente la parte con acqua contenente una moderata dose 
di potassa. Indi, mentre la parte è ancora bagnata si comincerà a soffregare con una piccola spazzola o con un pennello a pelli troncati intinto in una soluzione di acido acetico assai diluito, asportando con una spugna la poltiglia che verrà a provocarsi a cioè fino a quando il pezzo del dipinto risulterà sufficientemente scoperto ma non tutto, lavando poi con acqua pura per togliere ogni residuo di acido, È bene iniziare con una dose leggera che si potrà rinforzare gradualmente se risultasse non troppo attiva[.... $]^{146}$.

Quest'ultimo sistema descritto da Giovanni Secco Suardo, potrebbe essere stato usato nella Cappella Camuzio: il manuale del conte bergamasco, di grande successo, era stato rieditato nel 1918 dall'editore Hoepli e dalla Cisalpino Goliardica nel $1927^{147}$ ed era facilmente accessibile e conosciuto dagli studiosi come Berta. Come accennato precedentemente, nello stato di conservazione in cui si trova la cappella attualmente, è facile distinguere un importante degrado estetico e fisico dovuto alle abrasioni degli strumentali di pulitura ma anche, probabilmente, causato dall'azione di soluzioni come l'acido acetico capace di solubilizzare il carbonato di calcio superficiale dello scialbo ma allo stesso tempo di reagire con il supporto originale creando probabilmente sali solubili. 


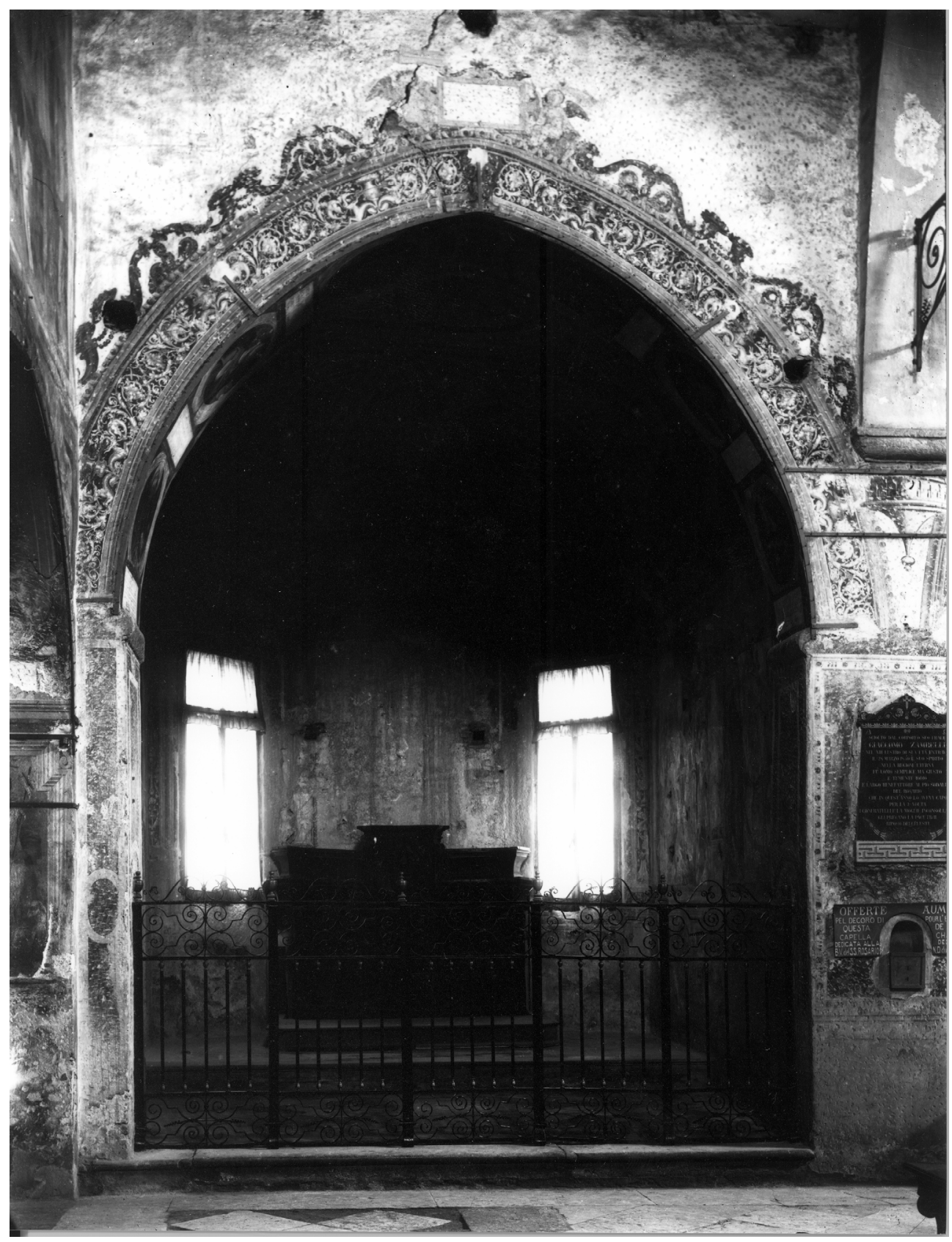

Figura 13 Carboni C., Cappella Camuzio prima del restauro, 1931 ant. Fotografia b/n. AUBCBZ, s.143 Lugano, S. Maria degli Angioli. 


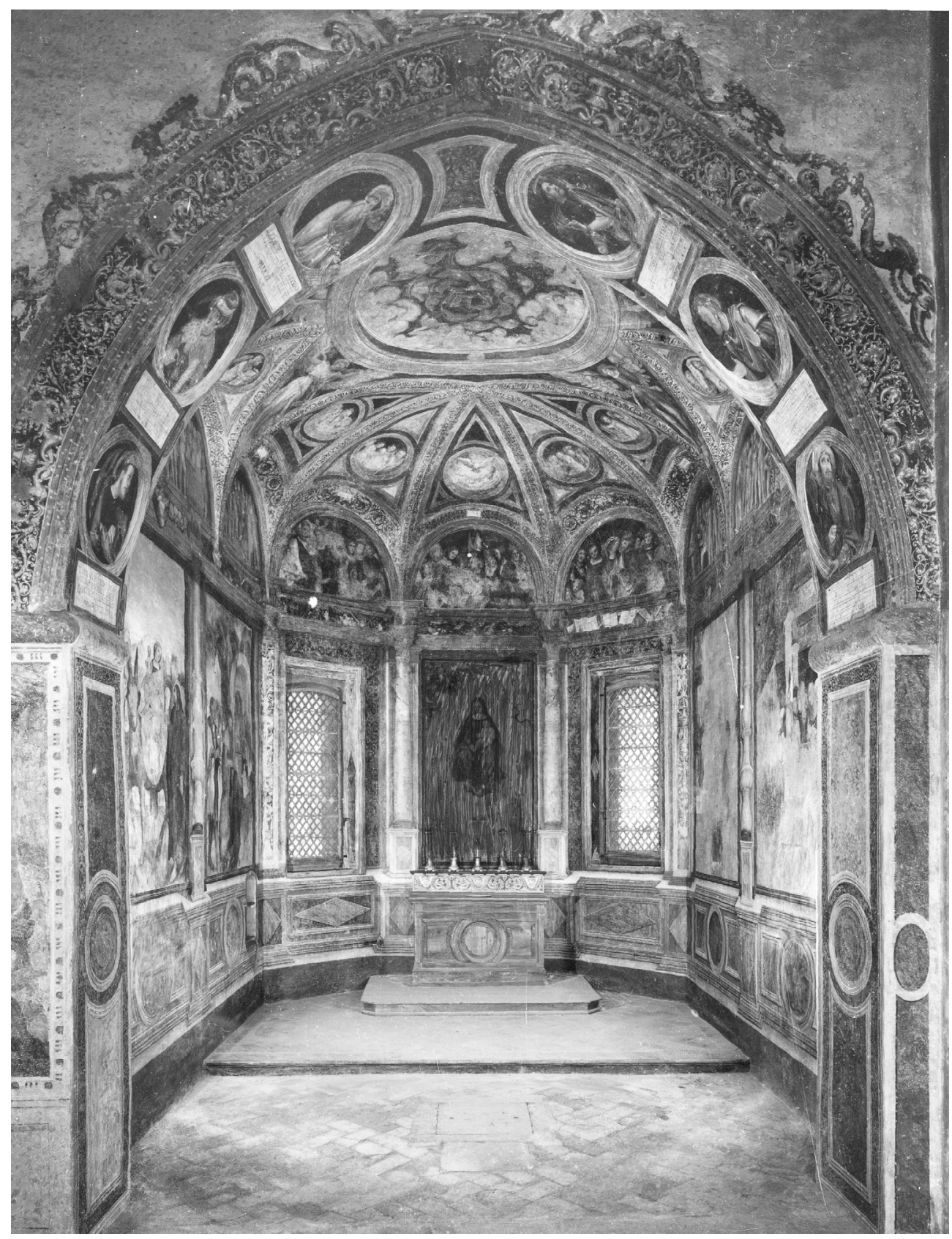

Figura 14 Carboni C., Cappella Camuzio dopo il restauro, 1931 ca. Fotografia b/n. AUBCBZ, s.143 Lugano, S. Maria degli Angioli. 


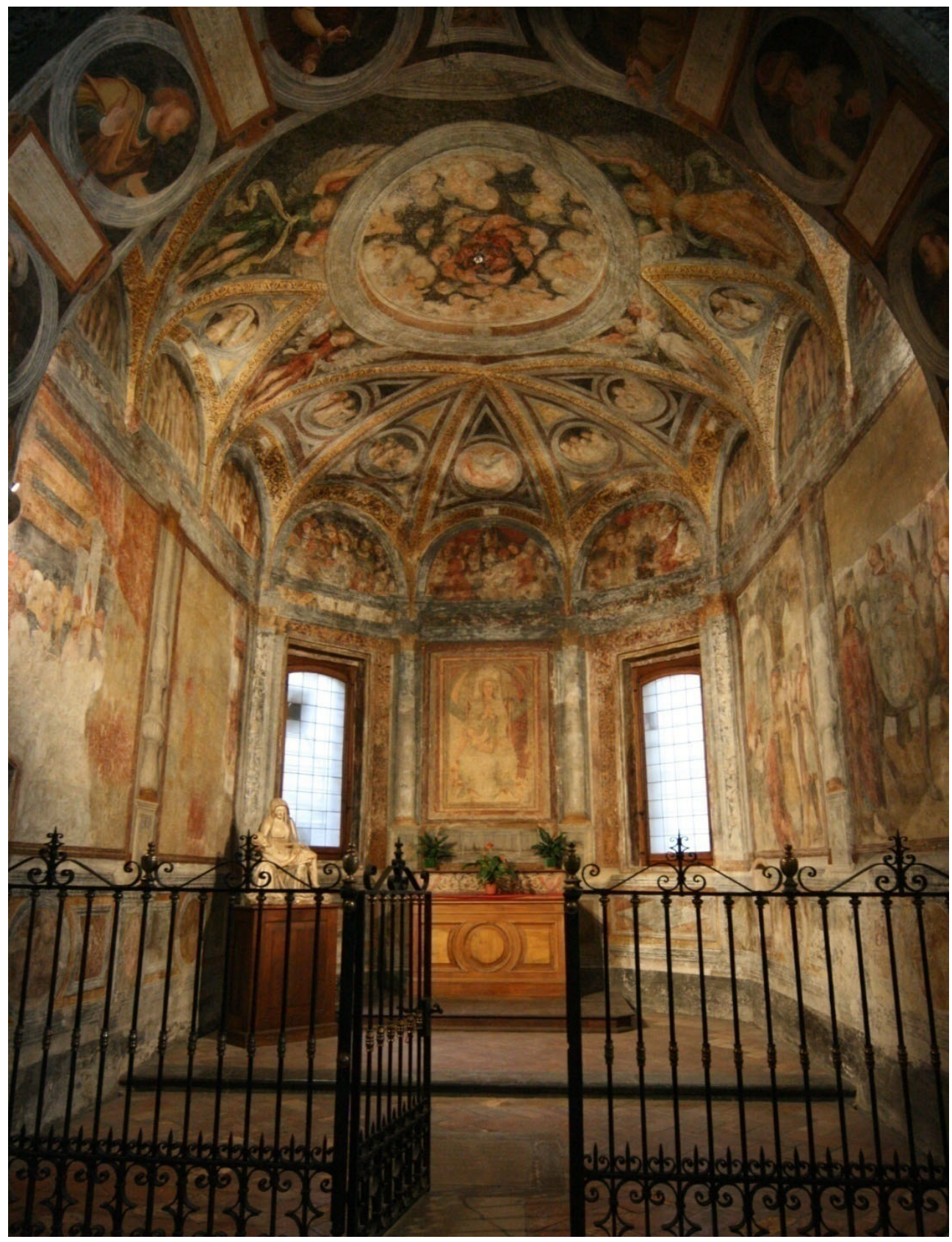

Figura 15 Giner Cordero E., Interno della Cappella Camuzio. Chiesa di S. Maria degli Angioli, Lugano. 2008. 


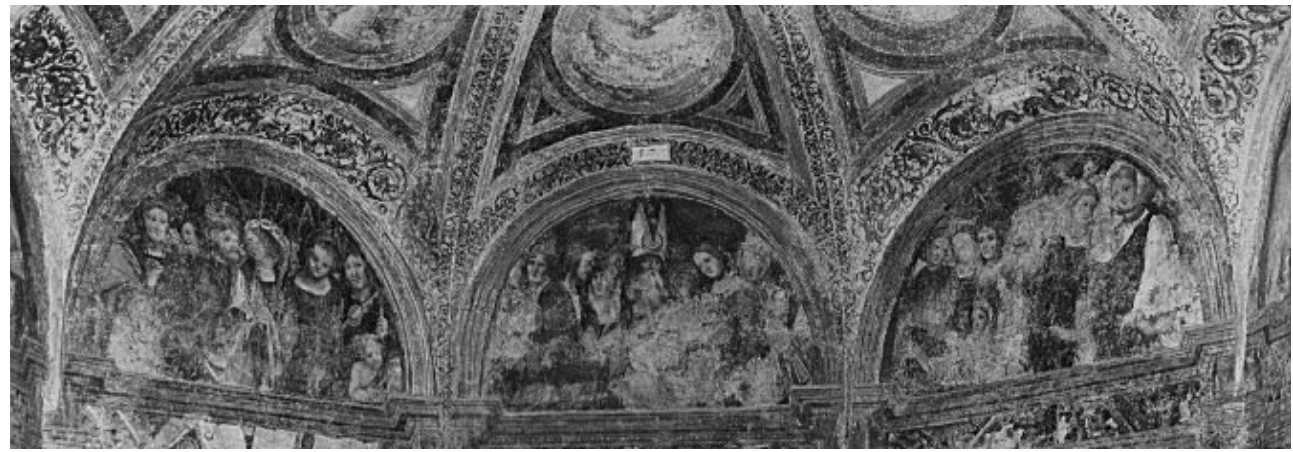

Figura 16 Particolare dei dipinti murali dopo il restauro, Chiesa di Santa Maria degli Angioli, Lugano TI, 1931 circa. Fotografia b/n. 17616b Archivio Federale dei Monumenti Storici. Collezione grafica, Biblioteca nazionale svizzera.

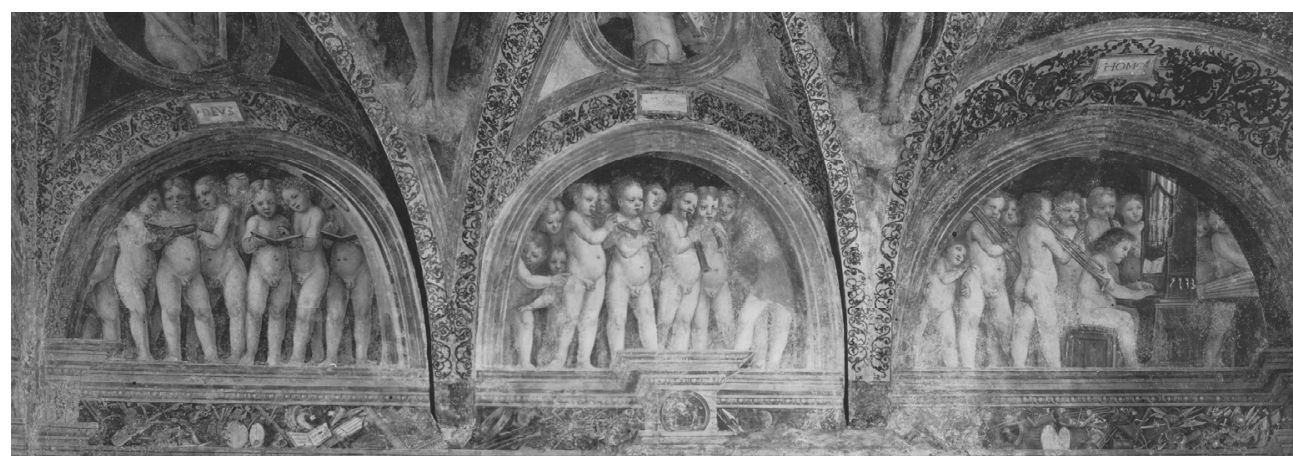

Figura 17 Particolare dei dipinti dopo il restauro, Chiesa di Santa Maria degli Angioli, Lugano TI, 1931 circa. Fotografia b/n. 17628b Archivio Federale dei Monumenti Storici. Collezione grafica, Biblioteca nazionale svizzera.

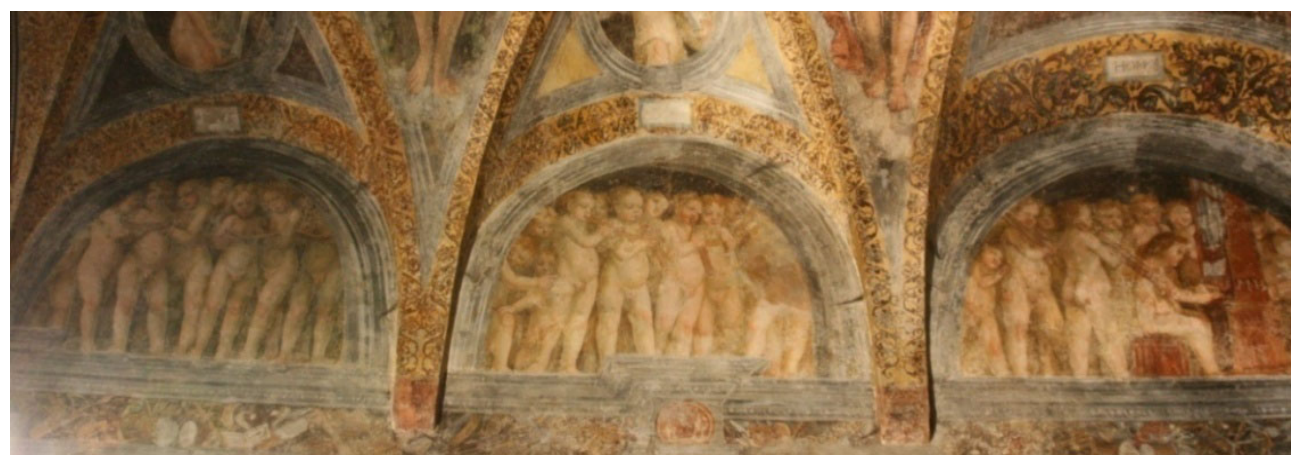

Figura 18 Giner Cordero E. , Particolare dei dipinti sulle lunette della volta della Cappella Camuzio. Chiesa di S. Maria degli Angioli, Lugano. 2008. 


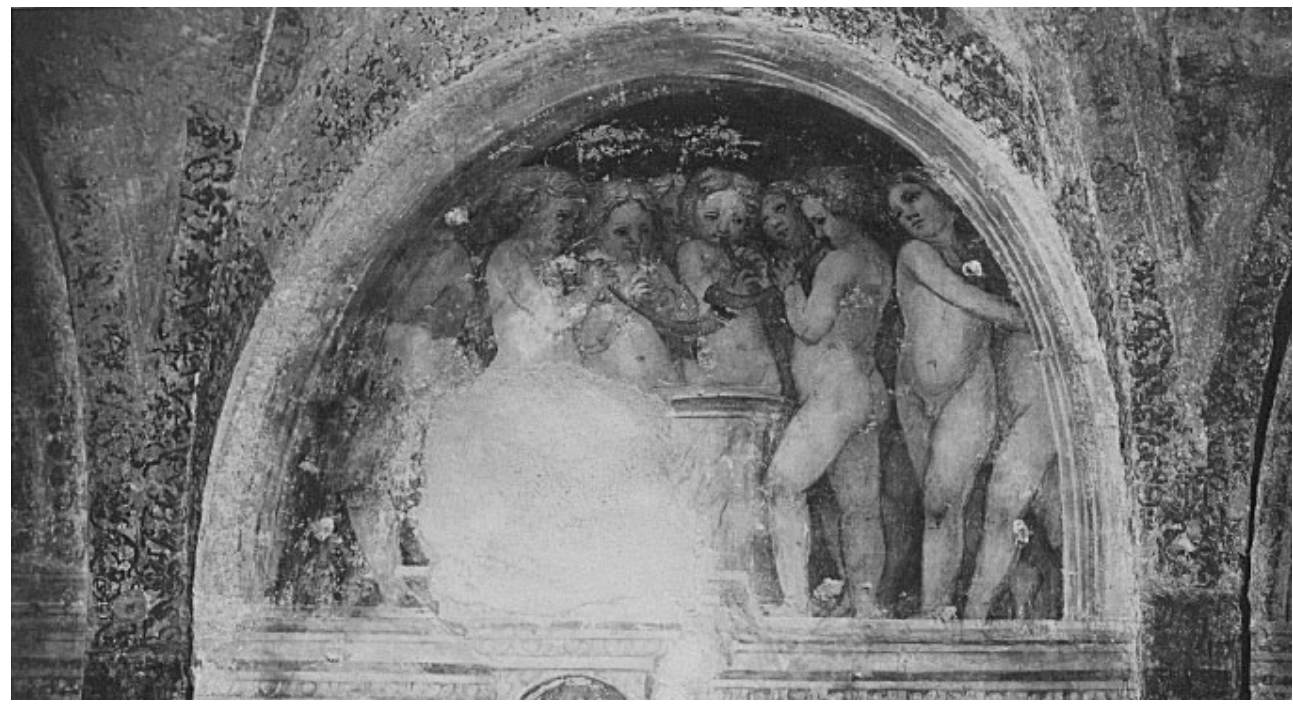

Figura 19 Particolare dei dipinti della cappella Camuzio durante i restauri, Chiesa di Santa Maria degli Angioli, Lugano, Fotografia b/n. TI. 14132 Archivio Federale dei Monumenti Storici. Collezione grafica, Biblioteca nazionale svizzera.

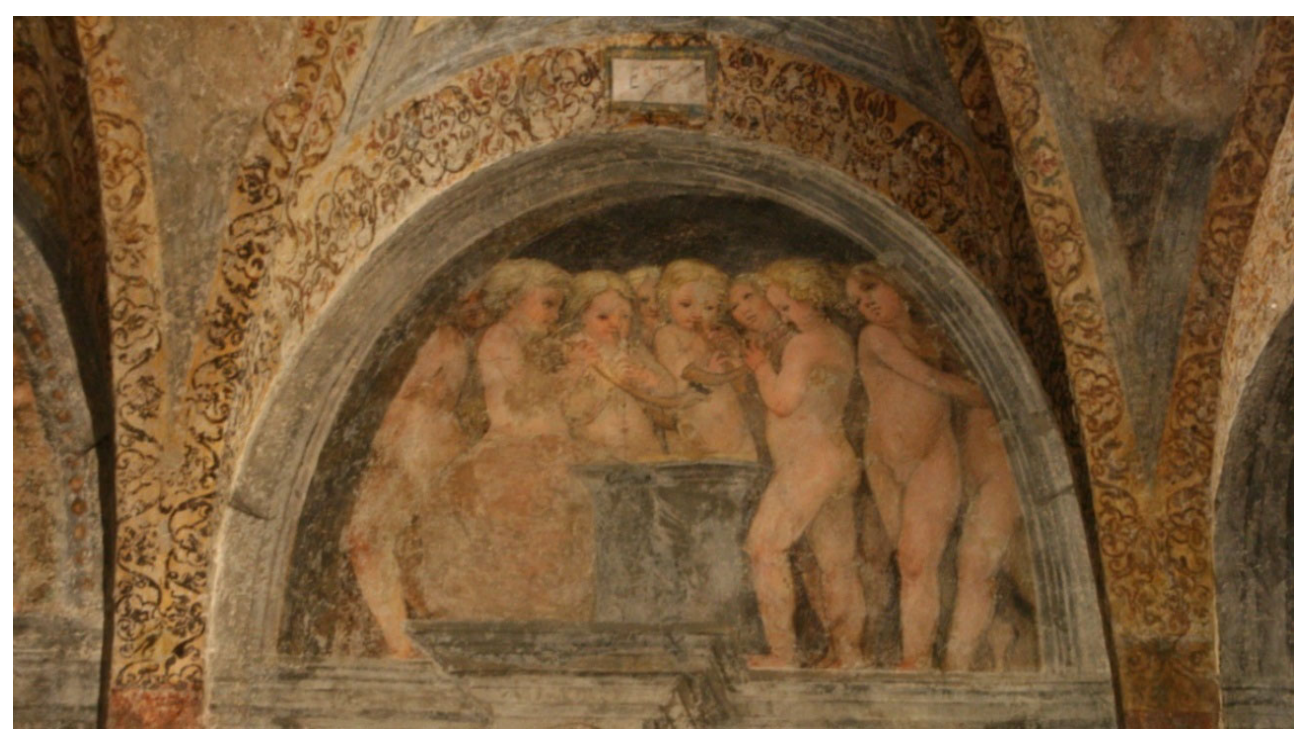

Figura 20 Giner Cordero E. Particolari dei dipinti della cappella Camuzio. Chiesa di Santa Maria degli Angioli, Lugano. 2008. 
Anche se le fotografie ad ultravioletto eseguite per questa ricerca non hanno evidenziato chiaramente la presenza di materiale ceroso, a causa delle condizioni di illuminazione poco idonee, le metodologie impiegate da E. Berta indicano invece come piú che fattibile questa possibilità. Nel 2003 una tesi di bachelor presentata alla Scuola Universitaria Professionale della Svizzera Italiana dalla restauratrice Simona del Pietro con il titolo "Reversibilità e irreversibilità negli interventi di restauro della prima metà del Novecento in Ticino" conferma, in parte, l'esistenza di cera nella sudetta Cappella Camuzio, mediante gli FTIR realizzati su due campioni analizzati dal Laboratorio tecnico Sperimentale (LTS). La cera fu applicata a modo di finitura del restauro come pellicola protettiva degli affreschi, ma anche per potenziare i colori di essi che dovevano avere una apparenza debole dopo i trattamenti di pulitura dello scialbo.

[...]Qualsiasi affresco che sia stato liberato dalla imbiancatura coi mezzi sopra accennati, appare sempre ricoperto, dopo che sarà ritornato allo stato asciutto, da un leggero velo bianco che offusca il dipinto. Questa velatura potrà venire completamente tolta unicamente attraverso le seguenti operazioni: prendere della "paraffina disciolta nella benzina"148 e bagnare sobriamente un grosso fiocco di cotone e con questo strofinarne tutto il dipinto, ripetendo questa operazione quanto occorra e sempre bagnandone il cotone. Dovrà usarsi una grande moderazione, per quanto le sostanze adoperate

\footnotetext{
${ }^{148}$ La ricetta di Secco Suardo consiste nel mescolare una parte di paraffina con dieci di benzina in un contenitore trasparente chiuso ed esposto per alcune ore al sole o a bagno maria fino alla dissoluzione. (Piva, 1988, p. 262).
}

(paraffina e benzina), appartenendo al regno minerale (si ricavano dal carbone fossile) "non siano da temere" come la cera e l'acquaragia che taluno adopera $e$ che purtroppo danneggiano il colore perché lo polverizzano. La semplice operazione di cui sopra varrà a ridonare al dipinto tutta la sua vivezza primitiva. $\mathrm{Nel}$ caso che il fresco appartenga alla categoria degli affreschi antichi, $i$ quali hanno una "particolare lucidezza", questa si potrà di nuovo realizzare ripetendo l'operazione quanto occorra $e$ (non appena il dipinto sarà ben asciutto) strofinando con una "pezza di lana" od una "morbida spazzola"[...] $]^{149}$.

Della cera sciolta in "acqua di ragia rettificata" ${ }^{150}$ che Giovanni Secco Suardo critica perché trova pericoloso per il dipinto, ne parla anche il restauratore Ulisse Forni nel suo trattato. Egli spiega la prassi esecutiva di Guglielmo Botti in 1870 per il consolidamento dei dipinti di Benozzo Gozzoli ${ }^{151}$ nel Camposanto di Pisa $^{152}$ : la cera, secondo il restauratore toscano, veniva sciolta ed applicata calda sulla superficie pittorica sopra la quale il restauratore ritoccava più tardi con dei colori sciolti nello stesso encausto. I risultati si consideravano equiparabili a quelli ottenutosi mediante il consolidamento con due mani di "tempera caseosa" spenta con l'acqua, dopo di ché

\footnotetext{
${ }^{149}$ (Piva, 1988, p. 219-220).

Mentre la benzina venne prodotta dalla distillazione del petrolio fra i 60 ed i 100 C, l'acquaragia è ottenuta da una miscela d'idrocarburi frutto da distillazioni frazionate.

${ }^{151}$ Gozzoli Benozzo (Firenze, 1420 ca. - 1497)

152 I dipinti eseguiti da Benozzo Gozzoli nel Camposanto di Pisa, datati nel 1484, conformano un ciclo di affreschi raffiguranti le Storie dell'Antico Testamento. Questa serie fu iniziata da Piero di Puccio fra il 1389 ed il 1391
} 
anche i ritocchi dovevano praticarsi con dei colori temperati con la caseosa.

Il restauro della Cappella Camuzio arriva a suo termine nell'anno 1924. Dopo questo lavoro, il più recente intervento sul "contenitore" delle pitture fu eseguito solo nel 1968 da Gianfranco Rossi. Il progetto dell'architetto ticinese interessava l'eliminazione dell'umidità di risalita, che danneggiava le pareti e così anche gli affreschi, mediante la creazione di un intercapedine interno ed un'altro esterno intorno ai muri per liberare la pavimen-tazione dal terreno umido. Non si ottiene invece nessun risultato soddisfacente nell'esecuzione poiché i muri in cemento dell'intercapedine limitano l'evaporazione d'acqua ad un livello inferiore al quale si trovano gli affreschi.

Lo stato di conservazione in cui si presenta oggi l'opera della Cappella Camuzio è molto grave, come si può osservare dalla documentazione fotografica allegata. Di fatto sono sconfortanti i paragoni fra le fotografie in bianco e nero conservate nell'Archivio dell'Ufficio dei Beni Culturali a Bellinzona ed a Berna, realizzate nel 1931 pochi anni dopo la finalizzazione del restauro, e quelle eseguitesi oggi. Lo zoccolo dipinto della Cappella Camuzio, nella maggior parte reintegrato, è praticamente perso o illeggibile e dentro della sala si respira tutt'altro che l'armonia tanto ricercata da Berta durante l'intervento; in cambio, entrando in essa si avverte l'abbandono in cui è stato nuovamente rilasciato il monumento.

Nel 18 luglio 1924 la Commissione dei Monumenti incarica Edoardo Berta il restauro architettonico ed artistico completo della chiesa. Questo ebbe luogo unicamente dopo il collaudo del preventivo presentato in 19 maggio 1927 per l'architetto Pietro Giovannini ed il sopracitato Berta.

Il preventivo concepisce due momenti operativi, dove si propone in primo luogo una ricostruzione architettonica con la proiezione del restauro della facciata principale della chiesa, lo scrostamento delle tre cappelle laterali, la riparazione dell'intonaco guasto, la ricostruzione di un rosone con lettere cristiane di $16.80 \mathrm{~m} 2$ ed il proseguimento delle ricerche archeologiche così come il restauro della pavimentazione e degli affreschi presenti sulle pareti della navata centrale, compreso il trasporto su tela di certi dipinti considerati da Berta di poco valore, secondo le sue parole [...]utile di levare perché non disturbino l'armonia dell'ambiente originario[....$^{153}$ - e lo stacco dell'affresco barocco della cappella di San Teodoro, in modo da permettere la visione di altre figure e decorazioni trovate sull'arco nella seconda cappella.

$\mathrm{Fu}$ questo un intervento significativo, ancora una volta, degli ideali dell'epoca sul ripristino dei monumenti storici in Ticino ma anche nel resto d'Europa: vengono cancellati gli eventi più recenti della chiesa per il rinnovamenti estetico in modo da fare rinvenire quelli medievali.

Testimoniano i due momenti storici, sull'esterno della fabbrica, una cartolina degli inizi del XX secolo dove di fondo si apprezzano le caratteristiche della facciata della chiesa, con il rosone predominante in facciata, ed una fotografia del 1932 dove il

(Berta, Edoardo. Preventivo delle spese per il restauro. AUBCBZ s. 143 Lugano, S. Maria degli Angioli). 
rosone condivide lo spazio con le due finestre barocche ritrovate e, mentre la parte inferiore del muro è rivestita da laterizi regolari, la zona superiore che reca il rosone, divisa mediante un cornicione, è intonacata.

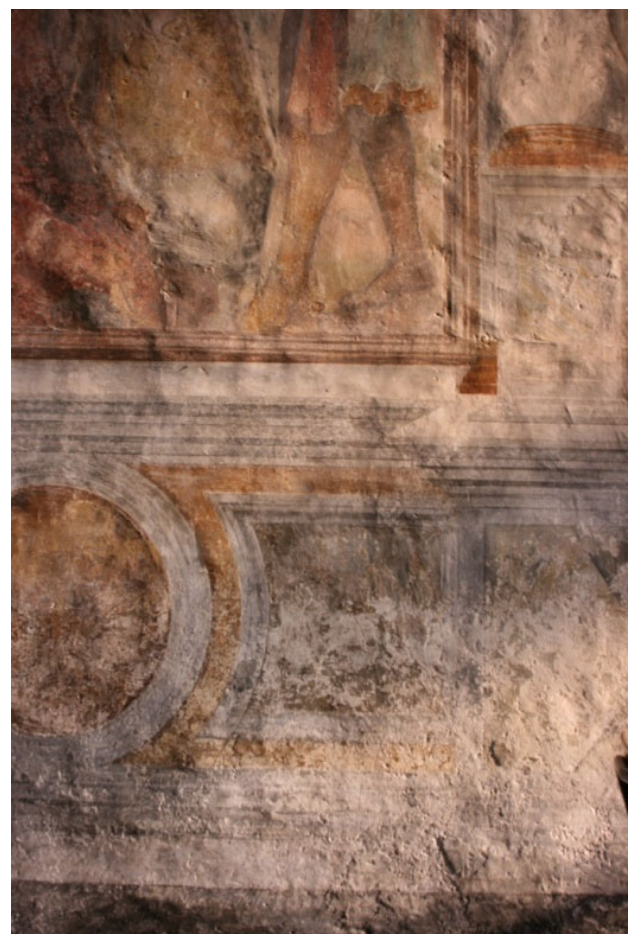

Figura 21 Giner Cordero, E. Cappella Camuzio,Chiesa di S. Maria degli Angioli, 2008. Fotografia di particolare a luce radente dello stato di conservazione attuale dei dipinti.

In un secondo periodo, nell'anno 1929, la ditta Tiefbohr und Baugesellschaft di Zurigo, sotto la direzione dell'architetto e professore del Politecnico Federale Gustavo Guhl, dell'architetto aiutante Americo Marazzi e dell'ingegnere Francesco Riva, realizza le operazioni di consolidamento murario della chiesa degli
Angeli, limitando i danni causati dall'umidità di risalita capillare.

Edoardo Berta propone inoltre il restauro del campanario, dell'abside del Coro dei Frati con la pulitura dell'intonaco di calce ed il ritocco delle decorazioni, dell'altare maggiore e del presbiterio, la pulitura ed il restauro degli affreschi del coro e di quelli sulla volta ed il trasferimento su tela dell'affresco barocco di $8.80 \mathrm{~m}^{2}$ esistente su quest'ultima.

La mano d'opera che intervenne nei due momenti operativi trattati si componeva principalmente, per il restauro pittorico, di allievi dell'Accademia di disegno, allievi del professore Berta, e pittori ticinesi. Fra $\mathrm{i}$ nomi che troviamo nei diversi contratti di lavoro sono da mettere in rilievo Emilio Ferrazzini ${ }^{154}$, Tito Tettamanti, Alfonso Cattaneo, Carlo Cotti e Basilio Rusca.

\footnotetext{
${ }^{154}$ Le fatture riguardo i lavori eseguiti da Ferrazzini dopo il 1930, ci confermano l'esecuzione del restauro nelle opere di restauro dei dipinti murali e su diverse tele. In un elenco elaborato dal Maestro Giuseppe Rovelli il 20 maggio 1931, si elencano le spese per materiale ed ore lavorative di Emilio Ferrazzini. Qui troviamo alcuni dei prodotti procurati dal medesimo restauratore per le operazioni di restauro: anacrosina, ceresina e colori. Sarebbe stato di grande interesse trovare le quantità in cui furono acquistati questi materiale, specialmente per l'anacrosina e per la ceresina. La prima, fortunatamente di uso isolato per la pulitura dei dipinti ad olio, è una miscela di saponi, ammoniaca, alcool ed acqua, simile ai prodotti attualmente in commercio come il Contrad 2000 ed il Vulpex. II rischio del suo impiego si trova nella basicità della miscela: a $\mathrm{pH}$ maggiore di 8,5 potrebbe generare la solubilizzazione delle vernici olio-resinose e degli oli successivamente. La ceresina o cera ozokerite purificata, le cui caratteristico vengono approfondite nel fu sicuramente usata nel ravvivamento dei dipinti murali, secondo la tecnica messa in pratica da Edoardo Berta sui dipinti della Cappella Camuzio e che più tardi il pittore Ferrazzini impiegasse nel completamento del restauro delle decorazioni pittoriche della chiesa di Santa Maria degli Angioli.
} 


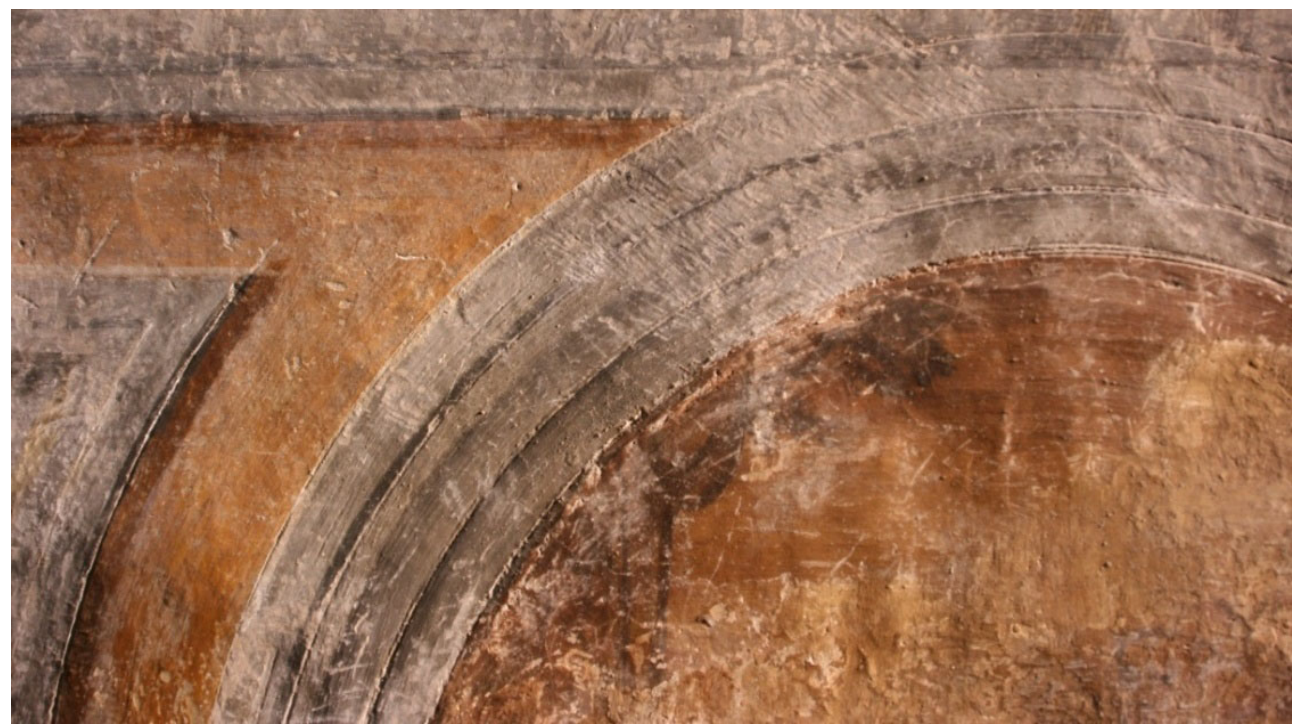

Figura 22 Giner Cordero, E. Particolare dei dipinti della Cappella Camuzio. Segni delle martelline utilizzate nelle operazioni di descialbo. Chiesa di Santa Maria degli Angioli, Lugano. Enero 2008.

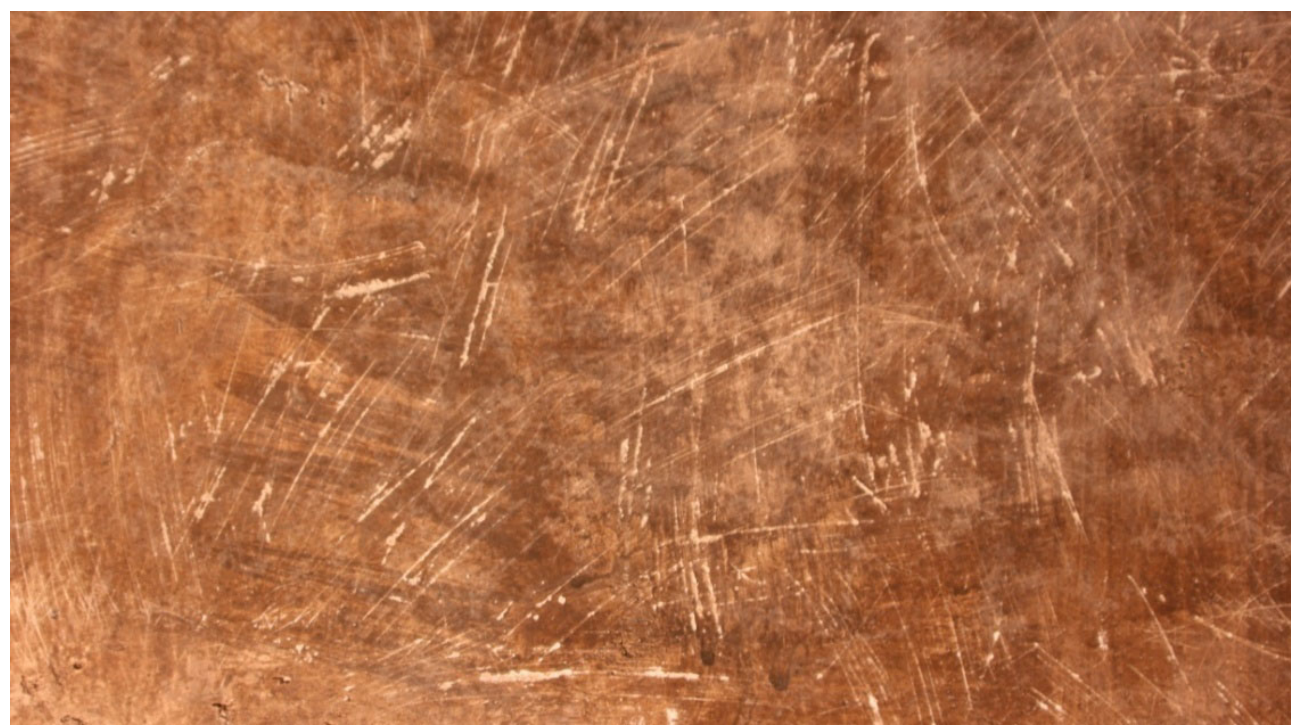

Figura 23 Giner Cordero, E. Particolare dei dipinti della Cappella Camuzio. Segni d'abrasione dai ferri utilizzati per le operazioni di descialbo. Chiesa di Santa Maria degli Angioli, Lugano. Enero 2008. 
Restauratori in Canton Ticino fra Ottocento e Novecento

Catalogazione e gestione dati

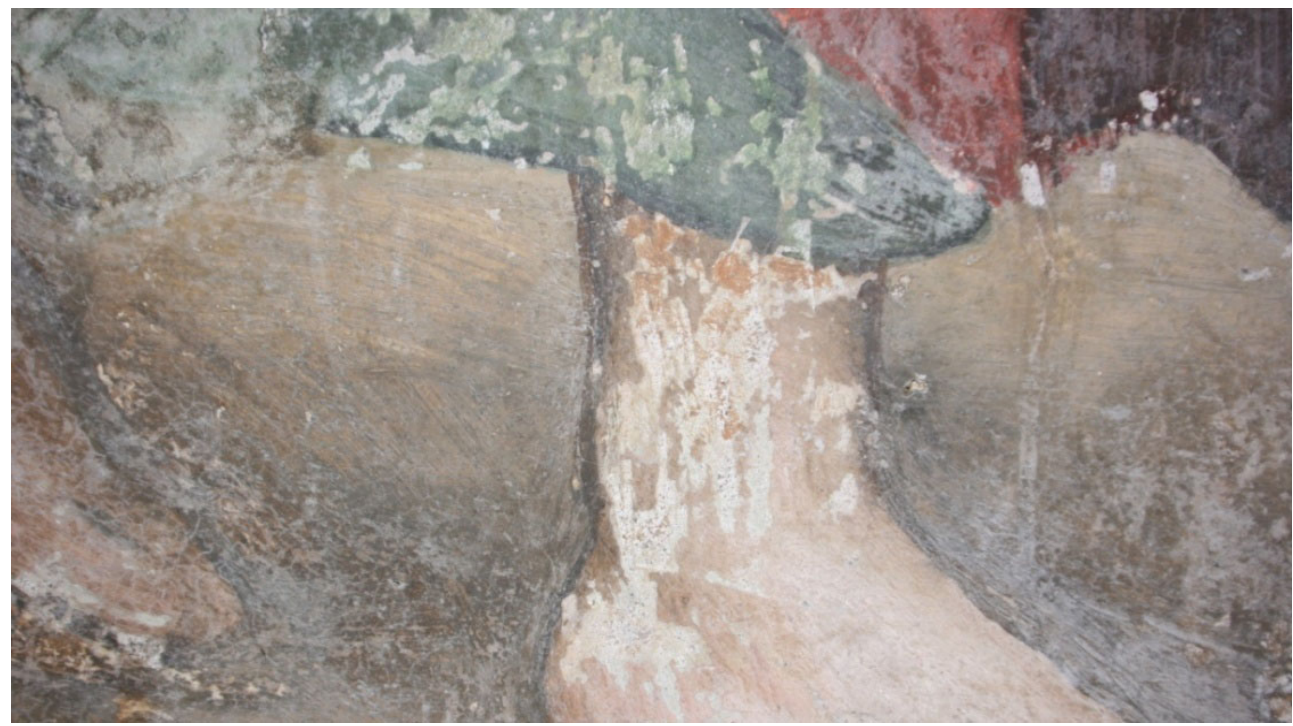

Figura 24 Giner Cordero E. Particolare dei dipinti della Cappella Camuzio. Segni delle martelline e dei ferri usati nello descialbo delle pitture. Chiesa di Santa Maria degli Angioli, Lugano. Gennaio 2008.

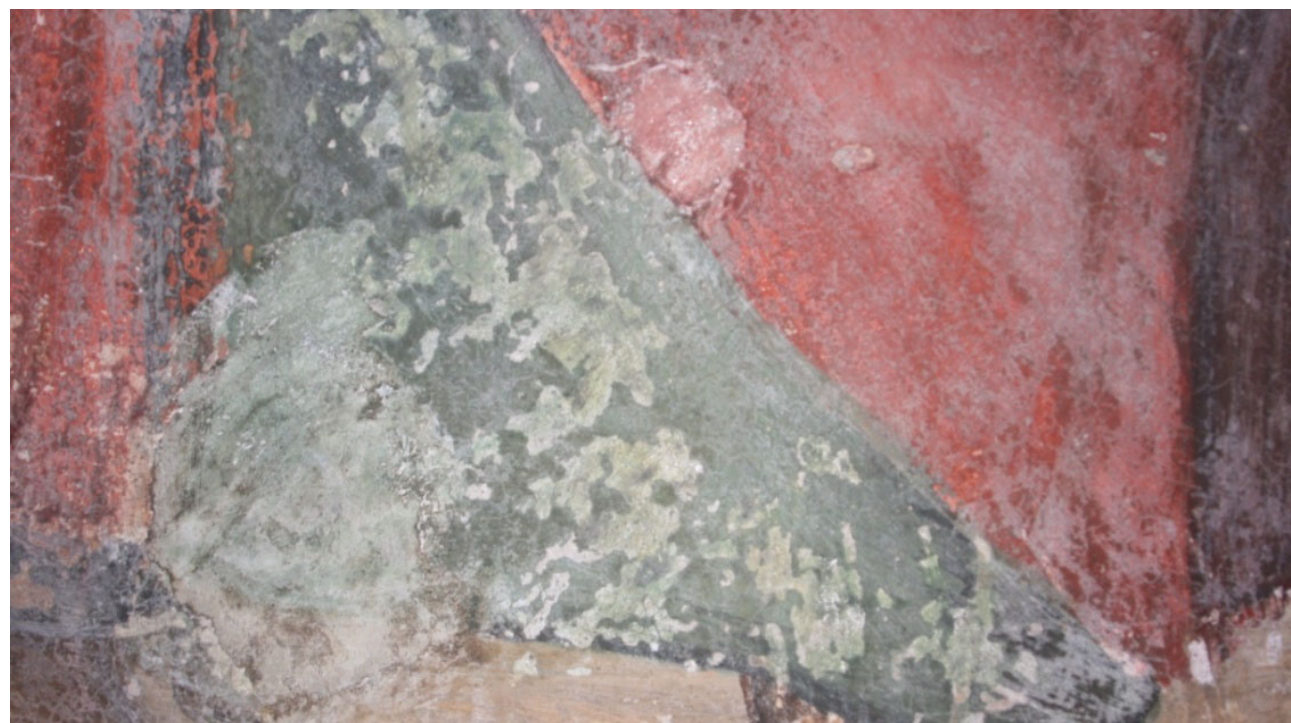

Figura 25 Giner Cordero, E. Particolare dei dipinti della Cappella Camuzio. Dettaglio del ritocco a basso tono delle lacune. Chiesa di Santa Maria degli Angioli, Lugano. Gennaio 2008. 


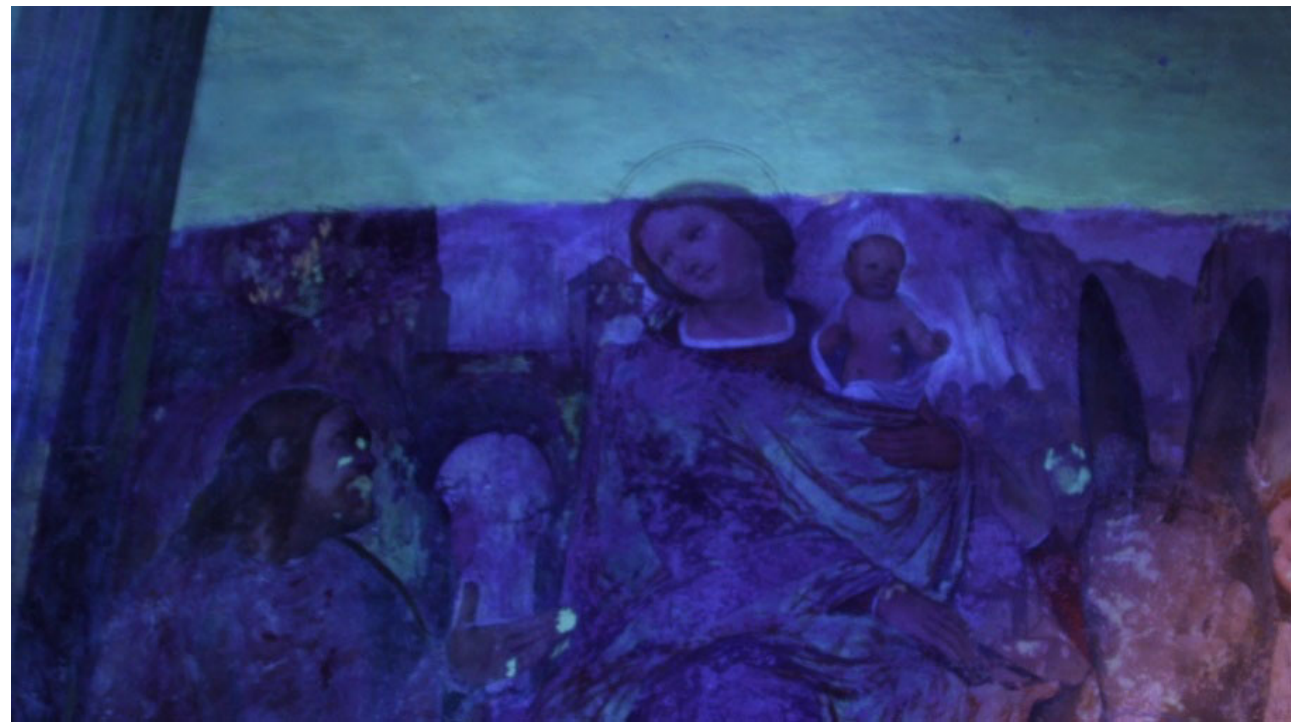

Figura 26 Giner Cordero, E. Particolare dei dipinti della Cappella Camuzio dove si evidenziano le aggiunte e ritocchi cromatici. Chiesa di Santa Maria degli Angioli, Lugano. Gennaio 2008. Fotografia UV.

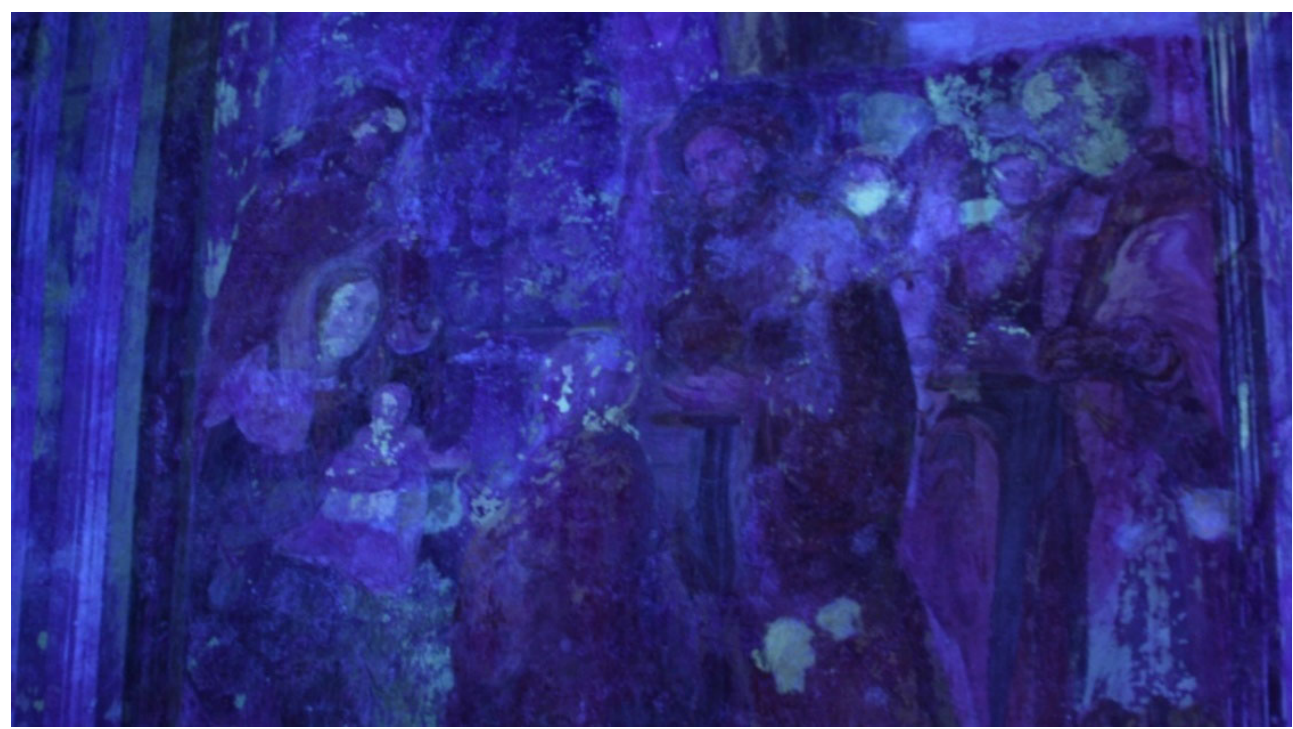

Figura 27 Giner Cordero, E. Particolare dei dipinti della Cappella Camuzio dove si evidenziano le aggiunte e ritocchi cormatici. Chiesa di Santa Maria degli Angioli, Lugano. Gennaio 2008. Fotografia UV. 


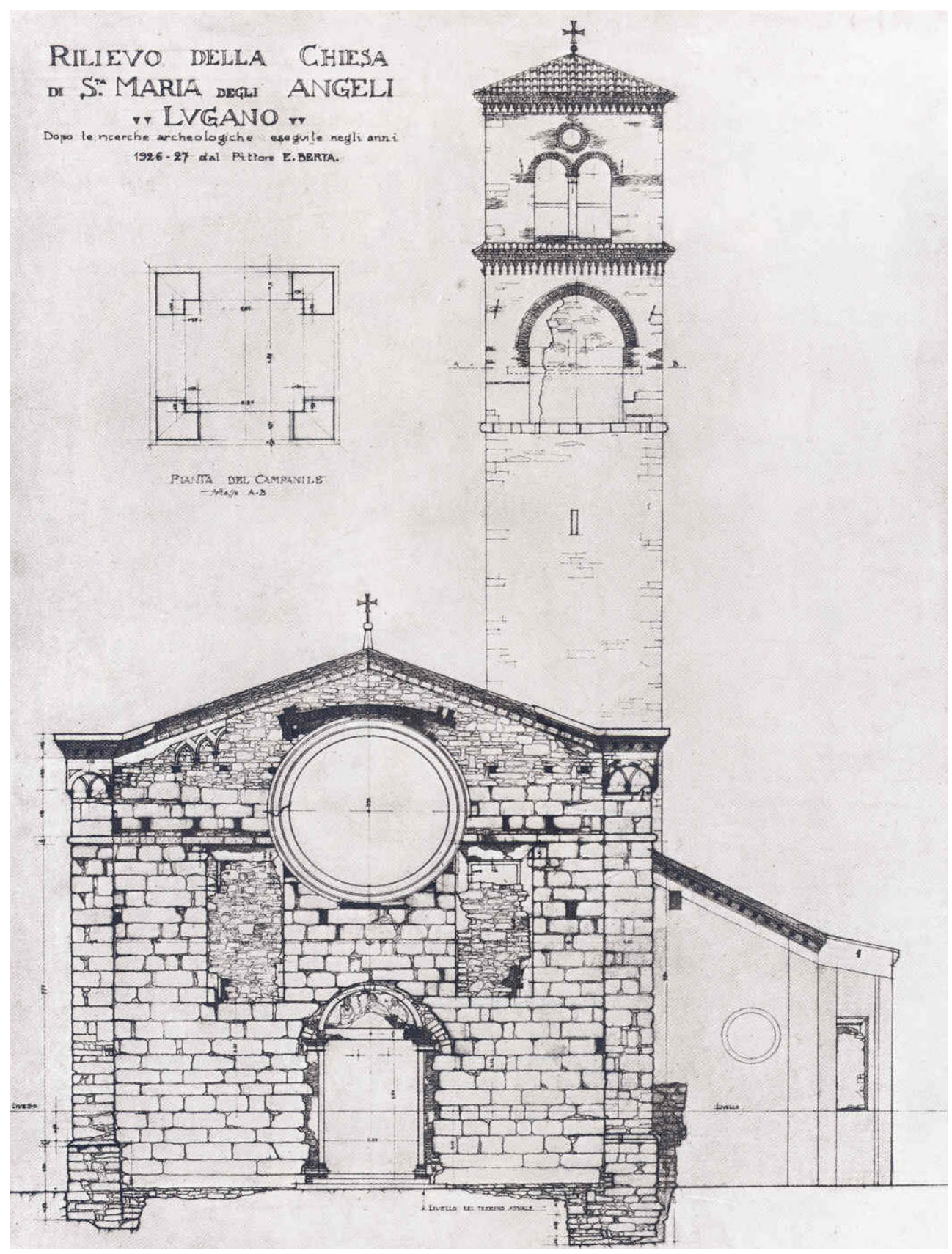

Figura 28 Giovannini Pietro, Rilievo della Chiesa di Santa. Maria degli Angeli a Lugano, studio della facciata dopo le ricerche archeologiche del 1926 al 1927 dal Pittore Edoardo Berta. (Marcionetti, 1975, p. Tav.90) 


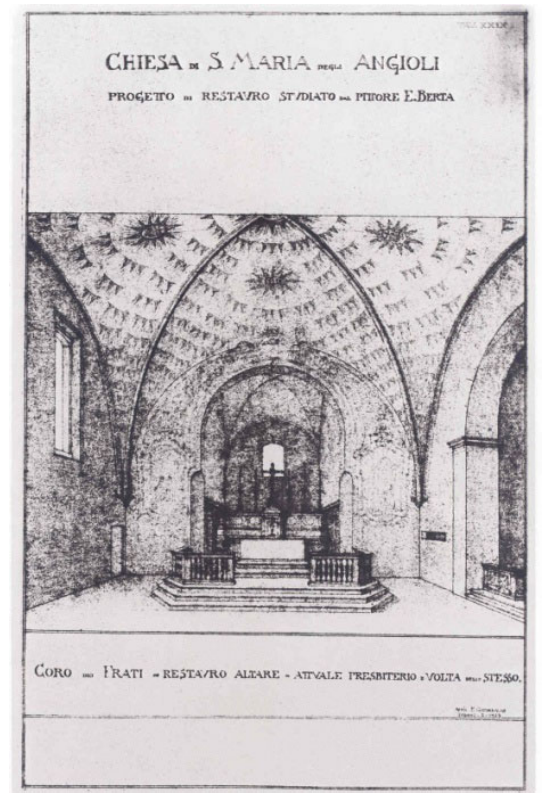

Figura 29 Berta Edoardo, Progetto di restauro studiato. Coro dei Frati. Restauro altare. Attuale presbiterio e volta dello stesso. 1926-1927. (Marcionetti, 1975, p. 96).

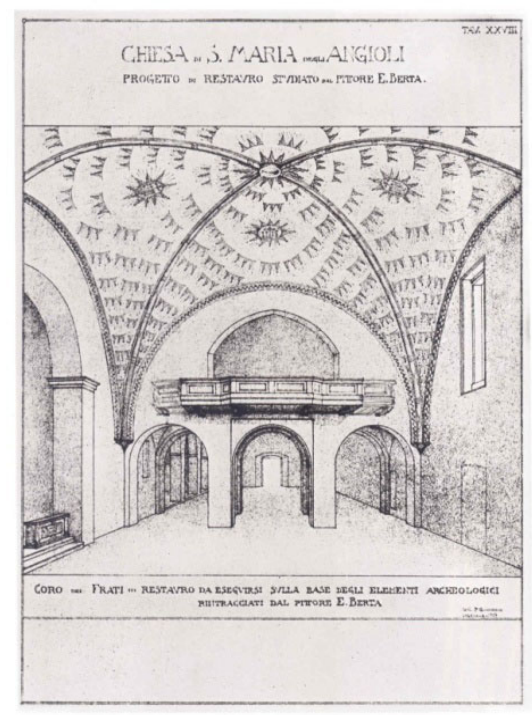

Figura 30 Berta Edoardo, Progetto di restauro studiato.Coro dei Frati. Restauro da eseguirsi sulla base degli elementi archeologici rintracciati. 1926-1927.

(Marcionetti, 1975, p. Tav.105). 


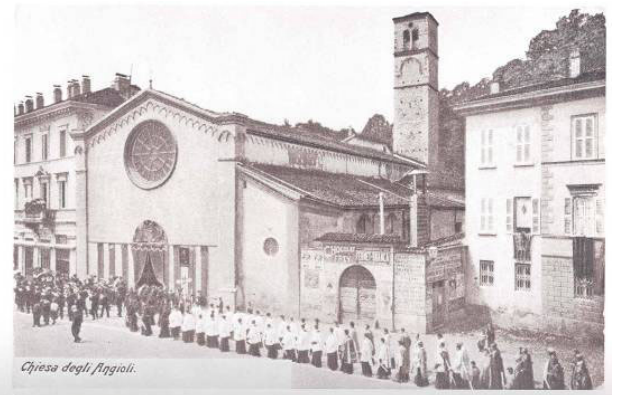

Figura 31 Chiesa di S. Maria degli Angioli, inizio XX secolo. Prima del restauro di Edoardo Berta. Stampa. (Marcionetti, 1975, p. Tav.87)

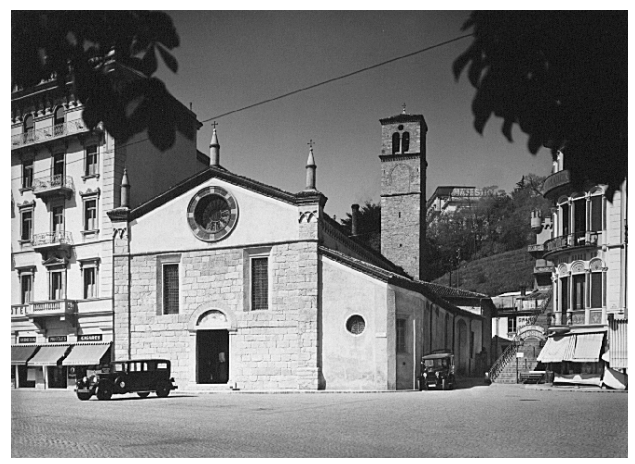

Figura 32 Sammlung Werhli, Chiesa di S. Maria degli Angioli., 1932. Negativo Num. B. 39399. AFMS, Berna.

All'Archivio dell'Ufficio dei Beni Culturali di Bellinzona si sono trovate fatture che ci dimostrano la collaborazione nel restauro con la ditta di Zurigo Christian Schmidt ${ }^{155}$, che non solo somministrava il materiale di restauro, pitture ed utensili vari ma anche mano d'opera. Creata dal pittore e decoratore Friedrich Christian Schmidt

\footnotetext{
${ }^{155}$ Ulteriore informazione riguardo la firma famigliare Chr. Schmidt, ancora oggi dedicata alla decorazione, c si trova in una esemplare tesi di diploma presentata dalla restauratrice Mirjiam Julien alla Scuola Superiore HKB Konservierung un Restaurierung di Berna, nel 2005. La copia della tesi di diploma è attualmente consultabile presso l'archivio della medesima scuola.
}

(1835 - 1911) in 1882, la firma era la distributrice ufficiale dei prodotti della “Keim Mineralfarben" in Svizzera ${ }^{156}$, ma fu dopo il primo intervento di restauro di Christian Jakob Schmidt figlio (1862 1937), nella "Weisser Adler" in Stein am Rhein, quando si farà del restauro la nuova specialità dell'impresa, la cui attività si svilupperà in tutto il territorio svizzero.

Particolarmente, nei lavori sulla chiesa di Santa Maria degli Angeli intervengono Christian Schmidt "sohn", (figlio) del fondatore della ditta, ed il pittore dipendente Emilio Dillena, domiciliato a Taverne. Invero una delle sopracitate fatture spedita da "Chr. Schmidt Dekorationsmaler, Zürich 5, Hafnerstase 47" al professore Berta il 15 Novembre $1930^{157}$ si calcola un totale di 182 ore pagati a Fr.4.50 per i rendimenti completi dal pittore Emilio Dillena nella chiesa di Santa Maria degli Angioli e dei materiali somministrati per il restauro, dove si elencano: mezzo chilogrammo di Speziallack a fr. 4.25 - che potrebbe trattarsi di gomma lacca-, due kilogrammi di Kasein (caseina) a fr. 4, 1 Schwamm (spugna) a fr. 2, Diverse Mineralfarben

\footnotetext{
${ }^{156}$ La conosciuta ditta tedesca pubblica nel 1928 un elenco delle opere realizzate in Svizzera utilizzando i suoi colori minerali. In Ticino si enumerano quindici edifici: a Locarno il Palazzo Pedrazzini (1915), Villa Mercedes (1915), Villa Bankdirektor Gianella (1917), Palazzo Immobiliare (1918), Palazzo Soc. Elettrica (1924), il Golf-Hotel di Murano (1925), il Kursaal (1926), la Chiesa di Sant'Antonio (1926), la Villa dell'arch. E. Cavadini (1926), Villa Dr. Varesi, Villa Lanfranchi a Tegna (1927) e Villa Regina e Villa Suter a Tenero, con ottimi risultati verificati dagli operatori. ${ }^{157}$ (Schmidt Christian, söhne. Conto. Chiesa di S. Maria degli Angioli in Lugano indirizzato a Edoardo Berta, Lugano. Zurigo 15 novembre 1930. AUBCBZ s. 143 Lugano, S. Maria degli Angioli).
} 
(pitture minerali) fr. 12.80, e Aetzflüssigkeit (liquido corrosivo, forse trattasi di acido acetico) a fr. 4.50, del quale non si specifica la quantità che potrebbe però facilmente riferirsi ad acido acetico, come citato prima dalle metodiche di restauro impiegate per la pulitura dei dipinti del carbonato di calcio superficiale.

La ditta Schmidt si dimostrerà in questa ricerca acerrima collaboratrice di Edoardo Berta nei più importanti restauri eseguiti in Ticino, come ad esempio il Castello Visconteo di Locarno e la Chiesa della Madonna delle Grazie a Bellinzona. Sono unicamente tre interventi, tra 170 casi circa, che la firma di decoratori specializzata portò a termine in Svizzera, e fra i cinque realizzati in Canton Ticino, sommandosi ai sopracitati: la Casa del Negromante a Locarno e Vaglio. II rappresentante principale della ditta Schmidt nei cantoni italofoni della Svizzera, cioè nel Ticino e nel Grigione italiano fu sempre il pittore Dillena, il cui intervento è facilmente distinguibile poiché era abituato a firmarlo e datarlo. Esso risulta in Santa Maria del Castello a Mesocco (Grigioni), dove lavora sulle decorazioni interne dell'edificio in modo lodevole, con l'utilizzo di un ritocco pittorico illusionista, d'accordo con la sua professione di decoratore $\mathrm{ma}$ in qualunque modo evitando la ridipinttura generalizzata dell'opera ma limitandosi a piccole aree d'intervento.

Nello specifico caso della chiesa di Santa Maria degli Angioli a Lugano, fra le attività svolte dalla ditta zurighese si può documentare inoltre l'elaborazione del telaio di sostegno per le strutture degli affreschi strappati, ad incarico di Berta.

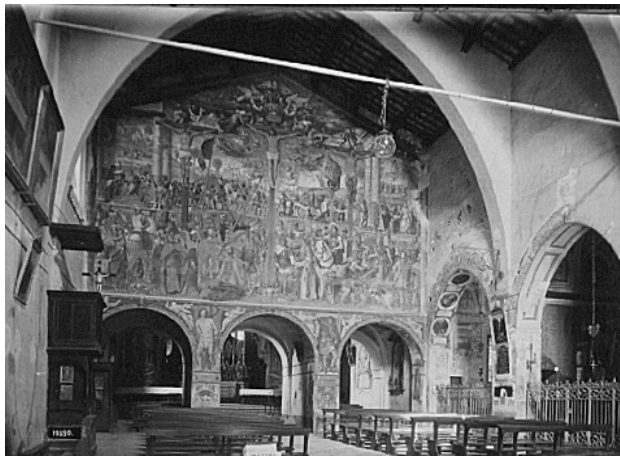

Figura 33 Sammlung Wehrli, Interno della chiesa di S. Maria degli Angioli dopo il restauro. Fotografia b/n. Negativo Num. A.10890, 1932. AFMS, Berna.

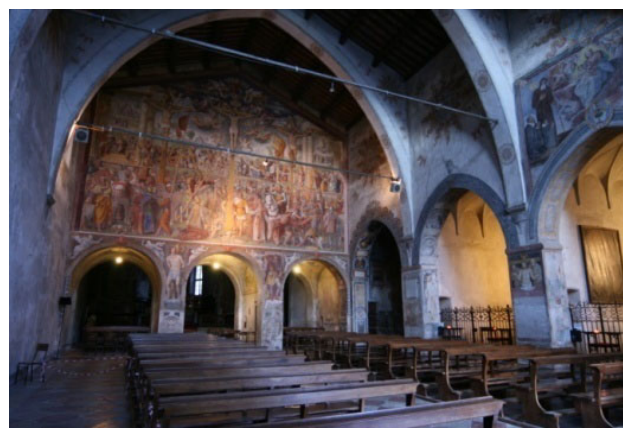

Figura 34 Giner Cordero, E . Interno della chiesa di S. Maria degli Angioli, Lugano 2007.

A questo eterogeneo gruppo si aggiungeranno, per i restauri della chiesa di S. Maria degli Angioli, i bergamaschi Michele Frana e Annibale Motterlini. II resoconto ${ }^{158}$ dell'Ispettore Cantonale dei Monumenti spedito al DPE di Lugano 28

\footnotetext{
158 Si segnalano le ore dedicate dai restauratori a questo intervento durante il mese di ottobre, suddivise in 112 ore Frana Michele, altre 112 ore Motterlini Annibale e 51 Mauro Pellicioli, con due visite da parte del Comm. E. Modigliani, una il 5 ottobre e un'altra il 18 ottobre. (Berta Edoardo. Conto pulitura Crocifissione nella Chiesa degli Angioli indirizzato al DPE. AUBCBZ s. 143 Lugano, S. Maria degli Angioli).
} 
ottobre 1930, ci conferma con esattezza il loro coinvolgimento nei lavori di pulitura dell'affresco della Crocifissione, operando sotto le indicazioni del restauratore Mauro Pellicioli e dalla sorveglianza di Ettore Modigliani, direttore della Reale Pinacoteca di Brera di Milano e direttore della Sovrintendenza all'Arte medioevale e moderna di Milano.

II contratto di lavoro elaborato per il Dipartimento della Pubblica Educazione del Canton Ticino, che Mauro Pellicioli firmò in data 8 febbraio $1927^{159}$ specificava che quest'ultimo, pittore restauratore domiciliato in Via Fiori Oscuri 9 di Milano, assumeva:

[...]il lavoro di pulitura generale, rinsaldamento e restauro del grande affresco della Crocifissione di Bernardino Luini, nella Chiesa di S. Maria degli Angioli secondo le norme e le direttive dal Sig. Comm. Ettore Modigliani, R. Sovrintendente all'Arte Medievale e in conformità della lettera 16 dicembre de 1926 diretta dal Cav. Pellicioli al Commissario Modigliani. Gli operatori che lavoreranno per conto del Cav. Pellicioli presumibilmente $i$ pittori bergamaschi sopracitati - riceveranno dallo Stato del Canton Ticino un salario L. 120 al giorno; - il Cav. Pellicioli riceverà per le sue prestazioni personali un salario di L.180 al giorno (durata normale di otto ore di lavoro). Lo Stato del Canton Ticino rimborserà le spese effettive di viaggio del sig. Pellicioli e dei suoi operatori da Milano a Lugano e viceversa, e rimborserà pure le spese di acquisto del materiale occorrente per i lavori. I lavori cominceranno nel prossimo mese di marzo $o$, al più tardi, nel venturo aprile, e si

${ }^{159}$ Fonte ARISTOS 52695.

http://aristos.mbigroup.it/search/index.php?entita=F onte\&id=52695 proseguiranno senza interruzione fino al compimento. $[. . .]^{160}$

I dipinti furono ispezionati in una prima visita nel 1926 da Mauro Pellicioli ed il 9 ottobre dello stesso anno l'ispettore Ettore Modiglione presentò al Consiglio di Stato del Canton Ticino ed alla Commissione cantonale dei monumenti storici una notevole descrizione sullo stato di conservazione dei dipinti e sulla proposta di restauro necessario per il recupero di essi, che fu studiato da lui stesso e da un [...]capo d'arte di mia assoluta fiducia[...] $]^{161}$, riferendosi al medesimo Mauro Pellicioli.

I risultati della sopraindicata perizia descrivono uno stato di conservazione generale buono, con limitati danni prodotti dalla presenza di sporcizia superficiale, polvere, grasso e puntuali zone decoese dovute ad infiltrazioni d'umidità localizzate sui pilastri dove vengono raffigurate le figure di San Sebastiano e di San Rocco distribuiti insieme ad altri segni di abrasione che comportano le mancanze di materiale pittorico: [...]guasti che purtroppo appaiono in ogni affresco nelle parti nelle quali giunge con facilità o la mano dell'uomo, o un bastone, o una canna, o un asse di scala[... $]^{162}$; il

\footnotetext{
160 (DPE. Contratto firmato da Mauro Pellicioli. 8 febbraio 1927. AUBCBZ s. 143 Lugano, S. Maria degli Angioli).

161 (Reale Pinacoteca di Brera, Milano. Esame dell'affresco della Crocifissione di Bernardino Luini nella Chiesa di S. Maria degli Angioli. Indirizzato a Francesco Chiesa, presidente della Commissione Cantonale dei Monumenti, Lugano, ed all'Illustrissimo Presidente del Consiglio di Stato del Canton Ticino, Bellinzona. AUBCBZ s. 143 Lugano, S. Maria degli Angioli).

${ }^{162}$ Le prossime citazioni riguardo la perizia di Ettore Modigliani realizzata sull'opera della Crocifissione di Bernardino Luini corrisponderanno alla fonte: (Reale Pinacoteca di Brera, Milano. Esame dell'affresco della
} 
degrado della zona all'estrema destra visto dal lato dello spettatore, nella parte centrale dell'affresco, con efflorescenze saline, polverizzazione della pitture e numerose perdite di essa, probabilmente causata da infiltrazione pluviali del tetto, la presenza di una rete di fessure che si estende per tutta la superficie dell'affresco è segno particolare dell'esecuzione di questa precisa tecnica, dove però alcune vengono differenziate per una maggiore profondità, dove la sporcizia si e depositata in modo più evidente scurendo e creando una disomogeneità nella percezione globale, per cui, come consiglia l'ispettore, dovrebbero essere pulite; altre zone presentano perdite dei ritocchi a secco realizzati dello stesso autore in determinate zone $o$ nell'uso d'un particolare pigmento incompatibile con la calce. Queste lacune sono state causate spiega Pellicioli - da tecniche di pulitura eseguite in passato; [...] Purtroppo quel tanto che è perduto - ma non è molto né è essenziale - è perduto, e non conviene certo pensare a rifare ciò che oggi non è più; ma si indicherà in seguito il mezzo di rendere meno sensibile all'occhio la perdita.[...].

A lavorare sul grande affresco luinisco fu Giuseppe Knoller il mese di settembre del 1851 sotto incarico e direzione di suo maestro all'Accademia di Brera, Ambrogio Nava. Knoller, non riuscì a compiere lo strappo dell'Ultima Cena quel stesso anno

Crocifissione di Bernardino Luini nella Chiesa di S. Maria degli Angioli. Indirizzato a Francesco Chiesa, presidente della Commissione Cantonale dei Monumenti, Lugano, ed all'Illustrissimo Presidente del Consiglio di Stato del Canton Ticino, Bellinzona. AUBCBZ s. 143 Lugano, S. Maria degli Angioli).

Fonte ARISTOS 52696

http://aristos.mbigroup.it/search/index.php?entita=F onte\&id $=52696$. per mancanza di mezzi, ma intervenne sulla Crocifissione mediante il ritocco pittorico limitato alle lacune con acquarelli. $^{163}$ I documenti dell'epoca dichiarano, di fatto, un restauro rispettoso che non sembra coincidere con gli argomenti del Pellicioli. Forse quelle perdite denunciate dal restauratore bergamasco fossero prodotto di un intervento precedente al 1851. Si sono riscontrati dai documenti i nomi di Francesco Hayez ${ }^{164}$ e del suo allievo Giovanni Airaghi, in un possibile restauro proposto nel 1838 non confermato.

Le operazione di restauro descritte da Modigliani e da Pellicioli nel 1926, dopo I'analisi macroscopica dell'affresco precedentemente presentata furono le seguenti:

1. Consolidamento delle zone decoese con la tecnica delle iniezione di malta [...]in cui non sia la più piccola molecola di calce non bene senta che possa produrre scoppi. Escluso in qualsiasi caso il cemento[...].

2. Ricostruzione dell'intonaco del margine destro, consolidamento dei bordi di quello rimasto con cemento e stuccatura dei piccoli buchi ed abrasioni con stucco romano, elaborato con calce e polvere di marmo $^{165}$. La reintegrazione delle

\footnotetext{
${ }^{63}$ (Martinola, 1944, p. 40).

Hayez Francesco ((Venezia 1791 - Milano 1882) Pittore romantico. Fu direttore dell'Accademia di Bella Arti di Brera nel 1850.

165 Questi stucchi, chiamati stucchi bianchi o marmorino, poiché la loro funzione era quella d'imitare la medesima roccia, venivano tradizionalmente composti come scrive Pellicioli da
} 
lacune doveva eseguirsi con la così chiamata "tinta locale" a tempera, cioè, un'acqua sporca realizzata dall'associazione dei colori circondanti la lacuna in sé, in [...]modo che, se da vicino il danno avvenuto possa essere testimoniato, da lontano l'occhio, nel variare delle figure $e$ dei colori, non avverta in alcuna maniera il danno e la riparazione[... $]^{166}$.

3. [...]Pulitura della sporcizia superficiale con spugne naturali inumidite [...]quasi fossero state di notte esposte a intensa umidità, ma non bagnate. Escluso in modo categorico qualsiasi sostanza atta a pulire, a cominciare dal sapone che sarebbe pericolosissimo per le parti a tempera, ed escluso financo la mollica di pane. In primo luogo distinguendo molto bene da quelle solo affrescate, le parti finite a tempera, nelle quale ultime conviene indugiare il meno possibile. In secondo luogo, tenendo presente che alcune di quelle velature a secco sono perdute $e$ che quindi l'affresco ha bisogno di smorzature; e perciò occorre non si spinga in quei luoghi la pulitura fino in fondo. $E$ così dicasi, in terzo luogo, per tutte quelle parti del dipinto le quali, per puliture passate o per qualità della pittura stessa, mostrano qua e là delle agrezze, sulle quali parti debbi, anche là, diciamo, dosare con cautela la pulitura per mantenere

calce, polvere di marmo, ma anche di polvere di calcari bianchi di diverso tipo, che potrebbe trattars della selenite o gesso di cui ci parla Secco-Suardo. (Piva, 1988, p. 253).

(Reale Pinacoteca di Brera, Milano. Esame dell'affresco della Crocifissione di Bernardino Luini nella Chiesa di S. Maria degli Angioli. Indirizzato a Francesco Chiesa, AUBCBZ s. 143 Lugano, S. Maria degli Angioli) l'armonia generale della pittura e darle così un equilibrio totale unico[.... $]^{167}$.

4. Velature con colori ad acquarello o a tempera in quelle zone dove è necessario ricuperare l'omogeneità estetica alle pitture, in modo speciale là dove i ritocchi a secco sono stati eliminati, ma in zone puntuali che necessitano di evidenziare alcuni elementi della composizione la cui mancanza danneggia la visione d'insieme. [...]Mi spiego con un esempio: in un luogo, l'altissima lancia di un guerriero, condotta a tempera sul fondo dell'affresco, e, per una zona di due o tre decimetri di lunghezza, caduta, cosicché nella mano del guerriero appare un'asta mozza $e$, in alto sul fondo un pezzo di lancia isolato che non ha alcun attacco con la parte di sotto. In questo caso, come è chiaro, risulta necessario un accenno di linee (che evidentemente non ha nulla a che fare con un rifacimento) il quale consenta la riunione dei due elementi e dia ancora un senso alla composizione $[. . .]^{168}$.

(Reale Pinacoteca di Brera, Milano. Esame dell'affresco della Crocifissione di Bernardino Luini nella Chiesa di S. Maria degli Angioli. Indirizzato a Francesco Chiesa, AUBCBZ s. 143 Lugano, S. Maria degli Angioli).

${ }^{168}$ (Reale Pinacoteca di Brera, Milano. Esame dell'affresco della Crocifissione di Bernardino Luini nella Chiesa di S. Maria degli Angioli. Indirizzato a Francesco Chiesa, AUBCBZ s. 143 Lugano, S. Maria degli Angioli). 


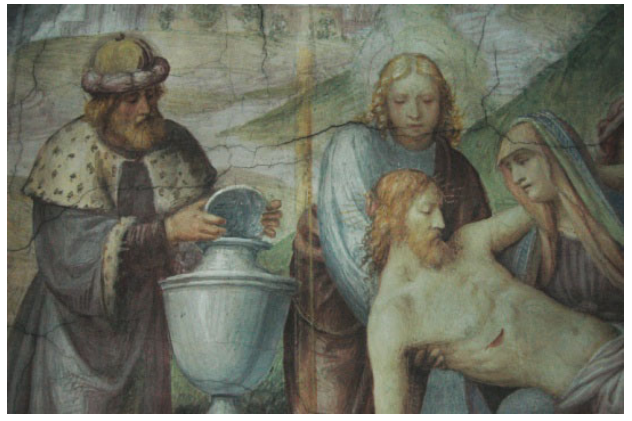

Figura 35 Particolare dell'affresco Crocifissione e passione di Cristo, dove si osserva il deteriore dei dipinti. Lugano 2006, Giner Cordero E.

II restauratore bergamasco si serviva allo stesso tempo dello sporco della pulitura diluito in acqua, per dare dei leggeri veli sui punti dove in un passato la pulitura fu poco rispettosa.

Come ultima osservazione Mauro Pellicioli evidenzia nella sua proposta di restauro l'assoluta disapprovazione dell'uso di qualsiasi materiale ceroso per il verniciato della superficie pittorica, facendo allusione alla conoscenza sull'utilizzo della menzionata tecnica all'encausto per la protezione degli affreschi, non solo di uso reiterato in Svizzera ma anche in Italia.

Le soluzioni trovate dal restauratore bergamasco e dal Sovrintendente della Reale Pinacoteca di Brera a Milano, Ettore Modigliani, risultano essere nella linea d'un restauro conservativo. Si apprezza un rispetto particolare per l'opera pittorica vietando la sua reinterpretazione ma assimilando la storia accumulata.

Dopo un'analisi preliminare molto esaustiva delle tecniche esecutive, dello stato di conservazione e delle cause dei diversi danni esistenti, si arriva a definire la metodica d'attuazione idonea che permetta all'opera di essere nuovamente leggibile dallo spettatore. Si tiene già in considerazione il fattore della "reversibilità" dei materiali utilizzati e del degrado che possono provocare certi prodotti come dimostrato dalla sperimentazione storica. Per questo motivo il rifiuto di materiali come i saponi che possono danneggiare i ritocchi a secco o l'uso delle cere per il "ravvivamento" dei colori.

Grazie all'intervista realizzatasi al restauratore bergamasco nel 1970 per la TSI, si è verificata la relazione diretta tra Pellicioli e Berta, Sembra che Pellicioli avessi avuto degli scontri con Berta durante l'esecuzione del restauro nella chiesa di Santa Maria degli Angioli. Chiaramente gli ideali di restauro di ambi due dovevano essere diversi. Secondo la parole manifeste per il reportage dal restauratore italiano, Berta [...] pretendeva che venissero alterati i toni; voleva creare quello che non è; per esempio gli episodi in piccolo, intendeva che andassero in lontananza[... $]^{169}$ ma Luini aveva differenziato le scene nella prospettiva facendo apparire le figure più grande in vicinanza. Quando Berta parla del lavoro sui dipinti di S. Maria degli Angioli, eseguito nell'ultimo momento operativo compreso dal 1926 al 1930, spiega di fatto avere realizzato un ritocco pittorico finale a base di velature. Queste vengono utilizzata dall'artista per dare all'opera quell'omogeneità persa con il passo del tempo o, perfino a causa degli interventi di restauro precedenti (descialbo, pulitura, consolidamento):

\footnotetext{
${ }^{169}$ (Televisione della Svizzera Italiana. Intervista a Mauro Pellicioli. 1970. RTSI, Archivi televisivi).
} 
[...] ho ripreso il restauro delle pitture [...] ricongiungendo con velature i segni assai vaghi alcuni avanzi troppo distante l'uno dell'altro in modo da ridare una pallida impressione dell'effetto decorativo d'assieme che in origine dovevano avere. I signori periti diranno a quel punto dovrò fermarmi e, se del caso anche di cambiare strada [...]. ${ }^{170}$

Malgrado le esplicite discrepanze anche da parte del Dipartimento Federale dell'Interno (DFI) riguardo al coinvolgimento nel lavoro sulla chiesa di Lugano del pittore-restauratore Mauro Pellicioli, procedente della vicina e concorrente Italia, le negative della Confederazione non riuscirono ad imporsi a ciò che era già stato accordato dalla Commissione Cantonale con l'ispettore Ettore Modigliani ed il suo collaboratore il medesimo Mauro Pellicioli. Tuttavia ciò il DFI rinunciò al sussidio dei lavori. Le osservazioni presentate dal medesimo Dipartimento dell'Interno nella lettera spedita al Governo del Canton Ticino il 27 maggio 1927, spiegava il suo obbligo a fare eseguire gli interventi ad uno specialista svizzero, che poteva concretizzare il progetto dell'ispettore Ettore Modigliani, la cui professionalità non si metteva in discussione poiché totalmente dimostrata e conosciuta, come sicuramente lo era per il Ministero anche Pellicioli. Qua non si voleva sottovalutare i professionisti italiani ma interessarsi delle necessità degli operai ticinesi, la cui realtà economica si trovava in dura difficoltà dopo la guerra. La Confederazione doveva a questo rispetto sentirsi responsabile di questi fatti e d'altra parte temeva,

\footnotetext{
${ }^{170}$ (Berta Edoardo. Relazione restauro, 1930. AUBCBZ s.143 Lugano, S. Maria degli Angioli).
}

sicuramente, le forte critiche che li potessero commettere.

[...]Se si pensa alle difficoltà che i nostri artisti hanno a guadagnarsi la loro vita, si comprende che il nostro Dipartimento non possa, senza esporsi a vivaci critiche, sussidiare un restauro eseguito da uno straniero residente all'estero $[\ldots]^{171}$

Lo stesso problema fu riscontrato dal Dipartimento del lavoro del Canton Ticino per il contratto degli operai:

[...]L'Ufficio centrale forestieri ci rimette per preavviso la domanda di soggiorno per un periodo di sei mesi di certo Frana Michele pittore restauratore, chiamato per il lavori della Chiesa degli Angioli di Lugano. Ci permettiamo rivolgerci a voi perché vogliate comunicarci se $i$ lavori in questione non possono per avventura essere assunti, con egual garanzia di esecuzione, da un artista ticinese o comunque già residente nel paese $[\ldots]^{172}$.

I lavori sul gran dipinto del transetto, in ogni modo, non furono iniziati fino ai primi del mese di maggio del 1930, quando Pellicioli ritorna da Londra dopo la sua partecipazione, insieme al Commissario Modigliani, nella vigilanza delle pitture di proprietà italiana esposte alla Mostra

(Dipartimento Federale dell'Interno. Restauro Chiesa di S. Maria degli Angeli indirizzato al Consiglio di Stato del Cantone Ticino, Bellinzona. Berna 27 maggio 1927. AUBCBZ s.143 Lugano, S. Maria degli Angioli).

${ }^{172}$ (Dipartimento del Lavoro del Canton Ticino, Lettera riguardo la domanda di soggiorno di sei mesi del pittore restauratore Frana Michele, chiamato per il lavori di restauro della Chiesa degli Angioli in Lugano indirizzata alla Lodevole Commissione Cantonale dei Monumenti Storici di Lugano. Bellinzona 24 giugno 1930. AUBCBZ s.143 Lugano, S. Maria degli Angioli). 
d'arte antica che ebbe luogo nella Royal Academy of Arts. ${ }^{173}$

Sabato 25 ottobre 1930 si riapre la chiesa di Santa Maria degli Angioli dopo diciotto anni di lavori, con una visita guidata realizzata da Edoardo Berta ed una cena nella Taverna di S. Luca nel quartiere di Molino Nuovo.

Le fotografie dell'intervento, prima e dopo il restauro furono eseguite dal fotografo $C$. Carboni come esplicito nel resoconto del 12 dicembre $1930^{174}$, e spedite in seguito alla Commissione Federale dei Monumenti Storici. L'architetto Cino Chiesa elenca un numero di ventitre negativi e copie fotografiche, presumibilmente per richiesta della Commissione Cantonale, che testimoniano la situazione del bene architettonico e dei beni artistici prima e dopo il restauro. ${ }^{175}$

Si sono trovate, tuttavia le abbondanti fonti analizzate in questo testo procedenti dall'Archivio dell'Ufficio dei Beni Culturali a Bellinzona, pochi dati riguardanti il lavoro meramente artistico, i materiali e le tecniche di restauro adottati, da parte del professore Berta o di qualcuno dei suoi collaboratori e colleghi della Commissione cantonale dei monumenti dove si descriva L'assenza dei citati documenti si chiarifica

\footnotetext{
${ }^{173}$ (Modigliani Ettore, Lettera indirizzata a Francesco Chiesa, presidente della CCMS in risposta alla lettera del 20 gennaio. Londra 28 gennaio 1930. in Milano, 3 maggio 1930. AUBCBZ s.143 Lugano, S. Maria degli Angioli).

174 (Ispettore Cantonale dei Monumenti. Lettera indirizzata al Lodevole DPE. 12 dicembre 1930. AUBCBZ s.143 Lugano, S. Maria degli Angioli).

${ }^{175}$ (Chiesa Cino, Elenco delle negative e relative copie fotografiche della Chiesa di S. Maria degli Angeli in Lugano, indirizzate alla Lodevole Commissione Federale dei Monumenti storici in Berna.3 settembre 1931. AUBCBZ s.143 Lugano, S. Maria degli Angioli).
}

in una lettera del 14 settembre 1932, che il DPE del Canton Ticino emise al Dipartimento Federale dell'Interno, quando questi, un anno dopo, richiedono certi rapporti di restauro. Scusandosi per non potere facilitare maggiormente le informazioni, il segretario del DPE scrive al DFI sul problema esistente nella chiesa di Lugano:

[...]nel 1931 morì, dopo sei mesi di malattia, il nostro Valente Ispettore dei monumento sig. Pittore Edoardo Berta, al quale era stato affidato il restauro dei nostri principali monumenti, fra cui S. Maria degli Angioli in Lugano, il Castello di Locarno e S. Maria delle Grazie in Bellinzona, tutti e tre sussidiati dalla Confederazione; A tutti è noto con quale senso d'arte e serietà d'archeologo e con quale passione il compianto Sig. Berta attendesse ai suoi lavori di restauro, $i$ quali si meritarono riconoscimento e lode anche dalla Commissione Federale. Disgraziatamente la salute già compromessa e il peso del lavoro addossato si gli impedirono talvolta, durante l'ultimo periodo della sua vita, di procedere con quella perfetta regolarità amministrativa e con quello scrupolo ossequio delle forme, che sarebbero stati desiderabili, cosicché, sopravvenuta la malattia e la morte, noi ci siamo trovati nell'impossibilità di documentare in modo assolutamente esauriente $i$ lavori eseguiti sotto la di lui direzione[...]. ${ }^{176}$
6 (Dipartimento della Pubblica Educazione lettera indirizzata al Dipartimento Federale dell'Interno. 14 settembre 1942, AUBCBZ s.143 Lugano, S. Maria degli Angioli). 
Restauratori in Canton Ticino fra Ottocento e Novecento

Catalogazione e gestione dati

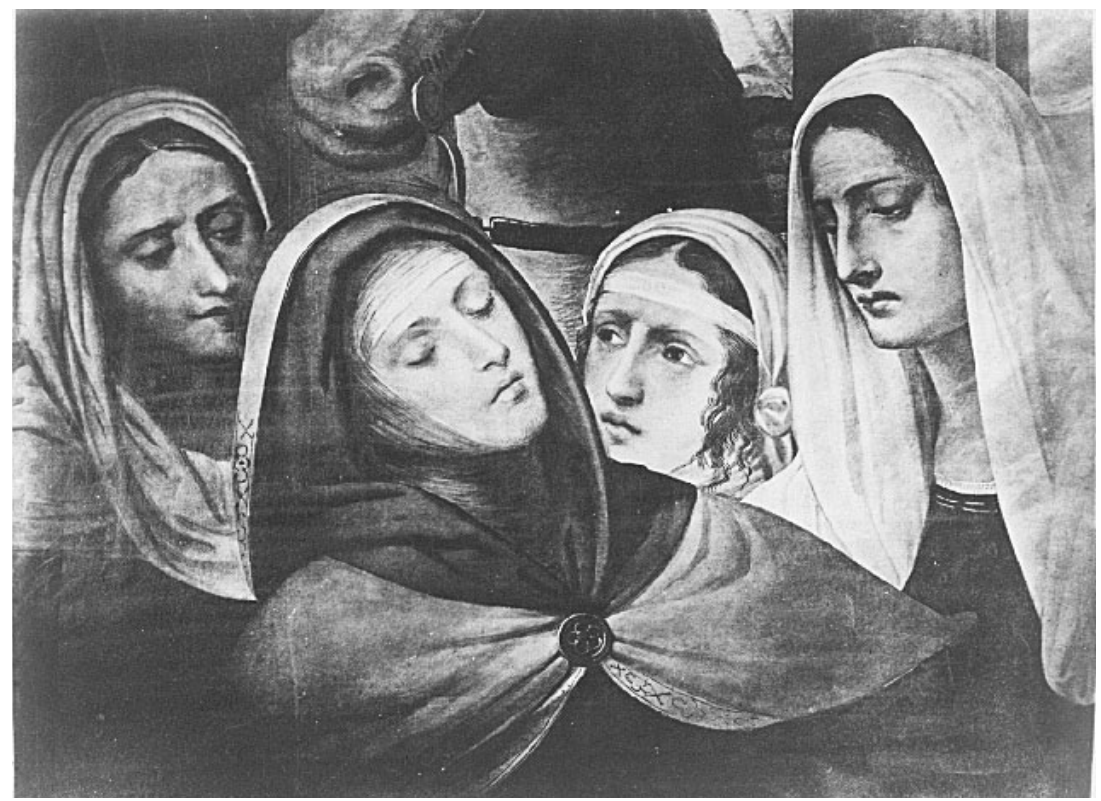

Figura 36 Particolare del dipinto della Crocifissione di Bernardino Luini. 1931 ca. Fotografia b/n. AUBCBZ Archivio grafico, 143 Lugano, S. Maria degli Angioli.

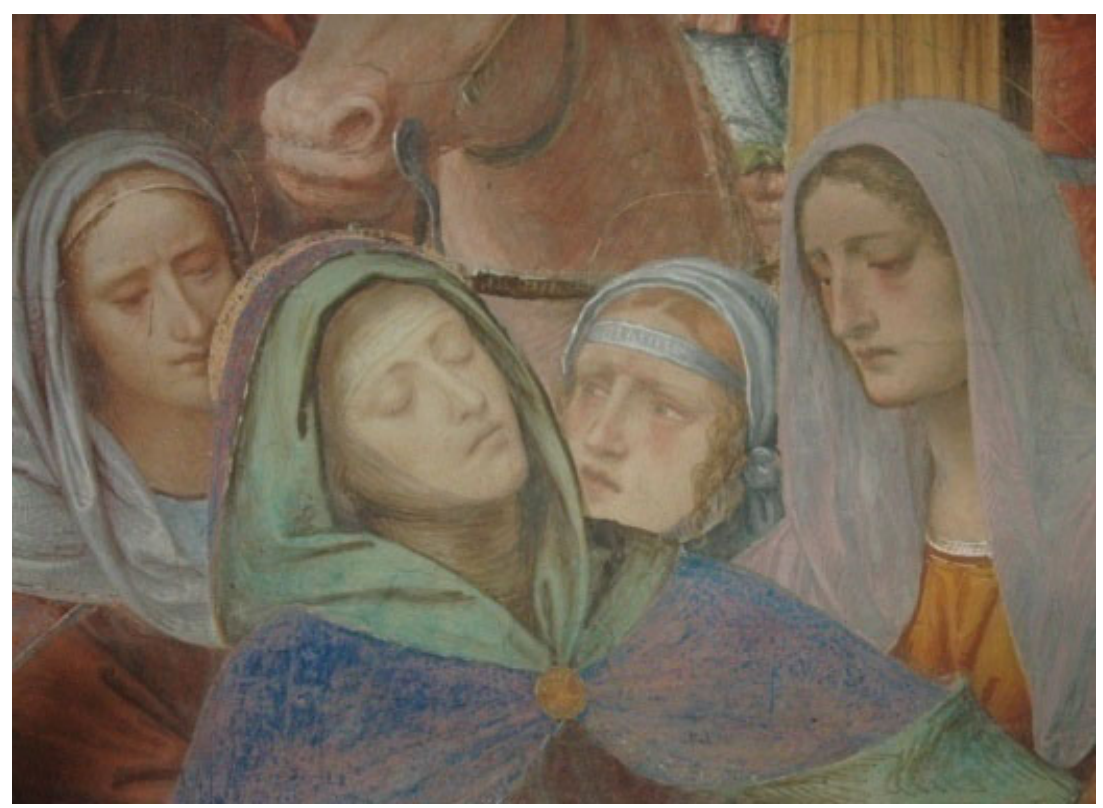

Figura 37 Giner Cordero E. Particolare del dipinto della Crocifissione di Bernardino Luini. 2006. 


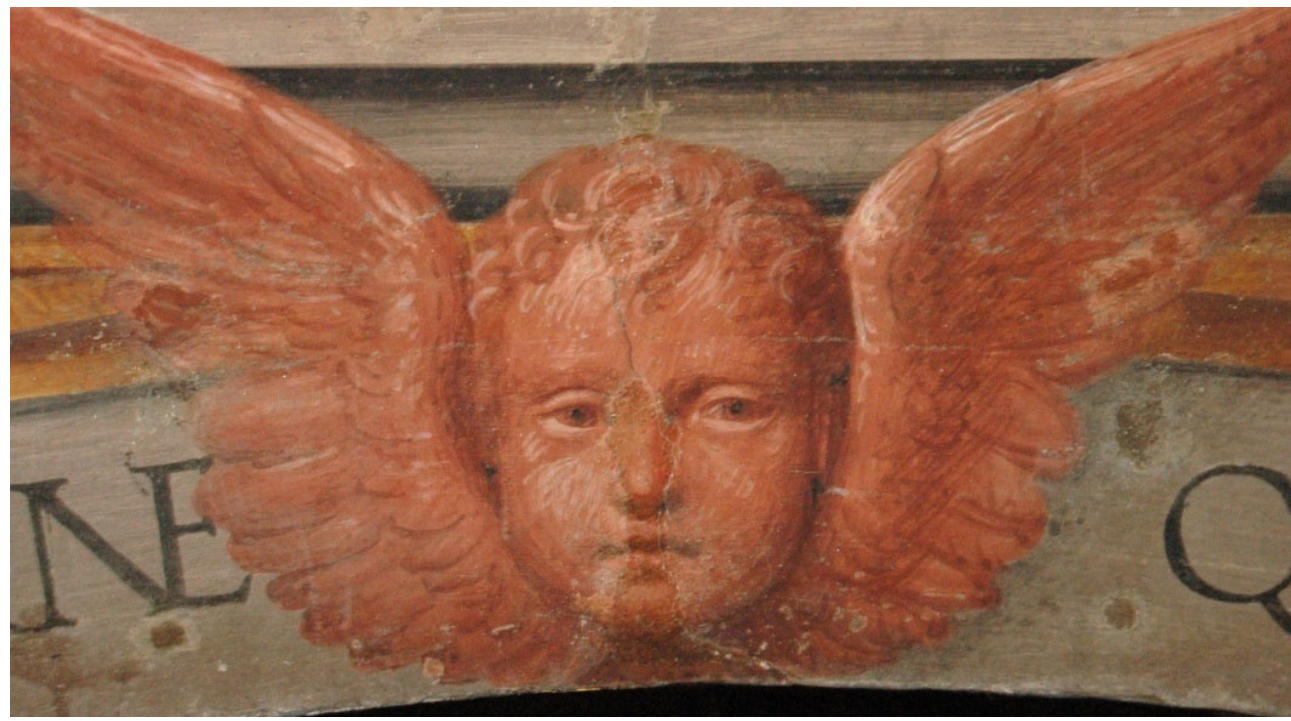

Figura 38 Giner Cordero E. Particolare del dipinto della Crocifissione di Bernardino Luini dopo il restauro, Lugano. 2006.

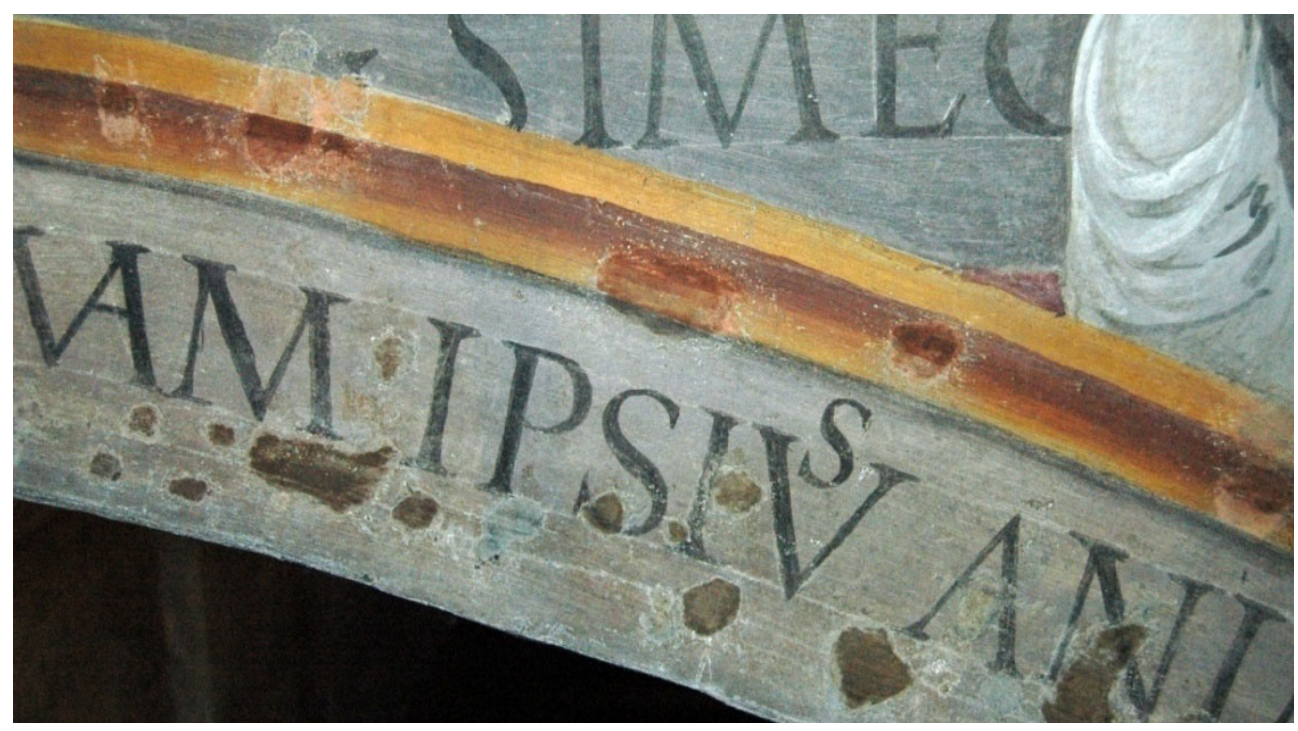

Figura 39 Giner Cordero E. Particolare del dipinto della Crocifissione di Bernardino Luini. Lugano 2006. 
La situazione attuale della chiesa è, in genere, in discreto stato di conservazione. Se da una parte possiamo osservare l'opera della Crocifissione e passione di Cristo di Bernardino Luini, apparentemente in buono stato, il suo continuo controllo, particolarmente oggigiorno, è necessario. Questo si deve alle opere di ricostruzione che recentemente stanno avendo luogo nel Ex-Hotel Palace, affiancato alla chiesa degli Angioli, con lo scopo di trasformare l'edificio nel futuro polo culturale della città di Lugano.

Il Cantone è stato presente dagli inizi dei lavori sull'Hotel, come supervisore delle possibili conseguenze provocate sulla fabbrica adossata, e nelle sue opere. In totale, dal 2004, sono stati eseguiti sette sopralluoghi consistenti nel controllo di eventuali distacchi dell'intonaco del grande affresco della Crocifissione. I rilievi sono stati realizzati dal Laboratorio Tecnico Sperimentale (LTS) della SUPSI a Lugano. In questi controlli si è potuto constatare il soddisfacente stato di conservazione dell'affresco del Luini sul transetto della
Chiesa di Santa Maria degli Angioli. Sono pochi, di fatto, i punti che si sono manifestati pericolanti, tanto è così che sarebbe giusto indicare in primo luogo, danni maggiori riscontrati sull'affresco come la consistente quantità di polvere e grasso accumulato sulla superficie del dipinto, così come l'importante pulverulenza in particolare di alcuni pigmenti, come il blu e il rosso, o la trasformazione cromatica dei ritocchi eseguiti nel 1930 da Mauro Pelliccioli, oggi specialmente scuriti.

A presentarsi evidentemente trascurata è invece la Cappella Camuzio. Come già descritto precedentemente, i danni occasionati dalle inopportune tecniche d'intervento impiegate, specie nel descialbo delle pitture e nel consolidamento con materiali cerosi, si sommano ora i deterioramenti causati dall'umidità di risalita, che si manifesta provocando il distacco degli intonaci e le trasformazioni cromatiche dei dipinti.

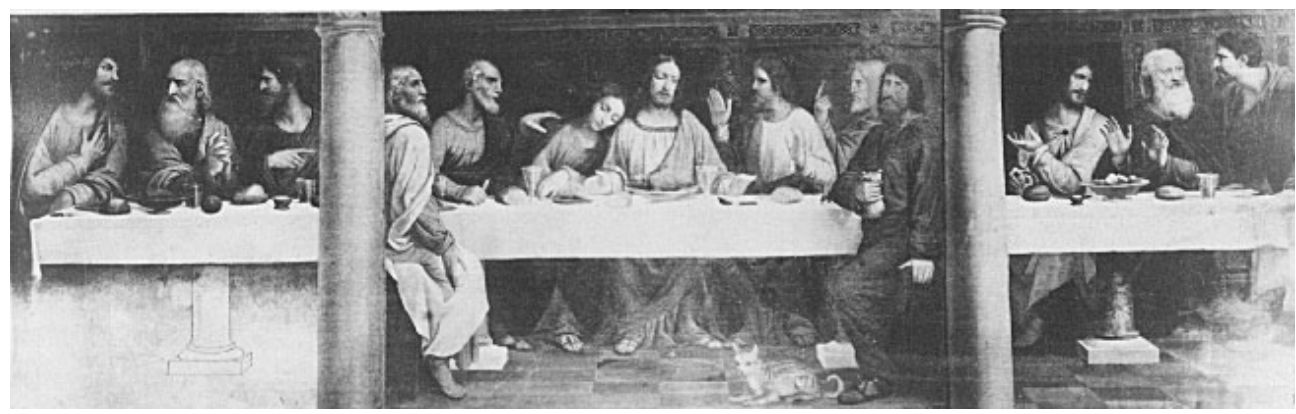

Figura 40 Cenacolo di Bernardino Luini nella Sala della Comunione.

Chiesa di Santa Maria degli Angioli. Lugano TI. 


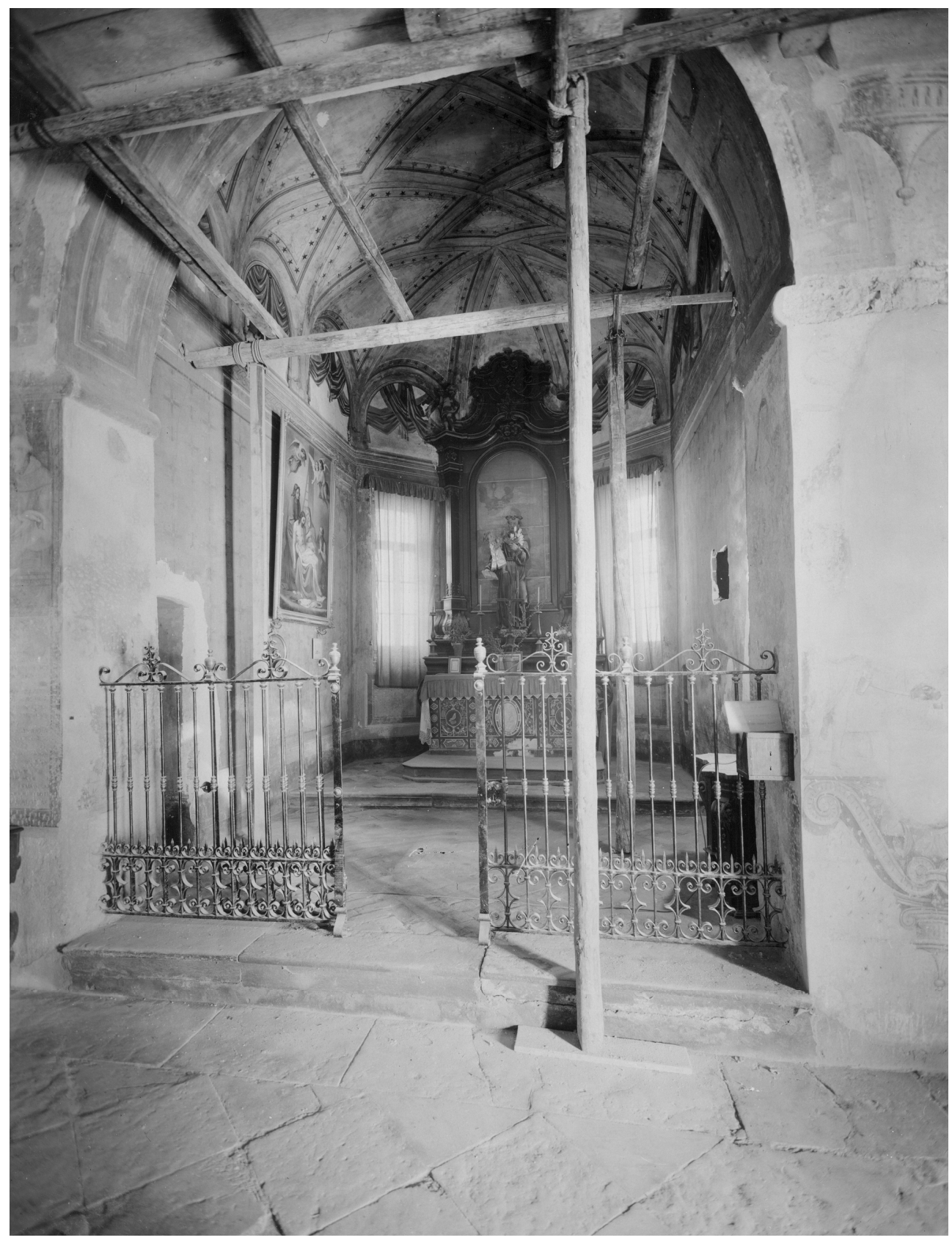

Figura 41 Seconda cappella durante i lavori di restauro. 1931 ant. Fotografia b/n AUBCBZ Archivio grafico, 143 Lugano, S. Maria degli Angioli. 


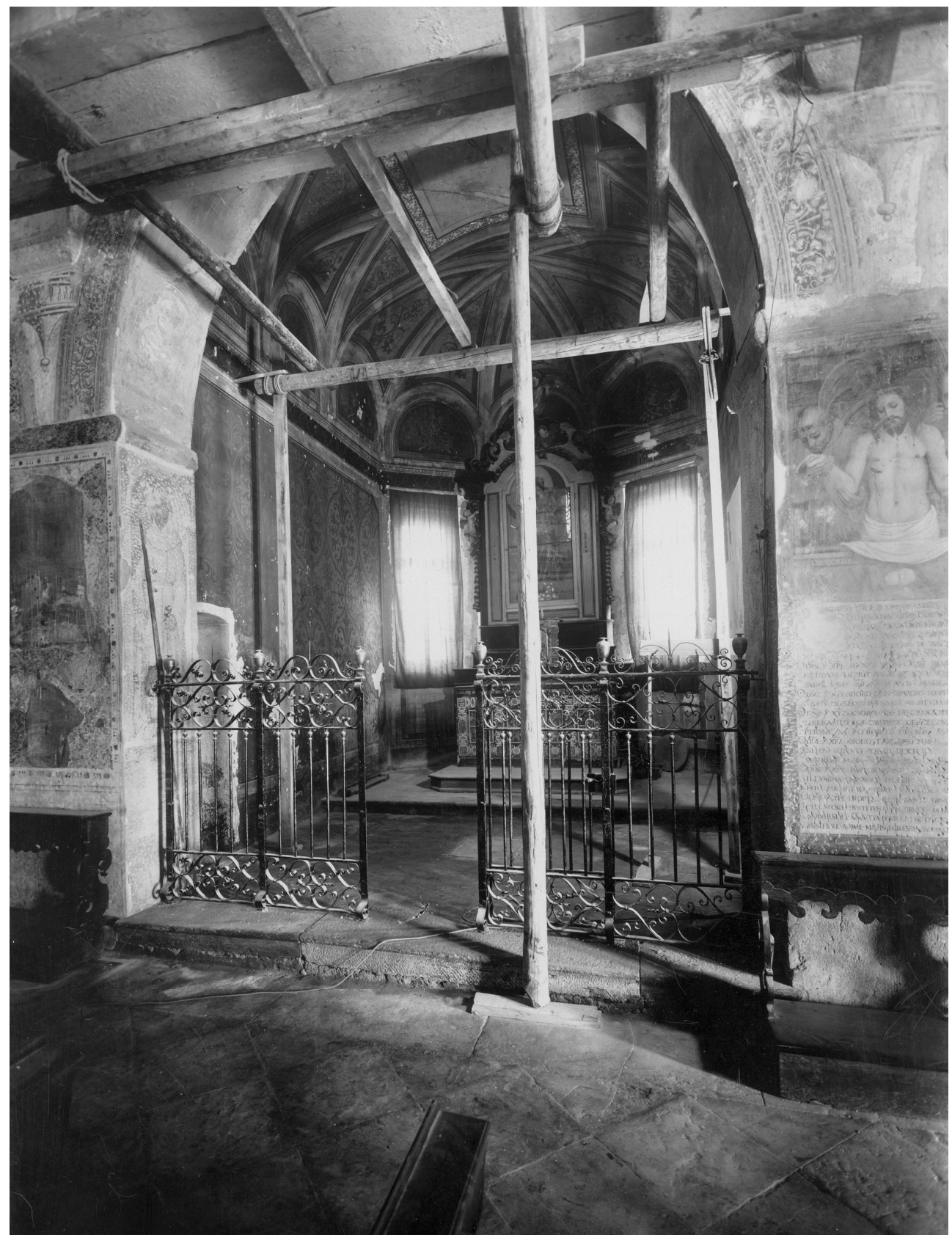

Figura 42 Terza cappella durante i lavori di restauro. 1931 ant. Fotografia b/n AUBCBZ Archivio grafico, 143 Lugano, S. Maria degli Angioli. 


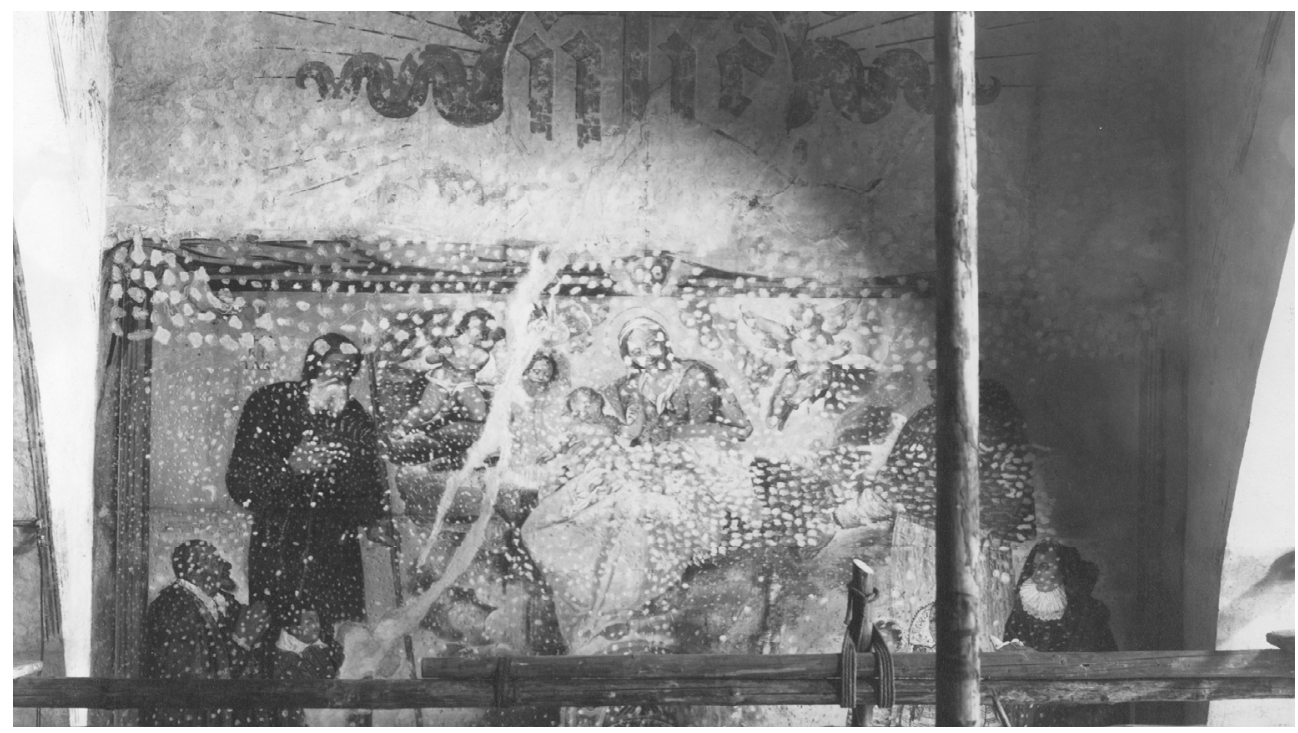

Figura 43 C. Carboni, Sacro orifiamma con le sigle di Cristo prima del restauro, 1931 ca. Fotografia b/n. AUBCBZ Archivio grafico, 143 Lugano, S. Maria degli Angioli.

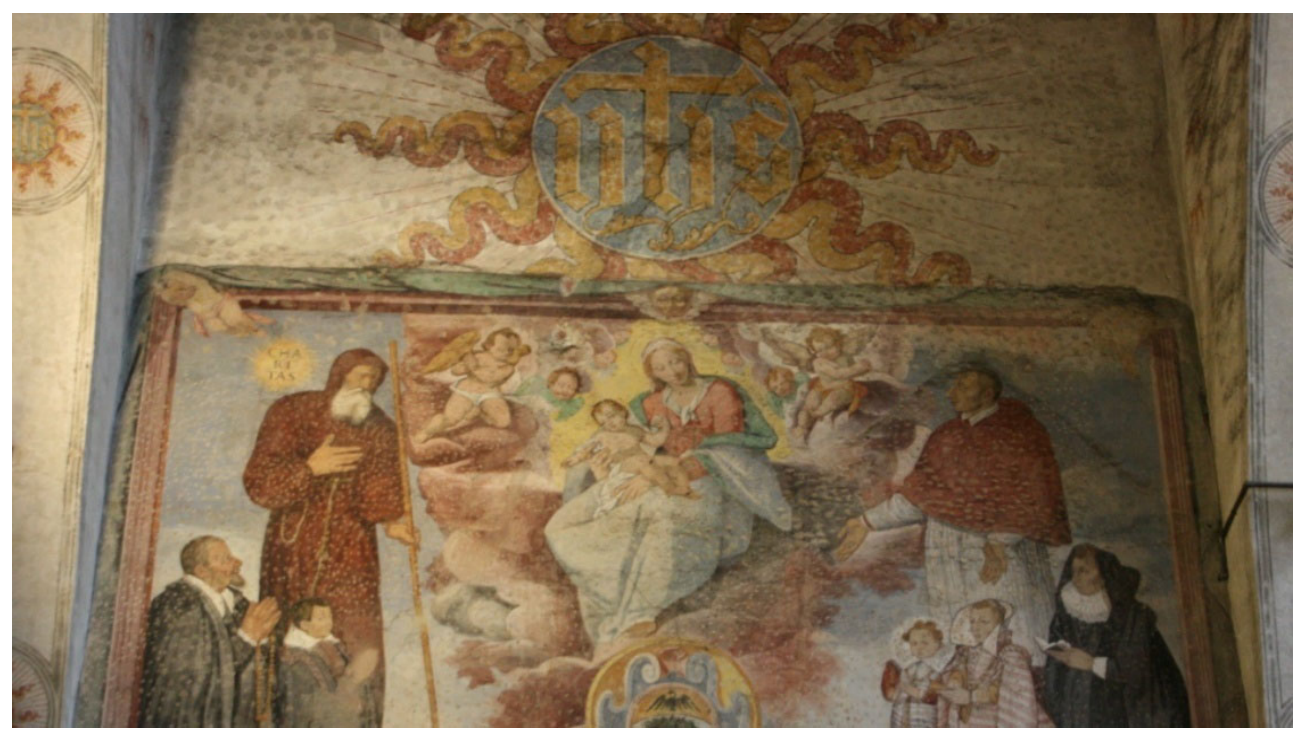

Figura 44 Giner Cordero E., Sacro orifiamma con le sigle di Cristo. Chiesa di S. Maria degli Angioli, Lugano. 2008. 


\section{Chiesa di S. Biagio, Ravecchia, (1913 - 1915)}

II 6 agosto del 1915 si pubblica la relazione sul restauro della chiesa di San Biagio a Ravecchia ${ }^{177}$, prodotta con lo scopo d'informare il lodevole Dipartimento della Pubblica Educazione sui lavori portati a termine nel medesimo monumento. L'intervento interessò le ricerche archeologiche dell'edificio ed il suo ricupero agli stili originari, e incluse non solo il restauro dei beni immobili ma anche quelli mobili, fra essi, la Pala di Altare raffigurante la Madonna tra i SS. Biagio e Girolamo, attribuita al pittore Domenico Pezzi ${ }^{178}$, firmata e datata 1520.

\footnotetext{
${ }^{177}$ (Berta, Relazione sui lavori di restauro della Chiesa di San Biagio in Ravecchia, 1915, p. 5).

${ }^{178}$ Pezzi Domenico, originario di Drano o di Sonvico, fu un pittore di formazione lombarda attivo a Genova e nel Canton Ticino. Lavora a Carona fra il 1581 ed i 1584, negli affreschi del coro della chiesa parrocchiale di S. Giorgio, raffiguranti la Crocifissione sul fondo, la Gloria del Paradiso sulla parete nord (ispirata alla Disputa di Raffaello in Vaticano), ed il Giudizio Universale sulla parte sud, copia del modello di Michelangelo alla Cappella Sistina, ved. (Simona Martinoli, 2007, p. 345); a Morcote, nella chiesa di S. Maria del Sasso nel 1513 con la realizzazione degl affreschi del coro: Costrizione al lavoro quale punizione per il peccato (quarto tondo) e le scene raffiguranti la Preghiera nell'orto degli ulivi e la Salita al Calvario e la Crocifissione, ved. (Simona Martinoli, 2007, p. 350); e probabilmente anche a Campione d'Italia, nella chiesa di S. Maria dei Ghirli affrescando le scene della Storia della Genesi e i santi patriarch Abramo e Mosé e gli apostoli Giacomo e Giovanni de 1514, strappati dal portico nord e trasferiti nel 1893 sull'ultimo pilastro del portale laterale ved. (Simona Martinoli, 2007, p. 361). II dipinto su tavola della chiesa di S. Biagio a Ravecchia, trovatos originariamente sull'altare antico è ora nella navata. La pittura ha influenze del Bramantino nella costruzione della volumetria, mentre i panneggi ed volti hanno le caratteristiche propria i primi dipinti di Bernardino Luini. La pala d'altare di S. Biagio è l'unica
}

Il lavoro su quest'ultima fu compiuto dallo "specialista" Francesco Annoni ${ }^{179}$ di Milano, che anni più tardi, esattamente nel 1938, si occupa di una altra tavola di grande importanza fra i beni culturali del Canton Ticino: la "Ancona della Pietà" nella chiesa della Madonna del Sasso di Orselina (Locarno) e che più avanti verrà trattata. La chiesa di San Biagio del XIV sec. fu costruita su un edificio già esistente, un monastero probabilmente, di epoca anteriore al $X$ sec. come svelarono le ricerche eseguite fra gli anni 1913 e 1914 con obiettivo del restauro generale della fabbrica. Infatti, questa datazione viene avvalorata nuovamente, come descrive Edoardo Berta, dalle notizie storiche: nel testamento dell' 11 novembre 1338 di Guglielmo de Rugario, citato negli atti di S. Carlo, pubblic, del Sac. Paolo D'Alessandri, Locarno 1909, questo appare come fondatore e donatore della Cappella di S. Bartolomeo, e lo storico Eligio Pometta ${ }^{180}$

opera firmata dall'artista: [...] Domenicus de Pe./ Dictus Sursnicus/ de Lacuu Lugani P.. 1520[...].. (Campagna, 1998, p. 33).

${ }^{179}$ I lavori di restauro sulla Pala d'Altare terminarono nel 1912, con l'aprovazione degli esperti federali Albert Naef e R. Durrer. II sussidio da parte della confederazione fu di un $40 \%$; inoltre parteciparono economicamente all'itervento il Patriciato di Bellinzona, la Confraternita del SS. Sacramento della Parrocchia di Ravecchia ed il vescovo Monsignore Peri Morosni.

180 Pometta Eligio (Cerentino 1865-Faido 1950) Fu principalmente storico ed scrittore, dopo frustrate esperienze negli studi di diritto, cominciati però mai conclusi a Berna e Monaco, inoltre ocupò il posto di segretario. del governo ticinese nel 1889 lasciando il carico solo un anno più tardi a causa delle rivoluzioni radicali lasciò la carica dopo la rivoluzione radicale dell'11.9.1890, divenendo redattore di fogli conservatori e deputato al Gran Consiglio ticinese (1901-11, dimissionario). Accanto a un percorso professionale variegato, fu tra l'altro segretario 
parla in uno dei suoi scritti su "Come il Ticino venne in potere degli Svizzeri" ${ }^{181}$ di documenti dell'Archivio Capitolare di Bellinzona dove si potrebbe confermare l'appartenenza della Chiesa di S. Biagio, già nel 1244 al Convento di Disentis nel Lucomagno ${ }^{182}$.

L'interesse per la chiesa avviene grazie agli scavi e lavori diretti da Edoardo Berta, e specialmente dopo la certezza sull'esistenza di importanti dipinti murali al suo interno. Come promosse alla fine del XIX secolo Viollet-le-Duc, Berta, [...] progetta e dirige un lavoro di restauro, deve agire come il chirurgo accorto ed esperto, che tocca un organo solo dopo aver acquisito una completa conoscenza della sua funzione ed aver previsto le conseguenze immediate o future dell'operazione.[... $]^{183}$ Fu nel 1885, come spiega l'ispettore Edoardo Berta nella relazione di restauro, quando insieme al pittore Michele Carmine ${ }^{184}$ si trovarono

traduttore della direzione delle FFS a Lucerna (191629) - si dedicò a studi storici spec. come redattore del Bollettino storico della Svizzera it. (1921-41). Fu autore di una vasta e ineguale produzione, fra cui si distinguono Come il Ticino venne in potere degli Svizzeri (in tre volumi, 1912-15) e Storia del cant. Ticino (in collaborazione con Giulio Rossi, 1941), a lungo unica opera complessiva sulle vicende cant. P. evidenziò in particolare i legami del Ticino con l'Italia e si distanziò dall'interpretazione elvetista della storia cant.

${ }^{181}$ (Pometta, 1912-1915).

${ }^{182}$ (Berta, Relazione sui lavori di restauro della Chiesa di San Biagio in Ravecchia, 1915, p. 5).

${ }^{183}$ (Galli).

184 Carmine Michele (Bellinzona 1854 -1894) Fu pittore di paesaggi, nudi, ritratti, temi allegorici e religiosi. Studiò nel 1871 all'Accademia di Brera, allievo di pittori come Hayez e Casendi. Ritornando in Svizzera insieme alla pitture si dedica alla decorazione religiosa in diverse chiese del Canton Ticino. Alcuni esempi gli troviamo alla nuova chiesa di Airolo ricostruita dopo l'incendio, alla chiesa di Sementina, di Gorduno, in Santa Maria di Valle Calanca, alla chiesa di Besazio dove lavora insieme al compagno scultore sotto la scialbatura di calce le iniziali traccia d'affreschi sul primo pilastro della navata sud. Questa raffigurava la figura di un vescovo di due terzi della grandezza naturale [...] ed altro ancora si sperava di ritrovare $[\ldots]^{185}$.

Le ricerche, purtroppo, non potettero continuare, in cambio però qualche benefattore finanziò in 1890 lavori di ristrutturazione della chiesa che consistevano nella chiusura delle finestre gotiche fino a quel momento conservate e nella tinteggiatura generale delle pareti della chiesa, per cui l'affresco ritrovato nelle indagini condotte da Berta, così come altri ancora sotto lo scialbo, furono distrutti. All'interno della chiesa si salvarono unicamente gli affreschi raffiguranti San Bartolomeo e Santa Agata, ed all'esterno il gran San Cristoforo della facciata per il quale non arrivarono i finanziamenti della tinteggiatura. Questi fatti ci dimostrano l'evidente assenza all'epoca di una legge sulla protezione dei monumenti in Canton Ticino che regolasse questo tipo di azioni, secondo parole di Berta, [...] i cosiddetti "restauri" per abbellire la Chiesa[...] $]^{186}$.

[...]La sorte toccata a questo monumento è la stessa che toccò a tanti altri nel nostro e negli altri Paesi in epoche nelle quali lo studio ed il rispetto per le opere d'arte antiche era meno sentito che nell'epoca nostra, tanto che avviene assai raramente di poter ritrovare un assieme

Spartaco Vela ed alla chiesa parrocchiale di Rancate. Carmine emigrò nel 1889 in Argentina tornando alla città natale solo nel 1894 dove pochi mesi dopo si spense.

${ }^{185}$ (Berta, Relazione sui lavori di restauro della Chiesa di San Biagio in Ravecchia, 1915, p. 5).

${ }^{186}$ (Berta, Relazione sui lavori di restauro della Chiesa di San Biagio in Ravecchia, 1915, p. 5). 
completo che ci indichi chiaramente le armonie estetiche che si crearono in altre epoche $d i$ civiltà $[\ldots]^{187}$.

È questa una nuova dimostrazione della predominanza degli ideali di ritorno agli stili architettonici originari della chiesa, poiché secondo le concezioni dell'epoca, e della persona di Berta specialmente, questi non potevano vivere in consonanza armonica con le aggiunte posteriori, anche se si dimostrava un riconosciuto valore artistico in questi ultimi. Per questo motivo sarà abitudine dell'ispettore ticinese eseguire delle rigorose ricerche storiche fondamentali negli scavi archeologici, come nel caso di S. Biagio, e che giustifichino la "necessità" di distruzione degli elementi più recenti. Oggi dobbiamo a questa metodologia la perdita di un gran numero di opere ottocentesche, ed allo stesso tempo l'inizio dello studio del manufatto storico e della sua rivalorizzazione.

Anche il rapporto di restauro scritto dalla Commissione Cantonale alla fine dei lavori, accentua il fatto di avvertire un aspetto che descrivono come "desolante" dei monumenti, a causa delle "alterazioni barocche" che attuavano come [...]una grave maschera su cui erano venute vegetando quei poveri abbellimenti che sono propri del decaduto uso chiesastico moderno[...]. Queste aggiunte si manifestavano nelle volte a crociera dell'età barocca, nei pilastri rivestiti, nelle finestre rettangolari della facciata dove anche il rosone era stato sostituito, nelle nicchie murate e nelle decorazioni a stucco.

Fra le indagine condotte da Edoardo Berta nei più importanti beni culturali del Ticino

${ }^{187}$ (Berta, Relazione sui lavori di restauro della Chiesa di San Biagio in Ravecchia, 1915, p. 5). si possono elencare i lavori eseguiti nella descritta chiesa di Santa Maria degli Angioli (1891), nella chiesa parrocchiale di San Martino a Malvaglia (1913), nella chiesa parrocchiale di San Bartolomeo a Medeglia (1915), nell'oratorio di San Ambrogio a Camignolo (1919), al Castello Visconteo di Locarno (1921) e nella chiesa di Santa Maria delle Grazie a Bellinzona (1922), in tutti loro con la riscoperta di pitture trovatesi nascoste sotto lo scialbo di calce.

[...]Per donare al nostro paese almeno un esempio di un piccolo tempio che fosse, oltreché pregevole opera d'arte, una genuina espressione del sentimento mistico dominante in un'epoca in cui la religione era così pura e sentita. Per raggiungere tale scopo, (sebbene sia grande in noi il sentimento di rispetto per tutte le nobili attività dei nostri padri) non abbiamo esitato ad ordinare la distruzione dei pochi avanzi di forme dell'epoca barocca e neoclassica (cantoria, pulpito, nicchie per statue di Santi ecc. $)[. . .]^{188}$.

Alessandro Conti, nella sua "Storia del restauro e della conservazione delle opere d'arte", parla di questo fenomeno come una museificazione delle chiese che nell'ultima metà del sec. XIX presenta i primi esempi europei di nudità nella presentazione degli edifici medievali in Italia quando nel 1834 si rimuove I'imbiancatura che nascondeva la muratura in pietra di Santa Maria Forisportam, nel 1841 nella chiesa di S. Giovanni e nel 1838 nel Tempietto del Volto Santo nella cattedrale di Lucca. La commissione fece in rimuovere le figure settecentesche per provvedere dopo con la doratura di esse secondo le ricerche documentali.

\footnotetext{
(Berta, Relazione sui lavori di restauro della Chiesa di San Biagio in Ravecchia, 1915, p. 6).
} 


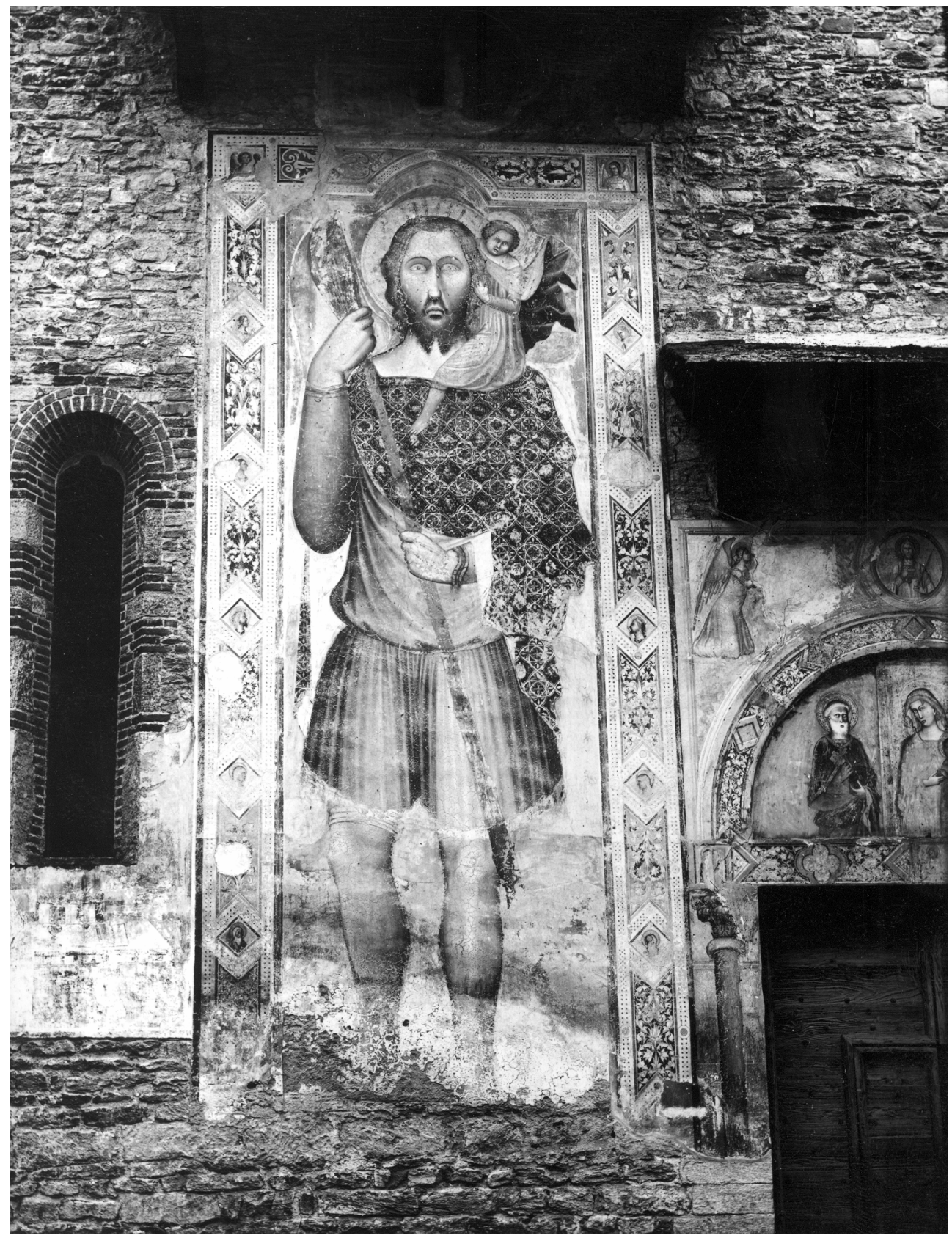

Figura 45 San Cristoforo sulla facciata della Chiesa di San Biagio di Ravecchia dopo il restauro. Bellinzona. 1913 ca. Fotografia b/n. AUBCBZ, Archivio grafico, Ravecchia, S. Biagio. 


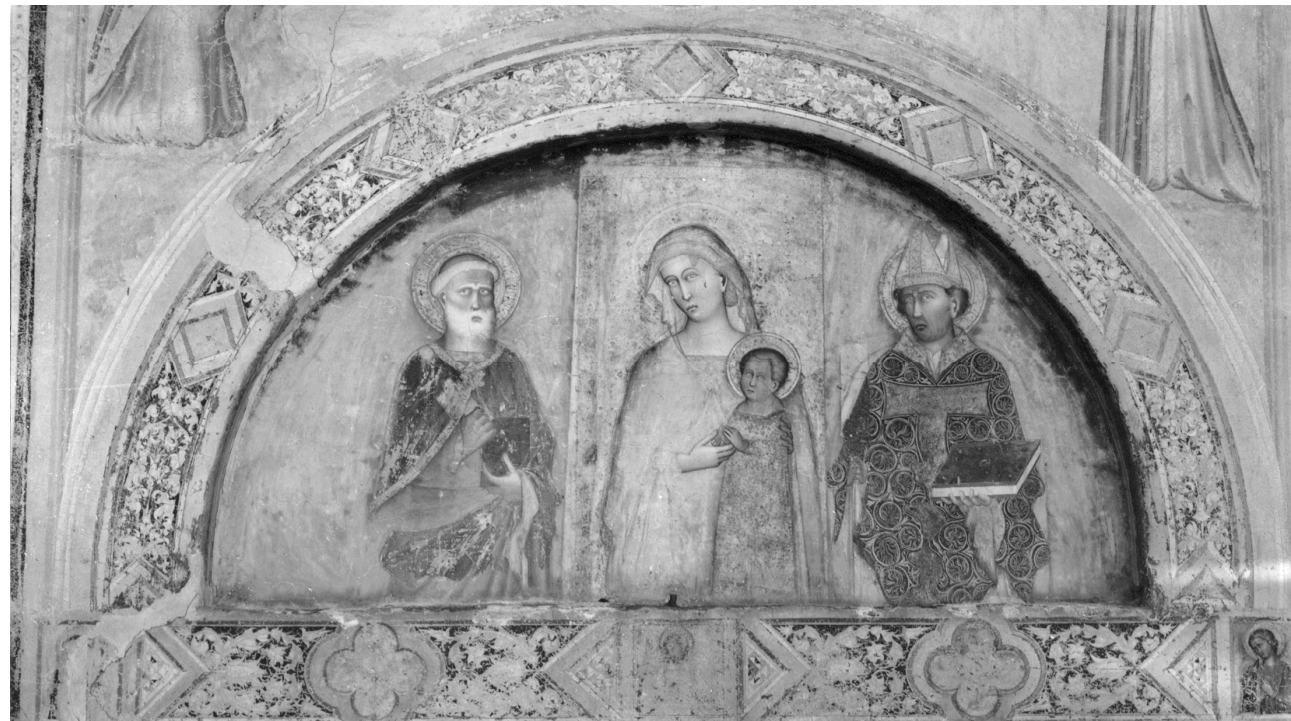

Figura 46 Maestro di San Biagio, Madonna con Bambino fra i Santi Biagio vescovo e Pedro, XII sec. Chiesa di San Biagio di Ravecchia dopo il restauro. Fotografia b/n. Bellinzona. AUBCBZ, Archivio grafico, Ravecchia, S. Biagio.

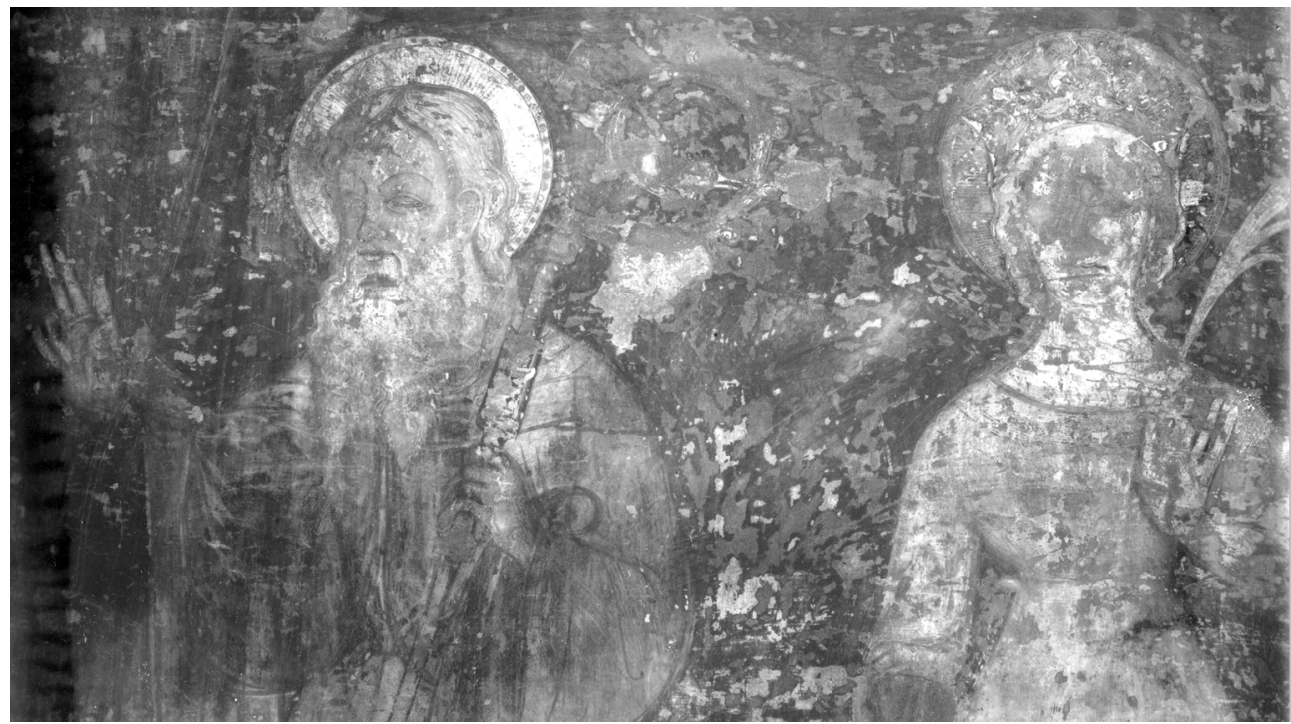

Figura 47 Maestro di San Biagio, Madonna con Bambino fra i Santi Biagio vescovo e Pedro, XII sec. Chiesa di San Biagio di Ravecchia dopo il restauro. Fotografia b/n. Bellinzona. AUBCBZ, Archivio grafico, Ravecchia, S. Biagio. 


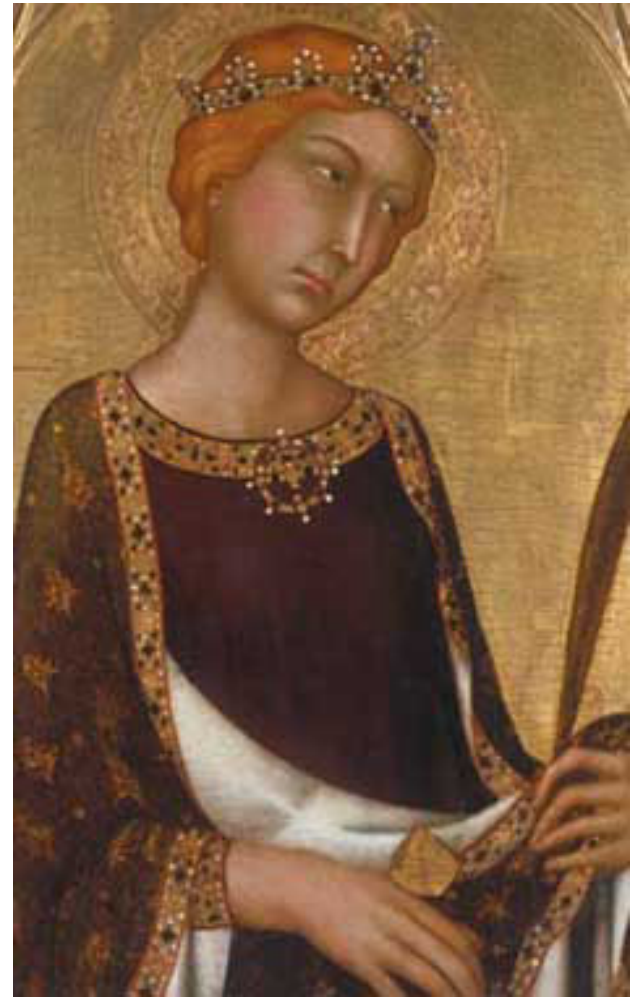

Figura 48 Martini Simone, Santa Caterina da Alessandria, Temple su tavola. XIV sec. National Gallery, Canada.

II progetto d'intervento sviluppato a Ravecchia con l'appoggio dell'architetto Silvio Soldati ${ }^{189}$, socio della compagnia di costruzione "Brenni e Soldini", fu accettato dalla Confederazione Svizzera con una lettera datata 14 luglio 1913, nella quale si considerava il finanziamento dei lavori da parte dello Stato con una somma di Fr. 9'840 sul totale di Fr. 29'200 del preventivo presentato. II Consiglio Parrocchiale offrì la

${ }^{189}$ (Soldati Silvio, Compiti metrici e perizia descrittiva delle opere ricorrenti per il restauro della Chiesa Parrocchiale di Ravecchia, Lugano 19 giugno 1913. AUBCBZ, s. 021 Bellinzona). cifra di Fr. 3'000 che doveva coprire i lavori per la ristrutturazione del tetto, e l'Amministrazione dell'Ospedale di $\mathrm{S}$. Giovanni offrì un totale di Fr. 784'17.

I lavori di ristrutturazione architettonica furono realizzati per la ditta di costruzioni dello stesso progettista, Brenni e Soldini, sotto la vigilanza degli esperti federali, I'architetto Albert Näf ed il Dottore Robert Dürrer, quest'ultimo presidente della Commissione Federale dei Monumenti Storici, e furono finiti nel mese di settembre del 1914. Lo stesso Edoardo Berta ci spiega i dubbi che presentarono i sopracitati responsabili della Confederazione, riguardo alle proposte di riadattamento della chiesa presentate; ma non fu disattesa l'approvazione del progetto, poiché ci conferma che anche l'ispettore federale Albert Naef difendeva una conservazione preventiva del monumento, non nel modo in cui oggi intendiamo questo concetto, ma per la conservazione delle parti originali del manufatto e la cancellazione dei restauri avvenuti, opposti all'omogenea visione della costruzione e delle decorazioni.

La chiesa fu riaperta al culto dopo i lavori il 27 settembre 1914, con notevole successo nel restauro pittorico che interessò la riparazione dei dipinti murali sulla facciata e degli interni coperti dalle scialbature di calce ed intonaci.

Furono puliti e restaurati gli affreschi sulla facciata raffiguranti: la Madonna con il bambino fra i Santi Biagio vescovo e Pedro, di stile giottesco XII sec., che il pittore Edoardo Berta identifica come opera di scuola lombarda con influenze toscane o più propriamente senese, attribuiti ad un artista conosciuto come il Maestro di S. 
Biagio. Secondo Francesco Chiesa, a Berta piaceva immaginare che fossero stati realizzati dal grande artista Simone Martini ${ }^{190}$ di Siena:

[...]si compiaceva d'immaginare che [...] in qualche suo viaggio in Francia fosse passato da Bellinzona e avesse dipinto quella deliziosa lunetta $[. . .]^{191}$.

Il resto delle opere oggetto di intervento furono l'Annunciazione ed il Cristo benedicente nella lunetta sopra la porta d'entrata, ed il gigante San Cristoforo con il Bambino sopra la schiena, il quale, come in tante altre chiese, si espande su tutta la facciata con la sua presenza, che facilita la sua visione dalla lontananza invitando ai viandanti, viaggianti, e pellegrini ad avvicinarsi all'orazione all'interno dell'edificio di culto. La protezione di questi affreschi dai danni medio ambientali fu realizzata con l'incorporazione di due tetti, concessi nel momento come il metodo meno invasivo. Anche se il Berta aveva ricevuto dei consigli riguardo la protezione dei dipinti murali della facciata, come il ricollocamento di un vetro sulla superficie di essi, il pittore confessa nelle sue relazioni di non aver trovato un modo più idoneo per isolare le pitture dall'azione degradante della pioggia e degli agenti

\footnotetext{
190 Martini Simone (Siena 1284 ca - Avignone 1344 ca) II pittore italiano fu promotore del gotico italiano che grazie alla sua presenza in Francia presso la corte papale diffonde internazionalmente con l'opera de miniatori. Fra le sue opere principali possiamo citare la Maestà del Palazzo pubblico di Siena del 1315, gl affreschi e le vetrate della cappella di San Martino sulla Basilica Inferiore di San Francesco ad Assisi nel 1317 ca., il politico per la chiesa conventuale di Santa Caterina a Pisa nel 1319 e l'Annunciazione a Firenze nel 1333.

${ }^{191}$ (Chiesa, In memoria di Edoardo Berta, 1931, p. 29).
}

biologici, ai quali le opere esterne sono pienamente esposti, che l'istallazione dei suddetti tetti.

Si tratta sicuramente del migliore metodo che il restauratore potesse scegliere per il riparo delle pitture, almeno in modo temporaneo, causando il minimo danno con il minimo intervento eseguito. In questo modo Berta, permesse che in un futuro, restauratori con meccanismi più idonei, ritrovassero la superficie pittorica originale. Tuttavia il ragionamento di Berta fosse sicuramente più estetico che conservativo, la sua attuazione concesse in qualunque modo quest'ultimo fattore, meglio di quanto lo avrebbe fatto l'applicazione di un vetro, come proponeva nella lettera in agosto del 1910 il restauratore di Milano Francesco Annoni al pittore, il quale potrebbe aver causato delle condensazioni sui dipinti.

[...] La cosa che più mi preme e sia mio dovere mettere fatto la sua considerazione è la pregiata lunetta centrale che osservandola bene mi è apparsa una pittura delicatissima è mi pare assolutamente necessario difenderla dalle intemperie del tempo studiando il mezzo di rimettere un vetro perché ho tanta paura che l'acqua a poco a poco possa operare sul dipinto in deperimento in quelle delicate mezze tinte che hanno finora affidato i secoli le faccio osservare che il dipinto e casi diventato come una spugna $[. . .]^{192}$.

L'interno della chiesa di San Biagio a Ravecchia ha una struttura di tre navate divise per colonne che si sviluppano in arcate verso i tre cori. La conservazione dei dipinti è, ancora oggi, come nel momento

\footnotetext{
192 (Annoni Francesco. Lettera indirizzata a Edoardo Berta. Milano 10 agosto 1910. AUBCBZ, s.021 Ravecchia).
} 
in cui arrivarono nelle mani di Berta, deplorabile, anche se in un passato dovevano coprire quasi tutta la superficie delle pareti e degli archi.

[...]l'effetto che in origine doveva scaturire da tutto questo assieme di freschi doveva essere certamente imponente ma gli stessi furono poi ridotti in uno stato miserando dalle picchiettature fatte per farsi aderire gli intonaci di calce sovrapposti nei vari rivolgimenti o restauri, eseguiti in varie epoche del Cinquecento. $[. . .]^{193}$.

Le pitture più antiche sono del 1340, si tratta della raffigurazione della Madonna della Misericordia che accoglie i fedeli fra angeli che avvolgono la composizione. Questo dipinto si trova sull'arco Trionfale e sono attribuiti al "Maestro di Sant'Abbondio", così chiamato in riferimento alle sue opere nell'omonima chiesa della città lombarda di Como. In basso a questa scena si trova la figura dell'Annunciazione dipinta con un vaso di fiori di gigli fra le mani.

Opera del "Maestro di San Biagio" precedentemente citato sono, insieme ai dipinti della facciata, le pitture dell'abside. Nella volta appaiono le figure dei quattro Evangelisti, una Crocifissione parzialmente leggibile e gli Apostoli, secondo Berta, di grande somiglianza con quelli degli Oratori di Lentate e Solaro (Lombardia) e che sicuramente influenzarono l'autore degli affreschi rappresentati sull'altare maggiore della Chiesa di S. Paolo di Arbedo.

Fra i dipinti di carattere votivo della parete meridionale si svela per la sua qualità il frammento di una Crocifissione del XII sec. Sulla controfacciata osserviamo le raffi-

193 (Berta, Relazione sui lavori di restauro della Chiesa di San Biagio in Ravecchia, 1915, p. 6). gurazioni di diversi santi, la Veronica e una Madonna fra santi tutti loro originari dei XIV-XV sec. A nord della basilica si trova uno strappo della Deposizione, in passato proprietà di un oratorio demolito, un Sant'Antonio Abate ed il Martirio di San Lorenzo.

Durante l'intervento diretto da Edoardo Berta si scoprirono, a parte dei dipinti già descritti, le figure di dodici Apostoli su sfondo azzurro di grandezza maggiore al naturale, decorazioni ornamentali sulle due finestre riaperte $e$ in alto delle lesene esterne del Coro, traccia di teste di un Santo e di una Santa, mentre nel coro nord furono unicamente ritrovati i resti delle decorazioni della finestra ad ornamentazione lineare nella volta e nel coro meridionale si scoprirono dei resti di una decorazione seicentesca con altre figure sulla parete nord a grandezza naturale.

[...]Non fu quindi che dopo un lungo, coscienzioso e paziente lavoro eseguito dall'operaio Fumagalli che potemmo ritornare a molte di tali pitture chiarezza della forma e la vivezza dei colori - per ottenere l'effetto d'assieme che le pitture dovettero avere nel 1300 era necessario togliere quelle lacune picchiettature - che impediscono al visitatore di ricomporre l'effetto pittorico ciò conseguimmo rifacendo con lavoro paziente e prudente l'intonaco mancante e distendendo sullo stesso delle velature in modo da ricollegare le sparse membra della composizione.[... $]^{194}$.

L'operaio citato, Fumagalli, era un muratore di cui si parla anche in una

\footnotetext{
194 In questa frase si fa riferimento allo scialbo che copriva tutte queste pitture prima della pulitura eseguita dell'operaio Fumagalli. (Berta, Relazione sui lavori di restauro della Chiesa di San Biagio in Ravecchia, 1915, p. 8).
} 
lettera che Francesco Annoni di Milano invia ad Edoardo Berta nell'Agosto del 1910. II restauratore milanese, invece, si era recato da poco a Bellinzona per esaminare la Pala d'Altare sopra la quale più avanti sarebbe intervenuto. Durante le indagini sulla tavola, Annoni controllò inoltre i lavori di ritrovamento dei dipinti nei muri di San Biagio. Ci risulta molto interessante l'avvertimento che il restauratore realizza successivamente a Berta sul metodo di descialbo mediante lavatura utilizzato dal Fumagalli, mentre egli consigliava per il contrario l'uso diligente del mezzo a secco, senza ulteriore impiego di disoluzioni. Si tratta di uno degli unici documenti che sono stati ritrovati, riguardanti gli interventi eseguiti nell'epoca sui dipinti murali, in cui si menzioni esplicitamente la tecnica "umida" per il descialbo, anche se come è stato approfondito nei capitoli sui lavori in Santa Maria degli Angioli, doveva trattarsi di una metodologia abituale.

II completamento del ritocco pittorico veniva eseguito con le menzionate velature, generalmente in tonalità grigie, considerato il colore neutro per ricreare la omogeneità visiva.

L'intervento descritto fu portato a termine dal pittore Arturo Ortelli ${ }^{195}$ sul coro

195 |l pittore Arturo Ortelli, i cui dati biografici non è stato al momento possibile ritrovare e ricostruire, partecipò attivamente a numerosi eventi di restauro, in qualità tanto di operatore come di progettista. Gli interventi catalogati grazie alla presente ricerca sono stati: San Biagio a Ravecchia (1910) dove il pittore scopre e consolida i dipinti del sec. XIV e sec. XV sotto lo scialbo di calce; la Chiesa Collegiata dei SS. Pietro e Stefano (1910) dove esegue la sorveglianza ai lavor lavori in corso per la parte architettonica e decorativa della facciata; Santa Maria delle Grazie a Bellinzona (1910) per i lavori di ricerca e di pulitura degli centrale, sulla Tribuna e sulla porta interna della facciata e si mandò copia della documentazione fotografica all'Archivio della Commissione d'Esperti del Dipartimento Federale dell'Interno.

Rimaneva anche in sospeso il progetto di pulitura della calce che ricopre le pietre delle facciate, che doveva essere fatta con "acidi", il rifacimento del gradino in granito di fronte all'entrata principale e la creazione di un selciato davanti al portale con ciottoli di colori che segnano la data del restauro ed il muro di cinta davanti alla facciata. II più radicale progetto eseguitosi in questo momento storico fu, tuttavia, la distruzione dell'Oratorio della Confraternita addossato alla chiesa. Questa costruzione doveva sembrare un disturbo per Berta nella visione dell'edificio nella sua entità primaria ed inoltre gli impediva di aprire le finestre originarie sulla facciata a nord, per cui il restauratore giustificò il sacrificio della medesima costruzione, il quale $\mathrm{fu}$ facilmente riparabile con I'indennizzo di una somma di Fr. 3'000 alla Confraternita $^{196}$.

affreschi coperti dallo scialbo di calce; Santa Maria Assunta a Brione Verzasca (1915) dove esegue la rimessa in luce sotto lo strato di calce degli affreschi quattrocenteschi; Battistero di Riva San Vitale (1920) con la rimozione degli intonaci, la ricerca degli elementi originari e quali successivi della costruzione, così come la scoperti di affreschi; Ossario di Balerna (1925) dove sorveglia i lavori di rimozione e ricostruzione dell'edificio; ed a Santa Maria degli Angioli a Lugano (1930). Un secondo contributo fondamentale da parte di Ortelli per la cultura e la salvaguardia della memoria fu realizzata nel 1955. Quest'anno il professore Arturo Ortelli donò un fondo di 325 volumi e opuscoli riguardanti il Risorgimento italiano che oggi fanno parte del fondo antico della Biblioteca Cantonale di Lugano (BCLu).

${ }^{196}$ (Berta, Relazione sui lavori di restauro della Chiesa di San Biagio in Ravecchia, 1915, p. 12). 
II commemorativo di restauro trovatosi ancora oggi nel monumento cita:

[...]La Chiesa di San Biagio in Ravecchia è stata restaurata del 1913 al 1915 dallo Stato del Canton Ticino con il concorso della Parrocchia di Ravecchia e con un sussidio federale. II 9 marzo 1913 la Parrocchia di Ravecchia ha assunto verso la Confederazione degli obblighi ratificati il 12 marzo 1913 dal Consiglio di Stato del Cantone Ticino[...].

In una lettera che Albert Naef dirige al Dipartimento Svizzero dell'Interno il 17 agosto 1915, dichiara la sua soddisfacente in fronte ai lavori eseguiti nella chiesa di Ravecchia [...]l'impression recueillie de ma visite ... est extrêmement favorable $[. . .]^{197}$. Dopo la sua visita del 4 agosto, Näf scrive un rapporto totalmente positivo: descrive un restauro [...]fort bien réussie, est harmonieuse $[. . .]^{198}$, dove le uniche critiche si rivolgono alle moderne dorature sulla cornice del quadro restaurato per il milanese Annoni, sospeso sulla parete sinistra della navata, il cui effetto ritiene si dovesse abbassare con una velatura.

Edoardo Berta chiamò a visitare nel 1909 la Pala d'Altare di Ravecchia. Si trattò di una delle prime collaborazioni fra il pittore Berta e la famiglia Annoni d'ebanisti milanese. La ditta fondata dal padre, Luigi, aveva vinto numerosi premi in qualità di creatori di opere su legno: nella Esposizione Universale di Parigi del 1867, nelle Esposizioni Riunite a Milano nel 1894 e nella Esposizione a Parigi nel 1900. Tutti

\footnotetext{
${ }^{197}$ Lo stesso giorni l'ispettore federale visita la Chiesa Rossa ad Arbedo e parte in serata a Lugano. (Berta, Relazione sui lavori di restauro della Chiesa di San Biagio in Ravecchia, 1915, p. 13).

${ }^{198}$ (Berta, Relazione sui lavori di restauro della Chiesa di San Biagio in Ravecchia, 1915, p. 13).
}

questi distintivi venivano segnati nell'intestazione delle lettere che oggi sono state ritrovate in Archivio all'Ufficio dei Beni Culturali di Bellinzona. Grazie in particolare alla figura di Francesco Annoni la bottega famigliare non si dedicherà unicamente alla creazione di nuove opere come anche al restauro di oggetti d'arte. Sarà lui a mantenere le relazioni con la Svizzera agli inizi del Novecento, specialmente con Edoardo Berta, ed operare oltre alla pala di San Biagio sulla famosa Ancona della Pietà nella chiesa della Madonna del Sasso ad Orselina (Locarno) nel 1923 e sulle decorazioni dell'altare di San Giuseppe nella medesima chiesa nel 1921.

In Italia invece, il restauratore aveva collaborato ad esempio nell'intervento sulla pala della Madonna col Bambino di Cima da Conegliano nella Pinacoteca di Brera nel 1903 che fu dalla azienda famigliare rintelata e pulita da Luigi Cavenaghi $^{199}$, e nella tavola della Madonna del Bosco della omonima chiesa milanese dove oltre il restauro esegue una copia a tempera esposta nel Santuario in sostituzione dell'originale come metodo di preservazione.

Il primo preventivo che Annoni presentò al professor Berta è datato 20 luglio del 1909 dopo la prima ispezione.

[...]Come all'incarico conferitomi nella prima riunione della Commissione Cant. Per la conservazione dei Monumenti Storici ed artistici, ho subito fatto una visita al quadro di Ravecchia e constatai infatti che urge d'essere consolidato, restaurato e collocato con un buona cornice in stile in una delle pareti laterali dell'altare

${ }^{199}$ (AA.VV., 2007, p. 180). 
maggiore sotto una luce che permetta di gustarne i suoi pregi[...]. ${ }^{200}$

II conto comprendeva i lavori per I'intervento sul dipinto e per la costruzione della nuova cornice di stile simile a quello originario. Le operazioni d'intervento dettagliate citano soltanto la pulitura che doveva essere eseguita sul posto, per un totale di fr. 95, l'elaborazione della suddetta cornice in legno per fr. 250, decorata con fondo blu, ornati a chiaro oscuro e dorata con oro fino per fr. 350 .

Un disegno di questa cornice si trova nella lettera che il restauratore spedisce ad Edoardo Berta un anno dopo la prima visita con i lavori sul quadro finiti. In questo disegno si osserva lo stile dell'opera in legno nella quale erano stati previsti due spazi, uno in alto ed un altro in basso segnati $B$ e A rispettivamente, che secondo Annoni nell'epoca si usavano per introdurre delle scritte sacre.

A lavorare nuovamente sui dipinti murali della chiesa di San Biagio sarà Emilio Ferrazzini quasi trenta anni dopo, esattamente nel 1944. II pittore luganese interverrà unicamente sulle pitture della facciata ma il suo approccio all'opera pittorica sarà meno rispettoso di quanto previsto dall'ispettore Berta, poiché indicherà l'utilizzo di consolidanti a base cerosa per la protezione degli affreschi agli agenti esterni, creando una pellicola impermeabile sullo strato pittorico e cambiando in questo modo le sue caratteristiche proprie di porosità e traspirazione talmente necessarie per una buona conservazione dei materiali inerenti all'affresco.

Attualmente i dipinti della facciata sono stati puliti dallo strato di cera applicato da Ferrazini mediante un rispettoso restauro che si è visto concluso nel 2000 , sotto la supervisione dell'Ufficio dei Beni Culturali di Bellinzona.

200 (Annoni Francesco, Preventivo di restauro della Pala d'Altare nella Chiesa di San Biagio in Ravecchia a Edoardo Berta, Lugano. Milano 20 luglio 1909. AUBCBZ, s. 021 Bellinzona). 


\section{Castello Visconteo, Locarno}

[...]Di due epoche sono l'impronte ben distinte[...] II piccone e lo scalpello hanno lavorato già per mesi e mesi a strappare al cimelio prezioso l'opera insolente dei barbari che alle loro aspirazioni di dominio in quest'angolo di terra italiana non seppero congiungere il rispetto dovuto all'impronte del genio. $[. . .]^{201}$.
[...]De dos épocas son los estilos bien diferenciados[...] II pico y el escalpelo han trabajado durante meses y meses para arrancar al preciado edificio, la obra insolente de los bárbaros que, con la aspiración de dominar este rincón de tierra italiana no supieron conjugar el respeto debido a los seños del genio[...].

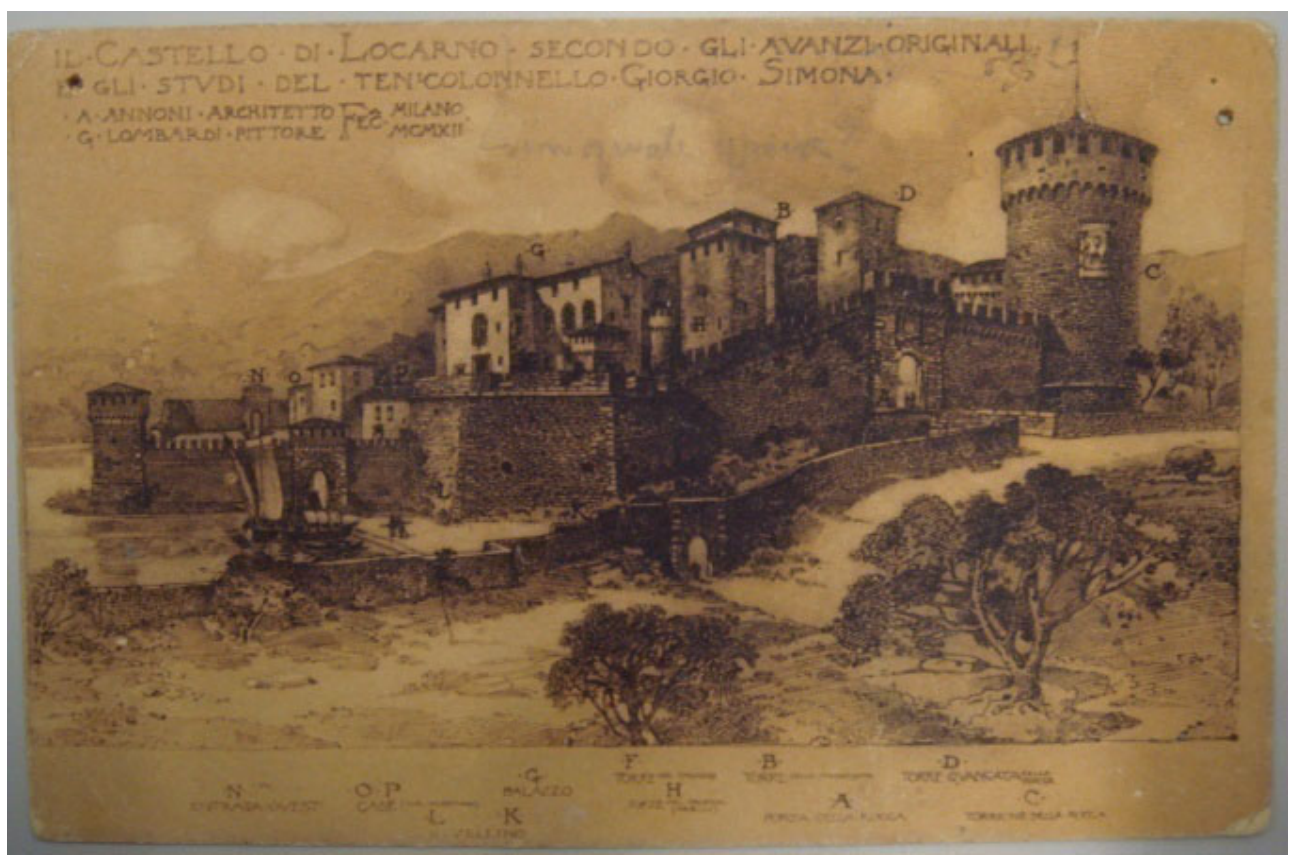

Figura 49 Lombardi G./Annoni A. I/ Castello di Locarno secondo gli avanzi originali e gli studi del Ten. Colonello Giorgio Simona. Disegno. Milano, 1912. AUBCBZ, s. 134 Locarno, Castello Visconteo.

201 (II nostro Castello, 1923). 
Così riferisce un giornalista per "Popolo e libertà" nel 1923 i risultati dei primi lavori che stavano avendo luogo nel Castello Visconteo a Locarno. La fabbrica, formata da un palazzo e da una fortezza che si erigono intorno ad un cortile centrale, è oggi sede del Museo Civico ed Archeologico di Locarno. Ha le origini nel IX secolo, con successivi cambiamenti: dagli anni 1342 al 1439 durante il dominio dei Visconti si realizza la costruzione delle mura, dal 1439 al 1512 con la dirigenza dei Rusca si conferisce l'estetica di maestosità alla cittadella e per ultimo, nel 1507 si innalza il rivellino oggi attribuito a Leonardo da Vinci e nascosto dalle numerose casa addossate.

Il castello rimasse abbandonato dopo il suo smantellamento nel 1531, essendo ancora sede dei landfogti dal 1513 al $1798^{202}$.

II 3 di novembre del 1922 il Dipartimento della Pubblica Educazione del Canton Ticino incarica a Edoardo Berta la direzione dei lavori di restauro progettati ed iniziati dall'architetto Otto Maraini ${ }^{203}$, già nel 1923

\footnotetext{
202 | landfogti vengono denominati anche con il termino di balivo.

${ }^{203}$ II progetto/preventivo stipulato dall'architetto Otto Maraini comprendeva soltanto una parte dei lavori di restauro. $\mathrm{Fu}$ elaborato in collaborazione con I'architetto Enea Tallone e compresse soltanto il ripristino del cortile e le sale adiacenti, la demolizione delle murature che nascondono gli archi a sesto acuto del porticato, mettendo a luce i capitelli e le arcate, i ripristino delle quattro finestre ogivali ed scoprimento delle finestre rettangolari aperte in epoca più recente e poi murate, il restauro della porta d'accesso a cortile, il ripristino dei soffitti in legno, la sostituzione delle moderne tegole dei tetti per piode di granito ed il restauro artistico della facciata e del cortile mediante lo scrostamento delle pareti per ricuperare dipinti murali ed i grafiti sottostanti. II preventivo fu di 26466,50 fr. (Maraini Luca, Progetto e preventivo di restauro parziale del Castello di Locarno, indirizzato al
}

proseguendo le ricerche archeologiche iniziate dai grandi architetti milanesi: Luca Beltrami $^{204}$ e Ambrogio Annoni ${ }^{205}$ nel $1910^{206}$. La magnitudine dell'impresa, di un valore di circa fr. 180 '000, fece che essa durasse fino al $1931 \mathrm{ca}$. Sette anni di lavoro, praticamente continuo, con l'obiettivo di ridare un'omogeneità estetica ragionabile alla fabbrica dal ritrovamento delle forme corrispondenti alle diverse epoche costruttive medievali alla conservazione di quelle più significative, ricostruendo i locali del palazzo per il suo uso effettivo come museo. L'intervento diretto da Edoardo Berta con una attualizzata concezione di restauro architettonico, non si basa nel riportare il Castello ad uno stato originario unico come si sarebbe aspettato [...] non ho mai sognato ricostruire parti che non esistono più

Lodevole Dipartimento della Pubblica Educazione. Lugano 11, 16 novembre 1921. AUBCBZ, s. 134 Locarno, Castello Visconteo).

204 Beltrami Luca (1854 - 1933). [...]Nel 1894 il deputato Beltrami aveva lamentato il "libero arbitrio dei restauratori, i quali non sempre usano le dovute cautele, e non sempre danno serio affidamento di compiere bene il lavoro affidato. II professor Beltrami, direttore dell'Ufficio per la conservazione dei monumenti lombardi, nonché deputato e quindi senatore del Regno, liberale - conservatore: un bell'intreccio tra politica, politica del restauro, e restaurazione. Per restare a Milano, e visto che Beltrami, oltre a inventarsi la bizzarra Torre del Filarete, ha messo mano anche a Piazza della Scala cominciando col demolire le "case rotte", ultimo ricordo della signoria dei Della Torre, viene in mente il Teatro che le aveva dato il nome, attualmente in fase di restauro[...] (Discorso del 2 giugno 1984, in Atti Parlamentari, Camera dei Deputati, Leg. XVIII, pag. 9646. Citazione tratta dalla tesi di laurea di S. Sicoli, Facoltà di lettere, Milano, AA 1975-76).

${ }^{205}$ Annoni Ambrogio (Milano 1882 -1954).

206 I lavori cominciati non furono cominciati a causa del cominciò pochi anni dopo della Prima Guerra Mondiale. 
affatto $[. . .]^{207}$, secondo le idee di Viollet-leDuc ma nel ricreare l'effetto d'unità mettendo in luce soltanto gli elementi medioevali più importanti e facendo convivere i diversi momenti storici fra $d i$ loro. I suoi ideali teorici si adattano all'opera in sé ed alle sue esigenze, ed il restauro avviene solo dopo fare congiungere arte e scienza con l'obiettivo di conservare ed avvalare ${ }^{208}$. Per restauro non si intenderà più né ricomposizione stilistica, né ricostruzione storica; ma conservazione, sistemazione, avvaloramento dell'edificio. Così lo manifesta Berta al Dipartimento della Pubblica Educazione in Bellinzona il 10 maggio del 1923: [...]Conosco un poco $i$ vari concetti informativi che sono oggetto di discussione tra gli Archeologi in punto ai principi che devono presiedere l'esecuzione di restauro dei Monumenti storici ed artistici, e apprezzo tutta la loro importanza e serietà sebbene siano sovente tra di loro discordanti. Ritengo però che $i$ principi teorici non debbano sempre essere applicati rigidamente, ma speciali del Monumento da restaurarsi che devono determinare la linea di condotta da adottarsi $[\ldots]^{209}$.

Nell'intervento eseguito al Castello Visconteo di Locarno leggiamo una chiara influenza delle teorie di restauro filologico di Camillo Boito, a chi Edoardo Berta aveva avuto l'opportunità di conoscere all'Accademia di Brera, ed in speciale del suo collega l'architetto Luca Beltrami, principale allievo a suo tempo del Boito. II

\footnotetext{
207 (Berta Edoardo, Lettera indirizzata al Sig. Tarabori. 12 maggio 1922. AUBCBZ, s. 134 Locarno Castello Visconteo).

${ }^{208}$ (Annoni, 1946, p. 14).

209 (Berta Edoardo, Lettera indirizzata al DPE. 10 maggio 1923. AUBCBZ, s. 134 Locarno Castello Visconteo).
}

Beltrami aveva finalizzato nel 1905 il restauro del Castello Sforzesco di Milano, riportandolo alle forme estetiche medievali che la fortezza ebbe prima della dominazione delle truppe napoleoniche, secondo un'analisi documentario approfondito che considerava tanto il monumento come il suo contesto, basi fondamentali del restauro storico. In questo progetto fu partecipe anche il pittore, decoratore e restauratore di Rancate, Ernesto Rusca ${ }^{210}$, che viene chiamato dall'architetto milanese per la realizzazione dei lavori sulla Sala delle Asse del Castello dipinta da Leonardo da Vinci nel 1495. Questi lavori procurarono il riconoscimento del restauratore che fu premiato con il titolo di "cavaliere della Corona d'Italia"211, malgrado oggi i suoi interventi risultino meno lodevoli. II pittore-restauratore di origini ticinesi esegue nella Sala medievale del Castello Sforzesco, un intervento molto prossimo alle dottrine dell'epoca apprese nel palazzo di Brera dal pittore Luigi Cavenaghi. Oggi ci si presenta un dipinto disturbato dai numerosi ritocchi realizzati nel tentativo di ricreare I'unità estetica primitiva. Essi interventi furono eseguiti contemporaneamente ai lavori nella

\footnotetext{
${ }^{10}$ Rusca Ernesto (Rancate, 1862 - Genova 1947) ॥ pittore formatosi all'Accademia di Belle Arti di Brera con i maestro Camillo Boito e Luigi Cavenaghi, lavorò principalmente in Milano come decoratore, dedicandosi al restauro dei monumenti storici, in particolare nella Lombardia e nel Canton Ticino, in collaborazione con Luca Beltrami.

211 L'Ordine della Corona d'Italia era destinato a premiare con le insigne di cavaliere, ufficiale, commendatore, grande ufficiale, Gran Croce o placca per Gran Croce ai cittadini italiani e stranieri che si fossero resi benemeriti verso la Nazione, la Corona o il Sovrano.
} 
Cattedrale di San Lorenzo a Lugano. Su quest'ultima fabbrica, il pittore Ernesto Rusca insieme all'architetto Augusto Guidini, realizzarono delle nuove decorazioni con elementi vegetali di marcato influsso leonardesco e dipinti murali raffiguranti figure di santi. Quarant'anni dopo l'intervento del Rusca a San Lorenzo a Lugano sarà descritto dalla propria CCMS come inappuntabile:

[...]Grandi certamente erano le difficoltà di restaurare in modo degno la Cattedrale di Lugano, per il concorso e la miscela degli elementi architettonici diversissimi che costituiscono la massa di quell'edificio. E riconosciamo volontieri che certi ostacoli furono superati con abilità e fortuna. Ma quella profusione di decorazioni pittoriche nelle volte delle navate suscita, in chiunque abbia buon occhio, un senso di cosa sovrapposta e non connaturata all'indole dell'edificio, né concordi con le preziose pitture antiche superstiti sui pilastri; e quegli enormi sfarzosi amboni non si saprebbe dire se siano più poveri di intrinseca bellezza o più disarmonici col carattere del tempio $[\ldots]^{212}$.

Ritornando alle dottrine filosofiche, da Boito e da Beltrami, bisogna ricordare che queste erano derivate nel Novecento nella teoria del "caso per caso" propugnato dall'architetto Ambrogio Annoni di Milano, il quale, come dagli scritti di Edoardo Berta precedentemente trascritti, propugna l'analisi di ogni manufatto che venne ritenuto il documento di studio fondamentale per la esecuzione di una proposta di restauro idonea.

Ambrogio Annoni, quando realizza nel 1910 le prime ricerche storiche in contributo al progetto di riordino del monumento locarnese, metterà a confronto questo con il sopracitato Castello Sforzesco di Milano restaurato dal Beltrami. Ques'ultimo diventerà prototipo di restauro ben riuscito:

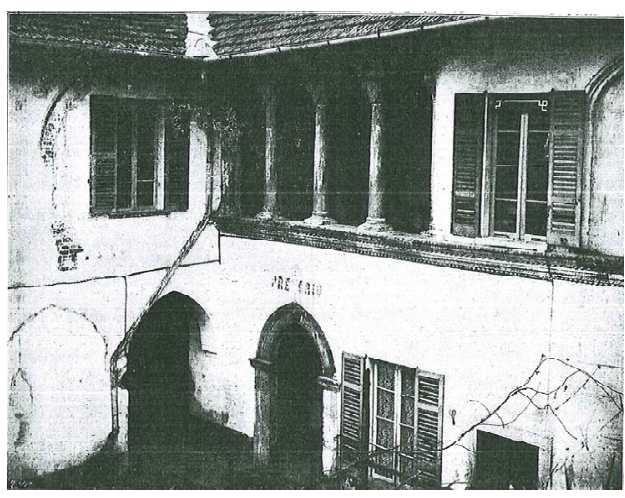

Figura 50 Fratelli Buchi. Castello Visconteo. Prima del restauro. Fotografia b/n. AUBCBZ, Archivio fotografico. Locarno Castello Visconteo.

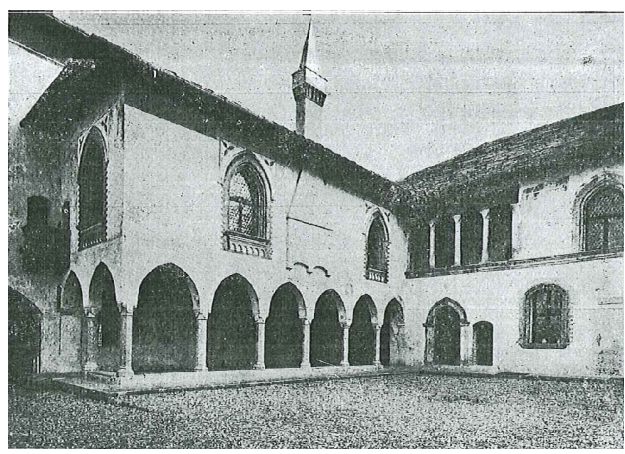

Figura 51 Fratelli Buchi. Castello Visconteo. Dopo il restauro. Fotografia b/n. AUBCBZ, Archivio fotografico. Locarno Castello Visconteo.

212 (DPE, 1959, p. 46). 
[...] Un magnifico esempio, in circostanze analoghe, mi soccorre: quello del nostro Castello Sforzesco. Considerato dapprima poco meno che un rudere ingombrante lo sviluppo cittadino, ricordo solo di tristi ultimi servaggi, ora per la oculata costanza di chi ne aveva ricercato $e$ rievocato con studi e disegni tutto lo splendore della vera sua storia e la fioritura della sua arte, per le cure, cioè, d'artista, di storico e di cittadino dell'Architetto Senatore Luca Beltrami, ora il Castello di Milano sta, fra il sorriso di un parco, che v'è nato attorno, in testa ad una delle più eleganti fra le arterie della Città moderna, visitato come uno dei principali monumenti con il Duomo e S. Ambrogio, meta e centro delle radunanze artistiche $e$ storiche, luogo di convegno e di ricevimento per le Autorità Cittadine nelle occasioni solenni. Di esso è stato conservato il nucleo costituente la residenza ducale, bastando gli avanzi mantenuti qua e là nel parco, con opportuna scelta e conveniente iscrizioni, a dove l'idea è a ravvivare il ricordo anche delle estesi prime propaggini fortilizie. Così appunto penserei e propongo di fare per il Castello di Locarno a decoro della Città, ed a vantaggio del suo stesso incremento edilizio. $[\ldots]^{213}$

Le proposte di restauro di Edoardo Berta per il Castello Visconteo, presentate il 28 marzo del $1923^{214}$, vengono accettate dal Municipio di Locarno e dal presidente della Commissione Federale dei Monumenti Robert Dürrer, il quale chiederà come unico dettame la conservazione di tutti gli stemmi Landfogti trovatesi sotto il portico del corpo sud del Castello, sulle pareti della scala che comunica il cortile e la loggia ed

\footnotetext{
${ }^{213}$ (Annoni Ambrogio. Rapporto indirizzato a Giorgio Simona. Milano, 28 ottobre 1910. AUBCBZ s. 134 Locarno Castello Visconteo).

${ }^{214}$ (Berta Edoardo, Proposta di restauro, indirizzata all'Onorevole Sig. Sindaci e Municipali. 28 marzo 1923. AUBCBZ, s. 134 Locarno Castello Visconteo).
}

in diverse sale. L'incarico di questo importante restauro artistico venne affidato ancora una volta alla compagnia zurighese "Chr. Schmidt Dekoratiosnsmaler", rappresentata in Ticino dal pittore Emilio Dillena.

Il primo preventivo di massima realizzato da codesta ditta venne presentato alla Commissione Cantonale dei Monumenti Storici in Canton Ticino il 14 giugno 1929. Su questo si elencano i seguenti processi d'intervento:

1. Nell'atrio: pulitura, consolidamento, ritocco degli stemmi e delle iscrizioni, ritocco e completamento delle lacune di sette elementi sull'arco.

2. Nella Sala XIII e nello scalone: restauro di due affreschi rinascimentali con il riempimento delle martellinature realizzate per l'applicazione di uno scialbo successivo e ricerche stratigrafiche per verificare la possibilità di ritrovamento di nuovi dipinti; restauro dei sette stemmi balivali, alcuni di loro composti e sovrapposti ad altre pitture dove si propone una eventuale eliminazione di quello più recente ed il successivo ritocco del dipinto primitivo.

3. Nella Sala XVII: restauro e completamente delle scritte negli stemmi.

4. Nella Sala X: ricostruzione della parte destra del dipinto rappresentato sul camino, rinfrescando questa zona fino a ritrovare l'intensità dei colori apparsi sulla parte sinistra; ricostruzione di un piccolo pezzo del fregio a graffito, esistente sotto il soffitto ligneo, per rendere visibile allo spettatore l'aspetto della decorazione originaria.

5. Nella loggia: restauro di dieci stemmi e ricostruzione delle scritte scomparse; 
ritocco delle decorazioni del soffitto di legno.

6. Nella Sala XVIII, restauro del gruppo di stemmi con la stuccatura delle martellinature sui dipinti e l'opportuno ritocco di questi punti con un leggero tono di colore che restituisca visivamente l'omogeneità.

7. Nella Sala XIX: ritocco delle pitture e completamento delle iscrizioni. Nella Sala $X X$ : consolidamento e completamento delle iscrizioni omogeneizzando l'aspetto completo dell'aula.

8. Nella Sala XXII ritocco di piccole traccie di stemmi.

9. Nella Sala XXIV: restauro di 66 elementi e rifacimento dell'intera decorazione della parte mediana del soffitto di legno [...] naturalmente fatti con discrezione $[. . .]^{215}$.

10. Nella Sala XXVII: restauro di due stemmi di Sury e Graf. Nella Sala XXXVII chiamata dei Sindacatori: eliminazione delle macchie sulla volta e ritocco degli stemmi seicenteschi evidenziando la differenzazione temporale fra gli elementi seicenteschi e le decorazioni in stucco di epoca posteriore.

$\mathrm{Fu}$ il restauro pittorico al Castello Visconteo, un intervento di "rifacimento" o di "completamento" decorativo come verifichiamo della proposta di restauro, la cui posteriore realizzazione è stata confermata dalle fonti d'archivio.

Sono particolarmente rappresentativi del lavoro realizzato dal pittore Emilio Dillena nel 1929, gli studi dei stemmi che vengono riprodotti su carta velina, su piccoli pezzi di tela o su cartone con pitture a tempera, alcuni conservati ancora nell'Archivio dei Beni Culturali di Bellinzona. Descrivono in modo particolare l'eredità di un insegnamento basato nelle arti applicate, che rivalutarono il concetto di "arti minori o artigianali" per la prima volta in Italia alla fine dell'Ottocento. Dillena lavora dal 1904 fino alla sua morte avvenuta nel 1934 per la firma di decoratori svizzera Chr. Schmidt, principalmente in solitario ed eccezionalmente insieme ad altri pittori come Eduard Gubler, Alexander Struker, Fritz Wittpennig, Ernst Morf, Jonh Schulze, Ernst Gutknecht ed Alfred Hartmann i quali non hanno nessuna relazione con gli interventi eseguiti dalla ditta zurighese in Ticino come ad esempio in Santa Maria delle Grazie a Bellinzona, Santa Maria degli Angioli a Lugano, la Casa del Negromante o il qui descritto intervento sul Castello di Locarno.

Una iscrizione, che la legge di tutela del 1909 del Canton Ticino prevedeva per ogni intervento, testimonia nel monumento i lavori di restauro eseguiti:

[...]Restaurato sotto la direzione del pittore Edoardo Berta dall'anno 1922 al 1926 per iniziativa ed a spese del Comune di Locarno, col concorso della Confederazione. In data 7 marzo 1924, il Comune di Locarno firmò verso la Confederazione gli impegni di legge relativi alla conservazione del Castello: impegni che furono ratificati il 27 marzo 1924 dal Governo del Cantone Ticino. $[\ldots]^{216}$.

\footnotetext{
(DPE del Cantone Ticino. Lettera indirizzata al Lodevole Municipio della Città di Locarno. Bellinzona 3 maggio 1928. AUBCBZ, s. 134 Castello di Locarno).
} 
Le date trascritte nell'iscrizione fanno riferimento unicamente al restauro architettonico, poiché quello artistico deve durare fino al 1932, ancora dopo la morte di Edoardo Berta, anno in cui avvengono i rapporti dell'ultima ispezione dei lavori, eseguita il 30 ottobre dall'ispettore federale Albert Naef ${ }^{217}$.

Solo cinquant'anni più tardi, gli stemmi dipinti sul porticato del cortile restaurati da E. Dillena, inizieranno a manifestare un stato di conservazione problematico. II Comune di Locarno, proprietario dal 1922 della fabbrica, chiedere nel 1972 al restauratore di Tegna, Carlo Mazzi, di realizzare una perizia per verificare la gravità della situazione e proporre degli interventi di restauro idonei per la futura conservazione degli stemmi.

Il rapporto redatto da Mazzi ci apporta alcune informazioni maggiori sull'intervento precedente. Egli denuncia l'impiego di una "tempera" usata in passato per consolidare le zone di colore staccate o pericolanti, che con il tempo invece a contribuito ad accrescere questo danno sulla superficie pittorica, e inoltre ritrova un ritocco pittorico realizzato direttamente su diversi strati d'intonaco senza eseguire una curata livellazione di essi. Il risultato dopo gli anni trascorsi era mediocre, da un opera specialmente lacunosa e di difficile lettura. Nella stessa perizia Carlo Mazzi realizzò dei disegni schematici che servivano a comprendere la estensione dei rifacimenti del $1929 \mathrm{ca}$. e

(Meyer Albert, Presidente del Dipartimento Federale dell'Interno. Rapporto indirizzato a Dipartimento della Pubblica Educazione del Cantone Ticino. Berna 11 aprile 1923. AUBCBZ, s. 134 Castello di Locarno). proponeva l'urgente consolidamento degli strati pittorici degli stemmi: [...]Si dovrà stare molto attenti nell'opera di consolidamento di non cadere nel precedente errore e cioè evitare un consolidamento che nell'andare degli anni faccia ancora da strappo[... $]^{218 .} \quad$ II metodo di consolidamento non poteva comunque essere definito, secondo il restauratore, senza previe sperimentazioni e prove fino ad ottenere il prodotto adeguato alle caratteristiche fisico-chimiche del materiale. Nel caso in cui i metodi di fissaggio non bastassero a frenare il degrado dei dipinti, e solo come ultima alternativa, il Mazzi proponeva lo strappo di essi. Quest'ultima sarà difatto la scelta eletta dalla Commissione Cantonale dei Monumenti Storici e dal Comune di Locarno come metodo d'attuazione, secondo le raccomandazioni nel 1977 del restauratore Luigi Gianola. Egli riteneva che un possibile consolidamento in situ mediante materiali moderni non potesse servire a fissare i diversi strati di pitture alla calce, mentre che il trasporto totale dei dipinti permetterebbe assolutamente il suo restauro "definitivo". ${ }^{219}$

I lavori, affidati finalmente a Luigi Gianola, furono compiuti nel 1978 mediante il trasporto degli affreschi su un supporto di vetroresina al giorno d'oggi specialmente degradato. $^{220}$

\footnotetext{
8 (Mazzi Carlo, Rapporto Riguardante gli affreschi decorativi, stemmi dei Landfogti, nella loggia del Castello Visconteo, indirizzato al Municipio di Locarno. 19 settembre 1972. AUBCBZ, s. 134 Locarno, Castello Visconteo)

${ }^{219}$ (Gianola Luigi, Rapporto, Corte del Castello Visconteo e la conservazione stemmi degli amministratori confederati, indirizzato al Municipio di Locarno. 1 agosto 1977. AUBCBZ, s. 134 Castello di Locarno).

${ }^{220}$ (Valentini, 2005, p. 89).
} 


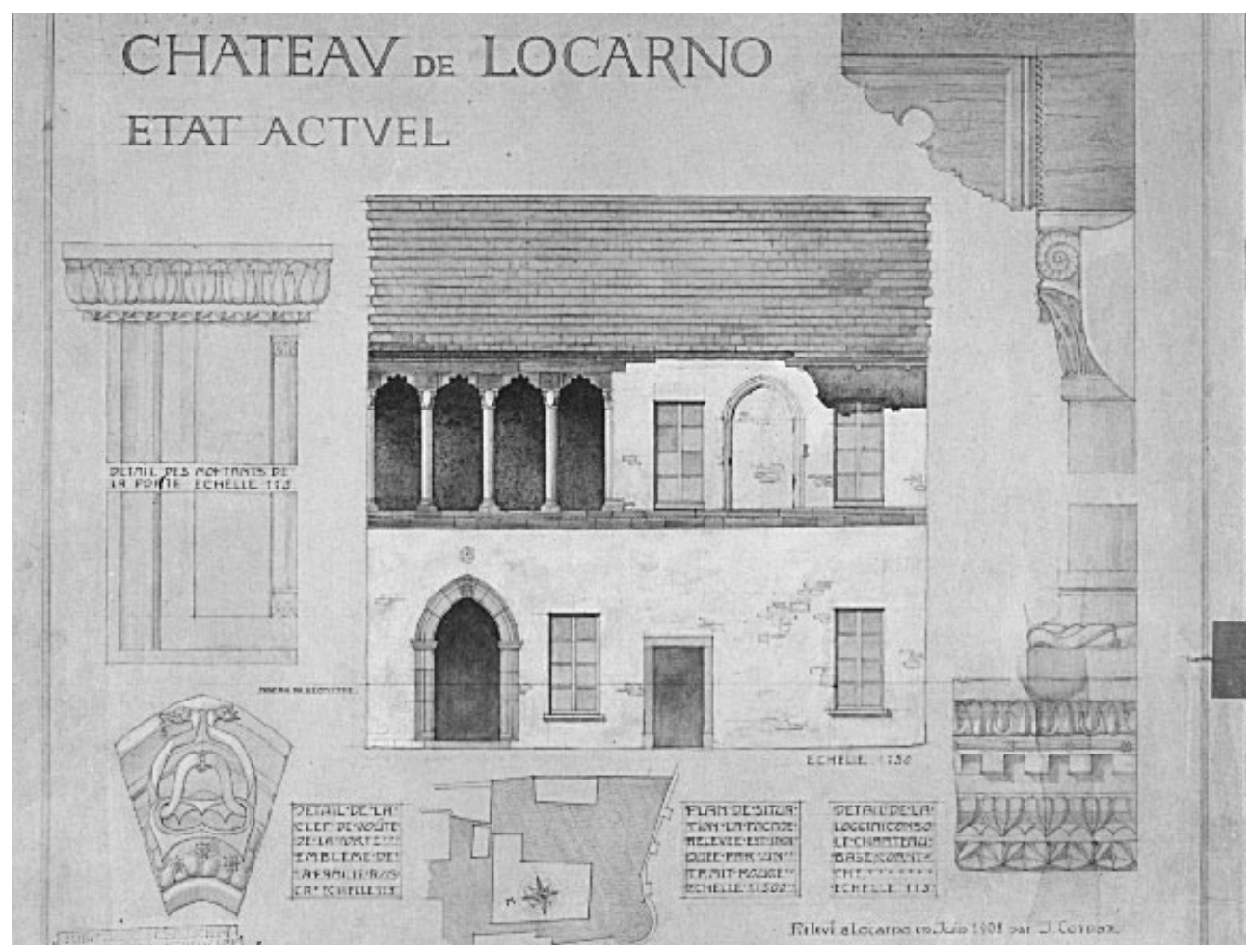

Figura 52 J. Cordoz?. Facciata sud verso il giardino con dettagli, Locarno 1808.

Fotografia b/n. 65685 B.4683 Plan Nr. 9504. ABN Berna.

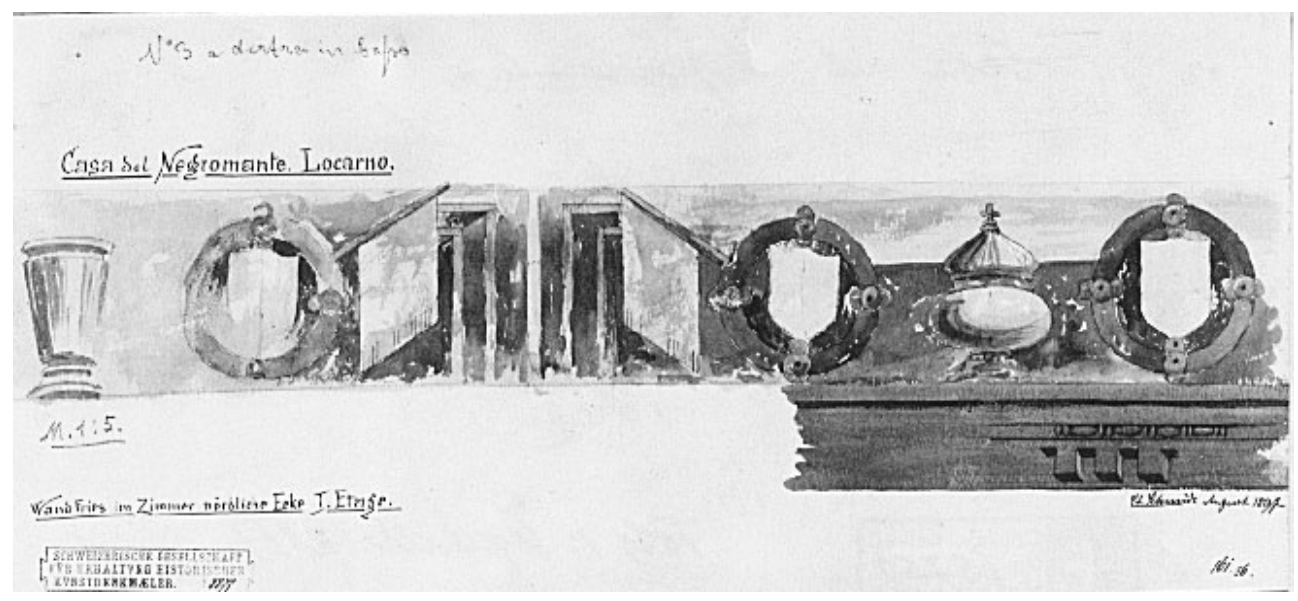

Figura 53 Chr. Schmidt, Casa del Negromante a Locarno. Studio delle decorazioni, Locarno 1897.

Fotografia b/n. 65685 B.4683 Plan Nr. 9504. ABN Berna. 


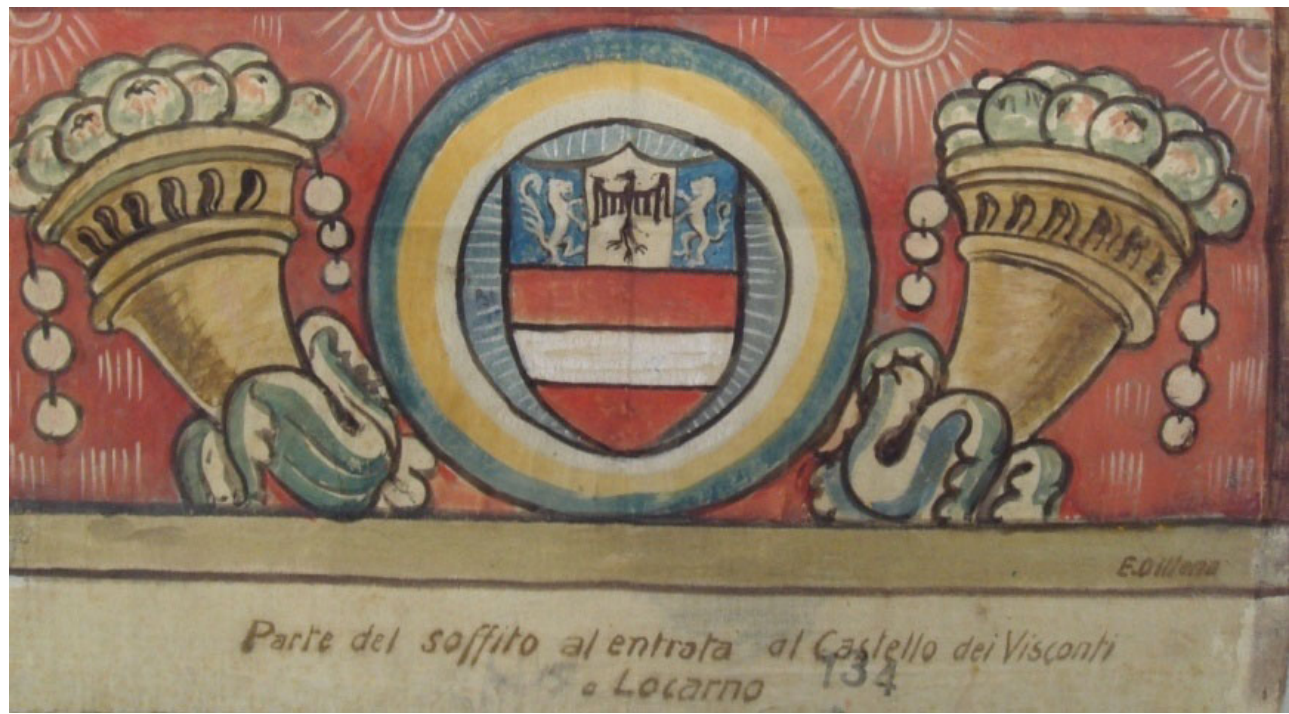

Figura 54 Dillena Emilio, Parte del soffitto all'entrata al Castello dei Visconti a Locarno. Dipinto; tempera su tela e cartone. Castello dei Visconti, Locarno. Ottobre 1929. AUBCBZ, s. 134, Locarno. Castello Visconteo.

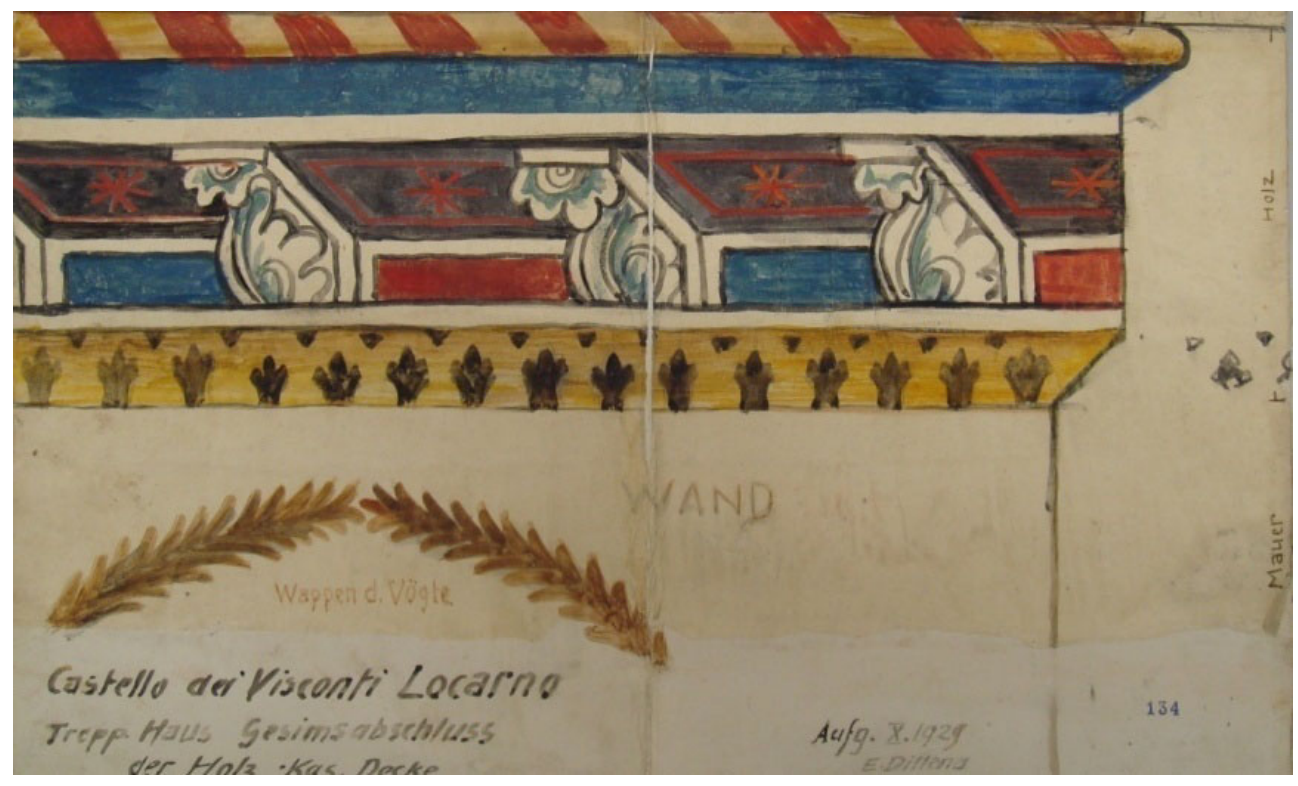

Figura 55 Dillena Emilio, Cornicione in legno. Dipinto; tempera su carta. Castello dei Visconti, Locarno. Ottobre 1929. AUBCBZ, s. 134, Locarno. Castello Visconteo. 


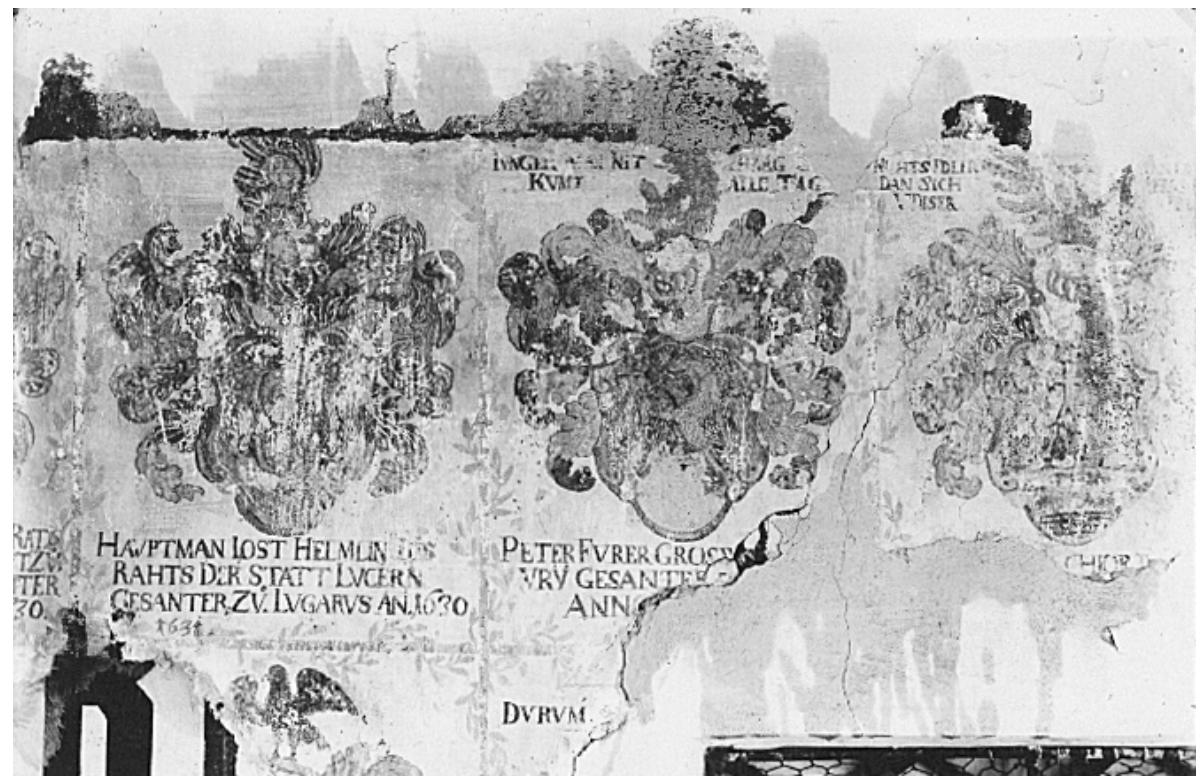

Figura 56 Fratelli Buchi. Castello, Locarno TI. Dipinti del cortile. Fotografia b/n. 2609 B.112. ABN Berna.

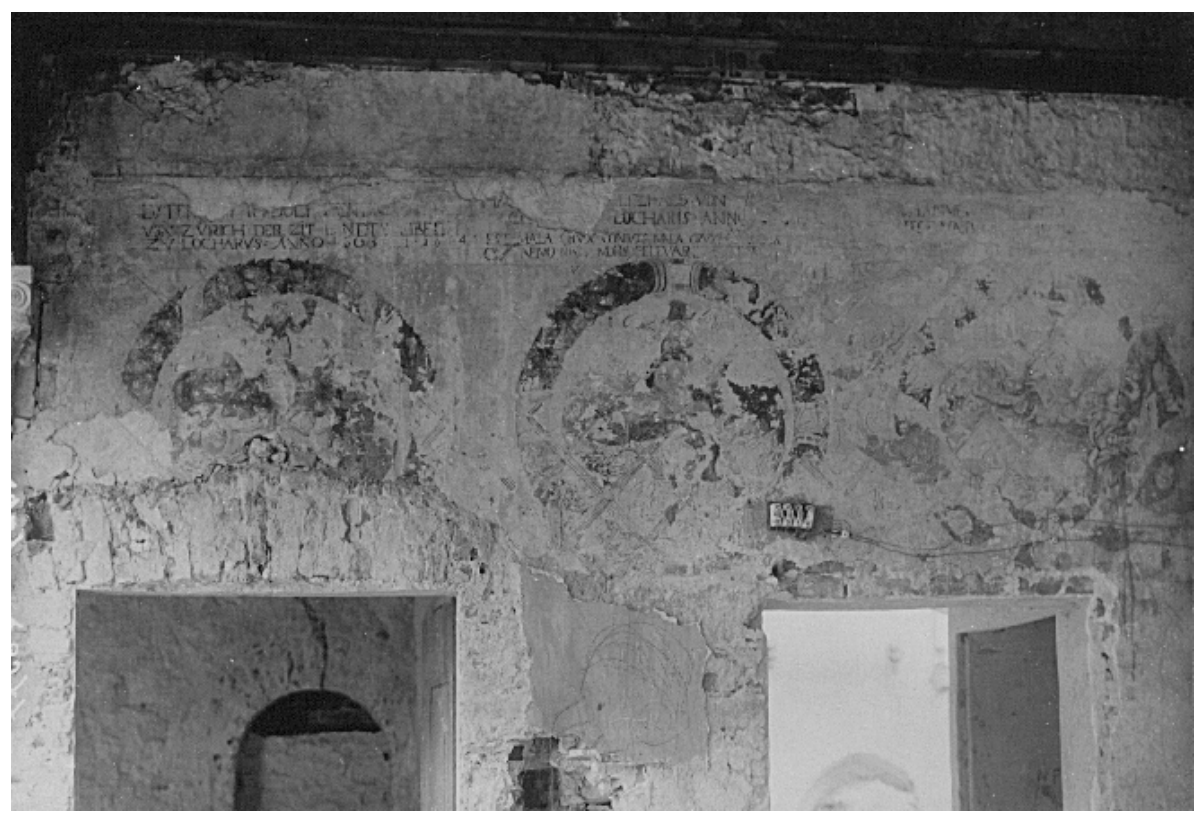

Figura 57 Fratelli Buchi. Castello, Locarno TI. Pitture sulla loggetta al primo piano (parete sud). Fotografia b/n. 21752 B.2180. ABN Berna. 


\section{Santa Maria delle Grazie, Bellinzona}

[...]Prima di passare alla diretta osservazione degli affreschi e delle altre pitture, è bene ricordare come negli anni 1911, 1912, 1926, 1928-1929, furono fatti alcuni restauri "curati e sorvegliati dallo Stato e promossi dal sacerdote Don Prada, allora rettore della chiesa. V. Gilardoni, 1956." Se oggi li possiamo ancora vedere e gustare, lo dobbiamo forse sopratutto, alla costanza di questo prete al quale era stata affidata la chiesa[...]. ${ }^{221}$

Di fatto, non si è voluto cominciare questo capitolo senza prima parlare della persona di don Giosuè Prada. Ad egli si deve il primo intervento documentato della chiesa di San Francesco a Locarno, avvenuto fra il 1922 ed il 1924 sotto il progetto dell'architetto Ambrogio Galli, ma non solo. Contemporaneamente, come sopracitato, il curato contribuì generosamente all'esecuzione di una delle più grandi campagne di restauro della chiesa di Santa Maria delle Grazie in Bellinzona, iniziata nel 1922 e conclusa nel 1931 sotto la direzione del pittore Edoardo Berta, che qui trattiamo.

Lo schema costruttivo di Santa Maria delle Grazie, come della già citata chiesa degli Angioli a Lugano, è di tipo conventuale dall'osservanza francescana lombarda della fine del XV sec. Altri esempi di architettura simile sono la Chiesa di Santa Maria delle

\footnotetext{
221 II complesso architettonico presenta una anomalia - liturgicamente non funzionale - divenuta caratteristica di alcune chiese contemporanee della Osservanza francescana lombarda (quella di Erba, Santa Maria degli Angeli; di Briñas presso Missaglia, Santa Maria della Misericordia; di Vercelli, Santa Maria in Betlem; di Maleo, Santa Maria delle Grazie; di Lugano, Santa Maria degli Angioli.
}

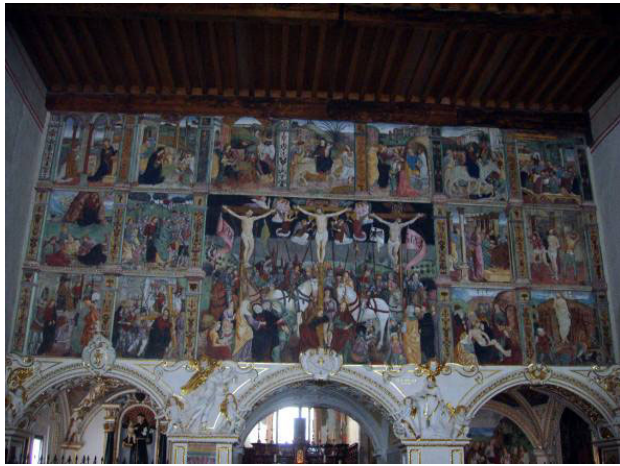

Figura 58 Giner Cordero, E. Transetto murale di S. Maria delle Grazie. 2006.

Grazie a Varallo e la Chiesa di S. Bernardino in Caravaggio a Ivrea. Un transetto murale centrale, di basto spessore contiene gli affreschi attribuiti a Gaudenzio Ferrari ${ }^{222}$ sulla Passione e Crocifissione di Cristo datati fra il 1495 e il 1505.

Nel transetto, in ambi i lati della crociera (unico legame fra il presbiterio-convento) e I'aula dei fedeli, si trovano le cappelle quadrangolari di Sant'Antonio e del Mortorio della Vergine, aperte verso I'aula di entrata a differenzia delle altre chiese dell'Osservanza lombarda.

Sopra queste cappelle la tribuna o cantoria, una volta chiamata "Paradiso" si apre unicamente verso la camera conventuale.

Nella parte settentrionale dell'aula dei fedeli, uscendo dal corpo degli edifici si aprono tre cappelle contigue e comunicanti come la già descritta S. Maria degli Angioli di Lugano, queste dedicate a San Bernardino, San Francesco ed all'Immacolata.

Dietro l'altare barocco del 1738 che nasconde l'accesso, si trova il coro

\footnotetext{
${ }^{222}$ (Venanzio Belloni, F. Genova, 1975 p.29).
} 
quadrato con volta a crociera a sesto acuto, formato da costoloni in laterizio.

Dal 1635 al 1636 furono dipinti gli affreschi delle lunette orientali e settentrionali del claustro, che raffigurano episodi della vita di San Francesco, mentre nel periodo compreso fra il 1696 ed il 1715, sotto la direzione del Padre Gaetano Lampugnani, furono terminati diversi interventi di ampliamento e finitura.

La chiesa ed il convento di S. Maria delle Grazie a Bellinzona rimasero in mani dei frati francescani fino alla nascita del Canton Ticino nel 1803. II 19 giugno di quell'anno il Gran Consiglio decreta che tutti i beni in proprietà delle corporazioni religiose sequestrate durante la Rivoluzione Francese fossero ritornati alle rispettive amministrazioni.

I primi lavori di ricerca e restauro sulle pareti della chiesa furono eseguiti nel 1911 dal pittore Augusto Ortelli e dal professore Eligio Pometta. Dopo I'abbandono della chiesa dai frati cappuccini, questa rimase chiusa al culto finché, per iniziativa di Don Giosuè Prada, appena nominato Rettore si inizia la campagna di esplorazione.

Lo scoprimento ${ }^{223}$ e pulitura dei muri della chiesa cominciò nel luglio 1922 nella parte nord essendo questa la più danneggiata dall'uscita del Dragonato nel $1768^{224}$.

\footnotetext{
223 Ne furono scoperti parecchi, se diamo fede a una breve notizia del 1929, ma ne furono strappati e recuperati soltanto due, attualmente appesi nella chiesa interna dei frati. (Gilardoni V. 1964. Le sinopie del Bramantino in Santa Maria delle Grazie di Bellinzona e le compagnie vaganti di pittore lombardi della fine del Quattro e dei primi decenni del Cinquecento, p.222).

224 L'alluvione avvenuto nel 1768 per l'uscita de torrente, distrugge numerosi edifici, chiese
}

I principali affreschi ritrovati furono La Dormizione nella Cappella del mortorio attribuita anch'essa a Gaudenzio Ferrari, La visita di Maria SS. a Elisabetta, ignoto, nella cappella di Sant'Antonio dedicata in origine ai Re Magi e ricoperta dallo strato di calce verso l'anno 1630 e, II Ciclo Mariano e la Pietà del Coro, entrambi ignoti ed una Santa Chiara. Ortelli spiega con molta precisione e carico di mistero come si scoprirono i citati affreschi:

[...]II 10 marzo 1923 dietro insistente voce interna rimosse l'urna della Madonna che poggiata su un altare di muratura semplice copriva tutta la parete centrale della Cappella ed apparve la meraviglia "Dormizione", meglio, della sepoltura della Vergine, attribuita in seguito a Gaudenzio Ferrari, scoperta questa che attirò per ben due messi continui visitatori, anche della lontana Inghilterra. Con questa scoperta acquistavano vita anche gli affreschi che ricoprono le pareti e la volta della medesima Cappella, altrimenti muti ed inspiegabili. In seguito ad un caso fortuito lo staccarsi di un po' d'intonaco sulla parete destra dell'abside, si scoperse il meraviglioso Ciclo mariano nonché la Pietà coi quattro Dottori della Chiesa, nonché $i$ medaglioni raffiguranti $i$ Profeti che ebbero relazione col mistero dell'Incarnazione. Poi venne scoperta la bellissima decorazione quattrocentesca della volta del transetto o parte superiore della Chiesa, in una colla rimessa in piena luce dell'Annunciazione ai lati dell'Altar Maggiore; tutti questi affreschi furono restaurati ai ben noti pittori Carlo Cotti ed Emilio Ferrazzini di Lugano A seguito poi della rimozione degli stalli del coro (costruito nel 1712) onde restaurarli si scopersero due affreschi che rilevati si possono ammirare sotto la cantoria e raffiguranti del Davide ed Isaia. Si scoperse pure, e fu l'ultima scoperta del genere,

conventuali, la chiesa dei SS. Giovanni Battista e Giovanni Evangelista, costruite a metà del XV sec. 
una Santa Chiara. L'opera più bella, almeno cosi mi pare, fu il restauro del grandioso affresco che arricchisce riempiendola cosi meravigliosamente bene la facciata interna della Chiesa eseguito dal pittore Dillena di Intragna, Bassetti Aldo.[...] 225

I primi veri lavori di ricostruzione e di restauro della chiesa si realizzarono fra gli anni 1922 e 1931 grazie ai sussidi cantonali e federali. L'esecuzione dei lavori fu affidata al pittore Emilio Ferrazzini mentre la direzione e supervisione veniva fatta dall'ispettore Edoardo Berta.

In questo momento si esegue la riparazione del tetto e del campanile, si aprono due finestre murate nel 1600 a sud della nave, si ricostruisce l'altare di San Bernardino, prima alla sinistra dell'entrata principale della chiesa, si restaura il coro, il pavimento, si recuperano cappelle fino ad ora chiuse e si restaura l'intonaco delle pareti interne ed esterne.

II restauro pittorico consiste nel consolidamento e pulitura dei dipinti murali sopra la cantoria, ai lati dell'altare maggiore, dell'affresco della Crocifissione nella parete trasversale e degli affreschi della Cappella della Immacolata, ai quali si sommano il restauro della Via Crucis delle decorazioni esterne del campanile e del trapasso a tela dei due medaglioni raffiguranti i profeti Isaia e Geremia trovati dietro gli stalli in legno del coro.

Questi ultimi dipinti murali furono strappati dal muro con l'obiettivo di traslocarli ad un posto più visibile e inoltre, per dargli la consistenza che l'intonaco, danneggiato dall'umidità, non riusciva ad offrirgli. I dipinti furono distaccati e riportati sulla tela successivamente

${ }^{225}$ (Bassetti A., 1943) applicata su un telaio di legno. Lo strappo raffigurante Isaia ha una misura di (156 x $160,5 \mathrm{~cm})$ e quello di Geremia $(81 \times 81 \mathrm{~cm})$. II dipinto di Isaia, più grande, comprende parte della decorazione dello zoccolo del muro con dei cornicioni che inglobano i medaglioni, mentre il secondo strappo, quadrato, contiene unicamente la figura del profeta, nemmeno centrato. Questi riaddattamenti, realizzati durante il trapasso dell'affresco alla tela, suggeriscono uno stato di conservazione del muro precario prima dell'intervento, o più difficilmente, un semplice capriccio dell'esecutore, il quale sconosciamo.

Grazie alle analisi realizzate dal restauratore Andrea Meregalli ed alla ricerca di diploma SUPSI elaborata nel 2004 dalla restauratrice Manuela Valentini, intitolata "La pratica dello strappo nel cantone Ticino. Modalità operative $e$ verifica degli interventi alla prova del tempo attraverso alcuni casi studio" possiamo oggi testimoniare la tecnica usata dagli operatori nel momento della esecuzione degli strappi. Ai dipinti, secondo il metodo tradizionale, si incollarono delle tele con colla forte, ancora presente sulla superficie pittorica nel momento dell'analisi, trovato in uno spessore di circa $300 \mathrm{~nm}$, staccando uno strato di intonaco di circa $800 \mathrm{~nm}$ composto da calce e sabbia di granulometria media-fine.

I quadri della Via Crucis furono restaurati nello stesso periodo dal pittore e professore della scuola di disegno di Bellinzona, Piero Tamò ${ }^{226}$. Mentre il resto

\footnotetext{
6 Tamò Piero (Gordola, 1899- Bellinzona, 1966). Pittore, poeta e professore; oltre a ciò l'artista fu un rilevante intelletuale della Bellinzona del dopoguerra.
} 
delle opere furono realizzate dai pittori Carlo Cotti, Christian Schmidtt ${ }^{227}$, Emilio Dillena, Angelo Bassi ${ }^{228}$, Bruno Nizzola ${ }^{229} \mathrm{e}$ il sopracitato Ferrazzini come incaricato principale.

Nel 1963 l'architetto Livo Doninelli ricostruisce il tetto della chiesa, mentre nel 1978 si ripara quello del convento insieme ai dipinti del claustro, restaurati prima da Baldo Carugo nel 1926.

Fra gli anni 1980 e 1982 il restauratore Luigi Gianola intervenne nel restauro del famoso affresco della Crocifissione ${ }^{230}$,

Suo figlio, Francesco Tamò (Cugnasco 1929 - Lugano 2002) è stato un importante artista contemporaneo e professore della Scuola del Canton Ticino. (Pettinatti, 2005, p. 30).

${ }^{227}$ Come nei restauri precedentemente trattati, il nome di Christian Schmidt sarà presente in tutti rapporti d'intervento in cui partecipa la sua ditta, però dalle fonti analizzate di archivio oggi possiamo confermare che in pochi casi egli fu presente ed esecutore diretto dei restauri nel Canton Ticino, ma consulente, direttore e capo del pittore Emilio Dillena, e distributore dei colori.

${ }^{228}$ Bassi Angelo (Loggio, 1878 - Lugano, 1957). Pittore d'affreschi, paesaggi e ritratti. Allievo di Antonio Barzaghi-Cattaneo.

${ }^{229}$ Nizzola, Aquilino, chiamato Bruno. (Loco, 1890 1963 Locarno). Pittore d'affreschi e mosaicista.

${ }^{230}$ L'affresco della parete centrale della chiesa delle Grazie, che separa la navata della chiesa interna, come nella chiesa degli Angioli a Lugano, si presentava assai danneggiato e qua e là ridipinto. Nel 1978 era stato richiesto un rapporto al restauratore Mario Rossi di Milano ed erano state fatte delle prove $d$ pulitura dal restauratore L. Gianola. Ved. (Fulvio Caccia, 1984, p. 11).

Il Comune di Bellinzona affrontò il problema nel 1979 proponendo a suoi periti: il prof. Cesare Brandi, la prof.ssa Noemí Gabrielli e il prof. Guido Fiume, restauratore, un progetto d'intervento. Con i sussid cantonali e federali, il restauro, affidato all'équipe di L. Gianola si svolse fra il 1980 e il 1982, seguito anche dal professore Schmidt e dal perito della C.F. O. Emmenegger. II restauro messe in evidenza in primo luogo lo stato inquietante delle superficie e le realizzando operazioni di consolidamento, pulitura dei ridipinti di 1931 e integrando pittoricamente a rigattino con acquarello.

\section{Le critiche. La Fuga di Egitto, Orselina.}

I lavori di restauro architettonico e pittorico eseguiti da Edoardo Berta furono in generale collaudati. Le uniche notizie inerenti le critiche verso le prassi esecutive del pittore in materia di restauro sono state datate in 1922 in riguardo all'intervento sulla tavola raffigurante la Fuga in Egitto del Bramantino alla chiesa della Madonna del Sasso ad Orselina. II restauro di questa opera, eseguito da Federico Bentz di Basile sotto la direzione del pittore Berta, fu causa di una serie di polemiche pubblicate sui giornali e critiche da parte dei Reverendi Padri Cappuccini del Santuario della Madonna del Sasso a cui fece largo eco la stampa ticinese.

A mettersi in dubbio fu precisamente la scelta di ridimensionamento del quadro al suo stato originale, quando dopo la pulitura si evidenziano diverse aggiunte lungo i lati orizzontali.

\footnotetext{
screpolature che alteravano i riquadri stessi. Queste ultime vennero mantenute in evidenza con fondi neutri. La pittura in genere venne ritenuta di notevole qualità ed eseguita da parecchie mani appartenenti alla stessa bottega. Questa si avvicina ad opere analoghe dell'ambiente di Martino Spanzotti (Ivrea) e dal suo allievo Gaudenzio Ferrari. Nel gruppo di affrescanti che lavoravano rapidamente sulla grande parete, in particolare nelle lesene a grotteschi, il prof. Brandi in una relazione presentata il 10 febbraio 1981 spiega che nei dipinti si potrebbe ritrovare la mano del Sodoma giovane (Monte Oliveto, Siena) considerando così una intuizione avanzata nel 1979 da Noemí Gabrielli.
} 
Le critiche, che offesero inoltre il Berta, discutendosi la sua professionalità, furono risposte da un autorevole studioso specialista ai restauri di quadri pittorici, il professor Paolo Ganz, già direttore dell'importante museo d'arte di Basilea. Egli collauda il lavoro del restauratore Basilese che aveva attuato secondo le idonee indicazioni di Edoardo Berta.

Solo nel 1947 si riscontrò la necessità di intervenire nuovamente sulla pregiata tavola. A questo scopo, fu chiamato il restauratore milanese Mario Rossi, chi eseguì una perizia presentata in data 17 maggio 1947 alla CCMS. II rapporto faceva allusione ai restauri precedenti eseguiti dal Berta, anche se le parole di Mario Rossi dimostrano che egli non conosceva I'autorità di questo anteriore intervento.

[...]1. Su tavola di cipresso (prov.) in tre tavole verticali unite con dodici cambre a farfalla, sostituite recentemente che sporgono dal piano di qualche centimetro $e$ non sono accuratamente incassate. A maggior resistenza furono applicate trasversalmente quattro spranghe di ferro dello spessore di oltre mezzo centimetro e larghe un paio di $\mathrm{cm}$, fissate da un numero considerabile di viti molto vicine una a I'altra (circa 60), che inibiscono il movimento di dilatazione e contrazione del legno. L'allungamento nella parte inferiore e fissato con un semplice innesto e con colla forte e quattro sbarrette di ferro fermate con viti. Sembra di recente esecuzione in sostituzione del vecchio allungamento o di una arbitraria aggiunta.

2. II colore sembra ad olio (indagine di laboratorio). In molte parti si scrosta dalla preparazione o con la preparazione dalla tavola.

3. L'intero dipinto è protetto da una vernice mastice semplice che comincia ad ingiallire ed a perdere la trasparenza perche principia il fenomeno dell'ossidazione. Si presenta friabile e debole. [... $]^{231}$

II restauratore italiano Mario Rossi propose la rimozione delle quattro spranghe di ferro applicate nell'intervento precedente, ricollocando a suo posto una grigliatura in rapporto alle giunture che costituiscono la tavola, alla qualità del legno, alla resistenza ed allo spessore dalla tavola ${ }^{232}$. Inoltre, denuncia la necessità di sostituire le farfalle, e di pulire la superficie pittorica ad olio, asportando le stuccature ed i vecchi ritocchi. Questi ultimi non vengono rispettati perché non solo sono stati realizzati ad olio invece di a vernice ma inoltre non si adattano alle tonalità originale.

La accurata descrizione di Mario Rossi nel suo rapporto ci serve a dimostrare i cambiamenti sviluppati nei venti anni di differenzia esistenti fra il primo ed il secondo intervento. Essendosi il primo realizzato ad ogni modo secondo gli ideali dell'epoca, e con la cura che solo Berta poteva dedicare alla salvaguardia dell'opera, il secondo esegue dei concetti moderni di "restauro" che inevitabilmente risultarono meno invasivi per il dipinto. Mario Rossi propose un intervento rispettoso, consapevole del significato di reversibilità dei materiali, del minimo restauro in favore della storia dell'oggetto, e dell'impiego dei metodi scientifici quale appoggio ad identificare le caratteristiche

\footnotetext{
1 (Rossi Mario, Perizia e proposta di restauro della tavola raffigurante la Fuga in Egitto alla chiesa della Madonna del Sasso ad Orselina, indirizzato alla CCMS. 17 maggio 1947. AUBCBZ s. 183 Orselina).

232 II restauratore chiede l'aiuto delle radiografie per conoscere le caratteristiche dell'opera.
} 
dell'opere e conseguentemente prospettare l'intervento più adeguato ad esse. ${ }^{233}$ Sulla medesima opera attuerà in seguito il restauratore bergamasco Mauro Pellicioli. L'intervento del Pellicioli seguirà le medesima prassi esecutive che Mario Rossi aveva proposto nel suo rapporto del 17 maggio 1947: [...]sistemazione della statica della tavola col chiudere le fessure ed impedire ogni modificazione del tergo chiuso entro una perfetta grigliatura [...] essere rimossi anche i restauri che deturpano la rarissima pittura. Documentazione fotografica e radiografica. [...] 1.Spese di documentazione e controllo radiografico del dipinto antecedente l'inizio dell'opera di restauro. (20fr); 2. Spese di documentazione fotografica antecedente l'inizio dell'opera di restauro (n.9 fotografie) (15fr); 3 . Opere meccaniche di risarcimento alla vecchia tavola. Risanamento dei due fili di congiunzione delle chiavi a farfalla di rinforzo ai medesimi. Registrazione dello spessore delle tavole. Applicazione a tergo di una grigliatura scorrevole di rinforzo. (200fr); 4.

Segue la perizie di Mario Rossi sullo stato conservativo della tavola, una secondo realizzata da una commissione di esperti internazionali costituita da Jean Verdier, sovrintendente a Parigi, e da Mauro Pellicioli di Milano, prima del 1950. Sarà il proprio Rossi, in conoscenza di queste circostanza a cedere il lavoro al collega Pellicioli, come testimonia una lettera informativa che la sovrintendente alla Pinacoteca di Brera, Fernanda Wittgens, dirige al Dipartimento della Pubblica Educazione del Canton Ticino il 9 agosto del 1950. Altre notizie ci permettono di conoscere ad esempio, che il restauro dell'opera avvenne all'interno della Pinacoteca di Brera, trasportato secondo le indicazioni di Aldo Crivelli, in una casa piombata ed imballata, contenente tanto la tavola originale come i due pezzi eliminati nell'intervento del Berta nel 1922. La spedizione dalla Dogana ebbe luogo il 10 aprile del 1951, secondo il rapporto dettagliato di Crivelli, del 11 aprile 1951 indirizzato al DPE. Inoltre il medesimo Pellicioli ne parlerà del restauro alla Radiotelevisione della Svizzera Italiana nell’intervista realizzata nel 1970.
Documentazione fotografica successiva a tale fase di lavoro (3 fotografie) $5 \mathrm{fr}$; 5.Consolidamento del colore nella parti pericolanti del dipinto. Liberazione del dipinto originale dalle larghissime ridipinture $e$ stuccature di epoche diverse, tra cui l'ultima estesissima dei primi del corrente secolo. Registrazione e revisione del piano della superficie dipinta e stuccatura della scrostature $e$ fenditure. Asportazione dal dipinto dei durissimi restauri del secolo XVIII. Rimesso in equilibrio tonale il dipinto col recupero dei colori originali. Conseguente restauro alle lacune $e$ abrasión, intonatura e vernice di finitura. $300 \mathrm{fr}$. Totale 540 fr. $[\ldots]^{234}$.

Questo cambiamento ideologico si riscontrerà ugualmente nel restauro dei dipinti murali in Canton Ticino, seguendo le correnti italiani. II primo ad introdurre particolarmente le metodologie di indagine scientifico e di restauro moderno sui dipinti murali, sarà il pittore-restauratore Ottorino Olgiati, intorno al 1947. Di fatto, come si tratterà nei testi successivi, in questo momento saranno confrontati nel Canton Ticino gli ideali di restauro tradizionale con quelli moderni, creandosi degli importanti scontri fra le personalità della cultura ed $\mathrm{i}$ responsabili nel campo della conservazione e della tutela del patrimonio storico artistico in Canton Ticino.

\footnotetext{
4 (Pellicioli Mauro, Fattura concerniente il lavoro di risarcimento e rinforzo della tavola e quello di pulitura e restauro del dipinto del Bramantino raffigurante la fuga in Egitto di proprietà del Santuario della Madonna del Sasso in Locarno. Indirizzato al DPE, 18 marzo 1952. AUBCBZ, s.183 Orselina).
} 


\section{Interventi di restauro}

\begin{tabular}{|c|c|c|c|c|c|}
\hline ANNO & LUOGO & OPERA & INTERVENTO & COLLABORATORI & FONTI \\
\hline $\begin{array}{l}1891 \text { - } \\
1930\end{array}$ & $\begin{array}{l}\text { Lugano, } \\
\text { chiesa di S. Maria } \\
\text { degli Angioli. } \\
\text { BA_2714. }\end{array}$ & $\begin{array}{l}\text { Cappella Camuzio, } \\
\text { affreschi } \\
\text { BAS_12918 }\end{array}$ & $\begin{array}{l}\text { Direzioni dei lavori } \\
\text { di descialbo e } \\
\text { restauro completo } \\
\text { delle pitture murali } \\
\text { presenti nella } \\
\text { cappella eliminando } \\
\text { gli elementi } \\
\text { seicenteschi per } \\
\text { fare visibili gli } \\
\text { affreschi primari. }\end{array}$ & $\begin{array}{l}\text { Emilio Ferrazzini } \\
\text { P_5484 } \\
\text { Emilio Maccagni } \\
\text { (Rivera) } \\
\text { P_4901 } \\
\text { Arturo Ortelli } \\
\text { (Lugano) } \\
\text { Emilio Dilena } \\
\text { (Taverne) } \\
\text { Christian Schmid } \\
\text { (Zurigo) } \\
\text { Tito Tettamanti } \\
\text { (Grancia) } \\
\text { Alfonso Cattaneo } \\
\text { (Manno) } \\
\text { Carlo Cotti } \\
\text { (Lugano) } \\
\text { P_5483 } \\
\text { Basilio Rusca } \\
\text { (Bedano) }\end{array}$ & $\begin{array}{l}\text { (Chiesa, Monumenti } \\
\text { storici e artistici del } \\
\text { Cantone Ticino } \\
\text { restaurati dal 1910 } \\
\text { al 1945, 1946, p. 32) } \\
\text { F. CHIESA (P_4903), } \\
\text { (DPE, 1959, p. 122) } \\
\text { (AUBCBZ, s. 143 } \\
\text { Lugano, S. Maria } \\
\text { degli Angioli) } \\
\text { (ASTi Fondo DPE, } \\
\text { Fascicolo XXVIII } \\
\text { cartella 1. } \\
\text { Monumenti Storici } \\
\text { (1848...). } \\
\text { (Marcionetti, 1975) }\end{array}$ \\
\hline $\begin{array}{l}1910- \\
1929\end{array}$ & $\begin{array}{l}\text { Ravecchia } \\
\text { (Bellinzona), } \\
\text { chiesa di San Biagio } \\
\text { a sec. XIII. } \\
\text { BA } 2778 .\end{array}$ & & $\begin{array}{l}\text { Direzione dei lavori } \\
\text { di ricerca } \\
\text { archeologica con la } \\
\text { realizzazione di } \\
\text { scavi ed } \\
\text { eliminazione degli } \\
\text { elementi stilistici } \\
\text { posteriori. }\end{array}$ & $\begin{array}{l}\text { Arch. Silvio Soldati } \\
\text { Albert Naef } \\
\text { P_4900 } \\
\text { Robert Dürrer }\end{array}$ & $\begin{array}{l}\text { (Chiesa, Monumenti } \\
\text { storici e artistici del } \\
\text { Cantone Ticino } \\
\text { restaurati dal } 1910 \\
\text { al 1945, 1946, p. 13, } \\
\text { 15) } \\
\text { (Berta, Relazione sui } \\
\text { lavori di restauro } \\
\text { della Chiesa di San } \\
\text { Biagio in Ravecchia, } \\
\text { 1915) }\end{array}$ \\
\hline $\begin{array}{l}1910- \\
1929\end{array}$ & $\begin{array}{l}\text { Ravecchia } \\
\text { (Bellinzona), } \\
\text { chiesa di San Biagio } \\
\text { a sec. XIII } \\
\text { BA } 2778 .\end{array}$ & $\begin{array}{l}\text { Scuola lombarda, } \\
\text { sec. XIV e sec. XV } \\
\text { influenze della } \\
\text { scuola di Siena } \\
\text { affreschi. }\end{array}$ & $\begin{array}{l}\text { Direzione dei lavori } \\
\text { di descialbo dei } \\
\text { dipinti murali. }\end{array}$ & Operaio Fumagalli & $\begin{array}{l}\text { (Chiesa, Monumenti } \\
\text { storici e artistici del } \\
\text { Cantone Ticino } \\
\text { restaurati dal } 1910 \\
\text { al 1945, 1946, p. 13, } \\
\text { 15) } \\
\text { (Berta, Relazione sui } \\
\text { lavori di restauro } \\
\text { della Chiesa di San } \\
\text { Biagio in Ravecchia, } \\
\text { 1915) } \\
\text { (AFMS BNS Bern K } \\
\text { Tessin) } \\
\text { Bellinzona n.82) } \\
\text { (AUBCBZ, s. 143 } \\
\text { Lugano, S. Maria } \\
\text { degli Angioli) }\end{array}$ \\
\hline
\end{tabular}




\begin{tabular}{|c|c|c|c|c|c|}
\hline 1910 & $\begin{array}{l}\text { Ravecchia } \\
\text { (Bellinzona), } \\
\text { chiesa di San Biagio } \\
\text { a sec. XIII. } \\
\text { BA } 2778 .\end{array}$ & $\begin{array}{l}\text { Affreschi del coro } \\
\text { centrale, Tribuna } \\
\text { ed sopra la porta } \\
\text { interna della } \\
\text { facciata. }\end{array}$ & $\begin{array}{l}\text { Direzione dei lavori } \\
\text { di consolidamento } \\
\text { dei dipinti murali. }\end{array}$ & Arturo Ortelli & $\begin{array}{l}\text { (Chiesa, Monumenti } \\
\text { storici e artistici del } \\
\text { Cantone Ticino } \\
\text { restaurati dal } 1910 \\
\text { al 1945, 1946, p. 13, } \\
\text { 15). } \\
\text { (AUBCBZ, s. } 143 \\
\text { Lugano, S. Maria } \\
\text { degli Angioli). }\end{array}$ \\
\hline $\begin{array}{l}1910- \\
1936\end{array}$ & $\begin{array}{l}\text { Bellinzona, } \\
\text { chiesa Collegiata } \\
\text { dei SS. Pietro e } \\
\text { Stefano. } \\
\text { BA_2779. }\end{array}$ & & $\begin{array}{l}\text { Vigilanza dei lavori } \\
\text { di ripristino } \\
\text { architettonico ed } \\
\text { artistico sulla } \\
\text { facciata. }\end{array}$ & Arturo Ortelli & $\begin{array}{l}\text { (Chiesa, Monumenti } \\
\text { storici e artistici del } \\
\text { Cantone Ticino } \\
\text { restaurati dal } 1910 \\
\text { al 1945, 1946, p. } \\
\text { 15). }\end{array}$ \\
\hline \multirow[t]{2}{*}{1913} & $\begin{array}{l}\text { Locarno, Orselina, } \\
\text { Chiesa della } \\
\text { Madonna del Sasso. } \\
\text { BA_2780. } \\
\text { BA_2781. }\end{array}$ & $\begin{array}{l}\text { Ancona della Pietà; } \\
\text { gruppo in legno } \\
\text { scolpito. } \\
\text { BAS_15264. }\end{array}$ & $\begin{array}{l}\text { Direzione dei lavori } \\
\text { di restauro. }\end{array}$ & Francesco Annoni. & $\begin{array}{l}\text { (AUBCBZ s.183 } \\
\text { Locarno, Orselina. } \\
\text { Madonna del Sasso) }\end{array}$ \\
\hline & $\begin{array}{l}\text { Locarno, Orselina, } \\
\text { Chiesa della } \\
\text { Madonna del Sasso } \\
\text { BA_2780. } \\
\text { BA_2781. }\end{array}$ & $\begin{array}{l}\text { Cappella della } \\
\text { Pietà. }\end{array}$ & $\begin{array}{l}\text { Formazione di una } \\
\text { nuova cappella } \\
\text { della Pietà, dove } \\
\text { saranno collocati il } \\
\text { famoso gruppo in } \\
\text { legno scolpito della } \\
\text { "Pietà", il quadro } \\
\text { La fuga in Egitto del } \\
\text { Bramantino } \\
\text { (BAS_15261), } \\
\text { I'Annunciata } \\
\text { (BAS_15262) di } \\
\text { Bernardino dei } \\
\text { Conti, e due altre } \\
\text { tavole annesse } \\
\text { come integrante a } \\
\text { quest'ultimo } \\
\text { dipinto. }\end{array}$ & & $\begin{array}{l}\text { (AUBCBZ s.183 } \\
\text { Locarno, Orselina. } \\
\text { Madonna del } \\
\text { Sasso). }\end{array}$ \\
\hline 1913 & $\begin{array}{l}\text { Malvaglia, } \\
\text { chiesa parrocchiale } \\
\text { di S. Martino } \\
\text { BA_2782. }\end{array}$ & & $\begin{array}{l}\text { Direzione dei lavori } \\
\text { di ricerca } \\
\text { archeologica con la } \\
\text { scoperta degli } \\
\text { affreschi. }\end{array}$ & $\begin{array}{l}\text { 1913, comitato } \\
\text { parrocchiale pro- } \\
\text { restauri. }\end{array}$ & $\begin{array}{l}\text { (Chiesa, Monumenti } \\
\text { storici e artistici del } \\
\text { Cantone Ticino } \\
\text { restaurati dal } 1910 \\
\text { al 1945, 1946, p. 41) } \\
\text { (DPE, 1959, p. 122) } \\
\text { (AUBCBZ s.149 } \\
\text { Malvaglia. S. } \\
\text { Martino). }\end{array}$ \\
\hline 1915 & $\begin{array}{l}\text { Medeglia, } \\
\text { Chiesa parrocchiale } \\
\text { di S. Bartolomeo } \\
\text { BA_2783. }\end{array}$ & $\begin{array}{l}\text { Affreschi, } 1478 \\
\text { Padre Eterno nella } \\
\text { mandorla } \\
\text { circondato da }\end{array}$ & $\begin{array}{l}\text { Ispezione dei lavori } \\
\text { che consistono } \\
\text { nella scoperta degli } \\
\text { affreschi e delle }\end{array}$ & & $\begin{array}{l}\text { (ACS BZ, Fascicolo } \\
\text { XXVIII cartella } 1 . \\
\text { Monumenti Storici }\end{array}$ \\
\hline
\end{tabular}




\begin{tabular}{|c|c|c|c|c|c|}
\hline & & $\begin{array}{l}\text { angeli musicante. } \\
\text { BAS_15270. } \\
\text { BAS_15271. }\end{array}$ & $\begin{array}{l}\text { decorazioni } \\
\text { ornamentali } \\
\text { ritrovate in agosto } \\
\text { del 1915, } \\
\text { ornamentale, in } \\
\text { pessimo stato di } \\
\text { conservazione a } \\
\text { causa dell'umidità. } \\
\text { Sigillature in } \\
\text { cemento dei bordi. }\end{array}$ & & $\begin{array}{l}\text { (1848...). Berta } \\
\text { Edoardo, lettera del } \\
28 \text { ottobre } 1915 \text { alla } \\
\text { Commissione dei } \\
\text { Monumenti Storici } \\
\text { ed Artistici ed al } \\
\text { DPE. }\end{array}$ \\
\hline $\begin{array}{l}9 \text { nov. } \\
1916\end{array}$ & $\begin{array}{l}\text { Semione, } \\
\text { Capella dei Morti } \\
\text { BA_2784. }\end{array}$ & $\begin{array}{l}\text { BAS_15272. } \\
\text { BAS_15273. } \\
\text { BAS_15274. } \\
\text { BAS_15275. } \\
\text { BAS_15276. }\end{array}$ & $\begin{array}{l}\text { Ispezione dello } \\
\text { stato di } \\
\text { conservazione } \\
\text { dell'opera per il cui } \\
\text { restauro richiama } \\
\text { allo Stato. }\end{array}$ & Operaio Fumagalli. & $\begin{array}{l}\text { (ASTi Fondo DPE, } \\
\text { Fascicolo XXVIII } \\
\text { cartella } 1 . \\
\text { Monumenti Storici } \\
\text { (1848...). }\end{array}$ \\
\hline $\begin{array}{l}1917- \\
1922\end{array}$ & $\begin{array}{l}\text { Locarno, Orselina } \\
\text { chiesa della } \\
\text { Madonna del Sasso } \\
\text { BA_2780. } \\
\text { BA_2781 }\end{array}$ & $\begin{array}{l}\text { Bramantino, Fuga in } \\
\text { Egitto, tavola sec. } \\
\text { XVII, } \\
\text { BAS_15261. }\end{array}$ & $\begin{array}{l}\text { Ispezione e } \\
\text { direzione dei lavori } \\
\text { di restauri } \\
\text { consistenti nel } \\
\text { ridimensionamento } \\
\text { alle misure originali } \\
\text { dell'opera } \\
\text { evidenziandosi } \\
\text { dopo la puliture } \\
\text { diverse aggiunte } \\
\text { lungo i lati } \\
\text { orizzontali della } \\
\text { pala. }\end{array}$ & $\begin{array}{l}\text { Federico Bentz, } \\
\text { Basilea (noto per i } \\
\text { restauri delle } \\
\text { pitture di Holbein in } \\
\text { quella città). }\end{array}$ & $\begin{array}{l}\text { (Chiesa, Monumenti } \\
\text { storici e artistici del } \\
\text { Cantone Ticino } \\
\text { restaurati dal } 1910 \\
\text { al 1945, 1946, p. 18) } \\
\text { (AUBCBZ s.183 } \\
\text { Locarno, Orselina) } \\
\text { (ASTi Fondo DPE, } \\
\text { Fascicolo XXVIII } \\
\text { cartella 1. } \\
\text { Monumenti Storici } \\
\text { (1848...). }\end{array}$ \\
\hline 1919 & $\begin{array}{l}\text { Maggia, } \\
\text { Chiesa di S. Maria } \\
\text { delle Grazie } \\
\text { BA_2785. }\end{array}$ & $\begin{array}{l}\text { Affreschi } \\
\text { BAS_15277/15291. }\end{array}$ & $\begin{array}{l}\text { Direzione dei lavori } \\
\text { di consolidamento } \\
\text { della statica della } \\
\text { chiesa avendo } \\
\text { riscontrato delle } \\
\text { crepe nella parete } \\
\text { dell'abside ed in } \\
\text { quella longitudinale } \\
\text { dove si trovano gli } \\
\text { affreschi più } \\
\text { importanti. }\end{array}$ & $\begin{array}{l}\text { Ottobre 1919, } \\
\text { arch. Ing. Cavadini, } \\
\text { Locarno }\end{array}$ & $\begin{array}{l}\text { (AFMS BNS . N.80 } \\
\text { KT M -P). }\end{array}$ \\
\hline 1919 & $\begin{array}{l}\text { Camignolo, } \\
\text { oratorio di S. } \\
\text { Ambrogio } \\
\text { BA_2786. }\end{array}$ & $\begin{array}{l}\text { Majestas Domini, } \\
\text { affresco XIV secolo; } \\
\text { muro a sud la figura } \\
\text { di S. Ambrogio. } \\
\text { BAS_15294/15298 }\end{array}$ & $\begin{array}{l}\text { Direzione dei lavori } \\
\text { di scoperta dei } \\
\text { dipinti. }\end{array}$ & & $\begin{array}{l}\text { (DPE, 1959, p. 119) } \\
\text { (AUBCBZ, s. } 054 \\
\text { Camignolo) }\end{array}$ \\
\hline 1919 & $\begin{array}{l}\text { Semione, } \\
\text { Capella dei Morti } \\
\text { BA_2784. }\end{array}$ & $\begin{array}{l}\text { Affreschi. } \\
\text { BAS_15273. } \\
\text { BAS_15274. } \\
\text { BAS_15275. } \\
\text { BAS_15276. }\end{array}$ & $\begin{array}{l}\text { Ispezione dei lavori } \\
\text { di restauro. }\end{array}$ & Attilio Balmelli. & $\begin{array}{l}\text { (ASTi Fondo DPE } \\
\text { Fascicolo XXVIII } \\
\text { cartella } 1 . \\
\text { Monumenti Storici } \\
\text { (1848...). }\end{array}$ \\
\hline
\end{tabular}


Vallone, Bellinzona. Affresco

Cappella già

Mariotti. quattrocentesco.

di strappo

dell'affresco e della

successiva

esposizione nella

chiesa di S. Biagio a

Ravecchia.

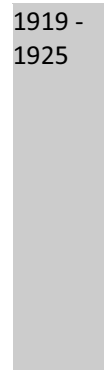

1921 -
Riva San Vitale,

Battistero,

sec. X.

BA_2787.

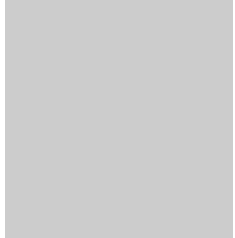

Locarno.

Castello Visconteo

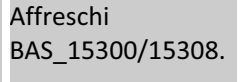

BAS_15300/15308.

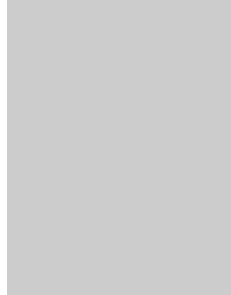

Stemmi XVI - XIX

sec. Dipinti murali.
Direzione dei lavori Arturo Ortelli

di esplorazione,

rimozione degli

intonachi, ricerca

degli elementi

originari, scoperta

di affreschi.

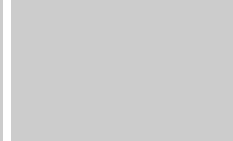

Direzione dei lavori Albert Naef

di ricerca e di Chr. Schmidt restauro.
Architetto Enea

Tallone.

Emilio Dillena.
(ASTi Fondo DPE

Fascicolo XXVIII

cartella 1.

Monumenti Storici

(1848...)

(Chiesa, Monumenti storici e artistici del Cantone Ticino restaurati dal 1910

al 1945, 1946, p. 20) (DPE, 1959, p. 125)

(Chiesa, Monumenti storici e artistici del Cantone Ticino restaurati dal 1910 al 1945,1946, p. 23) (DPE, 1959, p. 121) (AUBCBZ, s. 021, Locarno, Castello Visconteo).

$\begin{array}{ll}1922 \text { - } & \text { Bellinzona, } \\ \text { chiesa di S. Maria } & \text { Affreschi. } \\ \text { delle Grazie. } & \\ \text { BA_2788. } & \end{array}$

Cadro,

Chiesa parrocchiale

\begin{abstract}
Direzione dei lavori Emilio Ferrazzini. di ricerca e di Carlo Cotti. restauro: pulitura e Cristiano Schmid. consolidamento delle pitture sulla cantoria, sui lati dell'altar maggiore, dell'affresco della Crocifissione sulla parete trasversale, degli affreschi nella cappella dell'Immacolata, della Via Crucis, delle decorazioni esterne sul campanile e sulle pareti della Chiesa.

A. Bassi. Bruno Nizzola.$$
\text { la }
$$
Ispezione dei lavori Arch. Meneghelli
F. CHIESA, 1946, p. 26.

DPE, 1959, pp. 118. 021. 021.

(ASTi Fondo DPE Fascicolo XXVIII cartella 1.

Monumenti Storici (1848...). 


\begin{tabular}{|c|c|c|c|c|c|}
\hline 1922 & $\begin{array}{l}\text { Sonvico, } \\
\text { Chiesa parrocchiale }\end{array}$ & Affreschi. & $\begin{array}{l}\text { Ispezione dei lavori } \\
\text { di riscoperta degli } \\
\text { affreschi. }\end{array}$ & Arch. Meneghelli & $\begin{array}{l}\text { (ASTi Fondo DPE } \\
\text { Fascicolo XXVIII } \\
\text { cartella } 1 . \\
\text { Monumenti Storici } \\
\text { (1848...)AN Bern } \\
\text { n.80 KT M-P } \\
\text { Articolo, Echo del } \\
\text { Gottardo, Locarno } \\
12 \text { dicembre 1911: } \\
\text { ispezione del sig. } \\
\text { Naef e Durrer della } \\
\text { CFMS accompagnati } \\
\text { da Meneghelli per } \\
\text { l'approvazione del } \\
\text { sussidio dei restauri. }\end{array}$ \\
\hline 1925 & $\begin{array}{l}\text { Caslano, } \\
\text { Cappella di S. Maria } \\
\text { alla Magliasina } \\
\text { detta Cappella } \\
\text { Greppi. }\end{array}$ & $\begin{array}{l}\text { Affreschi } \\
\text { cinquecenteschi. }\end{array}$ & $\begin{array}{l}\text { Fotografie e } \\
\text { articolo nella Rivista } \\
\text { Storia ed Arte della } \\
\text { Svizzera Italiana. }\end{array}$ & & AUBC s.67 Caslano \\
\hline 1928 & $\begin{array}{l}\text { Bissone, } \\
\text { oratorio di S. Rocco. } \\
\text { Facciata con tracce } \\
\text { di pittura del XV } \\
\text { sec. }\end{array}$ & & $\begin{array}{l}\text { Direzione dei lavori } \\
\text { di restauro degli } \\
\text { stucchi. }\end{array}$ & $\begin{array}{l}\text { Stuccatore Frignoni } \\
\text { - Ussi. }\end{array}$ & $\begin{array}{l}\text { (F. CHIESA, 1946, p } \\
36 \text { ) } \\
\text { (DPE, 1959, pp.119) } \\
\text { (ASTi, Fondo DPE } \\
\text { Fascicolo XXVIII } \\
\text { cartella } 1 \\
\text { Monumenti Storici } \\
\text { (1848...). }\end{array}$ \\
\hline 1928 & $\begin{array}{l}\text { Lugano, } \\
\text { Palazzo Albertolli, } \\
\text { ora sede della } \\
\text { Banca Nazionale. } \\
\text { BA_ } 2789 \text {. }\end{array}$ & $\begin{array}{l}\text { Bernardino Luini, } \\
\text { affresco. } \\
\text { Proprietà Marco } \\
\text { Ghirlanda. } \\
\text { BAS_15309. }\end{array}$ & $\begin{array}{l}\text { Direzione dei lavori } \\
\text { di restauro. }\end{array}$ & $\begin{array}{l}\text { Emilio Ferrazzini. } \\
\text { Tita Pozzi. } \\
\text { Carlo Cotti. }\end{array}$ & $\begin{array}{l}\text { DPE, 1959, pp.122 } \\
143 .\end{array}$ \\
\hline 1930 & $\begin{array}{l}\text { Malvaglia, } \\
\text { chiesa parrocchiale } \\
\text { di S. Martino. } \\
\text { BA_2782 }\end{array}$ & & $\begin{array}{l}\text { 1926, Preventivo: } \\
\text { ing. Bagio } \\
\text { 1929, Esamine } \\
\text { Commissione: sig. } \\
\text { Weith. } \\
\text { 1930, la parrocchia } \\
\text { incarica uno studio } \\
\text { all'arch. Cino } \\
\text { Chiesa, approvato } \\
\text { dalla Commissione } \\
\text { con variante } \\
\text { proposte dall'arch. } \\
\text { Otto Maraini e dal } \\
\text { pitt. Berta. }\end{array}$ & $\begin{array}{l}\text { Luigi Bagio } \\
\text { Cino Chiesa. } \\
\text { Otto Maraini. }\end{array}$ & $\begin{array}{l}\text { AUBCBZ s.149 } \\
\text { Malvaglia. }\end{array}$ \\
\hline
\end{tabular}


Restauratori in Canton Ticino fra Ottocento e Novecento

Catalogazione e gestione dati

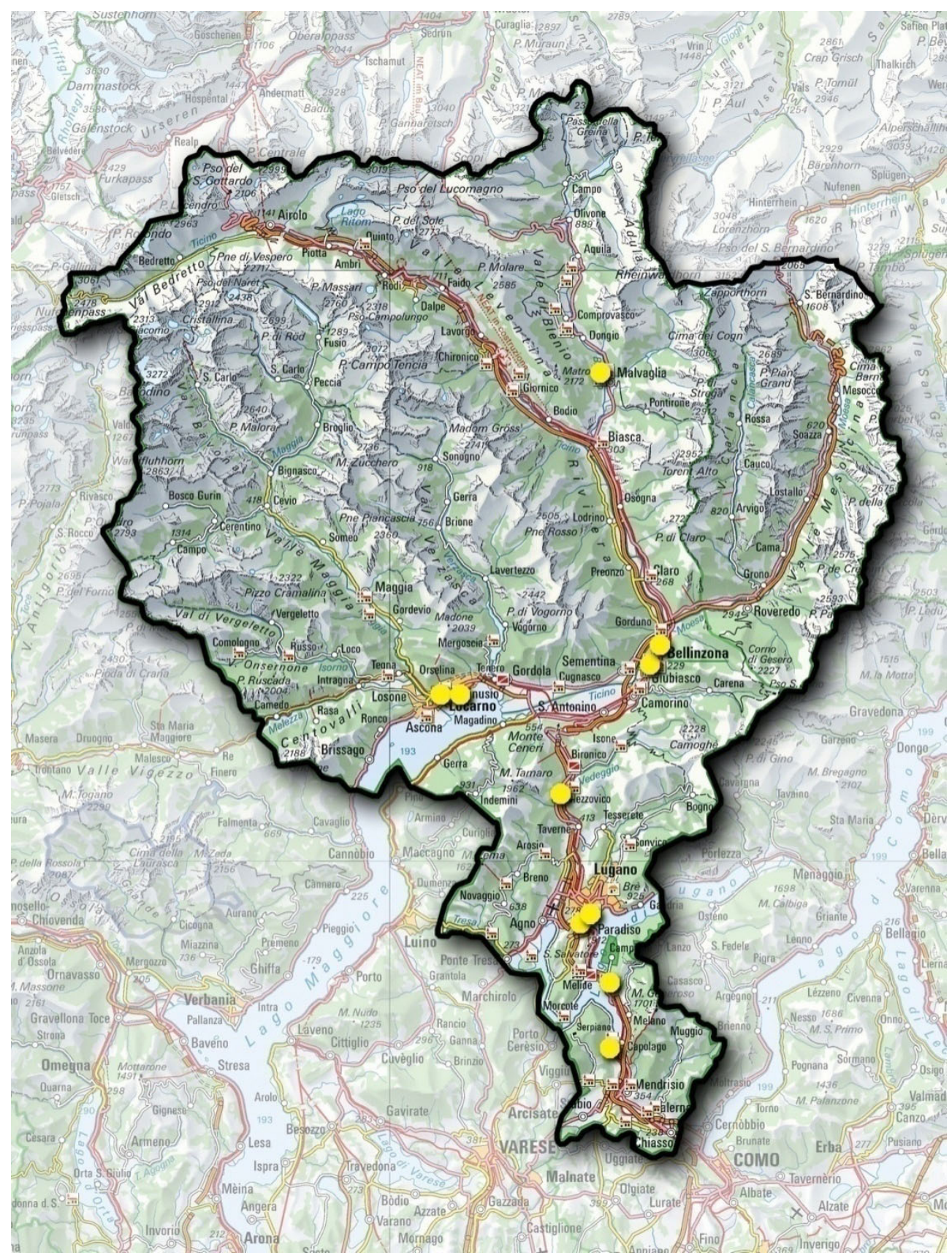

Figura 59 Cartina degli interventi documentati eseguiti da Edoardo Berta in Canton Ticino. 
instis

is 8

Dalla Chiesa di Sunta Maria degli Angeli in Ingano li 22 OPettoreber 1891
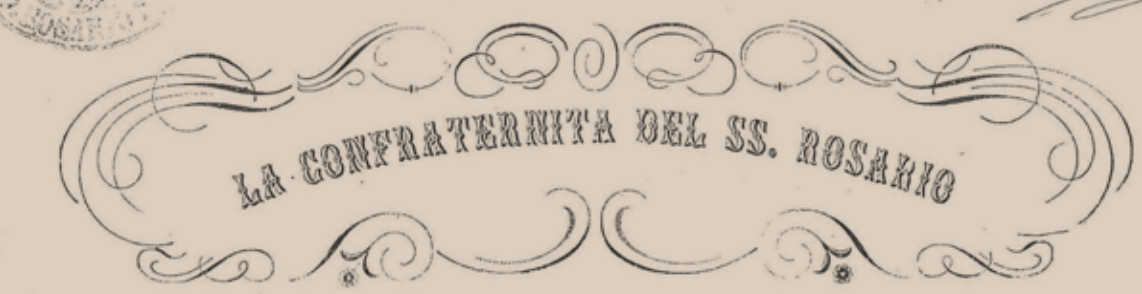

(0)

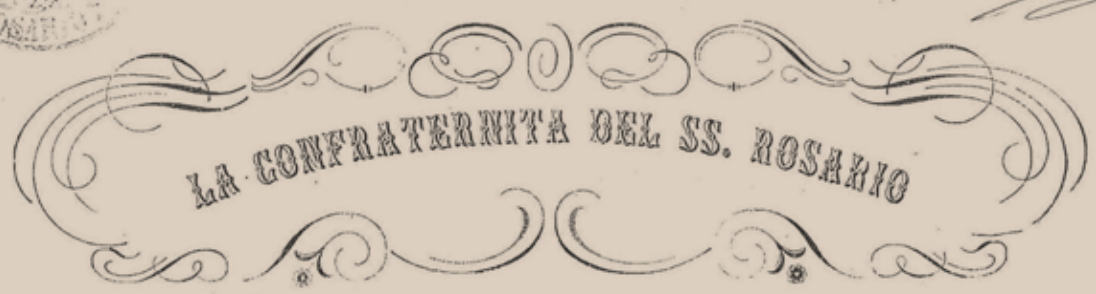
$\longrightarrow$

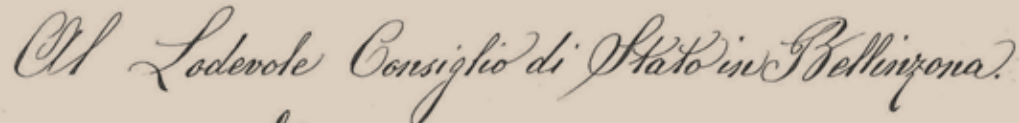

hy

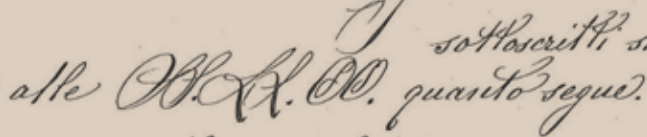

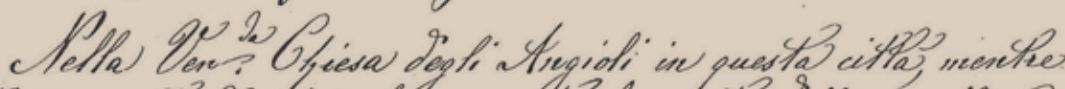

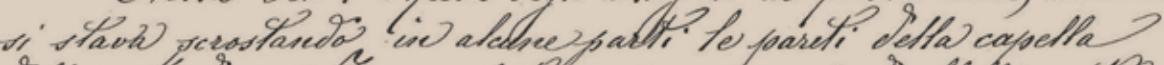

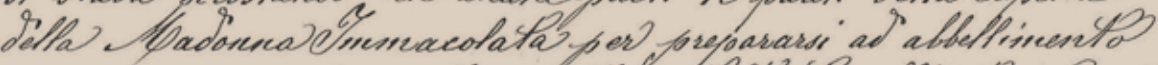

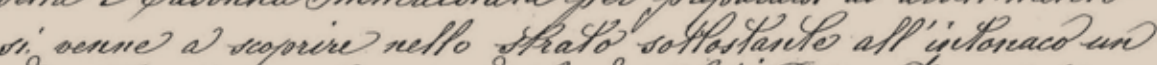

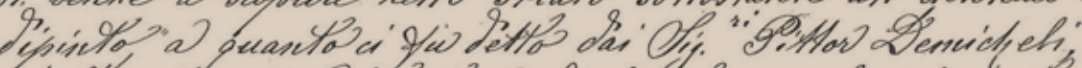

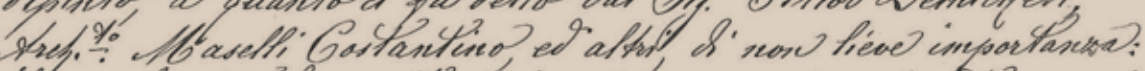

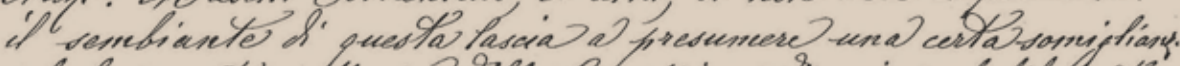

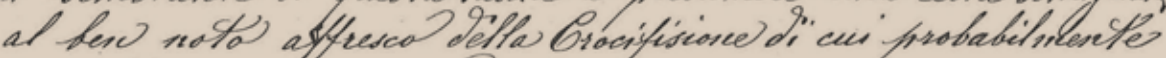
"sitien cortericharaned.

$$
\text { Gontinuaredo fo scrostamento conctivea pure }
$$
if nuovo affreseo if quate riscend o' interefrante, sie appourtos

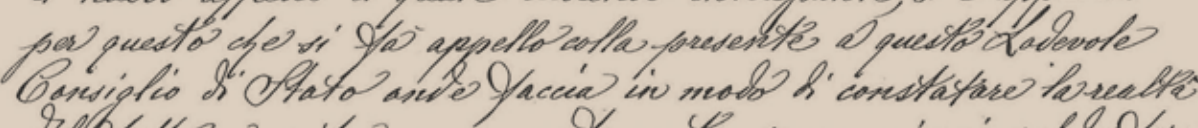

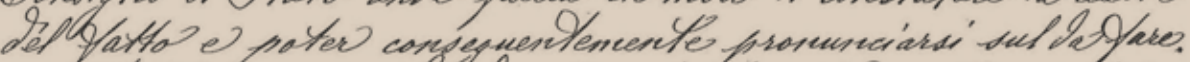

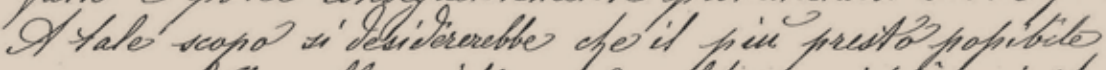
se ne frocectepe allas verifica con qualefje apposito incavicato

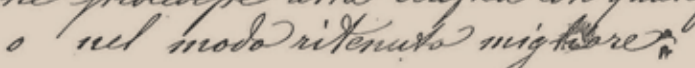

Figura 60 Confraternita del SS. Rosario; Lettera del 22 settembre 1891 indirizzata al Consiglio di Stato in Bellinzona. AUBCBZ, s. 143 Lugano, S. Maria degli Angioli. 


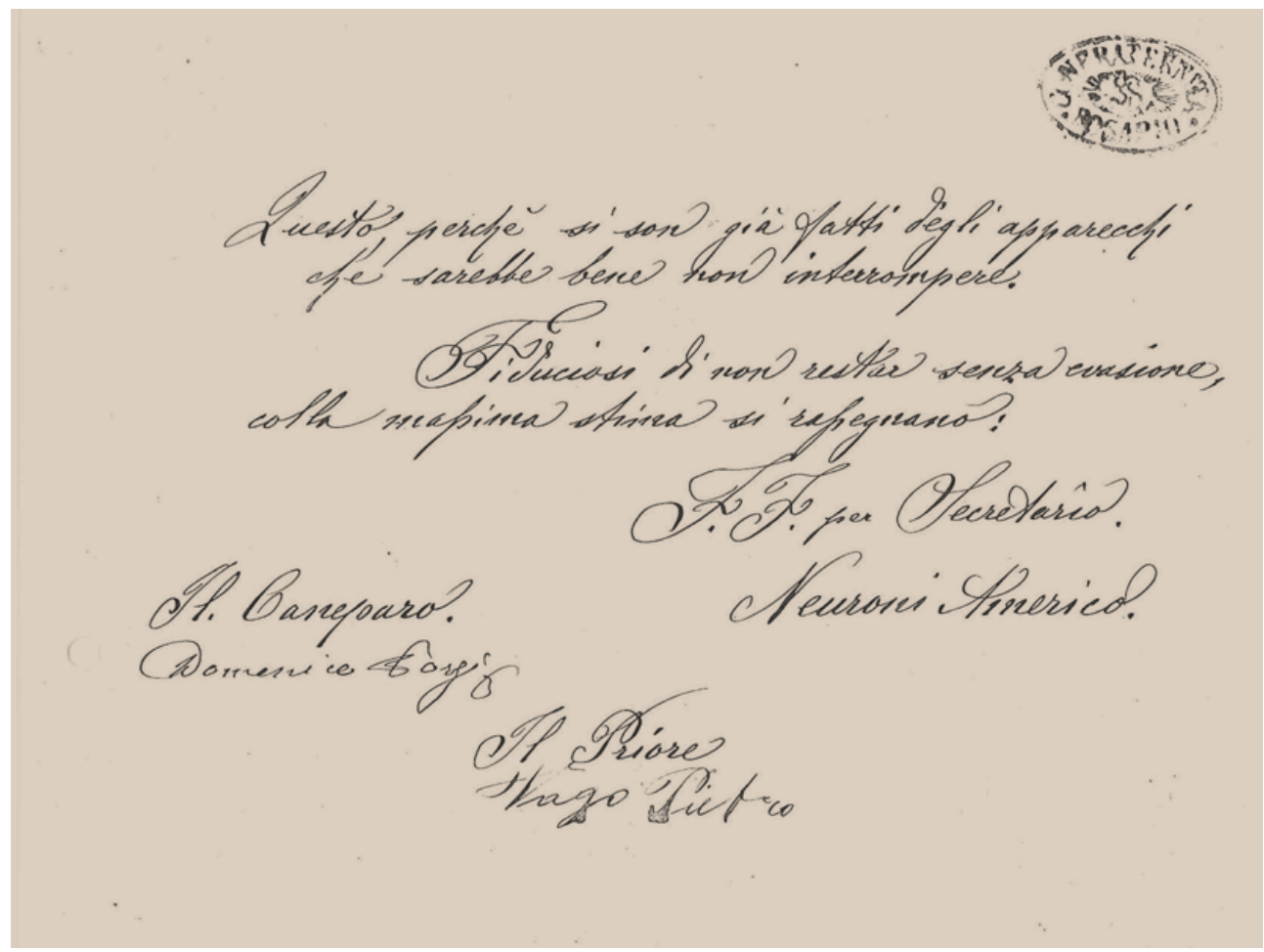

Figura 61 Confraternita del SS. Rosario; Lettera del 22 settembre 1891 indirizzata al Consiglio di Stato in Bellinzona. AUBCBZ, s. 143 Lugano, S. Maria degli Angioli. 


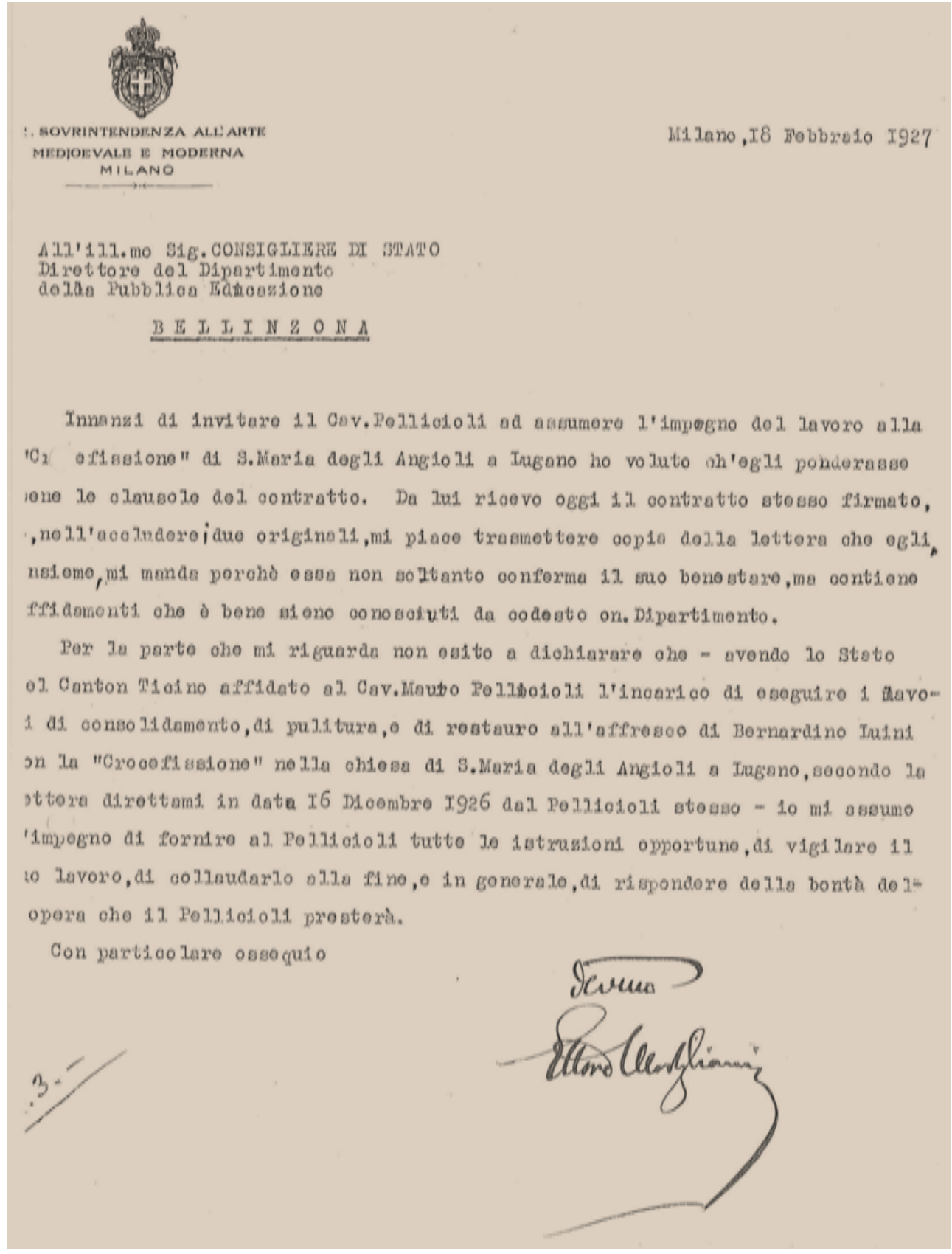

Figura 62 Ettore Modigliani, Milano. Lettera del 18 febbraio 1927 indirizzata al Consigliere di Stato. AUBCBZ, s. 143 Lugano, S. Maria degli Angioli. 


\section{CHR. SCHMIDT} DEKORATIONSMALER

TELEPHON SELNAU 3916 Telegr.: MALSCHMIDT ZORICH

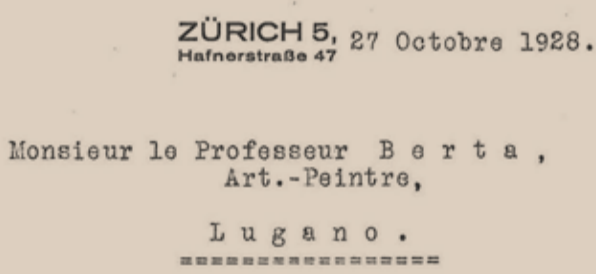

Monsieur,

Je viens de vous demander, s'il faut faire un cadre bombé pour la peinture murale au fond du coeur, qui couvrait la fenctre. Si vous percez la fenetre, il est évident, qu'il nous faut faire un cadro droit (non bombé). Il faudrait avoir une décision maintenant, parcequ' après on ne pourra plus bien bomber ou incliner la peinture transférée. Prière de me donner réponse aussit6t que possible.

Agréez, Monsieur, mes salutations sincères.
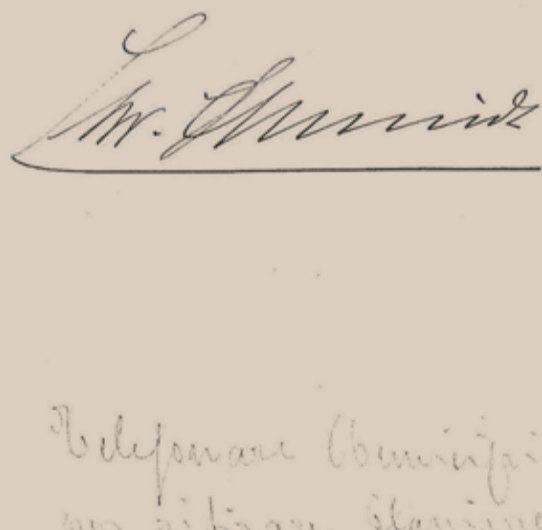

Figura 63 Chr. Schmidt, Zurigo. Lettera del 27 ottobrel 1928 indirizzata al pittore Edoardo Berta. AUBCBZ, s. 143 Lugano, S. Maria degli Angioli. 


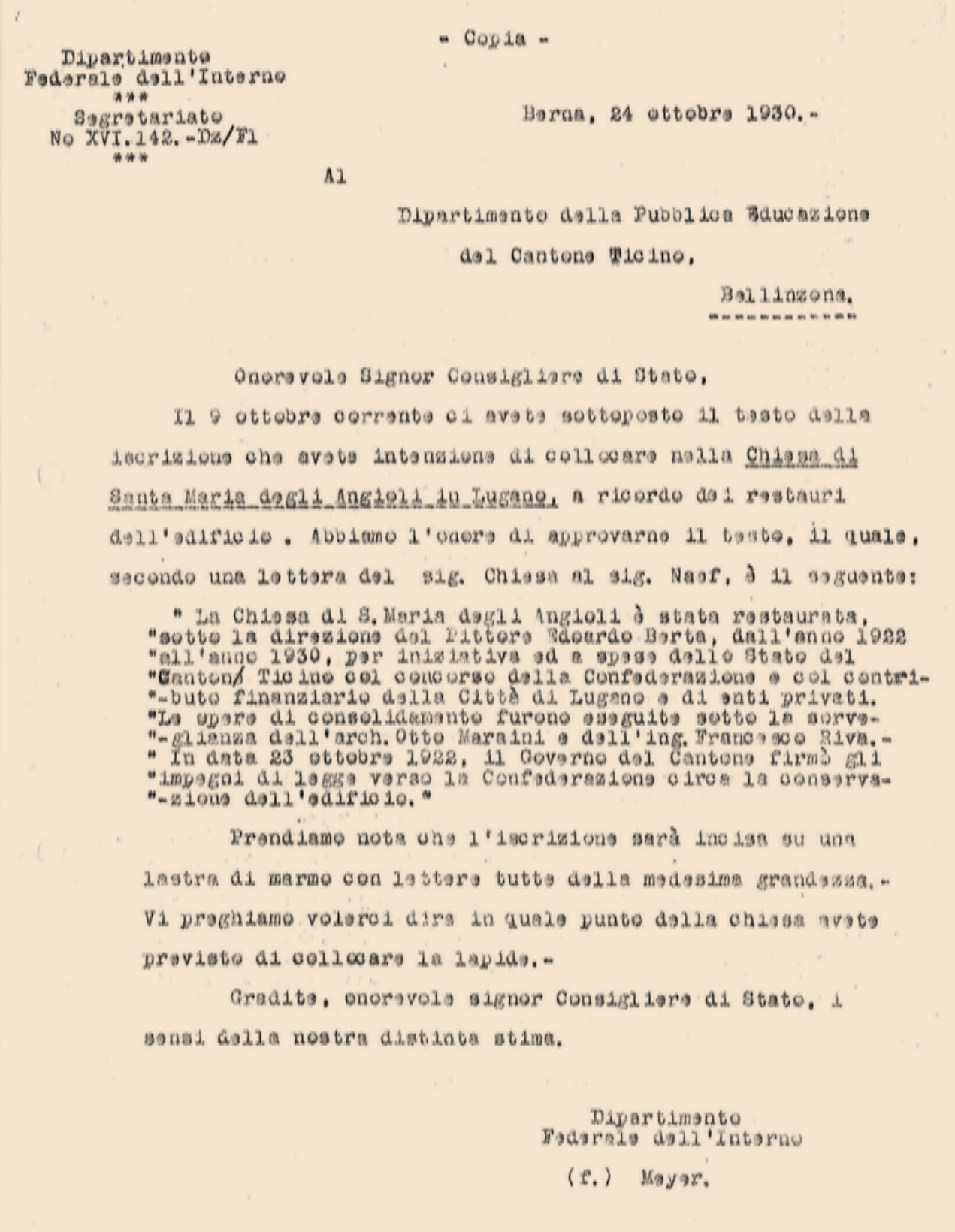

Figura 64 Dipartimento Federale dell'Interno, Berna, Lettera 24 ottobre 1930 indirizzata al DPE del Canton Ticino. AUBCBZ, s. 143 Lugano, S. Maria degli Angioli. 


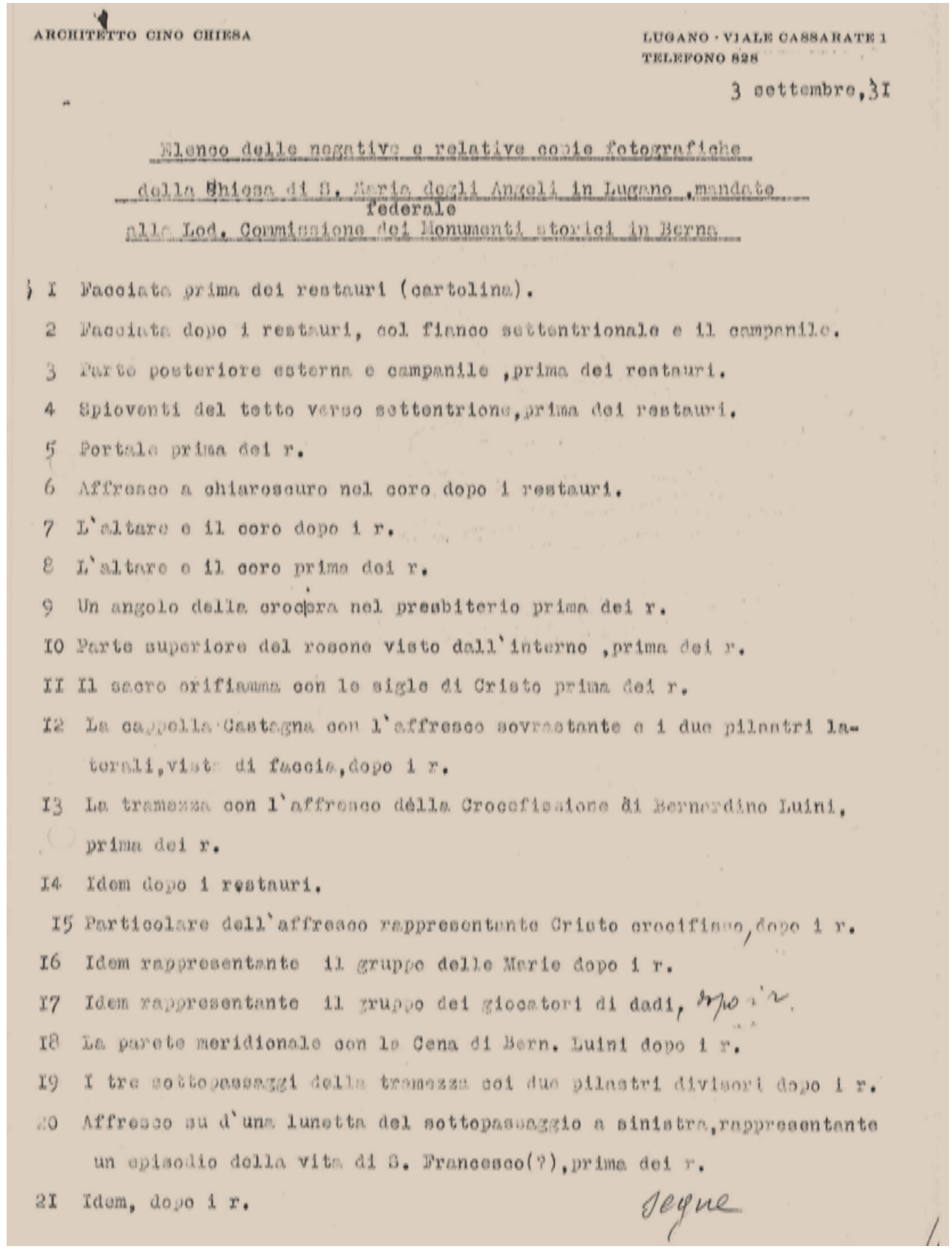

Figura 65 Cino Chiesa. Elenco numerato dei negativi e copie fotografiche di S. Maria degli Angioli indirizzato alla CMS, Lugano, 3 settembre 1931. AUBCBZ, s. 143 Lugano, S. Maria degli Angioli. 
ARCHITETTO CINO CHIESA

,

$+$

22 Afresco su d'un lunetta del sođtopassaggio centrale, rappresentante Gerusaleme, prima dei $r$.

23 Idem, dopo i restauri.

$\$$ non essendo stato possibile di trovare una fotografia della facciata prima dei restauri, ho dovuto includere una cartolina.Gia' 11 defunto pittore Berta 1 'aveva cercata Invano.

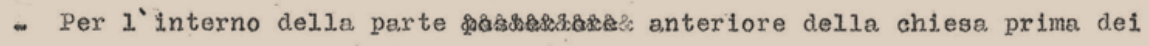
restauri, esiste la fotografia di un quadro nella racolta della Schweiz. Gesellschart für Erhaltung historischer Denkmäler.

Figura 66 Cino Chiesa, Elenco numerato dei negativi e copie fotografiche di S. Maria degli Angioli indirizzato alla CMS, Lugano, 3 settembre 1931. AUBCBZ, s. 143 Lugano, S. Maria degli Angioli. 


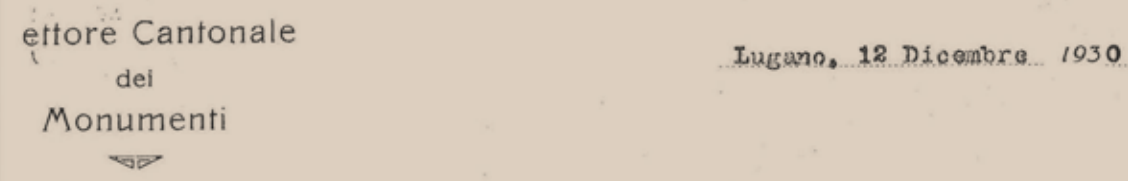

Figura 67 Edoardo Berta, Richiesta di materiale indirizzata al Economato dello stato. Lugano, 12 dicembre 1930. AUBCBZ, s. 143 Lugano, S. Maria degli Angioli. 


fillore Ruidis Fermorheni 


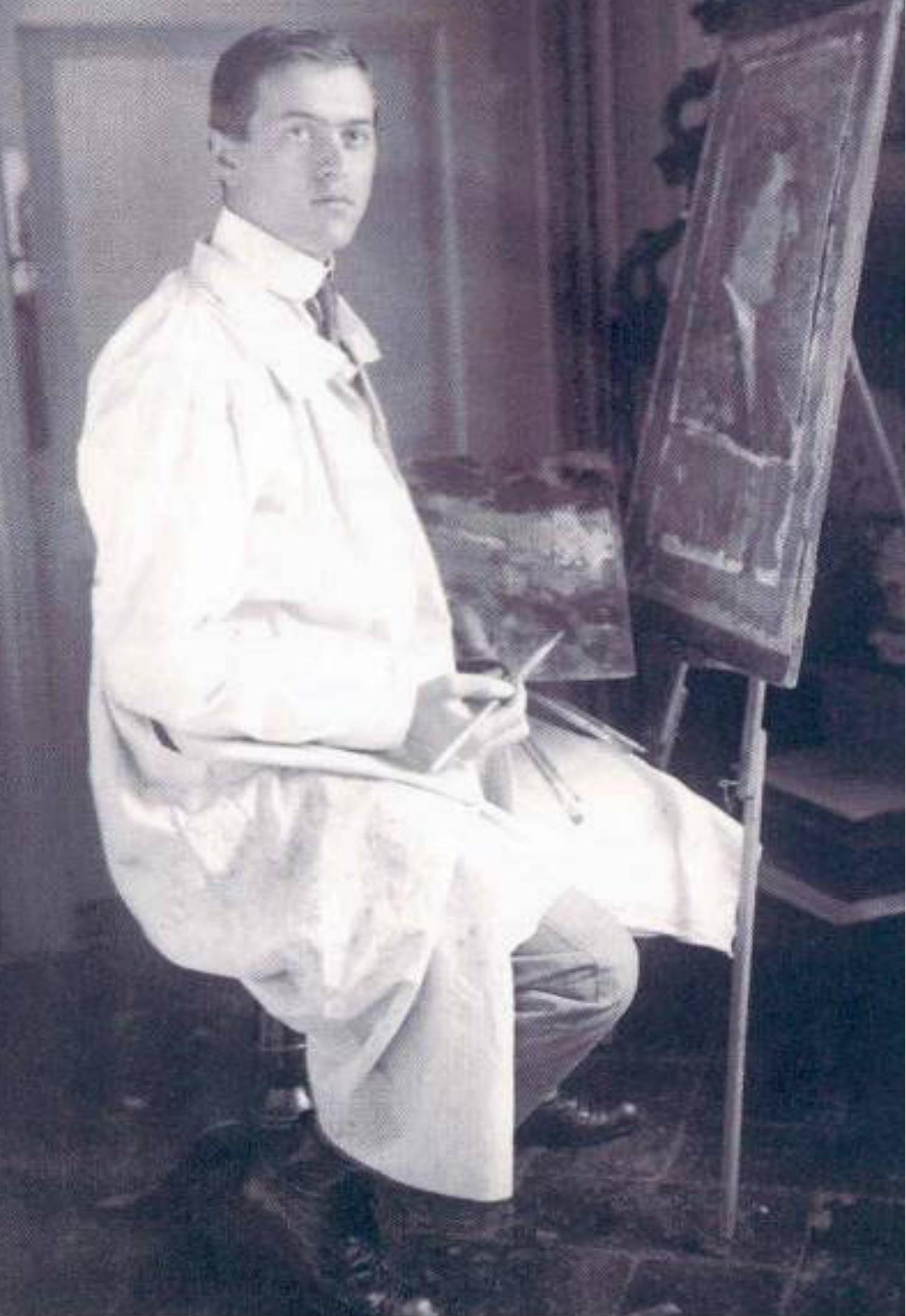




\section{EMILIO FERRAZZINI}

[...] Era uno dei campioni di un Ticino fervido che ora non c'è più: dove lavoravano, e spesso battagliavano in polemiche giornalistiche, vari personaggi cui ora si pensa con nostalgia...: Ugo Donati, Francesco Chiesa, Linus Birchler, Luigi Simona, Agostino Robertini, Arminio Janner, Piero Bianconi, Giuseppe Lepori, Virgilio Gilardoni, Aldo Crivelli, personaggi che non potevano certo dirsi in concordia concors, ma si spesso in concordia discors, poiché i lor cuori, in fondo, erano tutti rivolti, pur fra le polemiche, a un unico fine, il promovimento del patrimonio storico-artistico del paese $[. . .]^{235}$.

[...] Era uno de los campiones de un Ticino férvido que ahora ya no existe: donde trabajaban, y a menudo discutían en polemica periodísticas, varios personajes que ahora recordamos con nostalgia...: Ugo Donati, Francesco Chiesa, Linus Birchler, Luigi Simona, Agostino Robertini, Arminio Janner, Piero Bianconi, Giuseppe Lepori, Virgilio Gilardoni, Aldo Crivelli, personajes que no podían claro está, convivir en concordia concors, sino normalmente en concordia discors, ya que sus corazones, en el fondo, estaban todos revueltos, a pesar de las polemica, por una sóla finalina: promover el patrimonio histórico e artistico del país [...].

Pagina precedente

Figura 68 Emilio Ferrazzini. Autoritratto, Lugano 1922. (Dangio, Cima Norma, 2003)

35 (Agliati, 2003, p. 3-4). 


\section{Dati biografici}

Emilio Ferrazzini nasce il 25 settembre del 1895 a Lugano da Augusto Ferrazzini, discendente da un'antica famiglia di Mendrisio $^{236}$, e da Irene Nizzola, originaria di Chiggiogna in Valle di Blenio, il cui padre, Giovanni, ${ }^{237}$ fu un rispettato educatore e scrittore di Lugano curatore dell'ora biblioteca cantonale.

Ferrazzini inizia gli studi nel campo artistico nell'Accademia di Belle Arti di Monaco di Baviera $^{238}$, capitale tedesca dell'arte dalla fine dell'Ottocento, che deve abbandonare nel 1914 all'inizio della Prima Guerra Mondiale. Dopo aver lasciato la regione tedesca il pittore ritorna in Svizzera, esattamente a Ginevra, dove segue la

236 Nell'Archivio Comunale di Mendrisio si trova la raccolta dei documenti sull'attività commerciali che imprende nel XIX sec. la famiglia Ferrazzini.

Nizzola Giovanni; fra i suoi contribut all'insegnamento è di grande importanza il lavoro eseguito nella Libreria Patria di Lugano, nel momento più compromesso per questa poiché immersa nella marginale situazione economica del Cantone, quando come nuovo conservatore nel 1870 riordina la biblioteca con gli scarsi esemplari esistenti e tenta di moltiplicare gli acquisti elencando sulla rivista "L'Educatore della Svizzera Italiana" i nomi dei donatori. Nell'anno 1885 si raggiungono in deposito quasi 1500 volumi e vede la luce il primo catalogo delle opere acquistate. La medesima Libreria fondata dal Consigliere di Stato Luigi Lavizzari nel 1861 con il proposito di riunire tutte le pubblicazioni relative al Canton Ticino, formerà parte della Biblioteca Cantonale attraverso un atto stipulato nel febbraio 1913 tra Francesco Chiesa e Giovanni Nizzola. Sono anche di grande importanza le numerose pubblicazioni realizzate di libri ad uso scolastico ed il suo contributo alla "Società ticinese degli Amic dell'educazione del popolo" (Colombi, Bellinzona 1882).

${ }^{238}$ L'Accademia, situata nel quartiere degli artisti della città, Schwabing, fu centro di docenza e d insegnamento per illustri artisti come Franz von Stuck, Giorgio de Chirico, Paul Klee e Kandinskij. scuola di Ferdinando Hodler ${ }^{239}$, sicuramente spinto dallo spirito di modernità e rottura appreso nella scuola bavarese.

Finita la guerra il pittore si trasferisce in Italia (Roma), dove nell'Accademia di Belle $\mathrm{Arti}^{240}$ si istruisce nelle materie di pittura, architettura e restauro e si fece amico dell'architetto e restauratore ticinese Massimo Guidi, come appunta Aldo Crivelli nel testo commemorativo del 1972, ripubblicato con motivo di una mostra retrospettiva del pittore realizzata a Lugano nel $2003^{241}$.

Dopo l'apprendistato artistico Ferrazzini inizia la sua attività professionale a Lugano, conservando le relazioni con I'Italia, dove collabora con "Arti grafiche del Luganese Chiattone $^{242 "}$ di Milano e con la "Lithographisce Institut" di Laupen (Berna),

\footnotetext{
Hodler Ferdinand (Berna, 14 marzo 1853 Ginevra, 19 maggio 1918) Pittore di influenze simboliste e dall'Art Nouveau con introduzione all'espressionismo. Si trasferì a Ginevra dal 1871 dove ebbe lezioni dal pittore Bathélemy Menn, e comincia dal 1879 a realizzare mostre personali in circoli culturali svizzeri. Partecipa ai principali movimenti di Secessione come membro a Vienna, Berlino e Monaco, dopo il 1900 diventò una dei pittori più importanti di Europa. Nel 1914 fu presidente della Società di pittori, scultori ed architetti svizzeri.

240 L'Accademia di Belle Arti di Roma fondata alla fine dell' ottocento dai pittore Girolamo Muziano e Federico Zuccari con il nome di Accademia di San Luca è una delle più importanti e riconosciute Accademie di Belle Arti italiane e la prima ad essere riconosciuta dal ministero dell'istruzione italiano.

${ }^{241}$ (Crivelli, 2003, p. 9).

${ }^{242}$ La azienda fu fondata da Gabrielle Chiattone (1853-1934), a Milano nel 1899, membro della prima generazione di artisti della famiglia insieme agli scultori Antonio (1856-1904) e Giuseppe (1863-1954). La seconda generazione si componeva dall'architetto e pittore Mario (1891-1957), artista futurista in intima relazione con il pittore Boccioni, e Antonio Jr. (19041957), critico cinematografico e pittore autodidatta.
} 
entrambi studi d'arte litografica ed incisione dove completa la sua formazione.

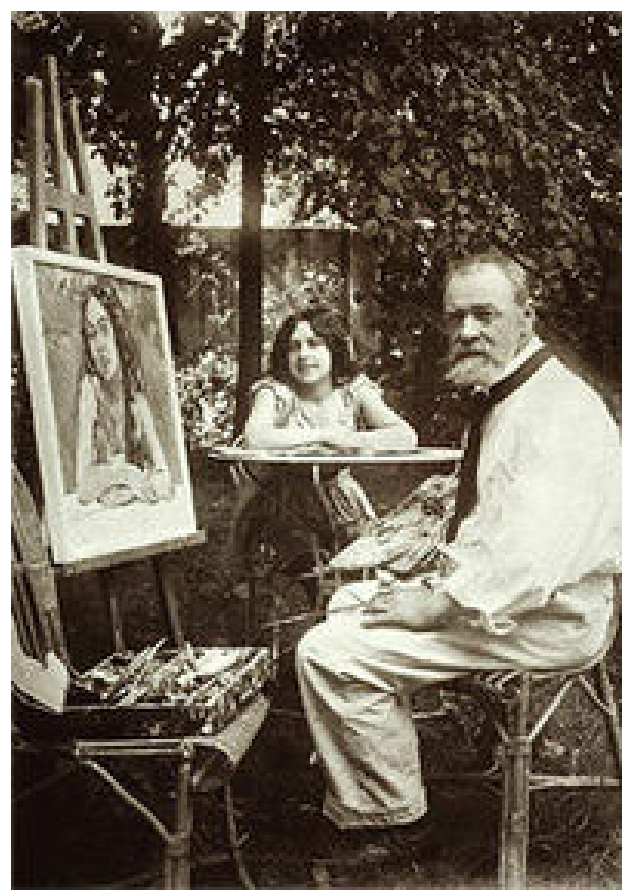

Figura 69 Bonzon Paul, Ferdinand Hodler, 1916. Fotografia b/n. [online]

Emilio Ferrazzini fu membro della "Società Pittori Scultori ed architetti Svizzeri" ${ }^{243 "}$ e della "Società ticinese di Belle Arti ${ }^{244 \text { " }}$

\footnotetext{
243 La SPSAS è la prima organizzazione professionale degli artisti svizzeri, pittori, scultori ed architetti, creata in 1866 dall'Associazione svizzera delle belle arti esistente da 1806, incaricata principalmente dell'organizzazione di mostre e della politica culturale. La rivista dell'associazione, Art Suisse, se pubblica per la prima volta in 1899. Separata dalla Associazione di donne pittrici, scultrice ed artigiane costituita in 1907 tutto e due si uniscono finalmente in 2001 anno in cui si fonda la giovane Associazione professionale delle arti visuali, VISARTE. Informazioni presso il sito www.visarte.ch.

${ }^{244}$ La Società Ticinese di Belle Arti è stata fondata il 29 settembre 1889 da un gruppo di artisti fra i quali lo
}

partecipando a mostre nazionali, specialmente alle annuali e itineranti Turnus $^{245}$.

Ferrazzini si offrì appassionatamente alla scoperta della pittura con quadri raffiguranti nature morte, nudi, ritratti e paesaggi, studiando costantemente i materiali ed i metodi tecnici d'espressione senza mai nasconderli ai suoi colleghi. Nonostante ciò la sua opere artistica non si limitò unicamente alla pittura di cavalletto ma anche alla decorazione murale, generalmente religiosa ed alla creazione di vetrerie: possiamo citare come esempi di quest'attività gli affreschi della facciata della chiesa dei SS. Martino vescovo e Giovanni evangelista a Bironico, cancellati durante i lavori di restauro del 1971-1974, l'affresco di San Cristoforo per l'Oratorio di Dangio, eseguito in collaborazione al collega di Attilio Balmelli, l'affresco raffigurante Santa Teresa sull'omonima cappella lungo la scalinata che porta alla Chiesa di Sant'Antonio Abate in Morcote, insieme ad altre cappelle dipinte tutte negli

scultore Antonio Chiattone che ne fu anche il primo segretario. Oggi, a più di un secolo dalla fondazione e con i suoi oltre mille soci, la STBA non è solo storicamente la più antica associazione culturale della Svizzera Italiana, ma anche quella con il maggior numero di iscritti. Lo scopo principale della Società è quello di promuovere l'apprezzamento della cultura artistica diffondendone la conoscenza nell'ambito della Svizzera Italiana. Ma se gli scopi attuali poco si discostano dalle intenzioni dei fondatori, profondamente mutati sono gli strumenti che continuamente si conformano alla mutevolezza della realtà artistica europea e svizzera in particolare.

${ }^{245}$ La Turnus promossa dalla Società Svizzera delle Belle Arti, fu la mostra annuale di maggior prestigio in ambito nazionali mediante la quale s'impostarono le basi del panorama artistico e museale nel territorio, realizzandosi dal 1840 in modo itinerante in tutti i cantoni, perfino nei rurali e nelle piccole città. 
anni Quaranta del Novecento da artisti come Pietro Chiesa (1876-1959), Ponziano Togni (1906-1971) e Felice Filippini (19171988). Sono anche opere sue le vetrate artistiche della chiesa della curia vescovile di Lugano.

Inoltre alla creazione artistica Ferrazzini invase anche il campo della conservazione ed il restauro, con la medesima prassi di sperimentazione che esercitasi in qualità $d i$ pittore. Secondo Aldo Crivelli ${ }^{246}$, mesi prima di morire, il pittore parlava di una ricetta che potrebbe essere stata utile nel campo della conservazione:

[...]che però non si sa se ha lasciato testimonianza scritta o se si potrà ricostruirla esaminando, attentamente, il voluminoso carteggio del suo archivio personale[... $]^{247}$.

Purtroppo oggi non si conserva l'archivio descritto dal letterato, che magari avrebbe apportato delle informazioni necessarie a soddisfare le nostre inquietudini e dove probabilmente trovare delle spiegazioni dettagliate circa le sperimentazioni del Ferrazzini e più approfonditamente i metodi e materiali di restauro impiegati nelle numerose opere da lui condotte.

Ma la consacrazione all'arte non si limitò alla pratica ma anche alla divulgazione di questa. II nonno materno, Giovanni Nizzola, era già stato un importante scrittore ed educatore luganese come precedentemente citato. II contributo più significativo di Nizzola all'insegnamento, assieme alle creazioni di molteplici pubblicazioni di uso scolastico, fu il riordino e l'incremento degli acquisti della Libreria Patria di Lugano dal 1870, in qualità di

\footnotetext{
${ }^{46}$ (Crivelli, 2003, p. 11).
}

(Crivelli, 2003, p. 11). conservatore della medesima. Questa passione per l'istruzione fu indubbiamente ereditata dal nipote Emilio, il quale dimostrò sempre motivazione al trasferimento delle proprie conoscenze attraverso scritti giornalistici. Gli articoli divulgati erano il risultato dei propri studi riguardo a monumenti e sulla loro conservazione e suscettibilità ad essere oggetti di restauro, tutti loro segnati da un ferreo carattere critico motivato dal sentimento di rivalorizzazione artistico. Fra i più notevoli articoli possiamo elencare gli scritti per la Rivista Storica Ticinese diretta da Aldo Crivelli: "Il S. Martino di Deggio ${ }^{248 \text { ", }}$ e "La chiesetta di S. Colombano di Scona ${ }^{249 "}$ (entrambi esponenti delle opere che lui stesso restaura nel 1939); "Gli stucchi bizantini di Corzoneso ${ }^{250 "}$ e "Sculture rustiche".

\footnotetext{
248 (Ferrazzini, II S. Martino di Deggio, 1939) L'articolo venne pubblicato alla fine dei lavori di restauro condotti dallo stesso Ferrazzini.

${ }^{249}$ (Ferrazzini, La chiesetta di S. Colombano di Scona, 1940) L'articolo pubblicato descrive il valore storico della fabbrica ed i lavori di restauro finalizzati nel mese di agosto 1939.

${ }^{250}$ (Ferrazzini, Gli stucchi bizantini di Corzoneso. Gli affreschi di Sorengo. La Madonna di Sommascona, 1938) e (Ferrazzini, Gi stucchi bizantini di Corzoneso, 1940) I due testi di Emilio Ferrazzini pubblicati nella Rivista Storica Ticinese rivelano la arricchita formazione artistica del pittore. È molto significativo in questo aspetto specialmente il secondo articolo sopracitato, ove il Ferrazzini analizza le critiche artistiche del padre Joseph Braun ( (Braun, 1924), realizzando un confronti fra gli stucchi trovati nella località ticinese di Corzoneso ed i frammenti dell'altare della Cattedrale di Santiago di Compostela in Spagna. Queste comparazioni servirono ad analizzare le funzioni degli stucchi di Corzoneso, i quali dopo il restauro condotto nella chiesa in 1949 vengono introdotti nella mensa dell'altare: [...]Confrontando gli stucchi esistenti a Santiago di Compostella ed i due preziosi frammenti di Corzoneso è facile notare la parentela di stile e di disegno
} 


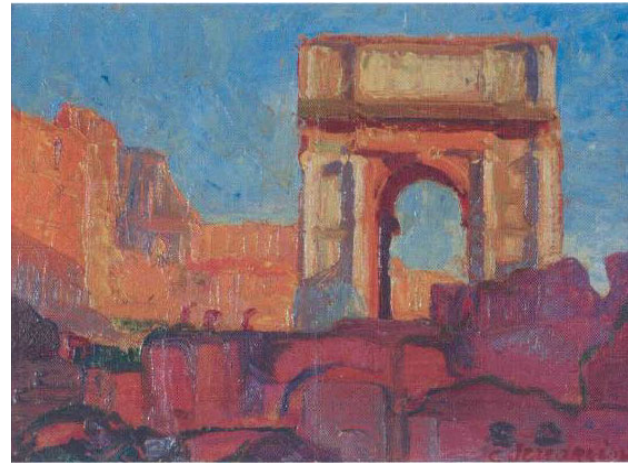

Figura 70 Emilio Ferrazzini, Roma. Olio su tela.

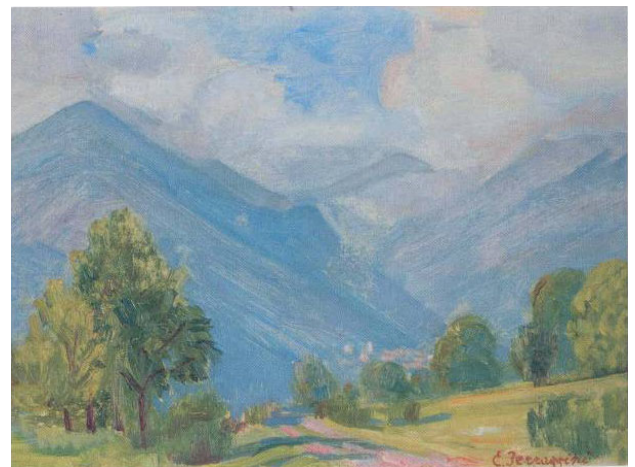

Figura 71 Emilio Ferrazzini, Paesaggio ticinese, 1927. Olio su tela.

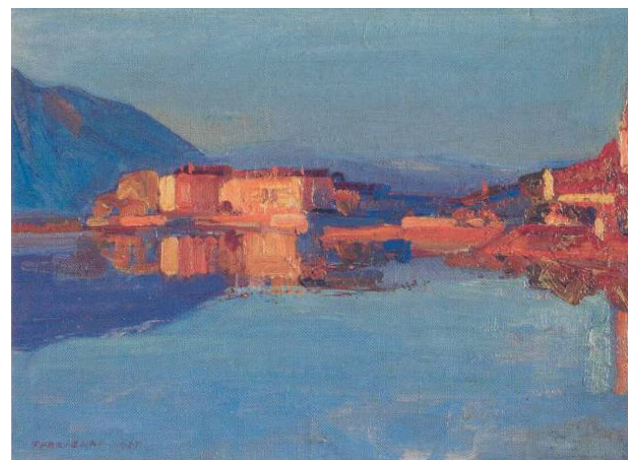

Figura 72 Emilio Ferrazzini, Bissone 1927. Olio su tela.

esistente fra quelle sculture. È da domandarsi se gli stucchi di Corzoneso (i frammenti sono alti circa 70 cent.) non avessero avuto una funzione analoga a quelli, così preziosi, esistenti a Compostella[...].
Si tratta della stessa motivazione che fece a Ferrazzini allestire nel 1960 alla Villa Ciani di Lugano una mostra ${ }^{251}$ dedicata al suo ammirato pittore Giuseppe Antonio Petrini di Carona ${ }^{252}$ al quale anni prima aveva omaggiato con un articolo intitolato "Affreschi di Giuseppe Antonio Petrini da Carona (1677-1759) nella Chiesa di Besano (Varese) ${ }^{253 \prime \prime}$ pubblicato in 1954 sulla "Rivista Archeologica" dell'Antica Provincia e Diocesi di Como.

\footnotetext{
${ }^{251}$ La mostra delle opere del pittore di Carona furono esposte al Palazzo di Villa Ciani di Lugano dal 3 ottobre al 6 novembre 1960. G. Cattaneo/ E. Ferrazzini a cura di, Catalogo della "Mostra delle opere del pittore Giuseppe Antonio Petrini da Carona (1677-1759)", Lugano, 1960, n. 36.

${ }^{252}$ Petrini Giuseppe Antonio (Carona 1677 - 1759) Pittore svizzero figlio dello scultore Marco Antonio e di Lucia Casella. È considerato il maggior rappresentante della pittura contemplativa ticinese del XVIII sec. Si forma nella pittura da cavalletto nella scuola di Bartolomeo Guidobono di Genova ed a Torino. Il suo stile caravaggesco sempre marcato da una intensa religiosità è influenzato da pittori come Giovanni Serodine di Ascona. Imiterà inizialmente i chiaro-scuri della pittura di Rembrandt rappresentando in generale figure di anziani e filosofi con uno splendido dominio del disegno, mentre man mano con l'arrivo della corrente rococò le sue pennellate si rivestiranno di un maggiore colorismo per influsso dei coetanei Carlo Innocenzo Carloni di Scaria e Paolo Pagani di Castello e Giambattista Tiepolo. Possiamo apprezzare le sue opere in numerose chiese ed oratori del Ticino ad esempio nel Santuario della Madonna d'Ongero nel paese natale del pittore, il San Francesco della seconda cappella della chiesa di S. Maria degli Angioli a Lugano del 1728, la tela di San Paolo alla chiesa di S. Giorgio a Carona del 1750, il quadro raffigurante la Vergine e i SS. Domenico e Caterina nel coro della chiesa di S. Abbondio a Gentilino e la Gloria di S. Antonio rappresentata sulla volta del coro della chiesa di S. Antonio Abate a Lugano.

${ }^{253}$ (Ferrazzini, Affreschi di Giuseppe Antonio Petrini da Carona (1677-1759) nella Chiesa di Besano (Varese), 1954-1955, p. 79-84).
} 


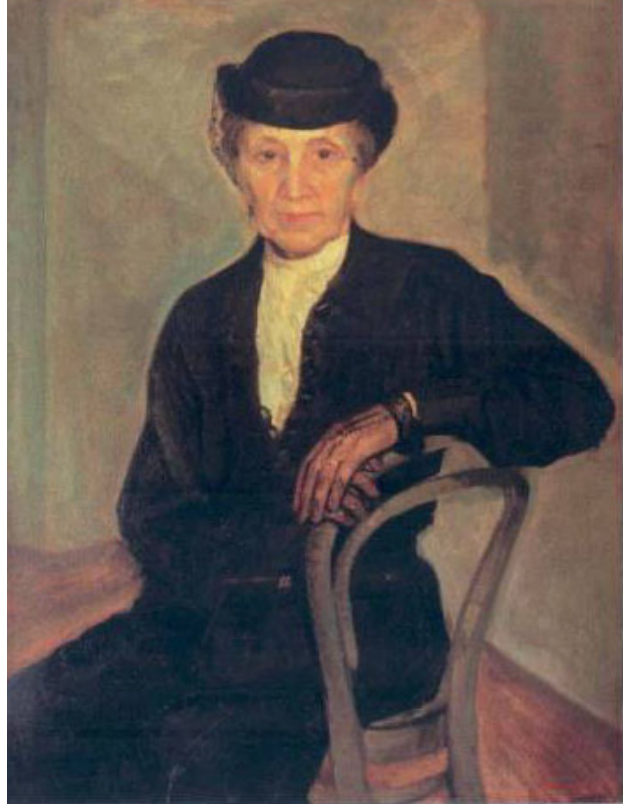

Figura 73 Emilio Ferrazzini, Irene Ferrazzini, 1933. Olio su tela.

Analogamente agli scritti, gli interventi di restauro eseguiti dal Ferrazzini furono numerosi a distinti dalle controversie che genereranno. Insieme a Tita Pozzi, egli fu il pittori-restauratore più prolifico in Canton Ticino; possiamo apprezzarlo nelle schede di catalogazione delle opere da lui restaurate, alla fine di questo capitolo.

Dopo la scomparsa di Edoardo Berta, a chi Ferrazzini ammirava, l'artista ebbe sempre il desiderio di sostituire l'amato maestro nel ruolo di esaltatore del proprio patrimonio.

Con Berta condivide gli ideali di restauro, che riflesse nella sua competenza ed esprime in numerosi scritti di corrispondenza con la Commissione Cantonale dei Monumenti Storici. Ricordiamo ad esempio il suo pensiero riguardo il ripristino dei monumenti, secondo le teorie di Ambrogio Annoni del "caso per caso", che Berta messe in pratica in opere come Santa Maria degli Angioli a Lugano, il Castello Visconteo di Locarno e Santa Maria delle Grazie a Bellinzona.

[...]Chissà perché oggi, nei restauri, ognuno vuol fare di sua testa quando la cosa è poi così semplice: si deve ubbidire al monumento il quale dice lui stesso come e cosa si deve fare per ripristinarlo e restauralo, secondo le epoche, gli stili e le tecniche con le quali è stato eseguiti $[\ldots]^{254}$.

Emilio Ferrazzini, come allo stesso tempo Berta, non fu un abile restauratore e la sua maestria manuale si esprimeva meglio nei quadri che sugli oggetti d'intervento; nonostante ciò sarebbe insensato giudicare questi interventi di restauro "scorretti" poiché partivano comunque e sempre da un enorme rispetto verso l'opera. Un rispetto che diventa fattore indispensabile nel momento d'affrontare il restauro e per la conoscenza del bene culturale, obiettivo difeso da Ferrazzini con energia fino alla sua morte il 30 maggio del 1975 e che speriamo, rendendogli omaggio, di divulgarlo anche noi.

\section{Le polemiche giornalistiche di Emilio Ferrazzini}

Come restauratore professionista Emilio Ferrazzini lavorò dagli ultimi anni Venti, dopo il suo ritorno in Svizzera, nei dipinti murali di chiese e cappelle a Corzoneso, Torre, Cavagnago, Dongio, Malvaglia,

\footnotetext{
${ }^{254}$ (Ferrazzini Emilio. Lettera indirizzata a Pierangelo Donati, Capo Ufficio della Commissione dei Monumenti Storici. Lugano 4 agosto 1971. AUBCBZ s. 143 Lugano, Madonna di Loreto).
} 
Sommascona, Negrentino, Deggio, Lugano, Bissone, Morcote, Gentilino, Croglio a Castelrotto, elencando qui solo una minima parte, e su numerose tele fra le quali le più importanti si trovano nell'altare maggiore della chiesa della Collegiata dei SS. Pietro e Stefano di Bellinzona, opera attribuita a Tintoretto o alla sua scuola; nella chiesa di Santa Maria degli Angioli a Lugano: San Domenico e San Francesco ai lati del Crocifisso; Madonna e Santi, il San Francesco ed il Sant'Antonio di Giuseppe Antonio Petrini, Angelo e Tobia ${ }^{255}$; nella chiesa di Santa Croce di Riva San Vitale dove consolida i dipinti su tela dei due altari nel 1940, e nella chiesa di San Nicolao a Giornico, dove intervenne sulla tela della Madonna delle Grazie della omonima cappella. Nel 1943 insieme al collega Tita Pozzi eseguirà una campagna di

\footnotetext{
${ }^{255}$ Oltre ai lavori nel restauro dei dipinti murale della chiesa di Santa Maria degli Angioli, nel 1930 Ferrazzini elaborò il preventivo di restauro per le sei tele in proprietà della Chiesa degli Angioli di Lugano. Propose i lavori di pulitura, sostituzione di telaio e pulitura della cornice del S. Domenico e S. Francesco ai lati del Crocifisso $(153 \times 130 \mathrm{~cm})$ per $130 \mathrm{fr}$.; la foderatura, sverniciatura, pulitura, stuccatura, ritocco pittorico, sostituzione del telaio e restauro della cornice della tela della Madonna e Santi $(165$ × $235 \mathrm{~cm})$ per $365 \mathrm{fr}$.; foderatura, consolidamento del colore, sverniciatura e pulimento dei vecchi ritocchi dell'opera di Giuseppe Antonio Petrini raffigurante S. Francesco (135 x 190 $\mathrm{cm}$ ) per $320 \mathrm{fr}$.; foderatura, consolidamento del colore, sverniciatura, pulimento dei vecchi ritocchi dell'opera, reintegrazione completa e sostituzione del telaio del S. Antonio $(145 \times 110 \mathrm{~cm})$ del Petrini, per $230 \mathrm{fr}$.; sverniciatura, pulitura, reintegrazione pittorica e sostituzione del telaio della tela di S. Francesco (200 x $160 \mathrm{~cm}$ ) per 230 fr.; per ultimo la ricucitura, rappezzatura, stiratura, pulitura e restauro pittorico della tela raffigurante Angelo e Tobia $(275 \times 165 \mathrm{~cm}$ ) per 200 fr. (Ferrazzini Emilio, Preventivo per il restauro di N.6 quadri, indirizzato al DPE del Canton Ticino. Lugano 20 ottobre 1930. AUBCBZ s. 143 Lugano. S. Maria degli Angioli).
}

conservazione degli autentici e preziosi trasparenti di Giovanni Battista Bagutti ${ }^{256}$, realizzati dall'autore per la cerimonia della Settimana Santa nella località di Mendrisio. Oggi si conserva una vasta raccolta di negativi fotografici elaborati dal restauratore con lo scopo di documentare ben diversi interventi o per mostrare alle istituzioni competenti (Commissione dei Monumenti Storici e Dipartimento della Pubblica Educazione) la testimonianza di quelle opere in deperimento che lui cercava insistentemente. Fotografie che, sebbene non sostituiscono la mancanza di documentazione cartacea, aiutano a ricomporre la storia del monumento ed a testimoniare, in alcuni casi, momenti unici. E. Ferrazzini formò parte dal 1931, anno in cui muore il maestro Edoardo Berta, al 1945, della "Commissione Cantonale dei Monumenti Storici" del Canton Ticino in difesa della sua ricchezza patrimoniale. Furono quattordici anni di dedicazione che il pittore non seppe separare dall'attività di

Bagutti, Giovanni Battista (Rovio 1742-1823). Figlio di Giovanni e Angela Caterina Vegezzi. Pittore di cavalletto, d'affreschi formatosi a Parma e Roma nella corrente neoclassicista che rappresenta nella tela “Deianira figlia di Eneo re di Etolia" del 1768 con la quale vince il premio dell'Accademia parmense. La sua pittura si riaprirà ad un dinamismo barocco dopo il suo ritorno in patria. In Ticino lavorerà per importanti famiglie locali, nella creazione di pale d'altare, pitture murali, ex-voto e "trasparenti", questi ultimi, opere uniche al mondo utilizzati per decorare le strade nella Settimana Santa nella località ticinese di Mendrisio. I quadri particolari per la trasparenza delle tele grazie all'abbondante applicazione di cera e di essenza di trementina, e dei colori. Vengono illuminati alla sera mediante un complesso sistema di luci. Troviamo alcune opere sue alla chiesa parrocchiale di Rancate del 1775, a Sala Capriasca, nelle tele di Santa Croce a Riva San Vitale del 1782 restaurate da Ferrazzini, alla chiesa di San Giovanni di Mendrisio del 1774 ed al Museo Cantonale d'arte di Lugano. 
esecutore diretto dei restauri che lui stesso doveva ispezionare, cosa che non gli era autorizzata. Forse per invidia, per complesso, per paura di perdere il prezioso lavoro difficilmente concesso a tutti $i$ pittori-restauratori, in un momento segnato dalla necessità economica, Ferrazzini si dichiara offeso in innumerabili lettere che dirige al direttore, Francesco Chiesa, "Peppo", che per lui fece più di quanto le leggi della Commissione Cantonale dei Monumenti Storici avrebbero permesso ${ }^{257}$.

\footnotetext{
${ }^{257}$ Dopo il 1948, le polemiche nei lavori di restauro si succedono. Queste, generate in inizio come una critica "costruttiva" hai lavori eseguiti, parte dagli articoli di Aldo Crivelli Rivista Storica Ticinese per svilupparsi man mano in una lotta fra i restauratori per l'ottenzione dei lavori, lotta che confronterà a diversi artisti ticinesi ed stranieri che lavoravano in quel campo per sopravivenza. Ferrazzini sarà una de primi, senza giustificazione, ad scrivere contro la Commissione, quando già non era membro di questa, e contro Francesco Chiesa. II pittore, pur consapevole che il presidente gli aveva aiutato sempre, ebbe i modo di lamentarsi per la mancanza di lavoro. Francesco Chiesa, in risposta ad un scritto che i pittore aveva fatto pubblicare sul Corriere del Ticino, scrisse [...]Ferrazzini, essendo membro della Commissione, e ricevendo come gli altri membri l'incarico di studiare questa o quella questione, s'immaginava d'acquistare ipso facto il diritto d diventare l'esecutore dei lavori proposti. Pretesa evidentemente indiscreta, poiché la qualità $d$ membro di vigilanza mal s'accorda con l'attività di ch eseguisce sotto vigilanza [...] ̇̀ bensì vero che, in altr tempi, incarichi per esecuzione di restauri important furono conferiti ad Edoardo Berta: ma bisogna tener presente il valore non comune di lui, l'impossibilità allora di sostituirlo [...] Poi dovrebbero sapere che ne Cantone esistono anche altri restauratori degni $d$ fiducia, che pur hanno il diritto di lavorare. Non più membro della Comm., il Sr. Ferrazzini ha avuto da no incarichi relativi alla chiesa del Carmelo a Villa Coldrerio.[...]. Però, avendosi confermato dalle fonti archivistiche che Emilio Ferrazzini lavorò in più di una occasione ancora come membro della Commissione,
}

Ma non solo il Chiesa lo proteggerà, anche il professore Linus Birchler ${ }^{258}$, presidente della Commissione Federale dei Monumenti dal 1942 al 1963, lo ammirerà e difenderà per la sua capacità di critica e capacità ad affrontare la polemica che negli anni successivi soffrirà da parte delle autorità cantonali e dei membri della commissione, come fu il caso dello scrittore Piero Bianconi ${ }^{259}$

Sono esempio del deciso carattere di Ferrazzini notizie come questa che cita Mario Agliati ${ }^{260}$ in una delle uniche monografie sull'artista:

[...]poco dopo la metà degli anni Trenta. Ugo Donati ${ }^{261}$ stava discettando con alcuni seguaci

crediamo che questo scritto sia soltanto un modo di nascondere i fatti, illegali, che lo avevano permesso. (Chiesa Francesco. Lettera indirizzata a A. Lucchini. Lugano 27 agosto 1949. AUBCBZ s.091 Corzoneso).

${ }^{258}$ Birchler Linus (24.4.1893 Einsiedeln - 2.1.1967 Männedorf, catt., di Einsiedeln) Scrive "Restaurierungspraxis und Kunsterbe in der Schweiz» "Principi per la tutela dei monumenti storici in Svizzera" (Zurigo 1948) dove si stabiliscono per la prima volta le norme per il trattamento del patrimonio storico. Dizionario storico della svizzera.

${ }^{259}$ Bianconi Piero (Minusio 1899 - 1984) scrittore laureato a Friborgo, realizza un breve soggiorno di studio a Firenze e Roma dove consoce intellettuali come Bargellini, Betocchi, Bo, don De Luca.

${ }^{260}$ Agliati Mario (Lugano 1922) Storico e scrittore Luganese formatosi a Locarno e Friburgo, è stato insegnante alla Scuola Professionale commerciale di Lugano fino al 1983 e ha collaborato nel settore giornalistico in special modo per la Gazzetta Ticinese. Fonderà la rivista "Il Cantonetto" nel 1953. Fra i suoi principali scritti si possono citare: "Lugano del buon tempo" (1963), il secondo volume della "Storia della Svizzera" (1969), Ottobre 1925: l'Europa a Locarno (1975), Lugano: racconto di ieri (1998-99), il romanzo L'erba voglio (romanzo, 1966), i libri di racconti I problemi del professor Pilati (1987) e La profezia del dottor Donzelli (1991).

${ }^{261}$ Donati Ugo (1891 Molinazzo di Monteggio - 1967 Lugano) Diplomato alla scuola di commercio di 
intorno a un certo quadro. Giusta il suo costume (grand'uomo, certo; ma ognuno è fatto di una particolare pasta) non mancava di sue critiche: "Mah, questi rossi, questi rossi...". Un aitante signore che stava, non veduto, alle sue spalle, intervenne imperioso: "Sono rosa, non rossi!" Era Emilio Ferrazzini, l'autore del quadro. Di carattere non sempre accomodante pur lui; nel segno di una generosità che fu una sua costante caratteristica[... $]^{262}$.

Ferrazzini discute ancora con lo storico Bianconi nel 1945. A egli controbatterà la fortunata scoperta del quadro della Sacra Famiglia di Giovanni Serodine ${ }^{263}$, poiché l'opera era già stata citata prima nel Dizionario "Thieme-Becker"264 (vol III,

Bellinzona nel 1910 si trasferì posteriormente a Londra fino al 1911, momento in cui si stabilisce a Roma per dedicarsi alla ricerca sugli artisti ticinesi del XVI e XVII sec. che darà frutto a due posteriori pubblicazioni, una nel 1936 intitolata Breve storia degli artisti ticinesi e nel 1942 per incarico del Cantone Ticino Artisti ticinesi a Roma. Durante il suo soggiorno in Italia si dedica all'antiquariato specializzandosi nel settore archeologico, e forma parte del gruppo dei Romanisti mantenendo la collaborazione con giornali e riviste fino al 1942 quando decide di rientrare nuovamente in Ticino dove continua l'attività d'antiquario. Lo stesso anno, dopo la scoperta del testamento di Francesco Borromini esegue la sua pubblicazione. Fu membro della commissione cantonale dei monumenti storici durante quattro anni $(1944$ - 1948) e si proclamò fino alla sua morte in difesa dell'italianità del Canton Ticino firmando numerosi articoli critici che diedero luogo a vivaci polemiche giornalistici.

${ }^{262}$ (Agliati, 2003, p. 3).

${ }^{263}$ Serodine Giovanni (Ascona, 1600 - Roma, 1631) pittore svizzero-italiano formato a Roma nella pittura di influenze caravaggesche.

${ }^{264}$ |l citato dizionario enciclopedico degli artisti fu fondato dagli storici d'arte tedeschi Ulrich Thieme (Liepzig 1865 - 1922), Felix Becker (1864-1928), e diretto dal 1923 da Hans Vollmer (1878-1964), dal 1907 al 1911 editato dalla casa Wilhelm a più tardi da E., A. Marinaio a Liepzig. Nel 1950 l'opera culmina con il 370 volume comprendendo in totale $148^{\prime} 180$ anno 1936) dove si descrive esattamente il quadro in quel momento trovatosi nella Villa Groccia-Luvini. Purtroppo la vendita di questa e di altre pitture della collezione, risultato dell'incompetenza della commissione del "Museo Caccia"265, avevano impedito a Ferrazzini di fotografare l'opera e di studiarla approfonditamente. $^{266}$ Questo tipo d'intrusioni ed iniziative accresceranno l'ostilità di tanti colleghi e finiranno per nascondere il suo valevole contributo al patrimonio, non come pittore ma come recuperatore, con violente denuncie e attacchi promossi specialmente contro $\mathrm{i}$ suoi metodi di restauro.

Fra i lavori più polemizzati che si effettuarono sotto la direzione di Emilio Ferrazzini e/o responsabilità di esecuzione si trovano la chiesa di San Carlo di Negrentino, dove Tita Pozzi lavora sotto il suo progetto di restauro, la chiesa di Sant'Ambrogio a Chironico, e la chiesa di San Nicolao a Giornico dove dopo la realizzazione delle analisi preliminari, delle ricerche archeologiche e delle proposte d'intervento, deve assentarsi per motivi di salute lasciando l'incarico ancora una volta al suo collega di Massagno. Altri

\footnotetext{
biografie d'artisti, artigiani d'arte, scenografi, tipografi e fotografi.

265 Museo Caccia. Con questa definizione Emilio Ferrazzini fa riferimento al Museo civico di belle arti di Lugano, la cui fondazione si deve allo scrittore e musicista Antonio Caccia (Trieste, 1829 - Lugano, 1893), patrizio di Morcote, il quale in 1893 dona la Villa Malpensata (attualmente sede del Museo di Arte Contemporanea) e tutte le opere trovatesi al suo interno, con lo scopo d'incentivare il Municipio per la formazione d'un Museo di Belle Arti. La sede del Museo si trova dal 1993 in Villa Ciani.

${ }^{266}$ (Ferrazzini, Un dipinto di G. Serodine. Nota d'arte, 1945, p. 10).
} 
collaboratori con cui il Ferrazzini si associava $\mathrm{o}$, più tardi, a chi coadiuvava come membro della Commissione Cantonale furono ad esempio i pittori Carlo Cotti, Nino Facchinetti, Tito Tettamanti, Ottorino Olgiati e Mario Moglia.

Gli ideali di restauro di Emilio Ferrazzini furono molto lontani dalle teorie puriste che in Italia aveva propugnato il teorico Giovani Battista Cavalcasselle, per cui è facile trovare negli interventi da lui condotti segni di puliture poco curate ed abrasive, abbondanti ridipitture 0 reintegrazioni illusionistiche, stuccature che sorpassano il livello dell'intonaco originale o che colmano gli incavi delle martellinatura eseguiti in passati rinnovamenti, saldatura dei bordi in cemento, e tecniche di consolidamento che cambiano esteticamente la tonalità dell'affresco. Emilio Ferrazzini ebbe enormi difficoltà ad imporre i suoi concetti di restauro, che riteneva i più idonei per la salvezza dei beni artistici, in lotta continua "di fronte a coloro che propugnano la "intoccabilità" degli affreschi":

[...]lo so per esperienza -spiegava- (ed il defunto pittore Berta ebbe a questo riguardo a sopportare aspre critiche, non sempre fondate) che per molti "toccare un affresco è quasi un delitto o, perlomeno un "vandalismo". [... $]^{267}$

Se nel sopracitato caso, esemplificato dal Ferrazzini, gli attacchi all'Ispettore Edoardo Berta arrivarono nel 1922 quando il restauratore Federico Bentz di Basilea intervenne sulla tavola del XVII sec. del

\footnotetext{
267 (Ferrazzini Emilio, Lettera indirizzata a Francesco Chiesa, presidente della Commissione Cantonale de Monumenti Storici in copia al Dipartimento della pubblica educazione. Lugano, 30 aprile 1940. AUBCBZ, s.240 Prugiasco).
}

Bramantino "la Fuga di Egitto", della chiesa della Madonna del Sasso, riportando l'opera alle sue forme originarie, e cioè, cancellando una delle aggiunte realizzate in precedenti restauri ${ }^{268}$, la causa principale di polemica nel lavoro di Emilio Ferrazzini fu l'uso delle cere come consolidanti e "ravvivatori" dei colori degli affreschi, in conflitto con le prescrizioni di Piero Bianconi, Ugo Donati e don Agostino Robertini membri dalla Commissione dei Monumenti.

Si tratta di una tecnica che era già stata ampiamente contestata e giudicata in Italia nella seconda metà del secolo XIX e che, malgrado i numerosi diffidenti, si stesse come metodo di finitura dei restauri durante un lungo periodo di tempo. Alessandro Conti ci lo spiega così:

[...] L'abitudine di ripassare con cera le superficie affrescate è molto antica, forse, si è trasmessa dall'antichità per tradizione diretta ed è rimasta in vita fino a ieri nell'esecuzione delle decorazioni a finti marmi. È stata usata per la manutenzione di affreschi che rivelassero di

\footnotetext{
68 Edoardo Berta aprì una querela contro il frate Leono Brughelli, Guardiano dei P.P. Cappuccini alla Madonna del Sasso e redattore del "Messaggero Serafico", per aver considerato di vandalico e mutilatore l'intervento di restauro della tavole del Bramantino alla chiesa della Madonna del Sasso in Orselina, per il quale furono tolti della tavole di pezzi aggiunti recentemente all'opera. La frase sul giornale del frate recava: [...] La diminuzione di esso quadro, e particolarmente la sua decapitazione fu un vero $e$ proprio vandalismo compiuto in nome dell'arte, era più che sufficiente ad esporre a Berta allo sprezzo del pubblico[...] secondo le notizie di archivio. Gli "Artisti Ticinesi" protestarono al signor Leone contro le parole di critica a Berta: fra essi gli scultori Foglia e Bernasconi ed i pittori Sartori, Maccagni, Soldati e Balmelli. (CCMS, Lettera indirizzata a Paolo Ganz 1922 post. (ASTi Fondo DPE, Fascicolo XXVIII cartella 1. Monumenti Storici $(1848 \ldots)$.
} 
aver avuto in origine la superficie così trattata $[. . .]^{269}$

Dall'ottimista sperimentazione sulle pitture pompeiane, la tecnica verrà utilizzata da restauratori come Daniele da Volterra sulla Deposizione del Duomo di Pisa o da Guglielmo Botti sui dipinti di Benozzo Gozzoli (ved. cap. 1, p. 20). G. Botti, nel 1857, accetterà inoltre l'alterazione estetica della tecnica a fresco occasionata dal trattamento ad encausto, con cera punica $^{270}$, anche se il manufatto finirà assomigliandosi ad una pittura ad olio, a cambio di permettere il consolidamento delle numerosissime finiture a secco originarie del dipinto. Secondo lo storico A. Conti, Botti criticherà infine i colleghi restauratori che rifiutano l'uso di questo prodotto e preferiscono il "latte scremato" o caseina $^{271}$.

Eppure l'uso dei metodi d'intervento che trasformano la fisionomia e le caratteristiche dell'opera, promossi dalla scuola e dalla tradizione di Giovanni SeccoSuardo, terminerà in Italia con la tutela di G. B. Cavalcaselle nella diffusione del [...]restauro che consolida senza ricorrere più al

\footnotetext{
${ }^{269}$ (Conti, 2005, p. 247).

${ }^{270}$ Cera punica, è il nome dato da Vitruvio alla cera d'api fatta bollire in una miscela d'acqua e carbonato sodico. L'origine della tecnica all'encausto venne attribuito ai dipinti romani su tavola da Al Fayum, sec I - IV d. C. (Conti, 2005, p. 291).

${ }^{271}$ Caseina lactica, prodotto organico naturale estratto dal latte, solubile negli alcali e nei sali solubili di basso $\mathrm{pH}$ e insolubile in acqua. Per farla solubile venne addizionata con ammoniaca, formando caseinato d'ammonio, o con calce, caseinato di calcio). Si tratta di un ottimo legante naturale e generalmente viene impiegata nella realizzazione di stucchi per affreschi, tele ed altre preparazione di colori alla caseina. Si presenta in commercio in polvere di colore bianco e crema. Ha un pH di 4,5, umidità dell' $11 \%$ circa ed una granulometria: $60-400 \mu$.
}

pennello, le lacune in vista, la rinuncia agli inganni del restauro amatoriale $[. . .]^{272}$, agli inizi del secolo XX.

In Ticino invece, le prime notizie dell'uso della cera le abbiamo documentate nel 1927, nei rapporti di Edoardo Berta sul restauro svolto all'interno della Cappella Camuzio alla chiesa di Santa Maria degli Angioli a Lugano, analizzato precedentemente.

Emilio Ferrazzini ne parlava a riguardo alla fine della sua carriera, in una lettera datata in 4 agosto del 1971, indirizzata al direttore dell'Ufficio dei Monumenti Storici, Pierangelo Donati. Dopo anni di litigio e di contestazioni da parte dei colleghi della Commissione Cantonale e degli esperti (Mauro Pellicioli, Jean Verrier, Ambrogio Annoni), il pittore luganese riconosceva ancora nella tecnica di consolidamento al "encausto" il metodo più adatto agli affreschi o dipinti murali. Per di più, nella medesima fonte, il restauratore giudicherà la mancanza di testimonianze scritte sui lavori di restauro eseguiti nel Canton Ticino dal 1909 al 1959 ca. poiché la stessa Commissione Cantonale dei Monumenti Storici, come da lui provato durante gli anni in cui fu membro di essa, non esige mai delle informazioni dettagliate che descrivano i metodi e i materiali di ripristino dell'opera artistica:

[...]alla mia lettera del 10 giugno u.s. Le avevo accluso fotocopia di un mio scritto del 21 settembre 1962 diretto alla C. M. S., nel quale trattavo [...] la questione della tecnica dell'encausto nel restauro degli affreschi. Una tecnica da noi sconosciuta, ma importante per la conservazione ed il restauro degli affreschi.

\footnotetext{
${ }^{272}$ (Conti, 2005, p. 247).
} 
Un giorno che Lei potrà disporre di una mezz'ora di tempo qui a Lugano, Le spiegherò perché si adoperò quella tecnica per il restauro nella cappella Camuzio (attribuiti al Bramantino) nella chiesa degli Angioli.

Dato che nei 14 anni della mia appartenenza alla C.M.S. non si volle mai registrare, annotare, le tecniche dei lavori di restauri pittorici eseguiti, sono certo che nessuno oggi più si ricorderà la tecnica usata nel 1928-1930 per il restauro degli affreschi nella cappella Camuzio degli Angioli.

Eppure è una cosa importante quella di annotare con precisione la tecnica usata per un certo restauro pittorico. E se potremo trovarci un momento alla chiesa degli Angioli, Le spiegherò perché oggi quegli affreschi sono appannati, e cosa bisognerebbe fare per renderli freschi e ben leggibili anche nei dettagli[...]. ${ }^{273}$

Il metodo di consolidamento con materiale ceroso non fu coscientemente giudicato in Canton Ticino fino agli ultimi degli anni Quaranta quando la concorrenza fra i restauratori si fece maggiore e più convinti i commissari delle nocive conseguenze avvenute da certi restauri, contemporaneamente ai nuovi eventi avvenuti in Italia con la creazione dell'Istituto del Restauro a Roma e la diffusione internazionale delle teorie brandiane che nel Canton Ticino non si applicheranno fino agli inizi degli anni Cinquanta.

Tuttavia l'analisi sulle controversie generate, non oserò qui sancire la qualità degli interventi eseguiti da Ferrazzini, poiché ritengo sarebbe troppo facile dalla attuale prospettiva, ma evidenzierò a continuazione tutto ciò che sarebbe andato perso se non fosse stato grazie alle ricerche

273 (Ferrazzini Emilio. Lettera indirizzata a Pierangelo Donati, Lugano 4 agosto 1971. AUBCBZ, s.143, Lugano). del pittore, incessanti, alla sperimentazione sui materiali, alla pressione generata attraverso gli articoli d'opinione ed all'emissione di numerose lettere oggi conservate all'Archivio dell'Ufficio dei Beni Culturali che riflettono questa sua tenacità.

\section{S. Maria di Loreto, Lugano}

La chiesa cinquecentesca di S. Maria di Loreto a Lugano, significò per Emilio Ferrazzini, agli inizi della sua attività come restauratore, motivo per proclamarsi di fronte alla Commissione Cantonale del Ticino. Troviamo ancora una volta un chiaro esempio della sua grande perseveranza critica, indifferente alle possibile inimicizie, con l'obiettivo di stimolare l'attenzione delle autorità pubbliche sui soggetti suscettibili ad essere conservati, o meglio, salvati. Così, le sue lettere avranno come primo destinatario il presidente della Commissione Cantonale dei Monumenti Storici, Francesco Chiesa, per poi raggiungere il Commissario Federale dei Monumenti nel caso in cui il primo ignorasse le sue petizioni.

$\mathrm{Fu}$ il 31 marzo 1928 quando Ferrazzini mandò il primo avvertimento sull'intenzione di procedere con il restauro degli affreschi della chiesa di Santa Maria di Loreto di Lugano e, contemporaneamente, dei dipinti esistenti nella casa del Signore Brivio in Via Nassa.

Si trattava, nel primo monumento, dei dipinti esterni corrispondenti al piano superiore del portico a cinque campate, raffiguranti scene della Passione di Cristo. Questi avevano subito diversi degradi dall'ultima perizia realizzata dall'architetto 
Augusto Guidini in $1907^{274}$, dove si elencavano le condizioni di stato conservativo favorevole dei dipinti, tanto esterni come interni, malgrado i diversi lavori avvenuti nella chiesa.

La Città di Lugano chiese alla CCMS ed alla Commissione del Museo Storico la loro opinione riguardo, prima di adottare una decisione, con una lettera emessa il 3 aprile del 1928 alle citate autorità. II 12 aprile la domanda viene contestata da Francesco Chiesa, che raccomanda allo Stato di accettare la realizzazione dei lavori di consolidamento e di restauro proposti dal pittore Ferrazzini [...] con l'obiettivo d'impedire il veloce degrado e non lontana scomparsa degli affreschi in Via Nassa nella casa di Brivio e nella chiesa di Santa Maria di Loreto[...].

Il preventivo fu elaborato da Ferrazzini e dal pittore Carlo Cotti nel 1928 ma i lavori non potettero realizzarsi subito.

Nel frattempo invece furono eseguiti altri lavori: il 23 Novembre $1934^{275}$ la Veneranda Cappellania Vescovile di Loreto $^{276}$, dell'omonima chiesa, chiede alla Commissione dei Monumenti Storici l'autorizzazione per l'esecuzione di una nuova cappella nella parete sinistra della chiesa il che significava, secondo il progetto dell'architetto Giacomo Alberti, lo strappo dell'affresco esistente nell'altare laterale

\footnotetext{
274 (Guidini Agusto. Lettera indirizzata ad Albert Naef 12 giugno 1907. AFMS, BNS, Bern K Tessin Bellinzona n.82).

275 (Veneranda Cappellania Vescovile di Loreto. Lettera indirizzata alla Commissione dei monumenti storici, Lugano 23 novembre 1934. AUBCBZ, s.143, Lugano).

276 Presiedeva in quel momento il M. Rev. Padre Materno Redertorff.
}

per essere conseguentemente riportato sulla nuova cappella.

Il progetto venne approvato dal Consiglio di Stato il 4 dicembre 1934 dando come unica condizione [...]che il trasporto dell'affresco, sia sopra tele, sia con rimozione dell'intonaco - cioè, stacco - venga eseguita da persona di riconosciuta competenza e sotto la responsabilità della Ven.da Cappellania[.... $]^{277}$.

Purtroppo nei cartacei dell'archivio dei beni culturali a Bellinzona ed a Berna non si è trovata maggiore informazione riguardo l'esecuzione dello strappo.

Nel gennaio del 1935 sorgerà il problema della riparazione del tetto, che eseguirà l'architetto Oscar Jauch di Lugano insieme alla rinnovazione della parete posteriore esterna del sotto-portico.

I lavori di tinteggiatura furono ispezionati da Emilio Ferrazzini come membro ora della CCMS mentre il restauro del campanile seicentesco fu realizzato dal collega Carlo Cotti, come spiega lo stesso restauratore:

[...]stando in casa con influenza mi sento con il dovere d'avvertirle che i lavori di restauro del campanario di Loreto stanno essendo eseguiti dal pittore Cotti che è stato con me lì settimana scorsa per le dovute spiegazioni. ${ }^{278}$

Finalmente nel 1938 i Frati Minori di Loreto richiedono il sussidio per l'esecuzione del restauro degli affreschi esterni della facciata ${ }^{279}$, che gli verrà concesso con un

\footnotetext{
7 (Risoluzione dal Consiglio di Stato della Repubblica e Cantone del Ticino, Bellinzona 4 dicembre 1934. AUBCBZ, s.143, Lugano).

${ }^{278}$ (Ferrazzini Emilio. Lettera indirizzata a Francesco Chiesa. Lugano, 6 de marzo de 1935. AUBCBZ, s.143, Lugano).

${ }^{279}$ (Padre Materno Rederstorff, Lettera indirizzata alla CCMS. Lugano 17 giugno 1938. AUBCBZ, s.143, Lugano).
} 
$5 \%$ del prezzo totale dal preventivo di Ferrazzini. Questo comprendeva il consolidamento degli affreschi esistenti nelle tre facciate della chiesa, delle cornici decorative, delle finestre ed il restauro dei fondi e dei brugnati negli angoli e negli archi, per un totale di Fr. 3600. I lavori furono finiti nel mese di giugno del $1939^{280}$.

[...] Se io non avessi insistito ed ottenuto che si fissassero e restaurassero gli affreschi delle facciate della chiesa a quest'ora credo che ben poco sarebbe rimasto e non più restaurabile! La commissione cantonale non faceva nulla di nulla $[\ldots]^{281}$.

Anni dopo, esattamente il 16 novembre 1941 , il restauratore presenta alla Commissione Cantonale dei Monumenti, di cui formava parte, un secondo progetto di restauro, in questo caso riguardante la Cappella di Sant'Antonio datata alla seconda metà del Seicento con decorazioni a stucco, e la Cappella delle Grazie dove si trova un affresco della fine del XV sec.

$\mathrm{Si}$ sollecitava l'esecuzione delle seguenti operazioni:

[...]a) apertura di un lucernario al disopra del corridoio situato superiormente e longitudinalmente sopra il porticato estero della chiesa. Detto lucernario, in parte apribile, permetterà [...] l'ariazione dell'interno della chiesa e della cappella della Madonna [...] attualmente quasi nulla e che ha provocato cosi evidenti danni alle pareti della chiesa. [...] e

\footnotetext{
${ }^{280}$ (Frate Colombano Fischerofur. Lettera indirizzata al Consiglio di Stato del Canton Ticino. Lugano 26 giugno 1939. AUBCBZ, s.143, Lugano).

281 (Ferrazzini Emilio. Lettera indirizzata a Aldo Crivelli, Lugano 10 ottobre 1959. AUBCBZ, s.143, Lugano)
}

l'illuminazione delle tre finestre di fondo della chiesa, ora completamente prive di luce $[. . .]^{282 .}$

Nella Cappella di Sant'Antonio s'intendeva rifare gli intonaci degradati dall'umidità, restaurare il pavimento in mattonelle, così come il restauro degli stucchi con successiva tinteggiatura a tempera e l'esecuzione di nuovi affreschi raffiguranti scene della vita del santo venerato, secondo le bozze presentate alla Commissione, disposti nei piccoli riquadri. Mentre nella Cappella della Grazie, detta della Madonna, l'intervento interessava, secondo la proposta del restauratore, la completa rinnovazione del pavimento in mattonelle di terracotta in sostituzione delle piastrelle in cemento ed il recupero delle decorazioni originarie delle pareti ad imitazione dei mattoni ancora presenti sotto lo scialbo.

La proposta approvata il 20 novembre 1941 dall'architetto Otto Maraini si completa il 12 dicembre dello stesso anno a causa di nuove necessità, includendosi al primo preventivo la pulitura degli affreschi dell'altare maggiore. Questi ultimi, attribuiti a Giulio Quaglio, raffigurano I'Assunta, i SS. Lorenzo e Stefano e l'Annunciazione, e sono datati del 1729 circa.

L'esecuzione dei lavori venne tuttavia ritardata a causa delle controversie esistenti fra la finanziatrice del restauro e Ferrazzini. Per diminuire gli inconvenienti il Dipartimento della Pubblica Educazione del Canton Ticino sceglie di intervenire stendendo una serie di clausole che la

\footnotetext{
282 (Ferrazzini Emilio, Proposta di restauro indirizzata a Francesco Chiesa, presidente della Commissione cantonale dei monumenti storici. Lugano 16 novembre 1941. AUBCBZ, s.143, Lugano).
} 
Curia Vescovile fece firmare alla signora. Su queste condizioni si accordava che la donatrice non aveva il diritto ad esigere nuovi cambiamenti sul progetto che tempo prima aveva autorizzato, e in questo modo, l'unica persona con le capacità e la responsabilità di richiedere delle ulteriori modifiche era il direttore dei lavori, in questo caso l'ispettore architetto Otto Maraini.

Nonostante tutto, pochi anni dopo si dovranno realizzare nuove operazioni sui dipinti. Nel 1948 venne assegnato il lavoro di restauro al pittore Mario Moglia il quale pulisce, consolida e reintegra le figure scoprendo i dipinti dallo strato di calce che li ricopriva con una pulitura abbastanza rispettosa $^{283}$, ed osserva i problemi dall'umidità di risalita causante delle lacune sulla superficie e delle fessure murarie, che furono colmate del restauratore con uno strato fino d'intonaco [...]granulometricamente troppo grosso rispetto all'originale così da rendere troppo visibile il ritocco $[\ldots]^{284}$.

Nel 1949, secondo il progetto dell'architetto Cino Chiesa, si demolisce e ricostruisce in modo identico il portale cadente sul ponente della chiesa di Loreto, approvato dal membro architetto Pietro Giovannini $^{285}$.

283 (Alberti Pierluigi, Loreto, Chiesa e Convento di Santa Maria. Conservazione e restauro dei dipinti sull'arco di trionfo e del dipinto nella nicchia sulla parete sinistra della navata sec. XVI. Relazione finale. Caslano, agosto 1999. AUBCBZ 134, Lugano).

${ }^{284}$ (Alberti Pierluigi, Loreto, Chiesa e Convento di Santa Maria. Conservazione e restauro dei dipinti sull'arco di trionfo e del dipinto nella nicchia sulla parete sinistra della navata sec. XVI. Relazione finale. Caslano, agosto 1999. AUBCBZ s.134, Lugano).

${ }^{285}$ (DPE. Risoluzione del 17 maggio 1949 indirizzata alla CCMS. AUBCBZ s.134, Lugano).
Ferrazzini opererà ancora per l'ultima volta in Santa Maria di Loreto alla fine di 1959, chiamato dai proprietari, per esaminare le condizioni di un affresco cinquecentesco situato nella cappella del 1524 incorporata dentro la chiesa. Temendo che la richiesta da egli inoltrata o da parte dei proprietari fosse rifiutata dalla Commissione [...]conosco troppo bene quei sistema per farmi delle illusioni![... $]^{286}$ - il pittore sollecita al professore Aldo Crivelli in una lettera emessa il 25 settembre 1959 ad essere lui a mettersi direttamente in contatto con le autorità, dopo previa ispezione della situazione che gli descrive:

[...]Soltanto sul posto ti farai una idea esatta della cosa. Perché assieme al restauro dell'affresco vorrei anche poter far rimettere in ordine il terreno davanti alla cappella (che si trova sul piazzale, di fronte alla chiesa) e cioè ritornarli come era, dopo i restauri da me compiuti circa 25 anni fa. Il terreno è stato abusivamente rimosso, la terra addossata alla costruzione (i basamenti delle colonne, di marmo d'Arzo) sono stati a metà interrati! L'umidità invade ora i muri circostanti, l'altare, la cappella e fa marcire tutto. Come si sia permesso questo non so: è il solito abbandono e disordine... Bisogna provvedere. $[. . .]^{287}$.

Per una maggiore informazione Ferrazzini invia ancora al professor Crivelli una lettera in data 10 ottobre dello stesso anno nella quale si succedono delle denuncie e delle critiche alle diverse autorità, caratterizzate per il tono ironico e duro del pittore; in primo luogo esprime la sua impotenza di

\footnotetext{
${ }^{286}$ (Ferrazzini Emilio. Lettera indirizzata al Prof. Aldo Crivelli. Lugano 25 settembre 1959. AUBCBZ s.134, Lugano).

${ }^{287}$ (Ferrazzini Emilio. Lettera indirizzata al Prof. Aldo Crivelli. Lugano 25 settembre 1959. AUBCBZ s.134, Lugano).
} 
fronte alle contestazione che ne erano state pronunciate alla Pro-Loreto in favore al restauro dell'affresco e richiama l'attenzione sui responsabili del degrado di questo come di tanti altri dipinti:

[...]i commissari governativi e competente possono distruggere affreschi antichi e preziosi per la storia del monumento e per il Paese (sala Orelli, chiesa di S. Antonio a Lugano, chiesa di Villa Coldrerio quattro affreschi e decorazioni a Comologno, ecc.) e sono retribuiti, onorati e sempre impuniti... Ma per chi vuol salvare dalla distruzione importanti affreschi c'è la loro prepotenza, il vile ostruzionismo, come nel caso di Preonzo, Loggia del comune a Carona, e quasi tutti gli affreschi da me elencati una quindicina di anni fa (lista che mi era stata richiesta) e che erano URGENTEMENTE DA SALVARE!![...] $]^{288 .}$.

L'Ispettorato dei monumenti storici artistici di Bellinzona ${ }^{289}$ assume gli interventi proposti da Ferrazzini ma esprime chiaramente il suo parere riguardo il modo in cui dovranno essere eseguite le operazioni di restauro. In particolare esprimono - il degrado percepito sull'affresco cinquecentesco non richiede un [...]rifacimento, intollerabile falso antico, delle parti mancanti, bensì una intonazione cromatica generale che lasci intatte le parti originali ancora conservate e con queste accordandosi $[. . .]^{290}$. Queste indicazioni evidenziano la concretizzazione delle teorie del restauro definite da Cesare Brandi nel 1948 e finalmente validate e assunte dalle

\footnotetext{
288 (Ferrazzini Emilio. Lettera indirizzata al Prof. Aldo Crivelli in Lugano 10 Ottobre 1959. AUBCBZ 134, Lugano).

${ }^{289}$ (IMSA. Lettera indirizzata alla Municipalità della Città di Lugano. Bellinzona, 14 ottobre 1959. AUBCBZ 134, Lugano).

290 (IMSA. Lettera indirizzata alla Municipalità della Città di Lugano. Bellinzona, 14 ottobre 1959. AUBCBZ 134, Lugano).
}

istituzioni Ticinesi, leggendosi concetti chiari come "falso antico". Questo corrisponde ai termini "falso storico" o "falso artistico" citati da Brandi, ai quali si dovrebbe rinunciare nel tentativo di riportare l'opera ad uno stato unitario. L'Ispettorato non ignorava quali erano le prassi di lavoro di Ferrazzini e dei restauri coetanei, e perciò decise di specificare le nozioni attuali da rispettare.

Nel 1971 si ritrovano nuovamente dei deterioramenti sulla cappella di Loreto causati principalmente dall'azione dell'umidità. Le parti più degradate sono l'affresco centrale raffigurante la Madonna ed i due oranti, con sfaldatura del colore nella parte centrale ed abbondanti lacune nella parte inferiore, così come la lunetta con la figura del Padre Eterno con decoesione della materia pittorica.

$\mathrm{Si}$ propongono i restauratori Adriano Bocchi e Cavallini per l'esecuzione del restauro, sorvegliati dall'Ufficio della Commissione dei Monumenti Storici nella persona di Giuseppe Martinola ${ }^{291}$.

Ferrazzini che era in contatto con il Padre Michele nella chiesa di Loreto, non perse le forze e la passione all'età di settantasei anni per interessarsi al lavoro, chiedendo autorizzazione al capo dell'allora Ufficio Cantonale dei Monumenti Storici l'archeologo Pierangelo Donati ${ }^{292}$ ad

\footnotetext{
291 (Martinola Giuseppe, CCMS. Lettera indirizzata al Municipio di Lugano. Lugano, 7 gennaio 1971. AUBCBZ 134, Lugano).

292 Donati Pierangelo (1937-1993). Archeologo la cui carriera all'interno dell'Ufficio Cantonale dei Monumenti Storici in Ticino cominciò in 1967 in qualità di archeologo cantonale come ispettore agli scavi ed ai Musei archeologici, fu dal 1969 per venticinque anni direttore della medesima istituzione promovendo gli scavi archeologici in una campagna di esplorazione simile a la realizzata da Edoardo Berta
} 
operare $^{293}$ nell'organizzazione dei lavori preparatori. Questi preliminari supponevano il ritrovamento delle parti originali che conformavano i dipinti della cappella con l'applicazione sul dipinto di una mano di "encausto moderno a fuoco". Con questo metodo di consolidamento la pittura autentica veniva differenziata dalle ridipitture e si poteva, a quel punto, scegliere il trattamento di restauro.

[...]E nello stesso tempo vorrei eseguire un campione d'encausto (di circa 50 cent. d'altezza) su una delle due colonne di marmo d'Arzo, che fiancheggiano I'affresco. Quel marmo delicato, esposto a nord, (come l'affresco), ha perso i toni ed $i$ colori, ed è in via di rapido disfacimento. Per ridare il colore primitivo alle due colonne di marmo non c'è nulla di migliore che quello di applicare l'encausto moderno.[... $]^{294}$.

\section{Chiesa Parrocchiale di S. Martino, Malvaglia}

Trovata in uno stato di totale rovina, la chiesa di San Martino ${ }^{295}$ a Malvaglia era stata oggetto di restauro nel 1910 per iniziativa del popolo. II progetto, realizzato dall'Ingegnere Baggio, consisteva principalmente nella liberazione dei problemi di umidità che minacciavano la conservazione dell'edificio.

agli inizi del XX sec., che coinvolge circa sessanta edifici di culto identificandosi più di trenta in stile romanico e diciannove di origine altomedievale.

${ }^{293}$ (Ferrazzini Emilio. Lettera indirizzata a Pierangelo Donati, Lugano 4 agosto 1971. AUBCBZ 134, Lugano)

${ }^{294}$ (Ferrazzini Emilio. Lettera indirizzata a Pierangelo Donati, Lugano 4 agosto 1971. AUBCBZ s.134, Lugano)

${ }^{295}$ La chiesa era attestata con il titolo di S. Benedetto dal 1207 al 1289, anno in cui riceve l'attuale nominativo. (Simona Martinoli, 2007, p. 83).

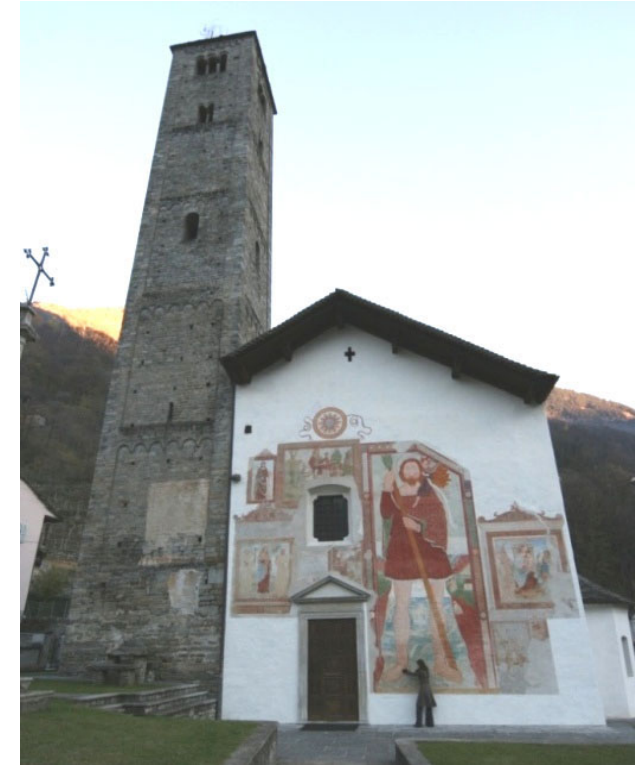

Figura 74 Giner Cordero, E, Facciata delle chiesa di San Martino. 2007.

Nello stesso periodo, dal 1912 al 1913, Edoardo Berta dirige le ricerche archeologiche mediante gli scavi interni che permettessero di avere una maggiore conoscenza del bene artistico. Durante queste operazioni si rivelano l'originaria costruzione della fabbrica a stile romanico a doppia navata orientata, descritta negli atti di San Carlo nel 1567, così come notevoli affreschi scoperti sotto lo strato di scialbo di calce.

I lavori interrotti per mancanza di finanziamento e per cause belliche si ripresero in un secondo periodo dal 1931 al 1934 grazie al Comitato "Pro Restauri", questa volta a cura dell'architetto Cino Chiesa e per incarico della Commissione Cantonale dei monumenti storici del Ticino. In questo momento si concludono i restauri pittorici eseguiti dai pittori Emilio 
Ferrazzini, Carlo Cotti, Tita Pozzi e Tito Tettamanti con un preventivo di fr. 4'500. II restauro interessò in concreto la pulitura ed il consolidamento degli affreschi sulla facciata del XVI sec. raffiguranti $S$. Cristoforo, la Madonna fra i SS. Francesco d'Assisi e Bernardino da Siena ed i SS. Barbara, Martino e Gerolamo ed all'interno le tracce di affreschi sulla vita di Cristo nella parete sinistra della navata del XVI sec. attribuiti al pittore Antonio da Tradate, il Cenacolo sulla parete opposta firmato dal pittore Cristoforo, il San Martino a cavallo e S. Bernardino da Siena con committente sulla parete del campanile, gli affreschi seicenteschi di S. Biagio, S. Stefano e la Madonna in Trono, gli affreschi sull'arco trionfale di Bernardino Serodine e la cappella della Madonna con gli affreschi dei Misteri del Rosario.

Fu esattamente il 13 luglio 1932, durante i sopraccitati restauri quando il pittore Ferrazzini realizza la prima prova di consolidamento con la tecnica ad encausto speciale, cioè con la cera minerale ozocerite, documentata qui per la prima volta in Ticino. L'obiettivo del restauratore con questa verifica era dimostrare le proprietà del prodotto che lui riteneva idoneo per la protezione della superficie senza produrre alterazione della stessa. Evidentemente questo non era stato ancora corroborato dal restauratore a più lungo tempo e ne parlava unicamente dalla conoscenza e dall'esperienza appressa dagli altri restauratori, probabilmente Edoardo Berta fra essi, o dalle tecniche impiegate a Roma durante il suo soggiorno come studente dell'Accademia di Belle Arti. L'Architetto Otto Maraini in qualità di membro della CCMS fu presente durante le prove del prodotto. Egli espose un parere favorevole sul metodo che lo stesso anno fu usata sull'affresco tardogotico frammentato raffigurante San Cristoforo nella parte destra della facciata della chiesa parrocchiale di Santo Stefano in Tesserete $^{296}$.

Ferrazzini avvertiva sulla difficoltà della tecnica, e sulla necessità di conoscere i materiali adatti ad ogni tipo di dipinto.

[...]Questa tecnica non è così semplice $e$ specialmente per ciò che concerne la scelta opportuna dei materiali, la loro purezza originaria, la loro stabilità; infine l'esatta conoscenza del loro uso a secondo delle loro proprietà particolari, e ciò affinché non abbiano a succedere poi inconveniente ed affinché il restauro sia duraturo[... $]^{297}$.

La cera ozocerite è una miscela di paraffina e idrocarburi ciclo-paraffinici trovata in stato naturale. Conosciuta anche con il nome di ceresina quando purificata, come lo stesso Ferrazzini la denominerà in altri documenti. La differenza fra ambedue definizioni è il loro punto di fusione, essendo maggiore quello della cera ozokerite che fonde fra i 140 ed i 200 gradi Fahrenheit mentre la ceresina lo fa fra i 130 ed i 160 F. Entrambe sono solubili nell'etere, nel petrolio, nella benzina, nel cloroformio, nel disolfuro di carbonio e simili agenti chimici, e l'ozokerite è stata usata inoltre, dal momento della sua industrializzazione, per la cura del corpo come lubrificante, in creme, pomate,

\footnotetext{
${ }^{296}$ La chiesa fu oggetto di restauro completo in 1935. ${ }^{297}$ (Ferrazzini Emilio. Lettera indirizzata alla CCMSA. Riguardo la seduta della Commissione il 21 luglio in cui il pittore spiega diffusamente e dettagliatamente la questione tecnico artistica del restauro con la tecnica all'encausto. Lugano 30 aprile 1940. AUBCBZ, s.149 Malvaglia).
} 
unguenti, nell'industria farmaceutica, cosmetica e in prodotti idrorepellenti, adesivi e vernici.

Come osserveremmo nei casi qui studiati il restauratore tentò sempre di creare una differenziazione nell'intervento fra le opere situate all'esterno, cioè esposti agli agenti atmosferici di degrado, e quelle interne. In questo modo Ferrazzini insisterà sull'impiego della cera "ozocerite" o "ozokerite" nei dipinti murali all'esterno come nei casi precedentemente citati o nella facciata della chiesa di San Biagio a Ravecchia, mentre la tradizionale miscela di paraffina e cera d'api è maggiormente utilizzata sui dipinti interni, come nei casi dei dipinti dell'Oratorio di San Martino di Deggio la chiesa dei SS. Ambrogio e Maurizio a Chironico e nella chiesa parrocchiale di Russo.

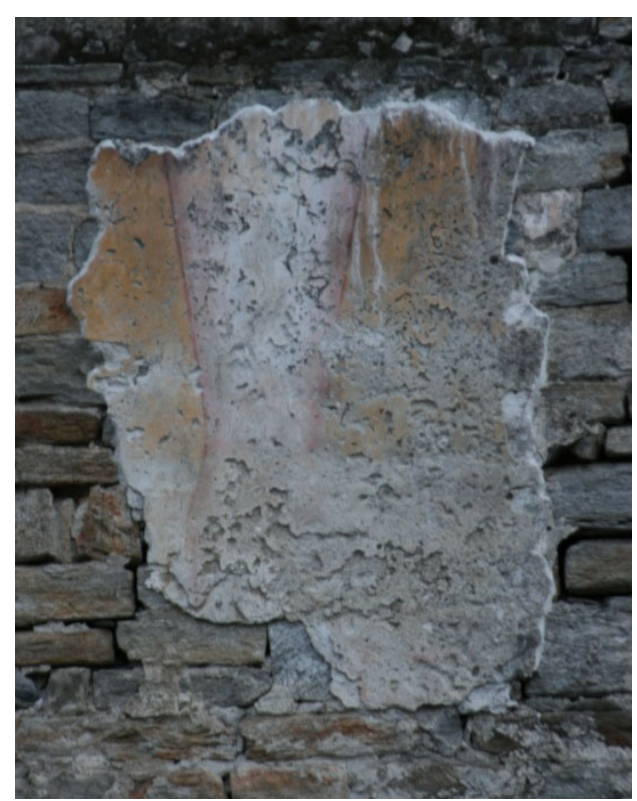

Figura 75 Giner E. Fragmento di affresco nella facciata principale della chiesa di S. Martino, Malvaglia. 2008.

\section{Oratorio di S. Martino, Quinto}

Una unica frase sopra la porta d'entrata all'Oratorio medievale di San Martino di Deggio (Quinto), attesta i lavori di restauro eseguiti fra il 1935 e il 1939. L'edificio eretto intorno al IX sec. come testimoniano oggigiorno le arcate cieche carolingie, è composto da un campanile a vela a sudovest di epoca posteriore. La fabbrica ad una sola navata è unita ad un coro quadrato, alzato su un gradino, con volta a botte. L'edificio è decorato con affreschi, tanto all'interno come all'esterno, di epoca quattrocentesca che non furono messi alla luce fino all'anno 1935 con il suddetto intervento eseguito dal pittorerestauratore Emilio Ferrazzini. I dipinti del XV sec. raffigurano, sulla parte esterna del portale sud, un San Cristoforo, ed internamente l'Annunciazione e due santi sull'arco trionfale, il Cristo con i simboli degli evangelisti e la Crocifissione nel coro, un San Martino che divide il mantello con il povero sulla parete sud della navata ed il Cenacolo con dipinti votivi sulla parte nord. Sotto gli affreschi del coro si osserva la sovrapposizione di almeno quattro strati diversi d'intonaci più antichi che confermano le origini antichi dell'edificio, su questi si distinguono dei dipinti facilmente identificabili raffiguranti un drago che sta per divorare un uccello ed un friso di pesci, sicuramente romanici.

L'intervento di ristrutturazione architettonica e di restauro pittorico realizzato da Ferrazzini fu eseguito grazie ai sussidi del Consiglio Parrocchiale e del Dipartimento della Pubblica Educazione del Canton Ticino. Il lavoro di restauro pittorico in particolare interessò il ritrovamento dei dipinti esistenti nella fabbrica e fu concluso 
il mese di agosto del 1939 e definitivamente approvato il 7 novembre dello stesso anno per il Consiglio di Stato del Canton Ticino, dichiarandosi un restauro condotto [...]a regola d'arte e con esito soddisfacente[...]. ${ }^{298}$ Questa è senza dubbio una interpretazione dei risultati molto diversa a quanto oggi si potrebbe giudicare, poiché l'intervento di Ferrazzini ha occasionato degli importanti danni sull'opera, oggigiorno evidentemente percettibili.

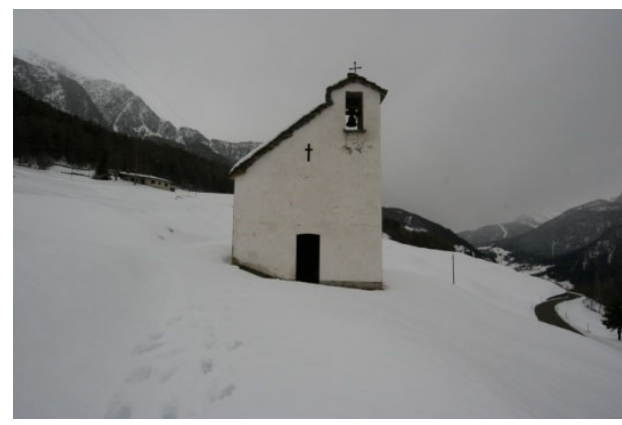

Figura 76 Giner Cordero E. Esterno dell'oratorio di S. Martino, Quinto. 2008.

Come descritto nella relazione di restauro che Emilio Ferrazzini dirige a Francesco Chiesa il 23 settembre 1939, i lavori dal punto di vista architettonico interessarono la sistemazione del tetto dell'edificio, la sostituzione della porta a ponente, nonché la riparazione della porta laterale ed il rinnovamento degli intonaci su tutte le pareti interne ed esterne dell'oratorio, specialmente degradati a causa della forte azione dell'umidità. Inoltre, per combattere questo grave agente

\footnotetext{
298 (Consiglio di Stato del Canton Ticino. Risoluzione del indirizzata alla CCMS. Lugano, 7 novembre 1939. AUBCBZ, s. 206 Quinto).
}

degradante fu creato, in questo stesso momento storico, un intercapedine lungo la parete nord della fabbrica. Attualmente possiamo riconoscere la scarsa efficacia della suddetta soluzione architettonica. Questo si deve principalmente al fatto che in contemporaneità con l'esecuzione dell'intercapedine i rivestimenti delle pareti dell'oratorio vengono completamente rifatti, all'esterno, con un sottofondo in cemento. Si tratta questa di una risoluzione contraddicente poiché, da una parte l'intercapedine venne realizzato con lo scopo di creare uno spazio che consenta l'areazione, fra il terreno ed il muro esterno della chiesa, e di traspirazione della facciata evitando l'umidità di risalita capillare; d'altra parte invece si applica in modo consapevole sul muro la malta cementizia che impermeabilizza il supporto evitando la traspirazione. Mentre all'esterno s'interveniva con i procedimenti descritti qui sopra, all'interno della fabbrica furono cancellate le modifiche realizzate nei secoli passati in modo da ridare l'oratorio di S. Martino le sua caratteristiche originarie di austerità: questo significò ad esempio la sostituzione del pavimento esistente per lastre di pietra, la costruzione d'un soffitto in legno d'abete, la riparazione degli intonaci guasti anche all'interno, nonché l'eliminazione dell'altare in muratura per applicare uno nuovo in granito, cioè lo stesso materiale lapideo che era stato impiegato in passato per la realizzazione del pavimento del coro.

Inoltre furono riaperte le due monofore romaniche originarie che oggigiorno 
illuminano l'abside [...](che il Rahn aveva disegnato dal vero, ancora aperte) $[. . .]^{299}$.

Tutte le operazione sopra elencate non fanno che parte dell'ideologia di restauro architettonico dell'epoca, come verificato nei casi precedenti, motivati dalla necessità di riportare l'opera artistica agli ordini stilistici del momento in cui fu concepita, perché le aggiunte posteriori, per i restauratori ed studiosi del Canton Ticino negli anni Trenta, non formavano ancora parte della storia del monumento ma se non altro "deturpavano" quest'ultimo. Secondo i documenti d'archivio, anche il rivestimento interno delle pareti dell'Oratorio di San Martino fu realizzato con sottofondo in cemento ed intonaco a base di calce. Queste malte però, applicate all'interno, non solo occasionarono l'alterabilità del muro oggi assai degradato dall'umidità, ma limitarono l'esecuzione di ulteriori future ricerche.

II restauro pittorico eseguito direttamente dal pittore Ferrazzini, avvenne principalmente nella scoperta dei dipinti

\footnotetext{
299 II professore Johann Rudolf Rahn visitò la chiesa nell'anno 1887. Questa venne descritta nella pubblicazione "I monumenti artistici del Medio Evo nel Cantone Ticino del 1881 (Rahn, 1881) tradotta all'italiano a Eligio Pometta nel 1899 (Rahn, monumenti artistici del Medio Evo nel Cantone Ticino, 1894). Nel Novecento come nell'attualità i testi ed $i$ disegni del Rahn sono una importantissima testimonianza della storia dei monumenti storici. Essi sono stati utilizzati come riferimento nel confronto sullo stato conservativo dei beni storici artistici in numerosi casi, ed in particolare nell'Oratorio di San Martino a Deggio consentirono di confermare l'esistenza delle due monofore sull'abside nonché della possibile presenza di affreschi sotto lo scialbo di calce nel coro e nella navata, e della buona conservazione per il contrario dell'affresco raffigurante il "S. Martino a cavallo che divide il suo mantello con il mendicante", già in parte ritoccato.
}

Quattrocenteschi descritti all'inizio del capitolo, fino al momento dell'intervento nascosti sotto lo strato d'intonaco e calce.

[...]L'unico affresco esistente prima dei restauri, raffigurante un San Martino era in buono stato di conservazione: solo la cornice che racchiude il dipinto era stata ricoperta e guastata con imbiancature e venne ripristinate $[. . .]^{300}$.

Queste pitture, secondo il preventivo presentato da Ferrazzini l'11 gennaio del 1937, per un totale di Fr. 1'600, furono pulite, consolidate e reintegrate, con delle tecniche che oggi possiamo definire come le meno adatte, al riportarci oggi un bene artistico completamente danneggiato. Di fatto i metodi di restauro impiegati dal pittore non hanno fatto che incrementare l'azione degli agenti di degrado estrinsechi quali ad esempio l'umidità per risalita capillare, per condensazione e per infiltrazione, tutti e tre agenti ritrovati sugli affreschi dell'Oratorio di S. Martino a Deggio.

In primo luogo, l'intervento più dannoso sugli affreschi è stato senza dubbio il rinsaldamento dei bordi degli intonaci con cemento. In paragone al modo in cui furono riparate le murature dell'edificio, Ferrazzini impiegò anche per il consolidamento dell'intonaco il cemento. In questo modo la problematica di conservazione che il pittore ritrovò sui dipinti a causa dall'umidità, si è vista in successione accentuata dai rinsaldamenti che lui stesso realizza, e non solo: dopo alla pulitura possibilmente realizzata con soda caustica $^{301}$ e le sopracitate operazioni di

\footnotetext{
${ }^{300}$ (Ferrazzini, II S. Martino di Deggio, 1939, p. 273).

301 L'utilizzo di soda o potassa caustica per la pulitura degli affreschi potrebbe essere confermata dagli analisi spettrofotometrici realizzati nel 2005 dal
} 
consolidamento, il pittore esegue la così chiamata "tecnica dell'encausto" su ampie zone degli affreschi, com'era inoltre sua prassi di lavoro abituale.

Come nei casi precedenti l'encausto fu realizzato con lo scopo di proteggere, oltre ché omogeneizzare e migliorare la qualità cromatiche della superficie pittorica, sbiadita nel tentativo di rimuovere lo strato di calce soprastante nel processo di pulitura.

Questo metodo fu eseguito con I'impiego di cera paraffina, come confermano gli analisi. (ved. cap.x), stesa ad ampi rettangoli molto concreti.

Non sono state trovate ulteriori informazioni riguardo le operazioni di restauro eseguite che ci aiuti ad spiegare il perché della concretezza nelle zone di consolidamento, distinguibili mediante la semplice analisi autoptica poiché caratterizzate da una forte lucentezza ed spessore. II modo in cui venne steso il sopracitato encausto non è potuto essere confrontato con casi simili; potrebbero pensarsi che Ferrazzini volesse realizzare una cura puntuale sulle zone di maggiore rischio ma forse sarebbe più logico che si essi fossero semplici prove, malgrado sorprenda la ampia superficie in cui fu applicata la cera paraffina ${ }^{302}$.

Dottore Arcangelo Moles. Essi rivelano la presenza di nitrati di sodio e di potassio, che potrebbero supporre l'applicazione in passato delle suddette soluzion caustiche. Altre cause dei nitrati di sodio e potassio potrebbero aggiudicarsi al l'esistenza di detrit organici dagli uccelli che stazionano sul tetto o dall'antico cimitero, scoperto durante i lavori di restauro architettonico nel 1938.

302 Questa teoria è inoltre quella adottata dal restauratore ticinese Andrea Meregalli, direttore della ditta Arte e Tecnica del Restauro ATR a Canobbio (Svizzera). Egli eseguì nel 2005 una perizia sullo stato
Questo fu il caso, almeno dei dipinti della chiesa di San Biagio in Ravecchia, con la differenza che in questi ultimi l'applicazione del prodotto ceroso esegue le forme decorative e figurative, mentre sui dipinti dell'Oratorio di San Martino i rettangoli lucidi di strato ceroso spezzano in due aree ben determinate i personaggi della composizione pittorica.

L'aspetto di degrado dell'opera trattata viene rinforzato da profondi segni d'abrasioni a causa di una violenta pulitura eseguita con l'impiego di mezzi metallici quali la martellina, gli scalpelli ed $i$ raschietti nonché a causa dello scialbo rilasciato sulla superficie pittorica e dagli ampi ritocchi pittorici specialmente invasivi. I lavori di restauro realizzati nella chiesa nel periodo compresso tra il 1935 ed il 1939 furono completati con il tinteggio e la patinatura del soffitto in legno, delle pareti, dei banchi e della nuova porta e si accompagnarono della documentazione fotografica opportuna con un totale di 8 riprese, citate nella relazione di restauro.

\section{[...]Annessi: N.8 fotografie raffiguranti:}

N.1 - 2 - 3 - con vedute interne della chiesa prima dei restauri

N. $4-5$ due vedute interne della chiesa dopo $i$ restauri

N. 6 - 7- 8 vedute interne esterne dopo $i$ restauri $[\ldots]^{303}$.

\footnotetext{
di conservazione della chiesa sulla quale mi sono confrontata.

${ }^{303}$ (Ferrazzini Emilio, Relazione di restauro della chiesa di S. Martino di Deggio (Quinto), indirizzata a Francesco Chiesa. Lugano 23 settembre 1939. AUBCBZ, s.206 Quinto).
} 
Nonostante la grave situazione in cui è arrivato ai giorni nostri l'Oratorio di San Martino a Deggio - situazione che, secondo le circostanze, dovrebbe sicuramente essersi verificata pochi anni dopo i restauri negli anni Quaranta-, non sono state ritrovate in questo caso critiche a riguardo, pur essendo uno dei restauri più controproducenti tra tutti i casi qui analizzati. Questo dimostra che I'Oratorio mancasse di un certo valore all'epoca, ciò che potrebbe inoltre aver causato il suo completo abbandono nonché l'esecuzione di un intervento in definitiva trascurato e non terminato, malgrado Ferrazzini avessi tentato il suo riconoscimento non solo con il "restauro" del monumento ma anche mettendo a conoscenza del pubblico $i$ lavori eseguiti e le caratteristiche dei dipinti ritrovati mediante la pubblicazione di un articolo a riguardo sulla Rivista Storica Ticinese.

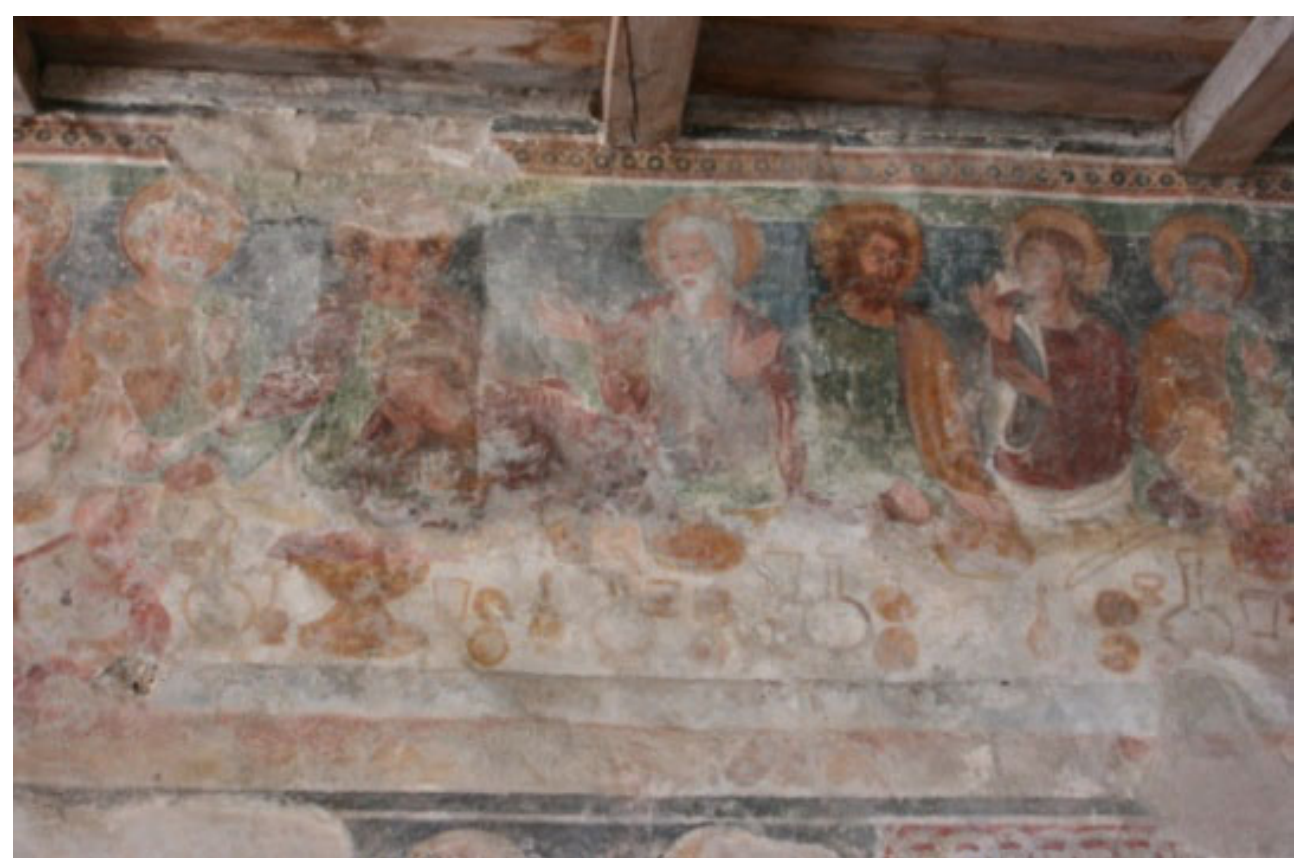

Figura 77 Giner Cordero E., Cenacolo. XV sec. Affresco. Deggio, 2008. 
Restauratori in Canton Ticino fra Ottocento e Novecento

Catalogazione e gestione dati

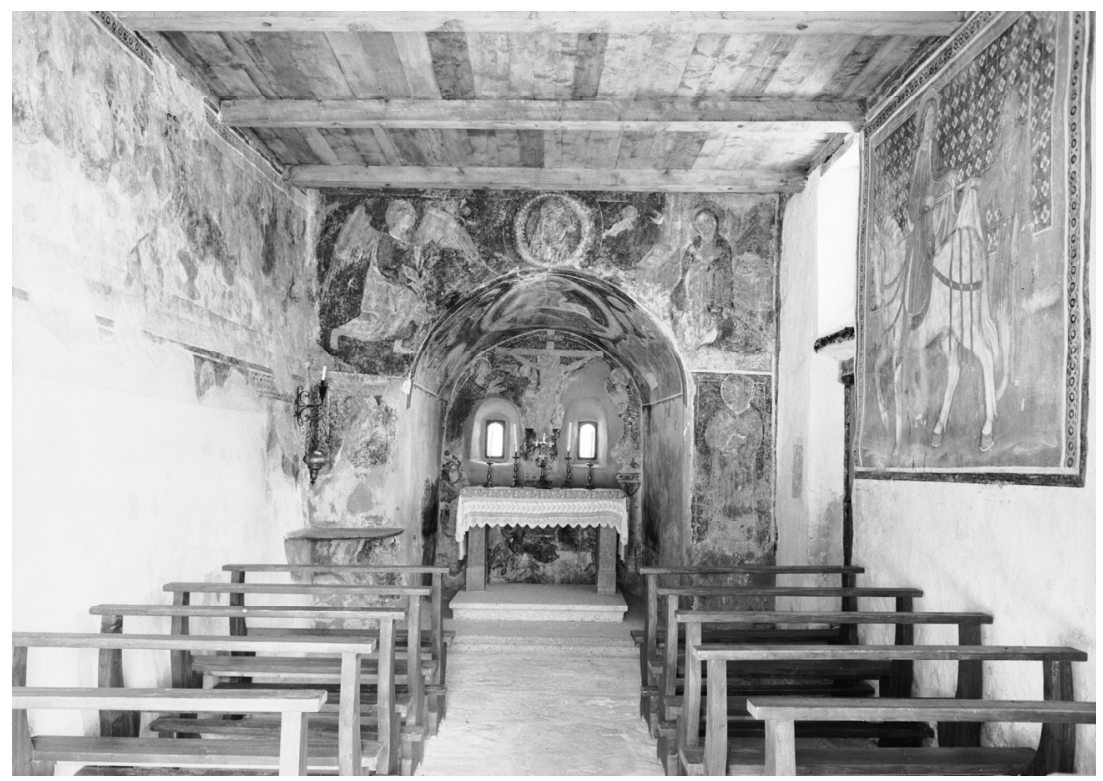

Figura 78 Interno dell'Oratorio di San Martino dopo il restauro. $1929 \mathrm{ca}$. XV sec.

Fotografia b/n, 1929 ca. AUBCBZ, Archivio grafico. Quinto, san Martino a Deggio.

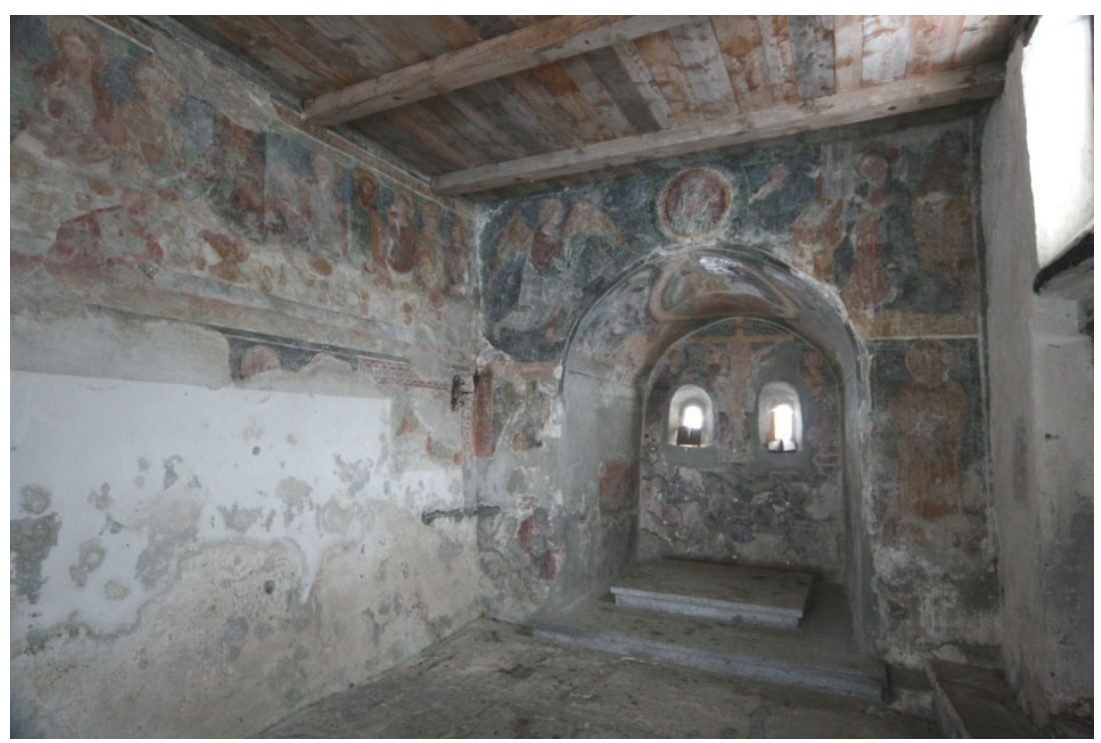

Figura 79 Giner Cordero E. Interno dell'Oratorio di S. Martino, 2008. 


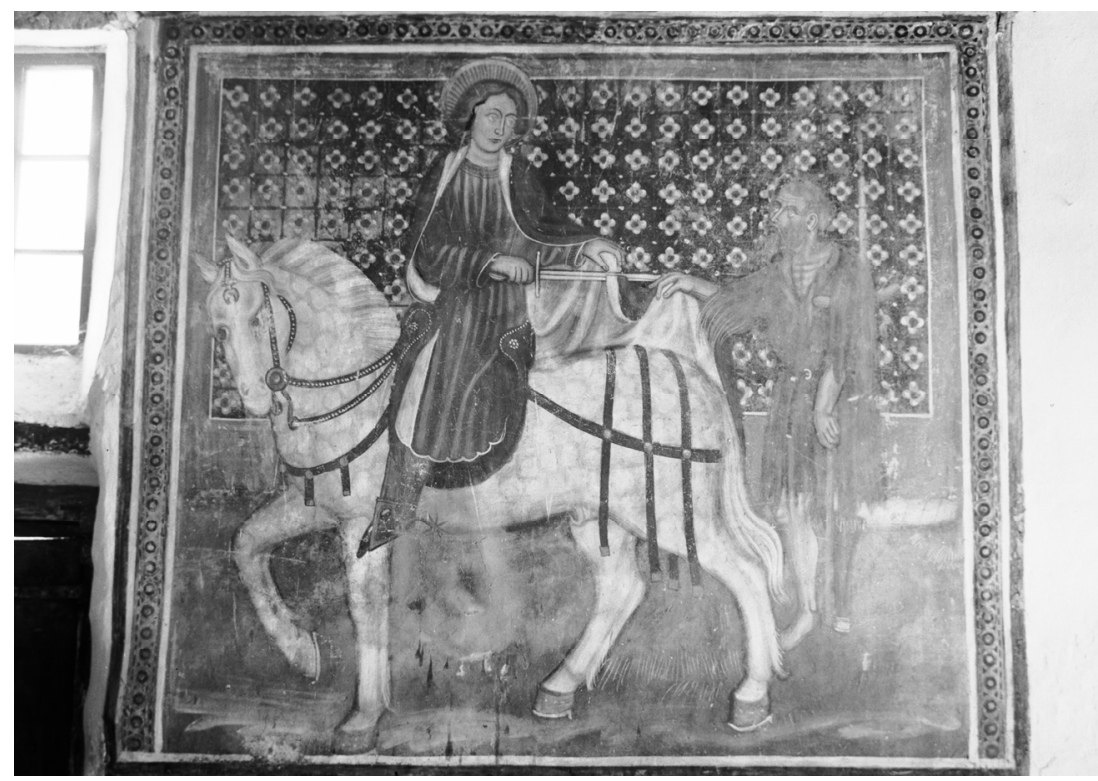

Figura 80 San Martino divide il mantello con il povero. XV sec. Fotografia b/n, 1929 ca. AUBCBZ, Archivio grafico. Quinto, san Martino a Deggio.

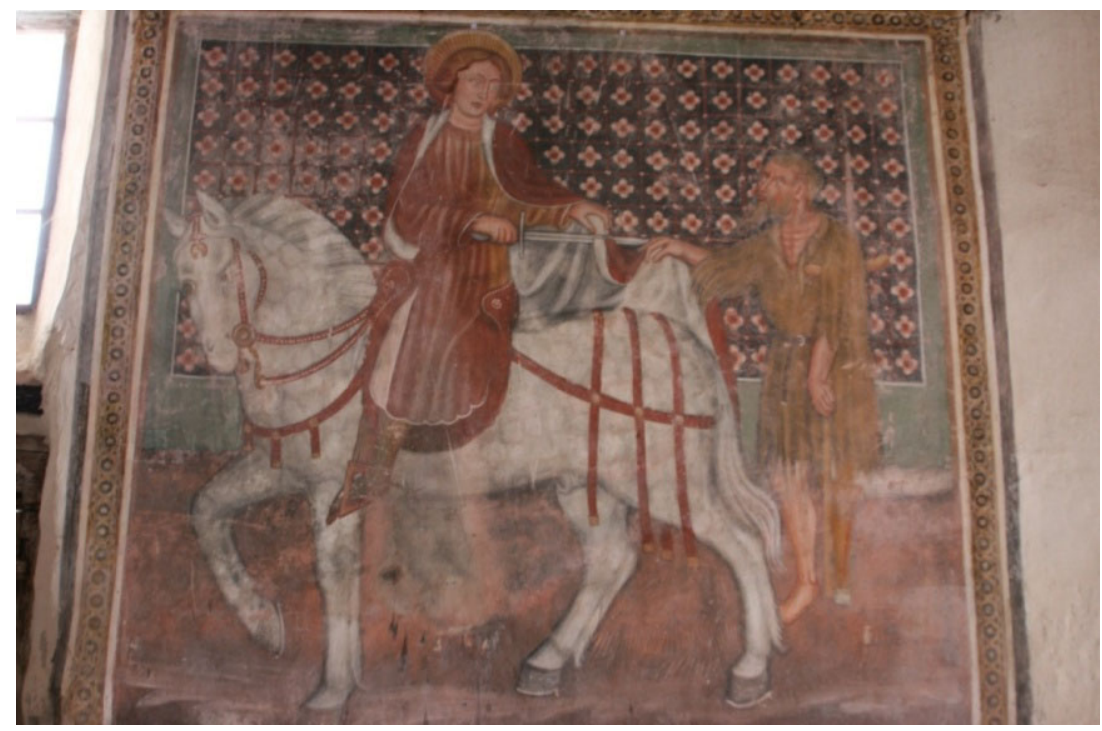

Figura 81 Giner Cordero E., San Martino divide il mantello con il povero, XV sec. Affresco. Deggio, 2008 
Restauratori in Canton Ticino fra Ottocento e Novecento

Catalogazione e gestione dati

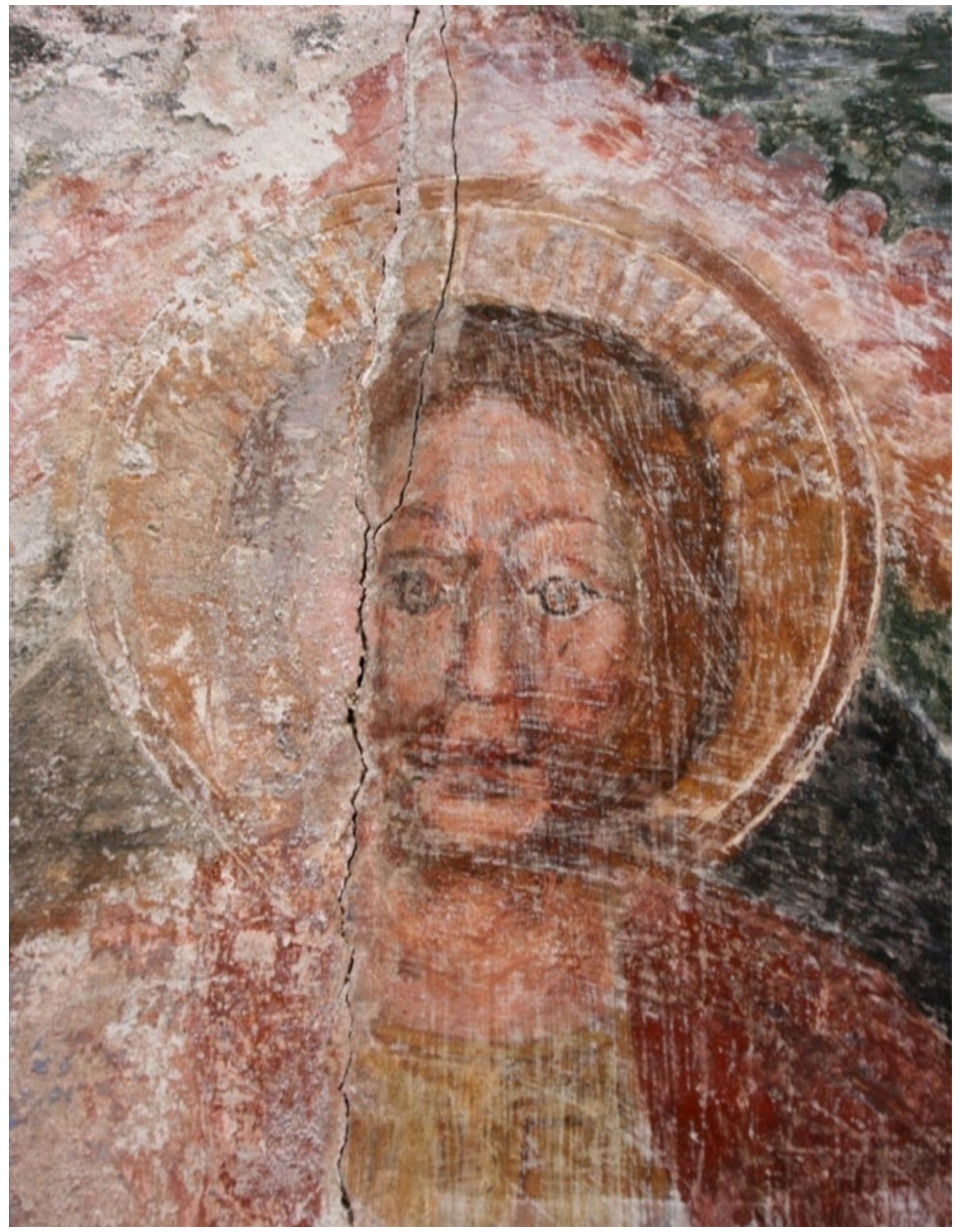

Figura 82 Giner Cordero E., Particolare del volto del Pantrocrator raffigurato sulla volta dell'abside dove si apprezzano dei segni dello descialbo, eseguitosi con mezzi meccanici abbrasivi, e segni della "verniciaturaconsolidamento" a cera sulla parte destra, Chiesa di san Martino a Deggio, Quinto, 2008. 


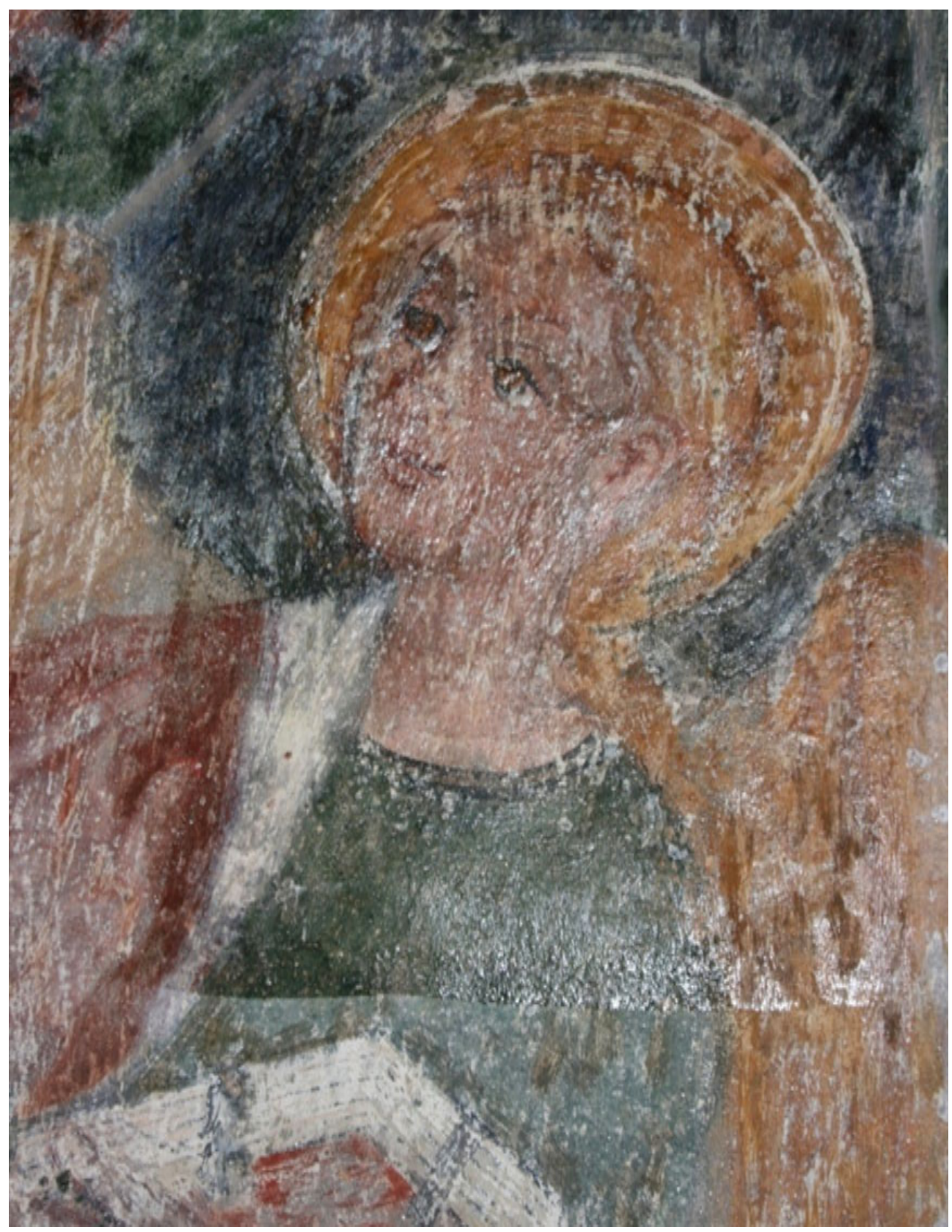

Figura 83 Giner Cordero E., Particolare dell'arcangelo raffigurato sull'abside dove si apprezzano dei segni dello descialbo, e una netta separazione fra la zona trattata con materiale cerosa-in alto- a la zona dove la pittura è rimasta intatta. Chiesa di san Martino a Deggio, Quinto, 2008. 
Restauratori in Canton Ticino fra Ottocento e Novecento

Catalogazione e gestione dati

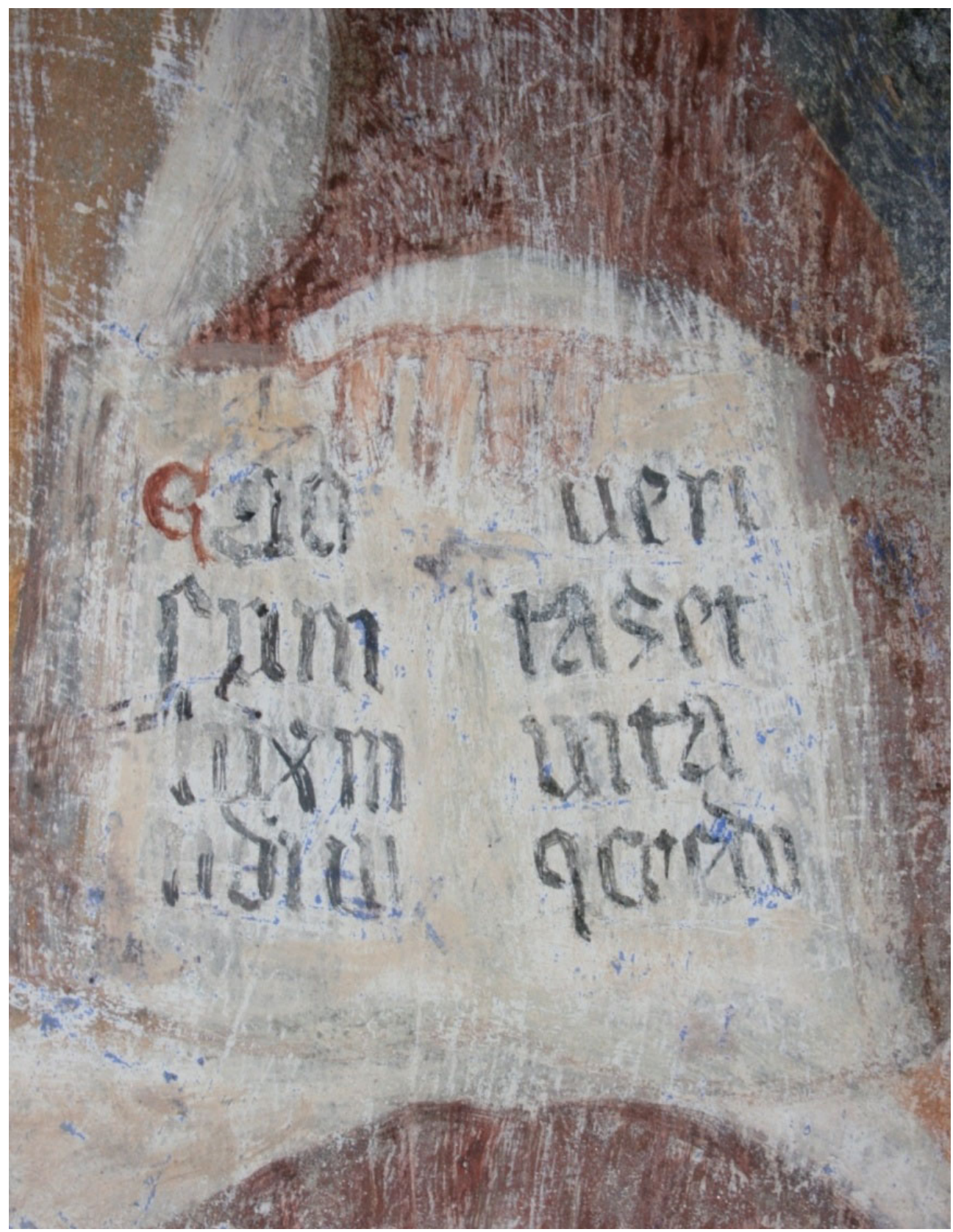

Figura 84 Giner Cordero E., Particolare dei dipinti raffigurati sullabside dove si apprezzano dei segni dello descialbo. Chiesa di san Martino a Deggio, Quinto, 2008. 


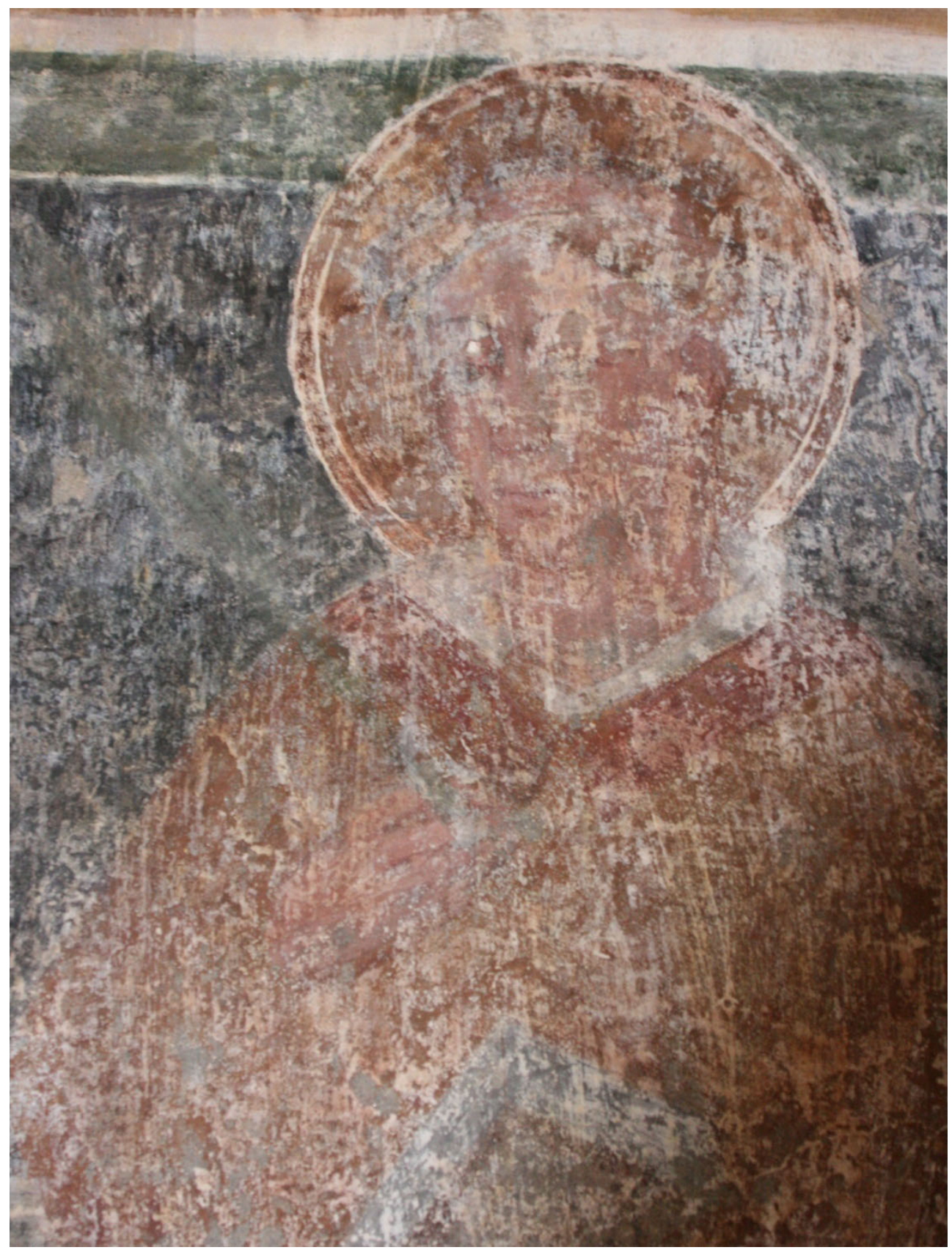

Figura 85 Giner Cordero E., Particolare dei dipinti raffigurati sullabside dove si apprezzano dei segni dello descialbo. Chiesa di san Martino a Deggio, Quinto, 2008. 


\section{San Biagio a Ravecchia}

Il caso specifico della chiesa di San Biaggio a Ravecchia, dimostra che il comportamento della cera poteva rivelarsi in poco tempo inaccettabile, creando dei veli biancastri sulla superficie del dipinto murale consolidato con questo metodo.

L'intervento di Ferrazzini arriva trenta anni dopo a quello di Edoardo Berta sull'edificio (ved. p.83) quando il 15 luglio 1940, chiamato dal Consiglio Parrocchiale, consolida una parte della lunetta e dell'architrave sopra il portone di entrata alla chiesa.

La materia pittorica molto degradata e polverulenta venne fissata con la tecnica all'encausto, con cera minerale ozokerite a $72^{\circ} \mathrm{C}$ di fusione sciolta in benzolo, e poi fatta penetrare nella superficie con un mezzo caldo.

Dalle prove d'intervento realizzate nella chiesa di Malvaglia a questo momento Ferrazzini ha acquisito una particolare sicurezza nell'utilizzo di questo metodo di consolidamento, che secondo le sue parole è frutto di una attenta, [...]continuata osservazione ed una pratica di molti anni.[...]Perché nel restauro, ogni colla, ogni cera, ogni tempera, ogni silicato, ogni ingrediente che si prepara e che si adopera è importante come ogni segno di pennello che vi si applica: e fa parte delle conoscenze, del bagaglio $e$ dell'esperienza del restauratore $[. . .]^{304}$.

La Commissione dei Monumenti, opposta a questo intervento ad encausto, valutò in contrapposizione alla proposta di Ferrazzini I'idea di Ugo Donati dell'incorporazione di un vetro di protezione davanti agli

\footnotetext{
304 (Ferrazzini Emilio. Lettera indirizzata alla CCMS Lugano 30 aprile 1940. AUBCBZ, s. 021, Bellinzona)
}

affreschi, ma davanti all'incapacità di decisione fra l'una o l'altra tecnica, nel 1943 si chiama il restauratore Henri Boissonas di Zurigo, considerato professionista nel genere, per esaminare gli affreschi e valutare l'intervento più idoneo. II 7 aprile 1943 egli collauda l'uso del metodo di consolidamento all'encausto di Ferrazzini senza adottare nessun ragionamento logico come invece si sarebbe aspettato.

[...]Prima di partire il Boissonas mi disse che egli di restauro e consolidamento di affreschi non se ne intendeva: mi pregò anzi di dargli schiarimenti $e$ di mandargli campioni del materiale usato a Zurigo. Ciò che feci premurosamente $[\ldots]^{305}$.

Henri Boissonas, qualificato nel restauro dei dipinti su tela e tavole, trasferì la proprie responsabilità al pittorerestauratore ticinese. Prima di attuare nel restauro completo delle pitture fu realizzata ancora una seconda perizia in data 5 settembre 1944 da parte dei committenti cantonali. II professore Francesco Chiesa e Ugo Donati concludono con sfavorevoli risultati sullo stato di conservazione del bene, specialmente deteriorato a causa dei fattori atmosferici, per cui la superficie intorno alla lunetta sopra il portale con la Annunciazione, il Cristo benedicente e la completa estensione del S. Cristoforo si trovavano gravemente sgretolate. Davanti finalmente a queste condizioni, Emilio Ferrazzini intervenne sollecitamente.

In data 18 agosto 1959, venne trasmessa la relazione del restauro effettuato nel 1944

\footnotetext{
305 (Ferrazzini Emilio. Lettera indirizzata alla CCMS. Lugano 30 aprile 1940. AUBCBZ, s. 021, Bellinzona).
} 
all'allora Ispettore dei Monumenti Storici, Aldo Crivelli.

In questa si specificano i passi eseguiti per il restauro che in primo luogo aveva interessato la pulitura del friso che incornicia le pitture della lunetta, illeggibile a causa delle numerose lacune nella superficie e dagli strati di calce bruno-rosso e giallastro, che una volta ricoprivano la superficie.

Dopo il citato descialbo la superficie venne consolidata mediante l'applicazione dell'encausto. Ferrazzini acquista la cera minerale dalla Germania, esattamente dalla grande ditta chimico-farmaceutica Schering- Kahlbaum ${ }^{306}$, A.G. di Berlino. L'uso del prodotto permette al pittore di riconoscere con maggiore precisione i piccoli resti di pittura blu e verde dei ritocchi a secco originali: alcune parti furono ritoccate ed una vecchia stuccatura fra la figura dell'Angelo Annunciatore ed il Cristo Benedicente fu attenuata con una tinta di tonalità grigia.

Sulla figura di San Cristoforo della facciata della chiesa si intervenne nello stesso modo, eliminando anzitutto la sporcizia superficiale e attenuando le macchie biancastre dalle lacune stuccate anteriormente, così come consolidando con cera certe zone pericolanti, in particolare i colori applicati a secco nella decorazione a mosaico del friso ed i fiori del manto del santo.

\footnotetext{
${ }^{306}$ C.A.F.Kahlbaum GmbH. Georg Wilhelm Kahlbaum (1823 Berlino - 1905 Basilea) prende in mano da 1847 la ditta del padre Carl August Ferdinand dedicato alla produzione di liquori che dal 1869 si convertirà in una della più grandi industrie chimiche della Germania. La firma, fusionata nel 1927 con la Schering A.G è comprata nel 2006 ed oggi si trova sotto la firma della Bayer Schering Pharma AG.
}

[...]L'encausto applicato ha fissato $i$ colori polverulenti. II sottile strato di ozokerite applicato sugli affreschi eviterà sopratutto il danno che le piogge apportano a queste pitture: quelle estive e temporalesche e quelle invernali, specialmente dannose perché il congelamento della superficie vagante produce il rigonfiamento, lo sfaldamento e 10 sgretolamento della superficie dipinta. $[\ldots]^{307}$.

Secondo le indicazioni del restauratore, gli effetti estetici del consolidante si dimostrarono in un [...]quasi insensibile cambiamento di tono[... $]^{308}$. Quello che Ferrazzini, invece, non ebbe in considerazione furono i veloci effetti negativi che appariranno sui dipinti, principalmente dovuti alla condensazione di umidità sulla superficie pittorica.

In una lettera del 16 aprile 1955 al Dipartimento della Pubblica Educazione del Canton Ticino il pittore Mario Rossi dirige l'ispezione realizzata sulle pitture restaurate dal Ferrazzini a San Biagio. I risultati di tale perizia parlano di una [...]conservazione discreta e nulla pericolante [...] ma chiama l'attenzione sui colori puntualmente biancastri e dall'ossidazione di una leggerissima vernice dell'ultimo restauro $[. . .]^{309}$.

\footnotetext{
${ }^{307}$ (Ferrazzini Emilio, Breve relazione sul consolidamento degli affreschi sulla facciata della Chiesa di San Biagio a Ravecchia. Lugano 20 novembre 1944. AUBCBZ, s. 021, Bellinzona).

(Ferrazzini Emilio, Breve relazione sul consolidamento degli affreschi sulla facciata della Chiesa di San Biagio a Ravecchia. Lugano 20 novembre 1944. AUBCBZ, s. 021, Bellinzona).

${ }^{09}$ (Rossi Mario, Concerne affresco di S. Biagio di Ravecchia. Madonna con Bambino fra due Santi, XIV sec. indirizzato al Lodevole Dipartimento della Pubblica Educazione, Bellinzona. Varese 16 aprile 1955.. AUBCBZ, s. 021, Bellinzona).
} 


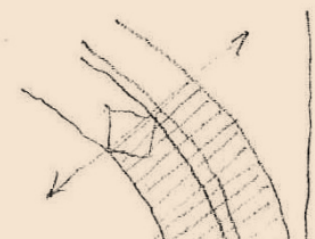

15 luolic

140

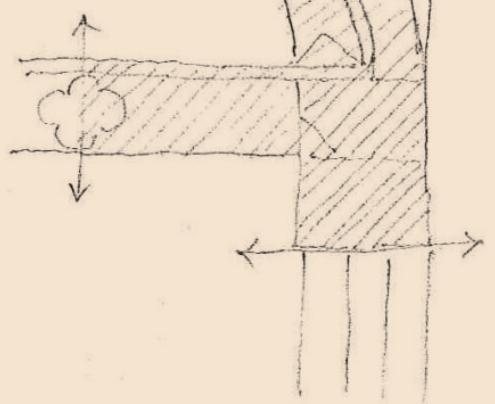

Figura 86 Ferrazzini Emilio, Disegno delle zone consolidate nel portale della chiesa di San Biagio il 15 luglio 1940. Lettera di Ferrazzini ad Aldo Crivelli in Lugano 18 agosto 1959. AUBCBZ Bellinzona.

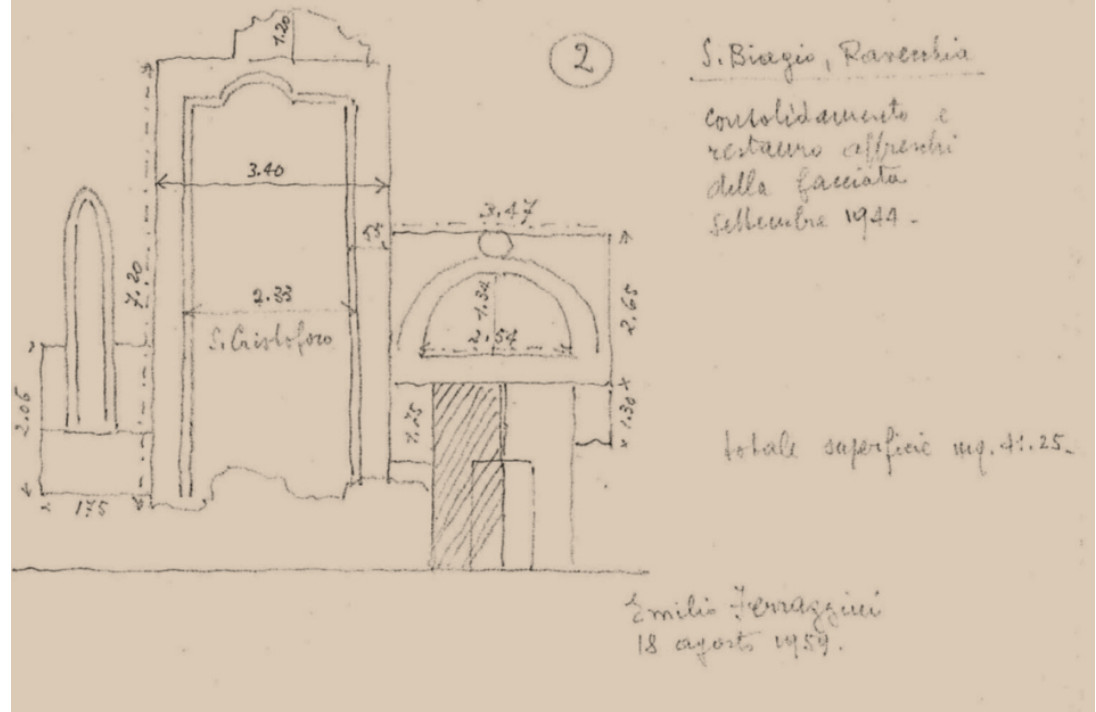

Figura 87 Ferrazzini Emilio, Disegno delle zone consolidate nella facciata della chiesa di San Biagio in settembre 1944. Lettera di Ferrazzini ad Aldo Crivelli in Lugano 18 agosto 1959. AUBCBZ Bellinzona. 
II pittore e restauratore di Varese propone nuovamente, come fecce anni prima il commissario U.Donati, l'uso di un vetro sovrapposto ai dipinti ad una distanza di un centimetro dalla superficie per garantire la sua durabilità nel tempo, isolati dall'azione degli effetti atmosferici ed evitando il fattore di condensazione.

Per Ferrazzini il problema dell'opacizzazione della ozocerite osservato dal pittore Rossi poteva essere facilmente risolto riscaldando nuovamente la superficie pittorica; [...]ma così non da fastidio; e sarà possibile nel futuro, un ulteriore intervento, usando anche altri materiali moderni[... $]^{310}$.

In una visita effettuata nel luglio 1967 a San Biagio, il professore e pittore Giuseppe Bolzani $^{311}$ membro della Commissione

310 (Ferrazzini Emilio, Breve relazione sul consolidamento degli affreschi sulla facciata della Chiesa di San Biagio a Ravecchia. Lugano 20 novembre 1944.. AUBCBZ, s. 021, Bellinzona).

311 Bolzani Giuseppe (Bellinzona 1921-Mendrisio 2002). Membro della Commissione Cantonale dei Monumenti Storici e Artistici dal 1960 al 1981, fu principalmente pittore, disegnatore, decoratore e insegnante. Diplomato nel 1947 all'Accademia di Brera, Milano, nel 1949 soggiorna a Parigi e poi all'Istituto Svizzero di Roma. Le sue opere si caratterizzeranno per lo stile naturalista d'impronta lombarda con tendenze all'astratto. Si occupa durante tutto il decennio degli anni Cinquanta della decorazione murale di diversi edifici pubblici. Gli esempi più noti sono il Palazzo delle Dogane di Chiasso in 1949 e la scuola superiore di commercio di Bellinzona in 1952. Questa attività venne ripresa negli anni 1983 - 1984 con la decorazione del Palazzo Mercurio di Chiasso e del Liceo cantonale di Mendrisio. Formò parte dal 1952 del Gruppo della Barca insieme ai pittori Mario Marioni, Pietro Salati e Alberto Salvioni con cui si presenta a diverse mostre a livello cantonale e nazionale. Come pittore di cavalletto partecipa assiduamente a mostre personali dal 1947. Fra questi possiamo citare le organizzate da Virgilio Gilardoni nel 1954 e nel 1962, le antologiche
Cantonale dei Monumenti in Ticino, accompagnato dal pittore Carlo Mazzi, riscontra sui dipinti della facciata di San Biagio una situazione di graduale perdita dei ritocchi a temple realizzati da [...]restauratori non perfettamente accorti $[. . .]^{312}$, con zone scurite nell'affresco raffigurante la Madonna in Trono con il Bambino a causa dell'applicazione della vernice cerosa. Le parti che sono rimaste intangibili alle mani di Emilio Ferrazzini erano invece in uno stato di conservazione ottimo, per cui il pittore Carlo Mazzi propose la rimozione della cera e dei ritocchi dal restauro del 1944.

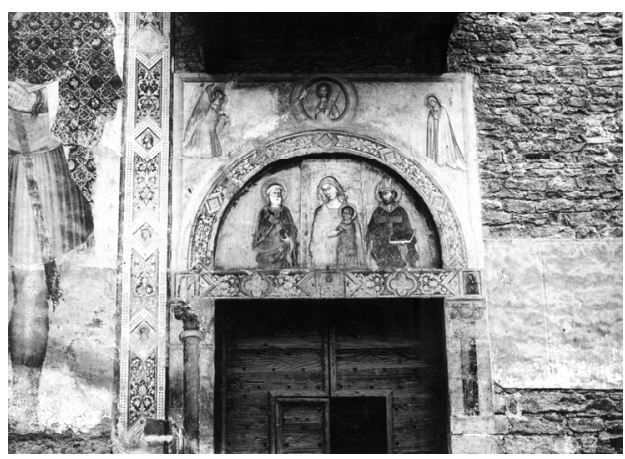

Figura 88 Facciata della Chiesa di San Biagio di Ravecchia dopo il restauro. Bellinzona. $1913 \mathrm{ca}$. Fotografia b/n. AUBCBZ, Archivio grafico, Ravecchia, S. Biagio.

del 1994 alla Civica galleria d'arte di Bellinzona, e del 2000, al Museo d'arte di Mendrisio A questo ultimo museo fu legato dal 1984 come membro della commissione d'arte.

${ }^{312}$ (Bolzani Giuseppe, Rapporto concernente l'affresco della lunetta del portale nella chiesa di San Biagio a Ravecchia indirizzato all'Ufficio Cantonale monumenti storici, Bellinzona. Locarno 3 luglio 1967. AUBCBZ, s. 021, Bellinzona). 
Il recente restauro sulla chiesa di San Biagio a Ravecchia, eseguito nel 2000 dalla ditta di restauro C.G.B. Restauri di Colombi P., Gallina S., e Bagutti M. a Bellinzona ha interessato l'eliminazione della cera cristallizzata sulla superficie pittorica mediante l'impiego di una resina di scambio ionico ${ }^{313}$ di tipo anionico ed ha consolidato successivamente le parti staccate degli affreschi con Paraloid $B 72^{314}$.

\footnotetext{
${ }^{313}$ La resine a scambio ionico sono polimeri organici di granulometria rivestiti di composti chelanti con la proprietà di scambiare ioni semplici (idrogenioni e ossidrili) con la soluzioni. Queste resine possono essere di tipo cationico, cioè quando scambiano ioni idrogenioni sottraendo ioni positivi; anioniche quando per il contrario hanno la capacità di sottrarre ioni negativi scambiando ossidrili; e per ultimo le chiamate "resine a letto misto" che raggruppano tutti entrambe proprietà. Esse vengono applicate sulla superficie pittorica o lapidea mediante impacchi umidificati in acqua distillata su la carta giapponese. L'azione della resine ad scambio ionico di tipo anionico applicata sugli affreschi della chiesa di San Biagio a Ravecchia attuarono sulla cera rigonfiandola, facilitando successivamente la sua asportazione.

314 L'appellativo di Paraloid B72 definisce un copolimero acrilico di durezza media, costituito a base di metacrilato di etile e metacrilato di metilo (70/30). Il prodotto sintetico fonde ai 150 으 ed è solubili negli esteri come l'acetato di etile e di amilo, il chetone, gli idrocarburi inorganici (toluene) e gli idrocarburi alifatici (tricoletilene), insolubile invece negli idrocarburi alifatici (white spirit). Può essere diluito negli alcoli, ed è compatibile con diverse resine acriliche e viniliche. Presenta un aspetto di cristalli particolarmente trasparenti ed una volta disciolto non applica viscosità. II Paroloid B72 ha trovato negli ultimi decenni impiego nel campo del restauro per la sua versatilità, il buon comportamento meccanico, l'effettività dell'adesione e la velocità in attuare. Malgrado questo la sue proprietà sono attualmente messe in discussione, principalmente per quanto concerne il fattore di "irreversibilità" dello stesso, poiché il materiale, con l'invecchiamento risulta difficilmente removibile.
} 


\section{SS. Ambrogio e Maurizio, Chironico}

Polemico e traumatico intervento di restauro fu allo stesso tempo effettuato sugli affreschi della chiesa dei SS. Ambrogio e Maurizio a Chironico, in Valle Leventina. La chiesa romanica a doppio abside della fine del XII secolo ed inizi del XIII' ${ }^{315} \mathrm{fu}$ ingrandita nel 1338 e modificata ampliamente nel 1897 con la realizzazione di un nuovo campanile eliminando l'originale a vela, l'eliminazione degli affreschi sulla facciata e del soffitto ligneo dipinto del Cinquecento, innalzando le pareti della navata e costruendo su questa una volta a botte caduta nel 1923.

Fra il 1940 e il 1952, sotto la direzione dell'architetto Alberto Camenzind ${ }^{316}$ e la supervisione dei commissari cantonali Pietro Salati e Piero Bianconi, si realizzarono dei lavori di restauro sulla fabbrica che riguardarono il recupero degli elementi originari persi nelle ristrutturazioni barocche, con il rifacimenti del soffitto ligneo e del campanile a vela, e la liberazione dell'apparato decorativo con l'eliminazione dello scialbo che ricopriva gli

\footnotetext{
${ }^{315}$ La prima datazione è dell'anno 1223.

${ }^{316}$ Camenzind Alberto (Lugano 1914 - Astano 2004) L'architetto studiò sei anni al Politecnico Federale di Zurigo prima di rientrare in 1942 a Lugano per aprire i suo studio d'architettura e di diventare lui stesso professore della prestigiosa scuola d'architettura zurighese. Fu socio dal 1959 al 1991 di Bruno Brocch e membro della Commissione Federale e Cantonale per la tutela dei monumenti storici e della Commissione Federale delle belle arti. L'architetto ha lasciato numerose ed importanti opere pubbliche, diminuendo l'attività alla fine degli anni Cinquanta dopo l'incarico per la realizzazione dell'Esposizione Universale Svizzera nel 1964 a Lausanne. Morì a novant'anni ad Astano, nel Malcantone, dove si era ritirato dal 1996.
}

affreschi trecenteschi così come le decorazioni in stucco del XVIII sec.

[...]II restauro Camenzind volle cioè- com'era prassi allora - ridare all'edificio il presunto aspetto medievale $[\ldots]^{317}$.

La scoperta degli affreschi, assai traumatica, fu opera di Emilio Ferrazzini e riguardava i dipinti murali sull'abside sud raffiguranti il Cristo in maestà, la Decapitazione di San Giovanni Battista, sull'abside nord l'Incoronazione della Vergine e la Crocifissione, sulla parete nord le Storie di Sant'Ambrogio, il Ciclo dei mesi, Storie di Sant'Anna e Gioacchino e Età dell'uomo ed il Giudizio Universale sulla controfacciata, eseguiti fra 1340 e 1341 dal maestro, "Magister Petruspaulus detto Soçus di Castello di Menaggio" secondo l'iscrizione sull'abside sud. ${ }^{318}$

II primo preventivo di restauro artistico eseguito che si è trovato documentato all'Archivio dell'Ufficio dei Beni culturali della città di Bellinzona e datato in Lugano 3 maggio 1941, parla degli interventi per realizzare sulla superficie affrescata di 258 $\mathrm{mq}$, calcolata in $70 \mathrm{mq}$ per la facciata sud, $58 \mathrm{mq}$ per la parete nord, $51 \mathrm{mq}$ per la parete a ponente, $23 \mathrm{mq}$ per la parete intorno agli absidi e $58 \mathrm{mq}$ per le due absidi.

Le operazioni comprendevano [...]il pulimento degli affreschi, la stuccatura delle crepe, dei buchi e delle pezze mancanti [...] dopo

\footnotetext{
(Cademario, Notiziario 2004 AUBCBZ, s.080, Chironico).

318 L'iscrizione venne firmata dal "magister Petruspaulus dictus Soçus pinctor de Castello de Menaxio" e attesta la riedificazione ed ampliamento della chiesa, come sopracitato nel 1338 e ampliata nel 1338.
} 
un diligente ricupero di tutta la superficie affrescata, attualmente ricoperta dalle imbiancature e dagli stucchi[... $]^{319}$, così come il tinteggio esterno, il distacco e la "rivoltatura" dell'affresco cinquecentesco nell'abside a destra della navata, per un totale di fr. 8'660.

Non fu chiaro all' inizio quali dovevano essere le persone capacitate per la realizzazione del lavoro e, malgrado il pittore scelto fu Emilio Ferrazzini sono stati trovati degli scritti, fra la corrispondenza in archivio, che proponevano la partecipazione in particolare di Ottorino Olgiati di Bellinzona, che aveva lavorato a Milano e Roma, il che significava avere un'esperienza nel campo ed una conoscenza tecnica molto più approfondita, con l'aiuto dei pittori Tita Pozzi, Mario Moglia, Mario Marioni e Ferrazzini. I lavori furono iniziati da quest'ultimo solo nel 1948.

II sopralluogo del 9 settembre dello stesso anno, realizzato da Pietro Salati e Piero Bianconi, descrive diverse considerazioni sugli interventi del pittore. Questi esigono una maggiore perfezione nella pulitura degli affreschi ritrovati sotto lo strato di calce, evitare i ritocchi sulle figure e nelle zone dove la pittura è staccata, al contrario di quanto risultava compiuto sulla raffigurazione della Morte, nella facciata, dove il ritocco si sovrapponeva all'originale; eseguire la reintegrazione pittorica delle martellature con un tono grigio neutro sulle zone figurative e con un colore velato sulle parte architettoniche e,

\footnotetext{
319 (Ferrazzini Emilio, Preventivo per le opere di restauro pittorico della chiesetta di S. Ambrogio indirizzato allo Spettabile Consiglio Parrocchiale $d$ Chironico. Lugano, 3 maggio 1941. AUBCBZ, s.080, Chironico).
}

per ultimo, ricostruire le fasce orizzontali che dividono architettonicamente le decorazioni della navata.

Bisogna specificare che la prima considerazione, sull'inadeguatezza della pulitura dello scialbo, è stata riscontrata su numerosi restauri dell'epoca; questa si doveva non solo ad un inabile intervento dei restauratori ma anche alla carenza di materiali e utensili idonei per l'asportazione della calce, che senz'altro continua ad essere uno dei processi più complicati e analizzati nel restauro.

Tutte queste indicazioni furono, secondo Francesco Chiesa, riconosciute dal pittore e restauratore Emilio Ferrazzini, per cui dopo le visite dei membri commissari si osservò una evidente miglioria dell'intervento con una pulitura degli affreschi apparentemente soddisfacente: mancarono al momento per compiere la riduzione dei rifacimenti pittorici sulle fasce decorative e la rielaborazione dei rappezzi di intonaco in modo da omogeneizzare i passaggi fra le aggiunte ed il materiale originale.

La relazione del 21 ottobre 1948 del pittore Emilio Maria Beretta ${ }^{320}$, membro delle Commissione, segnalò le difficoltà riscontrata nella valutazione del lavoro eseguiti da Ferrazzini, in primo luogo perché questo non realizzò un intervento

Beretta Emilio Maria (Muralto 1907, Ginevra 1974). II pittore eseguì gli Studio artistici al "Ècole des Beaux - Arts" di Ginevra per lavorare posteriormente nella Svizzera Francese, Ticino e Francia, come pittore e scenografo nelle tecniche dell'affresco, la pittura murale, il disegno, le vetrerie e l'illustrazione. Mantiene i contatti con il Ticino tramite Piero Bianconi, scrittore e critico d'arte nonché membro della commissione cantonale dei monumenti storici artistici del Ticino. www.sikart.ch. 
sistematico ed organizzato come si era richiesto e come si sarebbe aspettato, secondo il quale per idoneità dovrebbero essersi compiute in primo luogo tutte le operazioni di pulitura e in un secondo momento i consolidamenti degli affreschi; [...]il restauratore invece condusse innanzi saltuariamente il proprio lavoro, fino a completo restauro di questa o quella parte [...] ha reso impossibile fino ad oggi un esame esauriente $[. . .]^{321}$.

Per accrescere le difficoltà nel valutare l'idoneità dei metodi e dei risultati, venne a mancare inoltre una esaustiva documentazione fotografica che testimoniasi lo stato conservativo della fabbrica, prima e dopo il restauro effettuato dal Ferrazzini. Infatti la corrispondenza cartacea conservata in archivio $^{322}$ rileva una importante disarmonia ed incomprensione durante $i$ lavori, dovuta in parte alle discrepanze verificatesi fra il commissario Piero Bianconi ed il restauratore.

In una lettere emessa al consigliere Brenno Galli I'8 ottobre 1948, Ferrazzini conferma le differenze fra lui e l'ispettore.

[...]Ho affermato (e spiegato) che se vi furono lunghe discussioni fra me ed il sig. Bianconi circa il modo di condurre il restauro, è precisamente perché io non corrispondevo alle sue idee. (mi chiese perfino se avevo adoperato del silicato per... ravvivare i colori; stavolta non è la gomma arabica ma il silicato, secondo lui, sarebbe usabile per quel scopo!...) [...] Nella mia coraggiosa critica circa i restauri a San Remigio di Corzoneso [...] dimostrai servendomi di nomi

\footnotetext{
1 (Beretta Emilio Maria, Perizia indirizzata alla CCMS. 1948 post. AUBCBZ, s.080, Chironico).

${ }^{322}$ Si fa riferimento esattamente all'archivio morto dell'Ufficio dei Beni Culturali di Bellinzona, (AUBCBZ, s. 080, Chironico).
}

autorevoli come certe teorie (Bianconiane) sul restauro sono false e da abbandonare[... $]^{323}$.

Le suddette teorie "bianconiane", che Ferrazzini sottovaluta, erano quelle che si stavano adottando in quel momento dal restauratore di Bergamo, Mauro Pellicioli, nel restauro del Cenacolo Vinciano nel refettorio del convento domenicano di Santa Maria delle Grazie nella vicina città di Milano e senz'altro quelle che si stavano incentivando nel resto d'Europa partendo dall'Italia.

Come conseguenza della situazione di polemica sulla metodica d'intervento, di questa come di tante altre chiese restaurate dal 1909, la Commissione Cantonale organizzò un collegio d'esperti incaricato di valutare la qualità di diverse attuazioni. II mese di febbraio 1949 si dichiarano le perizie dei delegati l'architetto Ambrogio Annoni di Milano, il pittore-restauratore M. Pellicioli ed il professore Jean Verdier di Parigi. Sui lavori di Chironico questi denunciano l'uso deplorabile della cera - in questo caso una miscela di paraffina e cera d'api - i ritocchi arbitrari e gli sfumati d'intonaco e di colore che non permettono di distinguere chiaramente le pitture descialbate. Gli stessi esperti non diedero invece nessuna importanza alla cancellazione delle decorazioni barocche, poiché la sua carenza si assumeva dalla necessità di scoprire $\mathrm{i}$ dipinti primitivi. $\mathrm{Di}$ fatto potrebbero sembrare paradossali le teorie che in questo momento promuovevano il minimo intervento quando allo stesso

(Ferrazzini Emilio, Lettera personale riguardo i lavori di restauro della chiesetta di S. Ambrogio indirizzata al Consigliere Brenno Galli, Lugano. Lugano 8 ottobre 1948.. AUBCBZ, 080, Chironico). 
tempo si ammetteva la scomparsa o di più l'eliminazione di opere più recenti. Questo si giustifica solo nell'impossibilità di identificazione dimostrata nelle produzioni meno remote non riconosciute quali opere d'arte; i concetti estratti da un rapporto del 1946 della Commissione Cantonale Monumenti Storici ed Artistici riguardo il restauro dell'interno della chiesa parrocchiale di Verscio, esprimeva perfettamente gli ideali imposti per l'intervento ordinando di levare la decorazione ottocentesca per arrivare all'intonaco originale sopra il quale attuare con una limitata azione e ridare "decoro" all'edificio $^{324}$. Come abbiamo visto fino ad ora, la maggior parte dei monumenti restaurati in questo periodo esemplificano queste prassi.

Affrontate le osservazioni degli esperti nell'intervento sugli affreschi di $S$. Ambrogio, il Ferrazzini si dichiara comprensibile, come descrive Pietro Salati nella Relazione sugli ultimi lavori di restauro nella Chiesa di S. Ambrogio a Chironico del 19 settembre 1950. II restauratore corregge diversi aspetti dell'intervento eliminando i ritocchi illusionisti ed effettuando una successiva reintegrazione di differenziazione fra le parti originali e le aggiunte di restauro, armonizza le parti stuccate con toni neutri e accentua le martellinature in un primo momento coperte così come le fessure più grandi.

[...] Nel mese di giugno - così incaricato - ho seguito i lavori di restauro condotti dal pittore

${ }^{324}$ (Ugo Donati, Pietro Salati e Piero Bianconi. CCMS Lettera indirizzata al DPE riguardo il Restauro dell'Interno della Parrocchiale di Verscio. Minusio 28 marzo 1946. AUBCBZ, 246 Minusio).
Ferrazzini nella Chiesa di S. Ambrogio a Chironico, lavori ritenuti necessari in seguito alle critiche sollevate in un primo tempo dalla Commissione cantonale dei Monumenti storici ed artistici $e$, in seguito, dai signori Annoni, Pellicioli e Verdier.[...] Per completare il restauro si è cosi fissato come criterio base la netta divisione tra le parti intonacate con intonaco nuovo e quelle che ancora portano l'intonaco antico. Per questo il primo intervento è stato quello di eliminare fin dove era possibile (ma in molti punti era ormai difficile distinguere fin dove arrivassero $i$ ritocchi) le aggiunte fatte dal restauratore. In seguito per dare un'ossatura alla decorazione murale, si sono marcati $i$ contorni che definiscono i riquadri in cui sono divise le pitture, $e$, anche qui, per non lasciar dubbi, invece del tono rosso cupo originale si è preferito usare un grigio leggermente meno intenso. [...] Dove in alcune raffinatezze dei dipinti non permettevano l'uso del grigio si è usato il tono originale ma più basso. Risulti così chiaramente ciò che è e ciò che non è originale, senza, d'altra parte, che il complesso lasci l'impressione che il restauro sia stato condotto seguendo solo criteri puramente scientifici. [...] II restauro difficile come assieme causa le enormi zone vuote ora appare, a mio giudizio, discretamente organico $[. . .]^{325}$.

L'attenzione data sugli effetti negativi della stesura della cera sulla superficie murale, che non cita in nessun momento Pietro Salati, non impedirà che il pittore la riapplichi in un secondo intervento nel 1964 per ravvivare i colori ritrovati impalliditi, e contemporaneamente in diversi dipinti murali, come il caso degli affreschi della chiesa di Sant'Ambrogio in Ponte Capriasca, e sui dipinti esterni della Chiesa Parrocchiale di Santa Maria Assunta

\footnotetext{
(Salati Pietro, Relazione sugli ultimi lavori di restauro nella Chiesa di S. Ambrogio a Chironico, Viganello 19 settembre 1950. AUBCBZ, 080 Chironico).
} 
di Russo ${ }^{326}$. In questa ultima fabbrica il restauratore fu chiamato dal Consiglio Parrocchiale nel $1935^{327}$ dove realizza un primo consolidamento con cera d'api, che quindici anni dopo dovrà riprendere a causa delle alterazione. ${ }^{328}$

Analogamente successe nel restauro del Cenacolo a Ponte Capriasca ed a Novazzano.

Sugli affreschi dell'Ultima Cena ${ }^{329}$ e della Natività, all'interno ed all'esterno rispettivamente, nella chiesa parrocchiale di Ponte Capriasca, la superficie

\footnotetext{
${ }^{326}$ La restauratrice Nadia Fonti faceva riferimento nel preventivo presentato nel 1996 all'UCMS (Font Nadia. Relazione preventivo restauro. Ascona 1996. AUBCBZ, Russo) sui [...]residui di materiale consolidante; probabilmente caseato, dato che it colore giallognolo del residuo e una sostanza fissante a carattere ceroso[...] ritrovato sugli affreschi.

${ }^{327}$ (Ferrazzini Emilio. Lettera indirizzata alla CCMS Lugano, 10 novembre 1935. AUBCBZ, Russo). II consiglio parrocchiale avvertiva inoltre della possibilità d'intervenire sull'affresco con misure di "conservazione preventiva": [...]inoltre presente che sarà bene, avvenute che siano le necessarie riparazioni, fissare sull'affresco una retina metallica onde preservarlo dai colpi di frale di neve e di sassi che purtroppo vengono lanciati dai ragazzi che non sanno apprezzare il valore artistico[...] in (Consiglio Parrocchiale di Russo. Lettera indirizzata alla CCMS, Russo 26 settembre 1935. AUBCBZ Russo).

328 (DPE. Lettera indirizzata a Emilio Ferrazzini. Lugano, 1950. AUBCBZ, Russo).

${ }^{329}$ L'affresco cinquecentesco esistente nell'Oratorio di Sant'Ambrogio è sicuramente opera d'un allievo $d$ Leonardo da Vinci. Assieme al Cenacolo dei Tarilli nell'Oratorio di Novazzano è una delle copie di più grande fattura conservate nel Canton Ticino. Del supposto autore del dipinto scrive il pittorerestauratore Ottorino Olgiati in un articolo pubblicato nella Rivista Storica Ticinese nel 1939 [...]Sapevo che in quella regione vi erano delle opere d'arte, perché diversi pittori comacini vi lavoravano per i signori di Milano ai quali apparteneva la terra. Prova ne sia la famosa copia di Leonardo da Vinci, dovuta al suo discepolo Melzi (?).[...]. (Olgiati, 1939, p. 261).
}

consolidata nel 1929 con uno strato di cera minerale presentò, in poco tempo una patetica apparenza che lo scrittore Ugo Donati non ebbe dubbio di denunciare con un articolo al Giornale del Popolo del 20 giugno dello stesso anno. Le parole accusarono che [...]l'affresco era ridotto in uno stato lacrimevole in seguito a malaugurati restauri[.... $]^{330}$. La difesa di Emilio Ferrazzini alle proteste sembrò ragionevole; nel 1940 il pittore torna ad operare sull'affresco della Natività che effettivamente risultava esteticamente molto degradato, sporco, opaco e coperto da una patina verdastra che si era formata a causa del solfato di rame applicato dal parroco sul vicino vigneto. La pulitura di tutto questo sudiciume avvenne con successo ed il restauratore vuole con questo provare l'efficacia della cosiddetta "vernice cerosa" che, se non altro, aveva protetto i dipinti da un degrado maggiore.

Sul dipinto del Cenacolo all'Oratorio di Novazzano operarono i pittori-restauratore Nino Facchinetti e Carlo Cotti sotto la direzione di Emilio Ferrazzini nel 1943. Anche cui la superficie fu trattata con una miscela di cere per accrescere la vivezza delle tonalità cromatiche del dipinto, oltre che per consolidarlo e preservarlo degli agenti atmosferici. II dipinto in questione, analizzato dal Laboratorio Tecnico Sperimentale del Canton Ticino, elaborò nel 2006 un rapporto ${ }^{331}$ che dimostrava l'applicazione in più mani della cera, il

\footnotetext{
${ }^{330}$ Donati Ugo, Giornale del popolo. 20 giugno 1929. p. 26.

${ }^{331}$ (M. Beltrami, 2006, p. 113).
} 
quale dimostra l'uso abituale della tecnica come sistema di manutenzione ${ }^{332}$.

Ferrazzini, convinto delle ottime qualità del prodotto come consolidante dei dipinti murali oserà infine riutilizzarlo poco dopo le critiche degli esperti negli anni Cinquanta: [...]lo proporrei un lavoro di consolidamento fatto coraggiosamente (a due riprese) con encausto di cere minerali e con l'intervento del calore. (N.B. le cere minerali non appartengono alla serie degli oli e dei grassi = sono degli idrocarburi). Questa operazione specialmente per impedire alla pioggia di penetrare e di rompere il lavoro di distruzione dell'intonaco. Presento questa mia proposta non conoscendo un mezzo migliore. Sono però senz'altro disposto ad intervenire con altri mezzi tecnici qualora mi venissero indicati come più adatti a più sicuri[... $]^{333}$.

In S. Ambrogio a Chironico la cera, che a causa degli effetti dell'umidità stava deteriorando i dipinti, fu analizzata dal dottore A. Moles di Lucca (Italia) nel $2000^{334}$ motivato da una successiva proposta di restauro elaborata dal restauratore ticinese Andrea Meregalli. I campioni prelevati furono sottoposti a studi spettrofotometri di assorbimento infrarosso (FT-IR) ed estrazione in solventi organici, confermano i dati riscontrati in archivio che dichiarano la natura della cera

\footnotetext{
I campioni strati da diversi punti dell'affresco dell'Ultima Cena a Novazzano hanno evidenziato, dopo gli analisi con spettrometro ad infrarossi FT-IR, la presenza di una cera naturale. Alcune differenze fra gli spettri servono a supporre l'impiego di diverse cere a più riprese. (M. Beltrami, 2006, p. 113).

333 (Ferrazzini Emilio. Proposta di restauro indirizzata alla CCMS. Lugano 7 giugno 1950, AUBCBZ, Ponte Capriasca).

(Moles, Arcangelo. Chironico, Chiesa dei Santi Ambrogio e Maurizio. 3 Luglio 2000. Studio richiesto dall'Ufficio dei Beni Culturali di Bellinzona. AUBCBZ, s.080 Chironico).
}

impiegata nel consolidamento a base principalmente di paraffina con una minor parte di cera d'api. L'utilizzo abbondante di questo prodotto ha risultato peggiorare la presenza di sali, solfati di calcio e magnesio, ossalati e nitrati, sulla superficie pittorica mai risanata. Per la rimozione della cera, che risultò essere stata usata in quantità abbondante, si svolsero diverse prove per proporre definitivamente il sistema valutato di maggiore successo. Questo significò l'utilizzo di impacchi di sepiolite (10g), acqua deionizzata (100 cc), Arbocell (1 parte di 200/ 1 parte di 1000) lasciati agire durante circa due ore; poi impregnati con benzene leggero o ligroina per altre due ore dopodiché vengono tolti e tamponati con lo stesso solvente. La superficie asciutta si spalma con ammonio carbonato lasciato agire per circa 15 minuti, e poi lavata con una spugna impregnata in acqua deionizzata. lo stesso sistema si impiega nella pulitura delle decorazioni della chiesa di Sant'Abbondio a Mezzovico, capace di asportare non solo la cera ma anche i resti di calce dello scialbo che Emilio Ferrazzini non rimuove completamente nell'intervento di 1932 dai dipinti del XV sec. coperte in sec. XVII con cui si rompe l'omogeneità dell'edificio.

Le analisi realizzate nel 2000 rilevano anche la natura delle stuccature realizzate dal restauratore nel 1948, una malta pura a base di calce grassa e quarzo con tracce di silicati colorati e assenza di gesso per cui in quest'ultima proposta di restauro si decise di lasciarle, sostituendo unicamente le tinteggiature che creavano una certa disomogeneità nel complesso pittorico per altre maggiormente integrate. 


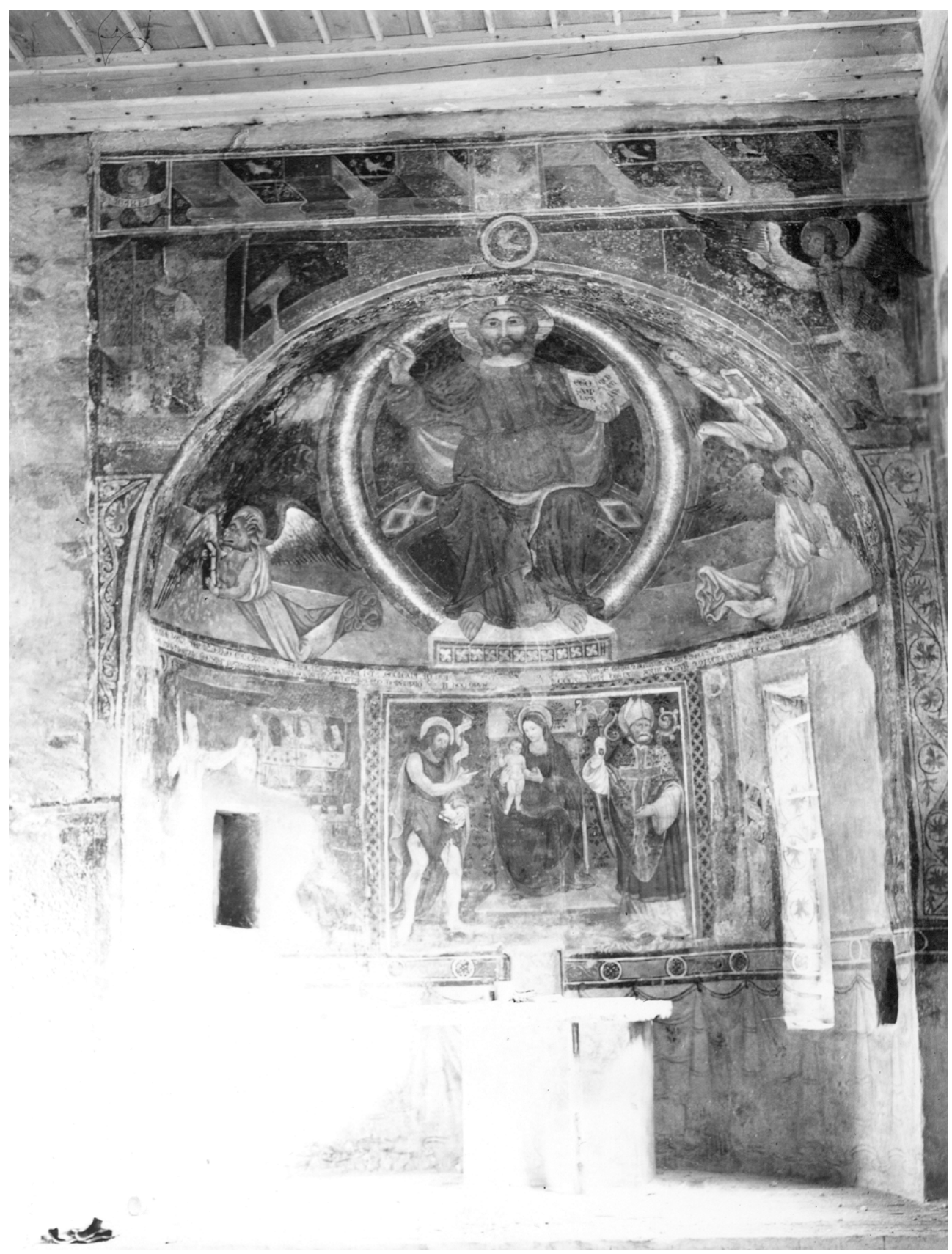

Figura 89 Fotografia dell'Interno della chiesa dopo il restauro. Abside Sud, 1950 ca. AUBCBZ. s. 80 Chironico. S. Ambrogio. 


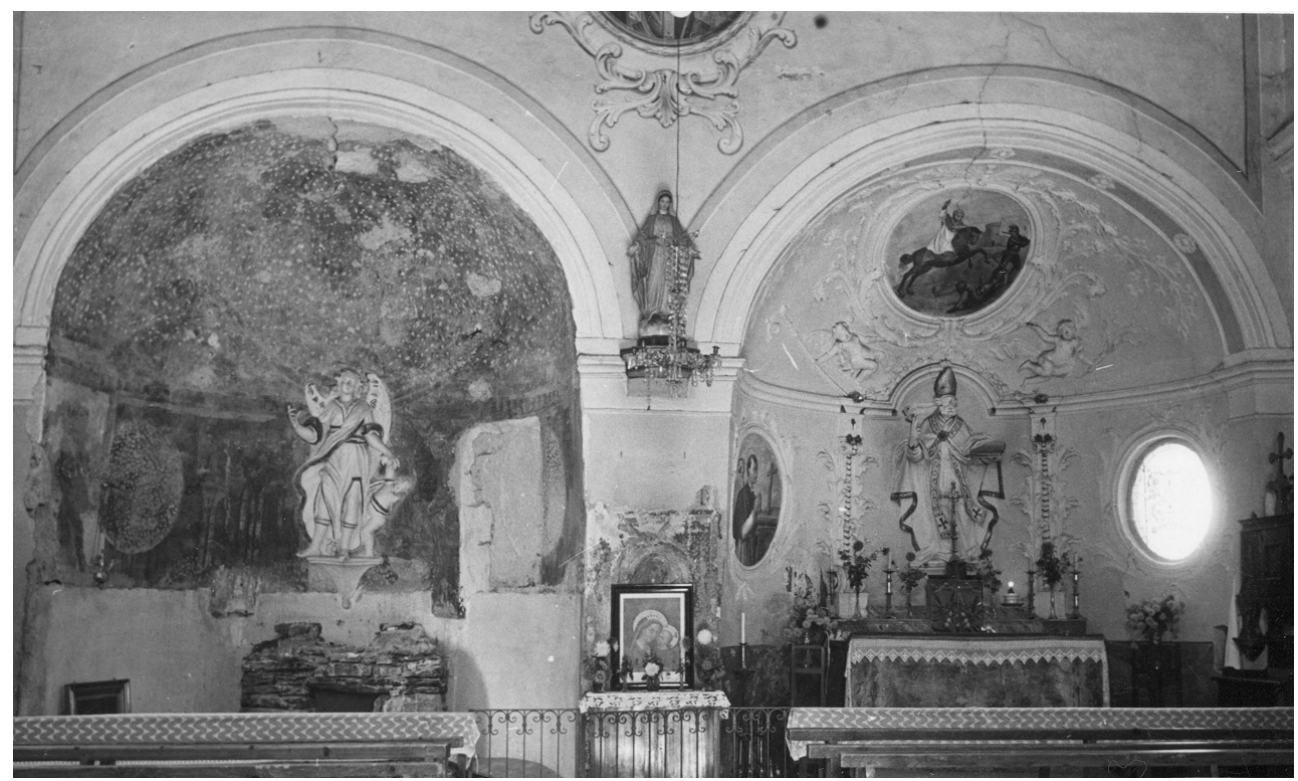

Figura 90 Fotografia dell'Interno della chiesa durante il processo di restauro, $1948 \mathrm{ca}$. AUBCBZ. Chironico. S. Ambrogio.

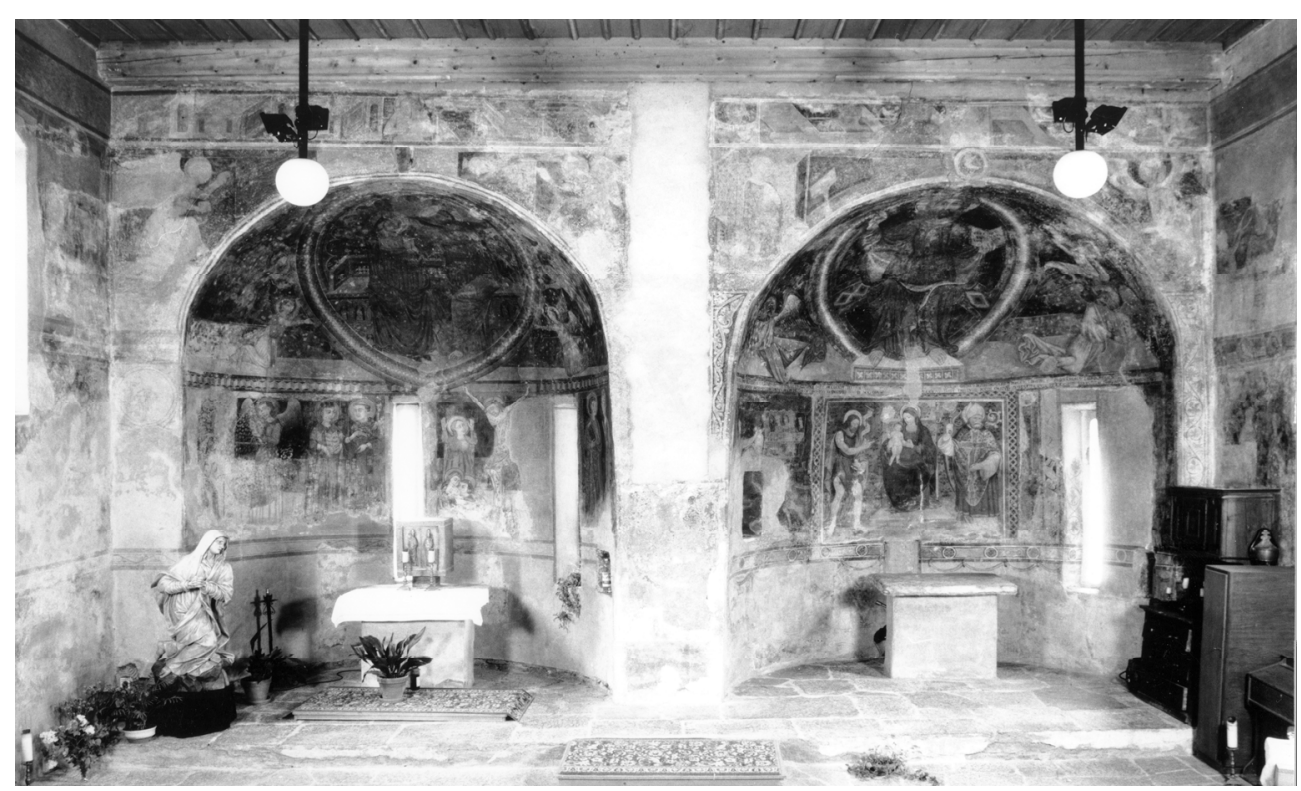

Figura 91 Fotografia dell'Interno della chiesa dopo il restauro, 1950 ca.

AUBCBZ. Chironico. S. Ambrogio. 


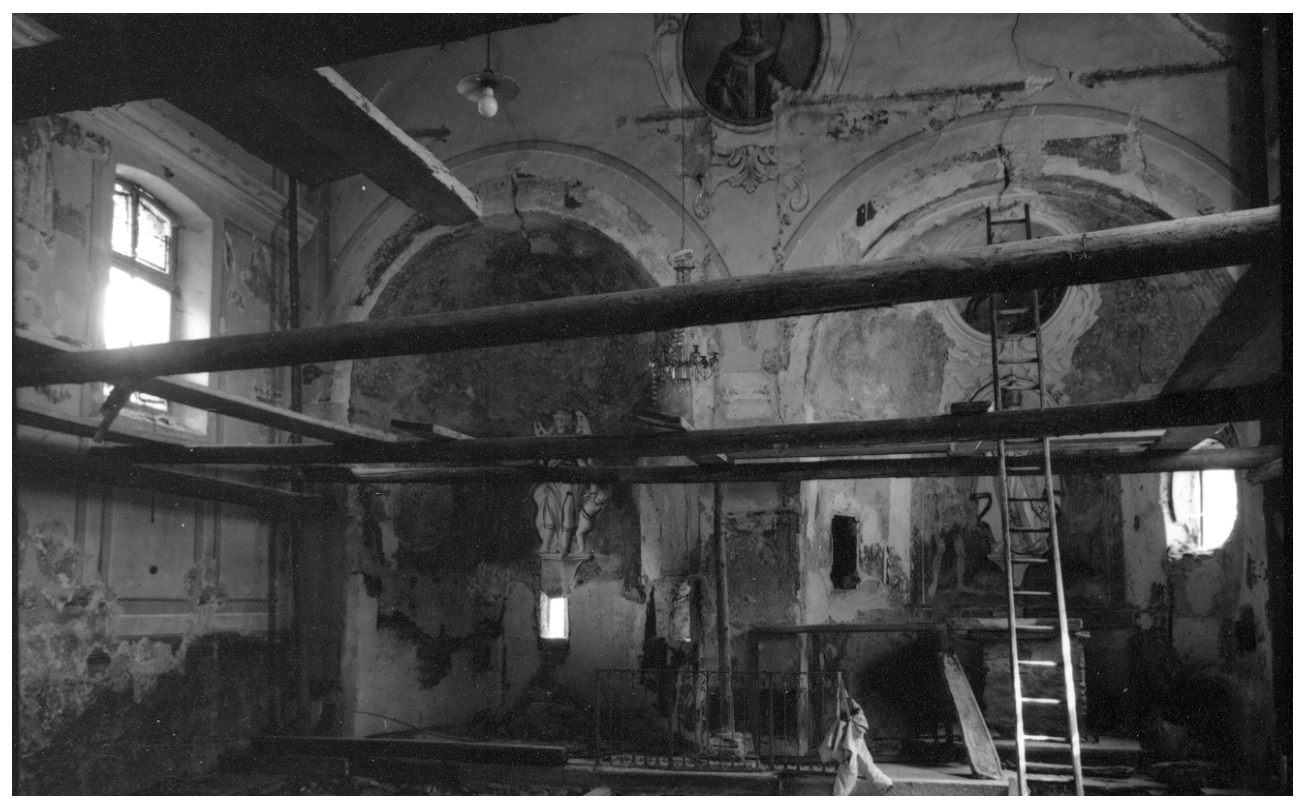

Figura 92 Fotografia dell'Interno della chiesa durante il processo di restauro, $1948 \mathrm{ca.}$ AUBCBZ. Chironico. S. Ambrogio.

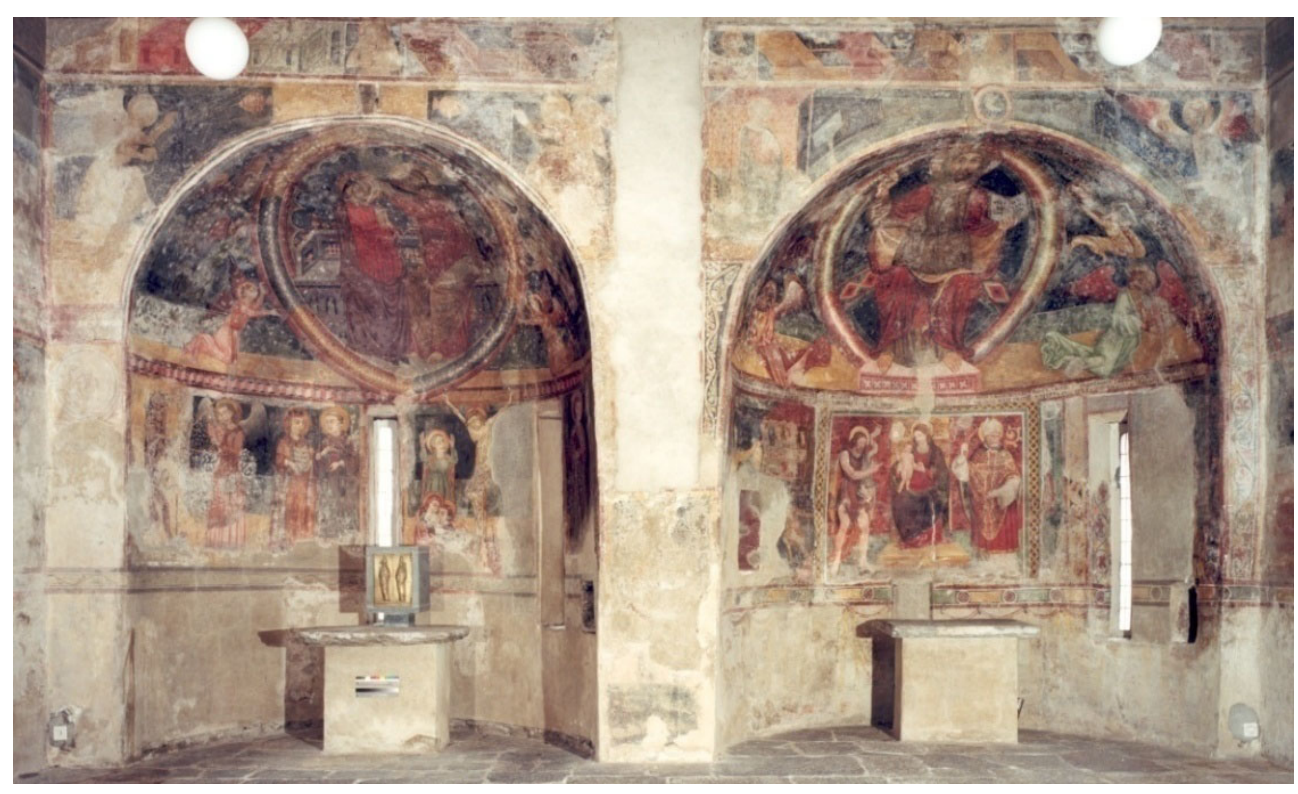

Figura 93 Mattei Francesco, Fotografia dell'Interno della chiesa dopo i lavori di restauro, 2004 ca. AUBCBZ. Chironico. S. Ambrogio. 


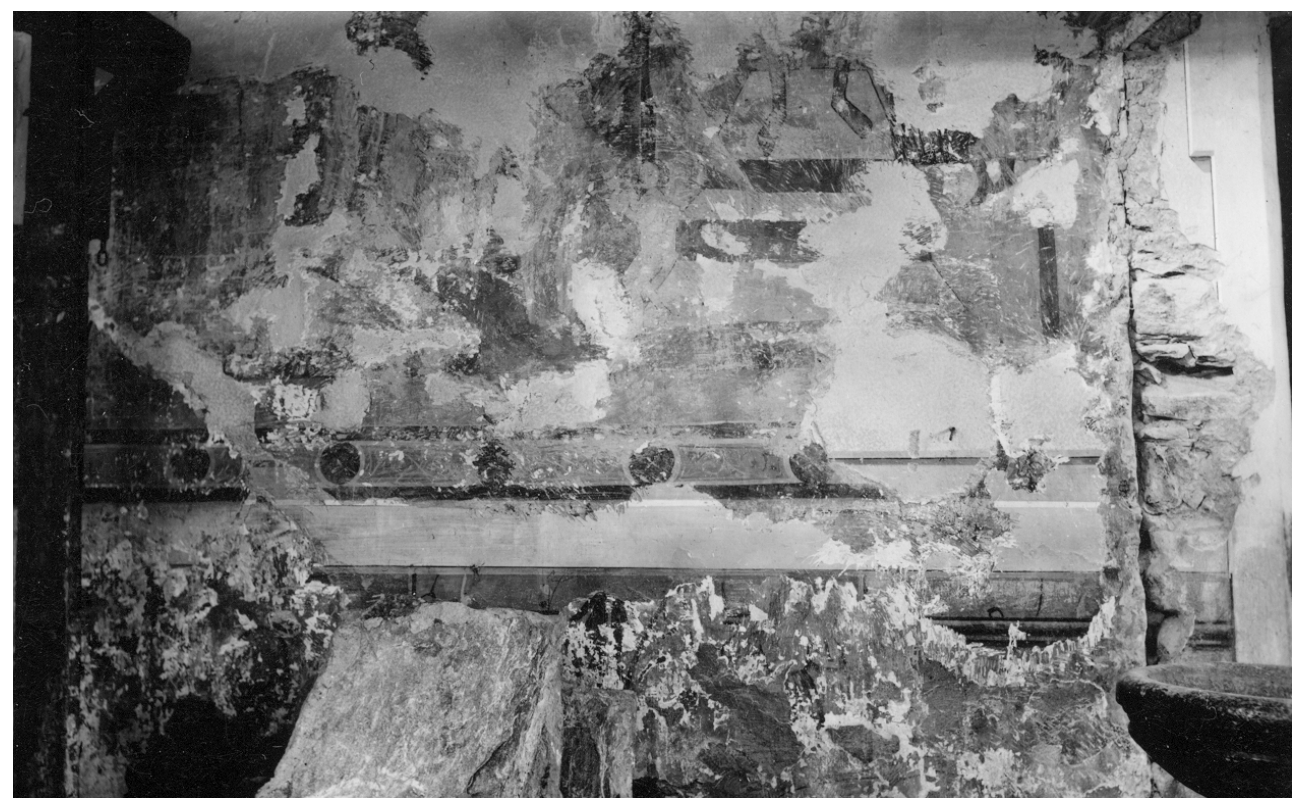

Figura 94 Fotografia dell'Interno della chiesa durante il processo di restauro, $1948 \mathrm{ca}$. AUBCBZ. Chironico. S. Ambrogio.

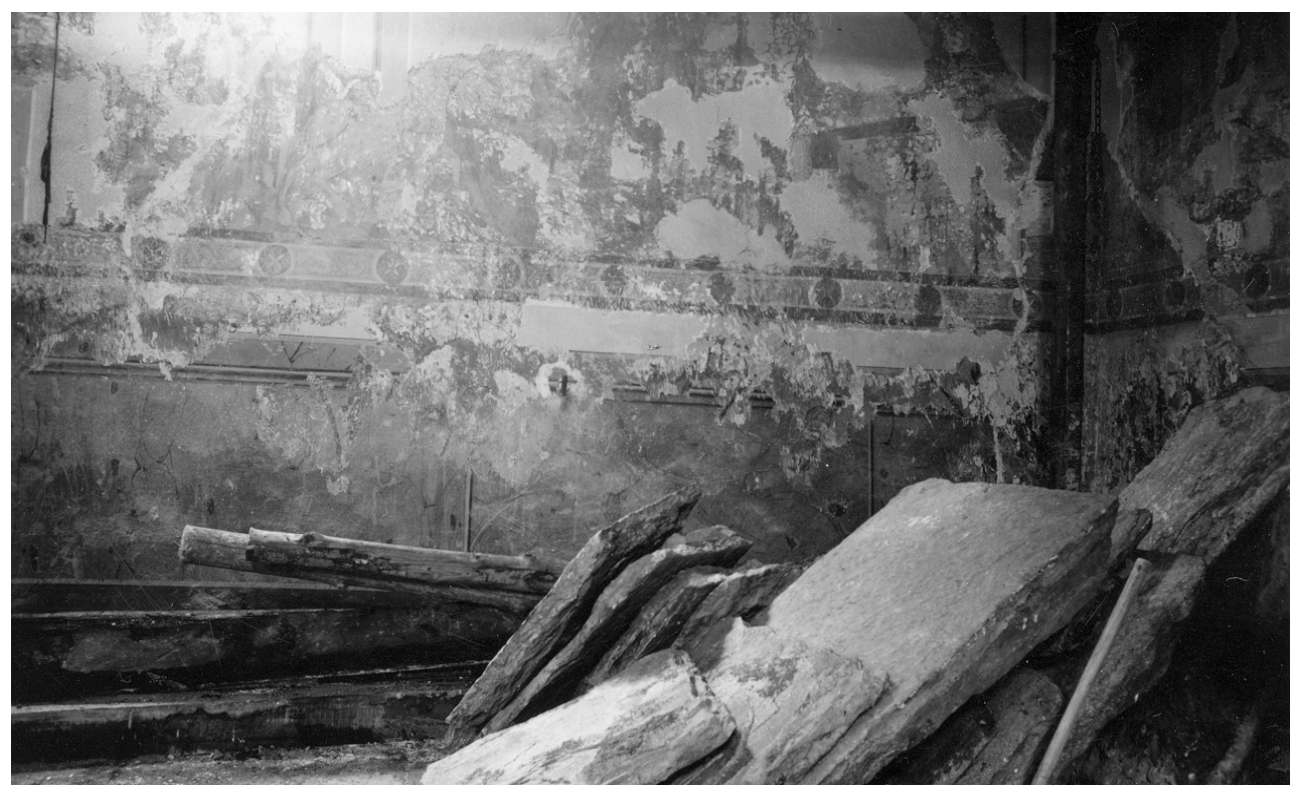

Figura 95 Fotografia dell'Interno della chiesa durante il processo di restauro, 1948 ca. AUBCBZ. Chironico. S. Ambrogio. 
A partire dai casi qui analizzati, oggetto di intervento del pittore-restauratore Ferrazzini, si può definire quella che fu la sua prassi di lavoro.

In definitiva il pittore prese come esempio i metodi di lavoro di Edoardo Berta, suo maestro nel campo del restauro dei monumenti, per cui gli ideali di restauro pittorico saranno condivise con quelli dal mancato professore.

Riassumendo i processi esecutivi che più ampiamente sono state descritti nei capitoli precedenti, si può confermare che lo scopo principali di essi fu in definitiva la ricostruzione dell'omogeneità del complesso decorativo mediante trattamenti di miglioria estetica non conservativa. Con questo si intende: I'uso della cera o della così chiamata tecnica all'encausto, utilizzata prima da Edoardo Berta ed sperimentata con nuovi materiali da Ferrazzini, che gli permetteva ridare vivezza ai colori dell'affresco; vivezza che in realtà non avevano mai avuta, però che gli faceva apparire con quella brillantezza propria delle superficie pulite, che poteva eliminare in vista ogni segno d'abrasione ed inoltre portare le tonalità cromatiche del dipinto ad uno stesso livello. Questa fu una fase di lavoro fondamentale in successione alla di pulitura della pittura murale, in generale aggressiva, con delle tecniche impiegate le stese dalla fine dell'Ottocento e fino alla metà del Novecento da tutti i pittori-restauratori: pulitura a secco con strumentale metallico per lo descialbo delle superficie, soda o potassa caustica nonché l'aggiunta di acido acetico per consentire la solubilizzazione della calce soprastante i dipinti.
L'ultimo processo, definito come "restauro" da Ferrazzini e dai suoi coetanei, consisteva alla reintegrazione pittorica, generalmente ricostruttivo. I ritocchi del pittore, nella maggior parte delle opere restaurate, sorpassano le lacune pittoriche invadendo la materia originale.

Potremmo concludere definendo Emilio Ferrazzini come un magnificò pittore ed storico dell'arte, la cui passione per essa creatività umana fecce di lui un perfetto curatore e combattente. La sua lotta per la conservazione dell'arte fu continua lungo gli anni di vita, nel territorio natale, anche se come dimostrato le doti di esecutore non furono le più idonei per $\mathrm{i}$ monumenti trattati.

Sotto la direzione di Ferrazzini altri pittori come Tita Pozzi e Carlo Cotti eseguirono perizie, preventivi e diversi interventi di restauro, anche se le prassi esecutive di questi ultimi furono in certo modo più prudenti, come a continuazione verrà dimostrato. Di fatto Carlo Cotti, che in inizio collaborò strettamente con Ferrazzini, alla fine della carriera non solo si separerà di egli a causa delle differenze personali come anche si distanzierà della metodica di restauro promossa dal pittore: rifiuterà l'impiego della cera per il fissaggio delle superficie pittoriche, i trattamenti invasivi in genere ed i ritocchi ricostruttivi.

Tita Pozzi invece eseguì le medesime prassi esecutive del Ferrazzini, apprese dai trattati di restauro italiani, ed impiegando quelli che vengono definiti come materiali di restauro "tradizionali", cioè i prodotti tramandati dai veterani sperimentati all'interno della loro bottega. La differenza fra un restauratore ed altro si è verificata 
nella cura con cui Pozzi realizzò questi interventi, di pregiata esecuzione, e nella consapevolezza che egli dimostrò, al contrario di Ferrazzini, del valore dell'opera originale: perciò i consolidamenti si limitavano alla zone di pericolo, la cera applicata sugli affreschi fu sempre stesa in minime quantità così che oggi è difficilmente ritrovabile $e$ in più non sembra avere creato ulteriori degradi sui dipinti, e per ultimo i ritocchi sono in genere contenuti, specie sulle parti figurative come nel capitolo successivo sarà documentato. 


\section{Interventi di restauro}

\begin{tabular}{|c|c|c|c|c|c|}
\hline ANNO & LUOGO & OPERA & INTERVENTO & COLLABORATORI & FONTI \\
\hline $\begin{array}{l}1891- \\
1930\end{array}$ & $\begin{array}{l}\text { Lugano, } \\
\text { chiesa di S. Maria } \\
\text { degli Angioli. } \\
\text { BA_2714. }\end{array}$ & $\begin{array}{l}\text { Affreschi della } \\
\text { Cappella Camuzio e } \\
\text { pitture nei sottarchi } \\
\text { della parete } \\
\text { trasversale. } \\
\text { BAS_12918. }\end{array}$ & $\begin{array}{l}\text { Liberazione e } \\
\text { pulitura degli } \\
\text { affreschi coperti } \\
\text { dell'intonaco. } \\
\text { Nella lettera al DPE } \\
\text { al Maestro } \\
\text { Giuseppe Rovelli il } \\
20 \text { maggio 1931, si } \\
\text { elencano le spese } \\
\text { per materiale ed } \\
\text { ore lavorative di } \\
\text { Emilio Ferrazzini } \\
\text { per ritocco e } \\
\text { sorveglianza } \\
\text { effettuate da } \\
\text { settembre a giugno } \\
\text { 1929. Nella stessa } \\
\text { fattura si elencano } \\
\text { dei materiali } \\
\text { procurati dal } \\
\text { restauratore: } \\
\text { anacrosina, } \\
\text { ceresina e colori. }\end{array}$ & $\begin{array}{l}\text { Pittori Emilio } \\
\text { Maccagni, Arturo } \\
\text { Ortelli, Emilio } \\
\text { Dillena, Christian } \\
\text { Schmidt e Tito } \\
\text { Tettamanti. } \\
\text { Diretti da } \\
\text { Edoardo Berta. }\end{array}$ & $\begin{array}{l}\text { (DPE, 1959, p. 122, } \\
\text { 143). }\end{array}$ \\
\hline $\begin{array}{l}1910- \\
1935\end{array}$ & $\begin{array}{l}\text { Bellinzona, } \\
\text { chiesa Collegiata } \\
\text { dei SS. Pietro e } \\
\text { Stefano. } \\
\text { BA_2779 }\end{array}$ & $\begin{array}{l}\text { Tela attribuita a } \\
\text { Tintoretto o alla sua } \\
\text { scuola sull'altare } \\
\text { maggiore. }\end{array}$ & $\begin{array}{l}\text { Pulitura e ritocco } \\
\text { pittorico. }\end{array}$ & & $\begin{array}{l}\text { (Chiesa F. , 1946, p. } \\
\text { 16). }\end{array}$ \\
\hline 1926 & $\begin{array}{l}\text { Bellinzona, } \\
\text { chiesa di S. Maria } \\
\text { delle Grazie. } \\
\text { BA_2788. }\end{array}$ & Affreschi. & $\begin{array}{l}\text { Lavori di ricerca e di } \\
\text { restauro } \\
\text { consistente nella } \\
\text { pulitura e } \\
\text { consolidamento. }\end{array}$ & $\begin{array}{l}\text { Pittori Carlo Cotti, } \\
\text { Christian Schmidt, } \\
\text { Emilio Dillena, A. } \\
\text { Bassi e Bruno } \\
\text { Nizzola } \\
\text { Diretti da, } \\
\text { Edoardo Berta. }\end{array}$ & $\begin{array}{l}\text { (Chiesa F. , 1946, p. } \\
27 \text { ). } \\
\text { (DPE, 1959, p. 118) }\end{array}$ \\
\hline 1929 & $\begin{array}{l}\text { Bellinzona, } \\
\text { chiesa di S. Maria } \\
\text { delle } \\
\text { Grazie } \\
\text { BA_2788. }\end{array}$ & $\begin{array}{l}\text { Profetti in } \\
\text { medaglioni. } \\
\text { Affreschi. }\end{array}$ & $\begin{array}{l}\text { Stacco di due } \\
\text { affreschi }\end{array}$ & $\begin{array}{l}\text { Pittori Carlo Cotti, } \\
\text { Christian Schmidt, } \\
\text { Emilio Dillena, A. } \\
\text { Bassi e Bruno } \\
\text { Nizzola } \\
\text { Diretti da, } \\
\text { Edoardo Berta }\end{array}$ & $\begin{array}{l}\text { (Chiesa F. , 1946, p. } \\
27) . \\
\text { (DPE, 1959, p. 118) }\end{array}$ \\
\hline $\begin{array}{l}1928 \\
\text { post/ } \\
1959 \\
\text { ante }\end{array}$ & $\begin{array}{l}\text { S. Maria di Loreto, } \\
\text { Lugano. }\end{array}$ & $\begin{array}{l}\text { Decorazioni } \\
\text { pittoriche murarie. }\end{array}$ & $\begin{array}{l}\text { Consolidamento e } \\
\text { restauro degli } \\
\text { incorniciamenti } \\
\text { decorativi delle } \\
\text { finestre e restauro }\end{array}$ & $\begin{array}{l}\text { Richiesto dal } \\
\text { Cappellano di S. } \\
\text { Maria di Loreto, } \\
\text { Padre Materno. }\end{array}$ & \\
\hline
\end{tabular}




\begin{tabular}{|c|c|c|c|c|c|}
\hline & & & $\begin{array}{l}\text { del fondo, delle } \\
\text { arcate. }\end{array}$ & & \\
\hline 1928 & $\begin{array}{l}\text { Lugano, } \\
\text { Palazzo Albertolli, } \\
\text { ora sede della } \\
\text { Banca Nazionale } \\
\text { BA_2789. }\end{array}$ & $\begin{array}{l}\text { Bernardino Luini, } \\
\text { affresco. } \\
\text { Proprietà Marco } \\
\text { Ghirlanda. } \\
\text { BAS_15309. }\end{array}$ & $\begin{array}{l}\text { Pulitura } \\
\text { dell'affresco }\end{array}$ & $\begin{array}{l}\text { Diretto da } \\
\text { Edoardo Berta }\end{array}$ & $\begin{array}{l}\text { (DPE, 1959, p. 122, } \\
143 \text { ). }\end{array}$ \\
\hline 1928 & $\begin{array}{l}\text { Lugano, } \\
\text { Palazzo Albertolli, } \\
\text { ora sede della } \\
\text { Banca Nazionale } \\
\text { BA_2789. }\end{array}$ & $\begin{array}{l}\text { Albertolli, } \\
\text { Decorazioni } \\
\text { murarie } \\
\text { neoclassiche nella } \\
\text { Sala del pian } \\
\text { terreno. }\end{array}$ & $\begin{array}{l}\text { Eliminazione dello } \\
\text { strato di calce e di } \\
\text { parati che } \\
\text { nascondevano le } \\
\text { decorazioni. } \\
\text { Pulitura, stuccatura } \\
\text { delle fessure e } \\
\text { ritocco pittorico } \\
\text { imitativo. }\end{array}$ & $\begin{array}{l}\text { Diretto da } \\
\text { Edoardo Berta. }\end{array}$ & $\begin{array}{l}\text { (Chiesa F. , 1946, p. } \\
\text { 35). }\end{array}$ \\
\hline $\begin{array}{l}\text { Ante } \\
1929\end{array}$ & $\begin{array}{l}\text { Ponte Capriasca } \\
\text { chiesa parrocchiale } \\
\text { di S. Ambrogio. } \\
\text { BA_2790. }\end{array}$ & $\begin{array}{l}\text { 1835?, affresco } \\
\text { dell'Ultima Cena } \\
\text { copia dell'opera di } \\
\text { Leonardo da Vinci. } \\
\text { BAS_15310. }\end{array}$ & $\begin{array}{l}\text { Pulitura e } \\
\text { consolidamento } \\
\text { dell'affresco. } \\
\text { Stuccatura delle } \\
\text { fessure e saldatura } \\
\text { dei bordi } \\
\text { d'intonaco staccato } \\
\text { in cemento. } \\
\text { Sulla superficie } \\
\text { pittorica } \\
\text { applicazione di uno } \\
\text { strato di cera } \\
\text { minerale per } \\
\text { consolidare e } \\
\text { proteggere } \\
\text { dall'ambiente. }\end{array}$ & $\begin{array}{l}\text { Diretto da } \\
\text { Edoardo Berta. }\end{array}$ & $\begin{array}{l}\text { (ASCT, DPE, Diversi. } \\
\text { Ferrazzini Emilio, } \\
\text { Fattura } 20 \text { maggio } \\
\text { 1931, al Maestro } \\
\text { Giuseppe Rovelli). }\end{array}$ \\
\hline $\begin{array}{l}1930- \\
1932\end{array}$ & $\begin{array}{l}\text { Malvaglia, } \\
\text { chiesa Parrocchiale } \\
\text { di S. Martino } \\
\text { BA_2782. }\end{array}$ & $\begin{array}{l}\text { Stucchi ed affreschi } \\
\text { interni ed esterni } \\
\text { BAS_15266/ } \\
15269 .\end{array}$ & $\begin{array}{l}\text { Restauro } \\
\text { consistente nella } \\
\text { pulitura e restauro } \\
\text { degli stucchi e degli } \\
\text { affreschi esterni ed } \\
\text { interni, con il } \\
\text { consolidamento di } \\
\text { questi ultimi } \\
\text { mediante } \\
\text { l'applicazione di } \\
\text { cera. } \\
\text { [...]Le vecchie } \\
\text { pitture rividero la } \\
\text { luce e giustificarono } \\
\text { i sacrifici fatti[...] } \\
\text { (Ingegnere G. } \\
\text { Baggio. Restauro } \\
\text { degli affreschi di St. }\end{array}$ & $\begin{array}{l}\text { I lavori di } \\
\text { ristrutturazione } \\
\text { architettonica } \\
\text { furono realizzati } \\
\text { dall'ingegnere } \\
\text { Baggio. } \\
\text { Coadiuvavano al } \\
\text { restauro i pittori } \\
\text { Carlo Cotti, Tita } \\
\text { Pozzi e Tito } \\
\text { Tettamanti, mentre } \\
\text { il restauro degli } \\
\text { stucchi venne } \\
\text { eseguito dal } \\
\text { gessatore Brignoni. }\end{array}$ & $\begin{array}{l}\text { (AUBCBZ, s.149 } \\
\text { Malvaglia). }\end{array}$ \\
\hline
\end{tabular}




\begin{tabular}{|c|c|c|c|c|c|}
\hline & & & $\begin{array}{l}\text { Martino. Lettera. } \\
\text { Malvaglia } 20 \\
\text { settembre } 1935 \text {, al } \\
\text { Comitato Pro- } \\
\text { Restauri. AUBCBZ, } \\
\text { Filza } 149 \text { Malvaglia) }\end{array}$ & & \\
\hline $\begin{array}{l}5 \text { ago. } \\
1931 \\
- \\
14 \text { ago. } \\
1931\end{array}$ & Giubiasco. & & $\begin{array}{l}\text { Restauro } \\
\text { [...]Ho aiutato } \\
\text { all'amico Cotti.[...] } \\
\text { Rimane ancora } \\
\text { qualcosa da finire } \\
\text { verso l'altare } \\
\text { Maggiore. Lavoro di } \\
\text { una giornata } \\
\text { circa[...] (Ferrazzini } \\
\text { Emilio, Lettera a } \\
\text { Francesco Chiesa } \\
\text { del } 19 \text { agosto } 1931 . \\
\text { AUBCBZ Filza } 206 \\
\text { Quinto). }\end{array}$ & Carlo Cotti. & $\begin{array}{l}\text { (AUBCBZ, s.080 } \\
\text { Quinto). }\end{array}$ \\
\hline 1931 & $\begin{array}{l}\text { Morbio Inferiore, } \\
\text { chiesa di San } \\
\text { Giorgio. } \\
\text { BA_2791. }\end{array}$ & $\begin{array}{l}\text { Affresco, XV sec. } \\
\text { della sagrestia. }\end{array}$ & $\begin{array}{l}\text { Pulitura e } \\
\text { consolidamento. }\end{array}$ & & $\begin{array}{l}\text { (Chiesa F. , 1946, p. } \\
43 \text { ) } \\
\text { (DPE, 1959, p. 124, } \\
\text { 170) }\end{array}$ \\
\hline 1931 & $\begin{array}{l}\text { Segno (Cavagnago), } \\
\text { oratorio di S. } \\
\text { Ambrogio } \\
\text { BA_2792 }\end{array}$ & $\begin{array}{l}\text { Ultima Cena ( } 2.80 \mathrm{X} \\
2.35 \mathrm{~m}), \mathrm{XVII} \text { sec. } \\
\text { Affresco, } \\
\text { parete sinistra della } \\
\text { navata } \\
\text { BAS_15311 } \\
\text { BAS_15317 }\end{array}$ & $\begin{array}{l}\text { Pulitura dallo strato } \\
\text { di calce superficiale } \\
\text { e consolidamento. }\end{array}$ & Carlo Cotti & $\begin{array}{l}\text { (Chiesa F. , 1946, p. } \\
44 \text { ). } \\
\text { (DPE, 1959, p. 120) } \\
\text { (AUBCBZ, 071, } \\
\text { Cavagnago). }\end{array}$ \\
\hline 1931 & $\begin{array}{l}\text { Segno (Cavagnago), } \\
\text { oratorio di S. } \\
\text { Ambrogio. } \\
\text { BA_2792. }\end{array}$ & $\begin{array}{l}\text { Quadro allegorico } \\
(2.10 \times 2.40 \mathrm{~m}) \text {; } \\
\text { Affresco, } \\
\text { sulla parete sinistra } \\
\text { della navata. } \\
\text { BAS_15311. } \\
\text { BAS_15317. }\end{array}$ & $\begin{array}{l}\text { Pulitura dallo strato } \\
\text { di calce superficiale } \\
\text { e consolidamento. }\end{array}$ & Carlo Cotti & $\begin{array}{l}\text { (Chiesa F. , 1946, p. } \\
44 \text { ) } \\
\text { (DPE, 1959, p. 120) } \\
\text { (AUBCBZ, 071, } \\
\text { Cavagnago). }\end{array}$ \\
\hline 1931 & $\begin{array}{l}\text { Segno (Cavagnago), } \\
\text { oratorio di S. } \\
\text { Ambrogio. } \\
\text { BA_2792. }\end{array}$ & $\begin{array}{l}\text { Transito di M. } \\
\text { Vergine, e } \\
\text { Adorazione dei } \\
\text { Magi (5.30 x 2.70). } \\
\text { Affresco, sulla } \\
\text { parete sinistra della } \\
\text { navata. } \\
\text { BAS_15311. } \\
\text { BAS_15317. }\end{array}$ & $\begin{array}{l}\text { Pulitura dallo strato } \\
\text { di calce superficiale } \\
\text { e consolidamento. }\end{array}$ & Carlo Cotti & $\begin{array}{l}\text { (Chiesa F. , 1946, p. } \\
44 \text { ) } \\
\text { (DPE, 1959, p. 120) } \\
\text { (AUBCBZ, 071, } \\
\text { Cavagnago) }\end{array}$ \\
\hline 1931 & $\begin{array}{l}\text { Barbengo, } \\
\text { Chiesa parrocchiale } \\
\text { di S. Ambrogio. }\end{array}$ & Dipinti murali. & $\begin{array}{l}\text { Sopralluogo per } \\
\text { verificare lo stato } \\
\text { degli affreschi }\end{array}$ & & $\begin{array}{l}\text { (AUBCBZ, 067, } \\
\text { Caslano). }\end{array}$ \\
\hline
\end{tabular}


BA_2794.

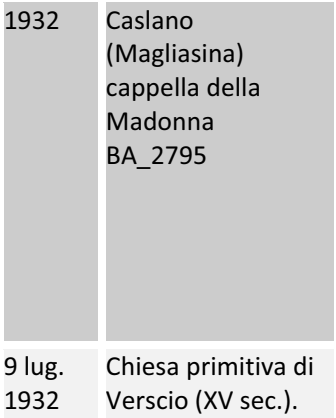

$\begin{array}{ll}13 \text { lug. } & \text { Malvaglia, } \\ 1932 & \text { Chiesa Parrocchiale } \\ \text { di S. Martino. } \\ \text { BA_2782. }\end{array}$

1931

Obino

Castel S. Pietro,

chiesa parrocchiale

$\begin{array}{ll}\text { 1932- } & \text { Bironico, } \\ \text { chiesa parrocchiale } \\ \text { dei SS. Giovanni } \\ \text { Evangelista e } \\ \text { Martino. } \\ \text { BA_2796. }\end{array}$

1934 Camignolo, cappelle della Via Crucis XVIII sec.

$\begin{array}{ll}\text { Bellinzona, } & \text { Dipinti barocchi } \\ \text { Oratorio di S. Marta } & \text { BAS_15328/ } \\ \text { BA_2797. } & \text { 15330. } \\ & \\ & \end{array}$

ritrovati sotto le

scialbature di calce.

$\begin{array}{llll}\text { Maestro Domenico } & \text { Pulitura e } & \text { Sorveglianza da } \\ \text { (Domenicus dictus } & \text { consolidamento. } & \text { Francesco Chiesa e } \\ \text { Fursnicus, le lacu } & \text { Ricollocamento } & \text { Cino Chiesa. } \\ \text { Lucani) attribuite al, } & \text { d'una pala d'altare } & \\ \text { 1534. Pitture. } & \text { nel vano } & \\ \text { BAS_15320. } & \text { dell'abside. } & \\ & \text { Restauro delle } \\ & \text { tinteggiature del } \\ & \text { portico e della } \\ & \text { cornice. }\end{array}$

Sopralluogo.

Descrive il

danneggiamento

delle pitture a

causa dall'umidità

d'infiltrazione del

tetto, il cui stato

era da verificare.

(Ferrazzini Emilio.

Lettera indirizzata

alla CCMS. Lugano

13 luglio 1932.

AUBCBZ, Verscio).

Prova di
consolidamento
con la tecnica
dell'encausto
(ozocerite $72 \circ$ di
fusione).

Sopralluogo.

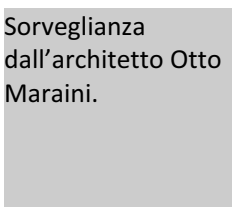

Progetto di

(AUBCBZ, s.067

restauro pittorico Caslano).

presentato a

Simone Gilardi.

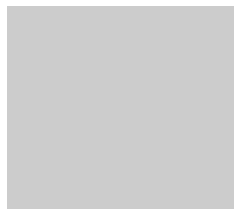

Preventivo di
restauro.

Pitture superstiti e Restauro a

sostituzione delle

pitture totalmente scomparse.

Sorveglianza ai
lavori di restauro.
Gli interventi
finanziati dal
Reverendo Don
Giosuè Prada
interessarono
l'eliminazione dei
problema
dell'umidità,

Sorvegliana air

Sorveglianza ai

lavori da Giuseppe

(AUBCBZ, s.149

Malvaglia).

Weith.

I lavori furono
eseguiti dal pittore
Tita Pozzi.

(AUBCBZ 021 ,

Bellinzona)

(Consiglio di Stato.

Lettera indirizzata al

Dipertimento della pubblica

Educazione.

Bellinzona 8 giugno

1934. AUBCBZ s.

021, Bellinzona). 


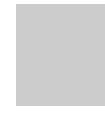

1935

Morcote,

chiesa parrocchiale di S. Maria del

Sasso.

BA_2798.

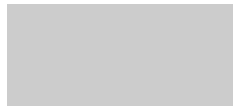

Pitture murale settecentesche della facciata BAS_15331/ 1533215360 .

\begin{tabular}{|c|c|}
\hline 1935 & $\begin{array}{l}\text { Morcote, } \\
\text { chiesa parrocchiale } \\
\text { di S. Maria del } \\
\text { Sasso. } \\
\text { BA } 2798\end{array}$ \\
\hline
\end{tabular}

SS. Tommaso,

Agostino e Gerolamo e il Padre Eterno sulla volta delle campata S-E. pulitura e

consolidamento dei dipinti.

Il restauro pittorico Comitato restauri: eseguito dal pittore A.T. Isella Emilio Ferrazzini Sindaco: Signor consiste nella Tamborini pulitura, sigillatura Rev. Arciprete Don dei buchi, raccordi Valentini diversi, ecc.
II restauro Sr. Vittorio Gianella architettonico Franchi e Sr.

eseguito secondo il Maspoli. progetto di Chiesa Cino, interessavano Diretto l'armonizzazione degli eclettici dall'architetto Cino elementi che conformavano la fabbrica di origine romanica, eliminando le pitture e stucchi che disturbassero il carattere di questa. Nella navata mediana vengono rimossi gli stucchi degradati e rifatte le parte mancanti, sistemate le lesene, capitelli e cornici, eliminate le decorazioni sulla volta e rifatti gli intonaci.

Nella cappella delle Madonna si esegue la pulitura degli stucchi ed il completamento di essi, la tinteggiatura a due tinte delle pareti e la ridora tura a foglia degli stucchi restaurati.

Pulitura, stuccatura Cino Chiesa.
e ritocco pittorico.

(Chiesa F. , 1946, p. 55).

(AUBCBZ s.172 Morcote).

Chiesa.
(Chiesa F. , 1946, p. 55). (AUBCBZ s.172 Morcote). 


\begin{tabular}{|c|c|c|c|c|c|}
\hline & & $\begin{array}{l}\text { Figure di sante nel } \\
\text { sottarco. }\end{array}$ & & & \\
\hline 1935 & $\begin{array}{l}\text { Morcote, } \\
\text { chiesa parrocchiale } \\
\text { di S. Maria del } \\
\text { Sasso. } \\
\text { BA_2798. }\end{array}$ & $\begin{array}{l}\text { Miracolo di S. } \\
\text { Francesco, } \\
\text { campata N-E. }\end{array}$ & $\begin{array}{l}\text { Pulitura, stuccatura } \\
\text { e ritocco pittorico. }\end{array}$ & Cino Chiesa. & $\begin{array}{l}\text { (Chiesa F. , 1946, p. } \\
\text { 55) } \\
\text { (AUBCBZ s.172 } \\
\text { Morcote). }\end{array}$ \\
\hline 1935 & $\begin{array}{l}\text { Morcote, } \\
\text { chiesa parrocchiale } \\
\text { di S. Maria del } \\
\text { Sasso. } \\
\text { BA_2798. }\end{array}$ & $\begin{array}{l}\text { Giovanni Battista } \\
\text { Tarilli attribuito a, } \\
\text { 1595. Stemma della } \\
\text { famiglia } \\
\text { committente } \\
\text { Fossati, campata N- } \\
\text { E. }\end{array}$ & $\begin{array}{l}\text { Pulitura, stuccatura } \\
\text { e ritocco pittorico. }\end{array}$ & Cino Chiesa. & $\begin{array}{l}\text { (Chiesa F. , 1946, p. } \\
\text { 55). } \\
\text { (AUBCBZ s.172 } \\
\text { Morcote). }\end{array}$ \\
\hline 1935 & $\begin{array}{l}\text { Morcote, } \\
\text { chiesa parrocchiale } \\
\text { di S. Maria del } \\
\text { Sasso. } \\
\text { BA_2798. }\end{array}$ & $\begin{array}{l}\text { 1614, San Carlo e la } \\
\text { Madonna di Loreto } \\
\text { sul registro } \\
\text { inferiore della } \\
\text { campata N-E. }\end{array}$ & $\begin{array}{l}\text { Pulitura, stuccatura } \\
\text { e ritocco pittorico. }\end{array}$ & Cino Chiesa. & $\begin{array}{l}\text { (Chiesa F. , 1946, p. } \\
\text { 55) } \\
\text { (AUBCBZ s.172 } \\
\text { Morcote) }\end{array}$ \\
\hline 1935 & $\begin{array}{l}\text { Morcote, } \\
\text { chiesa parrocchiale } \\
\text { di S. Maria del } \\
\text { Sasso } \\
\text { BA_2798 }\end{array}$ & $\begin{array}{l}\text { Pesca miracolosa; } \\
\text { 1614, SS. Giovanni } \\
\text { Battista e Antonio } \\
\text { abate. } \\
\text { campata N-O. }\end{array}$ & $\begin{array}{l}\text { Pulitura, stuccatura } \\
\text { e ritocco pittorico. }\end{array}$ & Cino Chiesa. & $\begin{array}{l}\text { (Chiesa F. , 1946, p. } \\
\text { 55). } \\
\text { (AUBCBZ s.172 } \\
\text { Morcote). }\end{array}$ \\
\hline 1935 & $\begin{array}{l}\text { Morcote, } \\
\text { chiesa parrocchiale } \\
\text { di S. Maria del } \\
\text { Sasso. } \\
\text { BA_2798. }\end{array}$ & $\begin{array}{l}\text { Madonna col } \\
\text { bambino, } \\
\text { parete N. }\end{array}$ & $\begin{array}{l}\text { Pulitura, stuccatura } \\
\text { e ritocco pittorico. }\end{array}$ & Cino Chiesa. & $\begin{array}{l}\text { (Chiesa F. , 1946, p. } \\
\text { 55). } \\
\text { (AUBCBZ s.172 } \\
\text { Morcote). }\end{array}$ \\
\hline 1935 & $\begin{array}{l}\text { Morcote, } \\
\text { chiesa parrocchiale } \\
\text { di S. Maria del } \\
\text { Sasso. } \\
\text { BA_2798. }\end{array}$ & $\begin{array}{l}\text { Medaglioni con la } \\
\text { colomba dello } \\
\text { Spirito Santo e } \\
\text { angeli, volta a N. }\end{array}$ & $\begin{array}{l}\text { Pulitura, stuccatura } \\
\text { e ritocco pittorico. }\end{array}$ & Cino Chiesa. & $\begin{array}{l}\text { (Chiesa F. , 1946, p. } \\
\text { 55). } \\
\text { (AUBCBZ s.172 } \\
\text { Morcote). }\end{array}$ \\
\hline 1935 & $\begin{array}{l}\text { Morcote, } \\
\text { chiesa parrocchiale } \\
\text { di S. Maria del } \\
\text { Sasso } \\
\text { BA_2798 }\end{array}$ & $\begin{array}{l}\text { Sibille e gli } \\
\text { evangelisti Giovanni } \\
\text { e Luca, sugli } \\
\text { intradossi degli } \\
\text { archi nella volta a } \\
\text { N. }\end{array}$ & $\begin{array}{l}\text { Pulitura, stuccatura } \\
\text { e ritocco pittorico. }\end{array}$ & Cino Chiesa. & $\begin{array}{l}\text { (Chiesa F. , 1946, p. } \\
\text { 55). } \\
\text { (AUBCBZ s.172 } \\
\text { Morcote). }\end{array}$ \\
\hline 1935 & $\begin{array}{l}\text { Morcote, } \\
\text { chiesa Parrocchiale } \\
\text { di S. Maria del } \\
\text { Sasso. } \\
\text { BA_2798. }\end{array}$ & $\begin{array}{l}\text { Giovanni Battista } \\
\text { Tarilli, 1595, firma } \\
\text { gli affreschi } \\
\text { raffiguranti i } \\
\text { quattro episodi } \\
\text { della vita di S. } \\
\text { Giovanni Battista } \\
\text { nelle vele della } \\
\text { volta nella campata } \\
\text { a S-O ed i Profeti }\end{array}$ & $\begin{array}{l}\text { Pulitura, stuccatura } \\
\text { e ritocco pittorico. }\end{array}$ & Cino Chiesa. & $\begin{array}{l}\text { (Chiesa F. , 1946, p. } \\
\text { 55). } \\
\text { (DPE, 1959, p. 124, } \\
\text { 172). } \\
\text { (Chiesa F. , 1946, p. } \\
\text { 55). } \\
\text { (AUBCBZ s.172 } \\
\text { Morcote). }\end{array}$ \\
\hline
\end{tabular}




\begin{tabular}{|c|c|c|c|c|}
\hline & & $\begin{array}{l}\text { sull'intradosso } \\
\text { dell'arco. }\end{array}$ & & \\
\hline 1939 & $\begin{array}{l}\text { Locarno, } \\
\text { chiesa di San } \\
\text { Francesco. }\end{array}$ & & $\begin{array}{l}\text { Richiesta di } \\
\text { restauro pittorico } \\
\text { dal Padre Francesco } \\
\text { Huninger, } \\
\text { aspettando un } \\
\text { adeguato sussidio. }\end{array}$ & $\begin{array}{l}\text { (Chiesa F. , 1946, p. } \\
\text { 29). }\end{array}$ \\
\hline $\begin{array}{l}18 \text { giu. } \\
1940\end{array}$ & $\begin{array}{l}\text { Ponte Capriasca, } \\
\text { parrocchiale di S. } \\
\text { Ambrogio. } \\
\text { BA_2790. }\end{array}$ & $\begin{array}{l}\text { Affresco della } \\
\text { Natività. } \\
\text { BAS_15362?. }\end{array}$ & $\begin{array}{l}\text { Pulitura } \\
\text { dell'affresco } \\
\text { degradato: sporco, } \\
\text { opaco e coperto da } \\
\text { una patina } \\
\text { verdastra a causa } \\
\text { del solfato di rame } \\
\text { applicato dal } \\
\text { parroco nel vicino } \\
\text { vigneto. } \\
\text { Questa pulitura è } \\
\text { stata possibile } \\
\text { grazie alla vernice } \\
\text { di cera minerale } \\
\text { applicata nel } 1924, \\
\text { la quale non ha } \\
\text { permesso la } \\
\text { penetrazione del } \\
\text { solfato di rame } \\
\text { nella superficie } \\
\text { colorata. } \\
\text { Ferrazzini si difende } \\
\text { in questo modo } \\
\text { dall'articolo scritto } \\
\text { da Ugo Donati sul } \\
\text { Giornale del Popolo } \\
\text { il } 20 \text { giugno } 1939 \\
\text { dove si accusava il } \\
\text { cattivo stato di } \\
\text { conservazione del } \\
\text { dipinto. }\end{array}$ & $\begin{array}{l}\text { (AUBCBZ, s.196, } \\
\text { Ponte Capriasca). }\end{array}$ \\
\hline 1936 & $\begin{array}{l}\text { Russo, } \\
\text { chiesa parrocchiale } \\
\text { della Madonna } \\
\text { Assunta. } \\
\text { BA_2800. }\end{array}$ & $\begin{array}{l}\text { S. Cristoforo sulla } \\
\text { facciata; affresco } \\
\text { sec. XV. Unica } \\
\text { pintura medievale } \\
\text { esistente in tutta la } \\
\text { Valle Onsernone. } \\
\text { BAS_15363. }\end{array}$ & $\begin{array}{l}\text { Consolidamento del } \\
\text { dipinto molto } \\
\text { degradato a causa } \\
\text { di corrosione e } \\
\text { stacco dell'intonaco } \\
\text { con cera d'api. } \\
\text { Stuccatura e } \\
\text { ritocco. } \\
\text { [...]Facciamo inoltre } \\
\text { presente che sarà } \\
\text { bene, avvenute che } \\
\text { siano le necessarie }\end{array}$ & $\begin{array}{l}\text { (Chiesa F. , 1946, p. } \\
\text { 56). } \\
\text { (DPE, 1959, p. 126, } \\
216 \text { ). } \\
\text { (AUBCBZ s. } 216 \\
\text { Russo). }\end{array}$ \\
\hline
\end{tabular}




\begin{tabular}{|c|c|c|c|c|c|}
\hline & & & $\begin{array}{l}\text { riparazioni, fissare } \\
\text { sull'affresco una } \\
\text { retina metallica } \\
\text { onde preservarlo } \\
\text { dai colpi di frale di } \\
\text { neve e di sassi che } \\
\text { purtroppo vengono } \\
\text { lanciati dai ragazzi } \\
\text { che non sanno } \\
\text { apprezzare il valore } \\
\text { artistico.[...] } \\
\text { (Consiglio } \\
\text { Parrocchiale di } \\
\text { Russo, Lettera. } 26 \\
\text { settembre } 1935 \text { alla } \\
\text { CCMS. AUBCBZ, } 216 \\
\text { Russo.) }\end{array}$ & & \\
\hline $\begin{array}{l}1937- \\
1941\end{array}$ & $\begin{array}{l}\text { Mendrisio, Ruderi } \\
\text { di torre medievale. }\end{array}$ & & $\begin{array}{l}\text { Direzione dei lavori } \\
\text { di manutenzione } \\
\text { sostenuti contro il } \\
\text { progetto di } \\
\text { demolizioni } \\
\text { dall'arch. Guglielmo } \\
\text { de Angelis d'Ossat. }\end{array}$ & & $\begin{array}{l}\text { (DPE, 1959, p. 122, } \\
\text { 157). }\end{array}$ \\
\hline $\begin{array}{l}1937 \text { - } \\
1938\end{array}$ & $\begin{array}{l}\text { Arzo, } \\
\text { oratorio della } \\
\text { Madonna del } \\
\text { Ponte. } \\
\text { BA_2801. }\end{array}$ & & $\begin{array}{l}\text { Vigila lavori di } \\
\text { restauro e di } \\
\text { tinteggiatura. }\end{array}$ & & $\begin{array}{l}\text { (Chiesa F. , 1946, p. } \\
\text { 57). } \\
\text { (DPE, 1959, p. 117). } \\
\text { (AUBCBZ, 010, Arzo) }\end{array}$ \\
\hline $\begin{array}{l}1938- \\
\text { ago. } \\
1939\end{array}$ & $\begin{array}{l}\text { Deggio (Quinto) } \\
\text { chiesa di S. Martino } \\
\text { BA_2802. }\end{array}$ & $\begin{array}{l}\text { Affresco } \\
\text { raffigurante S. } \\
\text { Cristoforo; alcuni } \\
\text { motivi decorativi } \\
\text { intorno alla } \\
\text { porticina sud ed alla } \\
\text { finestra } \\
\text { sovrastante. } \\
\text { Esterno. } \\
\text { BAS_15366. }\end{array}$ & $\begin{array}{l}\text { Ricerche. Pulitura } \\
\text { degli affreschi dallo } \\
\text { strato di calce } \\
\text { superficiale. }\end{array}$ & $\begin{array}{l}\text { Il progetto di } \\
\text { ripristino } \\
\text { architettonico } \\
\text { venne eseguito } \\
\text { dall'architetto } \\
\text { Paolo Mariotta. } \\
\text { Questo consisteva } \\
\text { nella sistemazione } \\
\text { del tetto, la } \\
\text { costruzione di un } \\
\text { nuovo soffitto in } \\
\text { legno d'abete, il } \\
\text { rinnovamento degli } \\
\text { intonaci interni ed } \\
\text { esterni, la } \\
\text { rimozione } \\
\text { dell'altare in } \\
\text { muratura e la } \\
\text { costruzione di uno } \\
\text { nuovo in granito; la } \\
\text { riapertura delle due }\end{array}$ & $\begin{array}{l}\text { (Chiesa F. , 1946, p. } \\
\text { 60). } \\
\text { (DPE, 1959, p. 125). } \\
\text { (AUBCBZ, s.206, } \\
\text { Quinto). } \\
\text { (AUBCBZ, s.149 } \\
\text { Malvaglia). }\end{array}$ \\
\hline
\end{tabular}




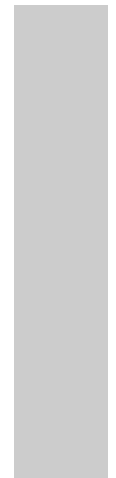

1938 -

ago. 1939.

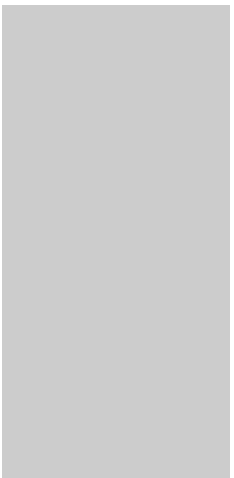

Deggio (Quinto) chiesa di S.

Martino. BA 2802.

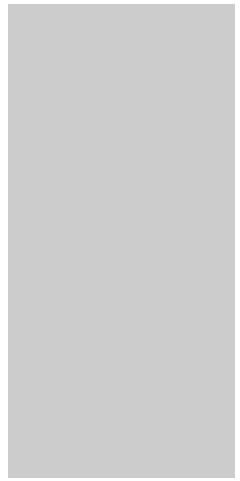

Pantocrator e simboli degli Evangelisti, nel coro. (BAS 15368).

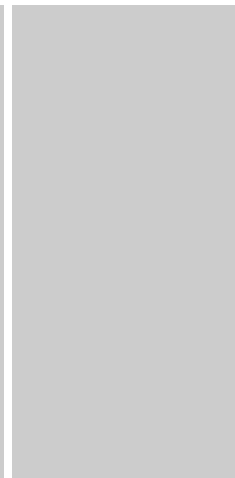

Put e consolidamento Ugo Tarabori con la tecnica dell'encausto. finestre romaniche del coro, la sostituzione della vecchia porta a ponente e riparazione di quella di levante e la costruzione di un intercapedine lungo le pareti nord della chiesa per evitare i problemi di umidità per risalita capillare.

(Ispettore

Cantonale).
(Chiesa F. , 1946, p.

60).

(DPE, 1959, p. 125).

(AUBCBZ, s.206,

Quinto).

(AUBCBZ, s.149

Malvaglia).

$\begin{array}{ll}\text { 1938 - } & \text { Deggio (Quinto) } \\ \text { ago. } & \text { chiesa di S. } \\ \text { 1939. } & \text { Martino. } \\ & \text { BA_2802. }\end{array}$

1938 - Deggio (Quinto)

ago. chiesa di S.

1939 Martino.

BA_2802.

\section{Deposizione di}

Ricerche. Pulitura
dello strato di calce superficiale e consolidamento con la tecnica dell'encausto.

Ricerche. Pulitura Arch. Paolo dello strato di calce Mariotta

\section{Salita nella tomba, parete destra dell'altare.} Gesù nella tomba, frammenti.

Affresco. Parete di sinistra dell'altare.

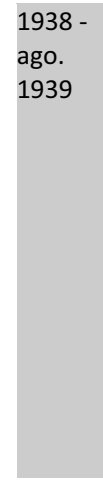

1938 ago.
Deggio (Quinto) chiesa di S. Martino. BA_2802.

\section{Deggio (Quinto)} chiesa di S.

\section{Cristo crocifisso figure di Maria e di S. Giovanni, sulla parete del fondo. Sullo zoccolo di questa parete interessante traccia di affreschi: un drago (BAS_15372) che sta per divorare un uccello, un fregio di pesci (BAS_15373).} (BAS_15369), con le rivelano la

Annunciazione (BAS_15367) superficiale e consolidamento con la tecnica dell'encausto.

Ricerche che

sovrapposizione di (Progetto). almeno quattro strati di intonaco diversi. Pulitura dello strato di calce sovrastanti ai dipinti più recenti e consolidamento con la tecnica dell'encausto.

(Progetto). Ugo Tarabori (Ispettore Cantonale).

Arch. Paolo
Mariotta
(Progetto).
Ugo Tarabori
(Ispettore
Cantonale).

Ricerche. Pulitura Arch. Paolo dello strato di calce Mariotta
(Chiesa F. , 1946, p. 60).

(DPE, 1959, p. 125). (AUBCBZ, s.206, Quinto). (AUBCBZ, s.149 Malvaglia).

(Chiesa F. , 1946, p. $60)$.

(DPE, 1959, p. 125). (AUBCBZ, s.206, Quinto).

(AUBCBZ, s.149 Malvaglia).

(Chiesa F. , 1946, p. 60).

(DPE, 1959, p. 125). (AUBCBZ, s.206, Quinto). (AUBCBZ, s.149 Malvaglia).

(Chiesa F. , 1946, p. $60)$. 


\begin{tabular}{|c|c|c|c|c|c|}
\hline 1939 & $\begin{array}{l}\text { Martino. } \\
\text { BA_2802. }\end{array}$ & $\begin{array}{l}\text { sull'arcosoglio e } \\
\text { traccia di un S. } \\
\text { Lorenzo. }\end{array}$ & $\begin{array}{l}\text { sovrastanti ai } \\
\text { dipinti più recenti e } \\
\text { consolidamento } \\
\text { con la tecnica } \\
\text { dell'encausto. }\end{array}$ & $\begin{array}{l}\text { (Progetto). } \\
\text { Ugo Tarabori } \\
\text { (Ispettore } \\
\text { Cantonale). }\end{array}$ & $\begin{array}{l}\text { (DPE, 1959, p. 125). } \\
\text { (AUBCBZ, s.206, } \\
\text { Quinto). } \\
\text { (AUBCBZ, s.149 } \\
\text { Malvaglia). }\end{array}$ \\
\hline $\begin{array}{l}1938- \\
\text { ago. } \\
1939\end{array}$ & $\begin{array}{l}\text { Deggio (Quinto) } \\
\text { chiesa di S. } \\
\text { Martino. } \\
\text { BA_2802. }\end{array}$ & $\begin{array}{l}\text { S. Martino, } \\
\text { Affresco } \\
\text { (BAS_15370). }\end{array}$ & $\begin{array}{l}\text { L'unico affresco } \\
\text { dell'Oratorio già } \\
\text { visibile prima dei } \\
\text { restauri fu ritrovato } \\
\text { in buono stato di } \\
\text { conservazione per } \\
\text { cui Ferrazzini } \\
\text { intervenne } \\
\text { unicamente nella } \\
\text { cornice che } \\
\text { racchiude il dipinto } \\
\text { eliminando le } \\
\text { diverse } \\
\text { imbiancature che } \\
\text { con il tempo la } \\
\text { avevano degradata } \\
\text { e realizzando il } \\
\text { ritocco pittorico. }\end{array}$ & $\begin{array}{l}\text { Arch. Paolo } \\
\text { Mariotta } \\
\text { (Progetto). } \\
\text { Ugo Tarabori } \\
\text { (Ispettore } \\
\text { Cantonale). }\end{array}$ & $\begin{array}{l}\text { (Chiesa F. , 1946, p. } \\
\text { 60). } \\
\text { (DPE, 1959, p. 125). } \\
\text { (AUBCBZ, s.206, } \\
\text { Quinto). } \\
\text { (AUBCBZ, s.149 } \\
\text { Malvaglia). }\end{array}$ \\
\hline $\begin{array}{l}1938- \\
\text { ago. } \\
1939\end{array}$ & $\begin{array}{l}\text { Deggio (Quinto) } \\
\text { chiesa di S. } \\
\text { Martino. } \\
\text { BA_2802. }\end{array}$ & $\begin{array}{l}\text { Nella parete di } \\
\text { fronte si ritrovò una } \\
\text { grande "Cena" } \\
\text { (BAS_15371). }\end{array}$ & $\begin{array}{l}\text { Ricerche. Pulitura } \\
\text { dallo strato di calce. } \\
\text { Consolidamento } \\
\text { con la tecnica } \\
\text { dell'encausto. } \\
\text { [...] altri avanzi di } \\
\text { dipinti si notano } \\
\text { ancora sulla parete } \\
\text { sottostante a } \\
\text { questo affresco e } \\
\text { più vicino } \\
\text { all'ingresso } \\
\text { principale alcuni } \\
\text { frammenti di } \\
\text { ornamenti e tracce } \\
\text { di un S. Michele.[...] } \\
\text { (Ferrazzini, 1939). }\end{array}$ & $\begin{array}{l}\text { Arch. Paolo } \\
\text { Mariotta } \\
\text { (Progetto). } \\
\text { Ugo Tarabori } \\
\text { (Ispettore } \\
\text { Cantonale). }\end{array}$ & $\begin{array}{l}\text { (Chiesa F. , 1946, p. } \\
\text { 60). } \\
\text { (DPE, 1959, p. 125). } \\
\text { (AUBCBZ, s.206, } \\
\text { Quinto). } \\
\text { (AUBCBZ, s.149 } \\
\text { Malvaglia). }\end{array}$ \\
\hline 1939 & $\begin{array}{l}\text { Scona, } \\
\text { S. Colombano. }\end{array}$ & $\begin{array}{l}\text { Re Davide e } \\
\text { Salomone, S. } \\
\text { Bernardo da } \\
\text { Mentone e S. } \\
\text { Antonio in } \\
\text { medaglioni, sul'arco } \\
\text { della cappella } \\
\text { primitiva. Affreschi } \\
\text { XVI sec. }\end{array}$ & $\begin{array}{l}\text { Ricerche. Furono } \\
\text { ritrovati dopo } \\
\text { l'eliminazione del } \\
\text { muro che divideva } \\
\text { la cappella primitiva } \\
\text { dedicata a S. } \\
\text { Colombano. }\end{array}$ & $\begin{array}{l}\text { Coadiuvato dal } \\
\text { pittore Perlasca. }\end{array}$ & $\begin{array}{l}\text { (Ferrazzini, La } \\
\text { chiesetta di S. } \\
\text { Colombano di } \\
\text { Scona, 1940). }\end{array}$ \\
\hline 1939 & $\begin{array}{l}\text { Scona, } \\
\text { S. Colombano. }\end{array}$ & $\begin{array}{l}\text { Evangelisti, volta } \\
\text { della cappella }\end{array}$ & Ricerche. & $\begin{array}{l}\text { Coadiuvato dal } \\
\text { pittore Perlasca }\end{array}$ & $\begin{array}{l}\text { (Ferrazzini, La } \\
\text { chiesetta di S. }\end{array}$ \\
\hline
\end{tabular}




\begin{tabular}{|c|c|c|c|c|c|}
\hline & & $\begin{array}{l}\text { primitiva. Affreschi, } \\
\text { XVI sec. }\end{array}$ & & & $\begin{array}{l}\text { Colombano di } \\
\text { Scona, 1940). }\end{array}$ \\
\hline 1939 & $\begin{array}{l}\text { Scona, } \\
\text { S. Colombano. }\end{array}$ & $\begin{array}{l}\text { Crocefisso con la } \\
\text { Madonna, S. } \\
\text { Giovanni e S. } \\
\text { Martino e S. } \\
\text { Colombano, sulla } \\
\text { parete in fondo } \\
\text { della cappella } \\
\text { primitiva. Affreschi, } \\
\text { XVI sec. }\end{array}$ & $\begin{array}{l}\text { Ricerche. Gli } \\
\text { affreschi si } \\
\text { ritrovarono molto } \\
\text { degradati, } \\
\text { rimanendo soltanto } \\
\text { piccole tracce. }\end{array}$ & $\begin{array}{l}\text { Coadiuvato dal } \\
\text { pittore Perlasca. }\end{array}$ & $\begin{array}{l}\text { (Ferrazzini, La } \\
\text { chiesetta di S. } \\
\text { Colombano di } \\
\text { Scona, 1940). }\end{array}$ \\
\hline 1939 & $\begin{array}{l}\text { Scona, } \\
\text { S. Colombano. }\end{array}$ & Affreschi. & $\begin{array}{l}\text { Ricerche. [....]Nel } \\
\text { corso dei lavori } \\
\text { sono stati ritrovati } \\
\text { avanzi d'un affresco } \\
\text { con diverse figure e } \\
\text { la dicitura "Coma } \\
\text { Uberto Consula } \\
\text { della vicinanza da } \\
\text { Arivoro Ha fato fare } \\
\text { questo... } 161 \text { Et } \\
\text { curatore della } \\
\text { Giesa..."[...] } \\
\text { (Ferrazzini, La } \\
\text { chiesetta di S. } \\
\text { Colombano di } \\
\text { Scona, 1940, p. } \\
\text { 316). }\end{array}$ & $\begin{array}{l}\text { Coadiuvato dal } \\
\text { pittore Perlasca. }\end{array}$ & $\begin{array}{l}\text { (Ferrazzini, La } \\
\text { chiesetta di S. } \\
\text { Colombano di } \\
\text { Scona, 1940). }\end{array}$ \\
\hline 1939 & $\begin{array}{l}\text { Scona, } \\
\text { S. Colombano }\end{array}$ & $\begin{array}{l}\text { S. Bartolomeo, S. } \\
\text { Giovanni e zoccolo } \\
\text { decorativo sulle } \\
\text { pareti laterali. } \\
\text { Affreschi. }\end{array}$ & $\begin{array}{l}\text { Ricerche. Pulitura } \\
\text { dello strato di calce } \\
\text { superficiale. Gli } \\
\text { affreschi si } \\
\text { ritrovarono molto } \\
\text { degradati e rotti da } \\
\text { due finestrine, } \\
\text { rimanendo soltanto } \\
\text { alcune tracce }\end{array}$ & $\begin{array}{l}\text { Coadiuvato dal } \\
\text { pittore Perlasca. }\end{array}$ & $\begin{array}{l}\text { (Ferrazzini, La } \\
\text { chiesetta di S. } \\
\text { Colombano di } \\
\text { Scona, 1940). }\end{array}$ \\
\hline 1939 & $\begin{array}{l}\text { Scona, } \\
\text { S. Colombano. }\end{array}$ & $\begin{array}{l}\text { S. Rocco e S. } \\
\text { Sebastiano, traccia } \\
\text { sulle pareti laterali. } \\
\text { Affreschi. }\end{array}$ & $\begin{array}{l}\text { Ricerche. Pulitura } \\
\text { dello strato di calce } \\
\text { superficiale e } \\
\text { consolidamento. }\end{array}$ & $\begin{array}{l}\text { Coadiuvato dal } \\
\text { pittore Perlasca. }\end{array}$ & $\begin{array}{l}\text { (Ferrazzini, La } \\
\text { chiesetta di S. } \\
\text { Colombano di } \\
\text { Scona, 1940). }\end{array}$ \\
\hline 1941 & $\begin{array}{l}\text { Riva San Vitale, } \\
\text { chiesa di } \\
\text { S. Croce. }\end{array}$ & $\begin{array}{l}\text { Petrini, } \\
\text { Tele degli altari. }\end{array}$ & $\begin{array}{l}\text { Riparazione e } \\
\text { consolidamento. }\end{array}$ & & $\begin{array}{l}\text { (Chiesa F. , 1946, p. } \\
63 \text {. } \\
\text { (DPE, 1959, p. 125). } \\
\text { (AUBCBZ, s. 209, } \\
\text { Riva San Vitale). }\end{array}$ \\
\hline
\end{tabular}


S. Maria di Loreto, Cappella di S. Lugano.

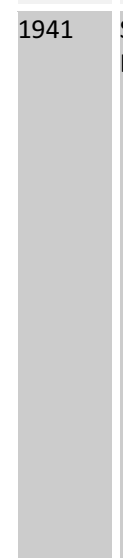

1941

$\begin{array}{ll} & \text { Lugano. } \\ 1942 \text { - } & \text { Negrentino, } \\ 1943 & \begin{array}{l}\text { Prugiasco, } \\ \text { chiesa di } \\ \text { S. Carlo. }\end{array}\end{array}$

1943

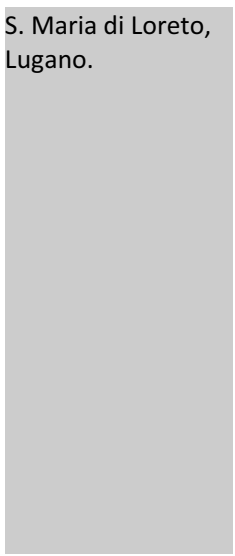

S. Maria di Loreto, Giornico, cappella delle Grazie.

Chiesa di

S. Nicolao.

BA 2777.
Rifacimento degli

intonaci guastati

dall'umidità,

restauro del

pavimento in

mattonelle,

restauro e tinteggio

(a tempera) degli

stucchi,

completamento

della decorazione

della cappella con

affreschi nei piccoli

riquadri,

raffigurazioni della

vita di S. Antonio

secondo i bozzetti

presentati alla

Commissione

Cantonale dei

monumenti.

\section{Rifacimento del}

pavimento in

mattonelle di

terracotta da

collocare al posto

del pavimento in

piastrelle di

cemento,

decorazione delle

pareti della

cappella con muri

ad imitazione dei

mattoni, come le

pitture originarie

ritrovate sotto le

imbiancature.

Restauro.

$$
\text { affreschi. }
$$

Affreschi.

Sorveglianza de

lavori eseguiti da

Pozzi di restauro

pittorico, pulitura e

consolidamento

degli affreschi.

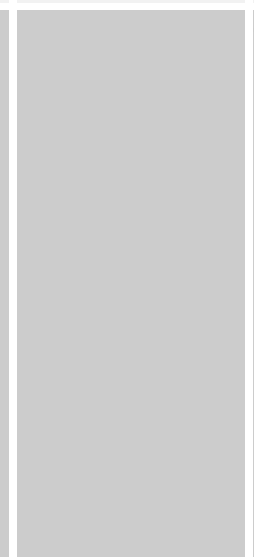

(AUBCBZ, s.143,

Lugano).

(AUBCBZ, s.143,

Lugano).

Titta Pozzi
(operatore).

(Chiesa F. , 1946, p.

75).

(DPE, 1959, p. 125).

(AUBCBZ, s.204,

Prugiasco).

(Chiesa F. , 1946, p.

73).

(DPE, 1959, p. 122).

(AUBCBZ, s. 143

Giornico). 


\begin{tabular}{|c|c|c|c|c|c|}
\hline 1943 & $\begin{array}{l}\text { Villa Luganese, } \\
\text { chiesa Parrocchiale } \\
\text { della B.V Assunta. }\end{array}$ & $\begin{array}{l}\text { Santa Martire con } \\
\text { angeli reggenti una } \\
\text { corono per } \\
\text { difendere l'anima di } \\
\text { un defunto dal } \\
\text { demonio e i SS. } \\
\text { Rocco, Lucia, } \\
\text { Antonio Abate } \\
\text { (prob.) e Giacomo } \\
\text { Maggiore. Nicchia } \\
\text { E. del portico. } \\
\text { Affreschi, inizio XVI } \\
\text { sec. }\end{array}$ & $\begin{array}{l}\text { Ricerche pittoriche } \\
\text { con esportazione } \\
\text { dello strato di calce } \\
\text { ritrovatosi molto } \\
\text { duro. }\end{array}$ & $\begin{array}{l}\text { Carlo Cotti } \\
\text { Mario Moglia, } \\
\text { Nino Facchinetti. }\end{array}$ & $\begin{array}{l}\text { (Chiesa F. , 1946, p. } \\
\text { 77). } \\
\text { (DPE, 1959, p. 126). } \\
\text { (AUBCBZ, s.251, } \\
\text { Villa Luganese). }\end{array}$ \\
\hline 1943 & $\begin{array}{l}\text { Villa Luganese, } \\
\text { chiesa Parrocchiale } \\
\text { della B.V Assunta. }\end{array}$ & $\begin{array}{l}\text { Madonna sulla } \\
\text { lunetta dell'antico } \\
\text { portale. Affresco, } \\
\text { inizio XVI sec. }\end{array}$ & $\begin{array}{l}\text { Ricerche pittoriche. } \\
\text { Pulitura dallo strato } \\
\text { di calce. }\end{array}$ & $\begin{array}{l}\text { Carlo Cotti, } \\
\text { Mario Moglia, } \\
\text { Nino Facchinetti. }\end{array}$ & $\begin{array}{l}\text { (Chiesa F. , 1946, p. } \\
\text { 77). } \\
\text { (DPE, 1959, p. 126) } \\
\text { (AUBCBZ, s.251, } \\
\text { Villa Luganese). }\end{array}$ \\
\hline 1943 & $\begin{array}{l}\text { Villa Luganese, } \\
\text { chiesa Parrocchiale } \\
\text { della B.V Assunta. }\end{array}$ & $\begin{array}{l}\text { Seregnesi, attr, S. } \\
\text { Giovanni Battista. } \\
\text { Portico. }\end{array}$ & $\begin{array}{l}\text { Ricerche pittoriche. } \\
\text { Pulitura dello strato } \\
\text { di calce. }\end{array}$ & $\begin{array}{l}\text { Carlo Cotti, } \\
\text { Mario Moglia, } \\
\text { Nino Facchinetti. }\end{array}$ & $\begin{array}{l}\text { (Chiesa F. , 1946, p. } \\
\text { 77). } \\
\text { (DPE, 1959, p. 126) } \\
\text { (AUBCBZ, s.251, } \\
\text { Villa Luganese). }\end{array}$ \\
\hline 1943 & $\begin{array}{l}\text { Villa Luganese, } \\
\text { chiesa Parrocchiale } \\
\text { della B.V Assunta. }\end{array}$ & $\begin{array}{l}\text { Madonna della } \\
\text { Misericordia, metà } \\
\text { XIV sec., e due } \\
\text { santi. }\end{array}$ & $\begin{array}{l}\text { Ricerche pittoriche. } \\
\text { Pulitura dello strato } \\
\text { di calce. Ritocco } \\
\text { pittorico. }\end{array}$ & $\begin{array}{l}\text { Carlo Cotti, } \\
\text { Mario Moglia, } \\
\text { Nino Facchinetti. }\end{array}$ & $\begin{array}{l}\text { (Chiesa F. , 1946, p. } \\
\text { 77). } \\
\text { (DPE, 1959, p. 126) } \\
\text { (AUBCBZ, s.251, } \\
\text { Villa Luganese). }\end{array}$ \\
\hline 1943 & $\begin{array}{l}\text { Villa Luganese, } \\
\text { chiesa Parrocchiale } \\
\text { della B.V Assunta. }\end{array}$ & $\begin{array}{l}\text { Visione di S. } \\
\text { Bernardo con il } \\
\text { Crocifisso, } 1577\end{array}$ & $\begin{array}{l}\text { Ricerche pittoriche. } \\
\text { Pulitura dello strato } \\
\text { di calce. Ritocco } \\
\text { pittorico. }\end{array}$ & $\begin{array}{l}\text { Carlo Cotti, } \\
\text { Mario Moglia, } \\
\text { Nino Facchinetti }\end{array}$ & $\begin{array}{l}\text { (Chiesa F. , 1946, p. } \\
\text { 77). } \\
\text { (DPE, 1959, p. 126) } \\
\text { (AUBCBZ, s.251, } \\
\text { Villa Luganese). }\end{array}$ \\
\hline 1943 & $\begin{array}{l}\text { Villa Luganese, } \\
\text { chiesa Parrocchiale } \\
\text { della B.V Assunta. }\end{array}$ & $\begin{array}{l}\text { Madonna col } \\
\text { bambino e S. } \\
\text { Giovannino, inizio } \\
\text { XVI sec. Lunetta del } \\
\text { portale principale. }\end{array}$ & $\begin{array}{l}\text { Ricerche pittoriche. } \\
\text { Pulitura dello strato } \\
\text { di calce. } \\
\text { Consolidamento. } \\
\text { Ritocco pittorico. }\end{array}$ & $\begin{array}{l}\text { Carlo Cotti, } \\
\text { Mario Moglia, } \\
\text { Nino Facchinetti. }\end{array}$ & $\begin{array}{l}\text { (Chiesa F. , 1946, p. } \\
\text { 77). } \\
\text { (DPE, 1959, p. 126) } \\
\text { (AUBCBZ, s.251, } \\
\text { Villa Luganese). }\end{array}$ \\
\hline 1943 & $\begin{array}{l}\text { Villa Luganese, } \\
\text { chiesa Parrocchiale } \\
\text { della B.V Assunta. }\end{array}$ & Decorazioni interne & $\begin{array}{l}\text { Patinatura } \\
\text { (armonizzazione del } \\
\text { colore) degli stucchi } \\
\text { ornamentali e } \\
\text { figurativi raschiati e } \\
\text { lisciati dagli } \\
\text { stuccatori Cantoni, } \\
\text { nella volta della } \\
\text { navata e nelle } \\
\text { pareti, nella parete } \\
\text { principale del coro } \\
\text { e su tutti i capitelli }\end{array}$ & $\begin{array}{l}\text { Carlo Cotti, } \\
\text { Mario Moglia, } \\
\text { Nino Facchinetti. }\end{array}$ & $\begin{array}{l}\text { (Chiesa F. , 1946, p. } \\
\text { 77). } \\
\text { (DPE, 1959, p. 126). } \\
\text { (AUBCBZ, s.251, } \\
\text { Villa Luganese). }\end{array}$ \\
\hline
\end{tabular}




\begin{tabular}{|c|c|c|c|c|c|}
\hline & & & $\begin{array}{l}\text { delle lesene. } \\
\text { Pulitura con } \\
\text { emollienti sino a } \\
\text { rintracciare lo } \\
\text { stucco lucido } \\
\text { esistente e restauro } \\
\text { pittorico di questo } \\
\text { (marmorizzato) } \\
\text { ritrovato nelle } \\
\text { lesene, basi e } \\
\text { zoccoli, nonché nel } \\
\text { fregio della } \\
\text { trabeazione, } \\
\text { sull'attico } \\
\text { sovrastante alla } \\
\text { stessa e nella } \\
\text { cornice delle } \\
\text { finestre (138 mq). } \\
\text { Lucidatura finale. } \\
\text { Restauro pittorico } \\
\text { delle quattro } \\
\text { finestre barocche } \\
\text { dipinte. }\end{array}$ & & \\
\hline 1943 & $\begin{array}{l}\text { Villa Luganese, } \\
\text { chiesa Parrocchiale } \\
\text { della B.V Assunta. }\end{array}$ & $\begin{array}{l}\text { Seregnesi, attr. } \\
\text { Affresco, XV sec. } \\
\text { ritrovato sotto la } \\
\text { pala d'altare nella } \\
\text { parete nord della } \\
\text { navata. }\end{array}$ & $\begin{array}{l}\text { Pulitura e leggero } \\
\text { restauro. Non era } \\
\text { possibile togliere la } \\
\text { tela (Assunta con i } \\
\text { SS. Giovanni } \\
\text { Battista e Caterina } \\
\text { inginocchiati, fine } \\
\text { XVI - inizio XVII } \\
\text { sec., con cornice a } \\
\text { stucco a raggiere di } \\
\text { finitura) per } \\
\text { mettere in luce } \\
\text { I'affresco. Si pensa } \\
\text { a uno strappo e } \\
\text { cessione al Museo } \\
\text { di Lugano. }\end{array}$ & $\begin{array}{l}\text { Carlo Cott,i } \\
\text { Mario Moglia, } \\
\text { Nino Facchinetti. }\end{array}$ & $\begin{array}{l}\text { (Chiesa F. , 1946, p. } \\
\text { 77). } \\
\text { (DPE, 1959, p. 126). } \\
\text { (AUBCBZ, s.251, } \\
\text { Villa Luganese). }\end{array}$ \\
\hline 1943 & $\begin{array}{l}\text { Mendrisio, chiesa di } \\
\text { S. Giovanni. }\end{array}$ & $\begin{array}{l}\text { Trasparenti dei } \\
\text { pittori Giovanni } \\
\text { Battista Bagutti. }\end{array}$ & $\begin{array}{l}\text { Consolidamento e } \\
\text { restauro. }\end{array}$ & Tita Pozzi. & $\begin{array}{l}\text { (Chiesa F. , 1946, p. } \\
\text { 78). } \\
\text { (DPE, 1959, p. 122). }\end{array}$ \\
\hline $\begin{array}{l}1943- \\
1945\end{array}$ & $\begin{array}{l}\text { Novazzano, } \\
\text { oratorio } \\
\text { dell'Annunciata. }\end{array}$ & $\begin{array}{l}\text { Pitture } \\
\text { dell'Oratorio. }\end{array}$ & $\begin{array}{l}\text { Consolidamento } \\
\text { ell'encausto. }\end{array}$ & $\begin{array}{l}\text { Nino Facchinetti } \\
\text { Vig. Ugo Donati. }\end{array}$ & $\begin{array}{l}\text { (Chiesa F. , 1946, p. } \\
\text { 78). } \\
\text { (DPE, 1959, p. 124). } \\
\text { (AUBCBZ, s.180, } \\
\text { Novazzano). }\end{array}$ \\
\hline 1945 & $\begin{array}{l}\text { Lottigna, } \\
\text { Chiesa parrocchiale } \\
\text { dei SS. Pietro e } \\
\text { Paolo. }\end{array}$ & & $\begin{array}{l}\text { Sopraluoghi del } 29 \\
\text { settembre } 1945 \text { e } 2 \\
\text { ottobre } 1945 \text {, } \\
\text { proponendo il }\end{array}$ & $\begin{array}{l}\text { Lavori a carico della } \\
\text { Sig.ra. Caterina } \\
\text { Pagani - Pedrazzini }\end{array}$ & $\begin{array}{l}\text { (AUBCBZ, s.140, } \\
\text { Lottigna). }\end{array}$ \\
\hline
\end{tabular}




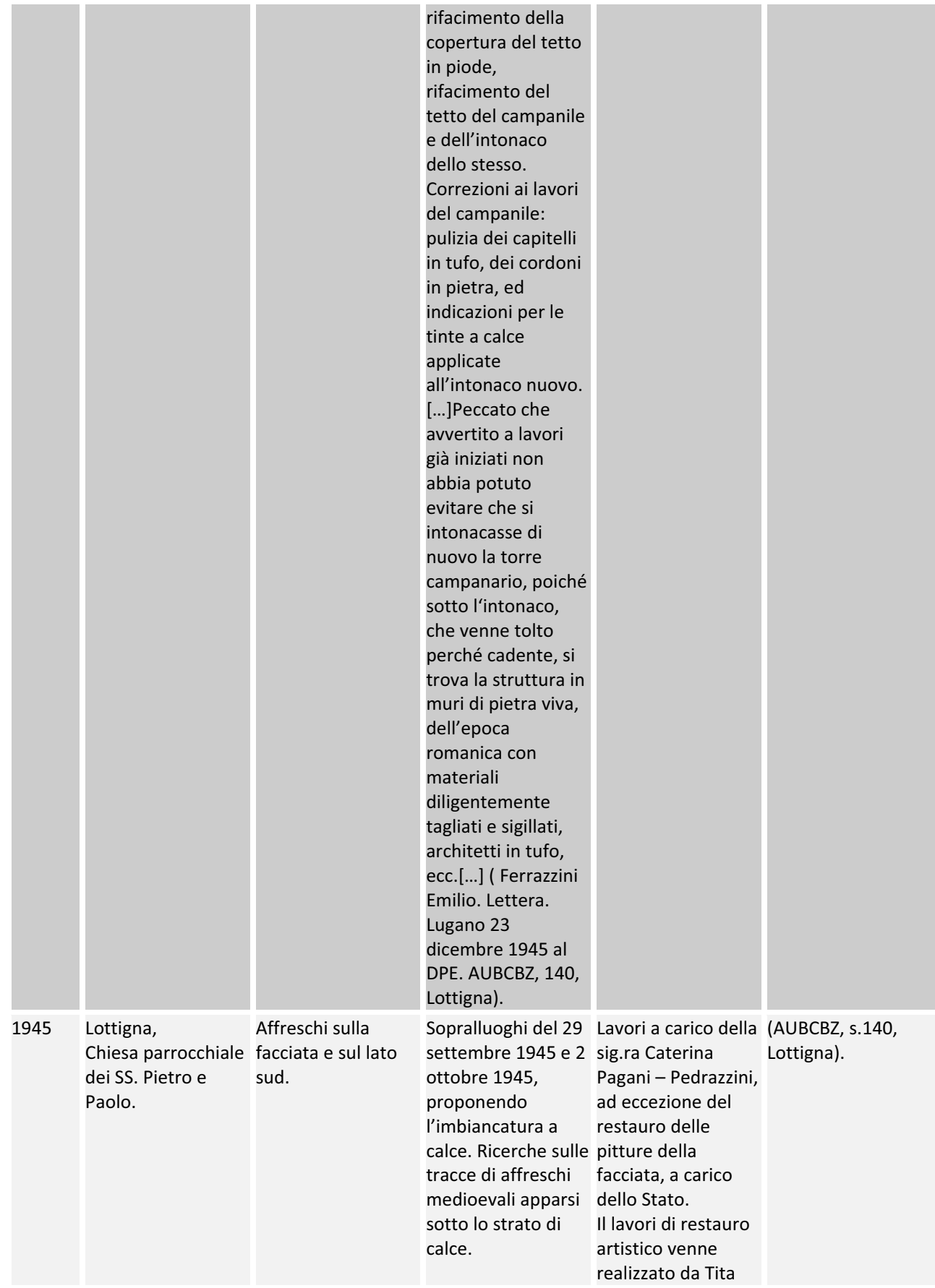


Pozzi

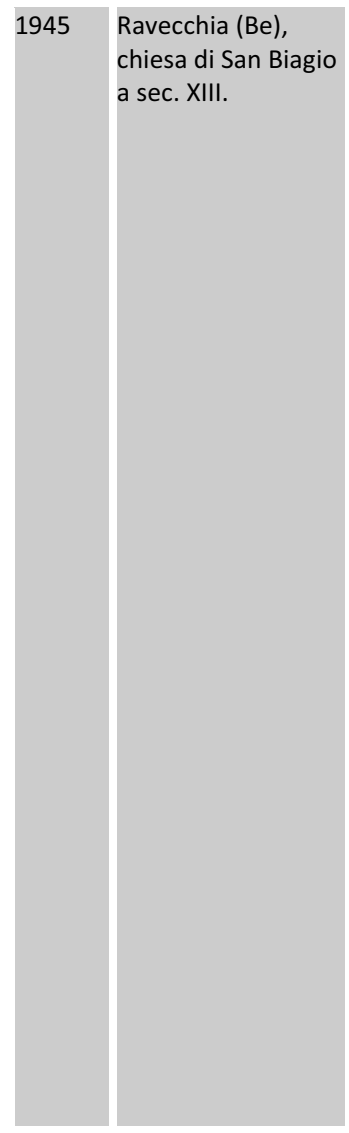

1940 - Chironico oratorio Affreschi. 1948 di S.'Ambrogio.

Affresco della
lunetta sulla porta
maggiore e San
Cristoforo.

Consolidamento di q 41,25 m con la tecnica dello encausto (cera ozocerite $72 \circ$ di fusione), stuccatura e reintegrazione cromatica. [...] ]usai cera minerale ad alto punto di fusione (ozokerite $72^{\circ}$ ) (da Kahlbaum, Berlino) sciolta in benzolo. La cera venne poi fatta liquefare e penetrare con il calore [...]

(Ferrazzini Emilio, Breve relazione sul consolidamento degli affreschi della facciata della chiesa di S. Biagio, Ravecchia, Lugano 18 agosto 1959 all'Egregio Sig. Aldo Crivelli Ispettore dei monumenti storici. AUBCBZ, 021, Bellinzona) .

$\begin{array}{ll}\text { Pulitura degli } & \text { I lavori furono } \\ \text { affreschi } & \text { inizialmente }\end{array}$

eliminando lo strato proposti a Ottorino di calce superficiale. Olgiati che avrebbe Consolidamento lavorato coadiuvato con miscela di cere dai pittori Emilio (paraffina e cera Ferrazzini, Tita d'api).

\section{"specialista" di Zurigo Henri-Paul Boissonas.}

(Chiesa F. , 1946, p. 15). (DPE, 1959, p. 118). (AUBCBZ,s. 021, Bellinzona).
(DPE, 1959, p. 120). (AUBCBZ, s.080, 


\begin{tabular}{|c|c|c|c|c|c|}
\hline $\begin{array}{l}23 \text { gen. } \\
1944\end{array}$ & $\begin{array}{l}\text { Corzoneso, } \\
\text { oratorio di } \\
\text { S. Remigio. }\end{array}$ & Affreschi. & $\begin{array}{l}\text { Preventivo di } \\
\text { restauro } \\
\text { interessato alle } \\
\text { ricerche sugli } \\
\text { affreschi ritrovati } \\
\text { nella parete } \\
\text { meridionale } \\
\text { (Crocifissione, } \\
\text { probabilmente } \\
\text { d'inizi XVI sec.), un } \\
\text { S. Cristoforo e una } \\
\text { figura di vescovo } \\
\text { (probabilmente del } \\
\text { XIII sec.). la pulitura } \\
\text { ed il } \\
\text { consolidamento di } \\
\text { tutti gli affreschi. }\end{array}$ & $\begin{array}{l}\text { I lavori saranno poi } \\
\text { eseguiti dal pittore } \\
\text { Tita Pozzi. }\end{array}$ & $\begin{array}{l}\text { (DPE, 1959, p. 120) } \\
\text { (AUBCBZ s.091 } \\
\text { Corzoneso). }\end{array}$ \\
\hline 1944 & $\begin{array}{l}\text { Ponto Valentino, } \\
\text { cappella di S. Anna. }\end{array}$ & $\begin{array}{l}\text { Antonio di Tradate, } \\
\text { attribuiti a. sec. XV- } \\
\text { XVI Affreschi. }\end{array}$ & $\begin{array}{l}\text { Sorveglianza ai } \\
\text { lavori di pulitura, } \\
\text { consolidamento e } \\
\text { reintegrazione } \\
\text { pittorica. }\end{array}$ & Tita Pozzi. & $\begin{array}{l}\text { (Chiesa F. , 1946, p. } \\
\text { 79) } \\
\text { (DPE, 1959, p. 125) } \\
\text { (AUBCBZ, s.198, } \\
\text { Ponto Valentino) }\end{array}$ \\
\hline $\begin{array}{l}1944- \\
1945\end{array}$ & $\begin{array}{l}\text { Croglio- Castelrotto, } \\
\text { oratorio di } \\
\text { S. Bartolomeo. }\end{array}$ & & $\begin{array}{l}\text { Sorveglianza ai } \\
\text { lavori di scoperta e } \\
\text { restauro pittorico. }\end{array}$ & $\begin{array}{l}\text { Nino Facchinetti } \\
\text { Vig. Ugo Donati. }\end{array}$ & $\begin{array}{l}\text { (DPE, 1959, p. 120) } \\
\text { (AUBCBZ, s.094, } \\
\text { Croglio). }\end{array}$ \\
\hline 1947 & $\begin{array}{l}\text { Coldrerio, } \\
\text { chiesa di S. Maria } \\
\text { del Carmelo. Villa } \\
\text { Coldrerio. }\end{array}$ & $\begin{array}{l}\text { Facciata e pala } \\
\text { d'altare } \\
\text { dell'Assunta. }\end{array}$ & Restauro pittorico & & $\begin{array}{l}\text { (DPE, 1959, p. 120) } \\
\text { (AUBCBZ, s.085, } \\
\text { Coldrerio). }\end{array}$ \\
\hline $\begin{array}{l}1945- \\
1946\end{array}$ & $\begin{array}{l}\text { Lugano, } \\
\text { Villa Ciani. }\end{array}$ & Facciata. & $\begin{array}{l}\text { Sorveglianza ai } \\
\text { lavori di } \\
\text { tinteggiatura della } \\
\text { facciata. }\end{array}$ & Prof. Poretti. & $\begin{array}{l}\text { (DPE, 1959, p. 122) } \\
\text { (AUBCBZ, s.143, } \\
\text { Lugano). }\end{array}$ \\
\hline $\begin{array}{l}7 \text { giu. } \\
1950\end{array}$ & $\begin{array}{l}\text { Russo, } \\
\text { chiesa parrocchiale. }\end{array}$ & $\begin{array}{l}\text { Affresco sulla } \\
\text { facciata. }\end{array}$ & Sopralluogo. & Pietro Salati. & $\begin{array}{l}\text { (AUBCBZ, s.216, } \\
\text { Russo). }\end{array}$ \\
\hline 1950 & $\begin{array}{l}\text { Russo, } \\
\text { chiesa parrocchiale. }\end{array}$ & $\begin{array}{l}\text { Affresco sulla } \\
\text { facciata. }\end{array}$ & $\begin{array}{l}\text { Consolidamento a } \\
\text { due riprese con } \\
\text { encausto di cere } \\
\text { minerali applicate } \\
\text { sulla superficie } \\
\text { pittorica a caldo. } \\
\text { [...]Residui di } \\
\text { materiale } \\
\text { consolidante; } \\
\text { probabilmente } \\
\text { caseato, dato che il } \\
\text { colore giallognolo } \\
\text { del residuo e una } \\
\text { sustanza fissante a }\end{array}$ & & $\begin{array}{l}\text { (DPE, 1959, p. 126) } \\
\text { (AUBCBZ,s. 216, } \\
\text { Russo). }\end{array}$ \\
\hline
\end{tabular}




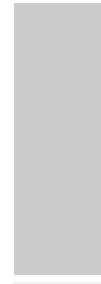

1950

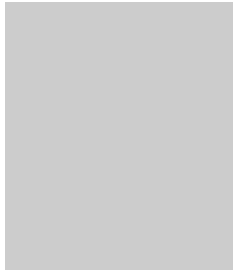

Chironico oratorio di

S.'Ambrogio.

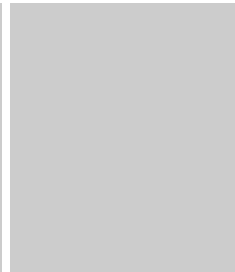

Affreschi.

carattere ceroso[...]

(Fonti Nadia.

Relazione

preventivo

restauro, Ascona

1996. AUBCBZ, 216,

Russo).

Perfezionamento

della pulitura degli

affreschi e

rimozione delle

strato di calce,

eliminazione dei

ritocchi realizzati

sulle parti figurative

così come le

velature sulle parti

abrase;

reintegrazione in

grigio neutro delle

stuccature delle

martellature nella

parte figurativa, ed

a basso tono sulle

raffigurazioni

architettoniche;

ricostruzione delle

fasce orizzontali

che dividono

architettonicament

e le decorazioni

della navata.

Secondo le

istruzioni dei

commissari dopo il

sopralluogo del 9

settembre 1950.
(DPE, 1959, p. 120)

(AUBCBZ, s.080,

Chironico). 


\section{Preventivi di restauro}

1928 S. Maria di Loreto, Decorazioni Lugano.

pittoriche.
Maestro Domenico

(Domenicus dictus

Fursnicus, le lacu

Lucani) attribuite al,

1534. Pitture.

BAS_15320.

25 mag. Bellinzona,

1934 Oratorio di S.

Marta.

Dipinti barocchi

BAS_15328/

15330.
Preventivo Richiesto dal

complessivo di fr. cappellano di S.

2'500 - 3'000 per il Maria di Loreto,

consolidamento e Padre Materno.

restauro degli

affreschi,

consolidamento e

restauro degli

incorniciamenti

decorativi delle

finestre e restauro

del fondo, delle

arcate.

\section{Preventivo di \\ Sorveglianza da}

restauro pittorico.

Cino Chiesa.

\section{Preventivo di Fr.}

2'200 per il

restauro pittorico.

II restauro fu

approvato dal

Consiglio di Stato

della Repubblica e

Cantone del Ticino

il 8 giugno 1934 e

s'incarica il pittore

Emilio Ferrazzini di

sorvegliare i lavori.

\section{Rev. Sac. Don}

Giosuè Prada (a

cura)

Vigilanza: Emilio

Ferrazzini e

Giuseppe Weith

(direzione e

sorveglianza)

Pittore Carlo Cotti

Pittore Tita Pozzi

(esecutore).

Caslano).

Lugano 25 maggio

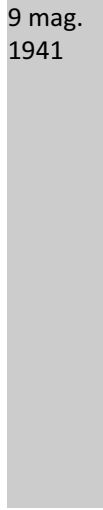

Giornico, chiesa di
Affreschi.

BAS_15378.

S. Nicolao.

BAS_15385.

BA_2777.

Preventivo di

restauro generale.

Considerato

incompleto dalla

Commissione

Cantonale dei

Monumenti Storici,

incaricare il

progetto ad una

sola persona, in

questo caso

all'architetto Paolo

Mariotta di

Locarno.

Preventivo per la si sceglie di

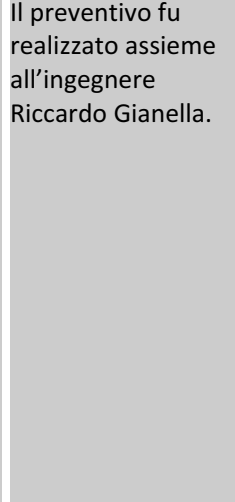

Ing. L. Andina
1934. AUBCBZ s.021

Bellinzona).

(AUBCBZ s.021

Bellinzona)

(Ferrazzini E. e Cotti

C. Preventivo del

Restauro completo

degli affreschi della

chiesa di S. Marta

eseguiti secondo le

direttive della CCMS

indirizzato Don

Giosuè Prada.

(Chiesa F. , 1946, p.

65).

(DPE, 1959, p. 121).

(AUBCBZ, s.112,

Giornico).

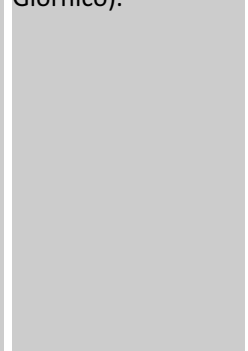

(AUBCBZ s.094, 
oratorio di

S. Bartolomeo.
(Cristo nella tazza, ristrutturazione ed Beata Vergine, S. il restauro pittorico Giovanni Battista e i dell'oratorio di S. dodici Apostoli. 1411, Thomas et Baldasar.

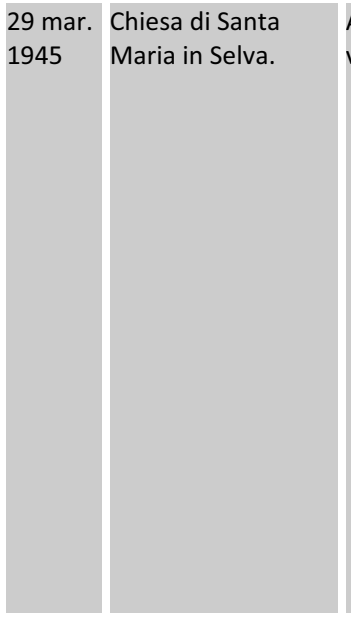

29 mar. Chiesa di Santa 1945 Maria in Selva.

$\begin{array}{ll}\text { Affreschi della } & \text { Preventivo restauro } \\ \text { volta. } & \text { pittorico } \\ & \text { consistente nel } \\ & \text { consolidamento } \\ \text { della parte } & \text { sgretolate e friabili } \\ \text { dell'intonaco a } & \text { mezzo di fluati, } \\ \text { consolidamento } \\ \text { della superficie } \\ \text { pittorica dove } \\ \text { necessario, } \\ \text { abbassamento con } \\ \text { toni grigli delle } \\ \text { macchie bianche } \\ \text { dell'intonaco per } \\ \text { omogeneizzare il } \\ \text { dipinto. }\end{array}$
Affreschi della Preventivo restauro navata.

\section{restauro} pittorico consolidamento della parte sgretolate e friabili della superficie torica dove abbassamento con oni grigli delle acchie bianche pittorico
Bartolomeo in

Croglio. Invita alla

Commissione

Cantonale dei

Monumenti Storici

a dare l'incarico del restauro al pittore

Tita Pozzi, ma i membri affideranno il lavoro ai pittori Carlo Cotti e Nino Facchinetti, sotto la sorveglianza di Ferrazzini.

Finalmente solo Facchinetti lavorerà ai restauri poiché Carlo Cotti si era rifiutato a collaborare con Ferrazzini. consistente nella pulitura generale dell'affresco della polvere e del sudiciume, esportazione delle stuccature e dei
Croglio).

\author{
(DPE, 1959, p. 122) \\ (AUBCBZ, 134, \\ Maria in Selva) \\ (Ferrazzini Emilio, \\ Pozzi Tita. \\ Preventivo. Lugano \\ 29 marzo 1945.
}

AUBCBZ, s. 134,

Locarno. S. Maria in Selva).

(DPE, 1959, p. 122)

(AUBCBZ, s.134,

Maria in Selva)

(Ferrazzini Emilio,

Pozzi Tita.

Preventivo. Lugano

29 marzo 1945.

AUBCBZ, s. 134,

Locarno. S. Maria in 
rifacimenti pittorici

Selva).

eseguiti in restauri

precedenti.

Consolidamento

delle parti friabili

(fluati), encaustica

ture di alcune parti

degli affreschi che

sono opache e

velate anche dopo

la pulitura. II totale

del preventivo

complessivo era di

fr. 3220. 
Restauratori in Canton Ticino fra Ottocento e Novecento

Catalogazione e gestione dati

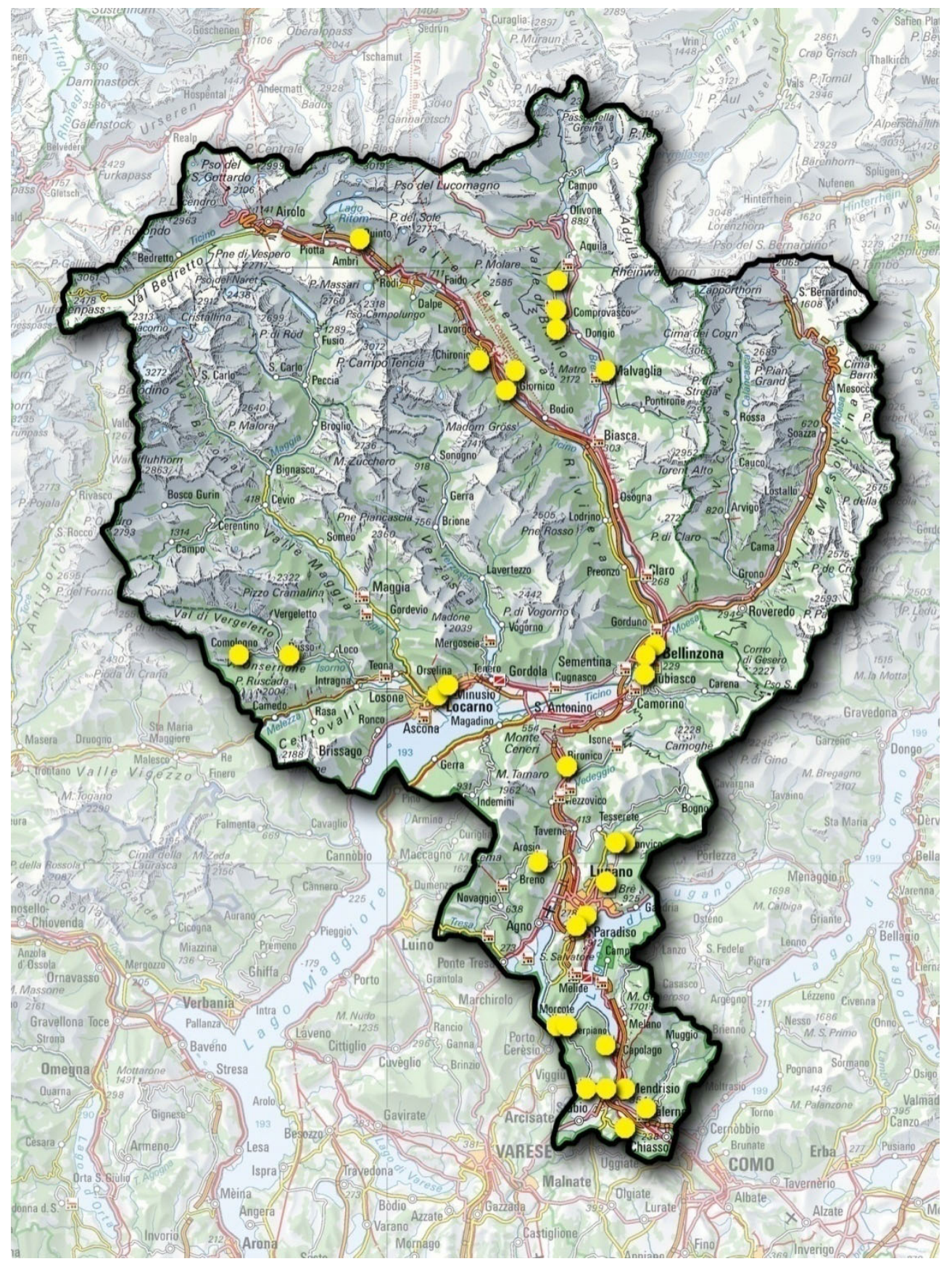

Figura 96 Cartina degli interventi eseguiti da Emilio Ferrazzini 


\section{Allegati}

(Copia)

Preventivo per il restauro di N.6 quadri di proprietà della Chiesa Degli Angioli in Lugano:

I) Tela S. Domenico e S. Francesco ai lati del Crocefisso I53 - I30 Pulitura, telaio nuovo. Pulitura cornice

2) Tela ladonna e Santi. I65-235 da foderare, sverniciare, pulire, stuccare. Restauro pittorico.Telaio nuovo, cornice da restaurare.

3) Tela S. Franceaco (Petrini) I35-I90.Foderatura, consolidamento del colore, sverniciatura, pulimento dei vecchi ritocchi.

4) Tela S. Antonio (Petrini) I45-IIo.Foderatura, consolidamento del colore, pulimento dei vecchi ritocchi, restauro completo. Telaio nuovo,

5) Tela S. Francesco e figura m.2-I60 Da sverniciare, pulire e restaurare completamente. Telaio nuovo

6) Tela Angelo e Tobia 275-I65 da ricucire, rappezzare,stirare, pulire, restaurare pittoricamente

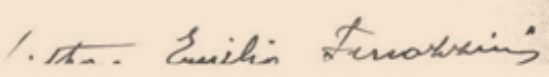

Figura 97 Ferrazzini Emilio, Preventivo di restauro delle tele di S. Maria degli Angioli. Lugano, 20 ottobre 1930. AUBCBZ, s.143, Lugano. 


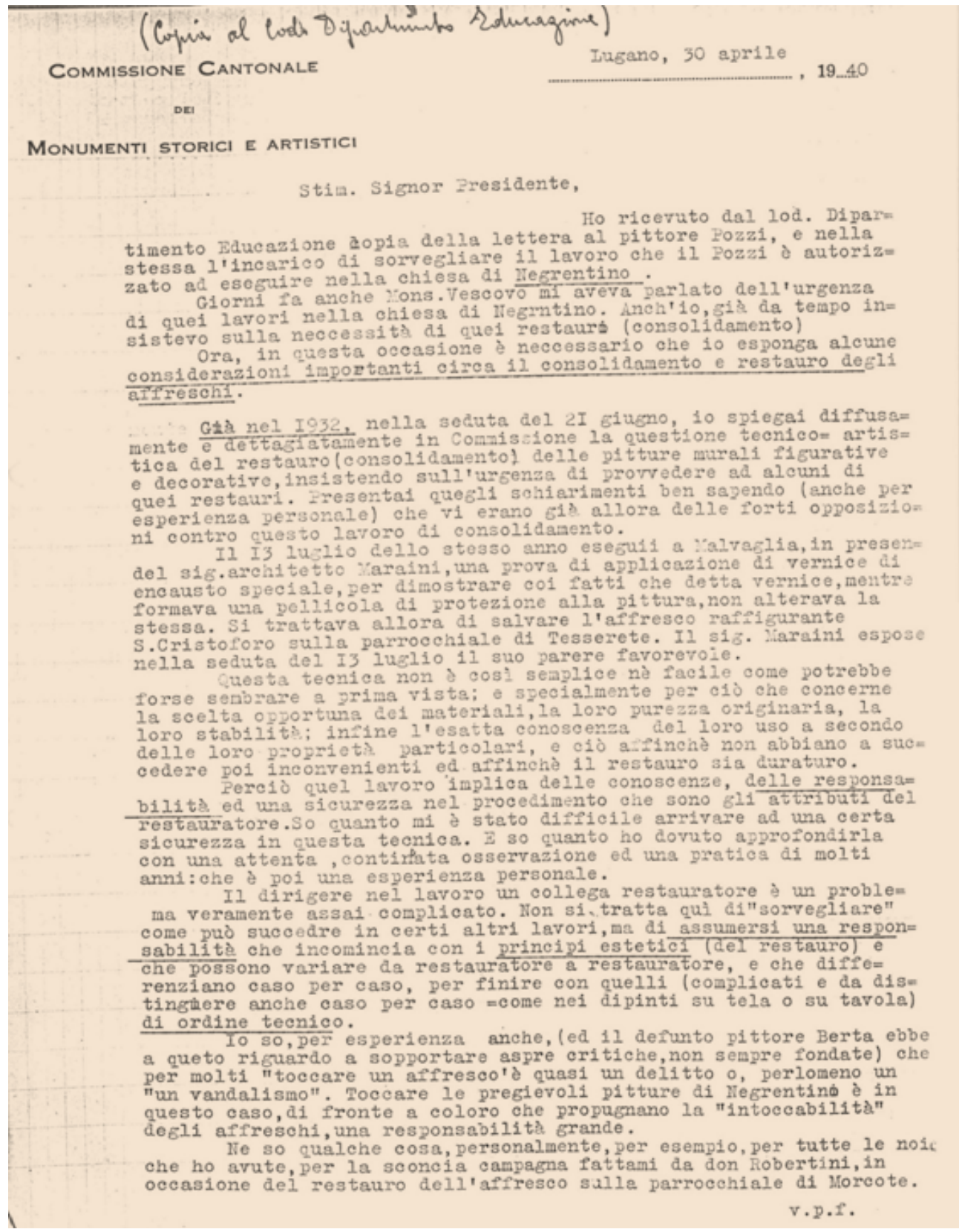

Figura 98 Ferrazzini Emilio, Lettera indirizzata alla CMSA, Lugano 30 aprile 1940. AUBCBZ, s.204, Prugiasco. S. Carlo di Negrentino. 


\title{
COMMISSIONE CANTONALE
}

\section{MONUMENTI STORICI E ARTISTICI}

\author{
St1a. S1 mor mesidente, ni sono recato ieri
}

a S.Ambrosto di Prugissco, col pittore Foz:1, yer esaainare quegli affreschi. Decole une relazione in proposito:

a) Parete con 1 s rafis surazione del Salvetoro:

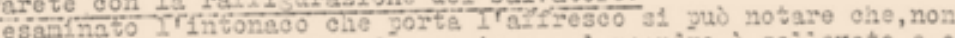
eso $M$ framento della decorazione col zeandro os solievato e cad to in Irantumi, as cice s ona jarto dell'intonaco s staceato dal fondo. C10 rovione dal fatto ole l'intonaco stosso, di spesnore

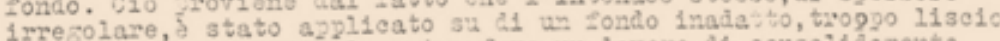

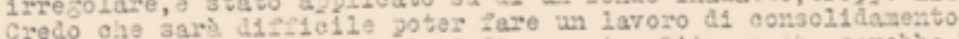

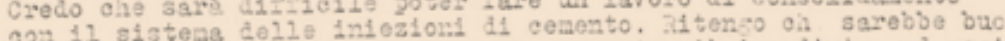
cosa provredere, dove 11 blsomo s1 yresenta 21 limediato, ad ass

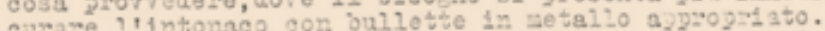

31 potro jol 2rocedsze: $7^{\circ}$ ) allo 30opriaento degli altri framenti di af?rasohi che st trova:so sulla stessa jaroto ed a ux

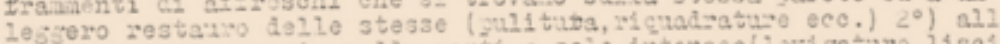

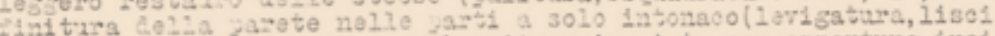

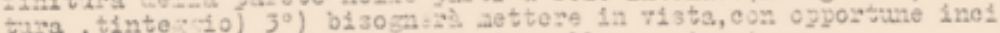

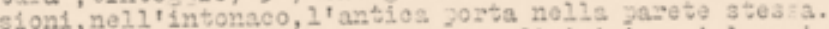

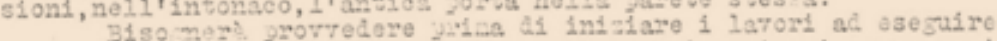

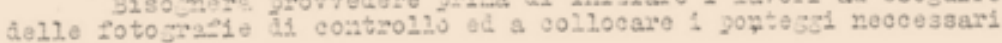

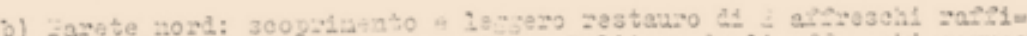

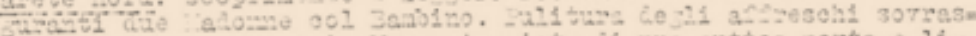

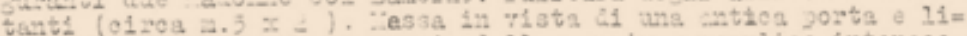

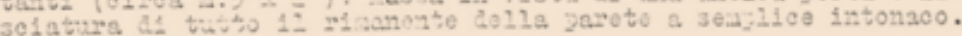

c) Abasde a nord : Kella tazza: conaol1danento del bla"z (Lap13lasul1) a

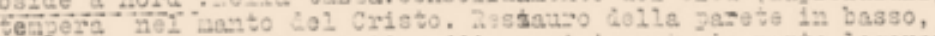

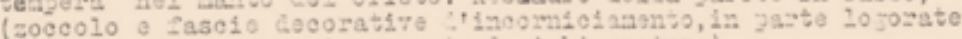
dalla undefte, in parto cozezto \&a labiameature)

a) Abside a suc : Conso:1Cas nto del uanto dol Colsto. Kestauro dello

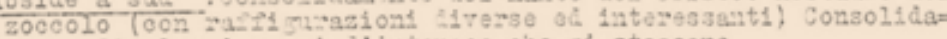
dento 21 a? ound jezsi d'intozaco che si staccano.

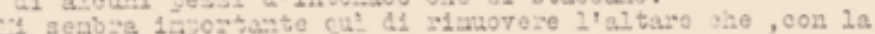
sua sassa copre jarte do 11 artreschi.

9) Afreschi gusli archi c1visori della chiesa: fa julive 1alla polver

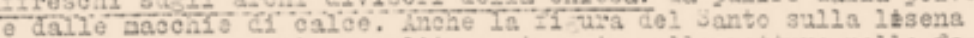
a monte (3.Antonio?) Ta pulita, reataurats nelle votture, nello la= sole oce.

1) defresco con S.Azoro-10 ed 1 zant1 Rrotas10 e Gervas1o: (os 3m. x 2) da jurve, jpecialnezte dalle sabattature fatte con la calce.

5) Parete a sud : pulitura degli affreschi dalle aschie di calce.

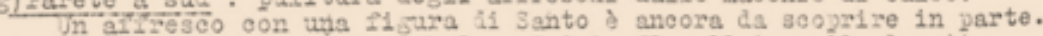
h) Afreschi estermi: S."ichele e Cristo llagellato nella lunetta sottostaite, oon serizione ed omat1.

occorre atoito: una rrondaia 11 protezione ed una iapermesbiliz= zazione degl1 affreschi e delle jarti dintonaso jal deteriorato.

Questo come indicsalone senerale sul lavoro di restauro alle pitture

$$
\text { v. } 2.1 \text {. }
$$

Figura 99 Ferrazzini Emilio/ Pozzi Tita, Proposta di restauro a Francesco Chiesa presidente della Commissione Cantonale dei Monumenti Storici e Artistici. Lugano 30 luglio 1942. AUBCBZ. s.204 Prugiasco, San Carlo di 
di S.Arorogio. D' risultato un po' difficile preparare un preventivo della spesa di restauro, anche solo per la parte come alla lettera (a) Il lavoro è delfeato e complesso. Il pittore Pozzi pensa di poter conpiere questa jarte del lavoro per Fr. $500=600 .=$ Ho dato dungue le indicasioni neccessarie al collega Pozzi ed egli attende ora un Suo cenno per iniziare il laroro (a). Per quento riguaria la jarte tecniea del restauro,e per cic che wi coneeme, ai riferisco alle spiegazioni date nella lettera a Lei spedita in data 30 aprile. (sui restauxi in generale)

$$
\begin{aligned}
& \text { Ie porgo distinti saluti e sono Suo }
\end{aligned}
$$

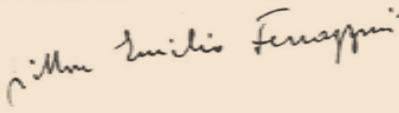<smiles>[Mg][Mg][Mg][Mg]</smiles>

Figura 100 Ferrazzini Emilio/ Pozzi Tita, Proposta di restauro a Francesco Chiesa presidente della Commissione Cantonale dei Monumenti Storici e Artistici. Lugano 30 luglio 1942. AUBCBZ. s.204 Prugiasco, San Carlo di Negrentino. 


Tita Po 22 


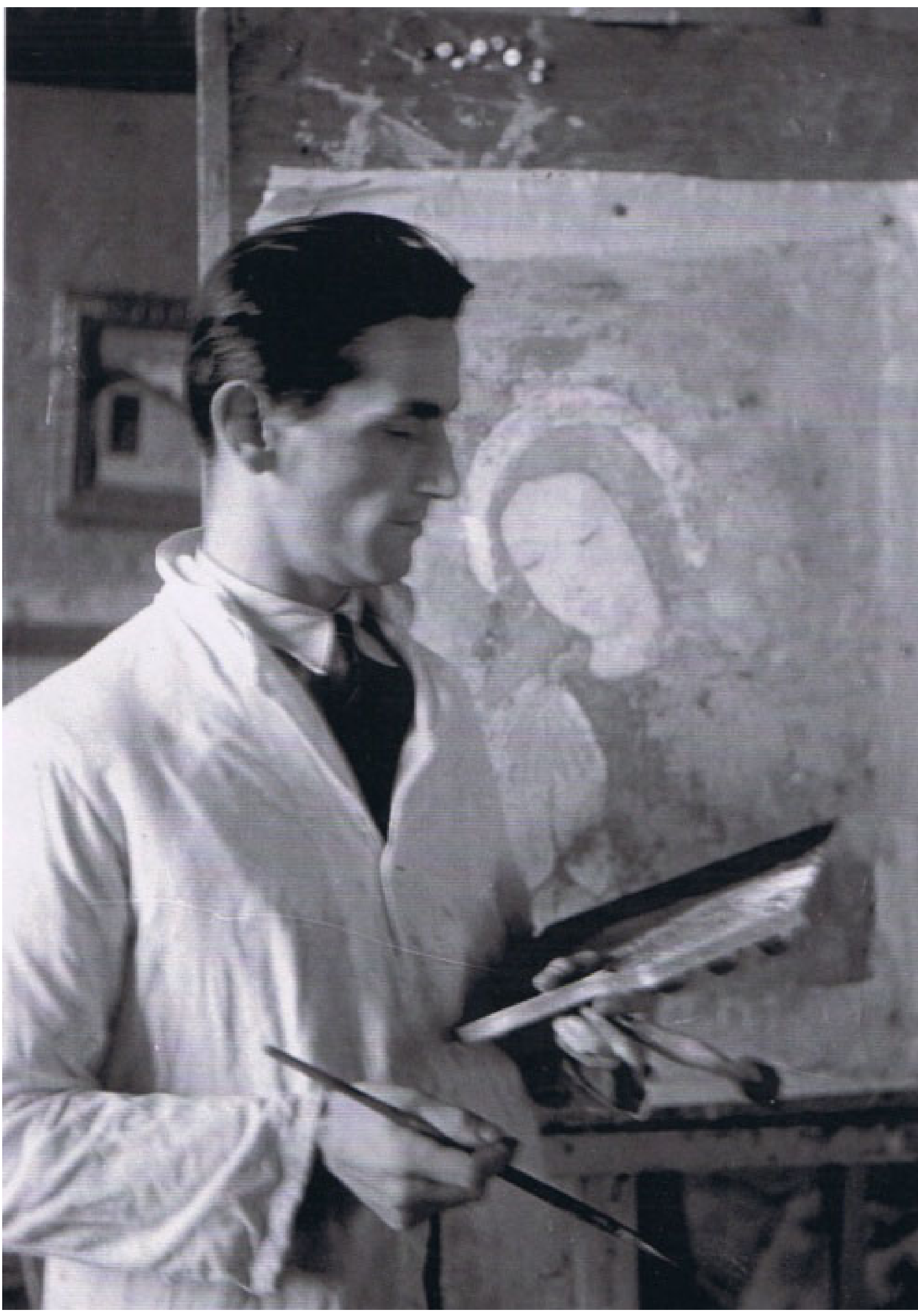




\section{POZZI BATTISTA "Tita"}

[...]Per noi Tita Pozzi è sempre stato un interprete sensibile, preciso, intelligente del paessaggio e degli ambienti di un Ticino contadino, che stava trovando una sua nuova dimensione e con essa una inedita collocazione culturale, un artista che - in un momento di passaggio e cambiamenti decisi - ha dedicato la sua vita per testimoniare il presente con la sua attività pittorica e preservare il passato con quella di restauratore $[. . .]^{335}$.

[...]Para nosotros Tita Pozzi ha sido siempre el interpretador sensible, preciso, inteligente del paisaje e del ambiente de un Ticino rural, que estaba encontrando su nuova dimensión y con ella una original identificación cultural, un artista que - en un momento de movimiento y de cambios decisivos - ha dedicó su vida a testimoniar el presente con su actividad pictórica y a preservar el pasado con la de restaurador[...].

Pagina precedente: Figura $101 \mathrm{Ch}$. Schiefer, Restauro di un affresco. 1932 ca. Archivio privato.

Blendinger Paolo, Una pittura ettica. Omaggio a Tita Pozzi nel 50.mo della scomparsa dell'artista e restauratore di Massagno. Rivista di Lugano, G.A.A. Lugano, Anno LXX, N.22. maggio 2008, pp. 6-7. 


\section{Dati biografici}

Battista Pozzi, detto Tita, nasce a Cadepiano di Barbengo il primo giugno del 1902, e morì prematuramente con appena cinquantasei anni il 19 maggio del 1958 a Massagno.

Questa ricerca ha permesso di avvicinarsi al medesimo artista anche dall'aspetto personale e dobbiamo ringraziare di questo la sua famiglia, in specialmodo il figlio Giovanni Pozzi. Lui e sua moglie che con un entusiasmo unico hanno permesso di riguardare le fotografie ed il materiale cartaceo dell'archivio personale del padre, riportando per ognuno dei fogli o delle riprese che lo compongono le rispettive storie.

Tita Pozzi fu uno dei principali difensori del patrimonio artistico e storico ticinese dalla prima metà del sec. XX, insieme al collega Emilio Ferrazzini di Lugano, erede spirituale del pittore-restauratore Edoardo Berta.

Fu introdotto all'arte grazie all'influenza di Attilio Balmelli ${ }^{336}$, ed eseguì in Ticino i corsi

\footnotetext{
336 Balmelli Attilio (Barbengo, 1887 - Corzoneso, 1971) Pittore e restauratore. Studiò alla Scuola d'arte di Lugano insieme a Emilio Maccagni e Emilio Ferrazzini dai maestri Edoardo Berta e Pietro Anastasio, e poi nella scuola d'arte del Castello Sforzesco di Milano e nel Palazzo di Brera nella medesima città frequentando i corsi di Giuseppe Mentessi e di Cesare Tallone. Qui lavorò dopo gli studi fino al 1910 specializzandosi nella professione de restauro. Come restauratore segue il percorso di altri colleghi italiani a Leningrado lavorando nel palazzo imperiale degli zar. Con l'esplosione della prima guerra mondiale ritorna in patria stabilendosi a Semione, e fu qui, nella Valle di Blenio dove lasciò i suo più grande legato artistico, decorando innumerevole cappelle e chiese. Decora l'interno della chiesa di Santa Maria Assunta a Semione (1919), dipinse il gigante San Cristoforo di Dangio in collaborazione con Emilio Ferrazzini, l'Oratorio di San Martino ad Olivone (1922), I'Oratorio dei Santi
}

di disegno della Scuola d'arte di Lugano, riaperta dopo il 1907 con l'intenzione di dare agli artisti ticinesi una sede di apprendistato in patria fino a quel momento assente, evitando la loro emigrazione nella vicina Lombardia. Tuttavia questo non impedisce che Pozzi si trasferisca a Milano all'età di diciannove anni per frequentare fino al 1925 l'Accademia di Belle Arti di Brera ${ }^{337}$, tradizionale scuola in questa competenza dal 1776, e la Scuola Superiore d'arte cristiana Beato Angelico ${ }^{338}$. Qui il pittore svilupperà l'amore per il patrimonio e per la sua conservazione, acquisendo il significato di "restauro" ed i metodi d'intervento che successivamente metterà

Nazario e Celso di Montagnola (1925). Fra i più importanti restauri invece, possiamo qui elencare gli affreschi del coro dell'Oratorio di San Carlo Borromeo di Semione, (1931), gli affreschi a Ponto Aquilesco, nel coro dell'Oratorio di Santa Caterina d'Alessandria, I'affresco di San Cristoforo nella chiesa parrocchiale di Giubiasco (1926), diverse cappella della Valle Maggia, a Marolta, Avegno, Bironico, la chiesa dell'Ospedale ad Acquarrosa e, fra gli ultimi lavori: la tavola nella chiesa parrocchiale di San Martino di Tours a Olivone, (1941), attribuita tutta ai Tarilli e l'Oratorio del Campaccio a Semione. (Agliati, 1987, p. 15-16) Informazione preso il sito http://www.sikart.ch.

${ }^{337}$ Dirige in questi anni I'Accademia il pittore Antonio Ambrogio Alciati (Vercelli 1878 - Bergamo 1929), successore della cattedra occupata prima dal suo maestro Cesare Tallone. Fu rappresentante del tardoverismo lombardo soprattutto come ritrattista. Si dedicò anche alla decorazione di chiese, cappelle e ville lombarde, come per esempio la Villa Pirota di Brunate (Como). (Foletti Giulio, Novembre 2002, p. 4) 338 La scuola fu fondata in 1921 dal monsignor Giuseppe Polvara (1884 - 1950) sacerdote della Diocesi di Milano, pittore e architetto, quale libera istituzione cattolica, il cui obiettivo ancora oggi è quello di rivalorizzare il patrimonio artistico e artigianale al servizio del culto mediante la sua conservazione e conoscenza. Informazione preso dal sito http://www.scuolabeatoangelico.it. 
in pratica dentro e fuori del Canton Ticino, parallelamente all'attività di pittore e silografo $^{339}$.

Formò parte della Società pittori, scultori ed architetti svizzeri, dove il pittore, stabilitosi finalmente in patria dopo gli studi, non dubitò ad esporre la sua impotenza riguardo alla situazione d'abbandono che ritrova nei monumenti di culto della sua terrà: nel giornale Arte Svizzera del 1929, insieme ad altri articoli di Pietro Chiesa, Aldo Patocchi ${ }^{340}$ e Giovanni Giacometti ${ }^{341}$, il contributo

339 In qualità di silografo Pozzi sviluppò delle collaborazioni maggiori e Milano, con le riviste letterarie "Comoedia" di Arnoldo Mondadori e "L'Eroica" di Ettore Cozzani.

340 Patocchi Aldo (Basilea 1907 - Lugano 1986), pittore e decoratore di ritratti, di temi religiosi e mitologici. Fu introdotto all'arte dallo scrittore Giuseppe Zoppi chi lo metterà in contatto con i direttore della rivista milanese l'Eroica con la quale collaborare dal 1925 in maniera discontinua. Ebbe una carriera artistica di enorme successo con la vincita di quattro borse di studi federali tra il 1925 e il 1931, una medaglia d'oro nel 1931 alla Triennale d'arti decorative di Monza. Nel 1934 sarà redattore capo d Illustrazione ticinese e farà parte di numerose giurie $\mathrm{e}$ commissioni artistiche. Sarà membro della fondazione Pro-Helvetia, presidente e vicepresidente rispettivamente della Società Ticinese per le belle arti e della Società dei pittori, scultori e architetti svizzeri, fondatore della Associazione d'incisori su legno Xylon Svizzera e presidente della Xylon internazionale a Friborgo, oltre che sovrintendente del Museo Caccia in Lugano.

${ }^{341}$ Giacometti Giovanni (Stampa, 1868 - 1933) Pittore neoimpressionista, studia nel 1886 nella Scuola di Arti Decorative di Monaco di Baviera dove conosce $\mathrm{i}$ pittore Cuno Amiet, la cui amicizia perdurerà negl anni successivi. Dopo un breve soggiorno a Parigi conosce l'arte impressionista e la pittura di Giovann Segantini; grazie ai guadagni ottenuti ad una prima mostra in Berna visiterà Roma e Napoli, ma presto ritornerà nuovamente in Svizzera dove raggiungere la maturità artistica ed i primi successi con le mostre realizzate nei primi anni del Novecento. dell'artista con Pitture e restauri di chiese nel Ticino manifestava un titolo apparentemente riservato ma denunciava intensamente la necessità di un'educazione artistica del popolo, oramai indifferente nei confronti dell'estetica, e di una nuova commissione ecclesiastica che proteggesse quegli edifici sacri dipendenti della Curia Vescovile, in cui:

[...] gli sconci aumentano a prima vista d'occhio; le nostre chiese che pure contengono $e$ rappresentano il patrimonio più caro e sacro a noi Ticinesi, lasciatoci in bella eredità dai nostri padri magari attraverso dure fatiche e sacrifici immani, sono sempre più deturpate e violate, mentre certi pittori mestieranti hanno sempre qualche cosa da fare o da brigare e certi altri importati dall'estero - trovano magari l'America in questo paese che pure è tutt'altro che in floride condizioni economiche $[. . .]^{342}$.

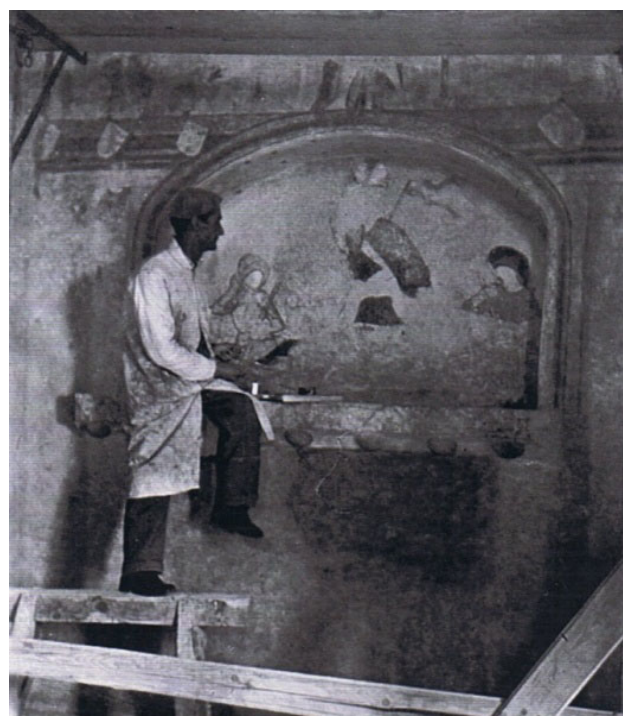

Figura 102 Tita Pozzi a Bubikon, 1943. Archivio privato.

\footnotetext{
${ }^{342}$ (Pozzi, 1929, p. 82)
} 
Lo stato delle chiese che il pittore intendeva difendere, come fece prima I'Ispettore Edoardo Berta e come tanti altri suoi colleghi, era quello della semplicità trovata negli antichi attributi. Così le nuove decorazioni artistiche che si sovrapponevano a essi rinnovando gli stili delle fabbriche non risultava altro, per Pozzi, che [...]un volgare $e$ ibrido raffazzonamento stilistico $[. . .]^{343}$.

I suoi ideali di restauro si basavano nel rinvenimento dei monumenti artistici dallo studio e l'analisi di essi, per poter idoneamente riportargli alle sue forme originarie. A tale scopo erano accettati, in questo periodo, la cancellazione degli apparati decorativi introdotti in epoche posteriori, gli strappi di affreschi che ricoprivano altri più antichi, e la pulitura dei dipinti nascosti sotto lo scialbo di calce, anche se essi non si trovavano nelle migliori condizioni né le tecniche per farlo fossero le più idonee.

L'artista collaborerà fra il 1929 e il 1931 nel gran restauro della chiesa di Santa Maria degli Angeli a Lugano, dove con i presupposti sopraccennati vengono ricuperate, fra altre, le importanti pitture della Cappella Camuzio sotto le direttive del professore Edoardo Berta, in quel momento Ispettore della Commissione Cantonale dei Monumenti Storici in Canton Ticino. Tuttavia la metodica di Tita Pozzi si allontanerà man mano dal restauro amatoriale per svolgere interventi più prossimi all'etica purista, usando le operazioni essenziali con il minimo materiale. Lo dimostrano i documenti dell'Archivio dell'Ufficio dei Beni Culturali, dove le sue perizie, preventivi e relazioni di

${ }^{343}$ (Pozzi, 1929, p. 82) restauro fra altri scritti, in chiarissima e armoniosa grafia, mostrano essere prodotto di meditati proponimenti.

Anche se le assidue polemiche che nell'epoca occupavano le pagine del Giornale del Popolo e la Rivista Storica Ticinese di Aldo Crivelli, compromettessero anche Tita Pozzi alla fine della sua carriera mettendo in dubbio la sua professionalità, come lo fecero a suo tempo con altri colleghi architetti e restauratori, oggi siamo certi di confermare la cura con cui si dedicò alla difesa dei beni artistici:

[...]fu tra coloro (e non erano molti) che ebbero in chiaro le necessità tecniche ed $i$ limiti interpretativi del mestiere di restauratore $[. . .]^{344}$.

A Lugano lavorò nelle decorazione murali del Palazzo Albertolli già Banca Nazionale Svizzera insieme a Carlo Cotti, (1928), negli strappi degli affreschi della casa già Ferrazzini (1948) prima della sua demolizione, nel restauro del ciclo d'affreschi di Giuseppe Antonio Felice Orelli presenti in Palazzo Riva (1951) e nella chiesa di Sant'Antonio (1954) fra altri, ma il suo apporto si stese dal Luganese alla Valle di Blenio: nell'Oratorio di Santa Marta a Bellinzona (1934) dove secondo Foletti ricomincia la sua carriera individuale dando a conoscere la sua professionalità ${ }^{345}$, in San Carlo di Negrentino (1942 - 1944), nella chiesa di San Nicolao in Giornico (1945), la chiesa di San Vigilio a Rovio e gli strappi dei dipinti seicenteschi dei fratelli Tarilli nel coro dell'Oratorio di S. Remigio in Corzoneso (1944 - 1946), fra i più importanti, con eventuali collaborazioni

\footnotetext{
4 (Foletti Giulio, Novembre 2002, p. 8).

5 (Foletti Giulio, Novembre 2002 p.45).
} 
fuori del Ticino, nel restauro dei dipinti del Castello dei Cavalieri di Malta a Bubikon dove fu chiamato dal restauratore specialista Henri Boissonas nel 1943, nella chiesa parrocchiale di Sant'Aubin nel 1950 e nella Niklauskapelle di Regensdorf in Müstair nel 1953. Un ricco lascito che oggi non sarebbe arrivato a noi se non grazie alla rispettosa professionalità e perseveranza di questo insigne pittore di Massagno al quale con queste righe vorremmo fare omaggio.

\section{San Carlo di Negrentino}

[...] Ora l'armonia è perfetta: il sacro tempio non è più soltanto delizia per l'occhio degli esteti, ma è elevazione per l'anima di chi crede.[... $]^{346}$

Così venne descritto nell'agosto del 1944 in Popolo e libertà il restauro della chiesa di San Carlo di Negrentino, eseguito da Tita Pozzi nel momento più prolifero della sua carriera come restauratore.

Se il primo intervento datato realizzato dal restauratore avviene in 1928 in Palazzo Albertolli insieme ai colleghi Carlo Cotti ed Emilio Ferrazzini, e fino al 1935 con il restauro all'Oratorio di Santa Marta in Bellinzona il suo lavoro non venne ancora riconosciuto. Finalmente negli anni Quaranta il pittore acquisirà la fama come professionista nel settore dei monumenti storici, specialista principalmente nella tecnica dello strappo.
Abbiamo considerato opportuno trattare in profondità quattro degli interventi di quest'epoca per essere appunto più identificativi delle prassi tecniche maturate dalle esperienze antecedenti, dal pittore e restauratore Tita Pozzi, e per significare anche dei punti di scontro con le teorie di restauro propugnate nell'epoca e specialmente all'interno della Commissione Cantonale dei Monumenti Storici.

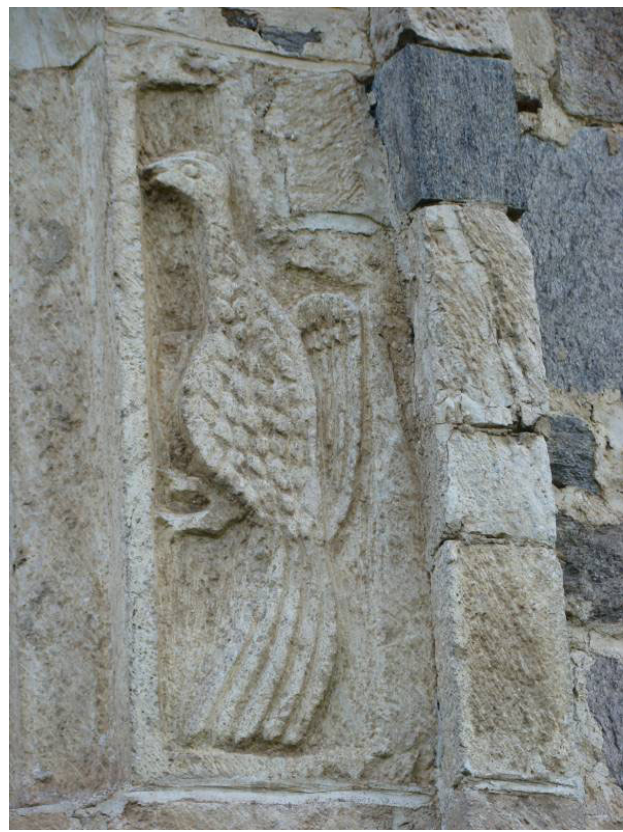

Figura 103 Giner Cordero E. Particolare del pavone raffigurato sull'abside. S. Carlo di Negrentino. 2006.

${ }^{346}$ Popolo e libertà, Bellinzona lunedì 21 agosto 1944. L'inaugurazione dei restauri di Negrentino. AUBCBZ, s.204 Prugiasco, San Carlo di Negrentino. 
Cominceremo con l'analisi dei lavori in San Carlo (1944) dove con i più vivaci plausi hanno luogo anche gli inizi delle prime polemiche giornalistiche, che avranno il suo climax con i restauri di San Nicolao di Giornico (1945). Lo stesso anno Pozzi realizza nell'Oratorio di San Remigio a Corzoneso (1944-1946), uno degli interventi di strappo più riusciti ed importanti in Ticino, e poco dopo, nel 1948, procede a realizzare il restauro dell'Oratorio di San Vigilio a Rovio, dove l'evoluzione professionale fece al restauratore scegliere per un trattamento meramente conservativo con il minimo intervento di ritocco.

La chiesa romanica di San Carlo del XI sec., denominata fino al 1702 di "Sant'Ambrogio Vecchio", si trova in Negrentino frazione di Prugiasco, in una zona privilegiata immersa nel verde della Valle di Blenio. Fu riscoperta nel 1870 dal professore Johann Rudolf Rahn

Prima del XIII sec. la costruzione era composta unicamente di una navata rettangolare ed un abside, ai quali fu aggiunta in epoca posteriore una seconda navata laterale dotata anche questa di un nuovo abside. Le due parti differenziate cronologicamente sono state fisicamente separate da un arco e da una robusta colonna centrale. Esternamente le caratteristiche stilistiche della fabbrica primitiva che ci permettono di datarla prima del XII sec. sono visibili dal pavone intagliato, simbolo della vita eterna, e degli archi ciechi che decorano l'abside.

II campanile del XII sec., è separato dalla fabbrica; ha pianta rettangolare e finisce in un tetto a piramide.
Troviamo diverse pitture all'esterno, quelle più distinguibili dalla lontananza si impongono sulla facciata est e rappresentano gli emblemi incrociati di Leventina, a cui Negrentino appartiene solo dal 1798, e del Canton Uri. Sulla porta d'entrata a sud invece, troviamo oggi due affreschi: il primo in alto all'interno di una lunetta, raffigura la Passione di Cristo mentre sotto si può osservare un gran San Michele opera del pittore Antonio da Tradate, XVI sec.

La decorazione pittorica all'interno della chiesa copre quasi per completo l'intera superficie muraria, sviluppandosi in tre periodi diversi, dove lavorarono due delle botteghe di pittori di maggiore produzione artistica in Ticino. Si tratta dei Seregnesi (Cristoforo e Nicolao) ${ }^{347}$ e del già citata Antonio da Tradate $^{348}$ con i suoi collaboratori.

\footnotetext{
Cristoforo (?-1493) ed il nipote Nicolao (14571500) furono degli artisti lombardi originari della località di Seregno residenti a Lugano ed attivi nel Ticino e nei Grigioni nell'ultima metà del XV sec. Fra le sue opere, gli affreschi della cappella di Lottigna (1455), ciclo di affreschi di Rossura (1463), la Deposizione sull'arco trionfale della chiesa di Santa Maria ad Ascona (1466) e gli affreschi della chiesa di San Nicolao di Giornico, che verrà trattato più avanti, del 1493, opera solo di Nicolao.

${ }^{348}$ Artista originario del paese lombardo di Tradate che visse a cavallo fra il XV ed il XVI sec., dirigente di una delle botteghe più importanti e produttive degli inizi del Seicento in Ticino dove ebbe la sua residenza. Suo figlio Giovanni Taddeo fu uno dei più brillanti pittori cresciuto nell'attività del padre, per chi lavorò nel ciclo di affreschi di S. Michele ad Arosio del 1508 e nell'affresco di Santa Maria in Selva; fra le opere più importanti prodotti dalla bottega si trovano $\mathrm{S}$. Michele a Palagnedra, le decorazioni del coro della chiesa di S. Martino a Ronco sopra Ascona la teoria di santi in S. Maria della Misericordia ad Ascona, e gli affreschi sopracitate a S. Ambrogio a Prugiasco.
} 


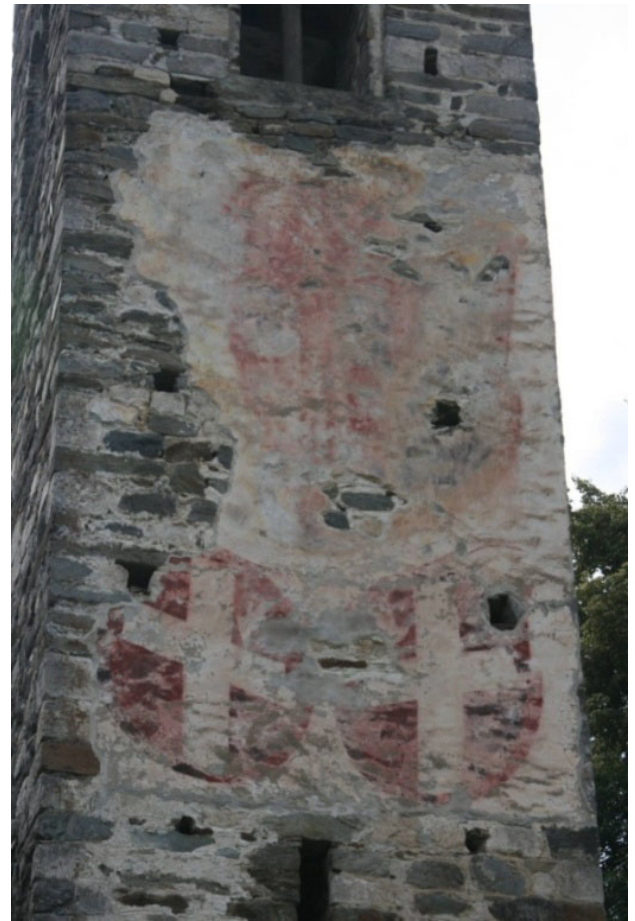

Figura 104 Giner Cordero E. Particolare degli affreschi sul campanile. S. Carlo di Negrentino. 2006.

I dipinti più antichi, probabilmente di tutto il Canton Ticino, sono del XI sec. ${ }^{349}$ e si trovano nel primitivo abside maggiore e nella controfacciata di questa chiesa. Rappresentano, sopra la porta antica, una complessa composizione con una potente simbologia dei motivi della Risurrezione, della Ascensione e del Giudizio Finale. II Cristo nel centro del dipinto è circondato da circoli concentrici equivalenti all'universo, con la lancia ed il bastone allegorie della Passione ed ai lati gli Apostoli. Sopra questi si svolge una greca dove si interpongono successivamente due angeli ed un animale marino. Della stessa epoca sono datati gli affreschi sottostanti ai dipinti quattrocenteschi nell'abside maggiore, dei quali si intravede da una lacuna nell'intonaco, la testa di un drago nello zoccolo in basso. I dipinti della scuola dei Seregnesi raffigurano i temi classici della chiesa medievale: il Pantocrator con i quattro Evangelisti e gli Apostoli. Nella parte inferiore il zoccolo venne decorato con la simulazione di un tessuto.

Ai lati dell'abside si trovano le figure di Santo Stefano e Santa Caterina. Nell'arco trionfale invece si raffigurano l'Annunciazione, il re ed i profeti. Nella parete settentrionale possiamo osservare una serie di dipinti votivi, un Sant'Ambrogio, al quale in un primo momento era stata destinata la chiesa, la Madonna in trono, la Madonna fra i SS. Antonio Abate e Bernardo, e la Crocifissione di Cristo.

Gli affreschi dell'altare minore datano del 1510 e furono opera di Antonio da Tradate. Nell'abside si succedono scene della vita della Vergine dalla sua Coronazione che occupa lo spazio centrale. Sugli archi divisori si trova l'Assunzione di Maria con gli angeli musicisti, e nella parete ad ovest, in opposizione all'altare, la scena del miracolo di Sant'Ambrogio che nella battaglia di Parabiago del 1339 appare in cavallo ai milanesi donandoli il suo appoggio. Ai lati gli affreschi di San Gervasio e San Protasio, fratelli martiri dei primi secoli del cristianesimo. dell'Università di Zurigo.

\footnotetext{
${ }^{349}$ Le date variano dal 1010-1030, 1050 o 1100 d. C.
} 


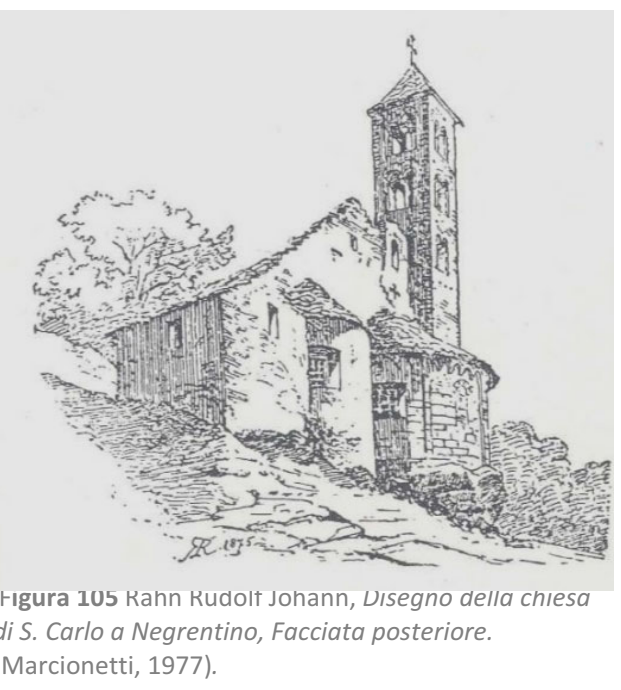

Era caduta in abbandono dagli inizi del XVIII sec. con la soppressione al culto, come tanti altri edifici religiosi, ed usata durante i due secoli come magazzino e come rifugio per gli animali, per cui lo stato di conservazione lasciava molto a desiderare. La cattive condizioni del tetto avevano provocato la infiltrazione dell'acqua piovana all'interno della fabbrica, danneggiando le travi in legno che si trovavano completamente deteriorate, mentre l'umidità di risalita capillare invece si era estesa ascendendo la muratura e degradando pesantemente gli affreschi.

Dopo la scoperta del bene artistico le autorità svolsero un urgente intervento di restauro per fermare i problemi causati dai fattori atmosferici, ma fra le operazioni realizzate ebbe pure luogo la cancellazione del campanile a vela esistente sulla vecchia facciata principale, la cui presenza oggi possiamo testimoniare unicamente dai disegni di studio realizzati dal professore Rahn alla fine del XIX secolo.
Dal 1897 il parroco Don Federico Ganna si fece carico della chiesa ed insieme a Johann Rudolf Rahn preparò una proposta d'intervento esclusivamente architettonico - [...]gli affreschi saranno ben ricoperti con tela durante i lavori di restauro e non devono essere per nulla ritoccati[... $]^{350}$ - con il sussidio della Confederazione, rappresentata dalla figura del presidente Josef Zemp ${ }^{351}$, d'un cinquanta per cento del totale del preventivo. Si susseguono da questo momento numerose proposte di restauro; il progetto dell'architetto Eugen Probst ${ }^{352}$ nel 1900 venne abbandonato all'uscita di questo del Canton Ticino e quattro anni dopo si autorizza invece l'esecuzione del preventivo dell'architetto Augusto Guidini di Milano.

L'impresa fu terminata nel 1907 con la pressione della Confederazione che negli ultimi anni presiede l'archeologo Albert Naef, senza essersi completati tutti i presupposti stipulati dal contratto. Di fatto già nel 1930 si rivelarono di nuovo problemi nella stabilità del tetto mettendo in pericolo i dipinti interni, principalmente sull'abside maggiore. Si dovranno aspettare ancora dieci anni per avere degli

(Ganna Federico, Lettera indirizzata al DPE. AUBCBZ, s.204 Prugiasco, San Carlo di Negrentino).

${ }^{351}$ Zemp Josef (1834 - 1908), il presidente della società svizzera per la conservazione dei monumenti dal 1897 al 1904 fu un politico affiliato al Partito Popolare Democratico Cristiano, oggi PPD, eletto consigliere federale nel 1891 e quattro anni dopo Presidente della Confederazione, carico che mantenne fino al 1905, tre anni prima della sua morte.

352 Probst Eugen (Basilea 1873 - ?) L'architetto restaura in collaborazione di Enea Tallone il Castello di Montebello nella città di Bellinzona in 1910, il Castello di Serravalle in Semione (1928), il Castello di Mesocco, 
appoggi economici che permettano un successivo intervento.

Finalmente nel 1940 si progettarono i lavori che incominciano nel 1942 e finiranno nel 1944. I progettisti furono I'Ingegnere Giovanni Baggio dell'Ufficio Tecnico Cantonale ed Emilio Ferrazzini, membro della CCMS. Linus Birchler era presidente, in questo momento, della Commissione Federale dei Monumenti, Francesco Chiesa rappresentava ancora alla Commissione Cantonale e Giuseppe Lepori $^{353}$ era capo del Dipartimento della Pubblica Educazione.

Le proposte definitive dei diversi progettisti furono presentate fra il mese di maggio e luglio dello stesso anno e l'autorizzazione dal Dipartimento della Pubblica Educazione $^{354}$ arrivò poco dopo, il 17 luglio 1942, notificando che i lavori di consolidamento della chiesa e del campanile sarebbero eseguiti sotto la vigilanza del progettista e del Ingegnere Riccardo Gianella, mentre i lavori artistici sarebbero realizzati dal pittorerestauratore Tita Pozzi sotto la sorveglianza e secondo le direttive di Emilio Ferrazzini, con i sussidi dello Stato del Canton Ticino.

\footnotetext{
353 Lepori Giuseppe (Lopagno 1902 - 1968). Membro del Partito Popolare Democratico l'insigne statista ticinese occupò il ruolo di Consigliere Federale in rappresentanza del Canton Ticino dal 1954 al 1959, e di capo del Dipartimento Federale delle Poste e della Ferrovie dal 1955 al 1959.

${ }^{354} \mathrm{Fu}$ il comitato pro-restauri formatosi in 1942 e composto dai membri del consiglio parrocchiale $d$ Prugiasco: Riccardo Gianella, Alfredo Gianela, don Ortensio Scapozza e Mario Scheggia a incitare i lavori.
}

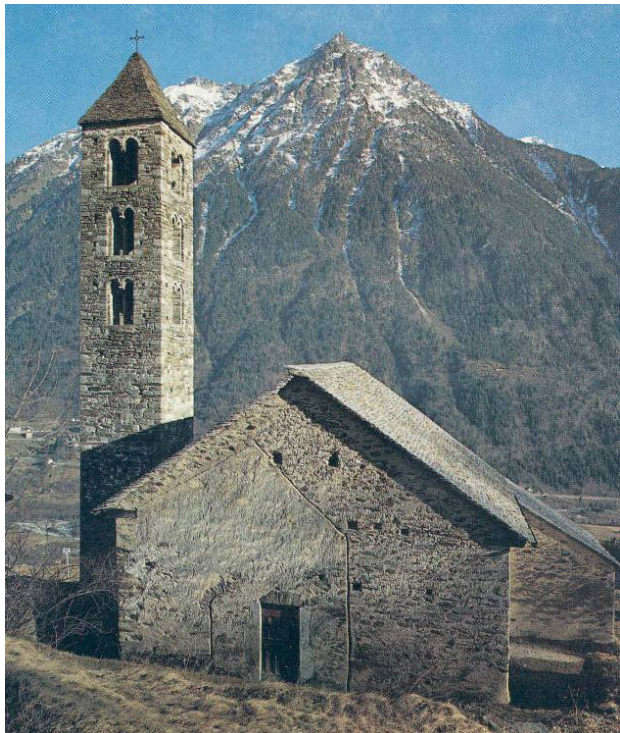

Figura 106 S. Carlo, Facciata principale. (Marcionetti, 1977).

L'inaugurazione dei restauri ebbe luogo la domenica del 20 agosto 1944 con una presentazione dall'Ing. Gianella sugli aspetti tecnici elaborati in quegli anni ed un discorso del professor Linus Birchler, seguito da una sua congratulazione con il restauratore dove affermava che [...]quello di Negrentino resterà un modello classico del modo con cui un restauro artistico deve essere fatto $[. . .]^{355}$.

La diversa documentazione d'archivio consultata ci ha permesso d'analizzare i principi eseguiti in un intervento di restauro distinto, tuttavia le parole sopraccitate, dalle numerose critiche.

\footnotetext{
${ }^{355}$ (Popolo e libertà, Bellinzona lunedì 21 agosto 1944. L'inaugurazione dei restauri di Negrentino. AUBCBZ, s.204 Prugiasco).
} 


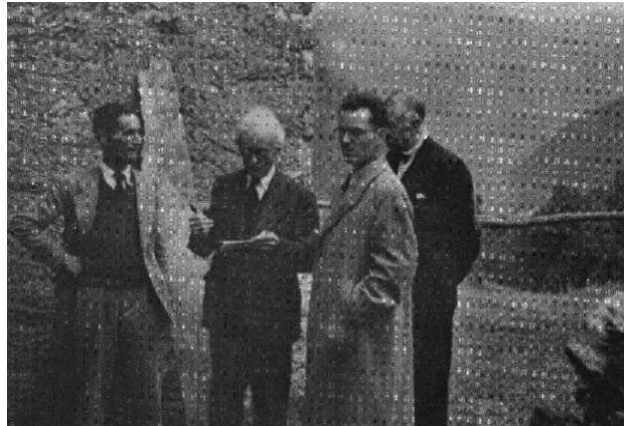

Figura 107 Da sinistra a destra: Tita Pozzi, Francesco Chiesa, Giuseppe Lepori, Ing. Baggio, 1949. Archivio privato.

In Aprile del 1940 quando Ferrazzini riceve informalmente l'incarico di sorvegliare a Tita Pozzi nei restauri della chiesetta di Prugiasco vuole, prima di accettare tale compito, far consapevoli le autorità della sua reticenza avvertendo sulle difficoltà e dei pericoli a cui solitamente il sovrintendente si vede esposto di fronte a restauri non bene compiuti. Si tratta d'una responsabilità che secondo il pittore dovrebbe cadere sul proprio esecutore e che [...]incomincia con i principi estetici [...]che possono variare da restauratore a restauratore, e che differenziano caso per caso, per finire con quelli di ordine tecnico[...]. ${ }^{356}$

\footnotetext{
${ }^{356}[\ldots .$.$] Personalmente ho dovuto, a poco a poco,$ abbandonare o rarificare l'aiuto dei colleghi, specialmente nel restauro di opere delicate, perché quella collaborazione utile nel risparmio del tempo, si confermava difficile nella pratica e nella distribuzione delle responsabilità tecniche.[...] Nell'attuale caso degli affreschi di Negrentino, (conosciuti per la loro importanza archeologica-artistica) faccio cortesemente osservare che ho ricevuto l'incarico di "sorvegliare" il lavoro, senza esserne stato interpellato[...] Preferirei per esempio, che nel futuro, questa parte fosse affidata al collega Sig. Bianconi che si interessa di molti di affreschi[...]. (Ferrazzini Emilio, Lettera a Francesco Chiesa, presidente della Commissione Cantonale dei Monumenti Storici e
}

Ferrazzini che ne aveva esperimentato tempo prima critiche negative al riguardo, assume l'incarico di sorveglianza poiché si tratta del pittore Pozzi, ma avverte la Commissione della necessità che nel futuro ogni restauratore sia obbligato a responsabilizzarsi dei suoi propri metodi di lavoro e che il Dipartimento scelga fra $i$ professionisti più capacitati. L'appoggio che il pittore offrì al collega restauratore dimostra che lo considerava fra questi professionisti anche se, come dimostreremo, le tecniche utilizzate da ambedue saranno leggermente differenziate.

Risalgono al 1942 le prime perizie sulla situazione conservativa degli affreschi della chiesa e le relazioni sull'esigenze di restauro ${ }^{357}$, dove venne stipulata la necessità di una precedente ed approfondita documentazione fotografica come testimonianza dello stato degli affreschi prima del restauro, dopodiché si considerava opportuno intervenire con il consolidamento delle zone staccate per proseguire posteriormente con il proprio intervento di pulitura, stuccatura e reintegrazione pittorica.

Sul dipinto bizantino della controfacciata si apprezzarono dei degradi nell'intonaco che si trovava decoeso praticamente in tutta la sua totalità, sicuramente dovuto - riteneva il pittore Emilio Ferrazzini - ad un fattore intrinseco alla propria tecnica d'esecuzione che permesse di estendere questo strato di

\footnotetext{
Artistici. Lugano 30 aprile 1940. AUBCBZ. S. 204 Prugiasco, San Carlo di Negrentino).

357 (Ferrazzini Emilio/ Pozzi Tita, Proposta di restauro a Francesco Chiesa presidente della Commissione Cantonale dei Monumenti Storici e Artistici. Lugano 30 luglio 1942. AUBCBZ. s.204 Prugiasco, San Carlo di Negrentino)
} 
spessore irregolare su una prima superficie troppo liscia da permettere l'adesione. Si scartò così il consolidamento con iniezioni di cemento che non sarebbe stato abbastanza tenace e si propose, invece, I'uso di bullette in metallo. Prima però, si stabiliva lo scoprimenti dei frammenti di affreschi ancora nascosti, seguito da un "leggero" restauro: la pulitura, la "rinquadratura" dei dipinti mediante la reintegrazione delle forme decorative che incorniciano le figure e per ultimo la levigatura, lisciatura e tinteggio della parete dove si trovava soltanto lo strato d'intonaco senza resti pittorici con evidenziazione dell'antica porta mediante un'incisione nella finitura del muro.

Le premesse operative per il resto dei dipinti murali si basavano nella pulitura della polvere e delle macchie di calce, consolidamento degli intonaci e dei colori, principalmente dei blu lapislazzuli applicati a secco sopra la superficie affrescata, e per ultimo l'impermeabilizzazione dei dipinti all'esterno della chiesa e la rimozione dell'altare dell'abside a sud poiché impediva la visibilità totale degli affreschi. Queste sono le indicazioni generali che vengono date al restauratore, Tita Pozzi, che nel preventivo di restauro pittorico le descrive più approfonditamente nei seguenti punti:

[...]1. A tutte le pareti non portanti dipinti: lisciatura alle parti irregolari; leggera tinteggiatura e patinatura.

2. Consolidamento, indurimento e - qua e là stuccatura a tutte le parti d'intonaco che sgretolano e sfaldano.

3. Fissaggio degli azzurri (tutti) che sfarinano e cadono. Pulimenti.

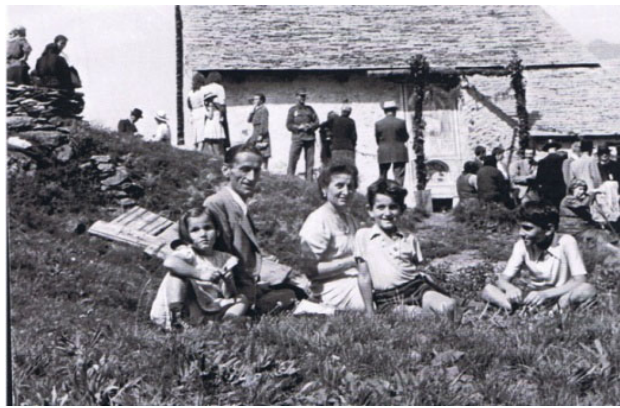

Figura 108 Tita Pozzi e famiglia nell'inaugurazione di

S. Carlo, 1944. Archivio privato.

4. Doratura "sgretolata" a foglia a quasi tutte le aureole (120 - 130 circa) per ripristinare l'effetto - in parte - scomparso a seguito la caduto dell'oro.

5. Leggera ed appropriata applicazione del blu ai fondi dove il colore è caduto.

6. Ricostruzione e restauro di quasi tutte le riquadrature, le fasce e di parte dei motivi architettonici; eventualmente anche delle stampinature ove il colore s'è alterato.

7. Ripassatura fasce verdi e panneggi (blu Madonna a nord) ove il colore s'è alterato.

8. Eventuale rinforzamento (colore caduto) delle parole nei nastri.

9. Sulla parete a nord: encausticatura di due figure per accrescere tonalità; pulimento della calce e leggero restauro di due Madonne in basso.

10. Nell'abside a nord: ricostruzione e restauro motivo mandorla; leggero restauro parte caduta nella tazza; leggero restauro (sgrigiolature) panneggi Apostoli e Santi; restauro parziale motivo a panneggi sullo zoccolo, motivi a medaglioni sull'intradosso e sulle parete frontale in alto.

11. Nell'abside a sud: restauro motivo architettonico al centro in basso della tazza; leggero restauro ai panneggi; idem motivo a medaglioni sull'intradosso e sulla parete 
frontale; fissaggio e restauro motivo sulla trave; leggero restauro fasce e fondali agli affreschi sullo zoccolo; lisciatura della parte frontale dell'altare lasciando in vista i pochi frammenti rimaste.

12. Parete a sud: restauro fasce e riquadri; restauro motivo architettonico e parte dei panneggi affresco Madonna a destra.

13. Affresco di S. Ambrogio, e sul pilastra a ponente: rifacimento e restauro fasce e riquadri, leggero restauro a sgrigiolature.

14. Archi divisori: restauro leggero di due medaglioni, dei nastri e delle fasce dei medaglioni sugli intradossi; leggero restauro a parti degli affreschi sopra gli archi; ricostruzione e restauro fasce e riquadri; restauro delle fasce $e$ alle architetture dell'affresco sul pilastra interabsidi.

15. Colonna portante archi-divisori: intonacatura - previo consolidamento - parti mancanti e lisciatura; tinteggio nell'attuale tonalità rossa, spruzzature e patinature; eventuale encausticatura.

16. Affreschi esterni sopra porta entrata: indurimento dell'intonaco sgretolato; encausticatura (per impermeabilizzare); applicazione del blu caduto nel fondo dell'Angelo; leggero restauro fasce dell'affresco in basso. 3.200 fr.Inclusi i necessari materiali idonei (fluati, silicati, solventi, caseine, oro in foglia, ecc.) $[\ldots]^{358}$

Sappiamo, grazie alla relazione di restauro che Pozzi scrive al Dipartimento della Pubblica Educazione il 22 settembre del 1942, che l'affresco bizantino sulla parete a

\footnotetext{
358 (Pozzi Tita, Comprovasco 20 luglio 1943. Elenco delle operazioni di restauro pittorico da eseguirsi nella Chiesa di Negrentino sopra Prugiasco; operazioni di restauro decise a seguito dei sopraluoghi: delle Lod. Comm. Cant. Monumenti della fine agosto 1942, e de pittori E. Ferrazzini e Tita Pozzi del 19 luglio 1943. AUBCBZ. s. 204 Prugiasco, San Carlo di Negrentino)
}

ponente non fu consolidato con delle bullette di metallo previste nel primo sopralluogo del 29 luglio insieme al pittore Ferrazzini, ma con il caseato ${ }^{359}$, che venne citato fra i materiali d'utilizzo nel preventivo. A questo consolidante, che abbiamo trovato nella documentazione riferito ugualmente mediante la qualificazione di "caseina insolubilizzata", venne descritto dal restauratore come un

[...]cemento tenacissimo, molto adesivo, insolubile nell'acqua (anche bollente) e negli alcali e resistente quindi all'umidità [...] usato, pochi anni or sono, per il consolidamento degli affreschi famosi delle volta della Cappella Sistina a Roma[...]. ${ }^{360}$

Quest'ultimo riferimento è molto importante, perché ci dimostra la considerazione che il restauratore ticinese faceva alle tecniche in quel periodo utilizzate nella vicina Italia. Questo mezzo fu ulteriormente usato nel consolidamento del resto dei dipinti e nelle stuccature che venivano prima trattate con il caseato e successivamente con la malta di calce e polvere di marmo.

La superficie dell'intonaco della controfacciata fu invece consolidata con silicati, pulita e in seguito reintegrata procedendo con una intensificazione del colore delle fasce rosse che incorniciano il dipinto e nascondendo con velature e patinature le macchie biancastre.

\footnotetext{
${ }^{59}$ Caseato o calcio caseinato. Si tratta d'un composto di caseina lactica e idrossido di calce $(\mathrm{Ca}(\mathrm{OH}) 2)$. II consolidante ottenuto è duro ed insolubile all'acqua una volta asciutto.

${ }^{360}$ (Pozzi Tita, Relazione di restauro, , indirizzata al Lod.le DPE, Bellinzona. Massagno-Lugano 22 settembre 1942. AUBCBZ. s. 204 Prugiasco, San Carlo di Negrentino).
} 


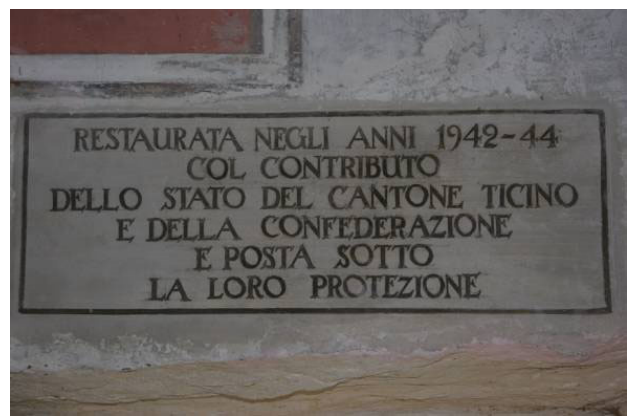

Figura 109 Giner Cordero E. S. Carlo, Particolare della targa commemorativa dei lavori di restauro. Giner. 2006.

L'uso dei silicati, in concreto del silicato di potassio, citato alla fine del preventivo del Pozzi $^{361}$, risale nel Canton Ticino al 1930 circa come materiale consolidante. Questo composto, conosciuto già nel medioevo con il nome di "liquor silicium", fu oggetto di numerosi studi eseguiti da Johann Wolfgang von Goethe dal $1768^{362}$, finché per iniziativa di Ludovico I Re di Baviera, il ricercatore Adolf Wilhelm Keim crea dei colori minerali a base di pigmenti inorganici che vengono legati con il silicato, ottenendo delle tonalità che potessero imitare le caratteristiche estetiche degli affreschi con dei materiali d'uso fattibile nel ferreo clima a nord delle Alpi. Alla fine del XIX secolo la ditta tedesca sperimenta i

\footnotetext{
${ }^{361}$ In questa ricerca sono stati già trattati i silicati quando si argomentava la proposta di restauro dei dipinti della chiesa di SS. Ambrogio a Maurizio a Chironico di Emilio Ferrazzini.

362 [...]Nell'ottavo libro della sua "Poesia e verità" scriveva: "Quello che mi ha tenuto occupato per la maggior parte del tempo è il cosiddetto 'liquor silicium' che si ottiene sciogliendo selce quarzifera pura con una parte appropriata di alcali. Si ottiene così una massa vetrificata trasparente che si scioglie all'aria formando un liquido chiaro e trasparente[...]" http://www.keim.it/.
}

colori in Svizzera con la tinteggiatura d'importanti facciate. II successo riscontrato si basava in risultati di grande protezione, durabilità e luminosità.

II silicato di potassio usato come consolidante degli affreschi trasforma il carbonato di calcio dell'intonaco in silicato di calcio insolubile, ciò che può permettere la coesione fra le parti.

La pulitura fu sicuramente realizzata con idrossido di sodio che frequentemente veniva adoperato in quel periodo; di fatto, anni dopo il restauro, l'apparenza spenta e vaporizzata dell'affresco sarà riconosciuta come conseguenza dell'utilizzo di questo prodotto commercialmente riconosciuto come soda caustica - [...]liquido che fece impallidire i colori naturali[.... $]^{363}$.

Tutti questi interventi furono compiuti nel mese di agosto, prima dei lavori architettonici, mettendo in sicurezza i dipinti ed evitando ogni pericolo avvenuto da questi ultimi.

\footnotetext{
363 Don Ignazio Pally del Comitato Pro-chiesa di Prugiasco con motivo di una retrasmissione della Televisione SI,.segnala in una lettera alla Commissione dei Monumenti Storici di Bellinzona del degrado degli affreschi del sec. XI raffiguranti il Cristo romanobizzatineggiante e gli Apostoli, i quali si trovano "assai sbiaditi e vaporizzati sicuramente causa dei sistemi di restauro usati negli anni 1942 - 1944, quali la soda caustica che corrose e fece impallidire i colori naturali. Inoltre risultano dei cuscinetti d'aria sotto l'intonaco che compromette la consistenza degli affreschi. (Comitato Pro-Chiesa di Negrentino Lettera Concerne affreschi romanici Cristo e Apostoli di Negrentino indirizzata alla CCMS. Bellinzona, 28 maggio $1969 .$. AUBCBZ. s. 204 Prugiasco, San Carlo di Negrentino).
} 


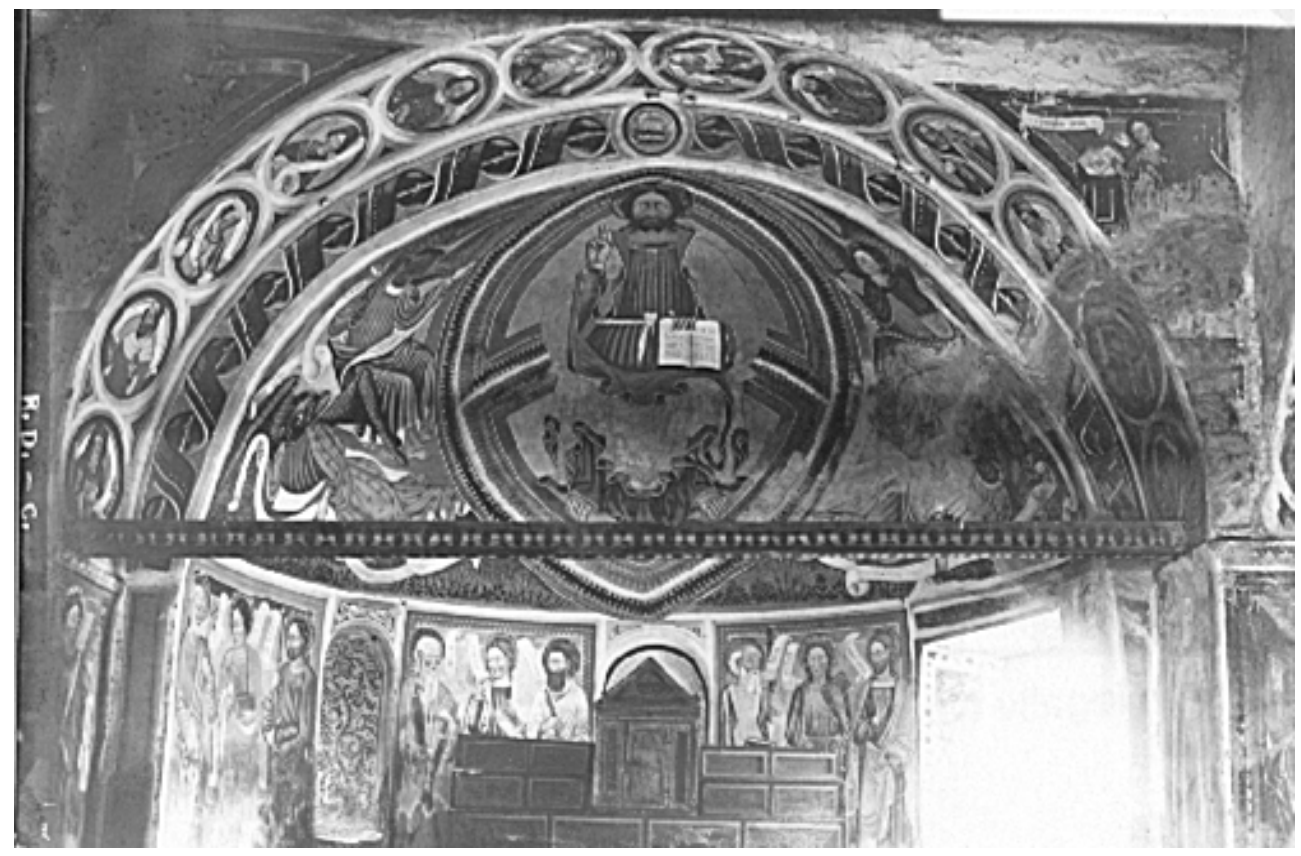

Figura 110 Abside nord prima dei restauri. Prugiasco, Chiesa di San Carlo di Negrentino, 1940 ca.AUBCBZ, Archivio fotografico. Prugiasco, San Carlo di Negrentino.

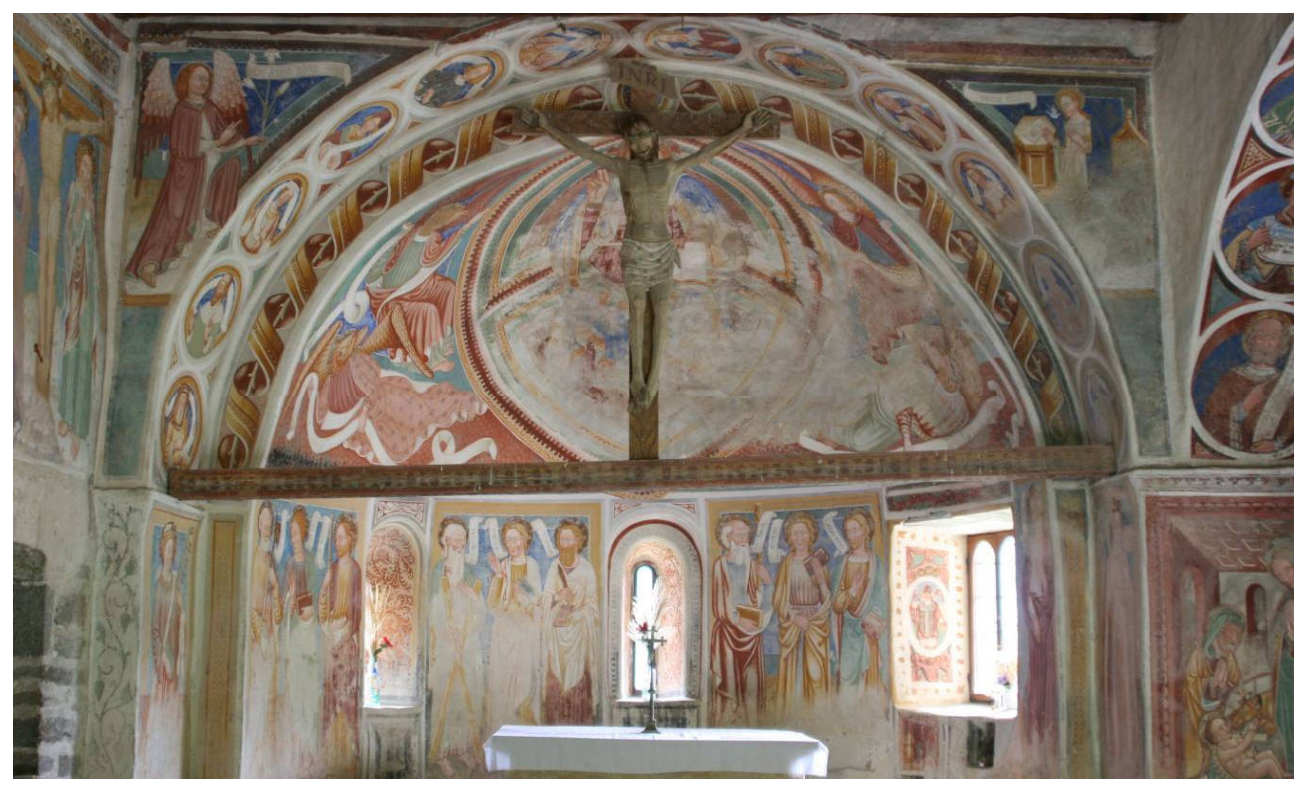

Figura 111 Giner Cordero, E. Abside nord. Prugiasco, Chiesa di San Carlo di Negrentino. 2006. 


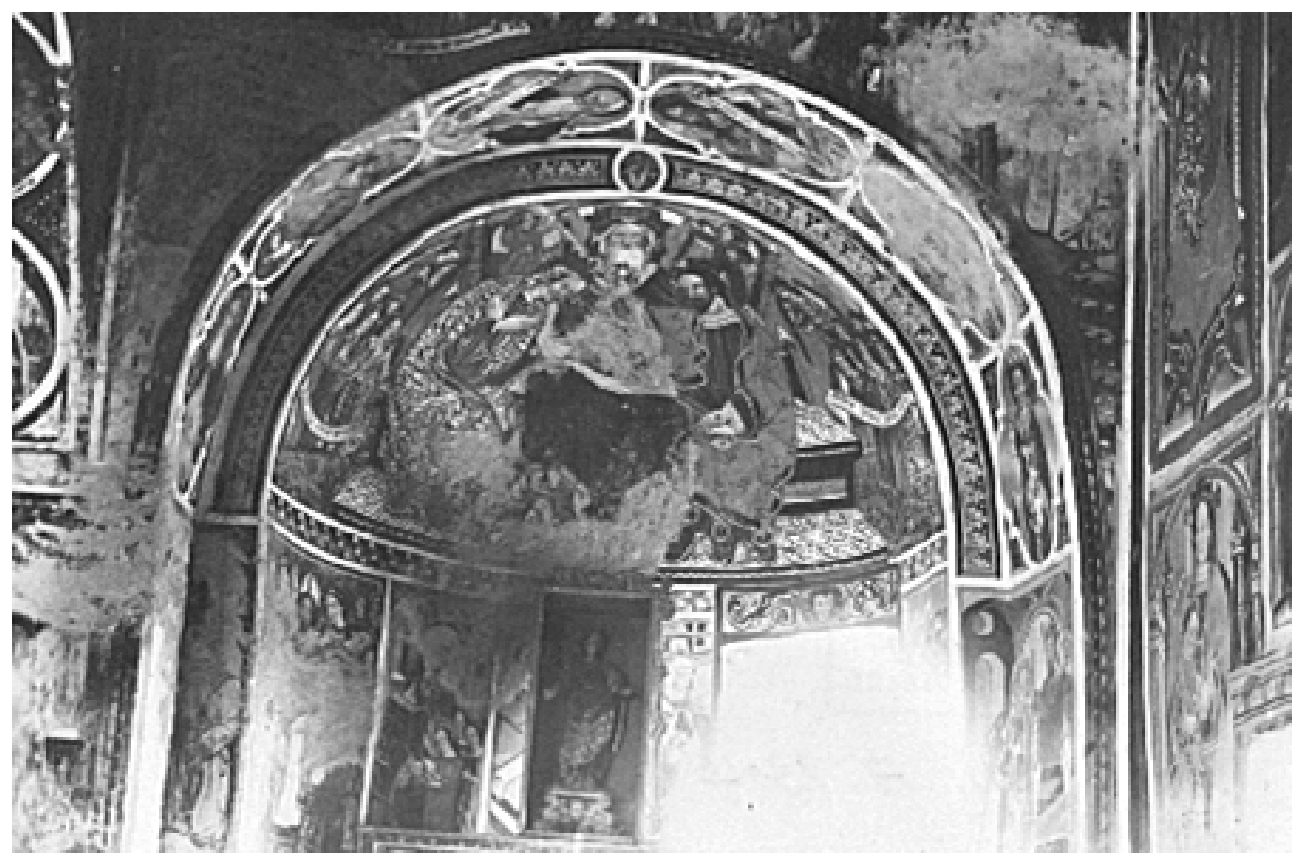

Figura 112 Abside sud prima dei restauri. Prugiasco, Chiesa di San Carlo di Negrentino, 1940 ca. 3410 A. 1304. Archivio Federale dei Monumenti Storici. Collezione grafica, Biblioteca nazionale svizzera.

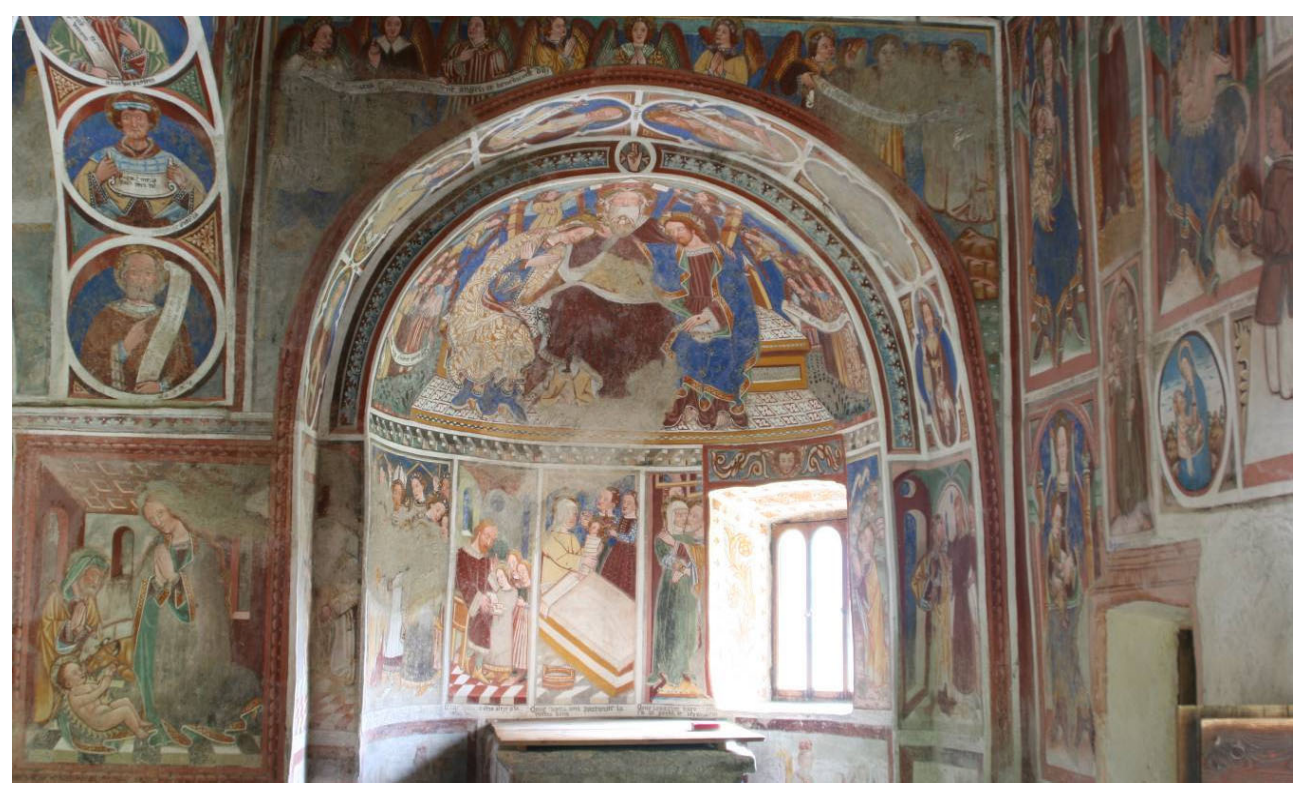

Figura 113 Giner Cordero E. Abside sud. Prugiasco. S. Carlo di Negrentino. 2007. 
Restauratori in Canton Ticino fra Ottocento e Novecento

Catalogazione e gestione dati

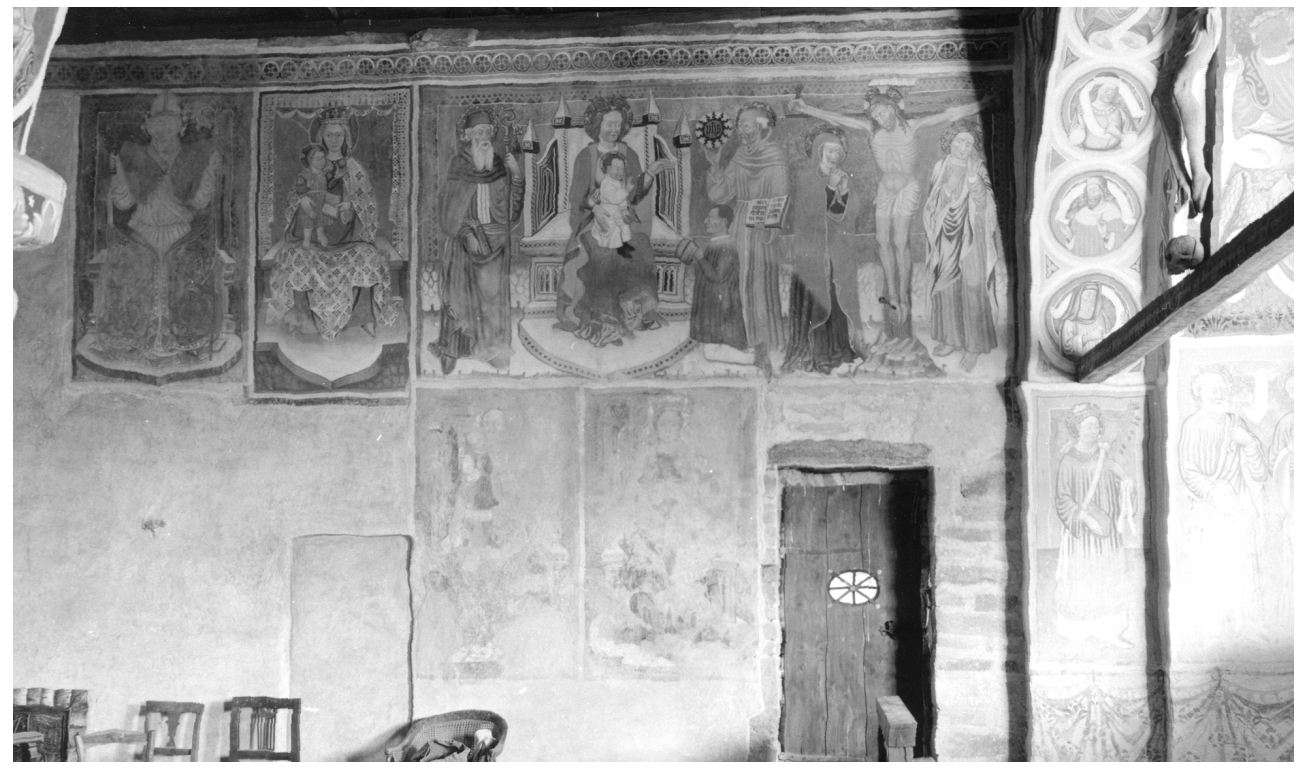

Figura 114 Affreschi della parete nord dopo i restauri. Prugiasco, Chiesa di San Carlo di Negrentino, 1944 ca. AUBCBZ, Archivio fotografico. Prugiasco, San Carlo di Negrentino.

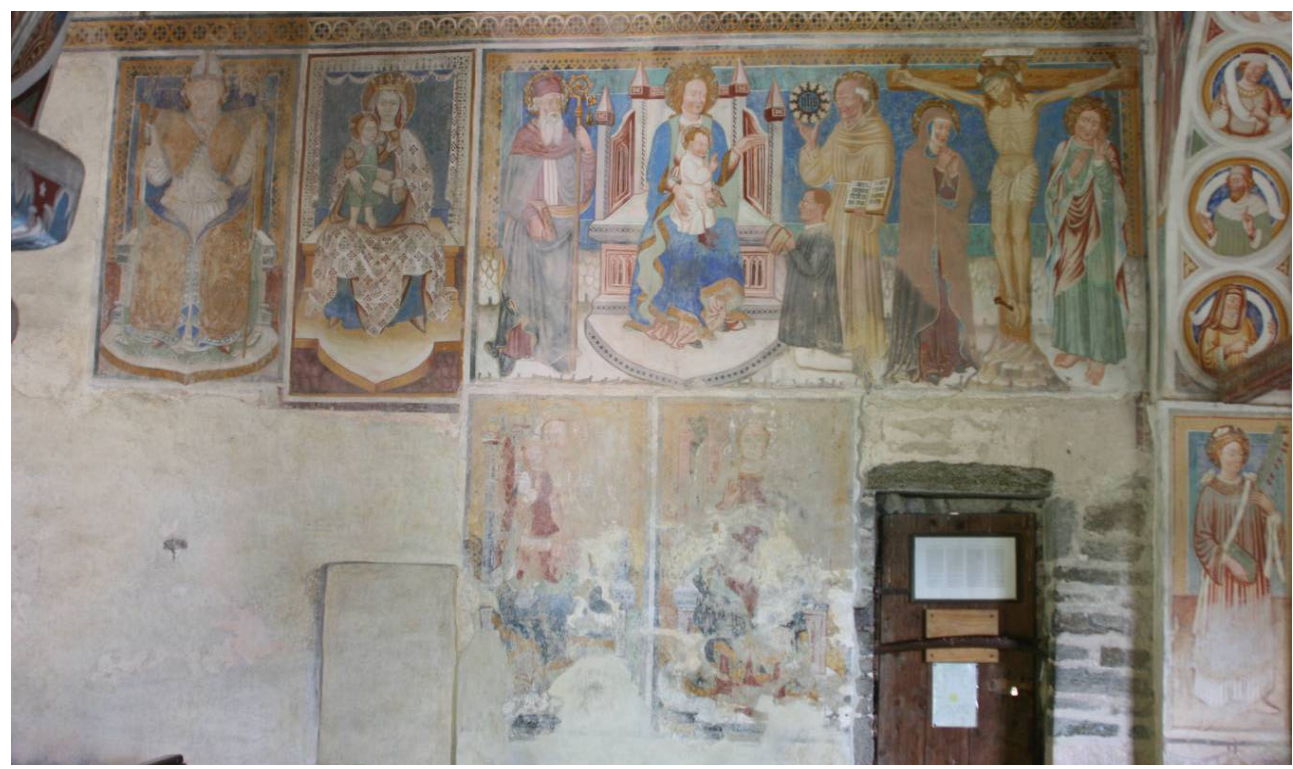

Figura 115 Giner Cordero, E. Affreschi della parete nord dopo i restauri. Prugiasco, Chiesa di San Carlo di Negrentino. 2007. 


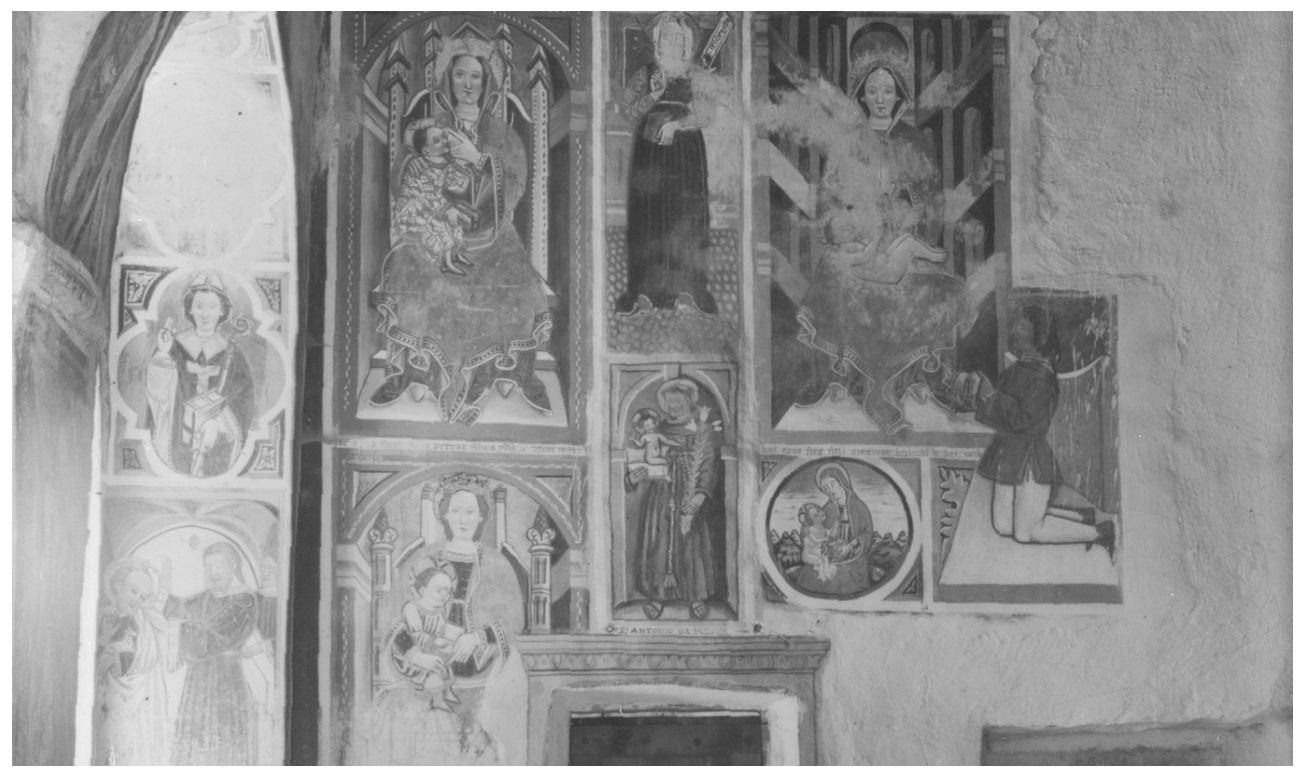

Figura 116 Affreschi della parete meridionale prima del restauro. Prugiasco, Chiesa di San Carlo di Negrentino, 1940 ca. AUBCBZ, Archivio grafico.

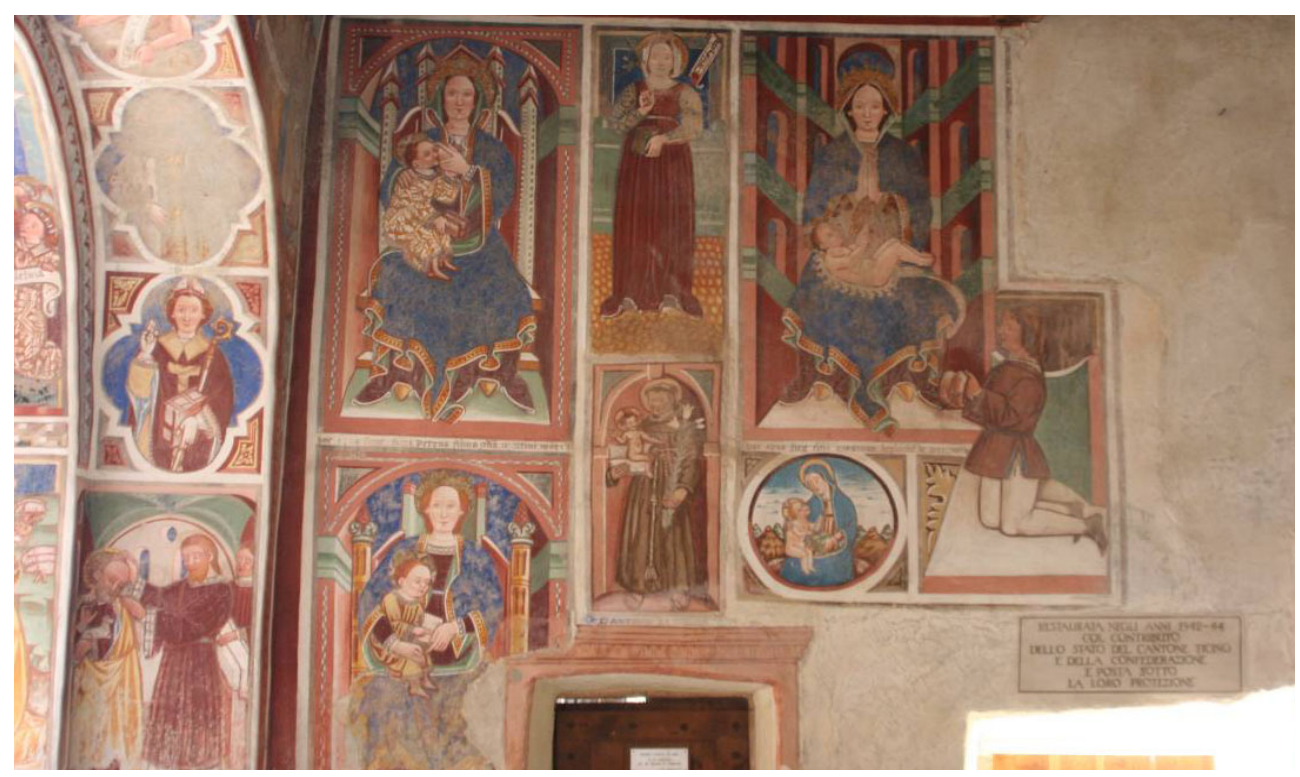

Figura 117 Giner Cordero, E. Affreschi della parete meridionale. Prugiasco, Chiesa di S. Carlo di Negrentino. 2007. 


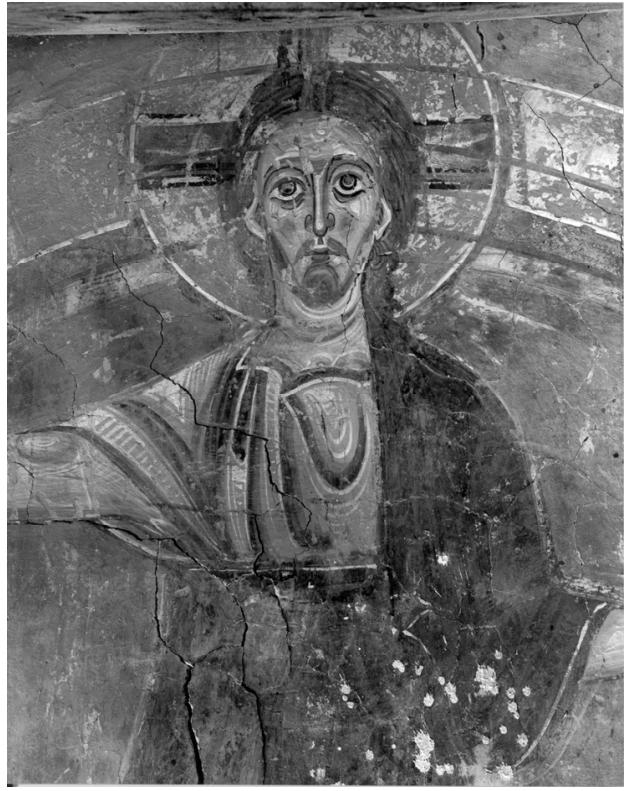

Figura 118 Particolare degli affreschi raffigurati sulla porta antica, nella contrafacciata della chiesa di San Carlo a Negrentino. Fotografia b/n, 1940 ca. Pubblicata nella Rivista Storica Ticinese (Crivelli, Restauri, bellezze naturali, tradizioni. Rettili preistorici., 1944, p. 963)

[...]Al consolidamento degli intonaci segui un'altra minuziosa e paziente fatica: il ripristino dei colori sbiaditi, il rifacimento delle aureole dorate e la completazione di parti mancanti con tinte neutre. $[\ldots]^{364}$

L'intervento qui eseguito determina le prassi di lavoro del restauratore, come riscontrato nei restauri successivi: negli affreschi cinquecenteschi attribuiti ad Antonio da Tradate nella Cappella di Sant'Anna in Ponto Valentino (1944), sugli affreschi Seregnesi della Cappella dei Morti

364 Gianella Riccardo A proposito dei restauri a San Carlo di Negrentino citato in (Crivelli, Restauri, bellezze naturali, tradizioni. Rettili preistorici., 1944, p. 963). in Semione (1944), nella Cappella Dionigi in Malvaglia (1944), nell'affresco della Madonna in Trono con i SS. Rocco e Sebastino in Largario (1944), nella Chiesa Parrocchiale, la Cappella in Piano ed il Pretorio di Lottigna (1946 - 1950), come esempi più importanti, dove si prevedevano puliture con idrossido di sodio e nel caso l'aggiunta di formaldeide come disinfettante, il consolidamento in profondità con iniezioni di cemento e dell'intonaco o del supporto sgretolato mediante fluatazione ${ }^{365}$, i rinzaffi con cemento e prodotti Sika ${ }^{366}$, l'otturazione dei buchi e delle crepe con stucco caseoso, consolidamento della superficie dipinta con soluzioni caseose e miscele di silicati, localizzate applicazioni di cera per ravvivare la tonalità dei colori, ritocco delle lacune stuccate con delle tonalità neutre o a basso tono, ricostruzioni discrete attenuate, e velature alle zone abrase o macchiate generalmente con colori a caseina o alla tempera, che lui descriveva come un [...]parco e cauto restauro pittorico $[. . .]^{367}$.

La percezione che oggi abbiamo della chiesa di San Carlo a Negrentino mostra chiaramente i numerosi ridipinti che il

\footnotetext{
${ }^{365}$ La fluatazione è un trattamento acido del muro che si realizza con dei prodotti a base di fluosilicati di alluminio e magnesio. Questo trattamento ha lo scopo di trasformare i sali solubili in acqua (per esempio in salnitro) in composti insolubili e quindi non dannosi per la struttura. In questo modo vengono bloccate ulteriori possibilità di sviluppo di efflorescenze saline e, nello stesso tempo, viene omogeneizzata la natura chimica del supporto garantendo una uniformità di reazione dello stesso con i rivestimenti minerali.

${ }^{366} \mathrm{http}: / /$ www.sika.it/.

${ }^{367}$ (Pozzi Tita, Relazione di restauro indirizzata al DPE Massagno - Lugano 15 ottobre 1944. AUBCBZ.s.091 Corzoneso, San Remigio).
} 
sopracitato preventivo prevedeva, con il rifacimento delle cornici, dei blu mancanti e di certi colori alterati; osserviamo la densità delle linee bianche che chiudono le scene figurative e perfino diverse macchie prodotte durante l'applicazione di questa tempera sovrastante il colore originale. Si verificano le stesse caratteristiche nei blu, anche se questi ritocchi non sono arrivati a noi a causa del deterioro nel tempo. Una delle prime critiche al metodo di restauro eseguito da Pozzi attaccherà giustamente questi ritocchi. Don Robertini scrive per il Giornale del popolo un articolo dove chiede:

[...] com'erano i blu dei fondi e come sono oggi?[...] Bleu il manto del Cristo, bleu il manto regale della Madonna, di tutte, anzi, le Madonne; bleu il cielo, e ce n'è molto, per angeli, apostoli e santi; bleu cobalto, bellissimo, nel brano della crocifissione dei Seregnesi, e bleu oltremare dappertutto, altrove[...] $]^{368}$.

A cui Pozzi si difenderà rispondendo:

[...] era per quasi la metà, caduto o scopato via, perché eseguito a tempera. D'accordo con la Commissione ed il Sovrintendente ho consolidato quello che ancora restava e ne ha rimesso parzialmente e parcamente dove era caduto e scopato via, e ciò per riottenere un parziale equilibrio armonico degli affreschi. E qui è questione di criterio di restauro: le teorie e le tendenze in materia sono molte e di tutte le gradazione $[. . .]^{369}$.

\section{E Riccardo Gianella:}

[...]II restauratore procedete [...] con appropriati scrostamenti, stuccature e tinteggi tali da conferire un aspetto armonioso all'insieme,

368 Don Robertini citato in Giornale del popolo 18 settembre 1945. Sbt, BCLU.

369 Pozzi Tita citato in Giornale del popolo 18 settembre 1945. Sbt, BCLU. senza nulla togliere e senza tentare di nulla aggiungere ai dipinti originali, né menomarne $i$ pregi $[\ldots]^{370}$.

Saranno gli specialisti chiamati a valutare la qualità dei restauri realizzati in Canton Ticino fino al 1949 ad osservare positivamente il restauro di Pozzi dichiarando che:

[...] il restauro è stato ben condotto, così che $i$ dipinti conservano - cosa molto rara! - l'azzurro originale, applicato talvolta anche a tempera. Poteva essere più limitata qualche ripresa dello stesso azzurro, ripresa eseguita però con molto buon senso. $^{371}$

Altre critiche si alimenteranno del fatto che I'intonaco della parete a ponente avrebbe necessitato d'essere consolidato nuovamente sei anni dopo, e accusava di questi interventi poco riusciti all'inefficacia del caseato usato dal restauratore. ${ }^{372} \mathrm{Ma}$ le spiegazioni con cui Pozzi si protegge di questi rimproveri dimostrano una assoluta consapevolezza della situazione, e che le sue attuazioni fecero tutto il possibile per la salvaguardia del materiale murario, colpito nel 1944 dai lavori architettonici con l'apertura dell'antica porta d'entrata e la realizzazione dell'intercapedine interna ed esterna.

\footnotetext{
${ }^{0}$ Gianella Riccardo citato in Giornale del popolo 18 settembre 1945. Sbt, BCLU.

${ }^{371}$ Rapporto 1949 DPE. (Marcionetti, 1977).

372 || 23 febbraio 1950 il DPE chiede a Tita Pozzi di sfruttare il suo soggiorno in Valle di Blenio con motivo del restauro della Cappella di Lottigna per intervenire sui dipinti nella parete di ponente lì dove il collegio d'esperti ha costatato la mancanza d'adesione dell'intonaco. AUBCBZ. s.204 Prugiasco, San Carlo di Negrentino.
} 


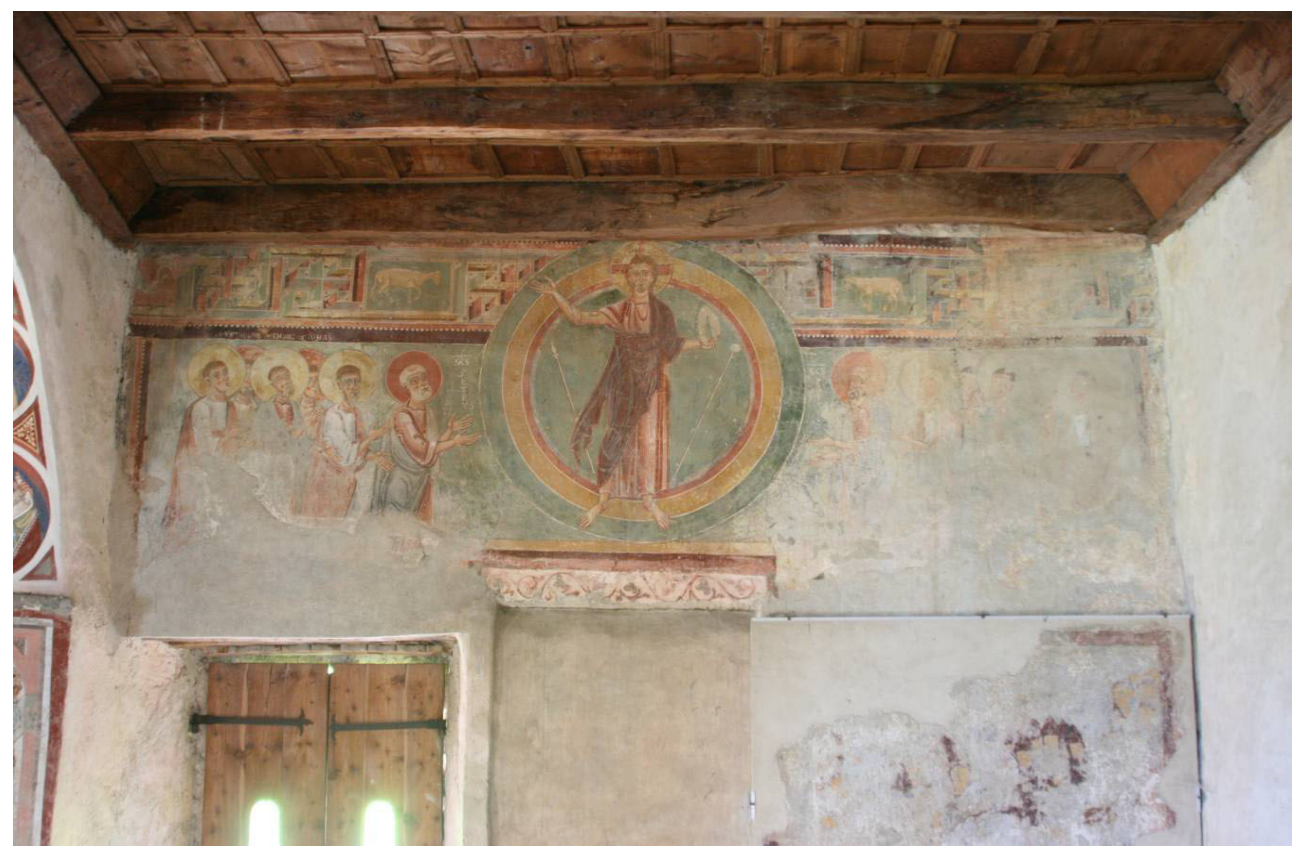

Figura 119 Giner Cordero, E. Affreschi della contrafacciata rafiguranti la Risurrezione, della Ascensione e del Giudizio Finale. XI sec. Chiesa di San Carlo, Negrentino (Prugiasco), 2007.

Chiamato ad intervenire nuovamente sull'affresco bizantino nel 1950, il restauratore operò questa volta forando di un centimetro di diametro il muro nei punti convenienti e successivamente iniettando attraverso questi, con siringhe adatte, lo stucco-cemento con gran violenza facilitando la sua penetrazione ed espansione nelle zone di stacco, dopo di ché si riapplicarono i fori di intonaco tagliati. Le parti più pericolanti previamente intelate come provvedimento di sicurezza, furono stuccate sul retro e posteriormente ricollocate e puntellate con assicelle e listoni. II lavoro finì con la sigillatura delle fessure e con la pulitura del dipinto $^{373}$.

Solo un anno dopo, il Dipartimento della Pubblica Educazione percepirà gli ultimi deteriori sul dipinto del XIII sec. restaurato dal Pozzi, e raccomandato dalla Sovrintendente alle Gallerie di Milano, Fernanda Wittgens ${ }^{374}$, chiederà al

\footnotetext{
373 (Pozzi Tita, Relazione tecnica rinsaldamente affresco del 1200 nell'interno della Chiesa di Negrentino, indirizzata alla CCMS. 1950 ca. AUBCBZ. s.204 Prugiasco, San Carlo di Negrentino).

${ }^{374}$ Wittgens Fernanda (Milano 1903- 1957). iniziò la sua carriera come docente di storia dell'arte ai licei, per passare poi nel 1928 a collaborare all'interno del Palazzo di Brera con Ettore Modigliani. Diventerà poco dopo, nel 1933 Ispettrice alla Gallerie e nel 1941 Direttore, proclamandosi la prima donna ad assumere
} 
restauratore Mario Rossi ${ }^{375}$ di Varese d'esaminare la situazione, avvertendo sulla possibilità di staccare il dipinto per rinsaldare completamente la superficie sottostante e il suo riattacco in sito in collaborazione con il pittore Mario Moglia di Lugano ${ }^{376}$. II 25 luglio del 1951 Rossi risponde $^{377}$ al Consigliere di Stato

il più alto carico all'interno dell'organo della Sovrintendenza a Milano, promovendo una significativa riforma dei musei e nella tutela del patrimonio artistico della città. Dal 1945 sarà assunta come Pro-Direttore e Commissario per l'Accademia delle Belle Arti di Brera. Appoggierà Modigliani nella ricostruzione della Pinacoteca dopo i bombardament nel 1950 lo stesso anno che venne nominata Sovrintendente alle Gallerie della Lombardia. Fra le opere di sovrintendenza più importanti in questi anni si troveranno la ricostruzione del Museo Teatrale della Scala e del Poldi Pezzoli, il restauro del Cenacolo di Leonardo, mentre che il suo progetto più ambizioso supponeva la trasformazione di Brera in un polo culturale e la creazione di un Laboratorio-Scuola del Restauro. Informazione presso il sito http://www.fondazionebadaracco.it/badaracco_test/ archivi/fondo fernanda wittgens/wittgens.htm

${ }^{375}$ II pittore-restauratore italiano Mario Rossi era già conosciuto in Svizzera per i suoi lavori di restauro in Locarno, in S. Maria en Selva ed in Ascona. (Wittgens Ferdinanda, Palazzo di Brera. Lettera indirizzata DPE del Canton Ticino. Milano 21 maggio 1951. AUBCBZ. s.204 Prugiasco, San Carlo di Negrentino).

376 (DPE, Concerne restauro pittorico. Lettera indirizzata al pittore-restauratore Mario Rossi di Varese, in copia alla CCMS, prof. Piero Bianconi, pittore Mario Moglia. Bellinzona 20 giugno 1951. AUBCBZ. s. 204 Prugiasco, San Carlo di Negrentino).

377 [...]Dopo un attento e scrupoloso esame posso tranquillamente affermare che la parete sulla quale è dipinto l'affresco è attualmente asciutta e non presenta tracce di umido o di salnitro. L'intonaco a sua volta è ben saldo al muro e solo due piccole zone di una diecina di centimetri quadri sono isolate, non aderenti e necessita la loro saldatura con le solite iniezioni di caseato di calce[...]. (Rossi Mario, Lettera al Consigliere di Stato Brenno Galli. Varese 25 luglio 1951. AUBCBZ. s. 204 Prugiasco, San Carlo di Negrentino).
I'avvocato Brenno Galli, da cui partiva il mandato, con una perizia che escludeva qualsiasi drastica operazione sulle pitture che riteneva fossero in uno stato idoneo di conservazione dopo il lavoro effettuato nel 1950. Necessitavano di consolidarsi con la stessa malta di caseato alcune zone molto puntuali e realizzare una finitura delle stuccature del Pozzi seguite da opportune tinteggiature.

$\mathrm{Nel}$ frattempo, senza conoscere la risoluzione di Mario Rossi dopo l'analisi del dipinto, Aldo Crivelli aveva pubblicato sulla rivista Svizzera Italiana ${ }^{378}$ un ironico articolo intitolato "Le desgrazie non vengono mai sole" diffondendo pubblicamente delle false condizioni in cui si trovava il dipinto bizantino della chiesa di San Carlo di Negrentino:

[...]il tenacissimo mastice adesivo ed insolubile, in meno di sette anni ha mollato disastrosamente ed ora si deve ricorrere al mezzo estremo: lo strappo del dipinto dal muro ed il riporto su tela[...].

La CCMS scrive a Crivelli negando quanto pubblicato nella rivista $^{379}$ e chiede di smentire le inquietudine diffuse che fra l'altro avevano offeso il proprio restauratore che vede in pericolo il suo prestigio:

[...] Lo scritto - con relativa vignetta - m'ha fatto veramente male[...] mi sarà data ancora fiducia nella mia opera di restauratore, oppure no?[...]. ${ }^{380}$

\footnotetext{
${ }^{378}$ (Crivelli, Le desgrazie non vegono mai sole, 1951)

${ }^{379}$ (Commissione Cantonale dei Monumenti Storici. Lettera indirizzata ad Aldo Crivelli. Lugano, 21 settembre 1951. AUBCBZ. s. 204 Prugiasco, San Carlo di Negrentino).

${ }^{380}$ (Pozzi Tita, Lettera indirizzata a Francesco Chiesa, presidente della CCMS. Massagno - Lugano 29 giugno
} 


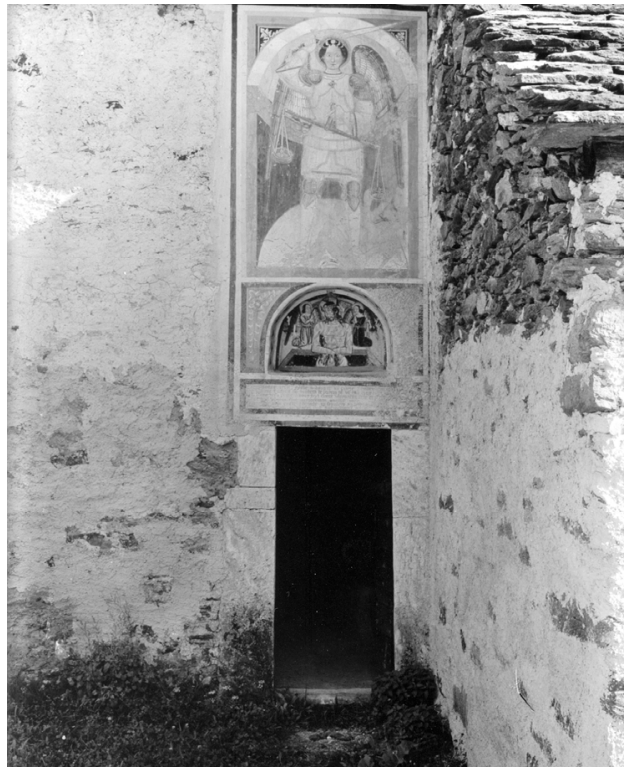

Figura 120 Porta d'acceso. Chiesa di San Carlo di Negrentino, 1940 ca. AUBCBZ, Archivio grafico.

Nuove perizie svolte nel 1957, descriveranno in modo più esaustivo il restauro eseguito all'altare maggiore che in quell'anno appariva alterato dagli effetti negativi dell'umidità.

Furono Don Robertini e Piero Bianconi a richiamare l'attenzione su questo fatto e chiedere consulenza al pittore Mario Moglia $^{381}$. Le parti che svelavano maggiormente il danno erano, secondo il restauratore, le reintegrazioni a tempera di Tita Pozzi del 1944 quali il blu del mantello del Pantocrator, il risvolto in terra verde e

1951. AUBCBZ. Filza 204 Prugiasco, San Carlo di Negrentino).

${ }^{381}$ (DPE, Concerne: chiesa di Negrentino. Lettera indirizzata al pittore Mario Moglia in copia al prof. Francesco Chiesa, prof. Bianconi, don Robertini, sac. Giuseppe Gallizia, scultore Giovanni Genucchi. Minusio, 13 luglio 1957. AUBCBZ. s.204 Prugiasco, San Carlo di Negrentino). dei ricami in terra rossa su sfondo di ocra gialla, mentre che il colore originale a fresco, sottostante ad alcuni ridipinti, conservava delle buone condizioni. Moglia dichiara perfino il degrado estetico creato da questi ritocchi in quanto dissimili dal originale:

[...]Tutta la ridipittura unitamente ad un ritocco più recente e di altra concezione lassieme a parti annerite, caratteristica dell'ossidazione del colore) con la loro accesa coloritura alterano il vero aspetto del colore dell'affresco, qui basta confrontare l'azzurro delicato adoperato dal pittore con il bleu oltremare sovraccarico messo a corpo nella ridi pittura[...]. ${ }^{382}$

Oggi l'umidità ha degradato per completo tutti i ritocchi realizzati sul dipinto dell'abside maggiore e perfino comincia a creare delle importanti perdite sulle parti ancora conservate di pittura originale. Nella pareti dell'altare minore dove il deterioramento è minore possiamo osservare ancora questi blu tanto criticati aggiunti dall'autore, che se bene dissimili dal colore originale, racchiudono le lacune delle figure in modo da riportarli una omogeneità. La scelta del restauratore al realizzare queste reintegrazione supponevano una leggera differenziazione fra le zone ritoccate e quelle originali per cui scartiamo che fosse la sua intenzione quella di compiere un restauro imitativo, fra l'altro lontano dagli interventi riscontrati su altri monumenti da lui eseguiti. II ritrovamento di ridipitture significava la scelta di Pozzi per l'applicazione di leggere velature su quelle parte maggiormente abrase.

\footnotetext{
382 (Moglia Mario, Perizia al DPE. Viganello, 22 luglio del 1957. AUBCBZ. s.204 Prugiasco, San Carlo di Negrentino)
} 
Nel 1957, Mario Moglia propone come rimedio al problema della penetrazione d'acqua nella tazza absidale, il distacco e la ricollocazione delle piastre del tetto, che nell'ultimo intervento furono, in modo controproducente, aderite con cemento.

Un altro preventivo fu elaborato da Carlo Mazzi $^{383}$ di Locarno nello stesso anno, suggerendo un intervento molto più aggressivo che supponeva lo strappo dei dipinti dell'abside ed il risanamento del supporto. Questo poteva permettere allo stesso tempo la messa in luce degli affreschi trecenteschi sottostanti, anche se si consigliava verificare la qualità di essi, prima di realizzare le opportune operazioni. Non si presero in considerazione né la prima proposta né, fortunatamente, la seconda.

Nel 1966 l'architetto Tita Carloni ${ }^{384}$, ispettore dell'Ufficio Cantonale dei Monumenti Storici di Bellinzona, esamina i problemi strutturali della chiesa e più recentemente nel 1996, Antonella Lentini $^{385}$ lo fa con quelli inerenti ai dipinti murali, ancora questa volta senza opportunità a fare eseguire i diversi progetti.
Sempre da Mario Rossi sarà realizzato, nel 1969, lo strappo dei dipinti esistenti nella parte bassa della parete a ponente, la cui pulitura venne affidata a Luigi Gianola ${ }^{386}$, e nel 1977, ancora dal restauratore italiano, venne eseguito l'intervento sui dipinti della facciata orientale.

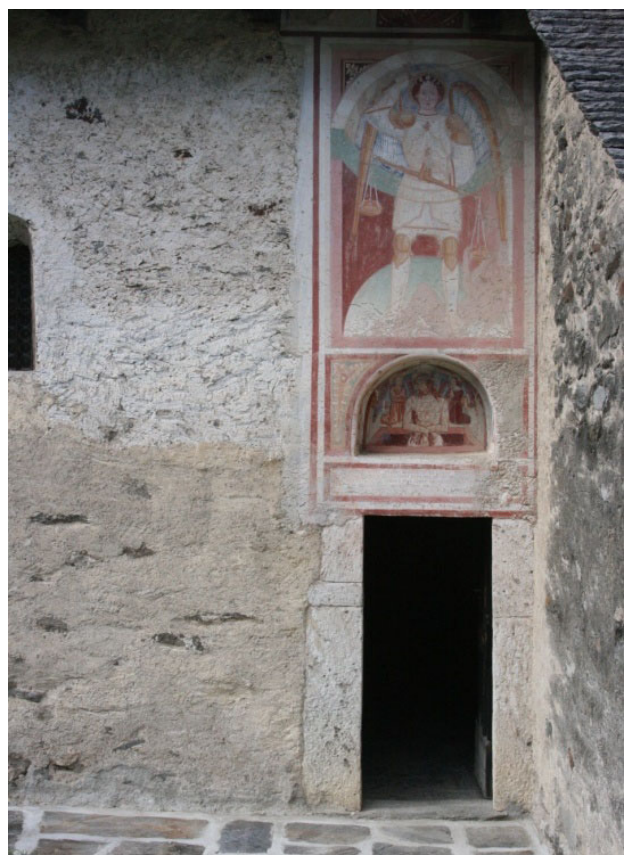

Figura 121 Giner Cordero E. Porta d'acceso. Chiesa di San Carlo di Negrentino. Prugiasco, 2007.

\footnotetext{
383 (Mazzi Carlo, Proposta d'intervento al DPE. Tegna-
Locarno, 28 ottobre 1957. AUBCBZ. s. 204 Prugiasco,

383 (Mazzi Carlo, Proposta d'intervento al DPE. Tegna-
Locarno, 28 ottobre 1957. AUBCBZ. s. 204 Prugiasco, San Carlo di Negrentino).

${ }^{384}$ Ispettore della sezione di pianificazione urbanistica del servizio per i monumento storici.

${ }^{385}$ (Lentini Antonella. Restauratrice diplomata ENAIP. Faido.Volta dell'abside sinistra, Chiesa Parrocchiale di Prugiasco. Studio dello Stato conservativo e ipotesi di intervento. Settembre 1996. AUBCBZ. s.204 Prugiasco, San Carlo di Negrentino).
} 


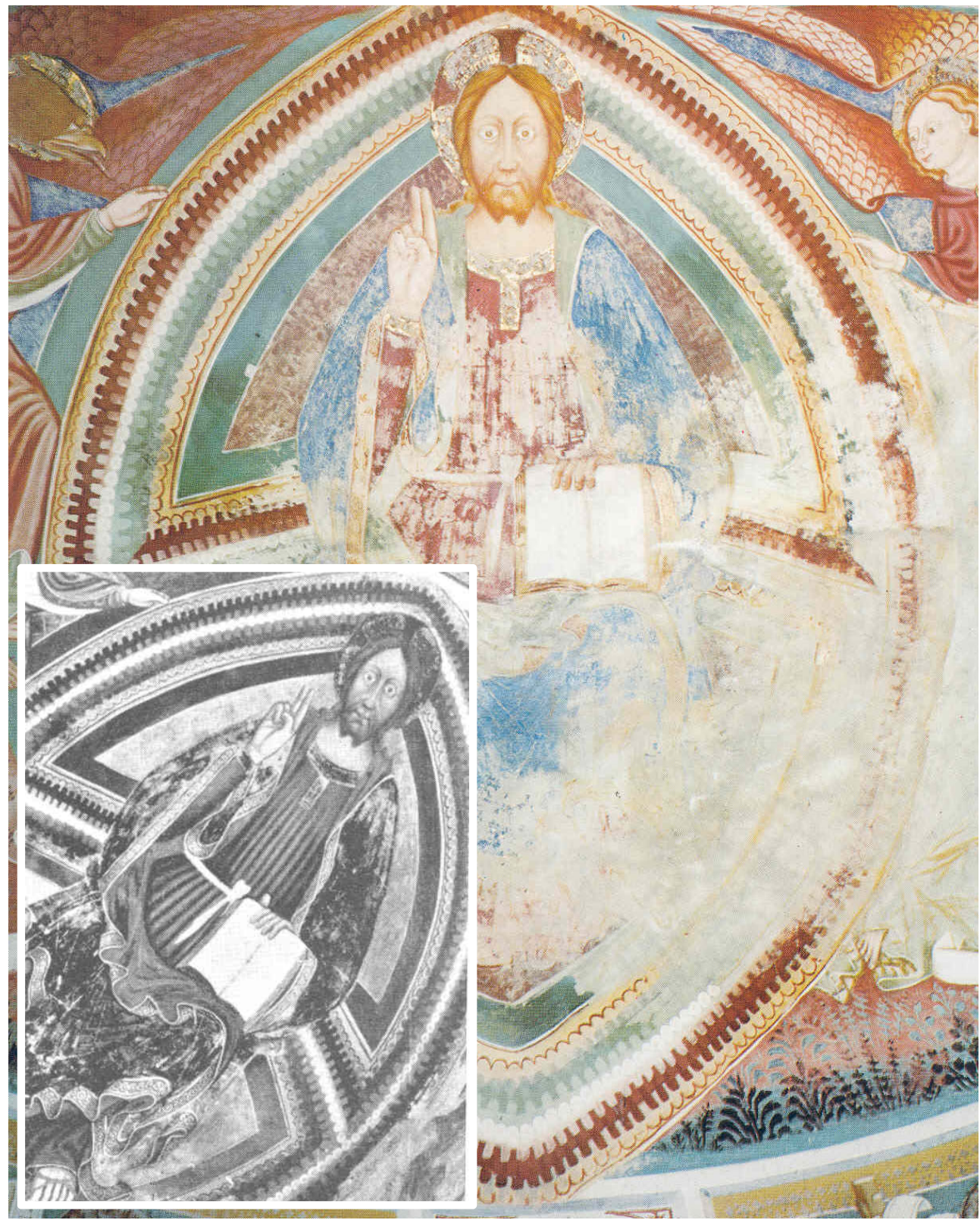

Figura 122 Confronto fra lo stato di conservazione dei dipinti murali dell'abside maggiore nei diversi momenti storici. Fotografia b/n in primo piano: particolare del Cristo redentore prima dei restauri $1940 \mathrm{ca}$. Pubblicata nella Rivista Storica Ticinese (Crivelli, Restauri, bellezze naturali, tradizioni. Rettili preistorici., 1944, p. 963). Fotografia a colore in secondo piano: particolare del Cristo redentore dopo i restauri. (Marcionetti, 1977). 


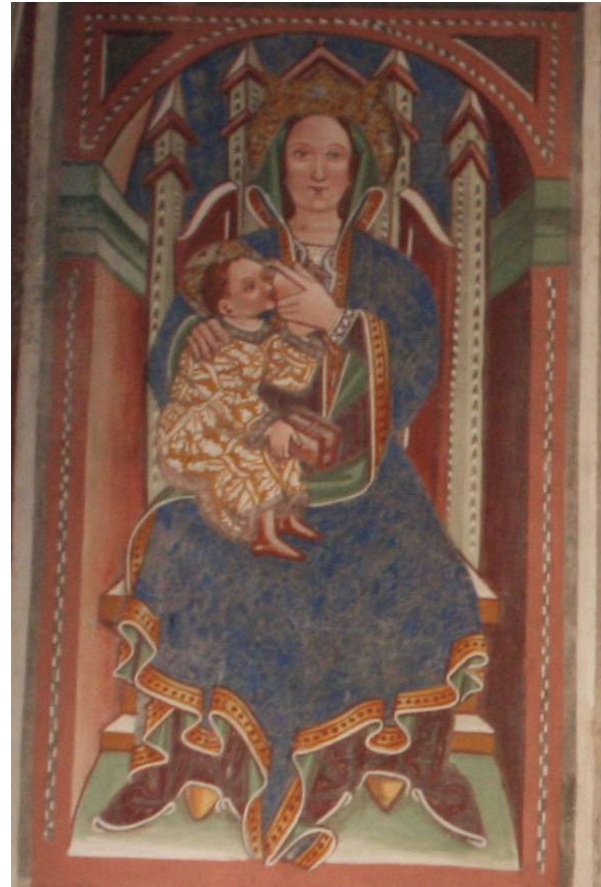

Figura 123 Giner Cordero E., Madonna del latte. Fotografia a luce naturale. 2007.

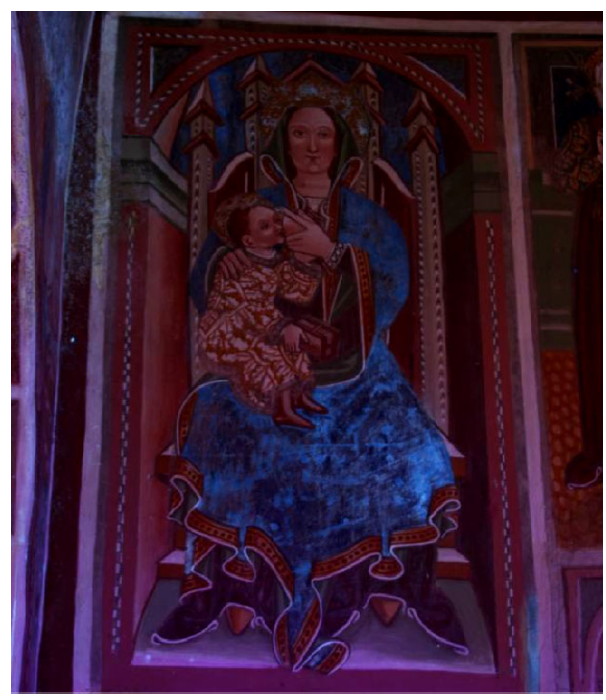

Figura 124 Giner Cordero E., Madonna del latte. Fotografia a luce UV. 2007.

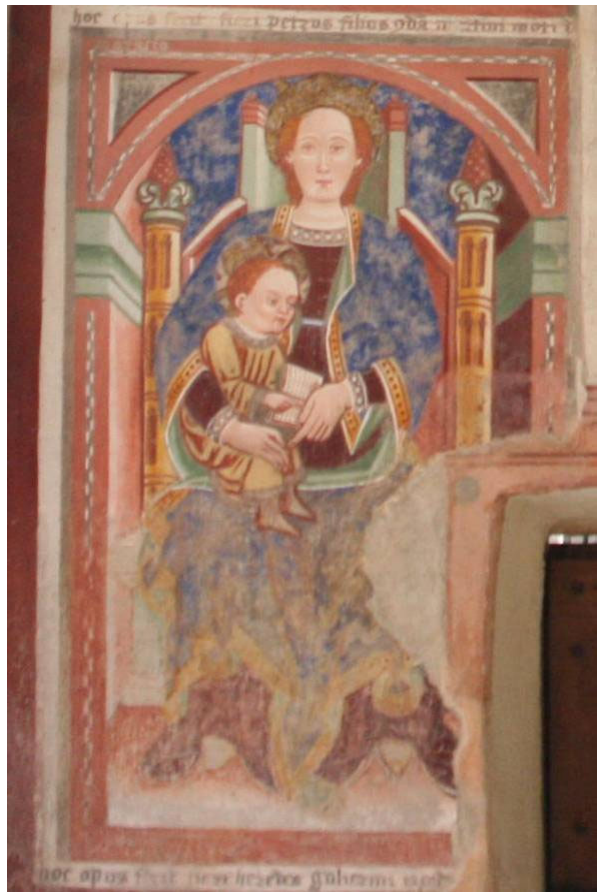

Figura 125 Giner Cordero E., Madonna con bambino. Fotografia a luce naturale. 2007.

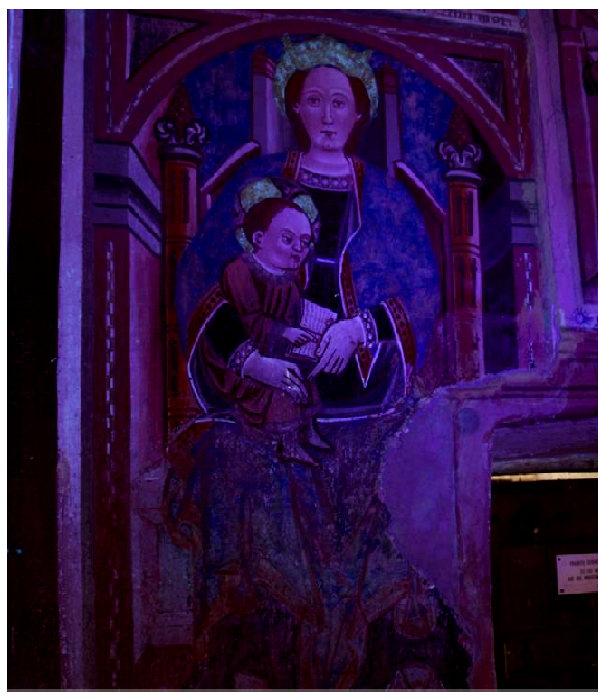

Figura 126 Giner Cordero E., Madonna con bambino. Fotografia a luce UV. 2007. 


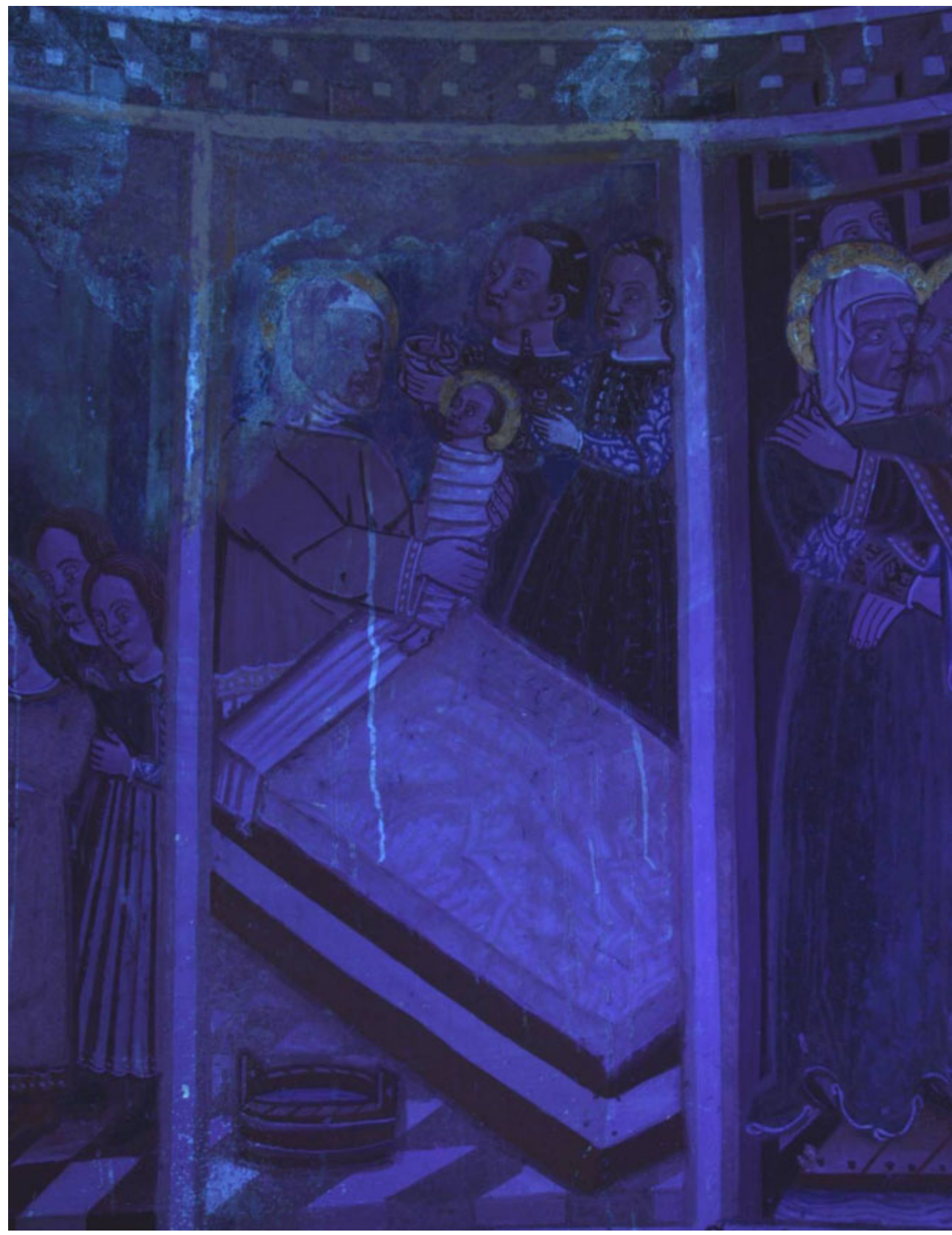

Figura 127 Giner Cordero E., Particolare degli affreschi dell'abside minore. Fotografia a luce UV dove si osservano fluorescenza delle gocciolature, così come delle dorature e delle lacune pittoriche. 2007. 


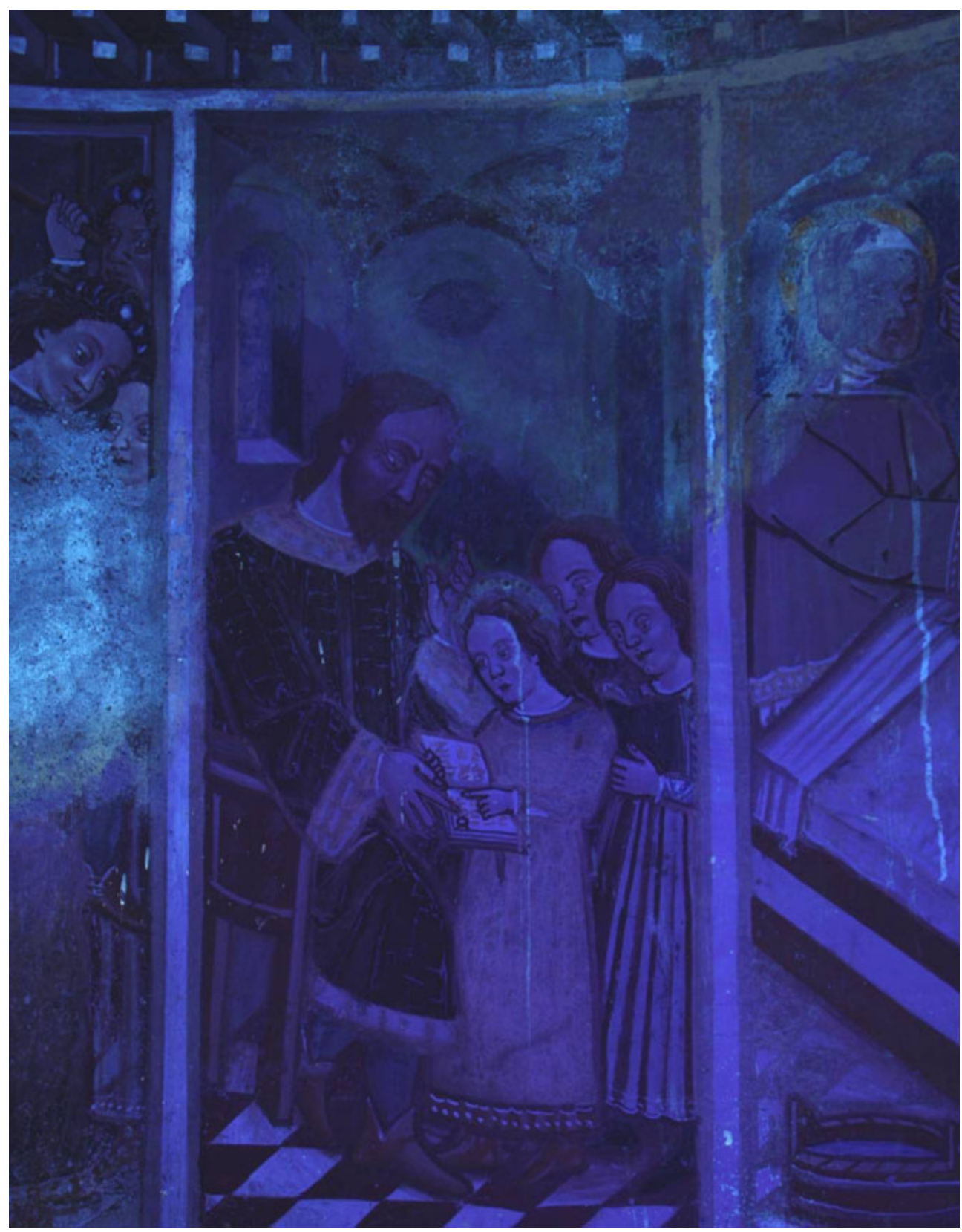

Figura 128 Giner Cordero E., Particolare degli affreschi dell'abside minore. Fotografia a luce UV dove si osservano fluorescenza delle gocciolature, così come delle dorature e delle lacune pittoriche. 2007. 
Restauratori in Canton Ticino fra Ottocento e Novecento

Catalogazione e gestione dati

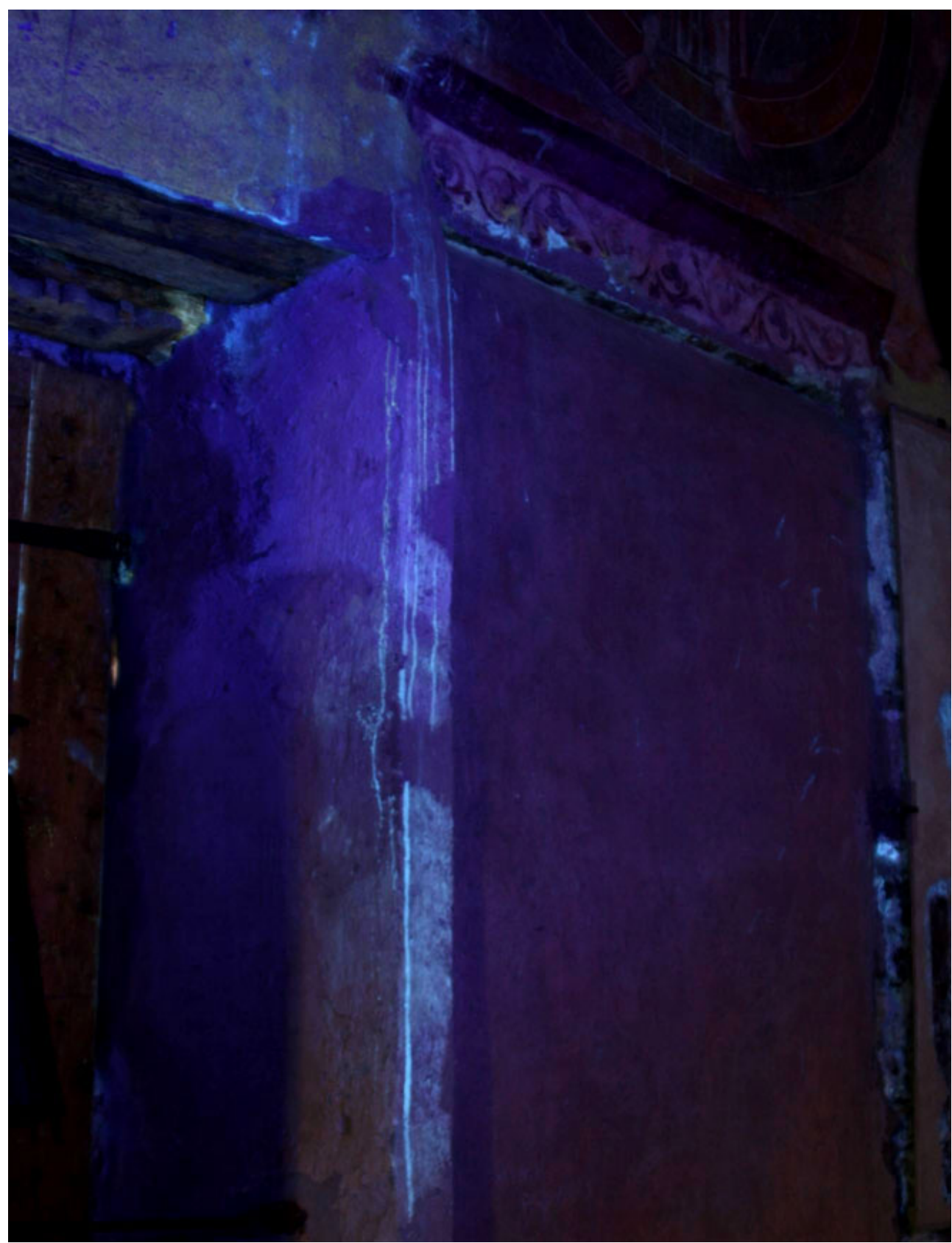

Figura 129 Giner Cordero E., Particolare della controfacciata della vecchia porta di entrata. Fotografia a luce UV. 2007. 


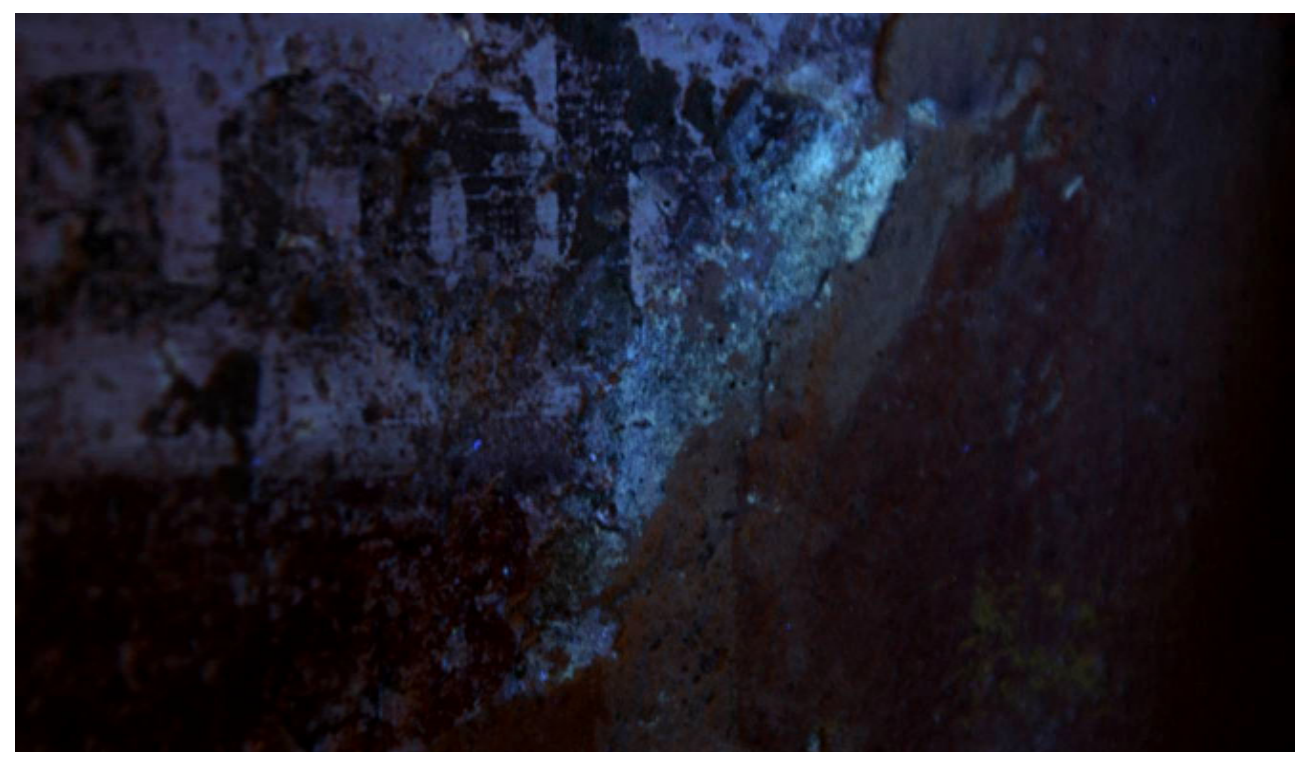

Figura 130 Giner Cordero E., Particolare di una reintegrazione e stuccatura sugli affreschi della navata con particolare fluorescenza ultravioletta che evvidenza la possibile presenza di sostanze proteiche sulla superficie. Chiesa di S. Carlo di Negrentino, Prugiasco. Fotografia a luce UV. 2007.

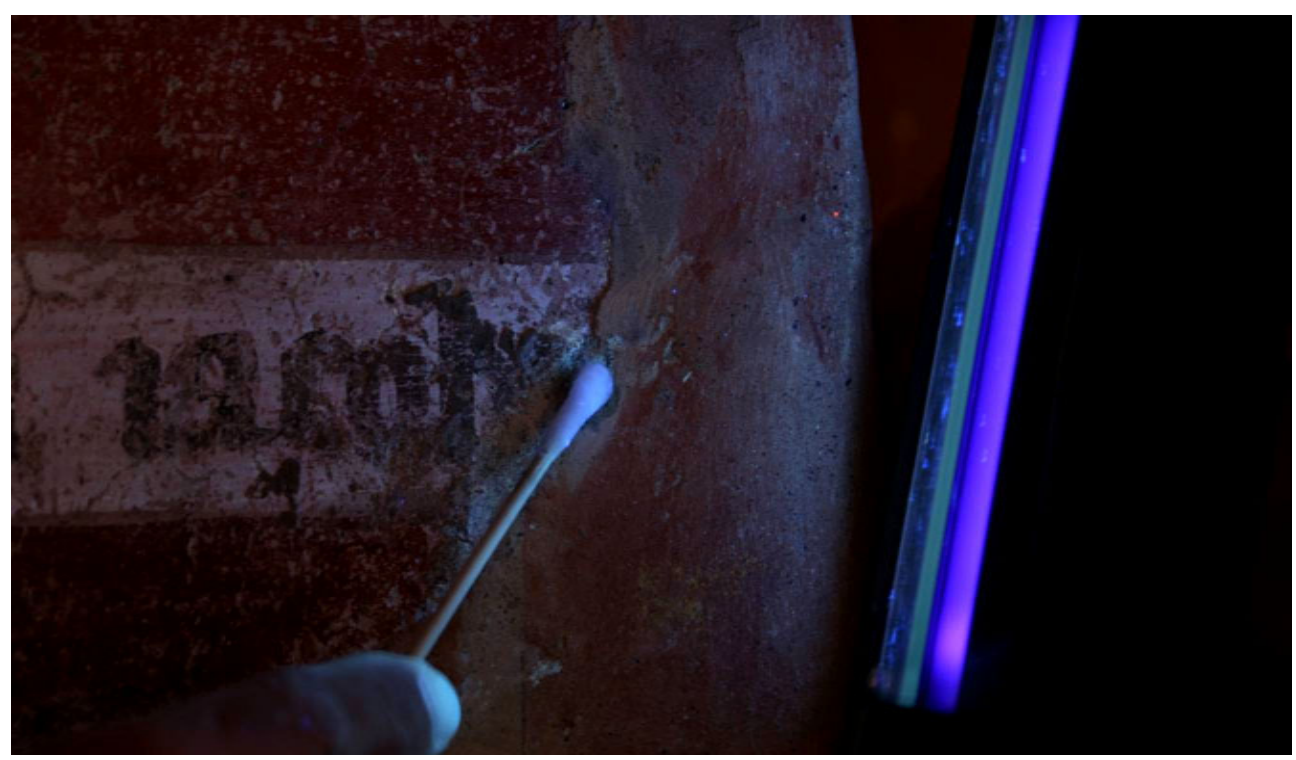

Figura 131 Giner Cordero E., Prova di pulitura per evvidenziare la presenza di sostanze cerose. Chiesa di S. Carlo di Negrentino, Prugiasco. Fotografia a luce UV. 2007. 
Restauratori in Canton Ticino fra Ottocento e Novecento

Catalogazione e gestione dati

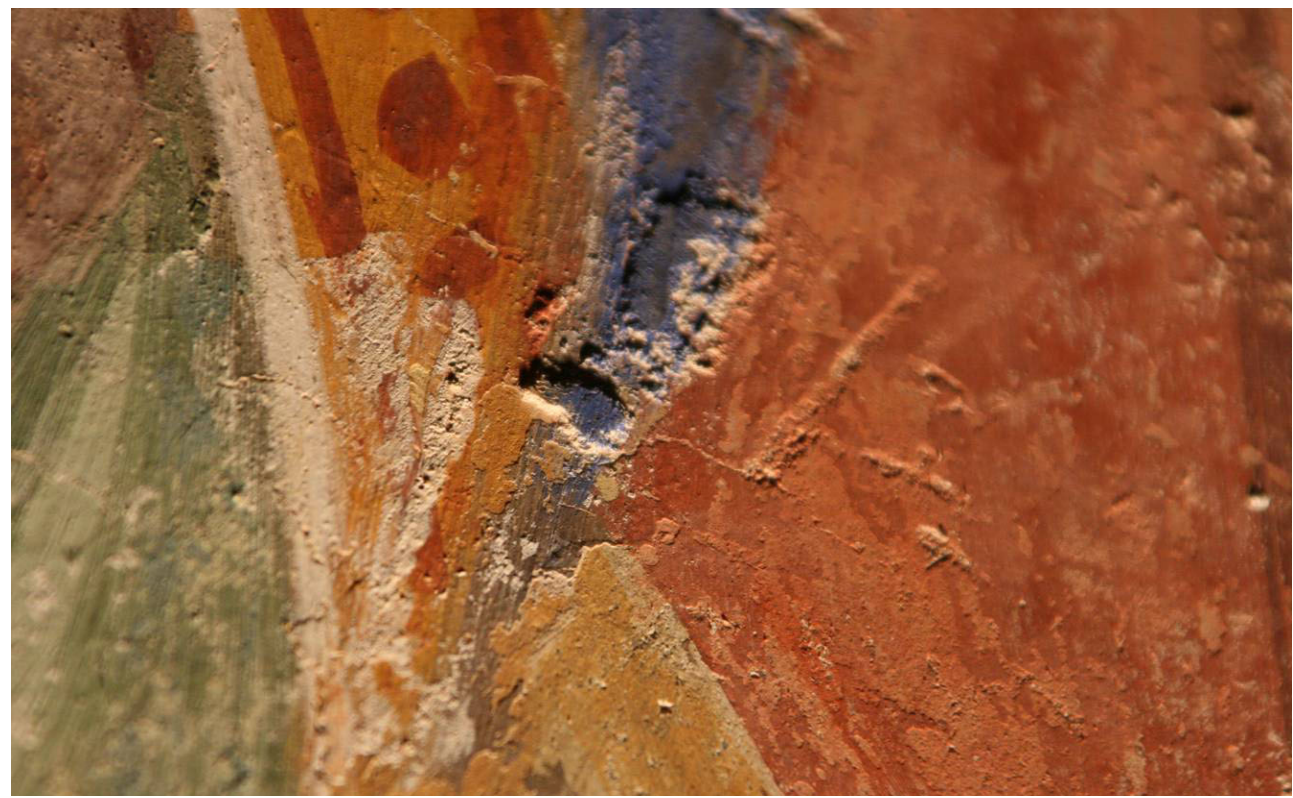

Figura 132 Giner Cordero E., Particolare di una reintegrazione e stuccatura sugli affreschi della navata. Chiesa di S. Carlo di Negrentino, Prugiasco. Fotografia a luce radente. 2007.

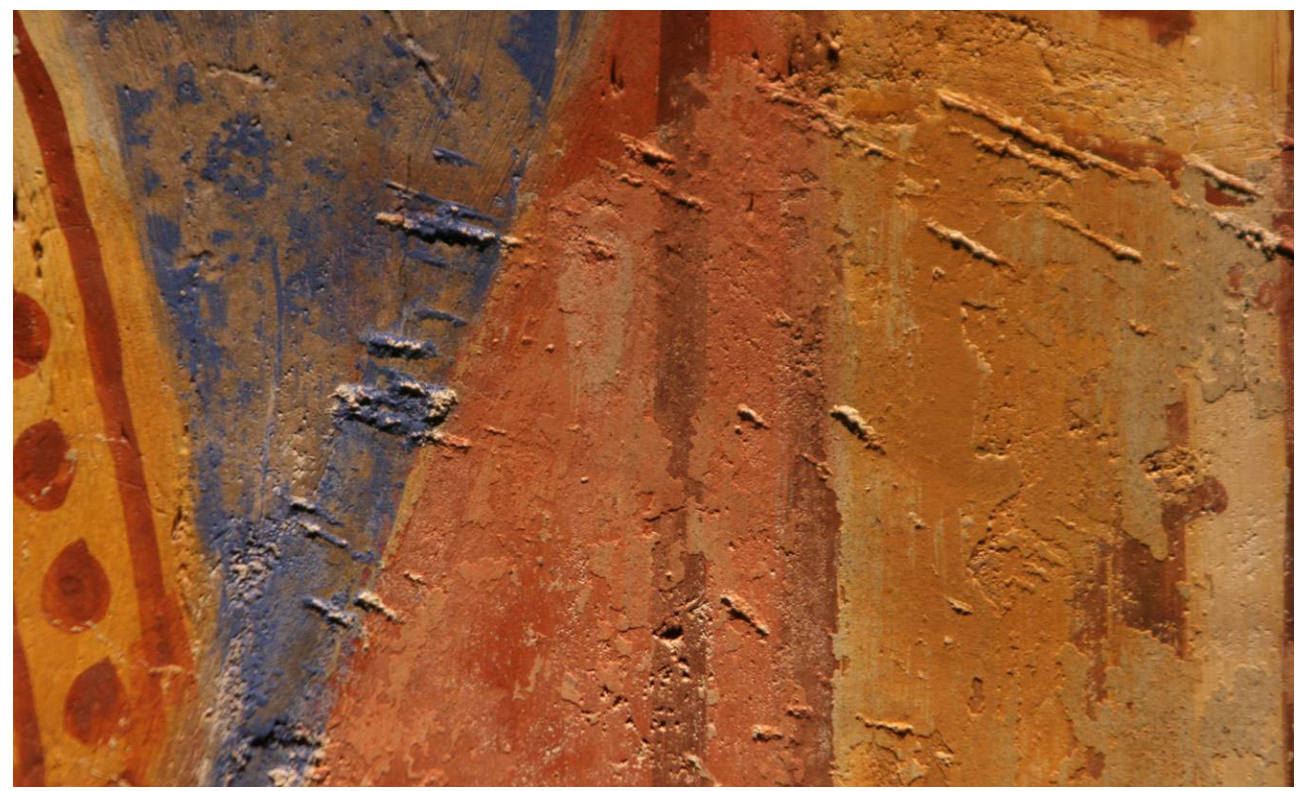

Figura 133 Giner Cordero E., Particolare delle abbrasioni provocate dagli strumenti impiegati per lo descialbo della superficie dipinta. Chiesa di S. Carlo di Negrentino, Prugiasco. Fotografia a luce radente. 2007. 


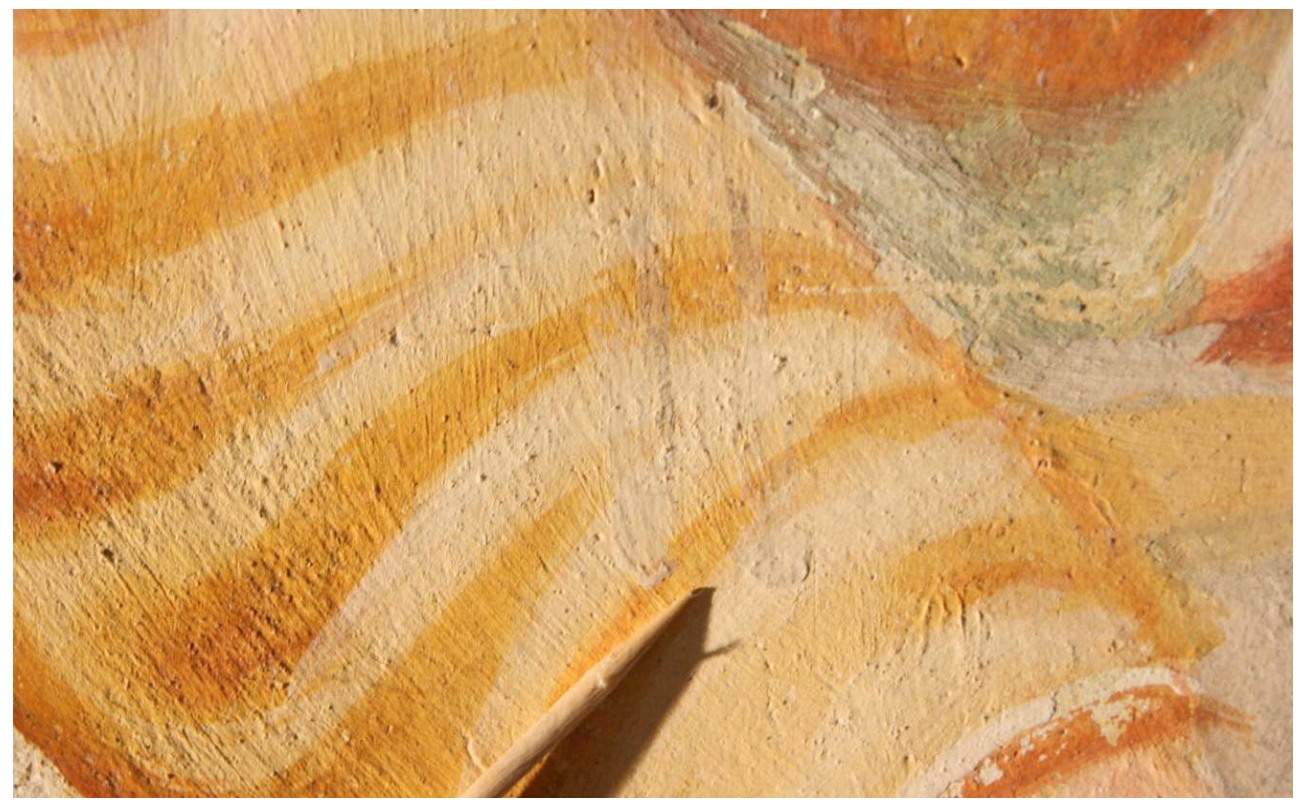

Figura 134 Giner Cordero E., Particolare di sostanza cerosa ritrovata sulla superficie affrescata nell'abside maggiore. Chiesa di S. Carlo di Negrentino, Prugiasco. Fotografia a luce radente. 2007.

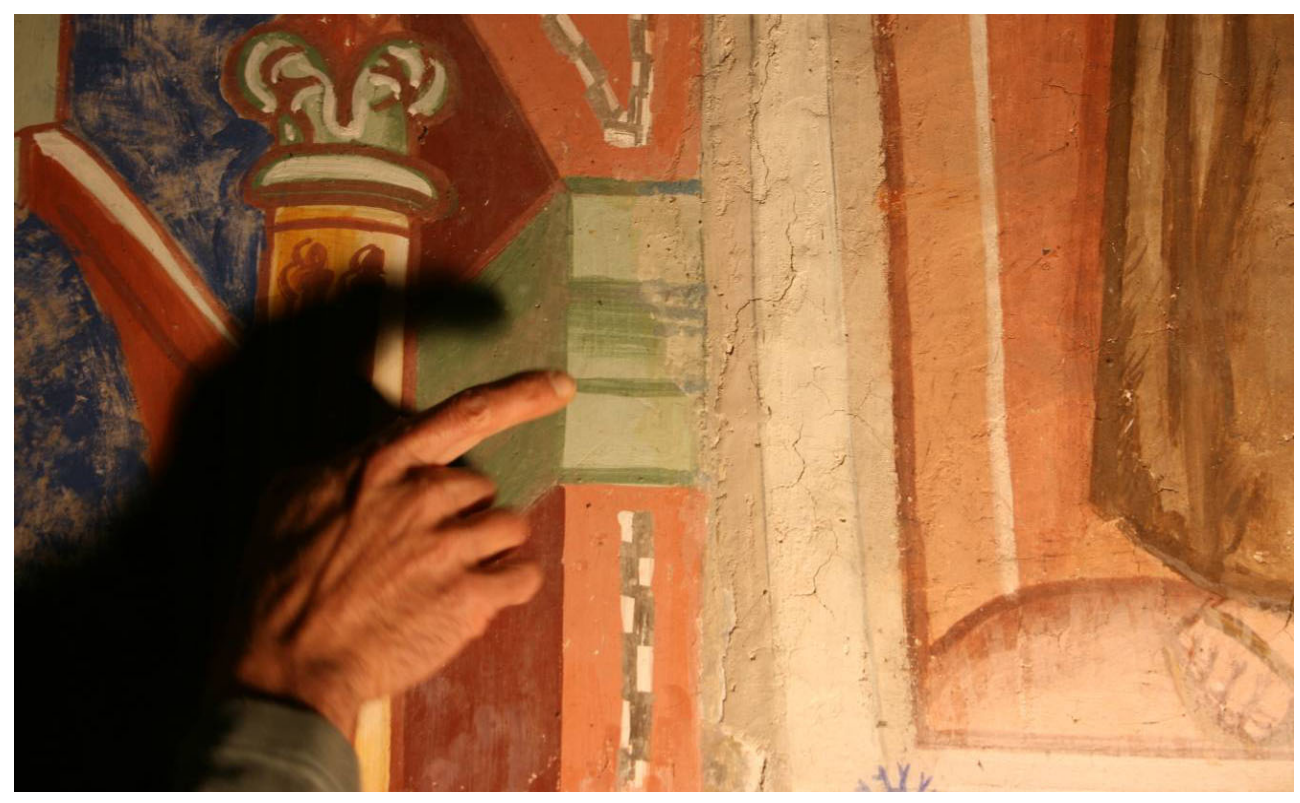

Figura 135 Giner Cordero E., Particolare di una reintegrazione e stuccatura sugli affreschi della navata. Chiesa di S. Carlo di Negrentino, Prugiasco. Fotografia a luce diretta. 2007. 


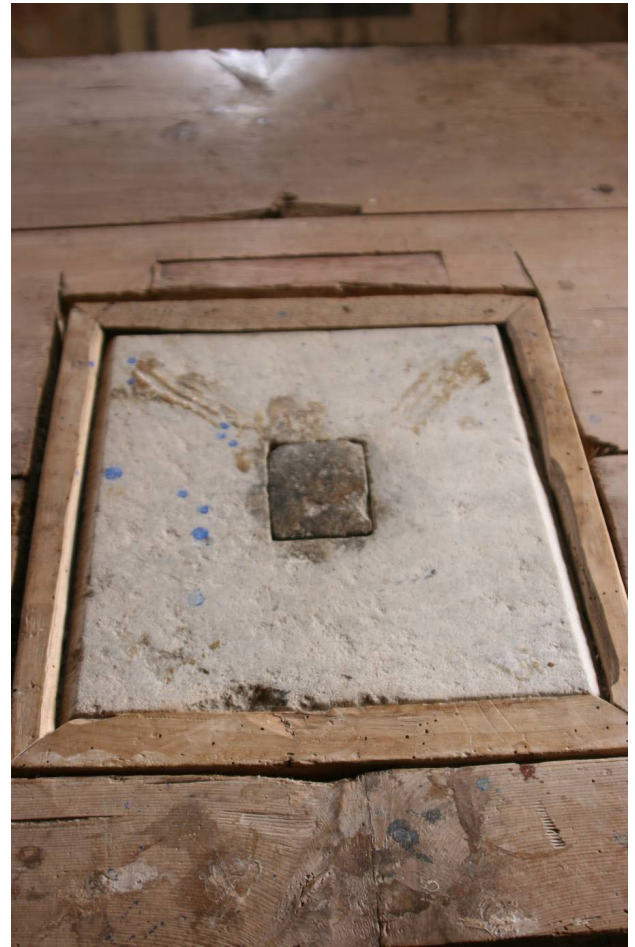

Figura 136 Giner Cordero, E. Particolare del pigmento blu ritrovato sopra l'altare dell'abside maggiore alla chiesa di s. Carlo di Negrentino, dimostrando i rifacimenti eseguitisi durante i lavori di restauro degli anni Quaranta. 2008.

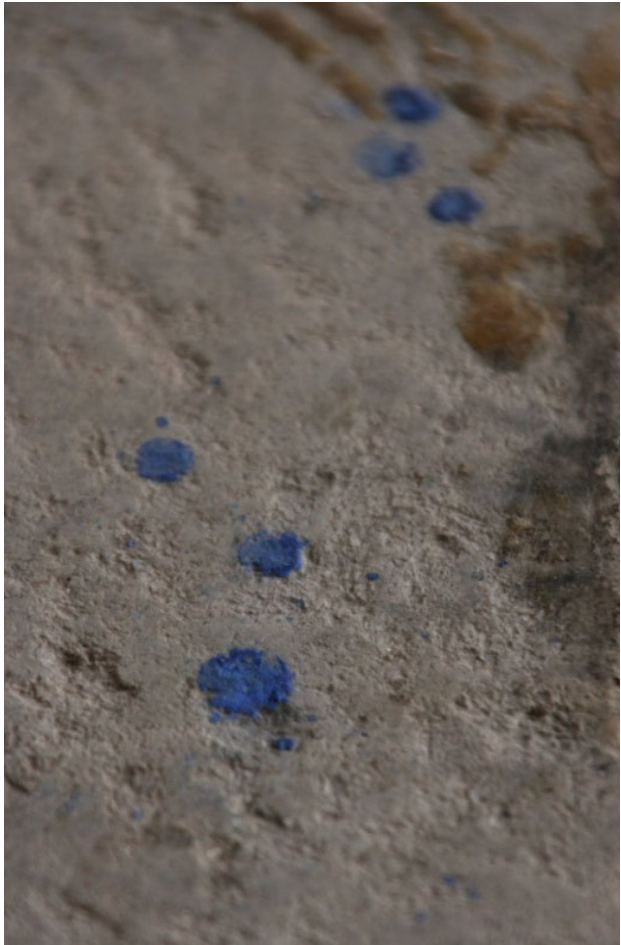

Figura 137 Giner Cordero, E. Particolare del pigmento blu ritrovato sopra l'altare dell'abside maggiore alla chiesa di s. Carlo di Negrentino, dimostrando i rifacimenti eseguitisi durante i lavori di restauro degli anni Quaranta. 2008. 


\section{San Nicolao di Giornico}

La chiesa di San Nicolao in Giornico, nella Val Leventina, è considerata dalla Guida d'arte della Svizzera Italiana a cura della Società di storia dell'arte in Svizzera ${ }^{387}$, il monumento romanico più importante del Canton Ticino. Sicuramente esso spiega quanto difficile fu il suo restauro già negli anni Quaranta, che come succede con la maggior parte di opere riconosciute nelle quali non pochi si sentono identificati, scatenò forte critiche da quelli che vedevano nei restauratori una minaccia. Era un sentimento sviluppato durante la campagna di restauri iniziata nell' anno 1909 , che adesso culminava con le voci esaltate d'una popolazione consapevole dei sui obblighi e dei suoi diritti a riguardo del patrimonio, cioè del loro modo di riconoscersi a sé stessi.

La fabbrica di S. Nicolao è databile al XII sec., ha pianta rettangolare e presenta un coro rialzato al quale si accede da una doppia scalinata settecentesca ed un'abside semicircolare. Inferiormente a questi appare la cripta a tre navate e volte a crociera, con otto colonne e semicolonne finiti in capitelli ognuno diverso dell'altro dove si mostrano forme geometriche, figurative o fitomorfi. Il campanile si trova a nord-est della navata.

Sono di grande importanza le decorazioni pittoriche che decorano la chiesa, in particolare quelle dell'abside firmate dall'artista Nicolao da Seregno nel 1478 e raffiguranti un Cristo in Maestà con i simboli degli evangelisti, i santi Gottardo, Vittore, Pietro e Nicola, la Trinità sopra la

${ }^{387}$ (Simona Martinoli, 2007). finestra mediana, la Crocifissione e le SS. Margherita e Maddalena.

Nel coro si trovano rappresentati l'Agnello di Dio sulla volta, la Madonna in Trono sulla parete destra, un Santo Vescovo su quella di sinistra e la Natività sull'altare. Nella parete nord della navata possiamo osservare l'affresco dell'Ultima Cena e sulla parete sud i resti d'una teorie di santi.

L'intervento, descritto da Francesco Chiesa $^{388}$ nella pubblicazione del Dipartimento della Pubblica Educazione per i Cinquant'anni della commissione dei monumenti nel Canton Ticino, fu compiuto fra i mesi di gennaio e di agosto del 1945. II monumento prima del restauro si trovava in pessimo stato di conservazione, dovuto principalmente alle condizioni d'abbandono sofferte durante gli anni dopo la abolizione del culto nel territorio. In tutto questo periodo la fabbrica, utilizzata come rifugio militare e come magazzino, aveva subito l'azione dell'acqua che era penetrata dal tetto e dall'umidità di risalita capillare, danneggiando particolarmente dipinti murali all'interno della chiesa descritti in precedenza.

Gli affreschi maggiormente degradati furono quelli disposti nell'abside maggiore, praticamente illeggibili.

I lavori cominciarono con la riparazione del tetto dell'abside per evitare ulteriori problemi di umidità pluviale. Ciò risulta fra il 1931 ed il 1935 grazie ad un finanziamento di Fr. 100 da parte dello Stato $^{389}$. Più tardi con l'arrivo di nuovi

388 (Chiesa, 1946, p. 64-70).

[...] al momento il tetto della Chiesa di San Nicolao è in buon ordine e non penetra acqua nella chiesa.[...]Purtroppo nell'affresco del coro appaiono manifesti i segni nefasti dell'acqua avvenuti prima del 1931.[...]. (Amministrazione Parrocchiale di Giornico. 
sussidi economici, sempre dalle autorità cantonali si può proporre il restauro completo della fabbrica e dei suoi beni collegati.

II 14 giugno del 1940 il Consiglio di Stato incarica il progetto, come si era già fatto con San Carlo in Negrentino, all'Ingegnere Riccardo Gianella per la parte tecnica ed archeologica, ed a Emilio Ferrazzini la pianificazione del restauro artistico. Ambedue proposte sono presentate nell'autunno dello stesso anno, ma una volta analizzate dalla Commissione vengono giudicate incomplete poiché omettono problemi fondamentali indispensabili per la qualità del restauro. Conseguentemente si decide d'affidare il progetto ad una sola persona. Fu scelto a tale scopo l'architetto Paolo Mariotta di Locarno $^{390}$. Egli esaminò le prime proposte con l'appoggio dell'archeologo italiano Ugo Monneret de Villard $^{391}$ e di Giuseppe Weith $^{392}$ di Bellinzona, riconosciuto per i lavori di ricerca eseguiti sui castelli della

Lettera a Francesco Chiesa, Giornico 12 gennaio 1933. AUBCBZ, s.112. Giornico, Chiesa di San Nicolao).

390 Mariotta Paolo. Architetto Diplomato B.S.A. (Mariotta, 1944).

391 Monneret de Villard Ugo (Milano 1881 - Roma 1954). Professore di Storia dell'Architettura fra il 1913 ed il 1924 presso il Politecnico di Milano. Fu oltre che archeologo ingegnere, specialista in storia medievale, storia dell'arte lombarda e storie ed arte orientali.

392 Weith Giuseppe. Fra i lavori più importanti dell'ingegnere si trovano la ricostruzione delle immagini d'epoca di Bellinzona frutto di ricerche storiche che nel 1925 il pittore Baldo Carugo dipinse in graffito sulle ventisei lunette nel cortile de municipio della medesima città, i restauri della Chiesa di San Rocco in Bellinzona (1926), il restauro del Castello medievale di Sasso Corbaro in Bellinzona fra i 1930 ed il 1935 ripristinando alcuni elementi costruttivi; il restauro della cappella barocca di San Martino all'interno del Castello Montebello (1934.) provincia ticinese. ${ }^{393}$ Dopo cinque anni di analisi, l'architetto presenta alla Commissione il progetto di restauro definitivo il 21 luglio 1943.

I lavori di restauro strutturale furono iniziati il 3 gennaio 1945 sotto la vigilanza del professore Piero Bianconi, con la finalità di adattamento alle condizioni stilistiche originarie mediante la liberazione dell'antica chiesa romanica dalle aggiunte e rifacimenti ${ }^{394}$. A questo scopo fu demolito il soffitto ligneo settecentesco, creandosi un nuovo sistema a capriate ad imitazione dai romanici lombardi ${ }^{395}$, consolidate le murature, chiusa l'entrata barocca nella facciata, rinnovato il pavimento con le stesse lastre dell'ultimo restauro scalpellate e ridotte a filo al livello primitivo; pulite le pareti interne dagli intonaci e dall'umidità, ricostruita la scala che da accesso alla cripta e dentro di essa riaperte le finestre romaniche e rimossa l'apertura barocca, trasportato l'altare ligneo barocco e creata una illuminazione d'accordo con il carattere austero dell'edificio ${ }^{396}$.

Gli affreschi, ilegibili e scuriti a causa delle infiltrazioni d'acqua, dal fumo e dalla polvere depositata in superficie, furono puliti e consolidati. Cade su Tita Pozzi la

[...]Avendo constatata la difficoltà di evitare interferenze e l'incompletezza del progetto Ferrazzini[...] si propose[...] che l'incarico ad un'unica persona, e precisamente ad un architetto; con l'avvertenze che il progettista avrebbe tenuto conto dei preziosi contributi forniti dai signori Gianella e Ferrazzini, e ch'egli fosse assistito da un archeologo d'alta autorità[...]. (Chiesa, 1946, p. 88).

${ }^{394}$ (Chiesa, 1946, p. 64-70).

395 Si adotta come esempio il sistema a capriate esistente nella chiesa di Castel San Pietro.

${ }^{396}$ L'altare fu restaurato da Hans Alexander? Fischer e collocato nella parte settentrionale della navata. 
responsabilità del restauro pittorico in fronte all'assenza di Ferrazzini, che interesserà anche lo strappo dei dipinti della cripta in cattivo stato di conservazione, per scoprire i dipinti sottostanti. Questo suo intervento, avvenuto appena dopo i restauri a San Carlo di Negrentino, si interromperà prima del previsto a causa delle differenze professionali che si scaturiranno nel lavoro con il compagno restauratore Bruno Abbiati e con l'Ispettore Piero Bianconi ${ }^{397}$.

Ad incrementare il risentimento di Tita Pozzi, avranno un ruolo fondamentale gli articoli pubblicati nella Rivista Storica Ticinese, nel Giornale del Popolo, Popolo e libertà e nell'Adula, fra tanti altri giornali e fascicoli di questo periodo. Essi furono utilizzati dai personaggi coinvolti nel progetto di restauro (politici, pittorirestauratori, architetti, ingegneri, uomini di cultura) per manifestare pubblicamente la diversità di criteri, che dopo si convertirono in più violente accuse e critiche offensive. Ed è grazie proprio a questi articoli che oggi riusciamo a ricostruire l'evento di restauro che ebbe luogo sui dipinti di San Nicolao in quegli anni.

Mentre un articolo di Aldo Crivelli nelle prime pagine della Rivista Storica del Ticino del mese di giugno del 1945 si pronunciava chiedendo di fermare le dispute sul restauro, poco tempo prima effettuato nella chiesa, con un "Basta":

\footnotetext{
397 [...]Gli affreschi non più leggibili, scuriti dalle infiltrazioni, dal fumo e dalla polvere, furono ripuliti e consolidati. Assente per ragioni di saluti il pittore Ferrazzini al quale si era affidato il restauro, il lavoro fu iniziato dal pittore Tita Pozzi, poi proseguito e condotto a termine dal pittore Abbiati che ripulì e consolidò anche gli affreschi della cripta e della navata. [...]. (Chiesa, 1946, p. 69).
}

[...]agli individui che non hanno il coraggio delle loro azioni e relative responsabilità; ai presunti competenti che non sentono il dovere di ritirarsi a più proficua meditazione dopo tutto quel rosario di fesserie che da anni vanno infilzando; basta col disonesto sistema di essere progettisti, esecutori e collaudatori del proprio lavoro[... $]^{398}$

Un chiaro riferimento ai membri che conformavano la Commissione Cantonale dei Monumenti Storici ed Artistici, appena un mese dopo nelle pagine del Giornale del popolo del 4 e del 16 luglio 1945 le denuncie continuano.

Dopo calorose discussioni circa la qualità dei lavori di ripristino architettonico, eseguiti grazie ai numerosi sopraluoghi e consulenze con professionisti procedenti dalla Commissione Federale dei Monumenti, arriva il momento della polemica al restauro artistico non meno problematico, in un momento nel quale gli ideali circa l'idoneità dei metodi si mettevano duramente a confronto. In questa occasione si discuteva la professionalità del pittore e restauratore Tita Pozzi, affermando I'utilizzo di tecniche e materiali dannosi per le pitture di San Nicolao.

Don Robertini aveva anticipato nuovamente la sua disapprovazione degli interventi che supponessero l'ingrassatura - con ciò s'intendeva l'applicazione di cera o qualsiasi altra vernice -, il ritocco e la ridipittura degli affreschi che erano, secondo lui, le prassi dei restauri fino al momento eseguiti dalla CCMS, e che sperava non fossero ripetute sui dipinti di Giornico. Poco tempo dopo le sue scritture rimproverano proprio I'utilizzo di puliture

\footnotetext{
8 (Crivelli, Basta. Polemica sui restauri di San Nicolao di Giornico, 1945).
} 
violente e l'impiego di sostanze protettive sul medesimo monumento:

[...]Andavano via i colori dai ritocchi anteriori [...] Invece dove era possibile bisognava levare il nerume grasso che velava particolarmente gli affreschi del lato sinistro [...] T. P provò qua tanto là, dispose della soluzione di cloro sugli affreschi e lasciò che vi lavorasse su per qualche settimana, ma il nero grasso fumoso non cedeva. Si chiarì qua e là qualche mano, qualche fronte, qualche mitra e nulla più. E allora si appoggiò a un altro sistema. Verniciare gli affreschi con certi materiali cerosi, per ravvivare $i$ colori e ottenere così quanto non era possibile con un'azione diretta. E successe il contrario, [...] affresco velato e nebbioso più di prima $[. . .]^{399}$.

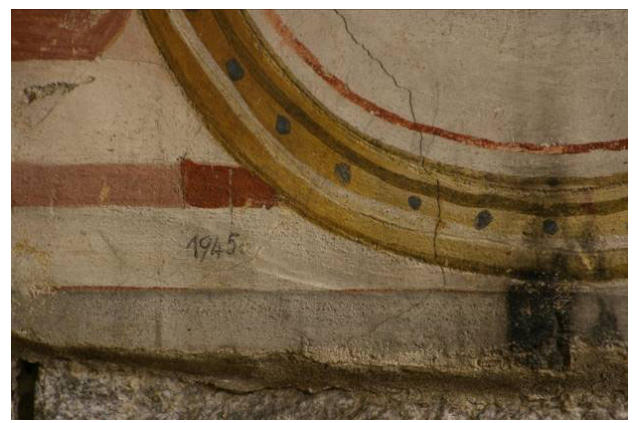

Figura 138 Giner Cordero E. Particolare degli affreschi dell'abside con la firma del restauro.
(Crivelli, II restauro della Chiesa di San Nicolao, 1945).
Alludendo all'intollerabile uso delle sostanze cerose, fu Don Robertini a dirigere una delle più grandi offese in questo caso ad Emilio Ferrazzini criticando il generoso impiego che egli fece della tecnica dell'encausto, il parroco scriveva :

[...]Gli affreschi di San Nicolao? Se fossero rimasti nelle prime mani cui furono affidati, sarebbero diventati ancora più opachi, nebbiosi di quanto non fossero già [...]. ${ }^{400}$

Non abbiamo potuto ancora verificare con certezza l'impiego della soluzione di cloro per la pulitura dei dipinti, ma abbiamo potuto confermare l'utilizzo da parte di Pozzi della cera. Codesta venne già considerata ed applicata nel precedente intervento nella Chiesa di San Carlo di Negrentino e venne proposta in tutti i preventivi da lui presentati dagli anni 1943 al 1950. Fu lo stesso pittore a difenderla in fronte all'uso della gomma arabica per fare rinvenire $i$ toni dei dipinti che in contrapposizione propugnava Bruno Abbiati. Secondo il restauratore l'encausto idoneamente realizzato non solo ravvivava i colori ma gli consolidava, mentre ché la gomma arabica non permetteva quest'ultimo, e perfino poteva lasciare dei brilli sulla superficie.

Secondo il parroco Don Robertini, i lavori di Abbiati non interessarono in nessun momento il ravvivamento dei colori ma il suo intervento suppose unicamente il lavoro di pulitura dei dipinti e di consolidamento senza ricorrere a nessun materiale addizionale.

L'appoggio del priore e conseguentemente dell'ispettore Piero Bianconi verso il

\footnotetext{
400 (Crivelli, II restauro della Chiesa di San Nicolao, 1945).
} 
restauratore italiano, suppose la vincita degli ideali conservativi verso le prassi svolte in Ticino dagli inizi del Novecento, ma significò per Tita Pozzi un duro colpo, in quanto ché ogni sua proposizione tecnica sul lavoro risultò rifiutata e più ancora si vede incolpato di ciò che non era responsabile. L'esempio più chiaro lo espose lo stesso restauratore in una lettera a Francesco Chiesa, e si produce durante un sopralluogo il 18 maggio del 1945 in cui il Bianconi lo accusa di rovinare la testa dell'Angelo, simbolo di San Giovanni Battista, sulla tazza dell'abside. A questo seguiranno le polemiche giornalistiche precedentemente discusse, che fortunatamente non incisero sull'attività professionale del pittore che in ogni momento trovò chi lo proteggesse da queste, elogiando in contrapposizione la qualità dei suoi precedenti restauri. Furono Henri Boissonas e Linus Birchler ad esaltare i lavori eseguiti al Castello di Bubikon:

[...]Nous avons employè Mr. T. Pozzi pour les travaux de restauration des peintures[...] nous avons surveillé de très près son travail d'un bout à l'autre et nous pouvons déclarer qu'il l'a fait avec beaucoup de soins, de con science et d'intelligence. Nous avons été entièrement satisfaits de lui.[...] Prof. Henri Boissonas e Johannes Meier Arch. B.S.A

[...]l lavori di restauro eseguiti dal sig. Pozzi a Bubikon furono per me una vera e grande sorpresa. Si tratta d'un pittore-restauratore di una esperienza rara in Svizzera.[...] Prof. Dott. Lino Birchler.

Mentre ancora il medesimo presidente della Commissione Federale dei Monumenti, insieme all'architetto Riccardo
Gianella valutarono positivamente l'esito a San Carlo di Negrentino.

[...]Gli affreschi murali di Negrentino parzialmente assai rovinati furono rimessi in ordine dall'eccellente restauratore ticinese [...] il quale fece un paziente lavoro, con scienza e tatto, senza permettersi anche la benché minima aggiunta o licenza artistica[...]. Prof. Dott. Linus Birchler ${ }^{401}$.

Senza dubbio le capacità di Tita Pozzi furono fra le più valide anche trattandosi di un "pittore" che fa il restauro e non di un proprio restauratore secondo le concezione brandiane, il suo carattere si distingue dalla onesta responsabilità e rispetto con cui cercò sempre di comunicare verso l'opera. Non si limitò unicamente ad apprendere $\mathrm{i}$ materiali tradizionali d'impiego descritti nel "Restauro dei dipinti murali" del maestro Secco-Suardo, quale uno dei capitoli dei trattati dell'epoca, ma s'interessò per capire la natura d'ognuno di loro per servirsi d'una capacità critica, minimamente scientifica, per adoperarli.

Rimproverò al suo collega italiano Bruno Abbiati, di essere un buon praticone, ma assolutamente ignorante per quanto a cognizioni tecniche [...] - perché - [...]chiama acido la soluzione di potassa caustica che ne è I'opposto; chiama etere l'acido acetico che nulla ha da vedere con gli eteri, non sa distinguere tra alcali e acidi; non sa nulla delle cera (animali, vegetali, minerali), dei solventi, ecc. ecc. $[. . .]^{402}$; ed alla Commissione Cantonale di Monumenti Storici di permettere di

\footnotetext{
${ }^{401}$ Tutte le citazioni sono stratte da Popolo e libertà, Bellinzona lunedi 21 agosto 1944

402 (Pozzi Tita, Relazione di sopraluogo allegato alla lettere indirizzata a Francesco Chiesa il 20 maggio 1945. Massagno 16 maggio 1945. AUBCBZ, s.112 Giornico).
} 
lavorare ad un straniero, quando la domanda occupazionali per i ticinesi era già scarsa. Di fatto, Abbiati con l'illimitato appoggio del parroco Don Robertini aveva già restaurato circa quindici opere su tela di chiese e cappelle in Gorduno, Giornico, Mosogno ed Ascona. II restauratore italiano finì individualmente sotto la sorveglianza di Bianconi la pulitura, consolidamento e reintegrazione degli affreschi dell'abside, della cripta e della navata della chiesa di San Nicolao.

Recenti analisi realizzati sui dipinti della chiesa di San Nicolao, hanno dimostrato l'impiego in passato di metodi di intervento che supongono stuccature proviste di un doppio stratto, il primo a base di calce aerea e inerti silicei, ed una secondo strato in superficie composto da leganti di natura idraulica e calcite, che conformano. Le stuccature sono state successivamente reintegrate differenziando l'originale dalle aggiunte ${ }^{403}$.

L'inaugurazione dei lavori avviene il 16 settembre 1945 .

Finalmente nel numero di dicembre dello stesso anno nella Rivista Storica Ticinese, il direttore di essa, Aldo Crivelli, pubblica la relazione di restauro in San Nicolao di Giornico curata dal presidente della CCMS Francesco Chiesa ${ }^{404}$, sperando di finire in questo modo l'appassionata polemica che se non altro aveva dimostrato l'interesse del popolo circa il suo patrimonio.

L'immagine delle opere realizzate dalla Commissione dei Monumenti storici fu in qualunque modo danneggiata da tutte queste dispute, fino al punto che il
Dipartimento della Pubblica Educazione del Canton Ticino decise nel 1949 dare fine a tutte le polemiche generate, convocando un collegio di esperti di alta competitività, di fuori del Cantone, capaci di valutare la qualità degli interventi nei monumenti più contestati.

Le persone chiamate a partecipare furono l'Architetto Ambrogio Annoni di Milano, il pittore-restauratore Mauro Pellicioli di Bergamo ed il professor Jean Verrier di Parigi, ispettore dei monumenti di Francia, partecipe a suo tempo dell'esame realizzate su Sant'Ambrogio di Chironico. Le visite ebbero luogo i giorni del 24, 25 e 26 di giugno accompagnati dal presidente della Commissione Cantonale Francesco Chiesa, e dai membri l'architetto Giovannini ed il professore Tarabori, che facilitarono l'eventuale informazione necessaria a capire meglio i particolari di ogni restauri.

Furono favorevoli i giudizi effettuati dagli esperti riguardo il restauro avvenuto all'interno della chiesa di San Carlo a Negrentino, dove l'unico attenzione fu lanciata ad una zona di distacco dell'intonaco e per la manifestazione di macchie biancastre sulla parte destra dell'affresco della parete a poniente, a conseguenza del [...]poco idoneo uso di sostanze a base di cera $[. . .]^{405}$.

Non conosciamo invece il giudizio dichiarato in merito ai restauri di san Nicolao di Giornico, poichè il ritrovamento dei verbali o del rapporto riguardo non è stato ritrovato.

\footnotetext{
${ }^{403}$ Simona del Pietro, p.74.

${ }^{404}$ (Crivelli, Sui restauri di San Nicolao di Negrentino, 1945).
}

\footnotetext{
${ }^{405}$ (Marcionetti, 1977) p.108
} 


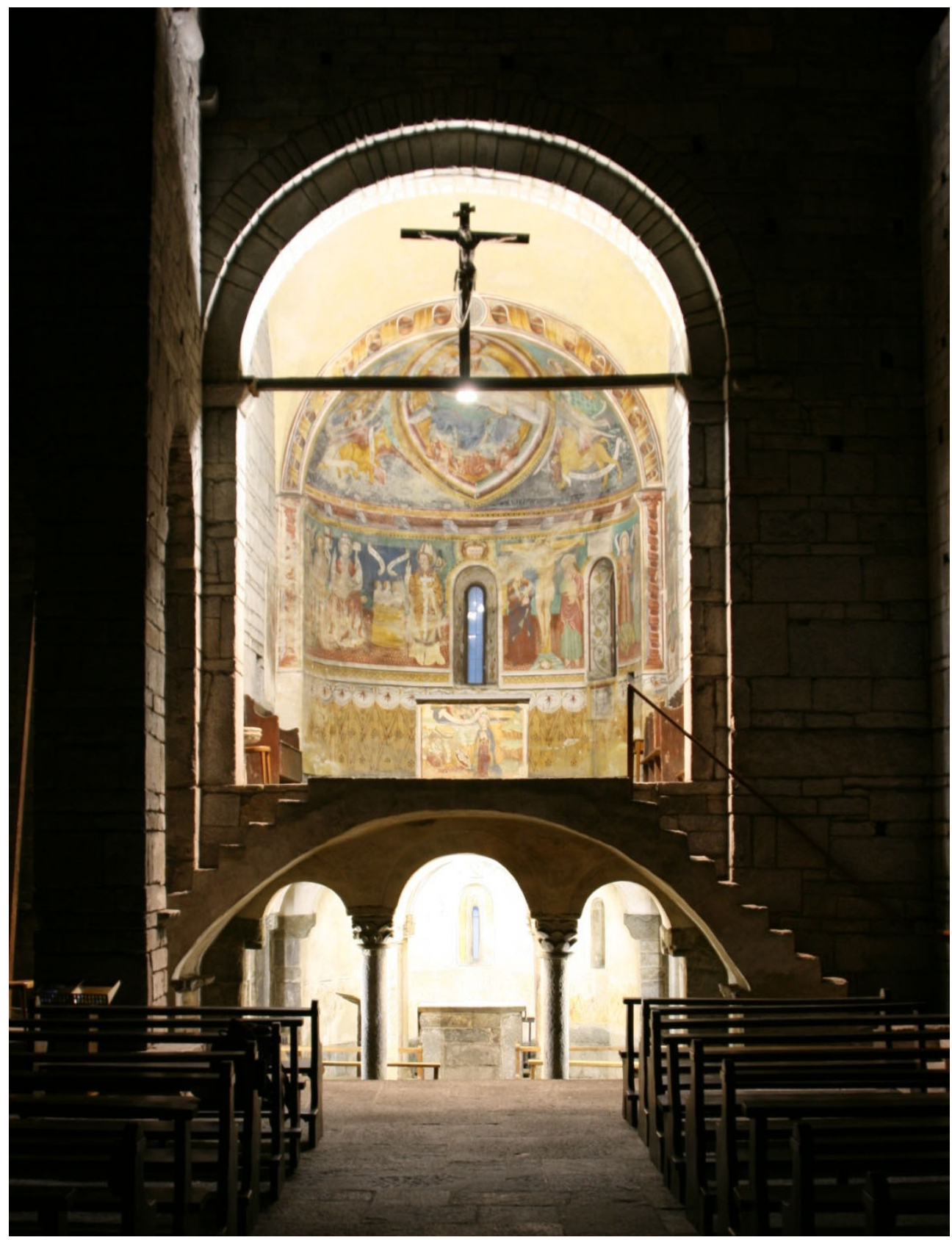

Figura 139 Giner Cordero E. Interno della chiesa di S. Nicolao, Giornico. 2007. 
Restauratori in Canton Ticino fra Ottocento e Novecento

Catalogazione e gestione dati

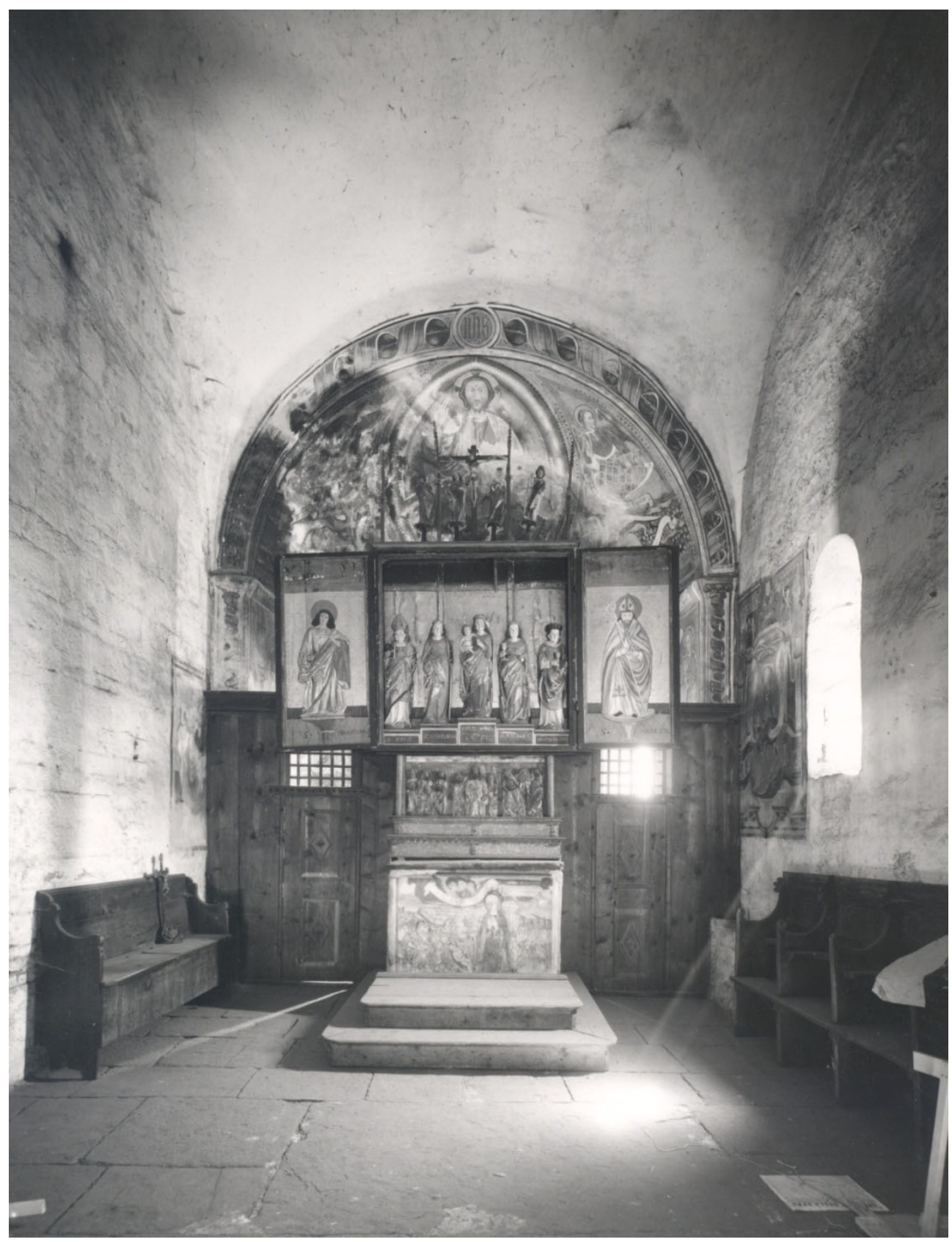

Figura 140 Abside della chiesa di S. Nicolao prima dei restauri, 1940 ca., Giornico. AUBCBZ, Giornico. 


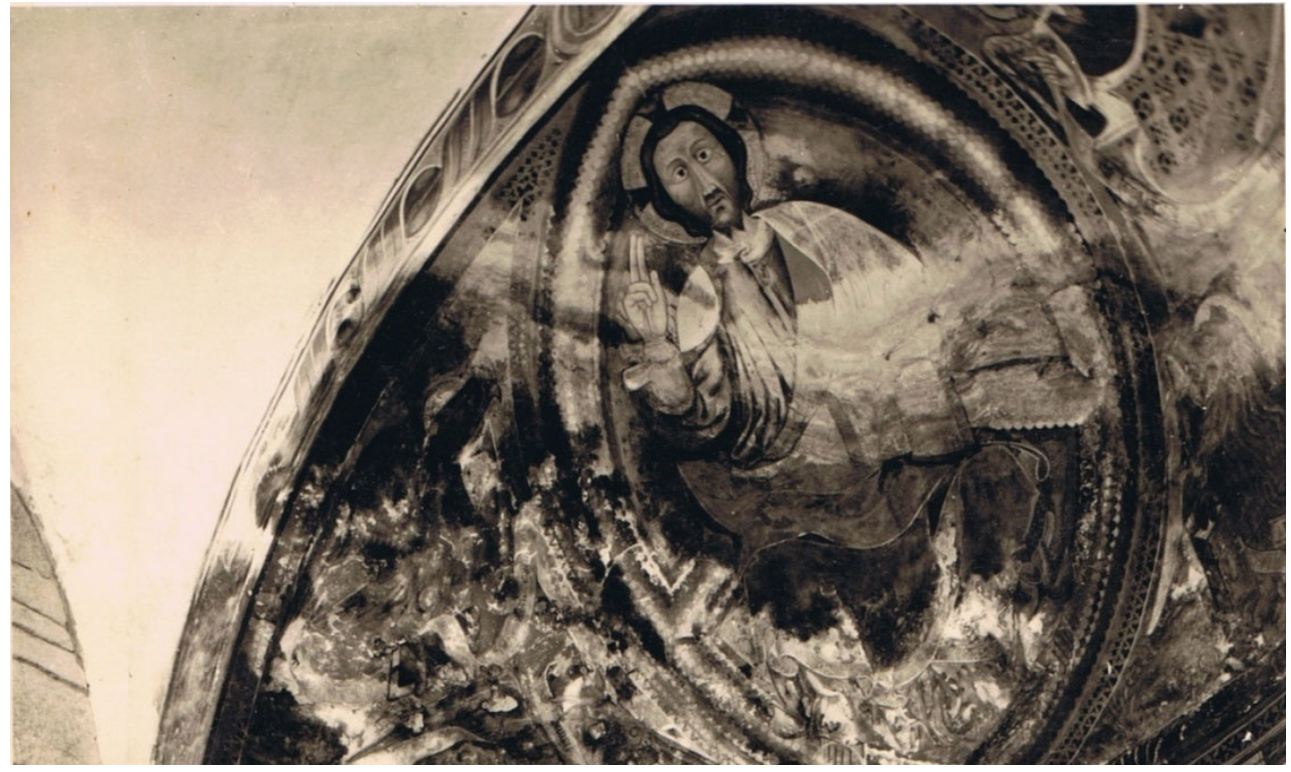

Figura 141 Abside della chiesa di S. Nicolao prima dei restauri, 1944 ca., Giornico. Archivio privato B.Abbiati.

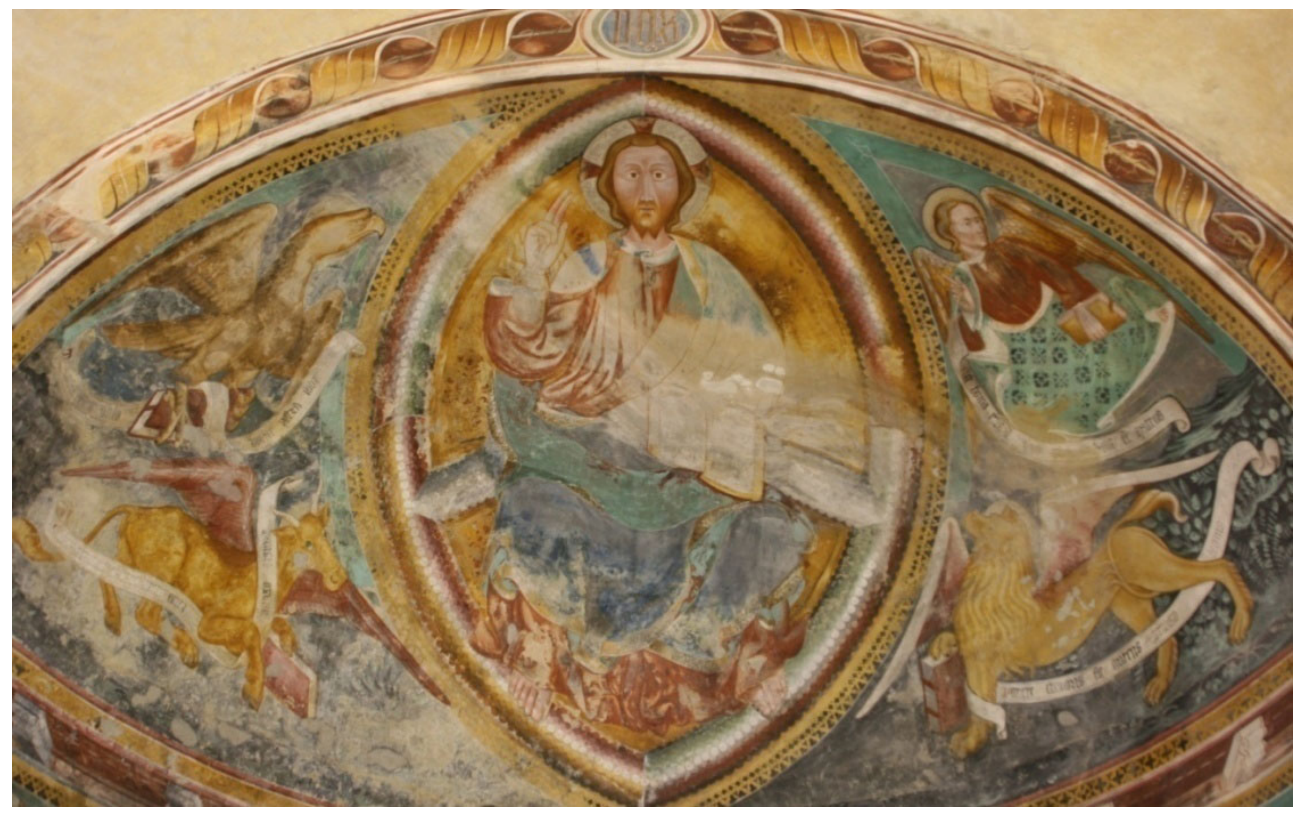

Figura 142 Giner Cordero E. Cristo in Maestà, 1478, Nicolao da Seregno. Abside della chiesa di S. Nicolao, Giornico 2007. 
Restauratori in Canton Ticino fra Ottocento e Novecento

Catalogazione e gestione dati

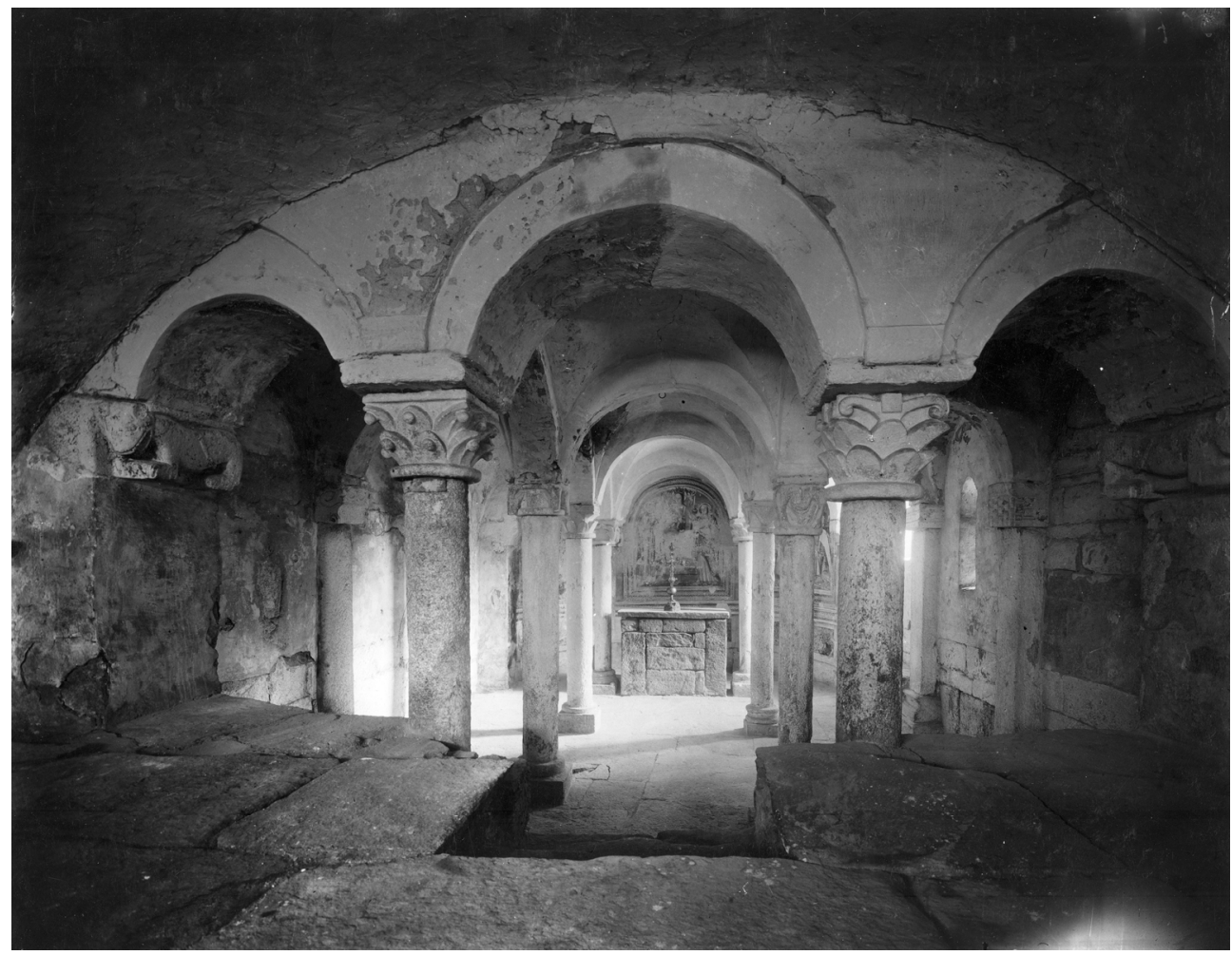

Figura 143 Cripta della chiesa prima del restauro, 1940 ca., Giornico, San Nicolao. AUBCBZ, Archivio grafico. 


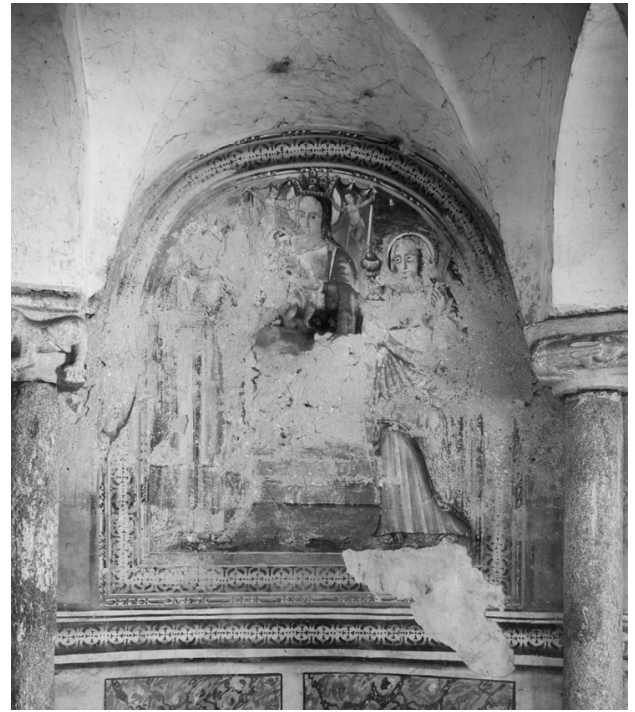

Figura 144 Affreschi dell'altare della cripta prima del restauro, 1940 ca., Giornico, San Nicolao. AUBCBZ, Archivio grafico.

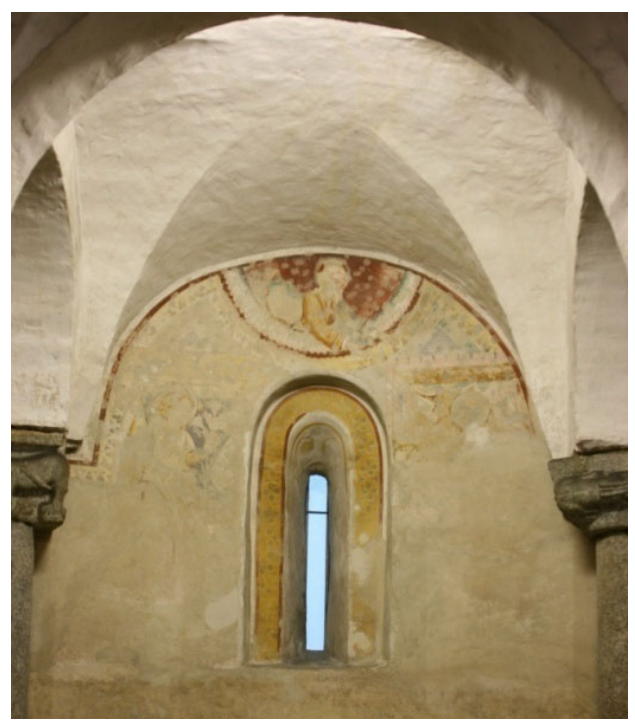

Figura 145 Giner Cordero, E. Affreschi dell'altare della cripta. Prugiasco, San Carlo di Negrentino. 2007.

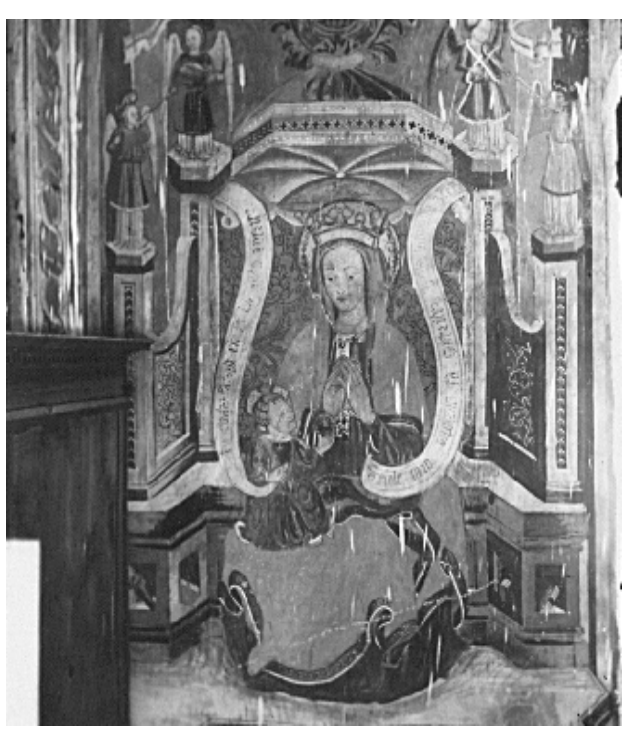

Figura 146 Affreschi dell'abside. S. Nicolao, Giornico prima del restauro, 1940 ca. A. 165. Archivio Federale dei Monumenti Storici. Collezione grafica, Biblioteca nazionale svizzera.

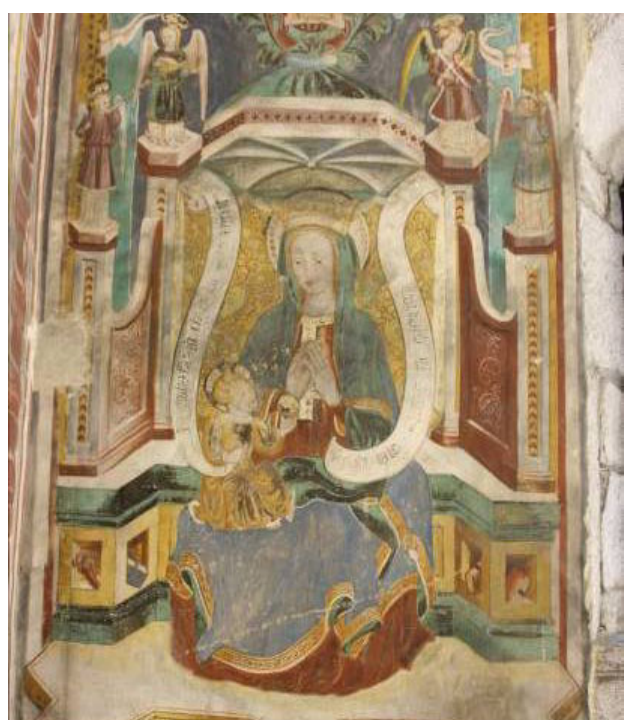

Figura 147 Giner Cordero E. Affreschi dell'abside. S. Nicolao, Giornico. 2007 


\section{Oratorio di S. Vigilio, Rovio}

[...] Coperta di campi ubertosi e vigneti in dolce declivio s'eleva la collina di S. Vigilio, e di là spiegarsi esteso panorama sul lago, sui monti che lo coronano e sui villaggi che rendono tanto vaghe le rive del Ceresio. Vi si erge una chiesetta che per l'organismo, per la struttura caratteristica e per l'armonia delle singole parti, può essere classificata fra gli esemplari più belli dell'arte romanica Lombarda in questa regione, giunta fino a noi abbastanza intatta[... $]^{406}$.

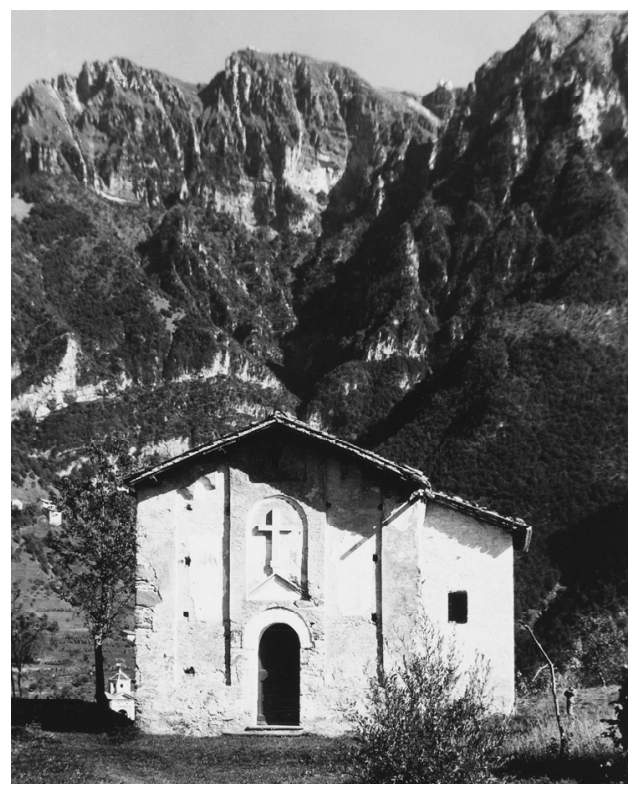

Figura 148 Veduta esterna. Facciata prima del restauro. In fondo il Monte Generoso. Rovio, S. Vigilio, Restauro $1949 \mathrm{ca}$. Archivio Federale dei Monumenti Storici. Collezione grafica, Biblioteca nazionale svizzera.
II restauro ed il consolidamento degli affreschi dell'Oratorio di San Vigilio a Rovio furono realizzati da Tita Pozzi fra il 1948 ed il $1949^{407}$, allo stesso tempo che si svolge la riabilitazione architettonica della fabbrica sotto la direzione del professor Taddeo Carloni e la vigilanza dell'architetto Giovannini.

Le prime notizie sulla necessità di studiare e ristrutturare l'edificio di culto risalgono all'anno 1911 quando la Signora Teresa Conza Rezzonico di Lugano incarica I'architetto Emilio Gusalli ${ }^{408}$ di realizzare un'ispezione dell'edificio. Le conclusioni della visita del soprintendente italiano vengono presentate e pubblicate il 30 marzo del 1912 insieme a sei tavole di disegni a colore e a un preventivo di circa Fr. 7'000, che tentava di coinvolgere le autorità cantonali, civili e religiose locali sull'importanza del restauro.

Gli assaggi ed analisi storici identificano l'architettura della fabbrica di tipo lombardo $^{409}$, di periodo compreso fra $\mathrm{i}$ secoli XI e XII, e negano l'ipotesi dichiarata dal professor Johann Rudolf Rahn ${ }^{410}$ nella pubblicazione I monumenti artistici del medio evo nel Canton Ticino, che considerava la chiesa come una aggiunta o innalzata su una cappella più piccola

\footnotetext{
407 (Chiesa, 1946).

Gussalli Emilio, Reale Soprintendenza ai monumento di Lombardia.

${ }^{409}$ Come scrive nella lettere diretta al Sig. Emilio Mazzetti, "in fatto questa, presumibilmente primigenia, pieve di Rovio appare, a chi ne studi l'ossatura architettonica, perfettamente logica in ogni sua parte ideata schiettamente in conformità della maniera lombarda in un'epoca che riteniamo più vicina al XI secolo che al XII sec."

${ }^{410}$ Rahn R. I monumenti artistici del medio evo nel Canton Ticino. 
sull'estremità orientale di origini anteriori, esattamente nel $X$ secolo circa.

Gli affreschi dell'abside sono considerati di grande importanza per la conoscenza della storia della pittura Lombarda. Sono d'epoca posteriore all'edificio, alla fine del XIII secolo circa, come atestiguano le forme romaniche delle decorazioni e le iscrizioni, e raffigurano sulla semitazza absidale il Cristo in Maestà nella mandorla che alza il braccio destro in atto benedicente mentre sulla mano sinistra mantiene il libro del Vangelo dove si legge: Ego sum, via, veritas et vita. La figura del Redentore è circondata dai Simboli degli Evangelisti con i nomi di ognuno scritti dall'alto in basso, e la Madonna orante affiancata dai dodici Apostoli accompagnati dai nomi di ognuno in verticale scritti con caratteri romanogotici, sulla parte bassa. Si trovano sui piedritti dell'arco trionfale le figure di due angeli, i più deteriorati prima dell'intervento del 1948, mentre sulla parte sinistra in prossimità all'abside due affreschi del XV sec. ca. rappresentano San Carlo (1618) dopo la sua canonizzazione e la Madonna. Tutti questi presentavano prima del restauro un cattivo stato di conservazione a causa dell'umidità e da invasive ridipitture.

I dipinti della facciata, anche essi del Seicento, sono distinguibili sugli sfondi della parete. Raffigurano a San Vigilio nella parte sinistra e a San Francesco d'Assisi sulla destra.

L'intenzione di ridare all'edificio le sue caratteristiche originali significava, secondo le analisi dell'ingegnere Gusalli, la demolizione della Cappella di San Rocco, [...]aggiunta vandalicamente nel 1854[...] in sostituzione dell'omonima cappella primaria alzata nell'antica calle di Melano a Rovio la cui esistenza si può osservare nell'illustrazione di Luigia Carloni Groppi ${ }^{411}$, nell'Almanaco Ticinese del 1922 e nella fotografia in bianco e nero qui annessa, con la ricostruzione del muro antico, degli archetti della cornice, delle lesene scendenti fino allo zoccolo e la riapertura delle finestre e della porta d'accesso alla navata; la sostituzione dell'altare barocco per un semplice blocco prismatico in muratura; la ricomposizione della pavimentazione originale conservata ancora in parte nell'abside che si estendeva allo stesso livello su tutta la navata, la ricostruzione del tetto in lastre di pietra in sostituzione dell'esistente in cotto del 1824 e del soffitto primitivo piano e le trave serventi da catena alle capriate come nelle coetanee basiliche, il restauro delle porte a imitazione di quelle conservate ad Arsago, la chiusura delle finestre, la pulitura delle pareti dagli strati aggiunti e la sigillatura delle pietre della muratura nella facciata. Si progetta la riabilitazione del terreno circondante, la sostituzione dell'altare barocco in stucco del secolo XVIII da un altro concorde allo stile della fabbrica e la pulitura degli affreschi anteriormente descritti, [...]opera che dovrà essere condotta con molta circospezione da un pratico operatore e sotto la immediata direzione dei competenti[...], specialmente degradati a causa dell'umidità e dell'abbandono in cui si trovava la fabbrica, assente di tetto dal 1708 ai restauri del 1817, secondo i documenti trovati nell'archivio parrocchiale.

\footnotetext{
411 Professoressa alla Scuole di Rovio tra la prima e la seconda metà del XX secolo.
} 
La chiesa venne curata e protetta da questo momento ma circostanze ignote impediscono l'esecuzione del progetto dell'architetto milanese E. Gusalli.

L'8 ottobre del 1929, Emilio Mazzetti, presidente del Consiglio Parrocchiale chiede al Lodevole Dipartimento della Pubblica Educazione in Bellinzona l'autorizzazione per la sostituzione della copertura attuale dell'abside da lastre di pietra di Osogna, più solide e resistenti, prima dell'epoche invernale, in modo d'impedire l'infiltrazione d'acqua che danneggerebbe i dipinti murali.

Nel 1938 si riprenderà il tentativo di ripristino della fabbrica, questa volta su progetto dell'architetto Giacomo Alberti ${ }^{412}$ di Bedigliora. Come aveva già fatto Gusalli, l'architetto propone la distruzione della cappella di San Rocco costruita nel 1824, conseguentemente al ritrovo d'una rottura fra la parete che unisce questa all'edificio primitivo $^{413}$.

Su questo progetto interverrà più avanti Taddeo Carloni ${ }^{414}$, proclamato direttore dei lavori strutturali e pittorici come membro della CCMS. Tita Pozzi progetta le proposte d'intervento artistico di cui lui stesso sarà l'esecutore. II preventivo presentato dal restauratore il 3 febbraio del 1943, a seguito del sopralluogo realizzatosi la sera

\footnotetext{
${ }^{412}$ Alberti Giacomo (Bedigliora, 1896-1973). Fra i suoi progetti più importanti si trovano la costruzione dell'Oratorio della Madonna della Provvidenza su Monte Generoso in 1946, la chiesa parrocchiale neoromanica di Santa Lucia in Massagno (1931) e I'Oratorio di San Mattia nella frazione di Certenago in Collina d'Oro.

${ }^{413}$ (Alberti Giacomo, Preventivo di spesa per il riordino della chiesa a norma dei piani allegati in scala 1:50. AUBCBZ, Rovio. 1938 ca. AUBCBZ, s. 213 Rovio). ${ }^{414}$ Carloni Taddeo (Rovio, Pittore-decoratore, insegnante alla Scuola di Arti decorative di Lugano).
}

prima, divide gli interventi possibile sull'abside, sulla navata e sul soffitto, con una somma totale di Fr. 3'250 che comprende:

[...]Abside: 1. Consolidamento e appropriata campitura del fondo giallo nella tazza;

2. Consolidamento ed eventuale leggera ricostruzione dei simboli Evangelisti;

3. Consolidamento ed eventuale restauro della Maestas Domini - parte caduta-;

4. Consolidamento e restauro della fascia della mandorla e fascia orizzontale;

5. Consolidamento dell'intonaco che si stacca e restauro delle due fascie decorative sullo spigolo dell'arco dell'abside;

6. Pulimento, consolidamento e leggerissimo restauro del semicerchio portante l'affresco della Madonna e quelli degli Apostoli (I'intonaco si stacca in qualche parte ed è da affrancare mediante stucco);

7. Consolidamento (parte dell'intonaco è staccato dalla parete portante) e restauro del motivo con meandro dell'intradosso dell'arcone; 8 Pulimento, ritrovamento di parte e leggero restauro della fascia a panneggi alla base della teoria degli Apostoli.

Navata: 1. Consolidamento, lisciatura di parti, pulimento e leggero restauro di due affreschi San Carlo e Madonna col Bambino - sulla parete a sinistra; 2. Pulimento e leggero restauro affresco di S. Ambrogio sulla parete a destra; Tinteggiatura generale - in grigio avorio - e patinatura delle pareti e della Cappella laterale a destra.

Soffitto: 1. Scrostamento totale delle mattonelle fino al colore naturale e qualche ritocco a tempera; 2 . Scrostamento totale delle travi e dei travetti e patinatura appropriata - a tempera degli stessi. $[\ldots]^{415}$

\footnotetext{
415 (Pozzi Tita, A seguito del sopralluogo a S. Vigilio e dell'abboccamento di ieri sera, eccole il preventivo per le opere di restauro pittorico da eseguirsi nella Chiesa di S. Vigilio a Rovio. Lettera a Taddeo Carloni. Massagno-Lugano 3 febbraio 1943. AUBCBZ, s. 213 Rovio).
} 
Malgrado non vengano specificati i materiali da impiegare possiamo presumere che essi fossero stati gli stessi adottati sugli interventi precedentemente analizzati, poiché eseguiti quasi in contemporaneità. Si avverte però un interesse da parte del restauratore a presentare il proprio lavoro come un'azione discreta e conservativa, dove il restauro si limita a zone puntuali e questo si compie con una leggera ricostruzione o un leggero restauro.

La documentazione fotografica anteriore e posteriore al restauro realizzata dal signor Vicari e dal signor Mazzetti, come descrive la lettera di Tita Pozzi a Francesco Chiesa il 21 febbraio $1950^{416}$, testimoniano il carattere dei lavori eseguiti, effettivamente privi di rifacimenti invasivi, limitati alla pulitura della sporcizia superficiali, dei ridipinti, della cera delle lampade che ancora oggi sono segnate sotto la Madonna ed ognuno dei dodici Apostoli, il consolidamento delle parti sgretolate, la stuccature delle lacune e la ricostruzione della decorazione architettonica mediante un ritocco con tempere a basso tono.

Le ampie zone mancanti di colore sulle parti figurative, che oggi credevamo sprovvisto di ritocco poiché perso a causa dell'azione dell'umidità, risultano essere nella maggior parte rimaste intatte da quest'ultimo restauro, specialmente purista, come può osservarsi dalle fotografie allegate. Questi minimi ritocchi saranno sufficienti ad omogeneizzare il decoro artistico dell'abside completamente spezzato dai degradi.
Una prima analisi autoptica ci ha permesso di distinguere due tipi di stuccature differenziate tanto per la materia (granulometria, colore) come per il metodo d'applicazione, che dimostra che i dipinti hanno subito un intervento puntuale, non documentato, su certe zone cadute. Si tratta d'un lavoro meno curato di quello di Tita Pozzi, che sopra il livello delle superficie pittorica originale e molto irregolare per cui risulta specialmente visibile, mentre le stuccature compiute alla fine degli anni Quaranta risultano specialmente mimetizzate ma rispettose con l'opera in quanto ché distinguibili in qualunque caso a luce radente o con altre tecniche assolutamente non invasive.

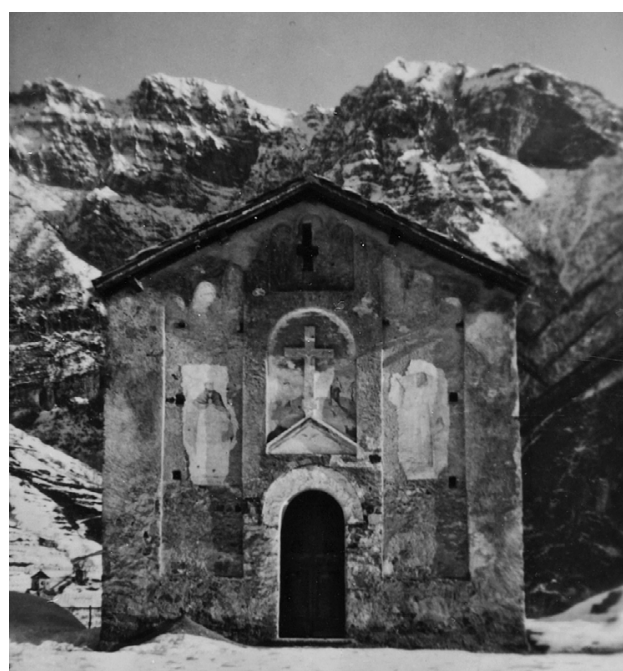

Figura 149 Facciata occidentale dopo il distacco della Cappella S. Rocco. Rovio, San Vigilio. Rivista storica ticinese 13 gennaio 1949 ca. EAD-63553. Archivio Federale dei Monumenti Storici. Collezione grafica, Biblioteca nazionale svizzera.

${ }^{416}$ (Pozzi Tita, Lettera a Francesco Chiesa, Massagno 21 febbraio 1950. AUBCBZ s.213 Rovio). 


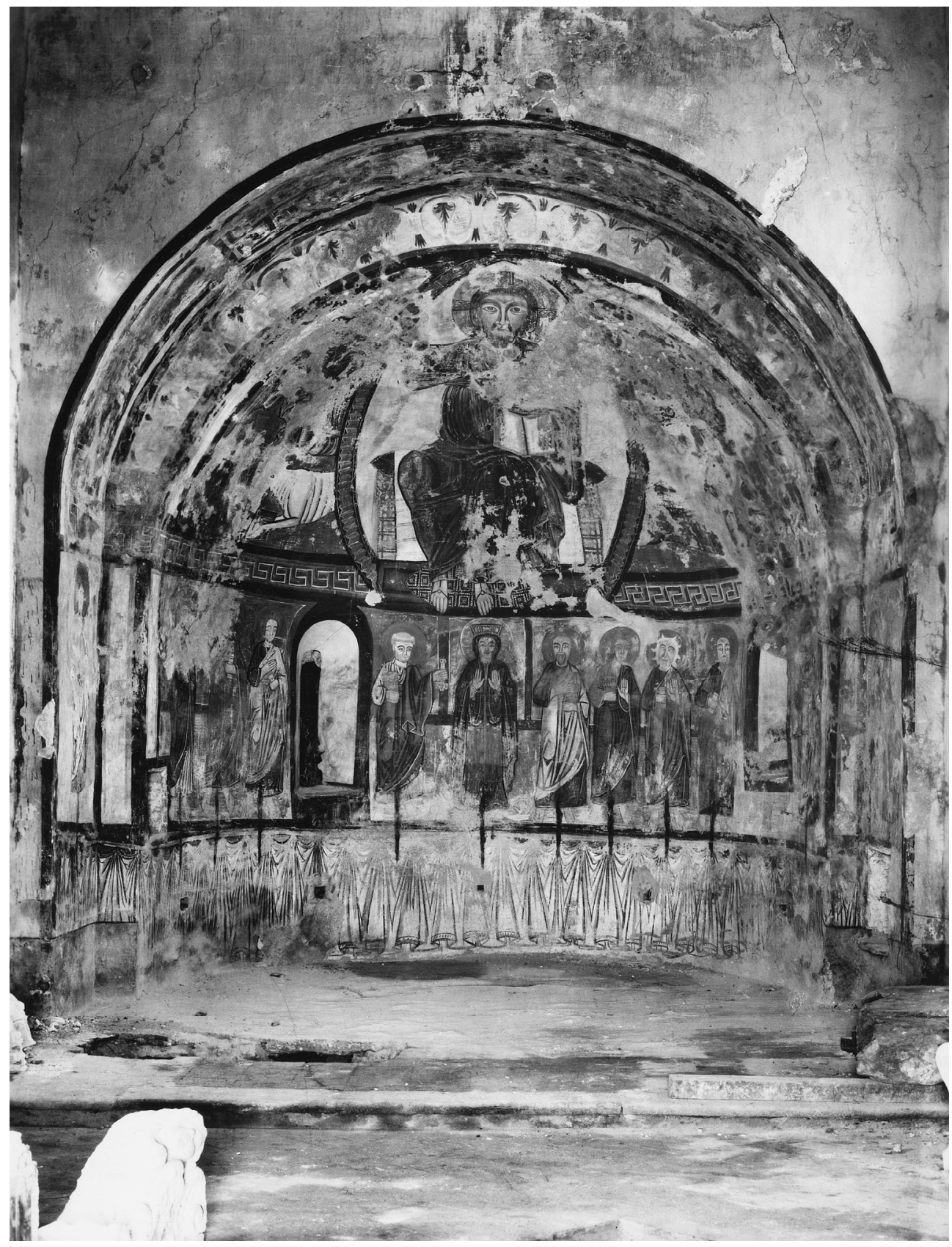

Figura 150 Affreschi dell'abside prima del restauro. Rovio, S.Vigilio. EAD-63553.

Archivio Federale dei Monumenti Storici. Collezione grafica, Biblioteca nazionale svizzera. 


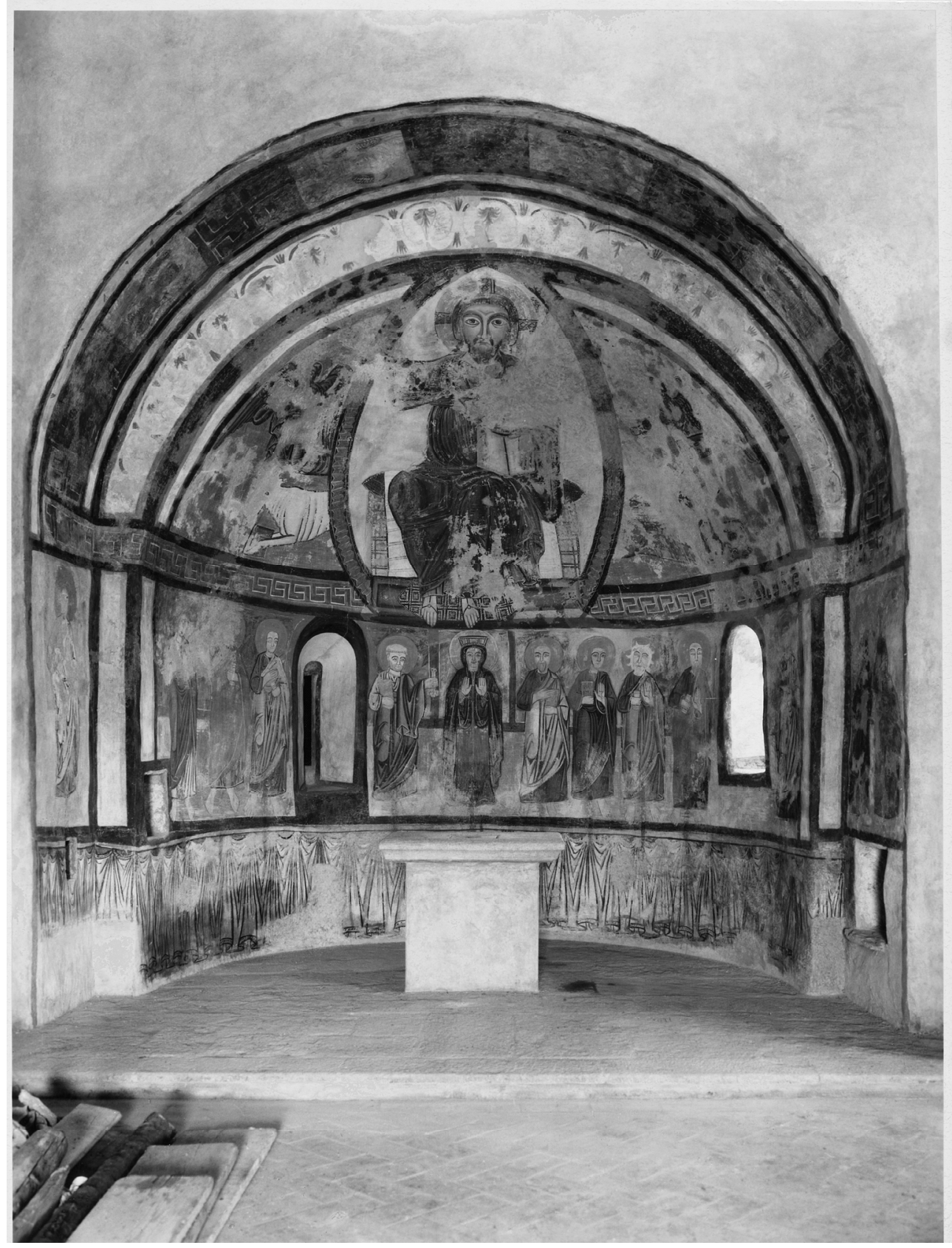

Figura 151 Veduta interna dell'abside con gli affreschi romanici dopo il restauro. Rovio, S. Vigilio. 1949 ca. EAD61097. Archivio Federale dei Monumenti Storici. Collezione grafica, Biblioteca nazionale svizzera. 


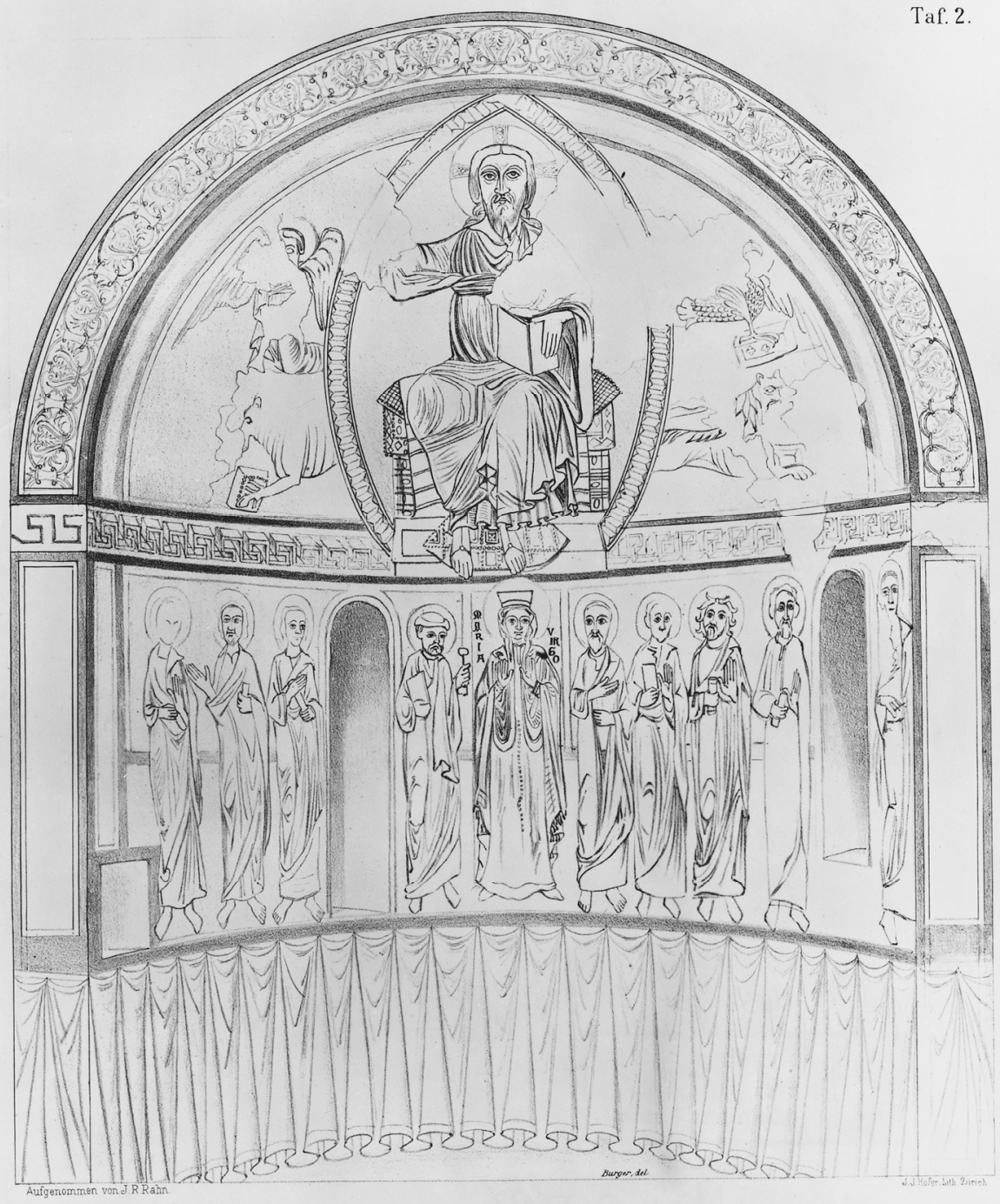

Figura 152 J.J.Hofer, Zürich, Litografia. Dipinti dell'abside disegnati da Johann Rudolf Rahn. Rovio, S. Vigilio. EAD65861 Archivio Federale dei Monumenti Storici. Collezione grafica, Biblioteca nazionale svizzera. 


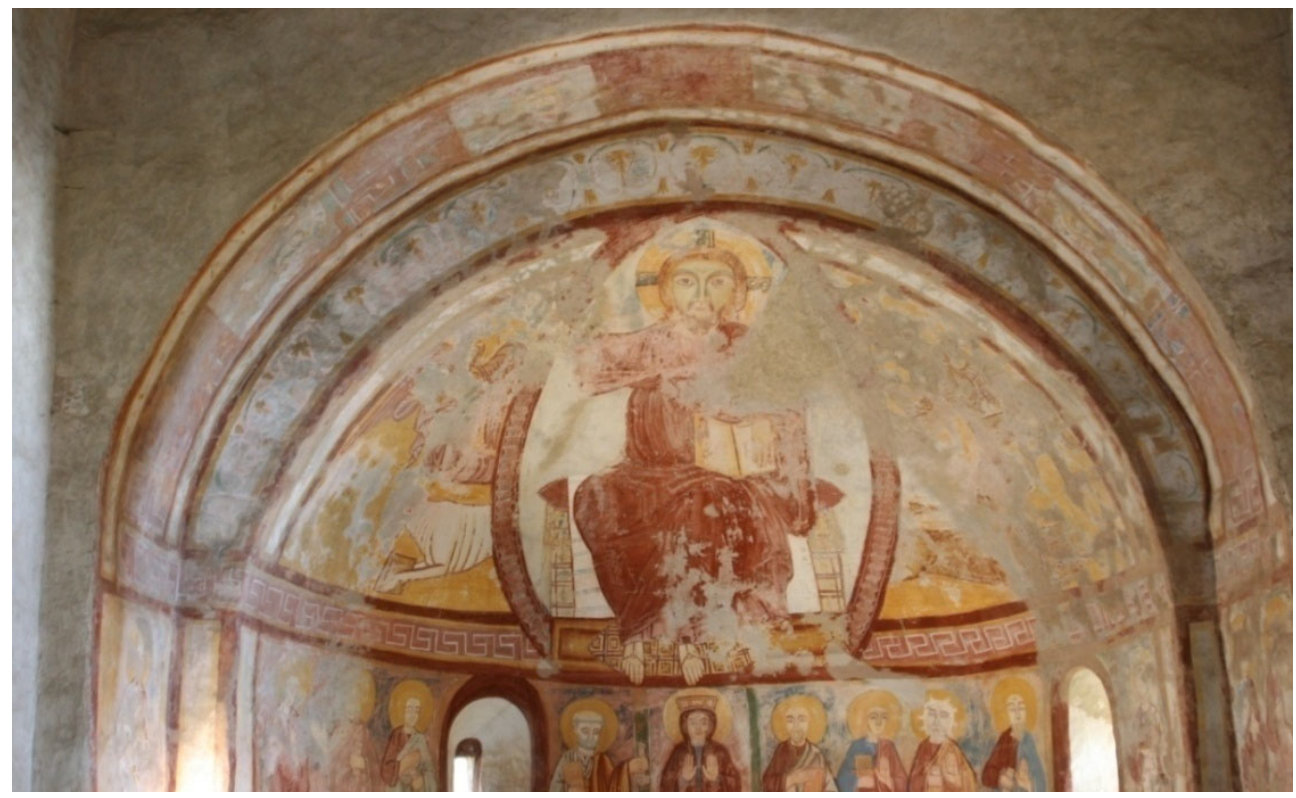

Figura 153 Giner Cordero E. Veduta interna della parte superiore dell'abside. Fotografia con luce naturale. Rovio, San Vigilio. 2008.

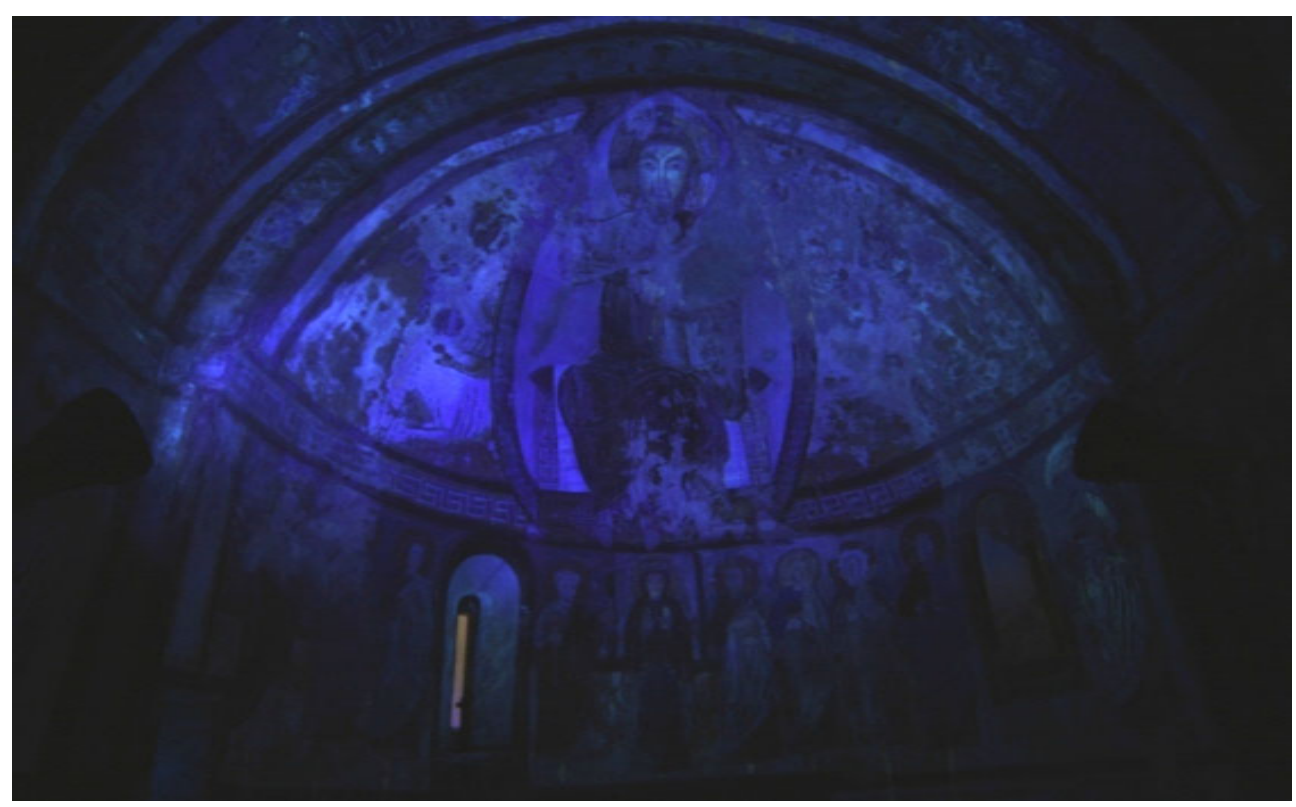

Figura 154 Giner Cordero E. Veduta interna della parte superiore dell'abside. Fotografia con luce UV. Rovio, San Vigilio. 2008. 
Restauratori in Canton Ticino fra Ottocento e Novecento

Catalogazione e gestione dati

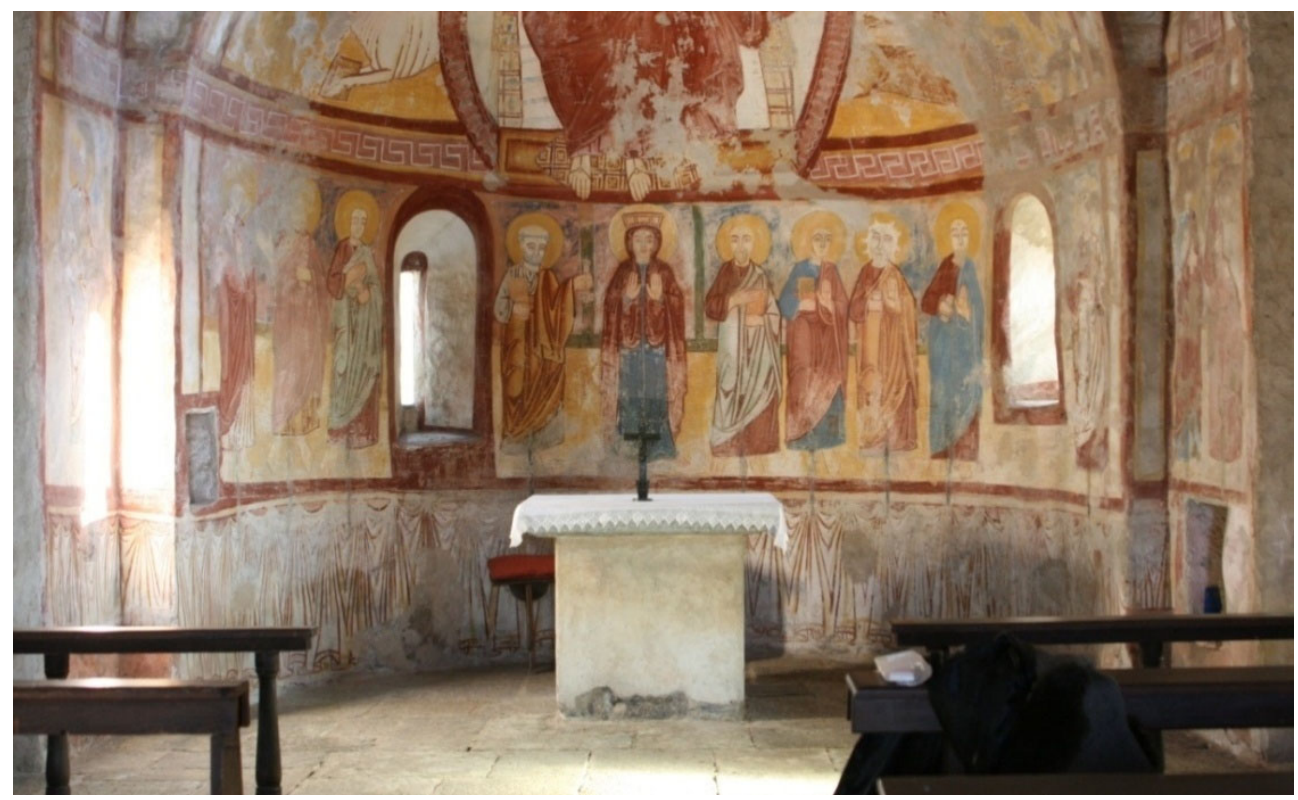

Figura 155 Giner Cordero E. Veduta interna della parte inferiore dell'abside. Fotografia con luce naturale. Rovio, San Vigilio. 2008.

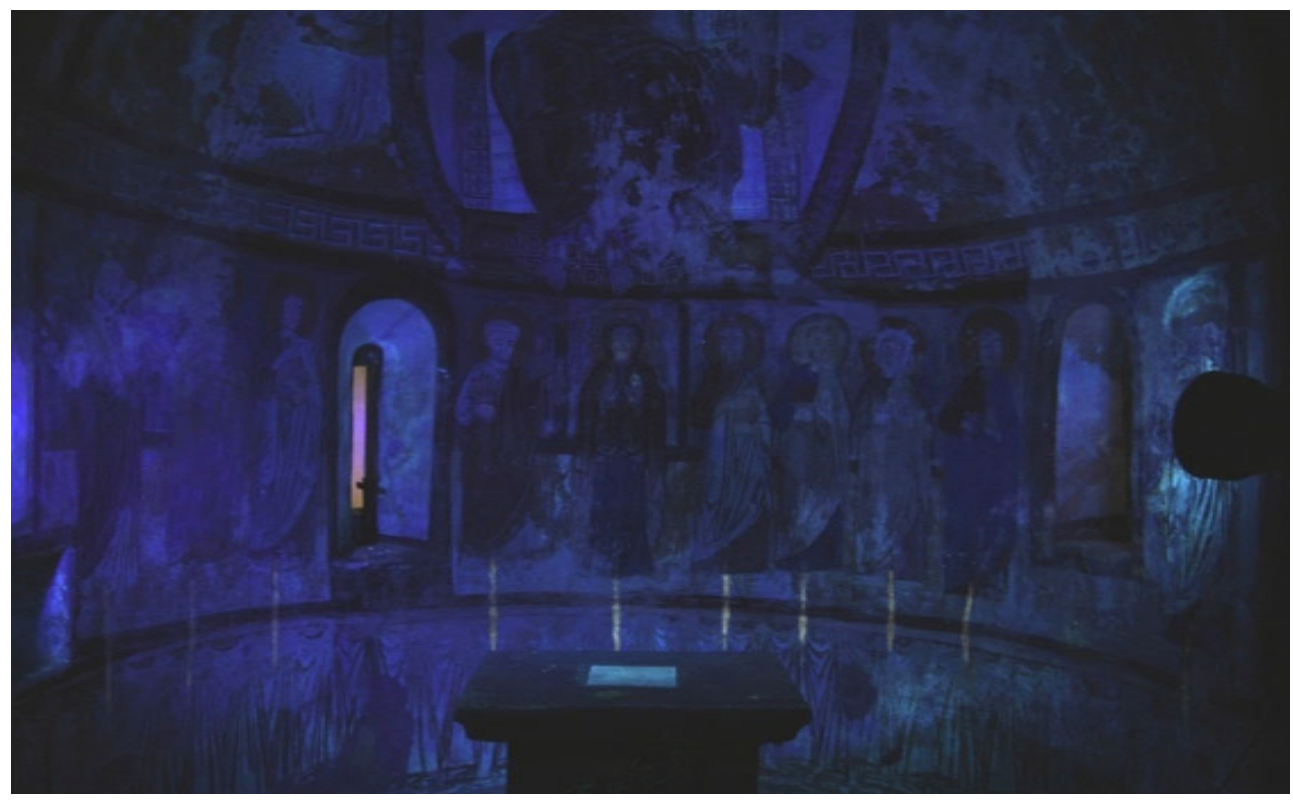

Figura 156 Giner Cordero E. Veduta interna della parte superiore dell'abside. Fotografia con luce UV. Rovio, San Vigilio. 2008 


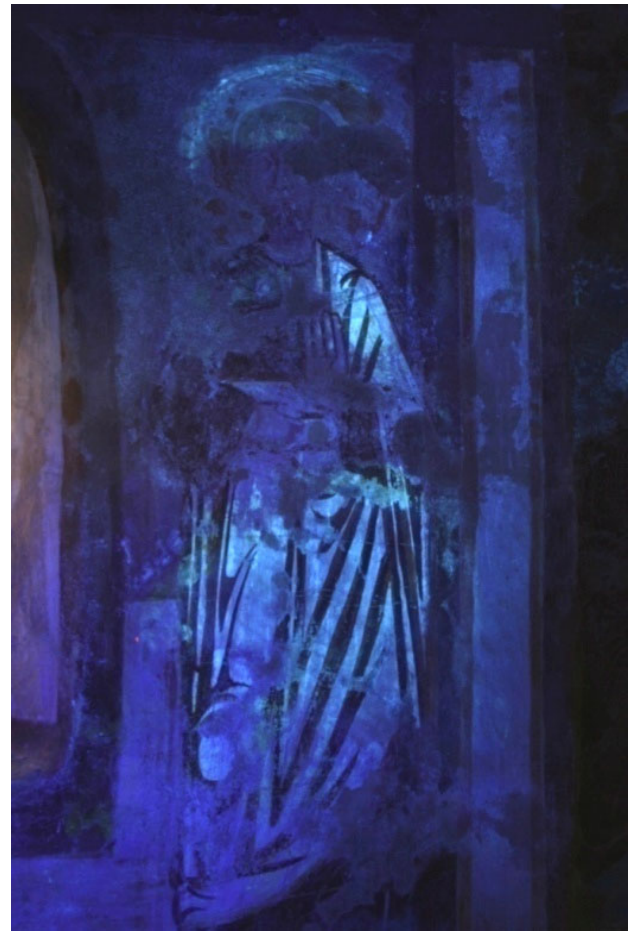

Figura 157 Giner Cordero E. Dettaglio delle pitture dell'abside. Fotografia con luce UV. Rovio, San Vigilio. 2008.

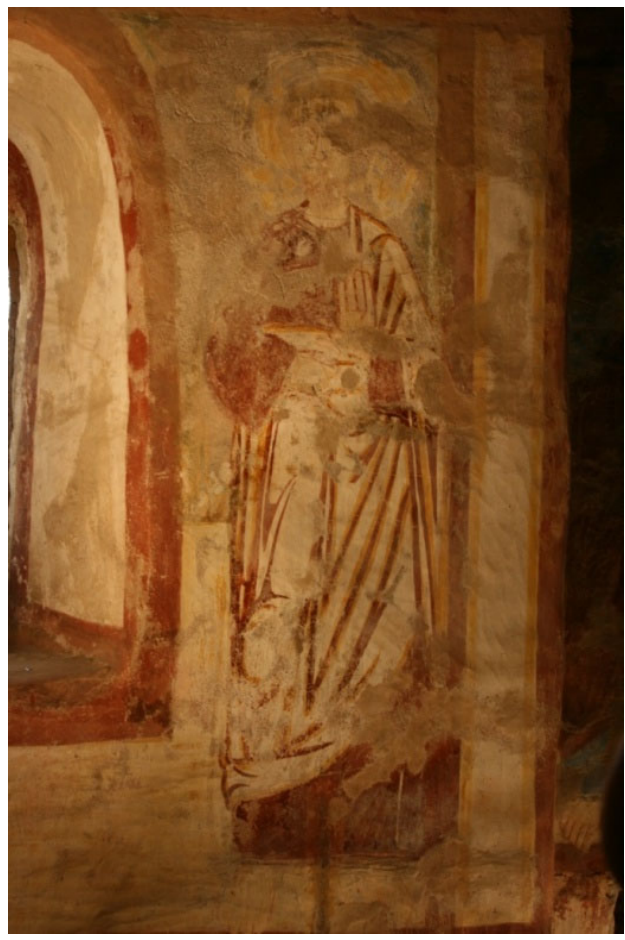

Figura 158 Giner Cordero E. Dettaglio delle pitture dell'abside. Fotografia con lucetrasmessa. Rovio, San Vigilio. 2008. 


\section{S. Remigio, Corzoneso}

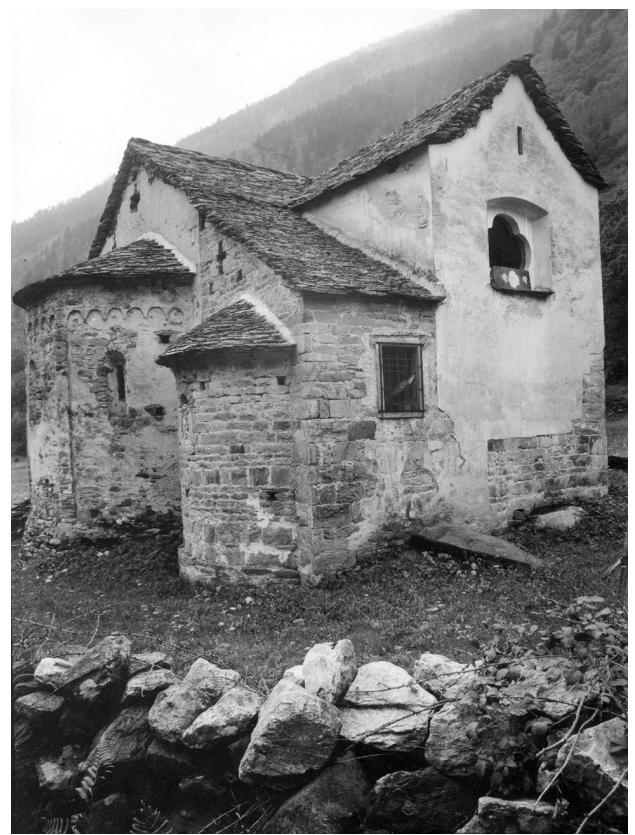

Figura 159 Veduta esterna dell'Oratorio di San Remigio. Fotografia b/n 1946 ant. Corzoneso, San remigio $A \cup B C B Z$, Archivio grafico.

Tita Pozzi fu uno dei pochi restauratori svizzeri ad impiegare la tecnica dello strappo in Canton Ticino in modo quasi specializzato, con dei risultati decisamente soddisfacenti. Una delle testimonianze più importanti del suo lavoro si trova all'Oratorio romanico di San Remigio a Boscero, frazione di Corzoneso, dove fra il 1944 e il 1946 utilizza la tradizionale tecnica di trasporto di affreschi sui dipinti manieristici dei Tarilli, soprastanti alle decorazioni medievali nell'abside maggiore, nell'arco trionfale e nei pilastri. Oscar Emmenegger, uno dei restauratori più importanti ancora attivo nella Svizzera tedesca, esprimeva pochi anni or sono in una delle sue ultime visite in Canton Ticino
all'Ufficio dei Beni Culturali ed al figlio del restauratore, l'avvocato Giovanni Pozzi, la sua ammirazione al ritrovare degli strappi eseguiti con tale professionalità.

La chiesa di S. Remigio fu in passato l'antica parrocchiale di Corzoneso. La fabbrica, trovatasi sulla riva destra del Brenno nelle vicinanze di Dongio, è di stile romanico. La chiesa è biabsidiata, composta da un'aula maggiore a sud costruita attorno alla metà dell'XI sec. e d'una seconda aula a nord, minore, del XII sec. II primo documento relativo all'edificio di culto è datato nel 1249. Nel XVII sec. l'edificio venne rinnovato e si procede con la costruzione del campanile a vela, l'apertura delle finestre sul lato sud e sulla facciata principale e per ultimo la suddivisione dell'aula minore in due spazi: la cappella della Madonna e la sagrestia dove rimane chiuso l'abside del XII sec.

I dipinti più antichi che decorano la fabbrica sono il grande San Cristoforo e Nicola di Bari affrescati sulla parte sud della navata e gli affreschi tardoromanici deI XIII sec. sull'abside principale e sull'arco trionfale che durante il rinnovo avvenuto nel seicento sono coperti dalla bottega di Giovanni Battista Tarilli con la medesima raffigurazione (Majestas Domini con i simboli degli evangelisti ed apostoli). Le seguono cronologicamente gli affreschi dell'abside minore sono posteriori, del XIV$\mathrm{XV}$ sec. con la rappresentazione di Cristo in Gloria e gli evangelisti, l'Annunciazione, il S. Remigio, sull'arco trionfale principale (XV sec.), la Madonna col bambino (XVI sec.) sulla porta della sacrestia e la Crocifissione con la Vergine ed i SS. Giovanni e Antonio Abate (XVI sec.) sulla parete sud. 


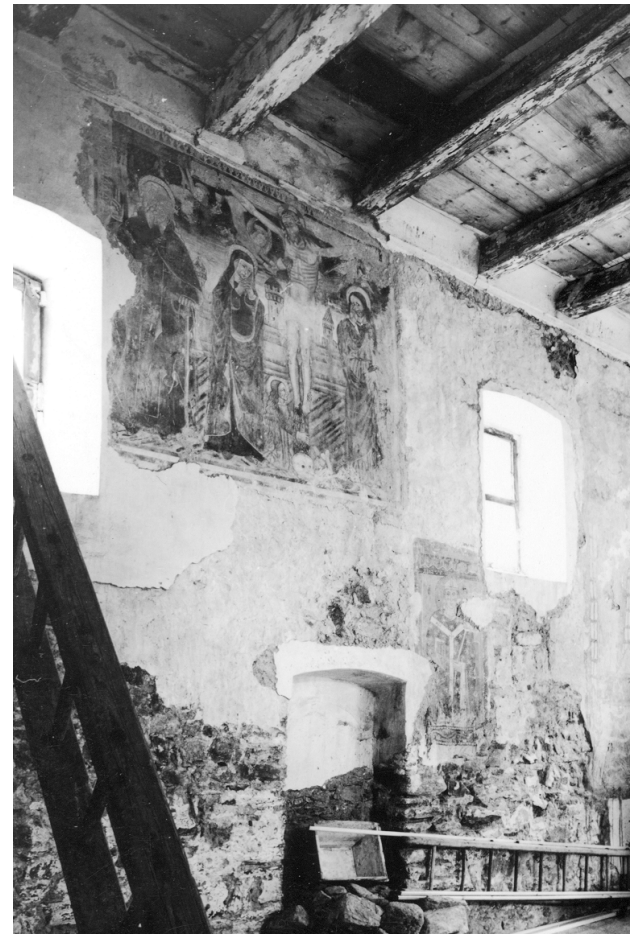

Figura 160 Veduta della parete meridionale durante i lavori di ricerca e di restauro. Fotografia b/n 1946 ant. Corzoneso, San remigio AUBCBZ, Archivio grafico.

L'interesse per la fabbrica risale al 1900 al ritrovarsi l'Oratorio in un deplorabile stato di conservazione; tuttavia questi i mezzi per riparare le struttura non sono a disposizione fino al 1943. Prima di quest'ultima data, anche Emilio Ferrazzini nei suoi costanti studi e ricerche, aveva pubblicato un articolo ${ }^{417}$ per la Rivista Storica ticinese dove analizzava le caratteristiche della fabbrica romanica ${ }^{418}$,

\footnotetext{
${ }^{417}$ (Ferrazzini, 1938, p. 76).

418 Secondo E. Ferrazzini, l'aula minore a nord, costituita dall'abside di $2 \mathrm{~m}$ di diametro, sarebbe primitiva all'aula maggiore finita nell'abside di $4 \mathrm{~m}$. d diametro. Contrariamente gli studi più recenti hanno dimostrato lo inverso. [...]G. Simona (Note d'Arte
}

gli affreschi esistenti al suo interno e, specialmente, i due frammenti di stucco raffiguranti apostoli. Era l'anno 1938.

Le sue osservazioni faranno chiamare l'attenzione sullo stato delle opere, come ad esempio, gli affreschi esterni della facciata dei quali erano rimasti unicamente dei frammenti difficilmente identificabili e l'affresco cinquecentesco della Crocifissione sulla parete della navata dove era perso abbondante materiale, nelle parti descritte dal Simona che raffiguravano un devoto e la Mandorla con la Madonna, non più esistenti.

[...] E ancora una volta bisogna constatare con rammarico con quanta rapidità se ne vanno questi preziosi dipinti quando non si provvede tempestivamente ad un loro consolidamento $[\ldots]^{419}$

Emilio Ferrazzini fu inoltre il primo ad eseguire delle indagini sull'abside

Antica) vide nello edificio una chiesa a due navate. Io credo invece che la chiesa, così com'è attualmente, è la risultante di tre costruzioni di epoche ben definite. La parte più antica è l'edificio che serve ora da sagrestia è comprende l'abside e parte, forse, della chiesa primitiva. II muro esterno dell'abside stesso è costruito in pietra viva ben tagliata, a strati di spessore irregolare. La seconda costruzione è formata dalla grande abside [...] che accoglie l'altare maggiore e dalla navata. La muratura esterna di questa seconda costruzione, che corrisponde a quella delle pareti sud ed ovest della chiesa, è di una fattura meno curata delle precedenti; anche se vi è una ricerca decorativa nella costruzione fatta con paraste ed archetti. L'edificio è leggermente sopraelevato dal piano, verso sud, essendo costruito sopra un masso roccioso $e$ presenta su quella facciata dei rifacimenti; si possono osservare infatti aperture chiuse od aperte in rottura nel muro. La terza costruzione è quella che forma la cappella della Madonna, decorata da un affresco del tardo rinascimento e da una ricca e tipica cancellata in ferro battuto[...] (Ferrazzini, 1938, p. 75).

${ }^{419}$ (Ferrazzini, 1938, p. 76). 
maggiore, ritrovando sotto i dipinti seicenteschi i dipinti primitivi che sembrarono completamente conservati. II pittore, aspettando di svegliare la curiosità delle istituzioni competenti attraverso la lettura dell'articolo, chiedeva: [...]Sarà possibile un giorno staccare l'affresco del $1600 \mathrm{e}$ rimettere in luce la decorazione primitiva, ottenendo così un singolare esempio di due affreschi ripetuti uno sopra l'altro, in epoche diverse?[... $]^{420}$

Perché succeda proprio quanto aveva previsto e proposto Ferrazzini bisognerà aspettare l'anno 1943. In questo momento il Consiglio Parrocchiale di Corzoneso, preoccupato per lo stato di conservazione in deperimento della chiesa di San Remigio, incaricherà le operazioni che accadono più urgenti per il ripristino della fabbrica al falegname Piero Borgna, ed un anno dopo sarà il Dipartimento della Pubblica Educazione del Canton Ticino a sussidiare il riordino completo dell'edificio.

I lavori di ristrutturazione architettonica furono affidati all'architetto Pietro Giovannini e le operazione artistiche al pittore Tita Pozzi, sempre con l'ispezione di Emilio Ferrazzini in qualità di membro della Commissione Cantonale dei Monumenti Storici. Dal primo verrà presentato un progetto di rinnovamento che interessava il rifacimento del tetto, la sostituzione degli intonaci degradati tanto all'interno come all'esterno della chiesa, il ripristino dei soffitti e delle sagomature della volta della cappella laterale ed il restauro di questa, fra i più importanti.

La fattibilità delle proposte venne confermata in seguito ai diversi sopralluoghi realizzati dal Ferrazzini. Egli

${ }^{420}$ (Ferrazzini, 1938, p. 76). indicherà assieme a Pozzi, in un primo rapporto presentato al Dipartimento della Pubblica Educazione il 16 novembre del 1943, le fasi che doveva comprendere il restauro artistico secondo un esame previo dell'opera. In questo analisi si confermò nuovamente l'esistenza degli affreschi primitivi sotto i visibili dipinti seicenteschi mediante "assaggi" nelle pitture, e si dimostrò lo stato di conservazione di essi, apparentemente in buono stato a carente delle abituali picchiettature usate per la adesione dell'intonaco recente.

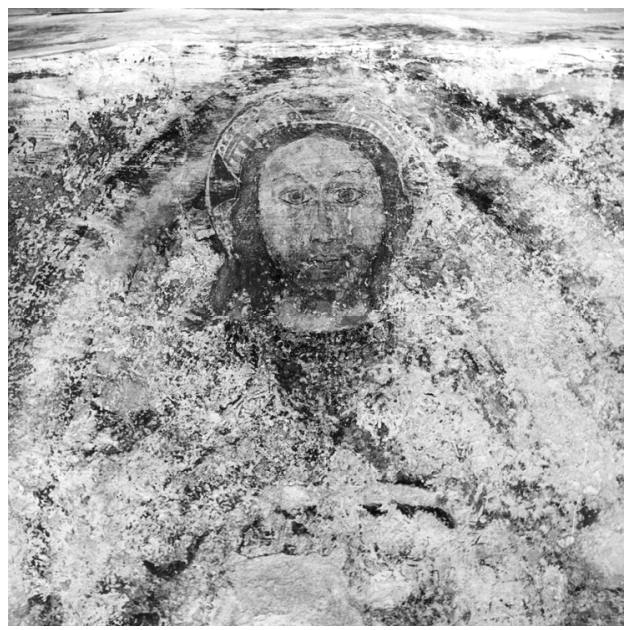

Figura 161 Affreschi dell'altare della cripta prima del restauro. Fotografia b/n 1946 ant. Corzoneso, San remigio $A \cup B C B Z$, Archivio grafico.

A questo punto i suggerimenti riguardo la scoperta degli affreschi medievali furono due. La proposta "1a", che era stata anticipata da Ferrazzini nel 1938, invitava al distacco dei dipinti della bottega di Giovanni Battista Tarilli raffiguranti gli Apostoli (cioè la parete verticale dell'abside di circa $10 \mathrm{mq}$ ), il Pantocrator nella parte superiore ( $2 \mathrm{mq}$ circa), le figure di San Remigio e San Michele sulla parete 
dell'arco trionfale ( $3 \mathrm{mq}$ circa) e finalmente il gruppo dell'Annunciazione $(3,50 \mathrm{mq}$ circa), per un prezzo complessivo di fr. 5'700 approssimativamente, che significava circa fr. 310 al metro quadro ${ }^{421}$.

La seconda proposta " $2 a$ " progettava, in maniera meno rispettosa riguardo l'opera, il distacco parziale degli affreschi del XVII sec. con l'eventuale cessione degli elementi strappati al Museo Civico di Belle Arti di Lugano. Lo scopo di questo invito, secondo il quale si sarebbero conservati unicamente le parti ritenute più belle del complesso pittorico come ad esempio le teste, il busto del San Remigio e il San Michele, [...] forse qualche figura intera da lasciare sul posto $[. . .]^{422}$, era decisamente quello di economizzare i lavori. Quest'ultima proposta esemplifica come lo strappo poteva convertirsi in un modo "facile" di portare l'opera ad un nuovo stato (dimensioni, supporto, luogo, funzione), in beneficio della sua mostra ravvicinata e della sua mobilità. Inoltre al restauro dei dipinti dell'abside nello stesso scritto si propose il restauro dell'affresco raffigurante la Madonna sopra la porta della sagrestia (XVI sec.) per un totale di fr. 150, della Madonna col bambino ed il pallio d'altare (XVIII sec.) nella cappella

Emilio Ferrazzini specificherà sul rapporto presentato al Dipartimento che il totale era stato preventivato secondo i prezzi d'anteguerra, il che significa che era sicuramente molto basso. Di fatti tre anni dopo quando Pozzi riprenderà il preventivo, egli si lamenterà sulla difficoltà di eseguire il lavoro a spese così ridotte poiché indubbiamente il costo della vita era aumentato. Tita Pozzi lavorerà finalmente per fr. 250 il mq.

${ }^{422}$ (Ferrazzini Emilio, Pozzi Tita. Proposta di restauro indirizzata al Dipartimento della Pubblica Educazione. Lugano 16 novembre 1943. AUBCBZ, s. 091 Corzoneso, S. Remigio). della Madonna, per Fr. 330 e Fr. 300 rispettivamente e finalmente il restauro degli affreschi dell'abside minore per fr. 550.

Lo stato di conservazione di questi ultimi era (è continua ad esserlo), precario poiché danneggiati dall'umidità infiltrata dalle fessure del tetto. II restauro di essi interessava unicamente l'esportazione dello strato di calce in superficie, che secondo le indagini si era dichiarato molto duro e tenace, per cui i lavori dovevano essere fatti con grande meticolosità evitando l' asportazione o graffiatura degli affreschi sottostanti lo scialbo. Non si citano invece operazioni di deumidificazione o di pulitura, neppure in successive fonti riscontrate.

Nel preventivo del 1943, Emilio Ferrazzini e Tita Pozzi indicavano l'intervento della "verniciatura" come ultimo processo da realizzare per raggiungere la completezza del restauro pittorico. Questo potrebbe accennare la possibile applicazione de cere sui dipinti dell'Oratorio di San Remigio a Corzoneso, come ancora prima avevamo trovato nei restauri di San Carlo di Negrentino, per esempio. Tuttavia si tratta della prima occasione che, in testi scritti dalla mano del Ferrazzini o del Pozzi, abbiamo trovato la parola "verniciatura" per definire la stesura di questo materiale. I processi esecutivi eseguiti dal pittorerestauratore Tita Pozzi dopo l'approvazione dei preventivi riguardarono ognuno dei punti precedentemente trattati, con alcune minime diversità. In primo luogo si trattarono gli affreschi della parete meridionale della navata, apparsi durante $\mathrm{i}$ lavori di saldature degli intonaci interni nel 1943. 
Restauratori in Canton Ticino fra Ottocento e Novecento

Catalogazione e gestione dati

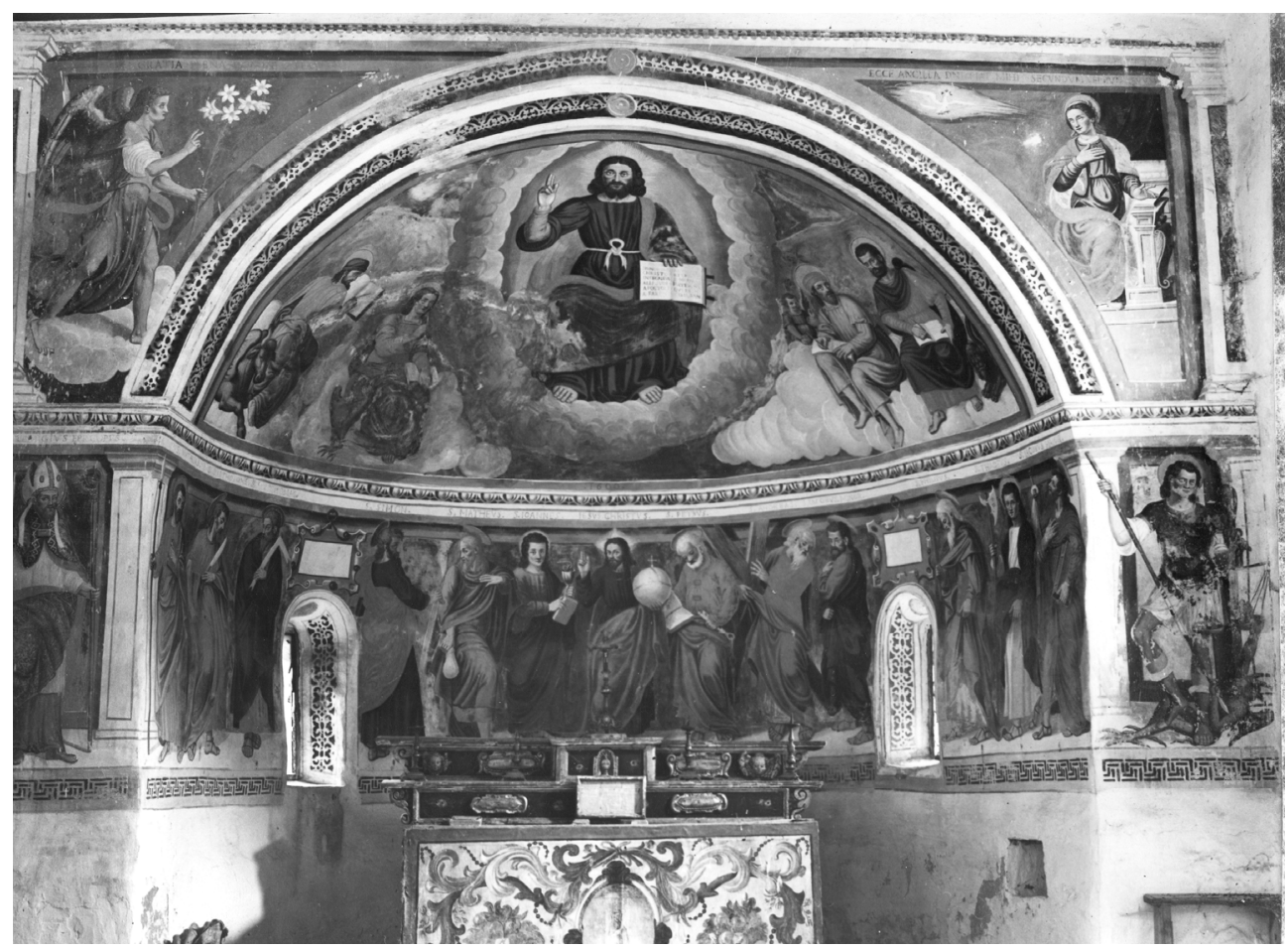

Figura 162 Veduta generale dei dipinti seicenteschi dell'abside maggiore, prima dei lavori di restuaro avvenuti nel 1944. Fotografia b/n. 1944 ant. Corzoneso, San remigio AUBCBZ, Archivio grafico. 


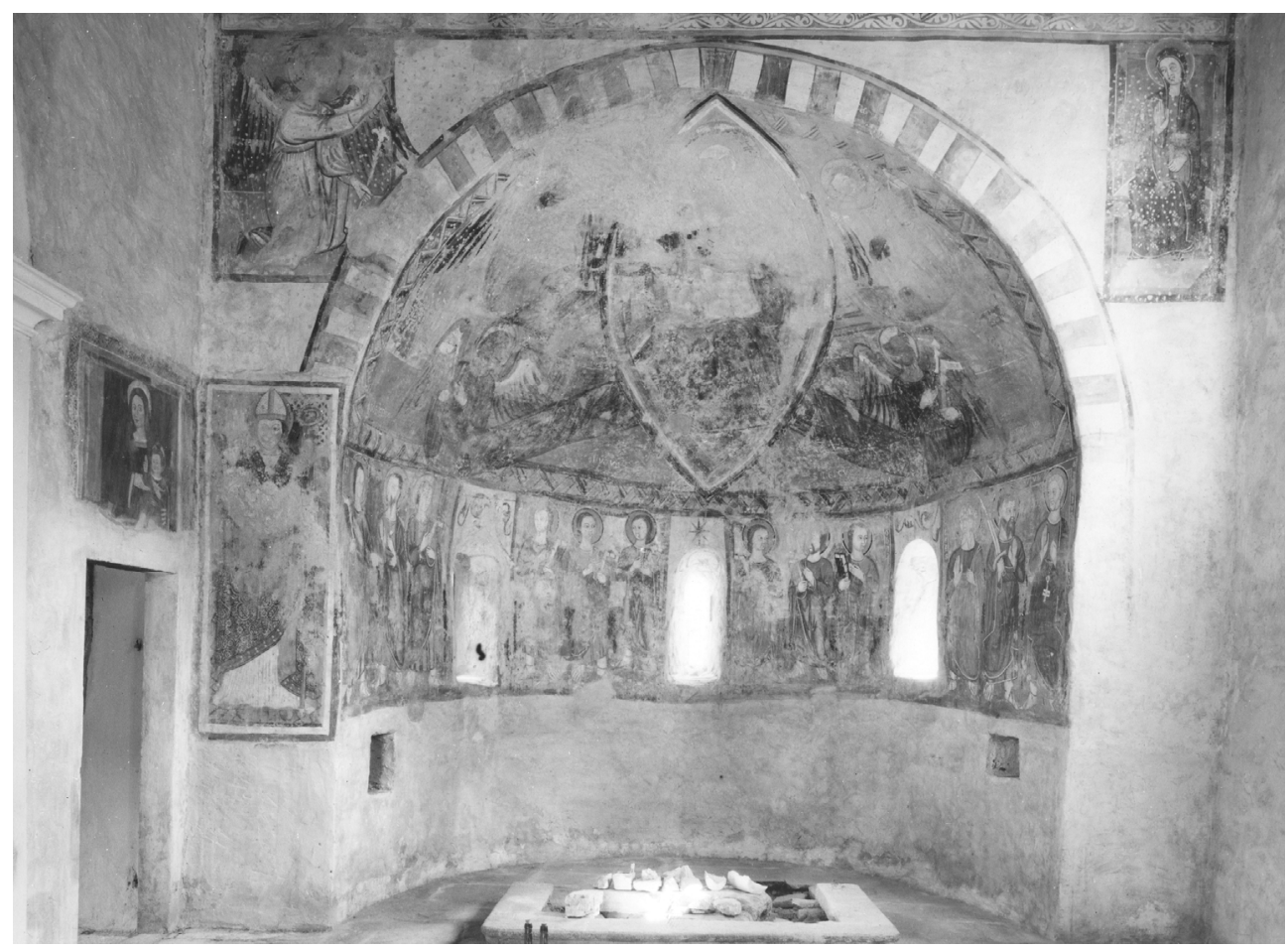

Figura 163 Veduta generale dei dipinti quattrocenteschi dell'abside maggiore, dopo i lavori di restauro avvenuti dal 1944 al 1946. Fotografia b/n. 1946 post. Corzoneso, San remigio AUBCBZ, Archivio grafico. 
Si tratta del dipinto raffigurante la Crocifissione (sec. XVI), e le figure tardoromaniche (sec. XIII ca.) di due santi: San Cristoforo ed un Santo Vescovo, attribuiti ambedue allo stesso autore della Crocifissione, poiché rappresentati con le stesse tecniche decorative riscontrate nei cromatismi, nella statiche figurativa, nella forza delle linee del disegno, e nell'esecuzione anatomica, fra altri fattori facilmente distinguibili. II Pozzi scoprì gli affreschi dallo scialbo di calce soprastante, e ritrovò un intonachino sano e solido, benché un arriccio friabile e pulverulento a causa dell'azione dell'umidità. A questo punto il pittore adopera consolidando per settori gli affreschi, dall'alto verso il basso, mediante abbondanti iniezioni di "stucco caseoso", ciò caseinato di calcio.

[...]stucco che iniettato poniamo, nelle parti alte degli affreschi - usciva da sotto l'intonaco uno e due metro più in basso[... $]^{423}$

Si avverte nella relazione di restauro presentata in ottobre del 1944 che il metodo impiegato non può essere definitivo e se lo avessero permesso i soldi si avrebbe dovuto intervenire mediante lo strappo delle due figure ed il trapasso sul posto originale dopo il rifacimento completo degli arricci degradati.

Dopo il consolidamento gli affreschi furono puliti dalle gocciolature e dalle macchie di calce e terra, stuccati e verniciati sicuramente con la tecnica dell'encaustico usata negli interventi contemporanei. Nella relazione il pittore dichiarava appunto che sui dipinti: [...]furono fatte rinvenire le tonalità $[. . .]^{424}$ espressione che abbiamo potuto ritrovare sul $90 \%$ delle proposte o relazioni di restauro presentate alla medesima Commissione dei Monumenti dalla fine dell'Ottocento e dagli anni Cinquanta del Novecento, sempre in riferimento alla suddetta tecnica. Per ultimo fu ritoccato lo scialbo della volta e realizzata la tinteggiatura dello zoccolo.

Nella semitazza absidale il restauratore, dopo essersi rassicurato sullo stato di conservazione delle pitture medievali sottostanti ai dipinti seicenteschi dei Tarilli, compie lo strappo di questi ultimi. Nell'agosto del 1945 le figure eseguite furono unicamente tre: gli Apostoli Filippo, Taddeo e Giacomo Maggiore, in un pezzo unico. II successo di questa operazione permette al Dipartimento della Pubblica Educazione d'autorizzare l'esecuzione sulla completa superficie.

I tredici dipinti strappati con la tecnica tradizionale a base di tele di diversa densità e colla animale, furono riportati su fondo rigido di legno "prevatex".

[...]Vi comunico che io non posso operare assunte informazioni e fatti i debiti calcoli - a meno di fr. 250 / 260 metro quadro- Alla Chiesa degli Angioli il restauratore di Zurigo, Dillena, operava, già quindici anni or sono - per $200-$ 220 franchi al metro. II sig. Pitt. Ferrazzini aveva calcolato per S. Remigio - nel preventivo da lui allestito - una media di fr. 350 - al metro. La colla è salita fa $2 \mathrm{fr}$. A $8 \mathrm{fr}$. La tela bambalina de $1.50 \mathrm{fr}$. A $6.50 \mathrm{fr}$. al metro, la caseina da $1.50 \mathrm{fr}$. A $7.50 \mathrm{fr}$. al $\mathrm{Kg}$, le opere di falegname (telai,

\footnotetext{
${ }^{424}$ (Pozzi Tita, Relazione di restauro indirizzata al DPE. Massagno - Lugano 15 ottobre 1944. AUBCBZ, s. 091

Corzoneso, S. Remigio).
}

${ }^{423}$ (Pozzi Tita, Relazione di restauro indirizzata al DPE. Massagno - Lugano 15 ottobre 1944. AUBCBZ, s. 091 Corzoneso, S. Remigio). 
tavole) sono aumentate del 40 al 50 per cento, e aumenteranno ancora $[. . .]^{425}$.

Gli strappi finiti furono collocati all'interno della stessa fabbrica, nella posizione dove ancora oggi possiamo ammirarli: sulla parete occidentale, sulla meridionale ed all'interno della cappella laterale, perdendosi logicamente parte dei dipinti e della sua disposizione originaria oggi riferita solo mediante la fotografia ripresa prima del restauro in 1944.

I dipinti medievali riscoperti risultarono essere iconograficamente simili a quelli seicenteschi, il che dimostra il senso letterale in cui furono adottate le leggi conformate dopo il Concilio di Trento, che richiedevano "rinfrescare le superficie pittoriche".

Tita Pozzi procede con la pulitura dello scialbo di calce sopra gli affreschi della chiesa primitiva, consolidando e ritoccando I'insieme. Lo stesso venne compiuto sulla Madonna dei Tarilli della cappella laterale, sull'affresco sopra la porta della sacrestia, sui dipinti dell'abside minore e sui resti delle pitture esterne la cui figurazione non è più riconoscibile.

Lo strappo delle tredici figure non causò nessun danno apparente al giorno corrente sulla superficie, dimostrando con questo che si trattò d'una esecuzione meticolosa e specialmente rispettosa. Di fatto lo stato di conservazione dell'opera risulta ancora al momento buono, e si sono ritrovate unicamente minime lacune realizzate durante il descialbo, alcuni residui superficiali dalle sostanze consolidanti, e

\footnotetext{
${ }^{425}$ (Pozzi Tita, Circa il trasporto degli affreschi de Tarilli all'Oratorio di S. Remigio in Corzoneso. Lettera indirizzata al DPE. Massagno 17 aprile 1946).
}

stuccature inadeguatamente eseguite con malta cementizia.

Possiamo confrontare questo lavoro di strappo con quello che più di sei anni più tardi il restauratore eseguirà alla chiesa parrocchiale di Sant'Ambrogio a Ponte Capriasca nel 1951. Anche in questa fabbrica i dipinti distaccati dal muro furono numerosi: dodici telai di legno servono da supporto ai sottili strati d'intonaco recanti i dipinti che oggi si possono ammirare, come a S. Remigio di Corzoneso, nella propria chiesa d'origine. La causa che motiva l'esecuzione di queste operazione così radicali sono i gravi deterioramenti delle pitture, assai danneggiate dall'azione dell'umidità. Già nel 1948 erano stati eseguiti nella chiesa di Sant'Ambrogio dei trattamenti di "deumidificazione" per risanare le pareti, ma non bastò a permettere la conservazione degli affreschi in sito mediante più semplici operazioni di consolidamento. La proposta eseguita fu lo stacco, cioè il prelevamento dell'affresco a massello, con tutti gli strati. Successivamente le pitture saranno, come apprezziamo attualmente, strappate e trasportate su un supporto ligneo. Non si è potuto verificare dai documenti il motivo per il quale Tita Pozzi debba cambiare l'impostazione dei lavori, ma si può pensare ad una disgregazione degli intonaci che impedi la manutenzione del completo supporto murario, tenendo in conto lo stato di degrado in cui erano arrivate le opere in mano del restauratore. I dipinti, oggetto di questo trattamento, furono l'Assunzione della Vergine con diversi frammenti di angeli in volo che potessero essere salvati (presenti ora nella parete della controfacciata); la Madonna 
della Misericordia, il Santo Domenico Martire e la Madonna in trono con bambino, sulla parete ovest della navata, e tre raffigurazioni diverse della Madonna del Latte (del XIV sec. e del XVI sec.) sulla parete est.

Lo stesso anno nella medesima fabbrica, il Pozzi restaura l'affresco dell'Ultima Cena del 1550, copia del Cenacolo leonardesco mediante una pulitura iniziale della polvere superficiale, il consolidamento dei bordi dell'affresco, degli sfondi delle teste, del zoofito e dell'angolo sinistro finendo con il ritocco pittorico limitato ai graffi occasionati per atti vandalici e nelle sgretolatture. (Libro Lalli)

Sarebbe interessante conoscere dove e da chi Tita Pozzi imparò la tecnica dello strappo: forse dal pittore-restauratore Attilio Balmelli ${ }^{426}$ con chi lavorò prima di iniziare gli studi all'Accademia di Belle Arti milanese o forse proprio qui, in Italia dove fu nata la suddetta tecnologia, nei corsi frequentati nella medesima scuola. Si deve avvertire sul fatto che tutti i pittori catalogati in questa ricerca, chi più e chi meno, dedicarono parte della loro attività al restauro, hanno avuto modo prima o poi di impiegare la tecnica dello strappo a scopi ben diversi. Questo significa che furono in tanti ad avere accessibilità alla conoscenza di questo metodo, anche se la difficoltà di tale prassi fece che solo in

\footnotetext{
${ }^{426}$ Ricordiamo che Attilio Balmelli fu attivo nel campo del restauro praticamente fino agli anni sessanta, e questo significa che le sue competenze potessero essere condivise con l'allievo e amico paesano Tita Pozzi. In ogni modo nessuno dei lavori documentati di Attilio Balmelli riferisce l'esecuzione dello strappo de dipinti murali, per cui le provabilità che quest'ultimo insegnasse una tecnica che, per quanto pare, non messe mai in pratica, sono minime.
}

pochi la impiegassero idoneamente. Tanto è vero che unicamente Pozzi, fra i pittorirestauratori ticinesi beneficiò della fiducia del Cantone e della Commissione dei Monumenti per l'esecuzione degli strappi. La prima opera di distacco che si è trovata documentata in Ticino fu il dipinto dell'Ultima Cena di Bernardino Luini (ved. cap. 1?) il quale venne realizzato dall'italiano, Bernardo Gallizioli. Più tardi gli Steffanoni (padre e figli), anche loro italiani di Bergamo, riconosciuti internazionalmente come propri professionisti in materia e definiti da loro stessi come "trasponitori di pitture", eseguono in Ticino gli strappi della Chiesa Rossa ad Arbedo (1899) e della Cattedrale di San Lorenzo a Lugano (1904). Altri strappi già citati vengono realizzati durante i restauri condotti nel 1929 da Edoardo Berta in Santa Maria delle Grazie a Bellinzona, ma senza indicare esattamente il/i professionista/i ai quali fu affidato l'incarico. Dopo questi minimi esempi documentati, sarà il nome del pittore Tita Pozzi a succedersi abitualmente nelle pratiche di restauro legate a codesta tecnica.

Nel 1932, quando inizia a farsi conoscere nel settore, il Pozzi esegue i primi strappi a Cadepiano e a Gordola (1934). I dipinti provenivano da case private e sarebbero trasportati con lo scopo di farli più visibili e decorare le chiese del paese. Seguiranno a essi gli strappi di Corzoneso (1944) per liberare le pitture sottostanti, gli strappi della casa già Ferrazzini che doveva essere demolita (1948) e della chiesa di S. Ambrogio a Ponte Capriasca (1951).

Contemporanei al pittore, solo Carlo Cotti, Teodoro Hallich e I'italiano Mario Rossi di 
Varese realizzeranno alcuni strappi nel Cantone: Hallich nel 1939 strapperà l'affresco quattrocentesco raffigurante la Madonna del latte fra i SS. Antonio Abate e Gottardo della maniere di Antonio di Tradate, ritrovato sotto il portico d'una casa privata di Lissoi che poi sarà collocato nella chiesa parrocchiale dei SS. Carpoforo e Gottardo ${ }^{427}$; analogamente lo strappo eseguito da Carlo Cotti a Brusino-Arsizio nella casa Polli nel 1953 sarà trasportato successivamente nella chiesa parrocchiale di San Michele ed i due strappi realizzati da Mario Rossi nel 1955 per mostrare i dipinti sottostanti nell'abside del Battistero di Riva San Vitale saranno poi collocati anch'essi nella parrocchiale di questa località.

Minori sono gli esempi documentati di trasporto di affresco con la tecnica dello stacco. I primi, realizzati nel 1851, furono le due opere di Bernardino Luini esistenti nella chiesa di S. Maria degli Angioli e nel Convento di S. Francesco a Lugano: la lunetta della Madonna col bambino e San Giovanni, oggigiorno nella prima cappella negli Angioli, e la Crocifissione trasportata dal Convento a Palazzo Albertolli. Poi, solo

\footnotetext{
427 La notizia sull'evento dello strappo e del restauro venne riportata da Aldo Crivelli sulla Rivista Storica Ticinese del 1 ottobre 1940. [...] sotto il portico di una comune casa colonica si trovava l'affresco [...] $\mathrm{Nel}$ novembre dello scorso anno, per interessamento della Commissione Cantonale dei Monumenti Storici, con una spesa di $100 \mathrm{fr}$., fu strappato dal muro e riportato su tela e restaurato. Si veda il "Rendiconto del Dipartimento Educazione della Gestione 1939". L'affresco si trova ora, se non erriamo, nella Chiesa Parrocchiale di Mergoscia. II lavoro, ben riuscito, è stato eseguito dal restauratore Teodoro Hallich di Locarno che ringraziamo per averci fornito la bella fotografia quando la pintura si trovava ancora al suo posto originale.[...]. (Crivelli, La Madonna di Mergoscia, 1940, p. 407).
}

nel 1919, il pittore Emilio Mazzetti distaccherà a massello il gruppo quattrocentesco del "Compianto" esistente nella cappella Marotti a Bellinzona, che sarà più tardi esposto nella chiesa di San Biagio di Ravecchia. Ventitre anni dopo, una Madonna col bambino del XV sec. ca. sarà staccata da Taverne e collocata nell'altare della cappella Camuzio in $\mathrm{S}$. Maria degli Angioli a Lugano, dove oggi si può apprezzare in uno stato di conservazione precario.

I dati qui sopra riportati, anche se limitati a causa dell'insufficienza di materiale documentario, bastano in ogni modo ad indicare quelle che furono le esigenze del cantone e della sua commissione dei monumenti; ritornando alla figura di Tita Pozzi, essi ci aiutano a ricordare che, se solo i lavori più significativi furono catalogati e segnati dalle istituzioni competenti, essi in sua maggior parte sono stati compiuti dal pittore e restauratore di Massagno.

L'analisi macroscopica delle opere strappate dal Pozzi hanno evidenziato I'impiego della tecnica dello strappo alla maniera degli insegnamenti del maestro Secco Suardo, cioè mediante il distacco minimo di intonaco in modo che l'affresco sia più flessibile e si adatti al nuovo supporto anche se, in questo modo, le caratteristiche fisiche ma innanzitutto estetico dell'opera cambiano.

Questo metodo fu idealizzato e diffuso dal conte bergamasco. Per mostrarlo ai professionisti del settore verrà chiamato nel 1864 dalle Regie Gallerie di Firenze. Alle sue lezioni sulla tecnica dello strappo assiste anche il secondo grande trattatista del restauro nell'Ottocento: Ulisse Forni, e i 
risultati furono esposti in una mostra aperta al pubblico, che sarà gradita non solo dai restauratori ma anche dai mercanti, antiquari e collezionisti.

Le opere murali, viste di solito nella lontananza, e proprietarie da tanti particolari impercettibili, potevano mediante lo strappo e lo stacco avvicinarsi all'appassionato d'arte, agli studiosi, e questo faceva il sistema ancora più suggestivo.

Ora, il nostro giudizio dell'opera e di ciò che significa oggi il rispetto verso questa, ci fa rifiutare qualsiasi intervento di trasformazione di essa. Strappare un dipinto è nell'attualità l'ultima considerazione per la conservazione di questo, e bene giustificato solo nel caso in cui la salvezza dell'opera non sia possibile mediante nessun altro metodo di restauro o per distruzione del luogo che questa occupa. Anche lo stacco, che ci permette almeno di mantenere le caratteristiche fisiche dell'affresco, la sua curvatura, il suo supporto originale, e aiutano senz'altro ad una migliore stabilità di questo, si sceglierebbe oggi come primo intervento prima dello strappo. Per motivare alla riflessione sui percorsi teologici qui analizzati, Cristina Giannini ricorda la Carta del restauro del 1972:

[...]quando si debba necessariamente orientarsi sulla remozione del dipinto dal supporto, fra i metodi da scegliere, con equivalente probabilità di riuscita, dovrà scegliersi lo strappo, per la possibilità che offre di recuperare la sinopia preparatoria, in caso di affreschi, ed anche perché libera la pellicola pittorica dai residui di un intonaco fatiscente o ammalato $[. . .]^{428}$.

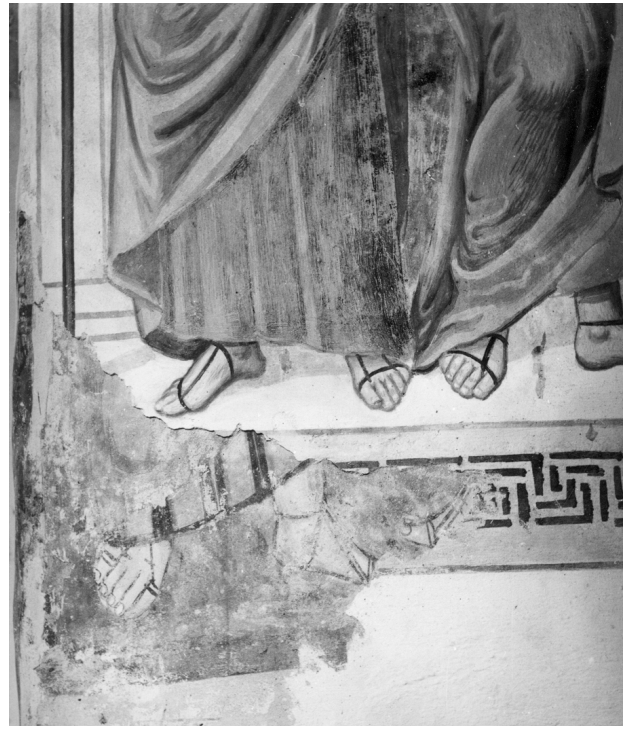

Figura 164 Particolare dei dipinti seicenteschi dell'abside maggiore, dove si osservano le pitture sottostanti primitive. Fotografia b/n 1946 ant. Corzoneso, San remigio AUBCBZ, Archivio grafico.

${ }^{428}$ (Gianini, 2006, p. 15). 


\section{Interventi di restauro}

\begin{tabular}{|c|c|c|c|c|c|}
\hline ANNO & LUOGO & OPERA & INTERVENTO & COLLABORATORI & FONTI \\
\hline $\begin{array}{l}1891- \\
1930\end{array}$ & $\begin{array}{l}\text { Lugano, } \\
\text { chiesa di S. Maria } \\
\text { degli Angioli. }\end{array}$ & $\begin{array}{l}\text { Affreschi della } \\
\text { Cappella Camuzio e } \\
\text { pitture nei sottarchi } \\
\text { della parete } \\
\text { trasversale. }\end{array}$ & Restauro. & $\begin{array}{l}\text { Emilio Ferrazzini, } \\
\text { Emilio Maccagni, } \\
\text { Arturo Ortelli, } \\
\text { Emilio Dillena, } \\
\text { Tito Tettamanti, } \\
\text { Alfonso Cattaneo, } \\
\text { Carlo Cotti, } \\
\text { Basilio Rusca, } \\
\text { Edoardo Berta. }\end{array}$ & $\begin{array}{l}\text { (DPE, Cinquant'anni } \\
\text { di protezione dei } \\
\text { monumenti storici } \\
\text { artistici della } \\
\text { repubblica e } \\
\text { cantone Ticino } \\
\text { 1909-1959, 1959, p. } \\
32 \text { ). } \\
\text { (DPE, Cinquant'anni } \\
\text { di protezione dei } \\
\text { monumenti storici } \\
\text { artistici della } \\
\text { reppubblica e } \\
\text { cantone del Ticino, } \\
\text { 1959, p. 122, 143). }\end{array}$ \\
\hline $\begin{array}{l}1930- \\
1932\end{array}$ & $\begin{array}{l}\text { Malvaglia, } \\
\text { chiesa Parrocchiale } \\
\text { di S. Martino. }\end{array}$ & $\begin{array}{l}\text { Affreschi interni ed } \\
\text { esterni. }\end{array}$ & Pulitura e restauro. & $\begin{array}{l}\text { Carlo Cotti e Tito } \\
\text { Tettamanti diretti } \\
\text { da Emilio Ferrazzini. }\end{array}$ & $\begin{array}{l}\text { (Chiesa, 1946, p. 42) } \\
\text { (DPE, Cinquant'anni } \\
\text { di protezione dei } \\
\text { monumenti storici } \\
\text { artistici della } \\
\text { reppubblica e } \\
\text { cantone del Ticino, } \\
\text { 1959, p. } 122,148 \text { ). }\end{array}$ \\
\hline 1931 & $\begin{array}{l}\text { Madrano, } \\
\text { Oratorio dei Santi } \\
\text { Gervasio e Protasio. }\end{array}$ & & Restauro. & & $\begin{array}{l}\text { (Foletti Giulio, } \\
\text { Novembre 2002, p. } \\
45 \text { ) }\end{array}$ \\
\hline 1932 & $\begin{array}{l}\text { Cadepiano, } \\
\text { casa di proprietà } \\
\text { Giuliani in Aquila. }\end{array}$ & Affreschi. & Strappo. & & $\begin{array}{l}\text { (DPE, Cinquant'anni } \\
\text { di protezione dei } \\
\text { monumenti storici } \\
\text { artistici della } \\
\text { reppubblica e } \\
\text { cantone del Ticino, } \\
1959, \text { p. } 80 \text { ). }\end{array}$ \\
\hline 1934 & $\begin{array}{l}\text { Gordola, } \\
\text { casa rustica } \\
\text { proprietà Flaminio } \\
\text { Signorotti. }\end{array}$ & $\begin{array}{l}\text { Madonna col } \\
\text { Bambino, XV sec. } \\
\text { frammento di } \\
\text { affresco. }\end{array}$ & $\begin{array}{l}\text { Strappo di affresco } \\
\text { riportato su tela e } \\
\text { collocato nella } \\
\text { chiesa parrocchiale } \\
\text { di S. Antonio di } \\
\text { Gordola. }\end{array}$ & & $\begin{array}{l}\text { (Chiesa, 1946, p. 50) } \\
\text { (DPE, Cinquant'anni } \\
\text { di protezione dei } \\
\text { monumenti storici } \\
\text { artistici della } \\
\text { reppubblica e } \\
\text { cantone del Ticino, } \\
\text { 1959, p. 81, 121) } \\
\text { (AUBCBZ s.117 } \\
\text { Gordola) } \\
\text { (Simona Martinoli, } \\
\text { 2007). }\end{array}$ \\
\hline
\end{tabular}




\begin{tabular}{|c|c|c|c|c|c|}
\hline $\begin{array}{l}1934 \text { - } \\
1935\end{array}$ & $\begin{array}{l}\text { Bellinzona, oratorio } \\
\text { di } \\
\text { S. Marta . }\end{array}$ & Affreschi. & $\begin{array}{l}\text { Pulitura, } \\
\text { consolidamento e } \\
\text { reintegrazione dei } \\
\text { dipinti interni, } \\
\text { secondo il } \\
\text { preventivo di } \\
\text { massima di fr. } 2200 \\
\text { e gli studi realizzati } \\
\text { dai pittori Emilio } \\
\text { Ferrazzini e Carlo } \\
\text { Cotti. } \\
\text { L'ultimo restauro è } \\
\text { stato eseguito nel } \\
1968 \text { dal pittore } \\
\text { Fabio Cavallini di } \\
\text { Campione d'Italia, } \\
\text { con un intervento } \\
\text { che interessò tutto } \\
\text { il complesso in stile } \\
\text { barocco autentico. } \\
\text { II lavoro fu eseguito } \\
\text { a secco, eliminando } \\
\text { i restauri } \\
\text { precedenti. }\end{array}$ & $\begin{array}{l}\text { I lavori furono } \\
\text { eseguiti a spese di } \\
\text { Don Giosuè Prada, } \\
\text { senza } \\
\text { partecipazione } \\
\text { finanziaria della } \\
\text { Confraternita. } \\
\text { Giuseppe Weith fu } \\
\text { incaricato di } \\
\text { dirigere e } \\
\text { sorvegliare assieme } \\
\text { a Emilio Ferrazzini i } \\
\text { lavori di restauro } \\
\text { pittorico. }\end{array}$ & $\begin{array}{l}\text { (Chiesa, 1946, p. 53) } \\
\text { (DPE, Cinquant'anni } \\
\text { di protezione dei } \\
\text { monumenti storici } \\
\text { artistici della } \\
\text { reppubblica e } \\
\text { cantone del Ticino, } \\
\text { 1959, p. 118) } \\
\text { (AUBCBZ s. 021, } \\
\text { Bellinzona). }\end{array}$ \\
\hline 1942 & $\begin{array}{l}\text { Melide } \\
\text { casa di } \\
\text { Domenico Fontana, } \\
\text { proprietà della S. A. } \\
\text { Immobiliare } \\
\text { Melide. }\end{array}$ & $\begin{array}{l}\text { Stemma di Papa } \\
\text { Sisto V. }\end{array}$ & $\begin{array}{l}\text { Consolidamento e } \\
\text { restauro. }\end{array}$ & & $\begin{array}{l}\text { (Chiesa, 1946, p. } \\
\text { 74). } \\
\text { (DPE, Cinquant'anni } \\
\text { di protezione dei } \\
\text { monumenti storici } \\
\text { artistici della } \\
\text { reppubblica e } \\
\text { cantone del Ticino, } \\
\text { 1959, p. 122). } \\
\text { (AUBCBZ, s.157. } \\
\text { Mendrisio). }\end{array}$ \\
\hline 1943 & $\begin{array}{l}\text { Negrentino, } \\
\text { Prugiasco, } \\
\text { chiesa di } \\
\text { S. Carlo. }\end{array}$ & $\begin{array}{l}\text { Affreschi parete di } \\
\text { ponente. Affreschi } \\
\text { XIII, XIV. } \\
\text { Seregnesi, da } \\
\text { Tradate. }\end{array}$ & $\begin{array}{l}\text { Restauro pittorico } \\
\text { consistente nella } \\
\text { ricerca e scoperta di } \\
\text { pitture sotto lo } \\
\text { strato di calce } \\
\text { (parete di ponente } \\
\text { e nella navata), } \\
\text { consolidamento, la } \\
\text { pulitura e la } \\
\text { reintegrazione } \\
\text { pittorica dell'intero } \\
\text { complesso } \\
\text { decorativo. } \\
\text { Il consolidamento }\end{array}$ & $\begin{array}{l}\text { Sorveglianza di } \\
\text { Emilio Ferrazzini in } \\
\text { qualità di membro } \\
\text { della Commissione } \\
\text { Cantonale dei } \\
\text { Monumenti Storici } \\
\text { in Ticino. } \\
\text { Per le operazioni di } \\
\text { consolidamento in } \\
\text { profondità } \\
\text { mediante iniezioni } \\
\text { intervenne il } \\
\text { muratore } \\
\text { "Antognoli" di }\end{array}$ & $\begin{array}{l}\text { (Chiesa, 1946, p. } \\
75 \text { ). } \\
\text { (DPE, Cinquant'anni } \\
\text { di protezione dei } \\
\text { monumenti storici } \\
\text { artistici della } \\
\text { reppubblica e } \\
\text { cantone del Ticino, } \\
1959, \text { p. 125). } \\
\text { (AUBCBZ, s. 204. } \\
\text { Prugiasco) } \\
\text { Popolo e libertà, } \\
\text { Bellinzona lunedì } 21 \\
\text { agosto } 1944 .\end{array}$ \\
\hline
\end{tabular}




\begin{tabular}{|c|c|c|c|c|c|}
\hline & & & $\begin{array}{l}\text { fu realizzato in } \\
\text { profondità } \\
\text { mediante il } \\
\text { caseinato di calcio, } \\
\text { e miscela di silicati. } \\
\text { I dipinti furono } \\
\text { consolidati } \\
\text { superficialmente } \\
\text { con caseina e } \\
\text { furono ravvivati } \\
\text { mediante la tecnica } \\
\text { dell'encausto. } \\
\text { I prodotti di } \\
\text { restauro: caseina, } \\
\text { silicati, caseato, } \\
\text { seringhe, ecc. } \\
\text { furono acquistati } \\
\text { dalla Farmacia } \\
\text { Federale di } \\
\text { Massagno. }\end{array}$ & Lottigna. & $\begin{array}{l}\text { "L'inaugurazione dei } \\
\text { restauri di } \\
\text { Negrentino" } \\
\text { Neu Zurcher } \\
\text { Nachrichten, } 22 \\
\text { settmbre 1944, } \\
\text { Christliche Kultur. } \\
\text { Negrentino. }\end{array}$ \\
\hline 1943 & $\begin{array}{l}\text { Bubikon, } \\
\text { Castello dei } \\
\text { Cavalieri di Malta. }\end{array}$ & Affreschi medievali. & $\begin{array}{l}\text { Restauro realizzato } \\
\text { in sostituzione di } \\
\text { Emilio Ferrazzini. }\end{array}$ & $\begin{array}{l}\text { Sorveglianza di } \\
\text { Henri-Paul } \\
\text { Boissonas, Zurigo. }\end{array}$ & $\begin{array}{l}\text { (Foletti, 2002, p. } \\
\text { 45). }\end{array}$ \\
\hline 1943 & $\begin{array}{l}\text { Mendrisio, chiesa di } \\
\text { S. Giovanni. }\end{array}$ & $\begin{array}{l}\text { Giovanni Battista } \\
\text { Bagutti, } \\
\text { Trasparenti. }\end{array}$ & $\begin{array}{l}\text { Consolidamento e } \\
\text { restauro. }\end{array}$ & $\begin{array}{l}\text { Lavoro eseguiti } \\
\text { assieme a Emilio } \\
\text { Ferrazzini. }\end{array}$ & $\begin{array}{l}\text { (Chiesa, 1946, p. } \\
78 \text { ). } \\
\text { (DPE, Cinquant'anni } \\
\text { di protezione dei } \\
\text { monumenti storici } \\
\text { artistici della } \\
\text { reppubblica e } \\
\text { cantone del Ticino, } \\
1959, \text { p. 122). } \\
\text { (AUBCBZ, s.157. } \\
\text { Mendrisio). }\end{array}$ \\
\hline 1943 & $\begin{array}{l}\text { S. Vigilio, } \\
\text { Rovio. }\end{array}$ & Affreschi. & $\begin{array}{l}\text { I lavori di restauro } \\
\text { interessarono il } \\
\text { consolidamento e la } \\
\text { reintegrazione del } \\
\text { fondo giallo nella } \\
\text { tazza dell'abside, il } \\
\text { consolidamento e la } \\
\text { leggera } \\
\text { ricostruzione dei } \\
\text { simboli degli } \\
\text { Evangelisti, il } \\
\text { consolidamento e la } \\
\text { reintegrazione a } \\
\text { basso tono delle } \\
\text { lacune della } \\
\text { Maestas Domini, } \\
\text { così come il }\end{array}$ & $\begin{array}{l}\text { Il restauro } \\
\text { architettonico e la } \\
\text { direzione dei lavori } \\
\text { pittorici fu } \\
\text { incaricata al } \\
\text { professor Taddeo } \\
\text { Carloni. }\end{array}$ & $\begin{array}{l}\text { (AUBCBZ, s. } 213 \\
\text { Rovio). }\end{array}$ \\
\hline
\end{tabular}




\author{
fissaggio \\ dell'intonaco \\ staccato e la \\ reintegrazione \\ cromatiche delle \\ due fascie \\ decorative nell'arco \\ trionfale. \\ Si realizzarono la \\ pulitura, il \\ consolidamento e la \\ reintegrazione a \\ basso tono degli \\ affreschi \\ raffiguranti la \\ Madonna e gli \\ Apostoli nella \\ navata, del S. Carlo, \\ della Madonna col \\ bambino e del $\mathrm{S}$. \\ Ambrogio nella \\ navata. \\ Le pareti della \\ navata furono \\ tinteggiate $\mathrm{e}$ \\ patinate in una \\ tonalità grigio \\ avorio.
}

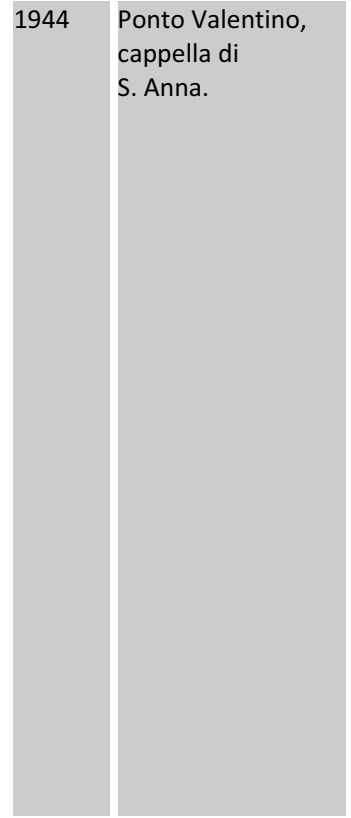

Antonio di Tradate, I lavori di restauro attribuiti a. sec. XV- eseguiti sugli XVI Affreschi.

affreschi, assai

danneggiati

dall'azione

dell'umidità

(sgretolamento

dell'intonaco,

formazione di buchi

e lacune di grande

dimensione),

interessò il

consolidamento in

profondità del

supporto murale

mediante

fluatazione

continuato da

operazioni di

impermeabilizzazio

ne con una miscela

di silicati.

Rinzafattura e

chiusura delle

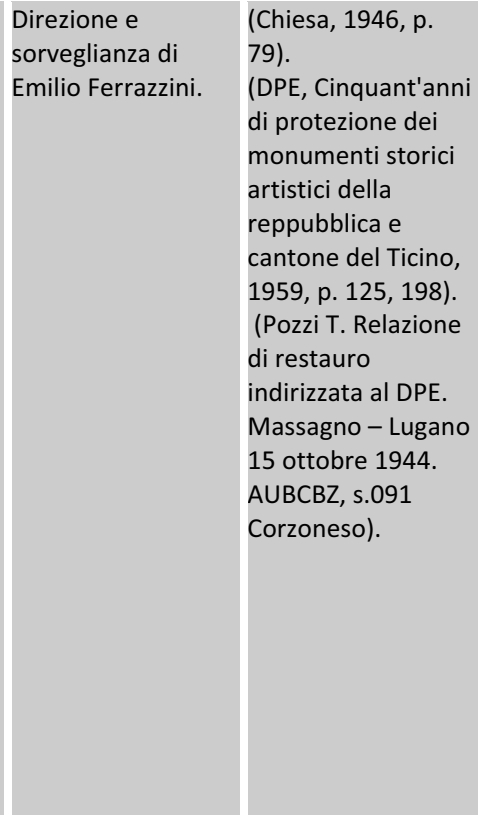




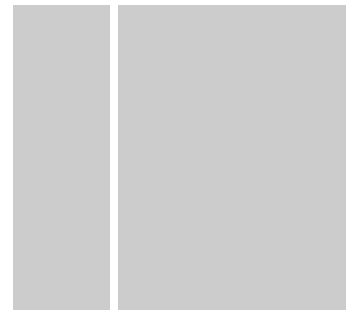

1944 1945

\section{Giornico, chiesa di} S. Nicolao.

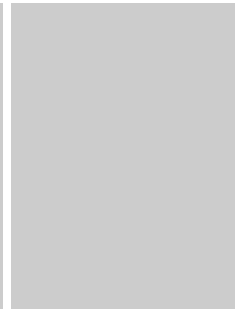

Affreschi del coro dell'abside e dell'altare della cripta.

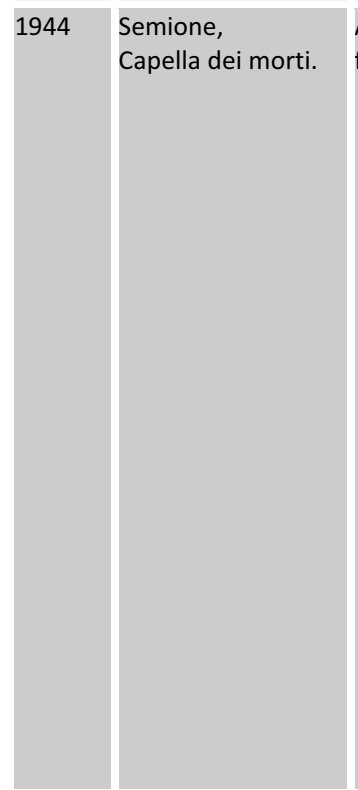

\section{Affreschi della} fronte (sec. XVIII). fessure eseguita dal

murature, ed

stuccatura dei buch

e lacune

dell'intonaco negli

affreschi realizzata

da Pozzi.

Reintegrazione pittorica.

Ricerche pittoriche, Sorveglianza dei pulitura del

sudiciume di

polvere e grasso

negli affreschi e

consolidamento

mediante la tecnica

rifiutata dai

commissari ed

lavori da Giuseppe

ispettori cantonali

così come dal

proprio collega

Bruno Abbiati che

propugnava un

consolidamento

mediante gomma

arabica.

I lavori di restauro
interessarono la
eliminazione dei
veli grigiastri
prodotti in
superficie a causa
delle emanazioni
salnitrose, così
come le larghe
macchie di nero
fumo e le scritte
vandaliche in
bianco, in
sanguigna ed a
carbone mediante
una prima pulitura
la quale fu seguita
dal consolidamento
delle parti
d'intonaco
sgretolato e dei
buchi con
fluatazione con la

I lavori di restauro

prodotti in

superficie a causa

delle emanazioni

vandaliche in

bianco, in

sanguigna ed a

carbone mediante

una prima pulitur

dal consolidamento

delle parti

fluatazione con la
Weith e

dall'Ispettore

Cantonale Piero

Bianconi.

Collaborò nel pittore Bruno 1959, p. 112)

(Chiesa, 1946, p.

68)F.

(DPE, Cinquant'anni

di protezione dei monumenti storici artistici della reppubblica e Abbiati.

(Foletti, 2002, p. 45)

(AUBCBZ s. 121,

Giornico).
(Chiesa, 1946, p. 79)

(DPE, Cinquant'anni di protezione dei monumenti storici artistici della reppubblica e cantone del Ticino, 1959$, p. 122,126$)$ (Bianconi Piero, Relazione sui restauri delle cappelle di San Dionigi a Malvaglia e dei Morti a Semione. Indirizzata alla CCM, Lugano. 20 agosto 1944. AUBCBZ, s. 149 Malvaglia). (Pozzi T. Relazione di restauro indirizzata al DPE. Massagno - Lugano 


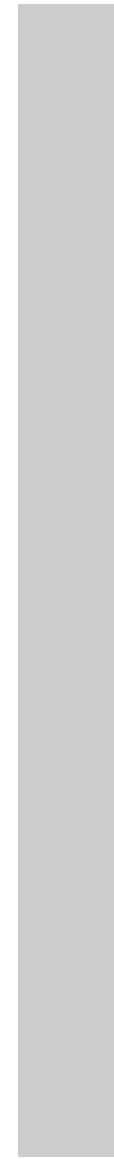

1944

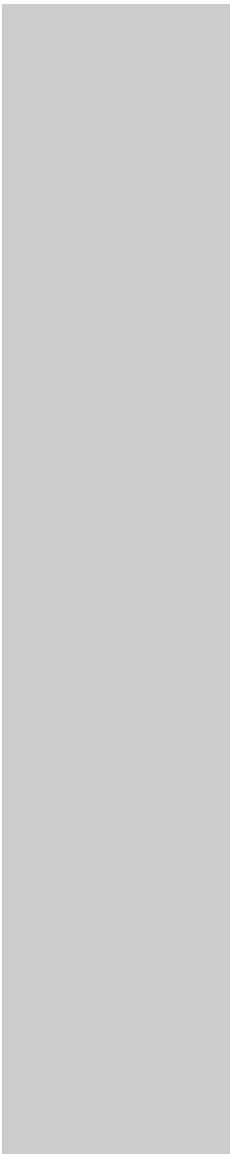

Malva di S. Dionigi. cura di non

sorpassare la

superficie pittorica.

I colori degli

affreschi furono

"ravvivati"

mediante la tecnica

dell'encausto, e si

reintegrarono a

basso tono le

lacune stuccate. Le

zone degli affreschi

dove il colore era

abraso o macchiato

furono riportate ad

una appropriata

omogeneità

mediante velature.

Sulla parete esterna

l'affresco

raffigurante la

Resurrezione fu

anche questo

pulito, consolidato,

stuccate le fessure

e ritoccate le lacune ricostruendosi $\mathrm{i}$

motivi

architettonici

mediante il

semplice accenno

delle partizioni

geometriche

scomparse.

Restauro degli

XVI sulle tre pareti e affreschi mediante

trigramma di Cristo la stuccatura

entro raggera sulla dell'intonaco con volta. caseinato di calcio, chiusura delle

fessure, pulitura

delle scritte fatte

sugli affreschi,

pulitura generale,

ripristino della

tonalità,

ricostruzione dei

motivi di

decorazione

architettonica e

reintegrazione

cromatica generale
15 ottobre 1944

AUBCBZ, s.091

Corzoneso).

(Chiesa, 1946, p. 80)

(DPE, Cinquant'anni di protezione dei monumenti storici artistici della reppubblica e cantone del Ticino, 1959, p. 122, 149)

(AUBCBZ, s.149.

Malvaglia)

(AUBCBZ, s.091

Corzoneso). 


\begin{tabular}{|c|c|c|c|c|}
\hline 1944 & $\begin{array}{l}\text { Gorduno, chiesa } \\
\text { parrocchiale. }\end{array}$ & $\begin{array}{l}\text { Affreschi medievali } \\
\text { nella parete interna } \\
\text { della facciata. } \\
\text { Attribuiti ad } \\
\text { Antonio da Tradate } \\
\text { ed ai Seregnesi. }\end{array}$ & $\begin{array}{l}\text { Pulitura dello strato } \\
\text { di calce soprastante } \\
\text { gli affreschi, } \\
\text { scoperte nella } \\
\text { primavera del } 1944 \\
\text { con motivo dei } \\
\text { lavori generali di } \\
\text { ripristino della } \\
\text { fabbrica. Essi erano } \\
\text { molto danneggiati a } \\
\text { causa delle } \\
\text { scialbature } \\
\text { applicate e dalla } \\
\text { costruzione di una } \\
\text { scala per cui si } \\
\text { saliva alla cantoria. } \\
\text { Gli affreschi furono } \\
\text { a continuazione } \\
\text { consolidati e } \\
\text { reintegrati. } \\
\text { (Nel } 1961 \text { con } \\
\text { motivo di nuovi } \\
\text { lavori di restauro } \\
\text { nella chiesa } \\
\text { vennero alla luce } \\
\text { degli affreschi } \\
\text { cinquecenteschi } \\
\text { raffiguranti sulla } \\
\text { facciata sud } \\
\text { dell'altare di St. } \\
\text { Antonio). }\end{array}$ & $\begin{array}{l}\text { (Chiesa, 1946, p. 80) } \\
\text { (AUBCBZ, s. } 118 \\
\text { Gorduno). }\end{array}$ \\
\hline 1944 & $\begin{array}{l}\text { Largario, Madonna } \\
\text { in Trono, } \\
\text { S. Rocco e } \\
\text { S. Sebastiano sec. } \\
\text { XVI. }\end{array}$ & Affresco. & $\begin{array}{l}\text { Consolidamento e } \\
\text { stuccature } \\
\text { dell'intonaco, } \\
\text { pulitura delle } \\
\text { abbondanti } \\
\text { macchie di calce e } \\
\text { terra sulla } \\
\text { superficie pittorica } \\
\text { e pulitura generale } \\
\text { eseguita } \\
\text { dall'applicazione } \\
\text { della cera per fare } \\
\text { "rinvenire" le } \\
\text { tonalità } \\
\text { dell'affresco. } \\
\text { Pulitura e } \\
\text { reintegrazione } \\
\text { pittorica della volta } \\
\text { e dello zoccolo. }\end{array}$ & $\begin{array}{l}\text { (Chiesa, 1946, p. 80) } \\
\text { (DPE, Cinquant'anni } \\
\text { di protezione dei } \\
\text { monumenti storici } \\
\text { artistici della } \\
\text { reppubblica e } \\
\text { cantone del Ticino, } \\
\text { 1959, p. 121) } \\
\text { (Pozzi T. Massagno } \\
\text { - Lugano } 15 \text { ottobre } \\
\text { 1944. Relazione di } \\
\text { restauro al DPE. } \\
\text { AUBCBZ, s.091 } \\
\text { Corzoneso) } \\
\text { (AUBCBZ, s.129 } \\
\text { Largario). }\end{array}$ \\
\hline
\end{tabular}




\begin{tabular}{|c|c|c|c|c|}
\hline 1944 & $\begin{array}{l}\text { Cademario, chiesa } \\
\text { di } \\
\text { S. Ambrogio. }\end{array}$ & $\begin{array}{l}\text { Affreschi della } \\
\text { facciata. }\end{array}$ & Consolidamento. & $\begin{array}{l}\text { (Chiesa, 1946, p. } \\
\text { 81). }\end{array}$ \\
\hline $\begin{array}{l}1944- \\
1946^{429}\end{array}$ & $\begin{array}{l}\text { Corzoneso, } \\
\text { Oratorio di } \\
\text { S. Remigio. }\end{array}$ & $\begin{array}{l}\text { Tarilli sec. XVI sec. } \\
\text { Affreschi nell'abside } \\
\text { maggiore, I'arco } \\
\text { trionfale e i pilastri } \\
\text { sottostanti. }\end{array}$ & $\begin{array}{l}\text { Strappo degli } \\
\text { affreschi dei Tarilli } \\
\text { (tre figure) per } \\
\text { scoprire affreschi } \\
\text { medievali. Strappo } \\
\text { in un pezzo unico } \\
\text { delle tre figure } \\
\text { d'Apostoli a destra } \\
\text { dell'abside: Filippo, } \\
\text { Taddeo e Giacomo } \\
\text { Maggiore; le due } \\
\text { prime a metà } \\
\text { figura, l'ultima } \\
\text { invece completa. } \\
\text { Strappo del S. } \\
\text { Remigio sul } \\
\text { piedritto sinistro } \\
\text { dell'arco trionfale. } \\
\text { Descialbo } \\
\text { del'intonaco per } \\
\text { scoprire pitture } \\
\text { anteriori. } \\
\text { Gli affreschi } \\
\text { strappati furono } \\
\text { riportati su fondo } \\
\text { rigido "prevatex" e } \\
\text { conservati nello } \\
\text { stesso oratorio. }\end{array}$ & $\begin{array}{l}\text { (Chiesa, 1946, p. 82) } \\
\text { (DPE, Cinquant'anni } \\
\text { di protezione dei } \\
\text { monumenti storici } \\
\text { artistici della } \\
\text { reppubblica e } \\
\text { cantone del Ticino, } \\
\text { 1959, p. 120) } \\
\text { (Ferrazzini E., } \\
\text { Relazione di } \\
\text { restauro del } 23 \\
\text { gennaio 1944 } \\
\text { indirizzata al DPE, } \\
\text { gennaio 1959. } \\
\text { AUBCBZ, s.091 } \\
\text { Corzoneso) } \\
\text { (Pozzi T., } \\
\text { Relazione di } \\
\text { restauro } \\
\text { indirizzata alla } \\
\text { CCMS. Massagno } \\
6 \text { agosto 1945. } \\
\text { AUBCBZ, s.091 } \\
\text { Corzoneso). }\end{array}$ \\
\hline 1944 & $\begin{array}{l}\text { Corzoneso, } \\
\text { oratorio di } \\
\text { S. Remigio. }\end{array}$ & $\begin{array}{l}\text { Affreschi sec. XIII } \\
\text { San Cristoforo e } \\
\text { una figura di } \\
\text { Vescovo (S. } \\
\text { Nicolao). }\end{array}$ & $\begin{array}{l}\text { Scoperta nella } \\
\text { parete meridionale } \\
\text { della navata } \\
\text { l'esistenza di } \\
\text { affreschi coperti } \\
\text { dall'intonaco, } \\
\text { pulitura e } \\
\text { consolidamento di } \\
\text { essi. } \\
\text { Malgrado lo stato di } \\
\text { conservazione } \\
\text { dell'arriccio recante } \\
\text { gli affreschi, molto }\end{array}$ & $\begin{array}{l}\text { (Chiesa, 1946, p. } \\
82 \text { ). } \\
\text { (DPE, Cinquant'anni } \\
\text { di protezione dei } \\
\text { monumenti storici } \\
\text { artistici della } \\
\text { reppubblica e } \\
\text { cantone del Ticino, } \\
\text { 1959, p. 120). } \\
\text { (Ferrazzini E., } \\
\text { Relazione di } \\
\text { restauro del } 23 \\
\text { gennaio } 1944\end{array}$ \\
\hline
\end{tabular}

\footnotetext{
429 DPE, Cinquant'anni di protezione dei monumenti storici artistici delle Repubblica e Cantone Ticino 1909-1959, Bellinzona, gennaio 1959, Edizione dello stato pp. 120091 se remite a una al 1946 como fecha de inicio de la intervención y el 1947 como fecha final, mientras que en F. CHIESA, Monumenti storici e artistici del cantone Ticino restaurati dal 1910 al 1945, Bellinzona 1946, p.82 se habla del 1944.
} 


\begin{tabular}{|c|c|c|c|c|}
\hline & & & $\begin{array}{l}\text { pulverulento, e la } \\
\text { proposta del Pozzi } \\
\text { di eseguire lo } \\
\text { strappo delle due } \\
\text { figure por riportarle } \\
\text { sullo stesso muro, } \\
\text { una volta rifatto } \\
\text { l'arriccio, le grande } \\
\text { spese economiche } \\
\text { che questo } \\
\text { comportava impedi } \\
\text { operare in questo } \\
\text { modo. II pittore Tita } \\
\text { Pozzi eseguì il } \\
\text { consolidamento } \\
\text { degli affreschi } \\
\text { mediante } \\
\text { numerose iniezioni } \\
\text { di caseinato di } \\
\text { calcio dal basso in } \\
\text { alto. } \\
\text { Posteriormente si } \\
\text { realizzò la pulitura } \\
\text { delle gocciolature, } \\
\text { di macchie e di } \\
\text { abrasioni, eseguita } \\
\text { da una pulitura } \\
\text { generale e } \\
\text { l'applicazione della } \\
\text { cera per "ravvivare i } \\
\text { colori dei dipinti. }\end{array}$ & $\begin{array}{l}\text { indirizzata al DPE, } \\
\text { gennaio } 1959 . \\
\text { AUBCBZ, s.091 } \\
\text { Corzoneso). } \\
\text { (Pozzi T., Relazione } \\
\text { di restauro } \\
\text { indirizzata alla } \\
\text { CCMS. Massagno } 6 \\
\text { agosto 1945. } \\
\text { AUBCBZ, s.091 } \\
\text { Corzoneso). }\end{array}$ \\
\hline 1944 & $\begin{array}{l}\text { Corzoneso, } \\
\text { oratorio di } \\
\text { S. Remigio. }\end{array}$ & $\begin{array}{l}\text { Affreschi sec. XVI } \\
\text { Crocifissione. }\end{array}$ & $\begin{array}{l}\text { Pulitura e } \\
\text { consolidamento. }\end{array}$ & $\begin{array}{l}\text { (Chiesa, 1946, p. } \\
82 \text { ). } \\
\text { (Ferrazzini E., } \\
\text { Relazione di } \\
\text { restauro del } 23 \\
\text { gennaio } 1944 \\
\text { indirizzata al DPE, } \\
\text { gennaio } 1959 . \\
\text { AUBCBZ, s.091 } \\
\text { Corzoneso). }\end{array}$ \\
\hline 1944 & $\begin{array}{l}\text { Corzoneso, } \\
\text { oratorio di } \\
\text { S. Remigio. }\end{array}$ & $\begin{array}{l}\text { Tarilli, } \\
\text { sec. XVI } \\
\text { Affresco di una } \\
\text { Madonna. }\end{array}$ & $\begin{array}{l}\text { Pulitura della } \\
\text { Madonna dei Tarilli } \\
\text { esistente nella } \\
\text { Cappella e } \\
\text { dell'affresco sulla } \\
\text { porta della } \\
\text { sacrestia. } \\
\text { Consolidamento } \\
\text { dell'affresco sulla } \\
\text { parte esterna }\end{array}$ & $\begin{array}{l}\text { (Chiesa, 1946, p. } \\
82 \text { ). } \\
\text { (Ferrazzini E., } \\
\text { Relazione di } \\
\text { restauro del } 23 \\
\text { gennaio } 1944 \\
\text { indirizzata al DPE, } \\
\text { gennaio 1959. } \\
\text { AUBCBZ, s.091 } \\
\text { Corzoneso). }\end{array}$ \\
\hline
\end{tabular}




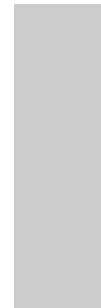

1944

\section{Corzoneso,} oratorio di S. Remigio.

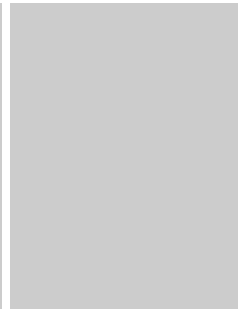

Affresco parte esterne affreschi del abside minore. settentrionale.

\section{Pulitura,}

consolidamento e restauro.

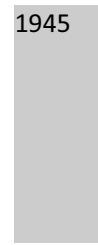

1946

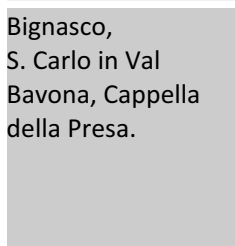

Lottigna, vecchio Pretorio già casa dei facciata. Landfogti.

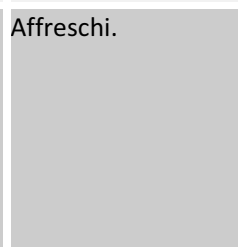

Affreschi sulla

\section{Scoprimento totale} e restauro degli affreschi sulla facciata del Pretorio, consolidamento dell'intonaco mediante fluatazioni e debite stuccature, pulimento dei dipinti, consolidamento dei freschi con idonee soluzioni caseose e miscele di silicati e qualche velatura opportuna e ripresa leggera in grigio dove sarà consentito. Le operazioni si stessero anche ai dipinti sotto il porticato di accesso per un totale cioè di
(Bianconi Piero, Invito a S. Remigio di Corzoneso.Corriere del Ticino Lugano 21 maggio 1948. Anno LVIII n. 115. AUBCBZ, s.091 Corzoneso).

(Chiesa, 1946, p. 82) (Ferrazzini E., Relazione di restauro del 23 gennaio 1944 indirizzata al DPE, gennaio 1959.

AUBCBZ, s.091

Corzoneso).

(DPE, Cinquant'anni di protezione dei monumenti storici artistici della reppubblica e cantone del Ticino, 1959, p. 119).

(DPE, Cinquant'anni di protezione dei monumenti storici artistici della reppubblica e cantone del Ticino, 1959, p. 122,140 ). (Pozzi T., Preventivo di restauro indirizzato al DPE. 12 novembre 1946. AUBCBZ, s.140. Lottigna). (Pozzi T., Preventivo di restauro indirizzato al DPE. 25 maggio 1948. AUBCBZ, s.140. Lottigna). 


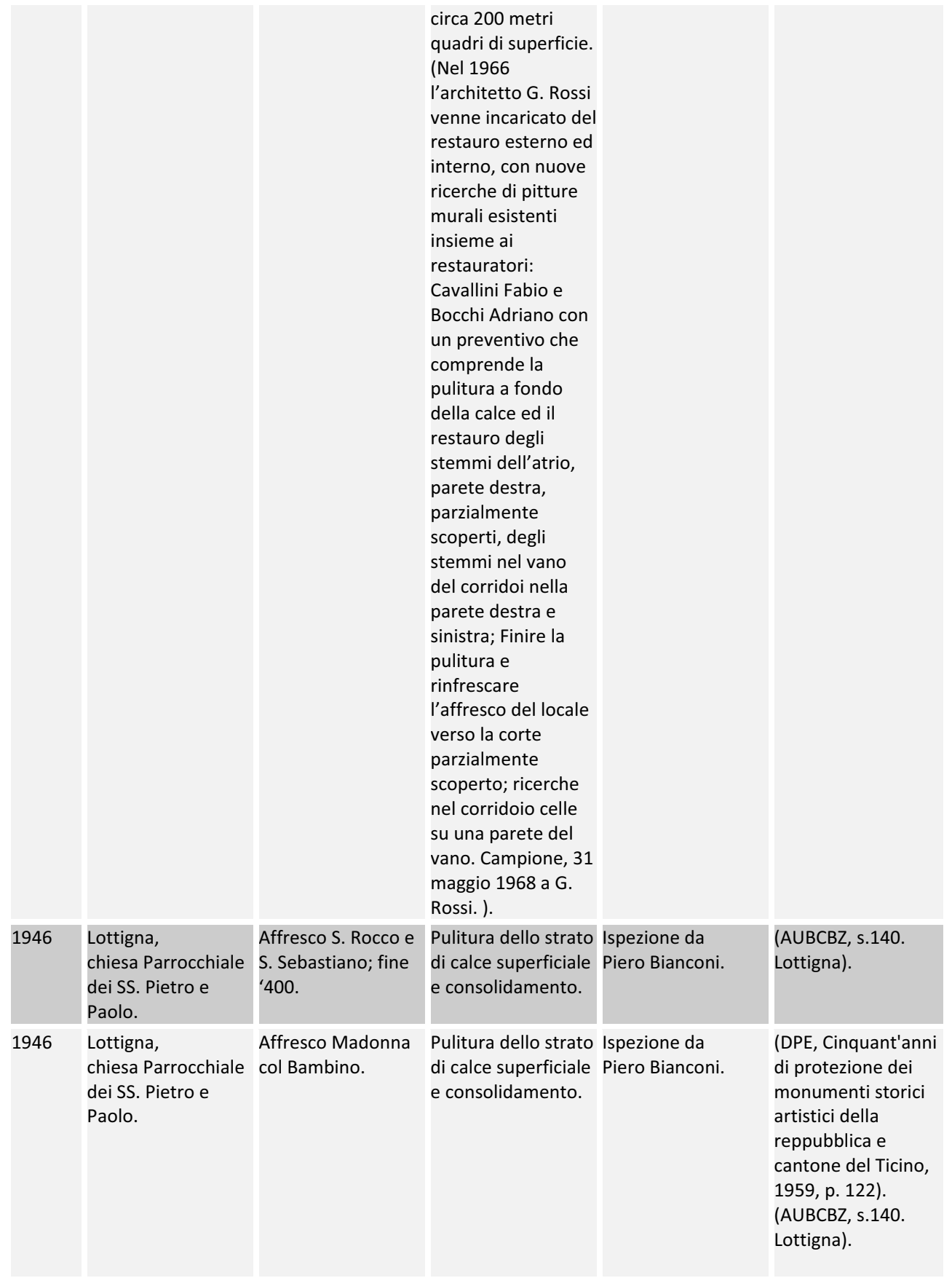




\begin{tabular}{|c|c|c|c|c|c|}
\hline 1946 & $\begin{array}{l}\text { Lottigna, } \\
\text { chiesa Parrocchiale } \\
\text { dei SS. Pietro e } \\
\text { Paolo. }\end{array}$ & $\begin{array}{l}\text { Affresco S. } \\
\text { Cristoforo. }\end{array}$ & $\begin{array}{l}\text { Pulitura dello strato } \\
\text { di calce superficiale } \\
\text { e consolidamento } \\
\text { dell'affresco molto } \\
\text { sbiadito e } \\
\text { frammentario. }\end{array}$ & $\begin{array}{l}\text { Ispezione da } \\
\text { Piero Bianconi }\end{array}$ & $\begin{array}{l}\text { (DPE, Cinquant'anni } \\
\text { di protezione dei } \\
\text { monumenti storici } \\
\text { artistici della } \\
\text { reppubblica e } \\
\text { cantone del Ticino, } \\
1959, \text { p. } 122 \text { ). }\end{array}$ \\
\hline $\begin{array}{l}1947- \\
1950\end{array}$ & $\begin{array}{l}\text { Lottigna, } \\
\text { Capella in Piano (in } \\
\text { campagna). }\end{array}$ & Affreschi 1455. & $\begin{array}{l}\text { Proposta di } \\
\text { realizzazione d'un } \\
\text { intercapedine per } \\
\text { allontanare } \\
\text { l'umidità. Restauro } \\
\text { e consolidamento } \\
\text { delle pitture } \\
\text { eseguite mediante } \\
\text { l'iniezione di } \\
\text { cemento in } 3 \text { grandi } \\
\text { fenditure nel muro } \\
\text { di prospetto, due } \\
\text { delle quali } \\
\text { attraversavano il } \\
\text { muro, così come in } \\
\text { diverse fessure e } \\
\text { nella fenditura } \\
\text { apparsa nel mezzo } \\
\text { della volta, facendo } \\
\text { calare il cemento } \\
\text { dalla parte } \\
\text { superiore della } \\
\text { volta. Rinzaffati con } \\
\text { cemento "Sika" i } \\
\text { muri privi } \\
\text { d'intonaco. } \\
\text { Consolidamento } \\
\text { dell'intonaco } \\
\text { recante i dipinti } \\
\text { mediante caseinato } \\
\text { di calcio } \\
\text { Gli affreschi esterni } \\
\text { frammentari } \\
\text { raffiguranti il Volto } \\
\text { del Cristo, aureola, } \\
\text { volto della figura in } \\
\text { piedi a sinistra e } \\
\text { San Sebastiano, } \\
\text { usati in passato } \\
\text { come materiale di } \\
\text { ripieno, furono } \\
\text { opportlocati dopo le } \\
\text { nel posto originario. } \\
\text { Lisciatura delle }\end{array}$ & $\begin{array}{l}\text { Ispezione da } \\
\text { Piero Bianconi, } \\
\text { Prof. Tarabori e } \\
\text { Pietro Salati. } \\
\text { Le operazioni di } \\
\text { muratore (fornitura } \\
\text { e trasporto } \\
\text { materiale, per } \\
\text { impianto punteggi, } \\
\text { opero murarie e } \\
\text { aiuti ) furono } \\
\text { realizzate dal } \\
\text { signore Antognoli. } \\
\text { Le fotografie furono } \\
\text { realizzate dalla casa } \\
\text { Von Moos. }\end{array}$ & $\begin{array}{l}\text { (Pozzi T., Fattura dei } \\
\text { lavori realizzati } \\
\text { entro i mesi di luglio } \\
\text { e agosto, controllati } \\
\text { e collaudati dalla } \\
\text { CCMS con } \\
\text { sopraluogo del } 31 \\
\text { agosto } 1950 . \\
\text { Indirizzata al DPE. } \\
\text { Massagno - Lugano } \\
10 \text { novembre } 1950 . \\
\text { AUBCBZ n.140 } \\
\text { Lottigna) } \\
\text { (Pozzi T., Relazione } \\
\text { tecnica } \\
\text { consolidamento e } \\
\text { restauro pittorico. } \\
10 \text { novembre } 1950 \text {, } \\
\text { Lugano -Massagno } \\
\text { al DPE. AUBCBZ } \\
\text { n.140 Lottigna) }\end{array}$ \\
\hline
\end{tabular}




\begin{tabular}{|c|c|c|c|c|c|}
\hline & & & $\begin{array}{l}\text { stuccature fino a } \\
\text { raggiungere il livello } \\
\text { dell'intonaco } \\
\text { originale. } \\
\text { Pulitura delle } \\
\text { macchie e segni } \\
\text { vandalici con matite } \\
\text { colorate, carbone, } \\
\text { sanguigna, sgraffi, } \\
\text { ecc., e delle muffe e } \\
\text { veli di salnitro } \\
\text { superficiali, } \\
\text { eseguito da una } \\
\text { pulitura generale e } \\
\text { disinfestazione. } \\
\text { Consolidamento dei } \\
\text { dipinti con silicati e } \\
\text { ritocco pittorico } \\
\text { con tonalità grigia. } \\
\text { Tinteggiatura dei } \\
\text { nuovi rinzaffi. }\end{array}$ & & \\
\hline 1947 & $\begin{array}{l}\text { Locarno, Orselina } \\
\text { Madonna del Sasso. }\end{array}$ & $\begin{array}{l}\text { Ciseri, } \\
\text { Trasporto di Cristo. } \\
\text { Olio su tela. }\end{array}$ & $\begin{array}{l}\text { Restauro realizzato } \\
\text { a spese del } \\
\text { Muncipio di } \\
\text { Locarno consistente } \\
\text { nella pulitura, } \\
\text { ammorbidimento } \\
\text { della tela secca la } \\
\text { cui ritrazione } \\
\text { causava numerose } \\
\text { lacune sulla } \\
\text { pellicola pittorica, } \\
\text { sverniciatura e } \\
\text { applicazione di } \\
\text { nuova vernice. }\end{array}$ & $\begin{array}{l}\text { Ispezione da } \\
\text { Piero Bianconi. }\end{array}$ & $\begin{array}{l}\text { (Pozzi T., Preventivo } \\
\text { per i lavori incaricati } \\
\text { dalla CCMS. } \\
\text { Massagno, } 8 \text { maggio } \\
1947 . \\
\text { AUBCBZ, s.183 } \\
\text { Orselina). }\end{array}$ \\
\hline 1947 & $\begin{array}{l}\text { Locarno, Orselina } \\
\text { Madonna del Sasso. }\end{array}$ & $\begin{array}{l}\text { Gruppo scultoreo } \\
\text { Cappella della } \\
\text { Pietà. }\end{array}$ & $\begin{array}{l}\text { Restauro realizzato } \\
\text { a spese dello Stato } \\
\text { del Canton Ticino. } \\
\text { Operazioni } \\
\text { consistenti nel } \\
\text { restauro delle } \\
\text { sculture e del } \\
\text { paliotto in scagliola } \\
\text { dell'altare. }\end{array}$ & $\begin{array}{l}\text { Ispezione da } \\
\text { Piero Bianconi. }\end{array}$ & $\begin{array}{l}\text { (Pozzi T., Preventivo } \\
\text { per i lavori incaricati } \\
\text { dalla CCMS. } \\
\text { Massagno, } 8 \text { maggio } \\
1947 . \\
\text { AUBCBZ, s.183 } \\
\text { Orselina). }\end{array}$ \\
\hline 1947 & $\begin{array}{l}\text { Locarno, Orselina } \\
\text { Madonna del Sasso. }\end{array}$ & $\begin{array}{l}\text { Tavola laterale, } \\
\text { Cappella della } \\
\text { Pietà. }\end{array}$ & $\begin{array}{l}\text { Proposta di } \\
\text { consolidamento e } \\
\text { verniciatura con } \\
\text { vernice "matt"). }\end{array}$ & $\begin{array}{l}\text { Ispezione da } \\
\text { Piero Bianconi. }\end{array}$ & \\
\hline 1947 & $\begin{array}{l}\text { Locarno, Orselina } \\
\text { Madonna del Sasso. }\end{array}$ & Tela di S. Francesco. & $\begin{array}{l}\text { Proposta di } \\
\text { restauro del dipinto } \\
\text { mediante la }\end{array}$ & $\begin{array}{l}\text { Ispezione da } \\
\text { Piero Bianconi. }\end{array}$ & \\
\hline
\end{tabular}




\begin{tabular}{|c|c|c|c|c|c|}
\hline & & & $\begin{array}{l}\text { riapplicazione dei } \\
\text { cinque pezzi } \\
\text { staccati che } \\
\text { compongono la tela } \\
\text { su fondo rigido o } \\
\text { tavola di } \\
\text { compensato. }\end{array}$ & & \\
\hline 1948 & $\begin{array}{l}\text { Lugano, } \\
\text { casa già Ferrazzini. }\end{array}$ & Affreschi & $\begin{array}{l}\text { Strappi di affreschi } \\
\text { a causa della } \\
\text { demolizione } \\
\text { dell'edificio. }\end{array}$ & & $\begin{array}{l}\text { (DPE, Cinquant'anni } \\
\text { di protezione dei } \\
\text { monumenti storici } \\
\text { artistici della } \\
\text { reppubblica e } \\
\text { cantone del Ticino, } \\
1959, \text { p. } 122,143 \text { ). }\end{array}$ \\
\hline 1948 & $\begin{array}{l}\text { Rovio, } \\
\text { oratorio di } \\
\text { S. Vigilio. }\end{array}$ & $\begin{array}{l}\text { Affreschi bizantino } \\
\text { romanico } \\
\text { XIII sec. }\end{array}$ & $\begin{array}{l}\text { Restauro e } \\
\text { consolidamento. }\end{array}$ & $\begin{array}{l}\text { Lavori diretti dal } \\
\text { professore Taddeo } \\
\text { Carloni e sorvegliati } \\
\text { dall'architetto } \\
\text { Giovannini. }\end{array}$ & $\begin{array}{l}\text { (DPE, Cinquant'anni } \\
\text { di protezione dei } \\
\text { monumenti storici } \\
\text { artistici della } \\
\text { reppubblica e } \\
\text { cantone del Ticino, } \\
1959, \text { p. } 125,215 \text { ). } \\
\text { (AN Bern n.80 KT M- } \\
\text { P). } \\
\text { Articolo, Echo del } \\
\text { Gottardo Locarno } 7 \\
\text { dicembre } 1911 \text {. Sui } \\
\text { rilievi dell'ing. } \\
\text { Emilio Gusalli peri I } \\
\text { restauro. }\end{array}$ \\
\hline $\begin{array}{l}1949- \\
1950\end{array}$ & $\begin{array}{l}\text { Lottigna, } \\
\text { cappella sotto il } \\
\text { villaggio. }\end{array}$ & & Riattazione. & & $\begin{array}{l}\text { (DPE, Cinquant'anni } \\
\text { di protezione dei } \\
\text { monumenti storici } \\
\text { artistici della } \\
\text { reppubblica e } \\
\text { cantone del Ticino, } \\
1959, \text { p. } 122,140 \text { ) }\end{array}$ \\
\hline 1950 & $\begin{array}{l}\text { Fribourg, } \\
\text { chiesa di Cordelier. }\end{array}$ & & Restauro. & & (Foletti, 2002, p. 45) \\
\hline 1950 & $\begin{array}{l}\text { Saint Aubin, } \\
\text { Chiesa parrocchiale. }\end{array}$ & Affreschi. & Restauro. & & (Foletti, 2002, p. 45) \\
\hline $\begin{array}{l}1950 \text { - } \\
1951\end{array}$ & $\begin{array}{l}\text { Negrentino, } \\
\text { Prugiasco, } \\
\text { chiesa di } \\
\text { S. Carlo. }\end{array}$ & Affreschi XII. & $\begin{array}{l}\text { Riconsolidamento } \\
\text { con caseinato di } \\
\text { calcio della parte } \\
\text { inferiore de la } \\
\text { parete. }\end{array}$ & & $\begin{array}{l}\text { (DPE, Cinquant'anni } \\
\text { di protezione dei } \\
\text { monumenti storici } \\
\text { artistici della } \\
\text { reppubblica e } \\
\text { cantone del Ticino, } \\
1959, \text { p. 125). } \\
\text { (AUBCBZ, s. 204, } \\
\text { Prugiasco) }\end{array}$ \\
\hline
\end{tabular}




\begin{tabular}{|c|c|c|c|c|c|}
\hline $\begin{array}{l}18 \text { dic. } \\
1950\end{array}$ & $\begin{array}{l}\text { Ponte Capriasca, } \\
\text { chiesa Parrocchiale } \\
\text { di S. Ambrogio. }\end{array}$ & Affresco Cenacolo . & Preventivo. & $\begin{array}{l}\text { Arch. Avv. } \\
\text { Camenzind. }\end{array}$ & $\begin{array}{l}\text { (Pozzi T., Lettera } \\
\text { indirizzata a } \\
\text { Francesco Chiesa, } \\
\text { Massagno-Lugano, } 1 \\
\text { novembre } 1951 . \\
\text { AUBCBZ, s.196 } \\
\text { Ponte Capriasca). }\end{array}$ \\
\hline $\begin{array}{l}18 \text { dic. } \\
1950\end{array}$ & $\begin{array}{l}\text { Ponte Capriasca, } \\
\text { chiesa Parrocchiale } \\
\text { di S. Ambrogio. }\end{array}$ & Affreschi & $\begin{array}{l}\text { Pulitura dello strato } \\
\text { di calce superficiale } \\
\text { sulla parte in basso } \\
\text { e strappo. } \\
\text { [...]Superficie totale } \\
\text { degli affreschi da } \\
\text { strappare e di } 11.50 \\
\text { m2 } 250 \mathrm{fr} / \mathrm{m} 2 \\
=3050 \text { totale[...]. }\end{array}$ & & $\begin{array}{l}\text { (Pozzi T., Lettera } \\
\text { indirizzata a } \\
\text { Francesco Chiesa, } \\
\text { Massagno-Lugano, } 1 \\
\text { novembre } 1951 . \\
\text { AUBCBZ, s.196 } \\
\text { Ponte Capriasca). }\end{array}$ \\
\hline $\begin{array}{l}18 \text { dic. } \\
1950\end{array}$ & $\begin{array}{l}\text { Ponte Capriasca, } \\
\text { chiesa Parrocchiale } \\
\text { di S. Ambrogio. }\end{array}$ & $\begin{array}{l}\text { Frammenti di } \\
\text { affreschi } \\
\text { raffiguranti angeli } \\
(80 \times 1000 \mathrm{~cm}) \text { e } 75 \\
\text { x } 70 \mathrm{~cm}) .\end{array}$ & Strappo. & & $\begin{array}{l}\text { (Pozzi T., Lettera } \\
\text { indirizzata a } \\
\text { Francesco Chiesa, } \\
\text { Massagno-Lugano, } 1 \\
\text { novembre } 1951 . \\
\text { AUBCBZ, s.196 } \\
\text { Ponte Capriasca). }\end{array}$ \\
\hline $\begin{array}{l}1951- \\
8 \text { ago. } \\
1951\end{array}$ & $\begin{array}{l}\text { Ponte Capriasca, } \\
\text { chiesa Parrocchiale } \\
\text { di S. Ambrogio. }\end{array}$ & $\begin{array}{l}\text { Affresco Cenacolo } \\
\text { leonardesco. }\end{array}$ & $\begin{array}{l}\text { Restauro. } \\
\text { Pulitura della polve- } \\
\text { re superficiale, con- } \\
\text { solidamento dei } \\
\text { bordi dell'affresco } \\
\text { così come delle } \\
\text { zone degli sfondi } \\
\text { delle teste, sul } \\
\text { soffito, e dell'an- } \\
\text { golo a sinistra oltre } \\
\text { il ritocco pittorico } \\
\text { dei graffi dovuti ad } \\
\text { atti vandalici e nelle } \\
\text { sgretolature. }\end{array}$ & & $\begin{array}{l}\text { (DPE, Cinquant'anni } \\
\text { di protezione dei } \\
\text { monumenti storici } \\
\text { artistici della } \\
\text { reppubblica e } \\
\text { cantone del Ticino, } \\
1959, \text { p. } 125 \text { ). } \\
\text { (AUBCBZ, s.196 } \\
\text { Ponte Capriasca). }\end{array}$ \\
\hline $\begin{array}{l}3 \text { set. } \\
1951\end{array}$ & $\begin{array}{l}\text { Ponte Capriasca, } \\
\text { chiesa Parrocchiale } \\
\text { di S. Ambrogio. }\end{array}$ & $\begin{array}{l}\text { Affresco XV sec. } \\
\text { venuto in luce ( } 150 \\
\text { x } 80 \mathrm{~cm})\end{array}$ & $\begin{array}{l}\text { Strappo } \\
\text { dell'affresco } \\
\text { scoperto nell'andito } \\
\text { a fianco } \\
\text { dell'affresco } \\
\text { raffigurante il } \\
\text { Cenacolo. }\end{array}$ & & $\begin{array}{l}\text { (Pozzi T., Preventivo } \\
\text { del } 9 \text { settembre } \\
\text { 1951. Indirizzato a } \\
\text { Francesco Chiesa. } \\
\text { Massagno-Lugano. } \\
16 \text { gennaio } 1952 . \\
\text { AUBCBZ } 196 \text { Ponte } \\
\text { Capriasca). }\end{array}$ \\
\hline 1951 & $\begin{array}{l}\text { Ponte Capriasca, } \\
\text { chiesa Parrocchiale } \\
\text { di S. Ambrogio. }\end{array}$ & $\begin{array}{l}\text { Madonna della } \\
\text { Misericordia } \\
(150 \times 186) \text {, Affresco } \\
\text { XVI sec. }\end{array}$ & Strappo. & & $\begin{array}{l}\text { (DPE, Cinquant'anni } \\
\text { di protezione dei } \\
\text { monumenti storici } \\
\text { artistici della } \\
\text { reppubblica e } \\
\text { cantone del Ticino, }\end{array}$ \\
\hline
\end{tabular}


1959, p. 108, 109).

\begin{tabular}{|c|c|c|c|c|c|}
\hline 1951 & $\begin{array}{l}\text { Ponte Capriasca, } \\
\text { chiesa Parrocchiale } \\
\text { di S. Ambrogio. }\end{array}$ & $\begin{array}{l}\text { S. Domenico } \\
\text { Martire. Affresco } \\
\text { XVI sec. }\end{array}$ & Strappo. & & $\begin{array}{l}\text { (DPE, Cinquant'anni } \\
\text { di protezione dei } \\
\text { monumenti storici } \\
\text { artistici della } \\
\text { reppubblica e } \\
\text { cantone del Ticino, } \\
1959, \text { p. } 108,109 \text { ). }\end{array}$ \\
\hline 1951 & $\begin{array}{l}\text { Ponte Capriasca, } \\
\text { chiesa Parrocchiale } \\
\text { di S. Ambrogio. }\end{array}$ & $\begin{array}{l}\text { Madonna in trono } \\
\text { col Bambino } \\
(146 \times 80) \text {. Affresco } \\
\text { XVI sec. }\end{array}$ & Strappo. & & $\begin{array}{l}\text { (DPE, Cinquant'anni } \\
\text { di protezione dei } \\
\text { monumenti storici } \\
\text { artistici della } \\
\text { reppubblica e } \\
\text { cantone del Ticino, } \\
\text { 1959, p. 108, 109). }\end{array}$ \\
\hline 1951 & $\begin{array}{l}\text { Ponte Capriasca, } \\
\text { chiesa Parrocchiale } \\
\text { di S. Ambrogio. }\end{array}$ & $\begin{array}{l}\text { Madonna del Latte } \\
\text { (78x161). Affresco } \\
\text { XVI sec. }\end{array}$ & Strappo. & & $\begin{array}{l}\text { (DPE, Cinquant'anni } \\
\text { di protezione dei } \\
\text { monumenti storici } \\
\text { artistici della } \\
\text { reppubblica e } \\
\text { cantone del Ticino, } \\
1959, \text { p. } 108,109 \text { ). }\end{array}$ \\
\hline 1951 & $\begin{array}{l}\text { Lugano, } \\
\text { Banca della Svizzera } \\
\text { Italiana. }\end{array}$ & Tarilli, affreschi. & Restauro. & & $\begin{array}{l}\text { (Foletti Giulio, } \\
\text { Novembre 2002). } \\
\text { p.45. }\end{array}$ \\
\hline $\begin{array}{l}1952- \\
1953\end{array}$ & $\begin{array}{l}\text { Ponte Capriasca, } \\
\text { chiesa } \\
\text { Parrocchiale.. }\end{array}$ & $\begin{array}{l}\text { Affreschi strappati } \\
\text { in } 1951 .\end{array}$ & Ricollocamento. & & $\begin{array}{l}\text { (DPE, Cinquant'anni } \\
\text { di protezione dei } \\
\text { monumenti storici } \\
\text { artistici della } \\
\text { reppubblica e } \\
\text { cantone del Ticino, } \\
1959, \text { p. } 125 \text { ). } \\
\text { (AUBCBZ 196 Ponte } \\
\text { Capriasca). }\end{array}$ \\
\hline 1952 & $\begin{array}{l}\text { Tesserete, } \\
\text { Chiesa di S. Stefano. }\end{array}$ & Affreschi. & Restauro. & & $\begin{array}{l}\text { (Foletti Giulio, } \\
\text { Novembre 2002, } \\
\text { p.45). }\end{array}$ \\
\hline $\begin{array}{l}1953- \\
1955\end{array}$ & $\begin{array}{l}\text { Müstair, } \\
\text { Niklauskapelle di } \\
\text { Regensdorf }\end{array}$ & Affreschi carolingi & Restauro & $\begin{array}{l}\text { Sorveglianza ai } \\
\text { lavori da Henri- } \\
\text { Paul Boissonas }\end{array}$ & $\begin{array}{l}\text { (Foletti Giulio, } \\
\text { Novembre 2002, } \\
\text { p.45) }\end{array}$ \\
\hline 1958 & $\begin{array}{l}\text { Ascona, } \\
\text { casa Abbondio. }\end{array}$ & Affresco. & $\begin{array}{l}\text { Stacco e } \\
\text { ricollocamento } \\
\text { nella chiesa } \\
\text { parrocchiale. }\end{array}$ & $\begin{array}{l}\text { Milani } \\
\text { Prof. Bianconi e } \\
\text { Salati. }\end{array}$ & $\begin{array}{l}\text { (DPE, Cinquant'anni } \\
\text { di protezione dei } \\
\text { monumenti storici } \\
\text { artistici della } \\
\text { reppubblica e } \\
\text { cantone del Ticino, } \\
1959, \text { p. 118) } \\
\text { (AUBCBZ, s.011, } \\
\text { Ascona). }\end{array}$ \\
\hline
\end{tabular}




\section{Preventivi di restauro}

\begin{tabular}{|c|c|c|c|c|c|}
\hline $\begin{array}{l}20 \text { lug. } \\
1943\end{array}$ & $\begin{array}{l}\text { Negrentino, } \\
\text { Prugiasco, } \\
\text { chiesa di } \\
\text { S. Carlo. }\end{array}$ & $\begin{array}{l}\text { Affreschi parete di } \\
\text { ponente. Affreschi } \\
\text { XIII, XIV. } \\
\text { Seregnesi, da } \\
\text { Tradate. }\end{array}$ & $\begin{array}{l}\text { Preventivo di } \\
\text { restauro pittorico. } \\
\text { Fr. } 3200 \text {. }\end{array}$ & $\begin{array}{l}\text { Elaborazione del } \\
\text { preventivo e delle } \\
\text { perizie assieme al } \\
\text { collega e } \\
\text { commissario } \\
\text { cantonale Emilio } \\
\text { Ferrazzini. }\end{array}$ & $\begin{array}{l}\text { (AUBCBZ, s. } 204 \\
\text { Prugiasco). }\end{array}$ \\
\hline $\begin{array}{l}3 \text { feb. } \\
1943\end{array}$ & $\begin{array}{l}\text { S. Vigilio, } \\
\text { Rovio. }\end{array}$ & Affreschi & $\begin{array}{l}\text { Preventivo } \\
\text { complessivo di } \\
\text { restauro pittorico. } \\
\text { Fr. } 3250\end{array}$ & $\begin{array}{l}\text { Presentato al } \\
\text { Professor Taddeo } \\
\text { Carloni. }\end{array}$ & $\begin{array}{l}\text { (AUBCBZ, s. } 213 \\
\text { Rovio) }\end{array}$ \\
\hline $\begin{array}{l}29 \text { mar. } \\
1945\end{array}$ & $\begin{array}{l}\text { Chiesa di Santa } \\
\text { Maria in Selva }\end{array}$ & Affreschi della volta & $\begin{array}{l}\text { Preventivo restauro } \\
\text { pittorico } \\
\text { consistente nel } \\
\text { consolidamento } \\
\text { della parte } \\
\text { sgretolata e friabile } \\
\text { dell'intonaco a } \\
\text { mezzo di fluati, } \\
\text { consolidamento } \\
\text { della superficie } \\
\text { pittorica dove } \\
\text { necessario, } \\
\text { abbassamento con } \\
\text { toni grigli delle } \\
\text { macchie bianche } \\
\text { dell'intonaco per } \\
\text { omogeneizzare il } \\
\text { dipinto. }\end{array}$ & $\begin{array}{l}\text { Emilio Ferrazzini } \\
\end{array}$ & $\begin{array}{l}\text { (DPE, Cinquant'anni } \\
\text { di protezione dei } \\
\text { monumenti storici } \\
\text { artistici della } \\
\text { repubblica e } \\
\text { cantone Ticino } \\
\text { 1909-1959, 1959, p. } \\
122 \text { ) } \\
\text { (AUBCBZ, 134, } \\
\text { Maria in Selva) } \\
\text { (Ferrazzini Emilio, } \\
\text { Pozzi Tita. } \\
\text { Preventivo. Lugano } \\
29 \text { marzo 1945. } \\
\text { AUBCBZ, s. 134, } \\
\text { Locarno. S. Maria in } \\
\text { Selva) }\end{array}$ \\
\hline $\begin{array}{l}29 \text { mar. } \\
1945\end{array}$ & $\begin{array}{l}\text { Chiesa di Santa } \\
\text { Maria in Selva. }\end{array}$ & $\begin{array}{l}\text { Affreschi della } \\
\text { navata. }\end{array}$ & $\begin{array}{l}\text { Preventivo restauro } \\
\text { pittorico } \\
\text { consistente nella } \\
\text { pulitura generale } \\
\text { dell'affresco della } \\
\text { polvere e del } \\
\text { sudiciume, } \\
\text { esportazione delle } \\
\text { stuccature e dei } \\
\text { rifacimenti pittorici } \\
\text { eseguiti in restauri } \\
\text { precedenti. } \\
\text { Consolidamento } \\
\text { delle parti friabili } \\
\text { (fluati), } \\
\text { encausticature di } \\
\text { alcune parti degli } \\
\text { affreschi che sono } \\
\text { opache e velate } \\
\text { anche dopo la }\end{array}$ & Emilio Ferrazzini. & $\begin{array}{l}\text { (DPE, Cinquant'anni } \\
\text { di protezione dei } \\
\text { monumenti storici } \\
\text { artistici della } \\
\text { repubblica e } \\
\text { cantone Ticino } \\
\text { 1909-1959, 1959, p. } \\
\text { 122). } \\
\text { (AUBCBZ, 134, } \\
\text { Maria in Selva). } \\
\text { (Ferrazzini Emilio, } \\
\text { Pozzi Tita. } \\
\text { Preventivo. Lugano } \\
29 \text { marzo 1945. } \\
\text { AUBCBZ, s. 134, } \\
\text { Locarno. S. Maria in } \\
\text { Selva). }\end{array}$ \\
\hline
\end{tabular}




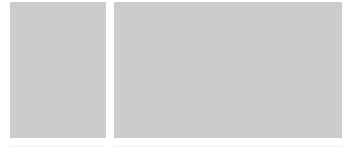

12 nov. Lottigna, vecchio Affreschi sulla 1946 Pretorio già casa dei facciata. Landfogti. pulitura. II totale

del preventivo

complessivo era di fr. 3220 .

\section{Preventivo di} restauro pittorico .

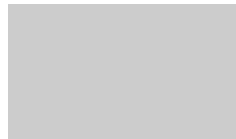

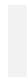

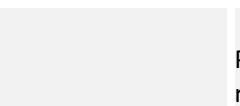

(Pozzi T. Preventivo di restauro indirizzato al DPE. MassagnoLugano, 12 novembre 1946

AUBCBZ, s. 140.

Lottigna)

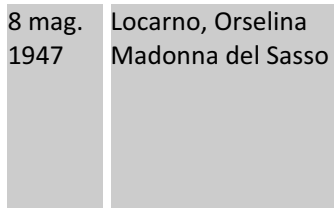

Ciseri,

Traporto di Cristo.

Preventivo di Olio su tela.

restauro pittorico .

\section{Gruppo scultoreo Preventivo di}

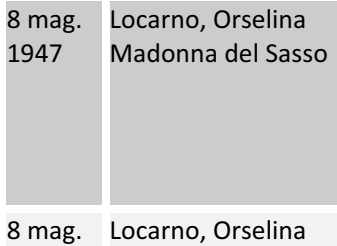

1947 Madonna del Sasso

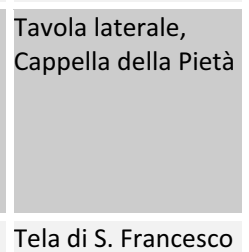

Preventivo di

restauro pittorico.

Preventivo di restauro pittorico

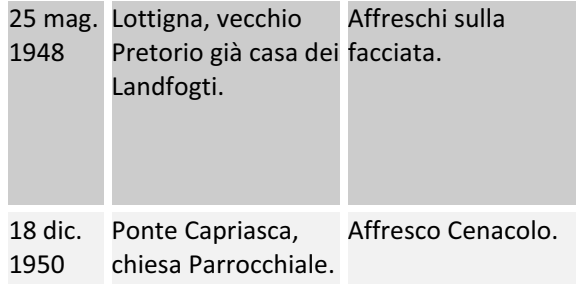

1950 chiesa Parrocchiale.

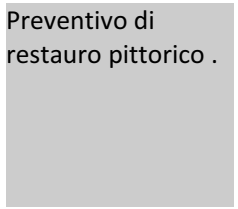

Preventivo di restauro pittorico. Fr. 450.
(Pozzi T.,

Preventivo.

Massagno-Lugano,

8 maggio 1947.

AUBCBZ, s. 183

Orselina).

(Pozzi T.,

Preventivo.

Massagno-Lugano,

8 maggio 1947.

AUBCBZ, s. 183

Orselina).

(Pozzi T.,

Preventivo.

Massagno-Lugano,

8 maggio 1947.

AUBCBZ, s. 183

Orselina).

(Pozzi T.,

Preventivo.

Massagno-Lugano,

8 maggio 1947.

AUBCBZ, s. 183

Orselina).

(Pozzi T., Preventivo

di restauro

indirizzato al DPE.

Massagno-Lugano,

25 maggio 1948.

AUBCBZ, Lottigna)

Arch. Avv.

Lettera del 1

novembre 1951 a

Francesco Chiesa,

AUBC, 196 Ponte

Capriasca. 


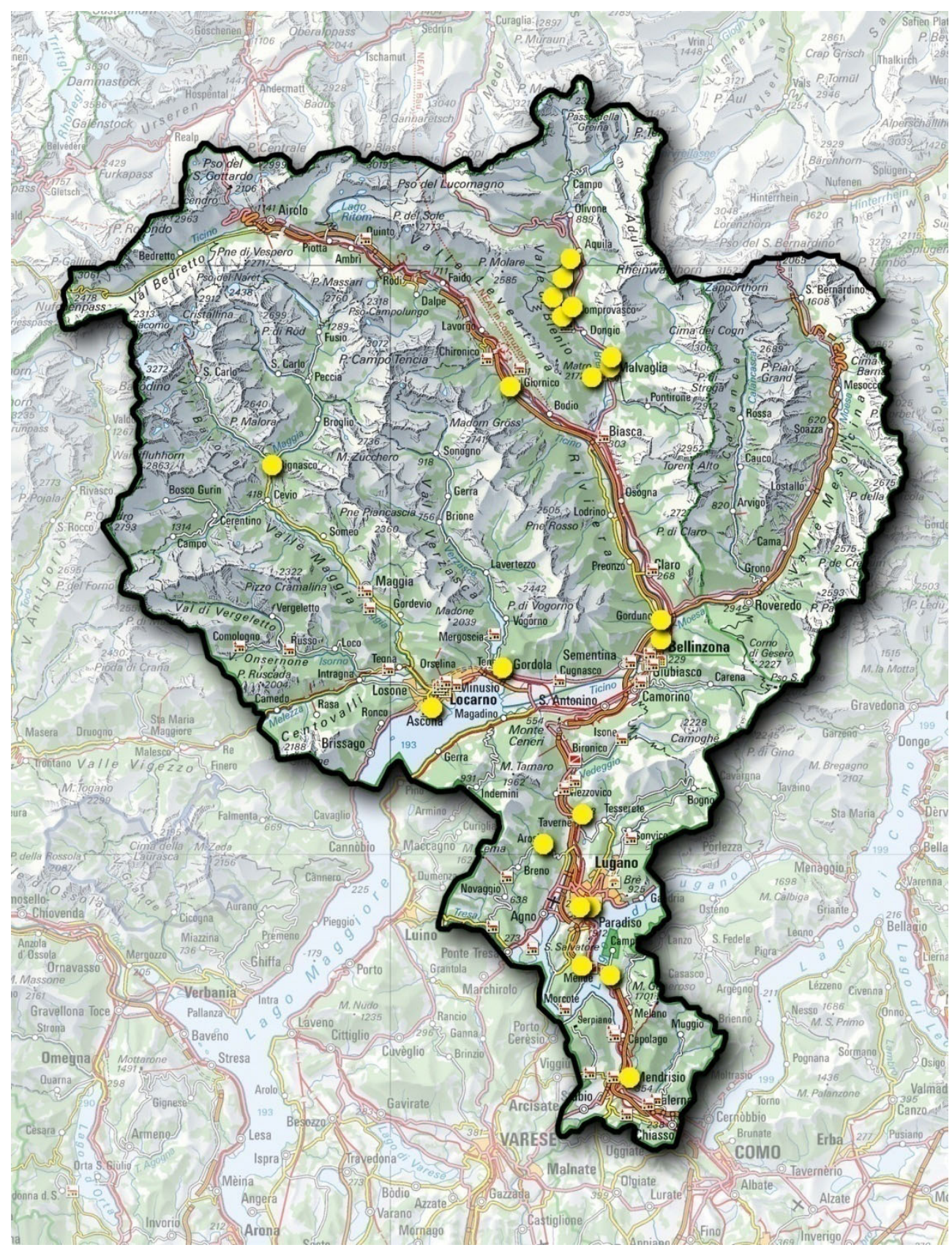

Figura 165 Cartina degli interventi di restauro eseguiti da Tita Pozzi in Canton Ticino. 
Restauratori in Canton Ticino fra Ottocento e Novecento

Catalogazione e gestione dati

\section{Allegati}

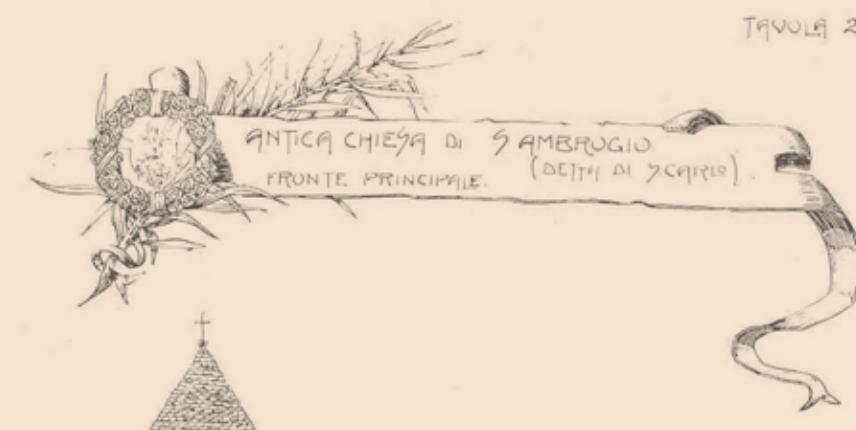

SCคLค Dค 1 A 100

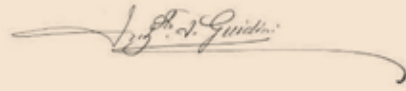

Figura 166 Augusto Guidini. Studio di restauro. 


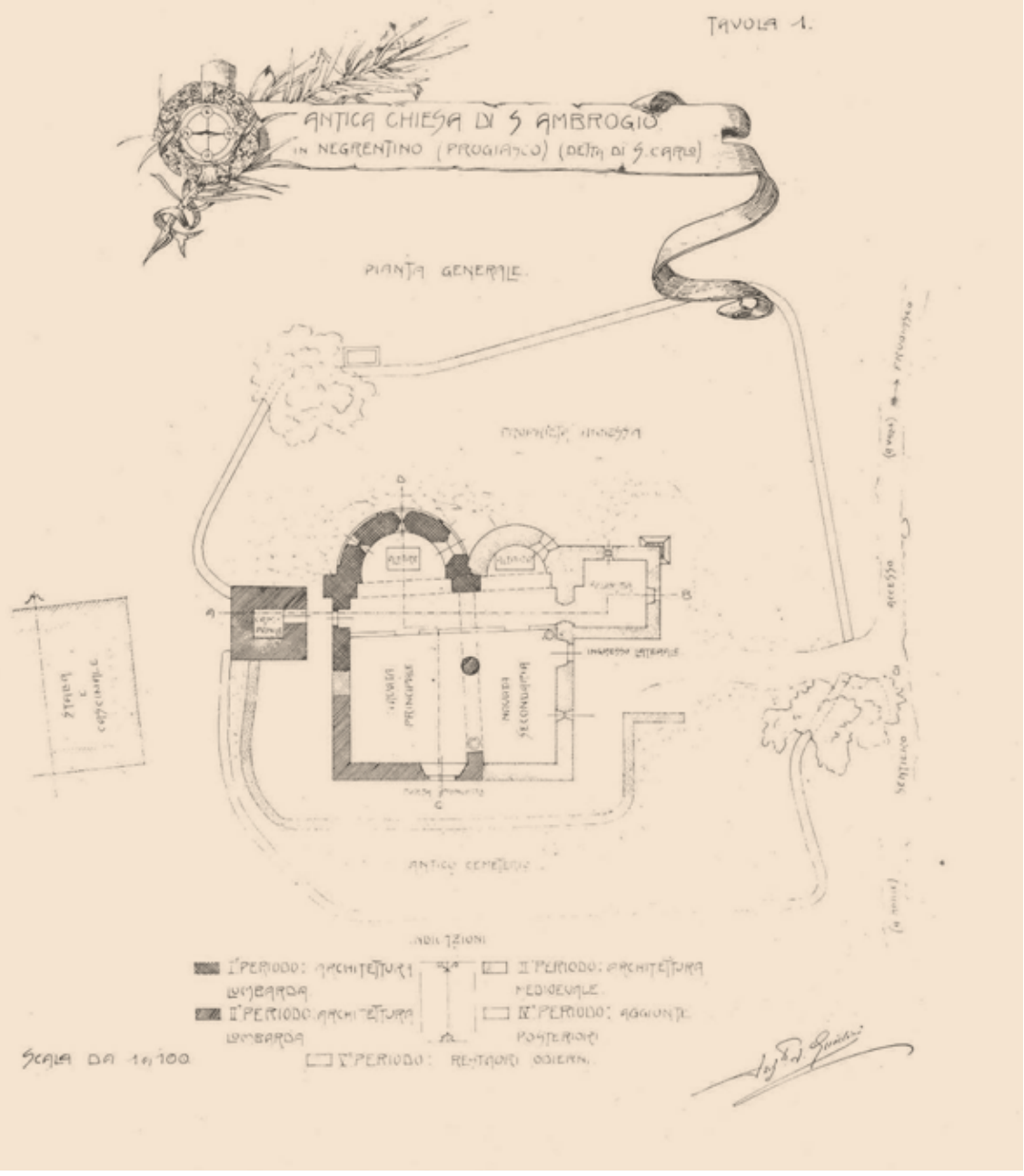

Figura 167 Augusto Guidini. Studio di restauro. 
Masurno- hugano, 22 sett. 1542 Sod.k Dipartionento della Pubblice Educazione,

\section{Bellinzona}

Sini nestauri piltorici - intrapresi nello scorso mese di agos. to - nella Chiesz di Negrentino sopra Prugizser, l'esecustore sottosinitto - pitt. Gitx Pozzi - trasmette a codesto lodevole Di: partimento la seguente relazione:

a). Paute a ponente con la rafficurzione del Palvator:

Errminato attentamente lo stato delle pittura e dell'into = naco eadente, if malanno zpparve molto piü grave di puanto fosse sembrats durzute it primo eszme (soprzluogs 29 buglia); impossibile juindi usare-quale partizle rimedio - le bullutle di inetallo come era otato proposto: dopo pari tentativi e prove if soltossitto fece ricorso al caseato- the im unasticecemcisto tenacissino, molto adekivo, insolubile nell'adqua (anche bollente) e negl. alcali e resiotente quiredi allinividitsIt detto mezzo fue quello usato, pochi anniorsono, pan il consolidamento degli afheschi famosi della volta della Pappells fistina a Roverz. Eon utendili epproprizti, patiente - attentione furono cosi consolidate tutte le parti staccate $e$ pericolanti dell'intanaco portante l'afhesco. Vi i ancora sualche breve scttore d'intanacs che "swona "e questo i" dovuto ad un sottostante inetonaco (sul quale si adagie quallo pontante if dipinto) che a brevi tratfi e di poco non aderisu completamente al muro. Dati i mesti e le possibilita fu ine possibile individuare esattamente questi brevi tralt. "sonanti.

Figura 168 Tita Pozzi, Proposta di restauro indirizzata al Dipartimento della pubblica educazione del Canton Ticino. Massagno, 22 settembre 1942. (AUBCBZ, s. 204 Prugiasco) - (segue). 
si poteva; non si pote ginugere pers fine sotto it softitto. Bisognerai quindi, guand si dasai mano alls nimo = zione det tetto a alla sistemazione del soffitto, usare molta attenzione - specialmente lungo il settore che corre al disoprz dei due archi che dividono la Chiessnel camminare il disoprz del soffitto e nel brigare. Ad ogni modo ora si potzanno intraprendere quei la = vori murari necesseri senza incomere nel pesicolo di

usare ai dipinti ulteviori novine gravi e irrepasabili. Care distints stime ed ossegui, pitl. Tilz Poz:

P.S. Copiz della presente è stata trasmessa at MII. Pev. Don fises. Galizia per il Eomitato Pro Negrestino in Prugizsco.

Tita Pozzi, Proposta di restauro indirizzata al Dipartimento della pubblica educazione del Canton Ticino. Massagno, 22 settembre 1942. (AUBCBZ, s. 204 Prugiasco). 


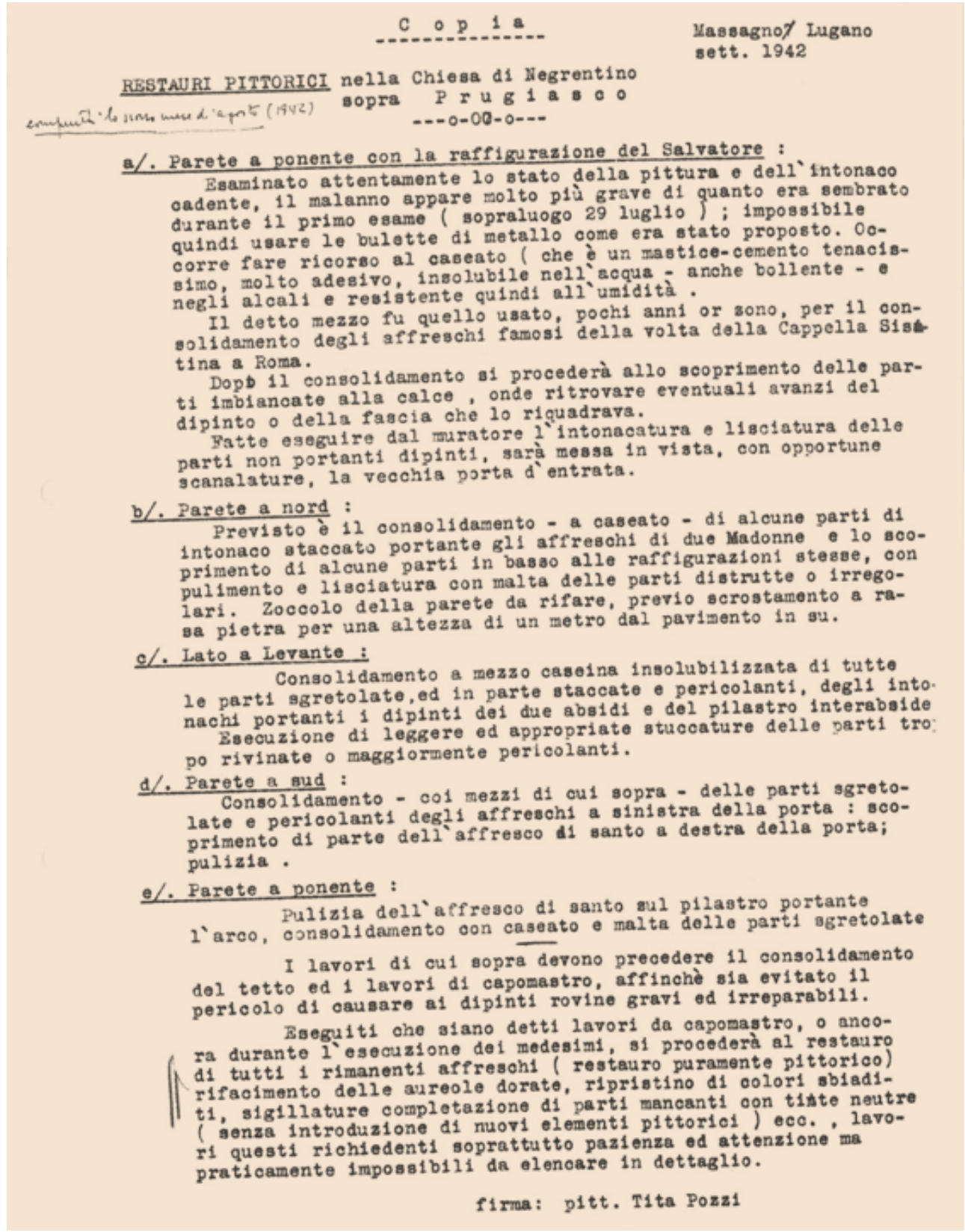

Figura 168 Tita Pozzi, (Copia) Proposta di restauro indirizzata al Dipartimento della pubblica educazione del Canton Ticino. Massagno, 22 settembre 1942. (AUBCBZ, s. 204 Prugiasco). 
PITONE TITA POZZI

LVGANO - MASBAONO

Dongis (valle Blenio), 28. VIII. 45

Lod. Dipartimento Pubblica Educazione,

Bellintona

$V$ i comunico che di guesti gionni mi somo necato a Negnentino, $e$, sacondo be vostre debite istruzioni, ho pnoceduto alla apposizione dalla iscrizione concernente i restauri e la tutela del monumento.

'́liscrizione - in caratteri confacente secondo il modello eseguito - eistata felt/2 a buan fussco e postz sopra la portina d'entrati literale.

Il testo e' quello dz voi a suo tempo indicatomi:

RESTAURATA NEGLI ANNI 1942-44 COL CONTRIBUTO

DELLO STATO DEL CANTONE TICINO

E DELLA CONFEOERAZIONE

E POSTA SOTTO

$\angle A$ LORO PROTEZIONE

Eon distintz stime edi mighiori saluti, pitt. T. Pozz:

Figura 169 Tita Pozzi, Lettera indirizzata al Dipartimento della pubblica educazione del Canton Ticino. Massagno, 22 agosto 1945. (AUBCBZ, s. 204 Prugiasco). 



$$
\text { (c) }
$$




\section{CARLO COTTI}

[...]L'arte, soprattutto in un contesto didattico, può essere indagata partendo dal campo più vasto delle manifestazioni del lavoro umano. Intendo questo come base di tutte quelle trasformazioni che l'uomo effettua nel tempo facendo interagire il proprio mondo interno, psicofisico, con il mondo esterno della natura e della cultura $[\ldots]^{430}$.

[...]El arte, sobretodo en un contexto didáctico, puede ser estudiada a partir de las manifestaciones de la creación humana más amplias. Es decir, como base de todas las trasformaciones que el hombre efectúa con el paso del tiempo, haciendo interactuar el propio mundo interior, psicofisico, con el mundo exterior de la naturaleza y de la cultura [...].

Pagina precedente:

430 Carlo Cotti, "Il Terzo Occhio", dispense per la Lezione di Critica d'Arte alla CSIA, Lugano. Archivio Olga Metalli. 


\section{Dati biografici}

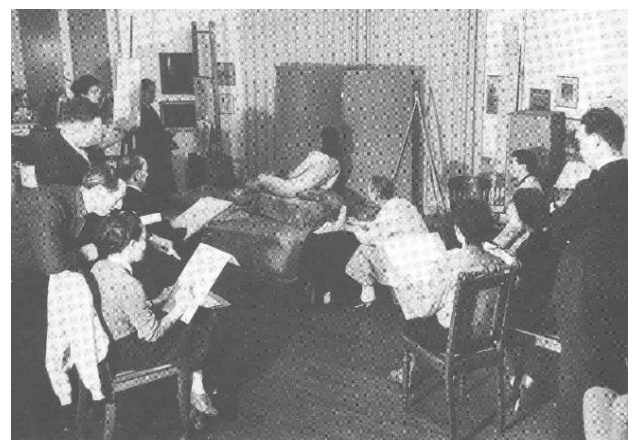

Figura 171 Carlo Cotti nella Scuola di Nudo di Lugano da lui fondata nel 1945.

Carlo Cotti, uno degli artisti più internazionali della metà del XX sec., nasce a Lugano il 27 maggio 1903 da Annibale Cotti di Bergamo e da Margherita Rösel di Weimar in Germania $^{431}$.

Si introdusse all'arte nel 1919, dopo aver terminato gli studi all'Istituto Mandriani di Collina d'Oro, sotto la guida del pittore Vespasiano Bignami ${ }^{432}$, chiamato "Vespa", professore nella prestigiosa Accademia di Belle Arti di Brera a Milano dove ancora impartiva lezioni a suo tempo il pittore Cesare Tallone. Qui conosce sicuramente artisti come l'italiano Pietro Verzetti ${ }^{433}$, ma

\footnotetext{
431 (Killer, 1979, p. 55).

${ }^{432}$ Bignami Vespasiano (Cremona 1841, Milano 1929). L'artista, docente all'Accademia di Brera, fu uno de rappresentati della scapigliatura lombarda più eclettici, il che dimostra il carattere aperto e curioso del pittore. $\mathrm{Fu}$ anche direttore di orchestra e violinista, e nel campo dell'arte si dedicò non solo alla docenza ma anche alla critica, collaborando con diversi giornali dove esibisce anche le sue doti come caricaturista e umorista.

Fu promotore di svariate iniziative culturali, tra cui la fondazione del sodalizio milanese "La Famiglia artistica" e organizzatore di numerose esposizioni e feste cittadine.

${ }^{433}$ Verzetti Pietro (Vercelli 1876 - Como 1955).
}

presto è costretto ad abbandonare la città per ritornare in patria (1923). In questo periodo risiederà a Zurigo e di nuovo a Lugano, ma solo due anni dopo si trasferisce per la seconda volta in Italia, precisamente a Roma. Qui nella capitale studierà al Regio Istituto di Belle Arti ed entrerà nel circolo dei pittori di Via Margutta ${ }^{434}$, convertita in centro di rifugio artistico. In questa città esporrà in una collettiva all'interno del gruppo Novecento italiano, assieme ai pittori Antonio Donghi $^{435}$, Achille Funi ${ }^{436}$ e Carlo Socrate ${ }^{437}$, e realizzerà la sua prima mostra collettiva come esponente del "Novecento Italiano" nel 1926.

Brevemente soggiornerà dal 1927 al 1928 a Lugano, Bergamo e finalmente a Zurigo dove vuole entrare in contatto con l'arte grafica. Finalmente, stabilito a Lugano, Carlo Cotti si dedica all'arte sacra come pittore di affreschi, mosaicista e come restauratore, quest'ultima la professione

\footnotetext{
434 Riconosciuta come centro di uso artistico ed espressivo per musicisti, artigiano, scultori, poeti, e pittore, la strada fu trasformata in proprio museo quando nel 1953 per iniziativa di diversi artisti si eseguono delle opere "al aperto" sulla suddetta strada. Attualmente è l'Associazione Cento Pittori Via Margutta a continuare questa tradizione artistica.

${ }^{435}$ Donghi Antonio (Roma, 1897-1963). Pittore.

436 Funi Achille (Ferrara, 1890 - Appiano Gentile 1972). Pittore futurista. Insegnante all'Accademia di Belle Arti di Brera (1930 - 1960) dove si era diplomato vent'anni prima ed all'Accademia di Belle Arti di Carrara a Bergamo.

${ }^{437}$ Socrate Carlo (Pavia, 1889 - Roma, 1967). Pittore italiano. Studia alla scuola libera del nudo a Firenze e più tardi a Roma dove abiterà definitivamente, ad eccezione di brevi soggiorni all'estero, in speciale a Parigi e Spagna (Barcellona e Madrid) che visita accompagnata dal grande artista cubista da Pablo Picasso. II suo sarà un arte di stile neoclassico tuttavia influenzato delle correnti di avanguardia sorte nell'epoca.
} 
meno conosciuta dall'artista anche se i lavori realizzati furono numerosi.

Nell'anno 1945 la prima Scuola del Nudo da lui fondata a Lugano diventerà uno dei centri più importanti di relazione dei giovani artisti ticinesi, ed ancora dopo gli anni Sessanta sarà un artista di riferimento nel Cantone come docente di critica d'arte al Centro scolastico industrie artistiche, sempre alla città di Lugano (1963-1969).

[...] Critica d'arte: esame, considerazione dell'arte nei suoi diversi aspetti per comprendere il significato interiore, profondo, dell'opera d'arte. Quasi una lente di esame microscopico posta, non sul pezzo di pittura, bensì rivolta allo spirito che ha mosso l'artista ad eseguirla $[\ldots]^{438}$.

Le sue opere come artista furono, fino agli anni Cinquanta, influenzate dallo stile impressionista, che lo seduce durante un suo viaggio a Parigi nel 1939 circa. Erano il risultato della viva motivazione dell'artista verso la ricerca di un nuovo linguaggio e modo di espressioni, che gli faranno mutare verso l'informalismo e finalmente nel costruttivismo astratto che svilupperà dal 1968 al 1980. La medesima strada adottata dal caro amico il pittore Gianni Metalli (1930- 2006).

Come contributo all'arte, a parte delle sue proprie produzione e della sua dedicazione alla didattica, Carlo Cotti donò la propria collezione di arte contemporanea al Museo civico di belle arti di Lugano con la condizione che il fondo creato per la sua iniziativa, Fondo $\operatorname{Cotti}^{439}$, avesse come

\footnotetext{
${ }^{438}$ (Cotti Carlo, Appunti per le lezioni di Critica d'arte. Capitolo: "Critica d'arte"; pag. 1 da 2. Archivio privato Sra. Olga Metalli, Lugano).

439 || Museo Cantonale di Lugano organizzò dal 15 febbraio all'11 maggio 2003 una mostra in omaggio
}

obiettivo l'acquisizione di opere di giovani artisti svizzeri.

Carlo Cotti, come prima si menzionava, fu anche restauratore, anche se il suo legame con questa professione fu molto meno intenso di quanto lo fosse stato per i suoi contemporanei Emilio Ferrazzini, Tita Pozzi e più tardi Carlo Mazzi.

La sua attività nel campo della protezione dei beni culturali iniziò dalla collaborazione con il pittore Ferrazzini, attraverso il quale esegue diversi interventi secondo le indicazioni del collega pittore o di altri membri della commissione cantonale, come ad esempio nel restauro degli affreschi della chiesa parrocchiale di S. Martino a Malvaglia (1931), alla chiesa della Madonna Assunta a Giubiasco (1931), alla Casa Clemente a Giornico (1932) ed all'Oratorio di S. Ambrogio a Segno (1932). Lavorare nel restauro non fu sicuramente per Cotti una necessità primaria per la "sopravivenza" come in tempi prima lo fu per artisti come Attilio Balmelli o Luigi Rossi. Malgrado questa disciplina appresa, deduciamo, all'interno del proprio cantiere, fosse per lui unicamente il modo per cercare una stabilità economica che non li era permessa come pittore, è certo che in qualche modo si sentì attratto: Cotti, artista moderno, era assolutamente incuriosito dall'arte sacro.

alla figura dell'artista ed al Fondo istituito dal medesimo Cotti, in commemorazione del centenario dalla sua nascita. La mostra ebbe lo scopo di presentare il rapporto esistente fra Cotti e l'interno che influisce sul suo arte: gli ambienti, le persone, la città di Lugano, rappresentati dal pittore in un gran numero delle sue opere. Dall'atra parte si mostrarono le più recenti acquisizione del Fondo Cotti come esempio dell'attività svolta dalla commissione scientifica, secondo gli obiettivi che Cotti aveva predi segnati nella fondazione del Fondo. 
Verso il 1945 il pittore si stacca professionalmente del collega Ferrazzini. Dai documenti analizzati sembrerebbe che questa separazione non fu di reciproco accordo ma che le differenze personali e lavorative causarono delle dispute fra entrambi. Non sappiamo esattamente il momento in cui scattarono i problemi, ma è proprio nell'Oratorio di Sant'Ambrogio a Croglio (Castelrotto) nel 1943 quando la corrispondenza fra Cotti e la Commissione Cantonale dei Monumenti Storici rileva i problemi. II pittore, chiamato dalla istituzione cantonale a lavorare assieme al collega Facchinetti sotto la direzione di Ferrazzini che era ancora membro della Commissione Cantonale dei Monumenti Storici, respinge senza esitazioni l'offerta. Da questo anno in poi, effettivamente, l'attività nel restauro di Cotti diminuisce considerevolmente. Se prima dal 1922 aveva lavorato nel settore senza interruzioni temporali: dal 1922 al 1930 nella chiesa di S. Maria della Grazie a Bellinzona sotto la direzione di Edoardo Berta, nel 1930 nella chiesa di S. Carlo a Lugano, nel 1931 a Malvaglia, Giubiasco e Segno, nel 1932 nella casa Stanga a Giornico e nella chiesa di S'Antonio a Lugano, nel 1933 nell'Oratorio di S. Anna a Giubiasco e nel 1935 nella chiesa della Madonna di Loreto a Lugano; dopo un lasso di tempo fino al 1942 senza notizie, quest'ultimo anno lavorerà ad Isona e Morcote e nel 1943 alla chiesa della Beata Vergine Assunta a Villa Luganese. Poi, gli interventi saranno intermittente e di poca importanza.

\section{San Pietro, Castel San Pietro}

Nell'ultimo periodo è notevole il restauro della chiesa di Castel San Pietro, detta chiesa Rossa ${ }^{440}$, eseguito assieme al collega Facchinetti dopo il ripristino architettonico della fabbrica eseguito sotto la direzione di Cino Chiesa dal 1944 al 1946.

L'intervento dell'architetto interessò, in questi due anni, l'eliminazione degli elementi barocchi che secondo gli ideali dell'epoca deturpavano la estetica medievale della fabbrica, eretta nel 1343 da Bonifacio da Modena, vescovo di Como. L'intervento pittorico concernette il restauro della ricca decorazione pittorica dell'edificio, realizzata in contemporanea alla costruzione della fabbrica fra il 1343 ed il 1345 dal Maestri di S. Abbondio. Questo artista di origini lombarde prende l'appellativo dalla omonima basilica di Como da lui affrescata fra il 1315 ed il 1325. Sono attribuibili al medesimo gli affreschi dell'arco trionfale della chiesa di S. Biagio a Ravecchia del $1340-1343$ ca. Gli affreschi della chiesa di S. Pietro raffigurano l'Annunciazione, la Madonna in trono e le Sante Agata, Caterina ed Agnese sull'arco trionfale; busti degli apostoli e di due oranti sull'intradosso; sei profeti e mezzo busto sui piedritti; la Majestas Domini e simboli degli evangelisti assieme alla scena della vita di S. Pietro nella calotta absidale e finalmente fasce decorative con clipei raffiguranti vescovi e martiri a mezzobusto lungo le pareti laterali. I processi d'intervento sull'opera furono il consolidamento delle zone pericolanti

\footnotetext{
L'appellativo si deve alla colorazione rossa della facciata, tinteggiata in questo modo nel 1599 per volontà del vescovo Archinti. (Simona Martinoli, 2007, p. 475).
} 
nell'intonaco e nella pellicola pittorica, staccata a causa dell'azione dell'umidità, la pulitura dei dipinti, limitandosi alle zone più danneggiate ed omogeneizzando così il tutto con un discreto ritocco pittorico.

Queste medesime premesse furono espresse da Cotti, in modo ancora più chiaro, nella proposta di restauro e preventivo presentato l'11 marzo del $1948^{441}$ alla Commissione Cantonale dei Monumenti Storici riguardo gli affreschi della chiesa di S. Maria in Selva a Locarno. Essi erano stati precedentemente studiati dai pittori-restauratori Emilio Ferrazzini e Tita Pozzi nel 1945, e contemporaneamente, come analizzavamo nel capitolo anteriore, da Ottorino Olgiati. La differenza fra la proposta da quest'ultimo presentata alla CCMS e di quella di Cotti si è trovata principalmente nella "finitura" del processo di restauro, o "verniciatura" della superficie dei dipinti murali.

Mentre Olgiati espone la necessità di "ravvivare" in generale il colore fra le ultime fasi di restauro prima del ritocco, Cotti prudentemente spiega come, utilizzando una metodica e ragionata pulitura del dipinto, si potrebbe raggiungere l'idonea omogeneità senza necessità di ricorrere all'impiego di prodotti per dare vivacità ai colori:

\footnotetext{
${ }^{441}$ (Cotti Carlo, Proposta e preventivo di restauro indirizzato alla Commissione dei Monumenti Storici. Lugano 11 marzo 1948. AUBCBZ, s. 134, Locarno. S. Maria in Selva).
}

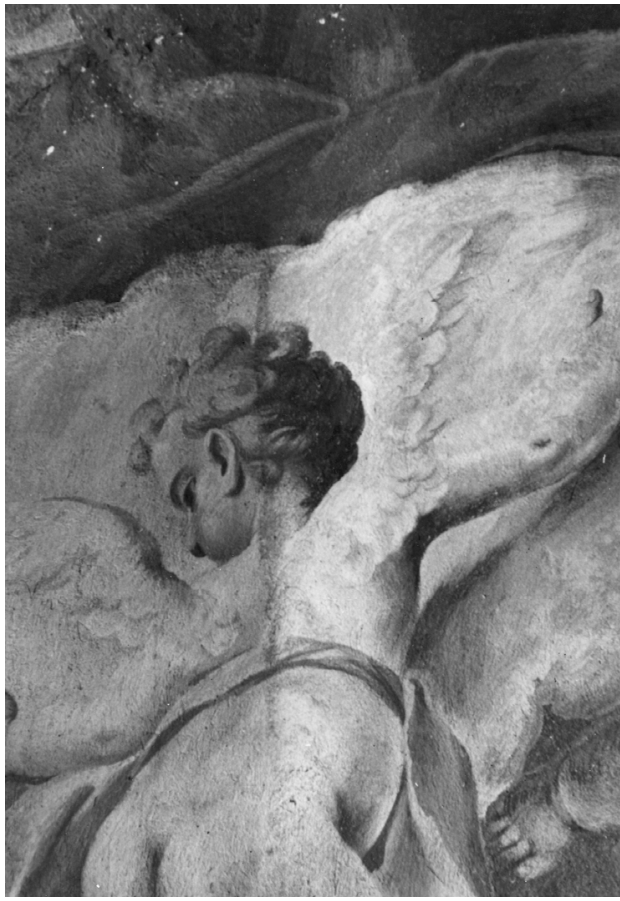

Figura 172 Pulitura degli affreschi della chiesa di S. Antonio, Lugano. Fotografia b/n. AUBCBZ Archivio grafico, Lugano, S. Antonio Abate.

[...]Ritengo di trattarsi di affreschi in condizioni molto simili a quelle in cui abbiamo trovato gli affreschi della chiesa di Castel S. Pietro, nei restauri eseguiti colla guida di cod. spett. Commissione. Il lavoro più urgente è anche cui il consolidamento di alcune parti dell'intonaco al quale seguire la stuccatura e correzione di piccole riparazioni fatte da muratori. Il lavoro di pulitura si presenta assai complicato perché deve variare secondo le diverse superficie coperte e talvolta intaccate dalla polvere $e$ dall'umidità. Avremmo allora un effetto d'insieme armonico grazie al quale si potrà omettere l'intervento sempre pericoloso di sostanze atte a ravvivare il colore $e$ che sacrificano il tono prezioso e caratteristico, leggermente polveroso, dell'affresco che ha sopra di sé secoli di vita. A questo punto, sé 
necessario, si potrà anche ricorrere a qualche ritocco, mentre lascerei come sono i fondi che specialmente sulla volta hanno perduto i colore $[. . .]^{442}$.

Prima che le polemiche riguardo l'utilizzo dei materiali cerosi o delle gomme per ravvivare le tonalità degli affreschi, Cotti si mostra contrario alla abituale pratica giustificando la necessità di conservare le qualità pittoriche dell'opera, ma non solo, poiché leggendo le sue argomentazione si apprezzano delle proposte di lavoro specialmente rispettose per l'epoca. Nemmeno il pittore più consapevole nel Canton Ticino come lo fu Tita Pozzi avrebbe ritenuto pericolosa l'applicazione delle sostanze di "verniciatura" dei dipinti murali e consentito la conservazione dei fondi pittorici sbiaditi dall'umidità, ma avrebbe pensato come minimo nella velatura di essi.

Sarebbe interessante scoprire come mai Cotti, la cui attività di restauro fu legata all'inizio a Berta e a Ferrazzini, ebbe una concezione così nuova di quanto doveva essere l'intervento sull'opera pittorica, e da chi apprese queste condiscendenti valutazioni.

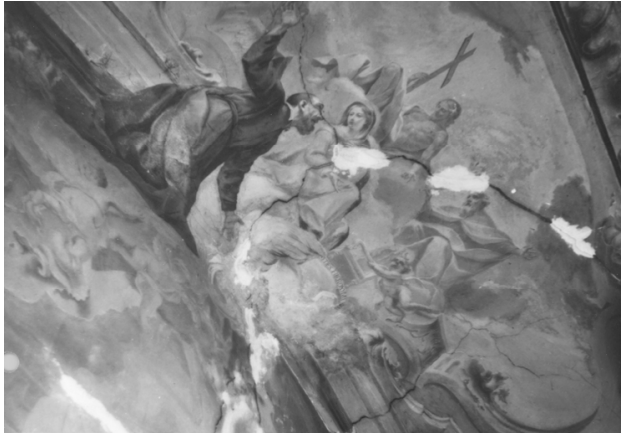

Figura 173 Stuccatura degli affreschi della chiesa di S. Antonio, Lugano. Fotografia b/n. AUBCBZ Archivio grafico, Lugano, S. Antonio Abate.

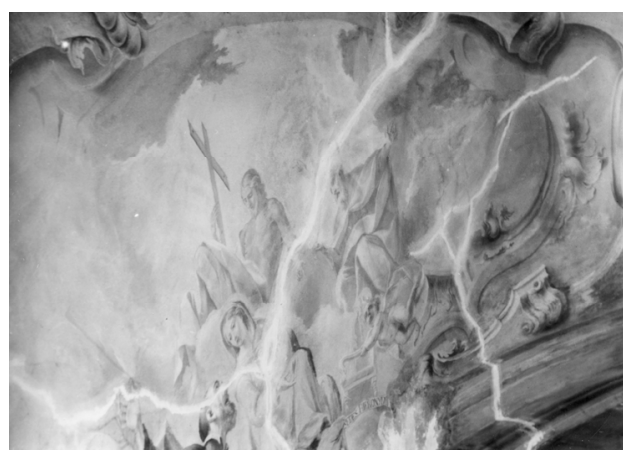

Figura 174 Stuccatura delle fessure. Chiesa di S. Antonio, Lugano. Fotografia b/n. AUBCBZ Archivio grafico, Lugano, S. Antonio Abate.

${ }^{442}$ (Cotti Carlo, Proposta e preventivo di restauro indirizzato alla Commissione dei Monumenti Storici. Lugano 11 marzo 1948. AUBCBZ, s. 134, Locarno. S. Maria in Selva). 


\section{Interventi di restauro}

\begin{tabular}{|c|c|c|c|c|c|}
\hline ANNO & LUOGO & OPERA & INTERVENTO & COLLABORATORI & FONTI \\
\hline $\begin{array}{l}1922- \\
1932\end{array}$ & $\begin{array}{l}\text { Bellinzona, } \\
\text { chiesa di S. Maria } \\
\text { delle Grazie. } \\
\text { BA_2788. }\end{array}$ & $\begin{array}{l}\text { Affreschi della } \\
\text { cantoria. }\end{array}$ & $\begin{array}{l}\text { Pulitura e } \\
\text { consolidamento. }\end{array}$ & $\begin{array}{l}\text { Pittori-restauratori, } \\
\text { Emilio Ferrazzini } \\
\text { Cristiano Schmidt } \\
\text { Emilio Dillena } \\
\text { A. Bassi } \\
\text { Bruno Nizzola. } \\
\text { Diretti da, } \\
\text { Edoardo Berta. }\end{array}$ & $\begin{array}{l}\text { (Chiesa, 1946, p. 27) } \\
\text { (DPE, 1959, p. 118) } \\
\text { (AUBCBZ, s.021, } \\
\text { Bellinzona, S. Maria } \\
\text { delle Grazie). }\end{array}$ \\
\hline $\begin{array}{l}1922- \\
1932\end{array}$ & $\begin{array}{l}\text { Bellinzona, } \\
\text { chiesa di S. Maria } \\
\text { delle Grazie. } \\
\text { BA_2788. }\end{array}$ & $\begin{array}{l}\text { Affresco della } \\
\text { Crocifissione } \\
\text { attribuita a } \\
\text { Tintoretto. }\end{array}$ & $\begin{array}{l}\text { Pulitura e } \\
\text { consolidamento. }\end{array}$ & $\begin{array}{l}\text { Pittori-restauratori, } \\
\text { Emilio Ferrazzini } \\
\text { Cristiano Schmidt } \\
\text { Emilio Dillena } \\
\text { A. Bassi } \\
\text { Bruno Nizzola } \\
\text { Diretti da, } \\
\text { Edoardo Berta. }\end{array}$ & $\begin{array}{l}\text { (Chiesa, 1946, p. 27) } \\
\text { (DPE, 1959, p. 118) } \\
\text { (AUBCBZ, s.021, } \\
\text { Bellinzona, S. Maria } \\
\text { delle Grazie). }\end{array}$ \\
\hline $\begin{array}{l}1922- \\
1932\end{array}$ & $\begin{array}{l}\text { Bellinzona, } \\
\text { chiesa di S. Maria } \\
\text { delle Grazie. } \\
\text { BA_ } 2788 .\end{array}$ & $\begin{array}{l}\text { Affreschi nella } \\
\text { cappella } \\
\text { dell'Immacolata. }\end{array}$ & $\begin{array}{l}\text { Pulitura e } \\
\text { consolidamento. }\end{array}$ & $\begin{array}{l}\text { Pittori-restauratori, } \\
\text { Emilio Ferrazzini } \\
\text { Cristiano Schmidt } \\
\text { Emilio Dillena } \\
\text { A. Bassi } \\
\text { Bruno Nizzola } \\
\text { Diretti da, } \\
\text { Edoardo Berta }\end{array}$ & $\begin{array}{l}\text { (Chiesa, 1946, p. 27) } \\
\text { (DPE, 1959, p. 118) } \\
\text { (AUBCBZ, s.021, } \\
\text { Bellinzona, S. Maria } \\
\text { delle Grazie) }\end{array}$ \\
\hline $\begin{array}{l}1922- \\
1932\end{array}$ & $\begin{array}{l}\text { Bellinzona, } \\
\text { chiesa di S. Maria } \\
\text { delle Grazie. } \\
\text { BA_2788. }\end{array}$ & Via Crucis. & Restauro. & $\begin{array}{l}\text { Pittori-restauratori, } \\
\text { Emilio Ferrazzini } \\
\text { Cristiano Schmidt } \\
\text { Emilio Dillena } \\
\text { A. Bassi } \\
\text { Bruno Nizzola. } \\
\text { Diretti da, } \\
\text { Edoardo Berta. }\end{array}$ & $\begin{array}{l}\text { (Chiesa, 1946, p. 27) } \\
\text { (DPE, 1959, p. 118) } \\
\text { (AUBCBZ, s.021, } \\
\text { Bellinzona, S. Maria } \\
\text { delle Grazie). }\end{array}$ \\
\hline $\begin{array}{l}1922- \\
1932\end{array}$ & $\begin{array}{l}\text { Bellinzona, } \\
\text { chiesa di S. Maria } \\
\text { delle Grazie. } \\
\text { BA_2788. }\end{array}$ & $\begin{array}{l}\text { Decorazioni esterne } \\
\text { sul campanile e } \\
\text { sulle pareti della } \\
\text { Chiesa. }\end{array}$ & Restauro & $\begin{array}{l}\text { Pittori-restauratori, } \\
\text { Emilio Ferrazzini } \\
\text { Cristiano Schmidt } \\
\text { Emilio Dillena } \\
\text { A. Bassi } \\
\text { Bruno Nizzola. } \\
\text { Diretti da, } \\
\text { Edoardo Berta. }\end{array}$ & $\begin{array}{l}\text { (Chiesa, 1946, p. 27) } \\
\text { (DPE, 1959, p. 118) } \\
\text { (AUBCBZ, s.021, } \\
\text { Bellinzona, S. Maria } \\
\text { delle Grazie). }\end{array}$ \\
\hline
\end{tabular}




\begin{tabular}{|c|c|c|c|c|c|}
\hline $\begin{array}{l}1922- \\
1932\end{array}$ & $\begin{array}{l}\text { Bellinzona, } \\
\text { chiesa di S. Maria } \\
\text { delle Grazie. } \\
\text { BA_2788. }\end{array}$ & $\begin{array}{l}\text { Affreschi } \\
\text { raffiguranti profeti } \\
\text { chiusi dentro } \\
\text { medaglioni venuti } \\
\text { alla luce dietro gli } \\
\text { stalli del coro. }\end{array}$ & $\begin{array}{l}\text { Stacco di due } \\
\text { affreschi. }\end{array}$ & $\begin{array}{l}\text { Pittori-restauratori, } \\
\text { Emilio Ferrazzini } \\
\text { Cristiano Schmidt } \\
\text { Emilio Dillena } \\
\text { A. Bassi } \\
\text { Bruno Nizzola. } \\
\text { Diretti da, } \\
\text { Edoardo Berta. }\end{array}$ & $\begin{array}{l}\text { (Chiesa, 1946, p. 27) } \\
\text { (DPE, 1959, p. 118) } \\
\text { (AUBCBZ, s.021, } \\
\text { Bellinzona, S. Maria } \\
\text { delle Grazie). }\end{array}$ \\
\hline 1930 & $\begin{array}{l}\text { Lugano, } \\
\text { chiesa di S. Carlo. }\end{array}$ & $\begin{array}{l}\text { Affresco } \\
\text { raffigurante la } \\
\text { Vergine con } \\
\text { Bambino. }\end{array}$ & $\begin{array}{l}\text { Tecnica } \\
\text { dell'encausto. }\end{array}$ & & $\begin{array}{l}\text { (Killer, 1979, p. 55). } \\
\text { (AUBCBZ, s. } 143 . \\
\text { Lugano. San Carlo). }\end{array}$ \\
\hline $\begin{array}{l}1930- \\
1932\end{array}$ & $\begin{array}{l}\text { Malvaglia, } \\
\text { chiesa Parrocchiale } \\
\text { di S. Martino. } \\
\text { BA_2782. }\end{array}$ & $\begin{array}{l}\text { Stucchi ed affreschi } \\
\text { interni ed esterni } \\
\text { BAS_15266/ } \\
15269 .\end{array}$ & $\begin{array}{l}\text { Restauro } \\
\text { consistente nella } \\
\text { pulitura e restauro } \\
\text { degli stucchi e degli } \\
\text { affreschi esterni ed } \\
\text { interni, con il } \\
\text { consolidamento di } \\
\text { questi ultimi } \\
\text { mediante } \\
\text { l'applicazione di } \\
\text { cera. }\end{array}$ & $\begin{array}{l}\text { I lavori di } \\
\text { ristrutturazione } \\
\text { architettonica } \\
\text { furono realizzati } \\
\text { dall'ingegnere } \\
\text { Baggio. } \\
\text { Coadiuvavano al } \\
\text { restauro Tita Pozzi e } \\
\text { Tito Tettamanti, } \\
\text { mentre il restauro } \\
\text { degli stucchi venne } \\
\text { eseguito dal } \\
\text { gessatore Brignoni. } \\
\text { Diretto da } \\
\text { Emilio Ferrazzini. }\end{array}$ & $\begin{array}{l}\text { (Chiesa, 1946, p. 42) } \\
\text { (DPE, 1959, p. 122) } \\
\text { (AUBCBZ, s. 148. } \\
\text { Malvaglia. San } \\
\text { Martino). } \\
\end{array}$ \\
\hline 1931 & $\begin{array}{l}\text { Giubiasco, } \\
\text { chiesa parrocchiale } \\
\text { della Madonna } \\
\text { Assunta, sec. XIII - } \\
\text { XIV }\end{array}$ & $\begin{array}{l}\text { Affreschi sec. XIV- } \\
X V \text { più alcuni } \\
\text { frammenti }\end{array}$ & $\begin{array}{l}\text { Ripulitura degli } \\
\text { affreschi scoperti } \\
\text { occasionalmente } \\
\text { durante i lavori in } \\
\text { chiesa. }\end{array}$ & $\begin{array}{l}\text { Pittori-restauratori, } \\
\text { Pitt. Augusto Sartori } \\
\text { Emilio Ferrazzini. }\end{array}$ & $\begin{array}{l}\text { (Chiesa, 1946, p. 43) } \\
\text { (AUBCBZ, s. } 113 . \\
\text { Giubiasco. } \\
\text { Sant'Anna). }\end{array}$ \\
\hline 1931 & $\begin{array}{l}\text { Segno (Cavagnago), } \\
\text { oratorio di S. } \\
\text { Ambrogio. } \\
\text { BA_2792. }\end{array}$ & $\begin{array}{l}\text { Ultima Cena ( } 2.80 \mathrm{X} \\
2.35 \mathrm{~m}), \mathrm{XVII} \mathrm{sec} . \\
\text { Affresco, } \\
\text { parete sinistra della } \\
\text { navata. } \\
\text { BAS_15311. } \\
\text { BAS_15317. }\end{array}$ & $\begin{array}{l}\text { Pulitura dallo strato } \\
\text { di calce superficiale, } \\
\text { consolidamento e } \\
\text { ritocco delle parti } \\
\text { danneggiate dai } \\
\text { lavori di restauro, } \\
\text { concretamente dal } \\
\text { descialbo, con } \\
\text { pittura a calce. }\end{array}$ & Emilio Ferrazzini. & $\begin{array}{l}\text { (Chiesa F. , 1946, p. } \\
44 \text { ). } \\
\text { (DPE, 1959, p. 120) } \\
\text { (AUBCBZ, s.071, } \\
\text { Cavagnago. } \\
\text { Sant'Ambrogio). }\end{array}$ \\
\hline 1931 & $\begin{array}{l}\text { Segno (Cavagnago), } \\
\text { oratorio di S. } \\
\text { Ambrogio. } \\
\text { BA_2792. }\end{array}$ & $\begin{array}{l}\text { Quadro allegorico } \\
(2.10 \times 2.40 \mathrm{~m}) \\
\text { Affresco, } \\
\text { sulla parete sinistra } \\
\text { della navata. } \\
\text { BAS_15311. } \\
\text { BAS_15317. }\end{array}$ & $\begin{array}{l}\text { Pulitura dallo strato } \\
\text { di calce superficiale, } \\
\text { consolidamento e } \\
\text { ritocco delle parti } \\
\text { danneggiate dai } \\
\text { lavori di restauro, } \\
\text { concretamente dal } \\
\text { descialbo con } \\
\text { pittura a calce. }\end{array}$ & Emilio Ferrazzini. & $\begin{array}{l}\text { (Chiesa F. , 1946, p. } \\
44 \text { ). } \\
\text { (DPE, 1959, p. 120) } \\
\text { (AUBCBZ, s.071, } \\
\text { Cavagnago. } \\
\text { Sant'Ambrogio). }\end{array}$ \\
\hline
\end{tabular}




\begin{tabular}{|c|c|c|c|c|c|}
\hline 1931 & $\begin{array}{l}\text { Segno (Cavagnago), } \\
\text { oratorio di S. } \\
\text { Ambrogio. } \\
\text { BA_2792. }\end{array}$ & $\begin{array}{l}\text { Transito di M. } \\
\text { Vergine, e } \\
\text { Adorazione dei } \\
\text { Magi (5.30 x } 2.70) \text {. } \\
\text { Affresco, sulla } \\
\text { parete sinistra della } \\
\text { navata. } \\
\text { BAS_15311. } \\
\text { BAS_15317. }\end{array}$ & $\begin{array}{l}\text { Pulitura dallo strato } \\
\text { di calce superficiale, } \\
\text { consolidamento e } \\
\text { ritocco delle parti } \\
\text { danneggiate dai } \\
\text { lavori di restauro, } \\
\text { concretamente dal } \\
\text { descialbo con } \\
\text { pittura a calce. }\end{array}$ & Emilio Ferrazzini. & $\begin{array}{l}\text { (Chiesa F. , 1946, p. } \\
44 \text { ) } \\
\text { (DPE, 1959, p. 120) } \\
\text { (AUBCBZ, s.071, } \\
\text { Cavagnago. } \\
\text { Sant'Ambrogio). }\end{array}$ \\
\hline 1932 & $\begin{array}{l}\text { Giornico, } \\
\text { casa Clemente } \\
\text { detta Casa Stanga }\end{array}$ & $\begin{array}{l}\text { Stemmi esterni e } \\
\text { pitture interne }\end{array}$ & Pulitura e restauro & Emilio Ferrazzini. & $\begin{array}{l}\text { (Chiesa, 1946, p. 45) } \\
\text { (DPE, 1959, p. 121) } \\
\text { (AUBCBZ, s. } 112 \\
\text { Giornico. Casa } \\
\text { Stanga) }\end{array}$ \\
\hline 1932 & $\begin{array}{l}\text { Lugano, } \\
\text { chiesa di } \\
\text { S. Antonio. }\end{array}$ & & $\begin{array}{l}\text { Restauro pittorico, } \\
\text { principalmente } \\
\text { nella cappella del } \\
\text { lato di levante. }\end{array}$ & & $\begin{array}{l}\text { (Chiesa, 1946, p. 18) } \\
\text { (DPE, 1959, p. 122) } \\
\text { (AUBCBZ, s. 143. } \\
\text { Lugano. } \\
\text { Sant'Antonio) }\end{array}$ \\
\hline $\begin{array}{l}1933- \\
1934\end{array}$ & $\begin{array}{l}\text { Giubiasco, } \\
\text { oratorio di S. Anna. }\end{array}$ & $\begin{array}{l}\text { Madonna col } \\
\text { Bambino sec. XV } \\
\text { con aggiunte } \\
\text { posteriore di figure } \\
\text { d'angioli. }\end{array}$ & Pulitura e restauro. & Sr. Giuseppe Weith & $\begin{array}{l}\text { (Chiesa, 1946, p. 51) } \\
\text { (DPE, 1959, p. 121) } \\
\text { (AUBCBZ, s. 113. } \\
\text { Giubiasco. } \\
\text { Sant'Anna). }\end{array}$ \\
\hline 1934 & $\begin{array}{l}\text { Giornico, } \\
\text { casa Clemente } \\
\text { detta Casa Stanga. }\end{array}$ & Pitture murali. & Restauro generale. & & $\begin{array}{l}\text { (DPE, 1959, p. 121) } \\
\text { (AUBCBZ, s. } 112 \\
\text { Giornico. Casa } \\
\text { Stanga). }\end{array}$ \\
\hline 1935 & $\begin{array}{l}\text { Lugano, Madonna } \\
\text { di Loreto. }\end{array}$ & Campanile. & $\begin{array}{l}\text { In sostituzione di } \\
\text { Emilio Ferrazzini. }\end{array}$ & Emilio Ferrazzini. & $\begin{array}{l}\text { (Ferrazzini Emilio. } \\
\text { Lettera indirizzata a } \\
\text { Aldo Crivelli, Lugano } \\
10 \text { ottobre } 1959 . \\
\text { AUBCBZ, } 143 \\
\text { Lugano). }\end{array}$ \\
\hline 1942 & $\begin{array}{l}\text { Isone, } \\
\text { casa di } \\
\text { Giovanni Guerra di } \\
\text { Filippo }\end{array}$ & $\begin{array}{l}\text { Affresco } \\
\text { Madonna in trono } \\
\text { col Bambino, XV. }\end{array}$ & $\begin{array}{l}\text { Consolidamento e } \\
\text { restauro }\end{array}$ & & $\begin{array}{l}\text { (Chiesa, 1946, p. 74) } \\
\text { (DPE, 1959, p. 121) } \\
\text { (AUBCBZ, s. } 127 \\
\text { Isone). }\end{array}$ \\
\hline $\begin{array}{l}\text { Ott. } \\
1942\end{array}$ & $\begin{array}{l}\text { Morcote, } \\
\text { chiesa parrocchiale } \\
\text { di S. Maria del } \\
\text { Sasso. }\end{array}$ & & $\begin{array}{l}5 \text { sopralluoghi } \\
\text { supplendo l'assenza } \\
\text { provvisoria del } \\
\text { pittore Ferrazzini. }\end{array}$ & Emilio Ferrazzini. & $\begin{array}{l}\text { (AUBCBZ, s.172 } \\
\text { Morcote. S. Maria } \\
\text { del Sasso). }\end{array}$ \\
\hline 1942 & $\begin{array}{l}\text { Isone, } \\
\text { casa M. Marcacci. }\end{array}$ & Affresco XIV. & $\begin{array}{l}\text { Riproduzione a } \\
\text { colori. }\end{array}$ & & $\begin{array}{l}\text { (Chiesa, 1946, p. 74) } \\
\text { (DPE, 1959, p. 121) } \\
\text { (AUBCBZ, s. } 127 \\
\text { Isone). }\end{array}$ \\
\hline 1943 & $\begin{array}{l}\text { Villa Luganese, } \\
\text { chiesa Parrocchiale } \\
\text { della B.V Assunta. }\end{array}$ & $\begin{array}{l}\text { Santa Martire con } \\
\text { angeli reggenti una } \\
\text { corona per }\end{array}$ & $\begin{array}{l}\text { Ricerche pittoriche } \\
\text { con esportazione } \\
\text { dello strato di calce }\end{array}$ & $\begin{array}{l}\text { Pittori-restauratori: } \\
\text { Emilio Ferrazzini; } \\
\text { Carlo Cotti }\end{array}$ & $\begin{array}{l}\text { (Chiesa, 1946, p. 77) } \\
\text { (DPE, 1959, p. 126) } \\
\text { (AUBCBZ, s. 251, }\end{array}$ \\
\hline
\end{tabular}




\begin{tabular}{|c|c|c|c|c|c|}
\hline & & $\begin{array}{l}\text { difendere l'anima di } \\
\text { un defunto dal } \\
\text { demonio e i SS. } \\
\text { Rocco, Lucia, } \\
\text { Antonio Abate } \\
\text { (prob.) e Giacomo } \\
\text { Maggiore. Nicchia } \\
\text { E. del portico. } \\
\text { Affreschi, inizio XVI } \\
\text { sec. }\end{array}$ & $\begin{array}{l}\text { ritrovatosi molto } \\
\text { duro. }\end{array}$ & $\begin{array}{l}\text { Nino Facchinetti } \\
\text { Mario Ribola. }\end{array}$ & $\begin{array}{l}\text { Villa Luganese, } \\
\text { Chiesa parrocchiale } \\
\text { della B.V. Assunta). }\end{array}$ \\
\hline 1943 & $\begin{array}{l}\text { Villa Luganese, } \\
\text { chiesa Parrocchiale } \\
\text { della B.V Assunta. }\end{array}$ & $\begin{array}{l}\text { Madonna sulla } \\
\text { lunetta dell'antico } \\
\text { portale. Affresco, } \\
\text { inizio XVI sec. }\end{array}$ & $\begin{array}{l}\text { Ricerche pittorico. } \\
\text { Pulitura dallo strato } \\
\text { di calce. }\end{array}$ & $\begin{array}{l}\text { Pittori-restauratori: } \\
\text { Emilio Ferrazzini; } \\
\text { Carlo Cotti } \\
\text { Nino Facchinetti } \\
\text { Mario Ribola. }\end{array}$ & $\begin{array}{l}\text { (Chiesa F. , 1946, p. } \\
\text { 77). } \\
\text { (DPE, 1959, p. 126) } \\
\text { (AUBCBZ, 251, Villa } \\
\text { Luganese). }\end{array}$ \\
\hline 1943 & $\begin{array}{l}\text { Villa Luganese, } \\
\text { chiesa Parrocchiale } \\
\text { della B.V Assunta. }\end{array}$ & $\begin{array}{l}\text { Seregnesi, attr, S. } \\
\text { Giovanni Battista. } \\
\text { Portico. }\end{array}$ & $\begin{array}{l}\text { Ricerche pittorico. } \\
\text { Pulitura dello strato } \\
\text { di calce. }\end{array}$ & $\begin{array}{l}\text { Pittori-restauratori: } \\
\text { Emilio Ferrazzini; } \\
\text { Carlo Cotti } \\
\text { Nino Facchinetti } \\
\text { Mario Ribola. }\end{array}$ & $\begin{array}{l}\text { (Chiesa F. , 1946, p. } \\
\text { 77). } \\
\text { (DPE, 1959, p. 126) } \\
\text { (AUBCBZ s.251, Villa } \\
\text { Luganese). }\end{array}$ \\
\hline 1943 & $\begin{array}{l}\text { Villa Luganese, } \\
\text { chiesa Parrocchiale } \\
\text { della B.V Assunta. }\end{array}$ & $\begin{array}{l}\text { Madonna della } \\
\text { Misericordia, metà } \\
\text { XIV sec., e due } \\
\text { santi. }\end{array}$ & $\begin{array}{l}\text { Ricerche pittoriche. } \\
\text { Pulitura dello strato } \\
\text { di calce. Ritocco } \\
\text { pittorico. }\end{array}$ & $\begin{array}{l}\text { Pittori-restauratori, } \\
\text { Emilio Ferrazzini; } \\
\text { Mario Moglia } \\
\text { Nino Facchinetti. }\end{array}$ & $\begin{array}{l}\text { (Chiesa F. , 1946, p. } \\
77 \text { ) } \\
\text { (DPE, 1959, p. 126) } \\
\text { (AUBCBZ, s.251, } \\
\text { Villa Luganese). }\end{array}$ \\
\hline 1943 & $\begin{array}{l}\text { Villa Luganese, } \\
\text { chiesa Parrocchiale } \\
\text { della B.V Assunta. }\end{array}$ & $\begin{array}{l}\text { Visione di S. } \\
\text { Bernardo con il } \\
\text { Crocifisso, } 1577 .\end{array}$ & $\begin{array}{l}\text { Ricerche pittoriche. } \\
\text { Pulitura dello strato } \\
\text { di calce. Ritocco } \\
\text { pittorico. }\end{array}$ & $\begin{array}{l}\text { Pittori-restauratori, } \\
\text { Emilio Ferrazzini; } \\
\text { Mario Moglia } \\
\text { Nino Facchinetti. }\end{array}$ & $\begin{array}{l}\text { (Chiesa F. , 1946, p. } \\
77 \text { ) } \\
\text { (DPE, 1959, p. 126) } \\
\text { (AUBCBZ, s.251, } \\
\text { Villa Luganese). }\end{array}$ \\
\hline 1943 & $\begin{array}{l}\text { Villa Luganese, } \\
\text { chiesa Parrocchiale } \\
\text { della B.V Assunta. }\end{array}$ & $\begin{array}{l}\text { Madonna col } \\
\text { bambino e S. } \\
\text { Giovannino, inizio } \\
\text { XVI sec. Lunetta del } \\
\text { portale principale. }\end{array}$ & $\begin{array}{l}\text { Ricerche pittoriche. } \\
\text { Pulitura dello strato } \\
\text { di calce. } \\
\text { Consolidamento. } \\
\text { Ritocco pittorico. }\end{array}$ & $\begin{array}{l}\text { Pittori-restauratori, } \\
\text { Emilio Ferrazzini; } \\
\text { Mario Moglia } \\
\text { Nino Facchinetti. }\end{array}$ & $\begin{array}{l}\text { (Chiesa F. , 1946, p. } \\
77 \text { ) } \\
\text { (DPE, 1959, p. 126) } \\
\text { (AUBCBZ, s.251, } \\
\text { Villa Luganese). }\end{array}$ \\
\hline 1943 & $\begin{array}{l}\text { Villa Luganese, } \\
\text { chiesa Parrocchiale } \\
\text { della B.V Assunta. }\end{array}$ & $\begin{array}{l}\text { Decorazioni } \\
\text { interne. }\end{array}$ & $\begin{array}{l}\text { Patinatura } \\
\text { (armonizzazione del } \\
\text { colore) degli stucchi } \\
\text { ornamentali e } \\
\text { figurativi raschiati e } \\
\text { lisciati dagli } \\
\text { stuccatori Cantoni, } \\
\text { nella volta della } \\
\text { navata e nelle } \\
\text { pareti, nella parete } \\
\text { principale del coro } \\
\text { e su tutti i capitelli } \\
\text { delle lesene. } \\
\text { Pulitura con }\end{array}$ & $\begin{array}{l}\text { Pittori-restauratori, } \\
\text { Emilio Ferrazzini; } \\
\text { Mario Moglia } \\
\text { Nino Facchinetti } \\
\text { Mario Ribola. }\end{array}$ & $\begin{array}{l}\text { (Chiesa F. , 1946, p. } \\
\text { 77) } \\
\text { (DPE, 1959, p. 126) } \\
\text { (AUBCBZ, s.251, } \\
\text { Villa Luganese). }\end{array}$ \\
\hline
\end{tabular}




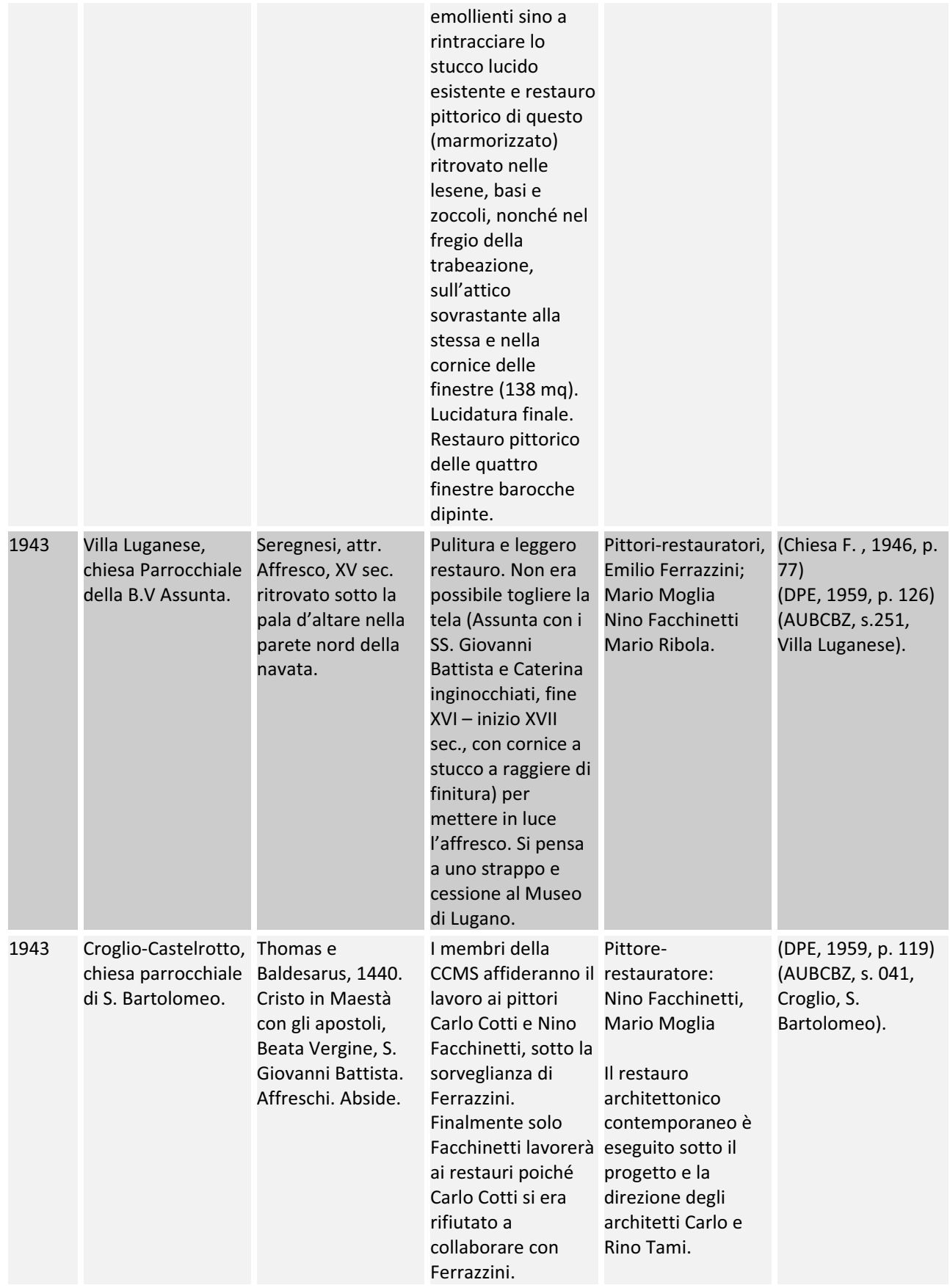




\begin{tabular}{|c|c|c|c|c|c|}
\hline 1946 & $\begin{array}{l}\text { Castel San Pietro, } \\
\text { chiesa di } \\
\text { S. Pietro(detta } \\
\text { Chiesa Rossa). }\end{array}$ & $\begin{array}{l}\text { Allievo del Maestro } \\
\text { di Sant'Abbondio, } \\
1343-1345 . \\
\text { Affreschi. }\end{array}$ & Consolidamento. & $\begin{array}{l}\text { Pittore- } \\
\text { restauratore: } \\
\text { Nino Facchinetti } \\
\text { Il restauro } \\
\text { architettonico } \\
\text { venne eseguito in } \\
\text { contemporaneità } \\
\text { secondo il progetto } \\
\text { dell'architetto Cino } \\
\text { Chiesa (1944-1946). }\end{array}$ & $\begin{array}{l}\text { (DPE, 1959, p. 120) } \\
\text { (AUBCBZ, s. 069, } \\
\text { Castel San Pietro). }\end{array}$ \\
\hline \multirow[t]{2}{*}{$\begin{array}{l}1948- \\
1949\end{array}$} & $\begin{array}{l}\text { Locarno, } \\
\text { chiesa parrocchiale } \\
\text { di S. Maria del } \\
\text { Sasso. }\end{array}$ & Affreschi. & $\begin{array}{l}\text { Ripresa restauri. } \\
\text { Consolidamento. }\end{array}$ & Nino Facchinetti & $\begin{array}{l}\text { (DPE, 1959, p. 124) } \\
\text { (AUBCBZ, s. } 172 . \\
\text { Locarno. S. Maria } \\
\text { del Sasso). }\end{array}$ \\
\hline & & & $\begin{array}{l}\text { Viganello } 19 \\
\text { settembre 1950, } \\
\text { Cotti Carlo?, } \\
\text { Relazione sugli } \\
\text { ultimi lavori di } \\
\text { restauro nella } \\
\text { Chiesa di S. } \\
\text { Ambrogio a } \\
\text { Chironico. }\end{array}$ & & \\
\hline 1953 & $\begin{array}{l}\text { Brusino-Arsizio, } \\
\text { casa di proprietà } \\
\text { Giuseppe Polli. }\end{array}$ & $\begin{array}{l}\text { Madonna in Trono } \\
\text { e S. Rocco. XV sec. } \\
\text { Affresco alla } \\
\text { maniera dei } \\
\text { Seregnesi. }\end{array}$ & $\begin{array}{l}\text { Strappo. } \\
\text { Ricollocamento } \\
\text { nella chiesa } \\
\text { parrocchiale di S. } \\
\text { Michele a Brusino- } \\
\text { Arsizio. }\end{array}$ & $\begin{array}{l}\text { Sig. Varoli. Governo } \\
\text { italiano DPE, } 1984 .\end{array}$ & $\begin{array}{l}\text { (DPE, 1959, p. 119) } \\
\text { (AUBCBZ, s. } 044 . \\
\text { Brusino-Arsizio). }\end{array}$ \\
\hline 1958 & $\begin{array}{l}\text { Carona, camera } \\
\text { stucchi. }\end{array}$ & & Restauro. & & $\begin{array}{l}\text { (DPE, 1959, p. 120) } \\
\text { (AUBCBZ, s. } 065 . \\
\text { Carona). }\end{array}$ \\
\hline
\end{tabular}

\section{Preventivi di restauro}

\begin{tabular}{|c|c|c|c|c|c|}
\hline 1928 & $\begin{array}{l}\text { Lugano, } \\
\text { Madonna di Loreto. }\end{array}$ & & Preventivo. & $\begin{array}{l}\text { Pittore- } \\
\text { restauratore: } \\
\text { Emilio Ferrazzini. }\end{array}$ & \\
\hline $\begin{array}{l}25 \\
\text { maggio } \\
1934\end{array}$ & $\begin{array}{l}\text { Bellinzona, } \\
\text { Oratorio di S. Marta } \\
\text { BA_2797. }\end{array}$ & $\begin{array}{l}\text { Dipinti barocchi } \\
\text { BAS_15328/ } \\
15330 .\end{array}$ & $\begin{array}{l}\text { Preventivo di Fr. } \\
\text { 2'200 per il } \\
\text { restauro pittorico. } \\
\text { Il restauro fu } \\
\text { approvato dal } \\
\text { Consiglio di Stato } \\
\text { della Repubblica e } \\
\text { Cantone del Ticino } \\
\text { il } 8 \text { giugno } 1934 \text { e } \\
\text { s'incarica il pittore }\end{array}$ & $\begin{array}{l}\text { Rev. Sac. Don } \\
\text { Giosuè Prada (a } \\
\text { cura) } \\
\text { Vigilanza: Emilio } \\
\text { Ferrazzini e } \\
\text { Giuseppe Weith } \\
\text { (direzione e } \\
\text { sorveglianza) } \\
\text { Pittore Tita Pozzi } \\
\text { (esecutore) }\end{array}$ & $\begin{array}{l}\text { (AUBCBZ } 021 \\
\text { Bellinzona) } \\
\text { (Ferrazzini E. e Cotti } \\
\text { C. Preventivo del } \\
\text { Restauro completo } \\
\text { degli affreschi della } \\
\text { chiesa di S. Marta } \\
\text { eseguiti secondo le } \\
\text { direttive della } \\
\text { CCMS. Lugano } 25\end{array}$ \\
\hline
\end{tabular}




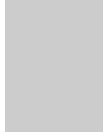

9 ago. Segno (Cavagnago), Affreschi.

1935 oratorio di S.

Ambrogio.

\section{Emilio Ferrazzini d}

sorvegliare i lavori.

Preventivo: rimessa

in luce degli

affreschi mediante

scrostamento degli

intonaci e delle

tinteggiature,

pulitura e restauro

pittorico definitivo.

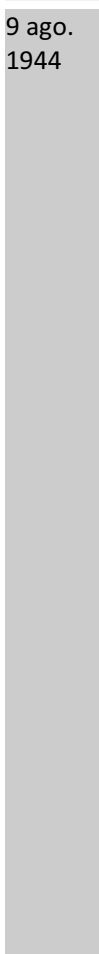

\begin{abstract}
Ponte Capriasca, chiesa parrocchiale di S. Ambrogio.
\end{abstract}

Statue sulla facciata Preventivo di restauro:

Pulitura delle statue togliendo il denso strato causato dalle diverse tinteggiature fatte con Bianca $(80 \mathrm{fr}$. ogni statua) Preparazione del legno con stucco necessaria per ogni successivo trattamento della statua.

Colorazione da eseguire in armonia col Crocifisso che si trova nella Sagrestia e per la quale verranno utilizzate, se ancora rintracciabili le tinte primitive. restauratore:

Emilio Ferrazzini.

(n)

44)

Giosuè Prada.

AUBCBZ s.021

Bellinzona).

(Chiesa, Monumenti storici e artistici del Cantone Ticino restaurati dal 1910 al 1945,1946, p. 44)

(DPE, 1959, p. 120)

(AUBCBZ, s.071,

Cavagnago.

Sant'Ambrogio)

(Cotti Carlo,

Preventivo

indirizzato al

Sacrestano A.

Codaghengo.

Lugano 9 agosto

1935. AUBCBZ,

s.071, Cavagnago.

Sant'Ambrogio).

(AUBCBZ, s. 196

Ponte Capriasca,

Sant'Ambrogio)

(Cotti Carlo,

Preventivo

indirizzato

all'Architetto Pietro

Giovannini. Lugano,

9 agosto 1944,

AUBCBZ, s. 196

Ponte Capriasca,

Sant'Ambrogio). 
11 mar. Locarno,

1948 Chiesa S. Maria in Selva.

$\begin{array}{ll}\text { Affreschi. } & \text { Preventivo e } \\ \text { proposta di } \\ \text { restauro } \\ \text { consistente nel } \\ \text { consolidamento } \\ \text { delle parte } \\ \text { d'intonaco staccate, } \\ \text { stuccatura delle } \\ \text { lacune e } \\ \text { livellamento. } \\ \text { Pulitura selettiva a } \\ \text { seconda delle } \\ \text { superficie e ritocco } \\ \text { limitati a piccoli } \\ \text { punti, scartando i } \\ \text { fondi. } \\ \text { Il totale del } \\ \text { preventivo per le } \\ \text { operazione } \\ \text { sopraccitate era di } \\ \text { fr. 3'000. }\end{array}$

(AUBCBZ, s.

Locarno. S. Maria in Selva)

(Cotti Carlo,

Proposta e

preventivo di

restauro indirizzato

alla Commissione

dei Monumenti

Storici. Lugano 11

marzo 1948.

AUBCBZ, s. 134,

Locarno. S. Maria in

Selva). 


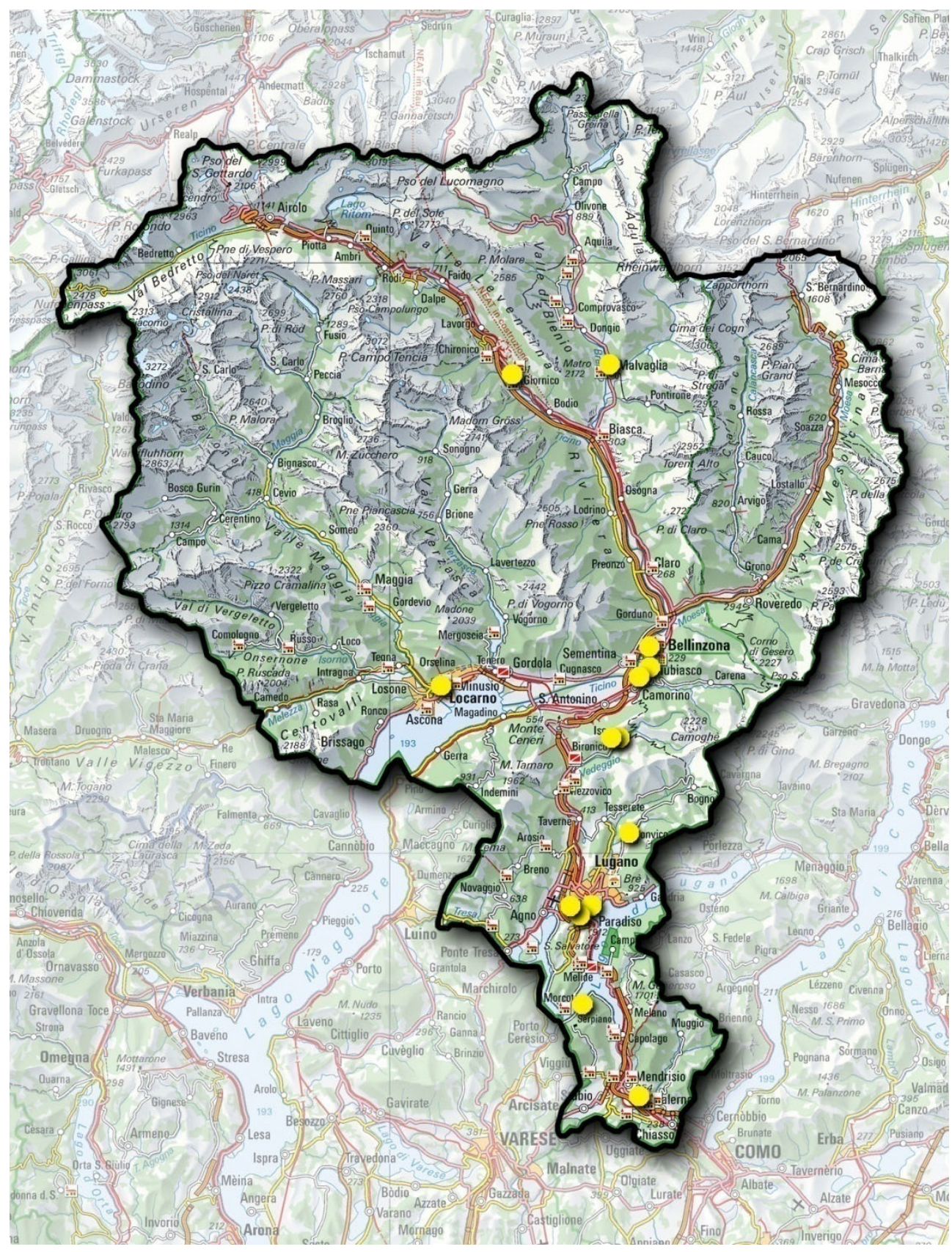

Figura 175 Cartina degli interventi eseguiti da Carlo Cotti in Canton Ticino. 

NINO FACCHINETTI 


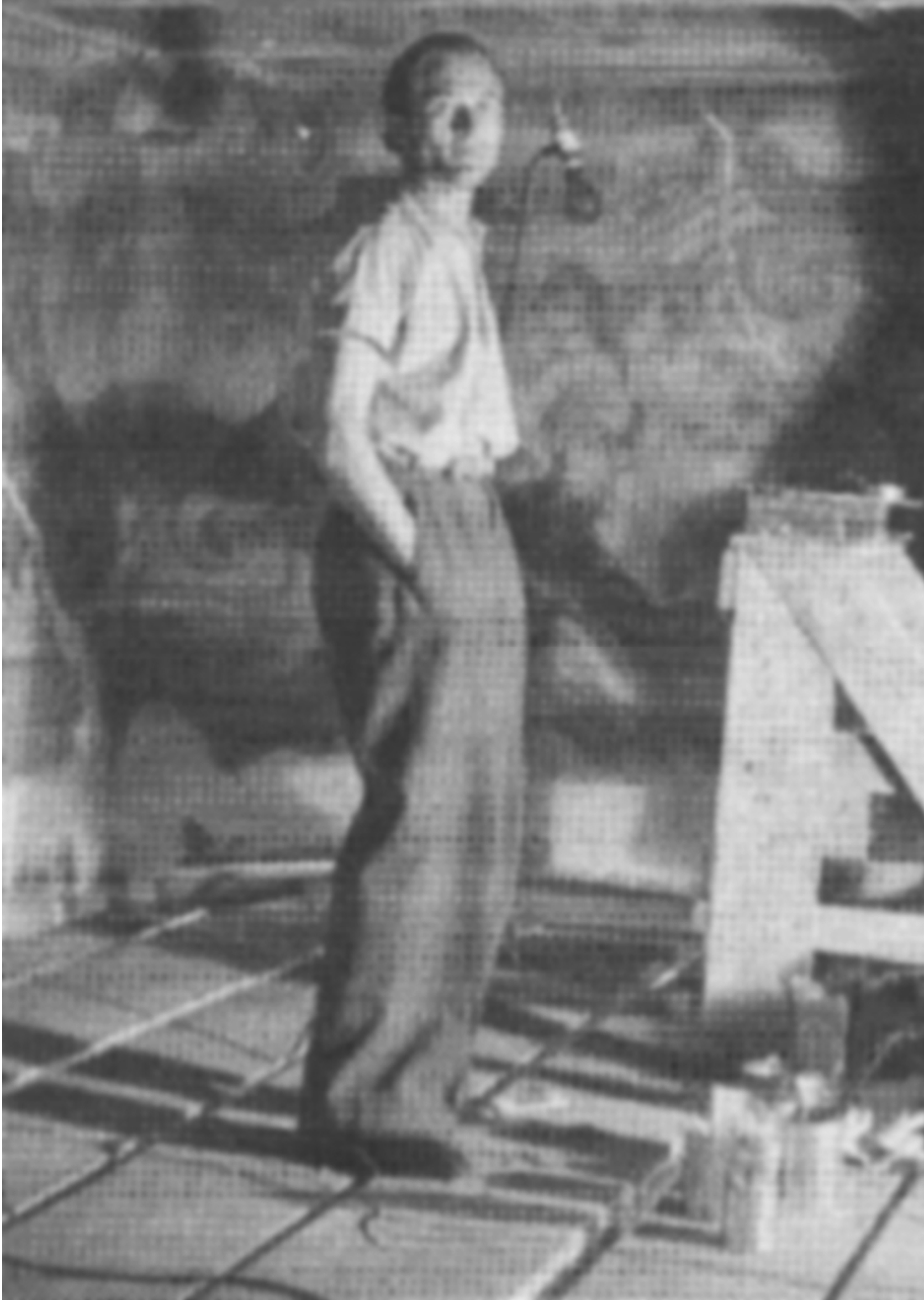




\section{NINO FACCHINETTI}

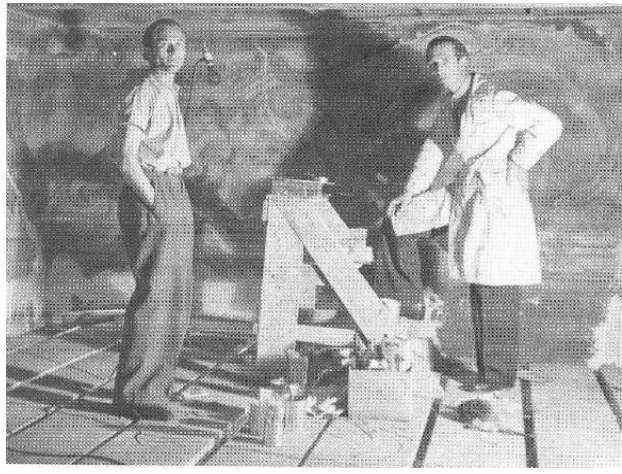

Figura 176 Nino Facchinetti (sinistra) assieme al pittore Carlo Cotti nella chiesa di S. Pietro, detta chiesa Rossa a Castel S. Pietro. 1946 ca.

Con dispiacere devo ammettere l'impossibilità di ottenere qualsiasi informazione riguardo la biografia del pittore-restauratore Nino Facchinetti, ritrovato in numerosi eventi di restauro catalogati nella presente ricerca, senza però completarla con dati che sarebbero stati fondamentali per una maggiore conoscenza della formazione artistica e capacità teoriche e pratica di egli.

Sicuramente, solo il fatto di riordinare I'attività che Facchinetti dedicò al restauro sia già stato un grande passo del quale sono molto orgogliosa, supponendo senza dubbio il riconoscimento di una personalità che ebbe, anche'essa, un suo ruolo non poco rilevante all'interno della protezione dei beni culturali nel Canton Ticino come operatore.

La sua presenza è stata svelata solo a partire dagli anni 1943 e fino al 1958; queste due sono per il momento le uniche date che ci permettono di contestualizzare la persona di Facchinetti. Il suo nome ci è 
apparso la prima volta nelle analisi del testo pubblicato dalla Commissione Cantonale dei Monumenti Storici per il cinquantesimo della sua esistenza, dove si riassumono i restauri eseguiti dal 1909 al 1959. Più avanti lo abbiamo trovato ancora citato fra la corrispondenza conservata all'Archivio dell'Ufficio dei Monumenti Storici, legato ai nomi di altri pittorirestauratori più importanti, che abbiamo trattato nei capitoli precedenti. Di fatto l'attività di Facchinetti nel restauro inizia, secondo le nostre indagini, circa nel 1943, con l'intervento alla chiesa della Beata Vergine Assunta a Villa Luganese accompagnando i colleghi Carlo Cotti, Mario Ribola, Mario Moglia e Emilio Ferrazzini. Lo stesso anno e fino al 1945 avrà luogo l'intervento sull'affresco del Cenacolo, copia dell'opera di Leonardo da Vinci, all'Oratorio dell'Annunciata, e contemporaneamente sugli affreschi quattrocenteschi della chiesa parrocchiale di S. Bartolomeo a Croglio, collaborando in entrambi i casi con il pittore Mario Moglia - come si è trattato nel capitolo dedicato a quest'ultimo. Assieme a Carlo Cotti, in cambio, lavorerà nel restauro delle decorazioni pittoriche all'interno della cosiddetta Chiesa Rossa a Castel San Pietro (1946) ed alla chiesa parrocchiale di S. Maria del Sasso a Locarno (1948), mentre con Carlo Mazzi intervenne nello scoprimento degli affreschi della chiesa parrocchiale di S. Siro a Mairengo (1955), nel restauro degli affreschi della chiesa parrocchiale di S. Maria Assunta a BrioneVerzasca (1956), ed allo strappo di un affresco ad Olivone lo stesso anno (1956). II nome di Facchinetti si è ritrovato singolarmente in poche occasioni, ad esempio: nello scoprimento dallo strato di calce del ciclo d'affreschi del 1508 firmati da Antonio da Tradate e figlio, presenti alla chiesa parrocchiale di S. Michele ad Arosio (1947), nel consolidamento e restauro degli affreschi del portichetto della chiesa di Vezio (1951) e nello strappo di un affresco della casa Orlandini ad Aquila (1955).

In totale si sono catalogati quindici interventi fra le date del 1943 e 1958 a cui Facchinetti partecipa, ma sicuramente ce ne sono tanti altri. La poca importanza data in passato alla conservazione della documentazione, così come la scarsa esigenza da parte degli organismi di più curate e dettagliate relazioni di restauro, lascia pensare alla parziale trasmissione della storia e con ella.

Per la inesistenza di informazione riguardo il pittore-restauratore Facchinetti, e perché ritenevamo fondamentale conoscere la sua relazione ai numerosi cantieri di restauro sopraccitati, abbiamo considerato fosse indispensabile dedicargli queste poche righe. Servano queste se non altro ad incentivare future ricerche che possano, al minimo, concretizzare gli aspetti anagrafici di Facchinetti. 


\section{Interventi di restauro}

\begin{tabular}{|c|c|c|c|c|c|}
\hline ANNO & LUOGO & OPERA & INTERVENTO & COLLABORATORI & FONTI \\
\hline 1943 & $\begin{array}{l}\text { Villa Luganese, } \\
\text { chiesa Parrocchiale } \\
\text { della B.V Assunta. }\end{array}$ & $\begin{array}{l}\text { Santa Martire con } \\
\text { angeli reggenti una } \\
\text { corona per } \\
\text { difendere l'anima di } \\
\text { un defunto dal } \\
\text { demonio e i SS. } \\
\text { Rocco, Lucia, } \\
\text { Antonio Abate } \\
\text { (prob.) e Giacomo } \\
\text { Maggiore. Nicchia } \\
\text { E. del portico. } \\
\text { Affreschi, inizio XVI } \\
\text { sec. }\end{array}$ & $\begin{array}{l}\text { Ricerche pittoriche } \\
\text { con esportazione } \\
\text { dello strato di calce } \\
\text { ritrovato molto } \\
\text { duro. }\end{array}$ & $\begin{array}{l}\text { Pittori-restauratori, } \\
\text { Emilio Ferrazzini; } \\
\text { Mario Moglia } \\
\text { Nino Facchinetti. }\end{array}$ & $\begin{array}{l}\text { (Chiesa, 1946, p. 77) } \\
\text { (DPE, 1959, p. 126) } \\
\text { (AUBCBZ, s. 251, } \\
\text { Villa Luganese, } \\
\text { Chiesa parrocchiale } \\
\text { della B.V. Assunta). }\end{array}$ \\
\hline 1943 & $\begin{array}{l}\text { Villa Luganese, } \\
\text { chiesa Parrocchiale } \\
\text { della B.V Assunta. }\end{array}$ & $\begin{array}{l}\text { Madonna sulla } \\
\text { lunetta dell'antico } \\
\text { portale. Affresco, } \\
\text { inizio XVI sec. }\end{array}$ & $\begin{array}{l}\text { Ricerche pittoriche. } \\
\text { Pulitura dallo strato } \\
\text { di calce. }\end{array}$ & $\begin{array}{l}\text { Pittori-restauratori, } \\
\text { Emilio Ferrazzini; } \\
\text { Mario Moglia } \\
\text { Nino Facchinetti. }\end{array}$ & $\begin{array}{l}\text { (Chiesa F. , 1946, p. } \\
\text { 77). } \\
\text { (DPE, 1959, p. 126) } \\
\text { (AUBCBZ, s. 251, } \\
\text { Villa Luganese, } \\
\text { Chiesa parrocchiale } \\
\text { della B.V. Assunta). }\end{array}$ \\
\hline 1943 & $\begin{array}{l}\text { Villa Luganese, } \\
\text { chiesa Parrocchiale } \\
\text { della B.V Assunta. }\end{array}$ & $\begin{array}{l}\text { Seregnesi, attr, S. } \\
\text { Giovanni Battista. } \\
\text { Portico. }\end{array}$ & $\begin{array}{l}\text { Ricerche pittoriche. } \\
\text { Pulitura dello strato } \\
\text { di calce. }\end{array}$ & $\begin{array}{l}\text { Pittori-restauratori, } \\
\text { Emilio Ferrazzini; } \\
\text { Mario Moglia } \\
\text { Nino Facchinetti }\end{array}$ & $\begin{array}{l}\text { (Chiesa F. , 1946, p. } \\
\text { 77) } \\
\text { (DPE, 1959, p. 126) } \\
\text { (AUBCBZ, s. 251, } \\
\text { Villa Luganese, } \\
\text { Chiesa parrocchiale } \\
\text { della B.V. Assunta) }\end{array}$ \\
\hline 1943 & $\begin{array}{l}\text { Villa Luganese, } \\
\text { chiesa Parrocchiale } \\
\text { della B.V Assunta. }\end{array}$ & $\begin{array}{l}\text { Madonna della } \\
\text { Misericordia, metà } \\
\text { XIV sec., e due } \\
\text { santi. }\end{array}$ & $\begin{array}{l}\text { Ricerche pittoriche. } \\
\text { Pulitura dello strato } \\
\text { di calce. Ritocco } \\
\text { pittorico. }\end{array}$ & $\begin{array}{l}\text { Pittori-restauratori, } \\
\text { Emilio Ferrazzini; } \\
\text { Mario Moglia } \\
\text { Nino Facchinetti. }\end{array}$ & $\begin{array}{l}\text { (Chiesa F. , 1946, p. } \\
\text { 77) } \\
\text { (DPE, 1959, p. 126) } \\
\text { (AUBCBZ, s. 251, } \\
\text { Villa Luganese, } \\
\text { Chiesa parrocchiale } \\
\text { della B.V. Assunta). }\end{array}$ \\
\hline 1943 & $\begin{array}{l}\text { Villa Luganese, } \\
\text { chiesa Parrocchiale } \\
\text { della B.V Assunta. }\end{array}$ & $\begin{array}{l}\text { Visione di S. } \\
\text { Bernardo con il } \\
\text { Crocifisso, } 1577 .\end{array}$ & $\begin{array}{l}\text { Ricerche pittoriche. } \\
\text { Pulitura dello strato } \\
\text { di calce. Ritocco } \\
\text { pittorico. }\end{array}$ & $\begin{array}{l}\text { Pittori-restauratori, } \\
\text { Emilio Ferrazzini; } \\
\text { Mario Moglia } \\
\text { Nino Facchinetti. }\end{array}$ & $\begin{array}{l}\text { (Chiesa F. , 1946, p. } \\
\text { 77) } \\
\text { (DPE, 1959, p. 126) } \\
\text { (AUBCBZ, s. 251, } \\
\text { Villa Luganese, } \\
\text { Chiesa parrocchiale } \\
\text { della B.V. Assunta) }\end{array}$ \\
\hline 1943 & $\begin{array}{l}\text { Villa Luganese, } \\
\text { chiesa Parrocchiale } \\
\text { della B.V Assunta. }\end{array}$ & $\begin{array}{l}\text { Madonna col } \\
\text { bambino e S. } \\
\text { Giovannino, inizio } \\
\text { XVI sec. Lunetta del } \\
\text { portale principale. }\end{array}$ & $\begin{array}{l}\text { Ricerche pittoriche. } \\
\text { Pulitura dello strato } \\
\text { di calce. } \\
\text { Consolidamento. } \\
\text { Ritocco pittorico. }\end{array}$ & $\begin{array}{l}\text { Pittori-restauratori, } \\
\text { Emilio Ferrazzini; } \\
\text { Mario Moglia } \\
\text { Nino Facchinetti. }\end{array}$ & $\begin{array}{l}\text { (Chiesa F. , 1946, p. } \\
\text { 77) } \\
\text { (DPE, 1959, p. 126) } \\
\text { (AUBCBZ, s. 251, } \\
\text { Villa Luganese, } \\
\text { Chiesa parrocchiale }\end{array}$ \\
\hline
\end{tabular}




\begin{tabular}{|c|c|c|c|c|c|}
\hline & & & & & della B.V. Assunta). \\
\hline 1943 & $\begin{array}{l}\text { Villa Luganese, } \\
\text { chiesa Parrocchiale } \\
\text { della B.V Assunta. }\end{array}$ & $\begin{array}{l}\text { Decorazioni } \\
\text { interne. }\end{array}$ & $\begin{array}{l}\text { Patinatura } \\
\text { (armonizzazione del } \\
\text { colore) degli stucchi } \\
\text { ornamentali e } \\
\text { figurativi raschiati e } \\
\text { lisciati dagli } \\
\text { stuccatori Cantoni, } \\
\text { nella volta della } \\
\text { navata e nelle } \\
\text { pareti, nella parete } \\
\text { principale del coro } \\
\text { e su tutti i capitelli } \\
\text { delle lesene. } \\
\text { Pulitura con } \\
\text { emollienti sino a } \\
\text { rintracciare lo } \\
\text { stucco lucido } \\
\text { esistente e restauro } \\
\text { pittorico di questo } \\
\text { (marmorizzato) } \\
\text { ritrovato nelle } \\
\text { lesene, basi e } \\
\text { zoccoli, nonché nel } \\
\text { fregio della } \\
\text { trabeazione, } \\
\text { sull'attico } \\
\text { sovrastante alla } \\
\text { stessa e nella } \\
\text { cornice delle } \\
\text { finestre (138 mq). } \\
\text { Lucidatura finale. } \\
\text { Restauro pittorico } \\
\text { delle quattro } \\
\text { finestre barocche } \\
\text { dipinte. }\end{array}$ & $\begin{array}{l}\text { Pittori-restauratori, } \\
\text { Emilio Ferrazzini; } \\
\text { Mario Moglia } \\
\text { Nino Facchinetti. }\end{array}$ & $\begin{array}{l}\text { (Chiesa F. , 1946, p. } \\
\text { 77). } \\
\text { (DPE, 1959, p. 126). } \\
\text { (AUBCBZ, s. 251, } \\
\text { Villa Luganese, } \\
\text { Chiesa parrocchiale } \\
\text { della B.V. Assunta). }\end{array}$ \\
\hline 1943 & $\begin{array}{l}\text { Villa Luganese, } \\
\text { chiesa Parrocchiale } \\
\text { della B.V Assunta. }\end{array}$ & $\begin{array}{l}\text { Seregnesi, attr. } \\
\text { Affresco, XV sec. } \\
\text { ritrovato sotto la } \\
\text { pala d'altare nella } \\
\text { parete nord della } \\
\text { navata. }\end{array}$ & $\begin{array}{l}\text { Pulitura e leggero } \\
\text { restauro. Non era } \\
\text { possibile togliere la } \\
\text { tela (Assunta con i } \\
\text { SS. Giovanni } \\
\text { Battista e Caterina } \\
\text { inginocchiati, fine } \\
\text { XVI - inizio XVII } \\
\text { sec., con cornice a } \\
\text { stucco a raggiere di } \\
\text { finitura) per } \\
\text { mettere in luce } \\
\text { l'affresco. Si pensa } \\
\text { a uno strappo e } \\
\text { cessione al Museo }\end{array}$ & $\begin{array}{l}\text { Pittori-restauratori, } \\
\text { Emilio Ferrazzini; } \\
\text { Mario Moglia } \\
\text { Nino Facchinetti. }\end{array}$ & $\begin{array}{l}\text { (Chiesa F. , 1946, p. } \\
\text { 77) } \\
\text { (DPE, 1959, p. 126) } \\
\text { (AUBCBZ, s. 251, } \\
\text { Villa Luganese, } \\
\text { Chiesa parrocchiale } \\
\text { della B.V. Assunta). }\end{array}$ \\
\hline
\end{tabular}




$\begin{array}{lll}1943- & \text { Novazzano, } & \text { Tarilli, Giovanni } \\ 1945 & \begin{array}{l}\text { oratorio } \\ \text { dell'Annunciata. }\end{array} & \text { Battista e bottega, } \\ & \begin{array}{l}1584 \text { ca. } \\ \text { Ultima Cena. } \\ \text { Affresco. }\end{array}\end{array}$

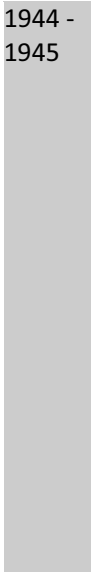

1945 1947

Riva San Vitale, cappella di

S. Bernardino, chiesa di S. Croce.

Croglio- Caste
oratorio di S. Bartolomeo.

Baldesarus, 1440. Cristo in Maestà con gli apostoli. Affreschi. Abside.

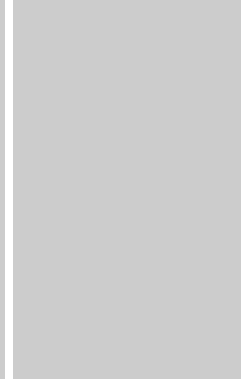

Fratelli Giovan

Pietro, Francesco e

Marc'Antonio Pozzi, 1591 - 1592.

Dipinti murali.

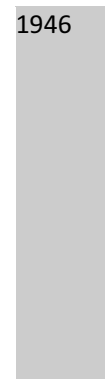

1946 Castel San Pietro,
chiesa di S. Pietro
(detta Chiesa
Rossa).

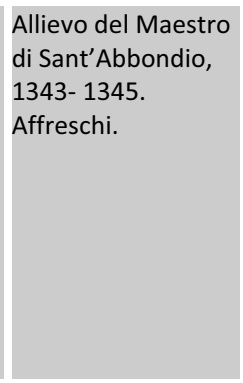

Arosio,

Antonio da Tradate chiesa parrocchiale e figlio, 1508.

\section{di Lugano.}

Pulitura e

Pittore-

consolidamento del restauratore:

dipinto mediante la Mario Moglia. tecnica ad encausto con la miscela di diverse cere $o$ Sorveglianza di Emilio Ferrazzini e I'applicazione di queste in diverse mani per rinnovare Ugo Donati.

le tonalità cromatiche.

(1)

\section{Pittore- \\ restauratore: \\ Mario Moglia. \\ Sorveglianza di \\ Emilio Ferrazzini e Ugo Donati. \\ II restauro \\ architettonico \\ venne eseguito da \\ Carlo e Rino Tami nel 1945, con la ricostruzione del tetto con orditura lignea a vista. \\ II restauro architettonico venne eseguito in contemporaneità secondo il progetto dell'architetto Cino Chiesa (1939 - 1947).} Consolidamento.

\section{Pittore-}

restauratore:

Carlo Cotti

II restauro architettonico venne eseguito in contemporaneità secondo il progetto dell'architetto Cino Chiesa (1944-1946)

DPE, gennaio 1959,

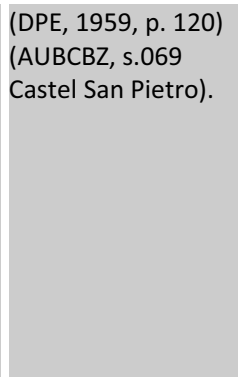

(Chiesa, Monumenti storici e artistici del Cantone Ticino restaurati dal 1910 al 1945, 1946, p. 75) (DPE, 1959, p. 124) (AUBCBZ, s. 080 Novazzano.

Oratorio dell'Annunciata).

(Chiesa, Monumenti storici e artistici del Cantone Ticino restaurati dal 1910 al 1945,1946, p. 84). (DPE, 1959, p. 120) (AUBCBZ, s. 094. Croglio. S. Bartolomeo).

(DPE, 1959, p. 209).

Rimozione dello scialbo di calce e 


\begin{tabular}{|c|c|c|c|c|c|}
\hline & di S. Michele. & $\begin{array}{l}\text { Episodi della vita di } \\
\text { Cristo e della } \\
\text { Passione. } \\
\text { Ciclo di affreschi. } \\
\text { Pareti nord e sud } \\
\text { del coro. }\end{array}$ & consolidamento. & & \\
\hline $\begin{array}{l}1946- \\
1947\end{array}$ & $\begin{array}{l}\text { Arosio, } \\
\text { chiesa parrocchiale } \\
\text { di S. Michele. }\end{array}$ & $\begin{array}{l}\text { Antonio da Tradate } \\
\text { e figlio, } 1508 . \\
\text { Crocifissione e } \\
\text { scene } \\
\text { dell'Adorazione dei } \\
\text { Magi e della } \\
\text { Circoncisione. } \\
\text { Parete est. } \\
\text { Ciclo di affreschi. }\end{array}$ & $\begin{array}{l}\text { Rimozione dello } \\
\text { scialbo di calce e } \\
\text { consolidamento. }\end{array}$ & & DPE, gennaio 1959 , \\
\hline 1947 & $\begin{array}{l}\text { Locarno, } \\
\text { chiesa } \\
\text { dell'Annunciata. }\end{array}$ & Affreschi. & $\begin{array}{l}\text { Lavori di ricerca } \\
\text { degli affreschi } \\
\text { scialbati. }\end{array}$ & $\begin{array}{l}\text { Pittore- } \\
\text { restauratore: } \\
\text { Teodoro Hallisch. }\end{array}$ & $\begin{array}{l}\text { (DPE, 1959, p. 122) } \\
\text { (AUBCBZ, s. } 134 . \\
\text { Locarno. Chiesa } \\
\text { del'Annunciata). }\end{array}$ \\
\hline 1948 & $\begin{array}{l}\text { Morcote, } \\
\text { Chiesa di S. Maria } \\
\text { del Sasso. }\end{array}$ & Affreschi. & $\begin{array}{l}\text { Incarico di } \\
\text { cooperare nel } \\
\text { restauro con Carlo } \\
\text { Cotti. }\end{array}$ & $\begin{array}{l}\text { Il restauro } \\
\text { architettonico } \\
\text { venne eseguito in } \\
\text { contemporaneità } \\
\text { secondo il progetto } \\
\text { dell'architetto Cino } \\
\text { Chiesa (1948-1949). }\end{array}$ & $\begin{array}{l}\text { (AUBCBZ, s.172 } \\
\text { Morcote, S. Maria } \\
\text { del Sasso). } \\
\text { (DPE, Lettera } \\
\text { indirizzata a Nino } \\
\text { Facchinetti } \\
\text { Bellinzona } 25 \\
\text { settembre } 1948 \text {, } \\
\text { AUBCBZ, s.172 } \\
\text { Morcote, S. Maria } \\
\text { del Sasso). }\end{array}$ \\
\hline $\begin{array}{l}1948- \\
1949\end{array}$ & $\begin{array}{l}\text { Locarno, } \\
\text { chiesa parrocchiale } \\
\text { di S. Maria del } \\
\text { Sasso. }\end{array}$ & Affreschi. & Consolidamento. & $\begin{array}{l}\text { Pittore- } \\
\text { restauratore: } \\
\text { Carlo Cotti. }\end{array}$ & $\begin{array}{l}\text { (DPE, 1959, p. 124) } \\
\text { (AUBCBZ, s. } 172 \\
\text { Locarno. Madonna } \\
\text { del Sasso). }\end{array}$ \\
\hline 1949 & $\begin{array}{l}\text { Morcote, } \\
\text { Chiesa della } \\
\text { Madonna del Sasso. }\end{array}$ & 4 Tele. & $\begin{array}{l}\text { Fissaggio nelle } \\
\text { relative } \\
\text { incorniciature a } \\
\text { stucco e far } \\
\text { completare la } \\
\text { cornici di legno } \\
\text { interrotte su due } \\
\text { brevi tratti. }\end{array}$ & & $\begin{array}{l}\text { (AUBCBZ, s.172 } \\
\text { Morcote, S. Maria } \\
\text { del Sasso). }\end{array}$ \\
\hline 1951 & $\begin{array}{l}\text { Vezio, } \\
\text { portichetto della } \\
\text { chiesa parrocchiale. }\end{array}$ & Affreschi. & $\begin{array}{l}\text { Consolidamento e } \\
\text { restauro. }\end{array}$ & & $\begin{array}{l}\text { (DPE, 1959, p. 126) } \\
\text { (AUBCBZ, s. } 248 . \\
\text { Vezio). }\end{array}$ \\
\hline 1955 & $\begin{array}{l}\text { Mairengo, } \\
\text { chiesa parrocchiale. }\end{array}$ & Affreschi. & Scoprimento. & $\begin{array}{l}\text { Pittore- } \\
\text { restauratore: }\end{array}$ & $\begin{array}{l}\text { (DPE, 1959, p. 122) } \\
\text { (AUBCBZ, s. } 148 .\end{array}$ \\
\hline
\end{tabular}




\begin{tabular}{|c|c|c|c|c|c|}
\hline & di S. Siro. & & & Carlo Mazzi. & $\begin{array}{l}\text { Mairengo). } \\
\text { (Belinelli, 1981, p. } \\
\text { 30). } \\
\text { (Belinelli, Carlo } \\
\text { Mazzi, 1911-1988 : } \\
\text { Galleria Carlo Mazzi, } \\
\text { Tegna, 14.VI - } \\
\text { 30.VIII.1998, 1998, } \\
\text { p. 29). }\end{array}$ \\
\hline 1955 & $\begin{array}{l}\text { Aquila (Dangio) } \\
\text { casa Orlandini, ora } \\
\text { proprietà della } \\
\text { signora Marianna } \\
\text { Fontana. }\end{array}$ & $\begin{array}{l}\text { Alessandro Gorla, } \\
\text { inizi XVII sec. Pietà } \\
\text { con il committente } \\
\text { inginocchiato. }\end{array}$ & $\begin{array}{l}\text { Strappo e } \\
\text { ricollocamento } \\
\text { nella sala della } \\
\text { Commissione del } \\
\text { Palazzo degli Studi, } \\
\text { Lugano dopo } \\
\text { appropriazioni dallo } \\
\text { Stato per fr. } 700 \text {. }\end{array}$ & & $\begin{array}{l}\text { (DPE, 1959, p. 117) } \\
\text { (AUBCBZ, s. } 005 . \\
\text { Aquila). } \\
\text { AA.VV., } 1984 \text { p.49. }\end{array}$ \\
\hline 1956 & $\begin{array}{l}\text { Brione Verzasca, } \\
\text { chiesa parrocchiale } \\
\text { di S. Maria Assunta. }\end{array}$ & Affreschi. & Restauro. & $\begin{array}{l}\text { Pittore- } \\
\text { restauratore: } \\
\text { Nino Facchinetti e } \\
\text { Mario Moglia. } \\
\text { Alberto Finzi } \\
\text { (architetto } \\
\text { direttore dei lavori). }\end{array}$ & $\begin{array}{l}\text { (Belinelli, 1981, p. } \\
\text { 30). } \\
\text { (Belinelli, Carlo } \\
\text { Mazzi, 1911-1988 : } \\
\text { Galleria Carlo Mazzi, } \\
\text { Tegna, 14.VI - } \\
\text { 30.VIII.1998, 1998, } \\
\text { p. 29). } \\
\text { (DPE, 1959, p. 119). } \\
\text { (AUBCBZ, s. 039 } \\
\text { Brione Verzasca). }\end{array}$ \\
\hline 1956 & Olivone. & Affresco. & Strappo. & $\begin{array}{l}\text { Pittore- } \\
\text { restauratore: } \\
\text { Carlo Mazzi. }\end{array}$ & (Archivio Mazzi). \\
\hline 1958 & $\begin{array}{l}\text { Castel San Pietro, } \\
\text { chiesa di S. Pietro } \\
\text { (detta Chiesa } \\
\text { Rossa). }\end{array}$ & Due tele. & Restauro. & & $\begin{array}{l}\text { (DPE, 1959, p. 120, } \\
\text { 122, 157). } \\
\text { (AUBCBZ, s. } 069 \\
\text { Castel San Pietro). }\end{array}$ \\
\hline
\end{tabular}




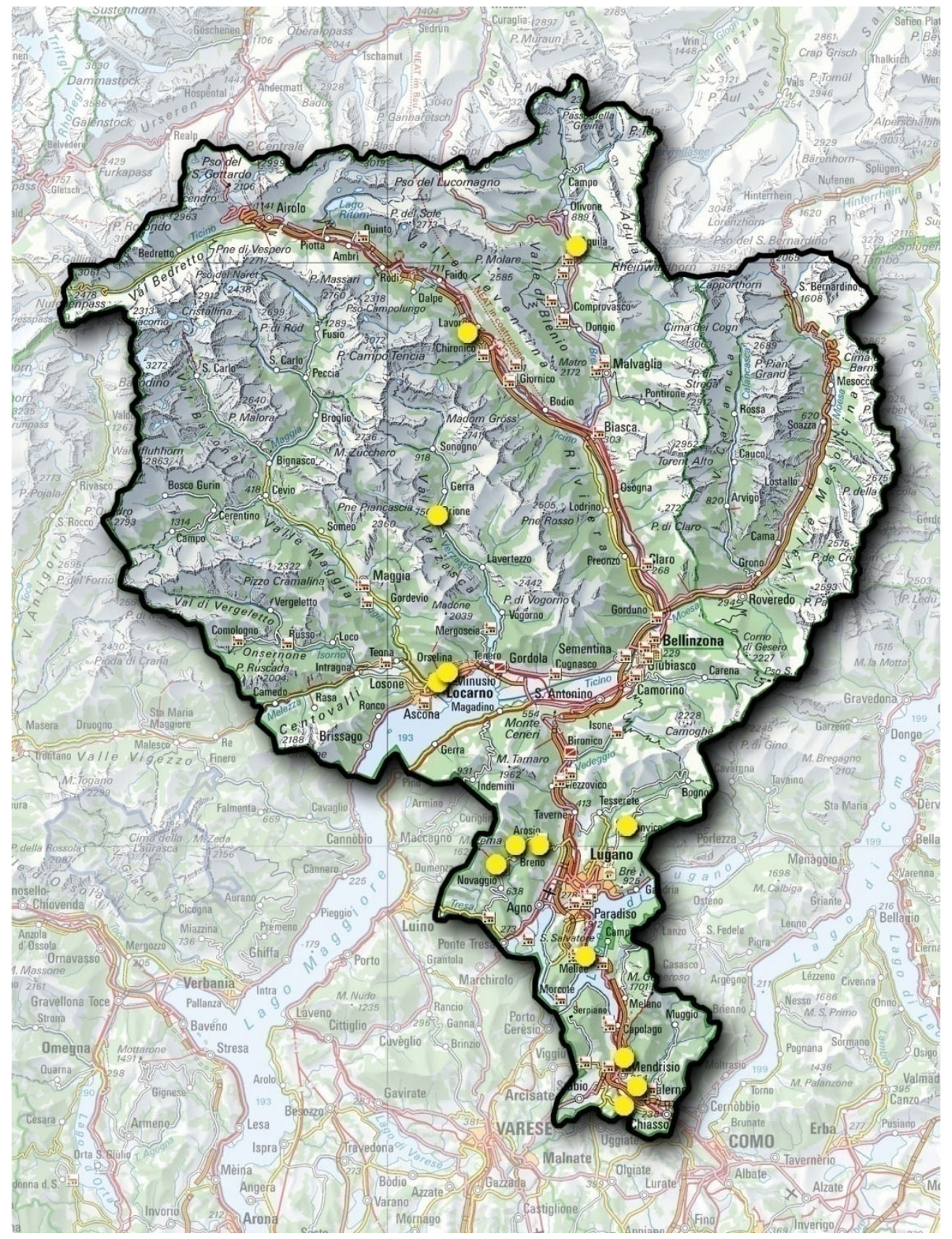

Figura 177 Cartina degli interventi realizzati da Nino Facchinetti. 


BRUNO ABBIATI 


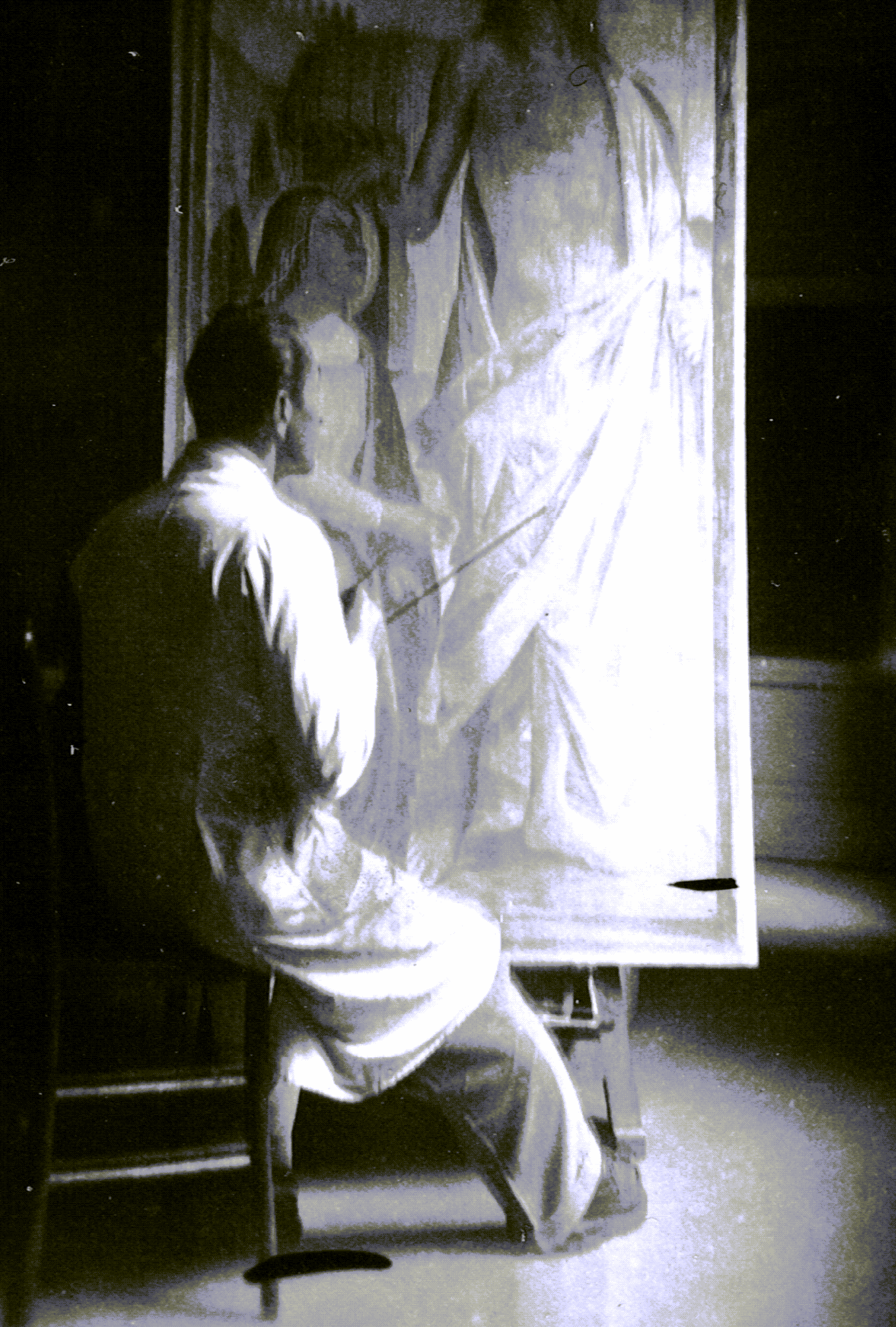




\section{BRUNO ABBIATI}

\section{Dati biografici}

Bruno Abbiati nacque il 31 gennaio del 1915 ad Arcisate (Varese) e morì il 31 agosto del 1966 a Zurigo. Carente di una particolare formazione artistica Abbiati iniziò l'attività professionale già da giovane assieme al fratello Dante, lavorando per il pittore-restauratore Mario Rossi nel suo atelier di Milano. In questo laboratorio eseguono durante gli anni Trenta numerosi interventi su opere di grande diversità provenienti da collezioni private $\mathrm{e}$ istituzioni pubbliche come ad esempio la Reale Pinacoteca di Brera o del Castello Sforzesco.

Il primo contatto che Bruno Abbiati ebbe con la Svizzera fu negli anni Quaranta quando decide di rifugiarsi qui dato l'inizio della Seconda Guerra Mondiale. Egli sarà accolto all'interno di una famiglia di Verscio, e poco più tardi riprenderà il lavoro in questo stesso paese grazie all'appoggio della potente figura del parroco Agostino Robertini ${ }^{443}$. Questo permesse ad Abbiati di concorrere con $\mathrm{i}$ restauratori ticinesi, Tita Pozzi, Emilio Ferrazzini, Nino Facchinetti, ecc. come nei capitoli precedenti si è analizzato. Per questi ultimi, pittori-restauratori, fu una grande offesa che la fiducia della Commissione Cantonale dei Monumenti Storici del Ticino cadesse sul collega italiano, essendo loro sostituiti; erano

\footnotetext{
443 Don Agostino Robertini fu parroco di Tegna e di Verscio per oltre cinquant'anni, oltre che membro della Commissione Cantonale dei Monumenti Storici dal 1955 al 1959, grazie alla sua conoscenza artistica. Fu proprio in quest'ultima località dove Bruno Abbiati risiede al suo arrivo in Svizzera.
}

momenti di difficoltà economica e questa situazione aveva provocato durante molti anni l'esecuzione di una curata protezione dal Governo Svizzero, del Cantone e dalle varie istituzioni, escludendo la concorrenza straniera anche se in nessun momento la legge stessa lo prevedesse.

Ritornato in Italia alla fine della Guerra, nel suo domicilio di Ponte Tresa, Bruno Abbiati aprì un piccolo laboratorio di restauro dove si pulirono, consolidarono e ritoccarono più di un centinaio di tele e tavole oggi documentate fotograficamente, provenienti da privati, chiese, cappelle e musei del Ticino. Nelle mani del pratico operatore, essi ricuperavano i valori estetici originari grazie alle tecniche tradizionali di intervento apprese da Mario Rossi all'interno della bottega italiana a Milano e Varese.

Purtroppo non abbiamo trovato fonti indirizzati da Abbiati in quantità sufficiente per conferirci delle indicazioni dirette riguardo le sue concezioni che avrebbero portato ad ottenere una ricerca sulla sua personalità più approfondita.

Questa possiamo ricrearla ora unicamente attraverso i ricordi del figlio, Attilio Abbiati, restauratore pure lui, e mediante la documentazione ${ }^{444}$ pertinente agli interventi che egli svolge.

Pagina precedente.

Figura 178 Fotografia b/n. Bruno Abbiati al Castello Sforzesco, Milano. Restauro d'un affresco. 1953. Archivio privato.

\footnotetext{
${ }^{4}$ L'assenza di lettere, rapporti o perizie, indirizzate dal proprio restauratore alle istituzioni tutelanti, si deve ad un incendio che distrugge l'archivio cartaceo famigliare.
} 
Si tratta in qualunque modo, malgrado le difficoltà, di una favolosa scoperta e sorprende ancora di più che nessuno prima, pur consapevole dell'esistenza del restauratore, se ne fosse interessato ad indagare nelle sue attività e competenze che furono specialmente significative nell'ambito ticinese.

Per comprendere il ruolo che Abbiati ebbe nel Canton Ticino bisogna analizzare il contesto in cui deve integrarsi già dai primi anni di lavoro.

Ci riferiamo alle polemiche generate dopo la campagna di interventi eseguiti dal 1909 ed alle diverse manifestazioni europee in divulgazione delle nuove teorie sul restauro e sulla formazione di restauratori divulgate in quei anni. Queste suggerirono le istituzioni dei beni culturali svizzere sulla necessità di avere professionisti qualificati nel settore, ma, in assenza di questi furono man mano gli stessi ispettori cantonali dei monumenti ad indirizzare gli operatori sulle basi richieste.

Particolarmente Abbiati venne sempre guidato dal suo protettore, don Robertini, verso un minimo intervento nelle numerose opere da quest'ultimo facilitate. Si può apprezzare chiaramente questa disciplina organizzativa nei restauri

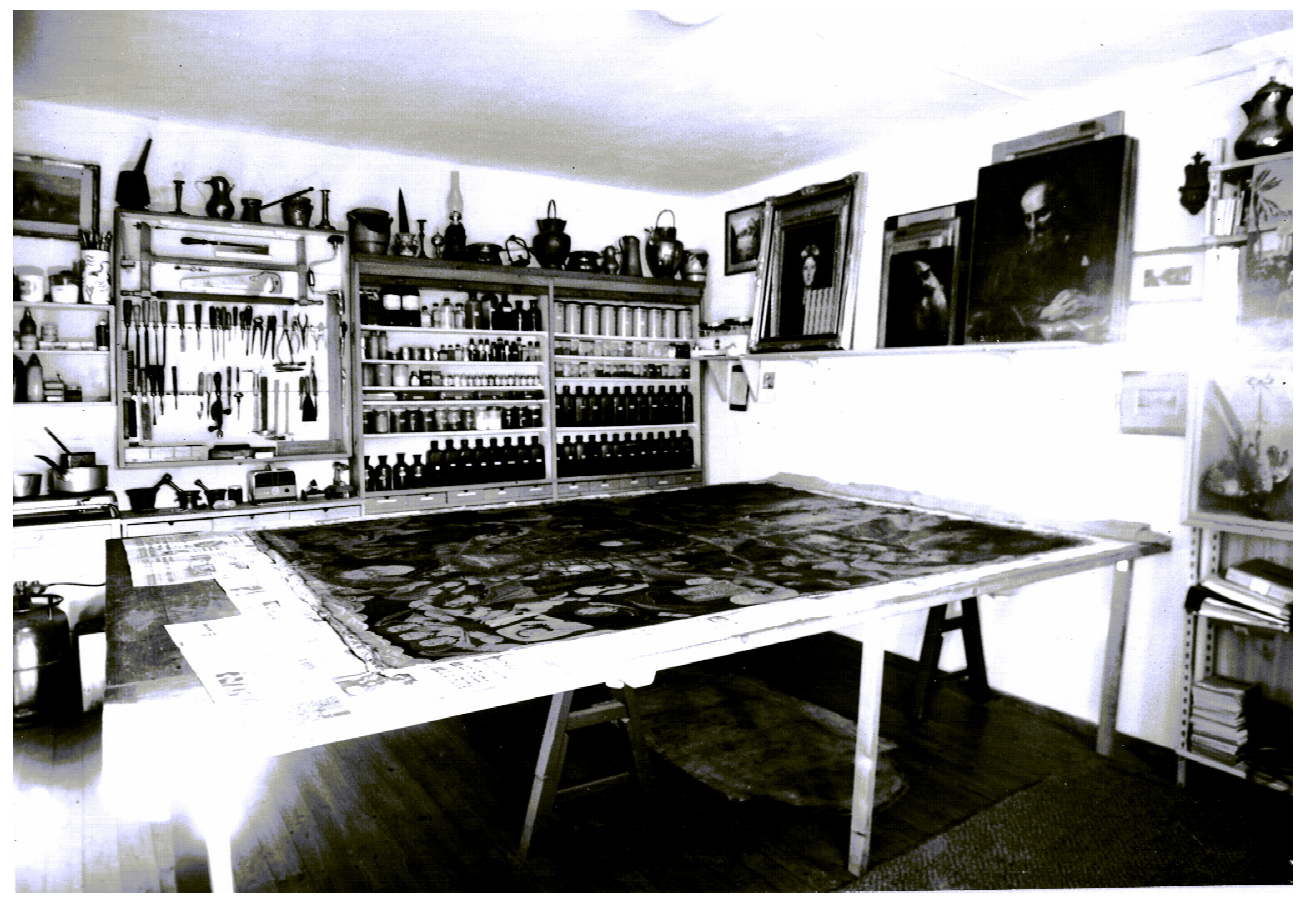

Figura 179 Abbiati Bruno. Fotografia b/n. Laboratorio di restauro a Ponte Tresa. Archivio Abbiati. 
condotti alla chiesa di S. Nicolao a Giornico, uno dei primi restauri murali eseguiti da Abbiati dopo il suo alloggio in Svizzera nel 1940 e più tardi nella chiesa primitiva di Verscio e nella chiesa di San Pietro a Biasca nel 1959, dove i lavori saranno condivisi con altri due colleghi restauratori.

Fu Tita Pozzi il primo ad avvertire che il suo collega italiano ai lavori di San Nicolao non aveva una istruzione scientifica, come invece si sollecitava dalla figura del restauratore secondo la Teoria del Restauro di Cesare Brandi, che si era già diffusa nel vicino territorio. $\mathrm{Ma}$ che formazione aveva Tita Pozzi? Per il magnifico pittore di Massagno, la conoscenza scientifica poteva significare unicamente poter distinguere fra gli "acidi e le basi", e conoscere le proprietà dei quattro materiali, come massimo, da lui impiegati (caseina, cera, caseinato di calce, silicati, ecc.) ai quali sicuramente era stato introdotto da Attilio Balmelli. Tita Pozzi ammetteva, tuttavia, che Bruno Abbiati era padrone d'una rispettosa dimestichezza ed esperienza nella professione, sebbene non sapeva descrivere le caratteristiche fisicochimiche dei materiali usati per una particolare pulitura o per un consolidamento. II restauratore italiano conosceva il prodotto che doveva utilizzare a dipendenze delle proprietà tecniche dell'opera e dei danni ritrovati in queste, ma il suo approccio ad essi erano limitati alla sola consuetudine.

Ciò nonostante gli ispettori cantonali Piero Bianconi, Ugo Donati, Pietro Salati ed anzitutto don Robertini dimostrarono di avere piena fiducia nelle facoltà pratiche del restauratore italiano ed è facile immaginare che essi vedessero in questa manualità, carente di ragionamento teorico, le possibilità per educare "ex novo" le moderne nozioni di restauro, che più difficilmente potrebbero apprendere pittori come Emilio Ferrazzini ancora combattente per i suoi propri ideali.

\section{San Fedele, Verscio}

II restauro artistico condotto nella chiesa parrocchiale di San Fedele a Verscio fu il primo esempio del cambiamento nei concetti teorici in Canton Ticino seguendo $i$ passi dell'Italia. Abbiati lavorò dal 1944 al 1946 nel ripristino artistico del monumento a spese dello Stato del Canton Ticino, dopo di ché fu realizzato il rinnovamento architettonico.

La odierna fabbrica, ricostruita fra il 1743 ed il 1748 su progetto di Francesco Antonio Bettetini ${ }^{445}$, è di pianta ottagonale allungata, contenente cinque altari barocchi, con bracci laterali poligonali e coro semicircolare. Si conservano ancora parti della primitiva navata consacrata nel 1214. L'interno della chiesa di San Fedele è decorato con pitture di Abbondio Brera eseguite nel 1852, con l'illusionistica Ancona nell'altare maggiore realizzata nel 1763 da Giovanni Antonio Caldelli ${ }^{446}$ che ora condivide il contesto spaziale con il dipinto contemporaneo raffigurante la

\footnotetext{
5 Bettetini, Francesco Antonio. Architetto. Studia all'Accademia di San Luca a Roma (1709 ca.).

Caldelli, Giovanni Antonio (Brissago 1721-1790). Figlio di Carlo Giorgio e di Maria Marta Zoppi. II pittore paesaggista e decoratore, del quale si ignora la formazione, fu influenzato dalle correnti illusionistiche e vedutiste italiane del XVIII sec. Opera in numerosi edifici di culto e profani del Canton Ticino (Brissago, Intragna, Locarno, Rasa, Verscio) e nei Paesi Basi dove ebbe sempre la considerazione del principe Carlo Alessandro di Lorena.
} 
Crocifissione (1963) dello spagnolo Joaquín Vaquero Turcios ${ }^{447}$, con la pala di altare raffigurante San Fedele, ora all'ingresso principale alla chiesa, ed un affresco nell'antica nicchia battesimale ambedue opere di Giuseppe Antonio Felice Orelli. I dipinti trecenteschi del coro raffigurano il Cristo in Maestà, gli evangelisti e l'Ascensione sulla volta a crociera, i profeti e i SS. Sebastiano e Rocco nell'intradosso dell'arco trionfale e traccia di figure d'apostoli e la rappresentazione dei mesi sulla parete destra. Nella parte sinistra invece si conservano frammenti di affreschi trasportati su tela che formavano un ciclo romanico $(1280-1290 \mathrm{ca}$.) con scene della Passione come il Bacio di Giuda e dell'Ultima Cena, opere attribuite ad un artista lombardo-piemontese. Altre opere della stessa epoca (XIII sec.) si trovano nella navata della chiesa.

\footnotetext{
${ }^{447}$ Vaquero Turcios, Joaquín (Madrid 1933). Figlio di Joaquín Vaquero Palacios, pittore di Asturias, vive la sua infanzia a Oviedo. Con la famiglia viaggerà in America Centrale e Messico prima di formarsi nella professione del padre come pittore ed scultore. Frequenterà l'Accademia di architettura di Roma dal 1950. In questa città rimarrà per sedici anni. Dopo gl studi vince diversi premi: in Italia nel 1952 guadagna il premio Internazionale Enit, e lo stesso anno in Spagna la Terza Medaglia di pittura nella Mostra Nazionale di Belle Arti di Madrid. La sua passione per la pitture murale che aveva conosciuto a Italia e in Messico, studiò le tecniche del fresco e dei mosaici. Le sue opere sono state esposte in molteplici musei di Spagna, Italia, Francia, Sudamerica, Germania ed Svizzera.
}

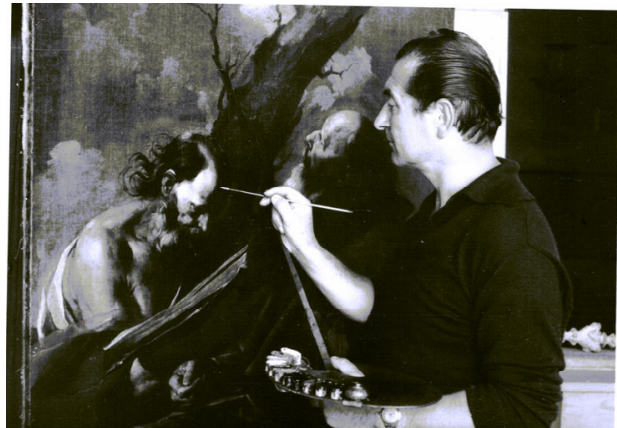

Figura 180 Bruno Abbiati nella fase di ritocco di una tela nel suo studio.

Per il restauro pittorico fu don Robertini in qualità di membro del Consiglio Parrocchiale di Verscio a proporre in novembre del 1944 l'Abbiati, alla Commissione Cantonale dei Monumenti Storici, per l'esecuzione dei lavori:

[...]Entro i prossimi mesi si vorrebbe anche procedere ad una pulitura, consolidamento, ecc. degli affreschi dell'antica abside, nell'ordine $e$ con criteri che la lod. Commis. Cant. Vorrà indicare. Nel caso favorevole noi avremmo da proporre un restauratore che dà ogni affidamento di buona riuscita[... $]^{448}$

I dipinti erano stati anni prima esaminati da Ferrazzini che aveva denunciato il degrado di essi a causa dell'umidità presente per infiltrazioni di acqua del tetto ${ }^{449}$. Purtroppo non è stato documentato da allora nessun intervento di riordino del tetto o di restauro dei dipinti, che possibilmente ebbe luogo prima dell'intervento da

\footnotetext{
448 (Robertini Agostino, Lettera indirizzata alla Lodevole CCMS. Verscio 16 novembre 1944. AUBCBZ, s.246. Verscio. San Fedele).

449 (Ferrazzini Emilio. Oratorio del 400 a Verscio. Perizia della Commissione Cantonale dei Monumenti Storici ed Artistici. Lugano, 13 luglio 1932. AUBCBZ, s.246. Verscio. San Fedele).
} 
Abbiati dodici anni dopo, nel 1944, giacché nessuna fonte riguardante questi ultimi lavori esprime lo stato di conservazione delle pitture danneggiate dall'umidità, ma sembra preoccupare unicamente lo scialbo di calce soprastanti alcune opere.

Il progetto di restauro che esegue Abbiati interessò in primo luogo il consolidamento urgente di un affresco del XII sec. esistente sulla parete a sud della navata, al quale eseguì una delicata pulitura "a secco" -così imposta dai commissari- degli scialbi in questo caso strati di calce e di scialbatura. L'eliminazione dello strato di calce fu compiuta anche sugli affreschi del coro primitivo precedentemente descritti e furono strappati anche in questo momento i frammenti dell'Ultima Cena e del Bacio di Giuda, che dai documenti di archivio non abbiamo potuto relazionare con il luogo che ebbero in origine.

[...] Per ora sarà meglio rinunciare a ulteriori tentativi di scoprire dallo scialbo l'affresco della parete di fondo con la Crocifissione; si potrà eventualmente riprendere il lavoro ripresentandosi l'occasione. Ma la parete sta bene com'è, col suo leggero gioco di colori. $[\ldots]^{450}$.

Si trattò di un restauro pittorico di massimo rispetto dove non si accoglievano in nessun modo le operazioni di ritocco, stuccature di crepe, strappi o buchi, e si accordava un consolidamento limitato al citato frammento della parete a sud, sempre sotto la vigilanza della Commissione Cantonale dei Monumenti. ${ }^{451}$

\footnotetext{
${ }^{450}$ (Bianconi Piero e Donati Ugo, Rapporto della CCMS sui lavori nella chiesa parrocchiale di Verscio. 3 febbraio 1946. AUBCBZ, s.246. Verscio. San Fedele). ${ }^{451}$ (Robertini Agostino, Lettera indirizzata alla CCMS, 4 dicembre 1944. AUBCBZ, s.246. Verscio. San Fedele).
}

Nonostante l'attesa, alla fine degli anni Cinquanta e con l'arrivo di Pierangelo Donati, in qualità di direttore dell'allora Ufficio dei Monumenti Storici, per presenziare dei cambiamenti tangibili di fatto nella chiesa parrocchiale di Verscio ancora una volta, come nella maggior parte dei monumenti intervenuti nel primo Novecento, gli stessi commissari cantonali che denunciarono le puliture eccessive e controllavano i limiti dei processi di consolidamento o di stuccatura dei dipinti primitivi, proponevano con normalità la cancellazione delle decorazioni ottocentesche per riportare la fabbrica al suo stile artistico primitivo. Con questo scopo fu realizzato anche il ripristino architettonico nel 1945 sotto progetto dell'architetto di Lugano Rino Tami, grazie al sussidio federale del $15 \%$ delle spese ${ }^{452}$. II lavoro interessò la sistemazione del tetto in piode, la ricostruzione del soffitto piano, la sistemazione del pavimento al suo livello primitivo e della finestre, la ripulitura e tinteggio delle pareti e del soffitto e la sistemazione dell'altare barocco. Compiuto questo, che era indispensabile nel processo integrativo dell'edificio, i commissari cantonali dei monumenti storici applicavano il concetto del "minimo restauro" alla possibilità di conservazione delle decorazioni "originali" settecentesche

\footnotetext{
(Dipartimento Federale dell'Interno, Lettera riguardo l'offerta di sussidio per i lavori di restauro nella primitiva chiesa di Verscio e le condizioni abituali regolamentari per la concessione di un sussidio federale, indirizzata al Reverendo don Robertini per il Consiglio parrocchiale e la Municipalità di Verscio (Ticino), in copia a I Dipartimento della pubblica educazione del cantone Ticino, Bellinzona, al sig. architetto Rino Tami, Lugano ed al sig. Birchler, Zurigo. Berna, 7 agosto 1945. AUBCBZ, s.246. Verscio. San Fedele).
} 
delle pareti, cappelle laterali e volta della navata della chiesa di San Fedele.

[...]Ill primo lavoro da compiere sarà di levar tutta la decorazione ottocentesca[...] Ritrovato cosi l'intonaco originale, si potrà decidere meglio sul di farsi: comunque è chiaro che bisognerà limitarsi a un minimo, qualche riquadratura $e$ due o tre tinte leggere, rispettando l'intento di chi ha costruito la chiesa. La navata sarà probabilmente da lasciar chiara e leggera, i punti di colore risultano il coro e i due altarini laterali; per la qual ragione si consiglia di eseguire il previsto affresco figurativi semmai sulla volta del coro e non sulla volta della navata[... $]^{453}$

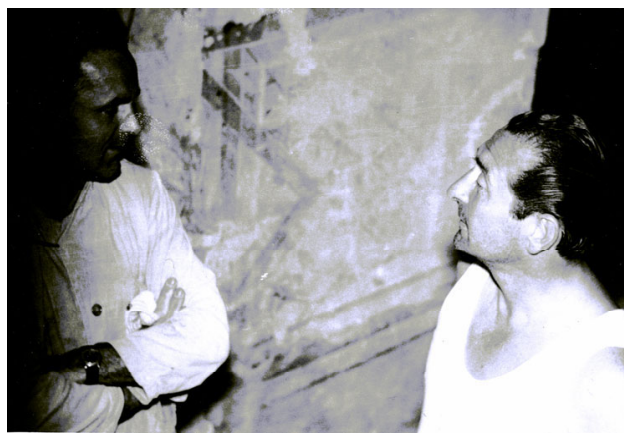

Figura 181 Bruno Abbiati (destra) durante i lavori di restauro a Biasca. Fotografia b/n, Biasca 1964 ca. Archivio privato Abbiati.

Oggi sappiamo, perché possiamo ancora gradire della loro presenza, che le decorazioni del XIX sec. opera di Abbondio Brera furono mantenute, però ancora una volta la mancanza di documentazione a riguardo limita lo studio dell'evento.

\footnotetext{
${ }^{453}$ (Donati Ugo, Salati Pietro e Bianconi Piero membr della CCMS. Restauro dell'interno della parrocchiale d Verscio. Minusio, 28 marzo 1946. AUBCBZ, s.246. Verscio. San Fedele).
}

\section{SS. Pietro e Paolo, Biasca}

All'interno della chiesa medievale dei SS. Pietro e Paolo a Biasca ${ }^{454}$, gli interventi di restauro generale della fabbrica ebbero luogo fra il 1955 ed il 1967 sotto il progetto dell'architetto Alberto Camenzind. Egli riordinò la fabbrica secondo le forme stilistiche romaniche (XI - XII sec.) sopprimendo l'ossario e la sagrestia a nord e le decorazioni aggiunte in epoca barocca. Contemporaneamente si concepì il restauro artistico iniziato nel 1959 e concesso ai restauratori Bruno Abbiati, Carlo Mazzi $^{455}$ e Mario Moglia e fu rifatto il soffitto ligneo a cassettoni delle tre navate, secondo il disegno del pittore e commissario cantonale dei monumenti storici artistici del Canton Ticino, Pietro Salati, ispirato all'originale soffitto quattrocentesco di cui si trovarono diversi frammenti nel corso dei restauri.

Trattandosi di un vasto manufatto, le aeree di lavoro all'interno della chiesa dei SS. Pietro e Paolo di Biasca furono divise in tre con lo scopo di facilitare le operazioni:

1. gli affreschi di Antonio da Tradate sull'abside semicircolare fino all'arco trionfale (Volto Santo e apostoli, due angeli inginocchiati e sopra S. Pietro, giovanetto con brocca d'acqua e sopra S. Paolo, nel sottarco; S. Ambrogio, S. Nicola, Pietro incontra Cristo alle porte di Roma, il diacono Filippo battezza Simon Mago e la Majestas Domini con gli evangelisti) furono assegnati all'Abbiati

\footnotetext{
${ }^{454}$ La chiesa odierna risale al tardo XI o inizi del XII. (Simona Martinoli, 2007, p. 71).

${ }^{455}$ Mazzi Carlo (Tegna 1911-1988).

http://www.ticino.ch/appendix/contempla/pdf/ita_co ntempla4.pdf.
} 
2. gli affreschi Seicenteschi (Scene della vita di S. Carlo Borromeo eseguite nel 1620 da Alessandro Gorla sulla navata a sud e S. Giovanni Battista e S. Antonio di Padova sul primo e secondo pilastro a destra rispettivamente) furono incaricati a Carlo Mazzi;

3. gli affreschi più antichi quattro e cinquecenteschi furono affidati al pittore Mario Moglia, mentre la proposta di lavoro veniva elaborata unicamente dai commissari cantonali dei monumenti storici, Pietro Salati e Piero Bianconi

[...]II 28 gennaio 1959 il prof. Piero Bianconi e il sottoscritto, dopo ulteriore e attento esame dei lavori riguardanti il restauro pittorico [...] hanno così dettagliatamente precisato l'intervento dei restauratori nella prima fase. $[. . .]^{456}$.

Percependo la complessità degli elementi le operazioni vennero divise in due fasi: la prima consistente nella pulitura e nel consolidamento degli affreschi e l'ultima interessata alla reintegrazione pittorica eseguita dai pittori, che in questo ruolo vengono chiamati "colorai-restauratori" ${ }^{457}$. La pulitura fu preceduta da indagini su tutte le pareti per verificarne lo stato dei dipinti coperti dalle pitture seicentesche e dall'eliminazione dello strato di calce o d'intonaco soprastante. A continuazione il consolidamento venne dedicato unicamente alle zone pericolanti, in questo caso solo piccoli frammenti sulla parete in fondo alla navata di destra.

\footnotetext{
${ }^{456}$ (Condizioni per il restauro pittorico. San Pietro di Biasca. Viganello 30 gennaio 1959. Firmato Pietro Salati.

${ }^{457}$ Così denominati nello scritto precedentemente citato; Salati Pietro, Viganello 30 gennaio 1959.
}

Qui Abbiati, incaricato unicamente dell'abside, ebbe il mandato di rimuovere la scialbatura di calce sulla parte inferiore e di esaminare le condizioni dei dipinti sottostanti le pitture seicentesche e settecentesche senza danneggiare questi ultimi. A continuazione venne richiesta la pulitura di tutti gli affreschi perfino quelli che successivamente sarebbero strappati del XVII sec. nel 1965.

La reintegrazione pittorica fu anche differenziata secondo lo stato di conservazione degli affreschi e lo stile, figurativo o decorativo. In questo modo i dipinti di cui rimanevano soltanto alcune tracce furono ritoccati a rigatino, seguendo le forme ed i colori; le lacune appartenenti alla figurazione furono reintegrate con una tonalità grigia alla quale si attribuiva il carattere "neutro", mentre le lacune delle parti decorative furono reintegrate a basso tono.

L'intervento eseguito dagli altri due pittorirestauratori, Carlo Mazzi e Mario Moglia, da quanto riportato nelle lettere di corrispondenza con la CCMS negli anni di lavoro, fu molto simile. Solo l'articolo di Mario Moglia 458 all'interno del libro "S. Pietro di Biasca" della Commissione ProRestauri pubblicato nel 1967 offre delle informazioni complementarie al materiale d'archivio. Di fatto le descrizioni riportate dal restauratore nel libro segnalano nuove operazioni di restauro applicate sui dipinti Quattrocenteschi della navata, dei pilastri e delle facciate esterne. Essi vengono consolidati da Mario Moglia con caseinato di calcio in modo da fissare gli intonaci pericolanti e furono puliti dal cemento e calce soprastante. Le fesure ed i segni delle

\footnotetext{
${ }^{58}$ (Moglia, 1967, p. 195-210).
} 
martellinature vengono chiusi e si ricostruirono l'arricicio e l'intonaco nelle parti mancanti, posteriormente reintegrate a fresco. Non fu possibile - spiega Mario Moglia - eliminare le macchie di nero fumo sulla superficie del dipinti, e fu ugualmente difficile il descialbo dello strato di calce, la cui operazione si realizzò con grande cura grazie all'aiuto di una lente per evitare lo stacco della pelicolla pittorica dei ritocchi a secco, che malgrado l'attenzione non fu possibile conservare. II "restauro pittorico", cioè la reintegrazione cromatica degli affreschi avvenne nel 1964. L'operazione venne realizzata da Mario Moglia e dal pittore Sergio Maino, secondo le premesse precedentemente elencate, adeguando la tecnica ad ogni situazione di stile e di stato di conservazione apparse nel complesso decorativo: le lacune ed stuccature furono reintegrate con tonalità neutre a fresco, mentre si ricostruirono i motivi geometrici che incorniciano $i$ dipinti. I fondi ed $i$ contorni delle figure si rittocarono parzialmente o totalmente a seconda dei casi con tempera all'uovo sulle parti originali danneggiate con lo scopo d'omogeneizzare l'aspetto generale. Negli affreschi esterni raffiguranti il gigante San Cristoforo (XII sec.) in facciata ed i frammenti della Pietà e la Madonna con Bambino (XV sec.) a destra della porta laterale furono reintegrati nelle parti originali fu compiuta con colori al silicato dalla Keim, e nel caso del S. Cristoforo questi rittocchi si sovraposero agli elementi di interveno precedente realizzato dal Ferrazzini nel 1944. Mario Moglia descrive come tutti i dipinti, in generale, si trattarono con acqua di calce che permette di consolidare le scaglie staccate di colore e inoltre, all'interno, le tonalità degli affreschi furono fatte rinvenire mediante l'applicazione di una soluzione di gomma arabica.

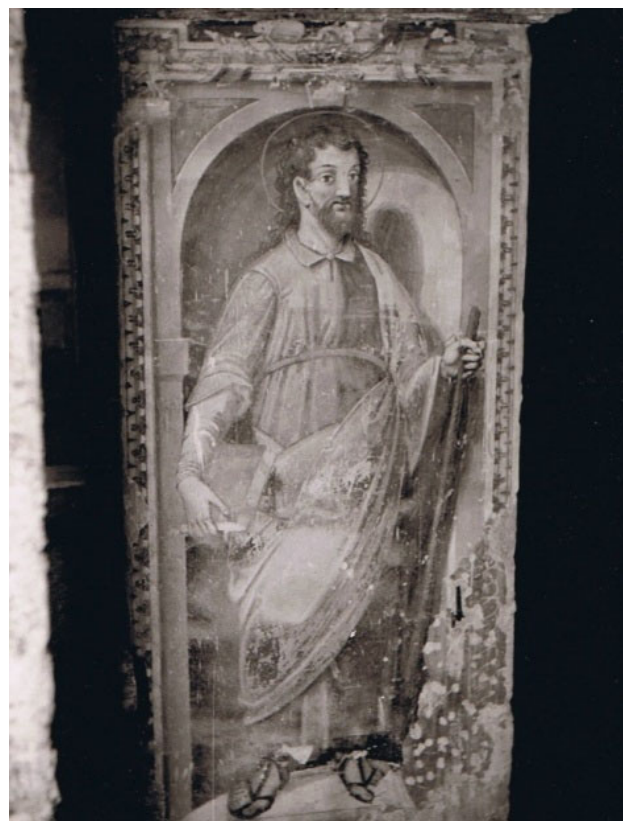

Figura 182 Bruno Abbiati, Particolare di affresco nella chiesa dei SS. Pietro e Paolo a Biasca, durante i lavori di restauro. Fotografia b/n. 1964 ca. Archivio privato. 
Cinque anni dopo, come sopraccennato, I'Ufficio dei Monumenti Storici decise di rendere visibili gli affreschi quattrocenteschi dell'abside restaurato dall'Abbiati. Con l'appoggio di Mario Moglia, il restauratore italiano strappa i quattordici $^{459}$ dipinti seicenteschi dalla tazza absidiale così come un frisio dello stesso periodo che denunciò l'esistenza di una decorazione sottostante simile però in cattivo stato di conservazione. Essi strappi si trasportarono su tela e sul supporto rigido si reintagrarono le lacune con malta a base di sabbia fine colorata, ricostruendo la decorazione del contorno. II rittocco pittorico delle figure fu eseguito con tempera all'uovo e, anche in questo caso, $\mathrm{i}$ dipinti si trattarono con la soluzione di gomma arabica che permise di ravvivare $i$ colori a fresco sbiaditi dopo la sucessione di operazioni di distacco, trasporto e pulitura. Finalmente i pannelli si collocarono sulle colonne verso la navata inferiore e sulla controfacciata.

Non fu considerato il distacco degli affreschi dell'arco trionfale e delle figure delle colonne. A continuazione le pitture scoperte furono pulite dallo strato di calce soprastante e si consolidarono le zone staccate prima di procedere alla reintegrazione cromatica delle lacune stuccate.

Si tratta di un momento molto importante che testimonia la convinzione nel testo divulgato dello storico d'arte italiano,
Cesare Brandi, anche in Ticino. Abbiamo scritto precedentemente termini come il "minimo restauro", "rigatino", "lacuna", queste non definiscono altro che la necessità di differenziare l'evento restauro per assimilazione dei concetti di rispetto e di originalità dell'opera.

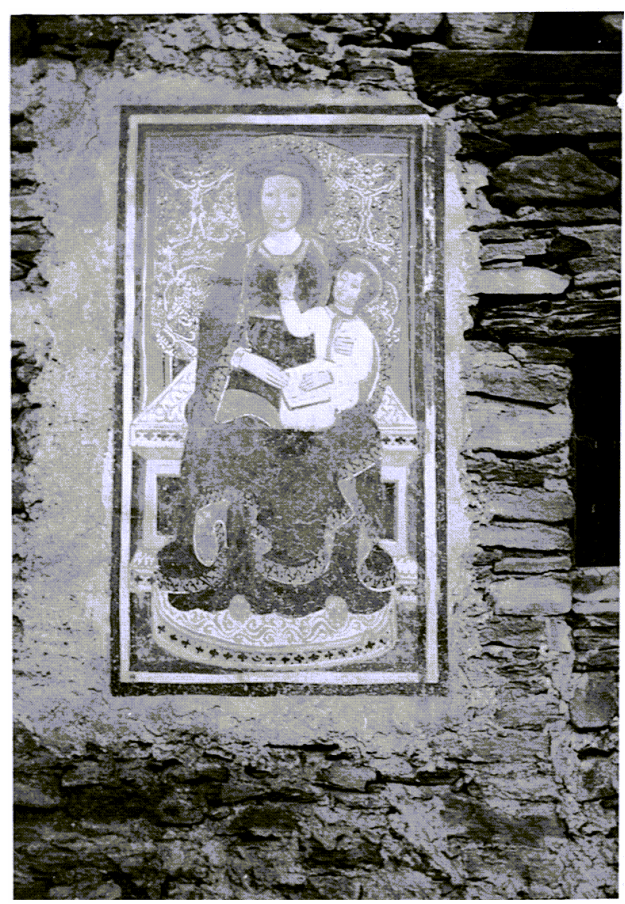

Figura 183 Bruno Abbiati. Particolare di affresco. Fotografia b/n senza identificazione. Archivio privato.

459 Le misure dei 14 elementi strappati sono state riportate nella tesi di bachelor della restauratrice Manuela Valentini (Valentini, 2005, p. 60): (91 x 200 $\mathrm{cm}),(91,5 \times 188,5 \mathrm{~cm}),(91 \times 182 \mathrm{~cm}),(41 \times 62 \mathrm{~cm})$, $(63 \times 102 \mathrm{~cm}),(85 \times 186 \mathrm{~cm}),(55 \times 82 \mathrm{~cm}),(71 \times 300$ $\mathrm{cm}),(88,5 \times 197 \mathrm{~cm}),(87 \times 100 \mathrm{~cm}),(87 \times 45 \mathrm{~cm}),(200$ x $150 \mathrm{~cm}),(71 \times 300 \mathrm{~cm}),(41 \times 62 \mathrm{~cm})$. 


\section{Interventi di restauro}

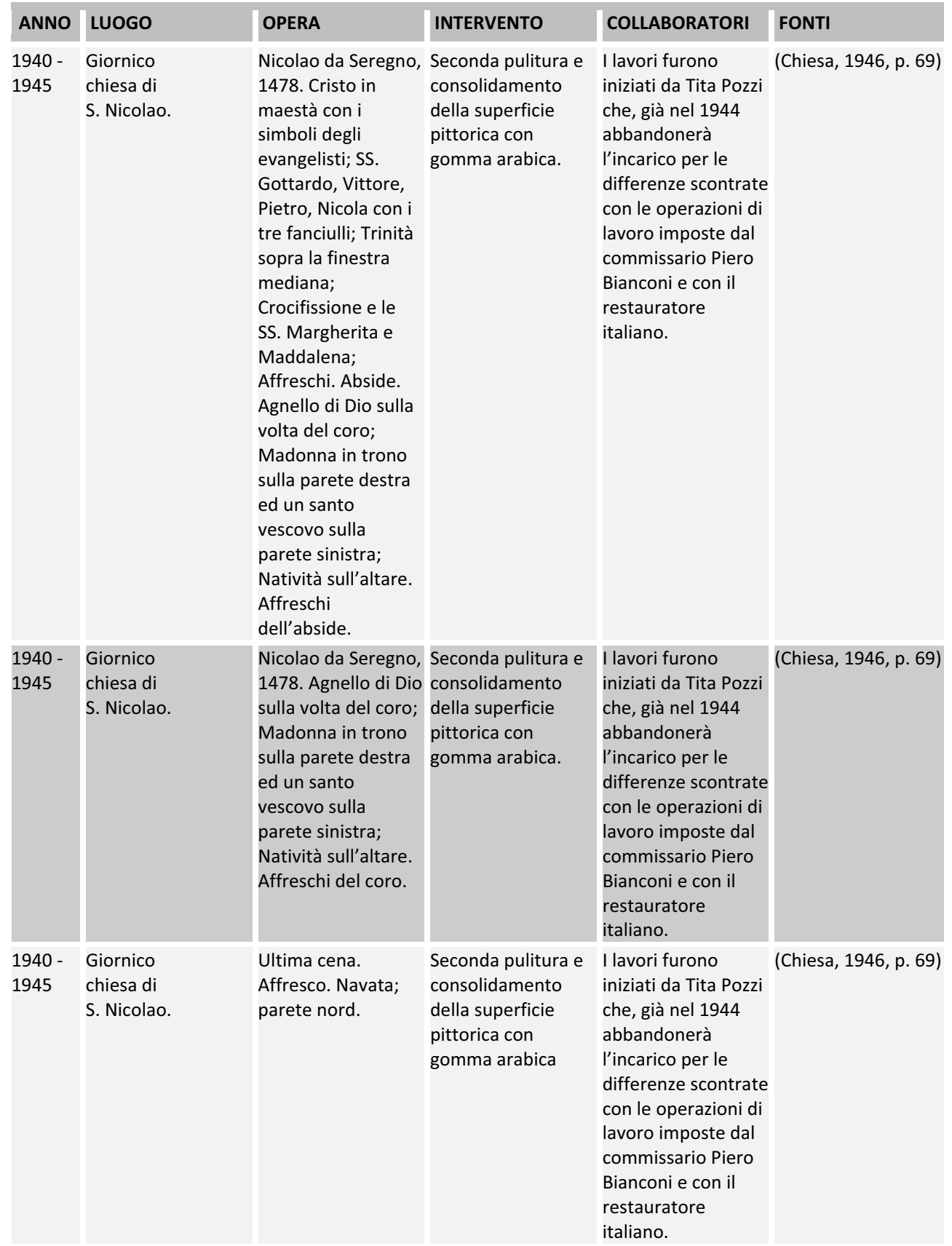




\begin{tabular}{|c|c|c|c|c|c|}
\hline $\begin{array}{l}1940 \text { - } \\
1945\end{array}$ & $\begin{array}{l}\text { Giornico } \\
\text { chiesa di } \\
\text { S. Nicolao. }\end{array}$ & $\begin{array}{l}\text { Frammenti di un } \\
\text { monaco e di un } \\
\text { vescovo di una } \\
\text { teoria di santi. XIII } \\
\text { sec. Affreschi. } \\
\text { Navata; parete sud. }\end{array}$ & $\begin{array}{l}\text { Seconda pulitura e } \\
\text { consolidamento } \\
\text { della superficie } \\
\text { pittorica con } \\
\text { gomma arabica. }\end{array}$ & $\begin{array}{l}\text { I lavori furono } \\
\text { iniziati da Tita Pozzi } \\
\text { che, già nel } 1944 \\
\text { abbandonerà } \\
\text { l'incarico per le } \\
\text { differenze scontrate } \\
\text { con le operazioni di } \\
\text { lavoro imposte dal } \\
\text { commissario Piero } \\
\text { Bianconi. }\end{array}$ & (Chiesa, 1946, p. 69) \\
\hline $\begin{array}{l}1944- \\
1945\end{array}$ & $\begin{array}{l}\text { Verscio, } \\
\text { chiesa parrocchiale } \\
\text { di S. Fedele. }\end{array}$ & $\begin{array}{l}\text { Cristo in maestà, gli } \\
\text { evangelisti e } \\
\text { Ascensione. XV sec. } \\
\text { Affreschi. Volta a } \\
\text { crociera del coro } \\
\text { primitivo. }\end{array}$ & $\begin{array}{l}\text { Rimozione dello } \\
\text { strato di calce } \\
\text { soprastante, } \\
\text { pulitura generale e } \\
\text { consolidamento } \\
\text { degli affreschi } \\
\text { rovinati } \\
\text { dall'umidità. }\end{array}$ & & $\begin{array}{l}\text { (Chiesa, 1946, p. 84) } \\
\text { (DPE, 1959, p. 126) } \\
\text { (AUBCBZ, s.246. } \\
\text { Verscio. San } \\
\text { Fedele). }\end{array}$ \\
\hline $\begin{array}{l}1944- \\
1945\end{array}$ & $\begin{array}{l}\text { Verscio, } \\
\text { chiesa parrocchiale } \\
\text { di S. Fedele. }\end{array}$ & $\begin{array}{l}\text { Profeti e SS. } \\
\text { Sebastiano e Rocco. } \\
\text { XV sec. Affreschi. } \\
\text { Intradosso dell'arco } \\
\text { trionfale. }\end{array}$ & $\begin{array}{l}\text { Rimozione dello } \\
\text { strato di calce } \\
\text { soprastante, } \\
\text { pulitura generale e } \\
\text { consolidamento } \\
\text { degli affreschi } \\
\text { rovinati } \\
\text { dall'umidità. }\end{array}$ & & $\begin{array}{l}\text { (Chiesa, 1946, p. 84) } \\
\text { (DPE, 1959, p. 126) } \\
\text { (AUBCBZ, s.246. } \\
\text { Verscio. San } \\
\text { Fedele). }\end{array}$ \\
\hline $\begin{array}{l}1944- \\
1945\end{array}$ & $\begin{array}{l}\text { Verscio, } \\
\text { chiesa parrocchiale } \\
\text { di S. Fedele. }\end{array}$ & $\begin{array}{l}\text { Frammenti di figure } \\
\text { d'apostoli e di } \\
\text { raffigurazioni dei } \\
\text { mesi. XV sec. } \\
\text { Affreschi. Parete } \\
\text { destra. Coro } \\
\text { primitivo. }\end{array}$ & $\begin{array}{l}\text { Rimozione dello } \\
\text { strato di calce } \\
\text { soprastante, } \\
\text { pulitura generale e } \\
\text { consolidamento } \\
\text { degli affreschi } \\
\text { rovinati } \\
\text { dall'umidità. }\end{array}$ & & $\begin{array}{l}\text { (Chiesa, 1946, p. 84) } \\
\text { (DPE, 1959, p. 126) } \\
\text { (AUBCBZ, s.246. } \\
\text { Verscio. San } \\
\text { Fedele). }\end{array}$ \\
\hline 1944 & $\begin{array}{l}\text { Tegna, oratorio } \\
\text { della Madonna } \\
\text { delle Scalate o di S. } \\
\text { Anna. }\end{array}$ & $\begin{array}{l}\text { Madonna con } \\
\text { bambino, metà XVI } \\
\text { sec. Affresco. }\end{array}$ & Restauro. & & \\
\hline 1958 & $\begin{array}{l}\text { Arzo, } \\
\text { oratorio della } \\
\text { Madonna del } \\
\text { Ponte. }\end{array}$ & $\begin{array}{l}\text { Madonna in trono } \\
\text { col bambino, XV } \\
\text { sec. Affresco. Altare } \\
\text { maggiore. }\end{array}$ & Restauro. & & $\begin{array}{l}\text { (DPE, 1959, p. 117) } \\
\text { (AUBCBZ, s. 010, } \\
\text { Arzo. Madonna del } \\
\text { Ponte). }\end{array}$ \\
\hline 1959 & $\begin{array}{l}\text { Biasca, } \\
\text { chiesa } \\
\text { prepositurale dei } \\
\text { SS. Pietro e Paolo. }\end{array}$ & $\begin{array}{l}\text { Affreschi, XVII sec. } \\
\text { Abside. }\end{array}$ & $\begin{array}{l}\text { Rimozione dello } \\
\text { strato di calce } \\
\text { superficiale e } \\
\text { consolidamento. } \\
\text { Indagini sui dipinti } \\
\text { cinquecenteschi } \\
\text { sottostanti. }\end{array}$ & & $\begin{array}{l}\text { (Renato, 1967) } \\
\text { (Moglia, 1967, p. } \\
\text { 201). }\end{array}$ \\
\hline
\end{tabular}


Biasca,

chiesa

prepositurale dei

SS. Pietro e Paolo.
Affreschi XVII sec. Abside.
Biasca,

chiesa

prepositurale dei SS. Pietro e Paolo.
Strappo di

quattordici figure e

frammenti.

Ricollocamento sui

pilastri della chiesa

verso la navata

inferiore e sulla

controfacciata. Gli

elementi distaccati

furono trasportati

su tela e

successivamente su

telai rigidi. Le

lacune furono

reintegrate con

malta a base di

sabbie fine

colorate.

Reintegrazione con

tempera all'uovo.

Trattamento con

gomma arabica per

ravvivare i colori.

Misure dei dipinti

strappati e

ridimensionati: (91

$x 200 \mathrm{~cm}),(91,5 \mathrm{x}$

$188,5 \mathrm{~cm}),(91 \times 182$

$\mathrm{cm}),(41 \times 62 \mathrm{~cm})$,

$(63 \times 102 \mathrm{~cm}),(85 \mathrm{x}$

$186 \mathrm{~cm}),(55 \times 82$

$\mathrm{cm}),(71 \times 300 \mathrm{~cm})$,

$(88,5 \times 197 \mathrm{~cm}),(87$

$x 100 \mathrm{~cm}),(87 \times 45$

$\mathrm{cm}),(200 \times 150 \mathrm{~cm})$,

$(71 \times 300 \mathrm{~cm}),(41 \mathrm{x}$

$62 \mathrm{~cm})$.

Antonio da Tradate

Cristo in maestà,

Scene degli Atti

degli apostoli. Ciclo.

Affreschi. Abside.

\section{Rimozione dello}

strato di scialbo di

calce soprastanti,

successiva allo

strappo degli

affreschi
Pittore-

restauratore:

Mario Moglia.

p. 71).

seicenteschi.

Pulitura generale,

consolidamento

degli intonaci con

caseinato di calcio,

e degli affreschi con

acqua di calce.

Stuccatura delle lacune e 

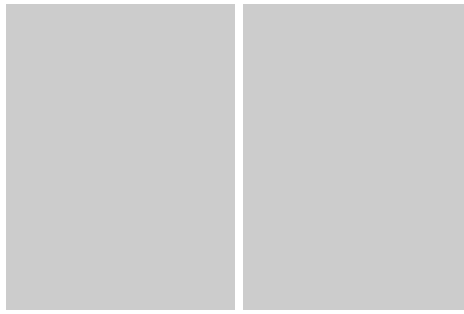

reintegrazione

pittorica con

tonalità neutre $a$

fresco nelle parti

mancanti, e

rigattino con

tempera d'uovo

nelle parti

figurative.

\section{Preventivi di restauro}

\begin{tabular}{|c|c|c|c|c|c|}
\hline ANNO & LUOGO & OPERA & INTERVENTO & COLLABORATORI & FONTI \\
\hline $\begin{array}{l}30 \text { giu. } \\
1960\end{array}$ & $\begin{array}{l}\text { Locarno, Orselina, } \\
\text { Madonna del Sasso. }\end{array}$ & $\begin{array}{l}\text { Calvario. } \\
\text { Tavola centrale } \\
\text { nell'altare della } \\
\text { Pietà. } \\
(129 \times 216 \mathrm{~cm}) .\end{array}$ & $\begin{array}{l}\text { Preventivo di } \\
\text { restauro. } \\
\text { La tavola esaminata } \\
\text { dal restauratore } \\
\text { divisa in due parti, } \\
\text { presentava tre liste } \\
\text { sul retro fissate con } \\
\text { viti. Lo strato } \\
\text { pittorico era } \\
\text { parzialmente } \\
\text { staccato a causa dei } \\
\text { movimenti della } \\
\text { tavola e si } \\
\text { osservavano } \\
\text { particelle di colore } \\
\text { già cadute. La } \\
\text { proposta di } \\
\text { intervento } \\
\text { presentata da } \\
\text { Abbiati interessava } \\
\text { una } \\
\text { documentazione } \\
\text { fotografica e messa } \\
\text { in sicurezza con } \\
\text { carta giapponese e } \\
\text { colletta della } \\
\text { pellicola pittorica } \\
\text { per il trasporto, la } \\
\text { rimozione delle liste } \\
\text { di legno sul retro a } \\
\text { la sostituzione di } \\
\text { queste per altre } \\
\text { mobili che } \\
\text { permettano il } \\
\text { naturale } \\
\text { movimento della } \\
\text { tavola di legno a }\end{array}$ & & $\begin{array}{lr}\text { (AUBCBZ, s. } & 183 \\
\text { Orselina, Madonna } \\
\text { del Sasso) } \\
\text { (Abbiati } \\
\text { Preventivo Bruno, } \\
\text { restauro indirizzato } \\
\text { al DPE. Ponte Tresa } \\
30 \text { giugno } 1960 . \\
\text { AUBCBZ, s. } 183 \\
\text { Orselina, Madonna } \\
\text { del Sasso). }\end{array}$ \\
\hline
\end{tabular}




\begin{tabular}{|c|c|c|c|c|}
\hline & & & $\begin{array}{l}\text { seconda } \\
\text { dell'umidità. } \\
\text { Iniezioni di colletta } \\
\text { nelle scaglie di } \\
\text { colore per } \\
\text { consolidarle al } \\
\text { supporto. Pulitura } \\
\text { del sudiciume di } \\
\text { polvere e } \\
\text { sverniciatura. } \\
\text { Stuccatura delle } \\
\text { piccole lacune e } \\
\text { reintegrazione } \\
\text { pittorica. Il totale } \\
\text { del preventivo } \\
\text { proposto era di fr. } \\
\text { 2'000. }\end{array}$ & \\
\hline $\begin{array}{l}30 \text { giu. } \\
1960\end{array}$ & $\begin{array}{l}\text { Locarno, Orselina, } \\
\text { Madonna del Sasso. }\end{array}$ & $\begin{array}{l}\text { S.M.Maddalena e } \\
\text { Pia Donna, tavole } \\
\text { laterali } \\
(49 \times 120 \mathrm{~cm}) .\end{array}$ & $\begin{array}{l}\text { Preventivo di } \\
\text { restauro } \\
\text { interessato nella } \\
\text { documentazione } \\
\text { fotografica previa al } \\
\text { lavoro, pulitura del } \\
\text { sudiciume e } \\
\text { sverniciatura, } \\
\text { stuccature delle } \\
\text { lacune e } \\
\text { reintegrazione } \\
\text { pittorica per un } \\
\text { totale di fr. } 500 \text {. }\end{array}$ & $\begin{array}{l}\text { (AUBCBZ, s. } 183 \\
\text { Orselina, Madonna } \\
\text { del Sasso) } \\
\text { (Abbiati Bruno, } \\
\text { Preventivo di } \\
\text { restauro indirizzato } \\
\text { al DPE. Ponte Tresa } \\
30 \text { giugno } 1960 . \\
\text { AUBCBZ, s. } 183 \\
\text { Orselina, Madonna } \\
\text { del Sasso). }\end{array}$ \\
\hline $\begin{array}{l}30 \text { giu. } \\
1960\end{array}$ & $\begin{array}{l}\text { Locarno, Orselina, } \\
\text { Madonna del Sasso. }\end{array}$ & $\begin{array}{l}\text { Angelo } \\
\text { Annunciante, tavola } \\
\text { davanti alla Mensa } \\
\text { dell'Altare. (112 X } \\
66 \mathrm{~cm}) \text {. }\end{array}$ & $\begin{array}{l}\text { Preventivo di } \\
\text { restauro } \\
\text { interessato nella } \\
\text { documentazione } \\
\text { fotografica, pulitura } \\
\text { del sudiciume e } \\
\text { sverniciatura, } \\
\text { stuccature delle } \\
\text { lacune e } \\
\text { reintegrazione } \\
\text { pittorica per un } \\
\text { totale di fr. } 600 \text {. }\end{array}$ & $\begin{array}{l}\text { (AUBCBZ, s. } 183 \\
\text { Orselina, Madonna } \\
\text { del Sasso) } \\
\text { (Abbiati Bruno, } \\
\text { Preventivo di } \\
\text { restauro indirizzato } \\
\text { al DPE. Ponte Tresa } \\
30 \text { giugno } 1960 . \\
\text { AUBCBZ, s. } 183 \\
\text { Orselina, Madonna } \\
\text { del Sasso). }\end{array}$ \\
\hline $\begin{array}{l}24 \text { dic. } \\
1961\end{array}$ & $\begin{array}{l}\text { Bellinzona, } \\
\text { Oratorio di S. } \\
\text { Marta. }\end{array}$ & & Preventivo. & $\begin{array}{l}\text { (Abbiati Bruno, } \\
\text { Preventivo di } \\
\text { restauro indirizzato } \\
\text { al DPE. Ponte Tresa } \\
24 \text { dicembre } 1961 . \\
\text { AUBCBZ, s. } 021 \\
\text { Bellinzona, Oratorio } \\
\text { di Santa Marta) }\end{array}$ \\
\hline
\end{tabular}




\begin{tabular}{|c|c|c|c|c|c|}
\hline $\begin{array}{l}16 \text { lug. } \\
1962\end{array}$ & $\begin{array}{l}\text { Caslano, } \\
\text { Capella della } \\
\text { Magliasina. } \\
\text { Cappella Greppi. }\end{array}$ & Affreschi. & $\begin{array}{l}\text { Perizia. II } \\
\text { restauratore } \\
\text { propone lo strappo } \\
\text { degli affreschi: } \\
\text { [...]Un restauro } \\
\text { pittorico non } \\
\text { risolverebbe niente, } \\
\text { lascerebbe il male, } \\
\text { che condurrebbe gli } \\
\text { affreschi a totale } \\
\text { distruzione entro un } \\
\text { periodo di non più } \\
\text { di } 50 \text { anni.[...]. } \\
\text { Prima di realizzare } \\
\text { un preventivo } \\
\text { raccomanda la } \\
\text { visita dei membri } \\
\text { della CCMS, per } \\
\text { constatare la } \\
\text { gravità dello stato } \\
\text { di conservazione e } \\
\text { l'impossibilità di } \\
\text { conservare sul } \\
\text { posto dette pitture. }\end{array}$ & $\begin{array}{l}\text { Tarabori } \\
\text { Salati. } \\
\end{array}$ & $\begin{array}{l}\text { (AUBCBZ, s. } 067 \\
\text { Caslano. Cappella } \\
\text { Greppi) } \\
\text { (Abbiati Bruno, } \\
\text { Perizia indirizzata } \\
\text { alla CCMSA. Ponte } \\
\text { Tresa, } 16 \text { luglio } \\
\text { 1962. AUBCBZ, s. } \\
\text { 067 Caslano. } \\
\text { Cappella Greppi). }\end{array}$ \\
\hline $\begin{array}{l}19 \text { mag. } \\
1962\end{array}$ & $\begin{array}{l}\text { Morcote, } \\
\text { Chiesa di S. Maria } \\
\text { del Sasso. }\end{array}$ & $\begin{array}{l}\text { Affreschi e tele ad } \\
\text { olio. }\end{array}$ & $\begin{array}{l}\text { Preventivo di } \\
\text { restauro di tutte le } \\
\text { opere. }\end{array}$ & & $\begin{array}{l}\text { (AUBCBZ, s. } 172 \\
\text { Morcote, S. Maria } \\
\text { del Sasso). }\end{array}$ \\
\hline
\end{tabular}




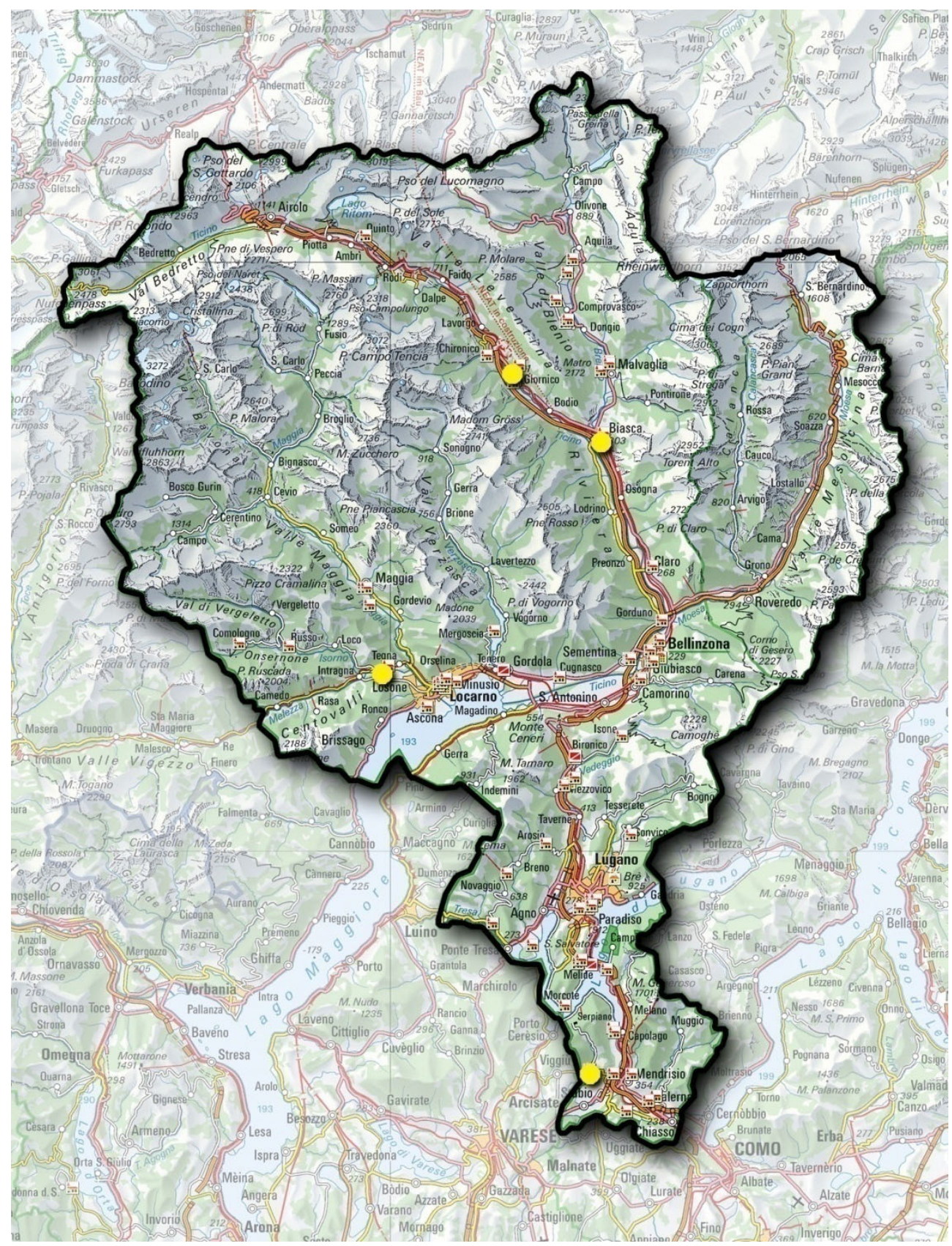

Figura 184 Cartina degli interventi eseguiti da Bruno Abbiati in Canton Ticino. 


Maris Mortis 


\section{MARIO MOGLIA}

\section{Dati biografici}

Mario Moglia è stato senza dubbio uno degli artisti meno studiati del Novecento ticinese. Contemporaneo ed amico dei pittori Carlo Cotti, Mario Ribola e Filippo Boldini $^{460}$, la sua persona fu ricordata singolarmente solo nel 1997, undici anni dopo la sua morte, in una mostra organizzata alla Gallerie Mosaico di Chiasso che ricompilava alcune incisioni da lui prodotte fra il 1944 ad il $1972^{461}$. Successivamente altre tre mostre al Museo d'Arte di Mendrisio (2002) $)^{462}$, alla Pinacoteca Cantonale Giovanni Züst a Rancate $(2003)^{463}$ ed al Museo Civico di Belle Arti a Lugano $(2004)^{464}$ hanno mostrato opere sue insieme ad alcuni colleghi coevi (Ottorino Olgiati, Pietro Salati, Emilio Maria Beretta) e precedenti

\footnotetext{
${ }^{460}$ Boldini Filippo (Paradiso 1900, Lugano 1989). Fu pittore, frescante e mosaicista. Introdotto all'arte dal pittore Antonio Barzaghi-Cattaneo (Lugano 1834 Paradiso 1922) e di Giuseppe Foglia (Lugano1888 Lugano 1950). Scopre a artisti come Giotto, Masaccio, Masolino, Piero della Francesca e specie il Beato Angelico, durante un breve soggiorno a Firenze nel 1924, che lo segneranno in futuro nella sua propria espressione artistica. Abitò dagli anni Trenta con la sua famiglia in via Cassarate a Lugano, quale Atelier dove dedicherà gli anni successivi alla realizzazione delle sue opere. Esse evoluzionarono da una prima fase che prova il naturalismo, il simbolismo, il postimpressionismo ed il cubismo lungo gli anni Venti, da una seconda negli anni Trenta, più matura $e$ intimista che si approssima all'arte del Novecento italiano, ad arrivare finalmente agli anni Cinquanta a riprendere le nature morte ed i paesaggi di ragionato cromatismo.

${ }^{461}$ (Mosaico, 1997).

${ }^{462}$ (Soldini, 2001).

${ }^{463}$ (Zeni, 2003)

${ }^{464}$ (Chiappini, 2003).
}

(Attilio Balmelli, Pietro Chiesa, Baldo Carugo o Mario Chiattone).

Moglia nasce en Bedonia (Parma) il 17 dicembre del 1915. La sua famiglia si trasferì quando lui ne aveva ancora l'età di quattro anni, nel 1919 in Svizzera, e sarà qui dove l'artista abiterà fino alla sua morte avvenuta il 9 marzo del 1986 a Viganello (Lugano). Studierà alla scuola diurna di Lugano e di Monza, dove otterrà il diploma di maestro d'Arte, anche se non abbiamo constatato il suo posteriore involucro nella docenza.

Moglia sviluppò la sua carriera come pittore, incisore ed scultore, specialista nella realizzazione dei mosaici e nella tecnica dell'affresco e membro degli "Incisori d'Italia".

Si possono elencare fra le opere più importanti da lui eseguite: diversi affreschi a Lugano al Banco di Roma, a villa Bossi, alla casa Boni, al mulino Bernasconi, al bar Federale ed al Kursaal, grafiti sulla facciata delle scuole di Morbio Superiore, un mosaico nel cimitero di Intragna, diversi affreschi a Ditto dove, particolarmente nella chiesa di S. Martino dopo il suo restauro in 1954, il pittore realizza una Deposizione sulla parete addossata all'antico altare; un mosaico cimitero di Mendrisio, un opera in terracotta nella villa Donati a Monteggio e due tondi con ritratti sulla facciata della Casa Isella a Morcote.

Le opere giovanili di Moglia furono esposte in diverse mostre a Milano, Roma, Venezia, Como e Lugano. Successivamente, con un linguaggio pittorico acquistato più maturo che tenterà in ogni momento il rinnovamento, la ricerca e l'approssimazione ed aggiornamento con le correnti del Novecento italiano, il pittore 
luganese esporrà a Soletta, Frauenfeld, Coira, e Weinfeld (1940), alla Galleria d'Arte della Collana (1944-1945) ed al Circolo di Cultura di Lugano (1946), ad Ascona (1949), alla XXI e XXIII Esposizione della S.P.S.A.S a Berna (1948 e 1953 rispettivamente), alla XXII a Zurigo (1950) all'Esposizione della Società Svizzerà di Belle Arti a Berna (1951), a Bruxelles, e per ultimo alle XXIV Esposizione della S.P.S.A.S. a St. Gallo (1955) e XXV Esposizione S.P.S.A.S. a Losanna (1957).

Moglia, come i contemporanei qui trattati (Pozzi, Cotti, Ferrazzini) intraprese l'attività di restauratore per motivazioni economiche, in un Cantone ancora risentito dalle due Guerre, povero e necessitato. Essa attività fu quasi continua dall'anno 1943 al 1972, intervenendo di modo pratico ed o con analisi e proposte di restauro su circa 20 opere (dipinti murali) catalogati nella presente ricerca, fondamentalmente di chiese e cappelle nel territorio ticinese.

Anche se non abbiamo potuto constatare quella che fu la sua formazione, possiamo immaginare che questa fosse avvenuta direttamente all'interno dei cantieri di lavori, iniziandosi nei restauri degli affreschi della chiesa della Beata Vergine Assunta a Villa Luganese nel 1943 assieme ai pittori Ferrazzini, Cotti, Facchinetti e Ribola per continuare nel fino al 1945 con il restauro degli affreschi Oratorio dell'Annunziata a Novazzano sotto la direzione di Emilio Ferrazzini e nel 1946 con gli affreschi della chiesa parrocchiale di S. Bartolomeo a Croglio (Castelrotto) in ambedue occasioni assieme a Facchinetti.

A questi due eventi succederanno altri restauri in cui Moglia lavorerà singolarmente; si tratta della chiesa parrocchiale dei SS. Fabiano e Sebastiano a Prato Sornico (1946), dello scoprimento e restauro degli affreschi della Madonna di Loreto a Lugano (1948), della chiesa dei SS. Anna e Cristoforo a Curogna (1948), della chiesa parrocchiale della Beata Vergine Assunta a Cimalmotto (1950), dell'antica chiesa parrocchiale di S. Ambrogio a Cademario (1953), dell'Oratorio di San Martino a Ditto (1954), della chiesa parrocchiale di S. Vittore Mauro a Moleno (1957), della chiesa parrocchiale dei SS. Simone e Giuda Taddeo a Vacallo, e della chiesa prepositurale di San Rocco a Bedigliora (1968).

Avrà l'opportunità di lavorare ancora assieme al collega Nino Facchinetti nel restauro degli affreschi della chiesa parrocchiale di S. Maria Assunta a Brione Verzasca nel 1956, e con Carlo Mazzi e Bruno Abbiati nel complessi pittorico della chiesa di SS. Pietro e Paolo a Biasca nel 1959. In quest'ultima fabbrica, come si era specificato in capitoli anteriori, ad ognuno dei pittori-restauratori scelti dalla Commissione Cantonale dei Monumenti Storici fu addetta una zona differente in cui lavorare per separato. Moglia fu incaricato del restauro degli affreschi seicenteschi, aiutato dal pittore Sergio Maino.

Il suo intervento interessò, come descritto nel capitolo riguardo il restauratore Bruno Abbiati, il consolidamento degli intonaci con caseinato di calcio, la rimozione dei cementi applicati in passato, la chiusura delle fessura e dei segni delle martellinature e reintegrazione pittorica a fresco in tonalità neutre sulle lacune mentre sul colore originale dei fondi e delle figure venne usata la tempera all'uovo, ed 
all'esterno con lo scopo di rendere le reintegrazioni resistenti furono impiegati $i$ colori ai silicati. Per ultimo gli affreschi tanto esterni come interni furono "ravvivati" con una soluzione di gomma arabica, che negli anni aveva sostituito all'abituale utilizzo delle cere con la così chiamata tecnica dell'encausto.

Inoltre a questi eventi sono stati catalogati due interventi di stacco, il primo fu eseguito fra il 20 marzo ed il 15 maggio del 1961 nella Cappella Greppi a Caslano, restaurata precedentemente nel 1845 e per ultimo nel 1932 dal pittorerestauratore Emilio Ferrazzini. II dipinto strappato fu la rappresentazione Seicentesca della Madonna col Bambino sul frontispizio della Cappella.

Le perizie preliminari al distacco elaborate da Moglia descrivono lo stato di conservazione dell'opera prima dell'intervento. In questo modo abbiamo potuto identificare le prassi che caratterizzano i restauri precedenti. Moglia ritrova numerose abrasioni nonché un forte strato di polvere e di cera "ossidata" sulla superficie del dipinto murale applicata sicuramente in passato per ravvivare le tonalità dell'affresco, e delle reintegrazione cromatiche eseguite a fresco che al momento erano apparse di colore diverso all'originale. Il lavoro di restauro di Moglia interessò la pulitura del sudiciume di polvere e cera soprastante le pitture, le stuccature delle lacune e delle fessure ed il livellamento degli intonaci rifatti.

Nel rapporto presentato all'Ufficio dei monumenti storici il 16 maggio del $1961^{465}$,

465 (Moglia Mario, Rapporto sul lavoro svolto dal 21 marzo al 15 maggio 1961.Viganello, 16 maggio 1961. AUBCBZ, s. 067 Caslano. Cappella Greppi).
Mario Moglia descrive come erano stati eseguite con cura le fasi di documentazione che all'epoca erano state finalmente imposte come indispensabile in materia di restauro. In questo modo il pittore testimonia lo stato conservativo dell'opera prima del restauro, dopo la pulitura, ed ancora una volta prima della esecuzione del restauro, con la superficie del dipinto idoneamente asciutta.

Il lavoro fu eseguito secondo le tecniche ed i materiali tradizionali per lo strappo:

[...]Materiali, $6 \mathrm{ml}$. Tela canapa; 5ml. Tela canapa sottile; $3 \mathrm{~kg}$. Colla forte; $16 \mathrm{dl}$ spirito; 1 scalpello lungo; 1 lampadina; 1 scatola chiodi tela; 2 secchi calce speciale; Ammoniaca; Latte; $1 / 2 \mathrm{~kg}$. Caseina in polvere; Telaio[... $]^{466}$,

Non sappiamo perché ragione, giacché il proprio restauratore ha eluso i motivi nel rapporto di lavoro, lo strappo non fu fattibile. L'applicazione delle tele sul dipinto con colla forte non consente il distacco del colore dell'affresco, e questo fa si che Moglia cambi procedimento adottando la tecnica dello stacco a massello della pittura. II lavoro si svolge mediante l'applicazione di un piano di legno sulla superficie evitando la perdite e rottura del materiale. Ad ogni modo, rispondendo agli ideali dell'epoca il dipinto venne pulito nel retro eliminando i primi strati fino a lasciare quello più superficiale contenente il colore, l'intonachino, in contatto con le tele di canapa applicate preliminarmente. II retro del dipinto fu stuccato con caseinato di calce per uniformare la superficie e fu applicata in successione la tela di supporto ed il telaio di sostegno. A continuazione furono

\footnotetext{
66 (AUBCBZ, s. 067 Caslano. Cappella Greppi).
} 
eseguiti i ritocchi pittorici con tempera all'uovo.

Il lavoro fu con molta sicurezza eseguito assieme al collega il pittore Sergio Maino, nel domicilio del quale fu ritirato il dipinto staccato dall'architetto Taddeo Carloni nel 1968 per il trasferimento di questo al Castel Grande di Bellinzona.

Il secondo lavoro di strappo che abbiamo potuto documentare fu svolto dal Moglia l'anno 1965 all'interno del cantiere di restauro alla chiesa di San Pietro e Paolo a Biasca, assieme ancora una volta a Sergio Maino. In totale furono eseguiti 14 strappi sugli affreschi del XVII sull'abside con lo scopo di mettere in luce i sottostanti dipinti medievali. II restauro si sviluppò con le medesime prassi impiegate per lo strappo dalla Madonna col bambino a Caslano. In questo caso però, il rapporto pubblicato da Mario Moglia ${ }^{467}$ aggiunge un procedimento che non era stato citato con motivo dello strappo a Caslano; questo è l'applicazione della gomma arabica sulla superficie pittorica distaccata.

Bastano i casi sopra descritti per confermare le prassi di restauro impiegate da Moglia: pulitura curate e rispettose, consolidamenti puntuali, reintegrazione pittorica con tempera all'uovo in corrispondenza ad un senso dell'irreversibilità dei materiali impiegati nel restauro, ed a seconda dei casi, uso della gomma arabica per la "finitura" o ravvivamento delle superficie. Questo serve a documentare in cambiamento avvenuto nelle ideologie della conservazione e del restauro delle opera artistiche e conseguentemente nelle tecniche e materiali idonei per compiere $\mathrm{i}$ valori di rispetto verso l'opera a metà del secolo XX istituiti in Italia grazie alle teorie di Cesara Brandi, e verso gli anni Sessanta del Novecento finalmente impiantati nel ambito ticinese.

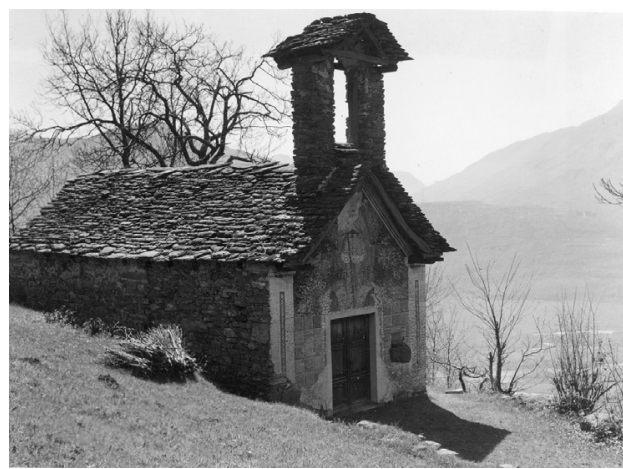

Figura 185 Cugnasco, Oratorio di Curogna. Chiesa dei SS. Anna e Cristoforo. AUBCBZ, Archivio grafico.

467 (Moglia, 1967) più informazione 


\section{Interventi di restauro}

\begin{tabular}{|c|c|c|c|c|c|}
\hline ANNO & LUOGO & OPERA & INTERVENTO & COLLABORATORI & FONTI \\
\hline 1943 & $\begin{array}{l}\text { Villa Luganese, } \\
\text { chiesa parrocchiale } \\
\text { della B.V Assunta. }\end{array}$ & & $\begin{array}{l}\text { Ricerca e } \\
\text { scoprimento degli } \\
\text { affreschi del } \\
\text { porticato e di } \\
\text { ripulitura delle } \\
\text { lesene dell'interno, } \\
\text { le quelli conservano } \\
\text { sotto il tinteggio in } \\
\text { intonaco finissimo a } \\
\text { stucco } \\
\text { Affresco scoperto } \\
\text { sotto una tela } \\
\text { dell'ancona lungo la } \\
\text { parete } \\
\text { settentrionale della } \\
\text { chiesa } \\
\text { Rifacimento del } \\
\text { tinteggio } \\
\text { conformemente } \\
\text { alla tracce } \\
\text { rinvenute } \\
\text { Manca } \\
\text { consolidamento } \\
\text { degli affreschi } \\
\text { scoperti e } \\
\text { correzione del } \\
\text { tinteggio interno in } \\
\text { certe parti } \\
\text { imperfetto } \\
\text { (proseguito nel } \\
\text { 1946 - 1953). }\end{array}$ & $\begin{array}{l}\text { Pittori-restauratori: } \\
\text { Emilio Ferrazzini; } \\
\text { Carlo Cotti } \\
\text { Nino Facchinetti } \\
\text { Mario Ribola. } \\
\end{array}$ & $\begin{array}{l}\text { (Chiesa, 1946, p. 77) } \\
\text { (DPE, 1959, p. 126) } \\
\text { (AUBCBZ, s. 251, } \\
\text { Villa Luganese, B. V. } \\
\text { Assunta). }\end{array}$ \\
\hline 1943 & $\begin{array}{l}\text { Villa Luganese, } \\
\text { chiesa Parrocchiale } \\
\text { della B.V Assunta. }\end{array}$ & $\begin{array}{l}\text { Santa Martire con } \\
\text { angeli reggenti una } \\
\text { corono per } \\
\text { difendere l'anima di } \\
\text { un defunto dal } \\
\text { demonio e i SS. } \\
\text { Rocco, Lucia, } \\
\text { Antonio Abate } \\
\text { (prob.) e Giacomo } \\
\text { Maggiore. Nicchia } \\
\text { E. del portico. } \\
\text { Affreschi, inizio XVI } \\
\text { sec. }\end{array}$ & $\begin{array}{l}\text { Ricerche pittoriche } \\
\text { con esportazione } \\
\text { dello strato di calce } \\
\text { ritrovatosi molto } \\
\text { duro. }\end{array}$ & $\begin{array}{l}\text { Pittori-restauratori: } \\
\text { Emilio Ferrazzini; } \\
\text { Carlo Cotti } \\
\text { Nino Facchinetti } \\
\text { Mario Ribola. }\end{array}$ & $\begin{array}{l}\text { (Chiesa, 1946, p. 77) } \\
\text { (DPE, 1959, p. 126) } \\
\text { (AUBCBZ, s. 251, } \\
\text { Villa Luganese, B. V. } \\
\text { Assunta). } \\
\text { (Emilio Ferrazzini, } \\
\text { Lettera indirizzata } \\
\text { all'On. Consigliere } \\
\text { G. Lepori . Lugano, } \\
13 \text { giugno 1943. } \\
\text { AUBCBZ, s. 251, } \\
\text { Villa Luganese, B. V. } \\
\text { Assunta). }\end{array}$ \\
\hline 1943 & $\begin{array}{l}\text { Villa Luganese, } \\
\text { chiesa Parrocchiale }\end{array}$ & $\begin{array}{l}\text { Madonna sulla } \\
\text { lunetta dell'antico }\end{array}$ & $\begin{array}{l}\text { Ricerche pittoriche. } \\
\text { Pulitura dallo strato }\end{array}$ & $\begin{array}{l}\text { Pittori-restauratori: } \\
\text { Emilio Ferrazzini; }\end{array}$ & $\begin{array}{l}\text { (Chiesa F. , 1946, p. } \\
\text { 77). }\end{array}$ \\
\hline
\end{tabular}


della B.V Assunta. portale. Affresco, di calce. inizio XVI sec.
Carlo Cotti

Nino Facchinetti

Mario Ribola.
(DPE, 1959, p. 126)

(AUBCBZ, s. 251,

Villa Luganese, B. V. Assunta).

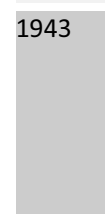

1943

Villa Luganese, chiesa Parrocchiale della B.V Assunta.

chiesa Parrocchiale della B.V Assunta.
Seregnesi, attr, S. Giovanni Battista. Portico.

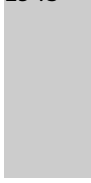

1943

Villa Luganese, chiesa Parrocchiale della B.V Assunta.

\section{Madonna della}

Misericordia, metà XIV sec., e due santi. della B.V Assunta.

Visione di S.

Bernardo con il

Crocifisso, 1577.

bambino e $S$.

Ricerche pittoriche. Pittori-restauratori: (Chiesa F. , 1946, p. Pulitura dello strato Emilio Ferrazzini; 77). di calce.

Carlo Cotti Nino Facchinetti Mario Ribola.

(DPE, 1959, p. 126).

(AUBCBZ, s. 251,

Villa Luganese, B. V. Assunta).

Ricerche pittoriche. Pittori-restauratori: (Chiesa F. , 1946, p.

Pulitura dello strato Emilio Ferrazzini; 77). di calce. Ritocco

Carlo Cotti. pittorico.

Nino Facchinetti Mario Ribola.

(AUBCBZ, s. 251,

Villa Luganese, B. V. Assunta).

Ricerche pittoriche. Pittori-restauratori: (Chiesa F. , 1946, p. Pulitura dello strato Emilio Ferrazzini; 77). di calce. Ritocco

Carlo Cotti

pittorico.

Nino Facchinetti

Mario Ribola.

(DPE, 1959, p. 126).

(AUBCBZ, s. 251,

Villa Luganese, B. V. Assunta).

Ricerche pittoriche. Pittori-restauratori: (Chiesa F. , 1946, p.

Pulitura dello strato Emilio Ferrazzini; 77).

di calce. Carlo Cotti

(DPE, 1959, p. 126).

XVI sec. Lunetta del Consolidamento.

Nino Facchinetti

portale principale. Ritocco pittorico. Mario Ribola.

(AUBCBZ, s. 251

Villa Luganese, B. V. Assunta).

Villa Luganese,
chiesa Parrocchiale interne.
della B.V Assunta.

\begin{tabular}{|c|c|c|}
\hline $\begin{array}{l}\text { Patinatura } \\
\text { (armonizzazione del } \\
\text { colore) degli stucchi } \\
\text { ornamentali e } \\
\text { figurativi raschiati e } \\
\text { lisciati dagli } \\
\text { stuccatori Cantoni, } \\
\text { nella volta della } \\
\text { navata e nelle } \\
\text { pareti, nella parete } \\
\text { principale del coro } \\
\text { e su tutti i capitelli } \\
\text { delle lesene. } \\
\text { Pulitura con } \\
\text { emollienti sino a } \\
\text { rintracciare lo } \\
\text { stucco lucido } \\
\text { esistente e restauro } \\
\text { pittorico di questo } \\
\text { (marmorizzato) } \\
\text { ritrovato nelle } \\
\text { lesene, basi e } \\
\text { zoccoli, nonché nel }\end{array}$ & $\begin{array}{l}\text { Pittori-restauratori: } \\
\text { Emilio Ferrazzini; } \\
\text { Carlo Cotti } \\
\text { Nino Facchinetti } \\
\text { Mario Ribola . } \\
\end{array}$ & $\begin{array}{l}\text { (Chiesa F. , 1946, p. } \\
\text { 77) } \\
\text { (DPE, 1959, p. 126) } \\
\text { (AUBCBZ, s. 251, } \\
\text { Villa Luganese, B. V. } \\
\text { Assunta). }\end{array}$ \\
\hline
\end{tabular}




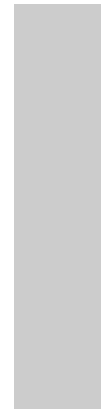

1943

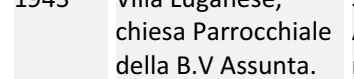

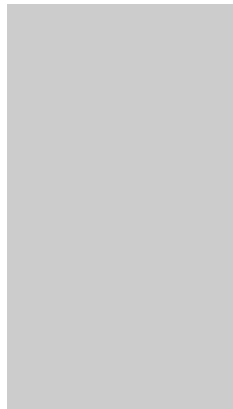

Seregnesi, attr. Affresco, XV sec. ritrovato sotto la pala d'altare nella parete nord della navata.

$\begin{array}{lll}1943 \text { - } & \begin{array}{l}\text { Novazzano, oratorio } \\ \text { dell'Annunciata. }\end{array} & \begin{array}{l}1945 \text { (a) } \\ \text { Battista e bottega, } \\ \text { Ultima Cena. } \\ \text { Affresco. }\end{array} \\ 1946 & \begin{array}{l}\text { Croglio-Castelrotto, } \\ \text { chiesa parrocchiale } \\ \text { di S. Bartolomeo. }\end{array} & \begin{array}{l}\text { Thomas e } \\ \text { Baldesarus, } 1440 . \\ \text { Cristo in Maestà } \\ \text { con gli apostoli. } \\ \text { Affreschi. Abside. }\end{array} \\ & \end{array}$

\author{
fregio della \\ trabeazione, \\ sull'attico \\ sovrastante alla \\ stessa e nella \\ cornice delle \\ finestre (138 mq). \\ Lucidatura finale. \\ Restauro pittorico \\ delle quattro \\ finestre barocche \\ dipinte.
}

\section{Pulitura e leggero}

restauro. Non era

possibile togliere la

tela (Assunta con $\mathrm{i}$

SS. Giovanni

Battista e Caterina

inginocchiati, fine

XVI - inizio XVII

sec., con cornice a

stucco a raggiere di

finitura) per

mettere in luce

I'affresco. Si pensa

a uno strappo e

cessione al Museo

di Lugano.

\begin{tabular}{|c|c|c|}
\hline $\begin{array}{l}\text { Pulitura e } \\
\text { consolidamento del } \\
\text { dipinto mediante la } \\
\text { tecnica ad encausto } \\
\text { con la miscela di } \\
\text { diverse cere o } \\
\text { l'applicazione di } \\
\text { queste in diverse } \\
\text { mani per rinnovare } \\
\text { le tonalità } \\
\text { cromatiche. }\end{array}$ & $\begin{array}{l}\text { Pittore- } \\
\text { restauratore: } \\
\text { Nino Facchinetti. } \\
\text { Sorveglianza di } \\
\text { Emilio Ferrazzini e } \\
\text { Ugo Donati. }\end{array}$ & $\begin{array}{l}\text { (Chiesa, Monumenti } \\
\text { storici e artistici del } \\
\text { Cantone Ticino } \\
\text { restaurati dal } 1910 \\
\text { al } 1945,1946, \text { p. } 75 \text { ) } \\
\text { (DPE, 1959, p. 124) } \\
\text { (AUBCBZ, s. 080 } \\
\text { Novazzano. Oratorio } \\
\text { dell'Annunciata). }\end{array}$ \\
\hline $\begin{array}{l}\text { Pulitura e } \\
\text { consolidamento } \\
\text { con miscela di cere } \\
\text { d'api e paraffina. }\end{array}$ & $\begin{array}{l}\text { Pittore- } \\
\text { restauratore: } \\
\text { Nino Facchinetti } \\
\text { Il restauro } \\
\text { architettonico } \\
\text { contemporaneo è } \\
\text { eseguito sotto il } \\
\text { progetto e la } \\
\text { direzione degli } \\
\text { architetti Carlo e } \\
\text { Rino Tami. }\end{array}$ & $\begin{array}{l}\text { (DPE, 1959, p. 119). } \\
\text { (AUBCBZ, s. 041, } \\
\text { Croglio, S. } \\
\text { Bartolomeo). }\end{array}$ \\
\hline
\end{tabular}

Pittori-restauratori: (Chiesa F. , 1946, p.

Emilio Ferrazzini; 77)

Carlo Cotti (DPE, 1959, p. 126).

Nino Facchinetti (AUBCBZ, s. 251,

Mario Ribola. Villa Luganese, B. V. Assunta). 


\begin{tabular}{|c|c|c|c|c|c|}
\hline 1946 & $\begin{array}{l}\text { Croglio, } \\
\text { chiesa parrocchiale } \\
\text { di S. Bartolomeo. }\end{array}$ & $\begin{array}{l}\text { Madonna in trono, } \\
\text { Incoronazione della } \\
\text { Vergine in } \\
\text { correlazione con la } \\
\text { Trinità, con accanto } \\
\text { S. Antonio abate. } \\
\text { XIV sec. Affreschi. } \\
\text { Parete sud. }\end{array}$ & $\begin{array}{l}\text { Pulitura e } \\
\text { consolidamento } \\
\text { con miscela di cere } \\
\text { d'api e paraffina. }\end{array}$ & $\begin{array}{l}\begin{array}{l}\text { Pittore- } \\
\text { restauratore: } \\
\text { Nino Facchinetti }\end{array} \\
\text { Il restauro } \\
\text { architettonico } \\
\text { contemporaneo è } \\
\text { eseguito sotto il } \\
\text { progetto e la } \\
\text { direzione degli } \\
\text { architetti Carlo e } \\
\text { Rino Tami. }\end{array}$ & $\begin{array}{l}\text { (DPE, 1959, p. 119) } \\
\text { (AUBCBZ, s. 041, } \\
\text { Croglio, S. } \\
\text { Bartolomeo) }\end{array}$ \\
\hline 1946 & $\begin{array}{l}\text { Croglio, } \\
\text { chiesa parrocchiale } \\
\text { di S. Bartolomeo. }\end{array}$ & $\begin{array}{l}\text { Madonna, XVII sec. } \\
\text { Affresco. Parete } \\
\text { nord. }\end{array}$ & $\begin{array}{l}\text { Pulitura e } \\
\text { consolidamento } \\
\text { con miscela di cere } \\
\text { d'api e paraffina. }\end{array}$ & $\begin{array}{l}\text { Pittore- } \\
\text { restauratore: } \\
\text { Nino Facchinetti } \\
\text { Il restauro } \\
\text { architettonico } \\
\text { contemporaneo è } \\
\text { eseguito sotto il } \\
\text { progetto e la } \\
\text { direzione degli } \\
\text { architetti Carlo e } \\
\text { Rino Tami. }\end{array}$ & $\begin{array}{l}\text { (DPE, 1959, p. 119) } \\
\text { (AUBCBZ, s. 041, } \\
\text { Croglio, S. } \\
\text { Bartolomeo). }\end{array}$ \\
\hline 1946 & $\begin{array}{l}\text { Prato Sornico } \\
\text { chiesa parrocchiale } \\
\text { dei SS. Fabiano e } \\
\text { Sebastiano }\end{array}$ & Affreschi & Pulitura & & $\begin{array}{l}\text { (DPE, 1959, p. 125) } \\
\text { (AUBCBZ, s. } 201 . \\
\text { Prato Sornico, SS. } \\
\text { Fabiano e } \\
\text { Sebastiano) }\end{array}$ \\
\hline 1947 & $\begin{array}{l}\text { Arogno, chiesa } \\
\text { parrocchiale di S. } \\
\text { Stefano. Cappella } \\
\text { della Trinità }\end{array}$ & & Commissione & & $\begin{array}{l}\text { (DPE, 1959, p. 117) } \\
\text { (AUBCBZ, s. 008, } \\
\text { Arogno, cappella } \\
\text { della Trinità) }\end{array}$ \\
\hline 1948 & $\begin{array}{l}\text { Lugano, Madonna } \\
\text { di Loreto. }\end{array}$ & Affreschi. & $\begin{array}{l}\text { Scoprimento e } \\
\text { restauro. }\end{array}$ & $\begin{array}{l}\text { Il restauro } \\
\text { architettonico } \\
\text { avvenuto fra il } 1948 \\
\text { - } 1949 \text { fu } \\
\text { progettato e diretto } \\
\text { dall'architetto Cino } \\
\text { Chiesa. }\end{array}$ & $\begin{array}{l}\text { (DPE, 1959, p. 122) } \\
\text { (AUBCBZ, s. 143, } \\
\text { Lugano, Madonna di } \\
\text { Loreto). }\end{array}$ \\
\hline 1948 & $\begin{array}{l}\text { Cugnasco, oratorio } \\
\text { di Curogna. Chiesa } \\
\text { dei SS. Anna e } \\
\text { Cristoforo }\end{array}$ & $\begin{array}{l}\text { S. Cristoforo, XVII } \\
\text { sec. Affresco. } \\
\text { Facciata. }\end{array}$ & $\begin{array}{l}\text { Restauro. } \\
\text { Testimoniato con } \\
\text { una iscrizione sotto } \\
\text { la pila } \\
\text { dell'acquasanta. }\end{array}$ & $\begin{array}{l}\text { Sorveglianza: } \\
\text { Piero Bianconi. }\end{array}$ & $\begin{array}{l}\text { (DPE, 1959, p. 120) } \\
\text { (AUBCBZ, s. 095, } \\
\text { Cugnasco, SS. Anna } \\
\text { e Cristoforo). }\end{array}$ \\
\hline 1948 & $\begin{array}{l}\text { Cugnasco, oratorio } \\
\text { di Curogna. Chiesa } \\
\text { dei SS. Anna e } \\
\text { Cristoforo. }\end{array}$ & $\begin{array}{l}\text { Majestas Domini, } \\
\text { XV sec. Affresco. } \\
\text { Abside. }\end{array}$ & $\begin{array}{l}\text { Restauro. } \\
\text { Testimoniato con } \\
\text { una iscrizione sotto } \\
\text { la pila } \\
\text { dell'acquasanta. }\end{array}$ & $\begin{array}{l}\text { Sorveglianza: } \\
\text { Piero Bianconi. }\end{array}$ & $\begin{array}{l}\text { (DPE, 1959, p. 120) } \\
\text { (AUBCBZ, s. 095, } \\
\text { Cugnasco, SS. Anna } \\
\text { e Cristoforo). }\end{array}$ \\
\hline
\end{tabular}


Cugnasco, oratorio Madonna del latte di Curogna. Chiesa fra i SS. Giovanni dei SS. Anna e Cristoforo.

Battista e Antonio, $\mathrm{XV}$ sec. Affresco. Parete sud.

1948 Cugnasco, oratorio
di Curogna. Chiesa
dei SS. Anna e
Cristoforo.

\section{8}

Cugnasco, oratorio di Curogna. Chiesa dei SS. Anna e Cristoforo.

Cristoforo da

Seregno, seconda metà XV sec. Imago Pietatis. Affresco. Altare.

Cristoforo da Seregno, seconda metà XV sec. Ultima Cena.
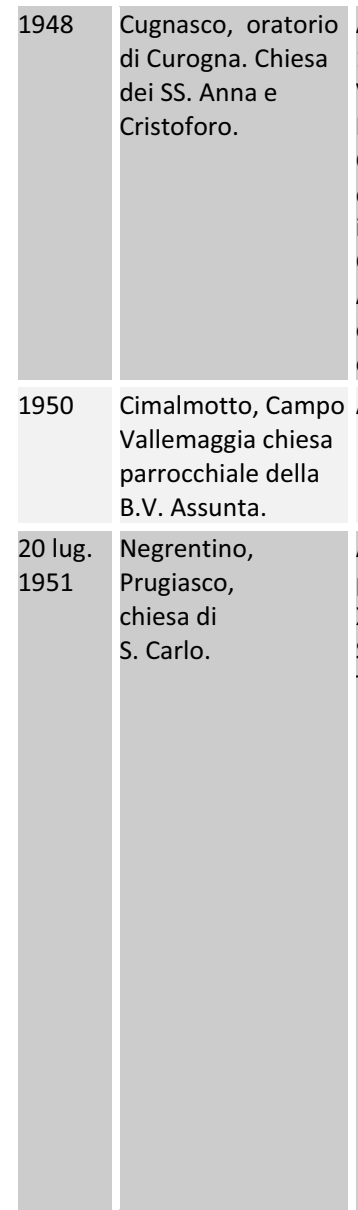
Affresco. Parete nord.

Cimalmotto, Campo Affreschi.

Vallemaggia chiesa parrocchiale della B.V. Assunta.

Restauro.

Testimoniato con una iscrizione sotto la pila dell'acquasanta..

Restauro.
Testimoniato con
una iscrizione sotto
la pila
dell'acquasanta.

\section{Restauro.}

Testimoniato con una iscrizione sotto la pila dell'acquasanta.

Cugnasco, oratorio
di Curogna. Chiesa dei SS. Anna e Cristoforo.

Alessandro Gorla,
1601, Nascita della
Vergine, la Morte,
Monogramma di
Cristo, Madonna
del latte, S. Pietro e
il gruppo della
Crocifissione.
Affreschi parte
ovest e
controfacciata.

Affreschi parete di ponente. Affreschi XIII, XIV.

Seregnesi, da Tradate.

\section{Restauro. \\ Testimoniato con \\ una iscrizione sotto \\ la pila \\ dell'acquasanta.

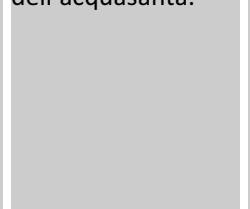

Restauro.

Perizia che constata Scultore: la presenza di umidità per infiltrazioni di acqua sulla volta dell'abside maggiore provocando il distacco delle reintegrazioni cromatiche realizzate da Tita Pozzi in 1944, sul mantello del Cristo in blu oltremare. Sotto questo strato di ritocco staccato appare la pittura originale. II restauratore Mario

\section{Sorveglianza: Emilio Maria Beretta. \\ Sorveglianza: \\ Piero Bianconi. \\ (DPE, 1959, p. 120) \\ (AUBCBZ, s. 095, \\ Cugnasco, SS. Anna \\ e Cristoforo). \\ (DPE, 1959, p. 119) \\ (AUBCBZ, s. 059. \\ Campo Vallemaggia. Cimalmotto).}

Sorveglianza:

(DPE, 1959, p. 120)

Piero Bianconi.

(AUBCBZ, s. 095,

Cugnasco, SS. Anna

e Cristoforo)

\section{Sorveglianza:}

Piero Bianconi.

Sorveglianza:

(DPE, 1959, p. 120)

(AUBCBZ, s. 095,

Cugnasco, SS. Anna

e Cristoforo).

(DPE, 1959, p. 120)

(AUBC Z, s. 095 ,

Cugnasco, SS. Anna e Cristoforo).

(AUBCBZ, s. 204, Prugiasco. S. Carlo) (Moglia Mario, Perizia indirizzata al DPE. Viganello Lugano, 22 luglio 1957. AUBCBZ, s. 204, Prugiasco. S. Carlo). 


\begin{tabular}{|c|c|c|c|c|c|}
\hline & & & $\begin{array}{l}\text { Moglia propose il } \\
\text { controllo del tetto } \\
\text { in piode. }\end{array}$ & & \\
\hline $\begin{array}{l}1953- \\
1954\end{array}$ & $\begin{array}{l}\text { Cugnasco, oratorio } \\
\text { di S. Maria delle } \\
\text { Grazie. }\end{array}$ & Affreschi. & $\begin{array}{l}\text { Scoprimento, } \\
\text { pulitura e } \\
\text { consolidamento. }\end{array}$ & & $\begin{array}{l}\text { (DPE, 1959, p. 120) } \\
\text { (AUBCBZ, s. 095, } \\
\text { Cugnasco, Oratorio } \\
\text { delle Grazie). }\end{array}$ \\
\hline 1954 & $\begin{array}{l}\text { Ditto, } \\
\text { oratorio di } \\
\text { S. Martino. }\end{array}$ & Affreschi. & $\begin{array}{l}\text { Pulitura e } \\
\text { consolidamento } \\
\text { Lascia una sua } \\
\text { opera raffigurante } \\
\text { una Deposizione } \\
\text { sulla parete alla } \\
\text { quale era addossato } \\
\text { l'antico altare. }\end{array}$ & & $\begin{array}{l}\text { (DPE, 1959, p. 120) } \\
\text { (AUBCBZ, s. 095, } \\
\text { Cugnasco - Dito, } \\
\text { Oratorio di S. } \\
\text { Martino). }\end{array}$ \\
\hline 1953 & $\begin{array}{l}\text { Cademario, antica } \\
\text { chiesa parrocchiale } \\
\text { di } \\
\text { S. Ambrogio. }\end{array}$ & Affreschi. & Restauro. & $\begin{array}{l}\text { Restauro } \\
\text { architettonico } \\
\text { eseguito da } \\
\text { Ferdinando Reggiori } \\
\text { hanno riportato la } \\
\text { chiesa agli ordini } \\
\text { stilistici romanici } \\
\text { con la chiusura del } \\
\text { coro barocco, la } \\
\text { riapertura } \\
\text { dell'abside } \\
\text { primitivo, la } \\
\text { ricostruzione delle } \\
\text { arcate divisorie } \\
\text { gotiche ed il } \\
\text { rifacimento di un } \\
\text { soffitto ligneo } \\
\text { ribassato. } \\
\text { Arch. Camenzind, } \\
\text { Giovannini, Salati. } \\
\text { Visita arch. Sulzer } \\
\text { della Commissione } \\
\text { Federale. }\end{array}$ & $\begin{array}{l}\text { (DPE, 1959, p. 119) } \\
\text { (AUBCBZ, s. 047, } \\
\text { Cademario. S. } \\
\text { Ambrogio). } \\
\text { Ved. Relazione } 1958 \\
\text { pitt. Salati. }\end{array}$ \\
\hline 1955 & $\begin{array}{l}\text { Miglieglia, chiesa di } \\
\text { S. Stefano. }\end{array}$ & $\begin{array}{l}\text { S. Antonio da } \\
\text { Padova, XVIII sec. } \\
\text { Dipinto murale. }\end{array}$ & $\begin{array}{l}\text { Progetto restauro } \\
\text { pittorico. }\end{array}$ & & $\begin{array}{l}\text { (DPE, 1959, p. 124) } \\
\text { (AUBCBZ, s. } 162 . \\
\text { Miglieglia, S. } \\
\text { Stefano). }\end{array}$ \\
\hline $\begin{array}{l}1956 \text { - } \\
1957\end{array}$ & $\begin{array}{l}\text { Brione Verzasca, } \\
\text { chiesa parrocchiale } \\
\text { di S. Maria Assunta. }\end{array}$ & Affreschi. & Restauro pittorico. & $\begin{array}{l}\text { Pittore- } \\
\text { restauratore: } \\
\text { Nino Facchinetti } \\
\text { Sorveglianza: } \\
\text { Piero Bianconi, } \\
\text { don Agostino } \\
\text { Robertini. }\end{array}$ & $\begin{array}{l}\text { (DPE, 1959, p. 119) } \\
\text { (AUBCBZ, s. 039, } \\
\text { Brione Verzasca,S. } \\
\text { Maria Assunta). }\end{array}$ \\
\hline
\end{tabular}




\begin{tabular}{|c|c|c|c|c|c|}
\hline & & & & $\begin{array}{l}\text { Restauro } \\
\text { architettonico fu } \\
\text { eseguito da Alberto } \\
\text { Finzi. }\end{array}$ & \\
\hline 1958 & $\begin{array}{l}\text { Torello, } \\
\text { chiesa parrocchiale. }\end{array}$ & Affreschi esterni. & Restauro. & & $\begin{array}{l}\text { (DPE, 1959, p. 120) } \\
\text { (AUBCBZ, s. 065, } \\
\text { Torello). }\end{array}$ \\
\hline $\begin{array}{l}1957- \\
1958\end{array}$ & $\begin{array}{l}\text { Moleno, } \\
\text { chiesa parrocchiale } \\
\text { S. Vittore Mauro. }\end{array}$ & & Restauro. & $\begin{array}{l}\text { Sorveglianza: } \\
\text { Piero Bianconi, } \\
\text { don Agostino } \\
\text { Robertini. }\end{array}$ & $\begin{array}{l}\text { (DPE, 1959, p. 124) } \\
\text { (AUBCBZ, s. } 165 . \\
\text { Moleno, S. Vittore } \\
\text { Mauro). }\end{array}$ \\
\hline $\begin{array}{l}1959- \\
1966\end{array}$ & $\begin{array}{l}\text { Biasca, } \\
\text { Chiesa di S. Pietro. }\end{array}$ & XV sec. Affreschi. & $\begin{array}{l}\text { Consolidamento } \\
\text { dell'intonaco con } \\
\text { caseinato di calcio } \\
\text { ed applicazione } \\
\text { provvisoria di } \\
\text { pressatoi. } \\
\text { Rimozione delle } \\
\text { zone coperte con } \\
\text { cemento e chiusura } \\
\text { delle fessure e dei } \\
\text { segni delle } \\
\text { martellinature. } \\
\text { Ricostruzione } \\
\text { dell'arriccio ed } \\
\text { intonacature delle } \\
\text { parti mancanti con } \\
\text { successiva } \\
\text { reintegrazione } \\
\text { pittorica a fresco. } \\
\text { Rimozione dello } \\
\text { strato di calce } \\
\text { sottostante e delle } \\
\text { macchie } \\
\text { occasionate delle } \\
\text { strutture murarie } \\
\text { aggiunte. Pulitura } \\
\text { della muffe } \\
\text { soprastanti gli } \\
\text { affreschi esterni. } \\
\text { Reintegrazione } \\
\text { pittorica a fresco } \\
\text { con tonalità neutre } \\
\text { sulle lacune, } \\
\text { ricostruzione } \\
\text { illusionistica degli } \\
\text { elementi geometrici } \\
\text { che incorniciano gli } \\
\text { affreschi. I contorni } \\
\text { delle figurazioni ed i }\end{array}$ & $\begin{array}{l}\text { Pittore- } \\
\text { restauratore: } \\
\text { Sergio Maino. } \\
\end{array}$ & $\begin{array}{l}\text { (Moglia, 1967, p. } \\
\text { 195-210) } \\
\text { (Renato, 1967). }\end{array}$ \\
\hline
\end{tabular}




\begin{tabular}{|c|c|c|c|c|c|}
\hline & & & $\begin{array}{l}\text { fondi furono } \\
\text { ritoccati con } \\
\text { tempera all'uovo. II } \\
\text { ritocco dei dipinti } \\
\text { all'esterno fu } \\
\text { compiuto con i } \\
\text { colori ai silicati della } \\
\text { marca Keim. Tutti } \\
\text { gli affreschi furono } \\
\text { consolidati con } \\
\text { acqua di cale e } \\
\text { posteriormente } \\
\text { ravvivati i toni con } \\
\text { l'applicazione di } \\
\text { una soluzione di } \\
\text { gomma arabiga. }\end{array}$ & & \\
\hline 1960 & Caslano. & Affresco. & $\begin{array}{l}\text { Distacco. In nota } \\
\text { del } 17 \text { maggio } 1960 .\end{array}$ & & $\begin{array}{l}\text { (AUBCBZ, s. } 067 \\
\text { Caslano). }\end{array}$ \\
\hline $\begin{array}{l}20 \text { mar. } \\
\text { al } 15 \\
\text { mag. } \\
1961\end{array}$ & $\begin{array}{l}\text { Caslano - } \\
\text { Magliasina } \\
\text { Capella della } \\
\text { Madonna o Greppi. }\end{array}$ & $\begin{array}{l}\text { Affreschi Madonna } \\
\text { col Bambino ( } 190 \text { X } \\
160 \mathrm{~cm} \text { ) } \\
\text { Dopo } 1534 \text { - Sec. } \\
\text { XVI. Scuola Luini. } \\
\text { Frontispizio. }\end{array}$ & $\begin{array}{l}\text { L'affresco era stato } \\
\text { restaurato insieme } \\
\text { al resto di pitture } \\
\text { del complesso } \\
\text { decorativo della } \\
\text { fabbrica nel } 1845 \text { e } \\
\text { successivamente } \\
\text { nel } 1932 \text { dal } \\
\text { pittore-restauratore } \\
\text { Emilio Ferrazzini. } \\
\text { Sopra la superficie } \\
\text { pittorica della } \\
\text { Madonna col } \\
\text { Bambino si ritrovò } \\
\text { un forte strato di } \\
\text { polvere e di cera } \\
\text { ossidata, } \\
\text { sicuramente } \\
\text { applicata nei } \\
\text { precedenti } \\
\text { interventi per } \\
\text { ravvivare le tonalità } \\
\text { dell'affresco. Anche } \\
\text { in passate si } \\
\text { realizzarono delle } \\
\text { reintegrazione } \\
\text { cromatiche a fresco } \\
\text { che apparsero di } \\
\text { colore diverso } \\
\text { all'originale. Inoltre } \\
\text { la perizia di Mario } \\
\text { Moglia denuncia le } \\
\text { numerose abrasioni }\end{array}$ & $\begin{array}{l}\text { Pittore- } \\
\text { restauratore: } \\
\text { Sergio Maina, di } \\
\text { Caslano } \\
\text { arch. Giovannini } \\
\text { direttore dei lavori } \\
\text { Rev. Don Quadri. } \\
\end{array}$ & $\begin{array}{l}\text { (AUBCBZ, s. } 067 \\
\text { Caslano. Cappella } \\
\text { Greppi). } \\
\text { (Moglia Mario, } \\
\text { Rapporto sul lavoro } \\
\text { svolto dal } 21 \text { marzo } \\
\text { al } 15 \text { maggio } \\
\text { 1961.Viganello, } 16 \\
\text { maggio 1961. } \\
\text { AUBCBZ, s. } 067 \\
\text { Caslano. Cappella } \\
\text { Greppi). }\end{array}$ \\
\hline
\end{tabular}




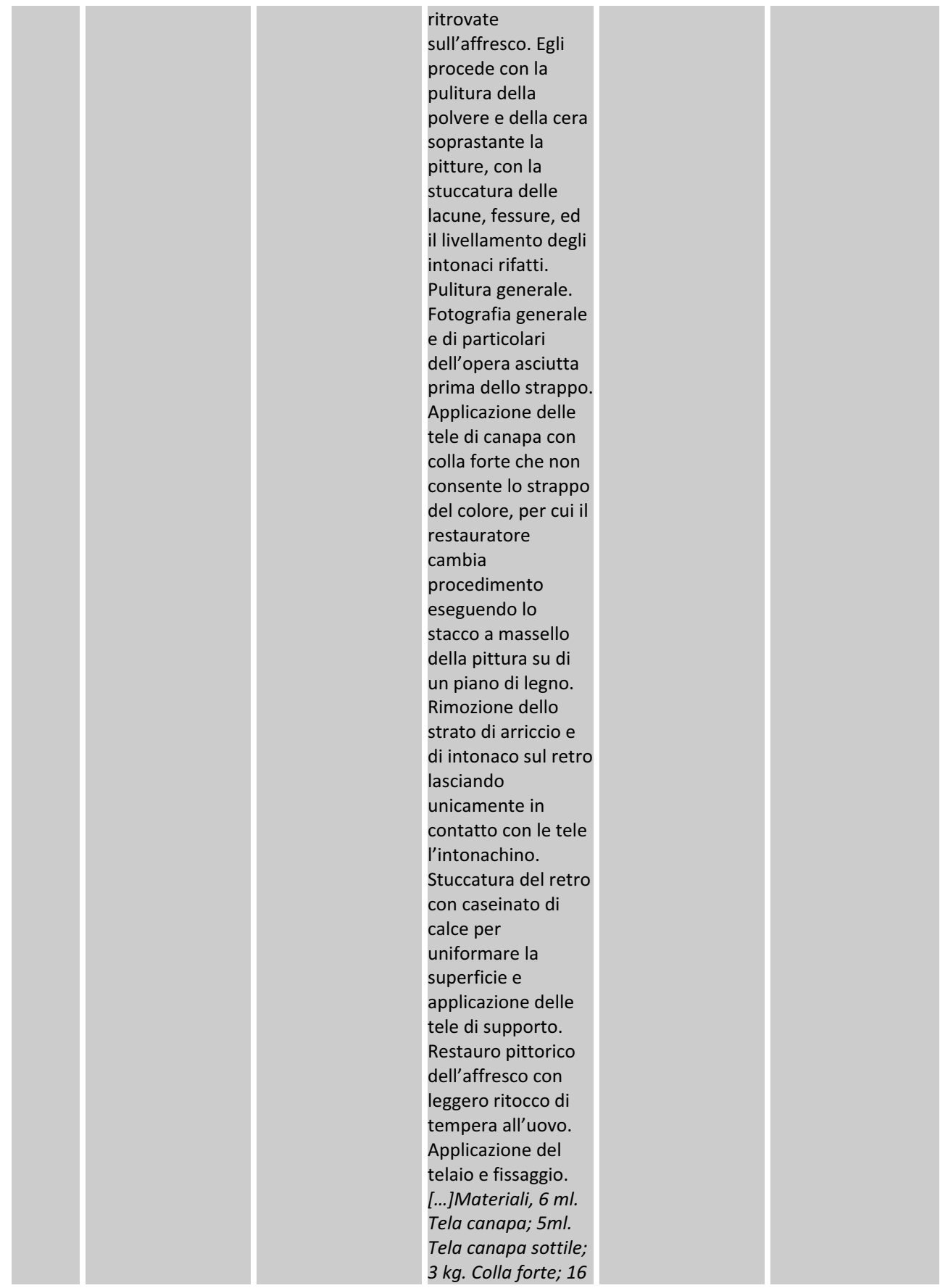




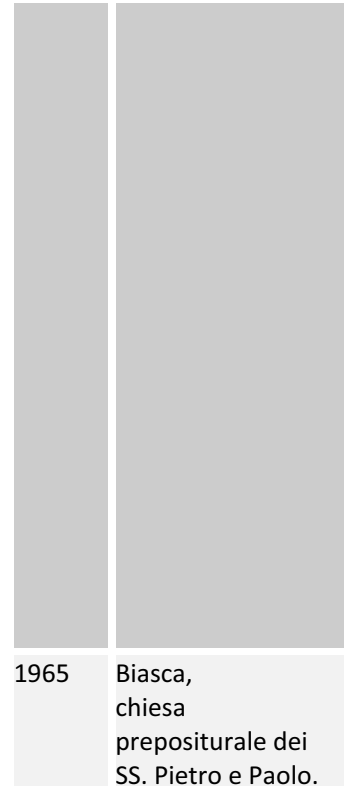

\section{Affreschi XVII sec.}

Abside.

SS. Pietro e Paolo. dl spirito; 1

scalpello lungo; 1

lampadina; 1

scatola chiodi tela;

2 secchi calce

speciale;

Ammoniaca; Latte;

$1 / 2 \mathrm{~kg}$. Caseina in

polvere; Telaio[...]

Lo strappo non fu

ritirato fino

all'ottobre del 1968

dall'architetto

Taddeo Carloni, al

domicilio del pittore

Sergio Maino, per

portarlo al Castel

Grande di

Bellinzona.

Strappo di

quattordici figure e

frammenti.

Ricollocamento sui

pilastri della chiesa

verso la navata

inferiore e sulla

controfacciata. Gli

elementi distaccati

furono trasportati

su tela e

posteriormente su

telai rigidi. Le

lacune furono

reintegrate con

malta a base di

sabbie fine

colorate.

Reintegrazione con

tempera all'uovo.

Trattamento con

gomma arabica per

ravvivare i colori.

Misure dei dipinti

strappati e

ridimensionati: (91

x $200 \mathrm{~cm}),(91,5 \mathrm{x}$

$188,5 \mathrm{~cm}),(91 \times 182$

$\mathrm{cm}),(41 \times 62 \mathrm{~cm})$,

$(63 \times 102 \mathrm{~cm}),(85 \mathrm{x}$

$186 \mathrm{~cm}),(55 \times 82$

$\mathrm{cm}),(71 \times 300 \mathrm{~cm})$,

$(88,5 \times 197 \mathrm{~cm}),(87$
Pittore- (Fulvio Caccia, 1984,

restauratore: p. 71).

Bruno Abbiati. (Renato, 1967).

(Moglia, 1967, p.

195-210). 


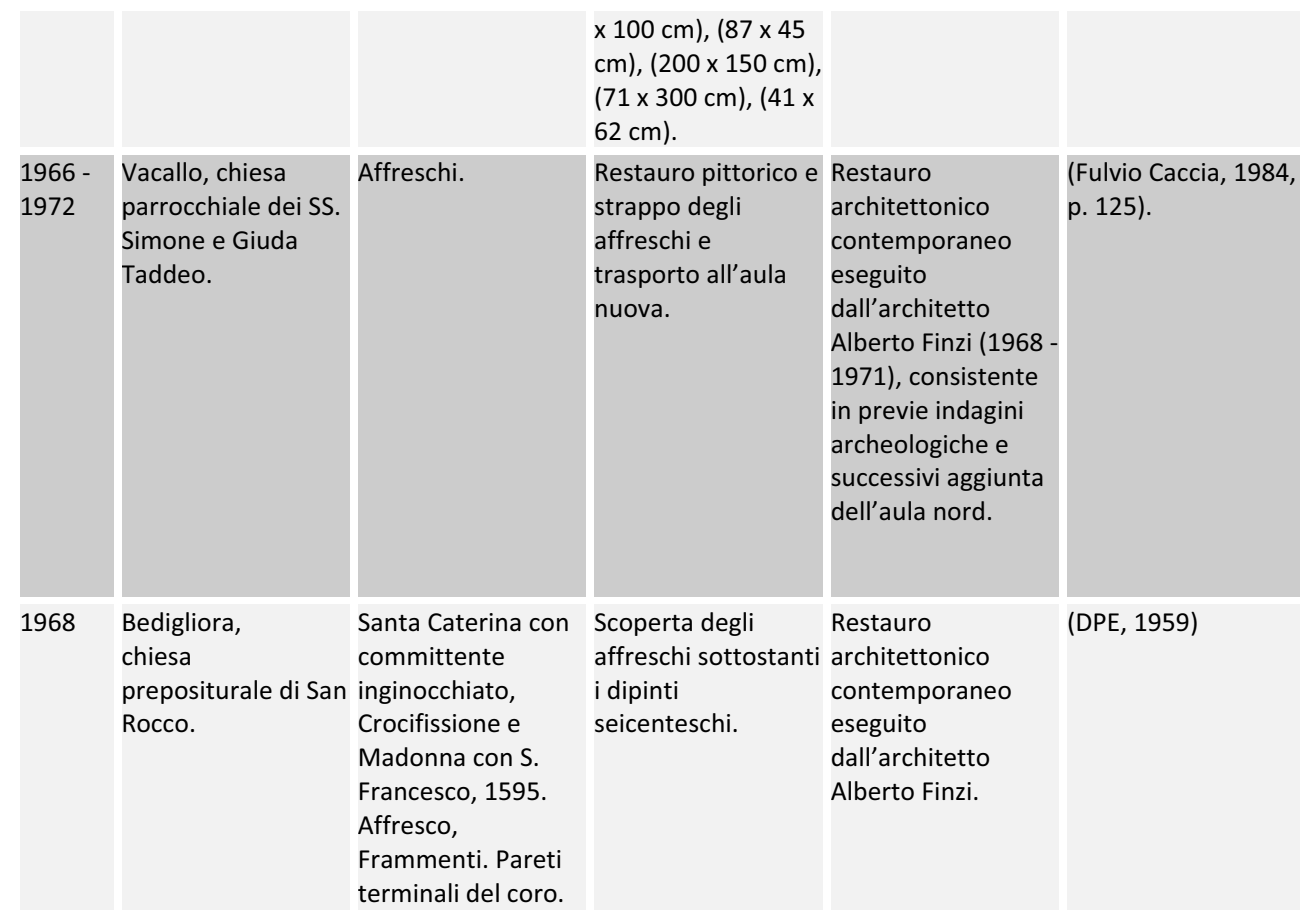




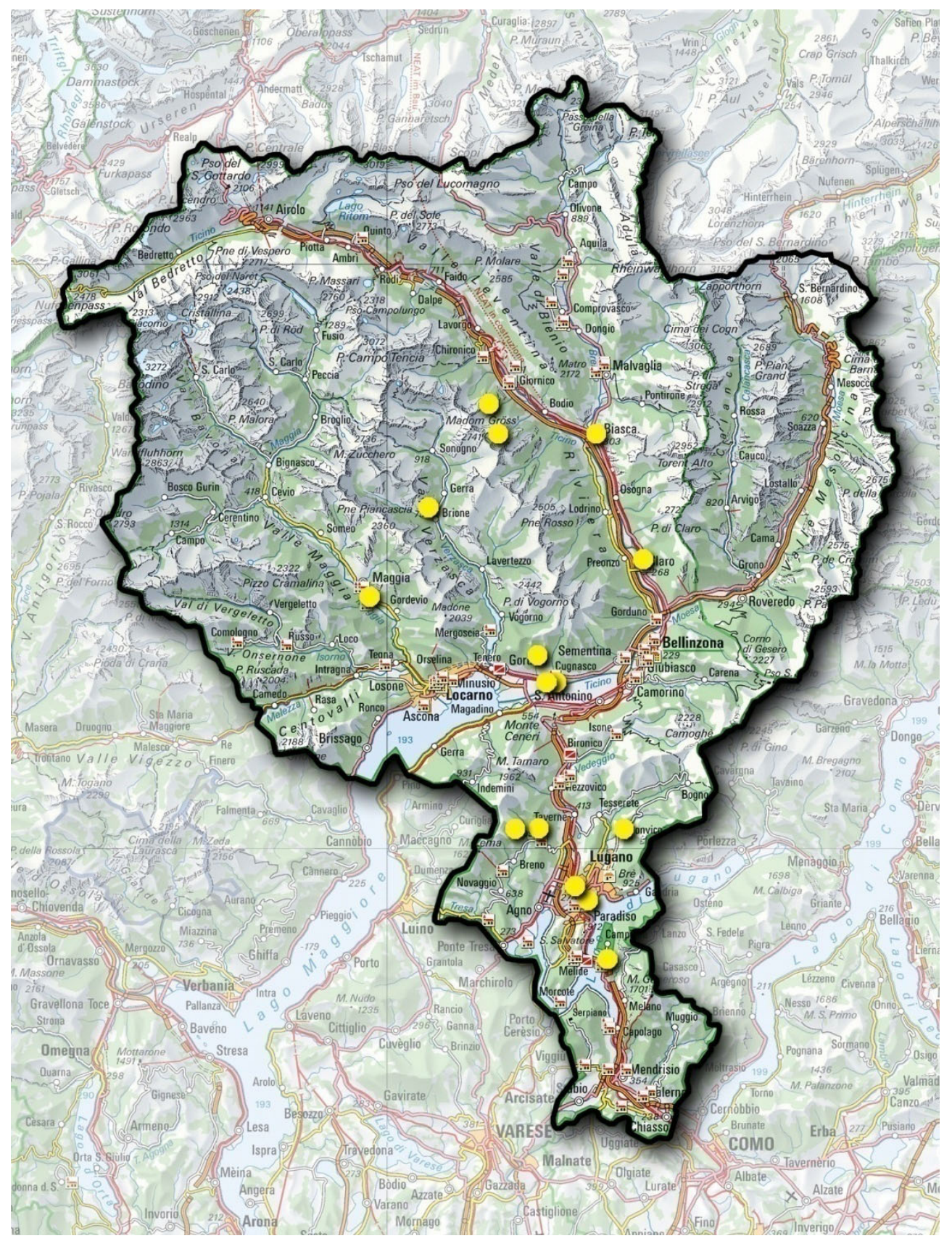

Figura 186 Cartina degli interventi eseguiti da Mario Moglia in Canton Ticino. 


glinati OAtrino. 


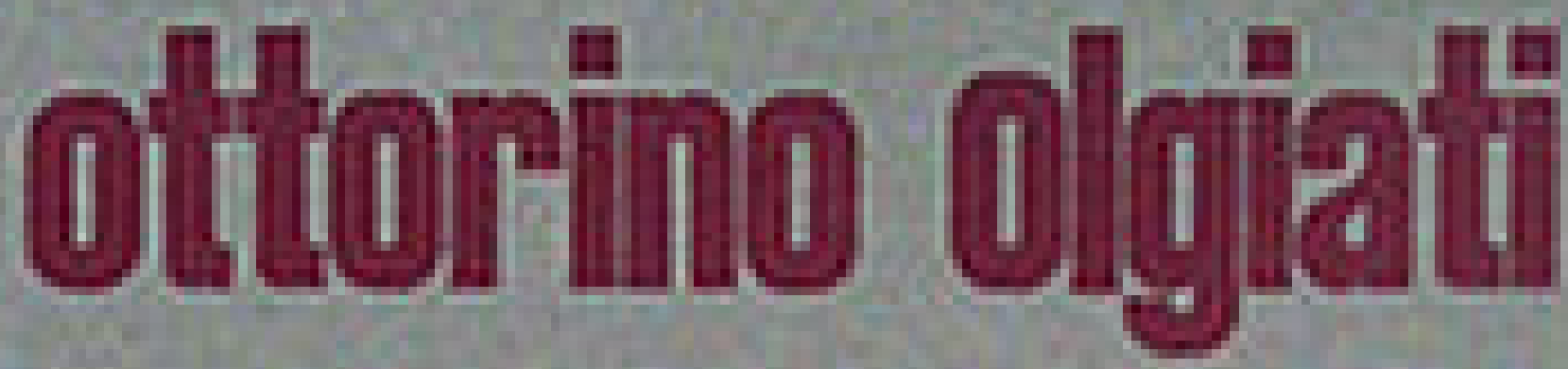

pelntures

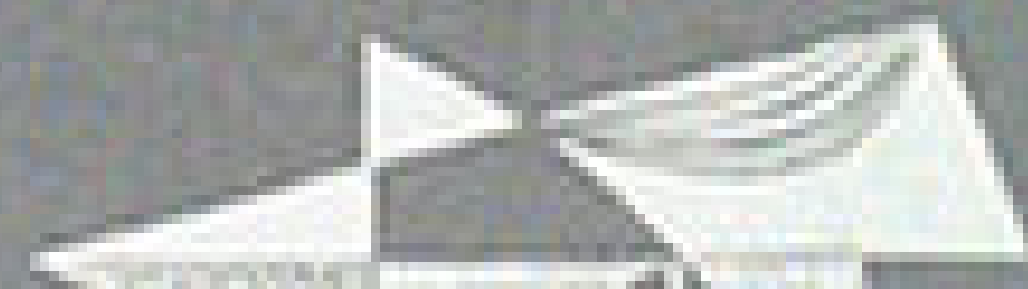

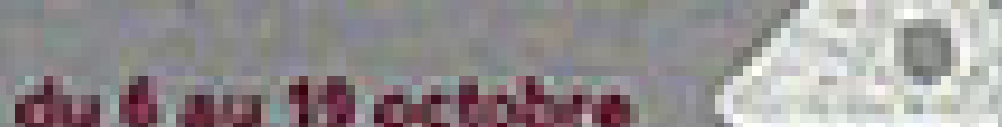

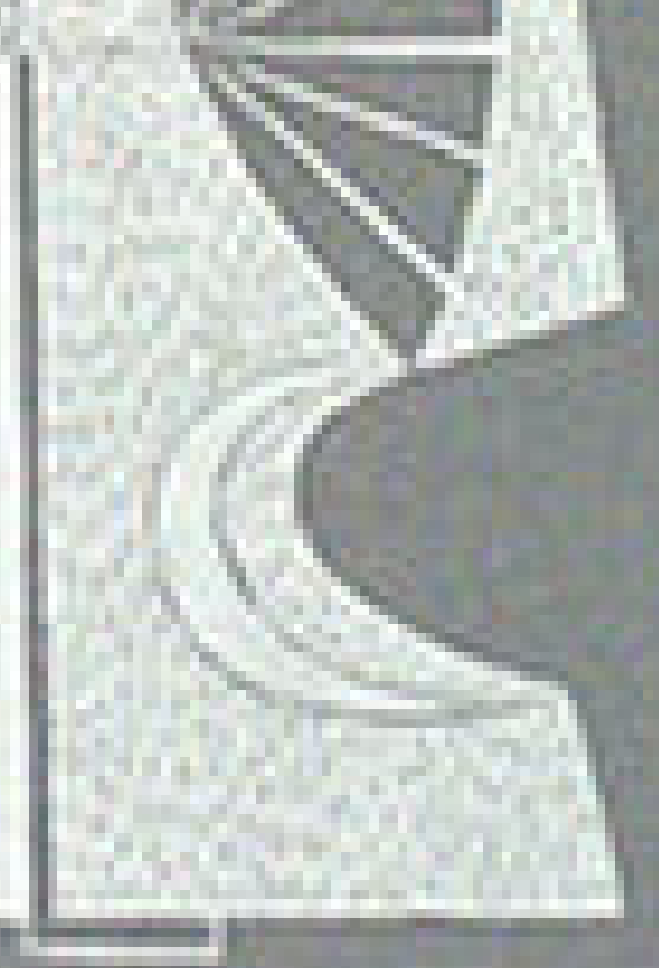

galeris amad

3 roedu four

paris 


\section{OTTORINO OLGIATI}

[...]Mi trovavo per il corso di ripetizione, nel giugno scorso, (copertura delle frontiere) a Ponte Capriasca. Sapevo che in quella regione vi erano delle opere d'arte, perché diversi pittori comacini vi lavoravano per i signori di Milano ai quali apparteneva la terra. [...] Nei momenti di libertà che il servizio mi permetteva, girovagavo per il paese osservando. Le case sono di costruzione tipicamente ticinese, bellissime, delle quali molte facciate lasciano intravvedere dei dipinti ormai consumati dalle intemperie. Durante una di queste mie peregrinazioni venni a trovarmi in una di queste case, dove, un'ispirazione (se cosi posso chiamarla) mi suggerì che scrostando il muro che mi stava di fronte, avrei rinvenuto qualche cosa molto interessante. Un primo colpo di baionetta (non avevo altro in quel momento) ed il vecchio intonaco si squarcia un pochino mettendo in luce delle tracce di colore. Incuriosito continuo lo scrostamento ed in poco tempo mi appaiono quattro bellissimi frammenti di affreschi. Sono cinque teste: un St. Cristoforo, un Cristo risorto, un Angelo (?) e due teste di santo. II S. Cristoforo è del 1300, gli altri sono del 1400. Parecchi giorni dopo in un'altra casa misi alla luce altre due bellissime teste. Un S. Giuseppe (?) ed un angelo pure del 1400. L'intonaco di circa 5 centimetri di spessore che li ricopriva trova la sua spiegazione nel fatto che nel 1500 infieriva in questi paraggi la peste. Per precauzioni d'igiene, $i$ muri delle case furono intonacati. Ecco in poche parole l'analisi e le sintesi del mio modesto lavoro, compiuto in parte durante le ore di libertà [...] Nell'ultima sua seduta, la Commissione Cantonale dei Monumenti Storici ha catalogato gli affreschi in parola.[... $]^{468}$.

${ }^{468}$ (Olgiati, 1939, p. 261).
[...]Me encontraba en el curso de repetición, el pasado mes de junio (control de las fronteras) en Ponte Capriasca. Sabía que en aquella región había obras de arte, porque distintos artistas $\operatorname{comascos}^{469}$ habían trabajdo en pasado en esta localidad para los señores de Milán a los que pertencían las tierras[...]. En los momentos de libertad que el servicio militar me permitía, vagabundeaba per el pueblo observando. Las casas de construcción típica "ticinese", preciosas, en las que numerosas fachadas muestran pinturas murales, la mayor parte desaparecidas por causas ambientales. Durante uno de estos paseos me encontré en frente a una de estas casas, donde, una inspiración (si así podemos llamarlo) me sugirió que rascando la pared de enfrente encontraría algo interessante. Un primer golpe con la vayoneta (no disponía de otr instrumento en ese momento) y el viejo intonaco se rompió mostrado restos de color. Intrigado continué la operación y en poco tiempo aparecieron cuatro preciosos fragmentos de afrescos. Se trata de cinco cabezas: un S. Cristóbal, un Cristo resucitado, un Ángel (?) y dos cabezas de santos. S. Cristóbal es del 1300, los otros son del 1400. El intonaco que cubría las pinturas, de 5 $\mathrm{cm}$ aproximadamente de profundidad, fue aplicado seguramente en el 1500 cuándo en la región amenazaba la peste. Per higiene los muros de las casas se encalaban. Éste ha sido, en pocas palabras, mi modesta labor ejercida durante las horas de libertad[...]. En su última asamblea, la CCMS ha catalogado las obras[...]

\footnotetext{
9 NdT. El término "comacini" en italiano, hace referencia a una corporación de artistas especializados en la edificación, activos desde el siglo VII aproximadamente en la zona del Cantón Ticino y del lago de Como. Seguramente el adjetivo "comacini" se deba de hecho al origen "comasco" de estos "maestros"; por esta razón se ha decidido emplear el gentilicio de "comasco" en la traducción.
} 


\section{Dati biografici}

Ottorino Olgiati nasce a Bellinzona il 13 settembre 1913, e muore il 3 giugno del 1972 a Locarno. Purtroppo della sua biografia sappiamo poco più che questi due dati anagrafici. Conosciamo la carriera formativa del pittore unicamente grazie ai documenti di archivio che ci indicano gli inizi dei suoi studi artistici all'Accademia di Belle Arti di Brera a Milano e successivamente presso l'Accademia di Belle Arti di Roma, città dove sviluppò la sua attività come pittore contemporaneamente alla professione di restauratore.

Senza dubbio la relazione di Olgiati con i laboratori di restauro aperti all'interno dei Musei e Gallerie Pontificie della Città del Vaticano, ancora dopo il suo ritorno in Ticino, furono per il restauratore uno splendido biglietto da visita. Questo lo aiuta ad essere scelto in maniera privilegiata fra i colleghi, pittorirestauratori che nell'epoca cercano di guadagnarsi la vita lavorando con questa carriera alternativa.

Roma, centro dell'arte e città dove nascono le concezione moderne di restauro poco prima dell'istituzione dell'Istituto Centrale del Restauro, era considerata per le istituzioni cantonali in Ticino il centro per antonomasia dello studio e della conoscenza delle tecniche in materia. Olgiati, che aveva studiato e lavorato nella medesima città era uno dei migliori professionisti al quale loro potevano affidare monumenti appartenenti al patrimonio storico del Cantone.

Anche se la partecipazione all'analisi di opere nel Canton Ticino catalogati nella ricerca sono unicamente tre di maggiore importanza, tutti svolti nella città di Locarno: la chiesa di S. Francesco, la Casa del Negromante e la chiesa di Santa Maria in Selva, dal 1947 al 1951. Inoltri, fra questi, solo il primo fu risolto con il proprio intervento di restauro.

Successivamente vedremo però che i metodi d'intervento del pittore, espressi nelle proposte di restauro di tutti e tre $i$ casi di studio locarnesi, non erano molto Iontani a quanto per il momento era stata prassi abituale di restauro nel territorio. La differenzia che la figura di Olgiati poteva supporre per la Commissione Cantonale dei Monumenti Storici in Ticino, in confronto con un Tita Pozzi, anche egli rispettoso e curato operatore, era unicamente il contatto diretto con il prestigioso laboratorio dei Musei Vaticani, con il quale l'Olgiati confrontava le proprie proposte di intervento così come i materiali che intendeva impiegare in ogni caso.

Le sue relazioni di restauro, complete e competenti, dimostrano l'approccio analitico con cui il pittore, formato in materia, descrive inizialmente l'opera: tecnica pittorica, materiali, stato di conservazione ed una argomentazione degli strumenti o meccanismi utilizzati per riconoscere essi. Finalmente i manoscritti si concludono con una proposta di intervento idonea ai casi accennati e con un elenco delle fasi di lavoro preventivato. Questo è quanto abbiamo trovato nella corrispondenza di archivio che l'Olgiati mantenne a lungo negli anni Quaranta: lunghe e precise relazione, quasi narrazioni, come l'articolo trascritti nella pagina precedente dove lui stesso si mostra come un ricercatore motivato nella 
scoperta e nella conservazione di opere che il tempo, la cultura e le mode, tolsero il valore primitivo.

La prassi di intervento di restauro del pittore $\mathrm{fu}$, in generale rispettosa, facendo uso tanto di materiali tradizionali (caseina, uovo, acquarello, calce) come di moderni che in quel momento si introducevano nei cantieri di lavoro italiani, allo stesso tempo che impiegava tecniche di reintegrazione come il rigattino, che in Ticino si impone solo dopo la fine degli anni Cinquanta.

Due casi esemplificativi del lavoro di Olgiati sono il restauro della Casa del Negromante a Locarno, eseguito dal pittore nel 1947, e la Chiesa di Santa Maria in Selva, anche essa a Locarno, la cui proposta di restauro venne presentata nel 1951.

\section{S. Maria in Selva, Locarno}

L'antica chiesa di Santa Maria in Selva risale al XV sec. Di quest'epoca oggi si conservano unicamente il coro e il campanile tardomedievale

La prima proposta di restauro dei dipinti murali esistenti alla chiesa di Santa Maria in Selva fu presentata dai pittori Emilio Ferrazzini e Tita Pozzi il 29 marzo del 1945. In questa relazione indirizzata a Francesco Chiesa gli artisti raccomandavano, nella volta, il consolidamento dell'intonaco sgretolato mediante la fluatazione, il fissaggio della pellicola pittorica nei punti pericolanti e la velatura delle macchie bianche nell'intonaco con una tonalità grigiastra, la pulitura generale del sudiciume di polvere e grasso superficiale, la rimozione dei rifacimenti pittorici e dell'intonaco, il consolidamento delle zone friabili e per ultimo l'encausticatura delle zona opacizzate e velate dopo la pulitura.
Inoltre si riteneva necessario il rifacimento dello zoccolo formato da diversi rappezzi e materiali diversi, trovato molto danneggiato a causa dell'umidità. Tutte queste fasi di restauro vengono preventivate per un prezzo complessivo di fr. 3'220.

Anni più tardi venne incaricato, prima a Carlo Cotti, poi a Mario Rossi ed a Ottorino Olgiati, di ispezionare le pitture e di presentare alla Commissione Cantonale dei Monumenti Storici Artistici una relazione riguardante lo stato di conservazione delle pitture ed una proposta di intervento idonea.

Olgiati fu, fra tutti loro, chi presentò la più completa relazione indicando con assoluta competenza non solo i degradi trovati nei dipinti murali ma anche la tecnica con cui $\mathrm{fu}$ eseguita la pittura argomentando seriamente $\mathrm{i}$ processi di deterioro dei materiali impiegati per la realizzazione dei dipinti e, conseguentemente dei metodi di restauro appropriati.

La tecnica artistica utilizzata per l'esecuzione delle pitture è la tempera forte (calce e uovo), applicata a secco su un solo strato di intonaco (arricciato) con uno spessore variabile dai $3 \mathrm{~cm}$ ai $6 \mathrm{~cm}$.

[...]Ho dovuto subito escludere che fossero eseguiti a buon fresco così pure come preparazione a fresco [...] l'intonaco che supporta queste pitture è di un solo strato (e noi sappiamo che una pittura a fresco necessita due strati l'arriccio e l'intonaco) [...] il colore poi presenta una opacità propria della tempera, il buon fresco essendo invece piuttosto lucido in più non ha trovato traccia alcuna degli attacchi dell'affresco (cioè le giunture dei pezzi d'intonaco delle giornate lavorative) che per 
quanto possano essere ben eseguite non si riesce mai a nasconderle. $[\ldots]^{470}$.

All'analisi della resistenza del colore, le carnazioni ed i colori chiari si manifestarono fra $i$ più resistenti allo sfregamento del batuffolo di cotone inumidito. La eccezione nella tecnica pittorica fu ritrovata nella scena della Crocifissione, nella quale il Cotti osserva la esistenza di due "giornate" per cui si tratta di una pittura a fresco che, inoltre, presenta la particolare lucentezza della tecnica.

Lo stato di conservazione osservato nell'analisi autoptica realizzata dal restauratore si dichiarava mediocre, con un intonaco e pittura disgregati e polverizzati a causa dell'umidità per infiltrazioni d'acqua dal tetto e per risalita capillare. Questo ha creato numerose lacune che lasciano a vista in diversi punti i sassi della muratura di supporto. Nel momento della perizia non si verificarono più infiltrazione perché il tetto e le pareti esterne erano state sistemate precedentemente. Un secondo agente di degrado si era verificato dalla presenza di tre finestre aperte (senza vetro) esistenti sulle pareti di Levante e di Sud e da dove passa l'acqua della pioggia ed il vento. Si osservarono diverse lesioni sulla volta e nelle pareti, chiuse durante la ristrutturazione del tetto con un intonaco di calce e sabbia eseguito, secondo il restauratore, da "mani inesperte" e con [...] poco rispetto alle pitture in quanto per una larghezza di cui $2 \mathrm{~cm}$ della lesione si mise un intonaco di $15 \mathrm{~cm}$ ricoprendo perciò buona

${ }^{470}$ (Ottorino Olgiati, Proposta d'intervento. AUBCBZ, s. 134 Locarno. S. Maria in Selva) parte della pittura sana $[\ldots]^{471}$, che poi si erano riaperte. II quadro raffigurante la Natività nella parete sinistra si trovò completamente sbiadito, mentre la mancanza di colore è generalizzata. Nella volta si ritrovò sullo sfondo, nel manto della Madonna e nel manto del Cristo incoronato un tono grigio dato a calce come preparazione del colore blu (forse lapislazzuli o cobalto) dato sopra a colla. Quest'ultimo si è completamente disgregato lasciando visibile unicamente il grigio sottostante. Si osserva uno strato di polvere e grasso superficiale rilevante, così come diverse macchie d'umidità che alterano l'omogeneità e la leggibilità dei dipinti.

Proposta di intervento interessato in due fasi: la prima consistente nel consolidamento del supporto, il distacco e riattacco di uno spanciamento dell'intonaco sulla volta, la stuccatura completa delle lacune, la pulitura generale dei dipinti, il consolidamento con nuovo glutine della pellicola pittorica, l'assorbimento delle macchie d'umidità ed il ravvivamento generale del colore, mentre la seconda fase interessa il ritocco pittorico, sperimentando il sistema in uso ai Musei e Gallerie Pontificie.

Il sistema di ritocco citato da Olgiati era il "tratteggio", così definito dal suo creatore Cesara Brandi, consistente nella giustapposizione e anche sovrapposizione di tratti verticali di colori differenti che ad una certa distanza venivano sintetizzati in un unico valore cromatico. II sistema permetteva omogeneizzare l'opera pittorica ma differenziare nella distanza le

${ }^{471}$ (Ottorino Olgiati, Proposta d'intervento. AUBCBZ, s. 134 Locarno. S. Maria in Selva) 
parti originali di questa delle parti aggiunte. Effettivamente, un anno prima che Olgiati scrivesse la proposta di restauro per il ritocco degli affreschi in S. Maria in Selva, il metodo del "tratteggio" fu utilizzato in primo luogo sui dipinti murali di Lorenzo da Viterbo alla Chiesa della Verità a Viterbo. Questo sistema di reintegrazione veniva descritto da Brandi nel seguente modo:

[...] ho escogitato un sistema di completamento che pur rimanendo sempre percettibile $e$ riconoscibile ad una visione ravvicinata [...] La tecnica consiste in tanti sottili filamenti ravvicinati, verticali e paralleli, che producono, all'acquarello, la plastica ed i colori come nel tessuto di un arazzo: se da vicino si staccano inequivocabilmente dalla stesura larga dell'affresco, da lontano l'immagine si coagula e rifiorisce $[. . .]^{472}$.

II metodo, adottato dai Musei e Gallerie Pontificie come accenna Olgiati, fu oggetto non solo della sua propria sperimentazione ma ancora a suo tempo della sperimentazione del suo creatore, Cesare Brandi chi insieme agli allievi dell'Istituto Centrale del Restauro di Roma dal 1945 al 1946 provò i diversi metodi di ricostruzione del colore non solo con il "tratteggio" ma anche con piccoli segni e figure geometriche come punti e cerchietti.

\footnotetext{
${ }^{472}$ La citazione di Cesare Brandi è stata estratta da testo di Maria Carolina Gaetani, La reintegrazione delle lacune attraverso la tecnica del tratteggio: considerazioni sul metodo all'interno degli atti del Convegno Internazionale di Studi tenutosi a Viterbo dal 12 al 15 novembre del 2003 (AA.VV., 2003, p. 277). A suo tempo la descrizione di C.Brandi sul restauro dei dipinti della Capella Mazzatosta è presente in Mostra dei frammenti ricostruiti di Lorenzo da Viterbo, a cura di C. Brandi, Roma 1946 e in II restauro. Teoria e pratica, di C.Brandi a cura di M. Cordaro, Roma 1994, pp.84-89.
}

Finalmente le linee risultarono essere quelle che maggiormente si adattavano alla riproduzione. La particolarità ritrovata nel metodo arricchiva il suo significato di differenziazione dell'originale anche dal fatto di dover usarsi l'acquarello per il suddetto ritocco, che permetteva una distinzione non solo visiva ma ugualmente per i materiai di impiego. Dalla messa a punto del sistema di ritocco Cesare Brandi lo introdusse in ogni restauro eseguito per conto dell'ICR, ed inevitabilmente questa tecnica sarebbe riconosciuta con la teoria di Brandi ed identificata con l'Istituto.

\section{Casa del Negromante, Locarno}

Le stesse prassi di lavoro qui sopra esaminate erano state proposte nel progetto di restauro che Ottorino Olgiati presentò al Dipartimento della pubblica educazione del Cantone Ticino il 7 novembre 1947 per l'intervento pittorico sulla casa del Negromante.

L'edificio, che prende l'appellativo dal suo proprietario nel XVIII sec. Giovan Battista Orelli, detto il Negromante, ha origini medievali, e conserva nell'atrio i dipinti decorativi della metà del XVI sec. Essi furono oggetto di analisi di Olgiati alla fine degli anni Quaranta. La perizia presentata alla CCMS svelava il sollevamento della pittura a causa dell'umidità, la presenza di un forte strato di calce sul fregio centrale, l'abbondante quantità di sudiciume superficiale, lo sgonfiamento ed il distacco del "retro intonaco" - utilizzando le parole di Ottorino Olgiati, osservando l'esistenza d'importanti lesioni nell'intonaco e nell'arricciato. L'intonaco presentava molte lesioni coperte e chiuse che attraversano il dipinto in senso verticale, lesioni non solo 
superficiali dell'intonaco ma pure dell'arricciato in profondità.

Il restauratore proponeva il consolidamento dell'arriccio in profondità mediante iniezioni di malta a base di cemento e sabbia, consolidamento dell'intonaco con caseato di calce ed il rifacimento della parti mancanti. $\mathrm{Di}$ seguito, secondo le pretese di Ottorino Olgiati, si sarebbero stuccate le lacune e chiuse le lacune, la "scoperta" sotto lo scialbo di calce dello strato di calce sul fregio, la pulitura generale della superficie pittorica, il ravvivamento del colore con miscela chimica adatta, il consolidamento della pellicola pittorica a tempera (sicuramente alla caseina), mediante l'applicazione di un nuovo "glutine", e per ultimo, il così chiamato "ravvivamento pittorico" che consisteva nel ritocco pittorico ad acquarello oppure a tempera a caseina delle piccole zone dove si delinea il disegno costruttivo, mentre la lacune stuccate ed i nuovi pezzi d'intonaco venivano velati da una tinta neutra in armonia con il complesso pittorico.

[...]NB. Mi permetto far notare che i restauri delle pitture murali a tempera sono i più delicati a trattarsi. Richiedono maggiori precauzioni e tempo. Tutti i materiali da me usati per il restauro sono stati analizzati dal Gabinetto Chimico del Laboratorio di Restauro di Musei e Gallerie Pontificie.[... $]^{473}$

\footnotetext{
${ }^{473}$ (Olgiati Ottorino. Relazione ed esame sullo stato di conservazione dei dipinti ornamentali situati nel porticato della casa dei "Nobili" in Locarno. Preventivo di spesa peri I restauro dei dipinti ornamentali situati nelle pareti nel porticato della casa dei Nobili cosidetta "Negromante" in Locarno. Indirizzata al DPE e CCMS. Bellinzona 7 novembre 1947. AUBCBZ, s. 134 Locarno. Casa del Negromante)
} 


\section{Interventi di restauro}

\begin{tabular}{|c|c|c|c|c|c|}
\hline ANNO & LUOGO & OPERA & INTERVENTO & COLLABORATORI & FONTI \\
\hline 1947 & $\begin{array}{l}\text { Locarno, Casa del } \\
\text { Negromante. }\end{array}$ & $\begin{array}{l}\text { Dipinti ornamentali } \\
\text { a tempera su } \\
\text { preparazione a } \\
\text { calce del colore } \\
\text { fundamentale ocra } \\
\text { gialla. }\end{array}$ & $\begin{array}{l}\text { Perizia e proposta } \\
\text { di restauro: } \\
\text { [...]In molti punti la } \\
\text { tempera si è } \\
\text { nervata causa } \\
\text { umidità staccando } \\
\text { così il colore. II } \\
\text { fregio centrale è } \\
\text { parzialmente } \\
\text { nascosto da un } \\
\text { forte strato di calce. } \\
\text { Sudiciume } \\
\text { abbastanza } \\
\text { abbondante. Per } \\
\text { quanto riguarda } \\
\text { l'intonaco il } \\
\text { processo } \\
\text { disintegrativo del } \\
\text { retro intonaco è } \\
\text { molto steso e in } \\
\text { molti punti il } \\
\text { fenomeno di } \\
\text { distacco causa } \\
\text { sgonfiamenti. } \\
\text { L'intonaco ha molte } \\
\text { lesioni coperte e } \\
\text { chiuse che } \\
\text { attraversano il } \\
\text { dipinto in senso } \\
\text { verticale, lesioni } \\
\text { non solo superficiali } \\
\text { dell'intonaco ma } \\
\text { pure dell'arricciato } \\
\text { in profondità. } \\
\text { Lavori di restauro } \\
\text { necessari: } \\
\text { press. Consolidament } \\
\text { o del retro } \\
\text { rifacimentonaco con } \\
\text { iniezioni di } \\
\text { caseato di calce e } \\
\text { nei punti più } \\
\text { profondi iniezioni } \\
\text { di cemento e } \\
\text { arena; messa in } \\
\text { onto dei }\end{array}$ & & $\begin{array}{l}\text { (AUBCBZ, s. } 134 \\
\text { Locarno. Casa del } \\
\text { Negromante) } \\
\text { (Olgiati Ottorino. } \\
\text { Relazione ed esame } \\
\text { sullo stato di } \\
\text { conservazione dei } \\
\text { dipinti ornamentali } \\
\text { situati nel porticato } \\
\text { della casa dei } \\
\text { "Nobili" in Locarno. } \\
\text { Preventivo di spesa } \\
\text { peri I restauro dei } \\
\text { dipinti ornamentali } \\
\text { situati nelle pareti } \\
\text { nel porticato della } \\
\text { casa dei Nobili } \\
\text { cosidetta } \\
\text { "Negromante" in } \\
\text { Locarno. Indirizzata } \\
\text { al DPE e CCMS. } \\
\text { Bellinzona } 7 \\
\text { novembre } 1947 . \\
\text { AUBCBZ, s. } 134 \\
\text { Locarno. Casa del } \\
\text { Negromante). }\end{array}$ \\
\hline
\end{tabular}




\begin{tabular}{|c|c|c|c|}
\hline & & & $\begin{array}{l}\text { pezzi d'intonaco } \\
\text { mancante } \\
\text { 2. Stuccatura } \\
\text { generale, chiusura } \\
\text { delle lesioni. } \\
\text { Scopritura del } \\
\text { dipinto centrale } \\
\text { dal forte strato di } \\
\text { calce } \\
\text { 3. Pulitura } \\
\text { generale della } \\
\text { superficie } \\
\text { colorata } \\
\text { 4. Ravivamento } \\
\text { del colore con } \\
\text { miscela chimica } \\
\text { adatta } \\
\text { 5. Rimessa di un } \\
\text { nuovo glutine che } \\
\text { fissa il colore } \\
\text { all'intonaco nella } \\
\text { parti dove il } \\
\text { colore tende ad } \\
\text { staccarsi } \\
\text { 6. Ravvivamento } \\
\text { pittorico:, } \\
\text { acquarella oppure } \\
\text { tempera a caseina } \\
\text { nei punti } \\
\text { principali dove si } \\
\text { delinea il disegno } \\
\text { costruttivo. Per } \\
\text { quanto riguarda i } \\
\text { nuovi pezzi } \\
\text { d'intonaco } \\
\text { consiglierò una } \\
\text { leggera tinta } \\
\text { neutra in armonia } \\
\text { con l'assieme } \\
\text { pittorico. }\end{array}$ \\
\hline 1947 & $\begin{array}{l}\text { Locarno, } \\
\text { Casa del } \\
\text { Negromante. }\end{array}$ & $\begin{array}{l}\text { Affresco. Piccolo } \\
\text { stemma nel centro } \\
\text { del fregio sulla } \\
\text { parete di sinistra. }\end{array}$ & $\begin{array}{l}\text { Relazione ed } \\
\text { esame sullo stato di } \\
\text { conservazione dei } \\
\text { dipinti ornamentali } \\
\text { situati nel porticato } \\
\text { della casa dei } \\
\text { "Nobili" in Locarno. } \\
\text { Sudiciume } \\
\text { abbastanza } \\
\text { abbondante. }\end{array}$ \\
\hline
\end{tabular}

(AUBCBZ, s. 134

Locarno. Casa del Negromante) (Olgiati Ottorino. Relazione ed esame sullo stato di conservazione dei dipinti ornamentali situati nel porticato della casa dei "Nobili" in Locarno. Preventivo di spesa 


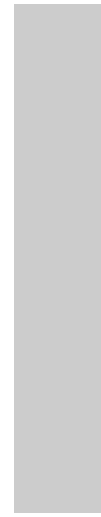

1950

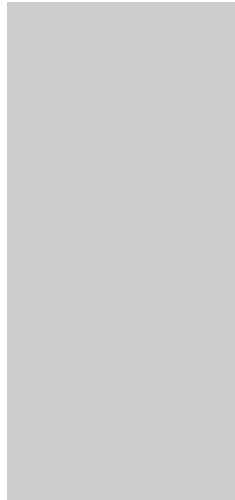

Locarno, chiesa di S. Affreschi, cappella Francesco.

dell'Immacolata.

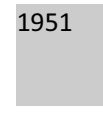

1951

ante

Locarno, chiesa di S.
Francesco.
Locarno,

Chiesa di S. Maria in Selva.

Dipinti murali.

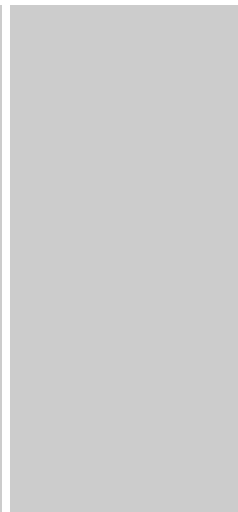

Annunciazione.
Affresco.

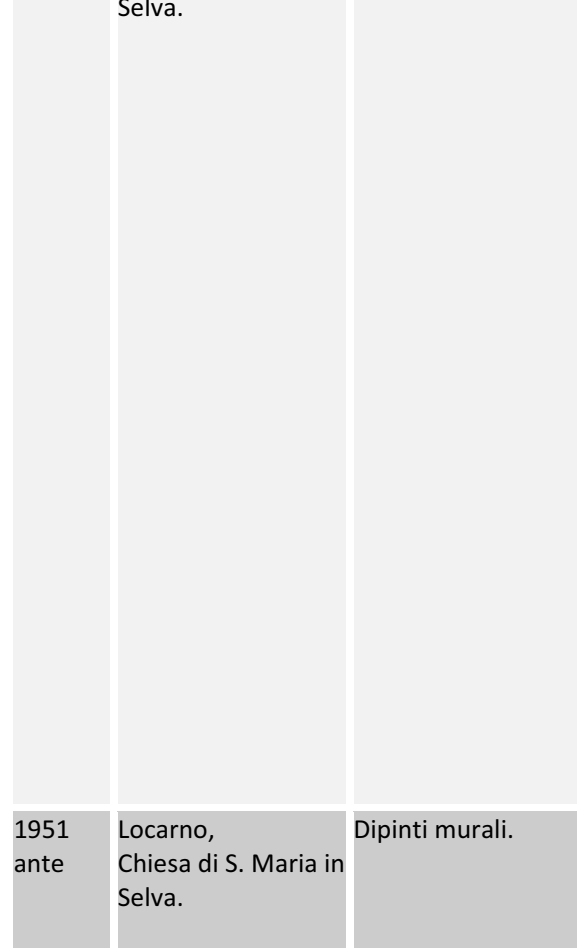

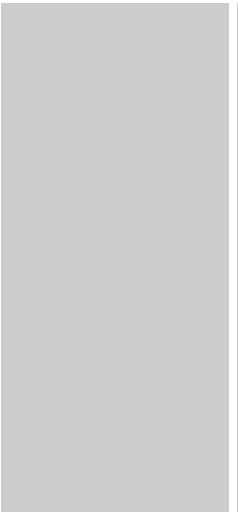

Restauro.

Restauro.

Perizia e proposta

di intervento

interessato in due

fasi: la prima

consistente nel

consolidamento del

supporto, il distacco

e riattacco di uno

spanciamento

dell'intonaco sulla

volta, la stuccatura

completa delle

lacune, la pulitura

generale dei dipinti,

il consolidamento

con nuovo glutine

della pellicola

pittorica,

l'assorbimento

delle macchie

d'umidità ed il

ravvivamento

generale del colore,

mentre la seconda

fase interessa il

ritocco pittorico.

Preventivo
provvisorio di
restauro
comprendendo la

peri I restauro dei

dipinti ornamentali situati nelle pareti nel porticato della casa dei Nobili cosidetta "Negromante" in Locarno. Indirizzata al DPE e CCMS. Bellinzona 7 novembre 1947. AUBCBZ, s. 134 Locarno. Casa del Negromante).

(AUBCBZ, s. 134

Locarno. S.

Francesco).

(AUBCBZ, s. 134 Locarno. S. Francesco).

(AUBCBZ, s. 134 Locarno. S. Maria in Selva).

(AUBCBZ, s. 134

Locarno. S. Maria in Selva). 


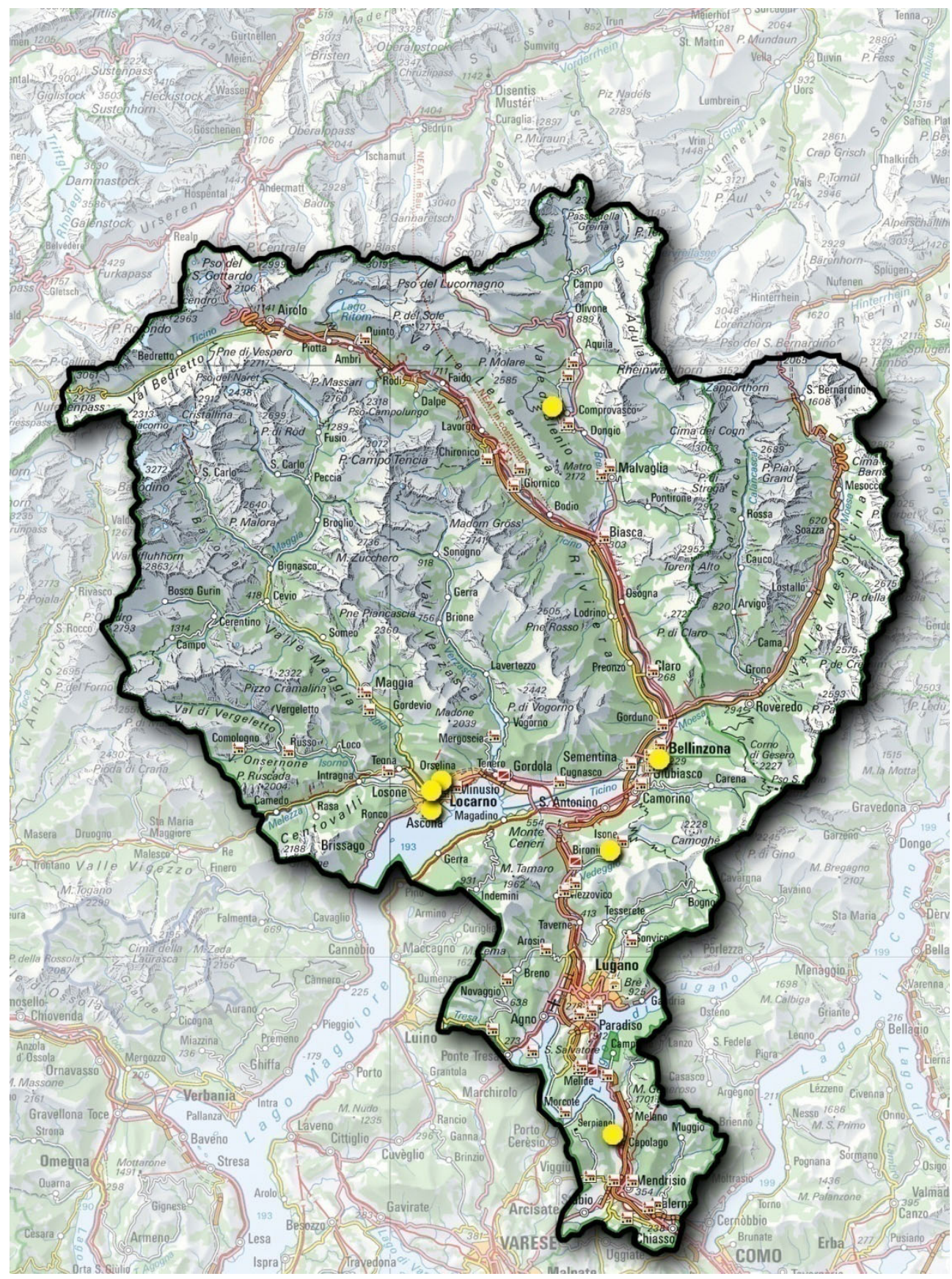

Figura 187 Cartina degli interventi eseguiti da Ottorino Olgiati in Canton Ticino. 

Qunlolinozis- 


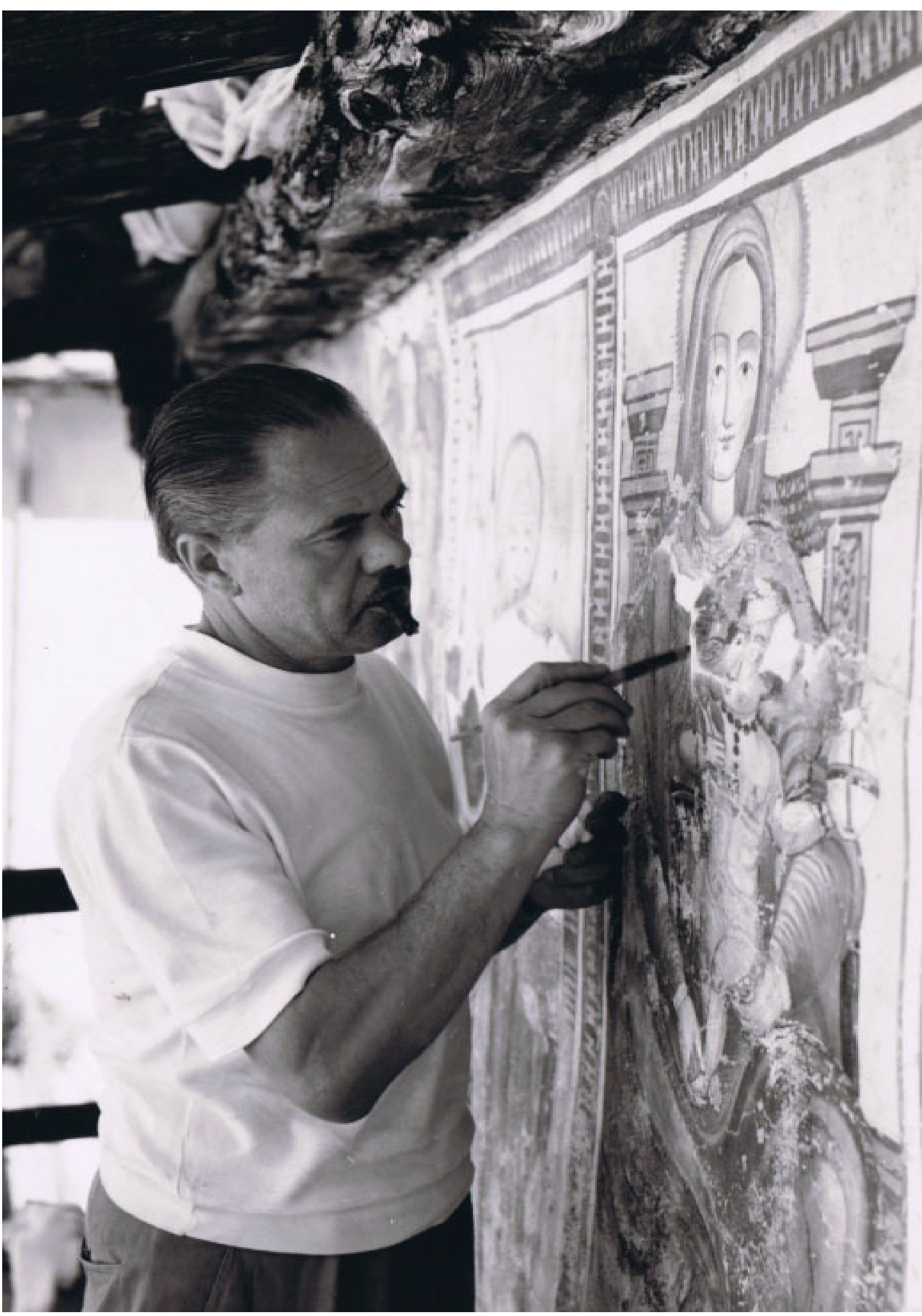




\section{CARLO MAZZI}

[...]II ventennale lavoro di restauratore è stato, per Mazzi, rilevante per il consolidamento della cultura che gli è propria, cattolica e contadina: ripassare gli affreschi religiosi è come ripassare il Vangelo e la Bibbia, evocare la profumata noia delle preghiere e ritrovarsi tu per tu con Cristo. [... $]^{474}$.

[...]El veintenale trabajo de restaurador ha sido, para Carlo Mazzi, relevante para la consolidación de la cultura a la que pertenece, católica y rural: repasar frescos religiosos es como repasar el Evangelio y la Biblia, evocar el perfume de las aburridas orazione $y$ reencontrarse tú a tú con Cristo[...]. 


\section{Dati biografici}

Carlo Mazzi, magnifico artista dell'ultima metà del Novecento, nasce nel piccolo comune di Tegna il 19 gennaio del 1911. Nella località locarnese abiterà e lavorerà fino alla sua morte, ad eccezioni di brevi soggiorni per studio nelle città italiane di Milano, Torino e Roma, a Parigi, a Zurigo e a Ginevra. In quest'ultima città della svizzera francese, dopo aver frequentato la Scuola di Commercio a Locarno dal 1929, l'artista inizierà la sua carriera artistica che esplorerà in modo autodidatta, nella pittura, dal 1930. I primi saranno quadri figurativi e rappresentazioni di paesaggi Ticinesi, che man mano muteranno in una splendida astrazione geometrica, risultato della maturità artistica conquistata dal Mazzi dopo gli anni Cinquanta. Le opere prodotte in quest'ultimo periodo (dipinti, disegni e incisioni) dimostrano una ricca conoscenza materica e tecnica, sicuramente influenzata della altre due attività da lui coltivate: la ceramica ed il restauro.

II mestiere di ceramista fu incominciato da Mazzi due anni dopo i primi assaggi con la pittura, nel 1932, e sarà sospeso nel 1965 a causa dei problemi di vista ad un occhio che la potente luce del forno gli aveva provocato. Cominciò in questo campo approfondendo la ceramica artigianale, per successivamente elaborare quella smaltata con grande successo, ottenendo preziose opere che oggi formano parte tanto di collezione private come del Museo di Ceramica di Faenza.

Visse in contemporaneità ad artisti come Carlo Cotti, Emilio Maria Beretta, Mario Moglia, Felice Filippini, Aldo Patocchi, Remo Rossi, Ottorino Olgiati, Mario Moglia,
Giovanni Genucchi e Pietro Salati, ma anche a grandi personalità riconosciute nell'ambito culturale, come il critico d'arte milanese Marco Valsecchi, Don Agostino Robertini, Aldo Crivelli o Piero Bianconi. Insieme a loro Mazzi interazionerà lungo la sua carriera nelle costanti manifestazioni e riunione dalle diverse associazioni artistiche, specialmente all'interno della SPSAS (Società pittori, scultori e architetti svizzeri) della quale fu membro dal 1952.

Con alcuni di essi le relazioni oltrepasseranno il settore puramente creativo per attuare inoltre nel settore della protezione dei beni culturali, al quale Mazzi si presta con completa competenza quasi per un ventennio (1952 - 1972).

L'artista locarnese fu introdotto alla professione di "restauratore" dal professor Mario Rossi di Varese, durante un tirocinio di due anni (1952 - 1954) in Italia. Con egli lavorerà al restauro della chiesa di Santa Maria in Selva a Locarno nel 1953 e nel Santuario della Beata Vergine dei Miracoli a Saronno nel 1954. Interverrà in quest'ultimo monumento sui magnifici affreschi di Bernardino Luini e di Gaudenzio Ferrari. Dopo questa pratica il Mazzi, pittore specializzato secondo la richiesta della propria Commissione Cantonale dei Monumenti Storici, incomincerà in solitario l'attività appresa in Italia lavorando su numerose ed importanti chiese, cappelle e palazzi in Canton Ticino, specie nel locarnese e nella leventina. Sarà Mario Rossi a raccomandare il pittore Mazzi al presidente della CCMS, Ugo Tarabori, in una lettera spedita alla fine del tirocinio il 27 marzo del 1954: 
[...]In questi giorni il Sig. Carlo Mazzi di Tegna ha finito, per suo desiderio, di collaborare nel restauro degli affreschi a me affidati in Italia. Le posso assicurare che ho trovato in Lui una persona di grande volontà, iniziativa e capacità di collaborazione. Naturalmente avrebbe bisogno di rimaner in esercizio con l'assegnazione di qualche lavoro di restauro per iniziarlo ad affrontare da solo i problemi che gli possono presentare; problemi che nell'arte del restauro sono infiniti e mai abbastanza conosciuti. Nella speranza, e con l'augurio, che questo tirocinio pur breve gli sia stato di valido contributo, anche per il vostro desiderio di avere in Ticino un pittore specializzato nei restauri, presento i miei migliori ossequi.[... $]^{475}$.

\section{II pittore ticinese "specializzato" in restauro}

Questa ricerca i ha permesso di catalogare i più importanti lavori di restauro eseguiti dal pittore Carlo Mazzi, circa vent'otto interventi, insieme ad altre piccole operazione generalmente di strappo di affreschi in diverse località del Ticino, che però non sono stati completamente identificati. Fra i restauri più importanti, solo due furono eseguiti su monumenti fuori del cantone: si tratta dei dipinti di Georges Dessouslavy ${ }^{476}$ nell'atrio della

\footnotetext{
${ }^{475}$ Rossi Mario. Lettera indirizzata a Carlo Mazzi, copia della medesima indirizzata al Sig. Tarabori. Varese, 27 marzo 1954. (Archivio C. Mazzi).

476 Dessouslavy Georges (La Chaux-de-Fonds 1898 Yverdon 1952). Figlio di Frédéric-Louis, commerciante di gessi. Frequenta dal 1914 al 1916 la scuola d'arte di La Chaux-de-Fonds e in seguito, fino al 1920, la scuola di belle arti di Ginevra. Dipinge figure e nature morte, paesaggista ed scenari monumentali, competendo anche nel settore della litografia. La sua pittura è influenzata dagli anni 1930 al 1940 dalla pittura postimpressionista trasformandosi improvvisamente al postcubismo come lo dimostrano le sue opere alle stazioni di Neuchâtel (1938) e di La Chaux-de-Fonds (1946-52)
}

stazione di Neuchâtel e la Chiesa di Bondo nei Grigioni.

Inoltre è stata fondamentale la scoperta dei preventivi e proposte di lavoro elaborati da Mazzi alla fine degli anni Sessanta, corrispondenti all'abside maggiore di San Carlo a Negrentino e alla chiesa della Madonna del Sasso a Morcote. Essi ci permettono di riconoscere gli ideali propri dell'artista in qualità di restauratore sicuramente influenzato dal professore italiano Mario Rossi, più di quanto possiamo rintracciare dal resto della documentazione cartacea esaminata all'Archivio dell'Ufficio dei beni culturali di Bellinzona. Questo si spiega dal fatto che i preventivi venissero redatti previamente alle riunioni ed ispezioni dei commissari dell'Ufficio cantonale dei monumenti storici, solo dopo un sopralluogo iniziale, e per tanto i pensieri di Mazzi non vengono nascosti dietro le possibili influenze o richieste istituzionali. Analogamente il ritrovamento fra la documentazione cartacea che conforma il suo archivio personale, scrupolosamente ordinato, di due quaderni con le precise spiegazione dei materiali, delle tecniche di lavoro, delle ricette e delle piccole provette usate per il restauro tanto degli affreschi come dei dipinti murali, ha facilitato enormemente questa ricerca. Si trattano degli appunti che Mazzi elaborò durante i primi anni di tirocinio (1953-1954) e, più avanti, nelle prime sperimentazioni di lavoro pratico. Questi scritti indicano le prassi di riferimento che successivamente saranno impiegate dal restauratore così come la bibliografia di riferimento alla quale si rivolgerà in numerosi scritti da egli redatti come i fondamentali testi di // Restauratore 
dei Dipinti, di Secco-Suardo ${ }^{477}$, di La tecnica della pittura dai tempi preistorici ad oggi di Leone Augusto Rosa ${ }^{478}$ e del Manuale pratico di tecnica pittorica : enciclopedia ricettario per tutti gli artisti, pittori dilettanti, allievi delle accademie di belle arti e delle scuole artistiche di Gino Piva $^{479}$.

Di fatto le tecniche che Mazzi impiega nei restauri sono le tradizionalmente usate dai trattatisti italiani della fine dell'ottocento, principalmente il sopracitato Secco-Suardo; seguendo inoltre le prassi avviate al laboratorio ed ai cantieri diretti dal restauratore Mario Rossi.

In un colloquio realizzato per la Radio Televisione della Svizzera Italiana (RTSI) nel 20 settembre 1967, all'interno del reportage Antichi monumenti, vecchi $e$ nuovi per lo spazio televisivo "Astrolabio" 480 , Mazzi spiega chiaramente queste sue idee:

[...] La tecnica che uso io in generale è la tecnica che ha usato il pittore per fare l'affresco, dato che io faccio il restauro in affresco; circa le tecniche nuove come mi ha chiesto ne esistono ma io sono malfidente, non le adopero, preferisco il sistema vecchio che hanno usato già cinquanta, cento anni fa perché ho le prove, vedendo altri restauri che hanno fatto altri pittori e restauratori che hanno usato le loro tecniche posso vedere i risultati, mentre se io uso i prodotti chimici venuti fuori adesso, che ce ne sono una infinità non ne ho tempo materiale per provarle. Posso fare le prove nel mio

\footnotetext{
477 Riferiamo la data dell'ultima edizione ritrovabile attualmente: (Secco Suardo, 1979).

478 (Rosa, 1937).

479 Riferiamo la data dell'ultima edizione ritrovabile attualmente (Piva, 1996).

${ }^{480}$ (TSI, Antichi monumenti, vecchi e nuovi in Astrolabio, 20.09.1967. Archivi televisivi. RTSI).
}

laboratorio ma per il momento non gli uso assolutamente[...].

Di fatto, nei lavori di Mazzi ritroveremo gli stessi materiali che lungo questa ricerca si sono analizzati più o meno in profondità. Cambieranno unicamente i limiti dei loro utilizzo, contenuto a le zone vulnerabili secondo la rispettosa e moderna concezione del minimo intervento di restauro.

Per la pulitura il Mazzi raccomanderà l'impiego della soda o potassa caustica, dell'ammoniaca e dell'acido acetico sciolti in acqua, l'ultima soluzione usata principalmente per il risciacquo finale o per il descialbo dello strato di calce sovrapposto all'affresco, con la cura di non intaccare il colore. I consolidamenti puntuali dei dipinti e degli intonaci venivano realizzati con caseina e caseinato di calcio rispettivamente i ritocchi il restauratore preferiva farli con i colori a calce o con gli acquarelli usando in genere la tratteggiatura.

Le stuccature erano, nel primo strato, conformate da una malta di sabbia (Mazzi indica la provenienza di essa nel fiume Maggia), pezzi di mattoni, e calce di almeno un anno, mentre insuperficie lo strato più fine era composto unicamente da calce e polvere di marmo. Per finalizzare il restauro Mazzi usava generalmente la gomma arabica diluita in acqua per, spiegava [...] rendergli maggiormente visibili $[\ldots]^{481}$.

Non abbiamo confermato l'utilizzo di cere nel restauro, anche se sappiamo che questo prodotto formava parte indiscutibile degli affreschi da lui elaborati,

(TSI, Antichi monumenti, vecchi e nuovi in Astrolabio, 20.09.1967. Archivi televisivi. RTSI) 
applicato come finitura della pittura, minimo due giorni dopo aver terminato di dipingere, e successivamente lucidato strofinando una pezza di lana. In particolare Mazzi scrisse nei suoi quaderni sulla cera bianca, che fa riferimento sicuramente alla paraffina: [...] finito un quadro diluire cera bianca con acqua ragia, buona $e$ ben calda; posare la miscela sull'affresco con pennellessa leggera. Appena asciutto lucidare $[. . .]^{482}$.

Le dosi in cui vengono utilizzati i diversi prodotti, si adatteranno alla necessità e qualità dell'opera, anche se come porzioni idonee il Mazzi nomina nei suoi scritti le stesse che definisce quasi un secolo prima Giovanni Secco Suardo nel suo Manuale. Ad esempio, la potassa sarà disciolta seguendo la ricetta di 8 parti d'acqua ed 1 di calce viva per 2 di potassa. Questa miscela acqua-potassa doveva essere bollita e fatta raffreddare prima d'aggiungere altra acqua e per ultimo la calce viva. II tutto si faceva riposare durante uno o due giorni, imbottigliando successivamente l'acqua limpida. La potassa caustica veniva impiegata nella pulitura degli affreschi, applicandola con un pennello di peli corti dopo di ché si risciacquerebbe bene la superficie con acqua pulita. Aveva, secondo il restauratore bergamasco, le proprietà di asportare il grasso, le macchie di cera, ed i microorganismi, così come lo scialbo di calce.

Sull'acido acetico Secco Suardo aveva già scritto la sua proprietà principale di togliere lo scialbo da [...]affreschi moderni con superficie ruvida[...], che con l'impiego di altri

\footnotetext{
${ }^{482}$ (Quaderni di studio. Archivio privato. Mina-Mazzi, Tegna.)
}

strumenti meccanici, quali i ferri, potrebbero danneggiare la superficie degli affreschi ed eliminare i molteplici ritocchi a secco di cui solitamente è conformato il dipinto murale che non è stato eseguito secondo la tecnica del buon fresco. Prima di servirsi dell'acido, il restauratore bergamasco consigliava realizzare una lavatura con la soluzione blanda di potassa sopracitata $^{483}$.

Anche nel caso del caseinato di calcio Mazzi si attenderà esattamente alle indicazioni del Manuale del Secco Suardo. Le dosi erano di 1 parte di caseina per 3 parti d'acqua e $1 / 4$ parte di colla forte. A questa miscela poteva aggiungersi del latte che rendeva più scorrevole il mastice e rallentava il tempo di presa facilitando il processo di riattacco degli intonaci e principalmente nell'adesione del dipinto strappato alle tele di supporto.

Lo strappo degli affreschi, per la cui operazione il pittore fu chiamato in numerose occasione ${ }^{484}$, veniva svolto anche questo nel modo tradizionale, secondo gli ideali instaurati in Italia, principalmente nella Lombardia. Gli affreschi trasportati alla tela venivano diminuiti ad una sottilissima lamina di colore, flessibile che però aveva completamente perso le sue caratteristiche proprie $^{485}$. Questo è quanto si voleva

\footnotetext{
483 (Giovanni Cecchini, 1995, p. 103)

${ }^{484}$ Nella presente ricerca sono riuscita a catalogare unicamente dieci casi in cui Mazzi esegue lo strappo degli affreschi, grazie alla documentazione presente nell'archivio personale e all'archivio dell'Ufficio dei Beni Culturali di Bellinzona, malgrado ci possano essere ancora piccoli interventi, eseguiti per privati, che il proprio restauratore potrebbe non aver avuto la cura, nella necessità di documentare.

${ }^{485}$ La prassi del distacco degli affreschi è stata una dei punti di maggiori controversie e discussione, specie
} 
nell'epoca. Mazzi descrive il processo da lui eseguito:

[...]1. Lavare bene l'affresco con acqua $e$ ammoniaca onde togliere qualsiasi traccia di grasso o unto del tempo, eventualmente passarlo dopo con acqua e soda leggera indi risciacquare con acqua pura. 2. Lasciare asciugare. 3. Preparare colla da falegname $e$ chicchi in dose di 900gr per $\mathrm{m} 2$ di affresco; la colla deve continuamente mantenersi caldissima a bagnomaria. La colla va fatta con circa metà colla secca e metà acqua. 4. Incollare con questa colla del calicot486 tagliato in quadratini allo scopo di poterli meglio tirare sull'affresco. Incollarli e cominciare dal basso verso l'alto allo scopo di evitare che goccioloni della colla si formino sull'affresco. Prima di cominciare l'applicazione della tela calicot passare una mano di colla più leggera sull'interno del dipinto - i quadratini di tela si devono sormontare l'uno a l'altro di circa $3 / 4 \mathrm{~cm}$ alla giuntura di ogni lato, a bordo del dipinto è importante piegare il calicot su sé stesso a mo di formare un bordo rinforzato allo scopo di facilitare alle manovre durante lo strappo. Questa $1^{\underline{a}}$ tela deve

per i due grandi trattatisti Italiani, il conte Giovanni Secco Suardo di Bergamo ed il restauratore Ulisse Forni annesso alle Regie Gallerie di Firenze. Mentre i Secco Suardo propugnava lo strappo degli affreschi seguendo la tecnica che manteneva unicamente lo strappo superficiale del dipinto, reso il più fine $\mathrm{e}$ flessibile possibile trasportato su tela, il Forni difendeva lo stacco degli affreschi con la tecnica a massello, conservando tutti gli strati che conformano l'opera murale. II Secco Suardo, fu chiamato dal senatore Morelli nel 1864 alle Gallerie fiorentine per fare un corso con lo scopo di [...]istruire dei giovani sull'arte di trasportare gli antichi dipinti dalla tavola e dalla tela[...] all'interno del dibattito sul distacco degli affreschi; vedi nota 21 in: (Giorgio Bonsanti, 2004, p. 267). Fu in questo evento quando si successero delle vive discussioni riguardo il metodo idoneo di trasporto dei dipinti.

${ }^{486}$ Si tratta di un tessuto di cotone chiamato anche cencio della nonna, che prende il nome della città di Calicut nell'India dove si elaborava. asciugare lo meglio possibile prima di passare all'applicazione della seconda (osservare ogni bollicina d'aria che si forma volentieri durante l'applicazione). Passare alla fine su tutto, o su ogni pezzo se grande, il pennello bagnato nella colla onde pareggiare le sgocciolature.[... $]^{487}$.

L'affresco strappato, descrive Mazzi, sarà posteriormente pulito e reso il più sottile possibile per poi riattaccarlo con caseinato di calce sul nuovo supporto.

[...]Per il riattacco pulire perfettamente la superficie dell'affresco dei detriti di calce $e$ renderlo più sottile possibile, ma spazzolare per bene e togliere la polvere la quale impedirebbe una buona adesione del caseinato di calcio (lavare pure con spugna). Più fine riesce più flessibile sarà e questo è importante.[...] II caseinato va sparso sull'affresco di rovescio. Strofinare sulla tela applicata perché aderisca bene. Riaggiungi caseato con una spugna piatta bagnata nel latte ben spremuta per levare quella colla eccedente che renderebbe solo duro l'intellaggio. Bagnare e stirare le tele. [... $]^{488}$.

Il caseinato di calcio del supporto poteva essere colorato. In questo punto gli appunti di Mazzi riferiscono al Manuale del Secco Suardo. (SS.p.244.). Infatti qui si parla del caseinato di calcio usato ugualmente come adesivo, come consolidante e come legante per temperare i pigmenti nei ritocchi di dipinti all'esterno o per rinforzare i colori del retro intonaco del dipinto strappato. Sono interessanti allo stesso modo altre osservazione allegate al medesimo quaderno, dove ad esempio il restauratore annota l'intervento eseguito in uno strappo a Biasca assieme al collega Nino

\footnotetext{
487 Quaderni di studio. Archivio privato. Mina-Mazzi, Tegna.)

${ }^{488}$ Quaderni di studio. Archivio privato. Mina-Mazzi, Tegna.)
} 
Facchinetti. Qui il dipinto distaccato fu aderito su una sola tela di supporto, e dopo averlo lasciato asciugare per tre giorni si attaccò direttamente al supporto rigido con il caseinato di calcio, prima di togliere le tele per lo strappo dello strato superficiale dipinto. Mazzi avverte la difficoltà di questo processo elaborato secondo la prassi di Nino Facchinetti, perché l'affresco non aderisce bene al supporto rigido formando dei vuoti d'aria.

II restauratore raccomanda l'impiego di un caseinato di calcio "pastoso", fatto riposare prima dell'uso per al meno un'ora ma preferibilmente durante ventiquattro ore. Le dosi idonee erano di 1 parte di caseina diluita più 3 parti di calce.

Alcuni indicazioni ci hanno sorpreso come ad esempio l'impiego dei detersivi di marca Persil e Well, a modo di saponi per la pulitura degli affreschi.

Un esempio degli interventi che Mazzi eseguì completamente sotto le istruzioni precise dell'Ufficio Cantonale dei Monumenti Storici lo abbiamo già analizzato parlando del restauratore Abbiati; si tratta dei lavori svolti alla chiesa dei Santi Pietro e Paolo a Biasca nel 1959. In questa fabbrica il restauratore lavorerà assieme al pittore ed amico Mario Moglia ed al sopracitato collega italiano Bruno Abbiati (anche egli allievo del maestro Rossi). Il compito di Mazzi nel suddetto intervento venne limitato in un primo tempo al lavoro di esplorazione nelle pareti e nelle volte, e di pulitura dei dipinti seicenteschi della navata.

L'istituzione cantonale, rappresentata dai membri Pietro Salati e Piero Bianconi, escludeva qualsiasi intervento di consolidamento generalizzato che, in qualunque caso sarebbe stata realizzata assieme alla reintegrazione pittorica, a rigattino, a neutro o a basso tono a dipendenza dei casi, da specialisti "ritoccatori". Tuttavia dagli scritti amministrativi questa definizione sembrava riferirsi a operatori diversi, nel 1965 furono ancora Bruno Abbiati ed il pittore Mario Moglia i professionisti incaricati non solo della meticolosa reintegrazione pittorica ma anche dello strappo delle pitture del XVII sec. sull'abside. Quell'anno Mazzi era occupato ai restauri degli affreschi di Antonio da Tradate nell'Oratorio di S. Maria di Loreto a Fosano e l'anno successivo nel restauro del coro primitivo della chiesa di S. Maria del Sasso a Morcote.

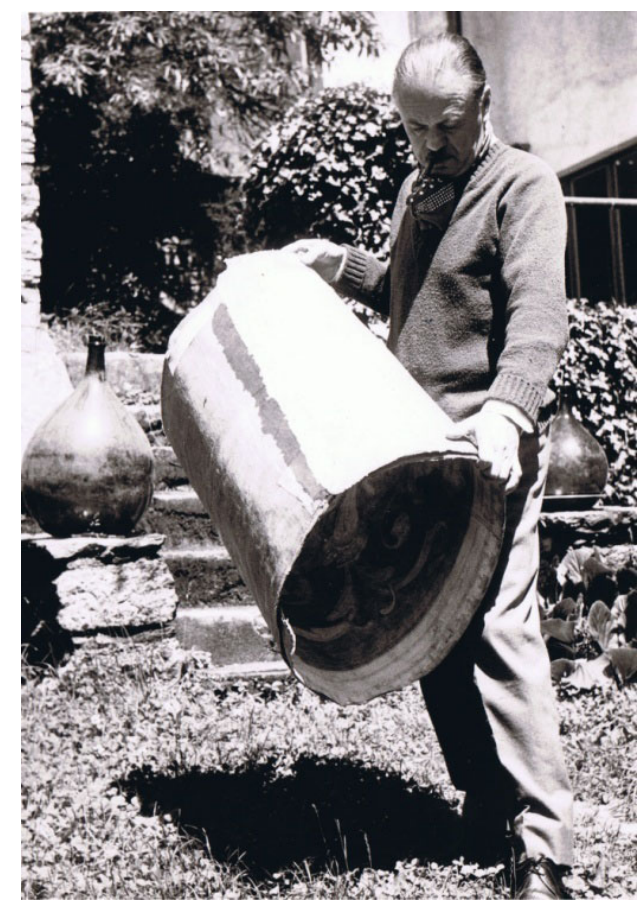

Figura 189 Carlo Mazzi mostrando uno strappo. Archivio Siliva Mina-Mazzi, Tegna. 


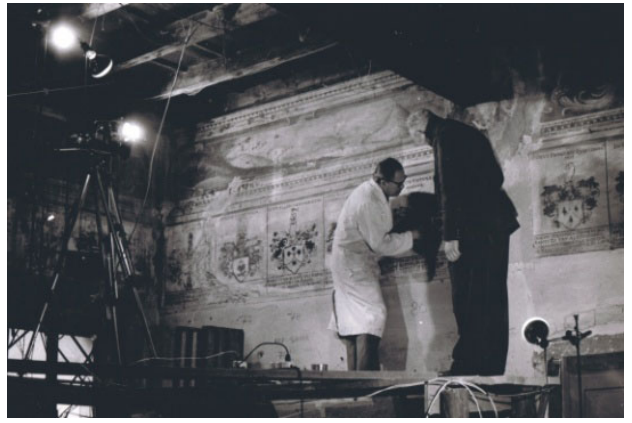

Figura 190 Carlo Mazzi insieme a Crivelli durante il restauro della casa dei Landfogti, Rivera. 1959 post. Archivio Silvia Mina-Mazzi, Tegna.

Quest'ultimo intervento avviene all'interno del progetto di ripristino architettonico diretto dall'architetto Guido Borella, al quale Mazzi fu estremamente legato e grazie a lui che il pittore riceve importanti lavori di collaborazione per la parte del restauro pittorico.

Con egli lavorò ad esempio nella chiesa della Madonna delle Grazie e nella chiesa dei Santi Martino e Rocco a Mendrisio $(1959)^{489}$, alla chiesa parrocchiale di Santa Maria della Purificazione a Comano (1963), alla chiesa di San Pellegrino a Giornico (1967), però sicuramente il più importante intervento realizzato in collaborazione con l'architetto fu il restauro della Casa dei Landfogti a Rivera. La costruzione eretta dalla famiglia Beroldingen, è conformata da due piani di logge nel cortile, con pareti decorate dal 1576 con più di cento stemmi dei balivi di Lugano e degli invitati dei cantoni. Il salone della casa, anche questo decorato con stemmi e con un fregio raffigurante paesaggi e figure allegoriche,

\footnotetext{
${ }^{489}$ Per più informazione sugli intervento architettonici vedi (Borella, 1964, p. 93-102) e (Borella, S. Martino dopo il restauro, 1964).
}

possiede un soffitto a cassettoni ed un camino in stucco con lo stemma della famiglia dei Beroldingen.

Dal 16 al 21 novembre del 1959 Mazzi inizia in quest'ultima fabbrica, datata prima del $1678^{490}$, i primi campioni con lo scopo di ritrovare possibili tracce di dipinti murali nelle pareti della casa, nel porticato e nei locali superiori, con risultato negativo. Nel 1960 il pittore realizzerà 14 strappi degli stemmi e 12 degli affreschi decorativi della casa Landfogti che assieme ad altri 3 dalla casa Peri a Lugano saranno provvisoriamente trasportati a Castel Grande a Bellinzona ${ }^{491}$. Solo nel $1964^{492}$ Mazzi procederà con il restauro degli affreschi della casa asportando le imbiancature soprastanti e con il ricollocamento dei suddetti strappi sulle pareti rintonacate seguendo un predeterminato piano di riordino.

Il pittore creò dei disegni che potessero servire in futuro a informare sul metodo di attacco dei dipinti ai muri del salone della casa dei Landfogti, per facilitare, se necessario, il loro trasporto. I pannelli supportanti gli strappi furono introdotti all'interno delle pareti conformando una superficie piana per creare nella misura del possibile lo stato primario dell'opera. Essi si fissarono a travetti di legno a sua volta

\footnotetext{
${ }^{490}$ In questo anno, il 1678 si stabilisce il giorno del 10 agosto per procedere ogni anno alla riunione fra i rappresentanti dei XII cantoni nei baliaggi italiani.

${ }^{491}$ Mazzi Carlo. Lettera indirizzata all'Ispettorato dei Monumenti Storici Artistici, Tegna 28 dicembre 1960. (Archivio Mazzi).

${ }^{492} \mathrm{Gli}$ affreschi furono riportati al restauratore Mazzi prima nel giugno del 1964. (Mazzi Carlo, Dichiarazione di ricevuta di 21 affreschi strappati per la Casa Landfogti depositati al Castel Grande, indirizzata al Dipartimento della Pubblica Costruzione, Bellinzona. Tegna, 19 giugno 1954. Archivio privato).
} 
fissati sul muro all'interno di uno spazio ricavato nella parete per circa $4 \mathrm{~cm}$.

Fu il 2 dicembre del 1966 quando Mazzi presentò, ancora assieme all'architetto Guido Borella, la sua proposta e preventivo di restauro per gli affreschi del coro primitivo della chiesa di S. Maria del Sasso a Morcote. Un primo esame macroscopico dell'opera denunciava, un buono stato di conservazione nell'affresco raffigurante la Crocifissione di Domenico Pezzi (1513), la presenza di ampie lacune e di rotture dell'intonaco e graffiature a causa della collocazione del coro in passato. II dipinto Gesù nell'Orto opera del medesimo Pezzi, assieme alle decorazioni della volta del XV sec., per il contrario era più danneggiato per l'azione dell'umidità e dall'invasione nella superficie pittorica di un fungo rosa, inoltre in parte scialbata e deturpata dai ritocchi realizzati in passato ${ }^{493}$.

\footnotetext{
${ }^{493}$ Le notizie precedenti al restauro di Carlo Mazzi sugli affreschi del coro della chiesa di Morcote gli abbiamo trovato all'Archivio federale dei monumenti storici a Berna Si tratta di una lettera che il restauratore italiano Luigi Cavenaghi indirizza alla commissione dei monumenti del DPE del Canton Ticino circa il 1891. Il documento espone la perizie che l'illustre restauratore aveva realizzato riguardo lo stato di conservazione degli affreschi: [...]Trovo valga la pena conservarli. Quelli delle due navate minori e della navata grande di mezzo attribuiti dai fratelli Silvestri al Sodoma mi paiono i più importante. II loro stato di conservazione però lascia molto a desiderare specie quelli centrali essendo anche coperti quasi interamente dall'organo. Quelli della volta a sinistra dell'altare Maggiore mi sembrano in maniera diversa dei primi, questi sono belli nella disposizione ornamentale e di un certo interesse. Sarebbe bene se si optasse levare l'organo dal posto dove si trova, per poter mettere in vista tutti i dipinti, quanto poi al restauro bisognerebbe limitargli a completare le parti decorative seguendo il motivo già esistente, pulire tutti i dipinti dal sudiciume che li copre, assicurare l'intonaco ove questo si stacca, e levare le
}

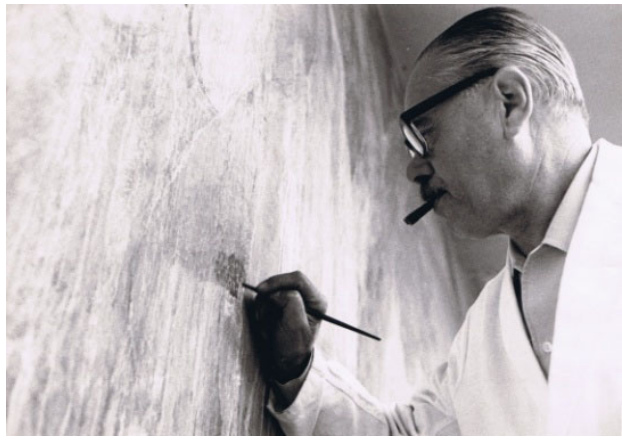

Figura 191 Carlo Mazzi durante il restauro dei dipinti murali a caseina, dell'atrio della stazione ferroviaria di Neuchatel. 1967 ca. Archivio privato Mina-Mazzi.

Il preventivo di Mazzi prevedeva la pulitura degli strati di calce soprastanti le pitture, il consolidamento localizzato alle zone pericolanti con caseinato di calcio ed il moderato ritocco pittorico.

imbiancatura che in molti punti ricoprono i dipinti. Almeno ciò sarebbe quanto io farei e ciò che ho sempre fatto in questi generi di restauro i quelli sono sempre limitati a quel poco che si può fare anche alle parti figurative. Ella mi chiede un preventivo[...] ma mi permetto però dirli che avendo capito che Morcote darebbe ostentieri l'incarico di tale lavoro al Sg. Pittore Silvestri, come quegli che per primo ne promosse la conservazione, pregherei questa On. Commissione a voler prima parlare al Sig. Silvestri, null'altro che per quei riguardi che io uso coi miei colleghi [...] (Cavenaghi L., Lettera indirizzata al Dipartimento della pubblica educazione riguardo il restauro del coro primitivo alla chiesa di Santa Maria del Sasso a Morcote, Milano Via Pontaccio, 12, 1891 ca. AFMS Bern, n.80 M-P). Grazie a questo documento si potrebbe dedurre che i ritocchi che Carlo Mazzi ritrova sulle pitture del coro siano del pittore Silvestri. 


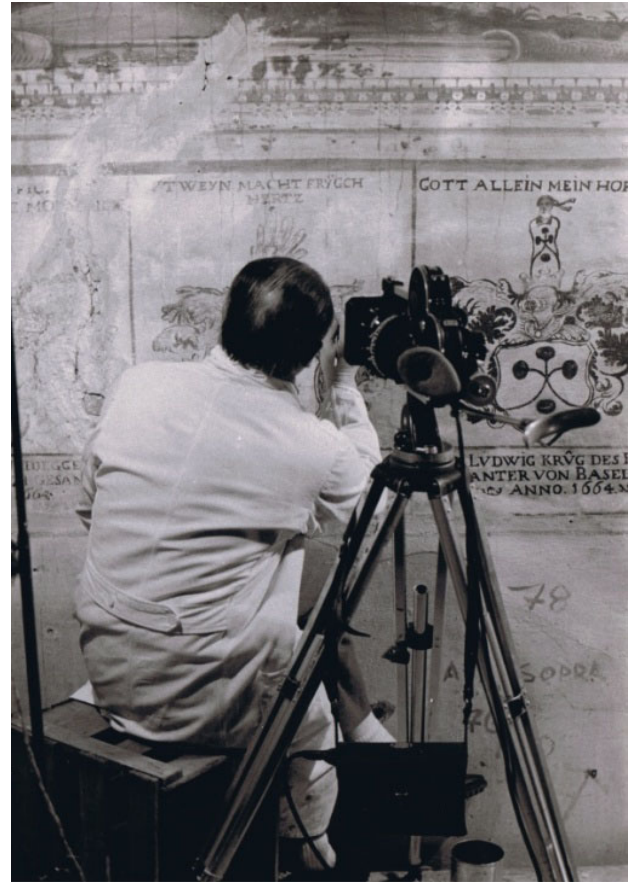

Figura 192 Carlo Mazzi durante il restauro degli stemmi alla casa dei Landfogti, Rivera. 1960 ca. Archivio privato.

Nel 1967, sui dipinti a caseina di Georges Dessouslavy presenti nell'atrio della stazione ferroviaria a Neuchâtel, Mazzi disse al giornale Ville de Neuchâtel: [...]Questo è il lavoro più difficile che mai avvia intrapreso[...] I colori a caseina sono ancora in buono stato ma si è formato in superficie uno strato causato dall'acqua di infiltrazione $[. . .]^{494}$. Lo stesso giornale affermava: [...]Sperato da molto tempo e tante volte richiesto[...] II restauro degli

\footnotetext{
494 II testo originale in francese cita [...]D'un pinceau délicat, la peintre tessinois $C$. Mazzi sort la fresque de l'oubli, l'arrache à cette dècaence auxquels mille palabres et le eaux de cuisine du buffet de la gare l'avaient condamnée[...] (La restauration d'une des fresques de Dessouslavy est enfin entreprise, 1967).
}

affreschi di Dessouslavy è finalmente cominciato[...] Con un delicato pennello, il pittore ticinese Carlo Mazzi, fa rinascere l'affresco, trascinandolo dalla decadenza a cui le mille discussioni e l'acqua infiltrata dal buffet di cucina della stazione lo avevano condannato[...].

Mazzi fece un intervento molto discreto consistente unicamente nell'asportazione delle incrostazioni di colature di acqua, a secco con bisturi e limitando l'uso dell'acqua. La fase successiva fu quella del ritocco, apparentemente dai documenti analizzati, senza necessità di consolidamento. Questa reintegrazione si realizza mediante una tempera alla caseina molto diluita, più leggera - spiega Mazzi di quello solitamente da egli impiegata. I ritocchi furono eseguito a rigattino, cioè [...] a piccoli tratteggi e a leggere sovrapposizioni di colore per non renderli pesanti e per potermi avvicinare in modo adeguato ai difficili toni del pittore. Rispettando in modo assoluto l'originale dove la velatura biancastra non era possibile asportarla.[...].

Le stesse operazioni erano state eseguite nel 1964 sugli affreschi della chiesa parrocchiale di S. Michele a Palagnedra, dove l'intervento interessò unicamente l'asportazione delle florescenze saline mediante pulitura a secco con bisturi, il rifacimento degli intonaci ed il ritocco a rigattino ad eccezioni delle ampie zone di lacuna dove non c'era informazione che giustificasse la ricostruzione, e furono unicamente intonate. Inoltre si fecero analizzare chimicamente due tipi diversi di funghi ritrovati negli affreschi, uno rosa apparse nella parte centrale, ed un secondo nero esistente sull'angolo sinistro. 
Una pratica moderna di ritocco fu messa in opera alla chiesa di S. Pellegrino a Giornico per Carlo Mazzi. Gli affreschi restaurati dal pittore nel 1967 denunciavano zone frammentarie come la raffigurazione della "Fede" e della "Speranza" le cui lacune furono intonate come nel sopracitato caso. Invece, la figura della "Carità", nella medesima chiesa di Giornico, evidenziava unicamente una zona puntuale mancante, dove I'Ufficio Cantonale dei Monumenti Storici decise di disegnare il "presunto" contorno della suddetta figura con una linea netta sopra un fondo di tinta unita ${ }^{495}$.

Ancora all'inizio della sua carriera come restauratore, Mazzi era stato chiamato ad effettuare una perizia ed una proposta di restauro nella chiesa di San Carlo di Negrentino, il cui abside maggiore si trovava specialmente attaccato dall'umidità di infiltrazione di acqua piovana. Fu il pittore Pietro Salati nel 1957 a suggerire un possibile strappo dei dipinti dell'abside, per salvargli da una perdita "sicura" a causa dell'azione dell'umidità, più di dieci anni dopo l'intervento architettonico che aveva rassicurato la stabilità del soffitto in piode e dalla pulitura e consolidamento degli affreschi realizzata da Tita Pozzi nel 1945. Mazzi esaminò suddetti dipinti esattamente il 27 ottobre del 1957 per richiesta della Commissione. Egli si limitò ad indicare le favorevoli condizioni del manufatto verso un probabile intervento con la tecnica dello strappo ed il prezzo approssimativo di questo lavoro in base alle misure dell'opera. Mazzi calcolò un totale di 24 mq

495 (Bolzani. Rapporto concernente i restauri della Chiesa di S. Pellegrino. Locarno 3 luglio 1967. Archivio Mazzi). di superficie dipinta sull'abside maggiore della chiesa ed un preventivo di fr. 400 al mq, ma avvertiva nello stesso scritto sulla necessità di aspettare la bella stagione in modo da evitare che l'intonaco fosse troppo bagnato e l'operazione rischiasse di fracassare. II fatto che la commissione affidasse un lavoro così difficile come quello dello strappo al pittore Mazzi prova la esperienza che egli aveva a riguardo. Tuttavia lo strappo non venne eseguito, e anche se questo potrebbe essere stato favorevole, purtroppo le pitture continuarono a degradarsi senza che nessun metodo alternativo di restauro fosse realizzato sugli affreschi dell'abside maggiore. Trascorsi cinquant'anni da allora, lo stato di conservazione degli affreschi è mediocre e necessità urgentemente degli opportuni lavori per asportare l'umidità che danneggia gravemente le opere.

In qualunque caso le conclusioni estratte dai diversi casi studiati sono chiare. Carlo Mazzi, uomo di grande sensibilità artistica e materica come le sue produzioni pittoriche e ceramiche dimostrano, segna nettamente una nuova generazione di restauratori. Restauratori in camice bianco, puliti, delicati, prudenti, attenti osservatori, il cui rispetto verso l'opera è condizionato dalle più recenti concezioni in materia di conservazione, anche se i materiali impiegati siano ancora quelli tradizionali.... L'opera artistica, per Carlo Mazzi, non più l'oggetto primario concepito dall'artista, sarà rigenerato culturalmente nella sua autenticità unica. In questo modo i suoi interventi saranno sempre percettibili poiché, a tal punto, non possono più alterare il bene "storico". 


\section{Interventi di restauro}

\begin{tabular}{|c|c|c|c|c|c|}
\hline ANNO & LUOGO & OPERA & INTERVENTO & COLLABORATORI & FONTI \\
\hline $\begin{array}{l}1952- \\
1953\end{array}$ & $\begin{array}{l}\text { Locarno, S. Maria in } \\
\text { Selva. }\end{array}$ & Affreschi. & Restauro. & Mario Rossi. & (Archivio Mazzi). \\
\hline $\begin{array}{l}1953- \\
1954\end{array}$ & $\begin{array}{l}\text { Saronno, } \\
\text { Santuario della } \\
\text { Beata Vergine dei } \\
\text { Miracoli. }\end{array}$ & Affreschi. & Restauro. & Mario Rossi. & (Archivio Mazzi). \\
\hline 1954 & $\begin{array}{l}\text { Orselina, } \\
\text { chiesa della } \\
\text { Madonna del Sasso. }\end{array}$ & Affreschi. & Restauro. & & $\begin{array}{l}\text { (Belinelli, 1981, p. } \\
\text { 30) } \\
\text { (Belinelli, Carlo } \\
\text { Mazzi, 1911-1988 : } \\
\text { Galleria Carlo Mazzi, } \\
\text { Tegna, 14.VI - } \\
\text { 30.VIII.1998, 1998, } \\
\text { p. 29) }\end{array}$ \\
\hline 1954 & Bedigliora. & Affresco & Strappo. & & (Archivio Mazzi) \\
\hline 1954 & $\begin{array}{l}\text { Gordevio, } \\
\text { cappella votiva. }\end{array}$ & $\begin{array}{l}\text { Giuseppe Antonio } \\
\text { Felice Orelli, } 1753 . \\
\text { Immacolata, S. } \\
\text { Vincenzo Ferreri e } \\
\text { un santo vescovo. } \\
\text { Affreschi. }\end{array}$ & Restauro. & & $\begin{array}{l}\text { (Belinelli, Carlo } \\
\text { Mazzi, 1911-1988 : } \\
\text { Galleria Carlo Mazzi, } \\
\text { Tegna, 14.VI - } \\
\text { 30.VIII.1998, 1998, } \\
\text { p. 29). }\end{array}$ \\
\hline 1954 & $\begin{array}{l}\text { Borgnone, } \\
\text { cappella della Cia } \\
\text { Crucis. }\end{array}$ & $\begin{array}{l}\text { Cristo che porta la } \\
\text { croce, XVIII sec. } \\
\text { Affresco. }\end{array}$ & Restauro. & & (Archivio Mazzi). \\
\hline 1954 & $\begin{array}{l}\text { Locarno, } \\
\text { chiesa } \\
\text { dell'Annunziata. }\end{array}$ & Affreschi. & $\begin{array}{l}\text { Restauro } \\
\text { consistente nella } \\
\text { pulitura e } \\
\text { scrostamento. } \\
\text { Finitura con leggera } \\
\text { mano di gomma } \\
\text { arabica. }\end{array}$ & & (Archivio Mazzi). \\
\hline 1955 & $\begin{array}{l}\text { Mairengo, } \\
\text { chiesa parrocchiale } \\
\text { di S. Siro. }\end{array}$ & Affreschi. & $\begin{array}{l}\text { Scoprimento e } \\
\text { restauro. }\end{array}$ & $\begin{array}{l}\text { Pittore- } \\
\text { restauratore: } \\
\text { Nino Facchinetti. }\end{array}$ & $\begin{array}{l}\text { (Belinelli, 1981, p. } \\
\text { 30). } \\
\text { (Belinelli, Carlo } \\
\text { Mazzi, 1911-1988 : } \\
\text { Galleria Carlo Mazzi, } \\
\text { Tegna, 14.VI - } \\
\text { 30.VIII.1998, 1998, } \\
\text { p. 29). }\end{array}$ \\
\hline 1955 & $\begin{array}{l}\text { Muralto, } \\
\text { chiesa di S. Vittore. }\end{array}$ & Affreschi. & Ricerche. & $\begin{array}{l}\text { Alberto Camenzind } \\
\text { (architetto direttore } \\
\text { dei lavori). }\end{array}$ & $\begin{array}{l}\text { (Belinelli, Carlo } \\
\text { Mazzi, 1911-1988 : } \\
\text { Galleria Carlo Mazzi, } \\
\text { Tegna, 14.VI - } \\
\text { 30.VIII.1998, 1998, } \\
\text { p. 29). }\end{array}$ \\
\hline
\end{tabular}




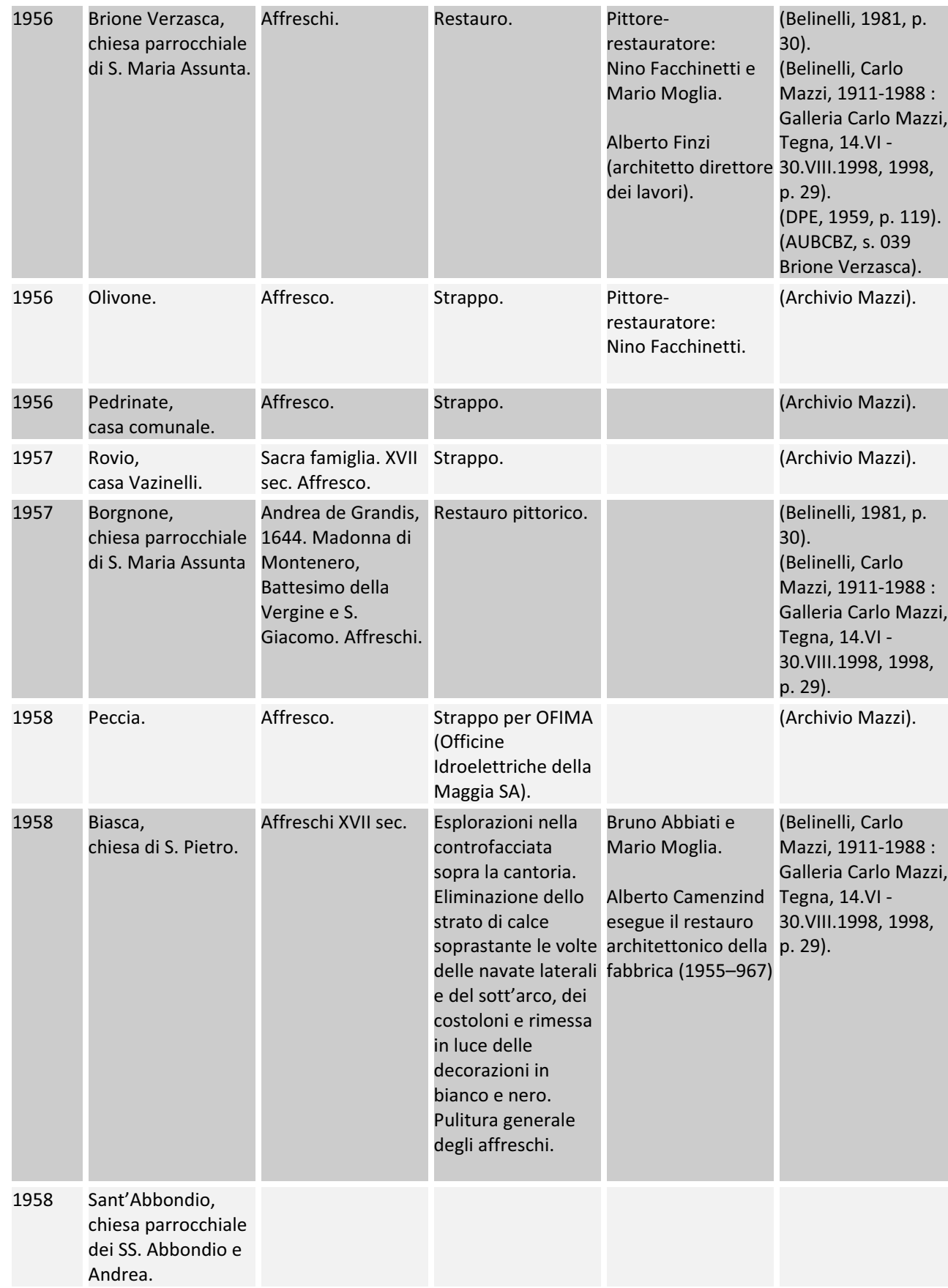




\begin{tabular}{|c|c|c|c|c|c|}
\hline 1959 & $\begin{array}{l}\text { Tegna, } \\
\text { oratorio della } \\
\text { Madonna delle } \\
\text { Scalate( S. Anna). }\end{array}$ & $\begin{array}{l}\text { Madonna, metà XVI } \\
\text { sec. }\end{array}$ & Restauro pittorico. & & (Archivio Mazzi). \\
\hline 1959 & Avegno. & $\begin{array}{l}\text { S. Antonio e } \\
\text { Teschio. Affreschi. }\end{array}$ & Strappo. & $\begin{array}{l}\text { Per ordine di don } \\
\text { Agostino Robertini }\end{array}$ & (Archivio Mazzi). \\
\hline 1959 & $\begin{array}{l}\text { Mendrisio, } \\
\text { oratorio della } \\
\text { Madonna delle } \\
\text { Grazie. }\end{array}$ & Affreschi. & $\begin{array}{l}\text { Ricerche e restauro } \\
\text { pittorico. }\end{array}$ & $\begin{array}{l}\text { Il restauro } \\
\text { architettonico della } \\
\text { chiesa venne } \\
\text { condotto nello } \\
\text { stesso anno da } \\
\text { Guido Borella, il cui } \\
\text { intervento } \\
\text { interessò la } \\
\text { rivalorizzazione } \\
\text { degli elementi } \\
\text { romanici presenti. }\end{array}$ & $\begin{array}{l}\text { (Belinelli, 1981, p. } \\
\text { 30) } \\
\text { (Belinelli, Carlo } \\
\text { Mazzi, 1911-1988 : } \\
\text { Galleria Carlo Mazzi, } \\
\text { Tegna, 14.VI - } \\
\text { 30.VIII.1998, 1998, } \\
\text { p. 29). }\end{array}$ \\
\hline 1959 & $\begin{array}{l}\text { Mendrisio, } \\
\text { chiesa dei SS. } \\
\text { Martino e Rocco. }\end{array}$ & $\begin{array}{l}\text { SS. Abbondio e } \\
\text { Fermo e la Colomba } \\
\text { dello Spirito santo. } \\
\text { Inizio XVIII sec. } \\
\text { Affreschi. }\end{array}$ & $\begin{array}{l}\text { Ricerche e restauro } \\
\text { pittorico. }\end{array}$ & $\begin{array}{l}\text { Le ricerche } \\
\text { archeologiche ed i } \\
\text { restauri } \\
\text { architettonici sono } \\
\text { eseguiti fra il } 1959 \\
\text { ed il } 1963 \text { dagli } \\
\text { architetti Guido } \\
\text { Borella e } \\
\text { Ferdinando } \\
\text { Reggiori. }\end{array}$ & $\begin{array}{l}\text { (Belinelli, Carlo } \\
\text { Mazzi, 1911-1988 : } \\
\text { Galleria Carlo Mazzi, } \\
\text { Tegna, 14.VI - } \\
\text { 30.VIII.1998, 1998, } \\
\text { p. 29). }\end{array}$ \\
\hline 1959 & $\begin{array}{l}\text { Mendrisio, } \\
\text { antico convento dei } \\
\text { Serviti (attualmente } \\
\text { Museo comunale } \\
\text { d'arte). }\end{array}$ & $\begin{array}{l}\text { Addolorato tra i SS. } \\
\text { Giovanni Battista e } \\
\text { Giuseppe, seconda } \\
\text { metà XVIII sec. } \\
\text { Affreschi. }\end{array}$ & Restauro pittorico. & & $\begin{array}{l}\text { (Belinelli, Carlo } \\
\text { Mazzi, 1911-1988 : } \\
\text { Galleria Carlo Mazzi, } \\
\text { Tegna, 14.VI - } \\
\text { 30.VIII.1998, 1998, } \\
\text { p. 29). }\end{array}$ \\
\hline 1959 & $\begin{array}{l}\text { Minusio, } \\
\text { chiesa parrocchiale } \\
\text { dei SS. Rocco e } \\
\text { Quirico. }\end{array}$ & $\begin{array}{l}\text { Misteri del Rosario, } \\
\text { XVII sec. } \\
\text { Dipinti murali. }\end{array}$ & Restauro pittorico. & & $\begin{array}{l}\text { (Belinelli, 1981, p. } \\
\text { 30). } \\
\text { (Belinelli, Carlo } \\
\text { Mazzi, 1911-1988 : } \\
\text { Galleria Carlo Mazzi, } \\
\text { Tegna, 14.VI - } \\
\text { 30.VIII.1998, 1998, } \\
\text { p. 29). }\end{array}$ \\
\hline $\begin{array}{l}1959- \\
1960\end{array}$ & $\begin{array}{l}\text { Rivera, } \\
\text { casa dei Landfogti. }\end{array}$ & $\begin{array}{l}\text { Stemmi dei balivi e } \\
\text { degli inviati dei } \\
\text { cantoni, seconda } \\
\text { metà XVII sec. } \\
\text { Dipinti murali sulla } \\
\text { pareti del cortile. }\end{array}$ & Restauro pittorico. & & $\begin{array}{l}\text { (Belinelli, 1981, p. } \\
\text { 30) } \\
\text { (Belinelli, Carlo } \\
\text { Mazzi, 1911-1988 : } \\
\text { Galleria Carlo Mazzi, } \\
\text { Tegna, 14.VI - } \\
\text { 30.VIII.1998, 1998, } \\
\text { p. 29). }\end{array}$ \\
\hline
\end{tabular}




\begin{tabular}{|c|c|c|c|c|c|}
\hline 1960 & $\begin{array}{l}\text { Lugano, } \\
\text { Casa Peri. }\end{array}$ & 3 Affreschi. & $\begin{array}{l}\text { Strappi, trasportati } \\
\text { a Castel Grande a } \\
\text { Bellinzona. }\end{array}$ & & $\begin{array}{l}\text { (Belinelli, Carlo } \\
\text { Mazzi, 1911-1988 : } \\
\text { Galleria Carlo Mazzi, } \\
\text { Tegna, 14.VI - } \\
\text { 30.VIII.1998, 1998, } \\
\text { p. 29). } \\
\text { (Fulvio Caccia, 1984, } \\
\text { p. 118). }\end{array}$ \\
\hline 1960 & $\begin{array}{l}\text { Moleno, } \\
\text { chiesa parrocchiale } \\
\text { di S. Vittore Mauro. }\end{array}$ & Affreschi. & Restauro pittorico. & & $\begin{array}{l}\text { (Belinelli, Carlo } \\
\text { Mazzi, 1911-1988 : } \\
\text { Galleria Carlo Mazzi, } \\
\text { Tegna, 14.VI - } \\
\text { 30.VIII.1998, 1998, } \\
\text { p. 29). }\end{array}$ \\
\hline 1961 & $\begin{array}{l}\text { Bondo (Gr)., } \\
\text { chiesa protestante. }\end{array}$ & Affreschi. & $\begin{array}{l}\text { Rilievo e restauro } \\
\text { pittorico. }\end{array}$ & & $\begin{array}{l}\text { (Belinelli, 1981, p. } \\
\text { 30) } \\
\text { (Belinelli, Carlo } \\
\text { Mazzi, 1911-1988 : } \\
\text { Galleria Carlo Mazzi, } \\
\text { Tegna, 14.VI - } \\
\text { 30.VIII.1998, 1998, } \\
\text { p. 29). }\end{array}$ \\
\hline 1961 & $\begin{array}{l}\text { Bellinzona, } \\
\text { soffitto salone Gran } \\
\text { Consiglio. }\end{array}$ & $\begin{array}{l}\text { Adelchi Maina, } \\
\text { 1899. Raffigurazioni } \\
\text { allegoriche. Dipinti } \\
\text { illusionistico neo- } \\
\text { barocco. }\end{array}$ & Restauro pittorico. & & $\begin{array}{l}\text { (Belinelli, 1981, p. } \\
\text { 30), (Belinelli, Carlo } \\
\text { Mazzi, 1911-1988 : } \\
\text { Galleria Carlo Mazzi, } \\
\text { Tegna, 14.VI - } \\
\text { 30.VIII.1998, 1998, } \\
\text { p. 29). }\end{array}$ \\
\hline 1961 & Bissone. & Affresco. & Strappo. & & (Archivio Mazzi). \\
\hline 1961 & $\begin{array}{l}\text { Rivera, } \\
\text { casa dei Landfogti. }\end{array}$ & $\begin{array}{l}7 \text { Stemmi dei balivi } \\
\text { e degli inviati dei } \\
\text { cantoni, seconda } \\
\text { metà XVII sec. } \\
\text { Affreschi. Cortile } \\
\text { esterno }\end{array}$ & Strappo. & & (Archivio Mazzi). \\
\hline 1962 & $\begin{array}{l}\text { Muralto, } \\
\text { chiesa di S. Vittore. }\end{array}$ & Affreschi. & Restauro. & & $\begin{array}{l}\text { (Belinelli, Carlo } \\
\text { Mazzi, 1911-1988 : } \\
\text { Galleria Carlo Mazzi, } \\
\text { Tegna, 14.VI - } \\
\text { 30.VIII.1998, 1998, } \\
\text { p. 29) }\end{array}$ \\
\hline 1963 & $\begin{array}{l}\text { Comano, } \\
\text { chiesa parrocchiale } \\
\text { di S. Maria della } \\
\text { purificazione. }\end{array}$ & Affreschi XVII sec. & Restauro. & $\begin{array}{l}\text { Il restauro della } \\
\text { fabbrica avviene a } \\
\text { carico } \\
\text { dall'architetto } \\
\text { Guido Borella fra il } \\
1960 \text { ed il } 1964 .\end{array}$ & $\begin{array}{l}\text { (Belinelli, 1981, p. } \\
\text { 30). } \\
\text { (Belinelli, Carlo } \\
\text { Mazzi, 1911-1988 : } \\
\text { Galleria Carlo Mazzi, } \\
\text { Tegna, 14.VI - } \\
\text { 30.VIII.1998, 1998, } \\
\text { p. 29). }\end{array}$ \\
\hline
\end{tabular}




\begin{tabular}{|c|c|c|c|c|c|}
\hline 1963 & $\begin{array}{l}\text { Losone, } \\
\text { chiesa di S. Giorgio. }\end{array}$ & $\begin{array}{l}\text { Cristo benedicente } \\
\text { con gli Evangelisti, } \\
\text { gruppo della } \\
\text { Crocifissione, i SS. } \\
\text { Giorgio e Vittore e } \\
\text { angelo } \\
\text { dell'Annunciazione. } \\
\text { Ultimo ventenio XV } \\
\text { sec. Affreschi } \\
\text { tardogotici } \\
\text { parzialmente } \\
\text { conservati } \\
\text { nell'antico coro. }\end{array}$ & Restauro & & $\begin{array}{l}\text { (Belinelli, Carlo } \\
\text { Mazzi, 1911-1988 : } \\
\text { Galleria Carlo Mazzi, } \\
\text { Tegna, 14.VI - } \\
\text { 30.VIII.1998, 1998, } \\
\text { p. 29) }\end{array}$ \\
\hline 1963 & Aurigeno. & Affresco. & $\begin{array}{l}\text { Strappo e trasporto } \\
\text { al Museo di } \\
\text { Valmaggia (Cevio). }\end{array}$ & & (Archivio Mazzi). \\
\hline 1964 & Soazza. & Affresco. & Strappo. & & (Archivio Mazzi). \\
\hline 1964 & $\begin{array}{l}\text { Rivera, } \\
\text { casa dei Landfogti. }\end{array}$ & $\begin{array}{l}\text { Stemmi dei balivi e } \\
\text { degli inviati dei } \\
\text { cantoni, seconda } \\
\text { metà XVII sec. } \\
\text { Affreschi. Salone. }\end{array}$ & Ripasso pittorico. & $\begin{array}{l}\text { Il restauro della } \\
\text { fabbrica avviene a } \\
\text { carico } \\
\text { dall'architetto } \\
\text { Guido Borella fra il } \\
1963 \text { ed il } 1966 . \\
\text { Alcuni dei lavori } \\
\text { consentirono la } \\
\text { costruzione del } \\
\text { portici della facciata } \\
\text { ad est a } \\
\text { continuazione di } \\
\text { una parziale } \\
\text { demolizione } \\
\text { avvenuta per } \\
\text { dell'allargamento } \\
\text { della strada. }\end{array}$ & (Archivio Mazzi). \\
\hline 1964 & $\begin{array}{l}\text { Palagnedra, } \\
\text { chiesa parrocchiale } \\
\text { di S. Michele. }\end{array}$ & Affreschi. & $\begin{array}{l}\text { Restauro } \\
\text { consistente nella } \\
\text { pulitura mediante } \\
\text { I'asportazione delle } \\
\text { florescenze saline } \\
\text { cristallizzate in } \\
\text { superficie in una } \\
\text { dura patina che fu } \\
\text { tagliata a filo del } \\
\text { dipinto con un } \\
\text { bisturi. Rifacimento } \\
\text { degli intonaci. Si } \\
\text { fece analizzare i } \\
\text { funghi rosa e neri } \\
\text { che invasero il } \\
\text { dipinto della } \\
\text { crocifissione la }\end{array}$ & & $\begin{array}{l}\text { (Belinelli, 1981, p. } \\
\text { 30). } \\
\text { (Belinelli, Carlo } \\
\text { Mazzi, 1911-1988 : } \\
\text { Galleria Carlo Mazzi, } \\
\text { Tegna, 14.VI - } \\
\text { 30.VIII.1998, 1998, } \\
\text { p. 29). }\end{array}$ \\
\hline
\end{tabular}




\begin{tabular}{|c|c|c|c|c|c|}
\hline & & & $\begin{array}{l}\text { parte centrale e la } \\
\text { parte alta } \\
\text { rispettivamente a } \\
\text { sinistra. } \\
\text { Reintegrazione } \\
\text { pittorica a rigattino } \\
\text { dove la } \\
\text { "ricostruzione" era } \\
\text { documentata e } \\
\text { intonazione delle } \\
\text { parti dove non } \\
\text { esisteva nessun } \\
\text { elemento } \\
\text { giustificativo. }\end{array}$ & & \\
\hline 1965 & $\begin{array}{l}\text { Fosano, } \\
\text { oratorio di S. Maria } \\
\text { di Loreto. }\end{array}$ & $\begin{array}{l}\text { Antonio da Tradate } \\
\text { attribuiti a. } \\
\text { Affreschi fine XV } \\
\text { inizio XVI sec. }\end{array}$ & Restauro. & & $\begin{array}{l}\text { (Belinelli, 1981, p. } \\
\text { 30). } \\
\text { (Belinelli, Carlo } \\
\text { Mazzi, 1911-1988 : } \\
\text { Galleria Carlo Mazzi, } \\
\text { Tegna, 14.VI - } \\
\text { 30.VIII.1998, 1998, } \\
\text { p. 29). }\end{array}$ \\
\hline 1966 & $\begin{array}{l}\text { Rivera, } \\
\text { chiesa parrocchiale } \\
\text { dello Spirito Santo. }\end{array}$ & Dipinti murali. & Restauro. & & (Archivio Mazzi). \\
\hline 1967 & $\begin{array}{l}\text { Gerra Gambarogno, } \\
\text { chiesa parrocchiale } \\
\text { dei SS. Rocco e } \\
\text { Sebastiano. }\end{array}$ & Affreschi. & Restauro. & & $\begin{array}{l}\text { (Belinelli, 1981, p. } \\
\text { 30). } \\
\text { (Belinelli, Carlo } \\
\text { Mazzi, 1911-1988 : } \\
\text { Galleria Carlo Mazzi, } \\
\text { Tegna, 14.VI - } \\
\text { 30.VIII.1998, 1998, } \\
\text { p. 29). }\end{array}$ \\
\hline 1967 & $\begin{array}{l}\text { Locarno, } \\
\text { chiesa di S. Maria } \\
\text { Assunta o chiesa } \\
\text { Nuova. }\end{array}$ & Affreschi. & Restauro. & & $\begin{array}{l}\text { (Belinelli, Carlo } \\
\text { Mazzi, 1911-1988 : } \\
\text { Galleria Carlo Mazzi, } \\
\text { Tegna, 14.VI - } \\
\text { 30.VIII.1998, 1998, } \\
\text { p. 29). }\end{array}$ \\
\hline 1967 & $\begin{array}{l}\text { Giornico - Altirolo, } \\
\text { chiesa a S. } \\
\text { Pellegrino. }\end{array}$ & Affreschi. & $\begin{array}{l}\text { Restauro } \\
\text { consistente nella } \\
\text { pulitura, } \\
\text { consolidamento e } \\
\text { reintegrazione } \\
\text { cromatica a } \\
\text { rigattino, che nella } \\
\text { parte mancante } \\
\text { della "Carità" si } \\
\text { limitò al disegno del } \\
\text { presunto contorno }\end{array}$ & $\begin{array}{l}\text { Il restauro della } \\
\text { fabbrica avviene a } \\
\text { carico } \\
\text { dall'architetto } \\
\text { Guido Borella dal } \\
1966 \text { e il } 1967 .\end{array}$ & $\begin{array}{l}\text { (Belinelli, 1981, p. } \\
\text { 30) } \\
\text { (Belinelli, Carlo } \\
\text { Mazzi, 1911-1988 : } \\
\text { Galleria Carlo Mazzi, } \\
\text { Tegna, 14.VI - } \\
\text { 30.VIII.1998, 1998, } \\
\text { p. 29) } \\
\text { (Bolzani. Rapporto } \\
\text { Locarno } 3 \text { luglio } \\
\text { 1967. Archivio }\end{array}$ \\
\hline
\end{tabular}




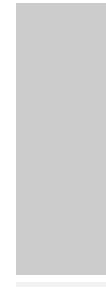

1967

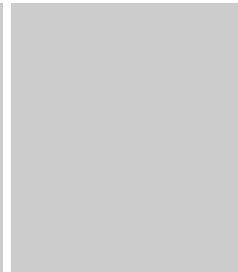

Neuchatel, atrio della stazion

\section{Dessouslavi}

Georges, dipinti a caseina.

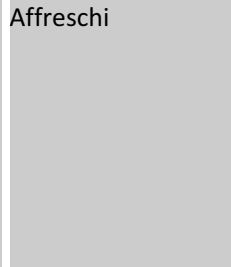

1971 Locarno,

Salone antica casa borghese Franzoni

Giovanni Antonio

Caldelli attribuiti a,

1780 ca. dipinti

murali illusionistici.

Restauro.

chiesa parrocchiale

di S. Bernardo

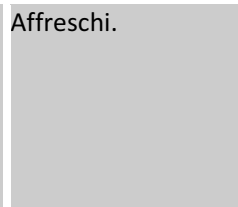

Affreschi.

Brissago, chiesa della Madonna del Ponte.

"Fede".

Restauro.

Restauro.

Restauro.

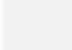

con linea netta su

fondo unito e

l'intonazione delle frammentarie

figure della

"Speranza" e della

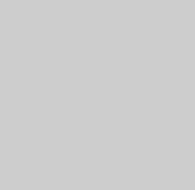

(Belinelli, Carlo

Mazzi, 1911-1988 :

Galleria Carlo Mazzi, Tegna, 14.VI -

30.VIII.1998, 1998, p. 29).

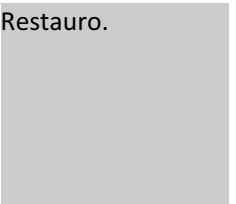

\section{(Belinelli, Carlo Mazzi, 1911-1988 : Galleria Carlo Mazzi, Tegna, 14.VI - 30.VIII.1998, 1998, p. 29).}

(Belinelli, 1981, p. 30)

(Belinelli, Carlo Mazzi, 1911-1988 :

Galleria Carlo Mazzi, Tegna, 14.VI 30.VIII.1998, 1998, p. 29).

(Archivio Mazzi) 


\begin{tabular}{|c|c|c|c|c|c|}
\hline $\begin{array}{l}1974- \\
1977 \text { ca. }\end{array}$ & $\begin{array}{l}\text { Avegno, } \\
\text { chiesa parrocchiale } \\
\text { dei SS. Luca e } \\
\text { Abbondio. }\end{array}$ & Affreschi. & Restauro. & $\begin{array}{l}\text { Questo stesso anno } \\
\text { ebbe luogo il } \\
\text { restauro } \\
\text { architettonico della } \\
\text { fabbrica ed il } \\
\text { rinnovamento } \\
\text { dell'arredo. }\end{array}$ & $\begin{array}{l}\text { (Belinelli, 1981, p. } \\
\text { 30). }\end{array}$ \\
\hline 1974 & Locarno. & Affreschi. & Restauro. & & (Archivio Mazzi). \\
\hline 1975 & $\begin{array}{l}\text { Lostallo, } \\
\text { casa Rosa. }\end{array}$ & Affresco. & Restauro. & & (Archivio Mazzi). \\
\hline
\end{tabular}

\section{PREVENTIVI}

\begin{tabular}{|c|c|c|c|c|c|}
\hline $\begin{array}{l}28 \text { ott. } \\
1957\end{array}$ & $\begin{array}{l}\text { Prugiasco, } \\
\text { S. Carlo. }\end{array}$ & & $\begin{array}{l}\text { Preventivo di Carlo } \\
\text { Mazzi al DPE per } \\
\text { strappo delle } \\
\text { pitture dei } \\
\text { seregnesi. }\end{array}$ & & (Archivio Mazzi). \\
\hline $\begin{array}{l}2 \text { dic. } \\
1966\end{array}$ & $\begin{array}{l}\text { Morcote, } \\
\text { chiesa parrocchiale } \\
\text { di S. Maria del } \\
\text { Sasso. }\end{array}$ & $\begin{array}{l}\text { Domenico Pezzi, } \\
1513 . \\
\text { Crocifissione. } \\
\text { Affresco } \\
\text { rinascimentale del } \\
\text { coro primitivo, } \\
\text { parete in fondo. }\end{array}$ & $\begin{array}{l}\text { Esame autoptico } \\
\text { dell'opera che } \\
\text { denuncia vaste } \\
\text { zone mancanti, } \\
\text { rotture dovute alla } \\
\text { posa dell'organo e } \\
\text { diverse graffiature. } \\
\text { Il preventivo } \\
\text { sviluppato dal } \\
\text { restauratore } \\
\text { comprendeva la } \\
\text { pulitura generale } \\
\text { dell'affresco ed una } \\
\text { discreta } \\
\text { reintegrazione } \\
\text { pittorica. }\end{array}$ & $\begin{array}{l}\text { Il restauro } \\
\text { architettonico } \\
\text { venne condotto fra } \\
\text { il } 1967 \text { e il } 1980 \\
\text { dall'architetto } \\
\text { Guido Borella di } \\
\text { Lugano. }\end{array}$ & $\begin{array}{l}\text { (AUBCBZ, 172, } \\
\text { Morcote) } \\
\text { (Mazzi Carlo, } \\
\text { Preventivo } \\
\text { indirizzato a Guido } \\
\text { Borella. Tegna, } 2 \\
\text { dicembre 1966. } \\
\text { AUBCBZ, s.172, } \\
\text { Morcote). }\end{array}$ \\
\hline $\begin{array}{l}2 \text { dic. } \\
1966\end{array}$ & $\begin{array}{l}\text { Morcote, } \\
\text { chiesa parrocchiale } \\
\text { di S. Maria del } \\
\text { Sasso. }\end{array}$ & $\begin{array}{l}\text { Domenico Pezzi, } \\
1513 . \\
\text { Gesù nell'orto. } \\
\text { Affresco } \\
\text { rinascimentale del } \\
\text { coro primitivo, } \\
\text { parete in fondo. }\end{array}$ & $\begin{array}{l}\text { Esame autoptico } \\
\text { dell'opera che } \\
\text { denuncia un cattivo } \\
\text { stato di } \\
\text { conservazione } \\
\text { dell'affresco, } \\
\text { danneggiato a } \\
\text { causa dell'umidità } \\
\text { e invaso da } \\
\text { [...] “fungo rosa”. } \\
\text { Contribuiva al } \\
\text { degrado la } \\
\text { scialbatura } \\
\text { generale } \\
\text { soprastante } \\
\text { l'affresco ed } i\end{array}$ & $\begin{array}{l}\text { Arch. Guido } \\
\text { Borella, Lugano. }\end{array}$ & $\begin{array}{l}\text { (AUBCBZ, 172, } \\
\text { Morcote) } \\
\text { (Mazzi Carlo, } \\
\text { Preventivo } \\
\text { indirizzato a Guido } \\
\text { Borella. Tegna, } 2 \\
\text { dicembre 1966. } \\
\text { AUBCBZ, s.172, } \\
\text { Morcote). }\end{array}$ \\
\hline
\end{tabular}




\begin{tabular}{|c|c|c|c|c|c|}
\hline & & & $\begin{array}{l}\text { ritocchi irrispettosi } \\
\text { realizzati in restauri } \\
\text { passati. Il } \\
\text { preventivo } \\
\text { elaborato } \\
\text { dell'artista } \\
\text { proponeva } \\
\text { l'eliminazione delle } \\
\text { scialbature, la } \\
\text { pulitura generale } \\
\text { dell'affresco, la } \\
\text { consolidazione } \\
\text { d'una piccola zona } \\
\text { e la reintegrazione } \\
\text { pittorica limitata e } \\
\text { l'intonazione delle } \\
\text { ampie lacune[...] }\end{array}$ & & \\
\hline $\begin{array}{l}2 \text { dic. } \\
1966\end{array}$ & $\begin{array}{l}\text { Morcote, } \\
\text { chiesa parrocchiale } \\
\text { di S. Maria del } \\
\text { Sasso. }\end{array}$ & $\begin{array}{l}\text { Domenico Pezzi, } \\
1513 . \\
\text { Salita al calvario. } \\
\text { Affresco } \\
\text { rinascimentale del } \\
\text { coro primitivo, } \\
\text { parete in fondo. }\end{array}$ & $\begin{array}{l}\text { Il preventivo } \\
\text { prevedeva la } \\
\text { stuccatura delle } \\
\text { ampie zone } \\
\text { mancanti, la } \\
\text { ripulitura generale } \\
\text { del sudiciume di } \\
\text { polvere, il } \\
\text { consolidamento } \\
\text { della parte bassa } \\
\text { quasi totalmente } \\
\text { distrutta, la } \\
\text { reintegrazione } \\
\text { pittorica limitata e } \\
\text { l'intonazione delle } \\
\text { lacune più grandi. }\end{array}$ & $\begin{array}{l}\text { Arch. Guido Borella } \\
\text { Lugano. }\end{array}$ & $\begin{array}{l}\text { (AUBCBZ, 172, } \\
\text { Morcote) } \\
\text { (Mazzi Carlo, } \\
\text { Preventivo } \\
\text { indirizzato a Guido } \\
\text { Borella. Tegna, } 2 \\
\text { dicembre 1966. } \\
\text { AUBCBZ, s.172, } \\
\text { Morcote). }\end{array}$ \\
\hline $\begin{array}{l}2 \text { dic. } \\
1966\end{array}$ & $\begin{array}{l}\text { Morcote, } \\
\text { chiesa parrocchiale } \\
\text { di S. Maria del } \\
\text { Sasso. }\end{array}$ & $\begin{array}{l}\text { Ignoto, } 1480 \text { ca. } \\
\text { Volta a spicchi. } \\
\text { Affresco } \\
\text { rinascimentale del } \\
\text { coro primitivo, } \\
\text { parete in fondo. }\end{array}$ & $\begin{array}{l}\text { L'esame autoptico } \\
\text { preliminare } \\
\text { denuncia un cattivo } \\
\text { stato di conserva- } \\
\text { zione occasionato } \\
\text { dall'imbiancatura } \\
\text { soprastante i } \\
\text { dipinti e dalla } \\
\text { possa dell'organo. } \\
\text { Il preventivo } \\
\text { elaborato da Mazzi } \\
\text { prevedeva la } \\
\text { ripulitura della } \\
\text { calce o imbianca- } \\
\text { tura che ricopre i } \\
\text { dipinti } \\
\text { (Pantocrator, } \\
\text { Evangelisti e }\end{array}$ & $\begin{array}{l}\text { Arch. Guido Borella } \\
\text { Lugano. }\end{array}$ & $\begin{array}{l}\text { (AUBCBZ, 172, } \\
\text { Morcote) } \\
\text { (Mazzi Carlo, } \\
\text { Preventivo } \\
\text { indirizzato a Guido } \\
\text { Borella. Tegna, } 2 \\
\text { dicembre 1966. } \\
\text { AUBCBZ, s.172, } \\
\text { Morcote). }\end{array}$ \\
\hline
\end{tabular}




\begin{tabular}{|c|c|c|c|c|}
\hline & & & $\begin{array}{l}\text { Angeli) Restauro } \\
\text { pittorico dove } \\
\text { possibile e } \\
\text { intonazione per il } \\
\text { resto. }\end{array}$ & \\
\hline $\begin{array}{l}5 \text { mag. } \\
1967\end{array}$ & $\begin{array}{l}\text { Locarno, } \\
\text { Orselina } \\
\text { Madonna del } \\
\text { Sasso. }\end{array}$ & $\begin{array}{l}\text { Otto lunette } \\
\text { affrescata nella } \\
\text { volta della capella } \\
\text { Cisera. }\end{array}$ & $\begin{array}{l}\text { Pulitura e } \\
\text { consolidamento. } \\
\text { Fotografie. } 2500 \mathrm{fr} \text {. } \\
\text { Restauro pittorico } \\
\text { degli stucchi, fondi } \\
\text { e pareti. } 5500 \mathrm{fr} \text {. }\end{array}$ & $\begin{array}{l}\text { (Mazzi Carlo, } \\
\text { Preventivo del } 5 \\
\text { maggio 1967, } \\
\text { Tegna. Archivio } \\
\text { privato). }\end{array}$ \\
\hline
\end{tabular}


Restauratori in Canton Ticino fra Ottocento e Novecento

Catalogazione e gestione dati

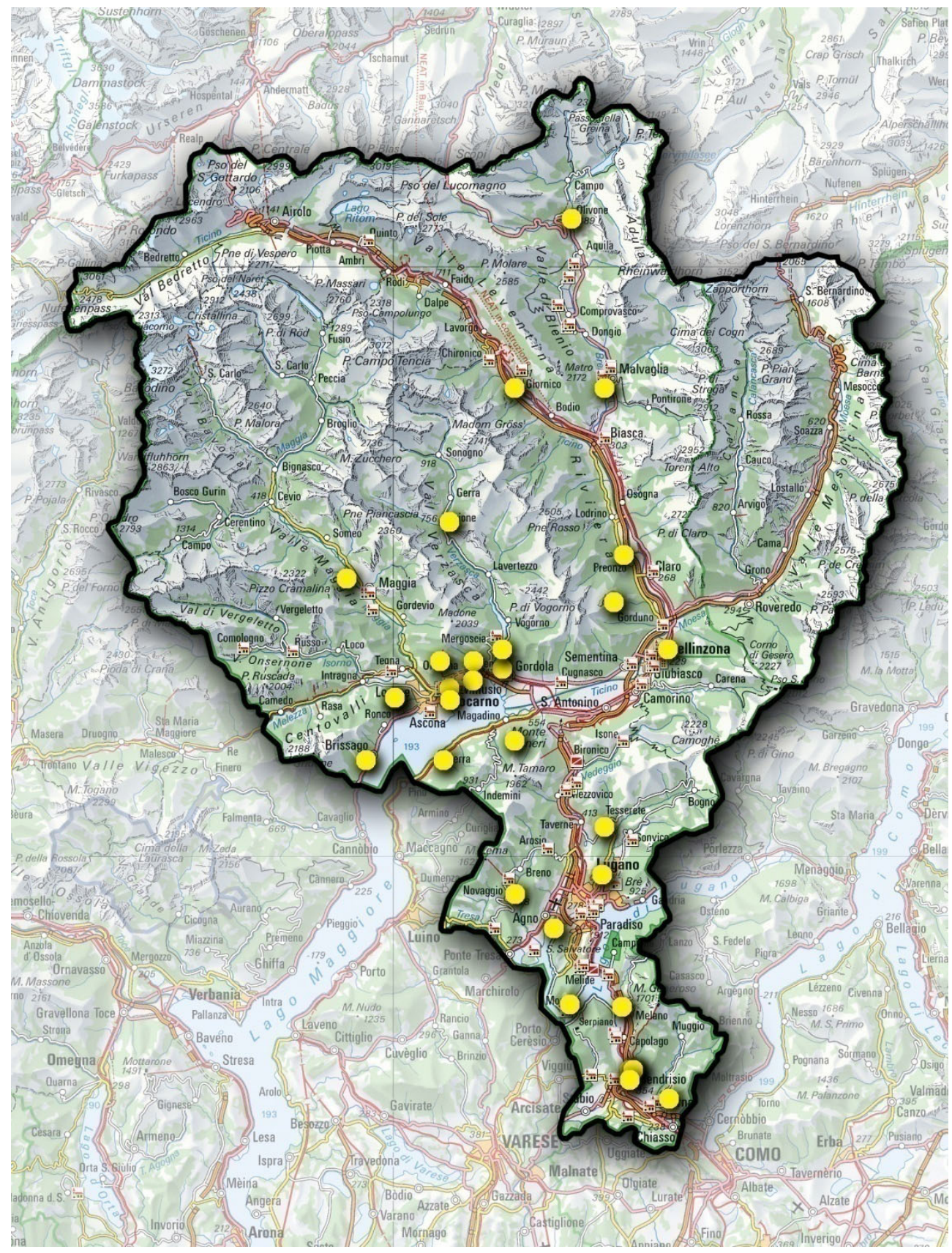

Figura 193 Cartina degli interventi eseguiti da Carlo Mazzi in Canton Ticino. 




$$
\ldots
$$




\section{GIUSEPPE STEFFANONI E FIGLI}

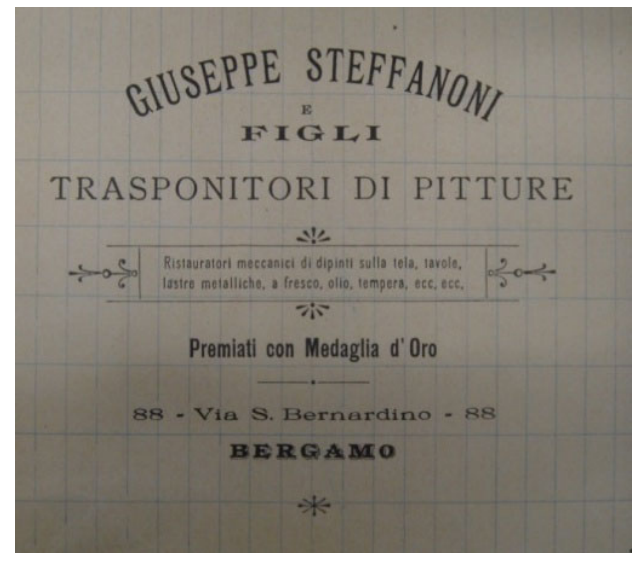

Si definivano "Trasponitori di pitture. Restauratori meccanici di dipinti sulla tela, tavole, lastre meccaniche, a fresco, olio, tempera, ecc, ecc." Così intestavano lettere e rapporti i restauratori di Bergamo, gli Steffanoni, domiciliati in Via San Bernardino 88. II metodo di distacco dei dipinti murali, in auge dagli ultimi anni del Novecento, fu iniziato dal padre Giuseppe e significò per la famiglia di restauratori un importante esito che gli diede inoltre la fama internazionale. Supponeva la messa in pratica degli ideali tecnici di strappo che già prima il conte Secco-Suardo aveva promosso ed altri sperimentato con grande successo, per la soddisfazione di studiosi dell'arte ed antiquari che vedevano in tale tecnica il modo per ravvicinarsi alle particolarità di un'opera solitamente osservata dalla distanza ed il guadagno nel mercato artistico per gli ultimi. Consisteva nel distacco degli affreschi con l'incollaggio di diverse tele alla superficie, in modo che la stessa colla all'asciugare si staccava dal muro portando con sé l'ultimo strato e con esso il colore. II retro del dipinto strappato veniva ridotto ad una fine lamina di pochi centimetri per rendere il materiali flessibile è adatto al nuovo supporto in tela. Era inoltre l'unico metodo ritrovato all'epoca per salvaguardare affreschi il cui supporto murario fosse gravemente danneggiato dall'umidità. Gli intonaci degradati venivano rifatti, dopo lo strappo, e successivamente riattaccato in situ al medesimo. Ma non solo, poiché la tecnica fu frequentemente utilizzata per scoprire dipinti primitivi, sottostanti le pitture fattibili allo strappo, o per riportare il complesso architettonico e decorativo della fabbrica restaurata alle sue forme stilistiche originarie. Gli strappi eseguiti venivano in alcuni casi collocati in posti diversi del proprio edificio o trasportati ad altri. Permette inoltre, la tecnica, la musealizzazione dell'opera, ma soprattutto la sua scontestualizzazione. II caso più esemplificativo di questo fatto è oggi ammirato al Museo Nazionale d'Arte di Catalunya che raggruppa all'interno della collezione d'arte romanica affreschi ed interi complessi decorativi provenenti di cappelle e chiesa della regione catalana. Essi furono realizzati con grande consenso pubblico da parte di Francesco, chiamato anche Franco, Steffanoni figlio ed allievo di Giuseppe.

In Svizzera, Giuseppe Steffanoni aveva già applicato il metodo dello strappo nel restauro della cattedrale di S. Pietro a Ginevra nel 1886. Più tardi, esattamente il 31 luglio del 1899, è incaricato degli strappi alla chiesa di S. Paolo, detta Chiesa rossa, ad Arbedo ed al Museo Civico a S. Michele 
(Zugo), e poi nel 1904 negli affreschi medievali della chiesa conventuale a Müstair e nella Cattedrale di San Lorenzo a Lugano.

Dalla corrispondenza mantenuta fra i restauratori bergamaschi ed il Dipartimento della Pubblica Educazione a Bellinzona negli anni 1899 con motivo del restauro della sopracitata chiesa rosa ad Arbedo, si può confermare la presenza di essi in interventi avvenuti a Zurigo e Berna, per l'esecuzioni di distacchi. L'informazione, in quanto scarsa, non ha potuto proporci maggiori dettagli che servirebbero a identificare le opere corrispondenti ed i luoghi precisi d'intervento.

La documentazione ritrovata all'Archivio Nazionale di Berna, sul restauro realizzato ad Arbedo, è invece più rica, con rapporti che spiegano esattamente le fasi operative corrispondenti.

\section{San Paolo, Arbedo}

La chiesa romanica di San Paolo fu oggetto di restauro architettonico fra il 1898 ed il 1900, per conto del Dipartimento della Pubblica Educazione del Canton Ticino e dei sussidi della Confederazione svizzera. Con lo scopo di riportare la fabbrica alle sue forme primarie, I'architetto Augusto Guidini $\mathrm{fu}$ incaricato dalle suddette istituzioni a dirigere il progetto di ripristino che interessò la cancellazione della parte superiore del campanile di epoca tardo barocca, il rinnovamento degli intonaci ed il restauro generale degli affreschi.

Giuseppe Steffanoni fu chiamato da Guidini ad intervenire sui dipinti, i quali presentavano un cattivo stato di conservazione a causa dell'umidità di risalita capillare e per infiltrazioni del tetto $^{496}$.

Gli affreschi conservati nella chiesa di San Paolo ad Arbedo sono, nella parte destra della facciata, l'affresco raffigurante $S$. Paolo (XV-XVI sec.) attribuito all'artista Antonio da Tradate, e la Passione di Cristo, raffigurata all'interno della lunetta sopra la parte d'accesso principale all'edificio, databile al 1543. All'interno della chiesa si osservano, sulla parete sud, le rappresentazioni di S. Antonio Eremita e San Sebastiano in basso, e la Trinità con il committente inginocchiato (1550) ed un Cenacolo realizzato probabilmente nel XV secolo per Nicolao da Seregno in alto. Sulla parete nord in cambio ci sono le raffigurazioni della Madonna, opere di Gian Giacomo Gorla del 1549, di Cristo e di una seconda Madonna con San Paolo del XV sec., Nel coro si trova la Crocifissione (1520-1530), ed un'Annunciazione assieme ad altri dipinti decorativi databili alla seconda metà del XV sec. attribuiti ad i Seregnesi.

II rapporto che l'architetto Guidini assieme al pittore Luigi Rossi ${ }^{497}$ presentò al DPE il 15 settembre del 1899 descrive le fasi di

\footnotetext{
496 Non risulta che questo fosse restaurato fino al 1950 da Giovannini.

${ }^{497}$ Luigi Rossi (-) È stata verificata la partecipazione del pittore alla protezione dei monumenti storici in qualità di professionista per il collauda mento dei lavori eseguitisi o da realizzarsi, con il documento citato del 14 settembre del 1899 e con un secondo rapporto presentato nel 1901 al DPE, nel quale si descrivono i controlli per la supervisione ai cantiere nel di Santa Maria degli Angioli a Lugano per il restauro della Crocifissione e passione di Cristo di Luini nel 1899; a Morcote, a Melide, alla Chiesa Rosa di Arbedo e per il Cenacolo alla chiesa di Pianezzo. (Guidini Augusto/ Rossi Luigi, lettera indirizzata al lod. DPE, Milano 6 gennaio 1901 AFMS BNS. Bern K Tessin A-M n.79)
} 
lavoro eseguite lo stesso anno da Giuseppe Steffanoni sui sopra descritti dipinti e sugli affreschi della chiesa parrocchiale dei SS. Giacomo e Filippo a Pianezzo ${ }^{498}$, secondo il contrato che il restauratore aveva accordato con la Confederazione. Si trattava di un lavoro specialmente di pulitura dello scialbo di calce soprastante le antiche pitture murali presenti in entrambe le chiese, nel consolidamento e nel ritocco pittorico di essi. II preventivo stipulava un totale complessivo di fr. 1350 e sarebbero pagati a Steffanoni solo al termine e dopo il collaudo del lavoro effettuato.

Secondo le parole di Guidini e Rossi riportate sul rapporto: [...] il lavoro [...] affidato allo specialista sig. Steffanoni [...] fu compiuto [...] e non esitiamo a dichiararlo, è lodevolmente compiuto $[. . .]^{499}$.

Oltre ai lavori di pulitura degli affreschi, nella chiesa di San Paolo le competenze dello Steffanoni interessarono anche lo strappo dell'affresco della Crocifissione dietro l'altare maggiore. Questo, trovatosi particolarmente danneggiato dall'azione dell'umidità, non permetteva il suo restauro in situ. Malgrado il riattacco degli elementi materici pericolanti fosse possibile, il restauratore bergamasco ammetteva che non poteva assicurare che $\mathrm{i}$

\footnotetext{
498 |l contratto di lavoro dettato dalla Confederazione in data 31 luglio 1899 per la concessione dei sussidi recava: [...]Le travaux serant exécutés par Mr. G. Stefannoni de Bergamo sour la diretion de Mr. Guidini di Milan.[...] in relazione ai restauri degli affreschi della Chiesa di San Paolo ad Arbedo ad a Pianezzo. AN Bern, KT A-M №79).

${ }^{499}$ (Guidini Augusto. Relazione e Collaudo. Chiesa di S. Paolo in Arbedo e di Pianezzo. (Antiche Pitture scoperte e restaurate). Indirizzato alla al Lodevole DPE, Bellinzona. Milano, 15 settembre 1899. AN Bern, KT A-M №79).
}

danni dall'umidità si fossero ridotte, ma sarebbe necessario ancora una continua manutenzione dell'affresco a brevi intervalli.

Steffanoni raccomandava per questo motivo lo strappo e trasporto del dipinti su un nuovo supporto, che dopo il ripristino degli intonaci nella parete frontale del coro, sarebbe su codesta idoneamente ricollocato.

La fasi di restauro a questo scopo furono:

- strappo dell'affresco della Crocifissione

- trasporto su tela e preparazione del telaio di sostegno di $525 \mathrm{~cm} \times 300 \mathrm{~cm}$ - ricollocamento sul posto originario

[...] Dopo un maturo esame ed approfondito studio del caso, nelle specialissime sue condizioni venne decisa una diversa, eccezionale ed indicatissima modalità di esecuzione: e cioè il completo prelevamento dell'affresco riportandolo ad una superficie opportunamente predisposta, applicata su telaio: così convertendo la materiale e primitiva condizione dell'affresco murale, minacciato di progressiva distruzione, in pittura isolata, solida $e$ di sicurissima e duratura conservazione. [...] $E$ l'esito fu davvero eccezionalmente favorevole: essendosi potuto integralmente staccare l'intero dipinto, anche nelle parti già ricoperte dell'altare, e nelle sue parti più danneggiate, restituendolo, meno le materiali lacune preesistenti, al suo pristino stato, e nella maggior evidenza.[... $]^{500}$.

Lo strappo della Crocifissione, come si spiegava precedentemente, fu idoneamente ricollocato nella sua ubicazione primitiva, però il progetto di

\footnotetext{
${ }^{500}$ (Guidini Augusto. Relazione e Collaudo. Chiesa di S. Paolo in Arbedo e di Pianezzo. (Antiche Pitture scoperte e restaurate). Indirizzato alla al Lodevole DPE, Bellinzona. Milano, 15 settembre 1899. AN Bern, KT A-M №79).
} 
restauro prevede in modo opportuno il distanziamento fra questo ed il muro di appena un centimetro, sufficiente a permettere la ventilazione retrostante.

La pulitura e descialbo degli affreschi cinquecenteschi consentì di mettere in luce nuovi elementi decorativi e di ammirare le qualità artistiche dell'insieme pittorico.

[...] Venne pure, oltre il segmento triangolare di volta, sopra la Crocefissione, scoperto l'antico fronte dipinto dell'arco acuto antistante fregiato da soggetti sacri[... $]^{501}$.

La reintegrazione pittorica fu sorprendentemente eseguita in accordo ai concetti di minimo restauro, che non sarebbero utilizzati così consapevolmente ed in modo generalizzato fino alla seconda metà del Novecento. A questo punto le lacune furono unicamente intonate con lo stesso stucco applicato per livellare le zone mancanti con l'originale. Questo venne impastato leggermente con terra d'ombra che diede la tonalità neutra capace di omogeneizzare cromaticamente il dipinto.

[...] In conformità del logico e moderno concetto della conservazione, e come è pure tassativamente prescritto dalle norme ufficiali in materia, nessuna applicazione e sovrapposizione di colore di sorta venne eseguita in alcuna parte del dipinto che ne avesse anche menomamente alterata l'intonazione e l'aspetto[... $]^{502}$.

\footnotetext{
${ }^{501}$ (Guidini Augusto. Relazione e Collaudo. Chiesa di S. Paolo in Arbedo e di Pianezzo. (Antiche Pitture scoperte e restaurate). Indirizzato alla al Lodevole DPE, Bellinzona. Milano, 15 settembre 1899. AN Bern, KT A-M №79).

${ }^{502}$ (Guidini Augusto. Relazione e Collaudo. Chiesa di S. Paolo in Arbedo e di Pianezzo. (Antiche Pitture scoperte e restaurate). Indirizzato alla al Lodevole DPE, Bellinzona. Milano, 15 settembre 1899. AN Bern, KT A-M №79).
}

\section{SS. Giacomo e Filippo, Pianezzo}

Nella chiesa parrocchiale dei SS. Giacomo e Filippo in Arbedo, il dipinto, oggetto di prudenti puliture degli strati soprastanti fu la raffigurazione della Cena degli Apostoli. II dipinto, attribuito ad un artista di scuola lombarda e databile al XVI secolo si trovava in un discreto stato di conservazione al momento del suo restauro. L'affresco di $2,90 \mathrm{~m} \times 6 \mathrm{~m}$ manifestava alcune piccole abrasioni causate dalle aperture di due porte, ed un zona mancante in corrispondenza con una delle dodici figure rappresentate, anche questa cancellata dai lavori di riordino architettonico, in particolare per l'applicazione della Cantoria in legno.

II dipinto fu pulito, e furono consolidate e reintegrate le parti st§accate cromaticamente. Inoltre nello stesso momento del restauro si scoprirono delle tracce di affreschi sottostanti la Cena. Secondo Guidini, gli elementi scoperti analizzati erano stati eseguiti su un intonaco di "stucco di marmo", cioè di stucco romano, più compatto e liscio di quello soprastanti con il dipinti della Cena, ma i disegni e colori dei medesimi erano di minore qualità artistica.

Nonostante, Guidini insisteva nel fatto che nessuno dei lavori realizzati in quel momento avrebbe evitato successivamente, se futuri professionisti lo credessero opportuno, il distacco del dipinto raffigurante la Cena degli Apostoli per rendere visibili gli affreschi primitivi.

[...]Quando in futuro si volesse scoprirla interamente, non resterebbe che asportare la Cena soprastante di facile distacco, col mezzo usato per la Crocifissione di Arbedo: destinando quella ad un futuro Museo Cantonale, rendendo 
così evidente, e restituendo al culto locale dei fedeli, la sacra e sottostante vetustissima pittura. Ma a questo ci penseranno se del caso, $i$ nostri posteri! [... $]^{503}$.

\section{Cattedrale di S. Lorenzo, Lugano}

Ancora una volta sotto la direzione dell'architetto Guidini, la famiglia Steffanoni (questa volta solo i figli) intervengono nello strappo di diversi elementi decorativi nella Cattedrale di S. Lorenzo, allo stesso tempo che l'architetto sviluppava il ripristino architettonico a egli incaricato, assieme al pittore Ernesto Rusca. La fabbrica era molto degradata a causa dello stato di abbandono a cui era stata sommessa negli ultimi anni. I restauri furono realizzati grazie alla promozione del Vescovo Monsignor Peri-Morosini fra il 1904 ed il 1910.

L'intervento architettonico con una concezione di restauro storico tentò di stabilire l'accordo stilistico fra i diversi elementi di epoche e manifatture diverse presenti all'interno dell'edificio di culto. Malgrado ciò il progetto permette la cancellazione delle cappelle laterali barocche di San Carlo Borromeo e di Sant'Abbondio. Inoltre si procede con la riparazione del campanile, il consolidamento delle volte e del coro, la riparazione del pavimento e del soffitto, e vengono restaurati gli affreschi assai danneggiati dall'umidità. A quest'ultimo scopo Guidini e Rusca progettano delle nuove decorazioni murarie mentre i fratelli Steffanoni realizzavano lo strappo delle vecchie decorazione la cui conservazione era ancora possibile, all'epoca solo mediante l'applicazione della suddetta tecnica.

In totale furono 14 elementi ad essere riportati su tela e successivamente su dei telaio elaborati per ognuna delle forme.

I dati ritrovati non hanno permesso di identificare l'origine precisa delle decorazioni distaccate, che dopo il loro restauro furono collocate all'interno della Curia Vescovile di Lugano.

\footnotetext{
${ }^{503}$ (Guidini Augusto. Relazione e Collaudo. Chiesa di S. Paolo in Arbedo e di Pianezzo. (Antiche Pitture scoperte e restaurate). Indirizzato alla al Lodevole DPE, Bellinzona. Milano, 15 settembre 1899. AN Bern, KT A-M №79).
} 


\section{Interventi di restauro}

\begin{tabular}{|l|l|}
\hline ANNO & LUOGO \\
\hline 1899 & Zurigo. \\
\hline 1899 & Berna. \\
\hline & \\
\hline
\end{tabular}

7 apr. Arbedo,

1899, Chiesa di S. Paolo

Milano detta Chiesa Rosa.

\section{OPERA}

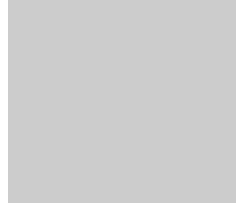

1520-1530,

Crocifissione,

Crocifissione

Cenacolo

Figure di santi

Dipinti della volta.

\section{INTERVENTO}

COLLABORATORI

FONT

(ASTI, Fondo DPEDiversi 1905 - 1955, Segnatura

1.1.4.2.12)

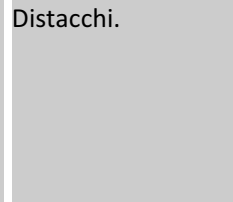

(a)

Contratto: Si spiega Augusto Guidini il lavoro eseguito da Luigi Rossi. Steffanoni. In modo particolare, sull'affresco della crocifissione assai deteriorato a causa dell'umidità, non si assicurava la conservazione di essi nel posto originale per ciò si raccomanda la pulitura e consolidamento (strappo)

\section{[...]Dopo un maturo} esame ed approfondito studio del caso, nelle specialissime sue condizioni venne decisa una diversa, eccezionale ed indicatissima modalità di esecuzione: e cioè il completo prelevamento dell'affresco riportandolo ad una superficie opportunamente predisposta, applicata su telaio... Venne pure, oltre il
(ASTI, Fondo DPEDiversi 1905 - 1955, Segnatura 1.1.4.2.12) .

(ASTI, Fondo DPEDiversi 1905 - 1955 , Segnatura 1.1.4.2.12). (Rossi Luigi, lettera indirizzata al lod. DPE, Milano 6 gennaio 1901. AFMS Bern, KT A-M №79) (DPE, Lettera indirizzata all'llmo. Sig. Arch. Comm. A. Guidini, Milano. Bellinzona, 28 giugno 1899. AFMS BNS.

Bern K Tessin A-M n.79). 


\begin{tabular}{|c|c|c|c|c|c|}
\hline & & & $\begin{array}{l}\text { segmento } \\
\text { triangolare di volta, } \\
\text { sopra la } \\
\text { Crocefissione, } \\
\text { scoperto l'antico } \\
\text { fronte dipinto } \\
\text { dell'arco acuto } \\
\text { antistante fregiato } \\
\text { da soggetti } \\
\text { sacri.[...] (Guidini } \\
\text { Augusto. Relazione } \\
\text { e Collaudo. Chiesa } \\
\text { di S. Paolo in } \\
\text { Arbedo e di } \\
\text { Pianezzo. (Antiche } \\
\text { Pitture scoperte e } \\
\text { restaurate). } \\
\text { Indirizzato alla al } \\
\text { Lodevole DPE, } \\
\text { Bellinzona. Milano, } \\
15 \text { settembre } 1899 .\end{array}$ & & \\
\hline $\begin{array}{l}7 \text { apr. } \\
1899, \\
\text { Milano }\end{array}$ & Pianezzo. & $\begin{array}{l}\text { Affresco } \\
\text { Cena degli Apostoli } \\
\text { Seconda metà XVI } \\
\text { sec. }\end{array}$ & $\begin{array}{l}\text { Contratto: } \\
\text { Pulitura e } \\
\text { consolidamento. }\end{array}$ & $\begin{array}{l}\text { Augusto Guidini } \\
\text { Luigi Rossi. }\end{array}$ & $\begin{array}{l}\text { (AFMS Bern, KT A-M } \\
\text { №79). }\end{array}$ \\
\hline $\begin{array}{l}1904- \\
1910\end{array}$ & $\begin{array}{l}\text { Lugano, Cattedrale } \\
\text { di S. Lorenzo. }\end{array}$ & & 14 strappi. & & $\begin{array}{l}\text { (AFMS Bern, KT A-M } \\
\text { №79). }\end{array}$ \\
\hline 1904 & $\begin{array}{l}\text { Müstair, } \\
\text { chiesa conventuale. }\end{array}$ & & Strappo. & & $\begin{array}{l}\text { (AFMS Bern, KT A-M } \\
\text { №79). }\end{array}$ \\
\hline 1916 & $\begin{array}{l}\text { Rovigo, Accademia } \\
\text { dei Concordi. IT }\end{array}$ & & & & $\begin{array}{l}\text { (Steffanoni F. } \\
\text { Lettera indirizzata } \\
\text { alla CCMSA. ASTI, } \\
\text { Fondo DPE- Diversi } \\
\text { 1905 - 1955, } \\
\text { Segnatura } \\
\text { 1.1.4.2.12) }\end{array}$ \\
\hline $\begin{array}{l}20 \text { lug. } \\
1916\end{array}$ & $\begin{array}{l}\text { Locarno, } \\
\text { Tavola del } \\
\text { Bramantino ed altri } \\
\text { affreschi nella } \\
\text { chiesa della } \\
\text { Madonna del Sasso. }\end{array}$ & & $\begin{array}{l}\text { Si compromette alla } \\
\text { visita, preventivo e } \\
\text { restauro. }\end{array}$ & & $\begin{array}{l}\text { (Steffanoni F. } \\
\text { Lettera indirizzata } \\
\text { alla CCMSA. ASTI, } \\
\text { Fondo DPE- Diversi } \\
\text { 1905 - 1955, } \\
\text { Segnatura } \\
\text { 1.1.4.2.12) } \\
\text { (Chiesa F. Lettera } \\
\text { richiedendo } \\
\text { autorizzazione per } \\
\text { chiamare i fratelli } \\
\text { Steffanoni a } \\
\text { controllare lo stato } \\
\text { di conservazione } \\
\text { della tavola del }\end{array}$ \\
\hline
\end{tabular}




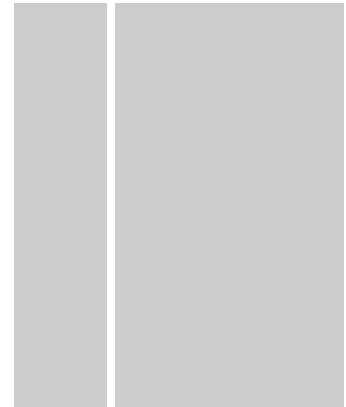

7 ago. 1916

Locarno,

Tavola del

Bramantino ed altri affreschi nella

chiesa della

Madonna del Sasso

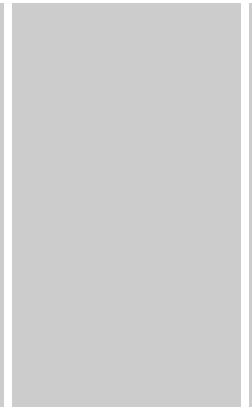

Chiede informazione realizzazione.
Bramantino alla

Chiesa della

Madonna del Sasso

di Locarno e la

chiesa di Santa

Maria in Selva.

Bernandino 31

Iuglio 1916. ASTI,

Fondo DPE- Diversi

1905 - 1955,

Segnatura

1.1.4.2.12).

(Steffanoni F.

Lettera indirizzata alla CCMSA. ASTI, Fondo DPE- Diversi 1905 - 1955,

Segnatura

1.1.4.2.12). 


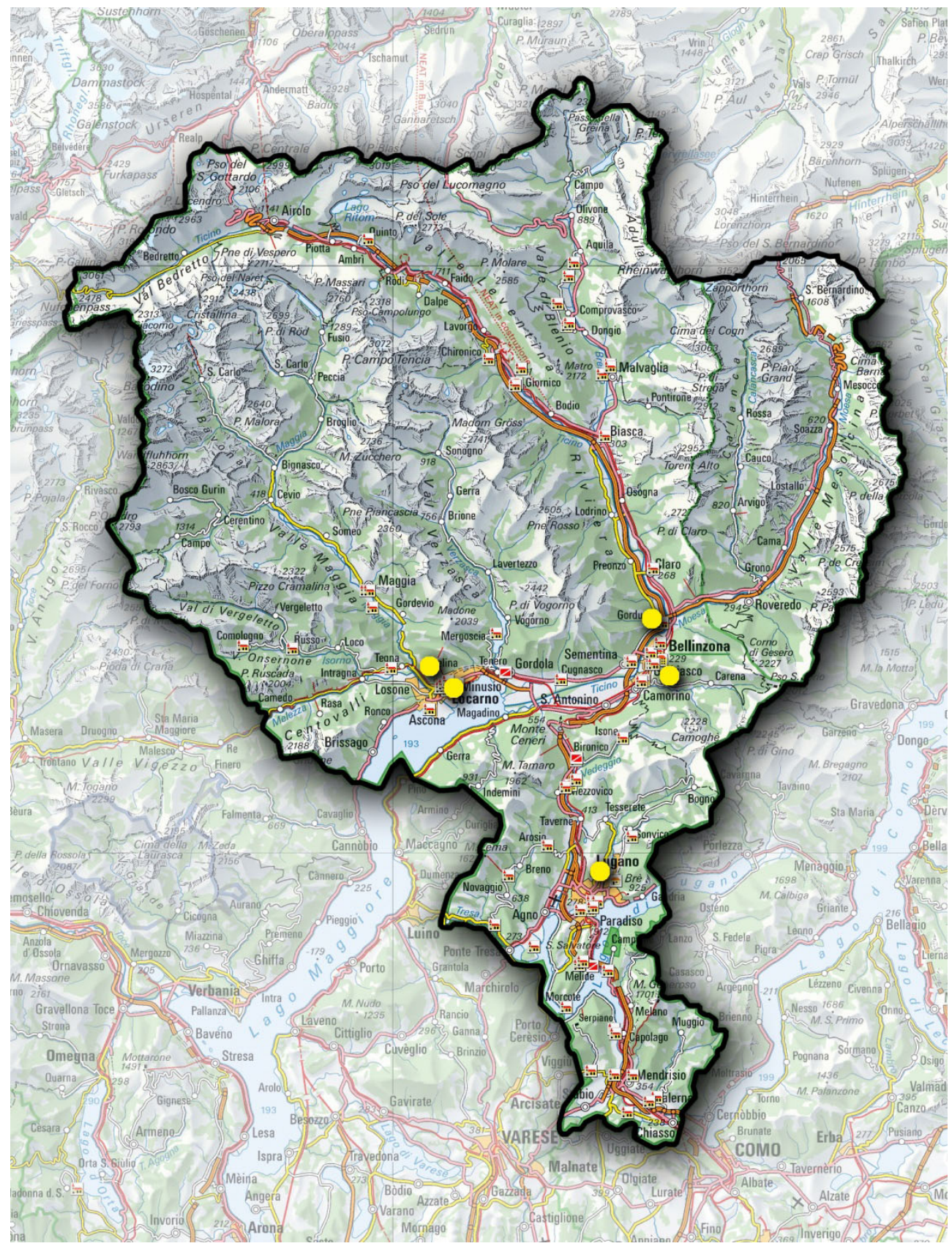

Figura 194 Cartina degli interventi eseguiti dai fratelli Steffanoni in Canton Ticino. 

MAURO PELLICIOLI 


\section{MAURO PELLICIOLI}

Pellicioli Mauro (Bergamo 1887 - 1974), è stato uno dei più prestigiosi pittorerestauratore italiano. Fra le opere più celebri restaurate nel paese natale si trovano il Cenacolo di Leonardo da Vinci eseguito in 1948 e la Cappella Scrovegni dipinta da Giotto a Padova nel 1953. Ma il Pellicioli ebbe anche un importante riconoscimento nel Canton Ticino dove, come abbiamo constatato nei capitoli anteriori, venne chiamato in numerose occasioni in qualità di professionista per realizzare, ispezionare o proporre un concreto intervento di restauro. Pellicioli ne parlava alla Televisione della Svizzera Italiana (TSI) nel 1970 circa, dei chiari ricordi che ancora ne conservava riguardo le sua attuazioni in Ticino. Inizialmente fu introdotto nel territorio svizzero grazie ad Ettore Modigliani, all'ora Sovrintendente per le Belle Arti e Antichità a Milano, sotto la cui direzione esegue il primo e più importante intervento per la Commissione Cantonale dei Monumenti Storici: questo fu senza dubbio il restauro dell'affresco della Crocifissione e Passione di Cristo sul transetto della Chiesa di S. Maria degli Angeli a Lugano (1929), del quale Pellicioli, nel colloquio televisivo realizzatosi quando lui aveva ora mai compiuto gli ottant'anni, ricordava perfino le misure: $110 \mathrm{~m} 2 . \mathrm{Ci}$ lavorò in questo caso insieme ad altri due dipendenti bergamaschi: i pittori Michele Frana ed Annibale Motterlini e ne sorvegliava direttamente, più che Modigliani, il pittore Edoardo Berta. A questo grande intervento, successero vent'anni dopo, il restauro della tavola del Bramantino raffigurante la Fuga in Egitto (sec. XVII) nella chiesa della Madonna del Sasso ad Orselina (Locarno) e diverse perizie e sopraluoghi realizzatosi assieme ad altri professionisti come l'architetto Ambrogio Annoni di Milano, il professore Jean Verdier o Cesare Brandi. Esattamente assieme quest'ultimo prestigioso teorico, Mauro Pellicioli esegue a Lugano diverse perizie intorno al 1952 ed il 1954, in concreto per il trasporto dell'affresco di Bernardino Luini (1528-1533) raffigurante la Crocifissione con due angeli che raccolgono il sangue di Cristo, la Madonna e San Giovanni. L'affresco, proprietà Marco Ghirlanda, era stato trasferito nel 1891 circa, dal convento di San Francesco a Locarno al Palazzo Albertolli a Lugano. Grazie alle indicazione dei due esperti esteri, Brandi e Pellicioli, il dipinto del Luini staccato fu trasportato idoneamente all'Oratorio di San Nazario a Dino.

In una intervista realizzata alla Radio Televisione della Svizzera Italiana il pittorerestauratore Mauro Pellicioli, nel 1970 spiega la sue collaborazioni cin la Svizzera, ma anche la sua concezione di restauro. Questa consisteva nell'osservazione analitica dello stato in cui arriva il paziente, il dipinto, alla sue mani, per procedere alla scelta idonea dei materiali e della tecniche scegliendo sempre in primo luogo le più evasive. Aggiunge a questo, come esempio da elogiare, le prassi di lavoro eseguite da Luigi Cavenaghi il quale puliva le tempere con panni appena umidi per eliminare dai dipinti il sudiciume di polvere superficiale, senza - dice Pellicioli nella suddetta intervista - l'impiego di materiali 
abrasivi $^{504}$. Da non imitare invece erano per il restauratore bergamasco il metodo utilizzato nella National Gallery di Londra, la cui prassi era completamente opposta agli ideali del Pellicioli del minimo restauro. Egli aveva avuto l'opportunità di conoscere il lavoro eseguitosi all'interno dei lavoratori del famoso museo e presto pronunciò la sua critica che si sviluppò in una intensa polemica enfatizzata dagli scritti di Cesare Brandi. Secondo le parole del restauratore bergamasco:

[...] i dipinti vengono pelati all'osso senza limiti di rispetto per quello che siano le velature ed il carattere che l'opera d'arte anche dopo la pulitura deve mantenere; il nostro compito è quello di fermarci al punto giusto $[. . .]^{505}$.

Pellicioli faceva uso come prassi abituale nel restauro dei dipinti murali, della pulitura con panni inumiditi in acqua con una piccola quantità di formaldeide, dello stucco romano e delle iniezioni di caseina per rinsaldare gli intonaci, della gommalacca (bianca, gialla e decerata a seconda dei casi diluita in alcol) per l'adesione delle scaglie di colore in pericolo di cadere, anche esso mediante iniezione, della gomma arabica come consolidante del film pittorico ed i colori a caseina per i ritocchi.

Secondo gli studi della ricercatrice Valeria Pracchi ${ }^{506}$, ci sono pochi interventi dove venne confermato l'uso d'una pulitura con materiali più tenaci. Questo è il caso ad esempio del lavoro sul Collegio del Cambio a Perugia (1940), dove il restauratore impiega la soda caustica ed una conveniente aggiunta d'ammoniaca, e l'applicazione dell'acido solforico sull'ossido di rame per trasformarlo in solfato di rame di tonalità più chiara. Per tutto questo l'Istituto del Restauro a Roma, e in speciale il direttore del medesimo istituto, Cesare Brandi, andranno in disaccordo con Pellicioli. I procedimenti del restauratore di Bergamo, anche se agirono sulle opere positivamente, non si basavano sull'analisi scientifico aspettato, ma sull'esperienza appressa durante gli anni di lavoro, e questo nella concezione del restauro moderno non era concesso.

Riassumendo ancora il suo ruolo all'interno del contesto ticinese, si deve insalzare anzitutto il carattere riconosciuto dalle autorità svizzere verso la sua persona, come professionista del restauro. Fu per questo motivo che la sua attività in Ticino non si limtò solo agli interventi di restauro, ma anche alle consulenze in qualità di esperto, come prima accennavamo. Di fatto, la sua opinione riguardo i diversi restauri che ebbe il compito di controllare, dopo le polemiche nate alla fine degli anni Quaranta, fu completamente rispettata ed influenzò future campagne d'intervento.

\footnotetext{
504 (Televisione della Svizzera Italiana. Intervista a Mauro Pellicioli. 1970. RTSI, Archivi televisivi).

505 (Televisione della Svizzera Italiana. Intervista a Mauro Pellicioli. 1970. RTSI, Archivi televisivi).

${ }^{506}$ (Pracchi, 2007, p. 457).
} 


\section{Interventi di restauro}

\begin{tabular}{|c|c|c|c|c|c|}
\hline ANNO & LUOGO & OPERA & INTERVENTO & COLLABORATORI & FONTI \\
\hline 1924 & $\begin{array}{l}\text { Lugano, } \\
\text { chiesa di S. Maria } \\
\text { degli Angioli. }\end{array}$ & $\begin{array}{l}\text { Bernardino Luini, } \\
\text { Crocifissione } \\
\text { Affresco. }\end{array}$ & $\begin{array}{l}\text { Pulitura e } \\
\text { consolidamento } \\
\text { dell'intonaco } \\
\text { sbollato con } \\
\text { iniezione di malta in } \\
\text { cui non ci sia la più } \\
\text { piccola molecola di } \\
\text { calce non bene } \\
\text { spenta che possa } \\
\text { produrre scoppi. } \\
\text { Stuccatura delle } \\
\text { lacune con stucco } \\
\text { romano (stucco e } \\
\text { polvere di marmo) } \\
\text { e intonatura con } \\
\text { velatura di tinta } \\
\text { locale a tempera } \\
\text { Intonaco antico } \\
\text { polverizzato, fissato } \\
\text { nei bordi con } \\
\text { cemento e } \\
\text { ricostruiri il pezzo } \\
\text { d'intonaco a } \\
\text { perfetta parità col } \\
\text { resto e intonarlo. } \\
\text { Pulitura e rimozione } \\
\text { del materiale grasso } \\
\text { depositato sul } \\
\text { dipinto con } \\
\text { morbidissimi } \\
\text { pennelli e piumini e } \\
\text { pannispugna } \\
\text { (spugne naturali } \\
\text { appena umide), } \\
\text { esclusa qualsiasi } \\
\text { sostanza per } \\
\text { pulire[... ]il sapone } \\
\text { sarebbe } \\
\text { pericolosissimo per } \\
\text { le tempere... } \\
\text { financo la molica di } \\
\text { pane[....]omogeneiz } \\
\text { are le zone con la } \\
\text { pulitura. } \\
\text { Reintegrazione con } \\
\text { qualche velo } \\
\text { d'acquarello o di } \\
\text { tempera. }\end{array}$ & $\begin{array}{l}\text { Com. Ettore } \\
\text { Modigliani } \\
\text { Sovrintendenza per } \\
\text { le Belle Arti e } \\
\text { Antichità, Milano. } \\
\end{array}$ & $\begin{array}{l}\text { DPE, gennaio } 1959 \text {, } \\
\text { pp.122 } 143 .\end{array}$ \\
\hline
\end{tabular}


Nessuna sostanza di protezione, dichiara - non paraffina.

Preventivo, Milano 16 Dicembre 1926, Pellicioli (pittorerestauratore; trasportitore di affreschi e quadri antichi) - 1500 lire di spese per il materiale occorrente per l'esecuzione dei lavori, come colori, panni, spugne morbidissime speciali, pelli di daino, pennelli, colori e spese diverse impreviste. Limpieza y consolidación Consolidación de la superficie desadherida con inyección de malta de cal.

$\begin{array}{cl}1949 & \begin{array}{l}\text { Negrentino- } \\ \text { Prugiasco } \\ \text { San Carlo, }\end{array} \\ 1950 \text { - } & \text { Locarno, }\end{array}$

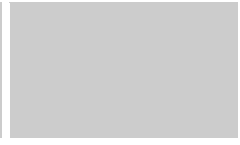

Isepzione

Rapporto 1949,

Bramantino, Fuga in Restauro:

sistemazione della statica della tavola col chiudere le Ugo Tarabori Brenno Galli Piero Bianconi fessure ed impedire "Famiglia artistica ogni modificazione locarnese", del tergo chiuso entro una perfecta grigliatura ma potranco essere rimossi anche i restauri che deturpano la rarísima pintura. Documentazione fotografica e radiografica.

II quadro era stato propone di fare una Lugano 1999. pp. 99 mostra al ritorno diverse opere del grande pittore lombardo: Ecce Homo della Collezione Thyssen, aráis delle serie dei mesi nel Castello Sforzersco con l'affresco del "Noli me tangere", due della pala con 


\begin{tabular}{|c|c|}
\hline $\begin{array}{l}\text { giudicato } \\
\text { precedentemente } \\
\text { da una Comm. } \\
\text { Composta da } \\
\text { Verdier, I Pellicioli e } \\
\text { di altri delegati } \\
\text { internazionali. } \\
\text { Mario Rossi, che } \\
\text { voleva questo } \\
\text { lavoro, dichiarò } \\
\text { dopo sapere } \\
\text { questo, } \\
\text { spontanemanete, } \\
\text { che preferiva che il } \\
\text { lavoro fosse fatto } \\
\text { da Pelliccioli. } \\
\text { Lettera Milano } 9 \\
\text { agosto } 1950 \text { di } \\
\text { Fernanda Wittgens } \\
\text { al DPE. }\end{array}$ & 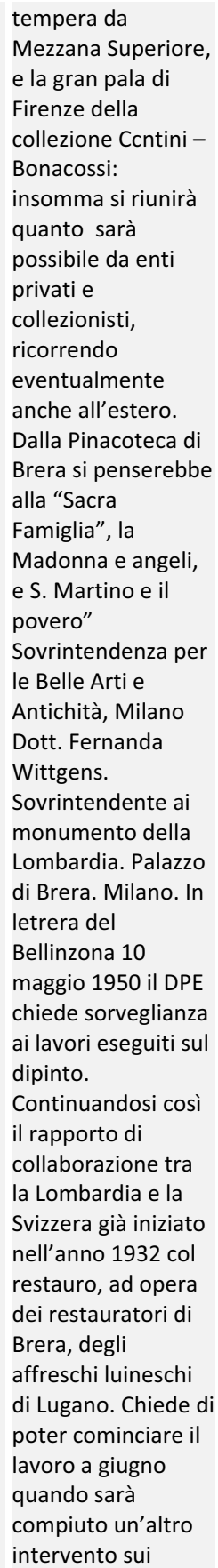 \\
\hline
\end{tabular}




\begin{tabular}{|c|c|c|c|c|c|}
\hline & & & & $\begin{array}{l}\text { dipinti } \\
\text { cinquecenteschi } \\
\text { della Pinacoteca. } \\
\text { Ditta Züst \& } \\
\text { Bachmeier S.A. } \\
\text { Trasporti } \\
\text { Internazionali, } \\
\text { Chiasso. Per } \\
\text { burocracia } \\
\text { doganale e } \\
\text { trasporto a Milano } \\
\text { della tavola, }\end{array}$ & \\
\hline 1952 & $\begin{array}{l}\text { Locarno, } \\
\text { Madonna del Sasso }\end{array}$ & $\begin{array}{l}\text { Fuga in Egitto, } \\
\text { Bartolomeo Suardi, } \\
\text { detto il Bramantino }\end{array}$ & $\begin{array}{l}\text { Fattura } \\
\text { concerniente il } \\
\text { lavoro di } \\
\text { risarcimento e } \\
\text { rinforzo della tavola } \\
\text { e quello di pulitura } \\
\text { e restauro del } \\
\text { dipinto del } \\
\text { Bramantino } \\
\text { raffigurante la fuga } \\
\text { in Egitto di } \\
\text { proprietà del } \\
\text { Santuario della } \\
\text { Madonna del Sasso } \\
\text { in Locarno. Al DPE, } \\
\text { 18 marzo } 1952 \\
\text { 1. Spese di } \\
\text { documentazione e } \\
\text { controllo } \\
\text { radiografico del } \\
\text { dipinto antecedente } \\
\text { l'inizio dell'opera di } \\
\text { restauro. (20fr) } \\
\text { 2. Spese di } \\
\text { documentazione } \\
\text { fotografica } \\
\text { antecedente l'inizio } \\
\text { dell'opera di } \\
\text { restauro (n.9 } \\
\text { fotografie) (15fr) } \\
\text { 3. Opere } \\
\text { meccaniche di } \\
\text { risarcimento alla } \\
\text { vecchia tavola. } \\
\text { Risanamento dei } \\
\text { due fili di } \\
\text { congiunzione delle } \\
\text { chiavi a farfalla di } \\
\text { rinforzo ai }\end{array}$ & & $\begin{array}{l}\text { AUBC n.183 } \\
\text { Orselina, }\end{array}$ \\
\hline
\end{tabular}




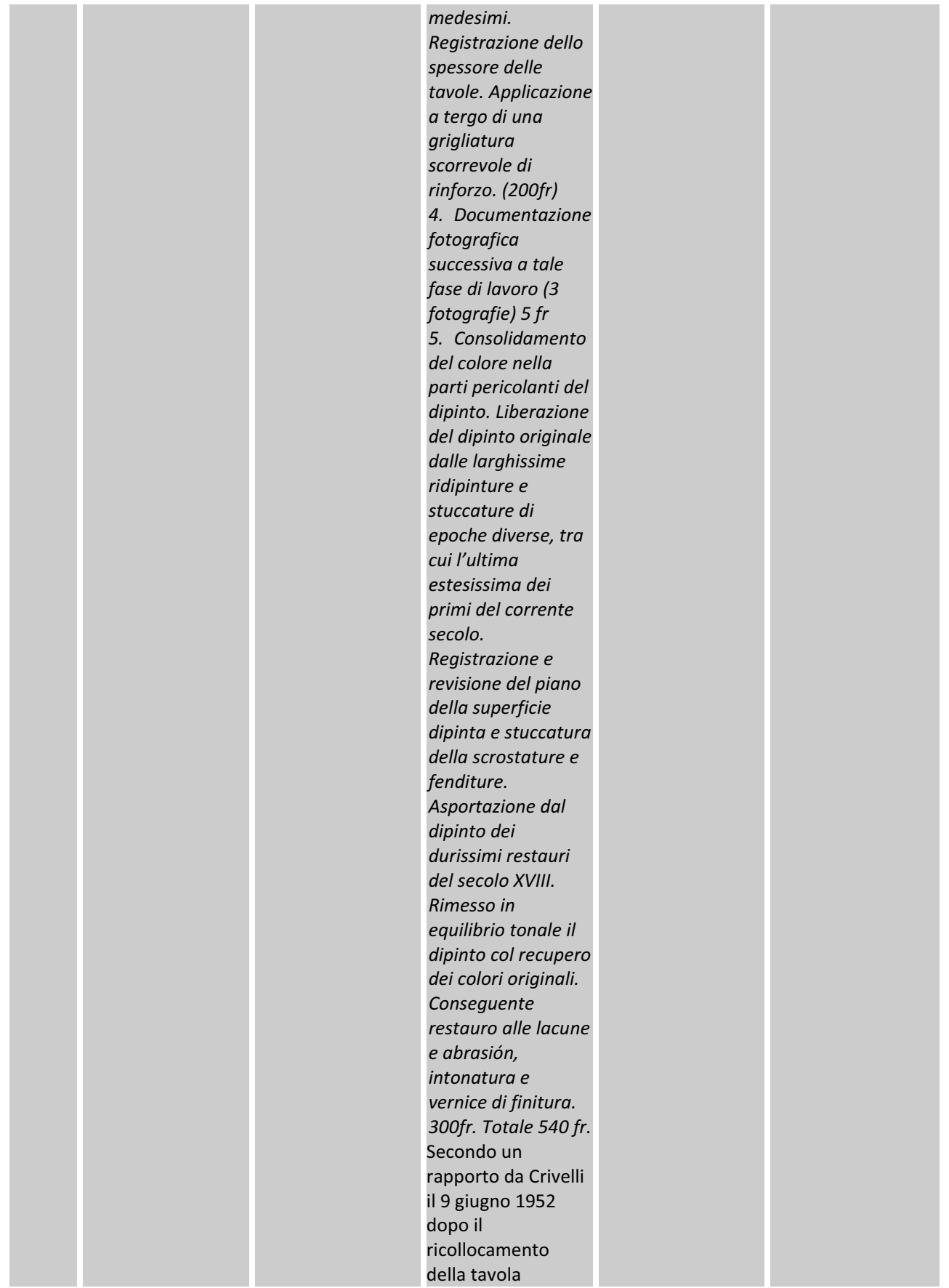




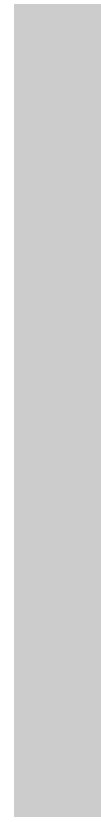

1952 1954

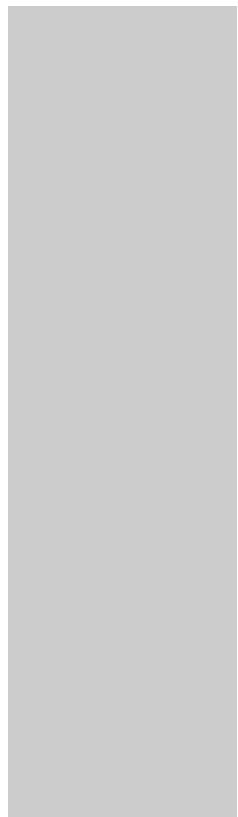

Lugano, Palazzo Albertolli, ora sede della Banca Nazionale.

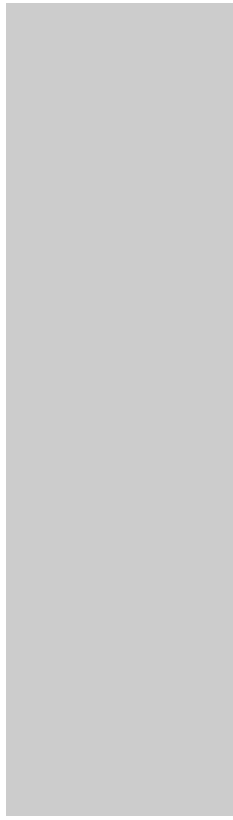

Bernardino Luini,

1528-1533.

Crocifissione con due angeli che raccolgono il sangue di Cristo, la Madonna e San Giovanni $(180 \times 220$ $\mathrm{cm})$. Affresco proprietà Marco Ghirlanda. nell'altare della

chiesa, una delle

antiche

screpolature in alto della tavola

centrale, della

lunghezza di $6 \mathrm{~cm}$. È leggermente

riapparsa, ma per 3 $\mathrm{cm}$ resta coperta dalla cornice.

Questo piccolo difetto non pe da attibuirsi ad inicdenti di trasporto. È probabilmente lo stucco del restauro che si è ritirato. La tavola nel suo complesso presenta ancora uan leggera curvatura nella parte centrale.

\section{Perizie per il} trasporto

dell'affresco

1954. L'affresco era

stato in 1851

trasferito

probabilmente

dallo stesso

Albertolli prima

della distruzione del

convento di S.

Francesco di

Locarno nel 1852

ca.

Trasporto avvenuto e collocamento nel oratorio di S.

Nazzario in Dino (scoprimento di affreschi in questa chiesa).

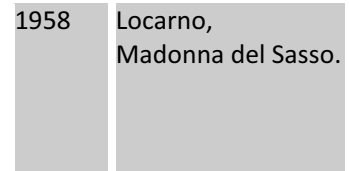

Fuga in Egitto,

Sopralluogo 30

gennaio 1958: i soli detto il Bramantino

piccoli danni al colore constatati, dovuti molto

\section{AUBC filza 143}

Lugano

(Chiesa, 1946, p. 35) (DPE, 1959, p. 110, 111, 122)

(Fulvio Caccia, 1984, p. 49,50$)$.
AUBC n.183

Orselina. 


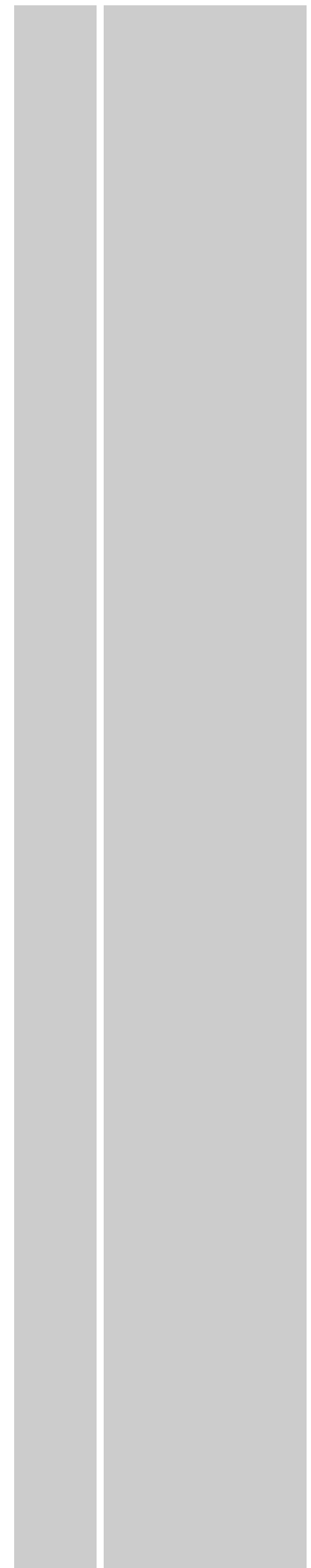

provabilmente al

sole che da una

finsetra laterale

batte directamente sulla tavola

specialmente

d'estate in alcune

ore del giorno, sono delle piccole zone del dipinto nella

parte alta che presentano delle piccole sbollature e scrostature. II fenómeno è dovuto exclusivamente al fatto che Bramantino in queste zone ha fatto largo uso di bitume il quale essicando si è raggrumato provocando quelle larghe screpolature. Il dipinto necesita di consolidamento a quelle piccole zone di colore sollevato, del restauro delle piccole scrostrature e di un buon strato di vernice di protezione. Si dovrà controllare meglio il ricollocamento in modo che la tavola sia ben fissata nel suo vanno in modo che non abbia posibilita di curvarse, pur rimanendo libero di dilatarse $o$

restringersi secondo il secco e l'umido dell'atmosfera e dell'ambiente. Sono pronto a questi piccoli restaurie controlli con l'aiuto di un mio assitente. 
15 febbraio 1958.

II lavoro venne

eseguito

dell'aiutante.

DPE. Servizio

Inventario delle

cose d'arte e di

antichità. Gilardoni?

Dichiara che nella

tavola, per effetto

della vernice o per

qual altra causa - il

colore si solleva e

minaccia di cadere

a squame nella

parte alta del cielo,

a sinistra, sul manto

della Madonna. In

altre parti,

parzilamente nei

bruni - il colore è

raggrumato; ma

può darsi che così

sia stato già prima

dei restauri. Fto?

Qualora si

chiamasse il prof.

Rossi, vorrei

pregare il

Dipartimento di

avvertirmi. Si

potrebbe

approfittare del

sopralluogo per far esaminare l'ancona di Osogna, in statu di avanzato

deperimento, $e$ altre cose minori che dovrebbero essere controllate da un restauratore di fiducia. Locarno 13 gennaio 1958.

Rimanda

interpellare a

Mauro Pelliccioli, restauratore della tavola. Anche se lui venne comunque chiamata ad esaminare gli 


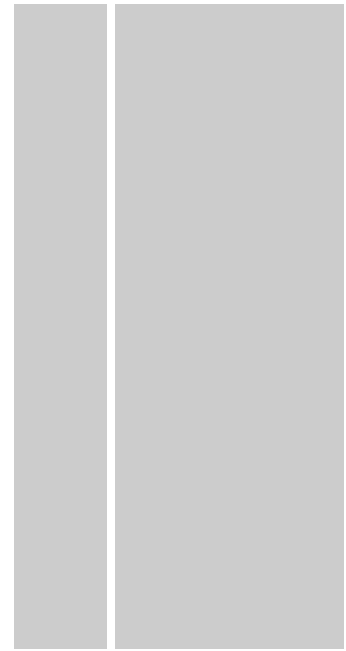

affreschi ad Osogna
e per discutre i
lavori che sono
necessari agli
affreschi strappati
al Battistero di Riva
San Vitale.
Nel 1968 si
apprezza
nuevamente la
necessità di un
urgente intervento
di restauro causa il
sollevamento del
colore nella parte
alta del cielo,
manto delle
Madonna, tunica di
S. Giuseppe.

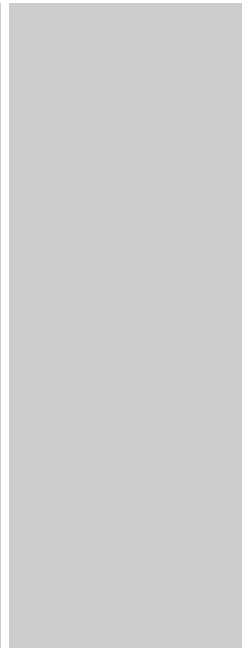




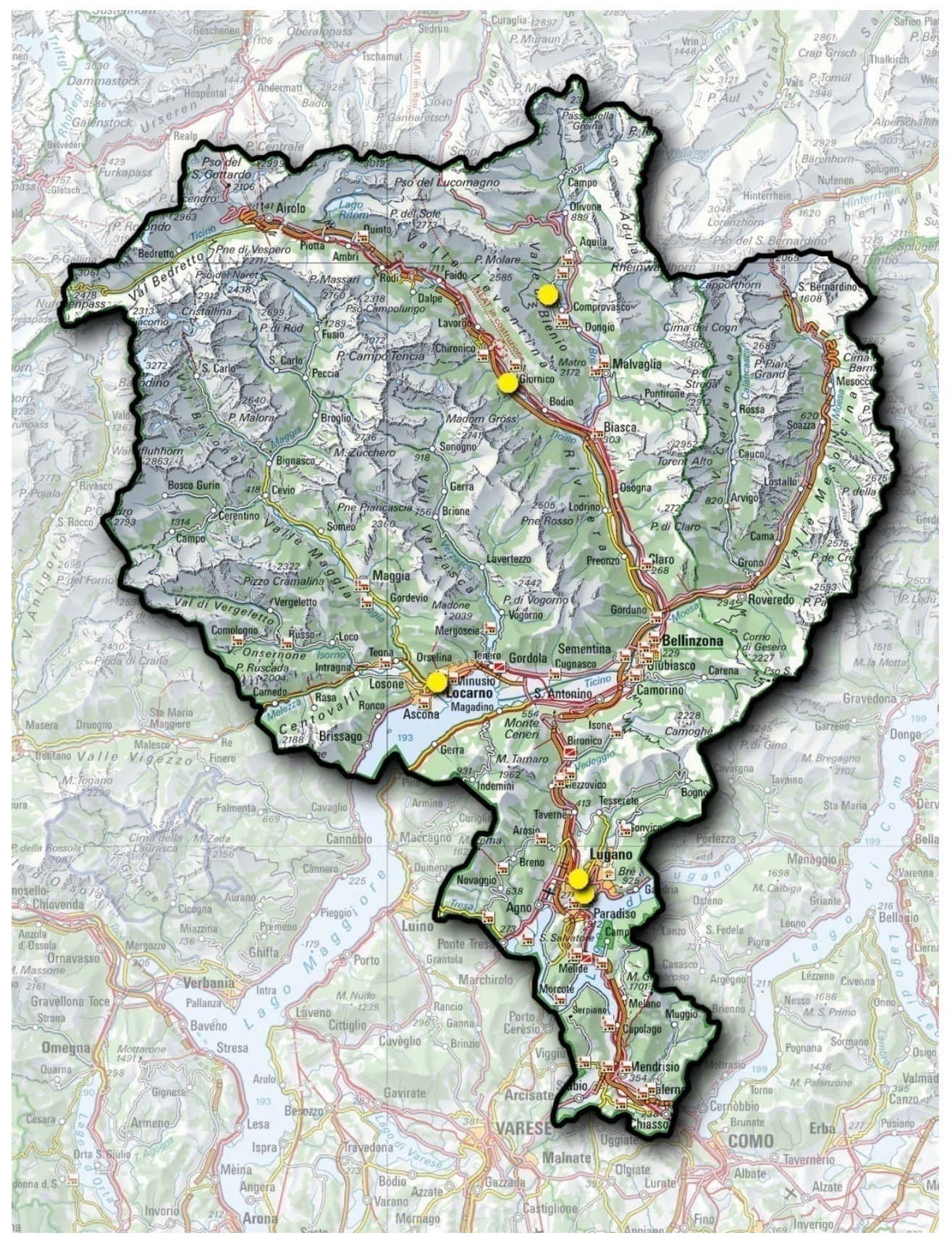

Figura 195 Cartina degli interventi eseguiti da Mauro Pellicioli in Canton Ticino. 


FRANCESCO ANNONI 


\section{FRANCESCO ANNONI, MILANO}

\section{Interventi di restauro}

\begin{tabular}{|c|c|c|c|c|c|}
\hline ANNO & LUOGO & OPERA & INTERVENTO & COLLABORATORI & FONTI \\
\hline $\begin{array}{l}20 \text { lug. } \\
1909\end{array}$ & $\begin{array}{l}\text { Ravecchia (Be) } \\
\text { chiesa di } \\
\text { S. Biagio. }\end{array}$ & $\begin{array}{l}\text { Domenico Pezzi, } \\
\text { detto Domenico del } \\
\text { lago di Lugano, } \\
\text { 1520. Vergine tra i } \\
\text { SS. Biagio e } \\
\text { Girolamo. } \\
\text { Dipinto ad olio su } \\
\text { tavola } \\
\text { Pala d'altare } \\
\text { (originariamente } \\
\text { sull'altare antico } \\
\text { poi trasferita alla } \\
\text { navata). }\end{array}$ & $\begin{array}{l}\text { Preventivo per } \\
\text { pulitura ( } 25 \text { lire), } \\
\text { elaborazione di una } \\
\text { nuova cornice ( } 250 \\
\text { lire) e doratura di } \\
\text { questa con oro fino } \\
\text { (350 lire). }\end{array}$ & & $\begin{array}{l}\text { (Chiesa, 1946, p. 13) } \\
\text { (AN Bern K Tessin } \\
\text { Bellinzona n.82) } \\
\text { (Berta, 1915, p. 2). }\end{array}$ \\
\hline 1910 & $\begin{array}{l}\text { Ravecchia (Be) } \\
\text { chiesa di } \\
\text { S. Biagio. }\end{array}$ & $\begin{array}{l}\text { Domenico Pezzi, } \\
\text { detto Domenico del } \\
\text { lago di Lugano, } \\
\text { 1520. Vergine tra i } \\
\text { SS. Biagio e } \\
\text { Girolamo. } \\
\text { Dipinto ad olio su } \\
\text { tavola } \\
\text { Pala d'altare } \\
\text { (originariamente } \\
\text { sull'altare antico } \\
\text { poi trasferita alla } \\
\text { navata). }\end{array}$ & $\begin{array}{l}\text { Restaurata e } \\
\text { collocata sull'altare } \\
\text { maggiore. }\end{array}$ & & $\begin{array}{l}\text { (Chiesa, 1946, p. 13) } \\
\text { (AN Bern K Tessin } \\
\text { Bellinzona n.82) } \\
\text { (Berta, 1915, p. 2). }\end{array}$ \\
\hline $\begin{array}{l}30 \text { ago. } \\
1910\end{array}$ & $\begin{array}{l}\text { Lugano, } \\
\text { chiesa di S. Maria } \\
\text { degli Angioli. }\end{array}$ & $\begin{array}{l}\text { Madonna col } \\
\text { bambino e santi } \\
(170 \times 230 \mathrm{~cm})\end{array}$ & $\begin{array}{l}\text { Preventivo di } \\
\text { restauro. } \\
\text { Sostituzione del } \\
\text { telaio. } 110 \mathrm{fr} \text {. }\end{array}$ & & $\begin{array}{l}\text { (Annoni Francesco, } \\
\text { Preventivo di } \\
\text { risarcimento } \\
\text { indirizzato alla } \\
\text { CCMS. Milano } 30 \\
\text { agosto } 1910 . \\
\text { AUBCBZ s. } 143 \\
\text { Lugano). }\end{array}$ \\
\hline $\begin{array}{l}30 \text { ago. } \\
1910\end{array}$ & $\begin{array}{l}\text { Lugano, } \\
\text { chiesa di S. Maria } \\
\text { degli Angioli. }\end{array}$ & $\begin{array}{l}\text { Seconda metà XVII } \\
\text { sec. San Michele. } \\
(175 \times 230 \mathrm{~cm}) \\
\text { Dipinto su tavola. } \\
\text { Pala d'altare della } \\
\text { cappella di S. } \\
\text { Teodoro. }\end{array}$ & $\begin{array}{l}\text { Preventivo di } \\
\text { restauro. } \\
\text { Sostituzione del } \\
\text { telaio. } 110 \mathrm{fr} \text {. }\end{array}$ & & $\begin{array}{l}\text { (Annoni Francesco, } \\
\text { Preventivo di } \\
\text { risarcimento } \\
\text { indirizzato alla } \\
\text { CCMS. Milano } 30 \\
\text { agosto } 1910 . \\
\text { AUBCBZ s. } 143 \\
\text { Lugano) }\end{array}$ \\
\hline $\begin{array}{l}30 \\
\text { agosto }\end{array}$ & $\begin{array}{l}\text { Lugano, } \\
\text { chiesa di S. Maria }\end{array}$ & $\begin{array}{l}\text { Petrini G. A. XVI sec. } \\
\text { Sant'Antonio di }\end{array}$ & $\begin{array}{l}\text { Preventivo di } \\
\text { restauro. }\end{array}$ & & $\begin{array}{l}\text { (Annoni Francesco, } \\
\text { Preventivo di }\end{array}$ \\
\hline
\end{tabular}


risarcimento

indirizzato alla

CCMS. Milano 30

agosto 1910.

AUBCBZ s. 143

Lugano).

\begin{tabular}{|c|c|c|c|c|}
\hline $\begin{array}{l}14 \text { lug. } \\
1913\end{array}$ & $\begin{array}{l}\text { Locarno, } \\
\text { Chiesa della } \\
\text { Madonna del Sasso. }\end{array}$ & $\begin{array}{l}\text { Ancona lignea della } \\
\text { Pietà, XVII - XVIII } \\
\text { sec. } \\
\text { Presente a sinistra } \\
\text { della cappella dello } \\
\text { Spirito Santo. }\end{array}$ & $\begin{array}{l}\text { Preventivo di } \\
\text { restauro } \\
\text { [...]1. Spese per } \\
\text { sorveglianza alle } \\
\text { operazione di } \\
\text { imballaggio, } \\
\text { restituzione e mano } \\
\text { d'opera (50 lire?), } 2 . \\
\text { opere di restauro } \\
\text { sagomatura degli } \\
\text { intagli e parti } \\
\text { mancanti } \\
\text { dell'assieme } \\
\text { architettonico (350 } \\
\text { lire?), 3. ritocchi } \\
\text { necessari di pittura } \\
\text { (80 lire?), } 4 . \\
\text { restauro di doratura } \\
\text { (200 lire), } 5 . \text { cornice } \\
\text { alla lunetta da } \\
\text { insieme colla } \\
\text { Ancona doratura } \\
\text { compresa (60 } \\
\text { lire)[...] }\end{array}$ & $\begin{array}{l}\text { (Annoni Francesco, } \\
\text { Preventivo di } \\
\text { risarcimento } \\
\text { indirizzato ad } \\
\text { Edoardo Berta/ } \\
\text { CCMS. Milano } 14 \\
\text { luglio 1913. ACS, } \\
\text { Bellinzona) } \\
\text { (AUBCBZ, s.183 } \\
\text { Orselina). }\end{array}$ \\
\hline $\begin{array}{l}14 \text { lug. } \\
1913\end{array}$ & $\begin{array}{l}\text { Locarno, } \\
\text { Chiesa della } \\
\text { Madonna del Sasso }\end{array}$ & $\begin{array}{l}\text { Bernardino de' } \\
\text { Conti, } 1500 \mathrm{ca} \text {. } \\
\text { Annunciazione. } \\
\text { Dipinto su tavola. } \\
\text { Pala d'altare. } \\
\text { Seconda cappella } \\
\text { della navata } \\
\text { laterale sinistra. }\end{array}$ & $\begin{array}{l}\text { Pulitura della } \\
\text { lunetta (20 lire). }\end{array}$ & $\begin{array}{l}\text { (Annoni Francesco, } \\
\text { Preventivo di } \\
\text { risarcimento } \\
\text { indirizzato ad } \\
\text { Edoardo Berta/ } \\
\text { CCMS. Milano } 14 \\
\text { luglio 1913. ACS, } \\
\text { Bellinzona) } \\
\text { (AUBCBZ, s.183 } \\
\text { Orselina). }\end{array}$ \\
\hline $\begin{array}{l}14 \text { lug. } \\
1913\end{array}$ & $\begin{array}{l}\text { Locarno, } \\
\text { Chiesa della } \\
\text { Madonna del Sasso }\end{array}$ & $\begin{array}{l}\text { Suardi Batolomeo } \\
\text { (detto Bramantino), } \\
1520 \text { ca. Fuga in } \\
\text { Egitto. } \\
\text { Dipinto su tavola. } \\
\text { Pala d'altare nella } \\
\text { navata laterale } \\
\text { destra. }\end{array}$ & $\begin{array}{l}\text { Risaldatura del } \\
\text { colore (30 lire). }\end{array}$ & $\begin{array}{l}\text { (Annoni Francesco, } \\
\text { Preventivo di } \\
\text { risarcimento } \\
\text { indirizzato ad } \\
\text { Edoardo Berta/ } \\
\text { CCMS. Milano } 14 \\
\text { luglio 1913. ACS, } \\
\text { Bellinzona) } \\
\text { (AUBCBZ, s.183 } \\
\text { Orselina). }\end{array}$ \\
\hline
\end{tabular}




\begin{tabular}{|c|c|c|c|c|}
\hline 1921 & $\begin{array}{l}\text { Locarno, } \\
\text { Chiesa della } \\
\text { Madonna del Sasso }\end{array}$ & $\begin{array}{l}\text { Altare di S. } \\
\text { Giuseppe }\end{array}$ & $\begin{array}{l}\text { Stile rinascimentale } \\
\text { con dorature un po' } \\
\text { affumicate, } \\
\text { decorazioni delle } \\
\text { lesene in graffito su } \\
\text { fondo oro, ecc. }\end{array}$ & $\begin{array}{l}\text { (AUBCBZ, s.183 } \\
\text { Orselina). }\end{array}$ \\
\hline 1923 & $\begin{array}{l}\text { Locarno, } \\
\text { Chiesa della } \\
\text { Madonna del Sasso }\end{array}$ & $\begin{array}{l}\text { Ancona lignea della } \\
\text { Pietà, XVII - XVIII } \\
\text { sec. } \\
\text { Presente a sinistra } \\
\text { della cappella dello } \\
\text { Spirito Santo. }\end{array}$ & $\begin{array}{l}\text { Restauro mediante } \\
\text { la sagomatura degli } \\
\text { intagli e parti } \\
\text { mancanti } \\
\text { dell'assieme } \\
\text { architettonico, } \\
\text { restauro delle } \\
\text { dorature, e ritocchi } \\
\text { pittorici. }\end{array}$ & $\begin{array}{l}\text { (Chiesa, 1946, p. 16) } \\
\text { (DPE, 1959, p. 122, } \\
\text { 134). } \\
\text { (AUBCBZ, s.183 } \\
\text { Orselina). }\end{array}$ \\
\hline
\end{tabular}




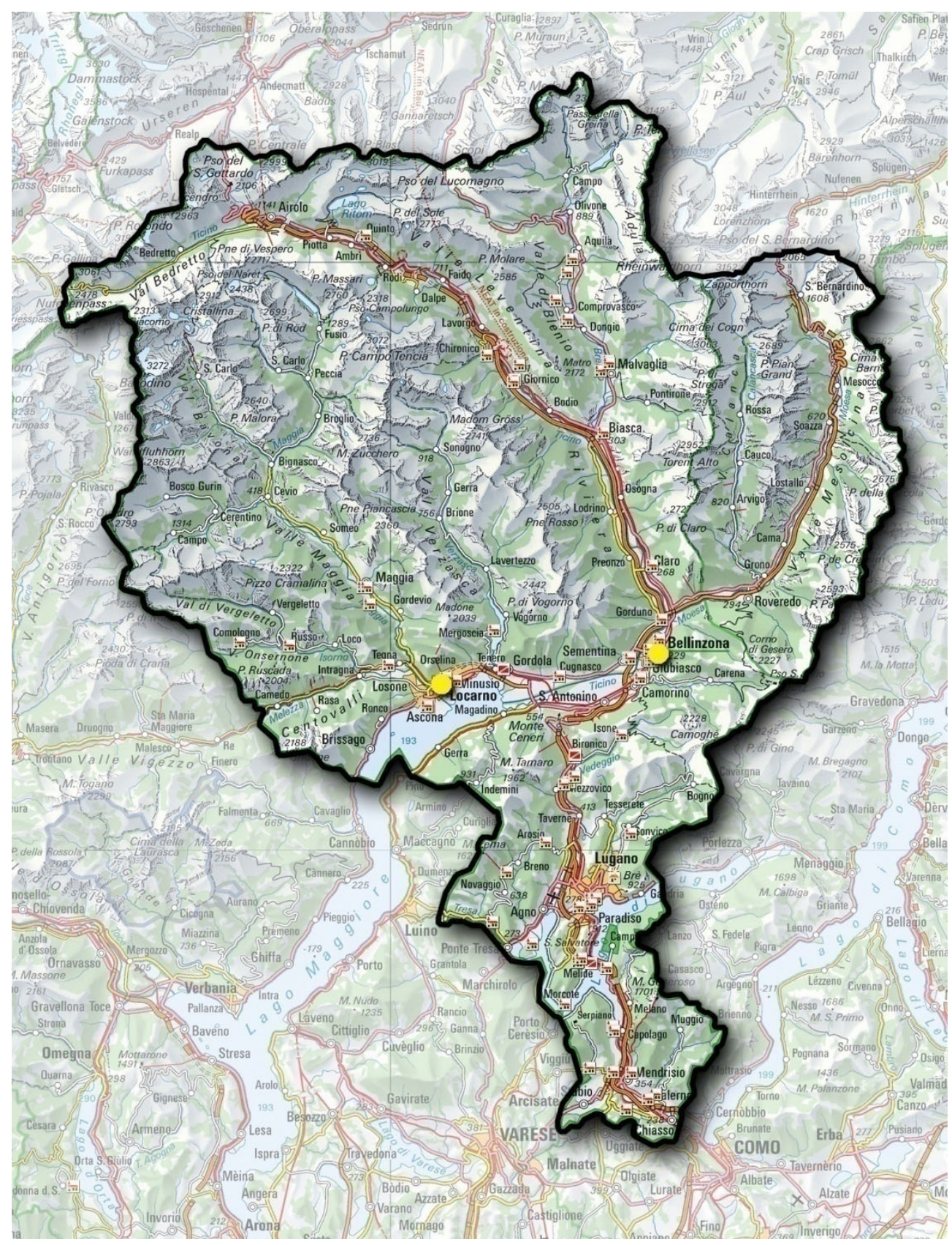

Figura 196 Cartina degli interventi eseguiti da Francesco Annoni in Canton Ticino. 


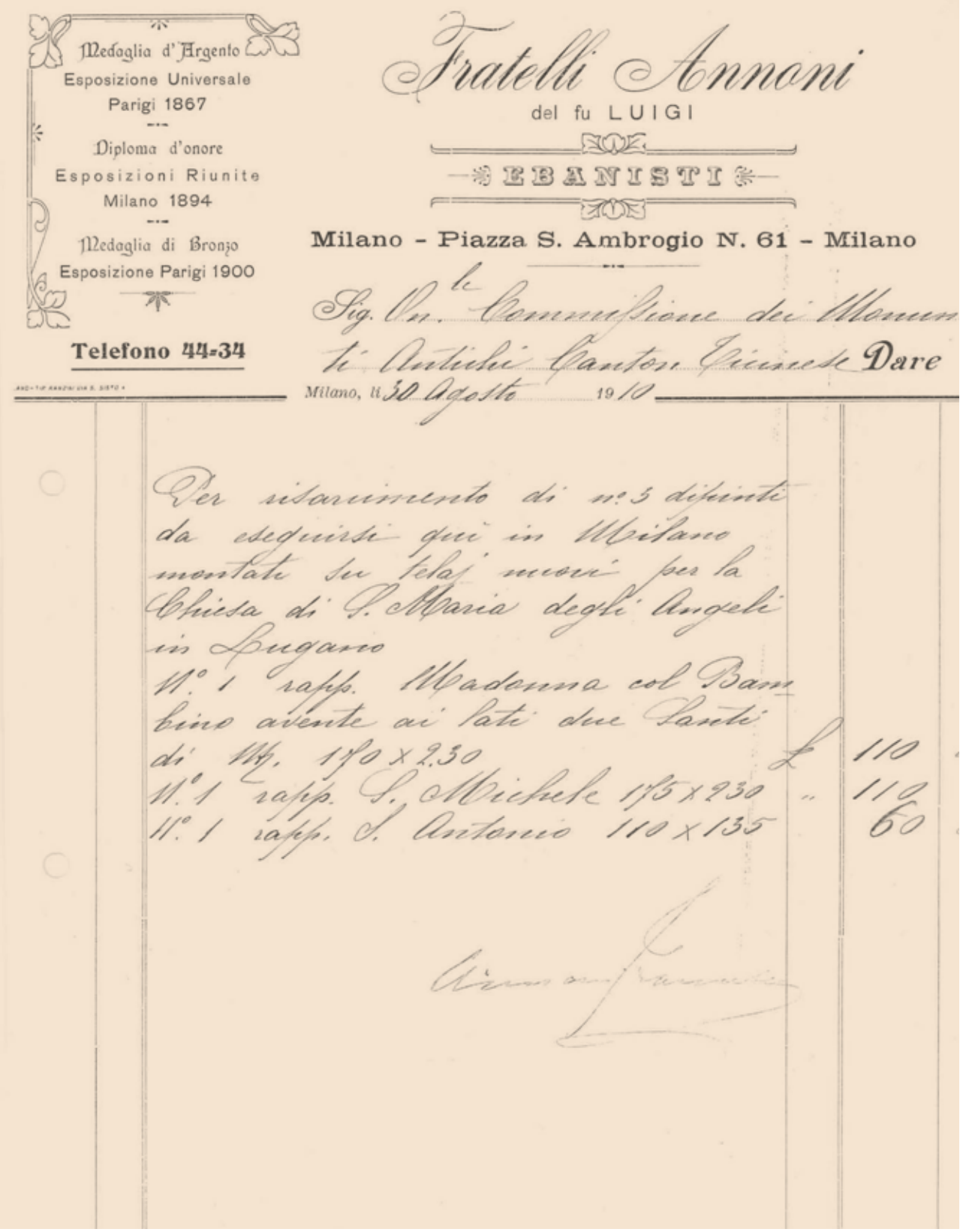

Figura 197 F. Annoni, Preventivo di restauro, indirizzato alla CMS, Milano 30 agosto 1910, AUBCBZ s. 143 Lugano, S. Maria degli Angioli 



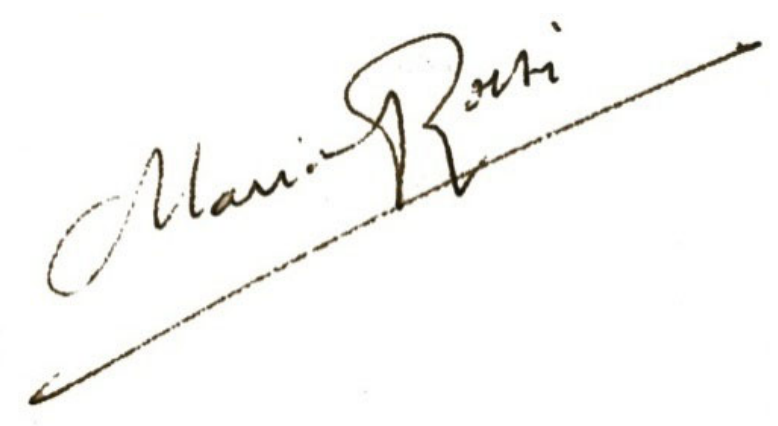




\section{MARIO ROSSI}

II restauratore Mario Rossi è stato una delle figure "estere" più attive nel Canton Ticino. Egli fu chiamato dalle istituzioni cantonali (Commissione cantonale dei monumenti storici e Dipartimento della Pubblica Educazione del Canton Ticino) per la sorveglianza, direzione ed esecuzione di lavori che riguardano il restauro non solo dei dipinti murali, ma anche di pitture su tele e tavole. Purtroppo non abbiamo potuto approfondire la sua figura e causa dell'assenza di fonti documentarie, mancando inoltre di notizie inerenti $i$ dati biografici.

Sappiamo dai documenti di corrispondenza conservati all'Archivio dell'Ufficio dei beni Culturali di Bellinzona che il pittore lavorava tanto a Varese come a Milano, in due atelier diversi della sue proprietà dove oltre che al restauro di opere d'arte dirige l'apprendistato di futuri restauratori, come ad esempio il ticinese Carlo Mazzi che esegue i corsi di Mario Rossi dal 1952 al 1954, e Bruno Abbiati, di Ponte Tresa, che studiò e lavorò più tardi assieme al restauratore milanese nella Pinacoteca di Brera con sede al Castello Sforzesco di Milano.

Sono tutte un complesso di notizie disperse che hanno consentito una minima ricostruzione dell'attività del restauratore. Collaboratore sicuramente preso la suddetta Pinacoteca di Brera, i suoi insegnamenti influirono le prassi di lavoro dei pittori-restauratori che nella seconda metà del Novecento eseguono i principali interventi di restauro nel Canton Ticino. Lo dimostrano ad esempio i restauri eseguiti dai suoi allievi Carlo Mazzi, Bruno Abbiati ed il coetaneo Mario Moglia.

Mario Rossi sarà uno dei professionisti ad introdurre nel Canton Ticino i metodi di indagini scientifiche, come appoggio ai processi di progettazione e di restauro, nonché i concetti instaurati negli anni Quaranta in Italia di "restauro" moderno.

Fra gli interventi più importanti eseguiti da Mario Rossi sui dipinti murali in Ticino, possiamo citare la pulitura degli affreschi della chiesa parrocchiale di Santa Maria in Selva (1950, 1956), il restauro pittorico della pitture e lo strappo di due scene Seicentesche, raffiguranti la Vita di San Giovanni al Battistero di Riva San Vitale (1953-1955), lo strappo degli affreschi della navata della chiesa parrocchiale di San Siro a Mairengo (1957-1958), e diverse perizie alla chiesa di San Carlo di Negrentino $(1951,1960)$ ed alla chiesa della Madonna del Sasso a Morcote (1969).

Riguardo agli interventi sulle opere mobili, sarà fondamentale la collaborazione di Mario Rossi nel 1948 per il restauro della tela Deposizione di Antonio Ciseri alla chiesa della Madonna del Sasso ad Orselina. II quadro assai deteriorato a causa dello stacco della pellicola pittorica fu oggetto di un tentativo di salvataggio pochi anni prima da parte del pittore Tita Pozzi ${ }^{507}$. Il fallimento dell'intervento di

\footnotetext{
507 Tita Pozzi, dopo la perizia di Mario Rossi deve soffrire le critiche del parroco don Robertini (Giornale del Popolo 14 dicembre 1948) che dichiara che il cattivo stato di conservazione del dipinti si doveva all'intervento eseguito anni prima dal pittore di Massagno. Pozzi, offeso scrisse al Consigliere di Stato Brenno Galli, il 16 dicembre 1948: [...] 1. Non è vero che io abbia detto di avere usato "soluzioni e vernici speciali", ho usato ciò che ogni restauratore deve e su
} 
Pozzi fece si che la Commissione cantonale dei monumenti contattasse la Sovrintendenza ai monumenti della Lombardia in cerca di un restauratore in grado di eseguire il consolidamento dell'opera. Ferdinanda Wittgens si rivolge da Milano alle istituzioni cantonali, proponendo uno dei restauratori al Palazzo di Brera, Mario Rossi.

L'intervento del restauratore milanese, consistente nello strappo del colore del dipinto su tela, e trapasso ad un nuovo supporto, significò uno dei primi eventi di questo tipo catalogati in Ticino, nonché un motivo di conflitto in particolare con il restauratore Emilio Ferrazzini, che vedeva la pericolosità dell'operazione. II pittore luganese aveva dimostrato la poca fiducia nel metodo proposto da Mario Rossi, come mostra la sua lettera indirizzata al Consigliere di Stato Brenno Galli il 18 dicembre 1948: il timore di Ferrazzini è creato da una recente commissione di 12 esperti, scelti fra le massime autorità europee ed americane in materia di restauro, creata per giudicare il metodo del

di poter usare. Anch'io avevo notato che il rimedio più sicuro sarebbe stata la trasponitura su un'altra tela, ma non osai farne la proposta in tale senso ben sapendo della pericolosità di tale operazione e da costo. Tuttavia l'affermazione del sig. Rossi, so comunque per esperienza fatta (parecchi trasporti da me eseguiti di pitture su tela dell'800) che $i$ dipinti $d$ questo periodo celano al restauratore, in cinquanta casi su cento, sgradevoli e amare sorpresa. II DPE ha agito nel modo ora noto.2. Non è vero che io abbia tentato "una specie di foderatura" perché non avevo nessun incarico per fare ciò e non avevo nemmeno alla mano il materiale idoneo.3. Non è vero che io abbia inumidito con soluzioni acquose poiché tutte le soluzioni usate erano a base alcooliche.[...](AUBCBZ s. 183 Orselina). trasporto dei dipinti. Secondo Ferrazzini queste autorità assicuravano che la "trasponitura" era una operazione molto complicata e poteva causare inevitabilmente gravi perdite.

Il lavoro di Rossi fu eseguito ad ogni modo senza inconvenienti, secondo le indicazioni delle istituzione cantonali responsabili di collaudare il lavoro (DPE, e CCMS). Rossi risponderà indirettamente alle critiche del restauratore Ferrazzini, nel rapporto presentato alla Commissione cantonale dei monumenti storici il 10 agosto 1949 con le seguenti parole:

[...]Perchè fu eseguita questa trasponitura che da alcuni deboli restauratori o incompetenti fu ripetutamente sconsigliata e considerata inutile, dannosa e rischiosissima operazione? Perchè il colore originale e la sua preparazione non avevano buona coesione alla tela sottostante; qua e la si erano già formate rigonfiature di colore che cominciavano a screpolarsi, aprirsi e conseguentemente staccarsi. Aggiungasi che la tela originale era di tessuto pesante e fitto $e$ qualsiasi sostanza adhesiva anche molto liquida non sarebbe paseata attraverso lo spessore della tela e non avrebbe mai raggiunto la preparazione fissandone il colore. Un precedente restauratore aveva tentato, per fortuna, su di una superficie ristretta, questo rimedio usando una colla molto liquida . La colla non è passata attraverso lo spessore della tela ottenendo solo il ritiro e la contrazione della tela per effetto dell'umido della colla, minacciando cosi di perdere tutto il colore che per sua natura non si restringe $e$ non si contrae. $[. . .]^{508}$.

(Rossi Mario. Rapporto indirizzato alla CCMS. Varese, 10 agosto 1949. AUBCBZ s. 183 Orselina). 


\section{Interventi di restauro}

\begin{tabular}{|c|c|c|c|c|c|}
\hline ANNO & LUOGO & OPERA & INTERVENTO & COLLABORATORI & FONTI \\
\hline $\begin{array}{l}14 \text { nov. } \\
1948\end{array}$ & $\begin{array}{l}\text { Locarno, Chiesa } \\
\text { delle Madonna del } \\
\text { Sasso. }\end{array}$ & $\begin{array}{l}\text { Tele di San } \\
\text { Francesco/ } \\
\text { Deposizione di A. } \\
\text { Ciseri } \\
(1.88 \times 2.73 \mathrm{~m})\end{array}$ & $\begin{array}{l}\text { Sopralluogo. } \\
\text { Proposta di distacco } \\
\text { del colore del } \\
\text { quadro e riattacco } \\
\text { su nuovo supporto. } \\
\text { [...]Non si può } \\
\text { pensare alla } \\
\text { saldatura locale del } \\
\text { colore con una } \\
\text { foderatura, perche } \\
\text { la cura sarebbe } \\
\text { peggiore del male } \\
\text { ed il dipinto } \\
\text { sarebbe } \\
\text { compromesso. Altri } \\
\text { applicativi non } \\
\text { esistono. Rimane } \\
\text { per salvarlo il } \\
\text { distacco del colore } \\
\text { dalla tela originale e } \\
\text { riapplicarlo } \\
\text { stabilmente ad altre } \\
\text { (tre) [...]. (DPE, } \\
\text { gennaio } 1959, \text { pp. } \\
122134 \text {.) }\end{array}$ & Piero Bianconi. & $\begin{array}{l}\text { DPE, gennaio 1959, } \\
\text { pp. } 122134 .\end{array}$ \\
\hline $\begin{array}{l}7 \text { mag. } \\
1947\end{array}$ & $\begin{array}{l}\text { Locarno, Chiesa } \\
\text { delle Madonna del } \\
\text { Sasso. }\end{array}$ & $\begin{array}{l}\text { La fuga in Egitto, } \\
\text { Bramantino. }\end{array}$ & $\begin{array}{l}\text { Perizia e proposta } \\
\text { di restauro. } \\
\text { Il restauratore } \\
\text { propone la } \\
\text { sostituzione dei } \\
\text { ferri sul retro della } \\
\text { tavola applicati nel } \\
\text { restauro } \\
\text { precedente (1922), } \\
\text { e la creazione in } \\
\text { cambio di una } \\
\text { grigliatura adatta } \\
\text { alle caratteristiche } \\
\text { dell'opera. Inoltre si } \\
\text { propone la } \\
\text { esecuzione di } \\
\text { radiografie, la } \\
\text { sostituzione della } \\
\text { farfalle sul retro } \\
\text { con altre nuove, la } \\
\text { sverniciatura del } \\
\text { quadro e l'asporto } \\
\text { dei vecchi ritocchi }\end{array}$ & & $\begin{array}{l}\text { AUBC n. } 183 \\
\text { Orselina. }\end{array}$ \\
\hline
\end{tabular}




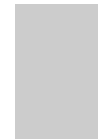

1949

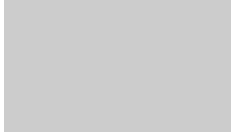

Locarno, Orselina, Madonna del Sasso.

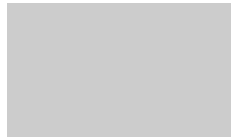

eseguiti ad olio. La

reintegrazione

sarebbe eseguita

con colori a vernice.

Varese 10 agosto

1949

Trasponitura:

CCMS

Dott. Fernanda

Wittgens.

stacco del colore Sovrintendente ai

dalla tela originale e monumento della

sostituzione con Lombardia. Palazzo

un'altra a sua volta di Brera. Milano. In

rinforzata da una lettera del

doppia foderatura: Bellinzona 10

velatura di

protezione con

applicazione di

adesivo olio

resinoso, lasciato

durante un mese.

Rinforzamento

dell'intellaggio con

una seconda garza

imbevuta di colla

ammorbidita da un

appropriato

plastificante. Un

paio di giorni dopo

fu riapplicata una

seconda colla

facendovi aderire

dei grandi fogli di

carta paraffinata.

Stacco del telaio.

Inumidimento della

tela originale sul

tavolo con acqua

tiepida. La tela si

staccò con facilità

senza la

preparazione

sottostante.

Ritrovamento nella

tela di un secondo

"Trasporto di

Cristo" alla rovescia

con disposizione

diversa delle figura

rappresentate.

Documentazione

maggio 1950 il DPE

chiede sorveglianza

ai lavori eseguiti sul dipinto.

Continuando così il rapporto di collaborazione tra la Lombardia e la

Svizzera già iniziato nell'anno $1932 \mathrm{col}$

restauro, ad opera dei restauratori di Brera, degli affreschi luineschi di Lugano. Chiede di poter cominciare il lavoro a giugno quando sarà compiuto un'altro intervento sui dipinti cinquecenteschi della Pinacoteca.

Vennero nel mio studio di Milano il sig. Aldo Crivelli, direttore dei Musei del Ticino, il sig.

Perucchini, secretario

comunale della città di Locarno e il M.to. R.do Don Agostino RObertini della Commissione fotografica. Pulitura Artistica Diocesana. del dipinto.

Sostituzione della
AUBC n. 183

Orselina. 


\begin{tabular}{|c|c|c|c|c|c|}
\hline & & & $\begin{array}{l}\text { tela originale per } \\
\text { una nuova tela } \\
\text { leggera applicata } \\
\text { alla preparazione } \\
\text { con colla di pasta. } \\
\text { Sostituzione del } \\
\text { telaio per uno } \\
\text { nuovo a crociera. } \\
\text { Ricollocamento. }\end{array}$ & & \\
\hline 1950 & $\begin{array}{l}\text { Locarno, } \\
\text { chiesa di S. Maria } \\
\text { in Selva. }\end{array}$ & Dipinti murali. & Pulitura. & $\begin{array}{l}\text { Pittore- } \\
\text { restauratore: } \\
\text { Carlo Mazzi. }\end{array}$ & $\begin{array}{l}\text { DPE, gennaio } 1959 \text {, } \\
\text { pp.122 } 134 .\end{array}$ \\
\hline 1950 & Ascona. & $\begin{array}{l}\text { Serodine, } \\
\text { Pala d'altare. }\end{array}$ & Restauro. & & $\begin{array}{l}\text { DPE, gennaio } 1959 \text {, } \\
\text { pp. } 118.011 .\end{array}$ \\
\hline 1950 & Ascona. & $\begin{array}{l}\text { Chiamata dei figli di } \\
\text { Zebedeo e Invito a } \\
\text { Emmaus, Giovanni } \\
\text { Serodine. }\end{array}$ & $\begin{array}{l}\text { Rifacimento } \\
\text { restauro del } 1946 \text { di } \\
\text { Boissonas. }\end{array}$ & & $\begin{array}{l}\text { DPE, gennaio 1959, } \\
\text { pp. } 118.011 .\end{array}$ \\
\hline 1951 & $\begin{array}{l}\text { Negrentino, } \\
\text { Prugiasco, } \\
\text { chiesa di } \\
\text { S. Carlo. }\end{array}$ & $\begin{array}{l}\text { Affresco della } \\
\text { parete occidentale. }\end{array}$ & $\begin{array}{l}\text { Perizia } \\
\text { Lettera Lugano } 21 \\
\text { settembre } 1951 \\
\text { CMS alla Direzione } \\
\text { della rivista } \\
\text { "Svizzera Italiana" } \\
\text { negando l'articolo } \\
\text { di Aldo Cirvelli del } \\
\text { maggio dello stesso } \\
\text { anno in cui } \\
\text { ironicamente } \\
\text { criticava il degrado } \\
\text { dei dipinti romanici } \\
\text { temendo il suo } \\
\text { distacco a causa di } \\
\text { un poco idoneo } \\
\text { restauro } \\
\text { precedente. Gli } \\
\text { affreschi, rilevati } \\
\text { dal pitt. Mario Rossi } \\
\text { (II DPE aveva fatto } \\
\text { domanda a giugno } \\
\text { di realizzare gli } \\
\text { strappi se necessari } \\
\text { a Mario Rossi } \\
\text { coadiuvato dal pitt. } \\
\text { Mario Moglia di } \\
\text { Lugano. Fu la } \\
\text { sovrintendenza alle } \\
\text { Gallerie di Milano } \\
\text { (Via Brera, 28) il } 21 \\
\text { maggio 1951, } \\
\text { Fernanda Willgens }\end{array}$ & & $\begin{array}{l}\text { DPE, gennaio 1959, } \\
\text { pp.125 } 204 . \\
\text { AUBC, Filza 204, } \\
\text { Prugiasco. } \\
\text { Fotografia Luglio } \\
1957 .\end{array}$ \\
\hline
\end{tabular}




\begin{tabular}{|c|c|c|c|c|c|}
\hline & & & $\begin{array}{l}\text { a segnalare il nome } \\
\text { del pittore milanese } \\
\text { il quale aveva } \\
\text { lavorato } \\
\text { anteriormente con } \\
\text { esito soddisfacente } \\
\text { in Svizzera.), non } \\
\text { presentavano } \\
\text { tracce di umido o } \\
\text { salnitro, } \\
\text { affermando il buon } \\
\text { consolidamento a } \\
\text { iniezioni di caseato } \\
\text { di calce. }\end{array}$ & & \\
\hline $\begin{array}{l}1953- \\
1955\end{array}$ & $\begin{array}{l}\text { Riva San Vitale, } \\
\text { Battistero. }\end{array}$ & Pitture. & $\begin{array}{l}\text { Restauro pittorico. } \\
\text { Tre anni dopo, } \\
\text { 1958, si osservano } \\
\text { delle alterazioni dei } \\
\text { ritocchi pittorici } \\
\text { eseguiti dal pittore. }\end{array}$ & $\begin{array}{l}\text { Dirige i lavori } \\
\text { I'architetto } \\
\text { Ferdinando Reggiori } \\
\text { secondo i propri } \\
\text { studi e progetto di } \\
\text { restauro del 1933- } \\
1935 . \\
\text { La sorveglianza ai } \\
\text { restauri è incaricata } \\
\text { all'architetto } \\
\text { Alberto Camenzind, } \\
\text { per conto della } \\
\text { Commissione } \\
\text { Federale, ed } \\
\text { all'ingegnere Guido } \\
\text { Borella di Lugano. }\end{array}$ & $\begin{array}{l}\text { DPE, gennaio } 1959, \\
\text { pp.125 } 209 \\
\text { In lettera Varese, } 25 \\
\text { luglio 1951. All'On. } \\
\text { Avv. Brenno Galli } \\
\text { Cons. di Stato - } \\
\text { Direttore. }\end{array}$ \\
\hline $\begin{array}{l}1953- \\
1955\end{array}$ & $\begin{array}{l}\text { Riva San Vitale, } \\
\text { Battistero. }\end{array}$ & $\begin{array}{l}\text { Vita di S. Giovanni, } \\
\text { XVII sec. }\end{array}$ & $\begin{array}{l}\text { Strappo in due } \\
\text { scene }(123 \times 130 / \\
135 \times 127)\end{array}$ & $\begin{array}{l}\text { Due collaboratori } \\
\text { (Abbiati e Mazzi?). }\end{array}$ & Lumia, 2004 \\
\hline 1955 & $\begin{array}{l}\text { Ravecchia (Be), } \\
\text { chiesa di San } \\
\text { Biagio.. }\end{array}$ & Affreschi esterni. & Esamine & & $\begin{array}{l}\text { DPE, gennaio } 1959 \text {, } \\
\text { pp. } 118.021 .\end{array}$ \\
\hline 1956 & $\begin{array}{l}\text { Santa Maria in } \\
\text { Selva, Chiesa } \\
\text { parrocchiale. }\end{array}$ & Pitture. & $\begin{array}{l}\text { Compimento dei } \\
\text { lavori di restauro. }\end{array}$ & & $\begin{array}{l}\text { DPE, gennaio 1959, } \\
\text { pp.122 } 134 .\end{array}$ \\
\hline $\begin{array}{l}1957- \\
1958\end{array}$ & $\begin{array}{l}\text { Mairengo, chiesa } \\
\text { parrocchiale di S. } \\
\text { Siro. }\end{array}$ & $\begin{array}{l}\text { Sant'Antonio e } \\
\text { Cenacolo. XV sec. } \\
\text { ca. }\end{array}$ & $\begin{array}{l}\text { Strappo degli } \\
\text { affreschi della } \\
\text { navata. }\end{array}$ & & (DPE, 1959, p. 116). \\
\hline 1958 & $\begin{array}{l}\text { Medeglia, } \\
\text { chiesa parrocchiale. }\end{array}$ & Pala di altare. & Restauro. & & $\begin{array}{l}\text { DPE, gennaio 1959, } \\
\text { pp.123 } 154 .\end{array}$ \\
\hline 1958 & $\begin{array}{l}\text { Locarno, } \\
\text { Madonna del Sasso. }\end{array}$ & $\begin{array}{l}\text { Fuga in Egitto, } \\
\text { Bartolomeo Suardi, } \\
\text { detto il Bramantino. }\end{array}$ & $\begin{array}{l}\text { Analisi dell'opera } \\
\text { constantandosi che } \\
\text { nella tavola, per } \\
\text { effetto della vernice } \\
\text { o per qualche altra } \\
\text { causa - il colore si }\end{array}$ & & $\begin{array}{l}\text { AUBC n.183 } \\
\text { Orselina. }\end{array}$ \\
\hline
\end{tabular}




\begin{tabular}{|c|c|c|c|c|c|}
\hline & & & $\begin{array}{l}\text { solleva e minaccia } \\
\text { di cadere a squame } \\
\text { nella parte alta del } \\
\text { cielo, a sinistra, sul } \\
\text { manto della } \\
\text { Madonna. In altre } \\
\text { parti, parzialamente } \\
\text { nei bruni - il colore } \\
\text { è raggrumato; ma } \\
\text { può darsi che così } \\
\text { sia stato già prima } \\
\text { dei restauri. } \\
\text { Rimanda } \\
\text { interpellare a } \\
\text { Mauro Pelliccioli, } \\
\text { restauratore della } \\
\text { tavola. }\end{array}$ & & \\
\hline $\begin{array}{l}\text { Lug. } \\
1960\end{array}$ & $\begin{array}{l}\text { Negrentino - } \\
\text { Prugiasco } \\
\text { San Carlo. }\end{array}$ & $\begin{array}{l}\text { Affresco primitivo } \\
\text { abside maggiore. }\end{array}$ & $\begin{array}{l}\text { Fu autorizzato a } \\
\text { ricerche per } \\
\text { rintracciare un } \\
\text { eventuale seguito a } \\
\text { questa figura } \\
\text { d'animale, ma, } \\
\text { fosse anche esistito, } \\
\text { adesso non ne } \\
\text { rimane traccia. }\end{array}$ & & $\begin{array}{l}\text { Marcionetti Isidoro, } \\
\text { San Carlo di } \\
\text { Negrentino, Lugano } \\
\text { 1977. p.22. }\end{array}$ \\
\hline $\begin{array}{l}8 \text { ott. } \\
1969\end{array}$ & $\begin{array}{l}\text { Morcote, } \\
\text { Chiesa di S. Maria } \\
\text { del Sasso. }\end{array}$ & & $\begin{array}{l}\text { Sopralluogo. } \\
\text { Raccomando } \\
\text { l'isolamento del } \\
\text { tetto, volta pareti e } \\
\text { pavimentazione } \\
\text { prima del resturo } \\
\text { degli affreschi. }\end{array}$ & $\begin{array}{l}\text { Prof. G. Martinola } \\
\text { Prof. P.A. Donati. }\end{array}$ & $\begin{array}{l}\text { (AUBCBZ, s. } 172 \\
\text { Morcote) } \\
\text { (Rossi Mario, } \\
\text { Lettera indirizzata } \\
\text { alla CCMS, } \\
\text { Bellinzona. Varese } 9 \\
\text { novembre } 1969 . \\
\text { AUBCBZ, s. } 172 \\
\text { Morcote). }\end{array}$ \\
\hline $\begin{array}{l}1969- \\
1970\end{array}$ & $\begin{array}{l}\text { Negrentino } \\
\text { (Prugiasco), chiesa } \\
\text { di San Carlo. }\end{array}$ & $\begin{array}{l}\text { Affresco (193 x } 160 \\
\mathrm{~cm}) \text { controfacciata, } \\
\text { parte inferiore. }\end{array}$ & Strappo. & Luigi Gianola. & \\
\hline
\end{tabular}




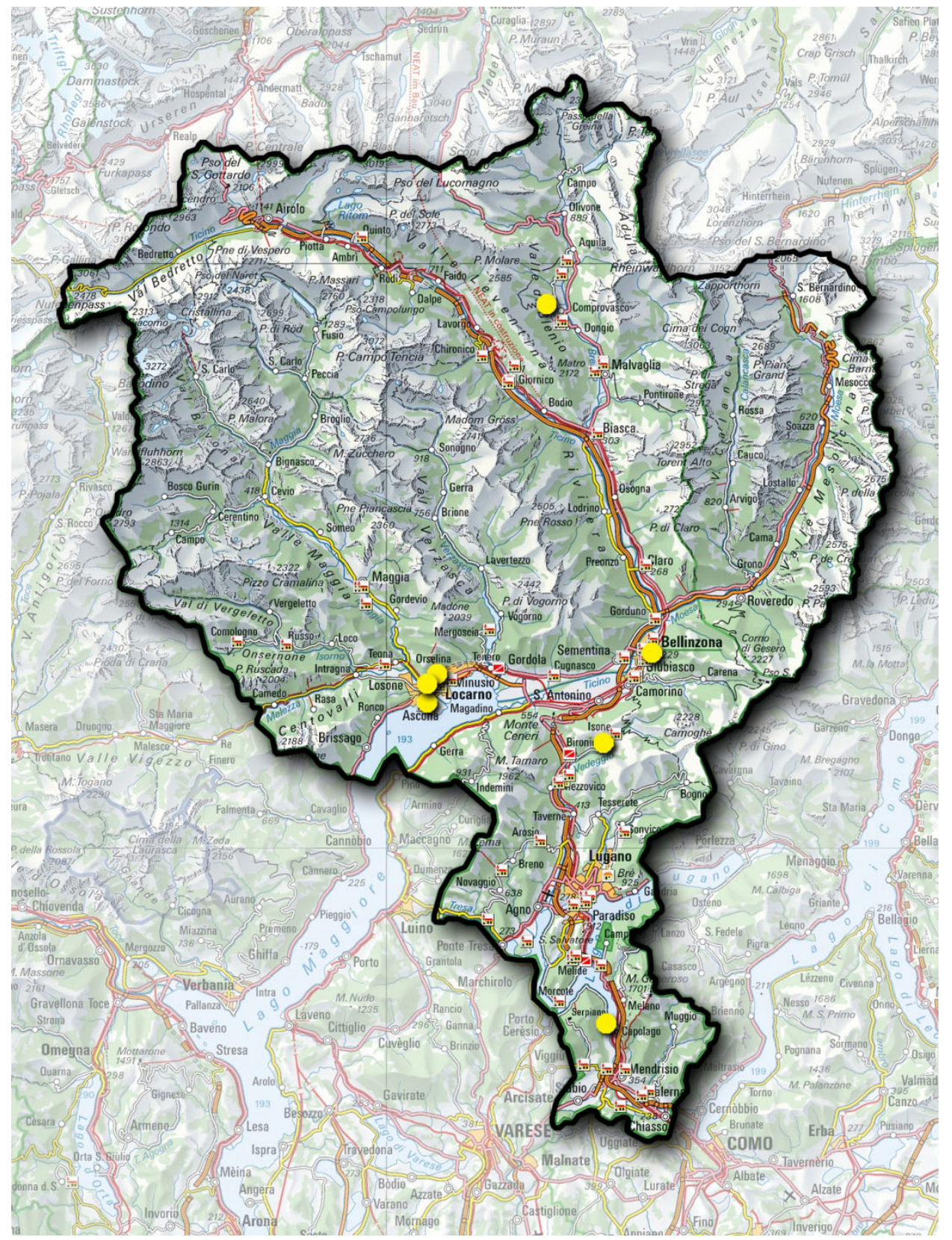

Figura 198 Cartina degli interventi eseguiti da Mario Rossi in Canton Ticino. 


BOISSONAS HENRI-PAUL 


\section{BOISSONAS HENRI- PAUL}

Henri-Paul Boissonas nasce a Ginevra il 24 giugno del 1894. Figlio del celebre fotografo Fred, Henri segue la strada artistica nel campo della pittura. Frequentò la scuola di belle arti a Ginevra, che dal 1909 al 1919 fu diretta dall'artista Daniel Baud-Body, compagno del padre. Qui conosce, probabilmente, la figlia di quest'ultimo, Valentine, che diventa non solo sua moglie ma anche sua collega ed assistente di lavoro. Henri viaggiò successivamente in Grecia nel 1919, in Asia nel 1921 ed in Romania dal 1930 al 1931. Dal 1924 al 1927 deve occuparsi dello studio fotografico famigliare, del quale si occuperà più tardi il fratello Paul, operando contemporaneamente in qualità di restauratore di opere d'arte. Questa professione fu sviluppata dall'artista con successo e con una grande professionalità, consapevole della necessità di documentare i propri lavori per farli a conoscere in futuro e di utilizzare la scienza come appoggio alle indagini sull'opera ma allo stesso tempo come base di conoscenza dei materiali suscettibili ad essere impiegati nel processo di restauro. Henri lavora come restauratore ai musei di Ginevra, Berna e per ultimo a Zurigo, dove si trasferisce nel 1934 e la professione di restauratore sarà condivisa con quella di pittore, acquarellista ed illustratore. In questa città l'apprezzato restauratore si spegne il 6 agosto del 1966. La sua preparazione $\mathrm{fu}$ riconosciuta anche in Ticino, di fatto sarebbe sempre descritto come "il professionista". Secondo le ricerche realizzate finora, la prima data di notizie del restauratore nel territorio della Svizzera italiana è del 1945. Questo anno fu incaricato di eseguire il consolidamento dell'olio su tela raffigurante la Chiamata dei figli di Zebedeo del XVII sec. opera di Giovanni Battista Serodine, collocato sulla controfacciata della chiesa parrocchiale dei SS. Pietro e Paolo ad Ascona. Lo stesso anno Boissonas fu interpellato per dei restauri nella chiesa di $\mathrm{S}$. Biagio in Ravecchia, che interessava il consolidamento degli affreschi esterni, assai danneggiati dall'umidità. La sua funzione dovrebbe essere stata di sorveglianza dei lavori condotti dal pittore E. Ferrazzini. Quest'ultimo aveva l'intenzione - e di fatto così intervenne- di impiegare cera minerale per il consolidamento dei suddetti affreschi. Boissonas diede la sua assoluta fiducia al pittore, pensando che Ferrazzini avrebbe avuto maggiore esperienza riguardo al trattamento dei dipinti murali di sé stesso. Purtroppo anni più tardi le polemiche per I'utilizzo dei materiali cerosi nel restauri attaccarono anche l'intervento di Ferrazzini. Nel 1948 Boissonas venne chiamato nuovamente, questa volta dal Dott. M. Winterhalter per il restauro di quattro tele e dell'affresco della Madonna presenti alla chiesa di S. Maria del Sasso a Morcote. Non si è approfondito ulteriormente questo personaggio dato che non ha voluto essere scopo della presente ricerca trattare il restauro dei dipinti su tele e tavole, sui quali il Boissonas si dedicò più ampiamente. In ogni caso vogliamo indicare nella tabella che segue, i principali interventi di restauro catalogati per incentivare future ricerche riguardo. 


\section{Interventi di restauro}

\begin{tabular}{|c|c|c|c|c|c|}
\hline ANNO & LUOGO & OPERA & INTERVENTO & COLLABORATORI & FONTI \\
\hline $\begin{array}{l}1945- \\
1946\end{array}$ & $\begin{array}{l}\text { Ascona, } \\
\text { chiesa parrocchiale } \\
\text { dei SS. Pietro e } \\
\text { Paolo. }\end{array}$ & $\begin{array}{l}\text { Giovanni Battista } \\
\text { Serodine, secondo } \\
\text { decennio XVII sec. } \\
\text { Chiamata dei figli di } \\
\text { Zebedeo. Olio su } \\
\text { tela. Controfacciata. }\end{array}$ & Consolidamento. & & $\begin{array}{l}\text { (DPE, 1959, p. 118). } \\
\text { (AUBCBZ, s. 011, } \\
\text { Ascona. SS. Pietro e } \\
\text { Paolo). }\end{array}$ \\
\hline 1945 & $\begin{array}{l}\text { Ravecchia, chiesa di } \\
\text { S. Biagio. affresco } \\
\text { della lunetta sulla } \\
\text { porta maggiore e } \\
\text { San Cristoforo. }\end{array}$ & $\begin{array}{l}\text { S. Cristoforo. } \\
\text { Affresco. }\end{array}$ & $\begin{array}{l}\text { Sorveglianza del } \\
\text { lavoro di } \\
\text { consolidamento } \\
\text { degli affreschi } \\
\text { mediante la tecnica } \\
\text { dell'encausto con } \\
\text { l'applicazione a } \\
\text { caldo della cera } \\
\text { ozokerite a } 72{ }^{\circ} \mathrm{C} \text { di } \\
\text { fusione. }\end{array}$ & $\begin{array}{l}\text { Pittore- } \\
\text { restauratore: } \\
\text { Emilio Ferrazzini. }\end{array}$ & (Chiesa, 1946, p. 15) \\
\hline 1945 & $\begin{array}{l}\text { Ravecchia, chiesa di } \\
\text { S. Biagio. affresco } \\
\text { della lunetta sulla } \\
\text { porta maggiore e } \\
\text { San Cristoforo. }\end{array}$ & $\begin{array}{l}\text { Lunetta sulla porta } \\
\text { maggiore. } \\
\text { Affresco. }\end{array}$ & $\begin{array}{l}\text { Sorveglianza del } \\
\text { lavoro di } \\
\text { consolidamento } \\
\text { degli affreschi } \\
\text { mediante la tecnica } \\
\text { dell'encausto con } \\
\text { l'applicazione a } \\
\text { caldo della cera } \\
\text { ozokerite a } 72{ }^{\circ} \mathrm{C} \text { di } \\
\text { fusione. }\end{array}$ & $\begin{array}{l}\text { Pittore- } \\
\text { restauratore: } \\
\text { Emilio Ferrazzini. }\end{array}$ & (Chiesa, 1946, p. 15) \\
\hline 1948 & $\begin{array}{l}\text { Morcote, } \\
\text { Chiesa di S. Maria } \\
\text { del Sasso }\end{array}$ & $\begin{array}{l}\text { Tele: } \\
1 \text { delle due grande } \\
\text { tele ai lati del coro, } \\
\text { e } 2 \text { tele degli altari } \\
\text { laterali }\end{array}$ & $\begin{array}{l}\text { Lavori arbitrari } \\
\text { senza supervisione } \\
\text { della Commissione. }\end{array}$ & $\begin{array}{l}\text { Dott.M. } \\
\text { Winterhalter. }\end{array}$ & AUBC 172 Morcote \\
\hline 1948 & $\begin{array}{l}\text { Morcote, } \\
\text { Chiesa di S. Maria } \\
\text { del Sasso. }\end{array}$ & $\begin{array}{l}\text { Affresco delle } \\
\text { Madonna. }\end{array}$ & $\begin{array}{l}\text { Rimozione del vetro } \\
\text { e della cornice che } \\
\text { chiudevano } \\
\text { l'affresco. }\end{array}$ & $\begin{array}{l}\text { Dott.Martino } \\
\text { O.Winterhalter. }\end{array}$ & AUBC 172 Morcote \\
\hline
\end{tabular}




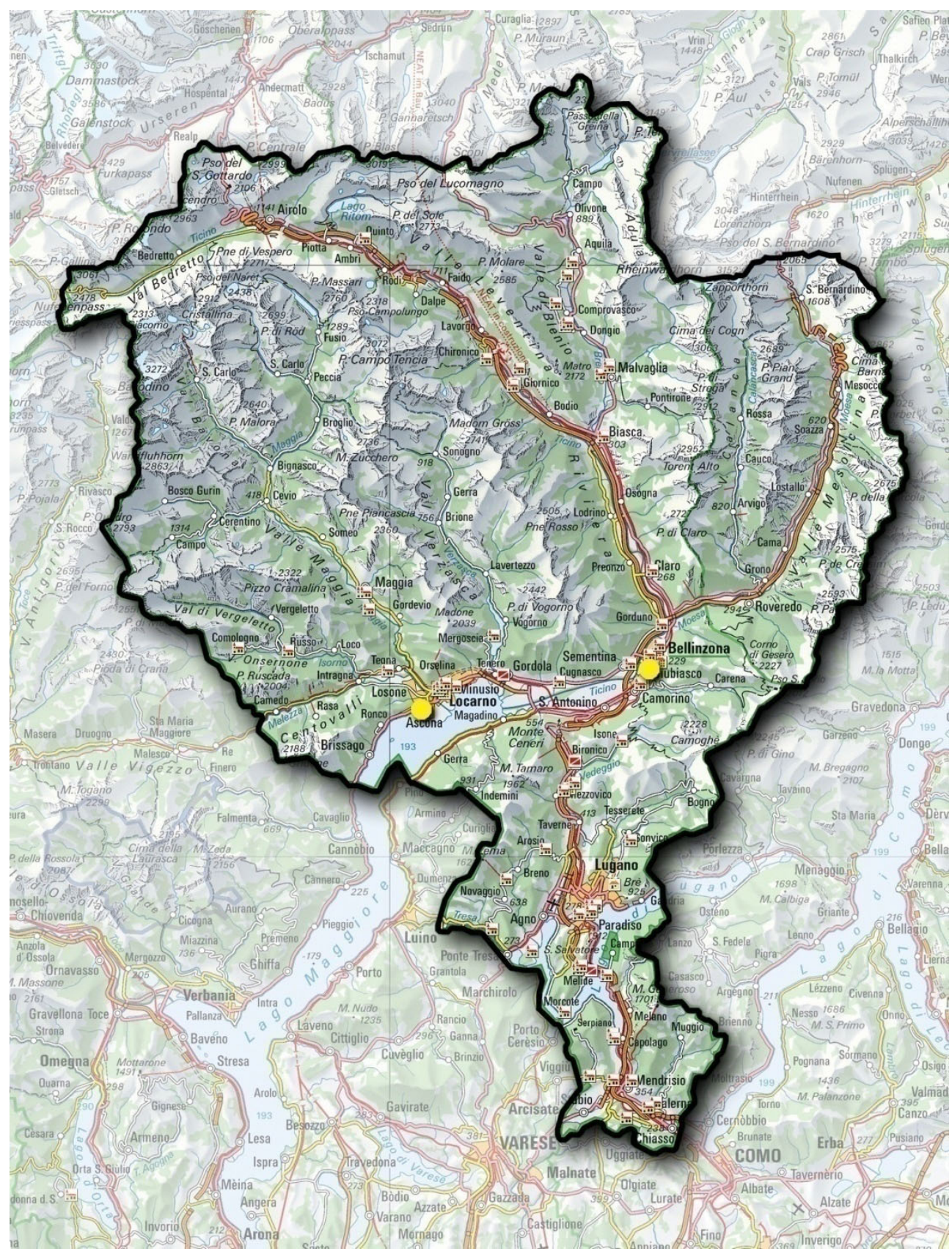

Figura 199 Cartina degli interventi eseguiti da Henri Boissonas in Canton Ticino. 

PARTE TERZA 


\title{
CATALOGAZIONE E SISTEMA DI GESTIONE DATI
}

\author{
Le fonti
}

[...]II libro e la biblioteca rappresentano un capitolo fondamentale della costituzione del sapere umano[...] di grande importanza per la storia della cultura[...] Lo stesso vale anche per gli $\operatorname{archivi[\ldots ..5}]^{509}$.

La base della presente tesi è stata la "fonte" di archivio, sia in formato cartaceo, audiovisivo, fotografico che informatico senza la quale sarebbe stato impossibile aggiungere dei dati allo studio bibliografico. La stessa "fonte" storica, recuperata in questo lavoro, vuole essere a suo tempo lo strumento di ricerca di futuri studi così come appoggiare chi dovrà tutelare od operare in futuro su uno dei beni culturali qui trattati, poiché il reperimento dei dati identificativi dell'opera e della sua storia è fondamentale nel momento preparatorio all'intervento sull'oggetto.

Gli archivi che custodiscono queste fonti, creati perché in un momento determinato la società è stata consapevole dell'importanza del documento in quanto testimonianza della storia, sono in Canton Ticino organizzati in diverse sedi a Bellinzona, a Locarno, a Lugano e a Mendrisio. La consultazione per la ricerca è stata effettuata principalmente all'Archivio di Stato e all'Archivio dell'Ufficio dei Beni Culturali a Bellinzona, all'Archivio della Curia Vescovile a Lugano, all'A Archivio della Società Storica Locarnese, e per ultimo all'Archivio Federale dei Monumenti Storici a Berna, capitale Svizzera.

Inoltre è stata una sorpresa molto gratificante, nonché di enorme valore, il ritrovamento di archivi personali di pittori-restauratori come quello di Tita Pozzi, custodito nella casa dell'artista dal figlio Giovanni Pozzi, l'archivio di Carlo Mazzi anche questo presente nella casa del pittore a Tegna dove inoltre c'è la Galleria Mazzi gestita dalla figlia Silvia MinaMazzi, o la documentazione fotografica degli interventi realizzati dal restauratore Bruno Abbiati, che il figlio Attilio conserva nel suo atelier di restauro e del quale il proprio Ufficio dei Beni Culturali del Canton Ticino non conosceva l'esistenza.

\section{Archivio Federale dei Monumenti Storici, Berna}

[...] Un cassetto dei grande armadi metallici grigi si apre silenziosamente. Lo sguardo si sofferma su una catasta di piani di formato e materiale diversi. Sfogliandoli ci si trova improvvisamente immersi nell'epoca dei pionieri della tutela dei monumenti storici in Svizzera. Accanto al timbro della "Gessellschaft für Erhaltung historischer Kunstdenkmäler" ed ai numeri d'inventario si possono leggere le firme autografe di Robert Dürrer (1867-1934), Josef Zemp (1869-1942), Albert Naef (18621936) o Eugen Probst (1873-1970)[... $]^{510}$

\footnotetext{
${ }^{509}$ Gian-Willi, V. L'Archivio federale dei monumenti storici (AFMS) offre rifugio a oltre due milioni di documenti. Giornale UFC. La memoria del passato (13), p. 4,5.

${ }^{510}$ (Amacher, 2004, p. 6).
} 
Doris Amacher è l'autrice del testo qui sopra citato riguardo le collezioni dell'Archivio Federale dei Monumenti Storici ${ }^{511}$ di cui lei stessa, nella sezione Patrimonio Culturale e Monumenti Storici è responsabile. Ho avuto il piacere di conoscerla durante il mio soggiorno di cinque giorni a Berna, per la raccolta di materiale pertinente alla tutela ed al restauro dei beni culturali in Canton Ticino alla fine del XIX secolo e inizio del XX. La Signora Amacher mi accompagnò a conoscere e consultare l'archivio da loro custodito nei due piani dell'ex-torre della Biblioteca Nazionale Svizzera, così come il locale apposito per i negativi fotografici, nella Halwylstrasse 15, accorpato al Gabinetto delle Stampe.

L'AFMS, fino al 1967 denominato Archiv für historische Kunstalertümer (Archivio dei Monumenti Storici) sotto la gestione del Museo Nazionale Svizzero (MNS), ha le origini attorno agli anni 1880, quando venne costituita la Società Svizzera per la Conservazione dei Monumenti Storici, che dal 1887 si dedica al restauro di monumenti ed agli scavi archeologici. II primo inventario di archivio realizzato nel 1898 constata che questo conteneva 808 voci, ma alla fine del 1940 esse diventarono 46'900, vent'anni più tardi 75'500 e già nel 1966, 100'000.

La fondazione dell'AFMS avviene unicamente dopo il 1917 quando la Commissione Federale dei Monumenti Storici, riconoscendo il valore dei documenti raggruppati negli anni precedenti, come testimonianza dell'attività di tutela eseguita fino al momento, provvide alla loro conservazione all'interno dell'Archivio.

Oggi l'AFMS è costituito da oltre due milioni di documenti di diversa tipologia: 110'000 piani, 700'000 negativi fotografici, 600'000 riproduzioni fotografiche, 350 metri lineari di raccolte di testi e 150 metri lineari di letteratura specializzata.

II materiale che conforma l'archivio viene schedato al suo arrivo, in modo di facilitare la ricerca, e provvisto di un numero di inventario. Oltre a ciò le riproduzioni fotografiche vengono ordinate pure alfabeticamente per Comune di provenienza, mentre i piani ed $i$ rapporti unicamente per ordine numerico.

Le raccolte ed i lasciti archiviati sono reperibili per nome del Comune di provenienza, mentre gli atti, taccuini e verbali della fondazione dell'archivio, al contrario, non hanno ricevuto nessuna segnatura in origine e dopo, a causa dei continui cambiamenti di sede e di personale dell'archivio dal 1975 al $2001^{512}$, questo è stato impossibile. Essi sono raccolti

\footnotetext{
${ }^{511}$ L'Archivio federale dei monumenti storici è gestito dalla Sezione patrimonio cultura e monumenti storici dell'Ufficio federale della cultura. È raggiungibile in Hallwystrasse 15, CH-3003 Berna. II sito Internet: www.bak.admin.ch/patrimonio.

512 L'AFMS ha sofferto numerosi trasferimenti dal 1966. In questo anno l'Archivio, affidato alla CFMS, fu traslocata alla sede della suddetta Commissione in Gasometerstrasse 9 a Zurigo. Nel maggio del 1975 il consigliere federale Hans Hürlimann decise di trasferire I'AFMS a Berna, assieme alla CFMS che si integra all'Ufficio degli affari culturali, recentemente autonomo dal Dipartimento federale dell'interno. L'Archivio trova sede in quel periodo nel nuovo edificio del Dipartimento federale di giustizia a polizia, Bundesrain 20, poiché la sede della CFMS in Thunstrasse 20 non aveva lo spazio sufficiente per ospitare i cresciuti materiali d'archivio. Nel 1996 l'AFMS venne traslocato in Halwystrasse 15, due anni dopo deve essere nuovamente chiuso per essere trasportato in Holzikofenweg 8, e dal maggio 2001 è ritornato alla sede in Halwystrasse 15 insieme alla BNS dove si trova ancor oggi. (Gian-Willi, 2003, p. 4,5)
} 
in fascicoli ed archiviati in classificatori o accatastati, alcuni per di più mischiati con altri documenti.

Dal 2004 invece I'AFMS sviluppa in progetto di conservazione preventiva del materiale archiviato e di inventariato esaustivo che suppone la ricerca sulla provenienza di certi documenti e sulla proprietà ed autorità, dopodiché i risultati potranno essere pubblicati su Internet.

[...]Nel suo importante ruolo di garante della memoria della cultura architettonica del nostro Paese, l'Archivio federale dei monumenti storici, assieme agli archivi degli uffici cantonali e comunali, è un punto fermo nel panorama degli archivi svizzeri e della politica nazionale della memoria[... $]^{513}$.

La visita all'AFMS fu realizzata con lo scopo di approfondire e completare l'informazione iniziale estratta dall'Archivio dell'Ufficio dei Beni Culturali del Canton Ticino e Bellinzona. $\mathrm{Fu}$ fondamentale a Berna la consultazione delle fonti documentarie ellaborate dal professore Albert Naef. L'archeologo scrisse dal 1909 al 1935 cinque volumi, Confederación Katalog der vom Bunde subventionerten historischen Baudenkmäler und Ausgrabungen ${ }^{514}$, di catalogazioni dei monumenti storici e siti archeologici da lui stesso ispezionati in qualità di ispettore della CFMS.

I dati stratti da questi volumi ci permettono di datare certi eventi, specialmente di inizio e di fine dei restauri, così come l'informazione riguardante gli aspetti finanziari.

\section{L'Archivio dell'Ufficio dei beni culturali, Bellinzona}

L'Archivio dell'Ufficio dei Beni Culturali del Canton Ticino si trova nella sede della medesima istituzione a Bellinzona, in Viale Stefano Franscini 30a, collegato alla Biblioteca Cantonale ed all'Archivio di Stato del Canton Ticino. Qui venne custodita la memoria della storia della tutela dei beni culturali nel territorio dalla creazione della CCMS, ma esattamente dal 1910 quando il 30 agosto venne concesso allo storico Emilio Motta di ispezionare gli archivi comunali, patriziali e parrocchiali asportando da essi i documenti di interesse per la esecuzione dei mandati legislativi riguardo la tutela dei monumenti storici. Un locale sotto il piano terreno dell'edificio dell'UBC, contiene in armadi mobili dei fascicoli con le fonti scritte, conservati idoneamente in scatole di cartone. Essi racchiudono documenti pertinenti all'attività e alla storia dei monumenti storici protetti dalla Commissione dei beni culturali ordinati alfabeticamente per Comune, e conservando

\footnotetext{
513 (Amacher, 2004, p. 7).

${ }^{514}$ Dal 1915 in poi, Näf cominciò a inserire aggiunte, riferimenti bibliografici e indicazioni relative all'ubicazione di diversi atti. Queste annotazioni successive, perlopiù inserite diagonalmente, marcate in rosso o in verde... II primo volume è rigidamente strutturato in tabelle. Le colonne distribuite su due pagine, contengono indicazioni sul luogo, il Cantone, il monumento e la sua classificazione. Seguono rubriche relative alle questioni finanziarie (finanziatori, preventivo, partecipanti della Confederazione, ecc.) e le perizie del comitato direttivo della Società Svizzera per la conservazione dei monumenti storici. Alla fine sono indicate le condizioni particolari per la concessione dei sussidi e riportate le osservazioni sulle ispezioni dei lavori eseguiti. Gli altri quattro volumi hanno una struttura più libera. I monumenti sono descritti sotto forma di testo corrente. Gurtner Kathrin, L'eredità di Albert Naef in Giornale UFC 13, 2004.
} 
la segnatura dalle origini della fondazione della CCMS e dei primi inventari. È il cosiddetto Archivio "morto", recentemente riorganizzato con motivo di un progetto del Servizio di Inventario che interessa l'informatizzazione dei dati presenti nel registro, con lo scopo di facilitare la catalogazione e di permettere in futuro la loro esplorazione via internet. Oltre a questo, in un locale del piano terreno si trova la raccolta fotografica dell'UBC, anche essa ordinata alfabeticamente.

Al primo piano dell'edificio si trova la biblioteca dell'UBC e lo spazio che dovrebbe servire per la consultazione del materiale archivistico e favorire lo studio, non solo dei ricercatori, ma anche ai restauratori che devono conoscere la storia che ha determinato l'opera su cui intervenire. Sappiamo però che questa non è una pratica abituale dei professionisti che attualmente lavorano in Canton Ticino, sia per mancanza di consapevolezza sull'importanza delle indagini e della documentazione, sia per l'infattibilità data dai mezzi fisici o economici.

Il materiale documentario estratto dai fascicoli dell'AUBC, dall'archivio fotografico e dalla biblioteca del medesimo Ufficio, sono stati oggetto del mio studio successivamente all'esecuzione del primo analisi bibliografico.

Prossimamente, la sopraccitata informatiz-zazione delle "schede informative" che riassumono i dati amministrativi, descrittivi e documentari di tutti i singoli edifici o oggetti inventariati dall'UBC saranno consultabili dagli interessati via internet, e permetterà senza dubbio di motivare alla ricerca a diffondere il materiale documentario presente in Canton Ticino anche fuori del territorio.

\section{Catalogazione}

Durante la lettura della tesi qui presentata si potrà osservare quale è stata la complessità della ricerca documentaria eseguita, con lo scopo di arrivare a individualizzare i nomi dei personaggi, pittori-restauratori del Novecento in Canton Ticino più rilevanti, gli interventi eseguiti, nonché definire nel modo più concreto le prassi esecutive ed i materiali usualmente impiegati da ognuno di loro.

In primo luogo, come descritto nell'introduzione a questa ricerca, lo studio ha richiesto un'analisi approfondita delle pubblicazioni bibliografiche fondamentali della storia della tutela dei beni culturali in Ticino e dell'arte dalla fine del XIX sec. al XX sec.: si trattava dei Monumenti storici e artistici del Cantone Ticino restaurati dal 1910 al 1945 di Francesco Chiesa $^{515}$, Cinquant'anni di protezione dei monumenti storici ed artistici della repubblica e cantone del Ticino del DPE ${ }^{516}$, i Settantacinque anni della Commissione dei monumenti storici ed artistici del Cantone Ticino di Fulvio Caccia ${ }^{517}$ e Arte nell'Ottocento di Giulio Foletti ${ }^{518}$. Mentre quest'ultimo mi ha illustrato il contesto artistico dell'epoca iniziale scelta per la ricerca, descrivendoci l'organizzazione educativa delle accademie di belle arti

\footnotetext{
(Chiesa, 1946).

(DPE, 1959).

517 (Fulvio Caccia, 1984)

${ }^{518}$ (Foletti, 2002).
} 
dentro e fuori dal territorio ticinese, il mercato dell'arte ed alcuni dei personaggi che più tardi troveremo, a suo tempo, legati all'ambito della tutela e restauro dei monumenti storici, come Luigi Rossi, Edoardo Berta o Ernesto Rusca e testi redatti dalla Commissione Cantonale dei Monumenti Storici e dal Dipartimento della pubblica educazione del Canton Ticino, negli anni 1946, 1959 e 1984 ci hanno permesso di classificare gli interventi di restauro ed associare le figure responsabili. A partire di questi primi dati, è stata mia premura la realizzazione di una serie di tabelle dove poter ordinare per data e per "restauratore" i diversi eventi di intervento estratti dalla bibliografia in modo di facilitare le ricerche documentarie successive più approfondite. Queste tabelle, per ogni pittorerestauratore, sono state divise in cinque colonne: Anno dell'intervento, Luogo dell'intervento, Intervento (sia questo una perizia, un sopralluogo, un restauro, un preventivo o una proposta di restauro ad esempio), Collaboratori e Fonti.

Con questa informazione preliminare, l'analisi avanzò verso lo studio dei documenti conservati all'Archivio "morto" dell'Ufficio dei beni culturali del Canton Ticino con sede a Bellinzona. Ho cominciato dai primi grandi restauri intrapresi dalla formazione della CCMS: la chiesa di S. Biagio a Ravecchia, la chiesa di S. Maria delle Grazie a Bellinzona e la chiesa di S. Maria degli Angioli a Lugano tutti diretti dal pittore Edoardo Berta. L'esplorazione meticolosa di essi diede gli indizi per cominciare la ricerca di nuove figure e restauri, che mi portò inoltre all'esplorazione dell'Archivio Federale dei Monumenti Storici ed a perseguire il ritrovamento degli archivi personali degli artisti. Presto l'informazione, ogni volta più complessa, ebbe bisogno di un riordino sistematico e pratico, completando sempre in ogni caso, le pratiche tabelle realizzate inizialmente.

Le nuove esigenze furono momentaneamente compensate con la realizzazione in Access di una banca dati che mi permise non solo di strutturare i dati ma di creare delle relazioni fra essi. Per esempio la tabella con i dati attribuiti ai "Pittori-restauratori" veniva collegata alla tabella "Opera" (in questo caso il bene storico artistico oggetto di restauro) attraverso altre tabelle che definivano l'intervento eseguito, la georeferenziazione e le fonti.

Le limitazione personali ed il tempo, impedirono di completare la banca dati con la complessità necessaria per rispondere a maggiori necessità, anzitutto l'esigenza e il desiderio di creare uno strumento che servisse non solo ad aiutarmi nella ricerca ma che come elemento innovativo suppose anche un istrumento di appoggio alla documentazione, investigazione e studio di altri ricercatori.

Per fare realistica questa aspirazione si doveva innanzitutto pensare a scavalcare le limitazioni presenti nella attualità degli archivi consultati, ed in concreto della documentazione recapitata. 


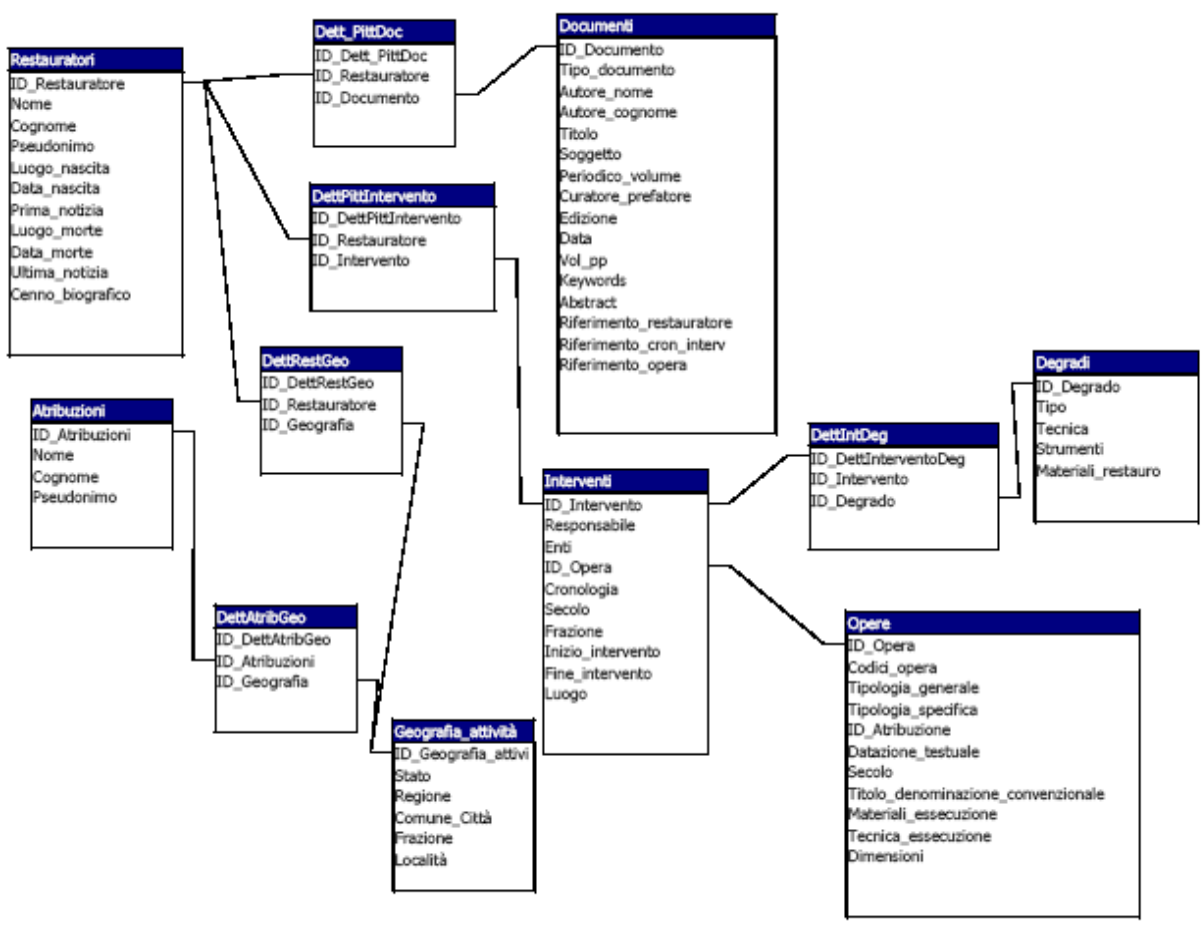

Figura $\mathbf{2 0 0}$ Schema delle relazioni creata all'interno della banca dati su Access 


\section{Sistemi di gestione dati}

Gli archivi sono fondamentali per la protezione, conservazione e divulgazione del patrimonio documentario e della memoria umana e collettiva dello Stato. Questi ci permettono di tornare al passato e scoprire persone, luoghi ed eventi che hanno formato la storia e la cultura del paese ed in definitiva la nostra società attuale, sebbene per rendergli vivi bisogna che qualcuno apra gli armadi, le cassette o le cartelle in cui sono conservati, li legga, li analizzi e li metta a pubblica disposizione attraverso nuove fonti d'informazione che saranno il frutto della propria interpretazione personale dell'autore.

Per comunicare nel modo più obiettivo possibile l'informazione presente negli archivi, idealmente essi dovrebbero essere messi alla luce, permettendo facilmente la visibilità ed accesso completo dei documenti che li formano, a tutte le persona. Questo è ovviamente un progetto che si dovrebbe trattare a lungo tempo: pensiamo ad esempio ai costi per digitalizzare gli oltre due milioni di documenti (piani, fotografie, negativi, rapporti di restauro e di scavo e documenti della storia dell'architettura o dei monumenti storici e della tutela del patrimonio) che formano in Svizzera l'Archivio Federale dei Monumenti Storici. Nonostante questo, le ultime innovazione nell'informatica ed in particolare della rete permettono non solo di sviluppare i sopraccitati interessi ma di creare degli strumenti contro l'amnesia. Diversi organismi lottano attualmente per la preservazione del "documento" e con sé della memoria umana testimoniata e tramandata grazie all'esistenza di questo.

Nel 1992 l'Organizzazione delle Nazione Unite per l'Educazione, la Scienza e la Cultura (UNESCO) ha iniziato il programma The Memory of the World ${ }^{519}$ con lo scopo di diffondere a livello mondiale ed allo stesso tempo conservare gli "oggetti" e le "parole" custoditi nelle collezioni di archivi, biblioteche, istituzioni culturali, musei e luoghi commemorativi e che formano il patrimonio documentario. Il programma parte dall'idea che l'eredità documentaria del mondo appartiene a tutti, e per questo, non solo deve essere conservato e protetto da tutti noi ma deve anche mettersi a disposizioni di chiunque senza limitazioni.

L'UNESCO con questo progetto sviluppa proposte che rispettano le limitazioni legislative in materia di divulgazione, di acceso e di tutela, così come i diritti delle proprietà private, ma promuovono la conservazione del materiale documentario, la sua diffusione motivando la digitalizzazione, la catalogazione accessibile da Internet, la pubblicazione e la distribuzione ampia di libri, CD, DVD ed altri prodotti, incrementando in questo modo a suo tempo la conoscenza e la trascendenza nella misura del possibile.

Con lo stesso scopo il progetto MICHAEL (Multilingual Inventory of Cultural Heritage in Europe) finanziato dalla Commissione Europea, ad esempio, è stato messo in funzione in rete da pochi anni or sono. Egli ha creato un servizio innovativo di digitalizzazione di

${ }^{519}$ || sito internet:

https://www.unesco.org/webworld/mdm 
collezioni di musei, biblioteche e archivi, attraverso un servizio multilingue per l'accesso e la promozione del patrimonio culturale europeo.

II programma MICHAEL si serve di una piattaforma software open source per le installazioni nazionali basata sui sistemi Apache, Tomcat, Cocoon, Lucene, XtoGen, XML, ecc. e di un portale europeo come strumento di ricerca nella banca dati europea, istituendo dei modelli per l'adesione dei dati in inventari multilingue di contenuti culturali digitali.

La Svizzera al contrario non è ancora addetta a nessuno dei sopraccitati programmi malgrado, in modo indipendente, possiamo trovare alcune banche dati on-line per la catalogazione come la "HelveticArchives" dove si registrano con il supporto di Access i fondi d'archivio della Biblioteca Nazionale Svizzera (BN), o la "ISplus" che riunisce il repertorio sommario dei fondi manoscritti conservati nelle biblioteche e negli archivi svizzeri e delle Istituzioni Svizzere di Salvaguardia della Memoria e dei Beni Culturali. La differenza fra questi sistemi e quelli internazionali ed europei precedentemente citati è che questi ultimi non solo permettono di catalogare i dati ma anche di essere visualizzati e consultati on-line in modo libero: i documenti degli archivi possono essere letti, studiati, analizzati dal proprio ufficio o da casa, mentre i sistemi svizzeri per il momento in vigore, danno accessibilità unicamente alla ricerca, ma la ulteriore consultazione del materiale deve realizzarsi nella biblioteca o archivio, limitando lo scambio d'informa-zione e la diffusione di essa.

Una delle premesse di questa ricerca è stata quella di analizzare le fonti consultate e comunicare i propri giudizi ed interpretazione della storia della tutela e del restauro in Canton Ticino dalla fine dell'Ottocento agli inizi del Novecento; a suo tempo però mi affliggeva pensare che anche essi rimasero limitati fisicamente e geograficamente nonché al giudizio ed alla obiettività. Per questo motivo il desiderio è stato far rivivere la storia della protezione dei monumenti nel Ticino mettendo in pratica e confrontando dei meccanismi fattibili al loro futuro tramando nel modo più obiettivo possibile, con sistemi analoghi ai precedentemente citati. II paragone di questi tipi di strumenti e l'impiego di uno di questi è stato fondamentale per capire le loro funzionalità, coprire le mie esigenze personali e studiare dei miglioramenti fattibili che potranno servire in futuro a questi stessi sistemi così come a nuovi progetti.

I requisiti basici che intendevo trovare per la gestione dei dati della ricerca e la sua trasmissione erano:

1. La catalogazione completa dei dati essenziali ottenuti dalle fonti di archivio consultate, usando le norme di termino-logia della International Organization for Standardization (ISO).

2. La visualizzazione on-line dei dati in modo libero da tutti gli utenti interessati (webdased/ open-source) poiché i documenti di archivi pubblici come l'AUBC sono d'interesse collettivo e per questo motivo non si dovrebbe limitare l'accesso a loro ma tuttavia accrescere l'interesse al suo studio, permettendo lo svolgimento di progetti che servano alla rivalorizzazione dell'identità. 
3. La stampa o l'acquisizione dei dati relazionati a dipendenza delle esigenze creando delle tabelle (ad esempio: un elenco della fonte in cui si trova citato un determinato personaggio, od un elenco degli interventi eseguiti dal pittore Emilio Ferrazzini nonché una lista dei restauratori che hanno partecipato al restauro di un determinato bene storico artistico, degli strappi sotto la tutela del comune di Lugano od i consolidamenti realizzati con l'impiego della caseina).

Si sono messi a confronto a questo scopo il sistema informatico per la catalogazione del Servizio inventariato all'Ufficio dei Beni Culturali a Bellinzona e due dei sistemi di banca dati attivi in Italia per la informatizzazione del materiale presente negli archivi della Sovrintendenze ai Beni Culturali Italiani (AR.I.S.T.O.S. Archivio Informatico on-line per la Storia della Tutela degli Oggetti Storici artistici) così come degli archivi dei restauratori italiani (RES.I) della Fondazione Secco-Suardo a Bergamo.

\section{RES.I}

La banca dati RES.I (Restauratori Italiani) è stata creata negli anni '90 da Matteo Panzeri per la Associazione Giovanni Secco Suardo di Bergamo con lo scopo di gestire i dati estratti dal progetto ASRI (Archivio Storico Nazionale e Banca Dati dei Restauratori Italiani) coordinato dall'Associazione in collaborazione con l'Istituto Centrale per il Restauro (I.C.R.).

La mia ricerca iniziò dalla conoscenza di questo originale programma italiano. Esso era riuscito fino al 2007 ad acquisire importantissime testimonianza dagli archivi di Giuseppe Arrigoni $^{520}$, Antonio Benigni ${ }^{521}$, Arnolfo Crucianelli ${ }^{522}$, Vito Mameli ${ }^{523}$, Andrea Mandelli ${ }^{524}$ e Mauro Pellicioli che ora formano parte del settore "Archivi conservati" (AR.CO) nelle fonti fondamentali per la storia del restauro, oltreché ad esaminare i principali archivi lombardi ${ }^{525}$.

Lo scopo della banca dati "Restauratori italiani" è quello di ricostruire i profili biografici di restauratori (in questo caso del paese italiano) e dei loro interventi di restauro, attraverso una sistema di base dati relazionale e multimediale sulle indagini delle fonti bibliografiche ed archivistiche.

\footnotetext{
${ }^{520}$ Arrigoni Giuseppe (Bergamo 1915-2006).

${ }^{521}$ Benigni Antonio (Bergamo 1939-).

${ }^{522}$ Crucianelli Arnolfo (Bahia Blanca, Argentina 1910 - Macerata 1991).

${ }^{523}$ Mameli Vito (Cagliari 1885-Roma 1977).

${ }^{524}$ Mandeli Andrea (Bergamo 1921-).

${ }^{525}$ Archivio della Soprintendenza ai Beni Artistici e Storici di Milano (sezione Archivio Vecchio, Archivio Nuovo, Sezione Archivio corrente - in parte); Archivio di Stato di Bergamo (indice delle Parti dell'Archivio Notarile); Archivio di Stato di Mantova (Scalcheria e Amministrazione di Palazzo Ducale, Magistrato Comunale Antico, Regia Intendenza Politica, Intendenza Provinciale di Finanza, Camera di Commercio, Archivio della Società Amici di Palazzo Ducale); Archivio Storico Comunale di Mantova (Atti secolo XIX, Atti secolo XX, Protocollo riservato); Archivio dell'Accademia Carrara di Bergamo (Sezione Archivio Storico - alcune Serie, Archivio di Deposito e Corrente - alcune Serie); Archivio della Biblioteca Civica "Angelo Mai" di Bergamo (Sezione Manoscritti Epistolario Lochis, Archivio Storico Comune di Bergamo - alcuni fascicoli); Archivio Storico della Provincia di Bergamo (Archivio Storico, Archivio di Deposito); Archivio dell'Accademia Virgiliana di Mantova.
} 
RES.I. è organizzata attraverso quattro schede ${ }^{526}$ principali: Restauratore, Intervento, Opera e Documento. La scheda Restauratore contiene a suo volta le informazioni: Numero identificativo, Onomastica, Nascita, Morte, Cenno biografico, Geografia dell'attività, Interventi, Fonti-Documenti-Bibliografia, Compilazione, Revisione e Pubblicazione; la scheda Intervento contenne: Numero identificato, Restauratore, Responsabile, Enti/Persone, Opera, Materia e tecnica, Dimensioni, Cronologia dell'intervento, Luogo, Degrado, Descrizione della procedure, Operazioni, Note critiche, Compilazione, Revisione e Pubblicazione; la scheda Opera si struttura in: Numero identificativo, Codice dell'opera, Codice univoco, Codice CdR, Altri codici, Tipologia, Ambito culturale, Attribuzione, Datazione, Altre attribuzioni, Soggetto, Altre identificazioni del soggetto, Titolo/Denominazione convenzionale, Materia e tecnica, Dimensioni, Collocazione, Precedenti collocazioni, Osservazioni, Interventi, Relazione all'immagine, Compilazione, Revisione e Pubblicazione; finalmente la scheda Documento si divide in: Numero identificativo, Tipo di documento, Autore, Titolo, Soggetto, Periodico/Volume, Specifica, Curatore/Prefatore, Edizione, Annata/Numero, Data, Volume/Pagine, ISBN/ISSN, Tecnica, Dimensioni, Collocazione, Keywords, Abstract, Riferimenti restauratore, Riferimenti intervento, Note, Relazioni multimediali, Compilazione, Revisione, Pubblicazione.

Questo dettaglio dimostra la complessità del sistema. Inoltre, ognuna di queste schede può gestire dei files allegati: immagini, filmati, registrazioni sonore, ecc.

Si tratta di un modello eccezionale sviluppato dal confronto del sistema progettato di banca dati con altri istrumenti analoghi. Esso adotta gli Standard archivistici nazionali ed internazionali (ISAD) per l'organizzazione di informazione di archivi, le norme dell'Istituto Centrale per il Catalogo e per la Documentazione (ICCD) per l'inventariato delle opere d'arte, la norme internazionali per i dati bibliografici, Internationale Künstlerdatenbank (IKD) per i dati biografici ed i modelli di schede sperimentati dall'istituto I.C.R. di Roma per I'elaborazione dei dati riguardanti gli interventi. Inoltre RES.I. assunse le difficoltà dell'internazionalizzazione del lessico e confrontò per lo sviluppo del sistema i progetti europei N.A.R.C.I.S.S.E. ${ }^{527}$, CRISTAL ${ }^{528}$ e LMCR $^{529}$.

\footnotetext{
${ }^{526}$ Questo sistema di schede è stato utilizzato per la presente tesi nella fase iniziale di catalogazione dei dati bibliografici e di archivi, senza però creare fra di essi una correlazione all'interno di un sistema di banca dati. Le tabelle "statica" sull'attività di ognuno dei restauratori qui studiati, offre i dati riguardanti a: Data dell'evento, Opera e luogo, Tipo di intervento, Collaboratore/i dell'evento, e Fonte/i fondamentale/i dove venne registrato l'intervento.

${ }^{527}$ N.A.R.C.I.S.S.E. (Network of Research Computer Image SystemS in Europe) Commission des Communautes Europeennes DG XIII , Glossaire Multilangue Glossario Multilingue, Arquivos Nacionais-Torre do Tombo, Lisboa, 1993.

${ }^{528}$ C.R.I.S.T.A.L. (Conservation Restoration Instititions for Scientific Terminology dedicated to Art Learning Network) Centre de Recherche et de Restauration des Musees de France, Institut Royal du Patrimoine Artistique, Regione Lombardia, Associazione Giovanni Secco Suardo e relativa pubblicazione Pittura Murale, proposta per un glossario a cura di Mara Nimmo, Associazione Giovanni Secco Suardo, Regione Lombardia - Direzione Generale Culture,Identità e Autonomie della Lombardia, 2001.

${ }^{529}$ L.M.C.R. (Lessico tecnico Multilingue di Conservazione e Restauro), Associazione Giovanni Secco Suardo (Italia), Istituto Centrale per il Restauro (Italia), Opificio delle Pietre Dure (Italia), Regione Lombardia - Direzione generale Culture e Identità della Lombardia (Italia), Ecole Nationale du Patrimoine (Francia), Université de La Sorbonne,
} 


\section{AR.I.S.T.O.S}

In confronto al sistema dell'Associazione Secco Suardo di Bergamo, si presentò recentemente un nuovo strumento per la catalogazione dei dati inerenti la storia della tutela e del restauro in Italia.

Si tratta della "giovane" banca dati AR.I.S.T.O.S., Archivio Informatico on-line per la Storia della Tutela degli Oggetti Storico artistici.

AR.I.S.T.O.S. è stato sviluppato all'interno del progetto per la digitalizzazione del patrimonio "ARTPAST"; si tratta di un programma del Ministero per i Beni e le Attività Culturali italiano di responsabilità della Direzione Generale per I'Innovazione tecnologica e la promozione che ha come fine la [...]conoscenza e la condivisione del patrimonio culturale tramite l'applicazione di tecnologie informatiche avanzata ai settori della catalogazione, del restauro e della circolazione delle opere d'arte $[. . .]^{530}$ attraverso il popolamento del Sistema Informativo Generale del Catalogo ( $\mathrm{SIGeC}$ ), la creazione di un Sistema informativo in rete degli Uffici Esportazione dove controllare la circolazione di opere rubate, e con il riempimento dei sistemi AR.I.S.T.O.S. per la storia della tutela e di SICaR web, sistema informativo per la documentazione georeferenziata di Cantieri di Restauro.

Fondamentali in queste ultime due banche dati sono le caratteristiche di web-dased, e di open source, che permettono che il riempimento dei dati o la ulteriore modifica siano realizzati via internet, e che ugualmente essi possano essere liberamente consultabili. I dati, attualmente in un server housing ${ }^{531}$, per la documentazione del restauro, saranno traslocati al termine del progetto sul server del Centro di Elaborazione Dati del Ministero per i Beni e le Attività Culturali, così da non perdere la possibilità di esponenziale arricchimento derivante dalla condivisione in rete della documentazione.

Il database AR.I.S.T.O.S. è nato dalla collaborazione dal 2001 della Soprintendenza BAPPSAE per le Province di Pisa e Livorno, la cattedra di Storia della critica d'arte della Facoltà di Lettere dell'Università di Pisa e la Scuola Normale Superiore di Pisa. Con questo programma si possono gestire, ripeto, le informazioni relative alla storia della tutela, ma specialmente del restauro e della catalogazione corrispondente alla gestione degli archivi della Soprintendenza italiana ed in risposta alla diverse necessità di gestione e amministrazione.

L'interesse per l'avvio di un sistema simile si deve alla rivalorizzazione del materiale presente all'Archivio Storico della Soprintendenza di Pisa, attraverso un progetto diretto dalla dottoressa Donata Levi sui restauri di dipinti e scultura nella Toscana nord occidentale dall'unità alla legge di tutela del 1939. Presto uno studio più approfondito

MST de Conservation et Restauration des Biens Culturels (Francia), Ministère de la Culture (Francia), Escuela Superior de Conservación y Restauración de Bienes Culturales (Spagna), Courtauld Institute of Art, University of London (Gran Bretagna), Université Libre de Bruxelles (Belgio), Hochschule fur Bildende Kunste Dresden (Germania)

${ }^{530}$ http://www.artpast.org/.

${ }^{531}$ L'housing venne chiamato al modo in cui all'utente di un determinato sistema è offerto un intero server connesso a internet, in confronto alla solita organizzazione di webfarm in cui all'utente è messa a disponibilità un gruppo di server web collegati ad un centro comune. 
delle fonti esistenti nel suddetto archivio esige la creazione di un sistema informatizzato dove gestire il materiale di informazione, eseguito grazie al finanziamento della Provincia di Pisa.

L'avanzamento del lavoro e delle sperimentazioni portato ad impiegare il sistema per la gestione della documentazione dei restauri contemporanei, consentendo un affinamento maggiore della struttura del sistema ha influito nella decisione di aprire la funzionalità verso una versione del programma on-line, dando come risultato l'attuale AR.I.S.T.O. $S^{532}$.

A definire la struttura del programma sono stati: le notizie trovatesi nelle fonti di studio, che sono inoltre la base di partenza del sistema, i personaggi e gli enti co-relazionati o citati nel documento che intervengono sui beni pertinenti, i medesimi beni divisi in Artistici (BA) o storico-artistici (BAS) su cui si agisce, e gli eventi che costituiscono il punto di interazione fra i personaggi, enti e beni sopracitati.

L'adozione della banca dati di tipo relazionale precedentemente descritta ha permesso di articolare le diverse connessioni dell'informazione evitando tuttavia la duplicazione dei dati e rendendo l'inserimenti di essi più velocemente. Così si è creata la "Pratica virtuale" che consente di unificare eventi relazionati tra loro che corrispondono ad un unico avvenuto generico.

[...] Ad esempio, una pratica virtuale di tipo "restauro" permette di unificare tutto l'insieme di eventi che riguardano un restauro: l'indagine conoscitiva dell'opera, la riproduzione fotografica dell'opera preliminare all'intervento, il trasferimento in laboratorio dell'opera, l'intervento di restauro vero $e$ proprio, la riproduzione fotografica dell'opera posteriore all'intervento ed infine la ricollocazione dell'opera nel contesto originario. L'entità "pratica virtuale" è quindi uno strumento molto utile, che permette di raccogliere e gestire un insieme di eventi, le cui correlazioni non sempre sono facilmente individuabili, amplificando e potenziando la possibilità di recuperare i dati e compiere ricerche

\footnotetext{
532 Fino al momento il sistema AR.I.S.T.O.S. è stato provato nei casi studi seguenti: La documentazione d'archivio a supporto della progettazione (Soprintendenza per i beni architettonici e il paesaggio e per il patrimonio storico, artistico e etnoantropologico di Pisa e Livorno); Indicizzazione e catalogazione degli archivi di restauro (Soprintendenze per i beni architettonici e il paesaggio e per il patrimonio storico artistico e etnoantropologico di Arezzo; di Napoli e la Soprintendenze per il patrimonio storico, artistico e etnoantropologico di Bari e Foggia; della Basilicata; della Liguria; delle Marche); Esperimenti di indagine storica e catalogazione analitica delle fasi di restauro. (Soprintendenze Speciali per il Polo Museale fiorentino; per il Polo Museale napoletano;Soprintendenze per i beni architettonici e il paesaggio e per il patrimonio storico artistico e etnoantropologico di Caserta e Benevento; di Sassari e Nuoro; del Friuli Venezia Giulia; Soprintendenza per il patrimonio storico, artistico e etnoantropologico della Calabria); Per costruire una storia della tutela: esplorazioni negli archivi storici (Soprintendenza Speciale per il Polo Museale veneziano; Soprintendenze per i beni architettonici e il paesaggio e per il patrimonio storico artistico etnoantropologico di Cagliari e Oristano, di Salerno e Avellino, di Venezia e Laguna, del Molise; Soprintendenza per i beni archeologici di Napoli e Caserta; Soprintendenza per il patrimonio storico artistico e etnoantropologico di Lecce, Brindisi e Taranto); Le fotografie come fonte per il restauro. (Soprintendenze per il patrimonio storico, artistico e etnoantropologico di Bologna, Ferrara, Forli-Cesena, Ravenna e Rimini; di Venezia (esclusa la città), Belluno, Treviso e Padova; di Verona, Rovigo e Vicenza; del Lazio); Progettare e comunicare i restauri.(Soprintendenza per i beni architettonici, il paesaggio e per il patrimonio storico artistico e etnoantropologico dell'Umbria; Soprintendenza per il patrimonio storico, artistico e etnoantropologico dell'Abruzzo).
} 
incrociate. I documenti che lo descrivono possono essere contenuti in diversi fascicoli, fondi, archivi e città $[\ldots]^{533}$.

Oltre a questo, il sistema consente anche l'introduzione della tipologia "fonte fascicolo" dove vengono indirizzati tutti i contenuti di una "Pratica virtuale" (persone, enti, beni, eventi citati). Questo significa che ogni singola fonte schedata sulla banca dati ARISTOS può a suo tempo essere compressa in una "pratica".

Il sistema permette: la configurazione di gruppi di lavoro e di singoli utenti; la gestione dei vocabolari con una terminologia controllata; la modalità di data-entry in multi-utenza, la modalità di ricerca per il compilatore e di visualizzazione delle fonti associate ad ogni entità possibile da ricercare e visualizzare tutte le fonti associate ad una entità, la modalità di inserimento dei dati con facilitazioni, la catalogazione degli interventi di restauro, la gestione delle fonti archivistiche, bibliografiche e/o iconografiche, la possibilità di allegare la scansione della documentazione a ciascuna fonte strutturata, possibilità di collegamento di tutte le fonti tra loro, la ricerca in full-text, avanzata o per indici, e la esportazione delle fonti strutturate in XML.

Per provare il meccanismo fu creato all'interno di AR.I.S.T.O.S. il gruppo "Lugano" e fui provvista di un nome utente e di una password per l'accesso all'inserimento dei dati.

Dietro una enorme complessità di moduli e relazioni, l'interfaccia del programma appare molto semplice. Uno schema nella parte sinistra ci permette di visualizzare le voci da riempire, sotto le quelli si trovano sottostrutture dove scegliere, a dipendenza dei casi, le definizioni o descrizioni che si adattino meglio ai dati da noi tratti dalla fonte. Ad un livello più basso si trova invece una clasificazione di elementi che aiutano a caratterizzare ogni modulo, dotando essi del significato richiesto dentro della banca dati che permetterà di diffarenziagli. La clasificazione è tanto ampie come i metodi di ricerca e di navigazione all'interno di AR.I.S.T.O.S. In questo modo si arriva ad una complessità tale da permettere le più varieta catalogazione e relazioni fra l'informazione allegata.

${ }^{533}$ http://www.artpast.org/. 


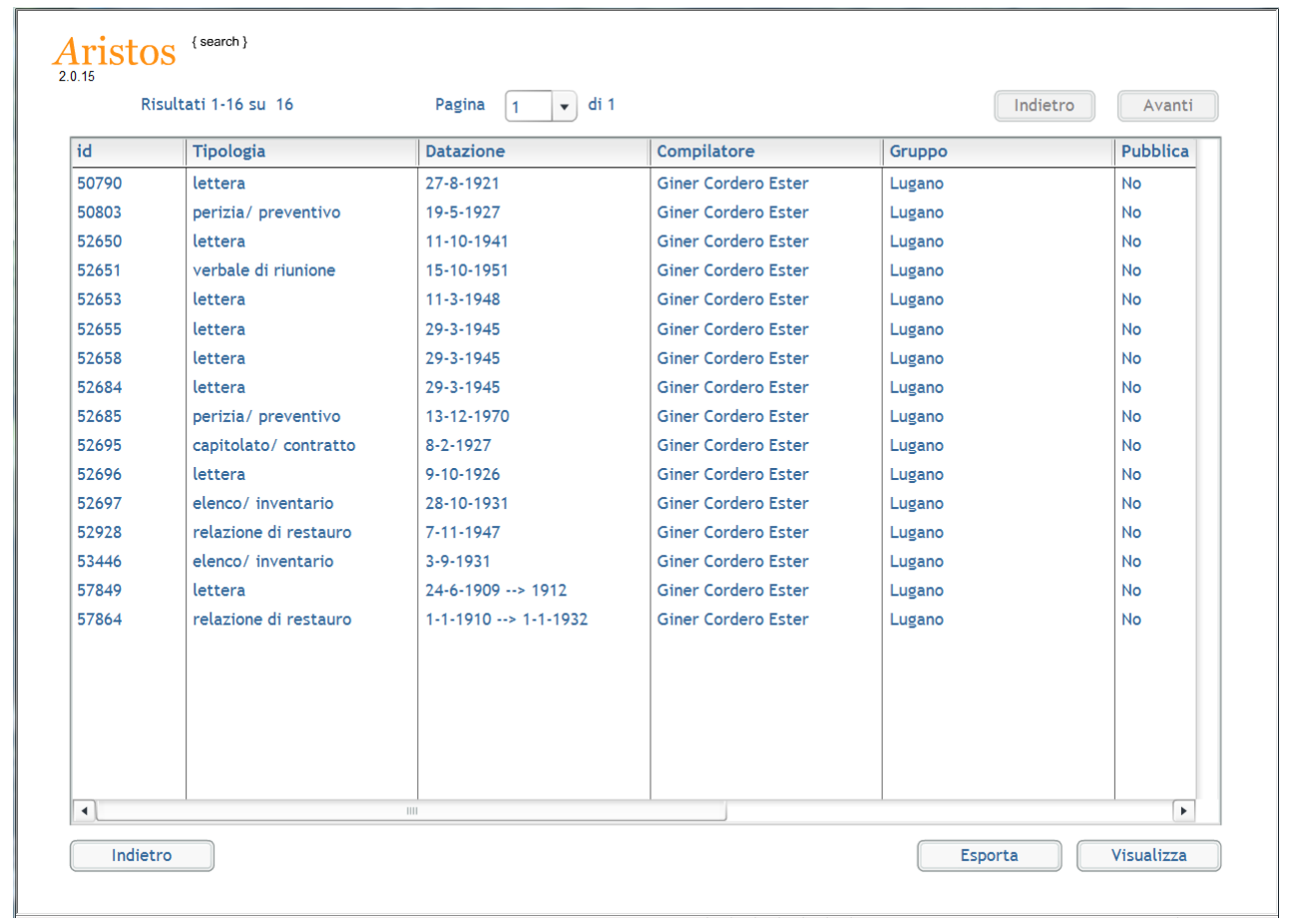

Figura 201 Interfaccia del sistema di consultazione dei dati AR.I.S.T.O.S. 2008. 


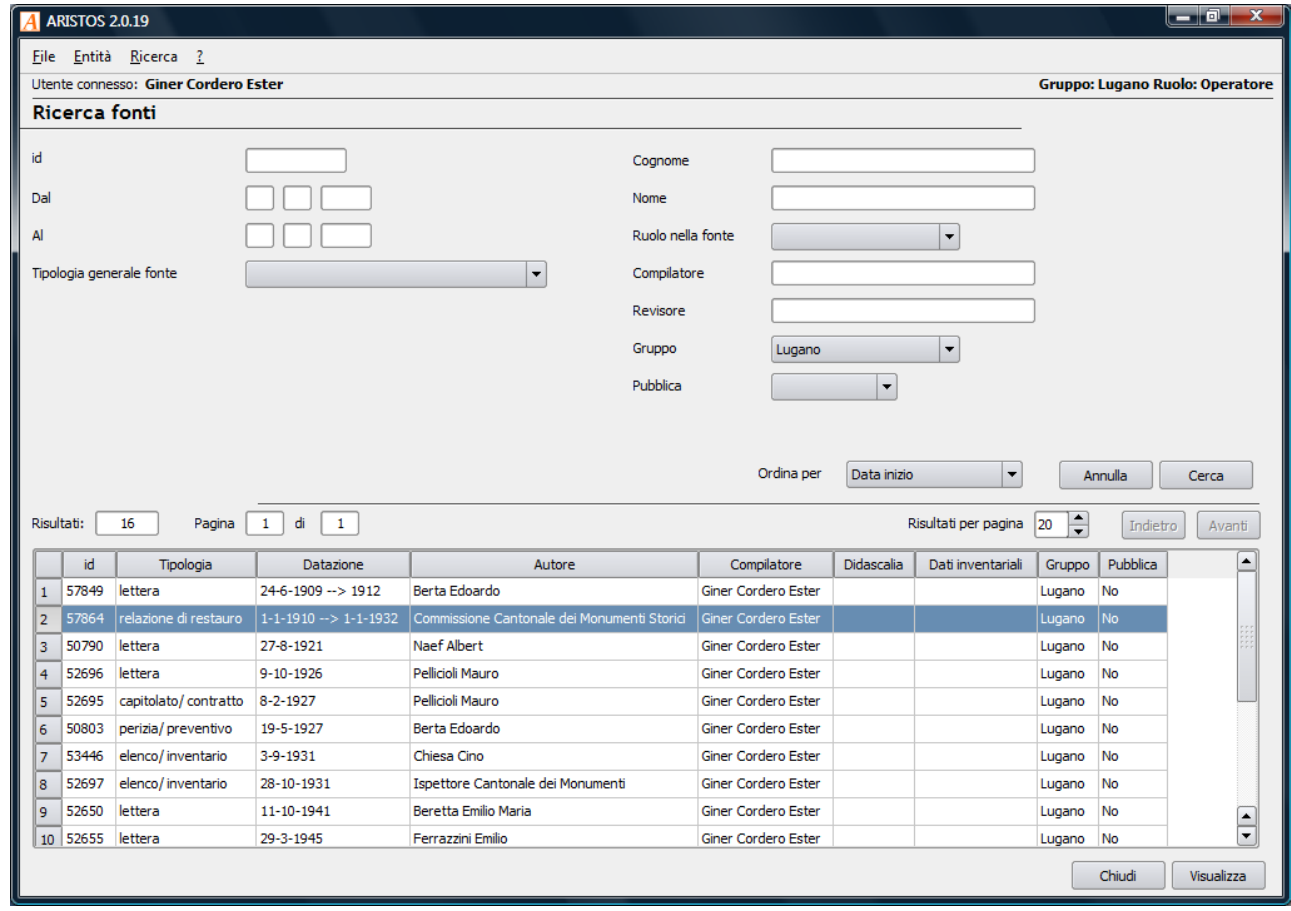

Figura 202 Interfaccia della banca dati AR.I.S.T.O.S. Fase di ricerca delle fonti associate al gruppo Lugano. 2008. 


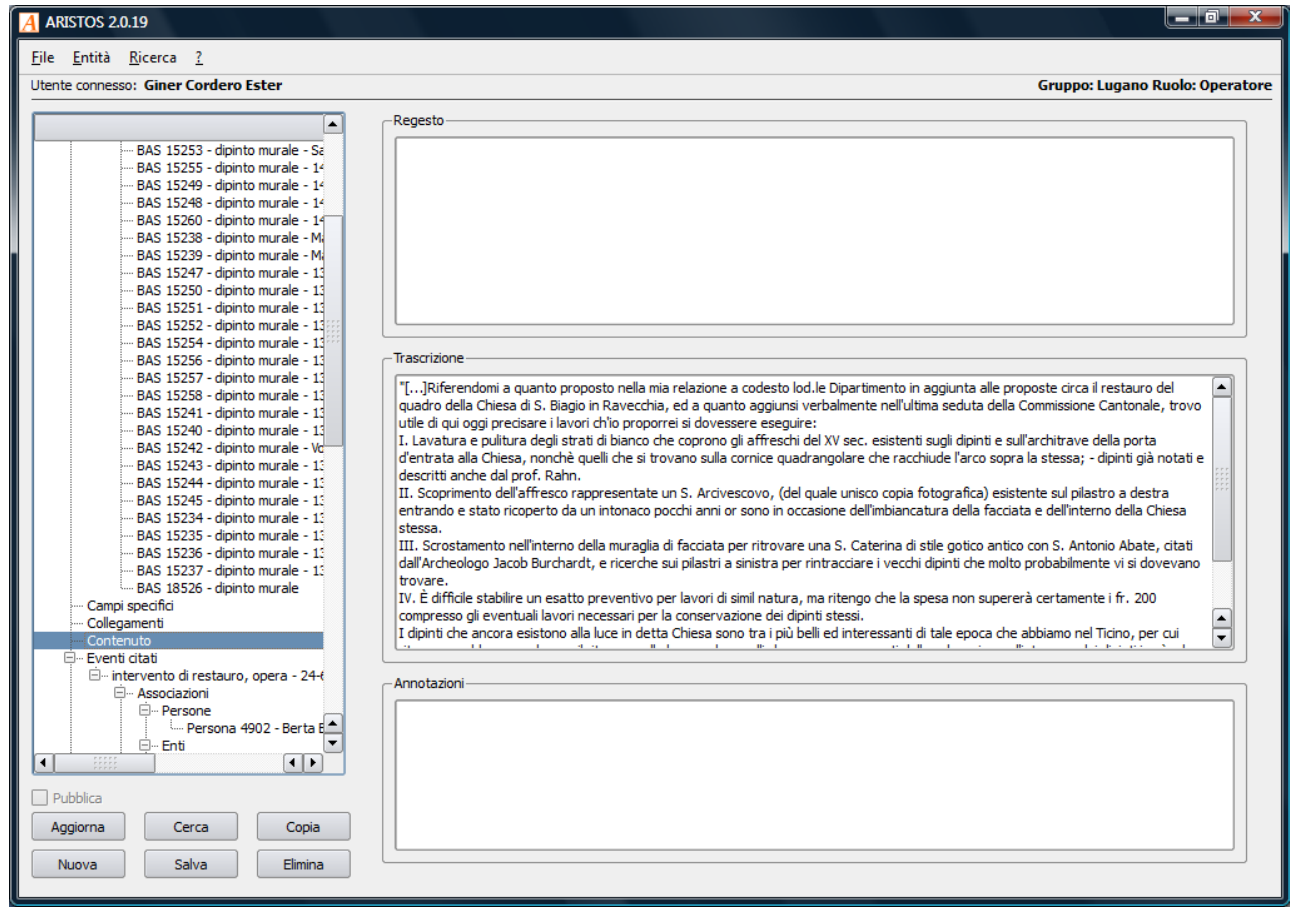

Figura 203 Interfaccia della banca dati AR.I.S.T.O.S. Fase di inserimento dei dati dalla fonte. 2008. 


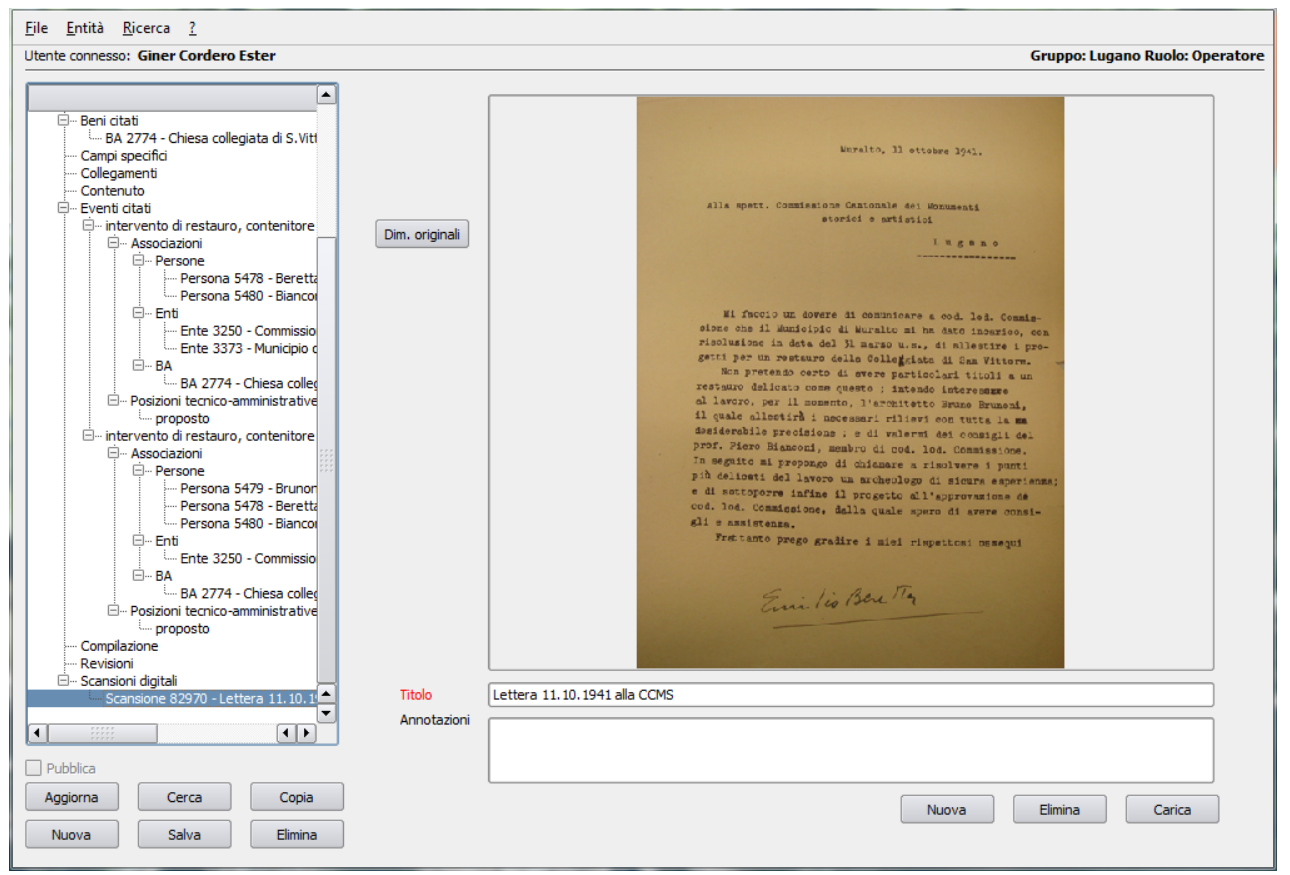

Figura 204 Interfaccia del sistema AR.I.S.T.O.S. Fase di inserimento della fonte scansionata. 2008. 


\section{Banca dati UBC, Bellinzona}

Il sistema di banca dati dell'Ufficio dei Beni Culturali del Canton Ticino è, come AR.I.S.T.O.S. di recente fattura. Si tratta di un sistema meno ambizioso, creato dal Centro Cantonale Sistemi Informativi (CCSI) per il Servizio Inventario dell'UBC, con lo scopo di riordinare e digitalizzare le fonti scritte e grafiche che conformano il loro archivio e metterlo a disposizione di tutte quelle persone interessate, dove i dati informativi riguardono unicamente la tutela dei beni culturali in Canton Ticino. Si tratta di un sistema georeferenziato inserito all'interno di un Sistema d'Informazione sul Territorio (SIT).

Le schede che conformano questa banca dati sono state create da un confronto con $i$ meccanismi di inventariato utilizzati in altri paesi, e finalmente messe a prova nel proprio territorio nella revisione dei "Piani regolatori" e per l'esecuzione degli elenchi della Protezione civile - Sezione Protezione dei Beni Culturali "PCi/PBC".

Questi moduli constano di una sezione descrittiva o segnatura (dati alfanumerici), una sezione iconografica (fotografia, documentazione di rilievo, ecc.) e una sezione geografica utile per la georeferenziazione (GIS) del bene storico-artistico schedato. La banca dati dei beni culturali protetti, circa 100'000 (mobili e immobili, sia di interesse cantonale, d'interessa locale, sia beni da proteggere in caso di conflitto armato o evento di catastrofe su cui interviene la Protezione civile).

La banca dati dei beni culturali del Canton Ticino offrirà agli interessati la raccolta di materiali verificati ed aggiornati sull'informazione amministrativa, storica e artistica. Inoltre per quanto riguarda lo strumento di lavoro della Protezione civile, la banca dati dovrà raggruppare l'informazione fondamentale per la pianificazione dell'intervento in caso di conflitto armato o catastrofe, come ad esempio le misure di sicurezza, di evacuazione o di protezione, in modo da evitare nella misura dello possibile i danni che $i$ menzionati eventi possano creare al bene culturale.

Si tratta quest'ultimo di un fattore di grande interesse che fa della presente banca dati un strumento molto eficace. II lavoro realizzato dalla Sezione Protezione dei Beni Culturali permette e consentirà in futuro l'aggiornamente continuo dei dati nel sistema nonchè renderlo effetivamente utile. 


\section{CHIESA SS FEDELE E SIMONE} VICO MORCOTE

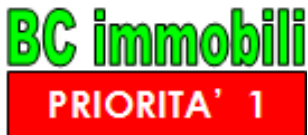

\begin{tabular}{|c|c|c|c|}
\hline foto dell'oggetto & $\begin{array}{c}\text { Iocalizzazione } \\
+ \text { no. bc }\end{array}$ & $\begin{array}{c}\text { cosa rappr.+ misure } \\
\mathrm{cm}\end{array}$ & $\begin{array}{l}\text { precauzioni da } \\
\text { adottare }\end{array}$ \\
\hline & 101 & $\begin{array}{l}\text { DIPINTO MURALE } \\
\text { (dietro scala chiocciola) } \\
\text { altezza: } 109 \\
\text { larghezza: } 94 \\
\text { profondità: } \\
\text { peso: }\end{array}$ & $\begin{array}{l}\text { livello } 1 \\
\text { dipinto murale } \\
\text { rovinato }\end{array}$ \\
\hline & 105 & $\begin{array}{l}\text { TRITIICO SCULTOREO } \\
\text { altezza: } 340 \\
\text { larghezza: } 225 \\
\text { profondità: } \\
\text { peso: }\end{array}$ & $\begin{array}{l}\text { livello } 1 \\
\text { da proteggeresul } \\
\text { posto }\end{array}$ \\
\hline & 146 & $\begin{array}{l}\text { DIPINTO MURALE } \\
\text { Ultima Cena } \\
\text { altezza: } 513 \\
\text { larghezza: } 575 \\
\text { profondità: } \\
\text { peso: }\end{array}$ & $\begin{array}{l}\text { LIVELLO } 1 \\
\text { Affresco piuttosto } \\
\text { rovinato nella parte } \\
\text { centrale }\end{array}$ \\
\hline & 143 & $\begin{array}{l}\text { DIPINTO MURALE } \\
\text { (lunetta) } \\
\text { Padridella Chiesa } \\
\text { altezza: } 300 \\
\text { larghezza: } 490 \\
\text { profondità: } \\
\text { peso: }\end{array}$ & $\begin{array}{l}\text { LIVELLO } 2 \\
\text { In buono stato }\end{array}$ \\
\hline
\end{tabular}

Figura 205 Schede elaborate dalla Sezione Protezione dei beni culturali (PCi/PBC). 

FUTURE LINEE DI RICERCA 
Con lo scopo di approfondire la conoscenza dei prodotti impiegati dai restauratori ticinesi in Canton Ticino sui dipinti murali tra la fine del Ottocento e l'inizio del Novecento, si sono valutate le cause che motivarono il loro utilizzo, la loro durabilità nel tempo e le conseguenze che hanno provocato sugli oggetti dove si è intervenuto, reinterpretandole e confrontandole con i materiali attualmente in uso, si è iniziata una ricerca analitica parallela, che purtroppo non è stata possibile finalizzare e includere nella presente tesi. A partire da circa centocinquanta opere di dipinti murali, restaurate in Canton Ticino dalla fine del XIX sec. alla prima metà del XX, sono stati selezionati dieci casi di studio come oggetti maggiormente esemplificativi delle tecniche d'intervento, impiegate nel suddetto periodo nel territorio svizzero. Le tecniche di analisi fisico-chimico di laboratorio sui casi di studio sopraccitati vogliono confermare o smentire la presenza di questi materiali, fornendo a future ricerche ulteriori dati riguardo agli oggetti da restaurare. I beni culturali scelti sono stati: l'affresco della Crocifissione sul porticato della chiesa di Santa Maria Assunta a Villa Luganese, i dipinti dell'abside della chiesa di San Vigilio a Rovio, gli affreschi interni della chiesa di San Carlo di Negrentino a Prugiasco, gli affreschi dell'abside della chiesa di San Remigio a Corzoneso, le pitture murali della Sala Albertolli nell'omonimo Palazzo di Lugano, gli affreschi della chiesa di San Martino a Quinto, le pitture della controfacciata della chiesa di Santa Maria Assunta a Gorduno, lo strappo raffigurante la Madonna con il bimbo e Santi trasportato alla chiesa di San Carlo a Lugano ed i dipinti della facciata della chiesa della Madonna del Sasso a Morcote, dato che su tutti i manufatti non sono stati eseguiti ulteriori restauri dal momento storico studiato. Finora sono state realizzate le prime indagini mediante tecniche di studio non invasive, alla quale segue un'analisi macroscopica visiva con l'appoggio della fotografia applicando luce riflessa, radente e ultravioletto. Per una maggiore garanzia dei risultati ottenuti da questo studio preliminare, l'analisi si completa con l'impiego di tecniche invasive attraverso il prelievo di micro campioni scelti da punti strategici dei dipinti. Questi campioni saranno oggetto di analisi al microscopio ottico con luce riflessa (MOLR), analisi istochimiche e analisi con lo spettroscopio ad infrarosso (FT-IR).

Un esempio significativo del lavoro di ricerca analitica iniziata, sono gli affreschi interni della chiesa di San Carlo di Negrentino, restaurati da Tita Pozzi fra il 1944 ed il 1945. Secondo la documentazione di archivio analizzata, il pittore attuò sui dipinti mediante il consolidamento degli intonaci con caseinato di calcio, il rifacimento delle dorature, il consolidamento pittorico con silicati, il ritocco pittorico degli azzurri scomparsi, la ricostruzione pittorica delle decorazioni architettoniche e l'impiego della cera per ravvivare le tonalità cromatiche. La documentazione fotografica di archivio consultata, confronta la situazione dei dipinti prima e dopo il restauro. Questa ha consentito di identificare i ritocchi pittorici eseguiti dal restauratore e le zone che hanno subito un ulteriore deterioramento successivo al restauro, con la perdita generalizzata della pittura di ritocco e della materia originale, particolarmente sui dipinti dell'abside maggiore dove l'azione dell'umidità d'infiltrazione pluviale ha creato più danni. Con l'appoggio dell'osservazione a luce radente è stata possibile l'identificazione delle diverse stuccature 
così come riscontrare delle zone lucide, che successivamente sono state confermate con la luce ultravioletta, relative a sgocciolature di una sostanza applicata durante il restauro, presumibilmente cera. Con lo scopo di identificare le caratteristiche e la presenza dei materiali applicati durante il restauro realizzato da Tita Pozzi, sono stati prelevati quattro campioni. Il Campione 1 è stato ottenuto da una zona lacunosa, a destra della figura della Madonna nella scena raffigurante la Natività di Gesù, fra le due absidi, dove la luce UV ha denunciato una particolare fluorescenza giallastra. Malgrado la sicurezza nel prelievo del campione la spettroscopia ad infrarossi (FT-IR) non ha segnalato in modo evidente la presenza di materiali proteici nei risultati dell'intervento di Pozzi. Gli spettri hanno confermato l'esistenza di calcite (carbonato di calcio) nell'intonaco, così come di gesso, ma i dati più interessati vengono dati dalla probabile presenza di ossalati, notata dalla lieve curva fra i $1620 \mathrm{~cm}-1$ ed i $1700 \mathrm{~cm}-1$, così come traccia di cere od oli fra il $2900 \mathrm{~cm}-1$ ed il $2800 \mathrm{~cm}-1$.

Il Campione 2, prelevato dalle tracce di colore blu sull'altare dell'abside maggiore, probabilmente gocciolato durante il ritocco della figura del Cristo redentore, raffigurato sulla tazza absidale, rispose più chiaramente all'analisi di spettroscopia ad infrarossi (FT-IR) con dei picchi intorno a $2918 \mathrm{~cm}-1$ ed a $2849 \mathrm{~cm}-1$, una leggera curva fra il $1370 \mathrm{~cm}-1 \mathrm{ca}$. ed un picco ai $719 \mathrm{~cm}-1$ in corrispondenza alle sostanze cerose.

Anche nel caso degli affreschi dell'abside della chiesa di San Vigilio a Rovio, restaurati fra il 1948 ed il 1949 dal medesimo restauratore, Tita Pozzi, non si è riscontrata documentazione di archivio in cui si menzionino in modo esplicito i materiali impiegati nell'intervento. Tuttavia, uno studio approfondito della prassi esecutiva del pittorerestauratore Tita Pozzi ci permette di approssimare con sicurezza i prodotti che potrebbero essere stati usati sugli affreschi della chiesa di San Vigilio a Rovio. In generale il pittore impiegava la soda caustica per la pulitura degli affreschi, in particolare per asportare il grasso superficiale; il caseinato di calcio veniva usato per il consolidamento dell'intonaco in profondità, così come dei silicati per fluatazione e la caseina per il consolidamento della superficie pittorica. La stuccatura delle lacune veniva di solito eseguita con stucco romano, la reintegrazione pittorica con pigmenti legati con caseina e la finitura dei dipinti mediante la tecnica dell'encausto, per rialzare la tonalità cromatica impallidita successivamente alle fasi di restauro precedenti.

La proposta di restauro che egli aveva realizzato per la presente opera implicava l'asportazione dello strato di calce soprastante gli affreschi, applicato in passato sullo zoccolo decorativo dell'abside, la pulitura dalla polvere e del grasso depositato in superficie e delle macchie di cera di candele ancora oggi presenti sotto le figure della Madonna e degli Apostoli. II consolidamento dell'intonaco staccato e pulverulento a causa dell'azione dell'umidità di infiltrazione d'acqua piovana, la stuccatura delle lacune e la reintegrazione cromatica consistente nella ricostruzione mimetica dei simboli degli Evangelisti, la Mandorla, le frange orizzontali che dividono le scene, i bordi dell'arco trionfale, il semicircolo che incornicia la figura della Madonna e la decorazione dello 
zoccolo. Tutti questi ritocchi furono eseguiti a basso tono. Invece, le lacune di maggiore stesura in corrispondenza alla aree figurative non furono ritoccate.

La prima indagine macroscopica realizzata sugli affreschi a luce diretta e luce radente ha affermato uno stato di conservazione in generale cattivo. Essi si trovano attualmente assai degradati, come lo furono già in passato, a causa dell'umidità. Questa ha originato diverse lacune sui ritocchi pittorici eseguiti negli anni Quaranta dal Pozzi così come della pittura originale. Inoltre i sali presenti nell'intonaco hanno originato delle macchie scure che complicano ancora di più la lettura delle pitture. Si sono verificati due tipi di stuccature in costituzione materica (granulometria degli inerti presenti nella malta e colore), come per il metodo di applicazione. La stuccatura di caratteristiche più simili all'originale è stata datata all'intervento di Tita Pozzi, mentre che il secondo tipo di stuccatura si è identificato come un intervento posteriore, non documentato, applicato in modo più irregolare al primo ed di conseguenza particolarmente visibile. L'osservazione con luce ultravioletto degli affreschi ha attestato la presenza di sostanze non originali. Hanno emesso una risposta particolarmente fluorescente alla illuminazione con le lampade di Wood le tracce di cera corrispondenti alle lampade che in passato furono sottoposte alle figure della Madonna e degli Evangelisti. In modo più discreto hanno risposto agli UV zone puntuali nelle figure assai deteriorate sotto l'arco trionfale.

Per confermare la presenza di sostanze proteiche o silicati, applicati da Tita Pozzi nel suo intervento di restauro, sono stati prelevati 4 campioni. Il Campione 1, è stato estratto da una zona visibilmente diversa in colorazione e lucidezza dal resto del dipinto nella manica di uno degli Evangelisti (non identificabile) a destra sotto l'arco trionfale. In questo punto I'osservazione con l'UV ha evidenziato la presenza di sostanze non originali. Lo spettro ad infrarossi (FT-IR) di questo campione è stato molto concreto, mostrando oltre alla presenza della calcite (carbonato di calcio) dell'intonaco ed alle tracce di gesso, picchi intorno a $2917 \mathrm{~cm}-1$ e $2849 \mathrm{~cm}-1$ corrispondenti alle sostanze cerose applicate sulla superficie pittorica e una lieve curva a circa $1000 \mathrm{~cm}-1$ relativa alla presenza di silicati.

Lo FT-IR del Campione 2, prelevato da una scaglia di pellicola pittorica staccata nelle vicinanze delle lacune di sinistra sulla veste del Pantocrator, al centro della tazza absidale, ha mostrato solo dei risultati riguardanti la presenza di carbonato di calcio e di gesso. I campioni 3 e 4, prelevati dalla veste azzurra della Madonna e dallo zoccolo rispettivamente, sono stati anch'essi sottoposti alle indagini di spettroscopia (FT-IR). II Campione 3 ha mostrato picchi in corrispondenza alla presenza di carbonato di calcio, di gesso e di ossalati, mentre il Campione 4, oltre ai picchi corrispondenti al carbonato di calcio ha evidenziato tracce di ossalati e di composti a base di silice con delle lieve curve fra i $1410 \mathrm{~cm}-1$ ed i $1500 \mathrm{~cm}-1$ (ossalati), e fra i $1000 \mathrm{~cm}-1$ ed i $1100 \mathrm{~cm}-1$. 


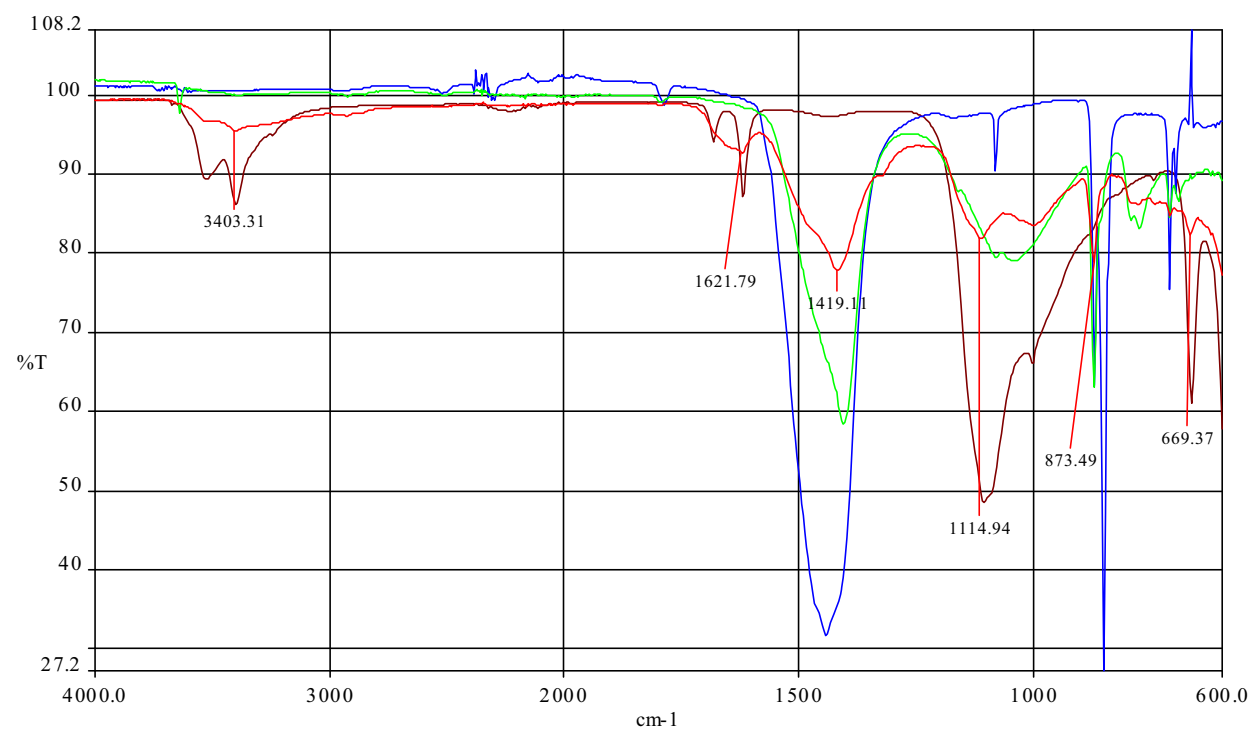

Figura 206 Confronto dello spettro FT-IR del Campione 1 San Carlo Negrentino con gli standards: campione 1, silicato di sodio $+\mathrm{CaCO}^{3}$, carbonato di calcio $\left(\mathrm{CaCO}^{3}\right)$, gesso.

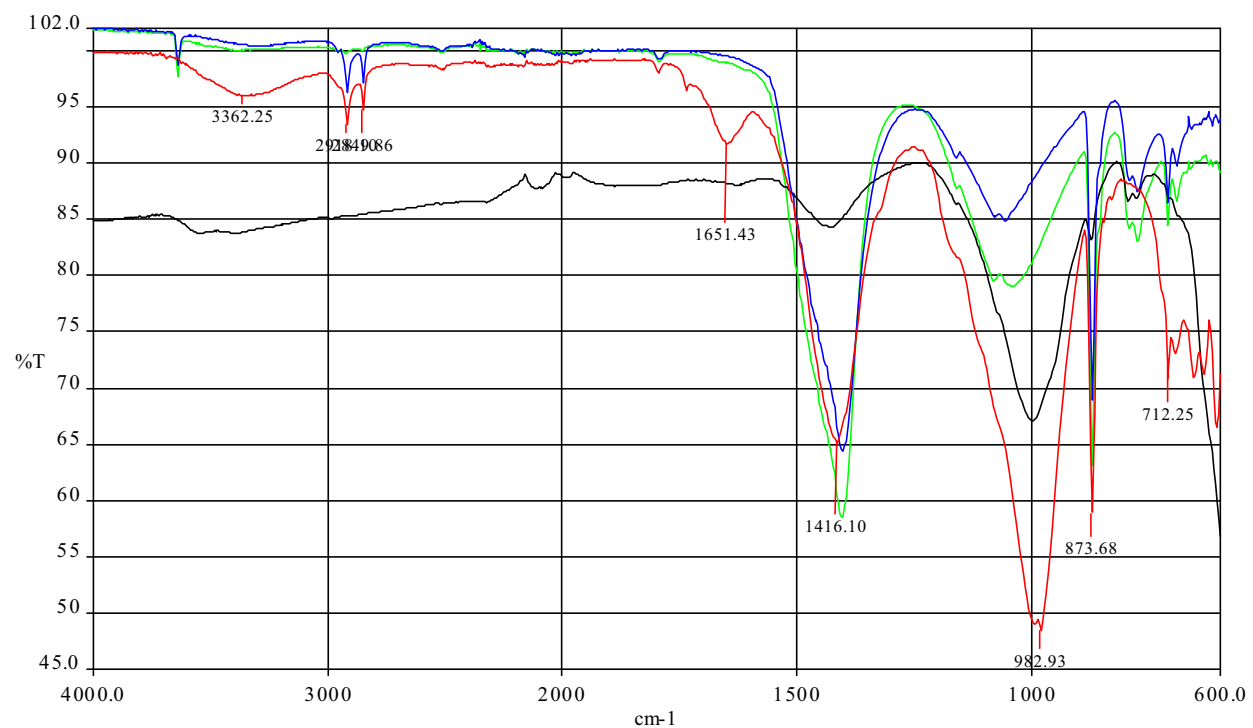

Figura 207 Confronto dello spettro FT-IR del Campione 2 San Carlo Negrentino con gli standards: campione 2, silicato di sodio $+\mathrm{CaCO}^{3}$, paraffina+benzina rettiicata, ossido di ferro 


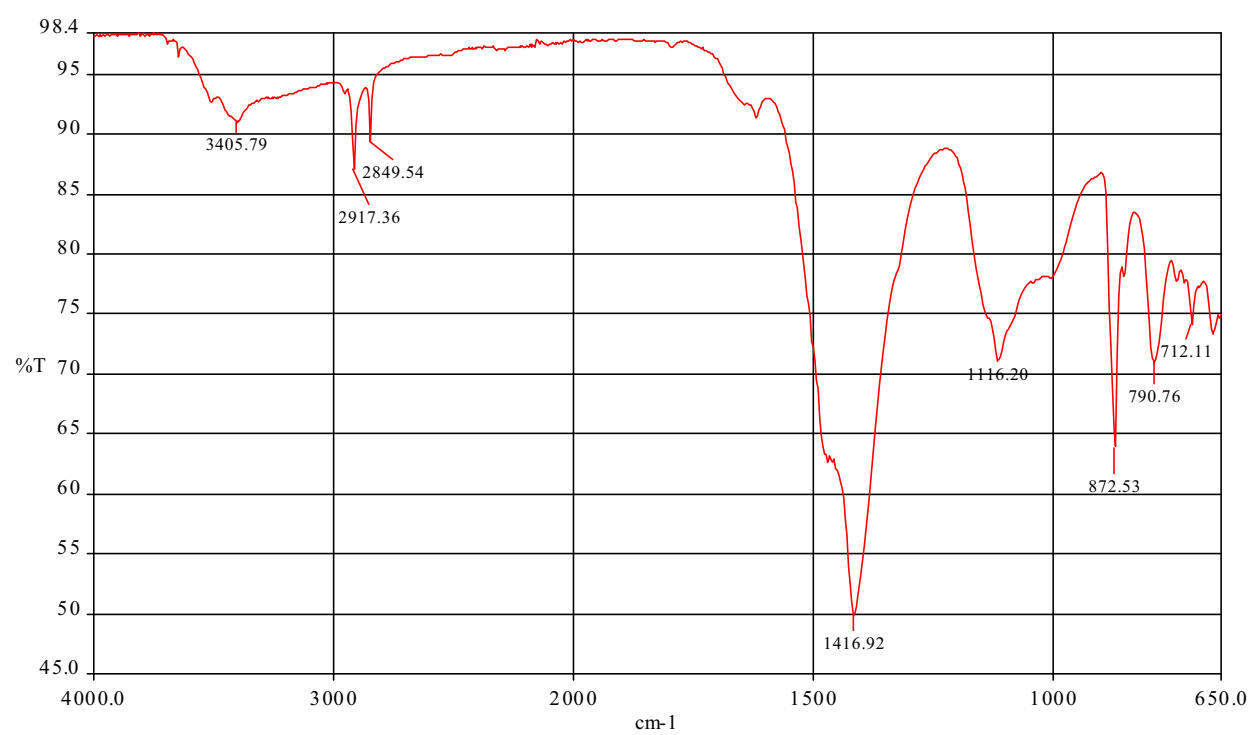

Figura 208 Confronto dello spettro FT-IR del Campione 1 Rovio con gli standards: campione 1

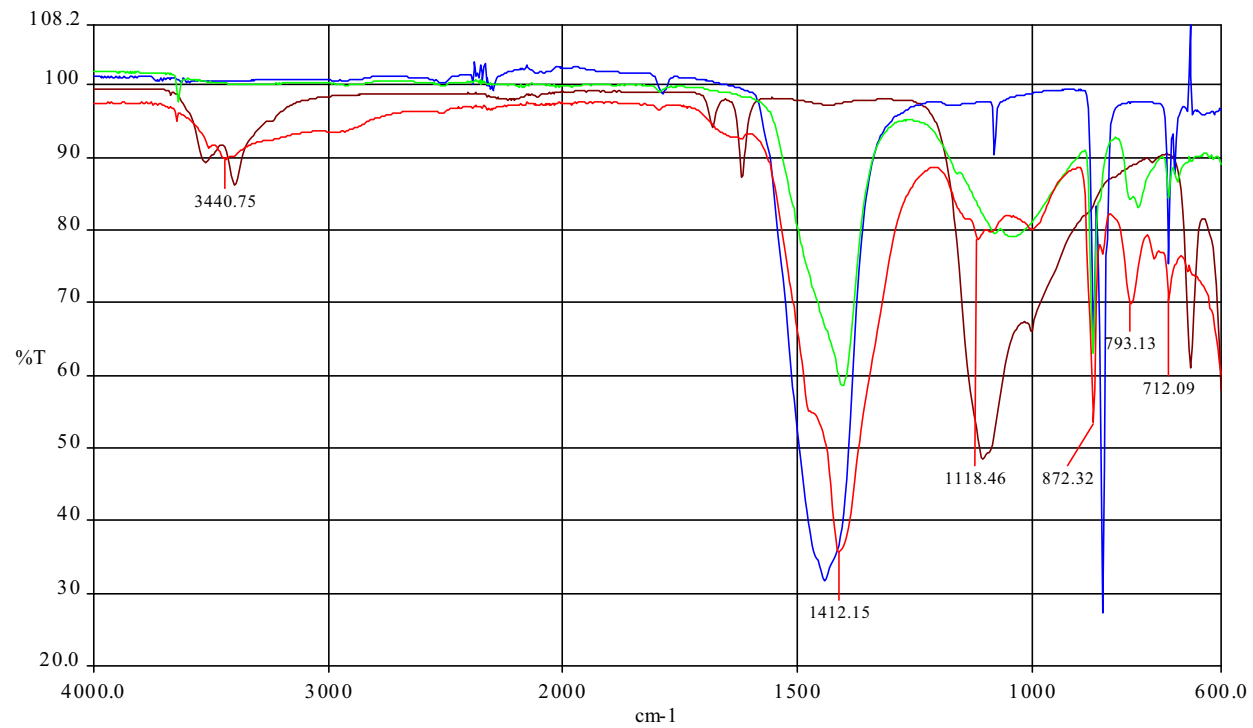

Figura 209 Confronto dello spettro FT-IR del Campione 4 Rovio con gli standards: campione 4, silicato di sodio $+\mathrm{CaCO}^{3}$, carbonato di calcio $\left(\mathrm{CaCO}^{3}\right)$, gesso 
Un terzo caso di studio, la cui analisi è stata conclusa, è il complesso pittorico dell'Oratorio di San Martino a Deggio, i cui affreschi furono puliti dello strato di calce superficiale dal pittore-restauratore Emilio Ferrazzini nel 1938. I dipinti che oggigiorno sono stati trovati in un mediocre stato di conservazione a causa dell'azione dell'umidità che agisce sull'intero edificio in maniera molto evidente, specie sul versante nord, ha originato dei forti degradi ancora più accentuati in conseguenza ai materiali di restauro impiegati nell'ultimo intervento eseguito dal Ferrazzini. Gli affreschi dell'abside sono completamente illeggibili: danneggiati da molteplici abrasioni create sulla superficie nel momento dell'asportazione dello strato di calce soprastante i dipinti durante l'impiego negligente degli utensili metallici, nonché non completamente puliti del medesimo scialbo dove questo strato si presentò più duro e difficile da levare o, semplicemente, trascurato per questioni di tempo e di economia. Il danno più grave è stato però causato dalla "saldatura dei bordi" realizzata in cemento e dal consolidamento della superficie pittorica o rinvenimento delle tonalità impallidite con cera. Inoltre "I'encausto", eseguito a quadri marcati, come se si trattassero di prove più che di un trattamento definitivo delle decorazioni, rende ancora più visibile, eterogeneo ed illeggibile il complesso pittorico dell'Oratorio. I ritocchi pittorici di ricostruzione imitativa, sorpassano la pittura originale. Le indagini macroscopiche con luce naturale (non fu possibile accedere alla corrente elettrica per esaminare con luce radente e con luce UV gli affreschi) prova facilmente le diverse zone di ritocco e di consolidamento con sostanze cerose in una di queste zone, esattamente sul libro rappresentato sulla parete sinistra dell'abside. Anche in questo caso, uno dei campioni prelevati (Campione 2) del libro raffigurato sull'abside è stato sottoposto ad analisi spettroscopici (FT-IR) che dichiarano in modo evidente la natura della cera applicata dal restauratore nel 1938. Si tratta in particolare di paraffina, come dichiarano i picchi ben pronunciati intorno ai $2955 \mathrm{~cm}-1,2916 \mathrm{~cm}-1,2848 \mathrm{~cm}-1,1472 \mathrm{~cm}-1,1462 \mathrm{~cm}-1$, a 729 cm-1 e $718 \mathrm{~cm}-1$ così come una leggera curva, in parte nascosta dai picchi della calcite (CaCO3), sui $1378 \mathrm{~cm}-1$ circa. Questi risultati, nel Campione 1 sono più discreti poiché la presenza di cera venne offuscata dal carbonato di calcio contenuto nell'intonaco trovatosi in maggior quantità nella campionatura prelevata. In ogni modo sono stati riscontrati pure su questo campione dei piccoli picchi intorno a $2917 \mathrm{~cm}-1,2849 \mathrm{~cm}-1$ ed un piccolo picco sui $1470 \mathrm{~cm}-1$ ed ai $1460 \mathrm{~cm}-1$ circa, corrispondente alle medesime sostanze cerose.

Purtroppo per il momento, ancora non si sono ottenuti risultati che confermino l'impiego di altre sostanze per il consolidamento, come la caseina o la gomma arabica, sperando che futuri analisi rafforzino le conclusioni riguardo le prassi esecutive del restauro dei dipinti murali in Canton Ticino a inizi del Novecento. 


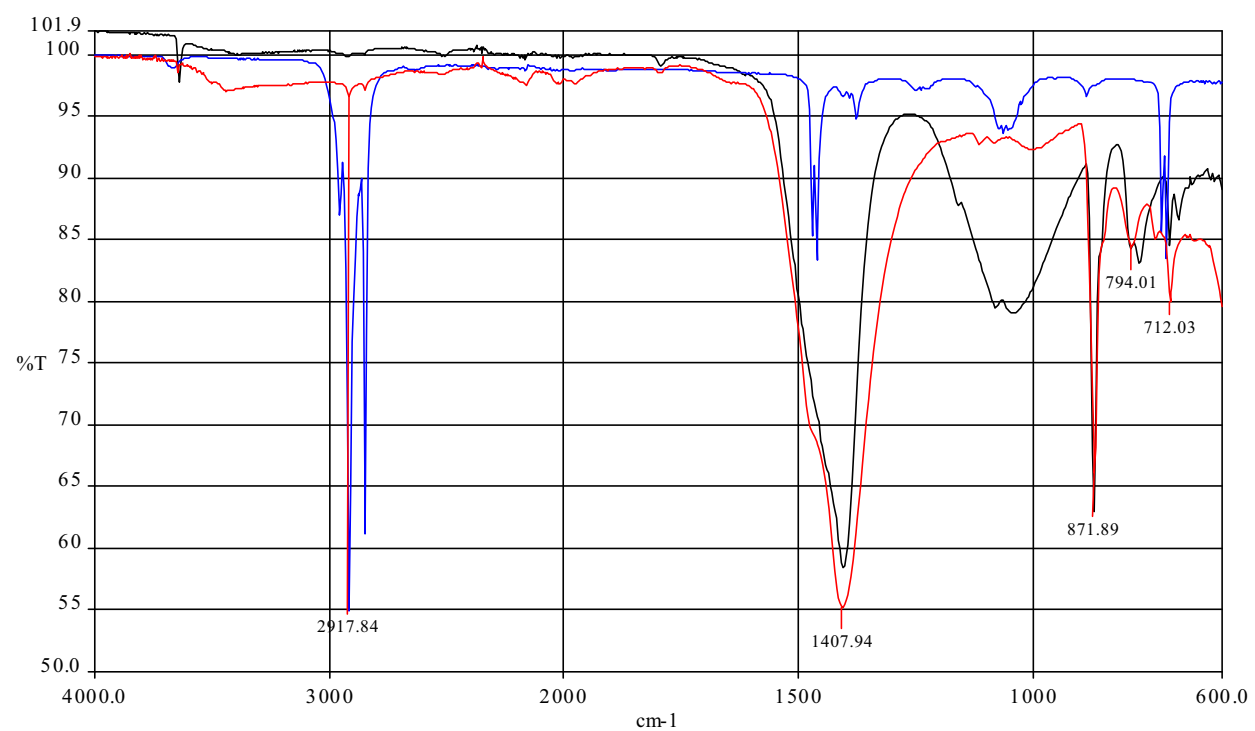

Figura 210 Confronto dello spettro FT-IR del Campione 1 Deggio con gli standards: campione 1, silicato di sodio $+\mathrm{CaCO}^{3}$, paraffina

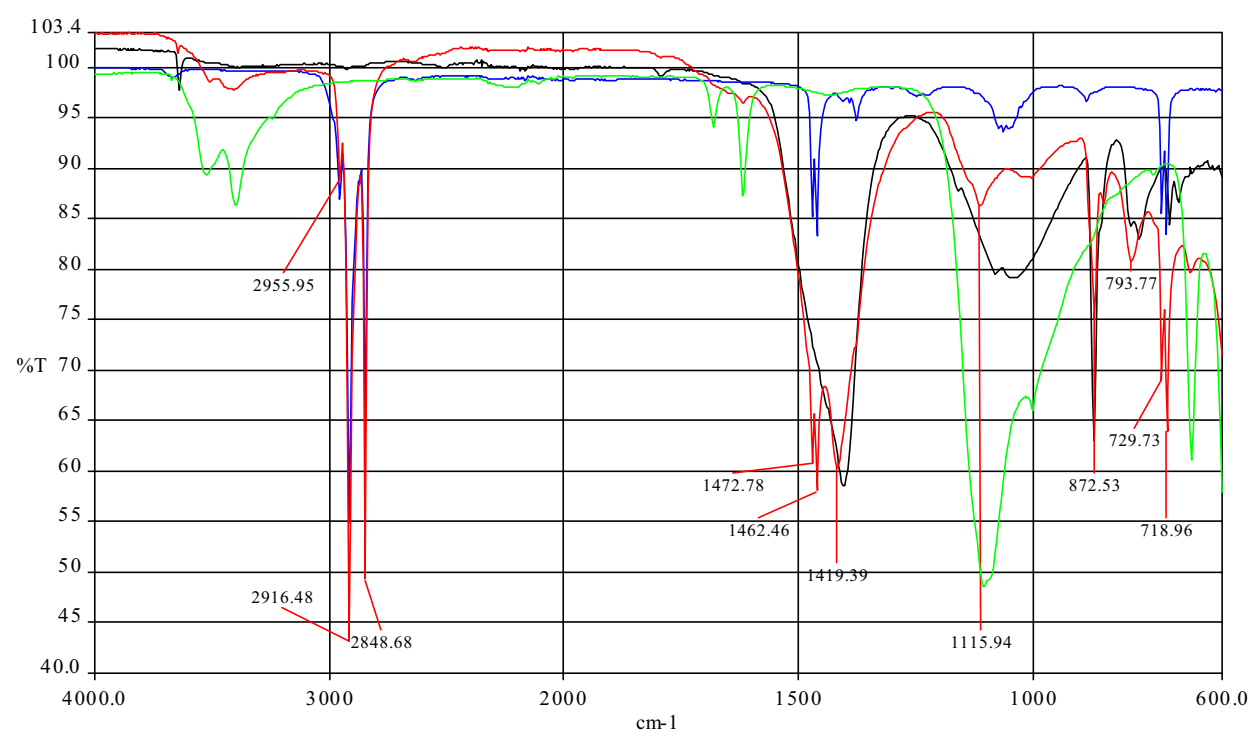

Figura 211 Confronto dello spettro FT-IR del Campione 2 Deggio con gli standards: campione 2, silicato di sodio $+\mathrm{CaCO}^{3}$, gesso, paraffina 
Restauratori in Canton Ticino fra Ottocento e Novecento

Catalogazione e gestione dati 
CONCLUSIONI 
Le prassi di lavoro impiegate lungo il periodo compreso tra la fine dell'Ottocento e la prima metà del Novecento in Canton Ticino, analizzato nella presente tesi dottorale, si sono concretizzati specialmente negli anni Trenta e Quaranta del XX secolo, quando gli interventi di restauro sono stati più numerosi. L'informazione ottenuta dall'intensa ricerca storica di archivio preliminare ha permesso di identificare alcuni dei più frequenti materiali usati nel restauro dei dipinti murali, ma non solo, poiché questa tesi è riuscita a definire le basi principali per future ricerche specifiche. In questo modo sono stati concretizzati $i$ momenti di inizio, di fine e di climax della storia del restauro in Canton Ticino, prima dell'epoca moderna. Si tratta di momenti decisivi, ottenuti dall'identificazione delle persone che hanno fatto possibile l'avvenimento di essi, così come le loro relazioni professionali e anche personali. In questo senso si può confermare con compiacenza che gli obiettivi prefissati per l'elaborazione di questa ricerca sono stati raggiunti con successo, anche se le difficoltà ritrovate, specie dalla scarsa e dispersa documentazione di archivio, sono state in certi momenti scoraggianti.

Ora che si redigono queste conclusioni, con relativa distanza dal momento in cui fu progettato il lavoro, si percepisce l'enorme valore che a volte una frase che permette di relazionare due persone, così come una fotografia che diventa testimone dello stato di un determinato bene artistico, o una fattura con l'elenco di alcuni materiali impiegati nel lavoro, può aiutare a concepire le circostanze storiche e che, per i restauratori così come per gli storici, chimici, architetti e ricercatori in genere, significa capire l'oggetto di studio in sé, procurando delle decisioni e analisi dei lavori da eseguire più adatte.

I documenti che in maggior misura hanno favorito l'arricchimento, nella conoscenza delle circostanze che influivano sulla concezione del restauro in Canton Ticino, particolarmente nella prima metà del Novecento, sono stati i diversi articoli di polemica pubblicati nelle riviste di attualità artistica ticinese, come ad esempio la Rivista Storica Ticinese e la Rivista Tecnica d'Arte. Essi, insieme all'eterogenea documentazione scritta e grafica che conforma gli archivi dell'Ufficio dei Beni Culturali del Canton Ticino, con sede a Bellinzona, e l'Archivio Federale dei Monumenti Storici a Berna, hanno permesso di sviluppare una ampia base di dati.

Riguardo l'attività dei diversi restauratori qui identificati, si è dimostrato come, anche se la formazione primaria di tutti loro (ad eccezione dell'italiano Bruno Abbiati) fu quella di pittori, alcuni di essi come Edoardo Berta, Emilio Ferrazzini e Tita Pozzi assumono un ruolo specialmente decisivo nella protezione dei beni culturali in Canton Ticino. Se l'architetto ticinese Augusto Guidini era stato il primo a lottare per la rivalutazione del patrimonio nel territorio, appoggiato da pittori come Ernesto Rusca, Luigi Rossi o da operatori specializzati come i fratelli Steffanoni da Bergamo, dopo di lui, con una recentemente imposta legge per la protezione dei monumenti e la nascita della Commissione Cantonale dei Monumenti Storici, fu Edoardo Berta ad imporsi eseguendo i primi e più ambiziosi progetti di restauro, affiancato dai giovani allievi della scuola di disegno. L'inizio del Novecento con Augusto Guidini era stato segnato dal restauro della Cattedrale di San Lorenzo a Lugano $(1902$ - 1910) con un importante progetto di decorazione murale 
all'interno del manufatto, ma a questo intervento proseguiranno fino al 1930 il ripristino architettonico e pittorico di importanti monumenti, come la chiesa di S. Maria degli Angioli a Lugano, la chiesa di S. Maria delle Grazie a Bellinzona, la chiesa di S. Biagio a Ravecchia ed il Castello Visconteo di Locarno, con la direzione di Edoardo Berta. Gli ideali dell'epoca erano: la riattazione dei beni storici agli ordini primari, medievali, cancellando così le testimonianze delle più recenti epoche a favore di una omogeneità stilistica e decorativa. A questo scopo le ricerche archeologiche furono numerose ed in concreto nell'ambito dei dipinti murali, Edoardo Berta assieme al collega pittore Augusto Ortelli ed agli studenti Emilio Maccagni, Emilio Ferrazzini e Mario Ribola, realizzano una fondamentale campagna di scoperta. In queste campagne di lavoro si instaurarono tre attività fondamentali come prassi per l'esecuzione del restauro pittorico: in primo luogo l'esaustiva ricerca storica che corroborasse l'interesse e l'importanza dei dipinti, secondo il processo di descialbo delle pitture con mezzi meccanici (spazzole di metallo, scalpelli e martelline) o "umidi" (acido acetico o soda caustica), poiché la maggioranza di loro si trovavano coperti da strati di calce e in terzo luogo, giacché le tecniche di pulitura precedentemente descritte provocavano solitamente lo sbiadimento dei dipinti, il consolidamento o meglio "ravvivamento" mediante sostanze cerose (cera d'api o paraffina) o con delle gomme (gomma arabica) per lucidare le superficie degradate. Le parti mancanti ed il consolidamento degli intonaci veniva effettuato in quasi tutti i casi studiati da muratori. Alcune volte essi furono responsabilizzati anche dalle fasi di descialbo.

I materiali e le tecniche impiegate nelle diverse attività di restauro dei dipinti murali furono sempre quelle tradizionalmente apprese, così come erano stati concepiti a metà dell'Ottocento in Italia dal primo trattatista in questo ambito: il conte bergamasco Giovanni Secco Suardo. Si tratta delle medesime prassi di lavoro che Edoardo Berta insegnò all'interno dei cantieri, uniche scuole di restauro all'epoca.

Dopo la morte di Berta fu il pittore Emilio Ferrazzini ad occuparsi della direzione di numerosi interventi di ripristino di monumenti e di restauri pittorici. Grazie ai documenti da lui scritti, è stato possibile provare l'impiego della cera fino agli anni Quaranta circa, come materiale di finitura principalmente usato in Canton Ticino. Solo dopo, nella seconda metà del Novecento, storici come Piero Bianconi e Aldo Crivelli criticarono questa tecnica, e richiederanno l'impiego della gomma arabica in sua sostituzione, secondo i consigli del rinominato restauratore Mauro Pellicioli.

Ferrazzini impiegò metodicamente le sostanze cerose in quasi tutti i restauri di dipinti murali: cera d'api e paraffina per le pitture in ambiti interni, e cera ozokerite o ceresina per gli esterni. Egli usa il termine "tecnica dell'encausto" per descrivere tale attività, che sarà adottata anche da altri collaboratori come Tita Pozzi e Nino Facchinetti. Questo sistema di ravvivamento fu di fatto duramente criticato alla fine degli anni Quaranta da quelli che la Commissione Cantonale dei Monumenti Storici del Canton Ticino riteneva esperti del restauro; si trattava di personalità provenienti dall'estero come Mauro Pellicioli da Bergamo, Jean Verrier da Parigi e Ambrogio Annoni da Milano. Però le prassi di 
"finitura" dei dipinti murali non venirono abbandonate fino agli anni Settanta circa, per quanto abbiamo potuto comprovare dai documenti. La cera venne sostituita a questo fine dalla gomma arabica, come accennavamo alcune righe prima, per essere considerata meno invasiva. Pochi sono stati i casi invece nei quali abbiamo potuto corroborare I'impiego della caseina per il restauro. Solo nei documenti di Tita Pozzi, particolarmente dettagliati, troviamo riferimento all'uso del caseinato di calce e dei silicati mediante fluatazione per il consolidamento degli intonaci.

La reintegrazione pittorica ebbe senza dubbio un carattere molto diverso all'epoca da quanto anni più tardi, con l'arrivo delle dottrine di Cesare Brandi e del restauro moderno in Canton Ticino, sarà adottato. Nei primi anni del Novecento, non esistevano le voci "reintegrazione" o "ritocco", e la fase di riempimento pittorico delle lacune veniva espresso con il semplice termine di "restauro". Questo consisteva nella ridipittura delle parti abrase e degradate mediante velature di colore a caseina o a tempera. Non si realizzava nessuna differenziazione particolare fra le parti originali e quelle reintegrate, anche se abbiamo potuto constatare in numerosi documenti come la Commissione Federale dei Monumenti Storici, nella persona di Albert Naef, richiedeva sempre un minimo intervento di ritocco pittorico sulle parti figurative ed il rifacimento invece nelle raffigurazioni architettoniche. Inoltre, sappiamo anche da fonti scritte, che già nei primi del Novecento si parlava di tonalità neutre per il ritocco delle lacune. II colore considerato neutro all'epoca era il grigio, ma l'impiego di questo è stato minimamente riscontrato nei casi catalogati.

In linea di massima queste erano le prassi per il restauro dei dipinti murali, fino agli anni Sessanta circa. Fu, dopo le polemiche originatesi negli anni Quaranta del Novecento, che la Commissione Cantonale dei Monumenti Storici decise di adottare delle nuove regole. II cambio provoca principalmente una scarsa partecipazione da parte dei restauratori nelle decisioni riguardanti i metodi ed i materiali di restauro da impiegare. L'esempio più chiaro lo troviamo nel restauro degli affreschi della chiesa dei SS. Pietro e Paolo a Biasca, dove lavorano Mario Moglia, Bruno Abbiati e Sergio Maino, dove I'intervento venne stabilito dalla CCMS.

Le nuove correnti influiranno in pittori-restauratori come Carlo Cotti, che a causa delle discrepanze professionali smette la sua collaborazione con Emilio Ferrazzini, a favore di un restauro conservativo. Anche Carlo Mazzi, allievo come Bruno Abbiati del restauratore milanese Mario Rossi, opta per interventi più curati e contenuti.

Il secondo obiettivo proposto dal presente lavoro è stato quello di indagare nei sistemi di catalogazione e gestione dei dati più innovativi, per confrontargli e analizzare le possibilità del loro sviluppo nella registrazione e divulgazione della storia del restauro. Fra questi, in particolare il sistema di database AR.I.S.T.O.S (Archivio informatico per la storia della tutela della opere storiche artistiche) impiegato dal Ministero per i Beni e le Attività Culturali in Italia e la banca dati del Servizio Inventario dell'Ufficio dei Beni Culturali del Canton Ticino in Svizzera, ha consentito di provare nella pratica le caratteristiche efficaci 
del suo utilizzo, risultando essere completamente soddisfacenti poiché soddisfano i requisiti richiesti da noi in anticipo: la catalogazione completa secondo le norme di terminologia internazionali (ISO) dei dati essenziali riguardo la tutela dei beni storici studiati, la visualizzazione on-line così come il libero accesso dagli utenti e la stampa o l'acquisizione dei dati relazionati a dipendenza delle esigenze. Sebbene altre banche dati sviluppate con le stesse premesse, come RES.I o la Banca dati dell'UBC del Canton Ticino, dimostrano essere dei sistemi altrettanto innovativi e complessi, il fattore dell'accessibilità illimitata al sistema che caratterizza in particolare AR.I.S.T.O.S., fa di quest'ultimo uno strumento con maggiori capacità e utilità per più persone, e soprattutto compie, più che un progetto conservativo della documentazione, un proposito di tramando della memoria con un dispositivo così prossimo a quasi tutti come è internet, che ci permette di consultare contemporaneamente diversi archivi senza trasferirci dal posto di lavoro.

La presente tesi dottorale ha consentito di iniziare, mediante I'impiego di AR.I.S.T.O.S., la catalogazione ed il riordino dei dati che interessano la storia della tutela dei beni culturali, ed in particolare del restauro dei dipinti murali in Canton Ticino, lavorando essenzialmente con delle fonti quali proposte di lavoro, preventivi, verbali, relazioni di lavoro e corrispondenza di ogni forma, con la quale si consente la interrelazione dei personaggi, enti, beni artistici, beni storico-artistici che permettono di concretizzare gli "eventi". Questi ultimi, entrando in dialogo all'interno della banca dati, permetteranno in futuro di possedere uno strumento informatico facilmente consultabile dove analizzare, studiare o completare con nuove ricerche, la ricostruzione della storia del restauro dei dipinti murali in Canton Ticino fra l'Ottocento e il Novecento. I dati inseriti e lavorati in futuro saranno relazionati con la già menzionata banca dati Cantonale dell'UBC, per la catalogazione dei beni culturali tutelati, mediante il riferimento all'interno di AR.I.S.T.O.S dei codici di identificazione dei beni culturali protetti dal Cantone.

Attualmente gran parte dell'area settentrionale dell'Italia si è unita al progetto ARTPAST, per la digitalizzazione del patrimonio, assieme a istituzioni come l'Università Politecnica di Valencia e la Scuola Universitaria Professionale della Svizzera Italiana. La collaborazione futura di più organismi sarà inoltre un beneficio di una miglioria nelle applicazioni della banca dati, ancora in fase di elaborazione, che con il tempo si riadatterà alle esigenze degli utenti. Sono ancora da incorporare ad AR.I.S.T.O.S, ad esempio, sistemi di georeferenziazione, come quello impiegato dall'UBC del Canton Ticino. Sarebbe anche interessante introdurre nel programma la possibilità di acquisire i dati introdotti nella piattaforma in formati digitali, idonei ad una successiva modifica prima dell'eventuale stampa. 
Senza dubbio, uno strumento informatico di questo tipo può risultare molto pratico per restauratori, storici e scienziati, ma permetterlo significa anche diffondere il suo utilizzo. Le banche dati possono sopravvivere soltanto grazie alla loro espansione ed al loro continuo aggiornamento, perciò in futuro dovrebbe essere previsto, se non l'utilizzo di un unico sistema, la riaggrupazione di quelli più vasti a livello nazionale ed internazionale. Questo consentirebbe inoltre, agli interessati alla tutela dei beni culturali, di avere un numero più ampio di informazioni suscettibili ad essere confrontate e senz'altro di rivalorizzare il patrimonio mediante una maggiore conoscenza della sua storia. 
Restauratori in Canton Ticino fra Ottocento e Novecento

Catalogazione e gestione dati 
APPARATI 


\section{FONTI DI ARCHIVIO}

\section{Archivio federale dei monumenti storici, Berna , AFMS BNS}

Sono stati analizzati sistematicamente i libri manoscritti di Albert Naef, tutta la collezione grafica riguardante il Canton Ticino ed i Grigioni italiani oltre che il Fondo Bern Kanton Tessin (fascicoli 7982). Le fonti più importanti citate nei testi della tesi sono:

\section{Bern K Tessin A-M n.79}

(San Paolo, Arbedo)

- Confederazione svizzera. Contratto di lavoro indirizzato a Mr. Guidini e Mr. G. Stefannoni. 31 luglio 1899.

- Guidini Augusto. Relazione e Collaudo. Chiesa di S. Paolo in Arbedo e di Pianezzo. (Antiche Pitture scoperte e restaurate). Indirizzato alla al Lodevole DPE, Bellinzona. Milano, 15 settembre 1899.

- Guidini Augusto/ Rossi Luigi, lettera indirizzata al lod. DPE, Milano 6 gennaio 1901.

\section{Bern K Tessin M-P n.80}

\section{(Santa Maria del Sasso, Morcote)}

- Cavenaghi L., Lettera indirizzata al Dipartimento della pubblica educazione riguardo il restauro del coro primitivo alla chiesa di Santa Maria del Sasso a Morcote, $1891 \mathrm{ca}$.

\section{Bern K Tessin Bellinzona $\mathbf{n . 8 2}$}

Schmid, arch. Ispezione del 2 aprile 1911 alla Chiesa di S. Biagio (Ravecchia) indirizzata al Prof. Ortelli a Bellinzona, 22 marzo 1911.

- Guidini Augusto. Lettera indirizzata ad Albert Naef. 12 giugno 1907.

- Annoni F. Preventivo di pulitura della pala d'altare a S. Biagio, Ravecchia, indirizzato a Edoardo Berta. 20 luglio 1909.

\section{Archivio di Stato del Canton Ticino, Bellinzona}

L'analisi dei due fascicoli che compongono la sezione Monumenti Storici del Fondo Dipartimento della Pubblica Educazione del Canton Ticino dell'Archivio di Stato del Canton Ticino con sede a Bellinzona, ha segnalato le seguenti fonti, fra le più interessanti del medesimo archivio, per la stesura del presente lavoro:

\section{ASTi, Fondo DPE. Monumenti Storici.}

Diversi 1905 - 1955, Segnatura

\subsection{2.}

- AA.VV. Lettera. Circa l'istituzione d'una scuola per le Belle Arti nel nostro Cantone. Milano 19 aprile 1887.

- Steffanoni G., Lettera indirizzata all'llmo. Sig. Arch. Comm. A. Guidini, Milano riguardo l'esecuzione dei lavori alla chiesa di S. Paolo ad Arbedo ed a Pianezzo. Bergamo, 28 giugno 1899. 
- Annoni Francesco, Preventivo di risarcimento dell'Ancona lignea della Pietà, indirizzato ad Edoardo Berta, chiesa dalla Madonna del Sasso. Milano 14 luglio 1913.

- Hoepli Ulrico. Cartolina indirizzata al Dipartimento della Pubblica Educazione di Bellinzona, Milano 2 febbraio 1915

- Stefannoni G., Lettera indirizzata alla CCMS in richiesta di informazione per i lavori di restauro della tavola del Bramantino nella chiesa della Madonna del Sasso a Locarno, richiesti dalla suddetta commissione con lettera in data 20 giugno 1916. Rovigo (Accademia dei Concordi), 20 luglio 1916.

- Steffanoni F. Cartolina indirizzata alla CCMS in richiesta di informazione per i lavori di restauro della tavola del Bramantino nella chiesa della Madonna del Sasso a Locarno. Rovigo (Accademia dei Concordi), 7 agosto 1916.

- Chiesa F. Lettera richiedendo autorizzazione per chiamare ai fratelli Stefannoni a controllare lo stato di conservazione della tavola del Bramantino alla Chiesa della Madonna del Sasso di Locarno e la chiesa di Santa Maria in Selva. Bernandino 31 luglio 1916.

- Naef Albert, Baptistère de Riva San Vitale. Rapport l'inspection du 23 août 1922. Lausanne 30 août 1922.

- Naef Albert, Eglise de Cadro (Tessin). Rapport de l'inspection di mardi 22 août 1922. Lausanne, le 28 août 1922. Visita precedente il 25 giugno 1920 con rapporto in data 20 luglio 1920.

- $\quad$ Naef Albert, Eglise de Ste. Marie des Anges, à Lugano. Rapport de I'inspection du mardi 22 août 1922. Lausanne 27 août 1922.

- Naef Albert, Eglise de Sonvico (Tessin). Rapport relatif à l'inspection du 22 août 1922. Lausanne 28 août 1922.

- Naef Albert, Oratoire de San Rocco, à Bissone (Tessin) Rapport de l'inspection du 23 août 1922. Lausanne 30 agosto 1922. Ispezione realizzata insieme a Francesco Chiesa ed Edoardo Berta.

- Ferrazzini Emilio, Fattura indirizzata al Maestro Giuseppe Rovelli. 20 maggio 1931.

- Beretta, Emilio Maria, Lettera indirizzata alla CCMSA, riguardo la richiesta dal Municipio di Muralto del 31 marzo del 1941 per l'allestimento del progetto di restauro della Collegiata di San Vittore a Muralto. Muralto 11 ottobre 1941.

\section{Fascicolo XXVIII cartella 1 Monumenti Storici (1848...).}

- $\quad$ Berta E., Lettera del 28 ottobre 1915 indirizzata alla Commissione dei Monumenti Storici ed Artistici ed al DPE riguardo i lavori di restauro alla Cappella dei Morti a Semione.

- Berta E., Lettera indirizzata al DPE riguardo il restauro della Cappella dei Morti a Semione, Bironico, 12 Novembre 1916 
- Naef Albert, Baptistère de Riva San Vitale. Rapport l'inspection du 23 août 1922. Lausanne 30 août 1922.

\section{Archivio Ufficio dei beni culturali, Bellinzona}

Sono stati sistematicamente analizzate per la stesura dei testi le filze: s.005 Aquila, s. 008 Arogno, s.010 Arzo, s.011, Ascona, s.021 Bellinzona, s.024 Biasca, s.026 Bignasco, s.029 Bironico, s.030 Bissone, s.039 Brione Verzasca, s.041 Croglio, s.044 Brusino-Arsizio, s.047 Cademario, s.054 Camignolo, s.059 Campo Vallemaggia. Cimalmotto, s.065 Carona, s.067 Caslano, s.069 Castel San Pietro, s.071 Cavagnago, s.085 Coldrerio, s.091 Corzoneso, s.095 Cugnasco, s.112 Giornico, s.113 Giubiasco, s.117 Gordola, s.118 Gorduno, s.127 Isone, s.129 Largario, s.134 Locarno, s.140 Lottigna, s. 143 Lugano, s.148 Mairengo, s.149 Malvaglia, s.162 Miglieglia, s.165. Moleno, s.172 Morcote, s.176 Muralto, s.180 Novazzano, s.182 Orselina, s.196 Ponte Capriasca, s.198 Ponto Valentino, s.201 Prato Sornico, s.204 Prugiasco, s.206 Quinto, s. 209 Riva San Vitale, s.213 Rovio, s.226 Semione, s.216 Russo, s.246 Verscio, s.248 Vezio, s.251 Villa Luganese.

Sono state citate nella presente tesi le fonti ritenutesi più importanti estratte dalle medesime filze di archivio:

\section{AUBCBZ, s.021 Bellinzona.}

(San Biaggio, Ravecchia)

- Annoni Francesco, Preventivo di restauro della Pala d'Altare nella Chiesa di San Biagio in Ravecchia a
Edoardo Berta, Lugano. Milano 20 Iuglio 1909.

- Berta Edoardo, Proposta di restauro indirizzata al Lodevole Dipartimento della Pubblica Educazione, Bellinzona. Lugano luglio 1909.

- Berta Edoardo, Proposta di restauro della Pala d'Altare della Chiesa di San Biagio a Ravecchia indirizzata al Lodevole Dipartimento della Pubblica Educazione, Bellinzona. Lugano luglio 1909.

- Commissione Cantonale Monumenti Storici e Artistici. Chiesa di San Biagio a Ravecchia. Bellinzona 19101914.

- Soldati Silvio, socio della compagnia di costruzione Brenni e Soldini. Compiti metrici e perizia descrittiva delle opere ricorrenti per il restauro della Chiesa Parrocchiale di Ravecchia, Lugano 19 giugno 1913. AUBCBZ, s. 021 Bellinzona.

- Berta Edoardo, Relazione sui lavori di restauro della Chiesa di S. Biagio in Ravecchia indirizzato al Lodevole Dipartimento della Pubblica Educazione, Bellinzona. Bironico 6 agosto 1915.

- Ferrazzini Emilio. Lettera indirizzata alla CCMS. Lugano 30 aprile 1940

- Ferrazzini Emilio, Breve relazione sul consolidamento degli affreschi sulla facciata della Chiesa di San Biagio a Ravecchia. Lugano 20 novembre 1944.

- Rossi Mario, Concerne affresco di S. Biagio di Ravecchia. Madonna con Bambino fra due Santi, XIV sec. indirizzato al Lodevole Dipartimento 
della Pubblica Educazione, Bellinzona. Varese 16 aprile 1955.

- Ferrazzini Emilio, Relazione sul consolidamento degli affreschi sulla facciata della Chiesa di San Biagio a Ravecchia. Annessi: due schizzi (del 1940 e del 1944), indirizzata ad Aldo Crivelli, Ispettore dei Monumenti storici. Lugano 18 agosto 1959.

- Bolzani Giuseppe, Rapporto concernente l'affresco della lunetta del portale nella chiesa di San Biagio a Ravecchia indirizzato all'Ufficio Cantonale monumenti storici, Bellinzona. Locarno 3 luglio 1967.

\section{AUBCBZ s.021 Bellinzona}

(Santa Marta, Bellinzona)

- Ferrazzini E. e Cotti C. Preventivo del Restauro completo degli affreschi della chiesa di S. Marta eseguiti secondo le direttive della CCMS indirizzato Don Giosuè Prada. Lugano 25 maggio 1934.

- Consiglio di Stato. Risoluzione, indirizzata al Dipartimento della pubblica Educazione. Bellinzona 8 giugno 1934.

- Ferrazzini E., Lettera indirizzata a Francesco Chiesa. 4 luglio 1934.

- Abbiati Bruno, Preventivo di restauro indirizzato al DPE. Ponte Tresa 24 dicembre 1961.

- Cavallini F., Lettera indirizzata agli architetti Pessina e Campi. 5 maggio 1968.

\section{AUBCBZ, s.024 Biasca}

(San Pietro e Paolo)
- $\quad$ Salati P., CCMS, Proposta di restauro pittorico della chiesa di San Pietro a Biasca, secondo il sopralluogo del 16 gennaio del 1959. Bellinzona, 30 gennaio 1959.

\section{AUBCBZ, s.067 Caslano}

(Cappella di S. Maria alla Magliasina detta Cappella Greppi)

- Abbiati Bruno, Perizia indirizzata alla CCMSA. Ponte Tresa, 16 luglio 1962.

- Moglia Mario, Rapporto sul lavoro svolto dal 21 marzo al 15 maggio 1961.Viganello, 16 maggio 1961.

\section{AUBCBZ, s.071 Cavagnago}

(Sant'Ambrogio, Segno)

- Cotti Carlo, Preventivo indirizzato al Sacrestano A. Codaghengo. Lugano 9 agosto 1935 .

\section{AUBCBZ, s.080. Chironico}

(Sant'Ambrogio)

- Ferrazzini Emilio, Preventivo per le opere di restauro pittorico della chiesetta di S. Ambrogio indirizzato allo Spett. Consiglio Parrocchiale di Chironico. Lugano 3 maggio 1941.

- Ferrazzini Emilio, Lettera personale riguardo i lavori di restauro della chiesetta di S. Ambrogio indirizzata al Consigliere Brenno Galli, Lugano. Lugano 8 ottobre 1948.

- Commissione Cantonale dei Monumenti Storici e Artistici, Rapporto d'ispezione nell'oratorio di S. Ambrogio a Chironico. Indirizzato al Lodevole Dipartimento 
della Pubblica Educazione, Bellinzona. Lugano 21 ottobre 1948.

- Beretta Emilio, Perizia indirizzata alla CCMS. 1948 post.

- Cotti Carlo, Relazione sugli ultimi lavori di restauro nella Chiesa di S. Ambrogio a Chironico. Viganello 19 settembre 1950.

- Salati Pietro, Relazione sugli ultimi lavori di restauro nella Chiesa di S. Ambrogio a Chironico, Viganello 19 settembre 1950.

- Fonti Nadia. Relazione preventivo restauro. Ascona 1996.

- Moles, Arcangelo. Chironico, Chiesa dei Santi Ambrogio e Maurizio. 3 Luglio 2000. Studio richiesto dall'Ufficio dei Beni Culturali di Bellinzona.

\section{AUBCBZ s.091 Corzoneso}

(San Remigio, Corzoneso)

- Ferrazzini Emilio, Pozzi Tita. Proposta di restauro indirizzata al Dipartimento della Pubblica Educazione. Lugano 16 novembre 1943.

- Pozzi Tita, Relazione di restauro indirizzata al DPE Massagno Lugano 15 ottobre 1944.

- Pozzi T., Relazione di restauro indirizzata alla CCMS. Massagno 6 agosto 1945.

- Pozzi Tita, Circa il trasporto degli affreschi del Tarilli all'Oratorio di S. Remigio in Corzoneso. Lettera indirizzata al DPE. Massagno 17 aprile 1946.

- Bianconi Piero, Invito a S. Remigio di Corzoneso.Corriere del Ticino
Lugano 21 maggio 1948. Anno LVIII n. 115.

- Ferrazzini Emilio, San Remigio di Corzoneso. Lettera al direttore nella Giornata del lettore. Corriere del Ticino, in risposta ad i numeri 115 e 132 dello stesso Giornale. Lugano venerdì 18 giugno 1948.

- Chiesa Francesco. Lettera indirizzata a A. Lucchini. Lugano 27 agosto 1949.

- Pozzi Tita, Relazione tecnica rinsaldamente affresco del 1200 nell'interno della Chiesa di Negrentino, indirizzata alla CCMS. $1950 \mathrm{ca}$.

- Wittgens Ferdinanda, Palazzo di Brera. Lettera indirizzata al DPE del Canton Ticino. Milano 21 maggio 1951.

- DPE, Concerne restauro pittorico. Lettera indirizzata al pittorerestauratore Mario Rossi di Varese, in copia alla CCMS, prof. Piero Bianconi, pittore Mario Moglia. Bellinzona 20 giugno 1951.

- Pozzi Tita, Lettera indirizzata a Francesco Chiesa, presidente della CCMS. Massagno - Lugano 29 giugno 1951.

- Rossi Mario, Lettera indirizzata al Consigliere di Stato Brenno Galli. Varese 25 luglio 1951.

- Commissione Cantonale dei Monumenti Storici. Lettera indirizzata ad Aldo Crivelli. Lugano, 21 settembre 1951.

- DPE, Concerne: chiesa di Negrentino. Lettera indirizzata al pittore Mario Moglia in copia al prof. Francesco Chiesa, prof. 
Bianconi, don Robertini, sac. Giuseppe Gallizia, scultore Giovanni Genucchi. Minusio, 13 luglio 1957.

- Moglia Mario, Perizia al DPE. Viganello, 22 luglio del 1957.

- Mazzi Carlo, Proposta d'intervento al DPE. Tegna-Locarno, 28 ottobre 1957.

- Ferrazzini E., Relazione di restauro del 23 gennaio 1944 indirizzata al DPE, gennaio 1959.

- Rossi Mario, Concerne: Stacco affresco a Negrentino. Lettera all'Ufficio dei Monumenti Storici di Bellinzona. Varese 21 ottobre 1970.

- Lentini Antonella. Restauratrice diplomata ENAIP. Faido.Volta dell'abside sinistra, Chiesa Parrocchiale di Prugiasco. Studio dello Stato conservativo e ipotesi di intervento. Settembre 1996.

\section{AUBCBZ, s. 112 Giornico.}

(San Nicolao)

- Amministrazione Parrocchiale di Giornico. Lettera indirizzata a Francesco Chiesa assicurando la stabilità del tetto sistemato nel 1931, Giornico, 12 gennaio 1933.

- Pozzi Tita, Relazione di sopraluogo allegato alla lettere indirizzata a Francesco Chiesa il 20 maggio 1945. Massagno 16 maggio 1945.

- Weith G., Quaderno di note: Lavori di restauro alla chiesa di San Nicolao di Giornico. Rapporti giornalieri dell'assistente ai detti lavori. Dal 24 novembre 1944 al a settembre 1945.
- Galli B. Consiglio di Stato del Canton Ticino, Copia di lettera indirizzata al Dott. Filippo Etter, Capo del DFI, riguardo le polemiche giornalistiche verso l'attività della CCMS nei lavori di restauro della chiesa di San Nicolao di Giornico, originatesi in Canton Ticino. Bellinzona 19 marzo 1949.

- Gianola L., Giornico. Chiesa di San Nicolao. Rivestimenti della monofora centrale e della parete della campata centrale dell'abside della cripta. Sopraluogo effetuato il 13 settembre 1970. Indirizzato all'Ufficio Cantonale dei Monumenti Storici, Bellinzona. Massagno, 23 settembre 1970.

- Gianola L., Preventivo di spesa per il lavoro di consolidamento di parte degli affreschi dell'abside della cripta secondo le proposte contenute nel rapporto sulla loro condizione del 23 settembre scorso. Indirizzato all'Ufficio Cantonale dei Monumenti Storici, Bellinzona. Massagno, 13 dicembre 1970.

\section{AUBCBZ, s.134 Locarno.}

(Casa del Negromante)

- Olgiati O. Relazione ed esame sullo stato di conservazione dei dipinti ornamentali situati nel porticato della casa dei "Nobili" in Locarno. Preventivo di spesa peri I restauro dei dipinti ornamentali situati nelle pareti nel porticato della casa dei Nobili cosidetta "Negromante" in Locarno. Indirizzata al DPE e CCMS. Bellinzona 7 novembre 1947. 
AUBCBZ, s. 134, Locarno.

(Castello Visconteo)

- Annoni Ambrogio. Rapporto indirizzato a Giorgio Simona. Milano, 28 ottobre 1910

- Maraini Luca, Progetto e preventivo di restauro parziale del Castello di Locarno, indirizzato al Lodevole Dipartimento della Pubblica Educazione. Lugano 11, 16 novembre 1921.

- Berta Edoardo, Lettera indirizzata al Sig. Tarabori. 12 maggio 1922.

- Naef A., Perizia, Lausanne 5 settembre del 1922.

- Berta Edoardo, Proposta di restauro, indirizzata all'Onorevole Sig. Sindaci e Municipali. 28 marzo 1923.

- Meyer Albert, Presidente del Dipartimento Federale dell'Interno. Rapporto indirizzato al Dipartimento della Pubblica Educazione del Cantone Ticino. Berna 11 aprile 1923.

- Berta Edoardo, Lettera indirizzata al DPE. 10 maggio 1923.

- Naef A., Perizia, Lausanne 25 novembre 1924.

- Zemp G., Verbale, 30 settembre 1925.

- Zemp G., Verbale, 11-12 ottobre 1926.

- $\quad$ DPE, Relazione del ottobre 1927.

- DPE del Cantone Ticino. Lettera indirizzata al Lodevole Municipio della Città di Locarno. Bellinzona 3 maggio 1928

- Schmidt Ch. Preventivo di restauro indirizzato alla Commissione
Cantonale dei Monumenti Storici in Canton Ticino. Zurigo 14 giugno 1929

- Mazzi Carlo, Rapporto Riguardante gli affreschi decorativi, stemmi dei Landfogti, nella loggia del Castello Visconteo, indirizzato al Municipio di Locarno. 19 settembre 1972.

- Gianola Luigi, Rapporto, Corte del Castello Visconteo e la conservazione stemmi degli amministratori confederati, indirizzato al Municipio di Locarno. 1 agosto 1977.

\section{AUBCBZ, s. 134, Locarno.}

(Santa Maria in Selva)

- Ferrazzini Emilio, Pozzi Tita. Preventivo per il restauro pittorico degli affreschi della chiesa di $S$. Maria in Selva a Locarno. Lugano 29 marzo 1945.

- Cotti Carlo, Proposta e preventivo di restauro indirizzato alla Commissione dei Monumenti Storici. Lugano 11 marzo 1948.

- Olgiati O., Relazione e documentazione sullo stato di conservazione dei dipinti murali esistenti nella chiesa di Santa Maria in Selva in Locarno. 1948 post.

- Olgiati O. Preventivo provvisorio di spesa per il restauro di consolidamento (I fase) dei dipinti murali della chiesa di Santa Maria in Selva in Locarno. Divisione in sedici riquadri. Ordine dei lavori e prezzo. 1948 post.

- Bianconi P., CCMS, Conclusioni dell'incontro fra i restauratori Mario 
Rossi e Ottorino Olgiati con i membri della CCMS: Emilio Maria Beretta e Piero Bianconi, riguardo i lavori di restauro della chiesa di $\mathrm{S}$. Maria in Selva a Locarno. Locarno 15 ottobre 1951.

\section{AUBCBZ, s.176 Muralto}

(San Vittore)

- Bianconi Piero, Sulla storia di San Vittore di Muralto. Bellinzona, 1913 post.

- Beretta Emilio Maria, Lettera indirizzata alla CCMS riguardo la progettazione dei lavori di restauro sulla Collegiata di S. Vittore per richiesta del Municipio di Muralto del 31 marzo 1941. Muralto, 11 ottobre 1941.

\section{AUBCBZ, s.140 Lottigna}

(Vecchio Pretorio già casa dei Landfogti)

- Pro Lottigna. Richiesta di autorizzazione per l'esecuzione dei lavori di restauro, indirizzata al DPE. Lottigna, 6 giugno 1946.

- Pozzi T., Preventivo di restauro indirizzato al DPE per lo scoprimento totale ed il restauro degli affreschi sulla facciata del vecchio Pretorio. Massagno-Lugano, 12 novembre 1946.

- Pozzi T., Preventivo di restauro indirizzato al DPE. 25 maggio 1948.

- DPE. Autorizzazione per l'esecuzione del restauro pittorico, indirizzata a Chiesa F. e Pozzi Tita. 8 febbraio 1949.
- DPE, Lettera indirizzata al Municipio di Lottigna confermando l'idoneità dei lavori eseguiti dal pittore Tita Pozzi, secondo il sopralluogo del 31 agosto 1950. 2 settembre 1950.

- Pozzi T., Relazione di restauro. Massagno-Lugano 10 novembre 1950.

(Cappella in Piano)

- Pozzi T., Relazione tecnica consolidamento e restauro pittorico Cappella in Campagna sotto Lottigna (luglio-agosto 1950). Indirizzata al DPE. Massagno Lugano 10 novembre 1950.

- Pozzi T., Fattura dei lavori realizzati entro i mesi di luglio e agosto, controllati e collaudati dalla CCMS con sopraluogo del 31 agosto 1950. Indirizzata al DPE. Massagno Lugano 10 novembre 1950.

\section{(Chiesa parrocchiale)}

- Ferrazzini E., Sopralluogo eseguito il 29 settembre ed il 2 ottobre del 1945, trovandosi traccia di affreschi medievali sulla facciata e sul lato sud della chiesa, sotto lo scialbo di calce. Indirizzato alla CCMS. Lugano, 23 dicembre 1945.

- Pozzi T., Rapporto riguardo le ricerche effettuate nella chiesa parrocchiale di Lottigna. MassagnoLugano, 16 giugno 1946.

- Pozzi T., Preventivo di restauro indirizzato al DPE per il consolidamento, pulitura e 
restauro.

Massagno-Lugano, settembre 1946.

- Consiglio di stato. Risoluzione di versamento di fr.495 a Tita Pozzi per i lavori di consolidamento, pulitura e restauro effettuati alla chiesa parrocchiale di Lottigna. Lugano, 12 novembre 1946.

\section{AUBCBZ, s.143 Lugano.}

(Santa Maria degli Angioli)

- Confraternita del SS. Rosario. Scoperta affreschi sottostanti l'intonaco nelle pareti della cappella della Madonna Immacolata nella Chiesa di S. Maria degli Angioli indirizzato al Lodevole Consiglio di Stato in Bellinzona. Lugano 22 settembre 1891.

- Pretore della Confraternita degli Angeli. Lettera indirizzata al Dipartimento della Pubblica Educazione del Canton Ticino, Lugano 23 Novembre 1892.

- Comité de la Société suisse des Monuments historiques. Dossier: Eglise S. M. degli Angioli, Lugano. N.1534 indirizzato al Consiglio di Stato del Canton Ticino, Bellinzona. Losanna 2 novembre 1907.

- Annoni Francesco, Preventivo di risarcimento di 3 dipinti da eseguirisi qui in Milano, indirizzato alla CCMS. Milano 30 agosto 1910.

- Berta Edoardo. Schema di progettopreventivo per la ricostruzione della Cappella dei Camuzi nel Museo Civico di Lugano indirizzato alla Commissione dei Monumenti Storici ed Artistici del Cantone Ticino. Lugano 22 febbraio 1913.

- Dipartimento Federale dell'Interno, Conservazione e rilievi dei freschi della Cappella Camuzio in S. M. degli Angioli in Lugano indirizzato al Dipartimento della Pubblica Educazione del Canton Ticino, Bellinzona. Berna 21 giugno 1916.

- Naef Albert, Copia del rapporto d'ispezione Chiesa di S. Maria degli Angioli a Lugano. Losanna 20 luglio 1920.

- Dipartimento Federale dell'Interno, Lettera con due allegati dei rapporti di ispezione del Sig. prof. Naef sulla Chiesa di San Biagio a Ravecchia e su quella di Sta. Maria degli Angioli a Lugano nella loro traduzione indirizzata al Lodevole Dipartimento di Pubblica Educazione del Cantone Ticino, Bellinzona. Berna 6 agosto 1920.

- Naef Albert, Copia del rapporto d'ispezione Chiesa di S. Maria degli Angioli a Lugano indirizzato al Lodevole Dipartimento Federale dell'Interno, Berna. Berna 27 agosto 1921.

- $\quad$ Naef Albert, Eglise de Ste. Marie des Anges, à Lugano. Rapport de l'inspection du mardi 22 août 1922. Lausanne 27 août 1922.

- Reale Pinacoteca di Brera, Milano. Esame dell'affresco della Crocifissione di Bernardino Luini nella Chiesa di S. Maria degli Angioli. Indirizzato a Francesco Chiesa, presidente della Commissione Cantonale dei Monumenti, Lugano, ed all'Illustrissimo Presidente del 
Consiglio di Stato del Canton Ticino, Bellinzona. Milano 9 ottobre 1926.

- Pellicioli Mauro. Preventivo per il restauro della Crocifissione indirizzato a Francesco Chiesa, Lugano. Milano 16 dicembre 1926.

- Dipartimento della Pubblica Educazione della Repubblica e Cantone del Ticino. Contratto indirizzato a Mauro Pellicioli, Milano. Bellinzona 8 febbraio 1927.

- Modigliani Ettore. Riguardo il contratto per il restauro della Crocifissione di Bernardino Luini nella Chiesa di S. M. degli Angioli indirizzato al Consigliere di Stato, Direttore del Dipartimento della Pubblica Educazione, Bellinzona. Milano 18 febbraio 1927.

- Modigliani Ettore, Lettera indirizzata a Francesco Chiesa, presidente della CCMS in risposta alla lettera del 20 gennaio. Londra 28 gennaio 1930. in Milano, 3 maggio 1930.

- Berta Edoardo/Giovannini P. Chiesa di S. Maria degli Angioli in Lugano. Preventivo delle spese per il restauro indirizzato al Dipartimento della Pubblica Educazione, Bellinzona. Lugano 19 maggio 1927.

- Dipartimento Federale dell'Interno. Restauro Chiesa di S. Maria degli Angeli indirizzato al Consiglio di Stato del Cantone Ticino, Bellinzona. Berna 27 maggio 1927.

- Schmidt Christian, Peinture transférée indirizzato a Edoardo Berta, Lugano. Zurigo 25 ottobre 1928.

- Dipartimento del Lavoro del Canton Ticino, Lettera riguardo la domanda di soggiorno di sei mesi del pittore restauratore Frana Michele, chiamato per il lavori di restauro della Chiesa degli Angioli in Lugano indirizzata alla Lodevole Commissione Cantonale dei Monumenti Storici di Lugano. Bellinzona 24 giugno 1930.

- Schmidt Christian, söhne. Fattura. Chiesa di Santa Maria degli Angioli, Lugano indirizzata a Edoardo Berta, Lugano. Zurigo 23 agosto 1930.

- Annoni Francesco, Preventivo di restauro indirizzato alla Commissione Cantonale dei Monumenti Storici Artistici. Milano 30 agosto 1930 .

- Ispettore Cantonale dei Monumenti. Elenco degli invitati. Chiesa degli Angioli indirizzato al Lodevole Dipartimento della Pubblica Educazione, Bellinzona. Lugano 15 ottobre 1930.

- Ferrazzini Emilio, Preventivo per il restauro di N.6 quadri, indirizzato al DPE del Canton Ticino. Lugano 20 ottobre 1930.

- Dipartimento Federale dell'Interno, Testo per l'iscrizione commemorativa dei restauri nella Chiesa di Santa Maria degli Angioli in Lugano indirizzato al Dipartimento della Pubblica Educazione del Cantone Ticino, Bellinzona. Berna 24 ottobre 1930.

- $\quad$ Ispettore Cantonale dei Monumenti. Chiesa degli Angioli. Conto Pulitura Crocifissione indirizzato al lodevole Dipartimento della Pubblica Educazione, Bellinzona. Lugano 28 ottobre 1930. 
- Schmidt Christian, söhne. Conto. Chiesa di S. Maria degli Angioli in Lugano indirizzato a Edoardo Berta, Lugano. Zurigo 15 novembre 1930.

- Ispettore Cantonale dei Monumenti. Richiesta materiale indirizzato al Lodevole Economato dello Stato, Bellinzona. Lugano 12 Dicembre 1930.

- Ispettore Cantonale dei Monumenti. Conto Crocifissione indirizzato al Lodevole Dipartimento di Pubblica Educazione, Bellinzona. Lugano 12 Dicembre 1930.

- Ispettore Cantonale dei Monumenti. Richiesta materiale indirizzato al Lodevole Economato dello Stato, Bellinzona. Lugano 31 gennaio 1931.

- Dipartimento della Pubblica Educazione del Canton Ticino. Copia del primo conto inoltrato dal pittore E. Ferrazzini per i lavori alla Chiesa degli Angioli in data Lugano 29 novembre 1926 indirizzato al Maestro Giuseppe Rovelli, Lugano. Bellinzona 20 maggio 1931.

- Chiesa Cino. Elenco delle negative e relative copie fotografiche delle Chiesa di S. Maria degli Angioli in Lugano, indirizzato al Lod. Commissione federale dei Monumenti storici in Berna. Lugano 3 settembre 1931.

- Dipartimento della Pubblica Educazione del Cantone Ticino, A proposito dei restauri di S. Maria degli Angioli in Lugano e del Castello di Locarno indirizzato all'Alto Dipartimento Federale dell'Interno, Berna. Bellinzona 14 settembre 1932.
- Dipartimento della Pubblica Educazione lettera indirizzata al Dipartimento Federale dell'Interno. 14 settembre 1942.

(Santa Maria di Loreto)

- Città di Lugano, Concerne l'autorizzazione ai restauri della chiesa di Loreto, Lugano. Indirizzato a Francesco Chiesa presidente della Commissione dei Monumenti Storici e della Commissione del Museo Storico. Lugano 3 aprile 1928.

- Veneranda Cappellania Vescovile di Loreto. Lettera indirizzata alla Commissione dei monumenti storici, Lugano 23 novembre 1934.

- Consiglio di Stato. Risoluzione dal Consiglio di Stato della Repubblica e Cantone del Ticino, Bellinzona 4 dicembre 1934

- Ferrazzini Emilio, Lettera indirizzata a Francesco Chiesa, presidente della Commissione dei Monumenti. Lugano 6 marzo 1935.

- Padre Materno Rederstorff, Lettera indirizzata alla CCMS. Lugano 17 giugno 1938.

- Frate Colombano Fischerofur. Lettera indirizzata al Consiglio di Stato del Canton Ticino. Lugano 26 giugno 1939

- Ferrazzini Emilio, Relazione sui lavori di restauro alla chiesetta di $\mathrm{S}$. Martino a Deggio. Indirizzata a Francesco Chiesa, presidente della Commissione Cantonale dei Monumenti Storici, Lugano. Lugano 23 settembre 1939. 
- Consiglio di Stato, Risoluzione di versamento di fr. 1600 per il restauro delle pittore nella chiesetta di S. Martino di Deggio. Bellinzona 7 novembre 1939.

- Ferrazzini Emilio, Proposta di restauro indirizzata a Francesco Chiesa, presidente della Commissione cantonale dei monumenti storici. Lugano 16 novembre 1941.

- Ferrazzini Emilio. Lavori di restauro nella chiesa di Loreto. Domanda d'autorizzazione indirizzata a Francesco Chiesa, presidente della Commissione dei Monumenti. Lugano 16 novembre 1941.

- Ferrazzini Emilio. Lavori di restauro nella chiesa di Loreto. Domanda d'autorizzazione a Francesco Chiesa, presidente della Commissione dei Monumenti. Lugano 12 dicembre 1941.

- DPE. Risoluzione indirizzata alla CCMS. 17 maggio 1949.

- Ferrazzini Emilio. Lettera personale. Concerne restauro affresco e cappella nella chiesa della Madonna di Loreto indirizzata ad Aldo Crivelli. Lugano 24 settembre 1959.

- Ferrazzini Emilio. Lettera indirizzata al Prof. Aldo Crivelli. Lugano 25 settembre 1959.

- Ferrazzini Emilio. Progetto di restauro nella chiesa della Madonna di Loreto indirizzato ad Aldo Galli. Lugano 10 ottobre 1959.

- IMSA. Lettera indirizzata alla Municipalità della Città di Lugano. Bellinzona, 14 ottobre 1959.
- Ferrazzini Emilio. Sulle fotografie della chiesa della Madonna di Loreto. Ad Aldo Crivelli. Lugano 26 ottobre 1959.

- Martinola Giuseppe, CCMS. Lettera indirizzata al Municipio di Lugano. Lugano, 7 gennaio 1971.

- Ferrazzini Emilio. Lettera indirizzata a Pierangelo Donati, Capo Ufficio della Commissione dei Monumenti Storici. Lugano 4 agosto 1971.

- Alberti Pierluigi, Loreto, Chiesa e Convento di Santa Maria. Conservazione e restauro dei dipinti sull'arco di trionfo e del dipinto nella nicchia sulla parete sinistra della navata sec. XVI. Relazione finale. Caslano, agosto 1999.

\section{(Palazzo Albertolli)}

- Ferrazzini E., Lettera indirizzata ad Edoardo Berta riguardo i lavori di restauro della sala albertolliana. Lugano 31 ottobre, 1928 ant.

- Rossi Raimondo, Lettera indirizzata ad Edoardo Berta riguardo il preventivo di restauro della sala albertolliana presentato dal pittore Emilio Ferrazzini. Bellinzona 18 novembre 1928.

- Banca Nazionale Svizzera, Commissione costruzioni per o stabile a Lugano, Lettera indirizzata ad Edoardo Berta, riguardo l'accettazione dei lavori di restauro pittorico della sala albertolliana proposti. Zurigo 19 novembre 1928.

- Ferrazzini E., Lettera indirizzata a Francesco Chiesa a termine dei lavori di tinteggiatura delle facciate 
del Palazzo Albertolli. Lugano 22 giugno 1936.

\section{AUBCBZ, s.149 Malvaglia}

(San Martino)

- Commissione dei restauri, Consuntivo al 31 dicembre 1930. Restauri della Chiesa Parrocchiale di San Martino in Malvaglia. Malvaglia 31 dicembre 1930.

- $\quad$ Ingegnere G. Baggio. Restauro degli affreschi di St. Martino. Lettera. Malvaglia 20 settembre 1935, al Comitato Pro- Restauri.

- Ferrazzini Emilio. Lettera indirizzata alla CCMSA.. Riguardo la seduta della Commissione il 21 luglio in cui il pittore spiega diffusamente e dettagliatamente la questione tecnico artistica del restauro con la tecnica all'encausto. Lugano 30 aprile 1940.

- Bianconi Piero, Relazione sui restauri delle cappelle di San Dionigi a Malvaglia e dei Morti a Semione. Indirizzata alla CCM, Lugano. 20 agosto 1944.

\section{AUBCBZ s.172 Morcote}

(Madonna del Sasso)

- DPE, Lettera indirizzata a Nino Facchinetti Bellinzona 25 settembre 1948.

- Mazzi Carlo, Preventivo indirizzato a Guido Borella. Tegna, 2 dicembre 1966.

- Rossi Mario, Lettera indirizzata alla CCMS, Bellinzona. Varese 9 novembre 1969.

\section{AUBCBZ s.183 Orselina}

(Madonna del Sasso)

- Pozzi T., Preventivo. MassagnoLugano, 8 maggio 1947.

- Abbiati Bruno, Preventivo di restauro indirizzato al DPE. Ponte Tresa 30 giugno 1960.

\section{AUBCBZ, s.196 Ponte Capriasca}

(Chiesa Rosa)

- Cotti Carlo, Preventivo indirizzato all'Architetto Pietro Giovannini. Lugano, 9 agosto 1944.

- Pozzi T., Lettera indirizzata a Francesco Chiesa, MassagnoLugano, 1 novembre 1951.

- Pozzi T., Preventivo del 9 settembre 1951. Indirizzato a Francesco Chiesa. Massagno-Lugano. 16 gennaio 1952.

\section{AUBCBZ, s.204, Prugiasco}

(San Carlo, Negrentino)

- Ganna Federico, Lettera indirizzata al DPE. $1897 \mathrm{ca}$.

- Ferrazzini Emilio, Lettera indirizzata a Francesco Chiesa, presidente della Commissione Cantonale dei Monumenti Storici in copia al Dipartimento della pubblica educazione. Lugano, 30 aprile 1940.

- Ferrazzini Emilio. Riguardo l'incarico di sorvegliare i lavori di restauro alla chiesa di San Carlo in Negrentino. A Francesco Chiesa, presidente della Commissione Cantonale dei Monumenti. Lugano 30 aprile 1940. 
- Bianconi Piero, Sopraluogo nella chiesa di San Carlo di Negrentino. A Francesco Chiesa, presidente della Commissione Cantonale dei Monumenti. Orselina, 21 novembre 1940.

- Baggio G. Ufficio Tecnico Cantonale II Sezione. Riparto: Correzione Fiumi. Rapporto sullo stato di conservazione della chiesa di $S$. Carlo a Negrentino. Alla Commissione Cantonale dei Monumenti. Bellinzona 1 maggio 1942.

- Ferrazzini Emilio/ Pozzi Tita, Proposta di restauro a Francesco Chiesa presidente della Commissione Cantonale dei Monumenti Storici e Artistici. Lugano 30 luglio 1942.

- Dipartimento della pubblica Educazione del Canton Ticino, Sui restauri alla chiesa di San Carlo in Negrentino. Al Lodevole Consiglio Parrocchiale, Prugiasco. Bellinzona 29 agosto 1942.

- Pozzi Tita. Sui restauri pittorici intrapresi nello scorso mese di agosto nella chiesa di S. Carlo di Negrentino sopra Prugiasco. Al Lodevole Dipartimento della pubblica Educazione del Canton Ticino, Bellinzona; in copia al M. Rev. Don Giuseppe Gallizia per il Comitato Pro Negrentino in Prugiasco. Massagno-Lugano 22 settembre 1942.

- M. Rev. Don Giuseppe Gallizia per il Comitato Pro Negrentino in Prugiasco, Sugli svolgimenti dei lavori e dei restauri. Al Lodevole
Dipartimento Cantonale della Pubblica Educazione, Bellinzona. Prugiasco 9 luglio 1943.

- Pozzi Tita, Comprovasco 20 luglio 1943. Elenco delle operazioni di restauro pittorico da eseguirsi nella Chiesa di Negrentino sopra Prugiasco; operazioni di restauro decise a seguito dei sopraluoghi: delle Lod. Comm. Cant. Monumenti della fine agosto 1942, e dei pittori E. Ferrazzini e Tita Pozzi del 19 luglio 1943.

- Gianella Riccardo. Distinta dei lavori di restauro della chiesa di S. Carlo di Negrentino. Alla Parrocchia e comune di Prugiasco.

- Bellinzona 24 maggio 1944. Gianella Riccardo. Relazione tecnica circa il restauro della chiesa di S. Carlo di Negrentino. Alla Parrocchia e comune di Prugiasco. Bellinzona 24 maggio 1944.

- Pozzi Tita. Testo per l'iscrizione commemorativa dei lavori di restauro nella chiesa di S. Carlo in Negrentino. Al Lodevole Dipartimento della Pubblica Educazione del Canton Ticino, Bellinzona. Dongio 28 agosto 1945.

- Dipartimento della Pubblica Educazione del Canton Ticino. Richiesta di consolidamento alla chiesa di S. Carlo in Negrentino.Al pittore Tita Pozzi, Massagno, in copia alla Commissione Cantonale dei Monumenti Storici. Bellinzona 23 febbraio 1950.

- Pozzi Tita. Circa i restauri nella chiesa di S. Carlo in Negrentino. Al Lodevole Dipartimento della 
Pubblica Educazione del Canton Ticino, in copia al prof. Francesco Chiesa presidente della Commissione Cantonale dei Monumenti, Bellinzona. MassagnoLugano 17 aprile 1950.

- Pozzi Tita. Circa i restauri nella chiesa di S. Carlo in Negrentino e della Cappella sotto Lottigna. Al prof. Francesco Chiesa, presidente della Commissione Cantonale dei Monumenti, Bellinzona. Acquarossa 14 agosto 1950.

- Pozzi Tita. Relazione tecnica rinsaldamento Affresco del 1200 nell'interno della Chiesa di Negrentino. Al Lodevole Dipartimento della Pubblica Educazione del Canton Ticino, Bellinzona, in copia al prof. Francesco Chiesa presidente della Commissione Cantonale dei Monumenti, Bellinzona. LuganoMassagno 10 novembre 1950.

- Pozzi Tita. Conto per i lavori di rinsaldamento Affresco del 1200 nell'interno della Chiesa di Negrentino. Al Lodevole Dipartimento della Pubblica Educazione del Canton Ticino, Bellinzona in copia al prof. Francesco Chiesa presidente della Commissione Cantonale dei Monumenti, Bellinzona. LuganoMassagno 10 novembre 1950.

- Wittgens Fernanda. Chiesa di San Carlo di Negrentino. A S. E. il Consigliere Brenno Galli, Dipartimento della Pubblica Educazione, Bellinzona. Milano 21 maggio 1951.
- Dipartimento della Pubblica Educazione, Restauro pittorico. Al prof. Mario Rossi, Varese. In copia alla Commissione Cantonale dei Monumenti Storici, al prof. Piero Bianconi, al pitt. Mario Moglia. Bellinzona 20 giugno 1951.

- Pozzi Tita, Sulle polemiche giornalistiche. A Francesco Chiesa, presidente della Commissione Cantonale dei Monumenti, Bellinzona. Lugano-Massagno 29 giugno 1951.

- Pozzi Tita, Sulle polemiche giornalistiche. A Brenno Galli, Consigliere di Stato, Lugano. Lugano-Massagno 29 giugno 1951.

- $\quad$ Rossi Mario, Gli affreschi di S. Carlo di Negrentino. A Brenno Galli, Consigliere di Stato, Lugano. Varese 25 luglio 1951.

- Commissione Cantonale dei Monumenti Storici ed Artistici. Sulle polemiche giornalistiche. Alla Direzione della Rivista "Svizzera italiana", Locarno. Lugano 21 settembre 1951.

- Dipartimento della Pubblica Educazione, Chiesa di Negrentino. A Mario Moglia di Viganello in copia a Francesco Chiesa, Piero Bianconi, don Robertini, sac. Giuseppe Gallizia, scultore Giovanni Genucchi. Bellinzona 13 luglio 1957.

- Moglia Mario, Perizia nella Chiesa di San Carlo di Negrentino. Al Lodevole Dipartimento della Pubblica Educazione, Bellinzona. Viganello 22 luglio 1957.

- Dipartimento della Pubblica Educazione. Richiesta di preventivo. 
Al pittore Carlo Mazzi, Tegna. In copia a Francesco Chiesa e Pietro Salati. Bellinzona 18 ottobre 1957.

- Mazzi Carlo. Preventivo per 10 strappo degli affreschi dell'abside nella Chiesa di San Carlo di Negrentino. Al Lodevole Dipartimento della Pubblica Educazione, Bellinzona. Tegna 28 ottobre 1957.

- Comitato Pro-Chiesa di Negrentino Lettera Concerne affreschi romanici Cristo e Apostoli di Negrentino indirizzata alla CCMS. Bellinzona, 28 maggio 1969.

- Rossi Mario. Stacco affresco a Negrentino. All' Spett.le Ufficio Monumenti Storici, Bellinzona. Varese 21 ottobre 1970.

- Gianola Luigi, Preventivo per stacco e riporto di parti frammentarie di un affresco romanico sulla parete ovest in Sant'Ambrogio vecchio di Prugiasco. All' Spett.le Ufficio Monumenti Storici, Castel Grande, Bellinzona. Massagno 23 dicembre 1970.

- Gianola Luigi, Strappo di un frammento di affresco romanico nella chiesa di Sant'Ambrogio vecchio di Prugiasco. All' Spett.le Ufficio Monumenti Storici, Castel Grande, Bellinzona. Massagno 16 gennaio 1972.

\section{AUBCBZ, s. 206 Quinto}

(San Martino, Deggio)
- Consiglio di Stato del Canton Ticino. Risoluzione del indirizzata alla CCMS. Lugano, 7 novembre 1939.

- Ferrazzini Emilio, Relazione di restauro della chiesa di S. Martino di Deggio (Quinto), indirizzata a Francesco Chiesa. Lugano 23 settembre 1939.

\section{AUBCBZ, s. 213 Rovio}

(San Vigilio)

- Gusalli Emilio, Sovrintendenza per la conservazione dei Monumenti di Lombardia. Esame e progetto di restauro architettonico. A Emilio Mazzetti. Milano 1913 ca.

- Mazzetti Emilio. Chiesa di S. Vigilio. Richiesta d'autorizzazione per l'esecuzione dei lavori. Al Lodevole Dipartimento della Pubblica Educazione, Bellinzona. Rovio 8 ottobre 1929.

- Alberti Giacomo. Preventivo di spesa per il riordino della chiesa a norma dei piani allegati in scala 1:50. Al Consiglio Parrocchiale di Rovio.

- Alberti Giacomo, Preventivo di spesa per il riordino della chiesa a norma dei piani allegati in scala 1:50. $1938 \mathrm{ca}$.

- Pozzi Tita, A seguito del sopralluogo a S. Vigilio e dell'abboccamento di ieri sera, eccole il preventivo per le opere di restauro pittorico da eseguirsi nella Chiesa di S. Vigilio a Rovio. Lettera a Taddeo Carloni. Massagno-Lugano 3 febbraio 1943.

- Pozzi Tita. Preventivo per le opere di restauro pittorico da eseguirsi nella 
chiesa di S. Vigilio a Rovio. Al prof. Taddeo Carloni, Lugano. MassagnoLugano 3 febbraio 1943.

- Pozzi Tita. Sui restauri alla chiesa di San Vigilio, Rovio. Al Lodevole Dipartimento della pubblica Educazione, Bellinzona. Massagno 4 gennaio 1944.

- Pozzi Tita, Lettera a Francesco Chiesa, Massagno 21 febbraio 1950.

- Pozzi Tita. Sulle fotografie del prima e dopo i restauri alla chiesa di San Vigilio, Rovio. A Francesco Chiesa, presidente della Commissione Cantonale dei Monumenti, Lugano. Massagno 21 febbraio 1950.

\section{AUBCBZ, s.216 Russo}

- Consiglio Parrocchiale di Russo. Lettera indirizzata alla CCMS, Russo 26 settembre 1935.

- Ferrazzini Emilio. Lettera indirizzata alla CCMS. Lugano, 10 novembre 1935.

- DPE. Lettera indirizzata a Emilio Ferrazzini. Lugano, 1950

- $\quad$ AUBCBZ, s. 246 Minusio

- Ugo Donati, Pietro Salati e Piero Bianconi. CCMS. Lettera indirizzata al DPE riguardo il Restauro dell'Interno della Parrocchiale di Verscio. Minusio 28 marzo 1946.

\section{AUBCBZ, s.246. Verscio.}

(San Fedele)

- $\quad$ Ferrazzini Emilio. Oratorio del 400 a Verscio. Perizia della Commissione Cantonale dei Monumenti Storici ed Artistici. Lugano, 13 luglio 1932.
- Robertini Agostino, Lettera indirizzata alla Lodevole CCMS. Verscio 16 novembre 1944.

- Bianconi Piero e Donati Ugo, Rapporto della CCMS sui lavori nella chiesa parrocchiale di Verscio. 3 febbraio 1946.

- Dipartimento Federale dell'Interno, Lettera riguardo l'offerta di sussidio per i lavori di restauro nella primitiva chiesa di Verscio e le condizioni abituali regolamentari per la concessione di un sussidio federale, indirizzata al Reveredno don Robertini per il Consiglio parrocchiale e la Municipalità di Verscio (Ticino), in copia a I Dipartimento della pubblica educazione del cantone Ticino, Bellinzona, al sig. architetto Rino Tami, Lugano ed al sig. Birchler, Zurigo. Berna, 7 agosto 1945.

- Donati Ugo, Salati Pietro e Bianconi Piero membri della CCMS. Restauro dell'interno della parrocchiale di Verscio. Minusio, 28 marzo 1946.

\section{AUBCBZ s.251 Villa Luganese}

(Santa Maria Assunta)

- Ferrazzini Emilio, lettera indirizzata all'On. Consigliere G. Lepori, Lugano 13 giugno 1943.

\section{AUBCBZ 112 Giornico}

(Chiesa di San Nicolao, Giornico)

- Amministrazione parrocchiale di Giornico. Chiesa di San Nicolao. A Francesco Chiesa, presidente della Commissione Cantonale dei 
Monumenti, Lugano. Giornico 12 gennaio 1933,

- Gianella Riccardo. Chiesa di San Nicolao, Giornico. Progetto di restauro. Tav. I-VI. Al Dipartimento della pubblica Educazione del Canton Ticino, Bellinzona. Bellinzona 20 aprile 1941.

- Weith Giuseppe. Lavori di restauro alla Chiesa di San Nicolao di Giornico. Rapporti giornaliere dell'assistente ai detti lavori. Giornico, 1944.

- Pozzi Tita. Risultante del sopralluogo a San Nicolao di Giornico il 15 maggio 1945 per il pulimento $e$ consolidamento degli affreschi. A Francesco Chiesa, presidente della Commissione Cantonale dei Monumenti, Lugano. Massagno 16 maggio 1945

- Pozzi Tita. Risultante del sopralluogo a San Nicolao di Giornico il 18 maggio 1945 per il pulimento $e$ consolidamento degli affreschi. A Francesco Chiesa, presidente della Commissione Cantonale dei Monumenti, Lugano. Massagno 20 maggio 1945

- Galli Brenno, Consigliere di Stato Direttore. Sulle polemiche giornalistiche. Al Consigliere Federale Dott. Filippo Etter Capo del Dipartimento Federale dell'Interno, Berna. Bellinzona 10 marzo 1949

- Gianola Luigi, Sopralluogo effettuato il 13 settembre del 1970. All' Spett.le Ufficio Monumenti Storici, Castel Grande, Bellinzona. Massagno 23 settembre 1970
- Massagno 13 dicembre 1970, Gianola Luigi. Preventivo per i lavori di consolidamento degli affreschi della Chiesa di San Nicolao. All' Spett.le Ufficio Monumenti Storici, Castel Grande, Bellinzona.

\section{Archivio Tita Pozzi}

L'Archivio Tita Pozzi è custodito dal figlio, Giovanni, nella casa famigliare a Massagno.

I dati raccolti nell'archivio privato del pittore-restauratore riguardo i diversi interventi eseguiti sono in definitiva $i$ medesimi che conformano l'Archivio dell'Ufficio dei beni culturali a Bellinzona, nella corrispondenza che l'artista scambio con i diversi enti istituzionali. È stato fondamentale il contato diretto con la famiglia del restauratore, ed alcuni dei materiali documentali (fotografie e libri essenzialmente) da loro facilitati nonché il confronto dei risultati ottenuti nella ricerca.

\section{Archivio Carlo Mazzi}

L'Archivio Carlo Mazzi, custodito dalla figlia Silvia Mina-Mazzi nella casa natale dell'artista, è stato da me analizzato solo in parte. $\mathrm{Si}$ sono spogliati sistematicamente i quaderni di appunti con le ricette ed i metodi di lavoro nel restauro ed i fascicoli corrispondenti ai lavori di restauro da lui eseguiti dal 1953 al 1967, lasciando da parte la documentazione corrispondente all'attività artistica, che potrà essere oggetto di studio e di ricerche in un futuro. Le fonti estratte da codesto archivio citate nel presente lavoro sono: 
- Rossi Mario. Lettera indirizzata a Carlo Mazzi, copia della medesima indirizzata al Sig. Tarabori. Varese, 27 marzo 1954.

- $\quad$ (Casa Landfogti, Rivera)

- Mazzi Carlo. Lettera indirizzata all'Ispettorato dei Monumenti Storici Artistici, Tegna 28 dicembre 1960.

- Mazzi Carlo, Dichiarazione di ricevuta di 21 affreschi strappati per la Casa Landfogti depositati al Castel Grande, indirizzata al Dipartimento della Pubblica Costruzione, Bellinzona. Tegna, 19 giugno 1954.

- (San Pellegrino, Giornico)

- Bolzani. Rapporto concernente i restauri della Chiesa di S. Pellegrino. Locarno 3 luglio 1967.

- (Madonna del Sasso, Orselina)

- Mazzi Carlo, Preventivo del 5 maggio 1967, Tegna.

\section{Archivio Bruno Abbiati}

Il figlio del restauratore Bruno Abbiati, ha facilitato questa ricerca con un importantissimo e complesso materiale iconografico che documenta tutta l'attività professionale del padre. Purtroppo è stato impossibile la consultazione del materiale cartaceo poiché i documenti andarono persi in un recente incendio.

\section{Archivio della Radiotelevisione svizzera}

- TSI, Antichi monumenti, vecchi e nuovi in Astrolabio, 20.09.1967.

- TSI, Intervista a Mauro Pellicioli. 1970. 


\section{BIBLIOGRAFIA TEMATICA}

\section{Edoardo Berta}

(Scritti sull'artista)

\section{0}

Widmer, J. Die neue Malerei in der Schweiz, Kunst und Künstler. Bern.

\section{1}

Widmer, J. (8. aprile 1911). Edoardo Berta, der Maler des Tessin. Die Alpen, pp. $458,483-489$.

Pometta, E. (1911). Edoardo Berta: il pittore del Ticino. Popolo e libertà. pp.118 - 122 - 123.

\section{3}

Foglia, G. (16. Septiembre 1913). Gli artisti ticinesi. Edoardo Berta. Corriere del Ticino.

\section{6}

Janner, A. Pages d'Art. Octubre. pp.1-24

Moobs R., The modern Swiss school of alpin landscape art and the work of Edoardo Berta, The Studio, vol.67. pp.93-97.

\section{1}

Chiesa, F. Edoardo Berta conmemorato per Francesco Chiesa. Adula (pp.28-30). Lugano.

Giussani A., Edoardo Berta. In Rivista Archeologica dell'antica provincia $e$ diocesi di Como, fascicoli 102-104, pp.259-262.
Guidi M., Dizionario degli artisti ticinesi, Formiggini, Roma.

Chiesa, F. In memoria di Edoardo Berta. In Edoardo Berta. In memoria - 18671931 (pp. 29-31). Lugano.

DFI. Lettere e telegrammi di condoglianza di autorità e di privati. In Edoardo Berta. In memoria 1867 - 1931 (pp. 33-41). Lugano.

Foglia, G. (6. settembre 1931). Berta: un uomo, un'idea, un simbolo. Adula, p. 34-37.

\section{2}

Ventarini L., Amore di terra lombarda. Articolo in lode dell'artista restauratore del Castello Visconteo di Locarno. (22 marzo 1932). Popolo d'Italia.

Janner A., Edoardo Berta. Neujahrsblatt der Zürcher Kuntsgesellschaft. Kuntshaus, Zurigo. pp.1-8.

\section{4}

Comanducci A.M. I pittori italiani dell'Ottocento. Casa Editrice artisti italiani S.A., Milano. Pp.50, 51.

\section{4}

Monteverde M., Storia delle pittura italiana dell'Ottocento. Bramante editrice, Busto Arsizio. Vol.1, p. 210, vol.2, p.99.

1990

Ginex G. Opere dell'Ottocento in collezione. In Quaderni di Villa dei Cedri, 
Civica Galleria d'Arte, Bellinzona. pp.23, 24.

\section{7}

Sonderegger C., Edoardo Berta a Villa dei Cedri, Associazione Amici di Villa dei Cedri. Bolletino, n.4, pp.20,21.

\section{8}

Chiappini R., Soldini S., Opere d'arte della Città di Lugano. La collezione. Città di Lugano, Lugano. Pp. 122-129, 490491, 526, 527.

\section{0}

Bianchi M., Sonderegger C., Edoardo Berta (1867-1931), Citta di Bellinzona, Bellinzona.

\section{1}

Berta, E. Documenti di una amicizia artistica : il carteggio Edoardo Berta Giuseppe Pellizza (1887-1905). Archivio storico ticinese, Bellinzona.

(Scritti dell'artista)

\section{6}

Berta, E. Relazione sul Congresso artistico internazionale : tenuto in Venezia nel settembre 1905. Tipogr. Cantonale. Bellinzona.

Berta, E., Mentessi G., Relazione sulle scuole di disegno Annate 1905-1906, Tipografia cantonale, Bellinzona.

\section{2}

Berta, E. Monumenti storici ed artistici del Ticino. Ulrico Hoepli, Milano.
Berta, E. Pitture murali e decorazioni pittoriche : II cenacolo di Ponte Capriasca.

Janner, A. II metallo. (E. Berta, A cura di) Ulrico Hoepli, Milano.

Janner, A. La pietra : camini e caminiere gotiche, del rinascimento e barocche. (E. Berta, A cura di) Ulrico Hoepli, Milano.

Janner, A. Il legno : soffitti dei secoli XV, XVI e XVII. (E. Berta, A cura di) Ulrico Hoepli, Milano.

\section{3}

Berta, E. Case tipiche ticinesi : il Luganese. Milano, Ulrico Hoepli.

Berta, E. Pitture anteriori al rinascimento nel bellinzonese: san Biagio di Ravecchia, Milano. Ulrico Hoepli.

\section{4}

Berta, E. La pietra : sculture diverse, bassorilievi rappresentativi. Ulrico Hoepli, Milano.

\section{5}

Berta, E. Relazione sui lavori di restauro della Chiesa di San Biagio in Ravecchia. Bironico.

\section{4}

Berta, E. Casa e paessaggio nel Canton Ticino. Heimatschutz.

Berta, E. II Ticino nell sue costruzioni e costumi popolari: conferenza con 100 proiezioni originali . Tipografia Luganese Sanvito e C. Lugano. 
Berta, E. Pitture murali e decorazioni pittoriche : pitture del Rinascimento : la cappella Camuzio nella chiesa di S. M. degli Angioli in Lugano. Ulrico Hoepli, Milano.

\section{6}

Berta E. L'anima del Ticino nelle sue abitazioni e costumi popolari, L'Adula.

\section{7}

Hugelshofer, W. Il legno : altari a intaglio d'origine tedesca nel cantone Ticino e altari della Madonna del Sasso e di Ascona. (E. Berta, A cura di, \& E. Talamona, Trad.) Ulrico Hoepli, Milano.

1928

Berta, E. Guida del Castello di Locarno. Tipografia Pedrazzini, Locarno.

\section{9}

Berta E. Il Castello di Locarno, Rivista archeologica della provincia e antica diocesi di Como. Fascicoli 96, 97, 98.

\section{0}

Berta E. Il Castello di Locarno, Premiata tipografia editrice Ostinelli di Cesare Nani, Como.

1936

Donati U., Breve storia degli artisti ticinesi, Salvioni, Bellinzona. pp.153-155.

Foglia G., Edoardo Berta nel $V$ anniversario della sua morte. In Corriere del Ticino (3 luglio 1936).

\section{1}

Berta, E. Documenti di una amicizia artistica : il carteggio Edoardo Berta Giuseppe Pellizza (1887-1905). Archivio storico ticinese. Bellinzona.

\section{Emilio Ferrazzini}

(Scritti sull'artista)

\section{2}

Crivelli A., Emilio Ferrazzini pittore 18951975. Lugano marzo 1972. In Catalogo delle mostra del 2003 a Dangio. Cima Norma.

\section{3}

Agliati, M. Un caro valente pittore, un caro uomo. In A. C. Mario Agliati, Emilio Ferrazzini pittore 1895 - 1975. Cima Norma, Lugano.

(Scritti dell'artista)

\section{8}

Ferrazzini, E. Gli stucchi bizantini di Corzoneso. Gli affreschi di Sorengo. La Madonna di Sommascona. Rivista storica ticinese : archeologia, storia, belle arti (4), pp.75-77.

\section{9}

Ferrazzini, E. II S. Martino di Deggio. Rivista storica ticinese : archeologia, storia, belle arti (6), pp.272-273.

1940

Ferrazzini, E. Gli stucchi bizantini di Corzoneso. Rivista storica ticinese : archeologia, storia, belle arti (5), p.397. 
Ferrazzini, E. La chiesetta di S. Colombano di Scona. Rivista storica ticinese : archeologia, storia, belle arti (3), pp.315-316.

\section{2}

Ferrazzini E., La chiesa di San Carlo Borromeo in Lugano. II terzo centenario dalla sua erezione, 1642 - 1942. Carlo Grassi, Bellinzona.

\section{5}

Ferrazzini, E. Note d'arte. Un dipinto di G. Serodine. Giornale del popolo. (12. Ottobre 1945). p.10

\section{4}

Ferrazzini, E. Affreschi di Giuseppe Antonio Petrini da Carona (1677-1759) nella Chiesa di Besano (Varese). Rivista archeologica dell'Antica Provincia e Diocesi di Como, pp.79-84.

Ferrazzini, E. (1939). Relazione ad opera compiuta. Restauro pittorico dell'Oratorio di S. Martino a Deggio. Lugano 23 settembre 1939. Destinatario Sig. Prof. Francesco Chiesa Presidente delle Commissione dei Monumenti, Lugano. AUBC, Filza 206 Quinto.

\section{5}

Ferrazzini E., II pittore Cav. Giuseppe Petrini da Carona, 1677-1759. In Almanaco Ticinese.

\section{0}

Ferrazzini E., Cattaneo G., Mostra del pittore Giuseppe Antonio Petrini, catalogo. Lugano, Villa dei Cedri.

\section{Tita Pozzi}

(Scritti sull'artista)

\section{2}

Foletti, G. Tita Pozzi (1902 - 1958): pittore, xilografo, restauratore. Comune de Massagno, Massagno.

(Scritti dell'artista)

\section{9}

Pozzi, T. Pitture e restauri di chiese in Ticino. Arte svizzera (4), pp.80-82.

\section{Carlo Mazzi}

(Scritti sull'artista)

\section{1}

Belinelli, E. (1981). Omaggio a Carlo Mazzi pittore: Galleria SPSAS, Ex Casa Rusca, Locarno, 12 settembre - 10 ottobre 1981 / [testo: Eros Bellinelli] . SPSAS Società pittori scultori e architetti svizzeri, Sezione Ticino. Locarno.

\section{8}

Belinelli, E. Carlo Mazzi, 1911-1988 : Galleria Carlo Mazzi, Tegna, 14.VI 30.VIII.1998. Galleria Carlo Mazzi, Tegna.

\section{Mario Moglia}

Scritti sull'artista

\section{6}

Bossi, G. M. Omaggio a Mario Moglia. Incisioni dal 1944 al 1972. Galleria Mosaico, Chiasso. 


\section{7}

Mosaico, G. Omaggio a Mario Moglia : incisioni dal 1944 al 1972 . Galleria Mosaico. Chiasso

(Scritti dell’artista)

\section{7}

Moglia, M. Note sul restauro degli affreschi di San Pietro. In R. Amerio, San Pietro di Biasca. Comitato Pro Restauri San Pietro di Biasca.

\section{Carlo Cotti}

(Scritti sull'artista)

\section{9}

Killer, P. Carlo Cotti. Un costruttivista svizzero ancora da scoprire. In G. Volonterio, Carlo Cotti, mostra antologica Lugano. p. 55.

\section{BIBLIOGRAFIA GENERALE}

\section{2}

Collezioni degli atti delle solenni distribuzioni dei premi dell'industria fatti in Milano e Venezia 1840 - 1852 Tipografia di Gio. Cecchini, Venezia.

1892

Rahn Johann Rudolf, I dipinti del Rinascimento nella Svizzera Italiana [traduzione dal tedesco di Giorgio Simona]. Tip. E Litografia Eredi C. Colombi, Bellinzona.

1893

Rahn, J. R. Anleitung zur Erhaltung von Baudenkmälern und zu ihrer
Wiederherstellung. In Jahresbericht [der] Schweizerischen Gesellschaft für Erhaltung historischer Kunstdenkmäler. Druck F. Schulthess, Zürich.

\section{3}

Beltrami, L. La cappella Camuzio nella Chiesa di S. Maria degli Angioli in Lugano. Bollettino storico della Svizzera italiana : pubblicazione trimestrale fondata da Emilio Motta nel 1879 , pp.115.

\section{7}

Zemp, J. Das Restaurieren. Schweizer Rundschau Heft (IV).

\section{5}

Pometta, E. Come il Ticino venne in potere degli Svizzeri (Vol. I). Stabilimento tipo-litogr., Belinzona.

\section{3}

Il nostro Castello. (17. Aprile 1923). Popolo e libertà. Locarno e Muralto.

\section{4}

Braun, J. Der Christliche Altar in seiner Geschichtlichen Entwicklung. Alte Meister Guenther Koch \& Co. Munchen

\section{7}

Rosa, L. A. La tecnica della pittura dai tempi preistorici ad oggi. Società Editrice Libraria, Milano.

\section{8}

Mazzetti, E., Silvestrini D., La Millenaria Chiesetta di S. Vigilio in Rovio in Rivista Storica Ticinese (Febbraio 1938), pp.7-9. 


\section{0}

Zanetti-Ripamonti, Giuseppe. Piante medicinali nostre; tavole a colori di Ottorino Olgiati. Istituto Editoriale Ticinese Bellinzona.

\section{3}

Bassetti A., Santa Maria delle Grazie di Bellinzona (Tentativo Storico). Arti Grafiche Arturo Savioni \& Co., Bellinzona.

\section{4}

Martinola, G. II distacco e il restauro degli affreschi del Luini a Lugano nel secolo scorso. Bolletino Storico della Svizzera Italiana, pp.38-41.

Mariotta, P. Sul restauro di San Nicolao in Giornico. Edizioni Veladini \& C.

\section{6}

Annoni, A. Scienza ed arte del restauro architettonico. Idee ed esempi. Edizioni Artistiche Framar, Milano.

Chiesa, F. Monumenti storici e artistici del Cantone Ticino restaurati dal 1910 al 1945. (D. d. Educazione, A cura di) Grassi, Bellinzona.

\section{8}

Birchler, L. Restaurierungspraxis und Kunsterbe in der Schweiz. In Kultur- und Staatswissenschaftl. Schriften der ETH; Polygraph. Zurigo.

\section{6}

Chiesa, V. In memoria di Massimo Guidi. In Scritti di Virgilio Chiesa e Mario
Agliati. Tipografia Editoriale Luganese, Lugano.

Bianconi P. Colloquio con Francesco Chiesa. Edit. Grassi, Bellinzona.

\section{9}

DPE. Cinquant'anni di protezione dei monumenti storici artistici della repubblica e cantone del Ticino. Edizione dello Stato, Bellinzona.

\section{4}

Borella, G. Ricerche archeologiche a S. Martino di Mendrisio dall'agosto 1959 alla fine del 1961. In A. Noseda, "Comum": miscellanea di scritti in onore dell'Arch. F. Frigerio pubblicata a cura della Società Archeologica Comense (p. 93-102 ). Como.

\section{6}

Fondazione Dietler-Kottmann, Gli affreschi del coro della chiesa di San Michele a Palagnedra. Officine tipografiche Carminati, Locarno.

\section{7}

Travel, H. C. Künstler Lexikon der Schweiz. XX Jahrhundert. Verlag Huber \& Co. Aktiengesellschaft. Frauenfeld.

Renato, G. Quanto abbiamo vissuto nel corso dei restauri. Cronistoria del direttore dei lavori. In R. Amerio, San Pietro di Biasca. Comitato Pro Restauri San Pietro di, Biasca.

La restauration d'une des fresques de Dessouslavy est enfin entreprise. (21. 
settembre 1967). Ville de Neuchâtel. Dons le canton.

\section{0}

Televisione della Svizzera Italiana. Intervista a Mauro Pellicioli. RTSI, Archivi televisivi, Canobbio.

\section{5}

Belloni, F. V. Bellinzona, affreschi in Santa Maria delle Grazie. In Silva Vassallorum. Francescano, Genova.

Marcionetti, I. Chiesa e Convento di Santa Maria degli Angeli in Lugano. Pedrazzini, Locarno.

\section{6}

Rahn Johann Rudolf, I monumenti artistici del Medio Evo nel Cantone Ticino. [traduzione con aggiunte all'originale tedesco da Eligio Pometta]. Società ticinese per la conservazione delle bellezze naturali ed artistiche. Locarno.

\section{7}

Pini, A. e. San Remigio di Corzoneso (Vol. III). (E. t. Blenio, A cura di) Tipografia Stazione SA. Locarno.

Marcionetti, I. San Carlo di Negrentino. Gaggini-Bizzozzero SA., Lugano.

\section{9}

Secco Suardo, G. II restauratore dei dipinti (Reprint antichi manuali Hoepli ed.). Cisalpino-Goliardica, Milano.

\section{0}

Pozzi G. La Madonna del Sasso fra storia e leggenda. Armando Dadó Editore, Locarno.

\section{4}

Fulvio Caccia, I. M. Settantacinque anni della Commissione dei monumenti storici ed artistici del cantone Ticino. Bellinzona: Dipartimento dell'ambiente Ufficio e commissione cantonale dei monumenti storici.

Bianconi P., La chiesa e gli affreschi di Santa Maria in Selva a Locarno. Edizioni Pedrazzini, Locarno.

\section{7}

Agliati, C. N. Attilio Balmelli. Istituto grafico Casagrande SA., Bellinzona.

\section{8}

Piva, G. L'Arte del restauro. II restauro dei dipinti nel sistema antico e moderno. Secondo le opere di Secco-Suardo e del prof. R. Mancia. (Terza ed.). Ulrico Hoepli. Milano, Italia.

Campagna A, La chiesa di San Biagio a Ravecchia-Bellinzona.

\section{5}

Cecchini, G., Giordano G., Milani D., Materiali tradizionali per il restauro dei dipinti. Preparazione e applicazione secondo il manuales di Giovanni Secco Suardo. Associazione Giovanni Secco Suardo. Lurano (BG). 


\section{6}

Piva, G. Manuale pratico di tecnica pittorica : enciclopedia ricettario per tutti gli artisti, pittori dilettanti, allievi delle accademie di belle arti e delle scuole artistiche. Ulrico Hoepli. Milano, Italia.

\section{8}

Campagna, A. La chiesa di San Biagio a Ravecchia-Bellinzona. SSAS in collaborazione con la BSI, Berna.

Rüsch, E. Chiesa di Sant'Ambrogio vecchio in Negrentino. Guide di Monumenti Svizzeri SSAS. Società di Storia dell'Arte in Svizzera, Berna.

Matteini M., Moles A., Scienza e restauro. Metodi di indagine. Nardini Editore, Firenze.

\section{9}

Marcionetti I., Chiesa e convento di Santa Maria degli Angeli in Lugano. Diocesi di Lugano, Lugano.

Cordaro M., Cesare Brandi, II restauro. Teoria e pratica. Editori Riuniti dalle A.C. Grafiche, Cerbara, Città di Castello.

\section{0}

Soldini S., Gilardi A., Gilardi J., L'orazione nell'orto di Giovan Battista Bagutti. Storia e restauro di un trasparente. Museo d'arte di Mendrisio, Mendrisio.

Basile G., Teoria e pratica del restauro in Cesare Brandi. Prima edizione dei termini. II Prato Casa editrice, Saonara.

\section{1}

Soldini, S. Ticino 1940-1945 : arte e cultura di una nuova generazione. (S. Soldini, A cura di) Museo d'arte. Mendrisio.

Perusini G., Christian Köster. Sul restauro degli antichi dipinti ad olio. Forum, Editrice Universitaria Udinense Srl., Udine.

\section{2}

Foletti, G. Arte nell'Ottocento. La pittura e la scultura del Cantone Ticino (1870 1920). Armando Dadò, Locarno.

Chiesa, V. Lineamenti storici del Malcantone. Museo del Malcantone, Curio.

Perusini G., II restauro dei dipinti nel secondo Ottocento. Giuseppe Uberto Valentinis e il metodo Pettenkofer. Forum, Editrice Universitaria Udinense Srl., Udine.

\section{3}

AA.VV. La Teoria del Restauro nel Novecento da Riegl a Brandi. In Andaloro M. (A cura di), Convegno Internazionale di Studi. Nardini, Viterbo.

AA.VV. Restauratori e restauri in archivio. Volume 1. In Basile G. (A cura di), Profili di restauratori italiani tra XVII e XX secolo. Associazione Giovanni Secco Suardo. Nardini Editore. 
Chiappini, R. II confronto con la modernità, 1914-1953. Lugano, Museo civico di belle arti, 2003-04. (Arte in Ticino 1803-2003 3). Salvioni, Bellinzona.

Gian-Willi, V. L'Archivio federale dei monumenti storici (AFMS) offre rifugio a oltre due milioni di documenti. Giornale UFC. La memoria del passato (13), p. 4,5.

Ghigonetto, S. Ovidio Fonti: pittore, restauratore, insegnate a Torino e in Piemonte. In S. Ghigonetto, Maestranza Malcantonesi in Piemonte tra Barrocco e primo Novecento (p. 95 - 105). Museo del Malcantone. Curio.

Terribile C., II "nobile rigeneratore". I restauri di Giuseppe Uberto Valentinis. Forum, Editrice Universitaria Udinese Srl, Udine.

Zeni, M. A. II ritratto femminile nell'arte del Ticino, 1650-1970. Rancate, Pinacoteca cantonale Giovanni Züst. Ulivo, Balerna.

\section{4}

Amacher, D. Le collezioni dell'AFMS scrigno e vaso di Pandora. Giornale UFC. La memoria del passato (13), pp. 6-7.

Lumia, C. Scavo, isolamento e restauro nel Battistero di Riva San Vitale in Canton Ticino. Materiali e tecniche negli interventi degli anni '20 e degli anni '50. In AA.VV., Atti Convegno di Studi, Bressanone 13-16 luglio 2004. Scienza e Beni Culturali. Bressanone.
Giorgio Bonsanti, M. C. Ulisse Forni. Manuale del pittore restauratore. Studio per la nuova edizione. (G. B. Ciatti, A cura di) Edifir. Firenze, Italia.

Gubler Jacques. Johann Rudolf Rahn. Geografia e monumenti. Catalogo della mostra 11 settembre-31 ottobre 2004, Museo d'arte di Mendrisio. Museo d'arte, Mendrisio.

Gurtner, K. L'eredità di Albert Naef. Giornale UFC (13).

Mürner, J. Memoria schedata-archivi e politica della memoria. Giornale UFC (13).

AA.VV. Pietro Chiesa. Alla ricerca dell'identità. Società d'Arti Grafiche già Veladini \& co. SA, Lugano.

Pedrioli P., Restauri nel Canton Ticino: notiziario 2001-2002. Nota introduttiva in BSSI, serie IX, vo. CVII, fasc. I, pp.255287.

\section{5}

Conti, A. Storia del restauro e della conservazione delle opere d'arte. Mondadori Electa, Milano.

Valentini, M. La prattica dello strappo nel Canton Ticino. Diploma di Bachelor SUPSI. DACD, Corso di laurea in conservazione e restauro. Lugano.

Julien, M. Christian Schmidt, Dekorationsmaler und Restaurator. Diplomarbeit. Berna 
Pettinatti, P. (26. Novembre 2005). Visioni tra realtà e memoria. Franco Tamò alla Galleria I'Incontro di Lugano. Giornale la Regione Ticino, p. 30.

AA.VV. Restauratori e restauri in archivio. Volume 2. In Basile G. (A cura di), Profili di restauratori italiani tra XVII e XX secolo. Associazione Giovanni Secco Suardo. Nardini Editore.

Pedrioli P., Restauri nel Ticino: notiziario 2004. Nota introduttiva, serie IX, vo. CVII, fasc. II, 2005, pp.371-394.

\section{6}

Mazzarello, D. L'Illustradario. Vie e personaggi celebri del Canton Ticino. Fontana edizioni. Lugano-Pregassona.

Gianini, C. Giovanni Secco Suardo: alle origini del restauro moderno. EdifirEdizioni, Firenze.

M. Beltrami, G. C. II Cenacolo di Novazzano (Ticino, Svizzera). Materiali costitutivi originali e applicati nel corso di successivi interventi. In C. D'Amico (A cura di), IV Congresso Nazionale Aiar. Pàtron, Pisa. pp. 105 - 114.

Andaloro M., La teoria del restauro nel Novecento da Riegl a Brandi. Atti del Convegno Internazionale di Studio, Viterbo, 12-15 novembre 2003. Nardini Editore, Firenze.

Cardani Vergani R., Damiani Cabrini L., Riva San Vitale. II battistero di San Giovanni e la chiesa di Santa Croce.
Società di storia dell'arte in Svizzera SSAS, Berna.

\section{7}

AA.VV. Luigi Cavenaghi e i maestri dei tempi antichi. Pittura, restauro e conservazione dei dipinti tra Ottocento e Novecento. (A. C. Muzzin, A cura di) Lubrina Editore, Bergamo.

AA.VV. Interim meeting on conservation training. Grupo de trabajo. Formación de restauradores. Jornada Internacional: $A$ 100 anni della nascita di Cesare Brandi, 7 de mayo de 2007. Universidad Politecnica de Valencia. Editorial UPV., Valencia.

AA.VV. Guida d'arte della Svizzera italiana. (S. d. Svizzera, A cura di) Berna, Svizzera: Edizioni Casagrande.

Pracchi, V. Mauro Pellicioli, "Restauratore principe": note sugli interventi di consolidamento. Scienza e beni culturali XXIII. II Consolidamento degli Apparati Architettonici e Decorativi Arcadia, Bresannone. p. 457 - 466.

Basile G., Marabelli M., Leonardo, I'Ultima cena. Indagini, ricerche, restauro. Nardini editore, Firenze.

Commissione Federale dei Moumenti Storici, Principi per la tutela dei monumneti storici in Svizzera. Vdf Hochschulverlag AG an der ETH Zürich.

\section{8}

CdT. (16. maggio 2008). Palace, cantiere da monitorare. Corriere del Ticino. p.18. 


\section{Fonti stampate}

Con lo scopo di localizzare le notizie della polemica generata negli anni Quaranta intorno ai restauri in Canton Ticino, sono stati consultati i giornali ticinesi: Giornale del Popolo tra il 1935 e il 1960 e Corriere del Ticino tra il 1940 e il 1950. Non è stata possibile invece I'analisi completa dei giornali: Archivio storico della Svizzera italiana, Bolletino Storico della Svizzera italiana e L'Adula: organo svizzero di cultura ticinese, Popolo e libertà, II Dovere, fondamentale per l'approfondimento in futuro della presente ricerca.

Gli articoli più importanti in riferimento alla polemiche di restauro citati nei testi, sono:

- Crivelli, A. Restauri, bellezze naturali, tradizioni. Rettili preistorici. (A. Crivelli, A cura di) Rivista Storica Ticinese 1944(5), pp.961-968.

- Robertini A. (firma d.R.), Su San Nicolao di Giornico in Giornale del Popolo, Lugano Anno XX, no160. Mercoledi 4 luglio 1945. (pp.1, 2).

- Gianola R. (firma Ing. R.G.), Critica ad una critica in Giornale del Popolo, Lugano Anno XX, no169. Martedì 10 luglio 1945. (p.2).

- Robertini A. (firma d.R), Osservazioni ad una critica. Polemica archeologica. In Giornale del Popolo, Lugano Anno XX, no172. Venerdì 13 luglio 1945.

- Gianola R. (firma i.r.g.), Note fuori di polemica. A proposito di San Nicolao. In Giornale del Popolo,
Lugano Anno XX, no177. Mercoledì 18 luglio 1945. (p.2).

- Pozzi T. (firma pitt. T.P.), Restauri, critici e via dicendo. In Giornale del Popolo, Lugano Anno XX, no177. Mercoledì 18 luglio 1945. (p.2).

- Robertini A. (firma d.R.), Su San Nicolao. In Giornale del Popolo, Lugano Anno XX, no189. Giovedì 26 luglio 1945. (pp.1, 2).

- Viator, firmato. Una proposta modestissima in favore di San Nicolao di Giornico. In Giornale del Popolo, Lugano Anno XX, no189. Giovedì 26 luglio 1945. (p. 2).

- Donati U., Intorno ai restauri della chiesa di S. Nicolao a Giornico. In Giornale del Popolo, Lugano Anno XX, no.218 Giovedì 23 agosto 1945. (p. 2).

- Crivelli, A. Sui restauri di San Nicolao di Negrentino. Rivista Storica Ticinese 1945 (5-6), pp.1105-1109.

- Crivelli, A. Basta. Polemica sui restauri di San Nicolao di Giornico. Rivista Storica del Ticino 1945 (3), pp.1057-1062.

- $\quad$ Crivelli, A. II restauro della Chiesa di San Nicolao. Rivista Storica Ticinese 1945 (1), p.1025.

- Crivelli, A. Le desgrazie non vegono mai sole. Svizzera Italiana 1951 (14).

- E.G. firmato, L'inaugurazione dei restauri di S. Nicolao a Giornico. In Giornale del Popolo, Lugano Anno XX, no.235 Mercoledì 19 settembre 1945. (p. 2). 
Altre notizie citate nella ricerca, fuori degli argomenti di polemica sono:

- Rahn Johann Rudolf, Gli affreschi del Ticino di nuovo scoperti in $\mathrm{S}$. Maria degli Angioli in Lugano, nella chiesa del Collegio d'Ascona e nella chiesa di Mairengo [trad. dal tedesco di Giorgio Simona]. In Bollettino storico della Svizzera italiana, Anno 15(1893), p. 29-33, 49-54, Bellinzona.

- Olgiati, O. Gli affreschi di Ponte Capriasca. Rivista storica ticinese : archeologia, storia, belle arti 1939 , pp.261-262.

- Crivelli, A. La Madonna di Mergoscia. Rivista storica ticinese 1940 (5). 



\section{INDICE DEI NOMI DI PERSONA, DI LUOGO E DI ASSOCIAZIONI}

Abbiati Bruno; 277; 366

Accademia di Belle Arti di Brera; 244

Accademia di Belle Arti di Brera, Milano; 74

Accademia di Belle Arti, Parma; 79

Accademia di Carrara, Bergamo; 77

Anastasio Pietro; 78

Annoni Ambrogio; 280

Annoni Francesco; 473

Baggio Giovanni; 251

Bagutti Giovanni Battista; 79; 173

Balmelli Attilio; 244

Banca Nazionale Svizzera; 246

Baracchini Clara; 39

Barzaghi Cattaneo Antonio; 78

Bassi Angelo; 146

Battistero, Riva San Vitale; 152; 486

Beltrami Luca; 78

Beretta Modesto; 74

Bernasconi Ferdinando; 74

Berta Battista; 74

Berta Edoardo; 55; 73; 74; 76; 78; 87;

213; 246

Bertini Giuseppe; 76; 77

Bianchi Paolo; 74

Bianconi Piero; 167; 264; 276

Birchler Linus; 167; 251

Boissonas Frederic; 492

Boissonas Henri-Paul; 247; 279

Bonalina Luigi; 75

Borgna Piero; 299

Boscaino Ivan; 39

Boschetti Pietro; 74

Bossi Emilio; 79

Botta Rodolfo; 75

Botti Guglielmo; 177

Brandi Cesare; 146

Bruni Vincenzo; 74

Camuzio Cappella; 246
Capella Camuzio, S. Maria degli Angioli, Lugano; 88; 90

Capella dell'Immacolata, S. Maria degli Angioli, Lugano; 88

Cappella dei Morti, Semione; 260

Cappella della Magliasina, Caslano; 231

Cappella della Trinità, Arogno; 393

Cappella di Sant'Anna, Ponto Valentino; 260

Cappella Dionigi, Malvaglia; 260

Carloni Taddeo; 286

Carloni Tita; 265

Carugo Baldo; 146

Casa Abbondio, Ascona; 323

Casa del Negromante, Vaglio; 109

Casa del sig. Pietro Perini, Mergoscia; 438; 450

Casa di Giovanni Guerra di Filippo, Isone; 344

Casa M. Marcacci, Isone; 344

Casa Orlandini, ora Fontana, Dangio, Aquila; 360

Casa Stanga, Giornico; 344

Casella Giorgio; 75

Castello - Visconteo, Locarno; 80

Castello dei Cavalieri di Malta, Bubikon; 247

Castello Visconteo, Locarno; 152

Cattaneo Alfonso; 149; 308

Ceppi Dante; 74

Chiattone Antonio; 78

Chiattone Giuseppe; 78

Chiesa Bernardo; 74

Chiesa dei SS. Anna e Cristoforo, Curogna; 393; 394

Chiesa dell'Annunciata, Locarno; 359

Chiesa Francesco; 74; 76; 79; 91; 167; 251 
Chiesa parrocchiale della B.V Assunta, Villa Luganese; 390

Chiesa Parrocchiale della B.V Assunta, Villa Luganese; 225; 226; 344; 345; 346; 356; 357; 390; 391; 392

Chiesa parrocchiale di S. Martino, Malvaglia; 150; 153

Chiesa parrocchiale, Brione Verzasca; 395

Chiesa parrocchiale, Broglio; 346; 392; 393

Chiesa parrocchiale, Cimalmotto; 394

Chiesa parrocchiale, Gorduno; 314

Chiesa Parrocchiale, Lottigna; 318; 319

Chiesa parrocchiale, Medeglia; 486

Chiesa parrocchiale, Moleno; 396

Chiesa Parrocchiale, Ponte Capriasca; 322; 325

Chiesa parrocchiale, Prato Sornico; 393

Chiesa parrocchiale, Russo; 219; 229

Chiesa parrocchiale, Sant'Aubin; 247

Chiesa parrocchiale, Torrello; 396

Chiesa parrocchiale, Vezio; 359

Chiesa Parrochiale, Tesserete; 216

Chiesa Pietro; 74; 79; 245

Chiesa primitiva, Verscio; 376

Collegiata dei SS. Pietro e Stefano; 173; 213

Collegiata dei SS. Pietro e Stefano Belinzona; 150

Collegiata dei SS. Pietro e Stefano, Bellinzona; 150; 213

Cometta Augusto; 89

Conza Rezzonico Teresa; 286

Cotti Carlo; 149; 152; 213; 246; 308

Crivelli Aldo; 167; 263; 277

de Volterra Daniele; 177

Demicheli Andrea; 88

Demicheli Giovanni; 88

Dilena Emilio; 149; 152; 308; 342; 343

Dillena Emilio; 108; 109
Dipartimento della Pubblica Educazione; 251

Donati Domenico; 74

Donati Ugo; 167

Doninelli Livo; 146

Dürrer; 149

Emmenegger Oscar; 297

Emmenegger Oskar; 146

Facchinetti Ivano (Nino); 354

Feragutti Visconti Adolfo; 78

Ferrari Gaudenzio; 146

Ferrazzini Emilio; 132; 149; 153; 167;

168; 244; 276; 308

Ferroni Bernardo; 74

Fiume Guido; 146

Foglia Giuseppe; 73

Foletti Giulio; 76

Fontana Domenico; 309

Fontana Gottardo; 74

Franzoni Filippo; 78

Gabrielli Noemí; 146

Galbusera Gioacchino; 78

Galetti Adolfo; 74

Gallerie di Milano; 262

Galli Brenno; 55; 263

Gallizioli Bernardo; 86

Ganna Federico; 250

Garlani Ercole; 74

Ghirlanda Pietro; 74

Giacometti Giovanni; 245

Gianella Riccardo; 251; 261; 276

Gianini Giuseppe; 74

Gianola Luigi; 265

Gilardoni Virgilio; 167

Giornale del popolo; 261

Giornale del Popolo; 246

Giovannini P.; 101

Gobbi Achille; 74

Guggeri Luigi; 74

Guidi Massimo; 168

Guidini Augusto; 53; 89; 250 
Gusalli Emilio; 286

Hoepli Ulrico; 80

Hotel du Parc; 84

Hotel Palace; 84

Janner Arminio; 167

L'Adula; 277

Lampugnani Gaetano; 144

Lentini Antonella; 265

Lepori Francesco; 74

Lepori Giuseppe; 167; 251

Levi Donata; 39

Lubini Pietro; 74

Ludovico I Re di Baviera; 255

Luini Bernardino; 85; 464

Maccagni Emilio; 91

Madonna del Sasso; 151; 460

Madonna del SassoLocarno; 151

Madonna del Ponte, Arzo; 220; 376

Madonna del Sasso, Locarno; 412; 413; 483

Madonna di Loreto, Lugano; 393

Maffei Andrea; 39

Maggi Antonio; 75

Maggiori Enrico; 74

Maraini Otto; 55; 87

Mariotta Paolo; 276

Martignoni Angelo; 55

Maselli Costantino; 88; 89

Mazzi Carlo; 265

Meier Johannes; 279

Mentessi Giuseppe; 74; 78

Mercoli Giacomo; 74

Meyer Albert; 81

Moglia Mario; 263

Moles A.; 206

Monneret de Villard Ugo; 276

Monteverde Luigi; 78

Motta Emilio; 79

Museo cantonale d'arte, Lugano; 79

Museo Civico di Lugano; 90

Museo Civico, Lugano; 90
Museo d'arte, Mendrisio; 79

Museo Vela; 80

Naef Albert; 91; 149; 250

Niklauskapelle, Regensdorf; 247

Nizzola Aquilino; 146

Nizzola Bruno; 152; 213

Noseda Giovanni, Mons; 55

Oratorio dell'Annunciata, Novazzano; 226; 358; 392

Oratorio delle Grazie, Cugnasco; 395

Orelli Giuseppe Antonio Felice; 246

Ortelli Arturo; 149; 152; 308

Palazzo Albertolli; 246

Palazzo Albertolli, Lugano; 153; 214; 464

Palazzo Riva; 246

Patocchi Aldo; 245

Pellandini Augusto; 74

Pellicioli Mauro; 85; 113; 280; 458

Pellizza di Volpedo Giuseppe; 77

Pellizza di Volpedo, Giuseppe; 79

Petrini Giuseppe Antonio; 85

Popolo e libertà; 247

Pozzi Giovanni; 244; 297

Pozzi Tita; 175; 243

Pretorio, Lottigna; 260

Probst Eugen; 250

Quadri Ernesto; 74

Quadri Giovanni; 75

Rahn Johann Rudolf; 248; 286

Regazzoni Ammellio; 74

Regli Francesca; 74

Rivista Storica Ticinese; 246

Robertini Agostino; 167; 261; 278

Rossi Luigi; 78; 80

Rossi Mario; 146; 197; 263

Rovelli Giuseppe; 102; 213

Rusca Basilio; 149; 308

S. Giovanni, Mendrisio; 226; 310

S. Maria in Selva; 485

S. Ambrogio, Cademario; 315; 395

S. Ambrogio, Chironico; 175; 228; 230 
S. Ambrogio, Ponte Capriasca; 214

S. Ambrogio, Segno; 215; 343; 344; 348

S. Anna, Giubiasco; 344

S. Antonio, Lugano; 344

S. Bartolomeo, Croglio; 229; 358

S. Bernardino, Caravaggio; 143

S. Biaggio, Ravecchia; 196

S. Biagio, Ravecchia; 80; 122; 131; 228; 486

S. Carlo, Bignasco; 317

S. Carlo, Lugano; 343

S. Carlo, Negrentino; 224; 394; 460; 485

S. Croce, Riva San Vitale; 223

S. Francesco, Locarno; 219

S. Giorgio, Morbio Inferiore; 215

S. Giovanni, Mendrisio; 79

S. Maria degli Angioli, Lugano; 80; 83; 149; 246; 308; 459

S. Maria del Carmelo. Villa Coldrerio; 229

S. Maria del Castello, Mesocco; 109

S. Maria del Sasso, Locarno; 347

S. Maria del Sasso, Morcote; 217; 218; 344

S. Maria delle Grazie, Bellinzona; 81 ; $143 ; 152 ; 213 ; 342 ; 343$

S. Maria delle Grazie, Varallo; 143

S. Marta, Bellinzona; 246; 309

S. Martino, Deggio; 220; 221; 222

S. Martino, Ditto; 395

S. Martino, Malvaglia; 214; 308; 343

S. Nicolao, Giornico; 231; 246; 375; 376

S. Pietro, Biasca; 376; 396

S. Pietro, Castel San Pietro; 347; 358

S. Remigio, Corzoneso; 229; 246; 315

S. Rocco, Bissone; 153

S. Stefano, Miglieglia; 395

S. Vigilio, Rovio; 321

San Carlo, Negrentino; 246
San Sisinio, Mendrisio; 79

San Vigilio, Rovio; 246

Sant'Antonio, Lugano; 246

Sartori Augusto; 74

Schmidt Christian Jakob; 108; 149; 152; $342 ; 343$

Scuola di disegno, Mendrisio; 89

Scuola Superiore d'arte cristiana Beato Angelico; 244

Scuola Ticinese di Coltura italiana; 79

Secco - Suardo Giovanni; 177

Seregno, Cristoforo e Nicolao da; 248

Sesti Davide, Mons.; 55

Simona Luigi; 167

Società di storia dell'arte in Svizzera; 275

Società pittori, scultori ed architetti svizzeri; 245

Soldati Achille; 74

SS. Giovanni Evangelista e Martino, Bironico; 216

Tallone Cesare; 77

Tamó Piero; 145

Tarabori; 280

Tarilli Giovanni Battista; 246

Tradate Antonio da; 248

Turati Filippo; 79

Vassalli Luigi; 74; 78

Vela Spartaco; 80

Verrier Jean; 280

Via Crucis, Camignolo; 216

Villa Ciani, Lugano; 229

Weith Giuseppe; 276

Wilhelm Keim Adolf; 255

Wittgens Fernanda; 262

Wolfgang Johann; 255

Zanetti Enrico; 74

Zanini Paolo; 89

Zemp Josef; 250 


\section{INDICE DELLE FIGURE}

Figura 1 Guidini Augusto, Copertina di Studio della Ragioni dell'Arte e del Diritto Sociale. Milano 1905. Lo si trova nella "Libreria Patria" della Biblioteca Cantonale di Lugano.

Figura 2 S. Maria del Castello, Mesocco; Targa commemorativa della Confederazio dei monumenti storici (1924)

Figura 3 Cartina degli interventi di restauro murale esegiti fra il periodo 1909 - 1959 in Canton Ticino

Figura 4 Foglia Giuseppe, Edoardo Berta. Disegno a mattita su carta

Figura 5 Francesco Chiesa. Fotografia pubblicata in Del Bove Milko, II Ticino delle lettere $e$ nelle lettere, 14 dicembre 2006; [in linea] 76

Figura 6 Berta Edoardo, Ruscello. Olio su tela. Museo Villa dei Cedri, Bellinzona. 79

Figura 7 Cartolina da Ulrico Hoepli indirizzata al Dipartimento della Pubblica Educazione di Bellinzona, Milano 2 febbraio 1915. ASTi, Fondo DPE- Diversi 1905 - 1955, Segnatura 1.1.4.2.12.

Figura 8 Cartolina da U.Hoepli indirizzata al DPE, Milano 2 febbraio 1915. ASTI, Fondo DPE- Diversi 1905 - 1955, Segnatura 1.1.4.2.12. Nella cartolina si legge: [...]La Soprintendenza dei monumenti della Lombardia mi informa con sua lettera di ieri di essere stata autorizzata a ritirare gratis i fascicoli dei Monumenti Storici ed Artistici del Canton Ticino. Oggi stesso fanno la consegna; ma desidero per regolarità amministrativa che mi venga confermato l'ordine di consegna[...].

Figura 9 Foglia Giuseppe, Maschera di Edoardo Berta eseguita sul letto di morte, 1931. Fotografia Himmelsbach.

Figura 10 Luini Bernardino, Madonna con il Bambino e San Giovanni. Stacco. Chiesa di S. Maria degli Angeli, Lugano. (Marcionetti, 1975).

Figura 11 Studio su carta dei dipinti della volta della Cappella Camuzio. 1913 post. Lugano. AUBCBZ Archivio gráfico, Lugano, S. Maria degli Angioli.

Figura 12 Volta della Cappella Camuzio. Chiesa di Santa Maria degli Angioli, Lugano. Giner Cordero E. 2008.

Figura 13 Carboni C., Cappella Camuzio prima del restauro, 1931 ant. Fotografia b/n...... 95

Figura 14 Carboni C., Cappella Camuzio dopo il restauro, 1931 ca. Fotografia b/n. AUBCBZ, s.143 Lugano, S. Maria degli Angioli. 
Figura 15 Giner Cordero E., Interno della Cappella Camuzio. Chiesa di S. Maria degli Angioli, Lugano. 2008.

Figura 16 Particolare dei dipinti murali dopo il restauro, Chiesa di Santa Maria degli Angioli, Lugano TI, 1931 circa. Fotografia b/n. 17616b Archivio Federale dei Monumenti Storici. Collezione grafica, Biblioteca nazionale svizzera.

Figura 17 Particolare dei dipinti dopo il restauro, Chiesa di Santa Maria degli Angioli, Lugano TI, 1931 circa. Fotografia b/n. 17628b Archivio Federale dei Monumenti Storici. Collezione grafica, Biblioteca nazionale svizzera.

Figura 18 Giner Cordero E. , Particolare dei dipinti sulle lunette della volta della Cappella Camuzio. Chiesa di S. Maria degli Angioli, Lugano. 2008.

Figura 19 Particolare dei dipinti della cappella Camuzio durante i restauri, Chiesa di Santa Maria degli Angioli, Lugano, Fotografia b/n. TI. 14132 Archivio Federale dei Monumenti Storici. Collezione grafica, Biblioteca nazionale svizzera.

Figura 20 Giner Cordero E. Particolari dei dipinti della cappella Camuzio. 99

Figura 21 Giner Cordero, E. Cappella Camuzio,Chiesa di S. Maria degli Angioli, 2008. Fotografia di particolare a luce radente dello stato di conservazione attuale dei dipinti. 102

Figura 22 Giner Cordero, E. Particolare dei dipinti della Cappella Camuzio. Segni delle martelline utilizzate nelle operazioni di descialbo. Chiesa di Santa Maria degli Angioli, Lugano. Enero 2008. 103

Figura 23 Giner Cordero, E. Particolare dei dipinti della Cappella Camuzio. Segni d'abrasione dai ferri utilizzati per le operazioni di descialbo. Chiesa di Santa Maria degli Angioli, Lugano. Enero 2008. 103

Figura 24 Giner Cordero E. Particolare dei dipinti della Cappella Camuzio. Segni delle martelline e dei ferri usati nello descialbo delle pitture. Chiesa di Santa Maria degli Angioli, Lugano. Gennaio 2008. 104

Figura 25 Giner Cordero, E. Particolare dei dipinti della Cappella Camuzio. Dettaglio del ritocco a basso tono delle lacune. Chiesa di Santa Maria degli Angioli, Lugano. Gennaio 2008.

Figura 26 Giner Cordero, E. Particolare dei dipinti della Cappella Camuzio dove si evidenziano le aggiunte e ritocchi cromatici. Chiesa di Santa Maria degli Angioli, Lugano. Gennaio 2008. Fotografia UV. 105 
Figura 27 Giner Cordero, E. Particolare dei dipinti della Cappella Camuzio dove si evidenziano le aggiunte e ritocchi cormatici. Chiesa di Santa Maria degli Angioli, Lugano. Gennaio 2008. Fotografia UV. 105

Figura 28 Giovannini Pietro, Rilievo della Chiesa di Santa. Maria degli Angeli a Lugano, studio della facciata dopo le ricerche archeologiche del 1926 al 1927 dal Pittore Edoardo Berta. (Marcionetti, 1975, p. Tav.90). 106

Figura 29 Berta Edoardo, Progetto di restauro studiato. Coro dei Frati. Restauro altare. Attuale presbiterio e volta dello stesso. 1926-1927. (Marcionetti, 1975, p. 96). 107

Figura 30 Berta Edoardo, Progetto di restauro studiato.Coro dei Frati. Restauro da eseguirsi sulla base degli elementi archeologici rintracciati. 1926-1927. (Marcionetti, 1975, p. Tav.105). 107

Figura 31 Chiesa di S. Maria degli Angioli, inizio XX secolo. Prima del restauro di Edoardo Berta. Stampa. (Marcionetti, 1975, p. Tav.87) 108

Figura 32 Sammlung Werhli, Chiesa di S. Maria degli Angioli., 1932. Negativo Num. B. 39399. AFMS, Berna. 108

Figura 33 Sammlung Wehrli, Interno della chiesa di S. Maria degli Angioli dopo il restauro. Fotografia b/n. Negativo Num. A.10890, 1932. AFMS, Berna. 109

Figura 34 Giner Cordero, E . Interno S. Maria degli Angioli, Lugano 2007. 109

Figura 35 Particolare dell'affresco Crocifissione e passione di Cristo, dove si osserva il deteriore dei dipinti. Lugano 2006, Giner Cordero E.

Figura 36 Particolare del dipinto della Crocifissione di Bernardino Luini. 1931 ca. Fotografia b/n. AUBCBZ Archivio grafico, 143 Lugano, S. Maria degli Angioli.

Figura 37 Giner Cordero E. Particolare del dipinto della Crocifissione di Bernardino Luini. 2006

Figura 38 Giner Cordero E. Particolare del dipinto della Crocifissione di Bernardino Luini dopo il restauro, Lugano. 2006.

Figura 39 Giner Cordero E. Particolare del dipinto della Crocifissione di Bernardino Luini. 2006.

Figura 40 Cenacolo di Bernardino Luini nella Sala della Comunione. Chiesa di Santa Maria degli Angioli. Lugano TI.

Figura 41 Seconda cappella durante i lavori di restauro. 1931 ant. Fotografia b/n AUBCBZ Archivio grafico, 143 Lugano, S. Maria degli Angioli. 
Figura 42 Terza cappella durante i lavori di restauro. 1931 ant. Fotografia b/n AUBCBZ Archivio grafico, 143 Lugano, S. Maria degli Angioli....

Figura 43 C. Carboni, Sacro orifiamma con le sigle di Cristo prima del restauro, $1931 \mathrm{ca}$. Fotografia b/n. AUBCBZ Archivio grafico, 143 Lugano, S. Maria degli Angioli.....

Figura 44 Giner Cordero E., Sacro orifiamma con le sigle di Cristo. Chiesa di S. Maria degli Angioli, Lugano. 2008.

Figura 45 San Cristoforo sulla facciata della Chiesa di San Biagio di Ravecchia dopo il restauro. Bellinzona. 1913 ca. Fotografia b/n. AUBCBZ, Archivio grafico, Ravecchia, S. Biagio.

Figura 46 Maestro di San Biagio, Madonna con Bambino fra i Santi Biagio vescovo e Pedro, XII sec. Chiesa di San Biagio di Ravecchia dopo il restauro. Fotografia b/n. Bellinzona. AUBCBZ, Archivio grafico, Ravecchia, S. Biagio.

Figura 47 Maestro di San Biagio, Madonna con Bambino fra i Santi Biagio vescovo e Pedro, XII sec. Chiesa di San Biagio di Ravecchia dopo il restauro. Fotografia b/n. Bellinzona. AUBCBZ, Archivio grafico, Ravecchia, S. Biagio.

Figura 48 Martini Simone, Santa Caterina da Alessandria, Temple su tavola. XIV sec. National Gallery, Canada.

Figura 49 Lombardi G./Annoni A. Il Castello di Locarno secondo gli avanzi originali e gli studi del Ten. Colonello Giorgio Simona. Disegno. Milano, 1912. AUBCBZ, s. 134 Locarno, Castello Visconteo.

Figura 50 Fratelli Buchi. Castello Visconteo. Prima del restauro. Fotografia b/n. AUBCBZ, Archivio fotografico. Locarno Castello Visconteo. 136

Figura 51 Fratelli Buchi. Castello Visconteo. Dopo il restauro. Fotografia b/n. AUBCBZ, Archivio fotografico. Locarno Castello Visconteo. 136

Figura $52 \mathrm{~J}$. Cordoz?Facciata sud verso il giardino con dettagli, Locarno 1808. Fotografia b/n. 65685 B.4683 Plan Nr. 9504. ABN Berna.

Figura $53 \mathrm{Chr}$. Schmidt, Casa del Negromante a Locarno. Studio delle decorazioni, 1897. Fotografia b/n. 65685 B.4683 Plan Nr. 9504. ABN Berna.

Figura 54 Dillena Emilio, Parte del soffitto all'entrata al Castello dei Visconti a Locarno . Dipinto; tempera su tela e cartone. Castello dei Visconti, Locarno. Ottobre 1929. AUBCBZ, s. 134, Locarno. Castello Visconteo.

Figura 55 Dillena Emilio, Cornicione in legno. Dipinto; tempera su carta. Castello dei Visconti, Locarno. Ottobre 1929. AUBCBZ, s. 134, Locarno. Castello Visconteo. 
Figura 56 Fratelli Buchi. Castello, Locarno TI. Dipinti del cortile. Fotografia b/n. 2609 B.112. ABN Berna.

Figura 57 Fratelli Buchi. Castello, Locarno TI. Pitture sulla loggetta al primo piano (parete sud). Fotografia b/n. 21752 B.2180. ABN Berna.

Figura 58 Giner Cordero, E. Transetto murale di S. Maria delle Grazie. 2006

Figura 59 Cartina degli interventi documentati eseguiti da Edoardo Berta in Canton Ticino.

Figura 60 Confraternita del SS. Rosario; Lettera del 22 settembre 1891 indirizzata al Consiglio di Stato in Bellinzona. AUBCBZ, s. 143 Lugano, S. Maria degli Angioli.

Figura 61 Confraternita del SS. Rosario; Lettera del 22 settembre 1891 indirizzata al Consiglio di Stato in Bellinzona. AUBCBZ, s. 143 Lugano, S. Maria degli Angioli. 156

Figura 62 Ettore Modigliani, Milano. Lettera del 18 febbraio 1927 indirizzata al Consigliere di Stato. AUBCBZ, s. 143 Lugano, S. Maria degli Angioli.

Figura $63 \mathrm{Chr}$. Schmidt, Zurigo. Lettera del 27 ottobrel 1928 indirizzata al pittore Edoardo Berta. AUBCBZ, s. 143 Lugano, S. Maria degli Angioli.

Figura 64 Dipartimento Federale dell'Interno, Berna, Lettera 24 ottobre 1930 indirizzata al DPE del Canton Ticino. AUBCBZ, s. 143 Lugano, S. Maria degli Angioli. 159

Figura 65 Cino Chiesa, elenco numerato dei negativi e copie fotografiche di S. Maria degli Angioli indirizzato alla CMS, Lugano, 3 settembre 1931. AUBCBZ, s. 143 Lugano, S. Maria degli Angioli.

Figura 66 Cino Chiesa, elenco numerato dei negativi e copie fotografiche di S. Maria degli Angioli indirizzato alla CMS, Lugano, 3 settembre 1931. AUBCBZ, s. 143 Lugano, S. Maria degli Angioli.

Figura 67 Edoardo Berta,richiesta di materiale indirizzata al Economato dello stato. Lugano, 12 dicembre 1930. AUBCBZ, s. 143 Lugano, S. Maria degli Angioli.

Figura 68 Emilio Ferrazzini. Autoritratto, Lugano 1922. (Dangio, Cima Norma, 2003).... 167

Figura 69 Bonzon Paul, Ferdinand Hodler, 1916. Fotografia b/n. [online]. 169

Figura 70 Emilio Ferrazzini, Roma. Olio su tela. 171

Figura 71 Emilio Ferrazzini, Paesaggio ticinese, 1927. Olio su tela. 171

Figura 72 Emilio Ferrazzini, Bissone 1927. Olio su tela. 171 
Figura 73 Emilio Ferrazzini, Irene Ferrazzini, 1933. Olio su tela.

Figura 74 Giner Cordero, E , Facciata delle chiesa di San Martino. 2007

Figura 75 Giner E. Fragmento di affresco nella facciata principale della chiesa di S. Martino, Malvaglia. 2008.

Figura 76 Giner Cordero E. Esterno dell'oratorio di S. Martino, Quinto. 2008.

Figura 77 Giner Cordero E., Cenacolo. XV sec. Affresco. Deggio, 2008.

Figura 78 Interno dell'Oratorio di San Martino dopo il restauro. $1929 \mathrm{ca}$. XV sec. Fotografia b/n, 1929 ca. AUBCBZ, Archivio grafico. Quinto, san Martino a Deggio.

Figura 79 Giner Cordero E. Interno dell'Oratorio di S. Martino, 2008. 190

Figura 80 San Martino divide il mantello con il povero. XV sec.

Figura 81 Giner Cordero E., San Martino divide il mantello con il povero, XV sec. Affresco. Deggio, 2008

Figura 82 Giner Cordero E., Particolare del volto del Pantrocrator raffigurato sulla volta dell'abside dove si apprezzano dei segni dello descialbo, eseguitosi con mezzi meccanici abbrasivi, e segni della "verniciatura-consolidamento" a cera sulla parte destra, Chiesa di san Martino a Deggio, Quinto, 2008.

Figura 83 Giner Cordero E., Particolare dell'arcangelo raffigurato sull'abside dove si apprezzano dei segni dello descialbo, e una netta separazione fra la zona trattata con materiale cerosa- in alto- a la zona dove la pittura è rimasta intatta. Chiesa di san Martino a Deggio, Quinto, 2008

Figura 84 Giner Cordero E., Particolare dei dipinti raffigurati sullabside dove si apprezzano dei segni dello descialbo. Chiesa di san Martino a Deggio, Quinto, 2008.

Figura 85 Giner Cordero E., Particolare dei dipinti raffigurati sullabside dove si apprezzano dei segni dello descialbo. Chiesa di san Martino a Deggio, Quinto, 2008.

Figura 86 Ferrazzini Emilio, Disegno delle zone consolidate nel portale della chiesa di San Biagio il 15 luglio 1940. Lettera di Ferrazzini ad Aldo Crivelli in Lugano 18 agosto 1959. AUBCBZ Bellinzona.

Figura 87 Ferrazzini Emilio, Disegno delle zone consolidate nella facciata della chiesa di San Biagio in settembre 1944. Lettera di Ferrazzini ad Aldo Crivelli in Lugano 18 agosto 1959. AUBCBZ Bellinzona. 
Figura 88 Facciata della Chiesa di San Biagio di Ravecchia dopo il restauro. Bellinzona. 1913 ca. Fotografia b/n. AUBCBZ, Archivio grafico, Ravecchia, S. Biagio.

Figura 89 Fotografia dell'Interno della chiesa dopo il restauro. Abside Sud, 1950 ca. AUBCBZ. s.80 Chironico. S. Ambrogio

Figura 90 Fotografia dell'Interno della chiesa durante il processo di restauro, 1948 ca. AUBCBZ. Chironico. S. Ambrogio. 208

Figura 91 Fotografia dell'Interno della chiesa dopo il restauro, 1950 ca. AUBCBZ. Chironico. S. Ambrogio. 208

Figura 92 Fotografia dell'Interno della chiesa durante il processo di restauro, 1948 ca. AUBCBZ. Chironico. S. Ambrogio.

Figura 93 Mattei Francesco, Fotografia dell'Interno della chiesa dopo i lavori di restauro, 2004 ca. AUBCBZ. Chironico. S. Ambrogio. 209

Figura 94 Fotografia dell'Interno della chiesa durante il processo di restauro, 1948 ca. AUBCBZ. Chironico. S. Ambrogio.

Figura 95 Fotografia dell'Interno della chiesa durante il processo di restauro, $1948 \mathrm{ca}$. AUBCBZ. Chironico. S. Ambrogio. 210

Figura 96 Cartina degli interventi eseguiti da Emilio Ferrazzini 234

Figura 97 Ferrazzini Emilio, Preventivo di restauro delle tele di S. Maria degli Angioli. Lugano, 20 ottobre 1930. AUBCBZ, s.143, Lugano.

Figura 98 Ferrazzini Emilio, Lettera indirizzata alla CMSA, Lugano 30 aprile 1940. AUBCBZ, s.204, Prugiasco. S. Carlo di Negrentino.

Figura 99 Ferrazzini Emilio/ Pozzi Tita, Proposta di restauro a Francesco Chiesa presidente della Commissione Cantonale dei Monumenti Storici e Artistici. Lugano 30 luglio 1942. AUBCBZ. s.204 Prugiasco, San Carlo di Negrentino.

Figura 100 Ferrazzini Emilio/ Pozzi Tita, Proposta di restauro a Francesco Chiesa presidente della Commissione Cantonale dei Monumenti Storici e Artistici. Lugano 30 luglio 1942. AUBCBZ. s.204 Prugiasco, San Carlo di Negrentino.

Figura $101 \mathrm{Ch}$. Schiefer, Restauro di un affresco. 1932 ca. Archivio privato. 243

Figura 102 Tita Pozzi a Bubikon, 1943. Archivio privato. 245

Figura 103 Giner Cordero E. Particolare del pavone raffigurato sull'abside. S. Carlo di Negrentino. 2006 
Figura 104 Giner Cordero E. Particolare degli affreschi sul campanile. S. Carlo di Negrentino. 2006

Figura 105 Rahn Rudolf Johann, Disegno della chiesa di S. Carlo a Negrentino, Facciata posteriore. (Marcionetti, 1977).

Figura 106 S. Carlo, Facciata principale. (Marcionetti, 1977).

Figura 107 Da sinistra a destra: Tita Pozzi, Francesco Chiesa, Giuseppe Lepori, Ing. Baggio, 1949. Archivio privato.

Figura 108 Tita Pozzi e famiglia nell'inaugurazione di S. Carlo, 1944. Archivio privato. ... 253

Figura 109 Giner Cordero E. S. Carlo, Particolare della targa commemorativa dei lavori di restauro. Giner. 2006.

Figura 110 Abside nord prima dei restauri. Prugiasco, Chiesa di San Carlo di Negrentino, 1940 ca.AUBCBZ, Archivio fotografico. Prugiasco, San Carlo di Negrentino. 256

Figura 111 Giner Cordero, E. Abside nord. Prugiasco, Chiesa di San Carlo di Negrentino. 2006.

Figura 112 Abside sud prima dei restauri. Prugiasco, Chiesa di San Carlo di Negrentino, 1940 ca. 3410 A. 1304. Archivio Federale dei Monumenti Storici. Collezione grafica, Biblioteca nazionale svizzera. 257

Figura 113 Giner Cordero E. Abside sud. Prugiasco. S. Carlo di Negrentino. 2007. 257

Figura 114 Affreschi della parete nord dopo i restauri. Prugiasco, Chiesa di San Carlo di Negrentino, 1944 ca. AUBCBZ, Archivio fotografico. Prugiasco, San Carlo di Negrentino. 258

Figura 115 Giner Cordero, E. Affreschi della parete nord dopo i restauri. Prugiasco, Chiesa di San Carlo di Negrentino. 2007. 258

Figura 116 Affreschi della parete meridionale prima del restauro. Prugiasco, Chiesa di San Carlo di Negrentino, 1940 ca. AUBCBZ, Archivio grafico. 259

Figura 117 Giner Cordero, E. Affreschi della parete meridionale. Prugiasco, Chiesa di S. Carlo di Negrentino. 2007. 259

Figura 118 Particolare degli affreschi raffigurati sulla porta antica, nella contrafacciata della chiesa di San Carlo a Negrentino. Fotografia b/n, 1940 ca. Pubblicata nella Rivista Storica Ticinese (Crivelli, Restauri, bellezze naturali, tradizioni. Rettili preistorici., 1944, p. 963). 
Figura 119 Giner Cordero, E. Affreschi della contrafacciata rafiguranti la Risurrezione, della Ascensione e del Giudizio Finale. XI sec. Chiesa di San Carlo, Negrentino (Prugiasco), 2007.

Figura 120 Porta d'acceso. Chiesa di San Carlo di Negrentino, 1940 ca. AUBCBZ, Archivio grafico.

Figura 121 Giner Cordero E. Porta d'acceso. Chiesa di San Carlo di Negrentino. Prugiasco, 2007.

Figura 122 Confronto fra lo stato di conservazione dei dipinti murali dell'abside maggiore nei diversi momenti storici. Fotografia $\mathrm{b} / \mathrm{n}$ in primo piano: particolare del Cristo redentore prima dei restauri 1940 ca. Pubblicata nella Rivista Storica Ticinese (Crivelli, Restauri, bellezze naturali, tradizioni. Rettili preistorici., 1944, p. 963). Fotografia a colore in secondo piano: particolare del Cristo redentore dopo i restauri. (Marcionetti, 1977)..... 266

Figura 123 Giner Cordero E., Madonna del latte. Fotografia a luce naturale. 2007. ........ 267

Figura 124 Giner Cordero E., Madonna del latte. Fotografia a luce UV. 2007. 267

Figura 125 Giner Cordero E., Madonna con bambino. Fotografia a luce naturale. 2007. 267

Figura 126 Giner Cordero E., Madonna con bambino. Fotografia a luce UV. 2007 267

Figura 127 Giner Cordero E., Particolare degli affreschi dell'abside minore. Fotografia a luce UV dove si osservano fluorescenza delle gocciolature, così come delle dorature e delle lacune pittoriche. 2007 268

Figura 128 Giner Cordero E., Particolare degli affreschi dell'abside minore. Fotografia a luce UV dove si osservano fluorescenza delle gocciolature, così come delle dorature e delle lacune pittoriche. 2007

Figura 129 Giner Cordero E., Particolare della controfacciata della vecchia porta di entrata. Fotografia a luce UV. 2007.

Figura 130 Giner Cordero E., Particolare di una reintegrazione e stuccatura sugli affreschi della navata con particolare fluorescenza ultravioletta che evvidenza la possibile presenza di sostanze proteiche sulla superficie. Chiesa di S. Carlo di Negrentino, Prugiasco. Fotografia a luce UV. 2007.

Figura 131 Giner Cordero E., Prova di pulitura per evvidenziare la presenza di sostanze cerose. Chiesa di S. Carlo di Negrentino, Prugiasco. Fotografia a luce UV. 2007.

Figura 132 Giner Cordero E., Particolare di una reintegrazione e stuccatura sugli affreschi della navata. Chiesa di S. Carlo di Negrentino, Prugiasco. Fotografia a luce radente. 2007. 
Figura 133 Giner Cordero E., Particolare delle abbrasioni provocate dagli strumenti impiegati per lo descialbo della superficie dipinta. Chiesa di S. Carlo di Negrentino, Prugiasco. Fotografia a luce radente. 2007

Figura 134 Giner Cordero E., Particolare di sostanza cerosa ritrovata sulla superficie affrescata nell'abside maggiore. Chiesa di S. Carlo di Negrentino, Prugiasco. Fotografia a luce radente. 2007

Figura 135 Giner Cordero E., Particolare di una reintegrazione e stuccatura sugli affreschi della navata. Chiesa di S. Carlo di Negrentino, Prugiasco. Fotografia a luce diretta. 2007.

Figura 136 Giner Cordero, E. Particolare del pigmento blu ritrovato sopra l'altare dell'abside maggiore alla chiesa di s. Carlo di Negrentino, dimostrando i rifacimenti eseguitisi durante i lavori di restauro degli anni Quaranta. 2008.

Figura 137 Giner Cordero, E. Particolare del pigmento blu ritrovato sopra l'altare dell'abside maggiore alla chiesa di s. Carlo di Negrentino, dimostrando i rifacimenti eseguitisi durante i lavori di restauro degli anni Quaranta. 2008.

Figura 138 Giner Cordero E. Particolare degli affreschi dell'abside con la firma del restauro. 278

Figura 139 Giner Cordero E. Interno della chiesa di S. Nicolao, Giornico. 2007 281

Figura 140 Abside della chiesa di S. Nicolao prima dei restauri, 1940 ca., Giornico. AUBCBZ, Giornico.

Figura 141 Abside della chiesa di S. Nicolao prima dei restauri, 1944 ca., Giornico. Archivio privato B.Abbiati.

Figura 142 Giner Cordero E. Cristo in Maestà, 1478, Nicolao da Seregno. Abside della chiesa di S. Nicolao, Giornico 2007.

Figura 143 Cripta della chiesa prima del restauro, 1940 ca., Giornico, San Nicolao. AUBCBZ, Archivio grafico.

Figura 144 Affreschi dell'altare della cripta prima del restauro, 1940 ca., Giornico, San Nicolao. AUBCBZ, Archivio grafico.

Figura 145 Giner Cordero, E. Affreschi dell'altare della cripta. Prugiasco, San Carlo di Negrentino. 2007

Figura 146 Affreschi dell'abside. S. Nicolao, Giornico prima del restauro, 1940 ca. A. 165. Archivio Federale dei Monumenti Storici. Collezione grafica, Biblioteca nazionale svizzera. 
Figura 148 Veduta esterna. Facciata prima del restauro. In fondo il Monte Generoso. Rovio, S. Vigilio, Restauro 1949 ca.

Figura 149 Facciata occidentale dopo il distacco della Cappella S. Rocco. Rovio, San Vigilio. Rivista storica ticinese 13 gennaio 1949 ca. EAD-63553. Archivio Federale dei Monumenti Storici. Collezione grafica, Biblioteca nazionale svizzera. 289

Figura 150 Affreschi dell'abside prima del restauro. Rovio, S.Vigilio. EAD-63553. Archivio Federale dei Monumenti Storici. Collezione grafica, Biblioteca nazionale svizzera. 290

Figura 151 Veduta interna dell'abside con gli affreschi romanici dopo il restauro. Rovio, S. Vigilio. 1949 ca. EAD-61097. Archivio Federale dei Monumenti Storici. Collezione grafica, Biblioteca nazionale svizzera. 291

Figura 152 J.J.Hofer, Zürich, Litografia. Dipinti dell'abside disegnati da Johann Rudolf Rahn. Rovio, S. Vigilio. EAD-65861 Archivio Federale dei Monumenti Storici. Collezione grafica, Biblioteca nazionale svizzera. 292

Figura 153 Giner Cordero E. Veduta interna della parte superiore dell'abside. Fotografia con luce naturale. Rovio, San Vigilio. 2008.

Figura 154 Giner Cordero E. Veduta interna della parte superiore dell'abside. Fotografia con luce UV. Rovio, San Vigilio. 2008.

Figura 155 Giner Cordero E. Veduta interna della parte inferiore dell'abside. Fotografia con luce naturale. Rovio, San Vigilio. 2008. 294

Figura 156 Giner Cordero E. Veduta interna della parte superiore dell'abside. Fotografia con luce UV. Rovio, San Vigilio. 2008. 294

Figura 157 Giner Cordero E. Dettaglio delle pitture dell'abside. Fotografia con luce UV. Rovio, San Vigilio. 2008. 295

Figura 158 Giner Cordero E. Dettaglio delle pitture dell'abside. Fotografia con lucetrasmessa. Rovio, San Vigilio. 2008. 295

Figura 159 Veduta esterna dell'Oratorio di San remigio. Affreschi dell'altare della cripta prima del restauro. Fotografia b/n 1946 ant. Corzoneso, San remigio AUBCBZ, Archivio grafico. 296

Figura 160 Veduta della parete meridionale durante i lavori di ricerca e di restuaro. Fotografia b/n 1946 ant. Corzoneso, San remigio AUBCBZ, Archivio grafico. 
Figura 161 Particolare dei dipinti dell'abside minore. Fotografia b/n 1946 ant. Corzoneso, San remigio AUBCBZ, Archivio grafico.

Figura 162 Veduta generale dei dipinti seicenteschi dell'abside maggiore, prima dei lavori di restuaro avvenuti nel 1944. Fotografia b/n. 1944 ant. Corzoneso, San remigio AUBCBZ, Archivio grafico.

Figura 163 Veduta generale dei dipinti quattrocenteschi dell'abside maggiore, dopo i lavori di restauro avvenuti dal 1944 al 1946. Fotografia b/n. 1946 post. Corzoneso, San remigio AUBCBZ, Archivio grafico. 301

Figura 164 Particolare dei dipinti seicenteschi dell'abside maggiore, dove si osservano le pitture sottostanti primitive. Fotografia b/n 1946 ant. Corzoneso, San remigio AUBCBZ, Archivio grafico. 306

Figura 165 Cartina degli interventi di restauro eseguiti da Tita Pozzi in Canton Ticino. ... 325

Figura 166 Augusto Guidini. Studio di restauro. 326

Figura 167 Augusto Guidini. Studio di restauro.

Figura 169 Tita Pozzi, (Copia) Proposta di restauro indirizzata al Dipartimento della pubblica educazione del Canton Ticino. Massagno, 22 settembre 1942. (AUBCBZ, s. 204 Prugiasco).

Figura 170 Tita Pozzi, Lettera indirizzata al Dipartimento della pubblica educazione del Canton Ticino. Massagno, 22 agosto 1945. (AUBCBZ, s. 204 Prugiasco).

Figura 171 Carlo Cotti.

Figura 172 Carlo Cotti nella Scuola di Nudo di Lugano da lui fondata nel 1945. 336

Figura 173 Pulitura degli affreschi della chiesa di S. Antonio, Lugano. Fotografia b/n. AUBCBZ Archivio grafico, Lugano, S. Antonio Abate.

Figura 174 Stuccatura degli affreschi della chiesa di S. Antonio, Lugano. Fotografia b/n. AUBCBZ Archivio grafico, Lugano, S. Antonio Abate.

Figura 175 Stuccatura delle fessure. Chiesa di S. Antonio, Lugano. Fotografia b/n. AUBCBZ Archivio grafico, Lugano, S. Antonio Abate.

Figura 176 Cartina degli interventi eseguiti da Carlo Cotti in Canton Ticino

Figura 177 Nino Facchinetti (sinistra) assieme al pittore Carlo Cotti nella chiesa di S. Pietro, detta chiesa Rossa a Castel S. Pietro. 1946 ca. 353

Figura 178 Cartina degli interventi realizzati da Nino Facchinetti 360 
Figura 179 Fotografia b/n. Bruno Abbiati al Castello Sforzesco, Milano. Restauro d'un affresco. 1953. Archivio privato

Figura 180 Abbiati Bruno. Fotografia b/n. Laboratorio di restauro a Ponte Tresa. Archivio Abbiati.

Figura 181 Bruno Abbiati nella fase di ritocco di una tela nel suo studio.

Figura 182 Bruno Abbiati (destra) durante i lavori di restauro a Biasca. Fotografia b/n. Archivio privato Abbiati.

Figura 183 Bruno Abbiati, Particolare di affresco nella chiesa dei SS. Pietro e Paolo a Biasca, durante i lavori di restauro. Fotografia b/n. 1964 ca. Archivio privato.

Figura 184 Bruno Abbiati. Particolare di affresco. Fotografia b/n senza identificazione. Archivio privato.

Figura 185 Cartina degli interventi eseguiti da Bruno Abbiati in Canton Ticino. 380

Figura 186 Cugnasco, oratorio di Curogna. Chiesa dei SS. Anna e Cristoforo. AUBCBZ, Archivio grafico.

Figura 187 Cartina degli interventi eseguiti da Mario Moglia in Canton Ticino. 400

Figura 188 Cartina degli interventi eseguiti da Ottorino Olgiati in Canton Ticino.....

Figura 189 Carlo Mazzi a Peccia durante il restauro di un affresco. Archivio Silvia MinaMazzi, Tegna.

Figura 190 Carlo Mazzi mostrando uno strappo. Archivio Siliva Mina-Mazzi, Tegna. 425

Figura 191 Carlo Mazzi insieme a Crivelli durante il restauro della casa dei Landfogti, Rivera. 1959 post. Archivio Silvia Mina-Mazzi, Tegna.

Figura 192 Carlo Mazzi durante il restauro dei dipinti murali a caseina, dell'atrio della stazione ferroviaria di Neuchatel. 1967 ca. Archivio privato Mina-Mazzi.

Figura 193 Carlo Mazzi durante il restauro degli stemmi alla casa dei Landfogti, Rivera. 1960 ca. Archivio privato.

Figura 194 Cartina degli interventi eseguiti da Carlo Mazzi in Canton Ticino. 440

Figura 195 Cartina degli interventi eseguiti dai fratelli Steffanoni in Canton Ticino. 453

Figura 196 Cartina degli interventi eseguiti da Mauro Pellicioli in Canton TIcino 468

Figura 197 Cartina degli interventi eseguiti da Francesco Annoni in Canton Ticino. 476 
Figura 198 F. Annoni, Preventivo di restauro, indirizzato alla CMS, Milano 30 agosto 1910, AUBCBZ s. 143 Lugano, S. Maria degli Angioli

Figura 199 Cartina degli interventi eseguiti da Mario Rossi in Canton Ticino. 488

Figura 200 Cartina degli interventi eseguiti da Henri Boissonas in Canton Ticino 495

Figura 201 Schema delle relazioni creata all'interno della banca dati su Access 504

Figura 202 Interfaccia del sistema di consultazione dei dati AR.I.S.T.O.S. 2008. 512

Figura 203 Interfaccia della banca dati AR.I.S.T.O.S. Fase di ricerca delle fonti associate al gruppo Lugano. 2008.

Figura 204 Interfaccia della banca dati AR.I.S.T.O.S. Fase di inserimento dei dati dalla fonte. 2008.

Figura 205 Interfaccia del sistema AR.I.S.T.O.S. Fase di inserimento della fonte scansionata. 2008.

Figura 206 Schede elaborate dalla Sezione Protezione dei beni culturali (PCi/PBC).

Figura 207 Confronto dello spettro FT-IR del Campione 1 San Carlo Negrentino con gli standards: campione 1 , silicato di sodio $+\mathrm{CaCO}^{3}$, carbonato di calcio $\left(\mathrm{CaCO}^{3}\right)$, gesso.

Figura 208 Confronto dello spettro FT-IR del Campione 2 San Carlo Negrentino con gli standards: campione 2, silicato di sodio $+\mathrm{CaCO}^{3}$, paraffina+benzina rettiicata, ossido di ferro

Figura 209 Confronto dello spettro FT-IR del Campione 1 Rovio con gli standards: campione 1

Figura 210 Confronto dello spettro FT-IR del Campione 4 Rovio con gli standards: campione 4, silicato di sodio $+\mathrm{CaCO}^{3}$, carbonato di calcio $\left(\mathrm{CaCO}^{3}\right)$, gesso 525

Figura 211 Confronto dello spettro FT-IR del Campione 1 Deggio con gli standards: campione 1 , silicato di sodio $+\mathrm{CaCO}^{3}$, paraffina

Figura 213 Confronto dello spettro FT-IR del Campione 2 Deggio con gli standards: campione 2 , silicato di sodio $+\mathrm{CaCO}^{3}$, gesso, paraffina 


\section{ARTICOLI PUBBLICATI DALLA RICERCA DOTTORALE}
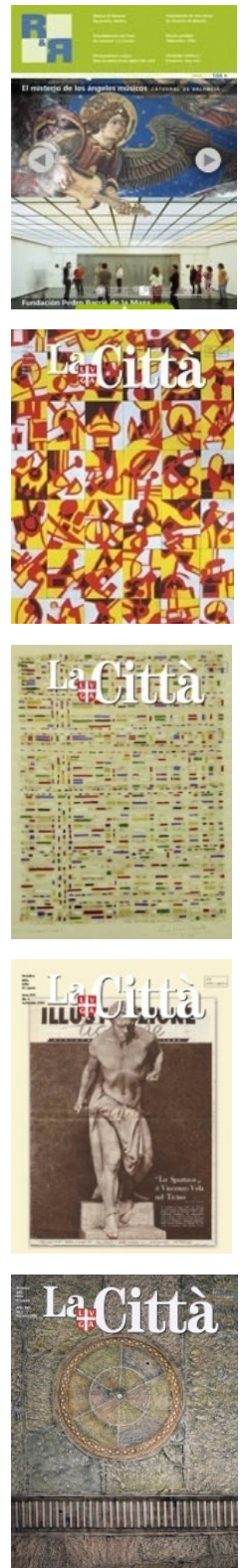

Giner Cordero E., Roig Picazo P., Zalbidea Muñoz Maria A., Jean G. Restauradores Suizos. Canton Ticino; Suiza. Bases de datos de los siglo XIX y XX, in "Restauración y Rehabilitación. Revista Internacional del Patrimonio Histórico". Edit. UPV. Gennaio 2008

Giner Cordero E. Restauratori ticinesi. Tita Pozzi (1902 1958), a cinquant'anni dalla morte, in "La Città", periodico d'informazione del Municipio di Lugano. Maggio 2008.

Giner Cordero E. Restauratori ticinesi. Rivalutare I'arte. Emilio Ferrazzini (1895 - 1975), in "La Città", periodico d'informazione del Municipio di Lugano.Luglio 2008.

Giner Cordero E. Restauratori ticinesi. Carlo Cotti (Lugano 1903-1980). Sedotto dall'arte sacra. in "La Città", periodico d'informazione del Municipio di Lugano.Settembre 2008.

Giner Cordero E. Restauratori ticinesi. Mario Moglia (19151986) in "La Città", periodico d'informazione del Municipio di Lugano.Settembre 2008. 



\section{ARTICOLI PUBBLICATI SULLA RICERCA DOTTORALE}
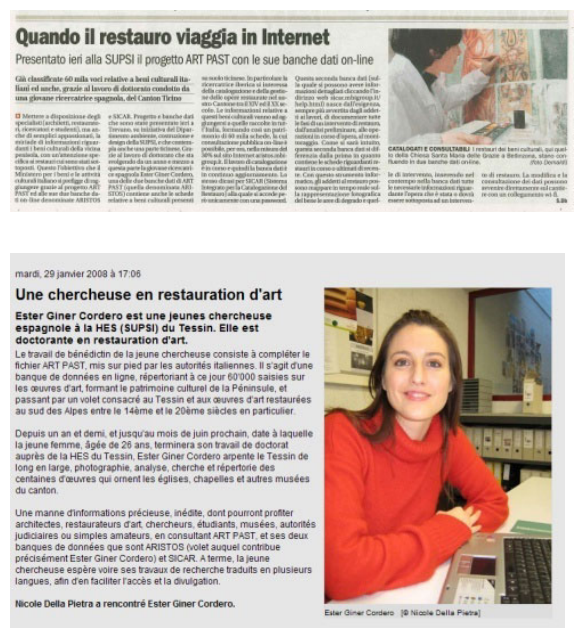

S. Db. Quando il restauro viaggia in Internet. Corriere del Ticino (CdT),

10 gennaio 2008

Nicole della Pietra, Une chercheuse en restauration d'art. Martedì 29 gennaio 2008. Colloquio per la Radio Suisse Romande (RSR)

\section{PARTECIPAZIONE A CONVEGNI}

\section{Ottobre 2007}

Restauratori in Canton Ticino tra Ottocento e Novecento. Catalogazione e gestione dei dati. presentazione inserita nel programma La tutela dei Beni Culturali: i cantieri, gli archivi e la comunicazione, promosso per il Ministero Beni Culturali. La Direzione Generale Innovazione Tecnologica e Promozione, la Scuola Normale Superiore di Pisa in collaborazione con l'Università di Udine ed il Laboratorio L.I.D.A. per il coordinamento del Progetto ARTPAST (3, 4, 5 ottobre nella Scuola Normale di Pisa).

\section{9 gennaio 2008}

Restauratori in Canton Ticino tra '800 e '900: catalogazione e gestione dei dati. Conferenza inserita nel programma Artpast - Applicazione informatica in rete per la tutela e la valorizzazione del patrimonio culturale. Presentazione generale C.Baracchini, D.Levi SICAR e ARISTOS: gli esordi e il progetto ARTPAST. Dimostrazione dei due sistemi informatici, F.Fabiani (SICAR), M.Mozzo e M.Visentin (ARISTOS). 



ESTER GINER CORDERO. Nata ad Aldaia (Valencia) nel 1982. Laureata in Belle Arti alla Facultà di San Carlos di Valencia. In possesso del "Diploma de Estudios Avanzados" (DEA), in conservazione e restauro del patrimonio storico-artistico, con la presentazione in novembre 2007 della ricerca di dottorato "Restauradores en Cantón Ticino entre Ottocento y Novecento. Catalogación y gestión de datos", all'Università Politecnica di València. Quest'ultimo anno, grazie ad una borsa di studio per studenti stranieri concessa dalla Confederazione Svizzera, la suddetta ricerca è stata continuata producendo la tesi dottorale qui esposta. 
